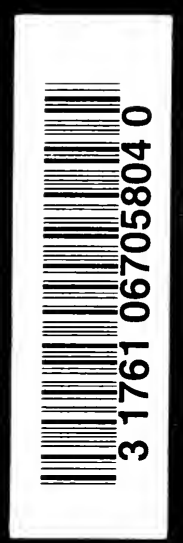





EPIDEMIOLOGY AND PUBLIC HEALTH 



\title{
EPIDEMIOLOGY AND PUBLIC HEALTH
}

\author{
A Text and Reference Book for Physicians, Medical \\ Students and Health Workers
}

\author{
IN THREE VOLUMES
}

BY

VICTOR C. VAUGHAN, M.D., LL.D.

CHAIRMAN OF THE DIVISION ON MEDICAL SCIENCES OF THE NATIONAL RESEARCH COUNCIL; EMERITUS PROFESSOR OF HYGIENE IN THE UNIVERSITY OF MICHIGAN

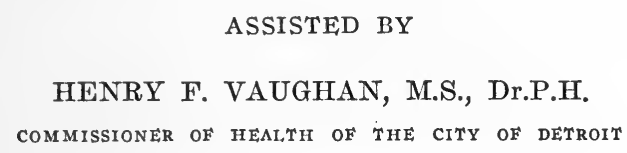

AND

GEORGE T. PALMER, M.S., Dr.P.H.

EPIDEMIOLOGIST FOR TIE DEPARTMENT OF HEALTH OF THE CITY OF DETROIT

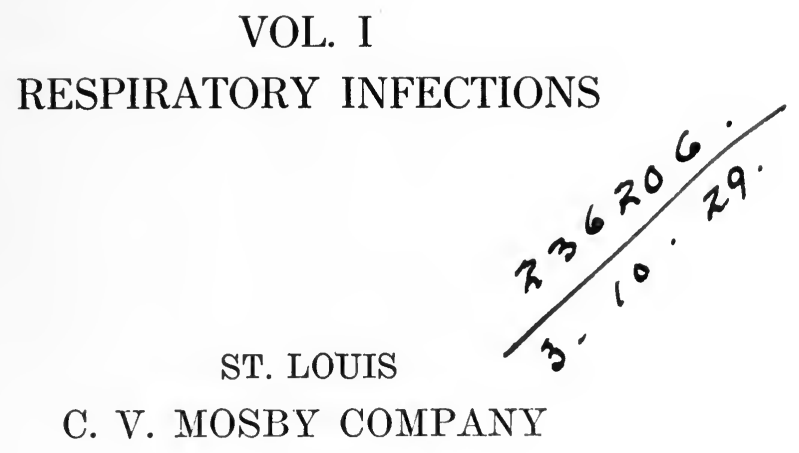

1922 
Copyright, 1922, By C. V. Mosby Company 


\section{DEDICATED}

TO MY WIFE

WHO HAS BEEN MY SYMPATHETIC HELPER AND WISE COUNSELOR FOR MANY YEARS

VICTOR C. VAUGHAN 


\section{Digitized by the Internet Archive in 2007 with funding from Microsoft Corporation}




\section{PREFACE}

The most vivid, and certainly the most distressing, pictures covering the walls of the memory chamber of my brain were painted by the invisible hand of epidemic disease. As a boy on my father's farm in Missouri, I was deeply and sadly impressed by the recurring epidemics of dysentery, or, as it was then and there designated "bloody flux." This disease was in evidence every summer and took toll from all ages, but most heavily from childhood. The Missouri farm of that time would not have a high rating from a present day sanitary inspector. The privy, or "back house" as it was known, with its accumulated contents and swarms of flies, was more frequently shunned than visited. Whites and blacks, old and young, deposited their excreta in fence corners, behind barns, in the weeds or shrubs, or in other places affording even a semblance of privacy, and these deposits were scattered by the bare feet of children, by chickens, turkeys, geese, pigs, and other living, walking, creeping, and crawling things, until their bacterial contents were widely and abundantly distributed, with the result that in bad years one or more little coffins were shaped and molded to receive the body of some playmate; perhaps a dearly loved brother or sister.

I spent the summer of 1865 in Southern Illinois, then known as Egypt, where at that time a frequent greeting was, "This is my chill day." The great majority of our friends and neighbors had a malarial paroxysm each alternate day. The more progressive heads of families provided capacious jugs of Peruvian bark and whiskey, daily rations of which were measured out for each member of the household and there was no shirking the nauseous draught. Thus, early in life I became practically acquainted with the prophylactic use of quinin as an antimalarial agent long before Celli adopted it as an efficient measure in Italy. Besides, the children of that time had no knowledge of, or experience with, the chocolate comfits with which the Italian sanitarian coated his bitter pills.

During my more than thirty years as a practitioner and as epidemiologist of the Michigan State Board of Health, I had to deal with epidemic disease almost continuously. Typhoid and scarlet fever and diphtheria were always with us, with cerebrospinal meningitis and infantile paralysis as occasional visitors. During the last quarter of the nineteenth century there was not a city, large or small, in the Great 
Lakes region in which typhoid fever was not endemic and in which epidemics of this disease did not occur from time to time. No city which takes its water supply from the Great Lakes system, including its bays, inlets, and rivers, has kept its own drinking water at all times free from specific infection. This is certainly true of Detroit, Chicago, Milwaukee, Duluth, and the smaller cities of Michigan, Indiana, Illinois, Wisconsin, and Minnesota. With the unrivaled pure water supply of Lake Superior at their command, Duluth, at one end, and Sault Ste. Marie, at the other, managed to mark the histories of their development with notable outbreaks of typhoid fever.

At the time of the World's Fair at Chicago (1893) the fact that the water supply of this city was badly and specifically polluted was so well known, both in this country and in Europe, that this ill repute threatened the success of the exposition. The management appointed a commission to make a survey and report. At that time thirty public, and innumerable private, sewers poured their contents into the city's water supply. The commission found the city supply so badly contaminated that it recommended the laying of a pipe line from a spring near Waukesha, Wis., to the Fair Grounds, and this was done. My fellows on this Commission were Professors Walter S. Haines and John H. Long. Since that time the construction and operation of the drainage canal, with other improvements in the prevention of water contamination, have reduced the typhoid death rate in Chicago until it compares favorably with that of any other great city in the world. The records of the small coast cities of this area tell like stories. These facts are recalled not with the intention of defaming the fair names of these corporations, but for the purpose of showing that the period of the development of procedures for securing wholesome drinking water for municipalities, even those with boundless supplies, naturally pure, at their doors, was marked by frequent, temporary, and at times costly, failures. Similar experiences in attempts for the eradication of water-borne typhoid fever marked the histories of many of the inland cities of the states mentioned. Most of these cities and villages are located on sand and gravel filter beds, constructed apparently in anticipation of man's coming by the forces of nature in the ice age and unsurpassed in efficiency by any of man's works, but for many years these natural filter beds were honeycombed with privy vaults and cesspools which contaminated the waters of the shallow wells from which the people drank.

My experience with water-borne typhoid extended to Duluth and smaller places in Minnesota and to Grand Forks, N. D. Some of the smaller cities on the Great Lakes, such as Benton Harbor, Muskegon, and Traverse City, and certain larger cities, as Chicago, Milwaukee, and 
Duluth, polluted their own intakes, while others, such as Port Huron and Detroit, poured their sewage into connecting rivers to poison the drinking waters of those lower down the stream. Similar conditions prevailed on many of the smaller lakes and inflowing streams. There is no body of fresh water, be it by nature ever so pure, which man may not render unfit for his own use. Nature has laid out no watersheds and constructed no filtration plants which man may not contaminate. While I read the discussions of Pettenkoffer and Frankland on the selfpurification of running streams, I learned more of this from practical observation in the large and varied area covered by my epidemiologic studies. While typhoid has been and is being satisfactorily reduced in the large cities in this territory, it still takes unnecessary toll from many small cities and villages and from the farms of this area.

In preantitoxin days diphtheria was a veritable scourge in the Northwest. In $1886 \mathrm{I}$ saw in a village of some two hundred families in a pine forest in Michigan diphtheria with every possible complication and sequel. The physician of the community had never seen the inside of a medical school and when a child died the body was taken to the church, the school children filed in and each kissed the corpse. This doctor had reported to the State Board of Health the prevalence in his community of a new noncontagious disease. The memory of my experience in this village is one of the distressing pictures to which $I$ have referred.

It was my good fortune to be present when Roux reported his experience with diphtheria antitoxin to the International Congress of Hygiene at Budapest in 1894. Twenty-seven years have passed since Roux read that paper and the hopes which it awakened in his hearers have come nearer realization than most promises for the relief of man-. kind from a distressing and highly fatal disease, but the complete eradication of diphtheria remains a problem for the future to solve.

There are epidemiologists of large experience who believe that the virus of scarlet fever is growing senile and will give us less trouble in the future. With more than forty years of experience with this disease, I am highly skeptical about this prediction and I have inserted in the proper place a statement made by Graves, the great clinician of Dublin, on this point nearly one hundred years ago. I am convinced from my own experiences with this disease that isolation and disinfection have markedly limited its spread. So far as treatment is concerned, we stand where Graves stood. As is shown in the chapter devoted to this disease, the virus from time to time and in place after place manifests an intensity of invasive power quite equal to its earlier reputation.

In the chapter on measles I have endeavored to impress upon the reader 
the following facts: (1) The only natural immunity to this disease is that acquired by one attack. (2) There is no increased resistance to this disease with advancing years. (3) Uncomplicated measles seldom or never causes death. (4) Measles is a serious disease, inasmuch as it opens avenues to other infections from without and gives opportunity for the development of latent infection already within the body. (5) Patients with this disease should be protected not only from those who are suffering from other infections, but from those with the same disease. (6) The ultimate result in measles depends largely upon the care given the patient. (7) Since measles has been transmitted to animals, a thorough search should be made for a practical and safe vaccine. (8) Epidemics of measles are serious in armies and other great assemblies of susceptible men because of the disorganization of the medical service by the large numbers falling ill and demanding hospitalization at one time. This fact was deeply impressed upon me in observing the progress of this disease at Camp Wheeler and other Southern eamps.

In Cuba in 1898 I saw the effects of tropical malaria on our soldiers. On our way to the Island I admired the appearance and bearing of both officers and enlisted men. They were of splendid physique and seemed mentally alert and morally clean. After a few weeks of residence in Cuba when I saw one of these men in the hospital he seemed not only a physical wreck, but his mental processes were slow and uncertain. This experience caused me to accept readily the suggestion made by Jones that malaria played an important part in the decline of Greek and Roman civilization.

In the Santiago campaign the medical officers had searcely finished the first dressings of the wounded when yellow fever was in evidence among us, although we had been assured that that part of the Island was at that time free from it. Immediately we faced two important epidemiologic problems. The first was, what should be done with the hundreds of wounded men who filled our tents. Fortunately, this, the more important of the two, was promptly and correctly solved. Every wounded man was carried to a transport and started home. Within twenty-four hours the surgical beds were emptied. Strange to say, there was not a case of yellow fever recognized, or at least reported, among these men. I often shudder at the thought of what would surely have happened had the more than 1600 wounded men been kept in the base hospital in Cuba.

Before the close of the first day of its recognition there were five or six cases of yellow fever, all from the front, diagnosed at the base hospital. The next day this number was multiplied by ten and within a week it exceeded 1000. By this time half the medical officers who had 
dressed the wounded, myself among the number, were patients in the yellow fever camp. Without laboratory assistance the medical officers were compelled to distinguish between malaria, typhoid, and yellow fever, and it is worthy of note that but few mistakes were made.

The second epidemiologic problem placed before the medical board at Siboney at the time of the recognition of yellow fever, was that of burning the village. This was done, with the approval of Gorgas, La Garde, Ireland, myself and others. We have often asked one another, how many infected mosquitoes probably lost their lives in that conflagration-what do you think of an epidemiologist who would burn a village for the purpose of eradicating yellow fever?

On my return from Cuba I was in charge of a ship which dropped anchor in New York Harbor with 330 patients convalescing from yellow fever and 30 cases of diphtheria. Diphtheria accompanied the two Michigan regiments from the time they left Fortress Monroe until their return to New York and we were improvident enough to sail without antitoxin. There was one death from this disease on the return voyage. Judging solely from this experience I can testify to the beneficial effect of a northward sea voyage on yellow fever patients. Men who were apparently moribund when we left Santiago were alert and full of energy before we reached New York. Possibly the abundant and varied food supply which we took aboard at Tampa (to which port we were ordered but where we were not permitted by the state health authorities to disembark) had something to do with the marked improvement of the yellow fever convalescents.

The army camps of 1898, with twenty per cent of the soldiers ill with typhoid fever, made an indelible impress upon my memory tablets. There was probably never more ample or varied opportunity for the study of every phase of the epidemiology of this disease. The members of the Commission saw thousands of cases and studied the records of quite 20,000. The lessons learned at that time will be discussed in the chapter devoted to typhoid fever.

When the report of this Commission was made I was the only surviving member, and in it I took opportunity to express my high appreciation of the excellent work done by my distinguished colleagues, Reed and Shakespeare. Dr. Shakespeare had some years before visited Spain, Egypt, and India, and made to our Government a most excellent report on cholera epidemics in those regions. As a member of the Typhoid Commission, he was untiring in his labor and much of the excellence of the report was due to him. Walter Reed, not only by his work as a member of the Typhoid Commission, but in his subsequent studies and solution 
of the problem of the transmission of yellow fever, placed his name high on the list of great benefactors to the race.

During the epidemic of poliomyelitis in New York in 1916, Dr. Haven Emerson, at that time Health Commissioner of the city, was kind enough to invite me, with others, to advise with him concerning restrictive and combative measures. In this way I had opportunity to study the epidemiology of this most elusive and interesting disease and to watch the methods employed so wisely and scientifically by Dr. Emerson in dealing with the most extensive and deadly epidemic of this disease known to history.

When we entered the World War, General Gorgas was kind enough to place me in charge of the section dealing with communicable diseases in the camps in this country. In the fall and winter of 1917-1918 I saw measles, epidemic pneumonia, and cerebrospinal meningitis, to say nothing of lesser epidemics, in the camps and was able, with the valuable assistance of one of my colleagues in the preparation of these volumes, to point out and offer an explanation of the unequal distribution of certain epidemic diseases in the camps. In my opinion, this work has opened up most important problems in epidemiology, some of which, such as that of nonspecific immunity, or, more properly, increase in natural immunity, or resistance to infection, are not yet solvable.

I went to Camp Devens as soon as influenza was reported and the realization of the utter helplessness of man in attempts to control the spread of this disease depressed me beyond words. We are inclined to boast that the age of pestilence has passed, but, with a fair acquaintance with the history of epidemics, I dare say that the world has never before known a pestilence more widespread, more intensive and appalling in its progress, or more destructive to life, than the epidemic of influenza which apparently came into being and grew in violence as the World War passed through its final stages. It seemed that Nature gathered together all her strength and demonstrated to man how puny and insignificant he and his forces are, with all his murderous machinery, in the destruction of his fellows. We have not passed beyond the age of pestilence. Much has been done in man's struggle against disease, but greater things are to be done. There has been no armistice signed between man and disease. Influenza, pneumonia, cerebrospinal meningitis, poliomyelitis, and tuberculosis, are still using weapons against which our defense is quite inadequate. They employ strategy in approach and attack which we do not fully understand. In this war against epidemic disease we must not permit the elation due to past victories to make us less careful and thorough in preparation for the battles of the future.

For forty years I gave annually to the students in Michigan Uni- 
versity a course of from forty-eight to eighty lectures on hygiene. These lectures were devoted largely to the infectious diseases and methods of their control. In the early years I talked to the medical students and then repeated my lectures, omitting, so far as possible, technical terms, to the collegiate students. Finding this irksome I combined the classes and continued my lectures keeping them as free as possible from technical terms. This was profitable to nonprofessional students and, so far as I could see, in no way detrimental to the information imparted to medical students. It will be seen that in these volumes I have continued, so far as possible, the avoidance of technical language. During the Spanish American War and the World War I met, among both line and medical officers, many of my old students and some of these were kind enough to say that they had profited by my instruction. With this incentive, as soon as I was released from army service in 1919 I began the preparation of these volumes. The compelling force which has led me to undertake this task is a desire to put the results of my labors in the field, laboratory, library, and lecture room in more orderly and permanent form. I have found this self-imposed task a most pleasant one. For the first time in my rather active life I have had opportunity to go quite fully into the history of epidemics and to correlate my own observations and experiences with those of others.

A minor impelling force which has led me to undertake this work is the desire to elaborate and develop my own ideas concerning the phenomena of infection. From time to time during the past forty years I have written upon these subjects and published in current scientific and medical literature. In 1913, with the aid of two of my sons, I went into detail concerning my views on the nature of infectious diseases in the volume on Protein Split Products in Relation to Immunity and Disease (Lea and Febiger). The present work gives me opportunity to make application of my own ideas in the discussion of each infectious disease. In the chapter on albuminal diseases I have endeavored to state the main points in my concepts of infection. It will be noticed, however, by the reader that $I$ have not confined my expression of these views to that chapter but have scattered my individual beliefs throughout the volumes.

I have dwelt quite largely upon the history of each infectious disease. Had I not been limited by time and space I should have been glad to have gone more deeply into these histories. In my opinion, the present day medical man, including the epidemiologist, is not as fully conversant as he should be with the history of epidemic diseases. As an illustration I may cite the fact that it took us many months to learn facts about the epidemiology of cerebrospinal meningitis that were demonstrated conclusively by French army surgeons in the first half of the nineteenth 
century. No one can claim to be learned in any specialty until he is fairly familiar with what has been done previously along the same line. I, therefore, make no apology for the length of some of my histories and I only regret that lack of space has prevented me from going more deeply into these subjects.

It may be thought that I have dwelt too largely upon the symptomatology and pathology of certain diseases. I believe that these subjects cannot be wholly divorced from epidemiology. The epidemiologist must be able to not only. trace disease from one community to another, from one place to another, but he must know the paths along which the infecting agent travels after it enters the body. Moreover, as I have pointed out in the chapter on cerebrospinal meningitis, it has been absolutely necessary to study symptomatology and pathology in order to determine whether or not certain epidemics reported in the past under various names were in reality epidemics of cerebrospinal meningitis.

It is not many years since we recognized the importance of healthy carriers in the distribution of epidemic diseases. We can now readily understand why certain diseases, like poliomyelitis, were not until recently regarded as contagious, or even infectious. As a rule, there is only one case in a family, while many are exposed. Until we knew something about healthy carriers it was quite impossible to explain the distribution of poliomyelitis on the ground that it is an infectious disease. This is equally true of many other diseases.

We are just now beginning to understand the nature of infection. A few years ago we thought that there is danger of acquiring diphtheria only when a susceptible person comes into direct contact with one actually sick from this disease. We now know that diphtheria, as well as many other infectious diseases, is distributed throughout communities and carried from one community to another by people who are apparently in perfect health. The man sick with typhoid fever, confined to his bed and under intelligent care, is of but little danger so far as the spread of this disease to his neighbors is concerned. It is the typhoid carrier who travels up and down the country and scatters the seeds of infection.

That a given microorganism may be pathogenic to certain tissues in man's body and without harm when growing in other localities is only recently being recognized. There can be no doubt that there are many bacteria which never harm man but which would prove highly fatal could they reach certain tissues in his body. It is desirable that the epidemiologist know much of pathology, but no amount of wisdom in pathology is sufficient of itself to qualify a man as an epidemiologist.

I have estimated that if I were to introduce a complete list of references in the discussion of each disease the amount of space required 
would be increased by at least one-third. I have, therefore, decided to limit my references to a few important ones attached to the discussion of each disease. The Index Medicus furnishes the profession with abundant facilities for looking up references. So far as possible in giving another's observation or opinion I have quoted his words.

In reviewing the literature I have been greatly aided by Miss Susan Biethan, in charge of the Medical Section of the University Library, who has placed on my table, properly marked, every book and pamphlet containing an article on the subject under investigation at the time. The illustrations in this volume have been prepared with skill and care by Miss Elsa Haberman and Hospital Sergeants Alvin D. Stultz and Edward H. Maitlen. My publisher has spared no expense in order to do well his part of this work. Miss Dorothy Donnelly has served as my secretary and I owe much to her untiring zeal and keen intelligence. I have called to my assistance two young men who have had, both in military and in civil life, large experience in the administration of health problems.

National Research Council, VICTOR C. VAUGHAN. 


\title{
CONTENTS
}

\author{
CHAPTER I \\ INTRODUCTION
}

Theory of Supernatural Origin, 17; Miasmatic Theory, 18; Theory of Contagion, 20.

\section{CHAPTER II}

\section{Albuminal Diseases}

Discussion, 25; Bacterial Proteins, 26; The Protein Poison, 31; The Action of the Living Bacillus, 32; Action of Cellular Proteins, 34; The Action of the Protein Poison, 34 ; Protein Fever, 36 ; Immunization with the Nonpoisonous Part of the Colon Protein, 41; Immunity to the Protein Poison, 43; The Parenteral Disposition of Foreign Proteins, 45; Anaphylaxis and the Infectious Diseases, 46; Vaceination, 50; Precipitins, 51; Agglutinins, 52; Bacteria, 55; Pathogenicity of Bacteria, 58; Toxins and Antitoxins, 60 ; Classification of Infectious Diseases, 60.

\section{CHAPTER III}

\section{Pollinosis}

Definition, 61; History, 61; Pollinosis; a Result of Protein Sensitization, 67; Cutaneous Test, 75; Desensitization, 76; Prevention, 78.

\section{CHAPTER IV}

\section{The Acute Coryzas}

Description, 79; Bacteriology, 79; A Filtrable Virus, 80; Influence of Chilling the Body Surface, 82; Conclusions, 83.

\section{CHAPTER V}

\section{The Pneumonitas}

Description, 85; History, 86; Recent Prevalence, 108; Pneumococcus Pneumonia, 111; Streptococeus Pneumonia, 118; Experimental Pneumonia, 126; Staphylococcus Pneumonia, 127; Spirochetal Pneumonia, 127; Typhoid Pneumonia, 128; Plague Pneumonia, 128; Anthrax Pneumonia, 129; Traumatic Pneumonia, 129; Other Pneumonias, 129; Predisposing Causes, 130; The Phenomena of Pulmonic Infections, 132; Nonspecific Immunity in Pulmonic Infections, 134; Pneumonia and the Weather, 139; Control of Pneumonia, 141.

\section{CHAPTER VI}

\section{Measles}

Definition, 144; History, 144; Present Prevalence and Virulence, 153; Measles in Our Army Camps in 1917-1918, 155; Periodicity of Measles Epidemics, 161; The Enanthem, 169; The Exanthem, 170; Experimental Measles, 170; The Virus, 174; Transmission, 175; Control, 176. 


\section{CHAPTER VII}

German Measles

Definition, 179; History, 179; Ineubation, 181; Invasion, 181; Exanthem, 181; Transmission, 182; Control, 182.

\section{CHAPTER VIII}

\section{Smallipox}

Definition, 183; History, 183; Inoculation, 185; Vaceination, 192; The Virus, 203; Incubation and Development, 203; Varieties, 205; Varioloid, 206; Presen̨t Prevalence, 207 ; Control, 209.

\section{CHAPTER IX}

\section{Chickenpox}

Definition, 212; History, 212; Incubation and Development, 213; Diagnosis, 214.

\section{CHAPTER $\mathrm{X}$}

\section{DIPHTHERIN}

Definition, 215; History, 215; The Bacillus, 216; Diphtheria Toxin, 218; Diphtheria Antitoxin, 220; Morbidity and Mortality in Recent Years, 223; The Schick Test, 227; Active Immunization with Toxin-Antitoxin Mixture, 232; The Period of Incubation, 235; Virulency Test, 237; Diphtheria Carriers, 238; Control and Eradication, 239.

\section{CHAPTER XI}

\section{ScARLet Fever}

Definition, 242; History, 242; Recent Prevalence and Virulence, 251; Transmission, 256; The Virus, 263; Susceptibility, 268; Animal Inoculation, 269; Incubation and Invasion, 270; Control, 271.

\section{CHAPTER XII}

\section{Mumps}

Definition, 275; History, 275; The Virus, 277; Susceptibility, 279; Control, 280.

\section{CHAPTER XIII}

\section{WhOOPING COUGH}

Definition, 282; History, 282; The Virus, 284; Susceptibility, 286; Transmission, 291; Vaccination, 293; Diagnosis, 294; Control, 295.

\section{CHAPTER XIV}

\section{INFLUENZA}

Description, 297; History, 300; Selective Action with Respect to Age, Sex and Race, 341; Physical Stamina, 355; Susceptibility and Immunity, 365; Transmission, 370; Bacteriology, 382; Combative Measures, 400.

\section{CHAPTER XV}

\section{TUBERCULOSIS}

Description, 409; History, 411; The Bacillus, 425; The Chemistry of Tubercle Bacilli, 429; The Bovine Bacillus, 432; The Avian Bacillus, 435; The Piscidian and Reptilian Types, 436; Other Acid-Fast Bacilli, 437; Avenues of Infection, 438; Latent 
Tuberculosis, 445; Individual Contact, 468; Infected Homes, 469; Garden Cities, 474; Home Care of the Tuberculous, 476; Dispensary Care, 478; Tuberculosis Sanatoria, 479; Tuberculosis Colonies, 486; Tuberculosis Hospitals, 490; Educational Propaganda, 495; Tuberculosis Surveys, 497; The Framingham Experiment, 500; The National Tuberculosis Association, 504; Open-Air Schools, 505; Present Schemes for Combating Tuberculosis, 506; Suggestions for Future Work, 507; Prevalence and Distribution of Tuberculosis in the U. S., 512; Effects of the War on Morbidity and Mortality Rates in Germany and Allied Countries, 513.

\section{CHAPTER XVI}

LEPROSY

Definition, 516; History, 516; Present Distribution, 517; The Bacillus, 520; Avenues of Infection, 521; Tubercular Leprosy, 523; Anesthetic Leprosy, 524; Eradication, 524.

\section{CHAPTER XVII}

\section{Cerebrospinat Meningitis}

Description, 528; History,.535; The Meningococeus, 565; The Path of Invasion, 572 ; Temporary and Permanent Carriers, 576; Period of Incubation, 577; Influence of Crowding, 578; Influence of Location, 582; Relation of Fatigue, 582; Diagnosis, 584; Sporadic Cases, 584; Distribution of Cases, 585; Age Incidence, 586; Vaccination, 588; Passive Immunization, 589; Control, 589; Antimeningococeal Serum, 593; Tubereulous Meningitis, 594; Pneumococic Meningitis, 595; Streptococeic Meningitis, 595; Anthrax Meningitis, 596; Syphilitic Meningitis, 596; Experimental Cerebrospinal Meningitis, 596; Meningitis Due to Other Organisms, 597.

\section{CHAPTER XVIII}

\section{Poliomyelitis}

Description, 599 ; History, 600 ; The Virus, 628; Transmission, 630 ; Path of Invasion, 631; The Schick Test in Acute Poliomyelitis, 634; Vaceination, 634; Transmission by Insects, 635; Domestic Animals as Carriers of the Virus, 636; The Cerebrospinal Fluid in Poliomyelitis, 636; Second Attacks, 637; Control, 637.

\section{CHAPTER XIX}

\section{Glanders}

Description, 641; History, 641; Bacillus, 642; Avenues of Infection, 642; Eradication, 643 .

\section{CHAPTER XX}

\section{Weather and Disease}

Discussion, 645; Climate and Disease, 648; The Climograph, 652; Season and Disease, 664; Influenza and the Weather, 671; What Starts Influenza Epidemies, 675; Daily Weather Changes and the Death Rate, 676; How Does Weather Influence Health, 678; Some Possible Uses of a Better Understanding of Weather and Disease, 680. 


\section{ILLUSTRATIONS}

FIG.

1. Acute Fatal Fever . . . . . . . . . . . . . . . . . . . . . . 38

2. Acute Fever Produced by Injections of Egg-White . . . . . . . . . . 39

3. Continued Fever Produced by Repeated Injections of Egg-White . . . . $\quad 39$

4. Production of Continued Fever with the Poisonous Group from the Typhoid Bacillus . . . . . . . . . . . . . . . . . . . . . . . . 41

5. Deaths in Army Camps According to Location . . . . . . . . . . . 94

6. Deaths in Army Camps According to Source of Men. . . . . . . . . 95

7. Death Rates of Rural and Urban Troops . . . . . . . . . . . . 97

8. Pneumonia Deaths by Age Groups in Michigan and Detroit in 1910 • . . $\quad .99$

9. Pneumonia Mortality in Urban and Rural Areas . . . . . . . . . 100

10. Pneumonia in the Largest Cities of the U. S. . . . . . . . . . . . 101

11. Measles and Pneumonia in 1917 and Influenza and Pneumonia in 1918 . .104

12. Pneumonia Among Males by Race and Age . . . . . . . . . . . . 106

13. Pneumonia Among Females by Race and Age . . . . . . . . . . 106

14. Deaths by Age Groups for Certain Respiratory Diseases . . . . . . . $\quad 109$

15. Pneumonia Mortality in U. S. Registration Area by Years . . . . . . 110

16. Deaths from Broncho and Lobar Pneumonia According to Age . . . . . 118

17. Causes of Death Among All Troops in U. S. by Seasons 1917-18 . . . . . 139

18. Measles Mortality in U. S. Registration Area by Years . . . . . . . 145

19. Measles at Camp Wheeler . . . . . . . . . . . . . . . . . . . 156

20. Measles Among Troops in U. S. and Expeditionary Force . . . . . . . 159

21. Measles Mortality in Urban and Rural Areas . . . . . . . . . . . 161

22. Measles in Detroit . . . . . . . . . . . . . . . . . . . . . 166

23. Measles Mortality Rates by Age Group in Michigan . . . . . . . . 169

24. Diphtheria Mortality in U. S. Registration Area . . . . . . . . . . . 223

25. Diphtheria in the Largest Cities of the U. S. . . . . . . . . . . . . 224

26. Diphtheria in Detroit . . . . . . . . . . . . . . . . . . . . 226

27. Diphtheria Mortality in Urban and Rural Areas . . . . . . . . . . 227

28. Diphtheria Mortality Rates by Age Groups in Michigall . . . . . . . 230

29. Scarlet Fever Mortality in U. S. Registration Area . . . . . . . . . 250

30. Racial Variation in Mortality from Communicable Disease . . . . . . 252

31. Scarlet Fever in Detroit . . . . . . . . . . . . . . . . . . . 254

32. Scarlet Fever Mortality in Urban and Rural Areas . . . . . . . . . 255

33. Scarlet Fever in the Largest Cities of the U. S. . . . . . . . . . . . 256

34. Scarlet Fever Mortality by Age Groups in Michigan . . . . . . . . . 259

35. Mumps Admission Rate Per 1000 Troops . . . . . . . . . . . . . 276

36. Whooping Cough Mortality in Urban and Rural Areas . . . . . . . . 283

37. Whooping Cough Mortality in U. S. Registration Area . . . . . . . 285

38. Whooping Cough Mortality Rates by Age Groups in Michigan. . . . . . 288

39. Influenza Mortality in Urban and Rural Areas . . . . . . . . . . 299

40. Influenza Mortality by Age Groups in Michigan . . . . . . . . . 299

41. Influenza Mortality in America and Europe in 18s9-90 . . . . . . . . 306

42. Influenza and Pneumonia at Camp Funston . . . . . . . . . . . . 316

43. Influenza Mortality in America and Europe in 1918-19 . . . . . . . 324

44. Influenza Mortality in New England Cities 1917-18-19 . . . . . . . . 326 
FIG.

45. Influenza Mortality in Middle Atlantic Cities 1917-18-19

46. Influenza Mortality in South Atlantic, East South Central, and West South Central Cities 1917-18-19 . . . . . . . • . . . . . . . . . . 329

47. Influenza Mortality in East North Central Cities 1917-18-19 . . . . . 330

48. Influenza Mortality in West North Central Cities 1917-18-19 . . . . . . 331

49. Influenza Mortality in Mountain and Pacific Cities 1917-18-19 . . . . . 332

50. The Progress of Epidemic Influenza in U. S. Army Camps . . . . . . 333

51. Influenza Mortality in Camp and City 1889-90 and 1918 . . . . . . . 335

52. Excess Mortality in U. S. Cities During Influenza Epidemic 1918-19 . . . 338

53. Mortality During the Influenza Epidemic in the Large Army Camps . . 340

54. Excess Mortality During Influenza Epidemics in U. S. Cities . . . . . 342

55. Morbidity and Mortality from Influenza by Age Groups in Baltimore . . . 347

56. Death Rates from Influenza and Pneumonia by Age in Boston . . . . 349

57. Influenza in Detroit in 1920 . . . . . . . . . . . . . . . . . 350

58. Influenza Among Troops in U. S., A. E. F., and Navy (1918) • . . . 368

59. Influenza Morbidity in Army Camps . . . . . . . . . . . . . . . 401

60. Mortality from Communicable Disease in Registration Area 1912-1916 . 410

61. Tuberculosis in the Largest Cities of the U. S. . . . . . . . . . . . 421

62. Tuberculosis Mortality by Age Groups in Michigan . . . . . . . . 460

63. Tuberculosis Mortality in Urban and Rural Areas . . . . . . . . . 472

64. Tuberculosis Mortality in U. S. Registration Area . . . . . . . . 511

65. Meningitis Annual Death Rate in U. S. Registration Area . . . . . . 551

66. Meningitis in Troops in U. S. and in Expeditionary Foree . . . . . . . 552

67. Meningitis Mortality by Age Groups in Michigan . . . . . . . . . 587

68. Poliomyelitis Death Rate in Registration Area . . . . . . . . . . . 609

69. Poliomyelitis Death Rate by Age Groups in Michigan . . . . . . . . 610

70. Distribution of Civilization . . . . . . . . . . . . . . . . . . . 646

71. Climatic Energy and Mortality in the U. S. . . . . . . . . . . . . 648

72. Mortality Among Soldiers in U. S. Army Camps . . . . . . . . . . 649

73. Comparative Death Rates in American Cities . . . . . . . . . . . 651

74. Comparative Weather Charts in Boston and Seattle . . . . . . . . . 653

75. Mean Temperature and Vital Processes. . . . . . . . . . . . . . 654

76. Deaths from Pneumonia in Detroit from 1900-1917 . . . . . . . . . 656

77. Pneumonia Deaths in Relation to T'emperature and Humidity in Detroit . 658

78. Climograph of the White Race in the Eastern U. S. . . . . . . . . 659

79. Yearly Fluctuations in Temperature in Detroit . . . . . . . . . . . 665

S0. Temperature, Humidity and Pneumonia in Detroit 1900-1917 . . . . . 666

81. Season and Disease Mortality 1911-1917 . . . . . . . . . . . . . 670

82. Daily Changes in Health and Mean Temperature . . . . . . . . . . 677

83. Comparative Prevalence of Colds Among New York City School Children . $\quad 681$ 


\section{EPIDEMIOLOGY AND PUBLIC HEALTH}

\section{CHAPTER I}

\section{INTRODUCTION}

The oldest and most persistent belief holds that disease is an infliction imposed on man by some supernatural power. This doctrine was handed down by tradition to the earliest civilizations, was incorporated in their records and is held by many today. Some have attributed disease to evil spirits and others have regarded it as a dispensation of their gods. The Babylonians regarded disease "as the work of demons which swarmed in the earth, air, and water, and against which long litanies and incantations were recited." In the hand of the Jehovah of the Jews disease was a whip of chastisement which fell at will on the chosen people or their enemies. "I will put none of those diseases upon thee which I have brought upon the Egyptians; for I am the Lord that healeth thee." There is no book of the middle ages down to the eighteenth century, describing an epidemic which does not attribute it to the wrath of God. Job ascribed his own pains to the "arrows of the Almighty." Luther wrote that "pestilence, fever and other severe diseases are naught else than the devil's work." Cotton Mather described disease as "flagellum Dei pro pecatis mundi." While the primitive Christian prayed his God to avert the pestilence, he did not neglect other measures in seeking protection. He established quarantine and practiced it in many cases in the most brutal manner.

A doctrine quite as old, but with a smaller following at present, taught that all the joys and ills of man are determined by the position of the heavenly bodies. Even within the present generation there are those who believe that famine, war, and pestilence are determined by the spots on the sun or by the juxtaposition of certain planets. Our great lexicographer, Noah Webster, wrote a book to prove that epidemics are due to earthquakes and other terrestrial disturbances.

Hippocrates taught that epidemies are due to a pestilential condition of the air. This theory dominated the medical profession for more than 2000 years, and still colors some of our conceptions of infection. Quite naturally, such a theory is liable to many modifications. How does the air become pestilential? This question has been answered in numberless ways. Some say it is made so by evil spirits; by an angered God; by the 
influence of heavenly bodies; by terrestrial disturbances; by prevailing winds; by emanations from earth or water; etc. Sydenham wrote:

"There are different conditions in different years. They originate neither in their heat nor their cold, their wet nor their drought; but they depend upon certain hidden and inexplainable changes within the bowels of the earth. By the effluvia from them the atmosphere becomes contaminated, and the bodies of men are predisposed and determined as the case may be, to this or that complaint."

It is natural for man to believe in the evil genius of the locality in which ills befall him. In 1898, intelligent army officers told with bated breath that the word "Chickamauga" means river of death. They believed that the epidemic of typhoid there prevalent was due, in part at least, to the miasm of the locality and the disease was called "Chickamauga fever." The word malaria (mal aria) owes its origin to this theory. In war and in pestilence, unburied bodies of men and animals were supposed to fill the air with deadly decomposition products. It was fear of this that secured burial of the numerous victims of the plague in the great epidemics of the middle ages. This fear had, in part at least, a religious basis, and burial was regarded not only as a necessary sanitary measure, but as a compliance with a divine command. The unburied dead became a reproach to the living. From the decomposing bodies noxious and fatal emanations polluted the surrounding air, and with this threatening weapon, the dead demanded the honor of Christian burial.

Lowlands and swamps were supposed to be places in which noxious vapors were generated and from which they spread, poisoning the air of the surrounding country. These localities were shunned, especially after sunset, and the fear of breathing night air became well nigh universal. We now know that the real truth in this belief was made plain by the discovery of the transmission of malaria by night flying mosquitoes. The latest scientific support of the theory of miasm appeared in the teachings of the great sanitarian of Munich, Pettenkoffer. When he began his work in the Bavarian capital, about the middle of the nineteenth century, that city was known as a hotbed of typhoid fever. Fecal matter was deposited in shallow vaults and the drinking-water was taken from shallow wells. The whole city was honeycombed with privies and wells and the people were drinking strong infusions of their own excrement. Pettenkoffer taught that the fecal matter undergoes a ripening process in the soil and thereby becomes the cause of typhoid fever. Responding to his teachings, the people of Munich introduced a complete sewer system and brought a pure drinking-water from a distant mountain lake. This resulted in the eradication of typhoid fever and Pettenkoffer's theory was justified by the result. We now know that the typhoid bacillus needs no ripening process during the interval which elapses from its passage from the bowels of one to its entrance into the alimentary canal of another. Pettenkoffer's theory did 
not assume that the noxious agent in the fecal matter is a living organism, but rather held that the ripening process consists of chemical changes. From this, there grew up the idea of the de novo origin of typhoid fever. The fecal matter of uninfected individuals was supposed to undergo ripening processes in the soil and the products of these changes, finding their way into either water or air, distributed the disease. This theory was not only plausible, but was in accord with the then known facts. It found ready support, especially among army medical officers. However healthy men appeared to be when they went into eamp, even in locations which could not have been contaminated previously, after a few weeks, typhoid fever appeared and spread in proportion to the filthiness of the camp and the inefficiency of the methods of fecal disposal. Men of the highest intelligence and widest experience became firm supporters of the de novo origin of typhoid fever which they regarded as a disease generated in filth. They believed the disease was spread by gases arising from any kind of filth, but more especially by those developed in fecal matter.

The theory of miasm was applied not only to typhoid fever and malaria; but to all diseases, especially those appearing in epidemic form. With sound sense, the Greek Father of Medicine said that when a large number of people fall ill simultaneously, the cause must be sought in that which is common to all, and what can that be save the air they breathe? It was soon observed, however, that while the whole community was breathing the same air, many remained well; some had eruptive diseases; others diarrheal disturbances; still others, respiratory affections. This necessitated the division of men into groups according to temperament. With the same factor in the air the result would vary with the temperament of the individual.

The study of the cholera epidemics of the nineteenth century removed the last scientific support of the theory of miasm. It had long been a matter of observation and record that epidemics travel from east to west. This was noted before the Christian era. It was frequently observed in classical times, and the cholera pandemies gave opportunity for collecting exact data on this matter. It was found that cholera, which is endemic about the delta of the Ganges, becomes pandemic on the occasion of certain religious pilgrimages. The faithful followers of Mohammed from all points of the compass gather at Mecca. Some come from the home of cholera, bringing the infection in their bodies. From their discharges others become infected, and as the great concourse disperses, bands of returning pilgrims carry the infection with them and the disease widens its area. It travels just as fast and no faster than man travels. It goes where he carries it and nowhere else. When an individual drops the infection, some one else must pick it up and carry it on or it ceases to spread. The general direction of the spread of infection has always been from east to west 
because the great lines of human travel have led from this point of the compass, but it was found in the study of cholera epidemics that whenever and wherever the paths of travel deviated from the general direction, the infection showed the same deviation; in other words, it became evident that infected man carried the infection in his own body. However, when the pandemics spread over Europe it was noticed that, while no place became infected unless visited by some infected individual, the disease established itself in certain localities and failed to do so in other places, This observation gave some support to the theory of miasm. In spme soils the seeds of the disease grew and multiplied, while in others they did not. When the water-supply of a locality became infected, the disease spread among its consumers. Budd, of London, was probably the first to detect the rôle of infected water in the dissemination of Asiatic cholera. Finally, the discovery of the specific bacteria of cholera and other diseases removed the last support of the theory of disease originating in miasmatic conditions of the atmosphere.

The "germ theory" of disease found lodgment in the brain of an occasional genius many centuries ago. Just who first suggested it and when are not easily determined, but the argument ran something as follows: Alexandria was free from the plague. A ship from a port where this disease prevailed came with sick on board. Those associated with the new arrivals acquired the disease and it spread in an ever widening circle until it prevailed throughout the city and extended in a similar manner to other cities and countries. The disease must be due to a poison of some kind. It cannot be a chemical poison, like arsenic, but it must be a living thing which finds its way from the body of the sick to those of the well where it grows and multiplies and in so doing causes the symptoms of the disease and death. Thus, man's reason, stimulated by exact observation, caused him to conclude that an epidemic must be due to a contagium vivum. He might theorize concerning this living thing, but at that time he could not demonstrate its existence. It was too small for him to see. In the first century of the Christian era, Varro wrote that there are swamps in which grow animals so small that they cannot be seen. They enter the body through the mouth and nose and cause disease. Others wrote to the same effect. Probably the first to see bacteria was the Dutch scientist and lens maker, Leeuwenhoek, who near the end of the seventeenth century described the "animalculæ" which he found under his microscope in the examination of tartar from the teeth and diarrheal stools. In 1849 a village doctor on the Rhine, with a crude compound microscope, saw rod-like bodies in the blood of animals sick with anthrax and failed to find them in the blood of healthy animals. The science of bacteriology may be said to owe its birth to these observations. Independently, this work was continued by Brauel, Davaine and others, 
but the founder of exact and systematic knowledge concerning the causal agents of epidemic disease was the great Frenchman, Louis Pasteur. This scientist overthrew the doctrine of spontaneous generation. Although Harvey, two centuries earlier, had laid down the dictum, onne vivum ex vivo, there were those who held that this does not apply to the lowest forms of life. As Abel says: "Homer wrote of autochthonous men who sprang from the soil, the sixteenth century saw recipes for the manufacture of mice and frogs, and in later days, it was claimed that lower forms of animal life must have developed spontaneously because the Bible makes no mention of their having been taken into the ark by Noah.' It was essential to the development of bacteriology, which depends so largely on sterilization, to dispose of spontaneous generation and to show that lower as well as higher, forms of life breed true. Pasteur showed that fermentation is due to the growth of living organisms, and that each kind of fermentation, like that in beer, wine, and milk, is due to specific organisms. Furthermore, he showed that a temperature high enough to kill these organisms arrests fermentation. By this process, now known as pasteurization, he preserved beer, wine, and milk. Along this line he went further still and demonstrated that putrefaction, like fermentation, is due to bacterial growth. Lister utilized this discovery in the development of antiseptic surgery from which aseptic surgery has come. If each kind of fermentation has its specific organism, why may not each disease have its specific bacterial cause? Exposure to smallpox does not develop typhoid fever. The answer to this question has been given by the discovery of the specific causal agents of many diseases, and when found and tested, their specificity is demonstrated. The anthrax bacillus grown through a hundred generations and then inoculated into susceptible animals induces anthrax still. Pasteur found that certain specific bacteria can be so attenuated by unfavorable conditions of growth that they will not develop the disease in susceptible animals, but do impart to them immunity to infection with virulent strains. In this way he prepared vaccines for chicken cholera, swine erysipelas, and anthrax. He also developed the successful treatment of hydrophobia, now used in every part of the world.

The great German scientist, Koch, extended and developed the work of Pasteur. His solid media and bacterial stains made the growth, identification, and separation of bacteria comparatively easy. His untiring labors led to the discovery of the specific bacteria of Asiatic cholera and tuberculosis and have placed in man's hands the possibility of completely eradicating these and other infectious diseases. The work so firmly established by Pasteur and Koch has been amplified by many workers in many lands and has already led to marked reduction in both morbidity and mortality from the infectious diseases.

The discovery of the bacterial and protozoal causes of disease merely 
constitutes the foundation upon which we must build. The foundations have been laid, but the superstructure awaits the energy, skill, and intelligence of this and coming generations. Forty years ago it was believed that by sanitation we would remove the danger from the infectious diseases. The sanitary engineer has accomplished great things. $\mathrm{He}$ has drained malarial marshes, sewered our cities, provided means for the destruction of our garbage, greatly reduced both morbidity and mortality, and made conditions of life more pleasing. The diagnostic skill of our physicians has been aided by the discovery and application of scientific instruments of precision. The fruits of physical, chemical, and biologic research have been abundant and nourishing. Notwithstanding all these advances, there is much to be done. Thirty years ago we believed that the infectious diseases are transmitted directly from the sick to the well. Then we were convinced that in order to stamp out an epidemic it was only necessary to isolate the infected. Now we know that most infections are distributed by those who are in apparent health. The man who is sick at home or in hospital with typhoid fever or cerebrospinal meningitis may be dangerous to those immediately about him, but there is little probability that he will scatter the seeds of the disease from which he suffers in the community at large. It is the carrier of diphtheria, typhoid fever, or other infectious disease, who is the greatest and most ubiquitous distributor of disease. Most of the bacteria which infect man grow naturally only in man's body, but they do not cause disease in all who carry them. The science of bacteriology wears quite a different aspect and its teachings are quite widely different from those which we learned even twenty years ago.

The pathologist has made weighty contributions to medicine; indeed, without him and his labor we could have never reached the advanced position we now hold. But he has only laid a corner stone in the great building of progressive medicine. In the dead house, whether the investigator study gross or microscopic changes, he is only investigating the final result. He is studying the result of disease rather than disease itself. Likewise, the bacteriologist is studying the causes of disease rather than disease itself. It remains for the epidemiologist to take up the work which lies between that of the student of etiology and the one who is devoting his time to pathology. The epidemiologist must trace the etiologic agent from the sick man through its often long and tortuous course to the one who becomes infected. He must ascertain, if possible, how the meningococcus leaves the sick man, under what conditions it can bear even a temporary extracorporeal existence, by what avenue it enters the body of the next man, where in that body it finds lodgment, why in only a small proportion of cases its introduction into the body is followed by disease, by what route it travels from the nasopharynx to the meninges, why so long as confined 
to the nasopharynx it is harmless, why when it reaches the meninges it is so deadly. The epidemiologist must study the reactions between the infecting agent and the infected body cell.

What are bacteria and by virtue of what constituent or constituents of their structures are they capable of causing disordered function or disease? Are there other protein bodies besides bacteria or protozoa which may cause disease? Is it essential that the infecting agent be a living, growing, multiplying, reproducing, particle of matter in order to cause disease? What are the reactions between body cells and bacterial cells? Are they physical, chemical, or vital, or are all these processes involved in the causation of disease?

Heretofore, works on epidemiology have been devoted almost exclusively to histories of past epidemics. This is essentially true and proper, because there is no way in which we can get information except through the study of things already accomplished. Now, however, the field of epidemiology has broadened and it includes everything that pertains to epidemic diseases. The epidemiologist must be a student of etiology, symptomatology, and pathology. The exercise of his function should not await the wide development of disease. Every case of an infectious disease is potentially the seed from which many cases are to develop. The bacteriologist need not know much about pathology or symptomatology in order to be useful and learned in his specialty. The pathologist may make valuable contributions to medical knowledge without himself having any wide acquaintance with etiology or symptomatology, but a knowledge of all these branches is required of the epidemiologist. It is to be hoped that in the future, as the knowledge and practice of epidemiology develop and increase in efficiency, epidemic disease may become more and more rare.

We may be asked for a definition of epidemiology. It is the science of epidemic diseases, and these may appear in a given community at any time singly or by the hundreds. The more of epidemiology we know and practice the smaller will be the numbers of those involved in an epidemic. Every practitioner of medicine owes it to his individual patient to do the best he can to restore him to health, but if the disease is an infectious one the same practitioner owes a greater debt to the community in which the patient lives, and that is to protect that community from the spread of the disease. It may be asked how many cases of a given disease must appear in a given community before the presence of an epidemic should be recognized. Every case of infectious disease should be regarded as a possible source of an epidemic.

We must not only study epidemic disease when it appears, but we must know this science so well that we shall be able to prevent the occurrence of epidemic disease. The epidemics of smallpox, typhus, and typhoid fever in the past have given us such full and detailed information concern- 
ing the nature and transmission of these diseases that now, whenever this knowledge is constantly and properly applied, they are negligible factors as causes of morbidity and mortality even in unusual assemblies of men. We must study the history of epidemics, because from this source we acquire information of the greatest value, but this information is useless unless we are able to make proper application of it. The aim of the medical profession is to reduce morbidity and mortality to a minimum. While all diseases are not infectious, there remains much to be done in the control of these agencies which, even up to the present time, contribute so largely to the sickness and death rates. 


\section{CHAPTER II}

\section{ALBUMINAL DISEASES}

\section{PROTEIN DISEASES; INFECTIOUS DISEASES}

Discussion.-Since all infecting agents owe their capability of inducing disease to their protein constituents and since it is not necessary for a protein body to be capable of metabolizing or of reproduction in order to cause disordered function, or disease, in man, it seems desirable that some inclusive word be found to cover all those diseases which are due to the introduction of a foreign protein into the animal. In 1913 we wrote:

"The designation 'protein diseases' might be used to cover the majority of bacterial and protozoal diseases, and many of those hitherto regarded as autogenous."

There are evident objections to the use of the word "protein" in this connection. To the ear it is not easily distinguished from the word "protean" which has a wholly different meaning. In seeking for a word suitable as a designation for all disorders which might arise as a result of the introduction of a foreign protein into the body, we believe the word albuminal to be the best. We are assured by classical authorities that it is etymologically correct and that it is broad enough to include all true proteins. It is evident that other students of etiology are thinking along this line. In a recent (1919) contribution Gay writes as follows:

"It would, perhaps, be more accurate to designate diseases or conditions which we propose to discuss in this section under the head of 'diseases due to foreign proteins.' We shall find that although the infectious diseases, strictly speaking, are due in each instance to the entrance, extension, and multiplication of some living microscopic plant or animal, a bacterium, a spirochete, a fungus, a mold, or a protozoan, many of the conditions they engender are due simply to the fact that these exogenous agents are foreign proteins. Inanimate protein, as well as the living tissue from which it is derived, can produce a disease and lead to reaction changes of immunity and hypersensibility."

\section{In his work on Public Health and Hygiene (1920) Park says:}

"In the production of disease by microorganisms there are four main factors involved, viz., on the part of microorganisms, the power to elaborate in the body poisons or proteins that may be split by ferments to yield poisons and the ability to multiply in the cells and fluids of the tissues; on the part of the cells of the body, the degree of sensitiveness to the poisons of the microbe, and the tendency of the eells which have absorbed the poisons to develop substances which neutralize similar poisons or attack the invading bacteria or protozoa. $* *^{*}$ Microorganisms produce serious mechanical injury only when they exist in such enormous numbers or are bunched together so as to interfere mechanically with the circulation of the 
blood or, together with fibrin, cause minute thrombi, and later emboli, which finally produce infarction and abscesses in different parts of the body. The injurious effects are almost wholly due to the chemical substances contained in the germ, which are directly poisonous or after being split by ferments. Some portion of the protoplasm of almost every variety of bacteria combines with some of the substance of the body cells, and acts as an irritant to the tissues."

Our English confrere, Teale, whose experimental work has been of great value in attempts to solve the problems of infection, is quite convinced that the poison which produces the symptoms of an infectious disease does not result from the proteolytic digestion of the invading bacteria. He also rejects the idea that the poison is due to the autodigestion of the blood plasma. He does not believe in a preformed specifie endotoxin, but he concludes that the poison formed in each infectious disease is due to an interaction, the nature of which is as yet obscure, occurring between the tissue and to a lesser extent the free antibodies and the infecting bacterial protein, and depending upon the intensity of this interaction between body proteins and invading proteins; the resulting intoxication varies within wide limits and the ultimate effects are determined largely by the amount of poison formed.

Recognizing the fact that the infecting agent is always harmful on account of its albuminous constituents, it is important in laying a basis for the study of infectious diseases that we first know something of the proteins of the infecting agents and the manner in which their introduction into the animal body leads to disturbances in the operations of body funetions. We use the words albuminal and protein as synonyms and understand that each includes all albuminous substances, particulate or in solution; organized or unorganized; living or dead.

Bacterial Proteins.-In 1900 we devised and put into operation the tanks for the massive culture of bacteria. By this method we were able to obtain bacterial cellular substance literally by the pound, and consequently have been able to study the chemistry and toxicology of this material. The fresh cellular substance taken from the tanks was extracted with dilute alcohol, then with absolute alcohol, and finally with ether. These extractions left the cellular proteins of the bacteria in an almost pure state. At first the colon bacillus was employed in this work, but subsequently the same procedure was applied to other bacteria, both pathogenic and nonpathogenic. Aqueous extracts of the dead colon bacilli made at any temperature up to $100^{\circ} \mathrm{C}$. were without effect upon animals; in other words, the poisonous group is not extracted from the cellular protein of the colon bacillus by water at or below the boiling point. The part insoluble in water when suspended in water or dilute salt solution and injected into animals subcutaneously, intravenously, or intraperitoneally, kills, and after death the same lesions found after inoculation with the living bacillus are in evidence. Boiling the cellular protein of 
the colon bacillus with a 0.2 per cent dilution of hydrochloric acid has but little effect upon the bacterial cell or its contained poison. Heating the cellular protein with from one to five per cent dilutions of hydrochloric acid slowly breaks up the albuminous substance and destroys the poison.

Digestion with artificial gastric juice slowly splits the bacterial protein into polypeptids and finally amino acids and destroys the poisonous constituent. That similar destruction of bacterial protein occurs in normal digestion was shown by the administration by the stomach of a thousand times the minimum fatal dose by intraperitoneal injection and the failure of this amount to produce any effect. This means that in the peptic digestion of bacterial proteins their poisonous constituent is rendered inert.

Heating bacterial proteins, suspended in water for 30 minutes in a sealed tube, at $184^{\circ} \mathrm{C}$. partially disrupts the cellular substance but does not destroy the protein poison. Filtration of the suspension which has been subjected to superheated steam and subsequent injection of the filtrate into animals show that the poison has been rendered more soluble by this process.

Disruption of the bacterial protein with a 2 per cent aqueous solution of sodium hydroxide carries the poisonous group into solution. When a dilute aqueous solution of alkali is employed, not only the poisonous group, but a large proportion of the other constituents of the bacterial protein, is carried into solution. For this reason we finally adopted the method of extracting the poisonous moiety from bacterial proteins with a 2 per cent solution of sodium hydroxide in absolute alcohol at boiling temperature, $78^{\circ} \mathrm{C}$.

In testing the action of bacterial protein on animals it was found that the more finely divided or pulverized this substance is the smaller the dose necessary to kill. The cellular substance of the colon bacillus coarsely ground in a porcelain mortar, suspended in water, boiled to insure complete sterilization, and injected intraabdominally into guinea pigs, killed constantly when the proportion was not less than 1-40,000 parts of body weight. When the proportion was reduced to $1-50,000$ and less none of the animals died. The same powder more finely ground in an agate mortar killed 15 out of 16 animals up to $1-75,000$; out of 28 guinea pigs it killed 9 at $1-100,000$; out of 8 it killed 5 at $1-200,000$; out of 34 it killed 4 at $1-2,000,000$. The finely ground powdered substance killed rabbits when injected intraabdominally, 2 out of 4 at $1-75,000 ; 4$ out of 7 at $1-100,000 ; 4$ out of 12 at 1-200,000; and 2 out of 18 at 1-2,000,000. One gram of the bacterial protein was incinerated and the whole ash injected intraperitoneally into a guinea pig without effect. The poisonous effect when subcutaneously employed was found to be practically the same as when introduced intraperitoneally, though death was somewhat delayed.

The reason that the fatal dose of bacterial protein decreases with the fineness into which it is ground is easily understood when we recognize 
that the protein has no poisonous action until its poisonous group is set free by the proteolytic enzymes in the body, and the rapidity of digestion is influenced and largely determined by the surface exposure of the bacterial protein which in this case is introduced in suspension. We shall find that surface exposure to the proteolytic enzymes in the animal body is a large factor in influencing the effects of the introduction of foreign proteins.

It has been generally assumed that bacteria are unicellular plants and it is well to still include them in the vegetable kingdom, but we were not able to find cellulose in any of the bacterial cellular substances with which we worked. Many years ago Hammerschlag estimated the percentage of cellulose in the tubercle bacillus as high as 28.1, but his analysis included much of the vegetable material in which the bacillus was grown. DeSchweinitz and Dorset reduced this amount to 6.95 per cent, but hardly accept their own figures since they conclude that cellulose is probably present in small amount in the tubercle bacillus and is not present in the bacillus of glanders. These and other investigators who have reported the presence of cellulose in bacterial substances have not properly distinguished between cellulose and other carbohydrates. Vincenzi, employing the proper tests, failed to find cellulose in the cellular substance of the bacillus subtilis but did find a nitrogenous carbohydrate. In our work, both Wheeler and Lynch made special search for cellulose in sarcina lutea and the colon bacillus, but were unable to find it. They did find a nitrogenous carbohydrate, undoubtedly chitin or a chitin-like substance, which did not reduce Fehling's solution even after prolonged boiling with dilute hydrochloric acid but did respond to the carbohydrate test with alphanaphthol. This substance causes none of the protein reactions and it is most probable that it is this chitin body which has been mistaken for cellulose by previous investigators. It is not soluble in Schweitzer's reagent and it gives no color with iodine even after treatment with sulphuric acid. When dried and heated with soda lime it gives off a gas which turns red litmus paper blue, thus indicating the presence of nitrogen which has been reduced to ammonia. We found in bacterial cell substance two carbohydrates; the one mentioned above combined with nitrogen, the other combined with phosphorus constituting a part of the nucleic acid group. The second carbohydrate does reduce Fehling's solution after prolonged boiling with dilute mineral acids.

There is no controversy concerning the presence of nuclein in bacterial cellular substance, since the xanthin bases have been demonstrated.among the disruption products, both in our own laboratory and elsewhere. Many years ago Klebs found in the tubercle bacillus a nuclein containing from 8 to 9 per cent of phosphorus. Later in the same organism Ruppel reported a nuclein containing 9.42 per cent of phosphorus. Leach obtained 
from the cellular substance of the colon bacillus a nuclein containing 7.33 per cent of phosphorus. In all bacterial cell substance we found xanthin bases.

In all bacterial proteins amino acids are present and may be obtained by disruption of the molecules by prolonged heating with dilute mineral acid. Not only are proteins found in all bacterial cells, but we have demonstrated that the cells of different species of bacteria contain different proteins. This is shown by the presence of different amino acids or by differences in the proportion of amino acids found in different cell proteins. The following figures indicate the distribution of certain amino acids in colon protein and in tuberculosis protein:

$\begin{array}{lll} & \cdot \text { CoLON } & \text { TUBERCULOSIS } \\ \text { Glutamic acid } & 3.00 \text { per cent } & 0.20 \text { per cent } \\ \text { Glycocoll } & 0.33 \text { per cent } & 0.00 \text { per cent } \\ \text { Alanin } & 1.00 \text { per cent } & 1.40 \text { per cent } \\ \text { Valin } & 1.60 \text { per cent } & 4.60 \text { per cent } \\ \text { Leucin } & 2.00 \text { per eent } & 1.82 \text { per cent } \\ \text { Phenylalanin } & 0.20 \text { per cent } & 0.50 \text { per cent }\end{array}$

Some years ago we summarized our findings in the chemical study of bacterial proteins as follows:

"The cellular substance of bacteria contains highly complex molecules. We have demonstrated the presence of the following groups among the split products: (a) A ehitin-like body consisting of a carbohydrate combined with nitrogen. It seems reasonable to infer that this exists in the cellular substance as a glycoprotein. (b) A carbohydrate group combined with phosphorus from which it is not easily detached. This group reduces copper after prolonged boiling with dilute mineral acid. The amounts as determined by the reduction of Fehling's solution and ealculated as xylose are large, but we are not sure that the reducing substance is all earbohydrate. Indeed, it might be better to speak of both of these groups as those responding to the alphanaphthol test rather than as carbohydrates and to distinguish between them as nonreducing and reducing bodies. However, it seems elear that the one now under consideration is a subgroup in the nucleinic acid constituent of the cell substance. (c) The presence of nucleinic acid is beyond doubt, as is shown by the high phosphorus content of some of the split products and by the demonstration of the xanthin bases. (d) That one or more protein groups exist in the cell substance. If all these groups exist in the same molecule the cell substance must contain a highly complex molecule which would be best designated as a glyconucleoprotein. The fact that these bodies are removed only by agents capable of causing molecular disruption inclines us to the belief that the molecules which make the cell substance are highly complex. Because bacteria are simple morphologically is no proof that they are made up of simple proteins. This certainly is not true even if it should prove that we have overestimated the size of these protein molecules."

We have gone somewhat into detail concerning the chemistry of bacterial protein, because it is frequently assumed that bacteria, being unicellular, are not highly organized. Our investigations have shown that the bacterial cell in the complexity of its organization compares well with 
the most highly developed cells in the animal body. Because bacteria are unicellular it should not be inferred that they have but limited function. Their activities are determined by the complexity of their molecular structure and in this particular they are quite equal to any cells in the animal body. Morphologically bacteria are simple structures, but chemically and physiologically they are highly complex.

It may be well to call attention to the fact that by the extractions with alcohol and ether to which our material was subjected, all coloring matter, fats, waxes, and other stored material not essential parts of the living cell, were removed. Even chromogenic bacteria after the extractions and after being pulverized form snow white powders. Organisms which in their natural state were "acid-fast" or "gram-positive," after these extractions still stained with anilin dyes but had lost those constituents to which acidfastness or gram-positiveness is due. These constituents are not essential parts of the protein structure of these organisms and consequently are not essential to the organism as a cause of disease.

We found the cellular proteins of all the bacteria with which we worked more or less harmful to animals when introduced parenterally. The amount of the protein necessary to produce a fatal result varies greatly with the bacterium from which it has been obtained. The cellular substance of bacteria to which in its living state an animal is highly susceptible does not kill that animal at all or does so only after large doses. We injected into the abdominal eavities of guinea pigs the cellular protein of the tubercle bacillus in quantities both small and large without causing death in a single instance, while, on the other hand, a fraction of a milligram of the protein from the bacillus prodigiosus kills. To kill a guinea pig one part of the cellular substance of the anthrax bacillus to 1700 parts of body weight is necessary, while with the colon protein one part to 75,000 kills all animals, provided the material is finely ground. In general it may be stated that the more highly susceptible a given animal is to infection with a given bacterium the more difficult it is to kill that animal with the cellular protein of that bacterium; on the other hand, the more highly immune a given animal to infection with a given bacterium the more readily does that animal succumb to injection of the cellular proteins of that bacterium. At first sight these statements seem wholly irrational, but when we reason we find that they are in strict accord with what we might have confidently predicted before hand. The guinea pig is highly susceptible to infection with the tubercle bacillus because the secretions of the body cells of this animal have no destructive action on that organism, while, on the other hand, the bacillus tuberculosis can feed upon certain substances found in the guinea pig's body with little or no hindrance so long as it is capable of multiplication. The infecting bacillus finds in this animal an abundance of suitable food almost with no resistance; on the 
other hand, the guinea pig is highly immune to infection with the bacillus prodigiosus, because the animal's body cells supply secretions which are immediately destructive to this organism and the first of these bacilli to find their way into the animal's body meet with immediate and complete annihilation, but when the prodigiosus is grown in vitro and a sufficient amount of its cellular substance, dead or alive, is thrown into the abdominal cavity of the guinea pig the same agency which has given the animal immunity to infection with this organism now causes it to fall a victim to the cleavage of the bacterial protein.

It should not be understood, however, because animals are not easily killed by certain bacterial proteins that the introduction of these into the animal body is without harm. When the dead protein of the bacillus tuberculosis is injected into the abdominal cavity of the guinea pig it has no recognizable effect so far as the behavior or external condition of the animal shows, but the dead bacilli are absorbed into the folds of the omentum and there develop local tubercles; in other words, the dead tubercle bacillus, the protein of the tubercle bacillus, develops in the guinea pig exactly the same histologic changes that are induced by the living bacillus. In short, the lesions which follow infections result also from the injection of dead bacterial protein. We conclude from this that the lesions of the infectious diseases are not directly due to the activity of the living bacilli, but result from reaction between the proteins of the bacterial cells and those of the cells of the animal body.

The Protein Poison.-As we have stated, bacterial cellular proteins may be split by boiling with a 2 per cent solution of sodium hydroxide in absolute alcohol into poisonous and nonpoisonous portions. This method of cleavage is undoubtedly crude and in the procedure there is great loss of protein, probably from both portions into which the original protein is split. That this cleavage follows along structural lines is believed to be true for the following reasons: (1) The poisonous part is freely soluble in absolute alcohol; the nonpoisonous part is insoluble in this menstruum. (2) The poisonous part contains no carbohydrate; the nonpoisonous part contains all the earbohydrate found in the original unbroken protein. (3) The poisonous part freely responds to the Millon test; the nonpoisonous part responds to this test in many instances not at all and in others very slightly. (4) The poisonous part contains no phosphorus, while the nonpoisonous part is rich in this element. (5) The poisonous portion is physiologically and largely physically and chemically the same, whatever the source of the protein from which it has been derived; the nonpoisonous part differs more or less markedly according to the protein from which it has been obtained.

Having obtained a poison by the disruption of the proteins of pathogenic bacteria, it was determined to test the proteins of nonpathogenic bac- 
teria for the same poison. As has been indicated, we found just as much or more of the protein poison in nonpathogenic, as we had found in pathogenic, bacteria. We are compelled to conclude from this that the pathogenicity of a given bacterium is not determined by its ability to construct a poison, because all bacterial cells contain a poison and this poison in all is practically the same so far as its gross physiologic effect is concerned.

Having found a poison in both pathogenic and nonpathogenic bacterial proteins, it was reasonable to suspect the presence of a similar poison in proteins of animal and vegetable origin. Such animal proteins as eggwhite, casein from milk, serum albumin, serum globulin, and the cells of various organs of the animal body, were tested and in all the protein poison was found. A like search in vegetable proteins, such as those obtained from grains and seeds, revealed the presence of the protein poison. These investigations have covered so many proteins of diverse origin that until it is otherwise proved we have the right to assume that there is a poisonous group in every protein molecule, whatever be the origin of that protein. It must be understood that in making this statement we are speaking only of true proteins. There are certain pseudoproteins, such as gelatin, which do not contain the poisonous group. If every protein molecule contains a poisonous group, then any protein introduced into the animal body in such a way as to avoid the destruction of its poisonous constituent may disorder the functions of the body and cause disease; in other words, it is not necessary that the infecting organism be a feeding, absorbing, excreting, reproducing, thing. It may possess none of these attributes of life and still it may cause serious disturbances in bodily function and may result in death. Under usual conditions the infecting agent is a living, multiplying, protein. These attributes are necessary in order to cause a continuous supply of the protein poison. There are, however, exceptions to this, as we shall see when we come to study pollinosis. Hay asthma is just as much a disease as measles and yet the former is caused by a nonliving, nonreproducing, protein, the continuous supply of which is kept up through the inhaled air. In this instance the continuous supply of protein poison is not secured by the multiplication of the pollen in the human body but through inhalation of fresh material.

The Action of the Living Bacillus.-In 1905 Vaughan (Victor C., Jr.) reported his studies on the comparative effects on guinea pigs of the living colon bacillus, its dead cellular protein, and the protein poison separated from the other constituents of the cellular substance. We quote:

"When a guinea pig is inoculated intraabdominally with a fatal dose of the living colon bacillus, practically no symptoms whatever are noticeable for a period varying from five to twelve hours, according to the size of the dose given. This may be considered as the period of incubation and is roughly proportional to the amount of the living culture injected. We have always worked with a bacillus 1 c.e. of a twelve hour or older, bouillon culture of which has invariably proved fatal 
to guinea pigs within 24 hours. If 1 c.e. of such a culture is given, no effects will be seen for a period of from ten to twelve hours. If, on the other hand, 2 e.c. of the same culture be injected, the animal will begin to manifest symptoms of illness in from eight to ten hours, and if larger doses are given the symptoms will become apparent in a shorter time. This period of ineubation undoubtedly represents the time taken for the bacillus to multiply and to be destroyed to such an extent that sufficient poison may be liberated through its disintegration to produce noticeable poisonous effects in the animal. This period of incubation is, therefore, in reality the crisis of the disease and the outcome depends solely upon whether all bacilli have been destroyed before a lethal dose of the poison has been set free or not. It is during this period that individual resistance and acquired immunity are important factors acting by causing increased bacteriolysis and the destruction of all bacilli before a fatal dose of the poison has been set free. During this time the temperature of the animal may rise to a greater or less extent or may remain stationary, the animal remains active, eats; its coat is not roughened and it appears in all respects as well as a normal animal. At the end of this period, however, the appearance changes, the animal becomes less active. It remains in one corner of its cage; its coat becomes roughened, it hangs its head and apparently enters into a state of stupor. At the same time the rectal temperature begins to fall abruptly; indeed, the fall of body temperature is often the first marked symptom, and, when occurring to a marked degree, it is invariably a bad omen. The body temperature will often fall from $101^{\circ}$ to $94^{\circ} \mathrm{F}$. or even lower within from two to four hours, and this fall is progressive and continuous until the animal's death, immediately preceding which a temperature as low as $87^{\circ}$ or $86^{\circ} \mathrm{F}$. is not uncommon. At the same time the animal shows signs of the most marked peritoneal inflammation, as is evidenced by rigidity and spasm of the abdominal muscles on pressure. At autopsy, the only gross lesion present is a marked hemorrhagic peritonitis with a large amount of bloody fluid containing intact red corpuscles and leucocytes in the peritoneal cavity. The parietal and visceral peritoneum are studded with minute punctiform hemorrhages. Hemorrhage is an especially prominent feature in the great omentum and is present to a less marked degree in the mesentery.',

In our opinion, this is a miniature of what happens in many infectious diseases. The intraperitoneal infection of the guinea pig is comparable with the development of a general peritonitis from a ruptured appendix. The period of incubation is short, and while there may be some elevation in temperature this is not marked or even constant. During the period of incubation when the bacilli are abundantly multiplying, the behavior of the animal in no way distinguishes it from its untreated fellows. When a man drinks water containing typhoid bacilli he does not develop the disease that day or the next. There is a period in typhoid fever of about ten days, the period of incubation, in which the typhoid bacilli are multiplying amazingly in the man's body, and still he is so well that he is absolutely unconscious of anything being wrong with him. At the end of the period of incubation the body cells begin to destroy the invading organisms and at this point the active disease appears. In an infectious disease there is always a period of incubation. It is not the bacteria which first found their way into the man's body that furnish enough poison to kill him. The few bacteria coming in must multiply and this they do 
during the period of incubation. There is no resistance at this time on the part of the body cells. These must be sensitized before they can begin the combat with the invading organisms and it is not until this point is reached that the active disease appears.

Action of the Cellular Proteins.-When a fatal quantity of the cellular protein of the colon bacillus is injected into the peritoneal cavity of a guinea pig the progress of events is exactly like that following infection with the living organism except that the period of incubation is shorter. There is no longer either opportunity or need for the multiplication of the bacillus. This has taken place in vitro and enough of the protein to kill has been introduced. One of the features that characterizes and marks the period of incubation has been withdrawn. It only remains for the body cells by means of their secretions to cleave the bacterial protein and set the poisonous group free. The period of incubation is reduced one-half or more. Then the evidences of poisonous action are exactly the same as in the inoculated animal. The temperature falls at the same rate and autopsy reveals exactly the same lesions. The chemotactic pull of the dead protein has proved just as strong and just as violent as that of the living protein; in fact, the pull in both instances is a chemical and not a vital one and the lesions result from a reaction between the proteins of the bacterial cells and those of the body cells.

The lesions that characterize the infectious diseases are due to the destructive warfare waged between the body cells and the cells of the invading organisms. Both of these are composed essentially of protein material and this on being disrupted liberates the protein poison. The poison may come from the bacterial cell or from the body cell, or from both; in any case it is a poison to the animal in whose body it is liberated. Having been set free, the protein poison endangers the life of the animal and may cause death without in and of itself inducing any recognizable lesion. The last supposition is true only when the protein poison has been fully and completely detached from the other constituent groups of the larger molecule to which it has belonged.

The Action of the Protein Poison.-It is due to the poisonous group which it contains that both the living and the dead bacillus have a deleterious effect upon the animal body. The effect of the isolated poisonous group on animals depends upon the extent and completeness with which it has been detached from the nonpoisonous group in the protein molecule. When the poisonous group is completely detached from the protein molecule by chemical agents and injected into animals, it is one of the most deadly poisons known. We have never obtained it in a chemically pure state. Every particle with which we have worked has been a more or less crude mixture, but in the purest form in which we have been able to obtain it, $0.5 \mathrm{mg}$. when administered intravenously kills a guinea pig 
within a few minutes with all the symptoms of anaphylactic shock. When given subcutaneously or intraabdominally, the evolution of symptoms proceeds more slowly and a larger dose is required in order to cause death. The following is a description of the symptoms that follow the subcutaneous or intraperitoneal administration of a minimum lethal dose: At first, after an interval of from five to ten minutes immediately following the injection, the animal appears restless, runs about the cage, and shows a great tendency to scratch itself, this undoubtedly being due to itching sensations in the skin caused by irritation of the peripheral nerves. The animal then begins to show evidences of lack of coordination, which is rapidly followed by partial paralysis, which is especially marked in the hind extremities. This stage lasts for from five to ten minutes, during the latter part of which the animal usually lies quietly on one side. From this state it passes into what one might term the convulsive stage. These convulsions are usually clonic in nature, and, as a rule, at first involve only the neck muscles, the head being momentarily drawn backward. At first these convulsions are but slight in degree and are separated by considerable intervals of time. Soon, however, they become much more frequent and of greater severity. Gradually they become more and more general in their extent, until all the muscles of the body become involved in violent clonic convulsions. This stage when present presages a fatal outcome; rarely an animal recovers after reaching the convulsive stage. During a convulsion, or occasionally in the interval of calm, respiration ceases. The heart, however, continues to beat, at first with perfect regularity and no acceleration; indeed, the rate seems to be somewhat slower than normal. Gradually the beat becomes more and more feeble, the rate and regularity being preserved to the end. It is usually only after an interval of from three to four minutes after the cessation of respiration that the heart ceases to beat. A fatal issue, if it occurs at all, results in one hour after injection and usually within from 30 to 40 minutes. This is to a large extent independent of whether the dose is the minimum lethal one or two or three times that amount. Autopsy reveals no gross lesions. In our opinion, this is very important and is in marked contrast to the hemorrhagic peritonitis found after injection of either the living or the dead colon bacillus. We believe that it is the distinguishing feature between the injection of the poison in a free and in a combined state. It must be clearly understood that the above is a description of the action of the protein poison fully detached from the other constituents of the protein molecule. This happens in anaphylactic shock, which we shall study later, but it probably never happens in the progress of an infectious disease. In the course of the disease there is no time that the protein poison in any dangerous quantity is completely detached from the other constituents of the protein 
molecule. When we employ the protein poison only partially detached from the other constituents of the protein molecule, progress of events is slower, death is less certain, and autopsy will reveal more or less marked lesions, depending upon the force necessary to complete the disruption of the protein molecule already begun in vitro. The isolated protein poison is decidedly acid in character and it may be combined with an alkali. This is best done by neutralizing it with bicarbonate of soda, making the solution decidedly alkaline. When such a preparation is kept at incubator temperature for a number of days and then injected into animals the symptoms develop more slowly and after death slight lesions, differing however only in degree from those following injection of the unbroken protein, are in evidence. A larger dose of the combined poison is necessary to kill, and even with the larger dose death may be delayed for hours while the free poison in smaller doses kills in minutes.

Protein Fever.-It has long been known that the parenteral introduction of proteins into the animal body in small amounts and especially in repeated injections leads to fever. The older literature on this subject, as well as an account of his own investigations, was given in 1883 by Roques. In 1888 Gamaleia showed quite clearly that fever results from and accompanies parenteral digestion of bacterial proteins. A year later Charin and Ruffer confirmed this work, extending it to nonbacterial proteins. In 1890 Buchner produced the characteristic phenomena of inflammation-calor, rubor, tumor, and dolor-by the subcutaneous injection of diverse bacterial proteins. In $1895 \mathrm{Krehl}$ and Matthes induced fever by the parenteral introduction of albumoses and peptones, but they did not obtain constant results, which we now know are secured only by regulation of the size and frequency of the doses. In 1909 Vaughan, Wheeler, and Gidley demonstrated that any desired form of fever (acute fatal, continued, intermittent, or remittent) can be induced by regulating the size and frequency of the doses of foreign protein administered parenterally, and in 1911, Vaughan, Cumming, and Wright extended the details of this work.

If egg-white could multiply in the blood and lymph it would be quite as dangerous to prick one's finger with a needle moistened with this bland protein as it would be to inoculate oneself with a virulent streptococcus. Since egg-white cannot grow and multiply in the animal body the truth of the above statement may be tested by frequent injections of this foreign protein into the animal, the frequency of the injection keeping up the supply in the absence of ability to grow. We found that when 0.05 c.c. of egg-white was filtered through cotton and injected intraabdominally into a rabbit every half hour from 8 A.M. until 4 P.M., the following results were obtained: 


$\begin{array}{ccc}\text { TIME } & \text { DOSE IN C.C. } & \text { TEMPERATURE } \\ 8: 00 \text { A.M. } & 0.05 & 102.8^{\circ} \\ 8: 30 & 0.05 & 101.0^{\circ} \\ 9: 00 & 0.05 & 102.6^{\circ} \\ 9: 30 & 0.05 & 102.9^{\circ} \\ 10: 00 & 0.05 & 102.6^{\circ} \\ 10: 30 & 0.05 & 103.2^{\circ} \\ 11: 00 & 0.05 & 103.4^{\circ} \\ 11: 30 & 0.05 & 103.5^{\circ} \\ 12: 00 & 0.05 & 103.4^{\circ} \\ 12: 30 \text { P.M. } & 0.05 & 104.4^{\circ} \\ 1: 00 & 0.05 & 104.8^{\circ} \\ 1: 30 & 0.05 & 105.1^{\circ} \\ 2: 00 & 0.05 & 105.3^{\circ} \\ 2: 30 & 0.05 & 105.3^{\circ} \\ 3: 00 & 0.05 & 105.8^{\circ} \\ 3: 30 & 0.05 & 105.8^{\circ} \\ 4: 00 & 0.05 & 105.8^{\circ} \\ 4: 30 & 0 & 106.6^{\circ} \\ 5: 00 & 0 & 106.6^{\circ} \\ 5: 30 & 0 & 106.1^{\circ} \\ 8: 00 & 0 & 104.1^{\circ} \\ 8: 00 \text { A.M. } & 0 & 103.0^{\circ}\end{array}$

In another experiment, the egg-white diluted with physiologic salt solution was injected intravenously each hour and the dose increased with each injection. The results are shown by the following figures:

$\begin{array}{ccc}\text { TIME } & \text { DOSE IN C.C. } & \text { TEMPERATURE } \\ \text { 8:00 A.M. } & 1 & 100.8^{\circ} \\ 9: 00 & 2 & 103.2^{\circ} \\ 10: 00 & 3 & 104.3^{\circ} \\ 11: 00 & 4 & 104.6^{\circ} \\ -12: 00 & 5 & 105.4^{\circ} \\ 1: 00 \text { P.M. } & 6 & 105.6^{\circ} \\ 2: 00 & 7 & 106.1^{\circ} \\ 3: 00 & 8 & 105.2^{\circ} \\ 4: 00 & 9 & 102.2^{\circ} \\ 4: 30 & 0 & 100.5^{\circ} \\ 5: 00 & 0 & \text { Death }\end{array}$

In still other experiments, fevers extending through weeks and accompanied by emaciation and increased nitrogen elimination were induced in rabbits by repeated injections of small doses of egg-white; also with other proteins, and especially with the poisonous portion of the typhoid bacillus. The intensity of the fever induced by the injection of proteins seems to vary markedly with the protein employed, the dose given, the frequency of administration, and the animal employed. In several instances we found that in order to induce fever it was necessary to decrease the size of 
the dose. The following are the conclusions which we formulated in 1913 after studying protein fever experimentally: (1) Large doses of unbroken protein administered intraabdominally, subcutaneously, or intravenously have no effect upon the temperature; at least, do not cause fever. (2) Small doses, especially when repeated, cause fever, the forms of which may be varied at will by changing the size and the interval of dosage. The effect of protein injection on the temperature is more prompt and marked in sensitized than in fresh animals. (4) The intravenous injection of laked blood corpuscles from either man or the rabbit causes in the latter, even in very small quantity, either in single or repeated doses,

\section{ACUTE FATAL FEVER PRODUCED IN A RABBIT BY REPEATED INTRAVENOUS INJECTIONS OF EGG-WHITE}

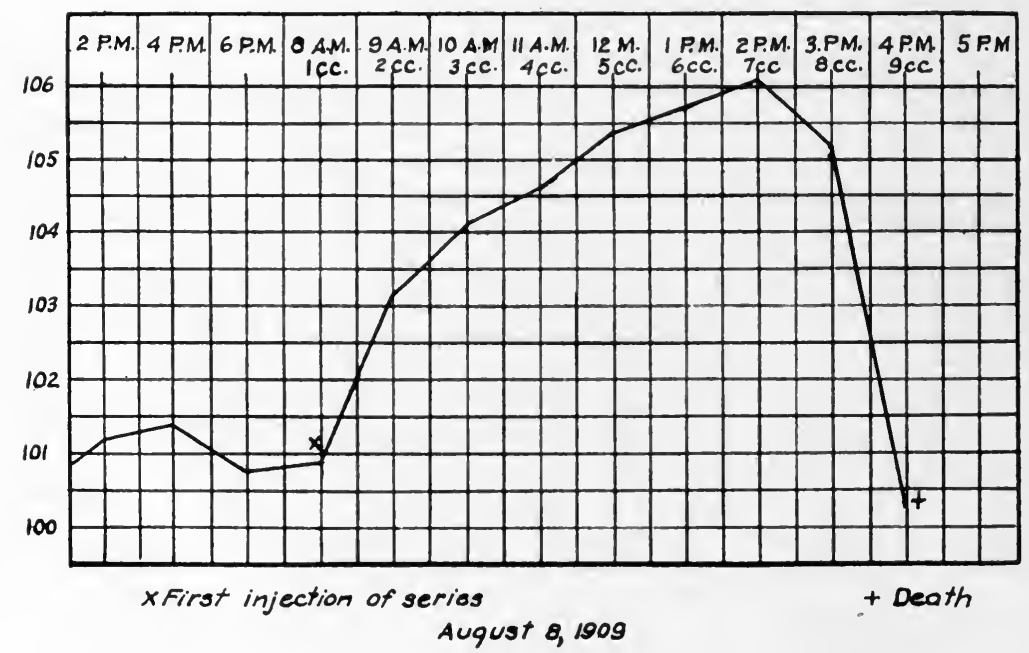

Fig. 1.

prompt and marked elevation of temperature. (5) Laked corpuscles after removal of the stroma by filtration have a like effect. (6) Protein fever can be continued for weeks by repeated injections, giving a curve which cannot be distinguished from that of typhoid fever. (7) Protein fever is accompanied by increased nitrogen elimination and gradual wasting. (8) Protein fever covers practically all cases of clinical fever. (9) Animals killed by experimentally induced fever may die at the height of the fever, but, as a rule, the temperature rapidly falls before death. (10) Fever induced by repeated injections of bacterial proteins and ending in recovery is followed by immunity. (11) The serum of animals in which protein fever has been induced digests the homologous protein in vitro. (12) Fever results from the parenteral digestion of proteins. (13) There 

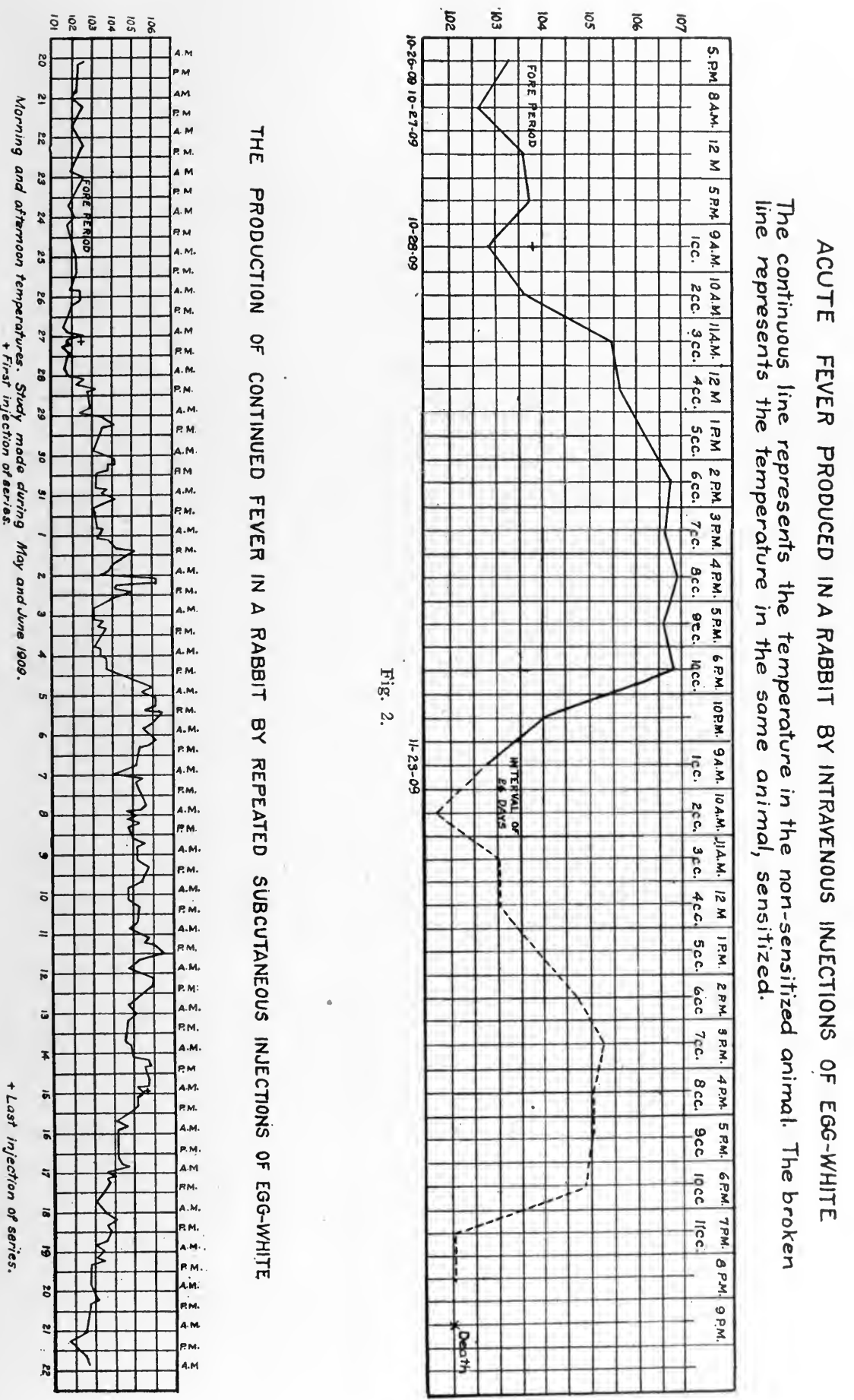
are two kinds of parenteral proteolytic enzymes, one specific and the other nonspecific. (14) The production of the nonspecific ferment is easily and quickly stimulated. (15) The development of the specific ferment requires a longer time. (16) Sensitization and lytic immunity are different manifestations of the same process. (17) Foreign proteins, living or dead, formed or in solution, when introduced into the blood soon diffuse through the tissues and sensitize the cells. Different proteins have predilection places in which they are deposited and where they are in large part at least, digested, thus giving rise to the characteristic symptoms and lesions of the different diseases. (Note-In our opinion, the foreign proteins in the exanthematous diseases are digested in the skin.) (18) The subnormal temperature which may occur in the course of the fever or at its termination is due to the rapid liberation of the protein poison, which in small doses causes an elevation, and in larger doses a depression of temperature. (19) Fever per se must be regarded as a beneficent phenomenon inasmuch as it results from a process inaugurated by the body cells for the purpose of ridding the body of foreign substances. (20) The evident sources of excessive heat production in fever are the following: (a) that arising from the unusual activity of the cells supplying the enzyme; (b) that arising from the cleavage of the foreign protein; (c) that arising from the destructive reaction between the split products from the foreign protein and the proteins of the body.

Thiele and Embleton make the following record of the effects of eggwhite and tubercle protein on fresh and sensitized animals:

\section{EGG-WHITE}

\begin{tabular}{|c|c|c|}
\hline Limits of & $\begin{array}{c}\text { NORMAL ANIMALS } \\
\text { grams }\end{array}$ & $\begin{array}{l}\text { SENSITIZED } \\
\text { grams }\end{array}$ \\
\hline Temperature fall & 0.05 & 0.005 \\
\hline Constant temperature & 0.02 & 0.0002 to 0.0001 \\
\hline Temperature rise & 0.01 to 0.001 & 0.0001 to 0.000002 \\
\hline
\end{tabular}

\begin{tabular}{|c|c|c|}
\hline Limits of & $\begin{array}{l}\text { RCLE EMULSION } \\
\text { NORMAL ANIMAI } \\
\text { grams }\end{array}$ & $\begin{array}{l}\text { SENSITIZED } \\
\text { grams }\end{array}$ \\
\hline Temperature fall & 0.005 to 0.002 & 0.0005 \\
\hline Constant temperature & 0.002 to 0.001 & 0.0001 \\
\hline Temperature rise & 0.001 to 0.00001 & 0.00001 to 0.000001 \\
\hline
\end{tabular}

Friedberger gives the following figures, showing the effect of lambs' serum intravenously administered to normal and sensitized guinea pigs:

Normal Animal

5.0 c.e. equal fatal dose

0.5 c.c. equals limit for fall in temperature

0.01 c.c. equals upper constant

0.005 c.e. equals fever plane

0.001 e.c. equals lower constant 


\begin{tabular}{ll} 
& \multicolumn{2}{c}{ Sensitized Animal } \\
0.005 & c.c. equals fatal dose \\
0.0005 & c.c. equals limit for fall \\
0.00001 & c.e. equals upper constant \\
0.000005 & c.c. equals limit for fever \\
0.000001 & c.c. equals lower constant
\end{tabular}

We have invariably found that small doses of foreign protein injected into animals parenterally cause elevation of temperature, while larger doses have no such effect. The explanation of this seems to us quite simple. We injected the whites of three eggs at one time into a rabbit and although the temperature of this animal was taken every few hours for a month it never rose above normal; on the other hand, 0.05 c.c. of eggwhite repeatedly injected into a rabbit causes a rapid elevation of temperature and if persisted in, speedy death. The readiness with which a foreign protein injected parenterally into the body is digested depends upon its surface exposure. The greater the surface exposure the more

THE PRODUCTION OF CONTINUED FEVER IN A RABBIT BY REPEATED SUBCUTANEOUS INJECTIONS OF THE POISONOUS GROUP FROM THE TYPHOID BACILLUS

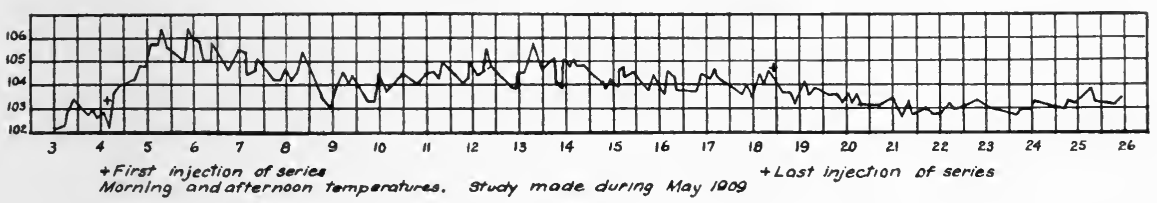

Fig. 4.

marked the digestive action, and in concentrated solutions there is no digestive effect. When massive doses of egg-white are thrown into the abdominal eavity of a rabbit the animal disposes of it by transferring it, largely through the liver, into the intestine where it is digested enterally and normally.

Immunization of Animals with the Nonpoisonous Part of the Colon Protein.-When colon protein has been disrupted by a 2 per cent solution of sodium hydroxide in absolute alcohol the part insoluble in the alcohol, after proper preparation, may be injected into the peritoneal cavities of guinea pigs in doses as high as 500 milligrams with apparently no effect on the animal. Rabbits and guinea pigs are easily immunized to the living colon bacillus by repeated treatments with this substance as the following results from our protocol illustrate: Rabbit No. 1 received on August 13 intraabdominally a solution of one gram of the residue. (We designate the nonpoisonous part of proteins the residue.) On August 20 a second injection of two grams was given. Eighteen days later this animal was inoculated with 5 c.c. of an 18-hour culture of the colon bacillus. From two to four hours after injection this rabbit was apparently very sick, the 
symptoms resembling those which are seen following the injection of the poisonous part. It then began to improve and eventually recovered completely. A control animal inoculated at the same time with an equal amount of the same culture died in five hours.

Rabbit No. 2 received doses of one gram of the residue on October 5, 10 , and 20. Four days after the last injection this animal was inoculated with 5 c.c. of an 18-hour culture from which it recovered. A control given the same dose at the same time died within five hours.

Rabbit No. 3 had the same treatment as did No. 2 and six days after the last dose of residue withstood 5 c.e. of an 18-hour culture. Eight days later this animal received 10 e.c. of a 16-hour culture and did not die.

Rabbits Nos. 4 and 5 had the same treatment as did Nos. 2 and 3, and seven days after the last dose each received 5 c.c. of a 24-hour culture of the living colon bacillus without a fatal result. A control which received the same amount of the same culture died within five hours.

When animals immunized with the residue are inoculated with the living colon bacillus and compared with companion nonimmunized animals which have received the same amount of the living organism certain differences in behavior in the two sets of animals are striking. The immunized animals soon become ill, as shown by their appearance and by the fall in temperature. So striking is this that when we first made the experiment and while watching the two sets of animals we were inclined to the opinion that the treatment with the residue had increased susceptibility to the living organism. It turned out, however, that as the nonimmunized animals began to fall ill and to show a drop in temperature the immunized ones began to improve and to show a rise in temperature. In the end the immunized animals recovered while the nonimmunized ones died. We demonstrated that animals immunized with the residue have no increased tolerance for the detached colon poison. They are killed quite as promptly and with the same dose that kills nonimmunized comrades. It follows from this that the immunity shown by these animals cannot be due to any increased tolerance for the colon poison. Theoretically, we explain these phenomena by supposing that the injections of the residue have stimulated certain body cells to the production of a bacteriolytic ferment and that when the living culture is injected this ferment destroys the organisms before they have time to greatly multiply. In this way we account for the early development of the symptoms in the immunized animals; indeed, although these experiments were made before we had heard of anaphylaxis, we believed at the time that if we could completely immunize an animal with the residue such an animal would be immediately killed by the injection of a large quantity of the living organism. The following quotation from a paper published in 1905 demonstrates the truth of this statement: 
"The difference in the behavior of the immune and the normal pig is seen to consist in the fact that in the first instance we have symptoms of the action of the free poison shortiy after the injection of the dead culture, which are entirely lacking in the second case. This shows beyond doubt that in the immune pig there is marked bacteriolysis of the dead bacilli and a consequent liberation of the contained poison shortly after the injection of the dead bacterial cell into the peritoneal cavity. Although we have as yet been unable to actually cause death in an immune pig at an early period, the animals are in every instance very ill within 30 minutes after the injection of the dead culture. In fact, several of them have shown signs of the commencement of the convulsive stage as evidenced by slight convulsive movements of the head separated by considerable intervals of time. We have been unable to secure a fatal result in these animals up to the present time simply because we have worked with pigs which did not possess a sufficient amount of bacteriolytic substance directly available to cause disintegration of enough bacilli to liberate a fatal amount of poison at one time. It is worthy of note that this behavior of animals immunized with the residue towards the dead bacterial substance furnishes additional proof of the fact that the poison of the colon bacillus is an intracellular one. If the poison existed free in the culture medium we should expect that the control would show evidences of its action at as early a period as does the treated animal. However, as has been stated above, this is not the case. The fact that the treated animal shows symptoms of poisoning to a much greater degree and at an earlier time than does the control can be explained only on the ground that the poison with which we are dealing is an intracellular one, and is set free only after the disintegration of the bacillus by bacteriolysis."

The immunity secured by treating animals with the nonpoisonous part of the cellular protein is strictly specific. Animals treated with this portion of the colon bacillus are immune to the colon bacillus only and not to the typhoid bacillus and vice versa. We were not able to obtain immunizing residues from any bacteria except the colon, typhoid, and tubercle bacilli. So far as our experiments have gone, the immunity secured with the nonpoisonous parts of these bacilli is not permanent, lasting in the case of colon and typhoid proteins for about 40 days at most. While this immunity holds, however, animals may be treated with the living organisms and in this way a more lasting immunity secured. It should be clearly understood that we are not recommending the nonpoisonous portions of bacterial proteins, at present at least, as suitable for general use. The evidence of their action as here adduced is for scientific purpose only, and especially in order that we may better understand the nature of the albuminal diseases and the protective procedures resorted to by the body cells.

Recently (1921) an English investigator, Thomson, has written on "Detoxicated Vaccines," in which he advocates the removal of the poisonous group from bacterial proteins with alkali and the employment of the nonpoisonous residue as a vaccine. In our opinion, this is a promising field, since we hold that the specificity of all proteins lies in this portion of their structure.

Immunity to the Protein Poison.-We found it easy to establish slight 
tolerance to the protein poison in animals. So rapid, however, is the action of this poison that one must proceed with caution or he will kill most of his animals. By beginning with nonfatal doses and gradually increasing the size with daily injections, guinea pigs may be brought to bear about twice the minimum fatal dose, but beyond this we have found that tolerance seldom goes. The tolerance thus established is nonspecific and no antibody is generated in its production; in other words, this part of the protein molecule is a poison and not a toxin. An animal which has been brought to a certain degree of tolerance with the poison obtained from the colon protein will bear just as much and no more of the poison obtained from any other protein, be it bacterial, vegetable, or animal. In no particular sense can this tolerance be designated as immunity. It is slight, transient, and nonspecific. The interesting discovery made in this part of our work lies in the fact that animals in which a tolerance for the protein poison has been established will bear, as a rule, the minimum fatal dose of a living culture. This has been tried only with colon and typhoid organisms. A tolerance may be established in an animal with the poison obtained from egg-white. Such a tolerant animal will bear the minimum fatal dose of either the colon or the typhoid bacillus and it is indifferent as to which is employed. These tolerant animals in a certain percentage of cases will bear twice the minimum fatal dose of the living organism, but beyond this, resistance seldom or never goes. It is evident that by establishing a tolerance for the poison and inoculating the animal at the time of the continuance of this tolerance active immunity may be secured. In the case of an animal which has been treated with the protein poison and has subsequently received a dose of the living bacillus which would surely have proved fatal for a normal animal, the symptoms induced are identical in every respect with those which follow a nonlethal dose in an untreated animal. The similarity of the symptoms in the two instances leads us to believe that in all probability we are dealing with a form of bodily resistance which is identical in character with that which is usually spoken of as natural immunity.

We venture to suggest that our experience in attempting to immunize animals with the protein poison may be the basis of a scientific explanation of the frequently observed fact that in epidemics of respiratory diseases in armies the recent recruit and the man from a rural community fall more ready victims to the epidemic than do his comrades among seasoned soldiers and among whose who have lived in crowded cities. The man who has inhaled for a long time and quite constantly a large number of bacteria whose proteins have been split in his body, setting the poisonous group free, has acquired a tolerance for the poison or an increased resistance to infection from any bacteria taken in through the respiratory organs. When we first studied the comparative freedom from pneumonia exhibited by the urban and the seasoned soldier in our camps as compared 
with the greater susceptibility of the rural and the recruit, we were inclined to believe that the former had acquired a degree of immunity by frequent exposure to those bacteria which cause the pneumonias. At that time we believed that the greater resistance shown by the seasoned soldier and the man from the crowded city was specific, but when the epidemic of influenza came along in the fall of 1918 we found that the same difference in susceptibility was observed as between the rural and the urban soldier. This means that the increased resistance possessed by the seasoned soldier and the urban is nonspecific. We are quite convinced that a nonspecific bacterial immunity, never reaching a high degree, but certainly affording protection beyond that usually present, is secured by repeated introduction of the protein poison into the body. Nonspecific immunity is of low grade and usually suffices to protect against only the minimum fatal dose, rarely against twice this amount. Elsewhere we have formulated our views upon this subject as follows:

"(1) When armies are recruited and men from all fields of life are crowded together, those from areas sparsely populated and who are unaccustomed to erowd life will prove most susceptible to infection and especially to those infections which are transferred through the respiratory organs. (2) That the man who has been long accustomed to crowd life and who has consequently inhaled particulate proteins (bacterial), whether they be pathogenic or nonpathogenic, acquires a nonspecific immunity which helps him in withstanding infection. (3) The seasoned soldier bears even newly imported infection better than his comrade who has recently joined the ranks.",

The Parenteral Disposition of Foreign Proteins.-When a foreign protein is introduced into the animal body intravenously, subcutaneously, or intraabdominally, there are three possible ways in which it may be disposed of: (1) It may be carried into the intestine and there digested. In this disposition the foreign protein may be carried to the intestine through the liver and with the bile. That this does frequently occur we have demonstrated by injecting foreign proteins parenterally, and subsequently sensitizing guinea pigs with the same protein by injecting into them a few drops of bile or of an extract from the liver. This method of disposing of foreign proteins introduced parenterally may be carried out by direct diffusion through the intestinal walls into the lumen of the intestine. That certain bacteria which have found their way into the blood, either by direct injection or by natural infection, do find their way into the intestine through the liver is an established fact. This is especially true of typhoid bacilli. (2) The foreign protein may be eliminated unchanged or in slightly altered form through the kidneys. This we have demonstrated by injecting egg-white subcutaneously into rabbits and subsequently sensitizing guinea pigs to egg-white by injecting into them small quantities of the urine. (3) The foreign protein introduced into the body parenterally in a short time disappears from the blood current and may be found by the sensitization test in certain tissues. Apparently, each pro- 
tein has its predilection tissue, in which it is most frequently and most abundantly deposited. When deposited in the tissue, the foreign protein sensitizes the body cells with which it comes in contact and these cells elaborate a specific ferment which digests the foreign protein. At one stage in the process of this digestion the protein poison is formed and may harmfully affect the animal and possibly cause its death. This is true whether the foreign protein be in solution and in the form of egg-white or be particulate in the form of typhoid bacilli.

Some years ago we summarized the results of a large number of experiments in which we studied the disposition of foreign proteins introduced into rabbits parenterally, as follows: (1) Egg-white injected into the stomach of a rabbit may be in part absorbed unchanged and can be detected by the sensitization test in the blood. (2) Egg-white injected into the rectum of the rabbit may be, in part at least, absorbed unchanged. (3) Egg-white injected into the peritoneal cavity may be taken into the blood unchanged. (4) Egg-white injected intravenously into rabbits quickly disappears from the cireulating blood. (5) Egg-white injected intravenously into rabbits may be detected in the peritoneal cavity after it has disappeared from the circulating blood. (6) Egg-white injected intravenously into rabbits may be detected in the bile. (7) Egg-white when injected intravenously into a rabbit may be detected by the sensitization test in certain organs after it has disappeared from the circulating blood. What is true of egg-white is, at least in a general way, true of other foreign proteins. It has been long known that bacteria injected into the blood rapidly disappear from the circulating fluid and may be detected later deposited in certain predilection tissues. Bacterial, as well as soluble, proteins are deposited in various organs and tissues. In many infectious diseases the characteristic symptoms and lesions are determined by the tissue in which the foreign protein is deposited and where an attempt is made by the tissue cells to digest it. The meningococcus is harmless so long as its presence in man's body is confined to the nasopharynx, but when it reaches the meninges of the brain and cord it becomes a deadly agent.

Anaphylaxis and the Infectious Diseases.-It has been long known that an animal to which a foreign protein has been administered parenterally is likely to behave in an extraordinary manner when subsequently treated with the same or some closely allied protein. In 1905 Richet and Portier reported that when they injected into an animal an extract obtained from the tentacles of a certain shell fish, allowing a certain time to elapse, and then made a reinjection of the same substance the reinjection was followed by symptoms of intense poisoning. Their interpretation of this phenomenon was that the first injection had robbed the animal of all its protective agencies against the constituents of the extract and, unfortunately, they coined the word "anaphylaxis"' as most suitable to cover 
this condition of increased susceptibility. Richet chose this word, understanding it to mean "without protection" and indicating that the first injection destroyed all natural resistance that the animal did possess against the poison. However unsuitable this term, it has come into general use, and with proper explanation it may be continued. Pirquet proposes to use the word "allergie," meaning altered energy. This is more suitable, inasmuch as it simply expresses a fact and binds no one to any theory. Other terms, such as "protein sensitization" and "hypersensitiveness" are used and are to be considered as synonyms with anaphylaxis and allergie. We do not intend to enter into any detailed discussion of this phenomenon, but shall content ourselves with pointing out what we believe to be the rôle played by anaphylaxis in the albuminal diseases.

The substance which produces the anaphylactic state, on being introduced parenterally into animals, is known as the sensitizer. Only proteins and only foreign proteins can sensitize an animal. One cannot sensitize a guinea pig with the blood serum of one of its own species, but it may be sensitized with the blood serum of another species. The guinea pig is especially suited for experimentation on anaphylaxis, since the amount of foreign protein necessary to produce the anaphylactic state in this animal is exceedingly small and on reinjection the guinea pig behaves characteristically and promptly. The dose of the sensitizer may be very small. Rosenau and Anderson found 0.000001 c.c. of horse serum sufficient to sensitize guinea pigs. Besredka places the minimum amount essential to secure uniform results as 0.001 c.c., while one-tenth of this proves sufficient in a considerable percentage of the animals. Usually the sensitizing dose of horse serum employed in experiments upon guinea pigs is 0.01 c.c. Larger doses sensitize but a longer time is required for the development of the anaphylactic state. According to Doerr and Russ, the proportion between the minimum sensitizing and minimum fatal dose is 1-1000. After the animal has been sensitized there is a period of incubation, which in the guinea pig is about eight days. After this period has passed, a reinjection of the same protein used in sensitizing is followed by symptoms identical with those which we have shown follow the injection of a fatal dose of the protein poison in normal animals. Many of the lower animals are not so easily sensitized as the guinea pig and repeated doses of the sensitizer may be necessary to develop the anaphylactic state. Man is easily sensitized, at least with certain foreign proteins. Unfortunately, this has been repeatedly demonstrated in the untoward results that sometimes follow the injection of horse serum in the form of antitoxin in the treatment of diphtheria when the patient has previously had an injection of horse serum for any purpose. In 1907 we formulated our theory concerning anaphylaxis and its relation to the infectious diseases and from this we make the following extract: . (1) Sensitization consists in developing in the animal a specific proteolytic ferment which 
acts upon the protein that brings it into existence and on no other.

This specific proteolytic ferment stored up in the cells of the animal as a result of the first treatment with the protein remains as a zymogen until activated by the reinjection of the same protein.

(3) Our conception of the development of a specific zymogen supposes a rearrangement of the atomic groups in the protein molecules of certain cells, or alteration in their molecular structure. In other words, we regard the production of the specific zymogen not as the formation of a new body, but as resulting from an alteration in the atomic rearrangement within the protein molecule and a consequent change in its molecular structure. (4) Some proteins in developing this specific zymogen produce profound and lasting changes in the molecular structure of the body cells, while the alterations induced by others are slighter and of temporary duration, the molecular structure soon returning to its original condition. (5) Bacteria and protozoa are living, labile proteins, while egg-white, casein, serum albumin, etc., are stabile proteins. The proteins of one group are in an active, while those of the other are in a resting, state; but both are essentially proteins made up of an acid or poisonous chemical nucleus, and basic, nonpoisonous groups. Bacterial immunity and protein sensitization, apparently antipodal, are in reality the same, and each consists in developing in the animal body the capability of splitting up specific proteins. If the living protein be split up before it has time to multiply sufficiently to furnish a fatal quantity of the poison, the animal lives and we say it has been immunized. If the stabile protein be introduced into the animal body it leads to the development of a specific proteolytic ferment, and if enough of it to supply a fatal dose be reinjected after this function has been developed, the animal dies. (6) Formerly we believed the structural changes or the lesions in the infectious diseases to be due wholly to the living, growing, feeding microorganism. For instance, we were sure that the intestinal ulcerations of typhoid fever are caused by the living bacilli. Now we know that these lesions follow the intravenous injection of dead proteins. As has been stated, each foreign protein has its predilection tissue in which it is largely deposited, whose cells it especially sensitizes, and where it is disrupted. This explains the characteristic lesions and symptoms of the different infectious diseases. Bacterial inflammation is essentially a chemical process, or is due to the disruption of cell molecules through the chemical affinity between certain groups in the bacterial cell and certain groups in the cell of the animal. So long as the bacterial cells are alive the chemism that holds the living molecule together tends to resist this process of disintegration. The pathogenic bacterium assimilates the relatively simple constituents of the fluids of the animal body and, building them into its own tissue, converts them into substances foreign to the host, and finally, when the bacterial cell goes to pieces either from spontaneous dissolution, or through the aggressive action of some 
animal cell, these reconstructed chemical groups are set free and poison the animal, inducing lesions in various tissues, and, in many instances, so interrupt the vital functions as to cause death. (7) It seems to be a physiologic law that the specific ferments elaborated by living cells are determined by the proteins brought into contact with them, but as yet we know but little concerning these bodies which we call ferments. That they are labile chemical bodies resulting from intramolecular rearrangement in the protein molecules of the cells seems a plausible theory, but at present it is only a theory. When the body cells find themselves in contact with, or permeated by, foreign proteins they tend to elaborate specific ferments which digest and destroy the foreign proteins.

Unfortunately, most people who have studied this subject, think of the phenomena of anaphylaxis only in their extreme manifestations. As we have seen, according to the computation of Doerr and Russ, the minimum killing dose in a sensitized animal on reinjection must be a thousand times the minimum sensitizing dose. Suppose the protein introduced on reinjection is not greater than the sensitizing dose or is anywhere from this to 500 times the sensitizing dose; there is no anaphylactic shock and the protein introduced on reinjection is split up without harm to the animal. The meningococcus finds its way from its normal habitat in the nasopharynx and is carried by the blood current to all parts of the body and like all other foreign proteins introduced into the blood current it rapidly disappears from this stream. Its predilection site is in the meninges of the brain and cord. Among the cells of these structures it takes up its residence. The meningeal cells after a period of incubation become sensitized and begin to destroy the invading cells. At this point the active disease begins and the lesions which result and which are found after death are due to the destructive chemical reactions between the proteins of the meningococcal cells and those of the body cells. The virus of smallpox finds its way into the body, is distributed by the blood current, and selects as the site of its development the epithelial tissues. Here it grows, multiplies, sensitizes the body cells, and destructive reactions take place. This is not altogether theoretical. We have demonstrated by the sensitization test the presence of foreign proteins in the skin long after they have wholly disappeared from the blood current.

We are fully aware of the fact that animals, especially guinea pigs, may be killed with all the symptoms of anaphylactic shock by primary injections of various substances, such as fine suspensions of agar, dilute incubated blood serum and tissue extracts, but so far as we can see these have nothing to do with anaphylaxis or protein sensitization as applied to the infectious diseases. An extract of an organ from a fellow of its own species injected intravenously or intracardially into a guinea pig will kill it with all the symptoms of anaphylactic shock, but this has nothing to 
do with protein sensitization and certainly has no bearing on the relation between anaphylaxis and the infectious diseases. As we have demonstrated, the guinea pig possesses a high degree of immunity to the prodigiosus, but when the prodigiosus is grown artificially and thrown in sufficient dose into the abdominal cavity of the guinea pig the animal quickly dies. In our opinion, it dies because the secretions of the body cells speedily break up the prodigiosus protein, setting free in a short time, a fatal dose of the protein poison. The more highly immune an animal is to a given bacterium the smaller the dose of the cellular protein of that bacterium necessary to kill that animal.

Vaccination.-Vaccine consists of the virus of smallpox modified by its passage through the cow. This virus consists of labile, growing, multiplying proteins which have been so modified by their cycle of life in the cow that they grow in man's body without harm and in doing so sensitize the body cells to destroy the specific protein of smallpox virus. When the vaccinated individual is exposed to smallpox and inhales the virus of the malignant form of this disease the body cells, sensitized by the previous vaccination, destroy the malignant virus before it has time to multiply and consequently the individual is protected; in other words, vaccination for smallpox is a form of protein sensitization. Vaccination for typhoid fever is another illustration along the same line. Dead typhoid bacilli are introduced into the body and the body cells trained to elaborate a specific ferment which dissolves the typhoid protein and no other. When the individual thus vaccinated swallows a few typhoid bacilli these are destroyed by the body secretions before they have time to multiply suffciently to do the body harm. When, however, there is massive infection the increased resistance of the body brought into existence by vaccination fails and typhoid fever results and in its symptomatology is not to be distinguished from that disease as it develops in the unvaccinated. In vaccination against Asiatic cholera the living organisms may be introduced subcutaneously, because when thus introduced they are incapable of causing the disease, but they do sensitize the body cells and the individual is protected. The same principle explains why one attack of certain diseases gives immunity. Vaccination never gives absolute immunity. There is always the opportunity for the reinfecting substance to be present in a quantity sufficient to overcome the increased resistance. Vaccination is most effective or effective in the largest percentage of cases when there is least chance for massive infection in the vaccinated individual. Neither does one attack of a disease give absolute protection against a second attack. Many people have typhoid fever more than once. A few have smallpox twice. Very few have yellow fever more than once. The chances of massive infection in typhoid fever are great. Those of massive infection in smallpox are small and those for massive infection in yellow fever 
do not exist, because in the last named disease the infection must always come through a mosquito and must be limited in amount.

Both body cells and bacterial cells consist of labile protein molecules whose structure and function can be influenced and altered by environment. All living things can be educated, i. e., their behavior responds to environment. Both body cells and bacterial cells may under the influence of altered environment develop new functions. When a man has smallpox or is vaccinated against this disease, the body cells respond to the environment and develop a new function, that of destroying, probably by digestion, the protein of the smallpox virus.

Precipitins.-In 1897 Kraus discovered that the blood serum of an animal which had been repeatedly treated with a given bacterium forms a precipitate when mixed with a filtered culture of that organism. Two years later Tchistowitsch and Bordet ascertained that this is a general protein reaction. A rabbit which has had several injections of horse serum yields a serum which precipitates horse serum; one treated with cow's milk furnishes a serum which precipitates cow's milk; one treated with the white of the hen's egg precipitates egg albumin. This test is now used in the identification of blood stains, in the detection of mixed meats, and in the study of the blood relationship of animals. If a blood stain be dissolved in salt solution and then mixed with the blood serum of a rabbit which has been repeatedly treated with human blood, a precipitate will be formed if the stain is human blood. If a Hamburger steak be extracted with water, the clear filtrate will be precipitated with the blood of an animal which has been treated with horse's blood, provided the steak contains horse meat. This reaction is not strictly specific. A rabbit treated with the blood serum of man will furnish a serum which will precipitate not only human blood, but the blood of the higher apes. Quantitatively the reaction is specific.

The protein which is injected into the animal is known as the precipitinogen (producer of the precipitin). Precipitinogens are bacterial, vegetable, and animal. A culture of the typhoid bacillus, preferably one several weeks old, is filtered through porcelain in order to free it from the bacterial cells, then every third or fourth day some of the filtrate is injected into a rabbit. Some days after the last injection, serum obtained from this rabbit precipitates the typhoid filtrate and that of no other bacterium.

The substance in the serum of the treated animal is known as a precipitin. In the sera of certain animals, which have had no treatment there are natural precipitins. The serum of the untreated ox, undiluted, precipitates many bacterial filtrates. Normal goat's serum gives precipitins with the sera of rabbits and dogs. These normal precipitins do not interfere with diagnostic tests because (1) they are not specific and (2) they do not give the precipitates in high dilution. Not every animal produces precipitins. The best laboratory animal for the production of precipitins is the rabbit. 
Administration by the mouth produces a precipitin only when for some reason the precipitinogen is absorbed undigested. This may result from overfeeding and easily happens in the young. Puppies, kittens, and rabbits up to ten days old frequently absorb unchanged proteins from the alimentary canal and this leads to the production of precipitins. It has been found in a few instances that the serum of infants precipitates cow's milk. It is possible that this is a factor in the causation of cholera infantum.

The precipitate results from a reaction between the precipitinogen and the precipitin. In case of bacterial filtrates the precipitate does not form directly on adding the precipitin to the precipitinogen and it is customary to allow the mixture to stand at incubator temperature for from two to twentyfour hours. The rapidity and abundance of the formation of the precipitate depends upon the richness of the fluids in their respective active constituents. With animal precipitinogens the precipitate may appear immediately on the mixture of the fluids. Precipitation takes place in neutral, feebly alkaline and feebly acid solutions. When the acidity is due to organic acid or acid salts the action is promoted. In the complete absence of inorganic salts the reaction fails. The precipitate consists essentially of proteins; it contains no carbohydrate group, is insoluble in dilute mineral acid and alkali, and is highly resistant to the digestive ferments. There scems no doubt that the bulk of the precipitate is derived from the precipitin. Welch and Chapman obtained with a precipitinogen which contained only one milligram of protein a precipitate which contained 25.1 milligrams of protein. Evidently in such a case the bulk of the precipitate must come from the serum. However, this is not always true, since the bulk of the precipitate produced with lactosera consists of casein. It is probable that in many instances substances other than the precipitinogen and the precipitin are carried down mechanically.

Zinsser makes the very reasonable suggestion that the function of the precipitin is to prepare the foreign protein which has found its way into the body for parenteral digestion. That even in perfect health we are more or less constantly absorbing from the alimentary canal undigested proteins is shown by the great number of us who respond to the intradermal test. As has been stated, the absorption of undigested proteins occurs frequently in young animals, and the probability is that it occurs with equal frequency in infants. This undoubtedly is one of the reasons that infants are much more susceptible to slightly decomposed milk than are adults.

Agglutinins.-In 1889 Charin and Roger, in studying the action of the serum of sick and inoculated animals on homologous bacteria, observed that the Bacillus pyocyaneus behaves peculiarly when placed in the serum of an animal which has been inoculated with this organism. In the serum of a normal rabbit this bacillus grows as it does in beef-tea, forming an opaque culture, while in the serum of an animal previously inoculated with this 
bacillus it forms floceules which soon subside leaving a clear supernatant fluid.

Two years later Metchnikoff noticed that the vibrio which bears his name behaves in a similar manner. He wrote as follows:

"In the blood and serum of nonvaccinated guinea pigs, the vibrio develops as it does in ordinary liquid media, the individual organisms retaining their motility and remaining distinct, one from the other. On the other hand, in the blood and serum of vaccinated animals the vibrios become immobile, and form smaller or larger floccules, which float in the liquid."'

In 1893 Issaeff, and later he and Ivanoff observed the same phenomenon and described it as follows:

"In the blood serum of healthy, nonimmunized guinea pigs the vibrio develops rapidly, and after from four to five hours at $37^{\circ} \mathrm{C}$. there is a uniform cloudiness throughout the fluid, while the surface is covered with a scum; but in immune serum, the microbes sink to the bottom of the tube, while the supernatant fluid remains clear. This condition continues for from eight to nine days, and it is not until the tenth day that the culture becomes cloudy and a scum appears on the surface."

In 1896 Gruber and Durham studied this phenomenon and suggested that it be designated as agglutination, believing it would be serviceable in the identification of bacteria. A few months later Widal demonstrated the value of this reaction in the diagnosis of typhoid fever.

It is evident that in such a reaction as that observed in agglutination there must be two factors. One of these, designated the agglutinin, is found in the serum, and the other, known as the agglutinogen, exists in the bacterial culture. The product of the reaction is known as the agglutinate. The serum of certain normal animals has slightly agglutinating action on certain bacteria, notably typhoid and colon bacilli. The normal serum of the horse, dog, donkey, and rabbit, in an undiluted state agglutinates typhoid cultures. Some effect may be observed when the serum is diluted 1-30. The serum of a man who has never had typhoid fever may agglutinate typhoid cultures when the dilution is not higher than 1-30. The period in the progress of typhoid fever in which marked agglutination manifests itself varies widely. Usually it is not before the seventh day, but it may occur as early as the second day, and it may be delayed until the second week and in rare cases even later. Likewise, the disappearance of the reaction after recovery is variable. It may fail within ten days after the loss of the fever, and it has been known to continue for years. In intensity the reaction is variable and bears no indication of the severity or seriousness of the disease.

Agglutinins exist not only in the blood, but in other fluids of the body. The agglutinating power of the urine in the same individual is variable not only from day to day, but from hour to hour, and is always feeble compared with that of the serum. Serum obtained by blisters has a rela- 
tively high agglutinating value. That of the bile is marked. Pus from typhoid abscesses markedly agglutinates bacilli. Agglutinins may pass from the mother to the fetus and to the nursing child. Typhoid serum may be treated with formaldehyde in a 40 per cent solution and kept quite indefinitely without loss of its agglutinating property. However, the use of antiseptics is not necessary to preserve agglutinating sera. A highly active serum will retain its agglutinating action after it has become putrid from bacterial contamination. Dilute mineral acids decrease the agglutinating property of typhoid sera, but when the contact has not been prolonged, full activity may be restored by neutralization. Alkalies act similarly. Apparently, the agglutinins are not altered by either pepsin or trypsin. In experimental animals, sera can be obtained which will agglutinate typhoid bacilli in dilutions as high as 1-1,000,000 and colon bacilli 1-2,000,000.

Quantitatively the agglutinins are specific, but in order to be so considered they must be active in high dilutions. A positive diagnosis of typhoid fever should not be made unless the serum acts decidedly in a dilution not less than 1-50 and even in this dilution there is a rare chance of mistake. A paratyphoid serum may agglutinate typhoid bacilli in a dilution of $1-1000$, or a typhoid serum may act in like dilution on a paratyphoid bacillus.

Agglutination does not destroy the viability or virulence of bacteria ; therefore, the reaction does not disrupt the living bacterial cell. Thoroughly washed typhoid bacilli are not agglutinable. When typhoid bacilli are thoroughly shaken in salt solution so as to remove the flagella and the bacilli are deposited in a centrifuge the emulsion of flagella is agglutinable. Cholera bacilli, as shown by Neufeld, when shaken with 1 per cent alkali, which does not dissolve the cells but washes away the adherent matter, are not inagglutinable, but when injected into animals they produce no agglutinin.

Agglutination and precipitation are like phenomena. When a bacterial culture is filtered, some of the proteins adherent to the bacterial cells pass into solution and constitute the precipitinogen. Some of the same class of near-cell proteins remain in close connection with the cells and constitute the agglutinogen. Inorganic salts are essential to agglutination. As Widal concluded years ago, agglutination is not a reaction of immunity, but a reaction of infection. It is most probable that both agglutination and precipitation are steps preparatory to the parenteral digestion of foreign proteins.

Agglutination is of value in distinguishing between closely related bacteria. A typhoid serum may agglutinate the Eberth bacillus or paratyphoid A or paratyphoid B, but it agglutinates in high dilution only that typhoid bacillus to which the infection is due. In like manner agglutination 
is of value in separating the strains of meningococcus and of colon bacilli, as well as other bacteria.

Bacteria.-Bacteria are living proteins capable of unlimited multiplication, provided they are furnished with sufficient food and come in contact with no adverse condition. Morphologically they are divided into cocci, bacilli, and spirilla. Chemically they consist, as we have already shown, principally at least, of glyconucleoproteins. The large amount of nuclear material in them is shown by their behavior to the anilin dyes. The chief function of bacterial cells is to multiply and their chemical composition is especialiy adapted to this function. As an infecting agent a living protein is much more effective than a dead protein. In the live state the protein keeps up a continuous existence and it does this by converting certain constituents of the body of its host into its own peculiar protein; in other words, the living protein is not only able to advance through the body of its host, but in doing so multiplies in numbers as it advances.

When bacteria reach a certain size which is fairly constant in the species, the cells divide. In bacilli and vibrios fission occurs at right angles to the long axis. In cocci the cleavage may occur in only one plane forming streptococci ; in two planes forming staphylococci; or in three planes forming sarcinae. The rapidity of multiplication and growth to maturity varies with conditions. It has been estimated that if fission occurs hourly without interruption, in 48 hours the descendants of a single individual would number more than 200,000,000. Such a rate of multiplication, however, can hardly occur, since growth would be checked by scarcity of food or by the accumulation of excreta from the bacteria themselves. The rate of multiplication under most favorable conditions is the best measure of the intensity of life processes, and in the case of pathogenic bacteria, of virulence. The "generation period" is the time interval between fissions in the same bacillus. This varies greatly with varying conditions, some of which seem inherent in the strain while others are more fluctuating. Under most favorable conditions the cholera bacillus has been found to divide about every half hour. As the culture grows older the rate of multiplication falls on account of the accumulation of metabolic products. In infection the rate of multiplication is partly determined by the number of bacteria introduced and more largely by the resistance with which the growth is opposed. One strain of the same species may multiply a thousand times as fast as another.

In the presence of untoward conditions, such as scarcity of food, the presence of deleterious agents, and the accumulation of metabolic products, some bacteria are capable of passing into a resting stage known as the spore form. Of those bacteria which are pathogenic to man, only anthrax, symptomatic anthrax, tetanus, botulinus, and malignant edema are known to be spore formers. The spores are of endogenous origin, are highly refractive to light, and resistant to heat and chemical disinfectants. No bacillus is 
known to form more than two spores, and this number is practically confined to saprophytic organisms. The appearance of two spores in an anthrax bacillus is rarely seen. The purpose of spore formation is not multiplication but preservation; it is a resting stage. Spore formation is a result of the concentration of the nuclear substance. The spore may be formed in any part of the cell, but the location in the same species is usually constant. In some bacteria the spore does not exceed the diameter of the cell; in others it is larger. A large spore located at one end of a bacillus gives it a "drum-stick" appearance; when in the middle, it forms a "spindle-shaped" organism. The bacterium passes into the resting state and awaits more favorable conditions. When these arrive, whether it be days or years later, the spore passes into the vegetative form and reproduction and multiplication begin anew.

Every living thing must feed, assimilate and eliminate. It thrives and multiplies when food is abundant and conditions of life are favorable; it hungers and dies when the food supply fails. These things are as true of unicellular as of multicellular forms of life. All bacteria, even the anaerobic, absorb oxygen and eliminate carbonic acid. In this gaseous exchange more oxygen is absorbed than is eliminated in the form of carbonic acid. This shows that some oxygen is used in nitrogen metabolism. When the food supply is suddenly withdrawn in the midst of rapid multiplication, the death rate is great, and greater among the young than among the older individuals. Fisher has shown that when a growth of cholera bacilli 24 hours old is suddenly transferred to salt solution, all the organisms die within 36 hours. With older cultures some live bacilli are found much later and with a seven-day culture more will be found to be alive after 50 days. When the food supply is gradually withdrawn the death rate is not so high and the more hardy individuals retain their vitality for months and even years, especially at low temperature. The respiratory quotient remains constant in the same species under identical conditions and is a measure of the rate of growth and multiplication. All living cells must depend on the pabulum within their reach. This must be digested or broken up into particles which fit into the cellular molecule. This is accomplished by agents which we designate as ferments or enzymes. Each species of bacteria elaborates its own specific enzymes, the functions of which are to cut the pabulum into proper blocks and to place these blocks in the structure of the cell. These enzymes are specific in two senses. First, they are specific in origin; the ferments produced by typhoid bacilli are not identical with those of anthrax bacilli. Secondly, they are specific in their action. Given the same pabulum, the shape and size of the blocks into which it is split differ with the ferment acting on it. Besides, a given ferment cannot act on all pabulum. What is food to one bacterium may be of no value or even a poison to another. 
All bacteria show passive movements when suspended in fluids and observed under the microscope. Such motion is known as molecular or Brownian and is common to all finely divided particles when suspended in fluid. Apart from this, certain bacteria are capable of active motion, which is accomplished by the contractions of flagella or whips. Those without flagella have no active motion. There may be only one whip at one end, as in the cholera bacillus; a single whip at each end, as in many saprophytic vibrios; a bunch of whips at one end, as in certain large saprophytic vibrios, and many whips distributed over the cell, as in the typhoid bacillus. The flagella seem to be outgrowths from the ectoplasm and they may be removed from certain bacteria by shaking and then by centrifuging, or by filtration through porcelain. Their presence favors agglutination, which however, is not wholly dependent on them.

Pasteur observed that some bacteria grow best when supplied with oxygen, while others have their optimum growth when this element is excluded. The former he designated as aerobic and the latter as anaerobic. Further study has divided bacteria according to their need of oxygen into the following classes: (1) Obligate aerobes are those which will not grow save in the presence of free oxygen. This does not mean that they must have an abundant air-supply, because some do grow when the oxygen tension is only one-hundredth that of the air. Among the pathogenic bacteria, plague and influenza bacilli and the pneumococcus and the gonococcus belong to this group. An excess of oxygen may prove harmful to even the obligate aerobes. (2) Facultative anaerobic bacteria are those that grow best in the presence of oxygen, but may grow when this element is wholly excluded. Many pathogenic organisms belong to this class, such as the cholera vibrio, the anthrax bacillus, the typhoid bacillus, and pus cocei. (3) Obligate anaerobes are bacteria which grow only in the absence of free oxygen. In this group are the bacilli of tetanus, symptomatic anthrax, botulinus, and malignant edema. These bacteria obtain their oxygen so far as they utilize this element from the combined oxygen in their pabulum. Pasteur found that fermentation is due to obligate anaerobes and he defined fermentation as life without oxygen. This, however, does not mean that all life without oxygen results in fermentation. The growth of bacteria of this group is favored by the presence of reducing agents in the medium. Anaerobic bacteria may grow in the presence of free oxygen, provided there are present aerobic organisms which absorb all the oxygen. The development of tetanus is favored by puncture wounds; also by the presence of aerobic bacteria.

As happens in all forms of life there is more or less antagonism between different species of bacteria. It is fortunate that this is true and man and other animals profit greatly by the conflict continually being waged between different species of bacteria. A drinking water supply becomes con- 
taminated with the typhoid bacillus which flourishes for a few days and is then completely destroyed by other bacteria. So. true is this that by the time the disease has developed among those who drink the water there may no longer be typhoid bacilli in it. A city's water-supply should be examined daily to be of value. The weapons used in this warfare among bacteria are many and varied. Some simply eat up the food supply and the invaders die of starvation. Others produce waste products which are harmful to other species. Anthrax bacilli planted in sterile cholera cultures are greatly weakened. Sterile cultures of the pyocyaneus dissolve anthrax bacilli. Some change the reaction of the common medium by the development of acid or alkali and their enemies die. The streptococcus kills the plague bacillus and thus the conflict of nations and races goes on among the unicellular organisms much as it does among the lords of creation.

Some species form allied armies and contend against a common enemy or overrun a foreign country. The pus bacteria are found in many combinations and most men die from diseases in which these cocci play an important part. After the tubercle bacillus has fed on one's lungs for years and prepared the soil some pus germ finds its way in before death and contributes largely to its coming. The cancer cell opens up ports of entry through which pus organisms enter. The lesions of syphilis are plundered and looted by the hordes of cocei. Thus, as Mayo has pointed out, sepsis plays an important rôle in the last acts of the three great tragedies of life, tuberculosis, cancer, and syphilis. Some pathogenic bacteria receive aid from organisms which by themselves are harmless. The tetanus bacillus is much more virulent when it enters the animal body in company with certain saprophytes. When alone, the phagocytes speedily fall on and devour it, while certain products of the growth of its friend repel the phagocytes.

Other bacteria are surrounded by mucilaginous capsules. When two or more individuals are attached, the capsule usually includes the group, and when many are imbedded in one large capsule, the whole is known as zooglia. The capsule is believed to be formed for protective purposes, since many bacteria develop them only when in the animal or when grown in animal fluids, secretions, or exeretions. The presence of deleterious agents, such as arsenic, seems to favor the development of capsules.

Pathogenicity.-Why is it that one bacterium is capable of inducing disease while another is harmless? The answer to this is quite clear. Some bacteria can feed only on dead matter. Their ferments will not prepare the fluid and tissues of the animal body for the sustenance of their cells, or it may be that the ferments of the body cells digest these bacteria and prevent their growth. These bacteria, which compose the great majority of existing species, are known as saprophytes.

There are other bacteria which can digest, absorb, and eliminate the con- 
stituents of the fluids and tissues of the animal body and which are not digested by the body cells. These are known as parasitic bacteria and parasitism is necessary in order that the bacteria may produce a continuous supply of foreign protein in the body of its host. Parasitism, however, is not the only requisite in order to make a given bacterium pathogenic. The meningococcus growing in the nasopharynx is a parasite, but so long as it is confined to this locality it is incapable of inducing disease. The pneumococcus is almost constantly present in the saliva of normal individuals, but so long as its presence in the upper respiratory tract is confined to the region above the glottis it does no harm. When it gets into the lungs, it may cause pneumonia. The diphtheria bacillus is frequently found in the saliva of normal individuals where it grows and multiplies without causing any disturbance of health. The typhoid bacillus may for years multiply in the gall bladder or in old abscesses without causing typhoid fever ; in fact, most of the bacteria which cause disease in man are carried by healthy persons. Under these conditions the parasitism of the bacterium is denominated commensal. (Inasmuch as the words, saprophytic and parasitic, are derived from the Greek, it would be more consistent to substitute a word of similar origin for the latin word commensal. We are assured by our classical colleagues that the word syssitic is suitable both in origin and in meaning. It means eating together in harmony.) The meningococcus in the nasopharynx eats at the same table with the local body cells, but since there is no contest between the bacterial cells and the body cells there is no disease. In order for a parasitic bacterium to become pathogenic it is necessary that there should be a reaction between its protein constituents and those of the animal body in which it exists. In nature the meningococcus is an obligate parasite and its only host is man. It becomes pathogenic only when it reaches the meninges of the brain and cord and in this tissue there is a reaction between meningeal proteins and meningococcal proteins. There is a tendency at present among bacteriologists to say that an organism growing in man's body without causing disease is living a saprophytic life. Unless we are willing to violate all rules of etymology this is gross error. There is, moreover, an important principle involved in this designation between syssitic and pathogenic parasitism. A bacterium may be an obligate parasite and may find in nature its only habitat in the body of man where it exists in most individuals without causing any disorder of health. It is only when there is a reaction between the proteins of the bacterial cells and those of the body cells that disease results. A given bacterium may be nonpathogenic so long as it is confined to one location in the body and it may be highly pathogenic when it finds its way into other tissues. This is a matter of prime importance in the proper understanding of the nature of infectious or albuminal diseases. The parasitic bacteria that are distributed by healthy carriers are in these 
people parasites, but they are not pathogenic. There must be a contest, a combat, between the body cells and the invading cells before lesions or symptoms of disease can result. The recognition of these important facts in recent years marks a great advance in our comprehension of the nature of infection.

Toxins and Antitoxins.-The discussion of these bodies will be reserved for those diseases, diphtheria, tetanus, and botulism, in which they play an important rôle; inasmuch, however, as both toxins and antitoxins are, so far as we now know, protein bodies we are justified in including the toxin diseases among the albuminal diseases.

Summary.-In our opinion, we have brought forward enough evidence to show that the reactions between invading bacterial cells and body cells are protein reactions. Asthma caused by the inhalation of pollen is just as truly a disease as is the asthma which is eaused by bacterial infection. The individual who is ill from serum disease is just as truly ill as is the one who has measles. The man who is suffering from the absorption of the proteins of clams is just as truly sick as is the man whose intestinal functions are disturbed by the bacillus of dysentery. All the reactions of the infectious diseases are protein reactions, and we are justified in calling these diseases albuminal.

Classification of Infectious Diseases.-In a work on epidemiology it is certainly best to classify the epidemic diseases according to the avenues through which the virus most frequently enters the body. Carrying out this idea, we have adopted the following classification: (1) Respiratory Infections. (2) Alimentary Infections. (3) Percutaneous Infections. (4) Venereal Infections. (5) Local Infections. The first volume of this book is devoted exclusively to the Respiratory Infections. It is hoped that the remaining infections may be condensed in a second volume and that a third volume be devoted to Public Health or the administration of those agencies by which disease may be reduced to a minimum.

\section{Bibliography}

Gay, Frederick P.: Oxford Medicine, Oxford University Press, 1919, i, Part 4. PArk, Wm. H.: Public Health and Hygiene, Philadelphia, 1920, Lea \& Febiger.

Teale, F. W.: Dobell Lecture, 1920.

'THomson, D.: The Lancet, 1921, i.

Vaughan, V. C.; Vaughan, V. C., JR.; and Vaughan, J. Walter: Protein Split Products in Relation to Immunity and Disease, Fhiladelphia, 1913, Lea \& Febiger. 


\section{CHAPTER III}

\section{POLLINOSIS}

\section{HAY FEVER; ROSE FEVER; SUMIMER CATARRH; AUTUMNAL CATARRH}

Definition.-Under the term "pollinosis" we include certain conjunctival, nasopharyngeal, and tracheobronchial congestive and eatarrhal conditions which are due to the inhalation of pollen and other particulate protein containing bodies.

History.-In medical literature extending back for nearly 400 years there may be found occasional mention of some individual who has been more or less seriously disturbed in health when brought in close contact with eertain plants during the season that these plants are in bloom. Botallus, of Pavia, writing in 1565, states that he had patients in whom inflammation of the conjunctivæ, running at the nose, sneezing, and headache were induced by the presence and odor of roses. Helmont (1607) observed a clergyman who suffered from asthma every summer but was completely free from it during the remainder of the year. Schneider (1662) describes a case in which the odor of roses caused sneezing and catarrh. Binningerus (1673) tells of a lady who suffered from an acute coryza when roses were in bloom but was free from it all the rest of the year. Ledelius (1684) had under his care a merchant who suffered from severe headache and eatarrhal inflammation whenever he visited the country in the summer time and especially when roses were in blossom. Rebeque (1691) describes his own case and says that for 13 years he suffered an acute coryza as long as roses were in bloom but lost this trouble with the deflorescence of these plants. He attributed it to the odor. Similar reports have been made by Riedlin in 1695 and by Heberden in 1802, and probably a thorough search of the literature would give other instances. In all these cases the report was given as a medical curiosity or as illustrative of some individual idiosyncrasy. So far as we can ascertain, no early author thought he had in hand a disease to which many are susceptible.

The man who placed hay fever in the list of clinical diseases was Bostock, an English physician, who, in 1819 reported his own case. From this report we make the following extracts:

"About the beginning or middle of June in every year the following symptoms make their appearance, with a greater or less degree of violence. A sensation of heat and fullness is experienced in the cyes, first along the edges of the lids, and 
especially in the inner angles, but after some time over the whole of the ball. At the commencement the external appearance of the eye is little affected, except that there is a slight degree of redness and a discharge of tears. This state gradually increases, until the sensation becomes converted into what may be characterized as a combination of the most acute itching and smarting, accompanied with a feeling of small points striking upon or darting into the ball, at the same time that the eyes become extremely inflamed and discharge very copiously a thick mucous fluid. This state of the eyes comes on in paroxysms, at uncertain intervals, from about the second week in June to the middle of July. The eyes are seldom quite well for the whole of this period, but the violent paroxysms never occur more than two or three times daily, lasting an hour or two each time; but with respect to their frequency and duration there is the greatest uncertainty. Generally, but not always, their invasion may be distinctly traced to some exciting eause, of which the most certain is a close moist heat, also a bright glare of light, dust or other substances touching the eyes, and any circumstance which increases the temperature. After the violent inflammation and discharge have continued for some time, the pain and redness gradually go off, but.a degree of stiffness generally remains during the day.

"After this state of the eyes has subsisted for a week or ten days, a general fullness is experienced in the head, and particularly about the fore part; to this succeeds irritation of the nose, producing sneezing, which occurs in fits of extreme violence, coming on at uncertain intervals. To the sneezing are added a further sensation of tightness of the chest, and a difficulty of breathing, and general irritation of the fauces and trachea. There is no absolute pain in any part of the chest, but a feeling of want of room to receive the air necessary for respiration, a huskiness of the voice, and an incapacity of speaking aloud for any time without inconvenience. To these local symptoms, are at length added a degree of general indisposition, a great degree of languor, an ineapacity for muscular exertion, loss of appetite, emaciation, restless nights, often attended with profuse perspirations, the extremities, however, being generally cold. The pulse is permanently quickened, from 80 , the average standard, to about 100 , and upon any considerable exertion it rises to 120 or more. This is an account of the complaint in its worst state, which, however, it does not assume in every season, and indeed its violence is generally less than is here described. The affection of the eye is recollected to have occurred when the patient was eight years old, and there has been more or less of it every, year since; the sneezings came on nearly at the same period, but the first attack of the chest was at the age of sixteen or seventeen. Generally speaking, the complaints have increased for the last twenty years, although not progressively. All the acute symptoms disappear about the end of July, but a considerable degree of weakness and languor is left, which remains a month or six weeks longer. It has happened that the most severe summer complaints have been experienced, after the patient had enjoyed the best of health during the preceding spring. On the contrary, it has been thought that after a severe summer attack, the patient has more completely and more rapidly regained his usual state of health and strength in the autumn.",

The title of the paper from which the above quotation is taken is, "Case of a Periodical Affection of the Eyes and Chest." In 1828 Bostock published a second paper under the title, "Summer Catarrh." In this contribution he gives an account of his efforts to escape or mitigate the disease. Evidently the disease was quite widely known at the time Bostock's contributions were made, and it was generally designated as hay fever. Bostock 
combats the idea that pollen has anything to do with the causation of the symptoms and states that he had found no relief during the summer of 1826 which he had passed on the Isle of Thanet where there was not an acre of meadow. In the year 1827, which was exceptionally cold and wet, he had comparative freedom from the disease at Kew where he walked daily in the gardens and was surrounded by hundreds of acres of grass.

The publications of Bostock aroused interest in this seasonal disease and led to the accumulation and study of data concerning it. In 1828, the year of Bostock's second publication, MacCulloch included hay fever in a book which he published under the title, "An Essay on the Remittent and Intermittent Diseases," and in which he stated his belief that summer catarrh is caused by some inhalation from plants, and especially by the odors or dust arising from hay fields. In 1829 Gordon wrote a paper, entitled, "Observation on the Nature and Treatment of Hay Asthma,"' in which he claimed that the odor of flowering grasses is the cause of the disease. He especially incriminated the sweet-scented vernal grass (Antozanthum odoratum) and stated that the disease should be called "grass asthma." In 1830 Elliotson made it quite clear that summer catarrh is due to the inhalation of pollen. He tells of a patient of his in whom the symptoms appeared and gradually increased as the grass comes more and more into flower, till at last they arrive at such a pitch of intensity that she is obliged to leave home and go to the seaside. That on one occasion when she was at a place where there either was, or where she thought there was, no grass at all, she was suddenly seized with the disease, and on looking out of the window found two stacks of hay "putting up" which had just been brought from a considerable distance. He observed in this patient that the handling of the flowers of grass caused swelling and inflammation of the hands. Elliotson writes:

"What sort of grass it is that produces it I do not know, there are so many sorts; but this would make it appear that with some persons it is only the sweet-scented grass. If the disease really prevails more now than formerly, and we observe it so frequently now, not merely because its existence is notorious, this might perhaps be ascribed to the large number of new grasses which have of late years been introduced."

It is interesting to note here that Elliotson reports the case of a lady who suddenly developed an acute coryza whenever her husband, who was a huntsman, brought a rabbit into the room. In reporting this case Elliotson says :

"There is no reason why some cannot bear a rabbit; than others a sucking pig, a cat, and so on. It is well known that some persons are so sensible to ipecacuanha, as to be seized with catarrh and asthma the moment it is near them.'

The most scientific and accurate investigator of the causation of hay fever in the early part of the nineteenth century was Blackley, a physician in Manchester, England, who, himself being a victim of this disease, made 
upon himself a great variety of most interesting experiments. He tested the pollen of more than 100 species of flowers and grasses, employed the pollen not only in its fresh state, but when dried and used as the basis of extracts. He found that aqueous or diluted saline washings from pollen placed in the eye, on the lips, tongue, in the nose or pharynx, induced an attack of the disease, and this occurred without reference to the season of the year when the experiment was made. Using the fresh or dried pollen he found that a minute quantity, one two-hundredth of a grain, and even less, was sufficient to induce a severe coryza. In his own case dried pollen from rye caused more serious symptoms than samples obtained from grasses. With the pollen of wheat and oats the effect produced differed but little from that following the employment of grass pollen. One drop of an extract of the pollen of gladiolus applied to the eye or to the pharynx caused marked congestion accompanied by intense itching and symptoms which lasted in some instances for a day or longer. Blackley was the first to make a skin test with pollen. To scarified surfaces of the forearm and leg he applied powdered pollen and in some instances extracts, thus inducing marked edema of the parts accompanied by intense itching with symptoms which lasted in some instances, at least, four days. He was able to induce the disease in himself with the pollen of all the plants with which he experimented, but we should say that these were included in the graminaceae. By eatching the pollen on glass plates smeared with some sticky substance, he was able to make a rough determination of the number of pollen grains deposited upon a given surface for a given time. He found in his home that the quantity of pollen in the air increased from June 1 to August 1, with a rapid decrease after the latter date. His own symptoms followed the same curve in increase and disappearance. More than 95 per cent of the grains deposited on his glass slides belonged to the graminaceae. He found that, although there may be a small amount of pollen in the air in England before June 1, it is not sufficient, in most susceptibles at least, to initiate the symptoms of the disease. Quite naturally, he found that the number of pollen deposited on his plates varied with the distance from the fields where the plants were growing, and also with the direction of the wind. For nearly 50 years Blackley, with most commendable perseverance, continued the experiments upon himself and it can be said that he fully established the causal relationship between pollen and so-called hay fever.

With the development of bacteriologic technic an attempt was made, especially in Germany, to show that summer catarrh is due to bacteria and not to pollen. It was easy to find in the abundant secretions from the nose varied kinds of bacteria. Helmholtz, Binz, Heyman, Weil, and others, for many years claimed that hay fever is of bacterial origin. In this country Beard, during the seventies of the last century, earnestly contested the 
idea that hay fever is caused by the inhalation of pollen. He believed that the disease is due to some instability of the nervous system, which of course, he was not able to properly define. In 1876 Beard wrote as follows:

"For the past half century or more, physicians and scientists, and sufferers have been looking for an objective cause of this disorder. They have studied hay, and grass, and flowers, and fruit, and the pollen of ragweed and corn, sunlight, and dust of every form and when any one of these substances has been found to excite the symptoms in any single ease, straightway the problem has been believed to be solved. An excellent illustration of this one-sided method of studying disease is found in the monograph of Blackley, of England, who, by a series of careful and admirably carried out observations, proved, what was well known before, that the pollen of certain. grasses may in some cases excite the paroxysms, and hence concluded that in this single and simple fact was the whole philosophy of the disease, and that it should be called 'pollen catarrh.' Consistently with this line of reasoning and the habit of looking at one side rather than all sides of a complex subject, observers in this country have proved that ragweed and the pollen of corn excite the paroxysms, and term the disease 'ragweed fever.' Others find that the paroxysms are excited by peaches, and call it 'peach cold;' others still by snow, and call it 'snow cold.'

"All these observers are right and all are wrong. In reality, hay fever, whenever and wherever occurring, is a subjective disease; it is from within, not from without, and all of these excitants, whose name is infinity, are but what friction is to a match. I have statistics of 54 cases in 12 families. I have notes of 25 cases, that had the disease from infancy. In Newport there is a man who has it, whose father and grandfather also were sufferers; at Bethlehem I presented to the assoeiation a child, five montlis old, with all the symptoms of hay fever inherited from its mother.

"Carrying out the iliustration of lighting a match, we may say that the tendency to hay fever may'remain latent for a long lifetime, until, under the irritation of some excitant, it suddenly blazes forth. Thus at Bethlehem there was a man who was first attacked at the age of 60 on removing from the West to Rhode Island; and another case of a man whose first attack was on exposure to ragweed and tomatoes at the age of 65 . A father and a son were first attacked at the same time while on a visit at a certain place in New Hampshire; thus demonstrating the relation of the latent tendency to the exciting causes, and also the hereditary character of the malady. All of these exciting causes, dust, hay, dried and fresh, pollen of all kinds, sunlight, gaslight, foul air, smoke, einders, mental influence, peaches, strawberries, ipecac, ete., etc., our ancestors in the last century were exposed to as much as we now are; but they did not in them exeite hay fever in any of its forms, and even now, not more than one out of five hundred or a thousand of our population are affected. None of these excitants can produce hay fever unless they find the nervous system prepared for them.",

When the time eame to study hay fever in this country it was found that the majority of eases observed here do not oceur at the same time of the year as do those seen in England. In England most eases of hay fever begin to show symptoms in June, some in May, and are well early in August. In this country most cases do not develop until about the middle of August and continue until a killing. frost eomes. The explanation for the differ- 
ence in seasonal appearance in the two countries is easily understood when we know that in England most of the cases are due to the pollen of the graminaceæ, monocotyledons, while in this country most cases are due to the pollen of the compositæ, dicotyledons. Quite naturally, there are some cases in this country due to the pollen of the graminaceæ and there may be cases in England due to the pollen of the compositæ. The common names of some of the common graminaceæ are as follows: wheat, rye, oats, timothy, June grass, red top, barley, foxtail, fescue, spear grass, sweet vernal grass, and ray grass. The common names of the compositæ most frequently inducing hay fever are the following: ragweed, oxeye daisy, common thistle, Canada thistle, blackeyed Susan, golden rod, and Indian corn. It should be clearly understood that the plants mentioned above are not the only ones whose pollen may induce hay fever. Hay fever, as we shall see later, is due to sensitization of the body cells to some foreign protein, and there is probably no plant whose pollen would not under certain conditions serve to sensitize man's body cells.

With the discovery and study of toxins and antitoxins, such as those of diphtheria and tetanus, the castor bean and the jequirity bean and the venoms of snakes, it occurred to some that the poison in pollen might be a toxin, and if this should prove to be the case, what could be more reasonable or more desirable than the preparation of a specific antitoxin? This theory has turned out to be all wrong, but the work to which it led has given us facts of fundamental importance in the study of the nature of infectious diseases. Dunbar, of Hamburg, undertook to prepare an antitoxin for hay fever. Quite naturally, the first step in this attempt was to ascertain the active constituent in the pollen and to prepare this in sufficient quantity and in suitable form to be employed in the immunization of animals in order to get the antitoxic serum. Roughly, pollen consists of protein and carbohydrate substances. It was soon demonstrated that the carbohydrates of the pollen are in no way concerned in the effects of pollen upon animals, including man. The active principles, so to speak, in the grains of pollen are its albuminous constituents. These may be extracted from the pollen grains by salt solutions which leave the carbohydrate and the husk of the pollen undissolved. It is not within the scope of this work to go minutely into the preparation or separation of the albuminous substances in pollen grains. It suffices to say that in these albuminous bodies lies the active constituent of the pollen. Is the protein in the pollen grain a toxin? A true toxin in solution is destroyed by a relatively low temperature; in other words, it is thermolabile. The pollen protein is thermostabile. When a true toxin is injected into an animal there is a period of incubation before there is any sign of its action; when the protein of pollen is injected into the body or applied to the mucous membrane, there is no period of incubation. A true toxin when injected in nonfatal, gradually 
increasing, doses, into a healthy animal stimulates the body cells to the production of antitoxin; there is no antitoxin produced when pollen albumin is injected into animals.

Dunbar treated horses over many months with gradually increased doses of pollen protein and, under the trade name of pollantin, placed on the market to be employed as an antitoxin the serum of the horses thus treated. It has been abundantly shown that this preparation is not specifically effective in the treatment of hay fever. A certain percentage of subjects treated with pollantin do improve, while many are not benefited and some are apparently quite harmed. Dunbar himself explains the harm done in some instances by supposing that the patients become sensitized to horse serum. This seems quite reasonable, inasmuch as Sewall has experimentally demonstrated that guinea pigs may be sensitized to horse serum by repeated application to the mucous membrane of the nose. Weichardt has prepared and placed on the market, under the trade name of graminol, another serum for the treatment of hay fever. This investigator supposes that cattle feeding on flowering grasses become immunized to the toxin believed to be in the pollen. Graminol is, therefore, nothing but ox serum taken from the animal after it has been feeding for weeks on timothy and other grasses. There is no proof that oxen in feeding on grasses do absorb the pollen any more than that the majority of people absorb pollen. In the second place, as we have seen, it is quite certain that the pollen protein is not a toxin. In the third place, it is still more certain that the serum of the ox which has fed upon grasses does not contain antitoxin; and in the fourth place, it has been demonstrated that graminol is not a specific in the treatment of hay fever. With all these facts before us, we must come to the conclusion that the protein present in pollen, whether it be from the graminaceæ or the compositæ, is not a toxin. Whether or not it is a poison will be discussed later.

Pollinosis; a Result of Protein Sensitization.-We are being forced more and more by recent investigations into the phenomena of infection to conclude that protein sensitization is an important factor in many infectious diseases. So long as our attention was confined to diseases caused by bacteria and other viruses we were quite naturally inclined to conclude that every infectious agent must, in order to be infectious, be possessed of the capability of growth and multiplication in the body. A study of pollinosis shows that this is not true. The pollen protein does not multiply after it finds its way into the body, but for a time a continuous supply is borne in with every inhalation and the function which, in ordinary bacterial diseases is performed by the invading organism itself, is now replaced by the continuous introduction of foreign protein. All men under like exposure take into their respiratory organs, under like conditions, like amounts of pollen. Most people digest this pollen while it lies on the mucous membrane of 
the upper respiratory passages. The pollen protein when digested by the secretions of the mucous membrane upon which it lies, is absorbed in the form of amino acids and has no harmful effect upon the body; indeed, the pollen protein supplies in this way an infinitesimal amount of food substance. When for any reason the digestion of the pollen on the mucous membrane of the upper respiratory passages is interfered with, the pollen protein, undigested and unchanged, is absorbed, passes into the blood, and after a time the body cells become sensitized to this protein and it is split up no longer outside the body on the mucous membrane of the respiratory passages, but now within the blood it undergoes parenteral instead of enteral digestion. The complete breaking down of the protein molecule into amino acids is not carried out and the protein poison is formed and causes the symptoms and lesions of pollinosis.

The individual who has developed hay fever is practically in the same condition as a guinea pig which has had one injection of egg-white or some other foreign protein. The man has been sensitized to pollen protein. We have seen that this protein is not a toxin, and there is no reason for believing it to be poisonous. Certainly, there is no more harmless substance than egg-white-no one would think of classing it among the poisons; but when egg-white has been introduced parenterally into a guinea pig that animal is sensitized to this protein, and when a subsequent injection of the same protein is given marked disturbances, and even death, follow. So far as we know there is only one case on record where an appreciable amount of pollen protein has been injected into a hay fever subject. Dunbar injected into his assistant, Praussnitz, a hay fever subject, a small amount of pollen protein. He describes the effects as follows:

"The results obtained were of extraordinary interest, but at the same time terrifying. When a small amount of the toxin was injeeted subcutaneously in the forearm, the first manifestation appeared in ten minutes and consisted of severe sneezing with plentiful secretion from the nasal mueous membranes and eonsiderable swelling of both nostrils; after 30 minutes a dry eough appeared with a slight tenacious expectoration, and at the same time the faee swelled and beeame very red and cyanotie. A marked injection of the conjunctivæ developed and later ehemosis. In both ears there was a feeling of tension objectively, however, no change could be pereeived in the tympanic membranes. One hour after the injection tormenting asthmatic disorders with audible stridor arose; an hour later an urtiearia-like eruption of large wheals appeared over the whole skin associated with violent itching; three hours after the injeetion the forearm began to swell. The edema spread during the following night to the whole arm. The edema of the arm, and a turgid appearance of the face remained for several days. All other objective phenomena had disappeared by the next morning. The temperature remained normal from the beginning. Nothing abnormal was found in the urine. For a week after the experiment the patient experienced a disturbing sensation of weakness and exhaustion, as well as oecasional attacks of palpitation of the heart. The same dose of pollen extract given to a normal person not subject to hay fever produced only a slight, somewhat itching eircumscribed edema around the place of injection." 
If there was nothing else in the literature bearing upon the subject, this experience alone would be quite suggestive at least, that hay fever is a condition of protein sensitization.

Why do some people become sensitized to pollen while other people do not? As already stated, we all inhale pollen when it is in the air, but under normal conditions the protein contained in this pollen is digested before absorption, and it is a well-known fact that digested proteins do not sensitize. One individual becomes sensitized or may become sensitized to pollen protein from any one of the following causes: In the first place, there may be some physical deformity in the nasal cavity, as an enlarged turbinated bone, which interferes with the normal digestion carried on by the secretions of these cavities. The inhaled pollen accumulates in pockets in the nasal cavity and not being readily digested, is gradually absorbed, taken into the blood, distributed throughout the body, and later sensitizes the body cells in general. It may be that without any physical obstruction, the quantity of pollen inhaled at any one time is too great to be digested even when the nasal secretions are acting normally. Again, in this case, undigested pollen protein is absorbed and sensitizes the body. It is a wellknown fact, demonstrated in the early work on protein sensitization by Arthus, that when proteins are absorbed through the skin there is more or less marked increase in the permeability of the tissue, and when subsequent injections are made in the same locality absorption goes on more rapidly; in other words, the permeability of the mucous membrane of the nasal cavities becomes abnormally great and subsequently it requires but a relatively small amount of pollen to be deposited upon these surfaces in order to be absorbed unchanged and lead to sensitization.

The explanation of protein sensitization as given by Vaughan, is as follows: When a foreign protein is carried into the blood and is distributed throughout the body it penetrates the body cells and leads these cells to produce a new specific ferment which digests the invading or foreign protein. This new function developed by the body cells may be permanent or it may be temporary. As long as it exists, whenever the cell subsequently comes in contact with this foreign protein the cell pours out its proteolytic ferment and splits up the foreign protein with the production of the protein poison. The symptoms of pollinosis are those induced by the reinjection of a given protein to which the animal has been sensitized. Some years ago Auer and Lewis in studying the phenomenon of protein sensitization suggested that it might lead to asthma. This has turned out to be true and has been confirmed by every one who has worked on the subject. We do not mean to say that every form of asthma is due to protein sensitization, but we do mean to say that the asthma of hay fever is a symptom of protein poisoning.

Pollen sensitization is not strictly specific, but belongs to what we now 
call a group reaction. An animal sensitized to one protein may be sensitized, though usually to a less degree, to a closely allied protein. A guinea pig sensitized to the protein of hemp seed is also sensitized to the protein of flaxseed; one sensitized to the protein of wheat is also sensitized, though to a less degree, to the protein of barley. According to Vaughan, the protein molecule consists of a primary group which he designates as the archon. Attached chemically to these molecular groups are secondary groups. The primary group is poisonous when stripped of its secondary attachments and is poisonous only when stripped of these attachments. In accordance with this view, which was promulgated nearly 20 years ago, it has been found that a hay fever-subject sensitized to ragweed is also sensitized, though possibly not to so high a degree, to other members of the compositæ; on the other hand, one sensitized to timothy is also sensitized to the pollen of other grasses.

The sensitizer may be any foreign protein. Sensitizers, for the most part, at least, are not in and of themselves poisonous. All proteins contain a poisonous group, it matters not whether they be bacterial, vegetable, or animal, and at the same time they contain sensitizing groups. The specificity of a protein lies in its sensitizing groups and not in its poisonous constituent. The sensitizing groups are just as numerous as the kinds of protein ; that is, they are innumerable, but the poison in each protein molecule, although there are probably as yet undiscovered chemical and physiologic differences, is in its gross physiologic effect the same.

It has been demonstrated that if the blood serum of a sensitized animal be transferred to a fresh animal, whether the two belong to the same species or not, sensitization is transferred along with the serum to the fresh animal. Acting on this principle, Koessler has passively sensitized guinea pigs with the blood serum of a hay fever subject. He says :

"From J. B., a patient who had severe asthmatic attacks due to hay fever, which in one season lasted for four weeks, we obtained a sufficient amount of blood to yield 20 c.c. of clear serum. Four guinea pigs were sensitized by the subcutaneous injection of 5 c.e. each and reinjected after 12 to 18 days intracardially with 1 c.c. of a solution of 1-10,000 ragweed pollen extract. Three of the four animals showed severe typical symptoms of anaphylaxis, but recovered. Control animals were not affected by the same dose of pollen extract. We must, therefore, conclude that the pigs had been sensitized with pollen protein which was present in the serum."'

According to White and Avery, the smallest sensitizing dose of pure crystallized edestin given intraperitoneally is $0.0001 \mathrm{mg}$. Koessler estimates that the amount of pollen protein inhaled per day in ragweed time is about $0.8 \mathrm{mg}$. Supposing that the sensitizing property of pollen protein is equivalent to that of edestin, it will be seen from these figures that the amount of pollen protein daily inhaled would be sufficient to sensitize several thousand guinea pigs, and there is no reason for supposing that man is sensitized with greater difficulty than the guinea pig. There can be no 
question about there being enough protein in pollen to sensitize man, provided that only a one-thousandth part of that inhaled is absorbed undigested. Rosenau and Anderson found 0.000001 c.c. of horse serum sufficient to sensitize guinea pigs, while Besredka places the minimum amount necessary to secure uniform results at 0.001 c.c. Taking any one of these estimates, it will be found that any individual inhaling air in the neighborhood of pollinating ragweed takes in enough pollen protein to thoroughly sensitize himself, provided this protein is absorbed undigested. Doerr and Russ place for proteins in general the minimum fatal dose on reinjection at a thousand times the minimum sensitizing dose. Vaughan and Wheeler found that in guinea pigs one-seventieth of the fatal dose employed in reinjection causes marked and even distressing symptoms. It follows from these figures that in the pollen inhaled daily there is enough protein to induce well marked symptoms, even when only a small part of that inhaled is absorbed unchanged.

Beard was right when he stated that a large number of substances may induce the symptoms which we classify as those of hay fever; he was wrong when he thought that the development of this disease is dependent upon some instability or abnormality of the nervous system. Any individual may be sensitized to pollen protein as well as to other proteins. There are people who show symptoms of pollinosis when they eat unpeeled peaches, and it has been shown that, in some instances at least, sensitization is due to the inhalation of the fuzz on the skin of the fruit. Some develop symptoms of pollinosis in the presence of withering flowers. Vaughan reports the case of a lady who never suffers from the symptoms of pollinosis in the presence of fresh flowers, roses for instance, but when these begin to wither. she is seriously affected. On a voyage to Europe friends supplied her stateroom with abundant roses. She developed no symptoms until these began to wither, which was about mid-ocean. Then she suffered an alarming attack of pollen asthma, which was speedily relieved when the roses were thrown overboard. The dust arising from sawing, planing, and working in certain soft woods, develops the symptoms of pollinosis. Pollinosis due to the inhalation of the hair and dandruff from horses is one of the most common forms of the disease. Some individuals are so speedily and characteristically affected that they can detect the presence of a cat, a guinea pig, or a rabbit, in the room even when the animal is not suspected and is hidden from sight. Bacteria, being protein bodies, may be inhaled, escape digestion on the mucous membranes of the upper respiratory passages, be absorbed, and sensitize the body cells. These bacteria are not necessarily pathogenic organisms. Sensitization with dead bacteria might result. The experiments of Sewall have proved that soluble proteins may sensitize and when absorbed into sensitized animals may produce perfect and typical cases of asthma. The existence of volatile sensitizers is still a 
matter of some doubt. Rosenau and Amoss sensitized guinea pigs by injecting into them water through which the exhaled air from men had been passed. Of 99 guinea pigs submitted to this test 26 manifested recognizable symptoms of shock and four of them died on the injection of human serum. These authors say :

"The logical conclusion from our results is that protein substances under certain circumstances may be volatile. It. seems unlikely that such a complex molecule should possess the power of passing into the air in a gaseous form. The volatility, however, now in question may resemble that solubility which deals with particles in suspension in a physiochemical state (colloidal suspension). The protein may simply be carried over in solution in the water vapor."

Later Rosenau announced that guinea pigs kept in stables with horses became sensitized to horse serum. Sewall was unable to confirm this statement, but did find that guinea pigs sensitized to horse serum do manifest symptoms of intoxication when placed in stables.

Koessler, after transferring pollen sensitization from a hay fever patient to guinea pigs, makes a statement concerning pollen protein sensitization which is so clear that at the risk of some repetition we quote:

"It is thus absolutely proved by rigid experiments that hay fever is a disease due to pollen protein sensitization and anaphylaxis and it is no longer permissible to speak of this established fact as a conception or hypothesis. On this basis not only do we eomprehend the pathogenicity of the pollen protein and the mechanism of reaction, but also the 'peculiar' individual disposition finds a satisfactory explanation. The pollen protein reaches at some season of the year the nasal mucous membranes of all persons. The human blood and all secretions of the mucous membranes of the body are digestive fluids and have normally very remarkable proteolytic properties. It has been known for a long time that the normal nasal secretion shows a decided baetericidal power; there is no doubt that this is due to proteolytic enzymes, whose presence has been experimentally established. This proteolytic enzyme of the nasal secretion in most people gradually splits the pollen like any other foreign protein, into harmless products, proteoses, and amino acids. This cleavage proceeds slowly step by step just as the cleavage of the protein material in the stomach. The poisonous group contained in every protein molecule is, therefore, at any one time, present only in small concentration, and since its diffusibility is low, it is rendered inert as cleavage proceeds. The absorption of protein through the nasal mucous membrane is under normal conditions exceedingly minute, practi. cally nil. Under various conditions, however, which interfere with the normal digestive function of the nasal mucosa, foreign proteins, e.g., pollen protein, will be resorbed in sufficient amount to lead to sensitization. Conditions of this kind are (1) a temporarily insufficient nasal secretion associated with a lowered quantity of normal proteolytic enzyme; (2) stenosis of the nasal canals through hypertrophied turbinates leading to excessive accumulation of inhaled matter and thus to increased resorption; (3) the accidental inhalation of an amount of pollen exceeding the protective function of the exposed mucous surface in the state of temporary nasal achylia mentioned under (1)."

It should be plainly understood that undigested, unaltered proteins may be absorbed from the alimentary tract quite as frequently as from the up- 
per respiratory tract. Protein sensitization, therefore, with all its symptomatology, may be due to the absorption of foods from the gastrointestinal tract. This is generally known as food sensitization and it has been found in the past few years that it is of frequent oceurrence. The most common sensitizer by way of the alimentary canal is egg-white, and instances of sensitization with this protein are eommon and sometimes very striking. Vaughan and Wheeler found that guinea pigs sensitized to egg-white lose their sensitization in about one year. They did find, however, and this is a matter of importance, that the continuance of the state of sensitization varies with the animal and with the sensitizer employed. There are people who cannot take any kind of food containing the minutest quantity of egg-white without being seriously disturbed. It has been demonstrated by laboratory experiments that when female guinea pigs are sensitized to eggwhite their young may, for a time at least, inherit this state of sensitization. It is quite certain that occasionally a child is born sensitized to eggwhite and the first time this food is given the child, more or less marked, sometimes alarming, symptoms speedily develop. Aceording to Vaughan's conception of sensitization, a body eell must be penetrated by the foreign protein and when this occurs there is a rearrangement of the atomic groups of the protein molecules in the cells; in other words, there is an alteration in the molecular structure of the protein constituting the cell. Some proteins in developing the speeific zymogen produce profound and lasting changes in the molecular structure, while the alterations induced by others are slighter and of temporary duration, the molecular structure soon returning to its original condition.

Alimentary sensitization, as well as that which results from penetration of the mucous membrane of the upper respiratory tract, may cause bronchial asthma. It is probably true that in the majority of instances of food sensitization the first passage of the unbroken protein through the alimentary wall into the blood is due to over eating. The child who gorges itself with some particular food is liable to pay for this in after life by an intolerance of that food. It is by no means uncommon to find persons who are sensitized simultaneously with several proteins. Talbot reports the case of a girl of seven years of age with frequent attacks of asthma who was found on the application of the skin test to be slightly sensitized to the proteins of wheat, rice, and peas, and more markedly sensitized to the proteins of rye, barley, sago, dog serum, hens' feathers, and chicken protein. Walker has reported on 400 cases of bronchial asthma which he has studied with reference to sensitization. So far as he can ascertain, nationality or race is not concerned. Oecupation does play a part; for instance, he finds that bakers frequently have bronchial asthma from the inhalation of flour. One man whose business it was to sift green coffee was sensitized to the proteins in the husk of this berry. Another who was a jewel polisher became sensi- 
tized to the protein in the dust from the boxwood with which he polished jewels. Asthma due to pollen sensitization is quite naturally seasonal and falls into two groups-estival and autumnal. That due to bacteria may prevail in winter as well as in summer. Of the 400 cases of asthma studied by Walker, 191, or forty-eight per cent, gave a positive skin test and were therefore sensitive to some protein. He found the relationship between the age of onset and the sensitization of the individual important. He says :

"Of the patients who began to have asthma under the age of two, 83 per cent were sensitive to some protein; of those whose onset was between two and five, 90 per cent were sensitive; of those beginning asthma between five and ten, 40 per cent were sensitive; of those beginning between ten and fifteen, 70 per cent were sensitive; of those having onset of asthma during the succeeding five-year periods between the ages of fifteen and thirty-five, 52 per cent were sensitive for each period; between the ages of thirty-five and fifty only 23 per cent were sensitive; and after the age of fifty no patients were sensitive. The above statements may be summarized as follows: 83 per cent of the patients who began to have asthma during infancy (under the age of two) were sensitive; during childhood or between the ages of two and fifteen, 66 per cent were sensitive; during young adult life or between the ages of fifteen and thirty-five, 52 per cent were sensitive; during middle life or between the ages of thirty-five and fifty, 23 per cent were sensitive; and none were sensitive when the age of onset of asthma was after fifty. Stated briefly, four-fifths of the patients who began asthma during infancy were sensitive; two-thirds who began during childhood were sensitive; one-half of those beginning asthma during young adult life were sensitive; one-fourth of those beginning asthma during adult life were sensitive; and none were sensitive that began asthma after the age of fifty. * * * It is noted that ninetcen patients, who began to have asthma under the age of two, were sensitive to animal hair proteins; of this number, ten were sensitive to the proteins of horse hair alone, one to cat hair alone, and the other eight patients were sensitive to the proteins of the hair of horse, cat and $\mathrm{dog}$, although they were more sensitive to the hair of horse than to the hair of cat or dog. Of the patients whose onset of asthma was between the ages of two and five and between five and ten, fourtecn and twelve patients, respectively, were sensitive to the proteins of animal hair, and in each instance eight of these patients were sensitive to horse hair proteins. Succeeding ages of onset of asthma show a gradual decrease in the number who were sensitive to animal hair protein. Sensitization to food proteins was by far most frequent among thosc patients who began to have asthma during infancy. Of the twenty-three patients, nine were sensitive to egg protein, eight to the cereal grains, and three to milk. Of the nine patients who began asthma between the ages of two and five and who were sensitive to food proteins, two were sensitive to egg, and five to cereal grain proteins. Succeding ages of onset of asthma show about a constant average of frequency in the sensitization to foods, but the frequency to egg, milk and cereal is much less than for other food proteins, such as fish, meat and potato."

So far as sensitization due to bacterial proteins is concerned, Walker found this to be about the same for all ages up to 40 years. More were found sensitive to the protein of Staphylococcus pyogenes aureus than to any other type of bacterial protein. In Walker's list, out of 92 patients who were sensitive to pollens, 17 were sensitive to the pollens of grasses, notably timothy, while 45 were sensitive to the pollens of the compositæ, 
notably the ragweed. The remaining 30 persons were sensitive to the pollens of both the grasses and the compositæ. Walker found sensitization to roses, red top, daisy, and golden rod, infrequent. He found multiple sensitization most frequent among those who began to have asthma during infancy. Vaughan reports the case of a lady who is most highly sensitized to clams. A single clam has caused in her more than once a severe attack of bronchial asthma and in one instance when she ate three clams she was unconscious for four or five hours. The same lady is sensitive to withering roses, and two grains of quinin administered to her will cause a scarlatinous rash which covers the entire body and which desquamates very much like the rash of scarlet fever. It is not infrequently found that persons sensitized through the alimentary tract are also sensitized through the upper respiratory tract.

Cutaneous Test.-The intradermal sensitization test, now so widely employed in tuberculosis, enables one to determine the protein to which a given individual is sensitized; in fact, the intradermal test is found to be too delicate. This means that most individuals have a slight degree of sensitization to a very great variety of proteins. Walker, who has had large experience, describes the test as he employs it as follows:

"A number of small cuts, each about one-eighth of an inch long, are made on the flexor surfaces of the forearm. The cuts are made with a sharp scalpel but are not deep enough to draw blood, although they do penetrate the skin. On each cut is placed a protein and to it is added a drop of tenth normal sodium hydroxide solution to dissolve the protein and to permit of its rapid absorption. At the end of a half hour the proteins are washed off and the reactions are noted, always comparing the inoculated cuts with normal controls on which no protein was placed. A positive reaction consists of a raised white elevation or urticarial wheal surrounding the cut. The smallest reaction that we call positive must measure $0.5 \mathrm{~cm}$. in diameter and any smaller reactions are called doubtful. Negative skin tests with protein rule out those proteins as a cause of asthma and all proteins which give a positive skin test should be suspected as a cause of asthma.',

It will be seen that this test here, as in tuberculosis, is one of sensitization; in other words, the epithelial cells of the skin have been sensitized to a given protein. That means that these cells supply, when stimulated by that protein, a ferment which splits up the foreign proteins and produces the free protein poison which acts upon the epithelial cells, injures them, and causes the reaction. It is as though the epithelial cells were able to pour out a ferment which would split sodium sulphate with the formation of free sulphuric acid, with the acid destroying the cells. As we have already stated, this is not strictly a specific reaction. When the cells are sensitized to a given protein they will split up not only that protein, but other biologically closely related proteins. When this test is made with the pollen of golden rod and is found to be positive, it does not mean that golden rod has furnished the pollen with which this individual has been 
sensitized. It simply means that some member of the order of compositæ has furnished the pollen with which the sensitization has been produced.

According to Goodale, cases of pollinosis in this country may be divided into three groups due to the season of their appearance. Cases occurring in April and May are generally due to the pollen of certain trees, such as the maple, willow, birch, or oak; those occurring in late May and through June are due to the grasses ; those occurring in August and September, constituting by far the greatest number, are due to the pollen of the compositæ, the most fruitful member of which is the ragweed. In applying the cutaneous test, the physician should be supplied with pollen from these three groups. When kept dry the pollen grains will preserve their proteins for many years, although the dryer they become the more difficult is it to extract the protein constituent. Goodale recommends the following method for the preparation of the protein from the pollen grains :

"One gram (of the pollen grain) is soaked in a small amount of normal salt solution for 48 hours and filtered. The filtrate, which contains albumin, proteose, and other proteins, is then treated with sufficient alcohol to bring the alcohol content up to 20 per cent, by which albumin is thrown down in the form of a floceulent precipitate. To this fluid is added enough 20 per cent aleohol to make a volume of 500 e.e. In this, which is termed our standard extract, we have the amount of protein extractable from one gram of pollen contained in 500 e.c. of 20 per cent aleohol. Dilutions of this are made in the preparation of 1-2000, 1-5000, and 1-50,000. Both in making these dilutions and in giving the injections, the bottle containing the material should be shaken suffieiently to distribute equally the suspended particles of albumin."

The intensity of the symptoms borne by a victim of pollinosis depends upon the abundance of the pollen in the air and the readiness with which the unbroken protein is absorbed and taken into the circulation. Even in the same neighborhood, in the different seasons or on different days during the same season, the number of pollen in the air varies greatly. Rain carries the pollen grains to the earth and at the same time, in part at least, washes out the proteins and these doubtlessly are largely absorbed in the earth. The hay fever sufferer may take advantage of the fact that the pollen proteins are readily soluble in water. Wet curtains hung at the windows will catch many pollen grains and dissolve out their protein constituents. Washed pollen grains do not sensitize. The sensitizer lies wholly in the soluble part of the grain.

Desensitization.-In the present state of our knowledge there is in reality no such thing as the desensitization of a sensitized animal. Such an animal owes its sensitization to rather radical changes in the molecular construction of the proteins of its cells. As we have seen, some proteins in sensitizing cause deep and permanent changes in the molecular structure of body cells, while others cause only slight and temporary effects. In either case science up to the present time knows no method by means 
of which a cell or a protein molecule which has developed a new function can be made to lose it. Notwithstanding these facts, we are justified, if we understand just what we mean, in speaking of desensitization. The proteolytic ferment poured from the body cells of a sensitized animal will act or fail to act according to the amount of the foreign protein present in the fluids of the body. A guinea pig which has been sensitized to eggwhite can be so treated that a reinjection of egg-white will have no apparent effect upon it. This condition we are in the habit of designating as a state of desensitization, but, that this is not true, properly speaking, can be shown by the fact that the blood taken from the animal in this condition and transferred to a fresh animal sensitizes that animal. The condition that we call desensitization is brought about by repeated injections of the protein body which has caused the sensitization; in other words, if we carefully increase the foreign protein in the blood of the sensitized animal up to a certain point the ferment does not act upon it. The action of a ferment upon a substrate depends, among other things, upon the proportion of the substrate in the menstruum. In dilute solution sugar easily undergoes fermentation. In concentrated solution sugar is to a certain extent a preservative. Large amounts of foreign protein injected into animals do not develop the state of sensitization either so quickly or so exquisitely as much smaller amounts. Vaughan has shown that the injection of the whites of three eggs at one dose into the peritoneal cavity of a rabbit has no effect upon the temperature of the animal, while the repeated injection of 0.05 c.c. of this albumin causes a rapid elevation in temperature and speedy death. The individual with incipient tuberculosis responds to the ophthalmic or cutaneous tests, but the advanced case of tuberculosis as a rule does not respond to either test. No perfectly satisfactory explanation has been given for these phenomena. Vaughan has suggested that when a large dose of egg-white is injected into an animal, no visible effect follows because the concentration of the foreign protein in the fluids of the body is such that the ferment will not act upon it; on the other hand, when 0.05 of a cubic centimeter of egg-white is injected, the surface tension of the foreign protein in the fluids of the body is multiplied greatly and the activity of the specific ferment is determined largely by the surface exposure of the substrate.

As we have seen, pollen protein has not proved to be a toxin and the proposed preparation and use of antitoxin have failed. The hay fever sufferer can in a great many instances, not in all, obtain partial relief by having the pollen protein to which he is sensitized injected in small increasing doses. The pollen protein employed in this way may be in solution, or it may be in suspension in 20 per cent alcohol as suggested by Goodale. The advantage in having the alcoholic suspension is that when application is made to the scarified skin the suspended protein is not so 
quickly diffused and the action is more local and more pronounced. Each specialist has his partieular method of desensitizing his hay fever patients. Generally, one begins with the subcutaneous injection of a few drops of an aqueous extract of pollen grains 1-50,000. After a few injections of this strength one advances to $1-5000$, later to $1-2000$, and so on. Better results are obtained when these injections are made some weeks before the annual onset of the symptoms. Practically in no instance is this method of treatment a permanent relief. It may enable the hay fever sufferer to pass through the season in greater comfort, but by the next season he is just as susceptible as he was before he received any treatment. We are aware of the fact that there have been reported exceptions, and we will admit that there may be exceptions-that there are some cases in which the condition of desensitization apparently extends through two or more years; but cases of this kind are so rare that they need not count. On this point Goodale makes the following statement:

"At the close of the hay fever season the patient may be assumed to have attained a relatively high degree of resistance to the pollen in question. With the omission of treatment the sensitization slowly and progressively returns, until at the beginning of the following year the skin tests show the same degree of intensity which they did originally. This fact is striking and has been brought out in all the eases under observation. An individual, for instance, who shows a No. 1 reaction will, year after year, show one of the same degree, and a No. 4 reaction will, each spring, appear as a No. 4."

Prevention.-Each sufferer from hay fever should ascertain by the cutaneous reaction to what pollen he has been sensitized. He should then take care not to be present in any locality during the pollination of that class of plants. The U. S. Public Health Service publishes yearly the time that each sensitizing plant is in pollination in each state in the union.

\section{Bibliography}

Beard, G. M.: Hay Fever or Summer Catarrh, New York, 1876.

Blackley, Charles H.: Experimental Researches on the Cause and Nature of Hay Fever, London, 1873; New Observations on Hay Fever, London, 1889; Brit. Med. Jour., 1898, i, 867.

Bostock, J.: Med. Chirur. Trans., London, 1819, x, 161.

Dunbar, W. P.: The Cause and Specific Treatment of Hay Fever, Munich, 1913.

Elliotson, J.: London Med. Gaz., 1831, viii, 411; Lancet, 1830-31, ii, 370.

Goodale J.: Boston Med. and Surg. Jour., elxxix, 293.

Gordon, W.: London Med. Gaz., 1829, iv, 266.

Koessler, K.: Forcheimer's Therapeusis of Internal Diseases, v, 671.

MacCulloch, J.: Essay on the Remittent and Intermittent Diseases, London, 1828, i, 394.

Walker, I.: Oxford Medicine, 1919, Part 1, pp. 115 and 143.

Wrman, M.: Autumnal Catarrh, New York, 1872. 


\section{CHAPTER IV}

\section{THE ACUTE CORYZAS}

\section{COMIMON COLDS; EPIDEMIC COUGHS}

Description.-Under these names we include certain frequent, exudative, eatarrhal conditions of the nasopharynx, pharynx, and larynx. The involvement may be strictly localized, as in the nasopharynx, or it may be more extensive, extending to all the above mentioned membranes and passing even into the larger bronchi. Although under ordinary conditions immense numbers of bacteria are inhaled through the nose and mouth, the upper respiratory passages are kept remarkably free from bacterial growths. In the nose, larynx, and bronchi, the action of the ciliated epithelium tends to throw back and to cast out all particulate substances. In all these tissues there is marked phagocytosis, which no doubt does much to keep these localities free from bacterial growth. Masses of foreign material are removed from the nose and nasopharynx by sneezing, from the pharynx by hawking, and from the larynx and upper bronchi by coughing. Epidemics in which one or all of these tissues may be involved are common. It is well known that a bad cold may go through a family, a school, a camp, or other assembly of human beings. In our cantonments during the World War we had instances almost innumerable of epidemic coughs and colds.

Bacteriology.-From time to time from the earliest beginnings of bacteriologic technic some one has reported the specific bacterial causal agent in the production of these epidemies. It would not be profitable to go into any large number of these reports. In the early eighties Pfeiffer discovered and named his Micrococcus catarrhalis, and as its name implies, he believed it to be the cause of catarrhal conditions. This organism has been subsequently studied in its etiologic relationship to bad colds, but except for its frequent presence on these mucous membranes there is no reason for believing that it is specifically related to catarrhal conditions. In 1894 Cautley discovered and described, under the name of B. coryzæ segmentosus, a diphtheroid bacillus found in seven out of eight cases of acute cold in the head. In 1906 Benham found the same bacillus in 20 out of 21 cases of coryza, but inasmuch as there were many other organisms present this investigator was careful not to ascribe specific properties to Cautley's organism. In 1902 Neumann concluded that colds are often caused by virulent diphtheria bacilli and pneumococci. The diphtheroid bacillus which he found was not virulent, but the pneumo- 
coccus was; and Neumann was inclined to attribute the pathogenic condition to one or both of these organisms. In 1913 Tunnicliff, working in Chicago, found a bacillus, to which she gave the name $B$. rhinitis, in 98 per cent of 82 cases of nasal cold and in 90 per cent of 20 cases of chronic rhinitis. By swabbing with cultures she produced in normal throats after from six to eight hours slight inflammation. The organisms were present in the nose and pharynx from 18 to 20 hours after inoculation and in some instances persisted in these localities for two or three days. Tunnicliff vaccinated two patients with this organism and thought she obtained from variations in the opsonic index some justification for claiming it as the causal agent. In 1907 Mathers, also working in Chicago, attributed an epidemic of acute infection of the upper respiratory passages to a virulent hemolytic streptococcus. A like finding has resulted from the work of Floyd in 1920. He says that almost invariably in acute pharyngitis the initial infection is produced by a member of the streptococcus group. Recently (1920) in the Boylston prize essay, Mudd, Grant, and Goldman, have collected the literature and discussed thoroughly the whole subject of infections in acute inflammations of the nose, pharynx, and tonsils. They say :

"That a state of confusion exists as to the etiology of the acute inflammations of the upper respiratory tract is evident upon casual excursion into the literature. A more patient search, however, reveals certain material, notably in the form of recent laboratory work, from which definite conclusions can be drawn, and in whose light the discussions of the 'common cold' in the current tests is clearly seen to be obsolete. The present dissertation is concerned with the etiology of the acute inflammations of the nose, pharynx, and fauces, particularly with reference to recent laboratory inquiries. * * * Since the early days of bacteriology, attempt has been made by the several proponents and opponents of the infectious theory to refer the 'common cold' on the one hand to the action of a specific microorganism, and on the other to various environmental and constitutional causes, such as exposure to changes of temperature, the 'lithemic diathesis,' and what not. Although perhaps laudable as philosophic ideals, such efforts to explain the many phenomena involved by a single cause are less deserving scientifically, and have met with just failure. The 'common cold' is, as a matter of fact, in most instances the result of a local infection, but there are many types of 'cold' and many infectious agents responsible for them; and the effect of various constitutional and environmental factors in determining infection is often of great importance. Furthermore there are many acute inflammations of the upper respiratory tract not primarily due to the local action of microorganisms, but rather the local expression of chemical or mechanical irritation, of thermal trauna, of nervous reflexes, of drug intoxication, of constitutional disease, or of anaphylaxis.",

A Filtrable Virus.-In 1914 Kruse took the nasal secretion from an assistant who was ill with coryza, diluted it with 15 times its volume of normal salt solution and passed it through a Berkefeld filter. The clear filtrate contained no bacteria so far as Kruse could ascertain, employing all known methods for their detection. A few drops of this filtrate placed 
on the nasal mucosa of 12 men produced in four an acute cold. The period of incubation varied from one to four days. The same investigator repeated the experiment and this time the filtrate from the nasal secretion was diluted 20 times. Thirty-six students were inoculated, forty-two per cent of whom developed symptoms of coryza within from one to four days.

In 1916 Foster repeated Kruse's experiments. He took the secretion from a member of his laboratory staff who had been ill with an acute coryza for two days and who complained of chilly sensations, sneezing, unilateral nasal stuffiness, dull frontal headache with a feeling of oppression over the eyes, impairment of smell and moderate aching pain in the extremities. This man showed the usual picture of an acute cold-injected conjunctivæ, considerable lacrimation, a copious, thin, mucoid nasal discharge which excoriated the upper lip and the alæ of the nose, and a very red, moist, swollen and boggy mucosa. The temperature was normal. Foster also took the secretion from his own nose in the first day of an acute cold and he obtained a third specimen from an employe in a large department store who had been ill for three days. These secretions were blown from the nose into sterile Petri dishes, mixed with 10 c.c. of sterile 0.8 per cent salt solution and carried to the laboratory in a sterile test tube. These mixed, diluted secretions were passed through a small Berkefeld $\mathrm{N}$ filter. Filtration was accelerated by suction from a Chapman water pump. The filtrates did not show any evidence of bacterial growth on human blood agar plates incubated at $37^{\circ} \mathrm{C}$. under either aerobic and anaerobic conditions. Into the nasal cavities of ten soldiers this filtrate was instilled. Nine out of the ten men inoculated showed symptoms of acute coryza in from eight to thirty hours.

"The first symptoms, as a rule, were dryness of the nose and throat and attacks of sneezing. These were followed in a few hours by nasal stuffiness and dull frontal headache with pain over the sinuses. Several of the men complained of alternate sensations of chilliness and flushing. There was copious rhinorrhea, usually on the second or third day in the majority of cases. Six of the men exhibited slight rise in temperature, from $99.2^{\circ}$ to $99.8^{\circ}$, and in these cases the pulse was accelerated. Tinnitus aurium and slight deafness were recorded in four instances; sore throat in five; cough in three, and aching pain in the back or extremities in three. Two of the men complained of parotid tenderness and difficulty in mastication; and, in another case a profuse crop of herpes labialis was noted. The duration of the symptoms varied from three to six days."

Although the filtered nasal secretion did not give bacterial growths, Foster found, under the dark-field microscope, myriads of extremely active, minute bodies occurring singly, in pairs, and in agglomerations of varying magnitude. Foster states his conclusions as follows:

"From the experimental evidence presented in this preliminary report, it seems logical to conclude that common colds-of a certain type at least-are infectious; that the causative virus occurs in the nasal secretions, and that this virus is capable 
of passing through a Berkefeld $\mathrm{N}$ filter which has proved impermeable to ordinary bacteria. The result of these observations as to the locus of the infective agent indicates that transmission of colds from the infected to others may occur through droplet infection or other means of direct or indirect contact. The cultural experiments suggest that, although its exact nature has not been proved, a virus from the nasal secretions of persons ill with acute colds has been cultivated in vitro, and that Berkefeld filtrates of subcultures of this virus are capable of producing symptoms of an acute cold in healthy persons. There are certain facts which favor such interpretation of the experimental results. The filtrable constituents of the original nasal secretions were in a dilution of 1:90,000 plus in the filtrates of subcultures which produced colds experimentally; and it is beyond the realm of imagination that any toxin, virus, or enzyme originally existing in the secretions could have been carried over mechanically and, in this high dilution, have exhibited pathogenicity. Such pathogenicity is possessed, so far as is known, only by organisms capable of growth and multiplication. Secondly, the eultures showed ehanges which ordinarily are interpreted as indicative of bacterial growth, and these changes were not observed in controls or in uninoculated tubes of medium incubated with the cultures. Again, the changes observed in the original cultures were intensified in successive subcultures, thus bearing an inverse relation to the dilution of the material originally introduced. This phenomenon frequently occurs during the continuous cultivation of known organisms as the result of acquired adaptability to the condition in an artificial medium. It is conceivable, if explanation of the changes is attempted from another point of view, that the cultural appearances described might be due to lysis of the tissue fragment (it should be stated that a small piece of sterile fresh rabbit kidney was placed in the bottom of each tube in the subcultures) with consequent clouding of the medium, this disintegration being induced by the action of proteolytic enzymes elaborated by an ultramicroscopic organism."

Although Foster's work was published in April, 1916, and announced as a preliminary report, we have been unable to find a subsequent communication from him concerning this matter; neither do we know of any other worker who has confirmed Foster's findings.

Influence of Chilling the Body Surface.-As the term "common cold" implies, it is a popular, well-nigh universal, belief, founded in many upon personal experience, that coryzas follow chilling of the body surface. In 1913 Hill and Muecke found that in a warm room, $80^{\circ} \mathrm{F}$., the mucous membrane of the upper respiratory passages flushes and swells, becomes turgid with blood and lymph and covered with a thick secretion. This causes a narrowing of the air passage, especially in case of a deflected - septum. The mucous membrane of the nose and throat, as the skin, is flushed and pours out a secretion in order to cool the body. When one in this condition passes from the warm to a cold atmosphere, the mucous membrane of the upper respiratory passages quickly becomes pale, owing to constriction of the blood vessels, but still remains swollen, as is shown by its appearance and by the fact that it pits deeply when touched with a probe. These investigators think that this condition of the mucous membrane facilitates local infection and accounts for one's susceptibility to 
common colds. In warm rooms, the turgidity of the mucous membrane may be avoided, provided the air in the room is kept in motion.

"Keeping the air in motion in erowded rooms by means of fans will not only keep the mucous membrane in better state, but also diminish the massiveness of the direct infection from mouth to mouth or nose to nose. We find that warm, dry air flushes the mucous membrane with blood, but produces less swelling and secretion than warm, moist air does. We find also that while convection heat, e.g., from an anthracite stove flushes and swells the nasal mucous membrane, radiant heat, e.g., from a modern gas fire, causes sweating from the skin and does not affect the nose. The cause of the headache and stuffiness felt in rooms heated by steam pipes, stoves, and hot air is due, we believe, to the swelling of the mucous membrane of the nose and its air sinuses. We find the change from convected heat to radiant heat relieves the stuffy feelings, and at the same time eauses the skin to become moist and the nose to return to the normal out-of-door appearance. As to the question of massive infection, this may be lessened greatly by inculcating the doctrine that those infected with colds and in the acute stage must sneeze and cough into handkerchiefs, and even speak with a paper held in front of the face. Or we can when interviewing those so infected protect ourselves from spray by holding a newspaper in front of our mouth and nose. If habits of this nature were established at the schools, great relief might be obtained from the present widespread discomfort and danger of epidemies of 'colds.' ,'

Mudd, Grant, and Goldman have shown by skilfully devised apparatus that chilling of the body surface causes reflex vasoconstriction and ischemia in the mucous membranes of the nasal cavity, postnasal space, palate, oropharynx, nasopharynx, and palatine tonsils. They say:

"It seems not improbable that the ischemia may be the means of lowering local resistance. In other instances the mechanism of Hill and Muecke, i.e., crowding in overheated places followed by emergence into a cold atmosphere is doubtless responsible for colds."

Conclusions.- In our opinion, the acute coryzas are most rationally explained on the ground that they are due to protein sensitization. They may be caused by any protein, living or dead, organized or unorganized, particulate or in solution. Omitting from consideration Foster's theoretical globoid bodies, suspected in his subcultures, his experiments and those of Kruse are most easily explained on the ground of protein sensitization. These experiments are practically duplications of the ophthalmic tuberculin test. A minute trace of tuberculin dropped into the eye of a patient in the early stages of tuberculosis causes an exudative inflammatory action. In all probability, Foster's soldiers had been previously sensitized to the same protein which existed in his nasal secretions and in those of his laboratory assistant. If this be true, it could not be otherwise than that an inflammatory reaction would result within a few hours after the application of this protein to the nasal mucous membrane of these soldiers. When the ophthalmic tuberculin test is made in one wholly free from tuberculosis, there is no reaction, but, if after a proper interval another 
application of the same material is made to the same eye, there is a reaction; in other words, the first application of tuberculin causes local sensitization of the cells with which it comes in contact. In our opinion, there can be no doubt that chilling the body increases liability to protein sensitization in the nasal cavity, because it interferes with the normal digestive processes in these cavities and at the same time increases the permeability of the mucous membrane. Some state that bad colds cannot be due to protein sensitization, because the skin reaction does not show that such sensitization exists. They admit that there are cases of coryza in which the skin reaction is positive, but in those cases in which the skin reaction is negative, they exclude the possibility of protein sensitization. This is due to a misconception of the fundamentals of protein sensitization. The skin reaction is positive when, and only when, there is a general sensitization of the body cells. In bad colds the sensitization is local. The individual free from tuberculosis, one of whose eyes has been artificially and locally sensitized with tuberculin, does not give the skin reaction with tuberculin; nor does the other eye give the reaction on the application of tuberculin. The sensitization is local. It is also stated that bad colds cannot be due to protein sensitization, because, in the first place, they may follow mechanical injuries to the nasal mucous membrane; for instance, from the inhalation of fine inorganic dust. Such inhalations wound the nasal mucous membrane and render the absorption of inhaled proteins easy. Furthermore, it is said that coryzas cannot be due to protein sensitization, because they result from the administration of certain medicinal agents, such as arsenic, mercury, quinin, iodid of potassium, etc. These are exactly the same agents that lead to general protein sensitization in many people, and if they ean produce general sensitization it is certainly not beyond the range of possibility that they may produce local sensitization. In Sewall's experiments on guinea pigs, to the nasal mucous membrane of which horse serum was applied, there occurred first a local protein sensitization and when administration was continued or the dose was increased sensitization became general. In our opinion, the fact that so many microorganisms have been believed to be the cause of acute coryzas is quite suggestive that any protein inhaled and, on account of an abnormal condition of the mucous membrane of the upper respiratory passages, absorbed unbroken, may eause local sensitization; and this means common colds, epidemic coughs, and acute coryzas, according to the locality and extent of the local sensitization.

\section{Bibliography}

Foster, George B.: Jour. Am. Med. Assn., April 15, 1916.

Hill, L., AND Muecke, F. F.: The Lancet, London, 1913, i, 1291.

KruSE, W.: München. med. Wehnschr., 1914, lxi, 1547.

Mudd, Stuart; Grant, S. B.; And Goldman, A.: Boylston Prize Essay for 1920; Jour. Lab. and Clin. Med., Jan. to Mar., 1921. 


\section{CHAPTER V \\ THE PNEUMONIAS PNEUMONIC FEVERS}

Description.-Under the general name of pneumonia, used in the singular, we include certain acute and subacute inflammatory diseases of the parenchyma of the lung. Pneumonia is not a specific disease in the sense that measles, scarlet fever, whooping cough, diphtheria, and typhoid fever are; i. e., pneumonia is not due to a specific virus or microorganism, but may be caused by any one of a number of bacteria which find their way into the lung tissue. It may be said that pneumonia may be caused by any microorganism which grows, multiplies, and sets up an inflammatory reaction in lung tissue. At present pneumonias are classified upon anatomic differences found in the lesions. In one form the inflammatory process involves entire lobes or the greater parts of lobes and the exudate poured out in the alveoli is largely fibrinous. This is called lobar, fibrinous, or eroupous pneumonia. In another form the inflammatory processes are most marked about the smaller bronchi and include lobules rather than entire lobes. The exudate into the alveoli consists of mucus and pus; in other words, the exudate is eatarrhal. This is known as lobular or bronchopneumonia. Some authorities make a third class, in which it is found that the inflammatory changes are most marked in the connective tissue constituting the framework of the lung. This is known as interstitial pneumonia.

All admit that the anatomic classification of the pneumonias is not altogether satisfactory. Two, or possibly three, forms may be found in the same lung after death. All attempts to satisfactorily connect the lesion with the etiologic agent have failed or at least have not been convincing. While some hold that the pneumococeus is at least the most frequent cause of lobar pneumonia and the streptococeus hemolyticus is the most common cause of bronchopneumonia, this contention has not been uniformly verified at autopsy. The same is true of the claim that influenzal pneumonia is always interstitial in type. While it does not fall into the province of the epidemiologist to go minutely into the lesions of any of the pneumonias, the above classification and its inadequacy must be borne in mind in the study of the transmission of all forms of pneumonia. It is highly desirable that a classification based on etiologic factors may result from further research. The time has not arrived, however, for dogmatic assertions in this field of investigation. 
History.-It is evident that Hippocrates was well acquainted with pneumonia. He devotes most of his discussion on this subject to treatment. In his introduction to the treatment, he says:

"If the fever be acute, and if there be pains on either side, or in both, and if expiration be attended with pain, if cough be present, and the sputa expectorated be of a blond or livid color, or likewise thin, frothy and florid, or having any other character different from the common, in such a case, the physician should proceed thus."

Elsewhere he says :

"Matter should be spit off easily and early in peripneumonia and pleurisies; the color of it should be a yellow well mixed with the spittle, or a concocted yellow matter that is tinged with some, but not too much blood;-if this happens in the beginning of the disease, it is very advantageous; but it is not so much to be depended upon after the seventh day. It is exceeding bad when there seems a great quantity, and rattling of matter, in the throat, and yet nothing is spit off. It is indeed, in all cases dangerous when nothing is expectorated; but that which is very viscid, small, and globular, or frothy, is unprofitable. The sincere yellow unmixed spitting is bad; when very bloody, or livid, it is dangerous; especially when this appearance is very early; but that which is quite black is worst of all; it is an ill sign also when it is very green. Whatever is spit up with great difficulty, violent cough, and no relief to the pain and oppression, shows the case to be bad."

We have introduced these quotations from Hippocrates to show that he knew and treated the diseases which we now designate under the term pneumonia. It may be well to add that this learned Greek connected the prevalence of the pneumonias with weather conditions, and in his aphorisms he states that cold northeasterly winds bring on disorders of the breast, sides, and lungs. Celsus and Galen followed the teachings of Hippocrates in their references to pneumonia.

During the dark ages there is no distinct reference to pneumonia so far as we know. In the fifteenth century pneumonia in some form seems to have been prevalent in Italy, as the following quotation from a book published by Savonarola in Venice in 1497 shows:

“In civitate nostra Paduae et Trevissi et Venetiae 1440 de mense Martii antea et post apparuit pleuresis mala, quae erat contagiosa, ex qua multi et plurimi moriebantur."

It is evident from this quotation that the author recognized the contagious character of the disease. It is not certain, however, with what form of malignant pleurisy or pneumonia he was dealing. It is possible that the disease described was the pulmonary form of the plague. There is further evidence that the contagious nature of pneumonia was recognized occasionally by early writers. In 1592 Dunns, an Englishmen, wrote that pneumonia, or, as he called it, peripneumonia, is a contagious disease, because he observed that it attacked many in the same family. In 1613 another Venetian, Ravicio, plainly stated that it constituted a malignant epidemic and was highly contagious. Again, we are not certain what 
form of pneumonia these authors were observing and describing. As we come to later dates in history, we are convinced that pneumonia was observed not only in life, but that the lesions after death were investigated. In 1761 Morgagni called attention to the liver-like condition of the lungs after death, and thus gave rise to the term hepatization. In 1779 Pirri, in an epidemic at Rome, evidently autopsied some of his patients and states that the lungs were degenerated and liver-like in their hardness.

In his great work on Geographical and Historical Pathology, Hirsch fills pages with data concerning epidemics of pneumonia. In the first edition of this work the author gives abundant evidence of the epidemic character of pneumonia, and is apparently disappointed that so little attention is paid to this subject, for he begins his section in the second edition as follows :

"Notwithstanding my detailed handling of the matter in the first edition of this work, it is only of recent years that the subject has received any considerable degree of attention; and even now it is discussed for the most part without regard to the past and with a one-sided preference for contemporary observations. I have judged it incumbent on me, therefore, to go through the whole of the published facts once more, so as to sketch the history of the epidemies of pneumonia as comprehensibly as may be.",

We must refer those readers who are interested in this subject to Hirsch's book for the numerous epidemics of pneumonia which have prevailed in both the old and the new world during the sixteenth, seventeenth, eighteenth, and a greater part of the nineteenth centuries. It is rather strange that with all the literature on this subject at our command we should occasionally, even now, hear surprise expressed at the statement that pneumonia is contagious and that it frequently manifests its infectious character in deadly epidemics. Pneumonia is endemic throughout the greater part of the world, certainly throughout temperate and tropical zones. In making this statement, we are not including those pneumonias which arise in the course of certain specific infections, such as the plague, typhoid fever, and tuberculosis.

Concerning epidemics in this country during the eighteenth century, Webster writes :

"In October, 1712, commenced a niortal sickness in the town of Waterbury in Connecticut, which raged for 11 months. It. was so general that nurses could scarcely be found to tend the sick. What the disease was, I am not informed; but not improbably it was that species of putrid pleurisy, which has so often made dreadful havoc in America.",

With reference to the same epidemic, Trumbull, in his History of Connecticut, says :

"In 1712, on the 15th of October, began a great sickness in the town (Waterbury) which continued until the 12 th of September, 1713, and was so general that 
there were scarcely a sufficient number well to attend the sick, and bury the dead. Between 20 and 30 persons died of the sickness."

We learn from the same authority that Waterbury was vested with town privileges in 1686 and that the number of freeholders at that time was about 28.

Webster speaks of a malignant pleurisy which prevailed with great mortality in Hartford in the winter of 1719-1720. This epidemic apparently spread widely throughout the colonies and is reported to have practically depopulated a then thriving village in Delaware known as Duck Creek. In 1743 an epidemic known as catarrh, possibly a form of influenza, prevailed in Europe and reached this country, there having died in the City of New York from July 25 to October 22, 217 out of a total population of about 7,000. Of this epidemic in England, Huxham writes:

"I well remember the catarhal fever, which spread through all Europe, under the name of influenza in the spring of 1743 , frequently becoming pleuritic or peripneumonic."

In 1753 the small town of Holliston in Massachusetts lost 43 of its citizens.

"The disease began with a violent pain in the breast, or side, not often in the head; then suceeeded a high fever, but without delirium. The critical days were the third, fourth, fifth, or sixth. Some of the patients appeared to be strangled to death. The town contained no more than 80 families."

In 1761 influenza spread over the northern parts of America, and Tufts has left us the following description of it as seen in Massachusetts :

"The distemper began in April, and in May ran into a malignant fever, which proved fatal to aged people. It spread over the whole country and the West India Islands. It began with a severe pain in the head and limbs, a sensation of sickness, shiverings succeeded by great heat, running at the nose, and a troublesome cough. It continued for eight or ten days, and generally terminated by sweating. In May, the aged who had before escaped, were seized with an affection like a slight cold; this, in a day or two, was followed by great prostration of strength, a cough, labor of breathing, pains about the breast, precordia, and in the limbs, but not acute. The countenance betrayed no great marks of febrile heat. The matter expectorated was thin, but slimy. As the disease advanced the difficulty of breathing inereased; the expectoration was more difficult; the matter thrown off more viscid; at length the lungs appeared to be so loaded with tenacious matter, that no efforts could dislodge it, and the patient sunk under it."

The following quotation from Webster describes what appears to have been an epidemic of influenza followed by mild pneumonias:

"In the spring of 1781 prevailed the influenza, or epidemic eatarrh. It began with a severe pain in the head, prostration of strength, coldness and chills, the pulse not quick nor tense. The pain in the head lasted about 24 hours, and was succeeded by a pain in the side, not pointed nor acute, extending to the hips, ac. companied with a soreness, and resembling a rheumatic pain. The cough was trouble- 
some, and full, and the matter discharged of the glandular kind, not well concocted. Respiration was difficult, and a considerable defluxion on the lungs. In a few cases, the disorder terminated in seven or eight days; but usually not till the thirteenth or fourteenth; although the patient was seldom confined to his bed. The disease left a soreness and weakness in the side, which continued after the strength was recovered. Venesection had little effect on the pain in the side. Epispastics applied to the part gave relief. The disorder was seldom fatal, but its effects were very visible in the multiplied cases of pulmonary consumption, in the following year."

In 1789 there was an epidemic of influenza followed by more or less pneumonia and which was believed to have come from Canada. Dr. Rush thought it was brought to Philadelphia by Members of Congress returning from New England and New York, it having appeared in New York City in September and in Philadelphia in October. Of this epidemic Webster writes :

"This disease pervaded the wilderness and seized the Indians-it spread over the ocean and attacked seamen a hundred leagues from land, and as to infection, entirely insulated-it appeared in the West Indies nearly at the time it did in the northern states. It overspread America, from the 15th to the 45th degree of latitude in about six or eight weeks; and how much farther it extended, I am not informed.'

This epidemic was followed by a great secondary wave in 1790 and in the northern states was more violent than during the preceding autumn.

In 1757 Sauvages described, under the name "bilious putrid pleuroperipneumonia," a recurrent epidemic observed among soldiers at Valenciennes. This disease was marked by severe pain and oppression in the chest; weakness ; small, feeble, rapid pulse ; marked dyspnea ; violent cough, sometimes dry; viscid, tenacious, yellowish sputa, expectorated with difficulty; coughing, sometimes followed by nausea and vomiting of bilious material; dry, coated tongue; light headache, increased by coughing, and in rare instances mild delirium. At autopsy suppurative changes may be found in all parts of the lungs; the thorax often contains either pure or gelatinous (fibrinous) serum; in some instances there is an exudate in the pericardium; the pleuræ may be found to be adherent to the walls of the chest; not rarely there are coagula in the heart.

A notable epidemic of pneumonia made its appearance among U. $S$. soldiers in the war with England in 1812. It was believed that this epidemic originated in Canada, which was invaded by our soldiers. We fail to find sufficient data to justify this claim. It is certain that the epidemic was first reported in New York State. Apparently it prevailed in the state, and especially at Albany, before it reached New York City. It gradually spread over the whole country and caused a marked increase in the death rate. Hosack, then Professor of Internal Medicine in the University of New. York, adopted the above given description of Sauvages as correctly describing the symptoms and lesions observed in the American epidemic. Low, who studied this epidemic at Albany in October, 1812, wrote as follows: 
"The onset is with a chill, prostration and a feeble pulse, horror and distress from pain in the head, back, and extremities. The tongue is furred, the breathing insupportably laborious even when the pain in the chest is not severe, and every muscle concerned in this function ceases to act until goaded on by volition. When the pain is acute, the anguish is inexpressible. Every cell in the lung seems infarcted and weighed down by the load. Restlessness, distressing dreams, sudden frights, and starting from sleep attend most cases. Many are unable to endure the horizontal posture from a sense of suffocation which attends that position. Coma and delirium ferox are frequent symptoms and there are aphthae and ulcers in the throat in some cases. The disease sometimes terminates in scarlet fever, measles, or in an anomalous rash, all of which prevailed, as was the case in other countries where the same epidemic has raged. Morgagni, Coiterus, and Wierus described the same in Italy and Germany, Cleghorn in Minorca, Sydenham, Huxham, and Home in England.',

From the above quotation it would appear that scarlet fever and measles followed the pncumonias, but Mann, deseribing the disease as he saw it among soldiers at Plattsburg, says that the pneumonia followed the measles. MacCallum has reviewed the literature of the epidemic of 1812 , and concludes :

"Nowhere are the pulmonary changes minutely described, but it is evident from the occasional clear description of definite hepatization and the vague description in other cases of congestion alternating with abscesses or with a form of consolidation from which one could squeeze pus from the bronchi that most of it perhaps was not definite lobar pneumonia but probably a form of bronchopneumonia with extensive pleural exudate."

The progress of this epidemic through the country was slow. It apparently disappeared from New England and New York in 1815, but was reported in certain parts of the South during the following ten years.

During our Civil War the morbidity and mortality rates from acute respiratory diseases, principally pneumonia, were high but received comparatively little attention because they were overshadowed by the greater prevalence and higher mortality of typhoid fever and dysentery. Pneumonia was much more prevalent among the Confederate than among the Federal troops. Among the former the annual pneumonia morbidity was 103 per 1,000, while among the white troops in the Federal Army it was 34. The morbidity from acute bronchitis and catarrhs among Confederate troops was 415 annually per 1,000 as against 192 in the Union ranks. The Confederate death rate from pneumonia was 20.6 per 1,000 , while among the white troops of the North it was 7.8. The average annual death rate from pneumonia among southern prisoners in the northern camps was 59.9 per 1,000, while the annual rate from the same cause at Andersonville was 27.4. Taking the two races that composed the Union Army it is found that the average annual morbidity from pneumonia among the white troops was 27.8 and the death rate 6.21. Among colored troops the annual morbidity was 87 and the mortality 27.29. It appears from the above figures that the mortality from pneumonia among Confederate soldiers 
(all white) was 20.6 per 1,000, while among the colored troops in the Union Army it was 27.29. It follows from these figures that the highest mortality from pneumonia among all troops in the Civil War was among the negro soldiers; next came the southern white soldier, and the least mortality was among the northern white soldiers. It is worthy of record that measles was also much more common among southern than northern troops; indeed, the wide prevalence of measles impeded to a marked extent the mobilization of the Confederate Army. It so diminished the effectiveness of the troops and proved so fatal in camps that companies, battalions, and whole regiments had to be disbanded for a time and the men sent home.

During the World War pneumonia from the beginning to the end continued the most potent cause of death. Taking the calendar year of 1917 there were in our Army 8,479 eases of pneumonia with 952 deaths, a fatality of 11.2 per cent. It will be understood that mobilization of the new army did not begin until October, 1917. During the winter months of 1917-1918 (September 29, 1917 to March 29, 1918) the cases numbered 13,393 with 3,110 deaths, a fatality of 23.1 per cent. During the summer months of 1918 (April 5 to August 30) the total cases of pneumonia were 8,912 with 1,679 deaths, a fatality of 18.8 per cent. During the autumn months of 1918 (the influenza period) the number of eases of pneumonia was 61,197 with 21,053 deaths, a fatality of 34.4 per cent.

During the fall and winter of 1917-1918 the number of deaths from pneumonia in our cantonments was twelve times as great as in the same age group of the civil population of this country. Only one other disease, meningitis, showed a higher case mortality than pneumonia, but the cases of pneumonia vastly outnumbered those of meningitis. So far did pneumonia in mortality overshadow all other diseases that the history of pneumonia is the medical history of our cantonments.

The method of mobilization employed in 1917 gave excellent opportunity for the study of some epidemiologic problems concerned with pneumonia. Individual camps were filled with men from certain sections of the country and this furnished opportunity for the study of the disease in the different eamps and at the same time among individuals from the same section. These facts are so important that we give herewith the name, site, and source of troops of the large cantonments (Table I).

It must be understood that at present we are confining our study to the prevalence of pneumonia in the above mentioned camps for the time indicated. It is evident that there were wide variations in morbidity among these camps. These variations both in morbidity and mortality are shown in Table II.

Why should 27,000 young men coming from Texas and Oklahoma anid encamped at Fort Worth, Tex., have an annual morbidity rate of 96 and 
TABLE I

Location of National Guard and National Army Camps, together with the States from Which Men Are Drawn, October, 1917, to March, 1918

\begin{tabular}{|c|c|c|}
\hline CAMP & SITE & SOURCE OF TROOPS \\
\hline \multicolumn{3}{|c|}{ National Guard } \\
\hline Beauregard & Alexandria, La. & Arkansas, Louisiana, Mississippi \\
\hline Bowie & Ft. Worth, Tex. & Oklahoma, Texas \\
\hline Cody & Deming, N. M. & Iowa, Minnesota, Nebraska, South Dakota \\
\hline Doniphan & Ft. Sill, Okla. & Kransas, Missouri \\
\hline Hancock & Augusta, Ga. & Penṇsylvania \\
\hline Kearny & Linda Vista, Calif. & $\begin{array}{l}\text { Arizona, California, Colorado, New Mexico, } \\
\text { Utah }\end{array}$ \\
\hline Logan & Houston, Tex. & Illinois \\
\hline MeClellan & Anniston, Ala. & $\begin{array}{l}\text { Delaware, District of Columbia, Maryland, } \\
\text { New Jersey, Virginia }\end{array}$ \\
\hline Serier & Greenville, S. C. & North Carolina, South Carolina, Tennessee \\
\hline Shelby & Hattiesburg, Miss. & Indiana, Kentucky, West Virginia \\
\hline Sheridan & Montgomery, Ala. & Ohio \\
\hline Wadsworth & Spartanburg, S. C. & New York \\
\hline Wheeler & Macon, Ga. & Alabama, Florida, Georgia \\
\hline \multicolumn{3}{|c|}{ National Army } \\
\hline Custer & Battle Creek, Mich. & Michigan, Wisconsin \\
\hline Devens & Ayer, Mass. & $\begin{array}{l}\text { Maine, New Hampshire, Vermont, Massachu- } \\
\text { setts, Rhode Island, Connecticut, New York }\end{array}$ \\
\hline Dix & Wrightstown, N. J. & Delaware, New Jersey, New York \\
\hline Dodge & Des Moines, Iowa & Illinois, Iowa, Minnesota, North Dakota \\
\hline Funston & Ft. Riley, Kan. & $\begin{array}{l}\text { Arizona, Colorado, Kansas, Missouri, Nebraska, } \\
\text { New Mexico, South Dakota }\end{array}$ \\
\hline Gordon* & Atlanta, Ga. & Alabama, Georgia, Tennessee \\
\hline Grant & Rockford, Ill. & Illinois, Wisconsin \\
\hline Jackson & Columbia, S. C. & Florida, North Carolina, South Carolina \\
\hline Lee & Petersburg, Va. & Pennsylrania, Virginia, West Virginia \\
\hline Lewis & American Lake, Wash. & $\begin{array}{l}\text { Alaska, California, Idaho, Montana, Nevada, } \\
\text { Oregon, Utah, Washington, Wyoming }\end{array}$ \\
\hline Meade & Annapolis Junetion, Md. & District of Columbia, Maryland, Pennsylvania \\
\hline Pike & Little Rock, Ark. & Alabama, Arkansas, Louisiana, Mississippi \\
\hline Sherman & Chillicothe, Ohio & Ohio \\
\hline Taylor & Louisville, Ky. & Illinois, Indiana, Kentucky \\
\hline Travis & Ft. Sam Houston, Tex. & Oklahoma, Texas \\
\hline Upton & Yaphank, L. I., N. Y. & New York \\
\hline
\end{tabular}

"This camp was occupied by the troops indicated less than two months, when these troops were sent to Wheeler and replaced at Gordon by draft men from many states.

a death rate of 20 , while the same number of men of the same ages from Pennsylvania encamped at Augusta, Ga., have from the same disease a morbidity rate of 6.7 and a death rate of 1.1 ? Comparisons might be made between any two camps in the above list and the same question might be asked. Are the young men from Texas and Oklahoma phys- 
TABLE II

Sickness and Deaths from Pneumonia in Army Camps

Six MON'THs' Period

September 29, 1917, то Мarch 29, 1918

\begin{tabular}{lccc}
\hline \hline & & \multicolumn{2}{c}{ ANNUAL RATE PER 1,000} \\
CAMP & ARMY & MORBIDITY RATE & DEATH RATE \\
\hline Bowie & N. G. & 96 & 20.0 \\
Wheeler & N. A. & 95 & 23.6 \\
Travis & N. A. & 78 & 10.6 \\
Pike & N. A. & 63 & 24.9 \\
Cody & N. G. & 52 & 9.7 \\
Beauregard & N. G. & 42 & 15.0 \\
Taylor & N. A. & 37 & 5.4 \\
Sevier & N. G. & 36 & 11.5 \\
Jackson & N. A. & 36 & 10.7 \\
Doniphan & N. G. & 33 & 9.0 \\
Dodge & N. A. & 29 & 5.3 \\
Funston & N. A. & 24 & 10.5 \\
Kearny & N. G. & 24 & 4.4 \\
Lee & N. A. & 24 & 5.5 \\
Shelby & N. G. & 21 & 4.7 \\
Meade & N. A. & 18 & 2.6 \\
Logan & N. G. & 16 & 1.0 \\
Gordon & N. A. & 15 & 5.3 \\
Sherman & N. A. & 15 & 2.5 \\
Upton & N. A. & 15 & 3.6 \\
Grant & N. A. & 14 & 1.5 \\
Lewis & N. A. & 11 & 1.5 \\
Devens & N. A. & 9.8 & 2.0 \\
McClellan & N. G. & 9.6 & 1.1 \\
Sheridan & N. G. & 9.3 & 1.7 \\
Wadsworth & N. G. & 9.1 & 1.1 \\
Dix & N. A. & 8.0 & 1.5 \\
Custer & N. A. & 7.0 & 1.5 \\
Hancock & N. G. & 6.7 & \\
& & & A. \\
\hline
\end{tabular}

ically inferior to those from Pennsylvania? This question is answered most positively in the negative by the findings of the draft boards. Pennsylvania demonstrated the physical inferiority of its young men by the high percentage of rejections. It was not, therefore, physical inferiority which led to the higher morbidity and mortality from pneumonia among young men born and reared in Texas and Oklahoma. The difference cannot be explained by change in accustomed climate. The Texas and Oklahoma boys were on their native heath and breathing their accustomed air at Fort Worth, while the Pennsylvania men had been transferred from their native state to Georgia. Since neither Texas nor Oklahoma, or any eity in either, was in the registration area in 1917, we 


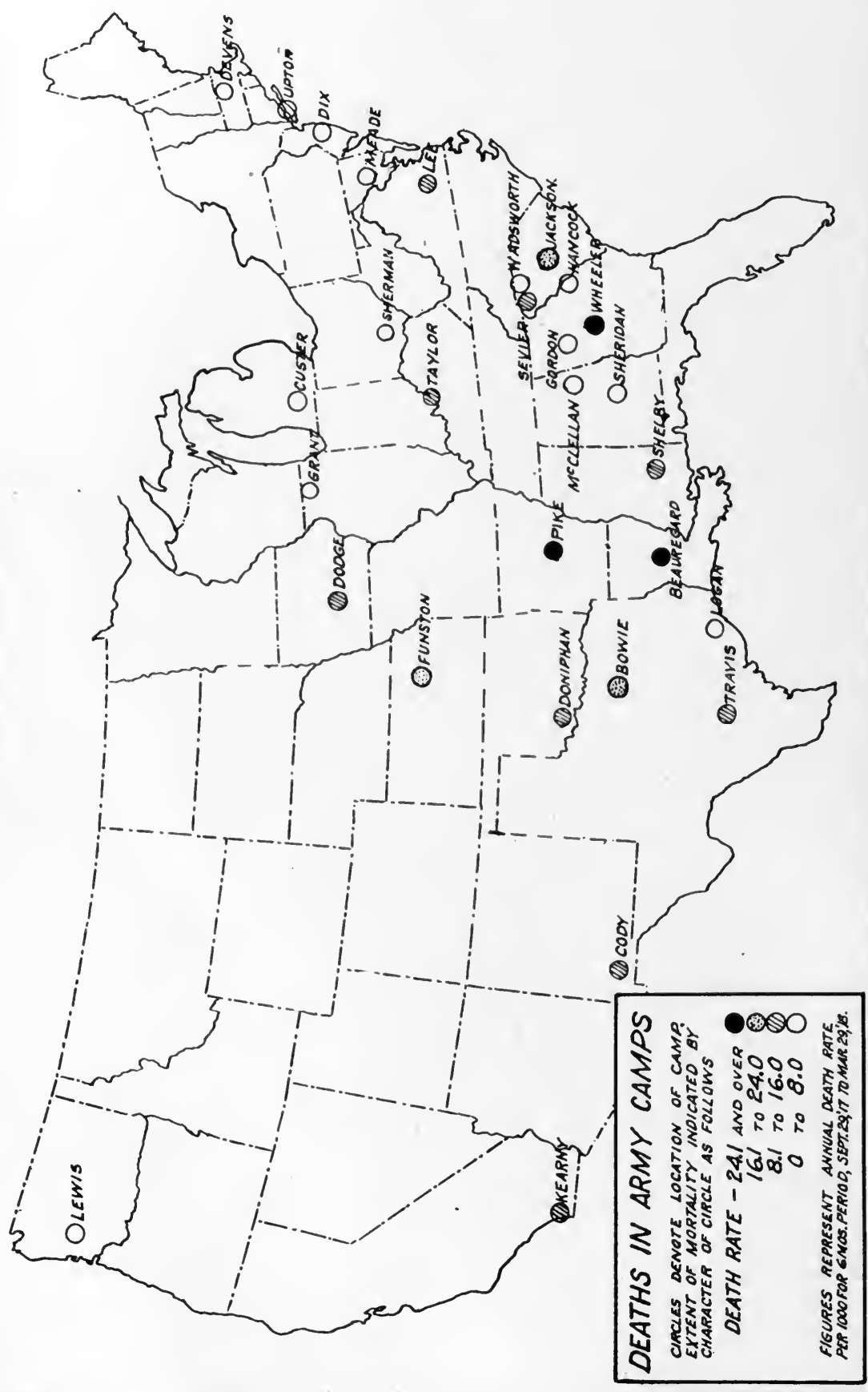




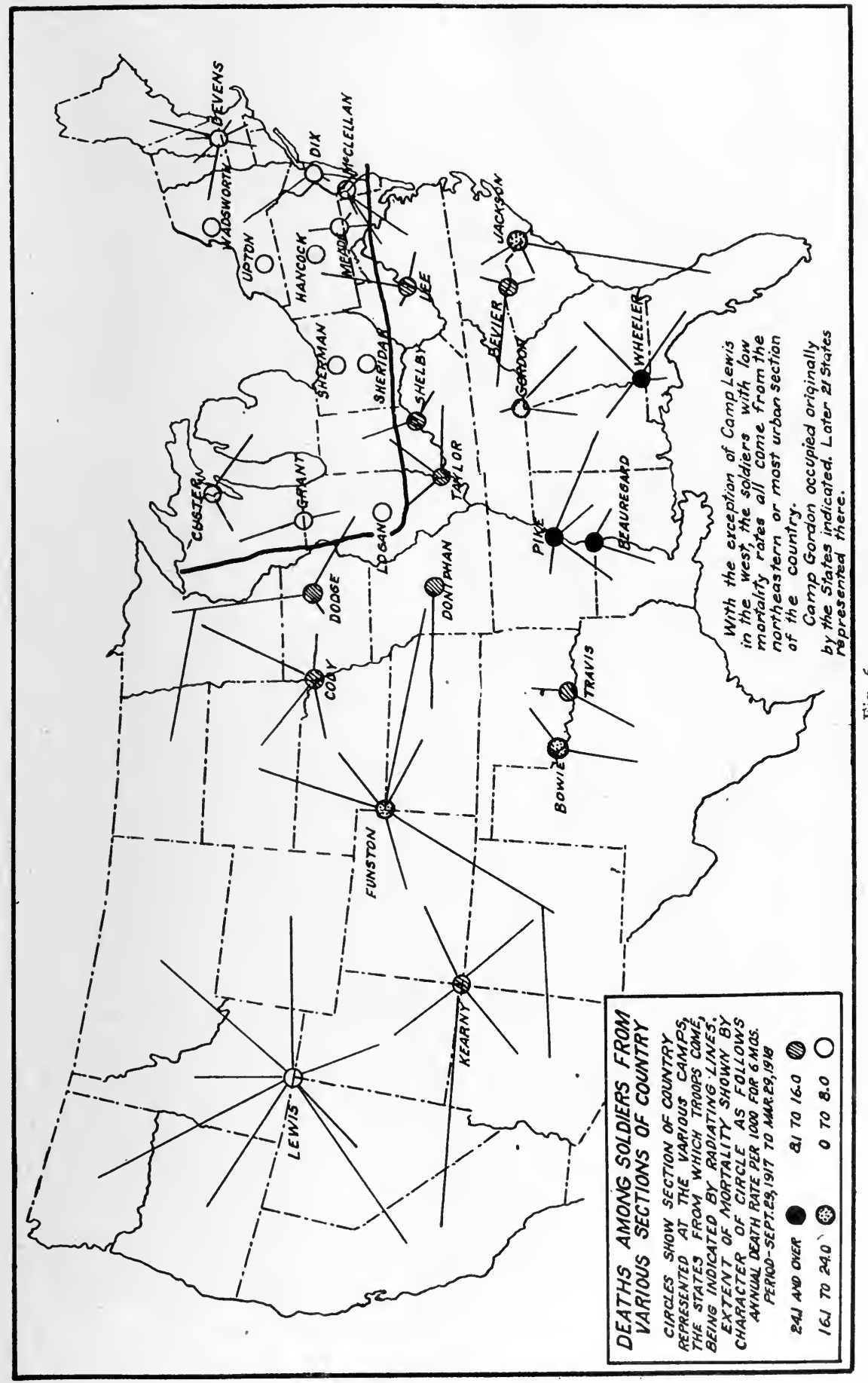


have no means of ascertaining the relative death rates in eivil life from this disease in the two sections. We do know, however, that the death rate from pneumonia in the State of Pennsylvania in 1917 was much higher than it was in the same year from the same disease in South Carolina, Tennessee, or Virginia, and when the death rate from pneumonia among the whites is compared with that in Pennsylvania the former is about one-half the latter. Furthermore, we learn from the mortality statistics that the death rate from pneumonia in Pittsburgh in 1917 was much higher than it was among the white population of any southern city in the registration area. It seems, therefore, that we are justified in concluding that in peace times both the morbidity and mortality rates from pneumonia are much higher in Pennsylvania than in Oklahoma and Texas.

The winter of 1917-1918 east of the Rocky Mountains was the coldest in 46 years. January, 1918, at San Antonio, Tex., was the coldest month in that region known, but it was far colder during the winter of 19171918 at Devens, Custer, Dodge, and other northern eamps than it was at Bowie, and still the latter exceeded all others in both morbidity and mortality from pneumonia. We must conclude, therefore, that while the unusually low temperature of that winter might have had, and probably did have, some influence upon morbidity and mortality from pneumonia in all camps, both North and South, it cannot account for the great. difference between the camps. It is possible that southern men were affected more by the unusual cold than were the northern men; indeed, we think it more than probable that the unusual cold of the winter of 1917-1918 was a factor in the wide prevalence and in the high morbidity and mortality in our cantonments, especially among southern troops. In a later section we hope to discuss in some detail the relation between pneumonia and the weather.

If we take a map of the United States and study the location of the camps with reference to the morbidity and mortality from pneumonia we find good and bad camps in the same geographical area. In the section covered by Georgia and South Carolina, we find Wheeler with an annual death rate per 1,000 from pneumonia of 23.6; Jackson with 10.7; Hancock with 1.1, and Wadsworth with 1.1. The great difference in morbidity and mortality from pneumonia in Wheeler and Jackson on the one hand and Hancock and Wadsworth on the other can hardly be explained by difference in temperature or any other climatic conditions. If we take a map of the United States and locate the camps not where they were but each near the center of the area from which it drew its population, we shall be struck by the fact that all the camps, with possibly two exceptions, having a death rate of less than 8 per 1,000 , are now in the same area. This area is that part of the United States which 


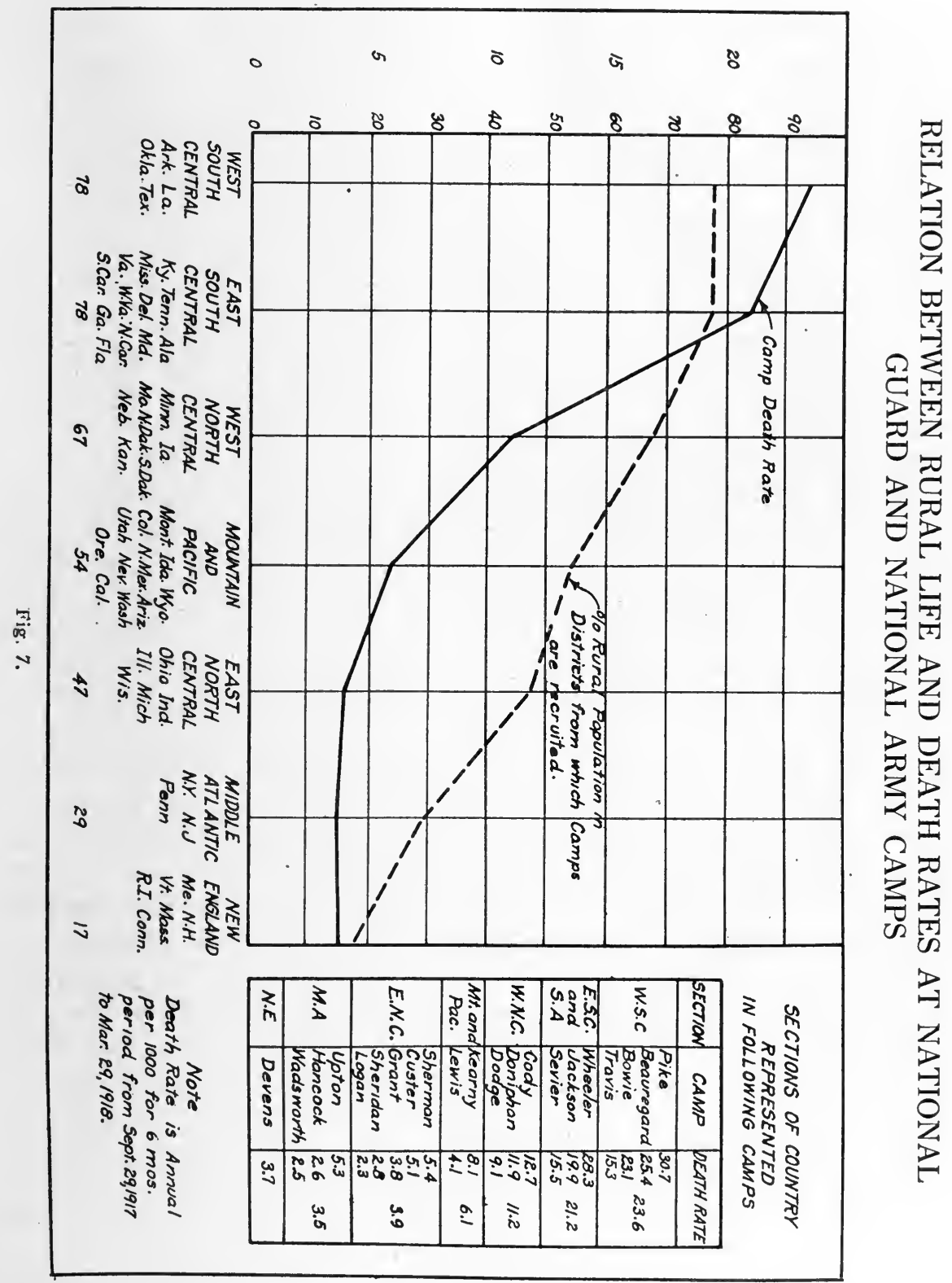

lies east of the Mississippi and north of the Ohio and Potomac Rivers. There is only one camp with an annual death rate less than 8 per 1,000 left in the southern states, and that is Gordon. We have called attention to the fact that, while Gordon was intended for draft men from Alabama, Georgia, and Tennessee, these men remained at Gordon less than two months and were replaced by draft men from many states, especially 
from the northeastern part of the country. The only other low death rate camp is Lewis, situated in the State of Washington. It is evident that in the winter of 1917-1918 both morbidity and mortality from pneumonia were much higher among southern soldiers than among those who came from the region east of the Mississippi and north of the Ohio and Potomac Rivers.

In 1917 the death rate from all forms of pneumonia for both sexes and races and all ages in North Carolina was 107.3 per 100,000. Among the white population of that state for both sexes and all ages it was 87.8, while among the colored population for both sexes and all ages it was 150.1. During the winter of 1917-1918 the mortality rate for pneumonia among southern boys from 21 to 31 years of age, selected on account of their physical fitness, was at Wheeler 23.6 per 1,000, which would be 2,360 per 100,000. In 1917 the death rate from pneumonia among both sexes and all ages in the State of Pennsylvania was 188.6 per 100,000, while at Hancock during the winter months of 1917-1918 it was, for a selected group of young men from Pennsylvania of ages from 21 to 31, 1.1 per 1,000 , or 110 per 100,000 . In other words, in the civil population of Pennsylvania the death rate from pneumonia is much higher than it is in North Carolina, notwithstanding the large negro population of the latter state. Taking into consideration only the white population of North Carolina and supposing the population of Pennsylvania to be wholly white, the death rate among civilians in Pennsylvania from pneumonia is more than twice that of North Carolina; in other words, the death rate from pneumonia among southern boys at Camp Wheeler was 22 times that among the civil population of North Carolina counting both sexes and races and all ages, while the deaths among Pennsylvania boys at Camp Hancock was only a little more than half that among the eivil population at home during the same time. In our opinion these figures, in part at least, answer the question why pneumonia was more prevalent among southern than among northern men in our eantonments. The former eame from communities in which the pneumonias are rare and consequently had had only slight opportunity to acquire immunity to these diseases, while the northern soldiers came from communities in which the pneumonias are more common and consequently had had greater opportunity to acquire immunity to these diseases.

We do not suppose that the southern soldier owed his greater susceptibility to the pneumonias wholly to the fact that he was born and reared south of the Mason and Dixon Line; on the other hand, we do not suppose that the northern soldier owed his greater immunity to the pneumonias wholly to the fact that he was born and reared north of this Line. It is a question of the density of population in which the individual is reared and,in which he lives. The South is more rural than the North. 


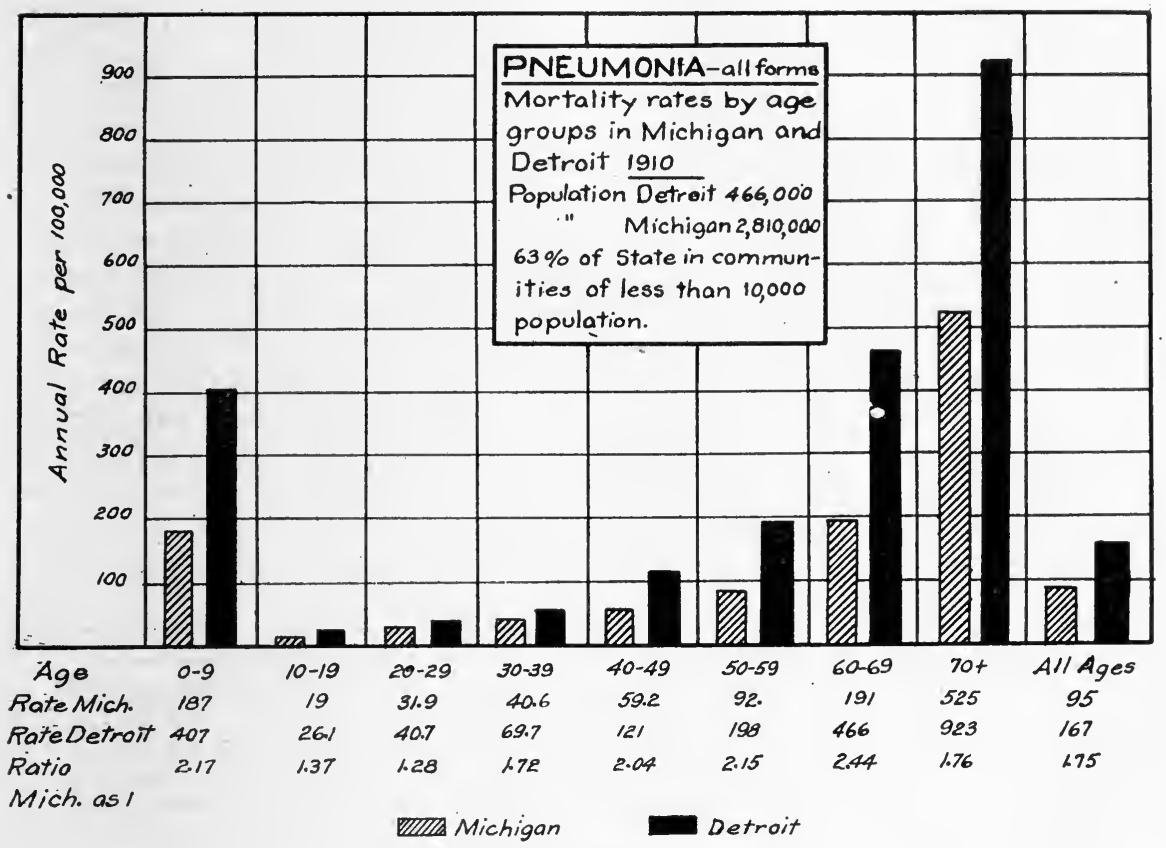

Fig. 8.

Lines drawn representing the death rate from pneumonia in our cantonments during the winter of 1917-1918 and showing the percentage of rural populations in the districts from which the camps were recruited are almost parallel. Vaughan and Palmer, in a study of the respiratory diseases in our cantonments during the winter of 1917-1918, make the following statements :

"It is a well recognized fact that respiratory disease is more common in centers of population where its means of transmission is so much readier than among the rural population. The table below illustrates this fact and these data are typical.

Urban and Rural Deati Rates, Annual Rate Per 100,000 1915

\begin{tabular}{|c|c|c|c|c|c|c|c|}
\hline & \multirow{2}{*}{ MEASLES } & \multirow{2}{*}{$\begin{array}{c}\text { SCARLET } \\
\text { FEVER }\end{array}$} & DIPHTHERIA & \multirow{2}{*}{ PNEUMONIA } & \multicolumn{3}{|c|}{ TUBERCULOSIS } \\
\hline & & & AND CROUP & & OF & THE & LUNGS \\
\hline $\begin{array}{c}\text { Cities in } \\
\text { registration } \\
\text { states }\end{array}$ & 7.1 & 4.0 & 17.5 & 156 & & 134 & . \\
\hline $\begin{array}{l}\text { Rural part of } \\
\text { registration } \\
\text { states }\end{array}$ & 3.4 & 3.1 & 12.9 & 106 & & 112 & \\
\hline
\end{tabular}

"Analyzing the situation in the army with this question of population in mind, we are brought face to face with some interesting facts. We have compared the death 
rates among the soldiers from different sections of the country with the percentage of rural population of that section. The association of high disease incidence and rural population is highly suggestive. The death rates are distributed roughly in three groups. Those representing the West South Central section, the East South Central section, and the South Atlantic stand first with the highest rates. Next with a rate just half that of the former group comes the West North Central. Next comes the other sections, the difference between them being slight. New England is the least rural and has next to the lowest death rate. New England is represented by Camp Devens which contains draft men. If New England were represented in this table by a larger proportion of eity men as are the other sections with their national guard units it is possible that New England's death rate would be lower than it appears. The similarity in these two curves is believed to be of the greatest significance.' It will be noticed that they are not parallel throughout. There are several explanations for this. In the first place the exactness of the two sets of data is not sufficient for a complete correlation, secondly it is felt that the disparity in the curves carries some significance. It means that factors other than sparseness of population are at work in the first two sections causing them to run unduly

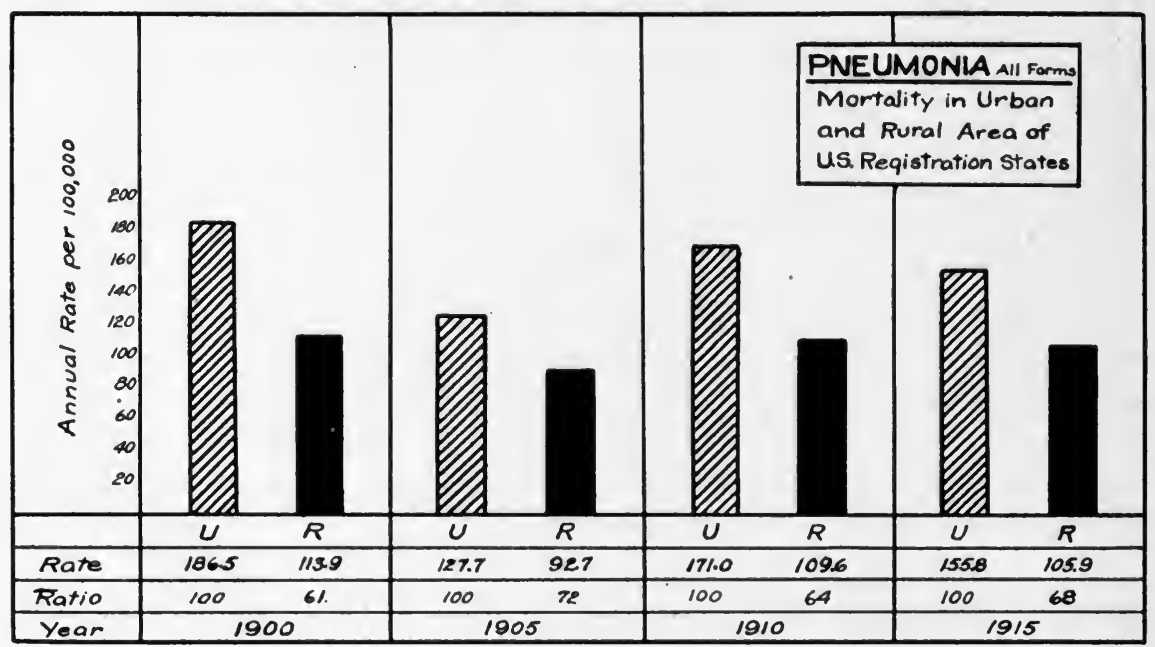

Fig. 9.

high. The first two sections which are more than twice the rate of the third are the only ones including southern territory. There is thus brought to light a secondary broad general influence which distinguishes different sections of the country. This is climate. It seems reasonable to explain the high position of the first two points then as due (1) to rural population and (2) to climate. This latter influence we shall discuss in a later paragraph.

"The influence of rural life on disease incidence as brought out in this table is further supported by the figures gathered from the city and rural units in the camp. Camp Cody reports that the respiratory disease incidence was forty-eight per cent higher in the 134th Infantry than in the 133rd. The latter is made up of troops from the larger cities of Iowa, while the former includes troops mainly from the smaller towns of Nebraska. Similarly disease incidence was fifty-one per cent greater in the 136th Infantry made up from the smaller towns of Minnesota than in the 135th Infantry made up of men from the larger cities of this state. 
"Camp Wadsworth reports that their division made up of Guardsmen from the larger cities of New York State was practically free from disease until March when about 1,500 draft men from the mountains of Tennessee and Kentucky were received. Their arrival had a marked effect upon the disease rate. The men soon developed meningitis, pneumonia, and the minor communicable diseases. Their noneffective rate was three times that of the original division.

"The epidemiologist at Camp Doniphan points out the unusually low disease incidence among eity troops as compared with those from the country. The 138th Infantry and the 128th Machine Gun Battalion were recruited from St. Louis, Mo. Their annual pneumonia morbidity rates from October to March were 15 and 25 respectively. The 137th Infantry and 129th Field Artillery were from small towns of Kansas. Their corresponding rates were 65 and 50 respectively."

\section{PNEUMONIA(all forms) IN THE LARGEST CITIES OF THE U.S. \\ (1910 RANKING) \\ AVERAGE ANNUAL DEATH RATE PER 100,000 FOR THE YEARS 19I1-17}

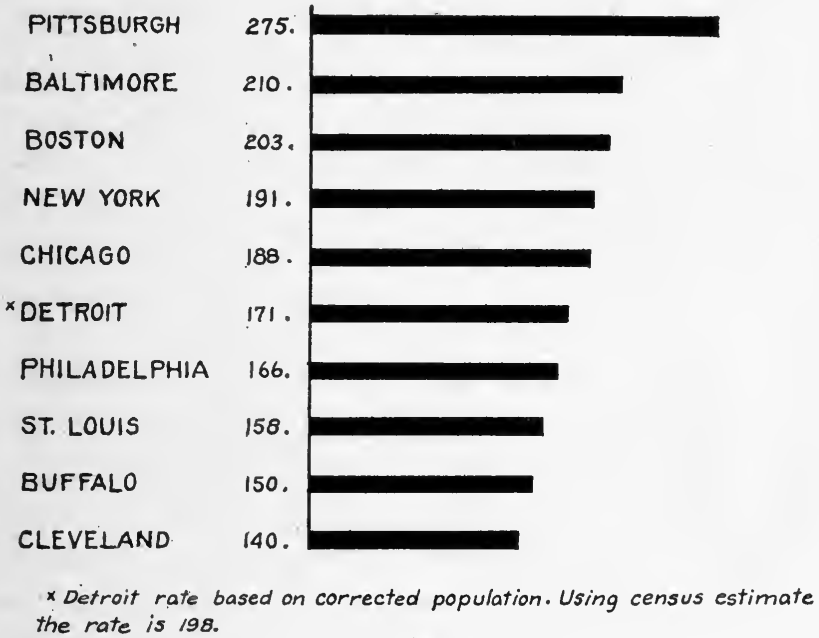

Fig. 10.

In studying the relative morbidity and mortality in urban and rural life we meet with many difficulties. In the first place, all attempts to draw definite lines between urban and rural communities are arbitrary and necessarily faulty. In many villages and even small cities assigned by the census authorities to rural districts the density of life and the closeness of individual contact among at least certain groups of the inhabitants may be as great as in large cities. Thousands of individuals having their legal residence in rural communities pass a large part of their daily lives in the dense crowds of great cities. We cannot, therefore, hope to draw a sharp line of demarkation between rurals and urbans. The figures which we present convince us that the greater susceptibility to pneumonia of the soldier from a rural district as compared with his comrade from an urban home is due to the fact that the former 
has not acquired the degree of resistance to this disease possessed by the latter.

That the wide prevalence of measles in our cantonments in the winter of 1917-1918 was a most potent factor in causing the high death rate from pneumonia there can be no question. In our article on measles we have discussed the relation between that disease and pneumonia and have shown that the man who has recently had measles is not only more likely to develop pneumonia, but is much more likely to die from the pneumonia than one who has not recently had measles.

As to the classification of the dominant pneumonias in our camps in 1917-1918, the epidemiologist is not competent to speak with authority. The pneumonias in our camps at that time were investigated by most competent bacteriologists and pathologists and from one of the latter, MacCallum, we make the following quotation:

"The relation of measles to severe and fatal pulmonary inflammations has always been recognized. So too the importance of measles as a predisposing disease in epidemies of pneumonia has been fairly accurately appraised since early times and is definitely mentioned by the writers who deseribe the pandemic of 1812 .

"It is not known, however, in just what way measles facilitates the invasion of the streptococcus. Very little is known of the actual changes produced by measles in the respiratory tract. In all the great epidemics of measles death appears to have depended largely upon pulmonary infection or else upon the fatal effects of exposure, starvation, and lack of care that accompanied several of the outbreaks among savage people.

"Such autopsies as have been performed upon eases of supposedly uncomplicated measles have left us with only very vague results, and we are in no better position to estimate the part played by measles in the production of the fatal lesions than were those who deseribed the epidemic during the war of 1812, except that we know that in our experience the anatomical changes in the bronchi and lungs are always associated with the presence of streptococcus hemolyticus. We know further that this streptococeus ean cause the same changes without any predisposing measles and that they are produced in practically identical form in children after whooping cough and diphtheria and, from at least one case in an adult, after searlet fever. We are, therefore, inclined to aseribe none of the actual anatomical changes which we can see to measles, but all to the secondary invader.

"Nevertheless, it is certain that measles plays an important part. In the Texas autopsy material, if we exclude seren cases of uncomplicated lobar pneumonia due to the pneumococcus, there were 28 cases of streptococcal pneumonic infection, and of these 15 had recently had measles or were still covered with the rash. At Camp Dodge, Iowa, of 23 cases of streptococeal pneumonia, 8 had just passed through measles and 3 had had German measles. In this epidemic, however, these particular figures are misleading since they depend upon a fresh outbreak of measles pneumonia at the end of a long epidemic in which measles had played no considerable part, the few cases of measles having been thoroughly isolated in a distant barrack.

"This accidental and sudden flare-up of streptococcal bronehopneumonia in a barraek of measles patients among whom there had previously been no pneumonia, although a great epidemic was prevailing in the general camp, brings out two important points. First, that in this eamp the streptococcus had gained a foothold independently 
of measles and produced an extensive and fatal epidemic of interstitial bronchopneumonia. Second, that when this infection did attack patients ill with measles the bronchopneumonia was much more severe and more quickly fatal. It was among these cases that we found the freshest lesions in patients who had died four to eight days after the beginning of the bronchial symptoms. It was almost exclusively among these patients too that we observed the hoarseness and loss of voice which was explained by finding deep ulcerations of the vocal cords and laryngeal walls.

" Therefore, it seems justifiable to conclude that measles brings about a most effective predisposition to infection with the hemolytic streptococcus, although the streptococcus can invade and even cause great epidemics without its aid. In addition it may be emphasized that the combination of streptococcus infection with measles seems to produce a far more intense affection than the streptococcus alone, but in this we cannot yet recognize any anatomical changes that are due to measles itself.

"At this point it may be said that these epidemics of streptococcal bronchopneumonia have not been confined to troops in camp but have existed in a more mild form in the civil population at many points. Among children the production of typical interstitial bronchopneumonia after measles and whooping cough has been recognized at autopsy in a number of cases."'

MacCallum thinks that, while lobar pneumonia due to the pneumococcus was present in our camps during the winter of 1917-1918, this form presented no unusual feature and that the dominant pneumonia was anatomically lobular and etiologically streptococcal. There can be but little doubt that the difference in fatality from pneumonia in the different camps was due to the kind and virulence of the organism acting as the dominant factor in the epidemic. At several camps the type of the dominant pneumonia was changed with the advent of highly infected accessions. In one camp with only a moderate number of cases of pneumococcus pneumonia the type of the disease was changed by the arrival of an organization badly infected with the streptococcus hemolyticus and the death rate even among the soldiers originally in the camp was markedly increased by the introduction of this more virulent organism.

Cole agrees with MacCallum as to the character of the pneumonias which existed in our camps during the winter of 1917-1918. He states that the lobar pneumonia at that time did not differ essentially from that seen in civil hospitals except perhaps in its relative mildness and that this is to be expected in a population composed of young adults. Some cases of lobar pneumonia were preceded by measles, but there was apparently no relation between the two diseases. The bronchopneumonia was more closely connected with the measles. Cole says:

"In certain cases of measles, the temperature does not fall with, or shortly after, the appearance of the rash, as is usually the ease, but the fever continues and becomes higher and the symptoms mentioned increase in severity. In other cases the temperature falls to normal and then after a period of from a few days to three wecks the temperature again becomes elevated and the symptoms mentioned become severe and characteristic of the disease. In none of the cases we have seen, however, nor in any of those of which we have histories, has there been a complete disappearanec of all the 


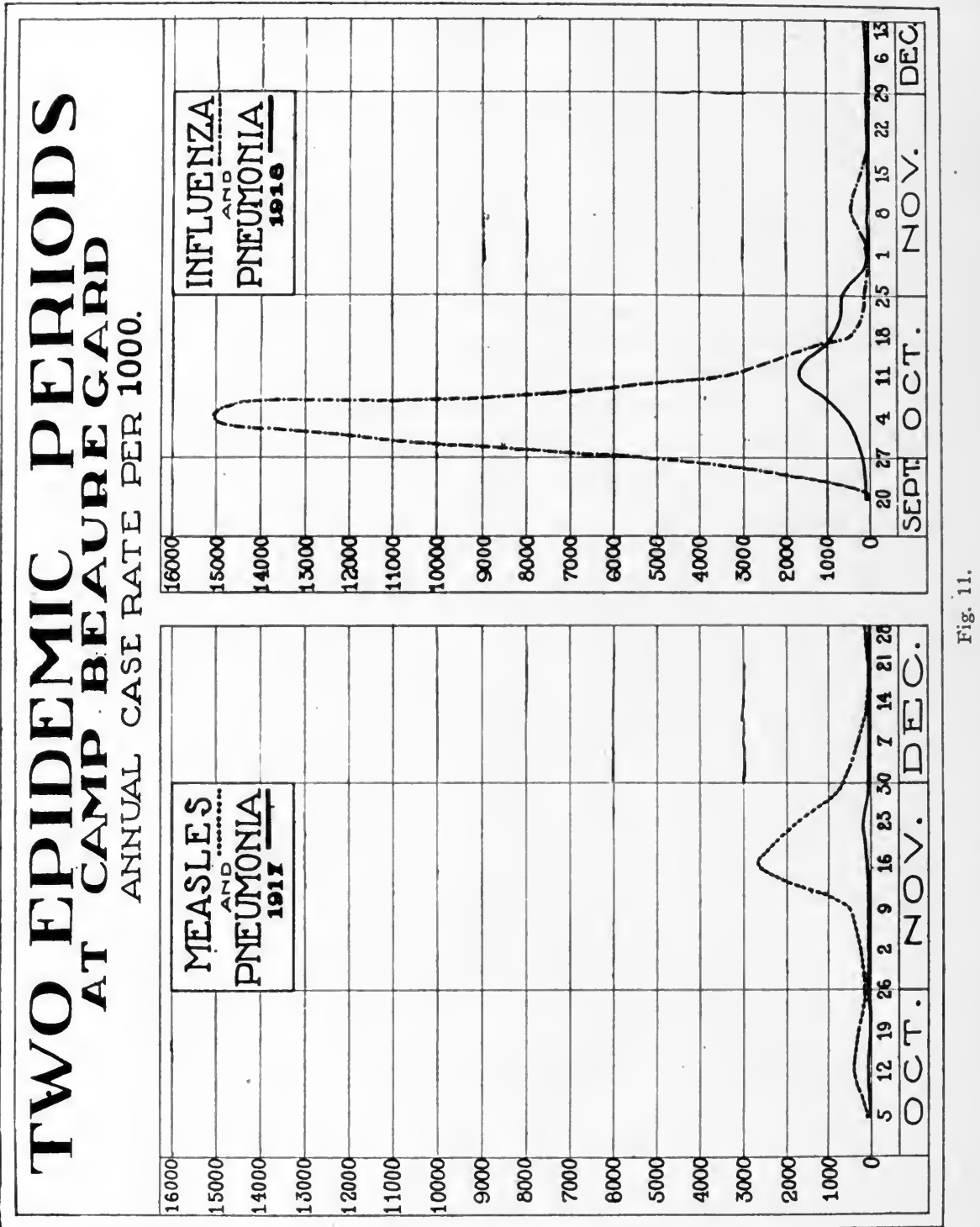

symptoms referable to the respiratory tract during the interval between recovery from measles and onset of bronchopneumonia. Even in the eases in which the onset has occurred following the discharge of the patient from the hospital, it is quite certain that cough or respiratory difficulty was present at the time of discharge and persisted up to the time of onset of the pneumonia."

Bronchopneumonia followed not only measles, but certain local diseases of the upper respiratory tract. Cole writes:

"These observations as well as the pathological studies, indicate that the infection, 
as well as the lesion, is probably a descending one, and that no sharp line can be drawn either in time or symptoms, between the occurrence of the pharyngitis, laryngitis, bronchitis, and finally the bronchiolitis and bronchopneumonia."'

In some cases Cole thinks that the bronchopneumonia was a complicating sequel to lobar pneumonia. The lobar pneumonias were due to the pneumococcus, while the bronchopneumonias were, for the most part at least, due to the streptococcus hemolyticus. Cole was convinced that in many instances measles patients became infected with the streptococcus while in the hospital being treated for the measles. He tested the throats of 44 cases of measles for the streptococcus hemolyticus on admission to the hospital and found only 11.4 per cent positive, while after from three to five days the per cent increased to 38.6 and after from eight to sixteen days to 56.8 per cent. Cole says:

"An analysis of this table indicates that a relatively small percentage of patients on admission to the measles wards harbor S. hemolyticus in their throats. However, as the time of residence in the ward increases, the number of carriers of S. hemolyticus increases, until finally the percentage of incidence becomes as high as that found in the measles wards in general. Because of these observations, there seems to be very little reason to doubt that a large per cent of measles patients acquire S. hemolyticus in their throats for the first time during their stay in the hospital wards. While this study is not extensive enough to be absolutely conclusive, it indicates strongly that the high incidence of bronchopneumonia during convalescence from measles is directly related to the transfer of the infectious agent from one patient to another in the wards of the hospitals."

This is certainly confirmatory of what we have said in our discussion of measles concerning the danger of infection in hospital with a more deadly virus than that of the measles. While the lobar pneumonia in our camps did not exceed in fatality that observed in civil life, the mortality in cases of bronchopneumonia was high and in most instances when this disease proved fatal it was complicated with empyema. There was, it is true, some empyema in cases of lobar pneumonia, but the incidence of empyema in this form of pneumonia was not high. Emphasizing again the danger that comes from infecting measles patients with the streptococcus hemolyticus in hospital wards, Cole adds:

"The work indicates that the high incidence of pneumonia in this hospital, and the resulting high mortality, has been due, to some extent at least, to infection occurring within the hospital itself. The conditions are not unlike those surrounding puerperal fever and surgical wound infections. While in measles, raw surfaces do not exist on which infection can occur, this disease renders the respiratory mucous membrane especially vulnerable to infection with streptococci. Possibly in other diseases, as searlet fever and even lobar pneumonia, similar conditions exist. When infection is once started in a ward in which the patients are closely associated, the streptococei become widely distributed; they probably gain in virulence with repeated transfer through the human subject, and serious and widespread infection results."

During the spring and early summer of 1918 most of the divisions 
which had been in camp during the preceding winter went to France and the vacated camps were filled with fresh men. During the five summer months (April 5 to August 30) the average strength of the army in this

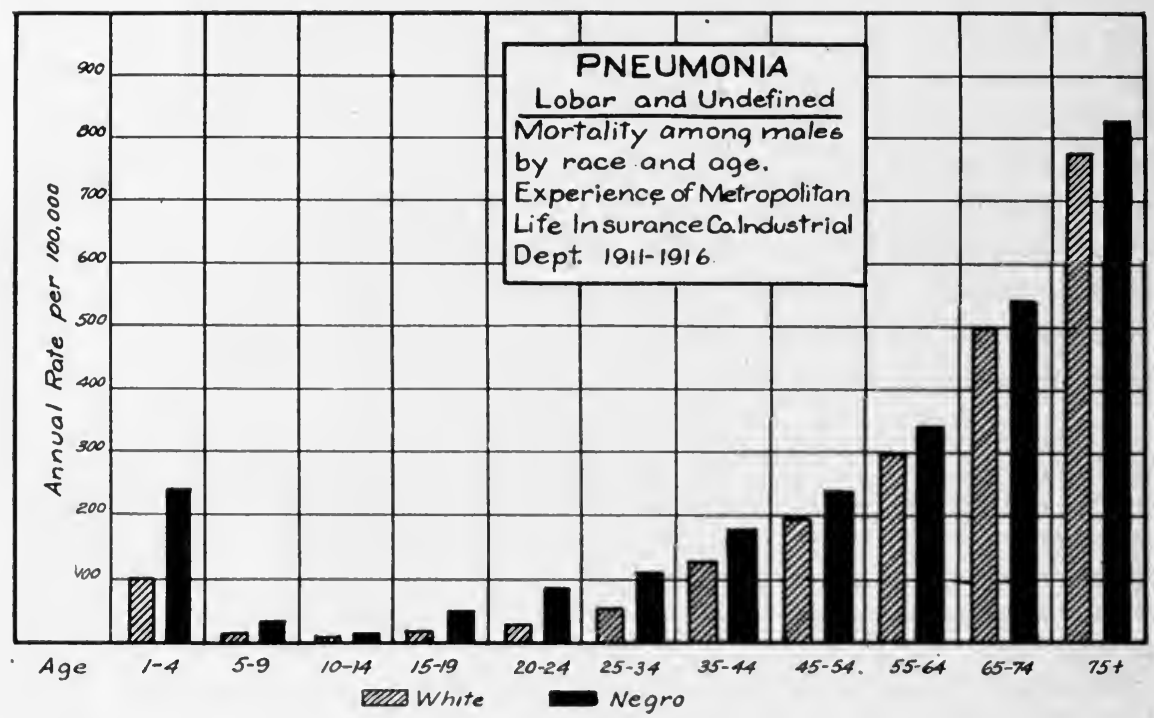

rig. 12.

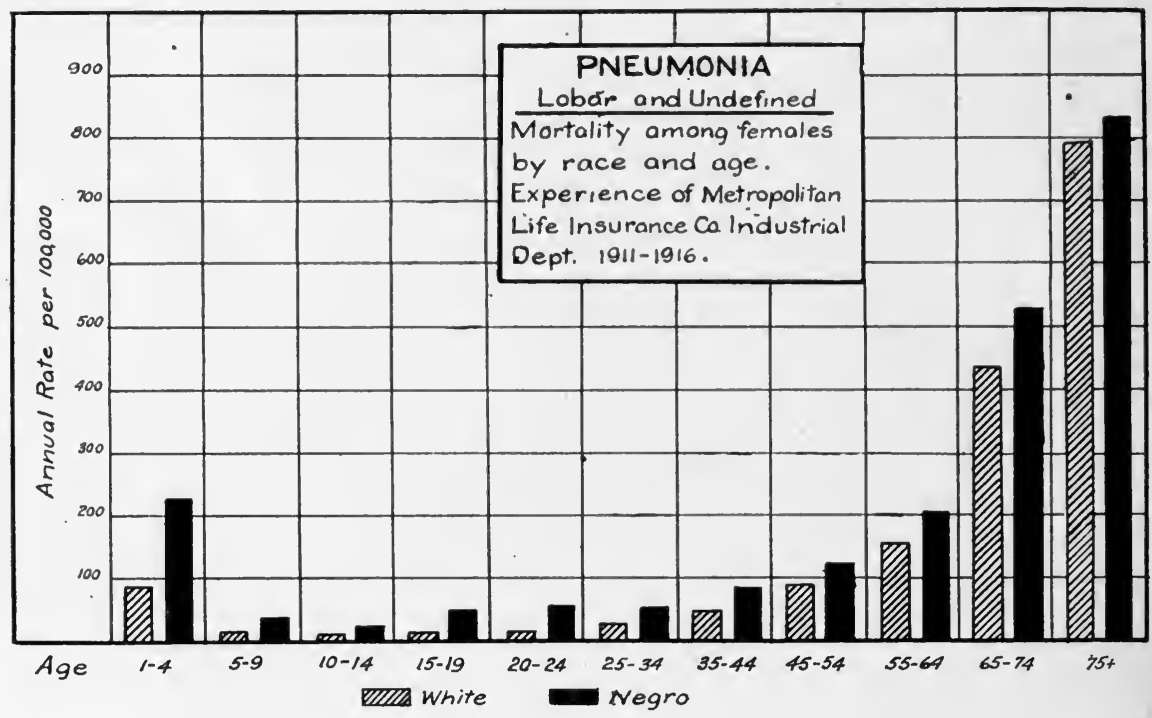

Fig. 13.

country was $1,420,000$ and the annual death rate from pneumonia per 100,000 was 279 . This showed a great improvement when compared with the death rate from this disease of 23.6 per 1,000 in the preceding winter. 
It was no longer possible to study disease among the camps with reference to the state or section from which the soldiers eame. One camp was devoted to field artillery, another to light artillery, etc., and their personnel was made up not from one section as formerly, but from many sections of the country. Pneumonia, although it continued the most potent cause of death and prevailed more largely in some camps than in others, manifested no new characteristics. The rural soldier continued more susceptible than his urban comrade. Both pneumococcus and streptococcus pneumonia continued in evidence. Camp Dodge (Iowa) had the greatest mortality with an annual death rate per 1,000 of 17 . This camp during this period was filled almost entirely with soldiers from rural areas including a large number of negroes from the South. Vaughan and Palmer, in their report upon communicable diseases in the army during this period, say:

"Pneumonia has continued to be throughout the entire period the chief cause of death. In our report of the winter of 1917-1918 we attributed the high death rate to the new recruit and especially to the new southern and new rural recruit. It was the nature of the eamp personnel far more than camp surroundings or camp management which determined sickness and death. With the experience of the past summer before us there is no reason to alter this judgment. Again the evidence points to the rural recruit as the camp's greatest liability."

A striking instance of the greater prevalence of pneumonia among negroes, especially those from southern rural districts as compared with white men, is shown by certain figures obtained from Camp Funston. In the June and July drafts there were received at Funston 27,000 white men who furnished four cases of pneumonia from June 15 to August 15. During the same period 12,000 colored draft men provided 83 cases of pneumonia. There were four times as many cases of pneumonia among Mississippi and Oklahoma negroes per 1,000 as there were among Missouri negroes. At Camp Devens from August 1 to 28 there were 20 cases of pneumonia among 34,406 white men and 68 cases among 3,950 negroes. The white incidence amounts to an annual rate per 1,000 of 7.6; that for the blacks to 226. The colored troops at Devens were mostly from Florida, while the whites were from New England. There were several factors accountable for this great difference in prevalence of pneumonia among whites and blacks at Devens. The blacks had experienced a change in climate and although this was the warm season, the changes from day to day and the change from warm days to cool nights were different from what the colored troops had been accustomed. Coupled with this factor was a shortage of bedding. Each man was furnished with a cotton comforter which was adequate for northern men, but not sufficient to keep the southern negro warm on the cool nights, of which there were many. The colored men were worked harder than had been their custom in eivil life and carelessness in cooling off after severe 
physical exertion was another factor that may have lowered their resistance.

We shall discuss the pneumonias of the autumn of 1918 in our chapter on influenza. At present we shall content ourselves with saying that highly competent bacteriologists in different camps report widely divergent findings as to the etiologic agent of both the influenza and the pneumonias that followed. At Devens, Shelby, Upton, Funston, Dodge, and McArthur, the Pfeiffer bacillus was found in such a large percentage of cases that it was believed to be the causative agent of both the influenza and the pneumonia. At Logan, Taylor, Grant, and Sherman, a pneumococeus was the dominant organism. The Pfeiffer bacillus was rarely found and when found believed not to be the chief agent, certainly in the causation of the pneumonias. In other camps, notably at Custer and Wadsworth, the pneumonias were attributed to the streptococcus hemolyticus, while at Jackson and Syracuse the staphylococcus aureus was believed to play an important rôle in the causation of the pneumonias. For the present we hold that the virus of influenza opened gateways to the lung tissues and that all organisms which are capable of growth and multiplication in these tissues and which were present in the locality at the time, availed themselves of the opportunity thus afforded to enter the field and to feed upon the soil. The pneumonias of the fall of 1918 differed, in our opinion, only in degree, both in symptomatology and pathology, from those of the winter of 1917-1918. Both measles and influenza prepare the way for the more deadly organisms which may eause pneumonia and which are variable in both form and function. It is a demonstrated fact that different strains of pneumococci and streptococci show wide variations in virulence, and that the same strain shows like variations with varying conditions of growth.

Recent Prevalence.-The figures in Table III, taken from the mortality statistics show the death rates from pneumonia in the registration area since 1911.

TABLE III

Death Rate from Pneumonia (All forms) per 100,000 Population

\begin{tabular}{llll}
\hline YEAR & RATE & YEAR & RATE \\
\hline 1911 & 133.7 & 1915 & 132.7 \\
1912 & 132.3 & 1916 & 137.3 \\
1913 & 132.4 & 1917 & 149.8 \\
1914 & 127.0 & 1918 & 284.3 \\
\hline
\end{tabular}

The death rate for 1918 is the highest for any year since 1900, the beginning of the annual reports. In 1918 the number of deaths from all forms of pneumonia in the registration area was 232,786 , of which 152,542 


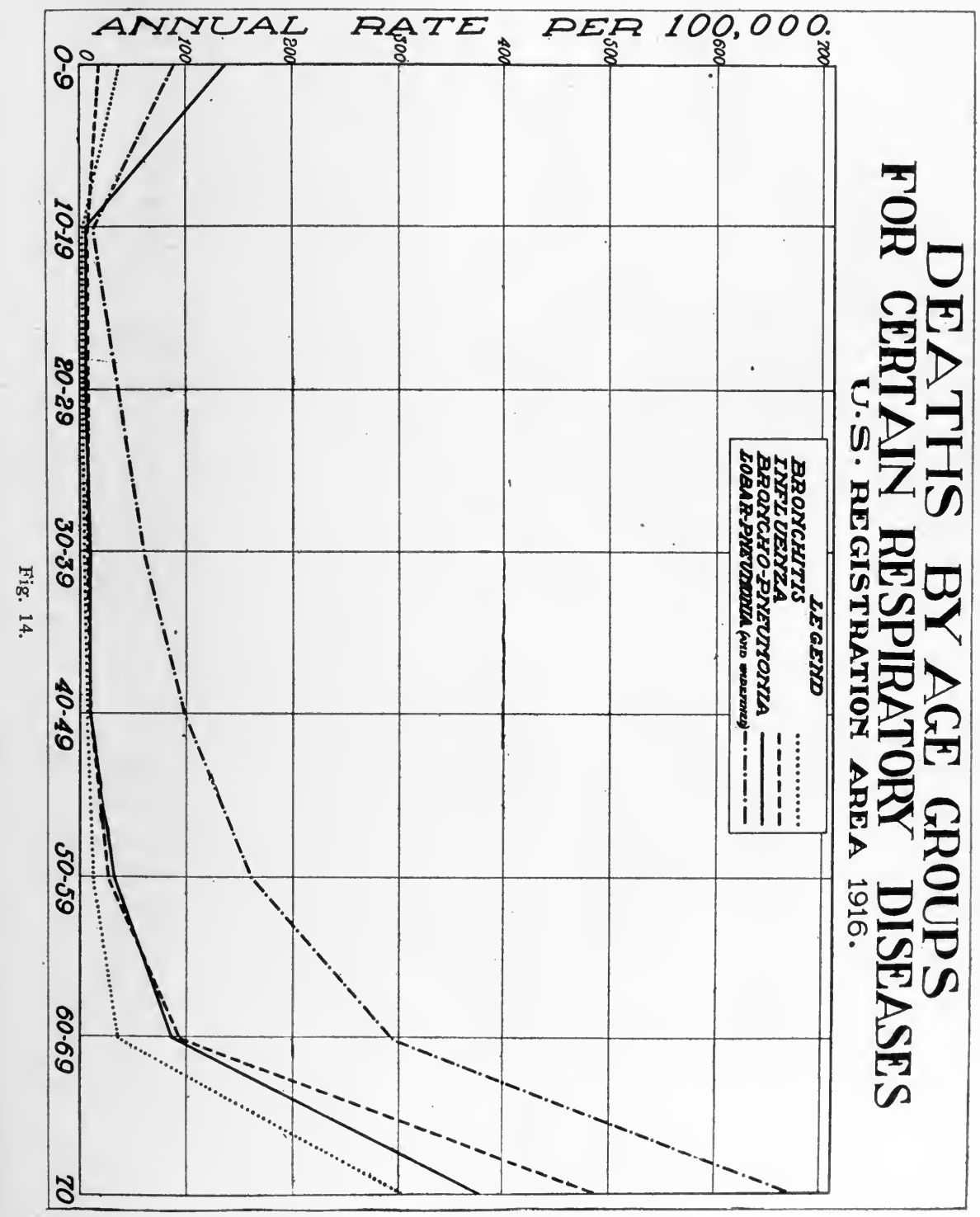


were reported as due to lobar, 56,966 to broncho, and 23,278 to pneumonia without qualification. The death rate among the total population in the North is much higher than among the white population in the South. In the South the death rate among negroes is as high on the whole and in many states higher than that among the total population in the North.

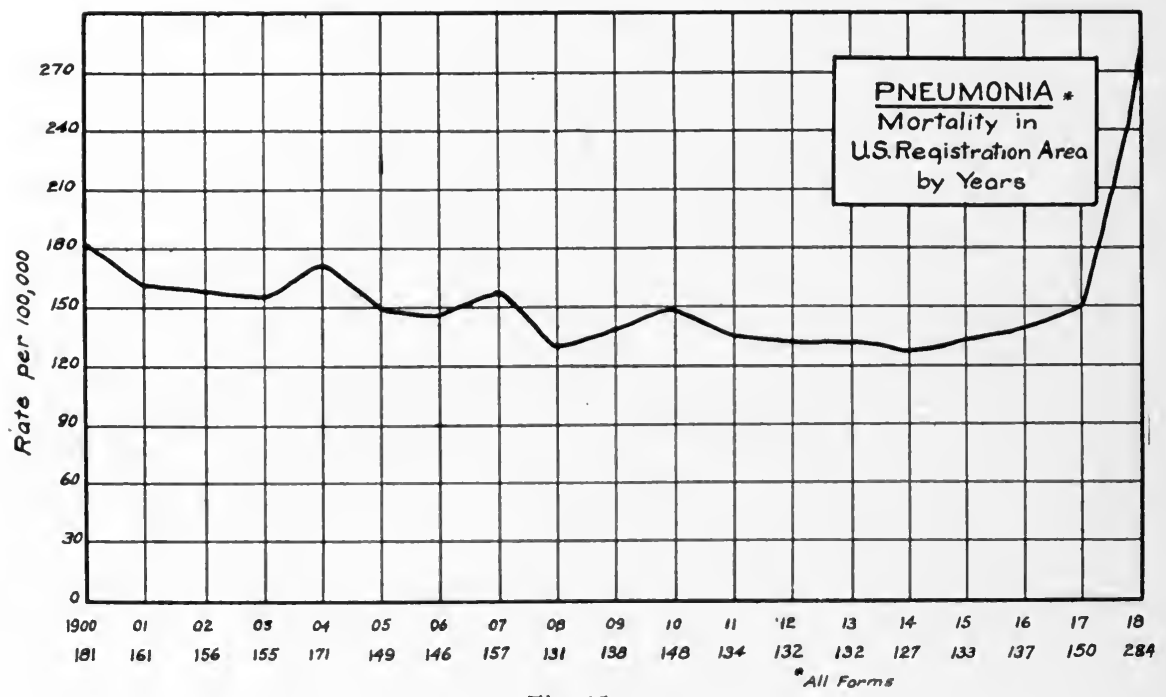

Fig. 15 .

TABLE IV

Death Rates from Pneumonia (All forms) per 100,000 Population in the Large Cities of the United States Since 1911

(Using Corrected Mid-year Population Following 1920 Census)

\begin{tabular}{lrrrrrrrrrr}
\hline \multicolumn{1}{c}{ CITY } & 1911 & 1912 & 1913 & 1914 & 1915 & 1916 & 1917 & 1918 & 1919 & 1920 \\
\hline Boston & 209 & 210 & 203 & 198 & 212 & 222 & 224 & 487 & 162 & 181 \\
New York & 212 & 198 & 200 & 186 & 204 & 190 & 195 & 418 & 198 & 178 \\
Philadelphia & 159 & 133 & 144 & 166 & 169 & 171 & 213 & 520 & 176 & 202 \\
Detroit & 132 & 157 & 171 & 133 & 139 & 169 & 192 & 261 & 157 & 237 \\
Chicago & 201 & 210 & 205 & 178 & 164 & 157 & 196 & 304 & 132 & 140 \\
St. Paul & 93 & 80 & 85 & 119 & 119 & 87 & 104 & 166 & 85 & 194 \\
Minneapolis & 113 & 93 & 105 & 124 & 115 & 115 & 120 & 171 & 92 & 88 \\
Si. Louis & 143 & 152 & 140 & 155 & 145 & 177 & 205 & 358 & 163 & 210 \\
San Francisco & 132 & 122 & 133 & 118 & 128 & 126 & 121 & 268 & 131 & 130 \\
New Orleans & 162 & 143 & 165 & 187 & 194 & 118 & 148 & 433 & 184 & 188 \\
Washington & 162 & 137 & 123 & 123 & 163 & 150 & 143 & 365 & 149 & 196 \\
Atlanta & 226 & 206 & 163 & 148 & 146 & 146 & 160 & 306 & 149 & 307 \\
Richmond & 155 & 163 & 152 & 168 & 205 & 195 & 187 & 301 & 154 & 184 \\
Pittsburgh & 208 & 263 & 287 & 243 & 250 & 336 & 364 & 889 & 330 & 381 \\
\hline
\end{tabular}

(As the distinction between pneumonia and influenza deaths is not clear cut, these figures tell but part of the story of the influenza epidemic.) 
In giving the rates for southern cities we have given total rates for both races. The following figures show the differences in death rate between white and colored in certain of the above given cities for 1917:

$\begin{array}{lcc}\quad \text { CITY } & \text { WHITE } & \text { COLORED } \\ \text { Richmond } & 103.7 & 343.5 \\ \text { Washington } & 109.5 & 302.2 \\ \text { Atlanta } & 75.7 & 330.9 \\ \text { New Orleans } & 96.1 & 294.7\end{array}$

From the figures given in Table IV, we see that pneumonia displays the characteristics of an epidemic disease. There are certain localities in which the death rate from this disease is constantly high. For instance, in Pittsburgh, it may be said that pneumonia has been epidemic for many years. In other places, while it is endemic at all times, it reaches epidemic proportions from time to time. For instance, in 1917 the death rate per 100,000 in Montana was 146.5, while at Great Falls, Mont., it was 494.7. During the same year for the State of New York the rate was 173.4, while for Middletown, N. Y., it was 352.4. In 1917 the rate for the State of Maine was 156.4, while for Augusta, Me., it was 453.8. Like measles, scarlet fever, whooping cough, and diphtheria, pneumonia is endemic the world over and, in this country at least, shows its epidemic character every year by its unusual prevalence in certain localities.

Pneumococcus Pneumonia.-In 1880 Sternberg, later Surgeon General of the U. S. Army, discovered and described certain micrococci found in his own saliva, which when injected into rabbits caused a septicemia with the blood teeming with the organisms. In the same year, quite independently, Pasteur described the same organism. In 1883 Talamon grew this micrococcus in bouillon and named it, on account of its shape, Micrococcus lanceolatus.' Later in the same year, Fraenkel went more minutely into the cultural, morphologic, tinctorial, and pathologic characteristics of this organism, and suggested it as the cause of pneumonia. Since that time it has been known as Fraenkel's pneumococcus and many have regarded it as the specific etiologic agent in acute lobar pneumonia. The pneumococcus is an oval or lanceolate body, usually arranged in pairs, sometimes in chains, the free ends being pointed. In vivo pneumococci are apparently surrounded by envelopes which consist of swellings and possibly partial detachments of the outer layers of the organism. It is supposed that the capsule, which may be stained by special methods, exists for protective purposes. The longer diameter of the coccus measures from 1 to 1.5 microns, while the lesser varies from 0.5 to 0.7 . The organism is stained by the ordinary anilin dyes. It is not inclined to grow abundantly upon ordinary laboratory media. It is impossible to obtain pure cultures from the sputum by plate methods. A bit of the sputum 
is injected into a white mouse, this animal being used as a plate. The other organisms in the sputum die out in the animal and the pneumococcus may be obtained from the heart's blood in pure culture. It grows fairly well on blood serum preparations; also in bouillon to which blood serum or ascitic fluid has been added. The pneumococeus is aerobic but may grow anaerobically and the temperature limits lie between $25^{\circ}$ and $43^{\circ} \mathrm{C}$., the optimum being that of the human body, $37^{\circ} \mathrm{C}$. It is easily and quickly killed by a temperature of $60^{\circ} \mathrm{C}$., by exposure to 5 per cent carbolic acid and 1 to 1,000 mereuric chlorid. In dried sputum, blood, or tissue this organism continues viable for many weeks. The pneumococcus is quickly and readily soluble in bile and to this fact man probably owes his protection against infection with this organism through the blood.

In artificial cultures the pneumococeus produces a small amount of acid by fermentation of carbohydrates, and Palmer has shown that in severe cases of lobar pneumonia the urine contains a large amount of organic acid which is freed at a hydrogen-ion concentration of 5.0. The acidosis determined by the combined carbon dioxid in the plasma is seldom, if ever, severe. The question of the formation of a soluble toxin by the pneumococeus has been repeatedly investigated. As early as 1891 Klemperer found that the filtrate from an old culture might kill small laboratory animals. This finding was later confirmed by other observers, but there is no proof that the filtrate in this case contains a toxin. The filtrates from old cultures of almost any bacterium will kill small laboratory animals. In 1907 Macfayden ground virulent pneumococei which had been frozen at the temperature of liquid air and extracted the powder with a dilute solution (1-1,000) of potassium hydrate, centrifuged this extract and filtered. The filtrate thus obtained killed guinea pigs when injected intravenously in doses of from 0.5 to 1 c.c. Later Rosenow obtained poisonous substances from pneumococei after simple autolysis. Cole found that he could obtain occasionally, not uniformly, salt extracts from pneumococei which killed guinea pigs with the symptoms of acute anaphylactic shock. The same investigator found that solutions of pneumoeocci in dilute bile salts injected into guinea pigs induces symptoms resembling those of anaphylactic shock; in other words, these investigators have shown that the cell substance of the pneumococcus contains the protein poison. In 1903 Vaughan and Wheeler prepared from powdered pneumococcus cell substance the protein poison and demonstrated its action on animals. In no way does the protein poison obtained from the pneumococeus differ from that which may be extracted from any true protein. The cell substance of the pneumococeus contains no toxin, using this word in the sense that it signifies a body which, when injected in nonfatal and inereasing doses into animals, eauses the elaboration of an antitoxin. Cole has found that solutions of pneumocoeci in bile salts 
may hemolyze the red blood cells of man, sheep, guinea pigs, and rabbits; but this does not indicate that the pneumococcus is an active hemolytic microorganism.

In about ninety per cent of all cases of lobar pneumonia the pneumococcus is found in the lungs after death. However, this organism. is found in other diseases as well. In a considerable percentage of cases of bronchopneumonia the pneumococcus is found in the lungs either to the exclusion of other microorganisms or in greatly predominating numbers. In pleurisy without pneumonia the pneumococcus is frequently the only bacterium found. It may cause meningitis and, indeed, is regarded as the active agent in many sporadic cases of this disease. In various suppurative processes, especially in children, such as middle ear disease, mastoiditis, and endocarditis, the pneumococcus is apparently the infecting agent. More rarely it is found in inflammatory and suppurative diseases of the joints.

Dochez and Avery have demonstrated that filtrates from young actively multiplying artificial cultures of the pneumococcus contain a specific precipitable substance; i. e., such filtrate gives the precipitin reaction with the blood serum obtained from animals inoculated with the pneumococcus. These authors conclude that this precipitable substance does not owe its origin to disintegration of the pneumococcal cells, but represents an extrusion of bacterial substance by the living organism. The only basis they have for this conclusion is that they have demonstrated the existence of this substance in filtrates from rapidly growing cultures and they have assumed that in such cultures there are no dying or dead pneumococcal cells. This is an assumption which, in our opinion, is wholly without warrant. Because bacterial cells in a given culture are rapidly multiplying is no proof that at the same time certain cells are not dying or not disintegrating and dying. This, however, is not a matter of great importance. The filtrates from many bacteria, both pathogenic and nonpathogenic, contain specific precipitable substances, the presence of which can be demonstrated by the addition to such filtrates of the blood serum of an animal treated with the living organism; in other words, Dochez and Avery have shown that the pneumococcus, like most other bacteria, elaborates or produces a specific precipitable substance. They have not shown that this substance is a secretion of the living cell, and if this were shown it does not prove that this specific substance is a toxin or, indeed, has any poisonous properties. The real contribution made by Dochez and Avery is that the urine in a certain percentage of cases of lobar pneumonia contains this specific precipitable substance, and this may be recognized on the addition to the urine of a specific serum.

The fact that the pneumococcus exists in the sputum of about 50 per cent of healthy persons quite naturally led to the conclusion that the 
development of pneumonia was due to autoinfection and that all that could be done to limit the disease was for each individual to keep himself in the best possible health. It was believed that if individual resistance was lowered by exposure to inclemency of weather or by other diverse circumstance the pneumococci present in the mouth took advantage of the opportunity and penetrated the lung tissue, causing the disease. In 1915 Dochez and Avery showed by immunologic reactions that pneumococci might be divided into several groups. Previous attempts at such grouping had been made by studies of differences in cultural, morphologic, and virulence characteristics, but none of these had led to any definite results. Cole and his coworkers at the Rockefeller Institute have devised methods for distinguishing and differentiating the types of pneumococci. In 1917 they reported on the types found in 454 cases of lobar pneumonia admitted to the hospital wards of the Institute.

TABLE V

Types of Pneumococcus Causing Lobar Pneumonia in 454 Cases

\begin{tabular}{ccc}
\hline \hline PNEUMOCOCCUS & INCIDENCE & PER CENT \\
\hline Type I & 151 & 33.26 \\
Type II & 133 & 29.29 \\
Type II & 6 & 1.32 \\
Type IIb & 4 & 0.88 \\
Type IIx & 9 & 1.98 \\
Type III & 59 & 12.99 \\
Type IV & 92 & 20.26 \\
\hline
\end{tabular}

On typing the pneumococci found in the saliva of normal individuals, they secured the results indicated by the figures in Table VI.

TABIS VI

Types of Pneumococcus Isolated from the Saliva of Normal Individuals Not in Direct Contact with Lobar Pneumonia

\begin{tabular}{|c|c|c|c|c|}
\hline PNEUMOCOCCUS & INCIDENCE & \multicolumn{2}{|c|}{$\begin{array}{l}\text { PER CENT DISTRIBUTION } \\
\text { AMONG } 187 \text { PEOPLE } \\
\text { WITH PNEUMOCOCCUS }\end{array}$} & $\begin{array}{l}\text { PER CENT DISTRIBUTION } \\
\text { AMONG } 297 \text { PEOPLE } \\
\text { EXAMINED }\end{array}$ \\
\hline Type I & 1 & \multicolumn{2}{|c|}{0.8} & 0.3 \\
\hline Type II & 0 & \multicolumn{2}{|c|}{0.0} & 0.0 \\
\hline Type IIa & 1 & \multicolumn{2}{|c|}{0.8} & 0.3 \\
\hline Type IIb & 7 & \multicolumn{2}{|c|}{5.8} & 2.4 \\
\hline Type IIx & 14 & \multicolumn{2}{|l|}{11.6} & 4.7 \\
\hline Type III & 34 & \multicolumn{2}{|l|}{28.1} & 11.4 \\
\hline \multirow[t]{4}{*}{ Type IV } & 64 & 52.9 & & 21.5 \\
\hline & \multirow{3}{*}{\multicolumn{2}{|c|}{$\begin{array}{l}\text { Pneumococcus present } \\
\text { Pneumococcus absent }\end{array}$}} & 116 & \\
\hline & & & 181 & \\
\hline & & & 297 & \\
\hline
\end{tabular}


By comparison of these tables it will be seen that Types I and II are rarely found in the saliva of normal individuals, while these types are found in the majority of cases of pneumonia. On the other hand, Type IV is most common in normal saliva, while it is responsible for only twenty per cent of the eases of pneumonia; and what is of more importance, the pneumonia caused by Type IV is mild and seldom fatal. These investigations completely changed the attitude of the medical profession towards the etiology and epidemiology of lobar pneumonia. Grave forms of this disease came to be regarded not as due to autoinfection, but as infections transferred from individual to individual by either direct or indirect contact. Fortunately, the methods of typing pneumococei were given to the world before our entrance into the great war and facilities for carrying out these procedures were furnished all laboratories in the base hospitals. The researches of Cole and his collaborators went further and developed a specific serum for the treatment of pneumonia due to Type I. The value of this specific treatment has been demonstrated, but it should be clearly understood that it is applicable only to those cases due to pneumococcus Type I. Unfortunately, it turned out in our base hospitals that the majority of lobar pneumonias were due to Type IV and the mortality in these cases was higher than Cole had found it in civil life. This, however, in no way detracts from the value and importance of the discovery of methods of differentiation between types of pneumococci. Under conditions of civil life the findings of Cole and his associates that most fatal lobar pneumonias are due to Types I and II will probably be confirmed. This fact is fully appreciated and all modern clinical laboratories are typing pneumococei found in the sputum of cases of lobar pneumonia. In 1917 Stillman, of the Rockefeller Institute, summarized the findings of that institution as follows:

"(1) Pneumococei of Type I and Type II are responsible for the majority of the cases of lobar pneumonia. (2) Among the pneumococei found in the mouths of healthy individuals Type IV predominates, Type III is frequent, and atypical organisms of Type II are occasionally found. (3) Healthy persons intimately associated with cases of lobar pneumonia may harbor in their mouth secretions the highly parasitic pneumococcus of Types I and II. (4) Occasionally a carrier of Type I or Type II pneumococcus is encountered in whom it is impossible to trace any contact with an infected patient. (5) From the dust of homes where cases of pneumonia due to Types I and II have occurred, pneumococei of the same type may be recovered."

Cole's serum, which has been successfully employed in the treatment of pneumonia due to Type I, protects laboratory animals against large amounts of a virulent culture. So far as we know, this serum has never been used in man for protective purposes. It probably never will be unless the contagiousness of pneumonia be demonstrated to be greater than it is now believed to be. It is not probable that if used for protective purposes it would have any value except in that form of pneumonia 
due to Type I. As the matter stands today (1921) every case of pneumonia should be regarded as a possible source of danger to others, should be isolated and the sputum deposited in special containers which should be burned. All eating utensils should be sterilized. Soiled pillow cases, sheets, blankets, etc., should also be sterilized. Stillman has shown that the dust swept from rooms occupied by pneumonia patients often contains virulent pneumococci and like demonstrations were made in certain base hospitals during the war. These facts point clearly to the necessity for a thorough cleansing of rooms occupied by pneumonia patients. This should be attended to daily during the illness of the patient and need not be postponed until the room is vacated by recovery or death. It has been found that individuals recovering from lobar pneumonia due to Types I and II may carry these bacilli in their mouths for from two to four weeks. We are inclined to the opinion that convalescents from these forms of pneumonia should be kept in quarantine until the sputum is found to be free from these types. In the case of pneumonia, as a rule this would be no great hardship, because the individual recovering from this disease needs a rest of at least two weeks' duration.

Type III of the pneumococcus, as will be seen by reference to the preceding tables, is found in about 11 per cent of normal individuals, 28 per cent of those having some form of pneumococcus, and eauses about 13 per cent of the deaths from pneumonia. Up to the present time there is no special agency for dealing with this type, either in prevention or cure. The only practical way to look at the matter is for health officials and physicians to regard all lobar pneumonias as contagious and demand that they be handled very much as are diphtheria and scarlet fever. It should be clearly understood that Type IV does not include homogenous strains; indeed, in Type IV may be found strains differing most widely in virulence. Type IV is a conglomeration and mixture of every strain which does not fall into one of the preceding types. Lister, typing pneumococci in South Africa, has made many more groups than those defined by the Rockefeller research workers. There remains much work along this line still undone. Some reports are fairly startling in their suggestions. As early as 1891 Kruse and Pansini reported upon a study of 84 strains of pneumococei obtained from the sputum in cases of pneumonia. In this study they eame to the conclusion that there is no sharp line to be drawn between pneumococei and streptococci. They concluded that they had in their collection a great many strains showing the intermediate links between these genera. More recently, Rosenow has reported that he has been able through animal passages to convert typical pneumococci into streptococci. These are matters about which dogmatic assertions are best not made.

Some years ago Lister, in South Africa, vaccinated large numbers of 
the laborers on the Rand against pneumonia. He employed a mixed vaccine consisting of his Types A, B, and C; Types B and C corresponding to Cole's Types II and I, respectively, while Type A has not been found in this country. Lister believed and has demonstrated by figures that this vaccination greatly reduced the prevalence of pneumonia among workers. In the winter of 1917-1918 Cecil and Austin prepared a vaccine of Types I, II, and III, and with this inoculated 12,519 men at Camp Upton (about 40 per cent of the camp's strength), most of the men receiving three or four inoculations at intervals of from five to seven days. The men remained under observation for ten weeks when they were ordered to France. During this time there were no eases of pneumonia of the three fixed types among the men who had received two or more injections of the vaccine. Among 20,000 men who served as controls, during the same time there were 26 cases of pneumonia of Types I, II, and III. Moreover, the incidence of pneumonia due to pneumococcus Type IV and of streptococcus pneumonia was much less among the vaccinated than among the unvaccinated troops. The final figures show only 17 cases of pneumonia of all types occurring among the 12,519 men who received vaccine, whereas among the unvaccinated troops during the same period there was a total of 173 cases of pneumonia of all types. The pneumonia death rate for vaccinated soldiers was only 0.83 per 1,000 ; for the unvaccinated it was 12.8 .

In the summer of 1918 Cecil and Vaughan (Henry) at Camp Wheeler, using a lipo vaccine containing Types I, II, and III, vaccinated 13,460 men, or about 80 per cent of the entire strength. This division remained under observation for about three months after vaccination. During this period there were 32 cases of pneumonia Types I, II, and III pneumococcus among the vaccinated, four-fifths of the camp; and 42 cases of these types among the unvaccinated or one-fifth of the camp. If, however, all cases of pneumonia that developed one week after vaccination are excluded from the vaccinated group there remain only eight cases of pneumonia due to the fixed types and these were all secondary to severe attacks of influenza. The weekly incidence rate for pneumonia among the vaccinated was conspicuously less than among the unvaccinated and the incidence rate during the time of the experiment was twice as high for unvaccinated recruits as for those vaccinated and nearly seven times as high for unvaccinated seasoned men as for vaceinated seasoned men. Influenza struck Wheeler before the full effects of the vaceination experiment could be ascertained and the demonstration cannot be regarded as leading to a final solution of the problem. Many reports have been published in the past few years lauding the value of mixed vaccines in the prevention of influenza and pneumonia. Inasmuch as none of these are founded upon a scientific basis, they are of no value to the epidemiologist. 
Streptococcus Pneumonia.-The streptococeus was first seen in pus by Klebs in 1872. In $1878 \mathrm{Koch}$, in his studies of wound infections, found and stained this organism. In 1880 Pasteur found streptococci in puerperal fever and differentiated them from staphylococci both by their morphology and by the lesions which they induce. These observations were confirmed one year later in England by Ogsten, whose contribution added materially to our knowledge of the morphology and activity of this microorganism. In 1883 Fehleisen isolated streptococci from the lesions of erysipelas and studied their cultural characteristics. One year later Rosenbach, studying wounds, added materially to the details of the characteristics of this micrococcus and gave to it the name streptococcus pyogenes.

As the name implies, streptococci consist of spherical or oval cells

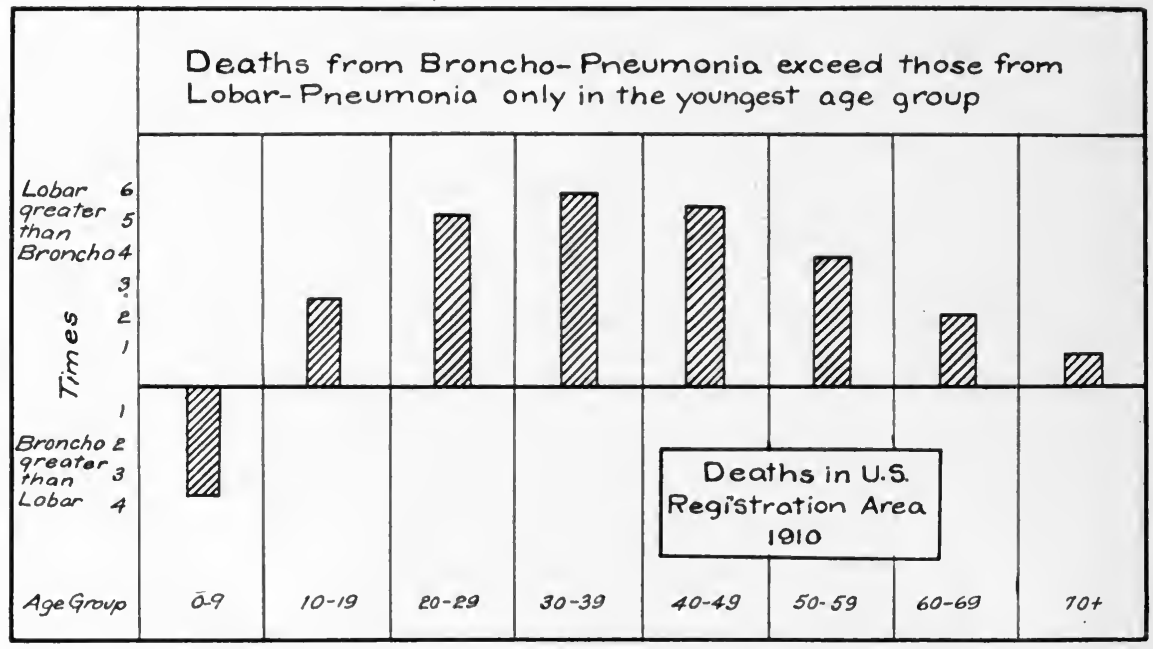

Fig. 16.

attached and forming chains. The diameter of the individual beads varies from 0.5 to 1 micron and the number of individuals in a chain is subject to great variation, usually running from four to twenty. Strains consisting of eight or more beads are usually designated as streptococcus longus, while the shorter chains are known as streptococcus brevis. It has not been shown that there is any difference in pathogenicity between strains forming long and those appearing in short chains; in other words, the length of the chain is apparently dependent upon environment and is not due to something inherent in the species. Streptococci stain readily with the ordinary anilin dyes and are usually gram-positive, though certain saprophytic strains may be found to be gram-negative. They are not soluble in bile and this is a feature by which they may be differentiated from pneumococci. 
Streptococei can be isolated from pus, sputum, and other secretions and excretions by plating on agar, especially agar containing dextrose. The streptococcus is aerobic, though it may grow anaerobically. Its range of temperature of growth lies between $15^{\circ}$ and $45^{\circ} \mathrm{C}$. It is destroyed by a temperature of from $60^{\circ}$ to $70^{\circ} \mathrm{C}$., depending upon the nature of the medium upon which it is growing. In dried sputum it will resist a temperature of $100^{\circ} \mathrm{C}$. for some minutes and at ordinary temperature, especially in the dark, will retain its viability and virulence for weeks. In blood, pus, and sputum kept at a low temperature and protected from direct light these organisms retain their virulence for some weeks, and possibly for months. The effect of chemical disinfectants upon these organisms depends upon the medium in which they are found. Unprotected, artificial cultures are killed in a few minutes by 5 per cent carbolic acid and 1 to 1,000 mercuric chlorid. When exposed to direct sunlight in thin layers they are quickly killed, but when surrounded by masses of albuminous material they retain their virulence for some days.

Streptococei are ubiquitous. Considering only those which feed upon man, they are to be found upon every square inch of the skin and in one form or another even more abundantly upon every exposed mucous surface. They are common, or at least are frequently found, in every part of the intestinal tract. They abound in water, milk, and soil. In small numbers they occasionally invade the blood tissues in healthy individuals. The streptococcus has been designated as an "opportunist" lying in wait on the cutaneous and mucous surfaces of man ready to enter any lesions which may open the gateway to richer pastures.

Scores of workers have devoted much time to attempts to classify streptococci. It has long been recognized that strains differ widely in virulence, and methods of identifying and separating strains are greatly needed. One of the first to study systematically the streptococcus was Marmorek who, in 1895, showed that all the strains with which he worked dissolved the red blood cells of infected rabbits, eausing hemorrhagic exudates. Moreover, he found that no strain which he employed would grow in the filtrate from an exhausted culture of another strain. In the third place, in his experiments it was shown that an antiserum against a given strain protected animals equally well against that and other strains. These investigations convinced Marmorek of the essential unity of streptococci and that all had hemolytic properties. Later it was shown by Bordet that streptococei taken from wounds and other infections in man dissolved red blood cells in vitro. This gave opportunity to determine whether or not all strains of streptococci are hemolytic. This test may be carried out either on solid or in liquid media. A common method is to streak the infected material over blood agar plates. If the strepto- 
coccus contained in the material is hemolytic, it dissolves the blood corpuscles and there appears a clear zone about each colony. By this method it has been found that streptococci may be divided into three groups. Strains of the first group are hemolytic; those of the second reduce oxyhemoglobin to metahemoglobin and in doing so cause a green color. Those of the third group have no action upon blood pigments. Strains of the first group are known as streptococcus hemolyticus, those of the second group as streptococcus viridans, and those of the third group as streptococcus nonhemolyticus. Of these groups the first is the most virulent. The second is possessed of less virulence but is capable of setting up inflammatory reactions and inducing focal infections.

In studying hemolytic streptococci and determining what strains belong to this group, some care must be given to the source of the blood used in the determination. It has been shown experimentally that the readiness with which blood corpuscles are dissolved by streptococci runs as follows: rabbit, man, horse, guinea pig, goat, and donkey. This means that strains which readily dissolve rabbits' corpuscles dissolve man's less quickly, etc. It is customary at the present time, and this is a matter of some importance, to use human blood in locating the strains of a streptococcus under investigation. Much time has been given to attempts to classify streptococci on the basis of fermentation of sugars. Nearly every known sugar has been used in this work, and while results reached are of scientific interest they are of but little if any practical importance. Most laboratory workers have found that all hemolytic streptococci ferment at least some of the sugars and they now limit the subdivisions by the action on lactose, mannite, and salicin.

Much work has been done in attempting to differentiate between strains of streptococci by agglutination, fixation, and anaphylactic tests. An animal is treated with successive doses of a given strain and after a time the blood serum of this animal is obtained and its agglutination titer on the strain employed and upon other strains is tested. Certain observers have also tested specific fixations. Davis has attempted to classify streptococci by anaphylactic reactions. He sensitized a number of guinea pigs with a certain strain and after a proper interval injected into these animals other strains. In a general way he found that animals sensitized with a hemolytic strain react to other hemolytic strains but do not react to nonhemolytic strains.

Rosenow has brought out a most interesting point in his work on tropism or the elective affinity of streptococci. He finds that a streptococcus taken from a suppurating appendix, for instance, and injected into an animal is prone to select the appendix of that animal as the locality for its future residence and activity. On this subject Rosenow has done a vast amount of work. He finds that streptococci obtained from 
erythema nodosum and injected into animals produce skin lesions in 90 per cent of the experiments, while streptococei taken from other localities produce skin lesions in only 2 pẹr cent. Gay speaks of Rosenow's work as follows :

"The specific reasons why localization of apparently similar organisms should take place now in one part of the body and now in another may be explained in two general ways. First, by a lowering of resistance of the affected part in the individual (locus minoris resistentiae) and secondly by specific affinity of the particular culture in question for a given tissue in the body. It is, of course, logically possible that both these factors may be operative in producing localization of lesions. Rosenow has been led to emphasize the organotropic effect of the bacteria rather than any particular diminished resistance in the part affected. In an elaborate series of experiments extending over several years, employing very large numbers of experimental animals, Rosenow has apparently been able to show that streptococei obtained from particular lesions and in all instances presumably the cause of those lesions have, when first isolated from the body, a particular affinity for the tissues whence they originated. Thus Rosenow and his coworkers have shown from the standpoint of etiology that streptococei are not only present in rheumatic fover, and in endocarditis, pericarditis and myocardial lesions as well as the joints affected in this disease complex, but also in such apparently diverse affections as appendicitis, ulcer of the stomach and duodenum, cholecystitis, erythema nodosum, herpes zoster, mumps, myositis, iritis, iridocyclitis. Each of the strains of streptococcus viridans isolated from one of these conditions will on injection intravenously in rabbits or dogs tend to localize in the same tissue and produce the same lesion in a relatively high percentage of animals as compared with the percentage of such lesions in animals inoculated with nonspecific strains of streptococcus."

Researches by Rosenow, Buerger, Rytenberg, and others, threaten to disturb seriously the whole structure of morphologic bacteriology. We refer to the question of mutation in bacterial species. Every bacteriologist is aware of the fact that in minor characteristics there are variations from time to time in well-defined species. A given organism may after long growth on certain food substances fail in ability to utilize a given food which nourished its ancestors. Ruediger found that hemolytic streptococci grown for two years on glucose broth no longer dissolved red blood corpuscles, and Anthony states that only 95 per cent of hemolytic streptococei remain true to type when earried through many generations. It is well known that practically all pathogenic bacteria vary more or less widely in pathogenicity from time to time and from generation to generation. When, however, Rosenow states that he has been able to transform 17 strains of streptococcus viridans into pneumococei and 21 strains of hemolytic streptococcus into viridans and then into pneumococei it seems that the stability of bacterial species is brought into question. We recognize the fact that the more closely we study a given species the more likely are we to find that it consists of varieties and strains which breed more or less true through many generations. This was our experience with the typhoid bacillus and we have now been compelled to recognize at least two varieties which are designated para- 
typhoid A and B, and we utilize these as well as the original Eberth bacillus in vaccination against typhoid fever. That there is more or less mutation in every form of life there can be no question. We also know that streptococci and pneumococci are large groups in both of which we find strains that give suggestions of having properties intermediate between the two, but that a typical streptococcus can be converted into a typical pneumococcus within a few generations is a suggestion that we are not ready to accept as a demonstrated truth. It is a popular saying that nothing lies like figures. The man who has given much time and energy to biologic experimentation might question this and say that nothing lies like experiments. Because a given species of streptococcus is injected into an animal and at autopsy we find a wholly different species, or even a pneumococcus, does not absolutely prove that we have found the exact cells or the descendants of the exact cells which we injected. Streptococei and pneumococei are too abundantly distributed not only in the outer world, but in the tissues of normal animals, for one to be sure that the bacteria isolated at autopsy are the descendants of those injected. There is another source of error which has been pointed out by Cole. The strain injected into the animal or planted in the new medium may in reality consist of two or more strains. One of these may be dominant in the material used, while this may entirely disappear and another come to the front or be the only representative growing in the new medium. It is now nearly 20 years since Vaughan first protested against the assumption that bacteria are low and simple forms of life and consequently unusually susceptible to wide variations in structure and function. Morphologically bacteria are unicellular and consequently low and simple in the scale of being, but, after all, the cell is the unit of life and as was shown by the researches of Vaughan and Wheeler, the bacterial cell is just as complex and complicated in structure and in function as is any cell in the animal body. Tubercle bacilli found in the bones of Egyptian mummies do not differ materially in size, shape, or chemical composition from those found today in the sputum of a consumptive; indeed, the tubercle bacillus can boast an unbroken and invariable ancestry of which no son of woman can boast. There is no reason, at least no accurate reason, for supposing that the streptococcus which fed upon the wounds of primitive man was radically different either in form or function from its descendant which is today feeding itself and begetting its kind at the expense of its human host.

Apart from the secretion by virtue of which the hemolytic streptococcus dissolves red blood cells, and which is known as hemotoxin, the streptococcus produces no toxin. Furthermore, there is no reason for believing that the hemotoxin plays any important part in the lesions 
induced in the body of man by the streptococeus. Some 25 years ago Marmorek labored earnestly to produce a serum which would prove of value in the treatment of streptococcic infections in man. Since his time many others have labored in the same field; in fact, these labors have been most strenuous and rather widely variable. Attempts to produce immune sera have been made along the following lines: single strain of streptococcus has been repeatedly injected into animals and the serum of these animals is characterized as monovalent.

Many strains from many disease conditions have been used in treating the animals and the serum thus obtained is called polyvalent. (3) Certain strain or strains are intensified in virulence as much as possible by frequent passage through some laboratory animal (usually rabbits or mice) and this highly virulent strain is used in immunizing animals. The results recorded by different investigators in the use of these sera in the treatment of streptococcic infections in man vary widely. These sera have been employed in the treatment of scarlet fever, poliomyelitis, streptococcic pneumonia, streptococeic sore throat and general streptococcic infections. Moser and Pirquet prepared a serum by treating animals with strains of streptococcus from scarlet fever. They claimed that this serum agglutinated streptococci from cases of scarlet fever and did not agglutinate streptococci found in other diseases. Like claims have been made by Rosenow and Mathers and Howell concerning serum obtained by immunizing animals against streptococci obtained from cases of anterior poliomyelitis. It has been pointed out by Zelenski and others that the blood serum of many healthy individuals agglutinates streptococei and that specific sera have no higher agglutination property than may be found, at least occasionally, with a serum obtained from healthy men. In our opinion, this is easily explainable since most men have at times without feeling any ill effects streptococei in their tissues and even in their blood. In a general way we can say that nothing very valuable has come from antistreptococcus sera. It seems to have been demonstrated that such a serum may protect mice and rabbits from minimum fatal doses of streptococcie cultures. Insofar as such sera have any beneficial effect it is not due to the presence in such sera of an antitoxin in any way comparable with that of diphtheria. Any good results that may come from the use of an antistreptococeus serum is probably due to increased phagocytosis, and this can never be raised sufficiently high to become a potent weapon in combat with virulent streptococcic infections.

Streptococcic vaccines have been largely used, much more frequently than they are justified either by scientific reasons or by practical results. It is true that in individual instances, especially in local infections, such as arthritis, otitis, and acne, brilliant results have from 
time to time been reported, but all the successes find their way into print while the failures, even the injuries done, are not so likely to be brought into the light. While the streptococcus, so far as we know, produces no soluble toxin and consequently can give rise to no antitoxin, the streptococcal cells do contain the protein poison and the conscientious physician will be careful about the doses of this which he injects into his patient. We would not discourage experimentation along these lines; indeed, an antitoxin, active and efficient in the treatment of streptococcic infections, would be a most valuable addition to the armament of the physician in his combat with disease and a boon to his patients, but man is not the proper animal upon which these experiments should be made.

In this chapter we are especially concerned with the part played by hemolytic streptococci in the causation of diseases of the respiratory organs. In the first place, it is desirable to know to what extent hemolytic streptococei are found in the upper respiratory passages. Many investigators have reported upon this subject and their findings run all the way. from zero to one hundred per cent. Ruediger found hemolytic streptococci in 59 per cent of normal throats. Smillie puts the number, including individuals with more or less abnormal conditions of the throat, at 50 per cent. We are justified in concluding that even in normal throats, which are rare, hemolytic streptococei may be present and the percentage increases with departures from health in the tissues.

Both in England and in this country since 1875 there have been numerous epidemics of septic sore throat in which the hemolytic streptococcus has been believed to be the active causative agent. In Boston in 1911 there was a sharp outbreak with 1,034 reported cases and 38 deaths. In December of the same year, according to Capps and Miller, there were 10,000 cases in Chicago in a few weeks. In 1912 there were between one and three thousand cases within a few weeks in Baltimore and about 30 deaths. This sore throat differs largely from ordinary tonsillitis and is marked by extreme prostration and is likely to be followed by many and varied severe complications to which death is due. In some of these epidemics there was good reason for believing that the streptococcus was distributed in the milk supply. At first it was believed that the virulent streptococcus was of bovine origin, because in some of the dairies supplying the infected milk mastitis was found among the cows. Even the best of milk contains streptococci, but usually these are of the nonhemolytic variety, although hemolytic streptococci are occasionally found in perfectly good milk and in that, the use of which is not followed by any disease. Later, and as a result of more exhaustive research, it was believed that the streptococcus of epidemic 
sore throat is not of bovine origin, but is a human strain which comes originally from the throats of milkers although it may be accidentally transferred to the udder of the cow. It is possible that a human strain of the hemolytic streptococeus may be greatly increased in virulence by passage through the tissues of the udder of the cow. There are questions connected with streptococcic sore throat which are awaiting solution.

During the wide and fatal prevalence of streptococcic pneumonia in our camps in 1917-1918 the question arose as to the possibility of strains of streptococci being increased in virulence by passage through horses. There is a disease quite common among these animals, known as shipping fever, or equine influenza, which was shown many years ago by Schutz to be due to a streptococcus and which often terminates in a fatal bronchopneumonia. In 117 cases of this disease in horses Mathers isolated hemolytic streptococci not only from the nasal discharges during life but from the lungs after death. At Camp Taylor, Alexander found a hemolytic streptococcus in nasal swabs from horses which were suffering from bronchopneumonia. Nothing definite came out of the attempt to trace relationship between streptococcic pneumonia in horses and in man. We may say that it is not likely that there was any such relationship, because streptococcic pneumonia was not more frequent among soldiers who were brought in close contact with horses than among those who seldom saw one.

Lamb, in his report on influenza-pneumonia at Camp Cody in the autumn of 1918 makes the following interesting statement:

"An unusual observation was made in connection with the guinea pigs housed in a small building near the laboratory. Soon after the epidemic of influenza reached the camp, these animals began to die and autopsy revealed unmistakable signs of pneumonia. The mucosae of the trachea and bronchi were deeply injected and covered with a glairy serofibrinous exudate. Cultures and smears from this exudate and from pieces of lung tissue revealed pneumococei in large numbers, and a few streptococei and small gram-negative bacilli, identical in every way with $\mathrm{B}$. influenzæ. The guinea pigs were sick from two to four days before dying. During this time the animals trembled with chills and sat with ruffled hair in the corners. Respiration was rapid and wheezing. Finally the animal would fall into a stupor which gradually deepened into death. All the pigs on hand, 30 in number, died within three weeks. A new batch of 50 was received from El Paso, four of which were dead on arrival, but the others were apparently healthy. These were placed in a room which had not been used for several months and which was thoroughly cleaned and supplied with fresh hay, changed daily. A heater was placed in the room and every effort was made to keep this new batch clean and healthful. Everything went well for about two weeks, when one pig was found dead, and within two weeks more all had died.'

We have already referred to the observation made by Cole that when measles cases are first brought into the hospital only a small percentage of them carry in their throats hemolytic streptococei but after from eight 
to sixteen days in the hospital, the percentage increases to more than fifty. Similar observations were made in many of the camps and measures were taken for segregating streptococcus carriers among the measles eases. At Camp Taylor it was found that 36.8 per cent of measles cases carrying streptococei developed pneumonia, while of those in whom streptococei were not found only 6.4 per cent developed pneumonia. At Fort Sam Houston, Cumming and others found that 49 per cent of those who carry during measles hemolytic streptococci in their throats develop complications, mostly bronchopneumonia. Of all the chiefs of medical service in the base hospitals in 1917-1918 no one, so far as we know, failed to recognize the importance of the hemolytic streptococcus as a causative agent in the bronchopneumonia which so distressed the medical officers and so greatly increased the death rate.

Experimental Pneumonia.-In 1888 Gamaleia injected pneumococcus cultures directly into the lung tissue of dogs through the chest wall and obtained pneumonic lesions. Two years later Tchistovitch resorted to intratracheal injections and succeeded in producing pneumonia in seven out of nineteen experiments. Later Monti, using intratracheal injections in rabbits, produced pneumonia in all instances. These experiments were for many years disregarded or dismissed with the statement that, while inflammatory lesions might have been induced, there was no proof that an acute lobar pneumonia had been established. In 1905 Wadsworth induced pneumonia in 11 rabbits by injecting intratracheally pure cultures of pneumococcus of moderate virulence. In 1912 Lamar and Meltzer concluded a report upon experimental pneumonia as follows:

"By intrabronchial insufflation of pure cultures of pneumococcus in dogs, we produced experimental pneumonia successively in 42 cases with a mortality of at least 16 per cent. The fatal cases resembled closely lobar pneumonia in man. In the nonfatal cases, the pathological and bacteriological findings were again in accord with the findings in man. Clinically, however, the cases of nonfatal experimental pneumonia run a milder and shorter course than in man. In a few instances, lobar pneumonia has been produced experimentally also with the pneumococcus mucosus and with Friedländer's pneumobacillus. The anatomical findings in these experiments have shown some characteristics agreeing with the findings in the pneumonias of man produced by these organisms. The quantity of the injected culture seemed to have a definite influence upon the outcome of the disease; in the fatal cases larger quantities of the cultures had been injected. The animals were neither selected nor prepared in any manner. The experimental success did not, therefore, appear to depend upon the degree of resistance of the individual hosts. It is suggested that the uniformly successful results of the experiments were due to the obliteration of a large number of bronchi by the injected culture, through which mechanical effect a favorable opportunity was provided the pneumococci to develop and display their pathogenic activities consisting in the calling forth of a characteristic local, more or less effective, widespread, inflammatory reaction of the lung tissue."

In 1913 Wollstein and Meltzer produced in dogs by intrabronchial in- 
sufflation of pure cultures of the streptococcus and of the Pfeiffer bacillus a bronchopneumonia which differs materially from the lobar pneumonia induced by similar treatment with the pneumococcus. They say:

"However, further investigation may show that the differences in the nature of the lesion are due rather to degree of virulence of the causative microorganism than to differences in the species; that is, that different lesions may possibly be produced by organisms of the same species, provided they possess different degrees of virulence. Further experimentation may also show that the condition of the animal and of the affected organ which, in the onset and development of the pneumonic disease, is perhaps, unimportant, may be the leading factor in determining the course and outcome of the disease."

The above-mentioned experiments show conclusively (1) that pneumonia may be induced in the lower animals by either the pneumococcus, the streptococcus, the Pfeiffer bacillus, or the Friedländer bacillus; (2) that the pathway of infection with these organisms is a descending one from the trachea into the bronchi and into the lung tissue. All attempts to induce pneumonia by injection subcutaneously, intravenously, and intraabdominally have failed. Furthermore, the above experiments justify the contention that pneumonia is not a specific disease in the sense that we use this term when we speak of measles, diphtheria, whooping cough, typhoid fever, and scarlet fever; i.e., pneumonia may be eaused by any microorganism which is capable of finding its way along the path above indicated and reaching the lung tissue and there setting up inflammatory processes.

Sewall suggests that atelectasis may play an important rôle both in natural and experimental pneumonia, and he asks whether atelectasis is a conservative or a pathologic phenomenon in the development of pneumonia.

Staphylococcus Pneumonia.-Although so far as we know experimental pneumonia has not been induced by the staphylococcus, bacteriologic examination of the lungs after death sometimes shows pure cultures of the staphylococcus pyogenes aureus. In a few of the camps in the fall of 1918, notably at Jackson and Syracuse, the staphylococeus was an important agent in the causation of pneumonia. Chickering and Park state that in 207 autopsies made at Camp Jackson, over 50 per cent showed pure cultures of staphylococcus, while the Pfeiffer bacillus was present in 16 per cent. Staphylococcus pneumonia is often exceedingly rapid in its progress and highly fatal in its results.

Spirochetal Pneumonia.-In 1904 Castellani, while on the Island of Ceylon, was consulted by a native who for two years had expectorated a bloody sputum. Test for the tubercle bacillus was negative, but the sputum was found to be filled with spirochetes. Since that time this infection has been reported from nearly every part of the world. It seems to be common in the tropies and sporadic in the temperate zone. A 
few cases have been found in the United States. This spirochete was first detected in Asiatic and African troops in France during the war, but French soldiers brought in contact with these auxiliaries contracted the disease. It is highly contagious, as is shown by the fact that men while examining patients and infected sputum in a laboratory at Khartoum contracted this disease. In the fall of 1918 Loygue in examining the sputa of 27 cases of supposed influenza found the spirochete in 14 . This disease may be acute, subacute, or chronic. As a rule the mortality is not high and when a fatal issue results a bronchopneumonia is found. In rare instances gangrene of the lungs results. The spirochetes may be detected and are best colored by the Leishman or the Giemsa stain. According to Castellani these organisms may be divided into the following groups: (1) Thick individuals from 15 to 39 microns in length with irregular coils which vary in number but are never numerous. (2) Individuals resembling S. refringens of Schaudinn and possessing graceful coils and pointed extremities. (3) Thin delicate spirochetes with numerous small uniform coils and tapering ends. This seems to be the most common type. (4) Extremely delicate organisms with very few irregularly shaped coils. This disease is likely, especially in its chronic form, to be confounded with tuberculosis. The latter disease is excluded by the absence of the tubercle bacillus and failure to infect guinea pigs. A résumé of the literature of this disease has been recently (1920) given by Salomon.

Typhoid Pneumonia.-As is well known, typhoid fever frequently terminates in a pneumonia. In these cases bacteriologic examination of the lung tissue usually shows typhoid bacilli either in pure culture or mixed with pneumococei or streptococei, one or both. However, just now we are not referring to the terminal pneumonia in typhoid fever. There are a few cases of this disease in which at the onset pneumonia is more marked than the typhoid fever and the former may mask the latter. Osler has reported cases of this kind in his own practice and in at least one of these instances typhoid fever was not recognized until autopsy showed the characteristic lesions. It is not infrequent in the beginning of typhoid fever for respiratory symptoms to be more or less in evidence. This is one of the arguments advanced by those who claim that this disease is often acquired by inhalation. We have discussed this in the chapter on typhoid fever.

Plague Pneumonia.-In this highly fatal disease the plague bacillus solely is responsible for the pneumonia, notwithstanding the fact that in the lung tissue pneumococci and streptococci may be found. Plague pneumonia belongs to the catarrhal type and the alveoli are often found filled with leucocytes. This form of pneumonia is discussed in the chapter on plague. 
Anthrax Pneumonia.-There can be no doubt that anthrax in man may be acquired by inhalation. What is known in England as "wool sorters' disease" is anthrax pneumonia. This disease has been induced in the lower animals by insufflation of anthrax spores mixed with lycopodium or pulverized charcoal. In this experiment only a small percentage of the animals died from pneumonia; in the majority the disease was a septicemia and the bacilli were found abundantly in the circulating blood.

Traumatic Pneumonia.-It occasionally happens that an acute pneumonia arises from injury to the chest wall. There may be fractured ribs resulting in the infliction of direct wounds in the lung tissue or there may be no fracture and not even a bruise on the outer surface. In Germany traumatic pneumonia figured rather prominently in insurance suits under the Workmen's Compensation Act. It seems proper to include under this head those pneumonias which result from wounds through the mucous membrane of the air passages, such as result from poisonous gases. In cases of this kind the bacteria found in the sputum during life and in the lungs after death depend upon those that are present in the upper air passages and which may be inhaled after the wounds have been received.

Other Pneumonias.-Some years ago Flügge stated:

"We are already acquainted with pneumonias which are caused by aspergillus and actinomyces. It is, a prior not improbable that also among bacteria there are several other species which can set up pneumonia."

Notwithstanding the evidence there have been those in recent years who have held that pneumonia is a specific disease. Netter makes the dogmatic statement: "There is no pneumonia without pneumococci." He evidently from the context means that there is no lobar pneumonia without pneumococei. But even this is not true. Furthermore, the pneumococcus does not always confine its operations to the production of typical lobar pneumonia. Some years ago Holt reported that in 270 cases of pneumonia in children under five years of age $75^{\circ}$ per cent were bronchopneumonia and still the causative agent was the pneumococeus.

That the more alert physicians of the eighteenth century recognized that pneumonia might be primary or secondary and that there are different forms of this disease is shown by the following quotation from Huxham, who wrote in 1750:

"A peripneumonia, in the largest sense, is a disease so common, either as an original malady, or consequent to some other, that its nature should be studied by every physician; as it is a morbid affection of one of the principal organs of life, is very frequently attended by the utmost danger, and requires very different treatment in its different stages. Besides there are very different degrees, I might say species of this disease, which demand a particular attention, and a method of cure peculiarly adapted to each." 
Predisposing Causes.-The death rate from pneumonia is constantly higher in eities than in rural communities. The following figures taken from the mortality statisties show that the excess of deaths in cities over those in rural populations has been, since 1911 at least, fairly constant and measures about 50 per 100,000 .

\section{TABLE VII}

Deati Rate from Pneumonia (All Forms) per 100,000 Population Since 1911

\begin{tabular}{ccc}
\hline & \multicolumn{2}{c}{ REGISTRATION STATES } \\
YEAR & CITIES & RURAL PART \\
\hline 1911 & 153.7 & 105.0 \\
1912 & 152.5 & 103.3 \\
1913 & 156.2 & 101.6 \\
1914 & 149.5 & 99.6 \\
1915 & 155.8 & 105.9 \\
1916 & 165.1 & 111.3 \\
1917 & 177.8 & 120.9 \\
\hline
\end{tabular}

The excess of pneumonia in cities is not due to any differences in age distribution of the population; in fact, pneumonia is indicated to be greater in the eity at all ages in comparing the City of Detroit with the State of Michigan for the year 1910. (See p. 99.)

This undoubtedly is due to the greater chance of infection in cities with the numerous microorganisms which eause the pneumonias. Other things being equal, it is probably roughly true that the greater the density of population the higher are both the morbidity and the mortality from the pneumonias. In our cantonments during the war the density of population and the intimacy of personal contact were greater than in any eity in the country. Add to this the fact that these cantonments were filled largely, some of them more than others, with highly susceptible men from rural districts and the explanation of the high death rate from the pneumonias among our soldiers is not difficult. Moreover, among the soldiers the death rate from the pneumonias was much higher in training camps where the men were densely crowded together than it was on the battle fields of France where the troops were more scattered. These observations are not isolated ones or peculiar to our own people, or to the late war. They are equally true of all armies at all times. It is, so far as we know, universally true that in the mobilization of armies both the morbidity and mortality from the pneumonias are increased. Men from crowded cities bearing in their respiratory organs many kinds of pathogenic bacteria to which they have acquired a high degree of resistance seatter these bacteria among their highly susceptible comrades from the rural districts. Fire and tinder are brought together and the conflagration necessarily results. It must not be un- 
derstood from what we have said that density of population is the only factor determining the prevalence of the pneumonias. It is important, as we have seen, to determine the relative number of susceptibles in the crowd. It is also essential before there can be a pneumonia epidemic that the pathogenic organisms which cause these diseases be introduced. Given the conditions which we have indicated, in our opinion there is today within the knowledge of man no procedure which would prevent pneumonia from becoming epidemic. If a vaccine as effective as that employed in the prevention of smallpox be discovered, then, and not until then, can we hope to mobilize great armies without increasing death rates from the pneumonias. Under ordinary conditions the pneumonias reap their richest harvests at the two extremes of life. During the first year there is only one other disease, infantile diarrhea, which is more fatal. Among the aged and those suffering from chronic diseases pneumonia is the kindly friend who, having prepared the bed for the body worn out with the work and worry of life's short day, administers the soporific which induces the sleep of the eternal night. On the whole, the old man who is rowed across the Styx by pneumonia is not compelled to pay a heavy toll in pain. Among the robust and vigorous of all ages pneumonia is the cowardly assassin who, watching for every ebb in the flow of life's forces, strikes at a vital part at the most opportune moment. It has long been a matter of observation that epidemics are most fatal among the most virile. An old writer, discussing typhus fever in England and Ireland, said that death went abroad among the community picking out the lustiest and the handsomest, even as you or I would go through a flock of sheep. The Typhoid Commission in 1898 found that more than 90 per cent of the men who developed typhoid fever had no preceding intestinal disorder. In the World War in the midst of the great epidemics of influenza and pneumonia it was a common observation that the most robust were most severely stricken and supplied the largest percentage of fatalities. Vaughan has offered an explanation of the high fatality among the physically robust in epidemics. He contends that the infectious diseases owe their lesions and symptoms to the destruction of the invading microorganisms by secretions from the body cells. The strong and vigorous man pours out these specific secretions in such abundance that enough bacterial cells are split up within a short time to set free a sufficient amount of poison to lead to a fatal result. Nature overreaches herself in the combat. Nothing worse could happen to a man with typhoid fever than the sudden disruption and destruction of all the bacterial cells in his body. Certainly this would be true if such disruption should occur after the invading cells had accumulated in sufficient numbers to furnish a fatal dose of the protein poison on their disruption. In epidemics the body cells of the strong 
and vigorous man go into action with energy and such a man either recovers promptly, or is more likely to die than his less robust comrade whose body cells destroy the invading organisms less abundantly and less rapidly. Epidemic diseases certainly do not improve the race by killing off the unfit, but like war, they destroy the flower of the race and in so doing rob future generations of the inheritance of certain virtues. Victory in combat with epidemic diseases is not always to the strong. .

The Phenomena of Pulmonic Infections.-Without going into the extensive literature of the subject we may say that bacteriologists generally agree on the following points: (1) In health all bacteria contained in inspired air are removed mechanically or killed in passing through the upper air passages. (2) The secretion of the nasal mucous membrane is markedly bactericidal. (3) Below the glottis the respiratory tract is in health practically sterile. (4) Expired air contains no bacteria either in health or in disease. (5) Bacteria which pass downward through the glottis in small numbers are rapidly destroyed. (6) Bacteria which pass downward through the glottis in numbers and are eapable of growing and multiplying in the lower part of the respiratory tract or in the parenchyma of the lungs may cause disease.

Hastings and Niles close a study of the bacteriology of the respiratory tract with the following conclusions:

“(1) In our examinations, only 38 per cent of the infections of the respiratory tract below the glottis were pure, and this percentage was reached only by earefully following Kitasato's method of handling sputa. (2) Lobar pneumonia may produce sputum free from pneumococei, and may undoubtedly be caused by organisms other than the pneumococcus. (3) There is found a marked yearly variation in the organisms which excite inflammation of the respiratory tract. (4) Micrococeus catarrhalis is usually considered a common secondary invader; but it may, and probably frequently does, assume pathogenic properties."

Recognizing that in health practically no living bacteria pass downward through the glottis, it is important that we give attention to the agencies in the upper respiratory tract which are concerned in the removal of bacteria from inspired air. This function is believed to be largely mechanical, aided somewhat by the bactericidal activity of the secretions of the parts. So far as we know the only study of the immunologic function of the nasal cavity has been the work of Sewall and Powell. These investigators have by their painstaking and thorough researches opened up practically a new field in the study of immunologic problems connected with the respiratory diseases. They have demonstrated that guinea pigs may be sensitized to horse serum by local application to the mucous membrane of the nose. They close one of their papers on this subject with the following summary:

"(1) Normal guinea pigs treated by four to six instillations of horse serum into the nose on alternate days become either hypersensitive or refractory to an intravenous 
injection of 0.38 c.c. of serum given 16 days after the last instillation. If the amount of serum in each instillation is as much as 0.2 e.c., anaphylactie death is caused by the toxie injection. If the amount of serum in each instillation is redueed to 0.04 e.c., the first intravenous injeetion is without marked effect, and a second injection and subsequent injeetions of the same amount of antigen are well tolerated in about half the eases. (2) The effect produced by a given dose of serum, whether protective or anaphylaetic, depends probably upon the extent of eontact with the mucous membrane of the nose. (3) Guinea pigs which, after nasal treatment, have beeome tolerant to a definite maximum intravenous injeetion of antigen appear to increase the degree of their tolerance, at least up to a resting period of more than four months. The same does not hold in animals immunized by the peritoneal route. (4) The first two or three instillations of a series probably determine the biologie eharacter, whether of hypersensitiveness or hyposensitiveness, of reaction towards the serum. (5) It is probable that, eontrary to the ease in parenteral sensitization, hypersensitiveness and protection, respectively, set up by nasal instillations and not followed by parenteral injections, gradually disappear in about 50 to 100 days. (6) We have failed in attempts to eliminate hypersensitiveness due to subeutaneous injeetion of serum, by nasal instillations which would protect the normal animal from the development of anaphylaxis. (7) It is suggested that the prineiples of prophylaxis evolved under these relatively simple eonditions should be applied in the study of infectious diseases."

Elsewhere Sewall reports that he has not been able to confirm Rosenau's statement that normal guinea pigs kept in stable air become sensitized to horse serum, but he has found that guinea pigs sensitized to horse serum may be desensitized by sojourn in a horse stable. Desensitization thus secured is transient and continues for only a few hours or at most one or two days. On this point Sewall says:

"Suffice it to say that a large proportion of guinea pigs sensitized subeutaneously to horse serum were desensitized by exposure to the air of a horse stable. There was a very obvious relation between the pungeney of the stable odor and the latent period of anaphylaetie disturbanee. When the smell was most intense the nervous symptoms of anaphylaxis eame on almost immediately."

Sewall has demonstrated that by the application of horse serum to the nasal mucous membrane of guinea pigs these animals may be sensitized to horse serum. The sensitization thus induced may be local or general. Calling attention for a few moments to the above given conclusions as stated by Sewall, we may say that in our opinion the different results obtained by the instillation of large and small doses of serum is a difference in degree rather than in kind. With the large doses a high degree of general sensitization was induced and when subsequently the horse serum was administered intravenously the proteins contained in it were split up so promptly that death resulted. In case of the small amounts of serum the proteins of the serum subsequently injected were split up slowly and no visible effect followed. That the serum injected intravenously in the latter set of animals did not remain undigested in the blood, is in our opinion, demonstrated by the fact that a second spaced injection in about half the cases was well tolerated. In his con- 
clusion No. 5 Sewall states a very important fact, and that is, that the sensitization induced by nasal instillations gradually disappears in from 50 to 100 days, while that induced by parenteral administration continues throughout life. If sensitization be an important immunologic phenomenon, and we believe that none now contest this statement, the researches of Sewall and Powell are at least highly suggestive and explanatory of the phenomena of pneumonic infections. We do not wish to go into a theoretical discussion of these points and we leave the reader to draw his own conclusions; and we hope that further work along this most promising line will be continued.

That certain infectious diseases may be induced by application of their specific viruses to the mucous membrane of the nose was apparently known and practiced by the Chinese in their methods of protecting against smallpox before the Christian era. In 1910 Dserzgowsky inserted plugs of cotton moistened with diphtheria toxin into his own nose and those of horses and claimed that by this method he induced both local and general immunity. This has been confirmed by Blumenau who, inserting cotton plugs moistened with diphtheria toxin diluted to one-third its strength into the nostrils of children, after 20 such treatments, detected antitoxin in the blood. These experiments need to be repeated since we now know that many children normally have antitoxin in their blood.

Some years ago Friedberger announced that he had been able to produce aseptic pneumonia in sensitized guinea pigs by inhalations of serum. Schittenhelm was unable to duplicate Friedberger's experiments, but Ishioka sprayed serum directly into the trachea of sensitized guinea pigs. Out of 42 animals thus treated two died at once in anaphylactic shock, while in a few there was induced an interstitial pneumonia with emphysema. In most of these animals there resulted a true lobar pneumonia as shown by patches of infiltration, some of which at least involved whole lobes. On this point Sewall makes the following remark:

"The statements of Friedberger, Ishioka, and Busson in regard to pneumonic changes in the lungs of sprayed animals were, to a degree confirmed. Such lungs at necropsy frequently revealed air-free hemorrhagic areas of limited extent. The affected portions when removed would sink in water, but they were soft and not resistant as in pneumonic consolidation. The postmortem change was by no means constant among sprayed pigs and was also found in animals dying from toxic injections which had not been sprayed at all.",

Evidently this matter needs further investigation.

Nonspecific Immunity in Pulmonic Infections.-We have already referred to the fact that in our camps during the war the soldiers from rural districts suffered more than their urban comrades in both morbidity and mortality from pneumonia. Did the city boy possess a specific immunity for each microorganism which may cause this disease? 
This seems highly improbable, but we accepted it as the best explanation until the influenza epidemic came in the fall of 1918. In the midst of this it was observed that the difference between the rural and the urban continued. Here was an infection new to both but it discriminated in the same way as did the older infections. In 1905 Vaughan, Jr., made a series of experiments upon rabbits and guinea pigs trying to induce immunity or establish tolerance by means of the poisonous protein which is common to all bacteria, and indeed to all true protein substances. He found that by carefully increasing the dose, starting with much less than the minimum fatal quantity and gradually inereasing the amount, he could establish in animals a small degree of tolerance to the protein poison. It mattered not what the source of the protein poison might be; whether it came from egg-white or the typhoid bacillus, it had the same effect. This tolerance was not high and the investigator chose to designate it as a tolerance and not as a form of immunity. Having established in animals this tolerance he found that such animals bore minimum fatal doses of typhoid and colon bacilli indifferently; in other words, the tolerant animals had an inereased resistance to both colon and typhoid bacilli. We make the following quotation from the original :

"From the foregoing experiments it becomes evident that animals which have been treated with the poisonous protein of the colon bacillus acquire a certain degree of immunity to the living germ. *** In the case of an animal which has been treated with poisonous protein and has subsequently received a dose of the living bacillus which would surely have proved fatal for a normal animal, the symptoms induced are identical in every respect with those which followed a nonlethal dose in an untreated animal. This is a very important fact and one upon which we have laid much stress. *** In both instances there is no appreciable fall in body temperature until from six to eight hours after inoculation. At this time the minimum temperature has been reached in each case, and within from ten to twelve hours it has returned to normal. The similarity of the symptoms in the two instances leads us to believe that in all probability we are dealing with an immunity which is identical in character with that which is usually spoken of as natural immunity. This idea has been upheld by the fact that we have been able to obtain from egg albumin and peptone poisonous substances which resemble the poisonous part of the colon bacillus in their action, and by the injection of single nonfatal doses of which it is possible to obtain the same transitory immunity to the living colon bacillus as is observed after the injection of the colon poison. That this poisonous group is common to certain bacteria and other protein bodies is not improbable, and this would furnish an explanation not only of the increased resistance to eertain bacterial infections occurring in animals treated with albumin and peptone, but of some phases of natural immunity as well.",

In our opinion it is evident that a nonspecific bacterial immunity, never reaching a degree comparable with that attainable in toxic immunity, but certainly affording protection beyond that usually present, is secured by repeated introduction of the protein poison into the body. Sewall has shown that guinea pigs may be immunized by the proteins 
of blood serum applied directly to the mucous membrane of the nose. That bacterial proteins may be absorbed from the same mucous membrane is well known. In our own laboratory it was found some years ago in the preparation of typhoid cellular substances that when it was necessary to grind the bacterial cells in a mortar the one who did the grinding, although wearing a mask, was in every instance acutely poisoned by the inhaled dust. This occurred to each new grinder once, after which he proved to be immune. Proteins absorbed from the mucous membrane of the upper air passages, whether they be those of blood serum or those of bacterial cells, are split in the body into poisonous and nonpoisonous parts and to the latter a certain amount of tolerance is established. This tolerance is nonspecific and is as effective against one organism as another, although there may be some difference in the degree of sensitization established at the port of entry. We mean by this that we would expect nonspecific immunity to be greater and more effective between those organisms which infect through the respiratory organs than it would be between these and those that infect through the alimentary canal. With these ideas we shall briefly review the epidemic of influenza, with accompanying pneumonia, that occurred at Camp Shelby. The division occupying this camp, consisting of National Guardsmen who had previously had some experience as soldiers, assembled at Hattiesburg, Miss., in October and November, 1917. In April, 1918, a well-defined characteristic epidemic of influenza appeared in this division. It will be understood that these men had been in camp since the preceding November and had become in an immunologic sense seasoned soldiers. When the influenza epidemic came the total strength was about 26,000 . There were less than 2,000 sick enough to be sent to hospital, while it is the testimony of the medical officers of the division that practically everybody was ill. This epidemic of influenza among 26,000 men was followed by only six cases of pneumonia, three of which died.

When the more virulent influenza struck Shelby in the fall of 1918 the men who had gone through the light attack of the preceding spring and had thereby acquired specific immunity were not to any recognizable extent affected and the fall epidemic was confined to recruits in the camp. We explain these facts by supposing that during the winter of 1917-1918 the soldiers of the Thirty-Eighth Division at Camp Shelby, living in a crowded condition, acquired a nonspecific tolerance or immunity which enabled them to easily withstand the epidemic of April. In passing through the April epidemic these men acquired a specific immunity which made them resistant to the more virulent form of influenza which came in the fall. In our opinion nonspecific immunity or tolerance, whichever we choose to call it, enables the 
individual to withstand a newly introduced infecting organism. It does not protect the individual from infection with this organism, but it gives to the individual increased resistance. He becomes infected, suffers lightly, and recovers in the possession of a specific resistance.

We shall compare influenza and pneumonia at Camp Shelby with the same diseases at Camp Sherman. When the influenza reached Camp Sherman on September 24, 1918, the strength of the division was 26,000 , the same that constituted the division at Shelby in April, 1918. Over 80 per cent of the men at Sherman were from Ohio, Tennessee, Pennsylvania, West Virginia, Alabama, Wisconsin, and Louisiana, and more than 46 per cent of the total strength had been in service less than one month. In this division, largely composed of raw soldiers, 33 per cent of its personnel were severely stricken with influenza, 6.6 per cent had pneumonia, and 3.3 per cent died. Fifty per cent of the pneumonia cases terminated fatally. The influence of the recruit is seen from the fact that of the first 4,269 cases of influenza 69 per cent occurred in men who had been less than one month in service. Although the recruit made up only 46 per cent of the population, he contributed 69 per cent of the admissions to hospital. At Sherman, bacteriologic studies showed no evidence of association between the Pfeiffer bacillus and either the influenza or the pneumonia. Early in the epidemic cultures were made from the throats of 2,500, at that time apparently healthy. men, and the pneumococcus was found widely prevalent. So rapidly were the men stricken that within a short time every available bed in the camp was filled and it was necessary to provide an annex of more than 400 beds. Smears and cultures of sputum from influenza patients uniformly exhibited the pneumococcus as the predominating organism and this was quite constantly Type IV. Swabbings from the throat and nasal pharynx exhibited pneumococcus in 54 per cent and hemolytic streptococcus in 4 per cent of all examined. In none of these cases was the influenza bacillus demonstrable. After the development of pneumonia the sputum showed pneumococcus of Type IV in 80 per cent of the cases. Cultural conditions were suitable for the propagation of Pfeiffer's bacillus, but in no instance was it detected. Blood cultures were made from 100 patients after the recognition of a complicating pneumonia. In only six per cent of these were growths obtained and in all Type IV was present. No other organism was detected in the blood cultures, the remaining 94 per cent being sterile. In cultures made at autopsy from various thoracic tissues and fluids the pneumococcus was found in 53.3 per cent and the streptococeus hemolyticus in 43.7 per cent. On being typed, the pneumococeus from autopsy cultures was classified as twenty-five per cent 'Iype III; 75 per cent Type IV. In only one instance were both pneumococcus and hemolytic streptococcus encountered in large numbers in the same case. 
We have contrasted Camps Shelby and Sherman as illustrating the difference in behavior of epidemics among seasoned and unseasoned soldiers. "We do not wish to leave the impression, however, that the low death rate in the Thirty-Eighth Division at Shelby was entirely due to the fact that the soldiers were seasoned men, neither do we wish to imply that the only factor in the high death rate at Sherman was the large percentage of raw soldiers. There was at Sherman widely distributed in the throats of the men a highly virulent pneumococcus when the influenza struek camp. We have no reason for suspecting that a similar condition existed at Shelby either in the spring or fall of 1918.

Love and Davenport have ealled attention to the fact of the greater prevalenee and mortality of infeetious diseases among rural compared with urban soldiers; in fact, they have demonstrated this mathematically by the method of multiple correlation. This finding holds good not only for measles and mumps-diseases which the urban recruits had probably had in ehildhood, but also for pneumonia, cerebrospinal meningitis, influenza, and scarlet fever, from which the majority had probably not suffered. This faet negatives the theory that urban recruits were less susceptible to the specifie infections because they had previously been subjected to each of them. There is one point in this connection which needs more careful study. We have already called attention to the fact that the death rate from the pneumonias in our urban population runs quite evenly about 50 per 100,000 higher than among the rural population. Undoubtedly the crowded life of cities has led to the elimination of those most susceptible to respiratory infections. Some mathematician should be able to find out whether the higher death rate from respiratory diseases in eities in times of peace compensates or balances in any way the higher death rate from these same diseases among rurals when armies are mobilized.

In our chapter on measles we call attention to the fact that quite a hundred years ago Watt, of Glasgow, thought the elimination of smallpox by vaceination among ehildren increased the death rate from measles in infaney and ehildhood; in other words, he had the idea that a child who had survived smallpox was for that reason more resistant to measles. He did not mean that the child who had had smallpox would not acquire measles on exposure, but that it was less likely to die from measles, having been fortified against the infection by its previous experience with smallpox. If there be anything in this, it of course means nonspecifie immunity or tolerance. Vaughan (Warren) and Sehnabel found that among 90 soldiers with bronchopneumonia who had had no previous history of speeific infections the mortality was 47 per cent, while among 243 who had averaged barely two previous infections the death rate was under 35 per cent. The general mortality among 234 cases of lobar 


\section{CAUSES oFDEATH AMONG ALLTROODS in U:S.}

\section{PERCENT OF TOTAL DEATHS BY SEASONS, 1917-18.}
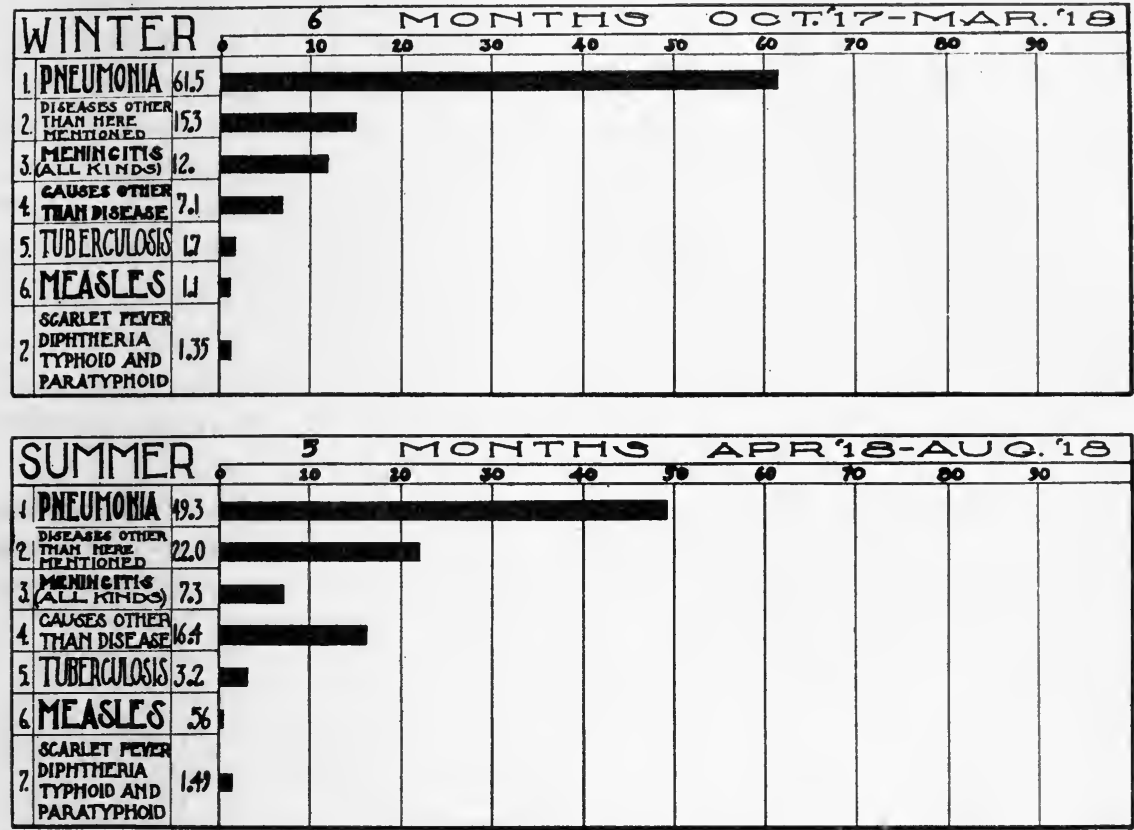

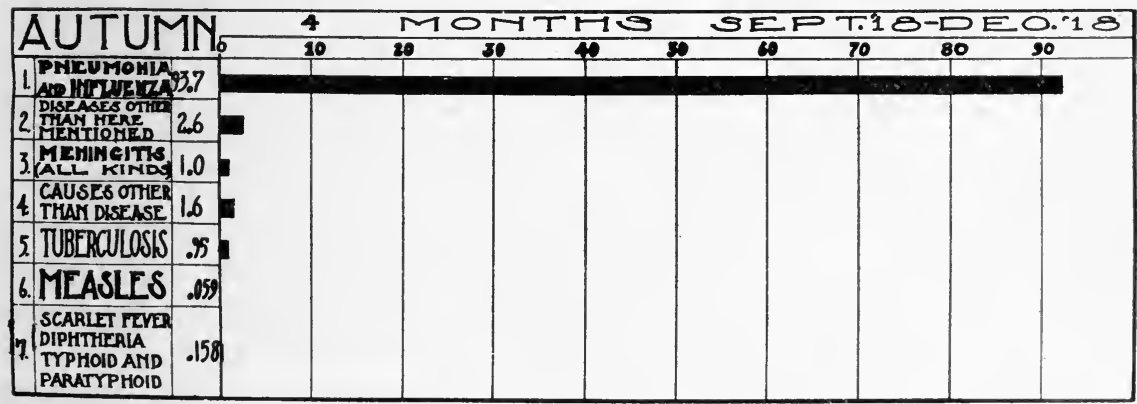

Fig. 17.

pneumonia was 14.9 per cent, while of 10 cases with entirely negative past histories for infectious disease 20 per cent died. These figures seem to indicate that the individual who has had other infections stood a better chance of winning out than the one who had never been called upon to fight a battle.

Pneumonia and the Weather.-Since the time of Hippocrates there has been a tendency to connect the prevalence of pneumonia with weather 
conditions. The father of medicine gave a bad reputation to the east wind and this name has clung to it through the centuries and in nearly every locality the east wind is regarded as especially harmful to man's health. During the seventies and eighties of the last century Baker, then Secretary of the Michigan State Board of Health, collected, through the physicians of the state, fairly satisfactory figures concerning morbidity from pneumonia. Lines drawn showing the prevalence of pneumonia and the average daily temperature were practically parallel when the temperature line was reversed; in other words, the lower the temperature the greater the number of cases of pneumonia in the state.

The prevalence of pneumonia increases a few weeks after the lowest temperature and after that as the temperature ascends the morbidity from pneumonia decreases. We make the following quotation from Baker's conclusions as stated in 1889, the figures concerning the prevalence of pneumonia and records for the temperature covering a period of eight years:

"From the accompanying diagram you will see that there was in January an average temperature of about $20^{\circ}$ and a large amount of pneumonia (following about a month later), in February it has become warmer and the line representing the amount of pneumonia soon falls, in Mareh it is still warmer and there follows still less pneumonia, and so on until the highest temperature is reached in July and the least amount of pneumonia in August-the disease following regularly about a month behind the cold."

How much cold per se has to do in the causation of pneumonia we cannot say. It is generally stated by Arctic travelers that there is but little pneumonia among the natives or those visiting that region. It seems also to be a matter of observation that when Eskimos and Laplanders come to our climate they are especially liable to the pneumonias and the fatality among them is great. Furthermore, all reports, none of which are official, coming from the Arctic regions concerning the late epidemic of influenza give high death rates. It would be interesting to know something about the flora of the upper air passages of the Arctic dweller in his home. It is well known that monkeys in this country are especially liable to pneumonia during our winters. It has been shown, however, that something more than lowering of temperature is necessary to induce the disease in these animals. More than 30 years ago Vaughan kept six monkeys in an air-tight room; all the air entering and leaving the room being filtered through eotton. The monkeys were kept in a room of this kind at a temperature of from $65^{\circ}$ to $70^{\circ}$ for six weeks and then one by one at different times were placed in a similar room, the temperature of which was kept near zero. The time of exposure to the cold varied from 30 minutes to two hours. Although these animals were frequently changed from the hot to the cold room, so long as the air was filtered no monkey developed pneumonia. Later, when the filtration of the air was neglected, and the 
monkeys were kept in a poorly ventilated cellar room, in the course of from six to ten weeks every animal developed pneumonia and died from it. It appears that if monkeys kept in confinement in cold countries be protected from infected air they do not develop pneumonia. As we have indicated above, travelers from temperate zones making expeditions into Aretic regions are not especially prone to respiratory diseases. We must take it for granted that they carry with them in their throats, at least some of them, Type IV pneumococcus, but still, even when subjected to extreme cold, they do not develop pneumonia. We are inclined to the opinion that it is the crowding that is necessarily a part of man's life which leads to the greater incidence of the pneumonias in the cold season of the year. Epidemics of pneumonia are not confined to cold or even temperate zones but are seen in the tropics quite frequently.

Other weather conditions besides temperature are supposed to favor the development and spread of the pneumonias. On these points, however, there is a great conflict in testimony; and in fact, a great diversity in observation. There are thousands of instances, and in fact so common is this observation that it comes in nearly everyone's personal experience, where men have gone cold, wet, and hungry for days without developing any respiratory disease; on the other hand, it is within the experience of nearly every one that exposure to a moderate draft for a few minutes may account for the development of a coryza, a bronchitis, or even a fatal pneumonia. There is promise of a closer and more scientific investigation of the relationship between weather and its complexities and the respiratory diseases. We have gone more thoroughly into these points in our chapter on weather and the respiratory infections.

Control.-If every individual practiced proper precautions and avoided to the utmost scattering among his fellow-men the diverse forms of life growing in his upper air passages, morbidity from the pneumonias would be materially reduced. If every physician and other attendant on the sick exercised well-known precautions and did not carry infection from one patient to another or permit such infection to be carried, the mortality from the pneumonias would be greatly reduced. We must realize that exchange of bacteria between individuals is not profitable, and indeed is in most instances accompanied by danger. A given pneumococcus or streptococcus resident in a given throat for many generations may have established a modus vivendi with the cells of the host and bacterial life and that of the body cells may proceed without any great disturbanee to either, but when the streptococeus or pneumococeus is transferred to the throat of some one else, there may be some trouble in establishing a modus vivendi with the cells of the new host. The mucous 
membrane of the nose in which a given streptocoecus has been growing more or less uniformly through some generations may have acquired methods of neutralizing any deleterious effeets of the microorganism, while like tissue in another individual to whom the bacteria might be transferred may not possess the same defenses.

In diseussing vaccination against pneumococeus pneumonia, we have brought out the results so far obtained by this proeedure. It would be unwise to attempt to predict what is to come from further investigations along these lines. Certainly the results thus far obtained are promising and justify further research.

All students of epidemiology reeognize that the pneumonias are infeetious diseases and that they are disseminated by spitting, sneezing, and eoughing; also by infected eating utensils. Lynch and Cumming deserve much credit for the very forceful way in which they have called attention to the distribution of respiratory diseases through infected tableware. They have also shown that infected hands undoubtedly play an important part in the dissemination of these diseases.

Although pneumococei of the more virulent Type I as well as those of the least virulent Type IV have been found in dust, it is not likely that the pneumonias are widely spread through this agency. In our opinion, air filled with dust may play an important part in the distribution of the pneumonias, but rather by increasing the cough of individuals inhaling such dust than by infection through the dust. It goes without saying that the sputum of all forms of pneumonia should be burned and that soiled linen, blankets, ete., should be disinfected. In hospitals one pneumonia patient should be protected against possible exchange of bacteria with his fellow-patients. With this in view, proper distance between beds and the employment of eubicals should be insisted upon; and what, in our opinion, is of greater importance, physieians and attendants should be sure that they are not the carriers in the transfer of bacteria from one patient to another. With proper precautions we do not believe that strict isolation is necessary in the care of pneumonia patients. There should be no possibility of direct bacterial transfer between patients by means of cough sprays, nor should the transfer be possible through the agency of a third person.

\section{Bibliography}

Avery, O. T.; Chickering, H. T.; Cole, Rufus, ANd Dochez, A. R.: Monograph of the Rockefeller Institute for Medical Research, No. 7, October, 16, 1917.

Baker, Henry B.: Report Michigan State Board of Health, 1889.

Colf, Rufus: Jour. Exper. Med., 1917, xxvi, 453.

Cole, Rufus, and Moore, Henry F.: Jour. Exper. Med., 1917, xxvi, 537.

Dochez, A. R., AND Avery, O. T.: Jour. Exper. Med., 1917, xxvi, 477.

GaY, Frederick P.: Jour. Lab. and Clin. Med., 1918, iii, 721.

Hastings, Thomas W., and Niles, Walter L.: Jour. Exper. Med., 1911, xiii, 638.

HippocRates, F.: Adams' Translation. 
Hirsch, August: Handbook of Geographical and Historical Pathology, New Sydenham Society, London, 1886.

Huxham, John: An Essay on Fevers, S. Austen, London, 1750.

Lamar, R. V., AND Melitzer, S. J.: Jour. Exper. Med., 1912, xv, 133.

Love, A. G., and Davenport, Charles B.: Arch. Inter. Med., 1919.

Palmer, Walter W.: Jour. Exper. Med., 1917, xxvi, 495.

Sewall, Henry: Arch. Inter. Med., 1914, xiii, 856.

Sewall, Henry, and Powell, Cuthbert : Areh. Inter. Med., 1915, xvi, 605.

Sewall, Henry, and Powell, Cuthbert: Jour. Exper. Med., 1916, xxiv, 69.

Stillman, ERnest G.: Jour. Exper. Med., 1917, xxvi, 513.

Trumbulu, Benjamin: A Complete History of Connecticut, Maltby, Goldsmith \& Company, New Haven, 1818.

Vaughan, V. C., and Palmer, G. T.: Jour. Lab. and Clin. Med., August, 1918, and July and August, 1919.

Vaughan, V. C., aNd Palmer, G. T.: Military Surgeon, January, 1920.

Vaughan, W. T., and Schnabel, T. G.: Arch. Inter. Med., 1918, xxii, 440.

Wadsworth, Augustus B.: Jour. Exper. Med., 1912, xvi, 54 and 78.

WeBster, NoAH: A Brief History of Epidemic and Pestilential Diseases, Hudson and Goodwin, Hartford, 1799.

WELSH, D. A.: Green's Encyclopedia of Medicine and Surgery, Wm. Green \& Sons, London.

Wollstein, Martha, and Mejutzer, S. J.: Jour. Exper. Med., 1912, xvi, 126. 


\section{CHAPTER VI}

\section{MEASLES}

\section{MORBILLI; RUBEOLA}

Definition.-Measles is a specific, highly contagious, febrile disease, ushered in by congestion and suffusion of the eyes accompanied by catarrhal manifestations of the upper respiratory tract and followed by a distinctive cutaneous eruption. . There is no other disease to which man is so universally susceptible and in the vast majority of instances one attack gives immunity throughout life. It is quite certain that this disease is due to a filtrable virus.

History.-We owe the first description of this disease to the accurate observations of Arabian physicians of the tenth and eleventh centuries. Undoubtedly measles is as old as the race, but before the Arabian period it was confounded with smallpox. This confusion continued even up to the time of Sydenham, who gave the following very accurate description of the disease:

"The measles generally attack children. On the first day they have chills and shivers, and are hot and cold in turns. On the second day they have the fever in full-disquietude, first, want of appetite, and white (but not a dry) tongue, slight cough, heaviness of the head and eyes, and somnolence. The nose and eyes run continually; and this is the surest sign of measles. To this may be added sneezing, a swelling of the eyelids a little before the eruption, vomiting and diarrhea with green stools. These appear more especially during teething time. The symptoms increase till the fourth day. Then-or sometimes on the fifth-there appear on the face and forehead small red spots, very like the bites of fleas. These increase in number, and cluster together, so as to mark the face with large red blotches. They are formed by small papulae, so slightly elevated above the skin, that their prominence can hardly be detected by the eye, but can just be felt by passing the fingers lightly along the skin.

"The spots take hold of the face first; from which they spread to the chest and belly, and afterwards to the legs and ankles. On these parts may be seen broad, red maculae, on, but not above, the level of the skin. In measles the eruption does not so thoroughly allay the other symptoms as in smallpox. There is, however, no vomiting after its appearance; nevertheless there is slight cough instead, which, with the fever and the difficulty of breathing increases. There is also a running from the eyes, somnolence, and want of appetite. On the sixth day, or thereabouts, the forehead and face begin to grow rough, as the papules die off, and as the skin breaks. Over the rest of the body the blotches are both very broad and very red. About the eighth day they disappear from the face, and scarcely show on the rest of the body. On the ninth, there are none anywhere. On the face, however, and on the extremities-sometimes over the trunk-they peal off in thin mealy squamulae; at which time the fever, the difficulty of breathing, and the cough are aggravated. In adults and patients who have been under a high regimen, they grow livid, and afterwards black." 
Sydenham not only gave the above quoted most excellent description of this disease, but he recognized that, while in and of itself measles is a mild disease, it induces as sequelae bronchitis and pneumonia and "destroys more than even smallpox itself." Morton, who was the great English authority immediately following Sydenham, confounded measles and scarlet fever, believing the latter to be a confluent form of the former. There was a highly fatal epidemic of measles in London in 1674 and, although the number of deaths attributed to this disease was relatively small, the total death rate ran high. The number of deaths attributed to the disease in each year of the eighteenth century is given in the mortality lists for London, but it is impossible to tell how many of these were actually due to scarlet fever. Webster says that in $\mathbf{1 7 7 2}$

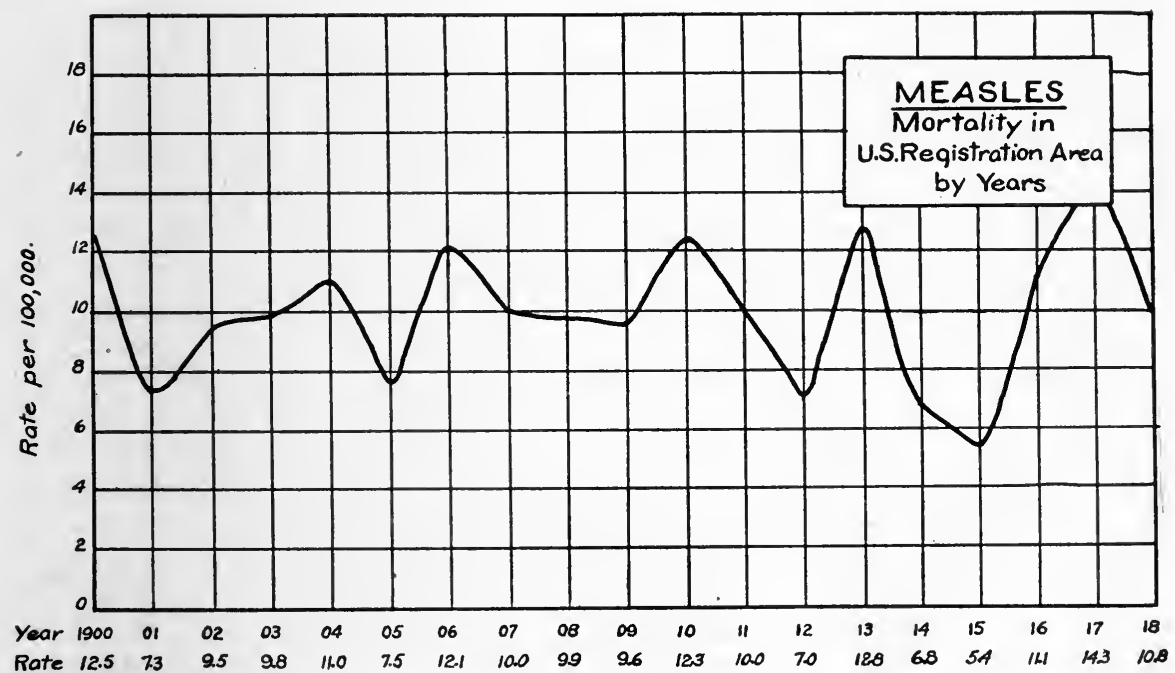

Fig. 18.

measles appeared in all parts of America with unusual mortality; that 800 or 900 children died in Charleston, and that in the following year measles "finished its course and was followed by a disorder in the throat." It was observed early in the nineteenth century especially in Scotland, that measles among adults is more frequently followed by pneumonia than happens in children. Furthermore, it was remarked that pneumonia following measles is highly fatal among recruits.

“The deaths from pulmonic complaints did not often happen among children, but among people somewhat advanced in life. Significant also was the outbreak in the Invernesshire Militia, which marched into Edinburgh in March while the epidemie was raging. Fifty men, all young recruits newly joined, were attacked in the course of a few days, the others escaping the disease though equally exposed to it; in some of those who died in the regiment there were found on opening the thorax, fibrinous pleurisy and pericarditis, with effusion of fluid, as well as evidences of bronchial catarrh.'” 
In 1808 and again in 1811 highly fatal epidemies of measles prevailed throughout England and Scotland, especially in the latter. Some believed that the new practice of inoculating children with cowpox was in some way responsible for the measles. It is worthy of note, however, that the better physicians, especially Watt, were able to show that the death rate among the vaccinated was no greater than among the unvaccinated. Watt did believe that the suppression of smallpox, which he correctly attributed to vaccination, largely increased susceptibility to measles. He wrote that formerly about nine-tenths of all children had both smallpox and measles; that as a rule the smallpox came first, and that children who survived smallpox were fortified by that disease against the measles. In regard to Watt's theory, Creighton writes as follows:

"While Watt's theory of the working of this prineiple of substitution is open to eriticism on some points of detail, the law itself, as enuneiated by him, remains to the present time one of the soundest and most instructive generalities in epidemiology. He based it upon a laborious search of the burial registers, such as no one before him in this country had undertaken. Next he saw eorrectly that a great rise in the deaths of infants by such a disease as measles could only be aceounted for by a great inerease in the rate of fatality. Thirdly he eonneeted the loss from measles with the saving from smallpox. Adopting an old opinion, which may be discovered in Willis, he argued that smallpox, when taken first, served to fortify children so that they passed easily through the measles afterwards; but in the following passage he indicated a better reason why the absence of smallpox gave measles the ehanee of proving more fatal: 'In this point of view we are not to consider the smallpox as so peculiarly fatal in their nature. They perhaps prove so fatal merely by having the start of other diseases. The measles, the ehincough, the eroup, the scarlet fever, and perhaps many others, would prove equally fatal had they occurred first.' The principle is true to this extent, that a certain proportion of weakly infants, or children of poor stamina, will suceumb to almost any disease-if not to smallpox, then to measles, and if not to measles directly, then to the sequelæ of measles. This was perceived in the form of a necessary truth by Haygarth in 1793. ' $A$ considerable number of those who now die of the smallpox would die in ehildhood of other diseases if this distemper were exterminated.' It was commonly believed that smallpox had at length found its real artificial eheck, not in the inoeulation of itself, but in the inoculation of cowpox. At all events it had declined greatly in Glasgow. During the three years before the measles epidemic of 1808 , there could hardly have been more than 1,000 children attacked by smallpox, or not one in ten of all the children born. During several years the infaney of the eity had been spared any great ordeal of infectious disease. The first epidemic that eame along happened to be measles, so that it fell to that infection to take off the weaklings. In the eeonomy of nature it is impossible to rear all the young of a species, nor would it be good for the species if it were possible. It is among the birds that the prineiple of population, or of the survival of the fittest, is seen working in the most admirable way; the annual migration of many speeies to breed in a remote country brings with it an ordeal for the birds of the year in finding their way to the winter feeding grounds-an ordeal which only the strongest come through. For some unexplained reason the young of the human speeies are peculiarly tried by infeetious diseases, which multitudes pass through safely, while many of poor stamina or of ill tending are cut off." 
As we understand him, Watt believed that the child who had survived smallpox was thereby better fitted to withstand measles. Does recovery from one infectious, disease increase resistance to other infectious diseases? This question, so far as we know first raised by Watt, is still without satisfactory answer. Is there such a thing as nonspecific immunity or increased resistance to infection? This is a matter of great importance to epidemiologists and we shall reserve a full discussion of it for a more opportune place.

The point made by Creighton is quite a different thing. He held that infectious diseases, especially among children, take off the weaklings. This is an idea which, as Creighton indicates, has prevailed more or less for many centuries. Even the great philosopher of modern science, Herbert Spencer, taught that infectious diseases, especially among children, were in the long run beneficial since they cut out in early life those who are unfit and give greater opportunity for the proper development of those who should live. Is there any basis in fact for this belief? Did smallpox during the centuries when it was uncontrolled by vaccination kill only weaklings and spare the robust? Do measles, scarlet fever, whooping cough, infantile diarrhea, play that function today? If these questions can be answered authoritatively in the affirmative, then we should hesitate in our attempt to eradicate the infectious diseases, especially those most prevalent and most fatal in infancy and childhood. No child acquires measles, whooping cough, or scarlet fever, unless exposed to the infection. The danger to the life of the individual child depends upon many conditions, especially upon the state of health at the time of the exposure and the amount and virulence of the infection transferred to the child at the time of exposure. Are these conditions determined or modified by the degree of fitness or unfitness to live possessed by the individual child? If a careless nurse carries articles of clothing soiled with discharges from a scarlet fever patient into a household where there are children, is there any reason for believing that the less fit of these children are more likely to come in contact, or are more likely to suffer from coming in contact, with the imported virus? An individual with open tuberculosis deposits bacilli-laden sputum on the floor of a schoolroom filled with children in all degrees of physical fitness and unfitness. By what law or by what chance will the less fit of these children be infected, while the more fit escape? Badly infected milk may be used in a children's school. Some will drink more of the poisonous milk and some will drink less, and possibly a few will drink none of it. All will suffer or escape as they fall into one of these elasses. What will lead all the unfit children to drink the poisonous milk and what will keep all the fit children from drinking any of it? If the fitness of a child to live is to be determined by passing through some such 
ordeal as exposure to a highly dangerous infectious disease, let us not leave this to nature but proceed to inoculate every child in a scientific and exact way. Suppose that we determine the minimum amount of white arsenic that may kill, say a weakling at one year of age; then on its first birthday we administer this dose of arsenic to every child. Are we to suppose that all those who might grow into inefficient or vicious citizens would die, while all those who are to develop into perfect manhood or womanhood are to live? If we are going to imitate nature in this experiment we must not weigh out the dose given each child, but measure it out, guess at it; and if we are going to imitate nature more accurately we shall select a blind person to measure out for each child its dose. The idea that the infectious diseases of childhood kill only the weaklings, or even kill the weaklings in larger proportion, is based upon a complete misunderstanding and misinterpretation of all epidemiologic science. It would be just as sensible to attempt to improve the race by mustering all the children in a community and have them pass down a street while blind-folded men with repeating rifles fired into the crowd. Fortunately, even the preachment of Herbert Spencer on this point has in no way abated or hindered the attempts of the sanitarian to control and eradicate the infectious diseases prevailing at any period of life.

It is true that children are born into the world who never should have been born. They come from stock so diseased or so impoverished that through the act of procreation they have not received vitality enough to carry them even over the first miles of the road of life. Fortunately, most of these children die. They die, as a rule, within the first month. But they do not die from the infectious diseases; they die because they are unfit to live. If the infectious diseases of infancy and childhood weed out the unfit and improve the race, then we should find in countries where the infantile death rate is high that the death rate at subsequent ages is low. This is not the case. Take those European countries in which vital statistics have been most carefully kept, and we find that where the infantile death rate is high the general death rate corresponds, and where the infant death rate is low the general death rate is low. The infantile death rates and the death rates for all ages in Italy, France, and Germany, have been higher than in Great Britain, Norway, and Sweden. The country that does not protect to the fullest extent possible the lives of its children fails to improve the quality of its people. Measles and other infectious diseases of infancy.and childhood kill both the fit and the unfit; probably in a manner as impartial in its operation as nature can make it, but they leave of both the fit and unfit for every one that they kill many who are to be cripples throughout life. No, the infectious diseases of infancy and childhood play no part in the up- 
lift of the race; on the other hand, they are evil and downward in their tendencies.

Some of the most valuable contribations to the epidemiology of measles have resulted from outbreaks of this disease either for the first time or after long intervals in isolated parts of the earth. In 1781 measles disappeared from the Faroe Islands. For 65 years thereafter there was not a case of this disease anywhere on any of the 17 islands constituting the inhabited parts of this group. During this period all imports and exports were handled by the Royal Danish Transportation Company and a successful quarantine seems to have been maintained; at least for 65 years there was not a case of measles among the inhabitants of the Faroe Islands. Fortunately, when measles did appear, the Danish Government sent a medical commission, one of whose members was Panum. His report forms one of the most important epidemiologic papers in existence. This man had the opportunity to study the death rates in a country where for 65 years measles, scarlet fever, and the other infectious diseases of childhood had been absent altogether or played a very subordinate rôle. Panum made a study of the average age reached by the inhabitants of the Faroe Islands during the 11 years preceding the reentrance of measles into that country and compared it with the average length of life in other countries for the same period, with the following results: average length of life among the Russians, 21.3 years: among the Prussians, 29.6 years; among the Swiss, 34.6 years; among the French, 35.8 years; among the Danes, 36 years; among the English, 38.5 years; among the inhabitants of the Faroe Islands, 44.6 years.

In addition to this comparison, Panum states that at that time the greatest mortality in Denmark fell between the sixtieth and seventieth years, while in the Faroe Islands the greatest mortality fell between the eightieth and ninetieth years. Panum was quite convinced that the longer life of the inhabitants of the Faroe Islands was not due to better food or climate, but was due to the absence of the infectious diseases of infancy and childhood. It is not over sanguine to claim that if measles, whooping cough, diphtheria, and scarlet fever could be entirely suppressed the average length of life would be increased by at least ten years.

Panum found in the Faroe Islands unique opportunities to study the epidemiology of measles and he availed himself of these opportunities. The total number of inhabitants at the time of the outbreak of measles in 1846 was 7,782. The people were distributed on 17 islands, and on these they were gathered in small villages of from 20 to 200 persons. Thorshavn, the administrative capital, was the only village with a population of more than 200, and its population numbered 800 . Measles was brought into the Islands by a cabinet maker who left Copenhagen March 
20 and reached Thorshavn on the twenty-eighth in perfect health. A few days before leaving Copenhagen he had come in contact with a case of measles and on the fourteenth day thereafter and some days after reaching Thorshavn he developed the disease. The fact that the population of the Islands was scattered on different islands and in small villages gave Panum an unusual opportunity to study the progress of the disease. He found that the period of incubation, counting from the hour of exposure to the first appearance of the rash, was invariably 13 or 14 days, almost certainly 14 . He was able to find no exceptions to this rule. Apparent exceptions, as related to him by others, turned out on more exact investigation to conform to the rule. It must be clearly understood that the termination of this period is indicated by the appearance of the rash. Panum says that if you wish to find out when an individual who is developing the disease was exposed you must not ask him when he began to be ill, but when he first observed the eruption, and then you can determine the date of exposure by counting back 14 days. In regard to the development of prodromal symptoms, Panum found considerable variation. There might be suffusion of the eyes, slight elevation of temperature, evidence of eatarrh, as long as six to eight days before the appearance of the exanthem. In many instances this period was from four to six days; usually two to four days, and in a small number one to two days. Ten men left a village and visited another, coming in contact with eases of measles. On returning to their own village every one of the ten showed measles eruption on the fourteenth day after exposure. In their native village all the inhabitants were exposed to them and the rule of 14 days was found to continue. In this way it happened that Panum found in a village of 100 inhabitants 80 ill with the measles at the same time, practically all of them showing the eruption for the first time on the same day.

In this population which had been free from the measles for 65 years no person who had had this disease 65 years or more previously developed it at this time; in other words, Panum could not find an instance of a second attack of measles, although the shortest possible time between the previous attack and the present exposure was 65 years. Panum concluded from this that the weight of evidence is in favor of the belief that one attack of the measles, practically in every case, protects against this disease throughout life. Most reports of an individual having measles twice were believed by Panum to be due to errors in diagnosis, although he admitted that such might occur. The man of 70 , and even of 80 , who had never had measles acquired the disease just as readily as the child of seven or eight. There is, therefore, no increased resistance to this infection developed with a oe. Under ordinary conditions measles is confined laroely to childhood simply because most adults have already had the disease. 
Panum was able to show that the virus of measles is most readily transmitted to others at the period of the development of the exanthem. He found pretty definite evidence that it might be transmitted during the prodromal period and before the first appearance of the rash, but he found no instance of its transmission during the period of desquamation. Panum leaves us somewhat in doubt about the transmissibility of the virus of measles through fomites. He tells of a nurse who had had measles many years before in Copenhagen and who nursed cases of the disease, on going to other places she apparently earried the virus with her. Panum reports that out of the 7,782 inhabitants of the Faroe Islands in 1846, 6,000 had measles and no one who was exposed escaped the infection, except those who had had it in 1781 or previously. Unfortunately, we have been unable to find any authoritative statement concerning the severity, complications, and mortality of measles in the Faroe Islands in 1846. In the article to which we have had access Panum makes no mention of these things.

So far as is known, measles had never touched the inhabitants of the Fiji Islands until 1875. The behavior of this disease in these islands has been described by Corney. The late King of the Fiji Islands, in the act of transferring his government to the British Empire, visited Sydney in New South Wales in 1874. Returning to his native country on a British man-of-war in January, 1875, his son and one of his servants who accompanied him developed measles. They landed at Levuka on January 12, 1875. The ex-King had called a conference of prominent men from all the islands. This conference was held during the second and third weeks after the arrival of the infected persons and all members of the conference were exposed. In this way the disease was spread throughout the entire population, which at that time numbered about 150,000 . It is officially stated that there were 40,000 deaths. In certain islands and villages where more exact information concerning the population and deaths can be secured, it appears that from 27 to 28 per cent of the people died from this disease. By the end of May the epidemic had burned out. This epidemic is usually referred to as an instance of the extreme virulence of measles when it finds its way into a virgin population. From this, it has been the custom to draw the inference that peoples, like ourselves, who have suffered from measles from generation to generation, have acquired a certain degree of immunity by inheritance, and the greater mortality among the Fiji Islanders is attributed to the absence of inherited resistance. After a careful reading of Corney's report; we are not quite sure that these explanations are justified. On this point Corney makes the following statement:

"I might quote numerous other instances from both official and private records of the epidemic, all of which go to show that the high rate of mortality, which, as I have 
already stated, amounted to no less than twenty-six per eent, was brought about mainly in consequence of all the people being infected at or about the same time, beeause none of them were protected by a previous attack of measles. The nation presented a virgin field for its insemination and deveiopment. Also, by the untutored and uncontrollable habits of the natives in giving up hope and reason, or acting purely in ignoranee, as manifested by their proneness to bathe in cold water, or expose their bodies to cooling influences, such as night air, drafts, or damp earth, when in a state of high fever; these inconsisteneies resulting very naturally in dysentery and pneumonia and death. Starvation was a secondary cause, brought about by the first I mentioned, there being no healthy people available to provide food and look after those that were sick. Want of cleanliness, speedy and efficient disposal of the dead, and other sanitary precautions, may be classed under the same eategory; and superstition and despair contributed also to the fatal results. The point which I wish to establish, therefore, is that whatever virulence there may have been in this particular epidemic-and we know that epidemics of measles vary very much in this respect-the excessively high rate of mortality which resulted was not only a direct consequence of the disease being implanted in a nation to whose ancestors, history, and traditions it had hitherto been a stranger, but that it occurred in great measure in a secondary manner, and was brought about in the ways $I$ have just now indicated. In by far the greater number of fatal cases, the cause of death was dysentery or pneumonia, or both together; and these diseases were mainly produced by causes which in a eivilized community, even if all new to measles, would not have operated. I know that it is usual to believe that a virgin community will suffer more, other things being equal, on a disease being introduced among them, than will one whose ancestors have been exposed to its influence during successive generations. It may be so, and probably is; but I am confident that, in the ease of this epidemic of measles in Fiji, more stress has been generally laid upon this hypothesis than the facts warrant us in believing to have been the fulfillment of it."

\section{In another place, Corney writes :}

"As a set-off to this state of affairs, we are reassured by learning, as an evidence of the good results where medical treatment and discipline could be enforced, that there was only a slight mortality amongst the members of the Armed Native Constabulary at Nasova. These men lived in so-ealled barracks, and were under English military discipline. Every one of them, 147 strong, as I have mentioned, took the disease; but only nine died. Of course, they were all male adults in the prime of life; but 6 per cent was their death rate, as against 26 per cent of the entire native population. And it happened that amongst the laborers indentured to planters and merchants, the mortality was comparatively small, and that in a few instances, even, the disease never reached them. This was owing to the isolation and discipline which their employers were able to insist upon, among the indentured working population on the various estates. These people were mostly natives of the Solomon Islands, New Hebrides, and Line Islands, who had emigrated to Fiji for a three years' term of indenture; and who were dependent upon their employers, not only for medicine and sanitary care, but for food and housing." "

Many of the Fiji Islanders believed that they had been poisoned by the English; others were quite convinced that the Supreme Being had stricken them with this curse because they had surrendered their government to the British; while in all cases there was no adequate care of the sick. Webb, a missionary on the Islands, testifies that the sick were 
left to themselves, subjected to every inclemency of weather, suffered from thirst and hunger, and that the strong winds and rains added to the horrors of the situation. The poor ignorant people suffering from the high fever dragged their bodies to streams of cold water in which they remained for hours. Pneumonia, dysentery, and other complications quite naturally arose and no doubt contributed largely to the high death rate. After carefully going over the records concerning the epidemic of measles in the Fiji Islands in 1875, we are convinced that the statement so frequently made that the high mortality in this epidemic was due to the fact that neither these people nor their ancestors had ever known this disease is not supported by the evidence in the ease; on the other hand, we believe that the greatest factor in causing the high mortality of this epidemic was the lack of eare provided for the sick. Study epidemies of measles wherever they appear and it will be found that the mortality rate is influenced by the care and attention given to the sick more than to any other agency. Measles, when the sick are ideally cared for, is a disease of low mortality; when the sick are inadequately taken eare of this disease may rank in its fatality among the great plagues of the world. When introduced into a population with a susceptibility of 100 per cent it strikes down so many at practically the same time that adequate care for the sick is impossible. The resistance of the body already reduced to a minimum by the virus of the disease is easily overcome by secondary infections, by exposure to cold, by thirst, and by hunger. This is true of measles, whether it appears for the first time in a virgin population as it did in the Fiji Islands in 1875 or whether it appears as it did in the camps in the United States in the winter of 1917-1918.

No less an expert on measles epidemics than Brownlee has given recently the following information concerning the virulence of this disease in virgin populations :

"I visited some years ago a Highland glen from which measles had been absent for more than 80 years. An epidemic had just ended; it swept the glen from the western end to the eastern, hardly any one-man, woman, or child-escaping the infection. Among the inhabitants whom I met was an old farmer of nearly 80 years of age who had just recovered from the disease. He discussed the matter with a curious mixture of pride and shame-pride that such an unusual experience had been his lot, shame that at his great age he had succumbed to such an infantile malady. It may be noted, by the way, in contradiction of the erroneous and quite common belief, that though the soil was practically virgin in this epidemic, not a single death occurred."

Present Prevalence and Virulence: The figures in Table VIII taken from the United States Census for 1917 show the death rates from this disease.

It will be seen from these figures that, while measles in the registration area fluctuates in its death rate from year to year, it holds out on the whole quite uniformly. 
TABLE VIII

Death Rate From Measles per 100,000 Population

\begin{tabular}{lrrrrrrr}
\hline \hline & 1911 & 1912 & 1913 & 1914 & 1915 & 1916 & 1917 \\
\hline Registration area & 10.0 & 7.0 & 12.8 & 6.8 & 5.4 & 11.1 & 14.3 \\
Registration states & 9.9 & 7.3 & 13.0 & 6.9 & 5.2 & 11.5 & 14.2 \\
Cities in registration states & 10.1 & 9.5 & 13.5 & 7.7 & 7.1 & 12.8 & 10.3 \\
Rural part of registration states & 9.7 & 5.0 & 12.4 & 6.1 & 3.4 & 10.3 & 17.7 \\
$\begin{array}{l}\text { Registration eities in non- } \\
\quad \text { registration states }\end{array}$ & 10.8 & 4.3 & 11.1 & 5.4 & 7.6 & 5.7 & 15.6 \\
All registration cities & 10.2 & 8.7 & 13.2 & 7.3 & 7.2 & 11.8 & 11.0 \\
\hline
\end{tabular}

TABIL IX

Death Rates from Measles per 100,000 Population in the Large Cities of the United States Since 1911

(Using Corrected Mid-year Population Following 1920 Census)

\begin{tabular}{lrrrrrrrrrr}
\hline \hline \multicolumn{1}{c}{ CITY } & 1911 & 1912 & 1913 & 1914 & 1915 & 1916 & 1917 & 1918 & 1919 & 1920 \\
\hline Boston & 10.4 & 16.2 & 11.1 & 8.5 & 5.8 & 15.3 & 14.7 & 15.8 & 3.9 & 8.6 \\
New York & 13.4 & 14.2 & 14.3 & 11.2 & 12.7 & 10.4 & 10.7 & 15.4 & 4.0 & 13.0 \\
Philadelphia & 18.3 & 3.3 & 12.5 & 4.4 & 11.2 & 6.5 & 4.7 & 7.7 & 2.3 & 9.0 \\
Detroit & 8.8 & 8.8 & 16.7 & 5.9 & 6.7 & 10.3 & 7.0 & 14.2 & 8.4 & 8.5 \\
Chicago & 5.8 & 5.3 & 11.8 & 3.2 & 10.5 & 5.4 & 9.8 & 2.3 & 7.7 & 3.3 \\
St. Paul & \multicolumn{1}{c}{. } & .5 & 8.1 & 1.3 & 5.3 & 7.9 & 5.6 & 1.7 & 7.7 & 5.5 \\
Minneapolis & 1.3 & 3.4 & 6.1 & 2.7 & 3.8 & 21.1 & 3.1 & 7.1 & 2.1 & 5.7 \\
St. Louis & 16.4 & 2.6 & 16.0 & 9.6 & 7.5 & 9.0 & 18.6 & 3.3 & 4.9 & 15.3 \\
San Francisco & 7.7 & 11.2 & 1.8 & 10.3 & 4.7 & 1.3 & 7.4 & 2.0 & 2.0 & 5.6 \\
New Orleans & 11.3 & .6 & 9.9 & 2.2 & .8 & 3.5 & 16.5 & 5.8 & 1.0 & 4.1 \\
Washington & 6.4 & 2.0 & 7.4 & .3 & 1.8 & 2.0 & 2.2 & 7.1 & .5 & .5 \\
Atlanta & 24.9 & 7.3 & 5.3 & 14.4 & 1.1 & 1.6 & 23.7 & 8.3 & 2.0 & 6.9 \\
Richmond & 8.3 & .7 & 18.3 & .7 & 0 & 26.3 & 3.7 & 4.9 & 2.4 & 5.7 \\
Pittsburgh & 9.4 & 26.6 & 27.6 & 9.5 & 13.1 & 24.1 & 11.5 & 17.6 & 2.1 & 16.6 \\
\hline
\end{tabular}

TABLE X

Death Rate from Measles per 100,000 Population in Certain Small Cities of the United States in Certain Years

\begin{tabular}{|c|c|c|c|c|c|}
\hline \multicolumn{2}{|r|}{ CITY } & & YEAR & \multicolumn{2}{|l|}{ DEATHS } \\
\hline \multirow{2}{*}{\multicolumn{2}{|c|}{$\underset{\text { Lackawanna, N. Y. }}{\text { Y }}$}} & & 1912 & \multicolumn{2}{|l|}{82.9} \\
\hline & & & 1913 & 172.7 & \\
\hline \multicolumn{2}{|r|}{ “6 } & & 1917 & 326.8 & \\
\hline \multirow{2}{*}{\multicolumn{2}{|c|}{$\begin{array}{l}\text { Webb City, Mo. } \\
\text { Duquesne, Pa. }\end{array}$}} & & 1916 & 188.1 & \\
\hline & & & 1913 & 167.8 & \\
\hline \multicolumn{2}{|c|}{ Steubenville, $\mathrm{O}$. } & & 1913 & 112.0 & \\
\hline \multicolumn{2}{|c|}{ El Paso, Tex. } & & 1913 & 127.5 & - \\
\hline "6 & 66 & & 1914 & 20.2 & . \\
\hline 66 & 6 & & 1915 & 66.9 & \\
\hline 6 & “6 & $\cdot$ & 1916 & 44.0 & \\
\hline & "6 & & 1917 & 102.7 & \\
\hline
\end{tabular}


It will be seen from the figures in Tables IX and $\mathrm{X}$ that even among us, whose ancestors for untold generations have paid toll to this well nigh universal disease, it does from time to time and from place to place show the heavy hand of a veritable plague. Unfortunately, we have no means of comparing the death rates in the last few years with those which occurred in either large or small cities 100 years ago, because even where we have the number of deaths for the earlier period we have no exact figures concerning the population, and consequently cannot calculate the death rate. In our opinion, we get enough information to make it quite certain that measles, as well as scarlet fever, is not showing any sign of senility but flares up from time to time with great malignancy.

That measles continues to be an important cause of death in England is shown by the following figures, given by Newman as the distribution of deaths in that country for the year 1917:

$\begin{array}{lr}\text { Smallpox } & 3 \\ \text { Measles } & 10,538 \\ \text { Scarlet Fever } & 768 \\ \text { Typhus } & 1 \\ \text { Plague } & 4 \\ \text { Influenza } & 7,289 \\ \text { Whooping Cough } & 4,509 \\ \text { Diphtheria } & 4,474 \\ \text { Cerebrospinal Fever } & 1,531 \\ \text { Enteric Fever } & 977 \\ \text { Infective Enteritis } & 3,095 \\ \text { Dysentery } & 897 \\ \text { Tetanus } & 179 \\ \text { Malaria } & 126 \\ \text { Anthrax } & 22 \\ \text { Syphilis } & 2,127 \\ \text { Puerperal Fever } & 873 \\ \text { Erysipelas } & 588 \\ \text { Other Infective Diseases } & 588 \\ & \\ \quad \text { Total } & 38,589 \\ \text { Tuberculosis (all forms) } & 55,934 \\ \text { All other Causes } & 404,399 \\ & \\ \quad \text { Total } & 498,922\end{array}$

Measles in Our Army Camps in 1917-1918.-Vaughan and Palmer have made a study of the communicable diseases in our large army camps in this country during the World War and have been able to make some important contributions to our knowledge of the epidemiology of measles and other acute infectious diseases. The plan of mobilization adopted in 1917 was, from a military standpoint, a necessity. We were 


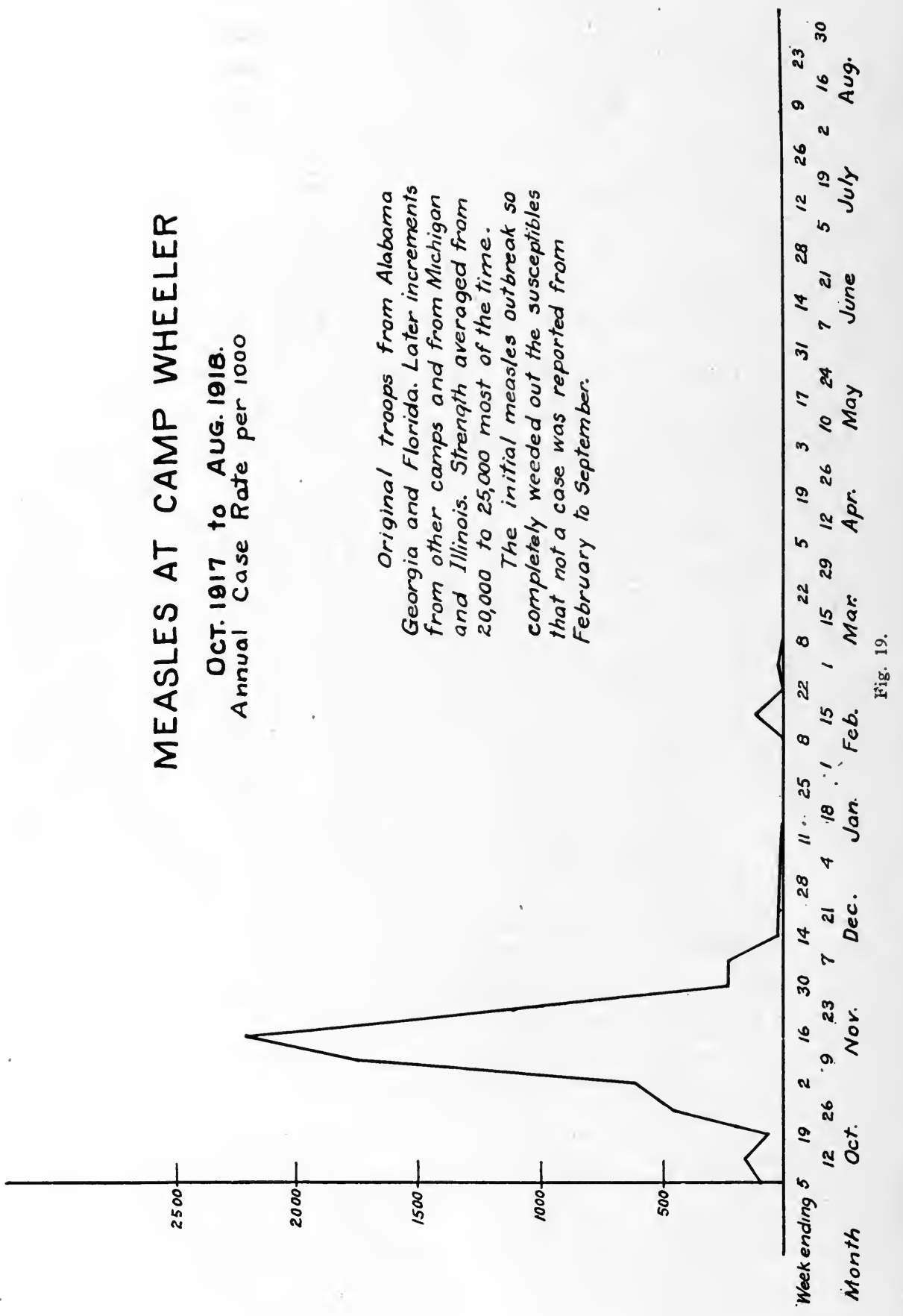


required to convert as speedily as possible the untrained youth of this country into efficient soldiers. The plan of mobilization, however, acted like a drag net and brought into all camps every infectious disease which existed in the communities from which the men eame. The extent to which measles or any other infectious disease prevailed in a given camp was determined by the number of susceptibles in that division. Naturally, there were more susceptibles to measles in divisions filled from the rural populations than in those made up of urban residents. During the winter of 1917-1918 there were 62 times as many eases of measles at Camp Pike, where the division consisted of country boys from Alabama, Arkansas, Louisiana, and Mississippi, as there were at Camp Upton filled almost entirely with draft men from New York City. This does not mean that measles at Camp Pike, Ark., was more virulent than the same disease at Camp Upton, N. Y. The greater prevalence in the former place was due to the greater number of susceptibles. Take all the camps; the measles curve ran practically parallel with the percentage of rural population in the camps. In the cities more than 90 per cent of those who had reached the draft age already had had this disease, while among the rurals of the same age the percentage protected by previous attack varied greatly. Into-some of the camps filled with boys from the rural sections, especially of the South, measles came in on every train; in fact, there was not a troop train that reached Camp Wheeler in the early fall of 1917 that did not have on its arrival at camp from one to six cases of measles already in the eruptive stage and at the very time when the infectivity of this disease is at its highest point. These men had been on the train from eight to forty-eight hours and during the journey practically every nonimmune had been exposed to the disease. To control the spread of measles throughout a camp under these-onditions was, in our opinion, practically impossible. The rate at which men at Camp Wheeler eame down with this disease in the fall of 1917 is indicated by the following official figures:

$\begin{array}{ccc}\text { FOR WEEK ENDING } & \text { ANNUAL MEASLES MORBIDITY RATE } \\ \text { PER } 1,000 \\ \text { October } & 19 & 83 \\ \text { October } & 26 & 428 \\ \text { November } & 2 & 615 \\ \text { 6 } & 9 & 1760 \\ \text { 6 } & 16 & 2200 \\ \text { “6 } & 23 & 1120 \\ \text { 6 } & 30 & 248 \\ \text { December } & 7 & 240 \\ \text { December } & 14 & 19\end{array}$

One of the chief difficulties in dealing with measles under the conditions present at Camp Wheeler and several other camps lies in the great 
number of eases thrown into the hospitals at the same time. It matters not how well equipped a base hospital may be or how well managed it is, when the demand for earing for three or four times its eapacity, of acutely sick men is thrown upon it, demoralization results. At Camp Wheeler hundreds of new cases of measles were brought in day after day, while at the same time the hospital foree in every branch was being reduced by the same disease; indeed, both in military and civil life, although more markedly in the former than in the latter, the problem which results from being overwhelmed by new cases is a most difficult one to solve. In most army camps its satisfactory solution is beyond human skill and endurance. At Camp Wheeler and other camps filled largely with susceptibles in 1917 measles continued until it burned itself out, until there were no more susceptible people. The efforts that were made to prevent its spread probably had some retarding influence and this is of great value in epidemics. There is no proof that anything that was done at Camp Wheeler lessened in the end the number of cases of measles in that division. These efforts, possibly in certain groups, retarded the rate at which the disease was spreading. They succeeded in beating out for the time being the fire here and there, but in the long run the fire continued and when measles ceased to appear on the daily admission reports at Camp Wheeler it was because there was not a susceptible in the camp. The truth of this statement is shown by the fact that during the spring and summer of 1918 there was not a case of measles reported at Camp Wheeler. Measles, probably more than any other disease, runs true to form in picking the nonimmunes. A previous attack is apparently the only effective guarantee against measles in later life. Measles permeated Wheeler so effectually as to pick every susceptible person. When the epidemic was over there was not one left untouched. In the following months there were practically no new increments entering Wheeler and consequently no fresh material was introduced. The experience at Wheeler was duplicated by a number of other camps where epidemics raged during the fall of 1917 . Bowie, with a rate of 374 in the winter of 1917-1918, dropped to a rate of 3.7 for the summer of 1918; in fact, during the summer of 1918, Las Casas (the camp in Porto Rico) was the only camp which showed an epidemic of measles even similar to those prevalent in southern camps in the fall of 1917 and Las Casas was filled with new recruits, largely susceptibles. In most of the camps during the summer months of 1918 there were only occasional eases of measles and these quite invariably were among recruits coming into eamp, especially those from rural populations. In our camps deaths due to noncomplicated measles were exceedingly rare; indeed, we are not certain that there were any. The part played by this disease in increasing the mortality lists lies in secondary infection, and especially in its 


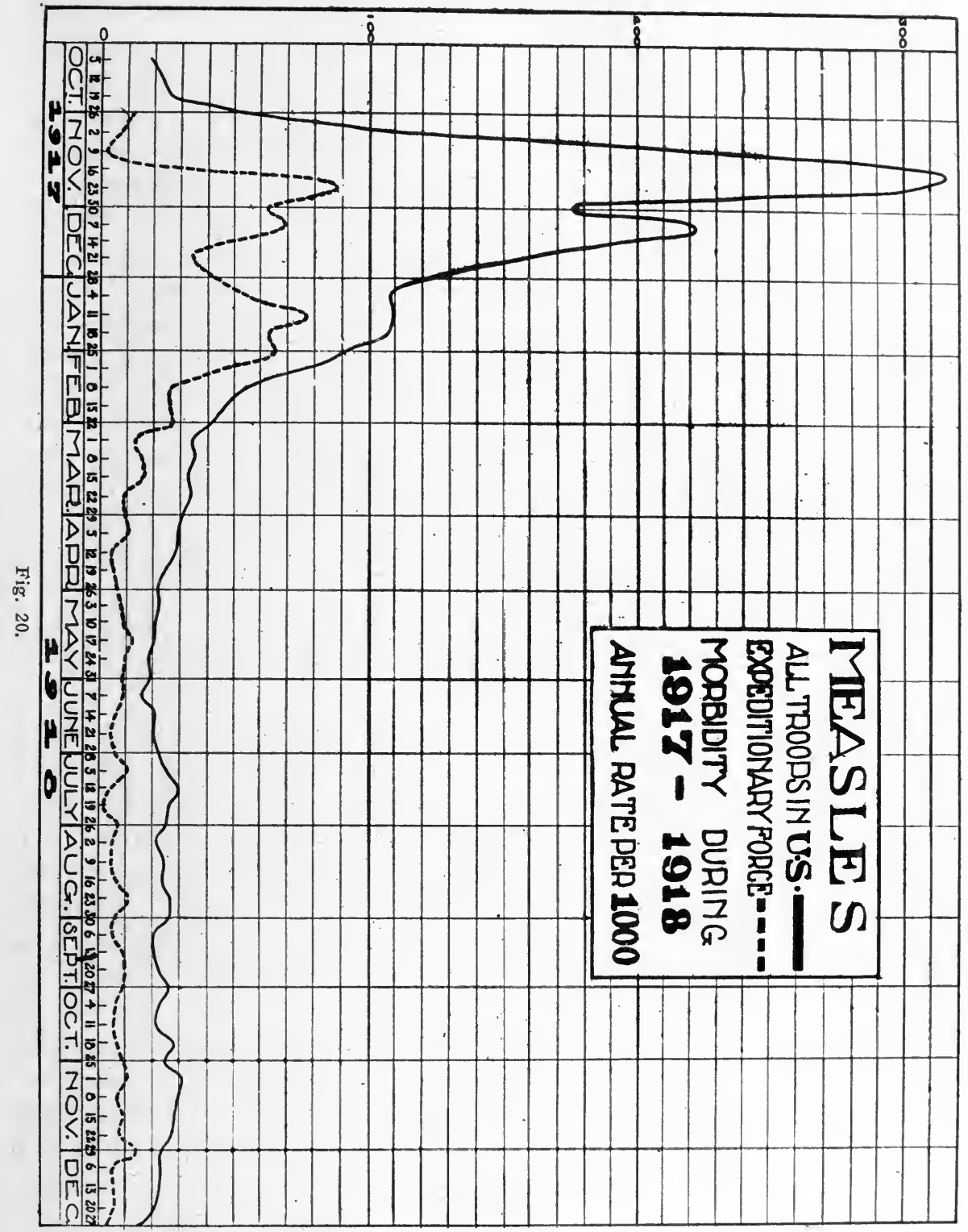

relation to pneumonia. Much has been said concerning the importance of measles as a predisposing cause of pneumonia, particularly bronchopneumonia. Of 235 cases of measles at Camp Cody during the winter of 1917-1918, 77, or 33 per cent, developed either bronchopneumonia or lobar pneumonia. Of these about 42 per cent died, which is a high pneumonia fatality. The Surgeon General's report for 1918 gives 48,900 measles admissions and 2,075 pneumonias resulting therefrom. This in- 
dicates that 4.25 per cent of measles cases terminated in pneumonia. This figure seems so small as to indicate a lack of association between the two diseases. Nevertheless, this association exists. Among 510,100 who did not have measles in 1918 there occurred 8,432 eases of pneumonia. The percentage pneumonia incidence is 1.65 . We may interpret these percentages as follows: where one man without measles has pneumonia, two to three (2.6) men with measles have pneumonia. Measles predisposes to pneumonia. Granted that measles does predispose to pneumonia, we must go further and ascertain the seriousness of it. It is much more than a ratio of 1 to 2.6 indicates. It is not merely that where one nonmeasles ease has pneumonia, there are two to three measles cases which have pneumonia. It is this; the nonmeasles case is more likely to recover from pneumonia once he has it than is the measles case. Thus, of 2,075 pneumonia cases following measles, 888 , or 42.7 per cent, died; of 8,432 pneumonia eases not following measles, 949, or 11.3 per cent, died. Measles not only predisposes to pneumonia, it predisposes to fatal pneumonia. The relationship of measles to pneumonia may be brought out in the following statements:

(1) Of every 1,000 men with measles, 44 had pneumonia and 19 died.

(2) Of every 1,000 men without measles, 17 had pneumonia and two died.

(3) A person who has recently had measles is ten times more likely to die from pneumonia than is the person who has not recently had measles.

The epidemiology of measles has been studied with more or less aceuracy in armies at different times, and it has invariably been found that this disease is more prevalent among rural recruits than among those from urban districts. It is also evident that both the morbidity and the mortality rates for this disease decrease the longer the soldiers are held in service. During the Civil War there were reported in the Union Army 67,763 cases of measles with 4,246 deaths, giving a case mortality of 6.27 per cent. It is more than probable that only a small part of this mortality was directly due to measles, but to certain sequelæ, notably pneumonia. The average annual morbidity rate of measles per 1,000 of strength was 30.41 , the maximum 77.57 during the first year, and the minimum 1.98 during the last year. Although there are no exact reports, it appears that measles prevailed in the Confederate Army and was much more highly fatal than in the Union Army. Eve says:

"In the Confederate Army measles prevailed extensively in the new regiments, especially in those from the country, and greatly impeded their organization. It so diminished the effectiveness of the troops and proved so fatal in camps that companies, battalions, and whole regiments had to be disbanded for a time and the men sent home.

"As the new men came within the influence of the contagious foci, the disease spread, giving a sudden elevation to the line of prevalence, which therefore fell until fresh accessions occasioned a corresponding rise in level. The highest rates occurred in the 
early months when the commands were small and unprotected by previous attacks. In subsequent periods, the increased prevalence of rates, if ealculated on the strength of the new regiments only, would probably have been equally high, but calculated, as they have been, on the mean strength, part of which had lost its susceptibility to the disease, they are necessarily lower than those of the earlier epidemic periods.

" Recruits from the city are more likely to have passed through the disease in childhood than those from rural districts. City regiments are, therefore, to be preferred in this connection."

The advisability of intentionally subjecting the men to measles under proper sanitary conditions was considered by medical officers in the Confederate Army, as is shown by the following quotation from Eve:

"Inoculation for smallpox was practiced before the discovery of vaccination. However, the efforts of sanitary officers have been so successful in controlling the spread of

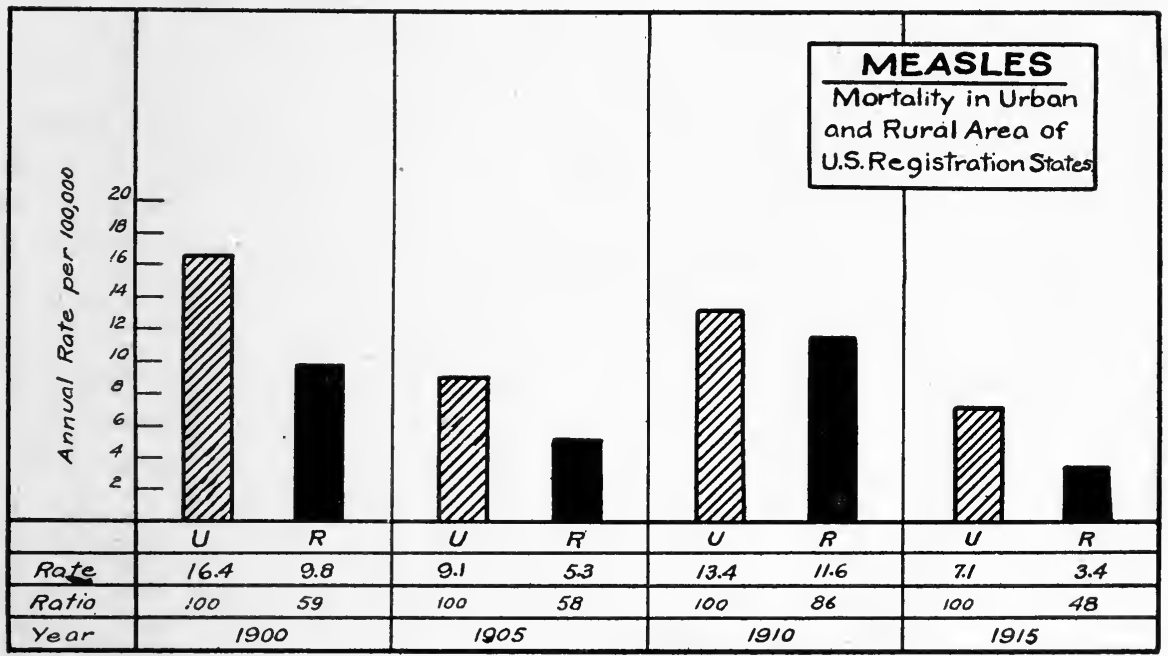

Fig. 21.

communicable diseases that iew medical men would counsel the intentional propagation of measles among large bodies of newly organized and susceptible troops. Isolation proved ineffective in restricting the disease during the war, but there is no record of its having been systematically earried out."

Periodicity of Measles Epidemics.-For many years epidemiologists in various parts of the world have reported the observation of a periodicity in epidemics of measles. It is generally believed that an epidemic of this disease is due about every two years. The figures furnished by the U. S. Mortality Statistics are of little value in trying to work out this problem. The registration area, registration states, and even registration cities cover areas that are too large. While one epidemic wave might, be at its crest in a certain community, the same wave might be at its lowest ebb in some relatively near by community and the result 
would be nore or less of a dead level. Moreover, death rates from this disease to be of value for this purpose must be presented for shorter periods of time than one year. For instance, we learn from the U. S. Mortality Statistics for 1917 that the death rate from this disease in Webb City, Mo., in 1916 was 188.1 and in 1917, 78.1 per 100,000. Both are abnormally high, but the majority of the deaths reported for 1916 may have occurred in the last months of that year, while the majority of those reported for 1917 may have occurred during the first months of that year; in other words, the figures for the two years might have been due, for the greater part at least, to the same epidemic. There is no reason for supposing that the virus of measles is controlled in any way by our calendar. In order to get anywhere in determining any law of periodicity in epidemies we must know the morbidity and mortality of the disease by days, or at least by weeks. In different parts of a large city there may be, and undoubtedly are, epidemic waves of measles on the flow or on the ebb at the same time. The best work that has been done along this line is that of Brownlee, who has figured out epidemic waves of measles, based on the weekly numbers of deaths, in London between 1840 and 1912. Brownlee concludes that there were three such waves operative in London during the period mentioned. One, the most dominant, had a periodicity of 97 weeks, while the others showed periodicities of 87 and 114 weeks respectively. The 97 weeks' strain showed its presence in all the districts of London, but especially in the West End. The 87 weeks' period was detectable only in that part of the city lying to the south of the Thames, while the 114 weeks' wave was less definitely located, though it showed most markedly in the central part of the eity. Brownlee himself seems to be somewhat in doubt as to the 114 weeks' wave and in some periods he has found it necessary to vary the length of the period somewhat. If it be true that there are two or more epidemics prevailing in the same city at the same time it must be evident that great care and skill are required in figuring out the results. For the sake of simplicity, suppose that there are two epidemics, one with a periodicity of 87 weeks and another of 97 weeks. On this supposition Brownlee makes the following statement:

"With the first recurrence, the maxima of these epidemics will be separated by an interval of ten weeks, while by the time the fifth or sixth recurrences are reached, the maximum of the one period will be coincident with the minimum of the other. A dead level of endemicity at this point is the phenomenon requiring explanation. As, however, it goes on the epidemics will again have their maxima simultaneously and epidemic outbursts will be associated with intervals free from the disease."

It is generally regarded as an established fact that each locality suffers from epidemic waves of measles and that the period is somewhere about two years. In certain relatively small localities in England where registration statistics have been kept for many years the health officer 
counts on an epidemic every two years. In some places the epidemic is expected to fall during the even years, while in others it occurs in the odd years. The usual explanation of this periodicity is the accumulation of a sufficient number of susceptibles to make an epidemic possible. These susceptibles are increased in number by births. Brownlee's conception of the cause of periodicity is quite different, and as this is a matter of great importance we make the following quotation from this authority:

"The meaning of the phenomena found now falls to be considered. The common explanation of the periodicity of epidemies of ehildren's diseases is that the susceptible children take the disease in sufficient numbers to limit the further spread. The epidemic thus dies out to recur when a further sufficient number of susceptible children have accumulated. This is quite a feasible theory and certainly explains the periodicity of epidemics. The forms of epidemic eurve which arise on this hypothesis are not unlike those actually found, the differences being no more than might be expected between a mathematical form based on a hypothesis and the natural conditions to which the hypothesis is only an approximation. This explanation, however, must fail if epidemies of different periods can be shown to exist in the same town at the same time, and I think this has been shown. In London, which on account of its size might be assumed deserving of special treatment, the existence of periods of different length have been demonstrated. In Edinburgh, Glasgow, and Birmingham also it has been shown that epidemics with periods in the neighborhood of 98 weeks and 110 weeks intermix. The same epidemicity even applies to districts in London. In the West End of London we have almost a replica of what occurs in Glasgow, Birmingham, and Edinburgh. The main period there is 97 weeks, the secondary period 1091/2 weeks. In the south of London one period is that of 97 weeks, but almost equally prominent is that of 87 weeks. The whole evidence, therefore, seems to point to some condition in the organism which produces the disease as the potent cause of the difference rather than to the number of sureeptible children. Compare the paramaecium which under natural conditions divides asexually for several hundred times and then dies out unless conjugation takes place. The resting stage following conjugation persists for some time.

"There is, however, one point of great importance which must be considered. If an epidemic begin in a definite locality and spread from that locality, and if there is no loss of infectivity on the part of the organism, it is demonstrable that a similar proportion of the population should be attacked in each zone as the epidemic spreads outward. On the other hand, if the organism lose the power of infecting with the lapse of time, in each additional zone invaded the proportion of susceptible persons infected should become smaller and smaller. Of course this might not be true for any one epidemic, as in many parts of the area invaded the population might be more or less insusceptible because of recent attack of the disease, but when an average of 20 outbreaks has been taken this effect should be eliminated, the number of times the invading organism comes into contact with an insusceptible population being balanced by the number of times which it meets one more susceptible than the average. The method of spread of epidemic on the average should thus give some indieation regarding the laws which determine the course of the phenomenon. Now with regard to London, the clearest facts refer to the 87 weeks', the 97 weeks', and the $109 \frac{1}{2}$ wecks' periods. The 97 weeks' period starts at the same time all over the eity and there is no evidence of any special center. The infection seems generalized. With regard to the 87 weeks' epidemie, however, the case is different. This seems to start in St. Saviour's 
Parish and to spread thence to Camberwell, Lambeth, etc. In this epidemic the rate of spread can be definitely measured. The maximum oceurs later and later as the distance from the center is increased and the percentage of children infected is also easily observed to fall as the time increases. With regard to the $109 \frac{1}{2}$ weeks' period epidemic the facts are similar though not quite so definite. This seems to show that for at least two strains of organism the epidemic ceases because the organism has lost its power of infecting. It may be inferred then that an epidemic ceases because the organism varies in its potency to cause infection. A cycle of epidemics now coinciding and now differing in their maxima ean thus be explained. Some kind of life cycle exists in the infecting organism. In this life cycle high powers of infecting are attained probably after a resting stage; a period of activity follows and gives place to a period of rest; the average length of the cycle is determined by the strain of the organism.",

The figures presented by Brownlee are of great value, and his theory is fascinating and has much in its favor not only in a study of epidemics of measles, but of the other infectious diseases of infancy and childhood, especially scarlet fever, whooping cough, and chickenpox. In order to solve the problem of periodicity in measles we must have more exact information than we now possess. Brownlee's figures pertain to deaths only. There are, so far as we know, nowhere in the world satisfactory statistics concerning morbidity in this disease. Newman (1919) tells us that in England only "first" cases in families are reported. Deaths from measles are so largely determined by the care bestowed upon the sick and upon the extent to which secondary infection is prevented that we are inclined to hesitate about the acceptance of a death rate or number of deaths from this disease as an index to the virulence of the organism causing the disease; in other words, we are not convinced that the death rate in a given outbreak of this disease is a measure of the virulence of the organism causing it. This involves the question whether measles per se is a disease of wide variation in malignancy or are the widely different death rates observed in different epidemics due to secondary infections. The streptococcus, a common invader of the body during the progress of a measles infection, is known to possess a most variable degree of malignancy. We are inclined to the opinion that if all cases of measles could be recognized before secondary infection occurs and could be cared for ideally, the death rate from this disease in different epidemics would be much more uniform than is now shown and would be low. The greatest danger to life in an attack of measles lies in the fact that the virus lowers the resistance of the body-cells and opens gateways to more deadly organisms, such as the streptococcus. We believe that there are demonstrated facts which support these ideas. Quite uniformly in measles there is a well marked leukopenia. As we now interpret it, this means a decrease in the number of the forces that naturally protect the body against the invasion of foreign cells. Again, as we interpret it, the failure of the body-cells to respond to the tuber- 
culin test during a course of measles or soon thereafter is evidence that the resistance of the body is lowered. If our interpretation on these points be correct we fail to see how deaths from measles can be properly employed as a standard in the measurement of the virulence of the organism of the disease. It is a generally accepted fact that when cases of measles are treated in hospitals the death rates are higher than when they are treated in their homes. We believe that this would not be true if the child could be isolated and protected properly in hospitals, but the high death rate in measles eases treated in hospitals, which certainly constitutes the history of the past, is explainable on the ground that in the hospital secondary infection is more common and more dangerous than in the home. Wilson, studying measles at Ellis Island, reports that of 1,675 measles admissions with poor isolation facilities 21 per cent suffered from serious complications, 3.2 per cent suffered from cross infection, and the case fatality was 10 per cent; while of 949 admissions with relatively good isolation facilities 10.5 per cent suffered from serious complications, 2.2 per cent suffered from cross infection, and the case fatality was 6 per cent.

During the latter part of the eighteenth century even the best hospitals in Paris were charnel houses. So mixed, varied, and deadly were the bacterial fauna of these buildings that cross infections, pus infections, and in short, many and varied kinds of infections were passed from bed to bed and from patient to patient until it happened that even the slightest wound made by the surgeon's knife or opened up by bacterial infection proved the gateway for the admission of all kinds of dangerous organisms. We fear that we have not yet fully realized the importance of secondary infection that may occur in hospitals, especially in the infectious diseases of infancy and childhood. The expert student of hospital construction is now recognizing this fact and already there are a few hospitals in which cases of measles may be cared for with no more danger of secondary infection than would happen to these cases in the best arranged homes. However, lest we be misunderstood, we hasten to add that hospital construction is not the most important matter in the prevention of secondary infections in these institutions. The most important things are the intelligence possessed and the care practiced by attendants upon these patients. The deadly secondary infecting agents are carried to the measles cases more frequently by attendants than through the air.

The histories of epidemics of measles in the Faroe and Fiji Islands and in our camps, notably Camp Wheeler, do not support Brownlee's theory. In these instances, when the lighted match was applied to the tinder piled high on the grate the flame flared up through the center and then 


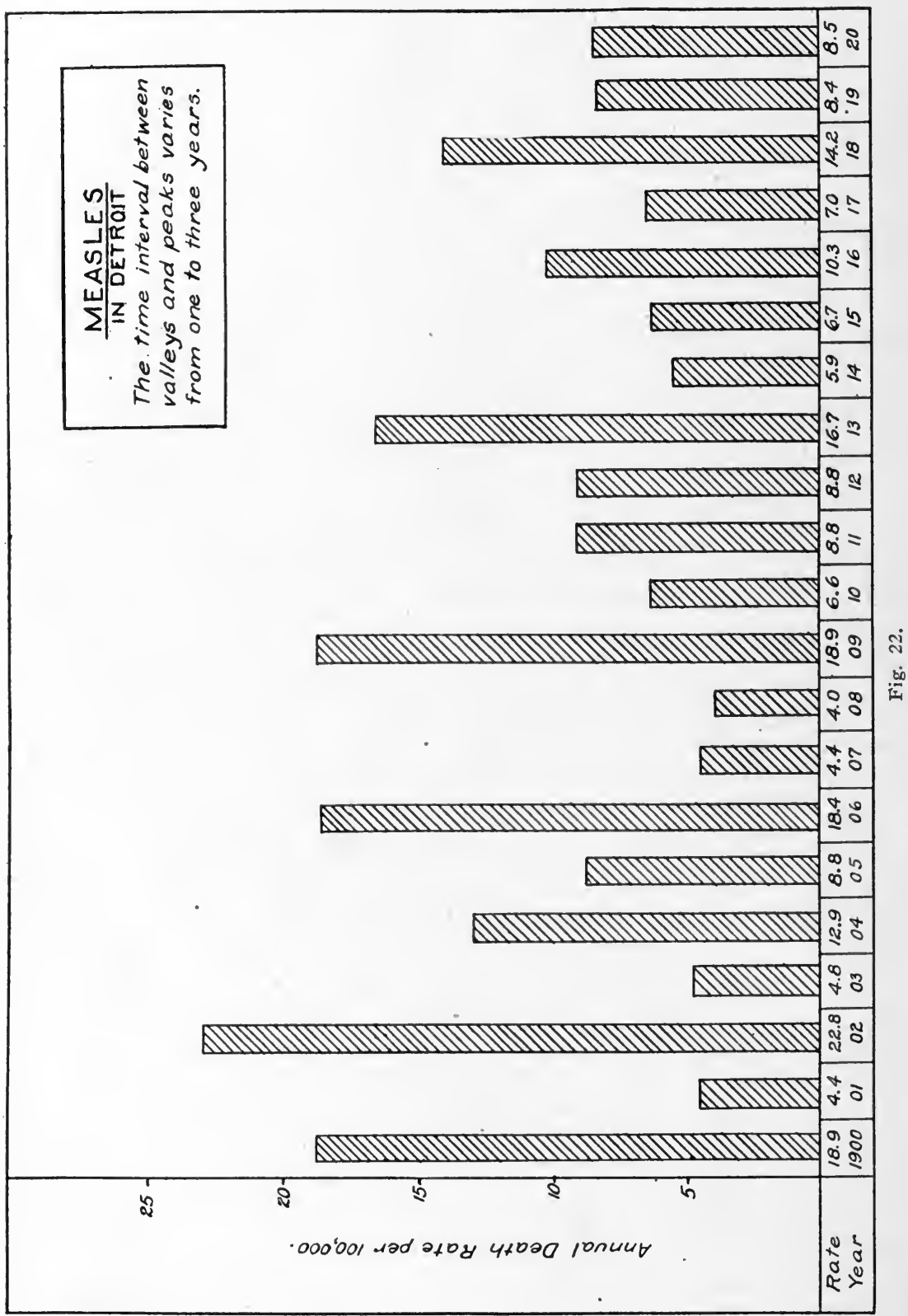

more slowly sought the outlying pieces until every shaving was consumed.

We have searched the literature for information concerning the case mortality in uncomplicated measles, and also in those epidemics in which secondary infections and complications have played important parts. 
Statistics taken from civil life are of but little value, because, so far as we know, there are no reliable morbidity statistics concerning this disease either in uncomplicated or complicated cases. We are of the opinion that in no community in civil life are all cases of measles reported. Besides, when deaths occur we are left uncertain as to whether or not there has been secondary infection. We are offering certain figures taken from army reports in our camps in 1917-1918.

TABLE XI

Case Mortality in Uncomplicated Measles at Different Periods of 1917-1918 All Troops in the United States

\begin{tabular}{lccc}
\hline \multicolumn{1}{c}{ SEASON } & CASES & DEATHS & PER CENT FATALITY \\
\hline 12 months 1917 & 44158 & 20 & 0.05 \\
Winter 1917-18 & 58035 & 52 & 0.09 \\
Summer 1918 & 13949 & 19 & 0.14 \\
Autumn 1918 & 11789 & 14 & 0.12 \\
\hline
\end{tabular}

TABLE XII

Case Mortality in all Measles (Complicated and uncomplicated) Ali Troops in the United States

\begin{tabular}{cccc}
\hline \hline YEAR & CASES & DEATHS & PER CENT FATALITY \\
\hline 1917 & 47573 & 947 & 1.99 \\
1918 & 38846 & 1144 & 2.9 \\
\hline
\end{tabular}

Accepting the figures in Tables XI and XII as correct and subtracting from the total cases the uncomplicated cases and from the total deaths the deaths in uncomplicated cases, we have the figures as shown in Table XIII.

TABLE XIII

Case Mortality in Complicated Measles

All Troops in the United States

\begin{tabular}{cccc}
\hline \hline SEASON & CASES & DEATHS & PER CENT FATALITY \\
\hline 1917 & 3415 & 927 & 27.1 \\
\hline
\end{tabular}

While these figures are probably not altogether exact, they give, in our opinion, a fair idea of the difference in death rates in uncomplicated and in complicated measles. It will be understood that the death rate in complicated measles depends upon the complication, and this upon the secondary infection. We have referred to the fact that the death rate in measles treated in hospitals is higher than in those cases treated in their homes. In substantiation of this we give below a table prepared by Brownlee. It is interesting to note that this table was prepared not for the purpose of showing the differenee in death rates between home- 
treated cases and those treated in hospital, but to show the death rate from measles at different ages. The cases reported from Glasgow were all treated in hospital, while those reported from Aberdeen were treated, some in hospital and some in their homes, most of them in their homes.

TABLE XIV

Age, Cases, Deaths, and Case Mortality in Measles in Aberdeen and Glasgow

\begin{tabular}{|c|c|c|c|c|c|c|}
\hline & ABERDEE & 3-1902 & & GLASGC & $85-1902$ & \\
\hline Age & & & Case & & & Case \\
\hline Months & Cases & Deaths & $\begin{array}{l}\text { Mortality } \\
\text { per } 100\end{array}$ & Cases & Deaths & $\begin{array}{c}\text { Mortality } \\
\text { per } 100\end{array}$ \\
\hline $0-3$ & 244 & 19 & 7.8 & 20 & 2 & 10.0 \\
\hline $3-6$ & 665 & 64 & 9.6 & 97 & 9 & 9.3 \\
\hline $6-9$ & 1263 & 186 & 14.7 & 366 & 78 & 21.3 \\
\hline $9-12$ & 862 & 157 & 18.2 & 387 & 79 & 20.4 \\
\hline Years & & & & & & \\
\hline $1-2$ & 5222 & 526 & 10.0 & 1227 & 321 & 26.5 \\
\hline $2-3$ & 5195 & 178 & 3.4 & 1545 & 240 & 15.5 \\
\hline $3-4$ & 5053 & 82 & 1.6 & 1718 & 179 & 10.4 \\
\hline 4-5 & 4336 & 43 & 0.9 & 1600 & 80 & 5.0 \\
\hline $5 \cdot 6$ & 5352 & 35 & 0.7 & 1503 & 65 & 4.3 \\
\hline $6-7$ & 4628 & 21 & $0 . \overline{5}$ & 1235 & 33 & 2.7 \\
\hline $7-8$ & 2818 & 14 & 0.5 & 796 & 11 & 1.4 \\
\hline $8-9$ & 1258 & 5 & 0.4 & 398 & 6 & 1.5 \\
\hline $9-10$ & 672 & 4 & 0.6 & 214 & 1 & 0.4 \\
\hline 10.11 & 406 & 1 & 0.2 & 110 & 4 & 3.6 \\
\hline 11.12 & 259 & - & - & 60 & 3 & 5.0 \\
\hline $12-13$ & 241 & - & - & 47 & - & - \\
\hline $13-14$ & 169 & 2 & - & 28 & - & - \\
\hline $14-15$ & 150 & - & - & 23 & 2 & 8.7 \\
\hline
\end{tabular}

There may be a fallacy in this table. It is possible that only the severer cases were carried to hospital. This seems to be indicated by the smaller number of both cases and deaths reported from Glasgow, which is the larger city.

In Chicago the total number of cases and deaths per month and per year have been reported from 1911. The ease mortality in these years has varied from 1.2 to 2.4 per cent. The highest mortality rate occurs in that year in which the smallest number of cases has been reported. This leads to the suspicion that all cases were not reported in that year. These figures include all cases and all deaths in Chicago whether treated in homes or in hospitals.

We have asked ourselves what is the cause of death in uncomplicated measles, and we are not able to satisfactorily answer this question. Brownlee makes the following statement: 
"When, on the fourth or fifth day of illness, the rash begins to appear in the bronchi, great respiratory distress and cyanosis are frequent. This condition I call 'suffocative catarrh' to distinguish it from bronchopneumonia. It is one of the curses of cellular pathology to gronp all sorts of diseases under one name. This suffocative catarrh is a part of the disease-measles--and has no relation to true bronchopneumonia, which is quite a different disease. The catarrh kills in a few days, the bulk of these deaths occurring within eight days of the first symptoms of measles. Bronchopneumonia runs a longer course, the bulk of the deaths occurring twelve days after the onset of the disease. It is a secondary infection to measles, while the catarrh is a part of the primary disease."

The Enanthem.-In 1896 Koplik, of New York, described an eruption confined to the mucous membrane of the cheek and lips occurring before the cutaneous eruption and which he believed to be pathognomonic of this disease. According to this author, this characteristic eruption con-

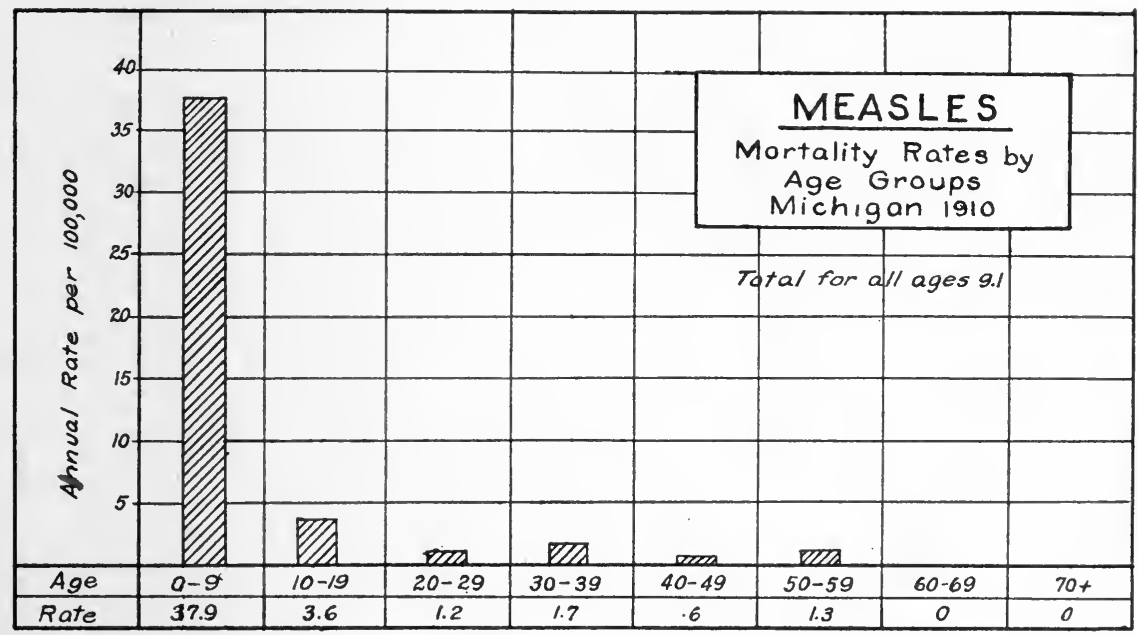

Fig. 23.

sists "of small, irregular spots of a bright red color; in the center of each red spot is seen a minute bluish-white speck. There may at first be only two or three or six such rose-red spots, with a bluish-white speck in the center. The combination of a bluish-white speck with a rose-red background on the buccal and labial mucous membrane is absolutely pathognomonic of the invasion of measles. Sometimes the bluishwhite speck is so small and delicately colored that only in a very direct and strong daylight is it possible to bring out the above effect, but the combination is always present."

Inasmuch as these spots occur in the preeruptive stage of the disease and are regarded as pathognomonic of the disease, special attention should be given to them in attempts to make an early diagnosis. It is a question, however, whether before or at the time of the appearance 
of these spots the disease is already transmissible. The New York Board of Health has issued (March, 1920) a bulletin dealing especially with the importance of these spots in making an early diagnosis. Sobel, who has written this report, says:

"Just as malaria can be diagnosticated upon finding the plasmodium in the bloodlet the history and symptoms be what they may - so can we diagnosticate measles by the presence of Koplik spots."

Physicians have been urged to pay especial attention to this diagnostic sign, with the hope that by the early recognition of the disease the infected child may be isolated and the spread of the disease curtailed. On the other hand, there are some noted and well experienced epidemiologists, Brownlee for instance, who hold that the disease is transmissible long before Koplik spots appear. This author says:

"The usual advice to look for Koplik spots is vanity. By the time these have apreared the damage is done."

The Exanthem.-The cutaneous eruption appears usually on the fourth day after the beginning of the febrile and catarrhal symptoms. It most frequently is first seen behind the ears, on the temples, along the border of the hair, and spreads rapidly over the face and neck where it is usually more abundant than on other parts of the body. In these locations it is likely to be confluent and to give to the skin a dusky appearance. The eruption consists of slightly elevated macules, the elevation being sufficient to be recognizable both by touch and by sight. When the lesions are circumscribed it may be distinctly papular, but when more diffuse it may appear as an erythematous efflorescence. As was first stated by Sydenham, it has the general appearance of flea bites and is of a dull red color, not so vivid as that of scarlet fever. There are various modifications of the exanthem in measles, but it is hardly within the province of a treatise on epidemiology to go into these points. The rash begins to disappear in the localities where it first made its appearance. The scaling is composed of minute flakes, is generally most easily seen on the face, and there is never the desquamation en masse that is so often seen in scarlet fever.

Experimental Measles.-In 1757 Monro wrote as follows:

"How successful inoculation of smallpox has turned out is known to all, but I regard it as altogether certain that inoculation of measles will be much more useful and successful. For it is well known how liable this disease is to infest the lungs, and how great destruction it causes there. This seems in the first place to be due in large part to the contagion which flies about in the air, and is drawn into the lung cells in breathing, and persistently clings to them, and causes a cough there, or in other words, excites an attempt by nature to drive off the noxious matter. If measles were really to be induced by inoculation artificially produced, it is very likely that the lungs would be more free from inflammation, and in general the disease would attack the skin only. If this should turn out so what a great profit and utility it would bring to man- 
kiud! The experiment ean bring about no inconvenience or loss. It is probable that inoculation can be performed, if only the pustules and spots of matter can be rubbed on cotton, and if this (either fresh or put on glass carefully covered and preserved) be applied to a little wound, exactly in the same way as variolous matter."

One year later, Home, of Edinburgh, reported that he had inoculated 15 persons with this disease and had succeeded in most instances in producing the disease in a modified and mild form. He made incisions in the thickest part of the measles eruption and soaked up in cotton the blood which came from these. He then made similar incisions on the arms of susceptible persons and bound over the wounds the blood-saturated cotton, keeping it in place for three days. About ten years later, Cook stated that many physicians had followed Home's method with success, but he recommended a somewhat different procedure, as follows:

"Dip a little bit of cotton or lint, on the watery humor that stands in the eyes of persons ill of the measles about the time of the crisis, make a slight scratch in the arm, above the elbow of the person to be inoculated, put the watery pledget upon the incision and cover it with a bit of sticking plaster to keep it on; and this without further trouble will produce the measles in a gentle and favorable degree, which during the whole course, will want no other care than that of keeping the patient moderately warm, nor any attendance but that of watching the fever, and encouraging the crisis, which, in a few days will carry off the infection, and complete the cure.",

Later still, Spry claimed that he had inoculated susceptible persons with measles by using linen threads which had been drawn through measles pustules. In this way he claimed to be able to induce a very mild form of the disease.

In 1822 Speranza reported as follows:

"A slight incision was made with a lancet upon a group of the more inflamed disease-spots, and with the point of the instrument charged with the bloody matter several incisions were made on the arm of a healthy person, the wounds being covered at once with a bandage. This operation was performed, with the greatest care and under our observation, upon six boys of different ages. The boys complained, a few days afterwards, of not feeling well; about the fifth or sixth days there appeared very slight traces of cold in the head, with cough and watery eyes, which remained after the appearance of a few exanthematic spots; there was very slight febrile irritation, in some cases a mild diarrhea, and by the ninth or tenth day after the inoculation measles had run its course without leaving any trace of secondary malady."

In 1842 Kantona, of Hungary, reported that he had inoculated 1,122 persons with positive results in 93 per cent. In 1850 McGirr, of Chicago, reported that he had inoculated children successfully by the method first employed by Home. In 1905 Hektoen reviewed the literature of this subject, including the reports to which we have referred and some others along the same line, and came to the conclusion that there is no adequate evidence that measles had ever been transferred by the methods claimed. Fortunately, Home gave the details of his experiment, and upon analysis Hektoen is quite certain that Home did not succeed in 
transmitting measles in a single instance. One of Home's cases had had measles two years previous to the inoculation and another had measles in the natural way six weeks after the inoculation. In the other instances, Hektoen found that the symptoms appeared too early and were not sufficiently characteristic of measles. All other inoculation experiments were made in the midst of epidemics and consequently are not convincing.

Hektoen made two inoculations with blood cultures. The blood was drawn from the measles cases and incubated for 24 hours in ascites broth consisting of two parts peptone broth and one part ascitic fluid. After incubation these cultures remained sterile, but on injection into susceptible persons induced the disease. Hektoen states his conclusions as follows:

"The results of these two experiments permit the conclusion that the virus of measles is present in the blood of patients. with typical measles some time at least during the first 30 hours of the eruption; furthermore that the virus retains its virulence for at least 24 hours when such blood is inoculated into ascites broth and kept at $37^{\circ} \mathrm{C}$. This demonstration shows that it is not diffieult to obtain the virus of measles unmixed with other microbes and in such form that it may be studied by various methods."

In 1911 Anderson inoculated nine rhesus monkeys with defibrinated blood from four cases of measles. The blood was drawn from the children within from 14 to 32 hours after the appearance of the eruption and was in all instances inoculated into broth cultures which appeared to remain sterile. The animals were inoculated intraperitoneally, subcutaneously, intracerebrally, and intravenously. Of the nine animals inoculated only four gave significant evidence of a reaction. Blood was taken from the hearts of the monkeys which appeared to react favorably and transferred to other animals. Of the five animals inoculated by passage, one gave anparently no reaction, one gave an ill-defined reaction, and three gave well-marked reactions. In those animals which gave a clearly defined reaction the neriod of incubation, that is the time elapsing between inoculation and beginning reaction, was from five to six days. Anderson states his conclusions as follows:

"The rhesus monkey is capable of being inoculated with measles with blood from a human case. This susceptibility appears at best not to be very great and is subject to considerable individual variation. The virus of measles gives no visible growth in standard glucose broth in a fermentation tube."

Recently (1921) Blake and Trask have experimentally made it quite certain that the virus of measles can be transferred from man to monkey with the production of a fairly typical disease in the animal. The material employed by these investigators consisted of the nasopharyngeal washings from patients in the preeruptive or early eruptive stages of the disease. These washings, both filtered and unfiltered, were introduced 
into the tracheas of monkeys (Macacus rhesus) and were followed after an incubation period of from six to ten days by typical enanthems and exanthems. The only symptoms lacking in comparison with those manifested in man were those of rhinitis and bronchitis. These authors make the following statement concerning this part of their work:

" The foregoing experiments have shown that monkeys injected intratracheally with nasopharyngeal washings from cases of measles in the preeruptive and early eruptive stages of the disease react after an incubation period varying from six to ten days with a constant and characteristic group of symptoms closely resembling those of measles in man. These symptoms in brief are as follows: (1) Onset with listlessness and drowsiness after a definite incubation period during which the animal appears well; (2) catarrhal conjunctivitis; (3) a well-defined and characteristic enanthem usually confined to the labial mucous membrane; (4) a definite exanthem consisting of discrete, red maculopapules constant in character though somewhat variable in extent and duration and comparable histologically with the exanthem of measles; (5) leukopenia coincident with the foregoing symptoms; (6) prompt and complete recovery after an illness of seven to ten days' duration. Variations that have occurred in this group of symptoms are ones of degree in the severity of the reaction rather than in character. In only two respects has the reaction shown any significant variation from the symptoms of measles in man; namely, in the inconstant occurrence of a definite elevation of temperature and in the entire absence of symptoms of rhinitis and bronchitis. The regularity with which this group of symptoms has developed and the close resemblance of the symptoms to the symptoms of measles are presumptive evidence that the reaction is due to the virus of measles. That the reaction is not due to ordinary organisms of the mouth flora inevitably present in unfiltered nasopharyngeal washings is shown by the occurrence of the same reaction following the inoculation of washings freed from ordinary bacteria by filtration. This, of course, does not exclude the possibility that the reaction might be caused by filtrable toxic substances contained in the nasopharyngeal secretions of measles patients rather than by the living virus of measles. This possibility, however, has been excluded by the successful transmission of the infection through a considerable series of monkeys.',

These investigators have furthermore transmitted measles from monkey to monkey by injecting the citrated blood of the infected animal into a fresh one. Equally successful results were obtained when extracts from the cutaneous and mucous tissue of infected animals were injected into fresh ones. Further evidence that the disease produced in the monkeys is identical with measles in man is shown by comparison of the microscopic examinations of the skin and buccal mucous membrane in the animal and in man. The incubation period in monkeys is shorter than that observed in man, but this is accounted for by the larger amount of infected material introduced into the animal compared with that which man is likely to receive on exposure to the disease. The onset in animals is marked by loss of appetite, drowsiness, and leukopenia. At this stage there may be a marked rise in temperature, but this is by no means constant. The conjunctivæ become hyperemic and more or less markedly injected. The onset is followed within from two to three days by a diffuse enanthem on 
the mucous membrane of the lips and cheeks, while from one to five days later still a maculopapular rash appears on the skin, coming out as a rule first on the face. This rash may spread to the skin of the neck, arms, chest, abdomen, and thighs, reaching its height in from two to three days. The exanthem then progressively fades and after from six to ten days all symptoms disappear. The claim of Blake and Trask that they have induced measles in monkeys by treating the latter with nasopharyngeal washings from human eases seems to be fully justified. Furthermore, they have rendered it quite certain that the virus is a filtrable one and that it exists during the early course of the disease in the blood, skin, and nasopharyngeal discharges, and that it is through the last mentioned excretions that the disease is transmitted from man to man.

The Virus.-It would be wholly without profit to review the literature of studies undertaken for the purpose of identifying the specific cause of this disease. We must content ourselves, for the present at least, with the recognition of the fact that the virus of measles is not known. According to Goldberger and Anderson it is present in the secretions of the nose and throat in the eatarrhal condition and may be passed through a Berkefeld filter. If we accept the work of Hektoen on two students and that of Anderson on a few monkeys, we must conclude that the virus of this disease is present in the blood and in the catarrhal secretions in the early stages of the disease; that this virus not only lives but multiplies for at least 24 hours in certain artificial culture media; that it does not belong to the ordinary bacteria, and that it is easily transmissible to susceptible individuals. This view is greatly strengthened by the recent studies of Blake and Trask already mentioned.

We are impressed by the resemblance in some respects between the phenomena of measles infection and those of protein sensitization (unfortunately called anaphylaxis). A susceptible individual rides behind a sweating horse or passes through a stable and soon thereafter develops catarrhal asthmatic symptoms. Rosenau published a few years ago some experiments in which he showed that guinea pigs kept in a stable were sensitized to horse serum. There are many instances on record where certain individuals inhaling powdered peptone are sensitized. The degree of sensitization in some of these instances seems almost unbelievable. It is reported that a highly susceptible man was sensitized to peptone whenever he came into a room where an open bottle of this preparation stood. Medical literature offers many instances of similar susceptibility to various organic preparations. We have seen scarlatinous rashes appear, accompanied by catarrhal symptoms, after the administration of very small doses of quinin. In cases of rose fever and hay fever the agents of sensitization, as we believe, are often carried through much longer distances than the measles 
infection can be carried. In all instances of this kind the only conclusion that we can come to is that minute particles are carried through the air; and still there are some who would insist upon the abandonment of all ideas implying that measles or any other infectious disease is ever air borne. We are convinced that the virus of measles is not transferred from distant hospitals or even from across the street, or possibly from an adjacent room, but we are satisfied that the infection may be and is transferred when a susceptible person comes into a room occupied by an individual in the most transmissible stage of this disease. The virus of measles must be a particulate living organism capable of passing through the air and probably of pervading the air of rooms of ordinary size. The similarity between measles infection and protein sensitization goes farther. After protein sensitization there may be, and often is, a period of incubation with prodromal symptoms and followed by cutaneous eruptions which are not unlike those of measles; in fact, the period of incubation, counting from exposure to the appearance of the cutaneous rash in measles, is not unlike that which occurs in delayed cases of serum sickness. These facts are put before the reader simply as suggestions. It is highly desirable that we should be able to identify the virus of measles and to study its life cycle; in fact, we must do this before we can hope to satisfactorily control this disease, which is so destructive not in and of itself possibly, but in its sequelæ.

Another striking resemblance between measles and protein sensitization found in the fact that the newborn child and up to six months of age is largely immune to measles: while the young of the sensitized guinea pig remains for a short time after birth sensitized to that same protein to which the mother has been sensitized. Some years ago we made experiments along this line and we found that the young of guinea pigs sensitized to a given protein responded to primary injections of this protein more or less energetically and that this sensitization in the young gradually disappeared. Similar observations have been made by other observers, but at the time we did this work we had no thought of its having any connection with the well-known comparative immunity of infants to measles. We do not know that it has, but this is certainly suggestive.

Transmission.-We have suggested that scarlet fever be regarded as "a neighborly disease," implying thereby that the most efficient factor in its spread lies in the exchange of things, fomites, between houses. In like spirit, we would suggest that measles be called "the visitor's disease," implying thereby that it is only necessary for a susceptible person to enter the room of one in the transmissible stage of measles to acquire the disease. We believe that the virus of measles is transferred, in the great majority of instances at least, from person to per- 
son and that direct contact is not essential; in other words, the contact between the individuals may be and usually is through the air. That the causal agent is sprayed into the air of his room by the coughing patient there can be scarcely any doubt. The length of time that the particulate bodies constituting the virus may remain afloat in the air of the room depends upon their physical properties and concerning these we have no exact information. It has been shown that certain bacteria thrown from the mouth and throat in coughing may remain for minutes and probably for hours suspended in the air. It is easy to conceive of the virus of measles consisting of particles so light that they may remain afloat in the air of a room for hours. There is, however, reason for believing that the virus of measles, whatever it may be, has a short extracorporeal existence. That it may be carried from patient to patient by fomites must be admitted, but that this is the usual method of transfer we cannot believe. In measles it is not necessary in order to acquire the infection that a susceptible person occupy the same bed or sit in the same chair or handle the same books as the infected individual; indeed, most casual visitation of a highly susceptible individual is sufficient to aequire the disease. The visitor need not bring anything to the infected house, nor need he carry anything, except that which he has inhaled, from the house. Fomites are not largely concerned in the spread of measles: As we have seen, with the possible exception of smallpox, measles is the most infectious of all diseases that afflict mankind. There is scarcely any condition of life, except having had measles, which gives immunity.

Control.-Chapin, in his most excellent work "The Sources and Modes of Infection," says :

"Measles is a disease which in eities it seems to be impossible to check to any appreciable extent by isolation. In Aberdeen this was faithfully tried for 20 years, 1883 to 1902 , but no apparent effeet was produced on the prevalence of the disease. Similar failures have been noted elsewhere. During the last half of this period in Aberdeen, when there was far more aceurate registration and better control than before, the number of cases rose to 24,254 , about 50 per cent more than in the first half of the period. A census of the children in eertain sehools indicated that from ninety to ninety-three per cent of children over ten years of age had had the disease. Restrictive measures which protect only 7 to 10 per cent of the population from attack were then wisely abandoned. The very exeellent report of the medical officer of health of Aberdeen diseusses the subject very fully. In New York measles was first isolated in 1896, but not until 1902 were the regulations very rigorously enforeed. The average death rate from 1895 to 1904 was 2.40 and the highest death rate since 1896 was reached in 1906 , when it was 2.69. There is no evidenee that the measures adopted in New York have had any more influenee on the prevalenee of the disease than did isolation and disinfection in Aberdeen. It seems in the highest degree probable that the disease prevails beeause of the unreeognized but infectious prodromal stage. No amount of isolation after the disease is reeognized can atone for the harm done before the diagnosis is made." 
We agree perfectly with the author of the above quotation and we are satisfied that the prevalence of measles is not likely to decrease until we are able to recognize the disease before it is transmissible or at least in the earliest stages of its transmissibility. Can this be done? We should at least attempt it, and we believe that it could be done under proper conditions in a large percentage of cases. The medical school inspector must be thoroughly convinced of the fact that every child coming to school with suffused eyes and a temperature above $99^{\circ}$ should be regarded as a probable victim of this disease and should be isolated for the protection of others and kept in bed for its own good. Nurses visiting homes within their jurisdiction should have the same training. It requires no great medical knowledge to recognize that the children in the condition which we have described are at least possible measles cases. We are sure that under present conditions, usually prevalent throughout the country, measles is not recognized in its earliest stages and that so far as the protection of others is concerned isolation under existing conditions has been futile and will continue to be so. We have stated elsewhere that not a troop train reached Camp Wheeler in the fall of 1917 that did not have on board from one to six cases of well developed measles. It was absolutely futile to isolate the men on reaching camp and expect to prevent those who accompanied these cases on the train from coming down with the disease.

Statistics from practically every part of the world show us that the mortality in measles from the sixth month of life to the fourth or fifth year is high. After the last-mentioned age has been reached measles, even with its complications, except under certain conditions, is not so highly fatal. We should, therefore, make every effort to protect children under six years of age from this disease. In doing so, as Brownlee has already pointed out, we must consider not only the child that is coming down with the disease, but especially those to whom this child is likely to carry the infection. We think it probable that if children who have reached six or seven years of age could be intentionally exposed to this infection at a time when the conditions are most favorable for their eare, the death rate from this disease would be greatly diminished. However, should this be put into practice a certain percentage of those exposed would have complications and die; therefore, we refrain from making any such recommendation. In attempting to control measles we should bear in mind that it is the older child, as a rule, who carries the infection into the home and that the danger to the younger members of the family is greater proportionately than it is to the one who brings it into the household.

In camps, especially in those filled with susceptibles, we are inclined to the opinion that even under conditions of the past, isolation has pos- 
sibly retarded the spread of measles and to this extent has been beneficial. As we have attempted to show, nothing done at Camp Wheeler lessened by one man the cases of measles in that division, but the isolation practiced retarded its advances, and to the extent that it did this such a measure was worth while.

\section{Bibliography}

Anderson, J. F.: Public Health Reports, June 9, 1911.

Blake, F. G., and Trask, J. D.: Jour. Exper. Med., 1921, xxxiii, 385.

Brownlee, John: Brit. Med. Jour., April 17, 1920.

Chapin, Charles V.: Sources and Modes of Infection, New York, 1912, John Wiley \& Sons.

Corney, Bolton G.: Transactions of the Epidemiological Society of London, 1884, iii. Creighton, Charles: History of Epidemies in Britain, 1891, Cambridge University Press.

Dunn, Charles H.: Pediatries, Southworth Co., Troy, N. Y., 1917.

Hektoen, L.: Jour. Infeet. Dis., 1905, ii, 238.

Panum,'P. L.: Virchow's Arch., i, 492.

Schamberg, J. F.: Diseases of the Skin and the Eruptive Fevers, Philadelphia, 1917, W. B. Saunders Co.

SYdenhaM, T.: Translation by Latham, London, 1847 .

Webster, Nonh: A Brief History of Epidemic and Pestilential Diseases, Hudson and Goodwin, Hartford, 1799.

Wilson, J. G.: American Association for Study and Prevention of Infant Mortality, 1918. 


\section{GERMAN MEASLES}

\section{LIBERTY MEASLES; RUBELLA}

Definition.-Rubella is a specific, mildly infectious disease, characterized by a cutaneous eruption, which may appear without prodromal symptoms, or these may be slightly in evidence. Both in infectivity and in mortality, this disease is of low grade.

History.-Capable students of the history of epidemiology claim that there is some evidence of the recognition of this disease as distinct from measles in the writings of Arabian physicians of the ninth and tenth centuries. We are also told that in the seventeenth century the learned epidemiologist of Sicily, Ingrassias, recognized that occasionally he had to deal with a disease resembling measles but to which an attack of measles gave no immunity. During the eighteenth and a large part of the nineteenth centuries there was much discussion as to the identity or the specificity of rubella and rubeola. These terms were used indiscriminately, and it is now quite evident that they were often transposed by certain authors. In the latter part of the eighteenth century certain German authorities, Selle, Ziegler, Fielitz, Sprengel, and others described highly malignant epidemies which they attributed to rubella. For instance, Formey states that between the years 1784 and 1796, 1,180 persons died from this disease in Berlin, while during the same time there were only 203 deaths from scarlet fever and 103 from measles. The description given of this malignant disease does not in any respect tally with what we now know as rubella. Formey states that the disease began in the throat where a white membrane could generally be found. Vomiting, followed by coma and delirium, was not uncommon. Others spoke of a diffused and flaming-red rash followed by desquamation en masse, and not infrequently by dropsy. Comparing this description with our present day knowledge we have no hesitancy in saying that the malignant disease here described was scarlet fever. In the early part of the nineteenth century some of the greatest German teachers, as Schönlein and Hildenbrand, taught that rubella is a hybrid of scarlet fever and measles and that transitional manifestations were in evidence in many epidemics. They evidently had some queer notions concerning the relationship of scarlet fever, rubeola, and rubella. Busche and others reported epidemics in which scarlet fever would at first be dominant, then would follow rubella, the hybrid, and as this disappeared, rubeola would take its place. This implies that one attack of any one of these 
diseases did not give immunity to the other two. Paaseh says that he attended families in which typical measles prevailed and after recovery from this disease the same children eame down with sore throat followed by a scarlatinous rash, and that some of them later developed rubella. Other authors believed that as scarlet fever lost its virulenee rubella appeared and marked the termination of the epidemic. About the middle of the nineteenth century some of the most eminent dermatologists in Germany, for instance, Hebra, Canstatt, Gintrac, and others, rejected entirely the idea that rubella is a disease sui generis. However, since 1860 the majority of German pediatrists have taught that rubella is a distinct disease, and Strümpel, in his great work on the Practice of Medieine, states that the existence of rubella eould be denied only by those who had never seen it.

The weight of opinion among great English clinieians since 1808 has been in favor of the specificity of rubella. In the last-mentioned year Willan, probably the greatest authority of the time, certainly in England, deseribed a rubeola sine catarrho which did not protect against measles. In 1815 Maton clearly pointed out the difference between searlatina, rubella, and rubeola. Rubella was given a variety of names; in fact, nearly every clinieian who wrote upon the exanthemata of infancy and childhood, for 100 years gave some new name to it. The term rubella was suggested by an English physician, Veale, in 1866 and soon found its way into the medical dictionaries. The name is espeeially appropriate, being the diminutive of rubeola and expressing at one and the same time the slight import of the disease and its relationship to measles; in other words, rubella means little or light measles.

The recognition of the specificity of rubella in France was first prominently urged by Alibert in 1832. In the first edition of their great work on diseases of children in 1853 Barthez and Rilliet named this disease roseola, but in the second edition they recognized its independent nature and stated that it bears the same relation to measles as varicella does to variola.

In our own country Homans, of Boston (1843), recognized the specific nature of this disease and described cases. His paper was followed by similar contributions by Cotting and Green. It remained, however, for the most prominent pediatrician of his time (1874), J. Lewis Smith, of New York, to bring out incontrovertible evidence of the specificity of this disease. He reported 48 cases, 19 of which to his knowledge had previously had measles. This paper was followed by others in which it was shown that large percentages of cases of rubella had previously had, or subsequently developed, measles and scarlatina. In 1887 Griffith and Atkinson independently worked up the history of this disease and established its identity beyond dispute. Griffith formulated the follow- 
ing propositions, upon which the theory of the independence of rubella rests:

“(1) It is an epidemic and contagious eruptive fever, and not a simple affection of the skin. (2) It prevails independently of the existence of measles or scarlatina. (3) Its incubation, invasion, eruption and symptoms differ materially from those of either of the other two fevers. (4) It attacks indiscriminately and with equal severity both those who had had measles or scarlatina and those who have not; nor does it protect in any degree from either of these diseases. (5) It never produces anything but rubella in those exposed to its contagion. (6) It occurs but once in the same individual.",

Incubation.-Nearly every one who has written upon this disease has been differently impressed as to the period of incubation. It is given as ranging all the way from five to twenty-one days. There is nothing like the definiteness in regard to incubation in rubella that there is concerning the same period in rubeola. This is notwithstanding the fact that many reports of outbreaks in children's homes and hospitals have been made. Griffith reported an epidemic in which a child appeared at a "home" on the day on which the rash developed. There had been no communication between the children in the home and the outside world. On the fifth day two children in the home exhibited the rash. This seems to be quite definite, and with similar reports from other observers, we think it fair to conclude that the shortest period of incubation if somewhere about five days. Where the incubation period is apparently much longer there is only one conclusion, so far as we can see, to draw, and that is that the virus has a relatively longer extracorporeal existence and that it is usually transferred from patient to patient through fomites. If this be true, rubella in period of incubation.and in transmission resembles scarlet fever more closely than it does measles.

Invasion.-As a rule, there are no prodromal symptoms or these are slight and of short duration. At one time Forcheimer believed that there exists a characteristic enanthem, about which he wrote as follows:

"It appears simultaneously with the exanthem, becoming evident about six hours after the first symptoms and lasting not to exceed 12 to 14 hours. It occurs on the soft palate, sometimes extending over on to the hard palate and is marked by the appearance of small, discrete, dark red, but not dusky, papules, which disappear in a short time, leaving no traces behind. The rest of the mouth may or may not be congested, the tonsils are more or less affected according to the nature of the epidemic."

Exanthem.-The cutaneous eruption generally begins on the face, along the border of the hair, extending down behind the ears and gradually spreading over the chest, and possibly extending to the limbs. It may involve the whole body, even including the soles of the feet and the palms of the hands. To the expert there is usually no.difficulty in recognizing the eruption of this disease. It has not the flaming-red color of scarlet fever, nor yet the pinkish dusky tint of measles. It is difficult 
to describe its exact appearance, but the color is usually denominated brown. The eruption is discrete, punctate, and macular, slightly elevated, and most distinctly colored in the center. It differs from the eruption of searlet fever not only in color, but in place of origin (always on the face) and the short time for maturation. In rubella the cutaneous eruption reaches its highest development in a short time and it lasts only a few days, not to exceed five. In the great majority of cases of rubella there is no recognizable desquamation. What there is consists of particles so fine that they escape observation. There is no defoliation en masse as there is in scarlet fever.

Transmission.-While we cannot speak with certainty, the evidence that we have leads us to believe that in the majority of instances this disease is transmitted through fomites. Evidently its infectivity does not in any way compare with that of measles and is in all probability much below that of scarlet fever. Only a small percentage relatively of the children exposed to it acquire the disease. We should state that our opinion concerning its infectivity does not correspond with that held by some eminent pediatricians. Forcheimer believed that rubella is a highly contagious disease, approximating measles in this respect. Since there are in many instances no prodromal symptoms, and in those in which they appear they are of short duration, we conclude that the period of the efflorescence of the cutaneous eruption is that of highest transmissibility.

Control.-Rubella has never been known to cause any important epidemic. It is mild and very rarely is there any complication or sequel which leads to serious results. Rather strange to say, respiratory complications are rare and at the worst seldom amount to anything more than slight evidences of laryngitis or bronchitis. The child with this disease should be isolated and kept in bed, or at least in its room. Even so mild a disease as this should be regarded as a departure from health in which bodily resistance to infection is lowered. For its own sake, therefore, the child with rubella should be withdrawn for the time being from exposure to inclemencies of weather and from the hazards of infection which always accompany mass life whether it be with children or adults. A healthy child may be carrying in its throat a bacterium which is perfectly harmless to its host, but it may prove serious when transferred to the throat of a child whose resistance is depressed even by so mild a disease as rubella. Believing as we do that this disease is usually transmitted by fomites, we must recommend that soiled articles be burned or otherwise disinfected.

\section{Bibliography}

Atkinson, J.: Am. Jour. Med. Se,. January, 1887.

Forchermer, F.: Twentieth Century Practice of Medicine, xiv, 175.

GrIFFTTH, J. P. C.: Med. Rec., New York, July 2, 1887. 


\section{CHAPTER VIII}

\section{SMALLPOX}

\section{VARIOLA}

Definition.-Variola is an acute, specific, infectious disease of unknown origin, characterized by sudden onset; usually with severe chill, accompanied by rapidly rising temperature and followed by a more or less general eruption which passes through papular, vesicular, and pustular stages, frequently leaving more, or less permanent scars.

History. - The most ancient medical chronicles of India testify to the great antiquity of the disease in that country. It is quite certain that inoculation for this disease was practiced in India before it was introduced into China, and this is believed to have been during the third century B.C. In one of the ancient Indian medical books there are special prayers which were repeated by the Brahman priests while performing the operation of inoculation for this disease. That the same or a very similar disease existed in Egypt about 1200 B.C. has been shown by Ruffer and Ferguson from eruptions found on the skin of a mumny belonging to this period. The question of the relation of Asiatic and African smallpox will be discussed later. According to the best information obtainable it appears that Indian smallpox spread first into China, then over and through western Asia into Turkey in Europe, and subsequently to America and the Islands of the Pacific Ocean.

Apparently neither Hippocrates nor Galen knew anything about this disease and we seem justified in concluding that it had not reached the Mediterranean Coast when these medical chroniclers wrote. Arabian physicians wrote about it in the ninth and tenth centuries. Abbas (980) was acquainted with its contagious character and Avicenna in the following century thoroughly distinguished between smallpox and measles.

The progress of this disease from the Orient was apparently slow, several centuries passing before it reached Europe, and still more before it became prominent at least in the British Isles. This disease must have been widely distributed in England during the sixteenth century, since Kellwaye wrote a treatise on it in 1593, in which he says:

"I need not greatly to stand upon the description of this disease, because it is a thing well known unto most people."

According to Creighton, the first recorded outbreak of epidemic proportion in London occurred in 1628. This was the year before the parish clerks began to print their annual mortality bills. Creighton writes: 
"The smallpox deaths in London in the week ending May 24, 1628, were 41, in the following week, 38, and in the third week of June, 58. Such weekly mortalities in a population of about 300,000 belong to an epidemic of the first degree; and it is clear from letters of the time that the London smallpox of 1628 made a great impression."

From 1629 to the present, with the exception of certain years, there is a record of the deaths from this disease in London. The death of Queen Mary in 1694 from the disease caused the historian, Macaulay, more than a century later, to write the following graphic description of this disease and its ravages:

"That disease, over which science has since achieved a succession of glorious and beneficent victories, was then the most terrible of all the ministers of death. The havoc of the plague had been far more rapid; but plague had visited our shores only once or twice within living memory; and the smallpox was always present, filling the churchyards with corpses, tormenting with constant fears all whom it had not yet stricken, leaving on those whose lives it spared the hideous traces of its power, turning the babe into a changeling at which the mother shuddered, making the eyes and the cheeks of the betrothed maiden objects of horror to the lover."

As Creighton points out, the distinguished essayist has undoubtedly overdrawn this picture. According to the record, there were in this year (1694) in London 1,683 deaths from smallpox, while the total deaths were 24,100. Creighton estimates from a study of advertisements for runaway boys and servants that there were about 16 in every 100 of the population of this class sufficiently marked by smallpox to make the markings of value in identification. Creighton's statement on this point is as follows:

"In the London Gazette, the first advertisement of a person 'wanted' appears in December, 1667; and thereafter until June, 1774, there are 100 such advertisements of runaway apprentices, of footmen or other servants who had robbed their masters, of horse-stealers, of highwaymen, and the like. There is always a description more or less full, and in the consecutive hundred I have included only such persons as are so particularly deseribed in feature that pock-pits would have been mentioned if they had existed. It is not until the ninth case that 'pock-holes in his face' occurs in the description, the eleventh case following close, with the same mark of identity. Then comes a long interval until the twenty-fourth and twenty-fifth cases, both with pock-holes, two of a band of highwaymen concerned in an attempt to rob the Duke of Ormond's coach near London, one of them having emerged from Frying-pan Alley in Petticoat Lane. Fifteen cases follow, all described by distinctive features, without mention of pock-marks, until we come to the fortieth, a boy of 12 or 13, who 'hath lately had the smallpox.' The next is the forty-ninth, a Yorkshire man, long-visaged and 'hath had the smallpox,' and close upon him the fiftieth 'marked with smallpox.' Then come four in quick succession, the fifty-sixth, fifty-ninth, sixty-first, and sixty-third; next the seventy-first; and then a long series with no marks of smallpox, until the ninetyfifth, ninety-seventh, ninety-ninth, and one-hundredth, three of these last four having been negroes."

Another newspaper advertisement of similar date may be of interest: 
"Wanted, a man between twenty and thirty years of age, to be a footman and under butler in a great family; he must be of the Church of England and have had the smallpox in the natural way. Also a woman, middle-aged, to wait upon a young lady of great fashion and fortune; the woman must be of the Church of England, have had smallpox in the natural way, etc."

In 1746 the first smallpox hospital in London, and so far as we know in the world, was opened. This served the rich as a place to which they could send their servants when stricken with this dreaded disease. Before that time these people had been cared for in private houses by nurses.

Inoculation.-As has already been stated, inoculation as a protective measure against this disease was employed in India at least three centuries before the Christian era. It seems that as the disease slowly and gradually spread over Asia the practice of inoculation followed and kept pace with its advance. According to Creighton, the first scientific information coming to England concerning this practice was in the form of a letter from a Greek physician, Timoni, who practiced medicine at Constantinople. This letter, written in December, 1713, was published in the Philosophical Transactions of the Royal Society early in the following year. Inoculation was practiced in Constantinople not by the Turks/ as is generally stated, but by Greeks, Circassians, and Georgians. According to the letter from Timoni, the practice was to take matter from the pustules in discrete cases of the disease, collecting it in a stoppered vial, and thus carrying it about from place to place in making the inoculations. A few punctures were made on the fleshy part of the arm deep enough to cause blood to appear. The smallpox matter was then rubbed in with the drop of blood and the part protected by a walnut shell bound over it. The pustules that resulted from this procedure were few and always discrete; commonly not more than twenty, sometimes only two or three, more rarely from fifty to one hundred. These vesicles filled with a thinner fluid than that generally found in the pustules of a naturally produced case. In many there was only a single pustule at the point of inoculation and it was found that persons showing only a single pustule were protected when subsequently exposed to smallpox. On one occasion fifty were inoculated at the same time. Of these four subsequently developed smallpox, but it was suspected that they had been infected before the inoculation. Timoni stated that he had never observed any untoward result of these inoculations. He said that he had heard rumors of ill effects, but on investigation he had found these reports to be without foundation. It seems that even at that early time svphilis was recognized as a complicating factor in inoculation. Timoni, in his letter, reports two fatalities in children following inoculation, but he states that these children were suffering from hereditary syphilis. It must be understood that he makes 
no reference to the possibility of transmitting syphilis in the process of inoculation for smallpox, but he calls attention to the undesirability. of inoculating children who are syphilitic.

The second paper on inoculation appearing in England was published in 1715 in the form of a monograph. The author was a Scotch physician, Kennedy, who had traveled largely in Greece, Turkey, and Persia. He states that in Persia the scabs from smallpox patients were powdered and taken internally. Unfortunately, he gives no detailed description of the effects of this form of administration. It is probable that in many of these cases pustules were formed on the mucous membrane of the lips and mouth. In Constantinople the matter was inserted by scarification upon the forehead, wrist, and ankle. In the same year, a paper on inoculation by Pylarini, an Italian physician, who had practiced in Constantinople, was published in the Philosophical Transactions. This author states that the practice of inoculation was introduced into Constantinople about the year 1701 by Greeks. Pylarini says that a wealthy Greek living in Constantinople had given him his first information concerning this procedure and that subsequently he observed its practice in the homes of Greek residents of Constantinople. In 1717 Lady Mary Wortley Montagu, living at Pera (her husband being British Ambassador to the Porte), learned that there was a set of old women who made it their practice to perform the operation of inoculation every autumn in the month of September when the heat of the summer had passed. Lady Montagu investigated these reports and had her five-year-old son inoculated by a Greek woman under the supervision of Maitland, the physician to the Embassy. On her return to London in 1718 Lady Montagu lent her influence to certain physicians who had been led by the publications referred to above to champion inoculation as a method of mitigating the effects of smallpox. The King, George I, was induced to remit the capital sentence of six Newgate felons on condition that they submit to inoculation. These convicts, three men and three women, were inoculated August 9, 1721, the matter being inserted on both arms and on the right leg of each. The greatest number of pustules that appeared upon any one of these convicts was 60 . Upon a seventh convict the Chinese method of placing a pledget of cotton dipped in smallpox matter in the nose was tried. This induced severe pain in the frontal sinuses, and there is no record that this experiment was repeated. After a repetition of the inoculation of six charity children the Princess of Wales had her children inoculated. The success of these experiments made inoculation popular for a short time. However, this popularity continued for only a short period. The method of inoculation was such that it caused in certain instances severe and even fatal forms of the disease. English physicians, while recognizing the fact that by inoculation they were 
transferring the disease, could not convince themselves that any adequate degree of immunity could be secured unless the inoculation was followed by numerous and well developed pustules. They could not believe that an inoculation which resulted in a single or even a dozen pustules could give immunity of any value. The more nearly inoculation was followed by all the symptoms and manifestations of true smallpox the more efficient it was believed to be; therefore, within a few years inoculation against smallpox fell into disrepute and was largely discontinued in England. During this time there was some division among English physicians. Maitland, who, as we have seen, had learned the procedure in Greece, continued this method, which was followed by the production of a single or small number of pustules. He taught that immunity was secured by the method. On the other hand, Nettleton and his followers made a long incision through the whole thickness of the skin of one arm and of the opposite leg and placed in the wounds cotton soaked in smallpox matter. It is not to be doubted that Nettleton's method not infrequently induced smallpox in aggravated and fatal form.

In 1721 a ship from the Barbados brought smallpox into Boston about the middle of April. The population of the city, as given at that time, was 10,565. In the course of the epidemic 5,989 persons, more than half the population, were attacked. The rival physicians in Boston at that time were Douglas and Boylston. Boylston practiced inoculation, but he employed the method of Nettleton. Douglas, while admitting that inoculation smallpox affords protection against the natural disease, did not believe that the practice of inoculation was justified, especially at a time when an epidemic prevailed. Moreover, he hinted rather bluntly that many of those who died in Boston during the epidemic were victims of the inoculations practiced by his rival. So clumsily and unscientifically was inoculation practiced, both in Old and New England that it was discarded and, according to Creighton, was not practiced at all in England from 1728 until about 1740.

The revival of inoculation had its impetus in the investigations of two American physicians, Mowbray and Kilpatrick, of Charleston, S. C. This eity, which had been free from the disease for 15 years, suffered in 1738 from an epidemic imported by slave ships. Mowbray and Kilpatrick began the method of inoculating from arm to arm, which continued in practice long after vaccination had been discovered. Kilpatrick published an essay in which the following statement occurs:

"Some persons were of the opinion that the pock of the inoculated would be too mild to convey the disease; or, at least, that it must become effete by a second or third transplantation. Experience manifested the contrary. I have inoculated from those who were infected by the matter taken from others of the inoculated, and found no defect. Mowbray, who inoculated many more than any other practitioner, assured me he had infused matter in the fifth or sixth succession from the natural pock, and ob- 
served no difference. * * * The smallest violation of the surface, if it was stained with blood, was a sufficient entrance for the matter, and the least matter was sufficient."

The exact number of persons thus inoculated in Charleston during the epidemic of 1738 is not known. Kilpatrick gives it as 800. Among the inoculated eight died. Of course, any method of inoculation practiced in the midst of an epidemic is quite certain to give unsatisfactory results, inasmuch as it must happen that some of the inoculated have acquired the contagion in the natural way. In 1743 Kilpatrick went to London, where apparently he changed his name to Kirkpatrick, and became "the most scientific inoculator in London." It seems that the success of vaccination from arm to arm was accepted and other methods were employed for attenuating the virus and introducing minimal quantities of it in the procedure. Tronchin, of Amsterdam, induced a small blister on the arm and passed a fine thread moistened with smallpox matter through the vesicle, securing good results. LaCondamine, a French mathematician, philosopher, and explorer, after witnessing the effects of inoculation in South America, wrote:

"The practice of inoculation was improved during the time of its disgrace. Neither the cruption is essential to the natural nor the pustules to the artificial smallpox; and perhaps art will one day come to effect what one hopes for and what Bocrhaave and Lobb have even tried-I mean a change in the external form of this malady without any increase of its danger."

Although the idea of attenuating the virus used for inoculation and of making the effects minimal was not his, the credit of this advance in procedure seems to have been bestowed upon an empiric, Sutton, who, from 1763 to 1766 inoculated many thousands of people and accumulated thousands of pounds. The most scientific writer on the subject at that time was Gatti, who practiced medicine in London, and at the same time conducted the foreign correspondence of the Royal Society and was principal librarian of the British Museum. He argued that inoculation could be made even more harmless without losing any efficiency; that the induetion of fever was unnecessary, and that a patient who had very few pustules or only one had had the smallpox as truly as one who has had 10,000 pustules. He wrote as follows:

"No reason can be alleged, why we should have the smallpox but once, that will not equally hold good for one as for 10,000 pustules. If one must have multiple pustules inoculate at multiple places."

Creighton says :

"From 1721 to 1727 the inoculations in all England were known with considerable accuracy to have been 857 ; in 1728 they declined to 37 ; and for the next 10 or 12 years they were of no account. The southern counties led the revival in the fifth decade of the century, so that before long some 2,000 had been inoculated in Surrey, Kent, Sussex, and Hampshire. Frewen, however, who could point to 350 cases done 
by himself in Sussex previous to 1749 , says that it gained but little credit among the common sort of people, who began to dispute about the lawfulness of propagating diseases, and whether or no the small-pox produced by inoculation would be a certain security against taking it by infection. In London, after the revival under Kirkpatrick's influence in 1743, inoculation became a lucrative branch of surgical practice, and was done by the heads of the profession-Ranby, Hawkins, Middleton, and others, and almost exclusively among the well-to-do. In 1747 Ranby had inoculated 827 without losing one; in 1754 his total, still without a death, had reached 1,200. In 1754 Middleton had done 800 inoculations, with one death. The operation was by no means so simple as it looked. It required the combined wits of a physician, a surgeon, and an apothecary; while the preparation of the patient to receive the matter was an affair of weeks and of much physicking and regimen. Thus innoculation was for a long time the privilege of those who could pay for it. As late as 1781, when a movement was started for giving the poor of Liverpool the benefits of inoculation, it was stated in the program of the charity that, as the matter now stands, inoculation in Liverpool is confined almost exclusively to the higher ranks, the wealthier inhabitants having generally availed themselves of it for many years."

Inoculation had but little effect upon the progress of smallpox in England. Epidemic after epidemic continued to occur and in London the disease was constantly endemic. It is found in the mortality reports for every year in the eighteenth century. In practice English physicians labored under several delusions. In the first place, the majority of them believed that smallpox never attacks the same individual twice; therefore, they held that one inoculation should give complete protection throughout life, and when this was not accomplished they pronounced the procedure a failure. It is interesting to point out that at least one physician, Kennedy, the Scotchman, did not fall into this mistake. He wrote as follows:

“The greatest objection commonly proposed is, whether or not it hinders the patient from being infected a second time. But in answer to this, it is advanced that we do rarely or never find any to have been troubled with this distemper twice in the same manner, or the same confluence of malignity. For when it happens the second time it generally proves that commonly called the bastard or hog pocks, which is empty or skinny, and very little matter or malignity contained in it."

It will be seen from this quotation that Kennedy recognized the fact that even one attack of smallpox in the natural way does not give complete immunity to the disease, but that a second attack is rare and when it does occur the disease appears in a modified and milder form. In the second place, the English physicians failed to recognize that inoculation smallpox is transmissible and that people aequiring it from the inoculated may have it in severe form. Even Maitland, who had learned the procedure in Constantinople, did not believe that the disease could be acquired by contact with those in whom it had been induced by inoculation. He inoculated a two-year old girl who developed 20 pustules and soon recovered. However, he writes: 
"But what happened afterwards was, I must own, not a little surprising to me, not having scen or observed anything like it before. The case was in short this: six of the domestic serrants, who all in turn were wont to hug and caress this child whilst under the operation, and whilst the pustules were out upon her, never suppecting them to be catching (nor indeed did I), were all seized at once with the right natural smallpox of several and very different kinds."

Notwithstanding these observations, inoculation, on the whole, gained in repute, and in 1754 the College of Physicians issued the following approval:

"The College, having been informed that false reports concerning the success of inoculation in England have been published in foreign countries, think proper to declare their sentiments in the following manner, viz:- that the arguments which at the commencement of this practice were urged against it have been refuted by experience; that it is now held by the English in greater esteem and practiced among them more extensively than ever it was before; and that the College thinks it to be highly salutary to the human race."

The reason that smallpox did not more frequently spread from inoculated cases was undoubtedly due, in part at least, to the precautions taken not for the purpose of preventing the spread of the disease, but for the good of the inoculated individual. For three weeks before the procedure the individual was placed upon a diet, restricted both in quality and in quantity. Meat and wine were prohibited and purgatives were administered at intervals of about three days. Fatigue and anxiety were avoided and an attempt was made to place the individual in the best physical and mental condition possible. While the methods of procedure differed greatly, the most customary was to make an incision about an inch long on each arm not deep enough to wound the cellular membrane. A thread carrying variolous matter was laid in each wound, covered with a plaster and held in place by a linen bandage. The dressings were not disturbed until the third day, when they were removed, and a whitish appearance was considered positive indication that the inoculation had taken. Usually on the seventh day after the operation there was some chilliness, with pain in the head, back, and limbs, and possibly nausea. On the second day after the appearance of constitutional disturbances a flea-bite-like rash, simulating that of scarlet fever, often appeared. After the fourth day the general symptoms began to disappear, while the variolous eruption protruded above the skin and gradually changed from a reddish to a yellowish hue. About the seventh day from the eruption on the face the pustules became charged with matter and then gradually shriveled and formed scabs. For some time longer the patient was kept in bed, carefully dieted, purged from time to time, and occasionally blistered and bled. Of course, there were some accidents; open sores with sloughs resulted and continued to discharge for days and weeks, but serious complications were unusual, although they did occur. Em- 
pirics, and occasionally qualified physicians, who practiced the art, were always ready to offer some explanation of all untoward results. Moore, writing of the practice, makes the following statement:

"An empiric never hesitates at making positive declarations, and is never at a loss for pretexts to cover failures. Should an infant at the accession of the variolous fever be carried off by convulsion, he denies with effrontery that the smallpox was the cause, and invents another upon the spot. Should the confluent smallpox and death ensue, he soon detects that his instructions were not strictly complied with, but some important error was committed in regimen; or, that the patient was too much or too little exposed to the air. In fine, the fault may be in the parents, in the nurse, or in the inoculated, but is never allowed fairly to fall upon the inoculator."

It is interesting to observe that it was in the period when inoculation for smallpox was being practiced in England that Haygarth suggested for the first time a plan for stamping out this disease. His first paper was written in 1777 and this was followed by a more elaborate statement in book form published in 1784. Haygarth stated the thesis upon which his plan was made as follows:

"Mankind are not necessarily subject to the smallpox; it is always caught by infection from a patient in the distemper, or the poisonous matter, or scabs that come from a patient, and may be avoided by observing the following rules of prevention: (1) Suffer no person who has not had the smallpox to come into the infectious house. No visitor who has had any communication with persons liable to the distemper, should touch or sit down on anything infectious. (2) No patient, after the pocks have appeared, must be suffered to go into the street or other frequented place. (3) The utmost attention to cleanliness is absolutely necessary during and after the distemper. No person, clothes, food, furniture, dog, cat, money, medicines, or any other thing that is known or suspected to be daubed with matter, spittle, or any infectious discharges of the patient, should go out of the house until they be washed, and till they have been sufficiently exposed to the fresh air. No foul linen or anything else that can retain the poison should be folded up and put into drawers, boxes, or be otherwise shut up from the air, but immediately thrown into water and kept there till washed. No attendants should touch what is to go into another family till their hands are washed. When a patient dies of the smallpox, particular care should be taken that nothing infectious should be taken out of the house, so as to do mischief. (4) The patient must not be allowed to approach any person liable to the distemper until every scab is dropped off, till all the clothes, furniture, food, and all other things touched by the patient during the distemper, till the floor of the sick-chamber and till his hair, face, and hands have been carefully washed. After everything has been made perfectly clean, the doors, windows, drawers, boxes, and all other places that can retain infectious air should be kept open until it can be cleared out of the house."

An English writer, Crookshank, states that similar rules and regulations were in force in New England long before Haygarth proposed them. If this be true, and there appears no reason for doubting it, the practice of controlling smallpox and other infectious diseases by isolation and disinfection originated in America.

Haygarth proposed to have general inoculations at stated times and 
places and under conditions where the spread of the disease might be controlled.

During the eighteenth century smallpox took heavy toll over the greater part of the civilized world. Making allowance for increase in population, there was but little decrease in its ravages in London from the beginning to the end of the century, with some slight decrease during the last quarter. On the whole, during the century, smallpox accounted for about 10 per cent of total deaths. In the year 1800 all deaths in the city numbered 23,068 , while 2,409 were attributed to smallpox. Certain years showed definite epidemic movements, but so far no one has been able to figure out any definite periodicity. The mortality bills give only the deaths and there is no way of estimating the morbidity. Years in which there was relatively a low mortality may have been years of marked morbidity. Speaking of the great variations in virulence, Wagstaffe, in 1722, wrote, as might be written of every infectious disease, "there were cases which a physician could not save and cases which a nurse could not lose." Inoculation for this disease continued for years after Jenner's discovery, and in fact, was not abandoned until declared a felony by Act of Parliament in 1840. The eighth clause of this Act provided that any person convicted of inducing smallpox by inoculation should be liable to imprisonment for not exceeding one calendar month. The chief argument used in securing the passage of this bill was that it tended to spread the disease.

Vaccination.-While inoculation smallpox protected individuals submitting to this procedure, it had no effect upon epidemics distributed in the usual way unless it possibly contributed to them. However this may be, it rendered one great service; it pointed the way to secure protection by inoculation with the material taken from cowpox. Indeed, cowpox fairly obtruded itself upon the attention of both the profession and the laity. There probably had been, even before inoculation for this disease was practiced in England, a popular belief that those who had been accidentally inoculated with cowpox were subsequently immune to natural smallpox. When inoculators were busy it happened not infrequently that they were called upon to inoculate those who had contracted cowpox, and in nearly all instances these persons were found to be resistant to inoculation. In 1781 Nash wrote as follows:

"It is rather remarkable that no writer should have taken notice of the cowpox. I never heard of one having the smallpox who ever had the cowpox. Cowpox certainly prevents a person from having the smallpox. I have now inoculated about 60 persons who have been reported to have had the cowpox, and I believe that at least 40 of them I could not infect with the variolous virus; the other 20 , or nearly that number, I think it very reasonable to presume (as they were no judges), had not the real cowpox. It is not my own opinion only, but that of several other medical gentlemen, that convinces me the cowpox is a prophylactic for the smallpox. I have not been able to 
discover that the human species get it from the cows in any other manner than by contact with the parts immediately infected, such as in milking; neither do I apprehend that one of the human species can communicate it to another but by the same means, as I have known some of the inhabitants of a house where it was eseaped, but none of those who lay in the same bed with the diseased person. *** When those who have had the cowpox are inoculated, the arms inflame, but never, or at least seldom, form an abscess, but some hard tumor in the muscular flesh. On cows, the cowpox usually appears at first in round pustules, afterwards in uleers upon the teats and udders, but principally upon the teats. They do not appear to have any sickness before it comes out. Their teats are so far injured by the inflammation it produces, that people are frequently obliged to open the tubes through which the milk passes with a knitting needle or some such instrument. One cow having it will communicate it to a whole dairy. * * * Cows have the disease but once. I have not been able to determine whether a person who has had the smallpox can receive this disease. In those who have had the cowpox, the arm on inoculation for smallpox is inflamed to a greater extent than in those who have not had it; but then there is little or no matter in the middle, where the puncture was made, nor does it fill as in those who have not had this disease, but soon heals and dries."

It will be seen from this quotation that Nash recognized the following important facts: (1) Cowpox gives immunity to smallpox. (2) An individual inoculated with cowpox does not transmit the affection to others unless it be through the closest contact and the transfer of some of the material from the pock to a wound in the second person. (3) That the inoculation of individuals with cowpox material is not followed by any alarming symptoms or dangerous effects. In addition to these facts, it should be stated that it was generally recognized that cowpox is spread from one animal in the herd to others by the hands of milkers and in no other way.

\section{About the same time Rolph wrote:}

"There is not a medical practitioner of even little experience in Gloucestershire, or scarce a dairy farmer who does not know, from his own experience or that of others, that persons who have suffered cowpox are exempt from the agency of the variolous poison. The late Mr. Grove was a very extensive smallpox inoculator, frequently having 200 to 300 patients at one time, and the fact of exemption, now asserted, had been long before his death abundantly established, by his experience of many scores of subjects, who had previously labored under the cowpox, being found insusceptible to the smallpox either by inoculation or by effluvia.'

Not only was the belief of the protection against smallpox secured by previous inoculation with cowpox widespread, but it had been purposely resorted to in order to secure protection against smallpox. The best and most commonly quoted illustration of this procedure, although by no means the only one recorded, is that of the farmer, Benjamin Jesty. This man had had in his employ two maidservants who, after having the disorder from the cows and knowing this to be a preventive of the smallpox, had attended, the one her brother, the other her nephew, in the natural smallpox without taking the infection. Moreover, Jesty himself 
had been inoculated with cowpox and had subsequently convinced himself that he was immune to smallpox. Consequently, in the spring of $\mathbf{1 7 7 4}$ when smallpox appeared in his village he determined to secure protection for his wife and two sons. He took them to a neighboring farm where cowpox existed and inoculated each with the virus taken from the cows. The children, who were two and three years of age, reacted in the normal way, but the wife's arm was much inflamed and no doubt gave great anxiety to the husband, and probably was the cause of condemnation of him and his procedure by the neighbors. The Jesty affair was investigated by the Jennerian Society after the announcement of Jenner's discovery and the evidence thoroughly convinced the members of this organization that the procedures had been carried out as claimed. Moreover, Jesty and his sons submitted to inoculation 31 years after they had been vaccinated and proved to be wholly resistant. Several other instances of intentional inoculation with cowpox as a protection against smallpox have been investigated and accepted as authentic.

Jenner, a country doctor, who had himself been inoculated for smallpox at the age of eight, had opportunity to inoculate several individuals who had previously had cowpox, and he found that it did not take in any of these. He, therefore, determined to inoculate some one with cowpox and subsequently with smallpox. On May 14, 1796, he took some matter from the sore of cowpox on the hand of a dairy maid and inoculated a healthy boy, James Phipps, of about eight years of age. The inoculation succeeded, the result being very much the same as that secured by inoculation smallpox. Jenner was exceedingly impatient to inoculate the boy with smallpox, and this he did on July 1 , only six weeks after vaccination. The inoculation was applied by means of slight incisions and punctures and induced no reaction. Jenner's enthusiasm, as the result of this one experiment, led him to prepare a paper for the Transactions of the Royal Society. It is worthy of record that the editor of the Transactions did not recognize the sufficiency of the proof of Jenner's claim and quite properly returned the manuscript, with the request that its publication await further experimental confirmation. It may be interesting at this point to briefly review the claims and theories advanced by Jenner at the time. Fortunately, the rejected paper was subsequently published and we have documentary evidence of the ideas which Jenner formulated at that time. He believed that an ulceration affecting the heels of horses and known to the farriers of the time as "the grease" was the source of cowpox. He wrote as follows:

"In this dairy country, a great number of cows are kept. The office of milking is here performed indiscriminately by both men and maid-servants. One of the former having perhaps been appointed to apply the dressings to the heels of a horse affected 
with the grease, and not paying due attention to cleanliness, incautiously bears his part in milking the eows, with some particles of the infectious matter adhering to his fingers. Should this be the case it commonly happens that a disease is communicated to the cows, and from the cows to the dairy maids, which pretty rapidly spreais until most of the cattle and domestics of the farm feel its unpleasant consequences.'

Having described the origin of cowpox, Jenner proceeded to deseribe this affection in cows :

"It first appears on the nipples of cows in the form of distinct pustules; very seldom white, but more commonly of a palish blue, or rather a color somewhat approaching to livid and are generally surrounded by more or less of an erysipelatous inflanmation."

The eruption and pustules that form on the hands of milkers are described as follows:

"Several inflamed spots appear on different parts of the hands of the domestics employed in milking, and sometimes on the wrists, which quickly run on to suppuration, first assuming the appearance of the small vesications produced by a burn. Most commonly they appear on the joints of the fingers, and at their extremities; but whatever parts are affected, if the situation will admit, these superficial suppurations put on a circular form, with their edges more elevated than their center, and of a color distinctly approaching to blue. Absorption takes place, and tumors appear in each axilla. The system becomes affected-the pulse is much quickened, shiverings with general lassitude, pains about the loins and limbs, with vomiting, come on. The head is painful, and the patient is now and then even affected with delirium. These symptoms, varying in their degree of violence (for they rarely attack so severely), generally continue from one day to three or four, leaving ulcerated sores abcut the hands which from the sensibility of the parts, are very troublesome, and frequently become phagedenic, like those from whence they sprung, commonly heal slowly. The lips, nostrils, eye-lids, and other parts of the body, are sometimes affected with sores; but these arise from their being heedlessly rubbed or seratehed with the patient's infectious fingers. No eruptions on the skin have followed the decline of the feverish symptoms in any instance that has come under my inspection, one only excepted, and in this a very few appeared on the arm. They were of a vivid red color, very minute, and soon died away without advaneing to maturation; so that I could not determine whether they have any connection with the preceding symptoms, but I am inclined to think they had not."'

In the rejected paper Jenner reports ten cases in which he had inoculated persons who had been exposed to cowpox and subsequently to smallpox. The time which had intervened between the cowpox and the smallpox inoculations in these cases varied from nine months to 38 years, and in all, the smallpox inoculation had been without effect. He reported smallpox inoculation in three persons who had previously been affected with "the grease." In one of these the inoculation produced its minimum effect, in a second eruptions followed, while the third subsequently caught smallpox in the natural way. It may be stated that Jenner never succeeded in showing that "the grease" had any relation 
to either cowpox or smallpox. It must be admitted that Jenner's paper contained more of theory than of demonstration and that much of this theory was never proved to be true. It may be of sufficient interest to give a further brief extract from this paper:

"It is curious to observe that this matter acquires new properties by passing from the horse through another medium, that of the cow; not only is its activity hereby increased, but those specific properties become invariable which induce in the human constitution symptoms similar to those of the variolous fever, and effect in that peculiar change which forever renders its insusceptibility of the variolous contagion. May wo not then reasonably infer that the source of smallpox is the matter generated in the diseased foot of a horse, and that accidental circumstances may have again and again arisen, still working new changes upon it, until it has aequired the contagious and malignant form under which we now commonly see it making its devastations among us? And from a consideration of the change which the infectious matter from the horse has undergone after it has produced a disease on the cow, may we not coneeive that many contagious diseases now prevalent among us, may owe their present appearance not to simple, but combined origin? For example, is it hard to imagine that the measles, the scarlet fever and the ulcerous sore throat with a spotted skin have sprung from the same source, assuming some variety in their forms according to the nature of their new combinations? The same question will apply respecting the yaws and the syphilis, and indeed many other diseases.",

Jenner's paper was not published until June, 1798, but during the interval between its rejection by the Royal Society and its publication, there was much discussion of the subject, and Jenner's friends allowed no opportunity to escape to repeat the experiment first made on the boy Phipps. At that time there was no constant supply of material for vaceination and the procedure could be resorted to only when cases of cowpox were available. In 1799 this disease was found in large herds of cows in and about London and from these sources enough material was secured to vaccinate great numbers of individuals, all of whom, or at least most of whom, were subsequently inoculated with smallpox and thus proved to be immune. Even Jenner's enemies took hold of the matter and for a time threatened to appropriate entirely both the business of vaccinating and the honor that might come from the discovery. Among the most active of these were Pearson and Woodville, who in about two months inoeulated 160 persons, 60 of whom were afterwards inoculated with smallpox, all with negative results. Pearson seems to have got hold of a goodly supply of the virus, because he sent quantities of it to France, Germany, Austria, and America; indeed, the success of vaecination, even a few years after its discovery, was recognized more universally in other countries than in England. At home there were conflicting parties among the vaccinators, each striving to get the business, win the money, and secure the fame, while all these were bitterly attacked by the antivaccinators. However, Jenner's friends succeeded in 
retaining for him at least the honor of the discovery. In 1802 Parliament granted him 10,000 pounds as a recognition of the worth of his discovery. After receiving this reward Jenner reluctantly consented to open an office in London, where his friends promised him a rich practice. However, in this adventure he failed absolutely and after a year's residence in this city retired to his village home in Gloucestershire. In 1806 Parliament granted him a further reward of 20,000 pounds and later Oxford conferred an honorary degree on him. Notwithstanding these pleasing marks of appreciation, Jenner's life was by no means free from anxiety. He claimed that vaccination gives absolute and life-long immunity against smallpox. From time to time cases were reported in which the disease appeared among the vaccinated. Jenner usually explained these failures by attributing them to imperfect and unskilful vaccination. However, in 1811 two cases of smallpox occurred in a prominent family and it turned out that both father and son had been vaccinated about ten years previously by Jenner himself. Fortunately, both revered and the recovery at least could be attributed to the vaccination. Even Jenner was compelled to admit that vaccination does not give absolute and life-long protection against smallpox, although it may modify the course and severity in the second attack. To a friend he wrote at this time as follows:

"Take a comprehensive view of vaccination, and then ask yourself what is the case? You will find it a speck, a mere microscopic speck on the page which contains the history of the vaccine discovery. In the very first thing I wrote upon the subject, and many times since, I have said the occurrence of such an event should excite no surprise; because the cowpox must possess preternatural powers if it would give uniform security to the constitution, when it is well known the smallpox cannot; for we have more than 1,000 cases to prove the contrary, and fortunately 17 of them in the families of the nobility. We cannot alter the laws of nature; they are immutable. But, indeed, I have often said it was wonderful that I should have gone on for such a series of years vaccinating so many thousands, many under very unfavorable circumstances, without meeting with any interruption to my success before. And now this single solitary instance has occurred, all my past labors are forgotten, and I am held up by many, perhaps the majority of the higher classes, as an object of derision and contempt."

It will be seen from this that Jenner finally recognized that both natural smallpox and vaccination by his method failed to give absolute and permanent immunity against the disease. As we have had occasion to remark, this is a block upon which many students of immunity have stumbled. Immunity to infectious diseases is always relative and never absolute. More than a century has passed since Jenner vaceinated the boy Phipps, and we have learned during that time that vacination and repeated vaccination are essential to the control of smallpox; and even these protective measures, as great and beneficent as they are, must be 
aided by isolation and disinfection. By the combined action of these agencies the disease which 200 years ago caused one-tenth of the total deaths in London and stamped upon its survivors more or less marked disfiguration, now stands near the small end of the mortality lists and the effects of its branding iron are seldom seen.

Arm to arm vaccination was practiced in many countries long after Jenner's discovery. However, this procedure made possible the criticism that certain diseases, especially syphilis, and possibly tuberculosis, might be transmitted with the vaccine virus. This criticism undoubtedly had some justification in fact, although the danger was greatly overestimated. In order to avoid any possibility of such a transfer, methods were devised for propagating the vaccine virus in the bovine species. This led to the establishment of "vaccine farms," as they are designated in this country. However, this gave rise to another form of eriticism. It was held that the long-continued transmission of the virus from calf to calf led to its attenuation and a consequent lessening in its protective power. This led to the enactment in certain countries of laws requiring that the virus be increased in potency in a certain number of generations by being passed through man. From Jenner's time until the present it has been generally held that vaccinia or cowpox is a modified smallpox. Numerous experiments have been made attempting to transfer the smallpox virus to the cow and in this way to secure a vaccine which would be both potent and mild in its action. In 1839 Theile, a Russian physician, succeeded in transferring the smallpox virus to a cow, and after several passages through calves, secured what was believed at that time to be an ideal vaccine. Similar results were obtained by Ceely and Badcock in England. Some of this material was sent to this country in 1852 and was used largely in New England. A French Commission, under the direction of Chaveau, succeeded in inoculating a calf with smallpox and finally produced lesions closely resembling those of vaccinia, but on subsequent passages through other animals the virus became inert. Chaveau held that smallpox could be transferred to calves but that it remains smallpox and is never converted into cowpox. The most successful experiment of this kind was made in 1881 by Voigt, of Hamburg, who succeeded in transmitting the smallpox virus to a calf, and after several passages, this strain has been employed in Hamburg for vaccination purposes up to the present time. Only twice since 1881 has this strain been inoculated into another species. In 1891 it was passed from calf to man and in 1902 from calf to rabbit. With this strain vaccinations have been successful in from ninety-eight to one hundred per cent of primary cases. It is claimed that under this form of vaccination the 
population of Hamburg has shown fewer cases of smallpox than any other community of its size in the world.

At the present time the bovine virus is used in most countries. However, Shera, writing in 1918, gives the following method for the preparation of vaccine employed in England:

"Female calves from two to four months old are taken. They are sometimes tested with tuberculin and always kept under observation for a few days, then elipped and thoroughly cleansed. The belly is completely shaved and prepared as for operation. About 100 small scarifications are now made under strict asepsis. Slight bleeding occurs, which is mopped up. The virus, which is obtained from a human case and preserved on sterile bone 'slips,' is inoculated on each area. The lesions are allowed to dry. Sterile gauze then covers the lesions. The animals are kept clean, excreta being promptly removed. The animal must not kick itself. Within 48 hours a reaction occurs, and the animal is killed after six days. Strict asepsis, as for operation, is observed. The field of papules is cleansed and curetted. After currettage serum exudes. 'Slips' are charged with this, and the pulpy exudate is made up thus:

$\begin{array}{lr}\text { Glycerin } & 50 \% \\ \text { Water } & 49 \% \\ \text { Phenol } & 1 \%\end{array}$

"The glycerin pulp is left standing three to four weeks, as it is always infected with bacteria. At the end of this time these should have undergone dissolution. The pulp is then triturated and put up in capillary tubes. The vaccine is tested bacteriologically and not put up till sterile. Also it is tested for tetanus and its potency estimated by the type of vesicle it produces."

According to Park, the most reliable seed virus is what he calls the human-calf-rabbit virus. Crusts from healthy children are collected on the nineteenth day after vaccination and worked into an emulsion with sterile water. With this material an area of about six inches square on the prepared abdomen of a calf is inoculated, while the remainder is planted with the ordinary vaccine. The virus from the limited space is separately collected and after being glycerinated is diluted with $121 / 2$ parts of a normal salt solution and planted on the properly prepared, shaved skin of the backs of rabbits. The pulp from the rabbit vaccination is used for vaccinating calves. Usually within 24 hours after vaccination the scratches become pink, within another day slightly raised and papular, and within from four to six days typical vaccinia vesicles are developed. In removing the material the scarified surface is washed with warm water and soap and then thoroughly with sterile water. The crusts are carefully picked off with sterile forceps and the contents of the vesicles removed with a curet. The mass of material thus obtained is placed in sterile beakers or tubes and treated with a mixture of water, glycerin, and carbolic acid, as given above in the English formula. According to Park, one calf should yield about ten grams of pulp-enough 
to vaccinate about 1,500 people. The efficiency of the virus is determined by inoculation on the shaved backs of rabbits. Dilutions of the virus, 1 to 10 and 1 to 100, are made, and a proper virus should produce vesicles on the rabbit in a dilution of 1 to 500 at least. The number of bacteria in the glycerinated preparation can be determined by the plate method. Usually, practically all bacteria disappear from the glycerinated preparation within a month. However, in all instances white mice should be inoculated in order to prove the absence of the tetanus bacillus.

About ten years ago manufacturers of vaccine virus in this country were led to believe that that supplied by the Japanese Government was the most efficient. Consequently, they secured this virus and for a short time it was used widely in our country. It was found to react more energetically than that which the manufacturers had been previously producing. Later it turned out that this Japanese virus was contaminated with that of the foot and mouth disease and its use was discontinued. It may be of interest to state that, although this vaccine was used on several thousand people in this country, the foot and mouth disease was transmitted to no human being and the only disadvantage was in the unnecessary extent of the pustule.

In practically all countries the manufacture of this and other vaccines is under government control. In the United States it is supervised by the U. S. Public Health Service. No one can manufacture a vaccine without a permit from this Service, and before such is granted an officer inspects the plant. Besides this, the director of the Hygienic Laboratory at Washington must go into the open market at stated intervals, purchase samples of all vaccines used in this country, and test them. All smallpox vaccines in the fresh state contain bacteria, but these usually die out in the glycerin preparation. Samples of vaccines are tested in the Hygienic Laboratory for number and kind of bacteria, special tests being made for the tetanus bacillus and for other pathogenic organisms. In Germany before the war vaccination was compulsory. This law went into effect as a result of the ravages of this disease in the Franco-Prussian War in 1870. Every child must be vaccinated during its first year and revaccinated in its twelfth year. In Austria since 1900 all children must be vaccinated before entering school. In France compulsory vaccination has been in force since 1902 . In the United States compulsory vaccination is not obligatory except in a roundabout way. The state laws provide that all children shall go to school and give the school authorities the right to exclude unvaccinated children at the time of the prevalence of an epidemic of smallpox in the community.

It is an interesting fact that, while vaccination protects against both 
revaccination and smallpox, the degree of protection against the latter is the greater. It is generally believed that when one responds typically to a revaccination he is susceptible to smallpox. While this may be a fair rule to go by, it is not necessarily true. On the other hand, there is more danger in the belief that failure to respond to revaccination is evidence that the individual is immune to smallpox. This does not necessarily follow. The failure to respond to revaccination may be due to any one of many causes, such as an inert vaccine and improper vaccination. In our attempts to eradicate smallpox we must realize that vaccination and revaccination are essential. No definite rule can be laid down as to how often revaccination should be practiced. However, it is safe to say that, while the majority of individuals are protected by proper vaccination against smallpox for a long time, probably for life, there are exceptions, and the only safe plan is to submit to revaccination every seven years. "The U. S. Public Health Service recommends that every child be vaccinated by the time it has reached the age of six months, that the operation be repeated at about six years of age, and whenever an epidemic of smallpox is present.

Too frequently vaccination is regarded as so simple a procedure that care and skill are not required in performing the operation. The skin over the insertion of the deitoid muscle should be washed with soap and water, then rubbed with sterile gauze moistened with alcohol or ether. After evaporation of the alcohol or ether a drop of the virus should be expelled from the capillary container on to the cleansed skin. A sterile needle should be drawn through the drop of virus and carried deep enough to break the external layer of skin but not deep enough to draw blood. This scratch should be parallel with the humerus and about one inch long. With the needle the virus may be assembled along the line of the scratch and rubbed in. Another method is to make six or eight oblique pricks or shallow punctures through the drop of virus and through the epidermis with the sterile needle held nearly parallel with the skin. A third method is to use a sterile drill, such as that devised by Pirquet for the cutaneous tuberculin test. This instrument is held between the thumb and middle finger and an abrasion made through the drop of virus. No blood should be drawn. Shields or other dressings should not be applied at the time of vaccination. Inspections by the operator should be made on the first, second, fifth, and seventh days after vaccination. If this is impossible the patient should report on the ninth day, or sooner if the vesicle, pustule, or crust breaks. Every effort should be made to prevent this. However, should such a break occur, daily moist dressings with some antiseptic, such as mereuric chlorid or dilute iodin, should be applied. Under no circumstances should any 
dressing be allowed to remain on the vacination wound longer than 24 hours, and no dressing should be applied so long as the natural protection is intact. Vaccination is preferably performed in cool weather when there is less danger of contaminating the wound by dust or by perspiration.

It will be of interest to briefly discuss the question of immunity to smallpox. Primitive man must have observed that one attack of certain diseases gives a certain degree of protection in subsequent exposure to the same disease. This phenomenon was observed in all countries in which smallpox became prevalent. The wise man, recognizing the fact that the chances were that he must have this disease sooner or later, decided to consult his own convenience as to when he should have it. As a result of this, the practice of inoculation smallpox followed the progress of the disease around the world. Then came the observation in England that milkers infected with cowpox were not liable to have smallpox even after prolonged exposure to the disease. Vaccination naturally suggested itself. Neither in inoculation smallpox nor in vaccination with cowpox was there any reasoning involved. During recent years, since vaccination for many diseases has come into operation, we are justified in endeavoring to ascertain whether or not there are any scientific principles involved in this procedure. We can now say, with at least a fair degree of confidence, that when a man has natural smallpox or has submitted to inoculation smallpox, or has been properly vaccinated, his body cells have been trained by the experience to destroy the virus of smallpox. The body cells have acquired by this training a new function. In making this acquisition these cells have undergone some modification in chemical structure, which is more or less permanent, and in the case of smallpox continues in a more or less efficient way throughout life. When a person protected by an attack of the natural disease, by inoculation, or by vaccination, is again brought in contact with the smallpox virus, his body cells utilize the acquired function and pour out some secretion which digests or otherwise destroys the invading organism before it has time to multiply. As Sternberg showed some years ago, the blood serum of a vaccinated ealf when mixed with the vaccine virus destroys this virus. Sternberg found that this destructive substance makes its appearance in the blood of the vaccinated calf about the ninth day after vaccination. About the fourteenth day after vaccination the destructive action of the blood on the virus reaches its maximum activity, after which it gradually declines. These observations have been confirmed by Kinyoun. This specific destructive substance can come only through the acquisition of a new function on the part of the body cells. Quite naturally, these cells do not uninterruptedly pour out this secretion 
which they have been trained to elaborate; but at any time subsequently when the stimulating agent, in the form of the smallpox virus, reaches these cells they are stimulated and pour out their specific secretion. This explanation of the chemistry of vaccination is supported by the results obtained by daily vaccination of the same individual. When this is done it has been found that the period of incubation grows shorter and shorter until at last a time is reached when the individual may be said to be sensitized to the smallpox virus, and so long as this continues he possesses some degree of immunity to the disease.

The Virus.-While the causative agent of smallpox has not been positively identified, the weight of evidence is that it is a protozoal disease and that the organism which causes it has been seen and imperfectly studied. In 1892 Guarnieri inoculated the cornea of rabbits with the virus of smallpgx and cowpox and observed changes which followed and which were the same in both instances. He chose this tissue because it gave him better opportunity to watch the evolution of the vaccinal lesions and to study the life history of the parasite. Soon after such inoculation he observed that a number of epithelial cells showed certain changes. There appeared in these cells small, round, highly refractory bodies lying in the protoplasm of the cells and surrounded by a clear zone. These bodies are spherical and vary in size from points barely visible under the microscope to the size of cell nuclei. Apparently these bodies manifest ameboid movements, and consequently change both in position and in shape. The observer could detect no limiting membrane about these bodies, nor could he discern a nucleus; in other words, they are without differentiation in structure. To these bodies Guarnieri gave the name Cytorrhyctes variolae. Soon after, these observations were confirmed by Pfeiffer, Clarke, and Wasielewski, independently. In 1903 Councilman, Magrath, and Calkins reported a full description of the life history of this parasite, and this work has been confirmed by DeKorte. This organism is apparently a homogeneous body, generally spherical, with a diameter of seven-tenths micron, although it may develop a diameter of three microns. It is believed that this parasite consists wholly of nuclear substance, and this accounts for its rapid growth.

Incubation and Development.-The period of incubation in smallpox is usually given as 12 days, though it may be as short as 8 and as long as 14. The period of invasion is ushered in by a chill, more or less pronounced, and this is accompanied and followed by a rapid rise in temperature, usually to $103^{\circ}$ or $104^{\circ}$, and in many instances higher. There may be nausea and vomiting, while the pulse is generally full and accelerated. Headache is one of the most common symptoms, while pain in the back occurs in about 50 per cent of cases. During this stage there 
may be prodromal rashes, the most common of which resembles measles. In some instances the rash is scarlatiniform. It will be understood that these prodromal rashes are not the characteristic eruptions of smallpox. With considerable regularity the characteristic eruption of the disease begins on the third day following the initial chill. In most instances the eruption first appears on the exposed parts of the body, notably on the face and wrists. From these regions the rash extends to distant parts of the body. The eruptions of smallpox pass through papular, vesicular, and pustular stages. As a rule the papules are a pinkish red and convey to the sense of touch a "shotty" feel; which is regarded as of diagnostic value. In the majority of instances the papules are discrete, though in the most severe forms of the disease they may become confluent. Usually on the third day of the eruption or the fifth day of the disease it will be seen that the eruptions contain clear serum, and vesiculation is generally completed by the fifth day after the first appearance of the eruption. The most characteristic feature of the smallpox vesicle is a minute central depression. This form is known as umbilication, and while occasionally seen in other cutaneous diseases it is quite characteristic in the natural development of the smallpox eruption. The vesicles, at first clear, become more and more turbid and finally reach, generally about the sixth day, the suppurative stage. The eruptions may continue discrete or they may become confluent, the latter condition indicating a grave form of the disease. The number of pustules varies within wide limits and generally is in proportion to the severity of the disease. Schamberg estimated the number on a patient as 26,701 and that each pustule contained three drops of pus, indicating that the patient carried in his skin about five quarts of pus. He states that he has seen more severe cases in which the number of pustules reached 40,000 , and says:

"With this prodigious amount of purulent material in the skin, the wonder is that any patient thus afflicted should recover."

As the patient recovers the pustules gradually dry up and pass through the stage of desiccation and this is followed by the casting-off of the crusts, or the stage of decrustation. The permanent scars, both in number and in depth, depend upon the abundance and size of the pustules. Unfortunately, smallpox pits or marks are usually most abundant and deepest on the exposed surfaces of the body, especially on the face. The usual duration of natural smallpox is about 40 days.

In inoculation smallpox the period of incubation is about eight days. The febrile and eruptive stages are similar to those manifested in the natural disease, though the temperature seldom goes so high, neither is the number of pustules so great. The average duration of inoculation smallpox is about 25 days. 
In vaccinia the incubation period is about five days, while the febrile period lasts from three to four days and the number of pustules is limited usually, provided the operation has been properly performed, to one or two. Desiccation occurs slowly and the duration of the infection is about 21 days.

Varieties.-Are there two closely related diseases designated by the name smallpox? As we have already stated, there is reason for believing that there are two endemic foci of this disease-Asia and Africa. Are the diseases coming from these sources identical, or are they different but closely related? The latter opinion has gained in weight and prevalence during the past 25 years. During the whole history of smallpox severe and mild epidemics have occurred not only in different localities, but in the same locality at different times. Under the name "alastrim" or "Kafir milkpox" Castellani and Chalmers recognize the African or mild form of smallpox/ They attribute it to the same cause, or at least to the Guarnieri bodies, although there must be some differences between the bodies as found in the two diseases, if there be two diseases. The authors just mentioned make the following statement:

"The causation would appear to be the same as ordinary smallpox, as it is generally agreed that Jenner's vaccination is protective, and Guarnieri bodies have been found, and the classical reaction in the inoculated cornea of the rabbit has been produced; after 60 hours the Guarnieri bodies have been recovered from the cornea, but it would appear to be due to an attenuated virus. The question which has been much debated is whether it is smallpox, chickenpox, or a new disease halfway between the two. In the first place, there are still some persons who disbelieve in the difference between variola and varicella, but their objection is usually disregarded. In the second place, alastrim differs from varicella because of-(1) Confluence of the vesicles in certain cases. (2) Its frequency among adults. (3) The partial protection by Jenner's vaccine.

"It differs from variola vera by-(1) Its low mortality (1 to 2 per cent). Because it is less severe in children than in adults, and is often found among babies, in whom the vesicles are often small. (3) There is no secondary fever in children. (4) Though Jenner's vacine is in some degree protective, the disease can oceur after recent successful vaccination. (5) Jenner's, vaccination can be successfully carried out shortly after an attack of alastrim. (6) According to the same authorities an attack of smallpox does give a lasting immunity to alastrim. Provisionally we may conclude that it is probably a slightly difierent form of disease from true variola."

Schamberg states that alastrim apparently first appeared in this country in Florida in 1896. He rejects the idea that it was imported from Cuba in 1898. He states that the period of incubation is longer than that of normal smallpox, being from 14 to 18 days. The initial stage is so mild that evidences of illness are not recognized even by the patient himself. The same author continues by saying that in this country hundreds of thousands of cases have developed and the disease 
has continued to maintain its mild character and is not reverting to the normal virulence of classical smallpox :

"Two phenomena of importance stand out in connection with this type of smallpox: (1) The disease occurs almost exclusively among the unvaccinated; a single vaceination, no matter how remotely performed, is protective in the vast majority of instances. Negroes vaccinated during the Civil War, living in the same household with persons suffering with this disease, were completely protected over 40 years later.

"This is a common circumstance. In Carbondale, $\mathrm{Pa}$., in 1912, 96 out of 97 patients who took this type of smallpox were unvaccinated, and there was some doubt about the vaceinal condition of the remaining patient. The patients who are immune against this type of smallpox are not necessarily immune against smallpox of normal virulence. (2) As has been stated, the symptomatology of this affection is mild and the disease is accompanied by a very low mortality, varying from $1 / 2$ to 2 per cent. In the epidemic referred to in Carbondale, Pa., there were no deaths. At the same time, smallpox of European importation was prevalent in Pittsburgh, Pa.; there were 114 cases with 31 deaths, a mortality of 27 per cent. We note, therefore, that the disease is characterized by a mild symptomatology and a mild infectivity. As to the cause of this deviation from classic smallpox, no definite statement can be made. Doubtless, as a result of some unknown eause, the parasite which produces this disease was attenuated in virulence; the phenomenon is what biologists would call a 'sport.' Whether or not this type of smallpox will continue to retain its present mild form no one can predict. It is possible that at some future time it may revert to the usual virulence of the disease."

Castellani has observed in Ceylon two mild smallpox-like diseases. In one the eruption reaches the vesicular stage and this form is now known as "vesicular fever." Pain develops in some part of the body and is soon followed by a slight rise in temperature, accompanied by an eruption which generally begins near the site of the pain but may extend to distant parts of the body. The vesicles are discrete and a few may become umbilicated. They are smaller than those of chickenpox and there is no inflammation about the base. The contents may become purulent. There is nothing known concerning the cause and the prognosis is good. Under the name "papular fever," the same author mentions another tropical disease. There is a prodromal period, during which the patient complains of malaise, depression, and pain in the back. After three or four days a morbilliform rash appears. Koplik spots in the mouth have not been observed. There are no eatarrhal symptoms of the eyes, nose, or throat. The eruption lasts from two to five days and then fades away without leaving any mark. It is distinguished from measles by the absence of Koplik spots and of eatarrhal symptoms. From German measles it is distinguished by its longer duration and from the diffuse type of the eruption. Nothing is known of its etiology and the prognosis is good.

Varioloid.-The name of this disease is unfortunate, inasmuch as it signifies a disease resembling but different from variola. Varioloid is smallpox and unprotected people who come in contact with it are liable 
to infection with variola vera. As we have seen, it was held for centuries that no one could have smallpox more than once. Then it was held that inoculation smallpox gave immunity for life, and with Jenner's discovery came the same claim for vaccination. We now know that none of these claims are true. One may have smallpox two or more times. Inoculation with smallpox does not give permanent and absolute protection. Even repeated vaccination does not secure this result, but when one has smallpox a second time or after inoculation, or after proper vaccination, the attack is a modified form of the disease; but it is still smallpox and it is still contagious. While varioloid is, as a rule, of but little danger to the person having it, it is quite as dangerous to the public as natural smallpox. In varioloid all the symptoms and lesions of smallpox are in evidence, but in a greatly mitigated form. The eruption does not differ except in number of pustules and depth of their penetration from that characteristic of geruine smallpox. It might be said that, while varioloid is not a matter of great concern either to the patient or to the physician in attendance, it may be a matter of grave concern to those who are exposed to it.

Present Prevalence.-It will be interesting to collect such information as we are able to find concerning the prevalence of smallpox during the first 20 years of this century. Vaccination by Jenner's method was fairly coming into operation in 1800 , this diseovery placing in the hands of man the most potent procedure for the prevention of a widely prevalent and loathsome disease. To what extent has man availed himself of this beneficent discovery? In 1900 the death rate from this disease in the registration area of the United States was 1.9 per 100,000. In 1902 it increased to 6.6 per 100,000 . Since 1910 it has never reached 0.5 per 100,000 . It is a noticeable fact, applicable to other infectious diseases as well, that the death rate from smallpox is much higher, in registration cities located in nonregistration states than it is in cities located in registration states. This is to be expected, because the order of admission of states to the registration area is the best possible index of the attention given by the state authorities to the control of infectious diseases. While smallpox is becoming a negligible factor in the death rate of our country at large, it has in certain localities and at certain times displayed a virulence and produced a mortality comparable with those of prevaccination times. At El Paso, Tex., in 1914, the death rate from this disease was 101 per 100,000, and in every year since 1910 there have been two or more outbreaks in localities, causing alarming death rates. As a rule, localities showing unusual death rates from this disease are those which, for the time being at least, include among their inhabitants relatively large numbers of ignorant people. In 11 minor cities showing a 
death rate of ten or more per 100,000 from smallpox from the years 1911 to 1917 , there has been a large negro population. The migration of this race in large numbers to northern cities, notably Chicago and Detroit, has been followed by startling outbreaks of smallpox. Along the Mexican Border the death rate from this disease is kept relatively high by Mexicans. During 1917 there were 110,073 persons vaccinated on the Texas-Mexican Border and even this energetic procedure did not succeed in preventing the introduction of the disease into this country, as is shown by the fact that in the same year the death rate from smallpox at El Paso was 5.8 per 100,000. The smallpox imported into this country from Mexico has been an especially malignant type of the infection. That vaccination and revaccination properly performed may reduce the mortality from this disease to the zero point is shown by the fact that during the World War in no army was this disease anything more than a negligible factor in the mortality lists. In the annual report of the Surgeon General for the year 1919 smallpox is not mentioned, and we fail to find evidence of a single death from this disease in our army or in any division of it whether located in the continental area, in the Philippines, in the Sandwich Islands, on the Canal Zone, or on the fighting fields of France.

The relation of vaceination to smallpox is well shown by recent statistics published in Detroit. From April 1 to June 30, 1920, there were reported 482 cases of smallpox. As determined by inquiry and arm examination,

382 had never been vaceinated $(79 \%)$.

21 had been successfully vacinated more than 8 years previously $(4.4 \%)$.

23 had been vaecinated but never successfully more than 8 years previously $(4.8 \%)$.

25 had been vaecinated but unsuccessfully within 8 years $(5.2 \%)$.

$S$ had been vaccinated successfully after exposure to the disease $(1.7 \%)$.

23 had been vaccinated unsuecessfully after exposure to the disease (4.8\%).

This experience may be summed up with the statement that no case of smallpox was discovered in which there had been a successful vaccination within 8 years.

From these 482 cases there resulted three deaths, an infant, an aged person and a young woman with a previous history of Bright's disease. Most all cases were mild, not over a dozen being of the confluent type.

Cases among white people amounted to 320 , colored 162 . The population of the city was about 950,000 white and 50,000 colored. The white rate per 100,000 people is 34.7 ; the colored rate 324 . Incidence among the colored is thus nearly ten times that of the white.

Divided by sex and color there were: 
178 white males

142 white females

144 colored males

18 colored females.

As the number of colored males in the eity is so much greater than colored females, the difference does not indicate a sex selection of the disease.

Age grouping among the white cases was as follows:

$\begin{array}{cc}\text { AGE } & \text { PER CENT OF CASES } \\ 0-9 \text { years } & 25 \\ 10-19 \text { years } & 18 \\ 20-29 \text { years } & 22 \\ 30-39 \text { years } & 16 \\ \text { Over } 39 \text { years } & 19\end{array}$

Control.-Should vaccination and revaccination be made compulsory? As we have already seen, this has been done in Germany since 1874 and has resulted in the practical annihilation of this disease in that country.

In the United States the state of Pennsylvania has had a very drastic vaccination law since 1895 which requires the exclusion from the public schools of children who have not been vaccinated. An amendment to this law in 1919 extends its jurisdiction to private and parochial, as well as public schools. The validity of the original act has on several occasions been upheld by the superior court of that state. An opinion rendered in 1916 has decreed that parents cannot escape the compulsory school attendance law by refusing to have their children vaccinated. In 1921 the municipal health authorities report that there has not been a case of smallpox in a native Philadelphian during the past fourteen years. In New York State the compulsory vaccination law for school children applies only to cities of over 50,000 population. In most states the law requires that children between certain ages shall attend school and it gives to the local school authorities permission to exclude children who are not vaccinated from the schools when smallpox exists in the community. This gives opportunity for outbreaks of the disease in the schools due to the exposure of a large number of unprotected scholars at times when the disease is not known to exist in the community. In Michigan University a few years ago a student who had never been vaccinated, returning from his vacation, developed the disease and exposed several hundred others. There were 17 cases in all and in no instance did the disease appear in one who had been vaccinated. By this time the university authorities, recognizing the prevalence of the disease, compelled each and every student to submit to vaccination or revaceination or 
to leave the university. Instances of this kind are constantly happening. The regents of the University of California require that all intrants shall show satisfactory vaccination sears or other evidence of immunity against smallpox or submit to vaccination. The laws of California sustain this wise provision, but there are many states in which it would not hold. In 1896, the one hundredth anniversary of the discovery of vaccination, there was a deadly epidemic of smallpox in Gloucestershire in the locality in which Jenner lived and where he did his work. Had compulsory vaccination been in effect this would not have occurred and many lives would have been saved, but unfortunately man needs painful reminders now and then of his failure to use his intelligence.

When smallpox occurs in an industrial population the intelligent health officer has, as it now stands, certain means by which to control the disease. He can notify the managers of manufacturing plants, mercantile companies, the owners of boarding-houses, and the keepers of places of amusement, that if they admit to their places the unvacinated their establishments will be quarantined. The health officer sends out a physician who is to do the vaccinating, a nurse who is to assist, and a policeman who is to be a reminder of the fact that the law will be enforced. This party visits, for instance, a boarding-house, a hotel, a manufacturing establishment, a dry goods store, and announees its readiness to vaceinate all persons who cannot show evidence of being protected. The health officer, through this agency, is ready to do the work, but there is no compulsion about it. Any one who objects will not be troubled, but the place will be quarantined if the request is not complied with. This method of dealing with the disease is much more costly both in money and life than compulsory vaccination. The individual who contracts it must be isolated, and the care of the ignorant, and we might say of the vicious, who still insist that they will not be vaccinated, costs the community large sums. Unfortunately the race as a whole, or at least a considerable part of it, fails to profit by the experience of the past and to listen to the wisdom of the more intelligent of their own time and needs the direct stimulation that comes to them in the form of disease.

\section{Bibliography}

Castellani, A., and Chalmers, A. J.: Manual of Tropical Medicine, New York, 1919, William Wood \& Co.

Councilman, W. T., Magrath, G. B., Calikins, G. N., Et AL.: Jour. Med. Research, 1904, xi.

Creighton, Charles: History of Epidemics in Britain, 1894, Cambridge University P'ress.

Crookshank, E. M.: History and Pathology of Vaccination, London, 1889, H. K. Lewis.

Haygarth, J.: An Inquiry How to Prevent the Smallpox, London, 1784, J. Johnson. 
Jenner, Edward: An Inquiry into the Causes and Effects of the Variolae Vaceinae, London, 1798, S. Low.

Park, Wm. H., and Williams, A. W.: Pathogenic Microörganisms, Philadelphia, 1920, Lea and Febiger.

Schamberg, J. F.: Diseases of the Skin and the Eruptive Fevers, Philadelphia, 1917, W. B. Saunders Co.

Shera, A. G.: Vaccines and Sera in Military and Civilian Practice, London, 1918, Oxford University Press. 


\section{CHAPTER IX}

\section{CHICKENPOX}

\section{Varicella}

Definition.-Varicella is a benign but highly contagious disease, characterized by a cutaneous eruption, which develops in successive crops. The eruption is confined to the superficial layers of the skin and is accompanied by only moderate disturbances in the general health. Death has never been known to result from this disease and one attack, in the majority of cases at least, protects for the remainder of life.

History.- In all probability varicella is one of the most ancient of diseases, but for centuries it was not distinguished from smallpox. So far as the records show an Arabian physician, Rhazes, of the ninth century, was the first to call attention to the fact that there is an eruptive disease resembling smallpox but in no way does one of these diseases protect against the other. This early observer, being well acquainted with the fact that a second attack of smallpox-is rare and that when it does occur develops in a modified form, noticed that a disease prevalent at times among his patients had no influence upon smallpox nor did smallpox have any influence upon the other disease. In 1553 a Sicilian physician, Ingrassias, described varicella so accurately that there is no question concerning the disease which he had under observation. Somewhat later, Vidius wrote of the same disease and gave it the name Variolæ crystallinæ and for centuries it was known as crystal or glasspox. In 1694 an English physician, Morton, wrote quite extensively on varicella under the medical name of Variolae admodum benignae, indicating that he believed it to be a benign form of smallpox. In Morton's writings there is for the first time the vulgar name of chickenpox, which has no reference to the barnyard fowl but was chosen in comparing the papules with the chicken-pea. In 1730 Fuller expressed his opinion concerning the relationship between this disease and smallpox, as follows:

"The pestilence ean never breed the smallpox, nor the smallpox the measles, nor they the crystals or chickenpox, any more than a hen ean breed a duck, a wolf, a sheep, or a thistle, figs, and therefore one sort cannot be preservative against any other sort."

The fact that chickenpox is entirely distinct from smallpox was emphasized and made prominent by Heberden in the first volume of the Transactions of the Royal College of Physicians (1767). During the latter half of the ninteenth century successive heads of the Department of Dermatology in the University of Vienna, notably Hebra, Kaposi, and 
Kassowitz, taught that chickenpox is a mild form of smallpox. Hebra wrote :

"I apply, then, the name variola vera to the most severe form of this disease, that in which the eruption is abundant and the fever intense, and in which a fatal result is often observed. On the other hand, I use the term varicella for cases in which the rash is very scanty and which run a favorable course and always terminate in recovery. * * * There is positive proof that varicella may generate variola or varioloid, and, conversely, variola may produce in another individual varicella."

It is evident that this distinguished dermatologist employed the term varicella for modified smallpox and not for chickenpox. Had he been dealing with true varicella he could scarcely have made the above quoted statement. Chickenpox cannot be even a modified smallpox, because an attack of one disease not only fails to give any degree of immunity, but fails absolutely to modify a subsequent attack of the other.

Numerous attempts have been made to inoculate children with varicella. Bryce of Edinburgh, in 1816 took fluid from the vesicles of varicella at all stages in the development of the disease and at different seasons of the year, inoculated children who had never had smallpox or cowpox and in all instances he failed to produce any eruptive disease. Like attempts followed by like results have been carried out by Delpech at the Hospital Necker in Paris. Hesse inoculated 113 children with varicellous fluid. In 87 there was no effect; in 17 a slight local manifestation, while in 9 a general eruption resulted. Smith, in New York, attempted to transfer chickenpox to children who had never had the disease and failed in all instances. The only successful record we can find is that of Steiner, who states that he inoculated 10 children and in 8 of these typical chickenpox developed. However, we hesitate to accept Steiner's conclusions on account of the universal failure of others, and it seems to us more probable that Steiner's apparent success was more likely due to natural infection than to the intentional transfer of the disease.

Incubation and Development.-The period of incubation in chickenpox is given by prominent authorities at widely different figures, ranging all the way from four to twenty-one days. This is not surprising, since it is quite impossible to more than guess at the time when the successful transfer from child to child occurs. There is one striking point about the development of this disease. In most other exanthematous diseases there is a distinct, and sometimes prolonged, prodromal period before the eruption occurs. This is not the rule, at least, in chickenpox. In the majority of instances the appearance of the papule is the first indication that the child has anything the matter with it. In adults, and rarely in children, there may be a period of malaise and general ill-feeling before the eruption appears, but, as a rule, this is not the case. In smallpox there is the chill, usually followed by threc days of fever before the eruption is in evidence, while in chickenpox, 
as has been stated, the eruption appears as a rule without previous chill or fever. In many instances the elevation of temperature throughout the disease is slight, not reaching $100^{\circ} \mathrm{F}$. There are exceptions and cases have been reported of temperatures above $104^{\circ}$ and even above $105^{\circ}$. Even such temperatures as these are not indicative in chickenpox of serious results. As a rule, they are of brief duration and are not necessarily accompanied by overabundant eruption.

While chickenpox is much more frequently observed among children than among adults, there is no justification in the claim made by some observers that a natural immunity comes at about the twelfth year of age. It is true that the disease is not common after twelve and still less common in adult life. This is due to the fact that most individuals have had the disease before reaching these ages. Chickenpox, while rare among adults, is by no means unknown at this time of life.

During the year 1919, the City of Detroit reported that eighty-three per cent of the cases coming to the attention of the Health Department were among children less than ten years of age. There were 27 eases over thirty years of age, three of these being over fifty.

The eruption usually appears first on the face, then on the back, and later successive crops develop on various parts of the body, though, as a rule, they are not in marked evidence on the extremities and most rarely do they appear on the palmar surfaces of the hands and feet. It is plainly seen that the eruption involves only the superficial layers of the skin, although sometimes destruction of tissue may go deeper and it is by no means uncommon for this disease to leave permanent and visible evidence of its former presence. The eruption reaches the vesicular stage quickly, usually within a few hours, and the vesicles seldom develop into pustules, though they may become infected and continue into ugly sores and even sloughs. The fact that the eruption appears in crops gives opportunity to study on different parts of the body at the same time the varying stages of accession and recession of the skin lesions. Individual vesicles disappear within a short time, usually six or eight hours.

Diagnosis.-The chief interest on the part of the epidemiologist in the study of chickenpox is to be sure that it is not confounded with smallpox. In the majority of instances the expert will have no trouble in positively determining the nature of the case before him. The absence of prodromal symptoms, especially the chill and high temperature, the presence of evidence of effective vaccination, and the absence of smallpox in the community, together with the appearance and rapid development of the vesicles, can leave no room for doubt. However, there are cases which, under certain conditions, trouble men of the widest experience. Of eourse, whether it be variola or varicella, the patient should be isolated. 


\section{CHAPTER X}

\section{DIPHTHERIA}

Definition.-Diphtheria is a specific, highly contagious disease caused by the Bacillus diphtheriæ (ordinarily known as the Klebs-Loeffler bacillus) which grows in the pharynx or other portions of the upper respiratory tract, forming a characteristic membrane and elaborating a soluble toxin, which is absorbed and to which the symptoms and lesions of the disease are due. The bacillus itself may find its way into the blood and tissues, but the essential factor in the causation of the disease is the soluble toxin. One attack does not give immunity.

History.-Under various names this disease is described with more or less accuracy in some of the most ancient medical records. It is the opinion of certain learned Jewish epidemiologists that a disease mentioned in the Babylonian Talmud is diphtheria. There is quite convincing proof that this disease existed in Greece long before the time of Hippocrates, and it was believed by the Greeks that it came to them from Egypt, to which country they were wont to ascribe most of their ills. The descriptions of Aretaeus of both the pharyngeal and the laryngeal manifestations of this disease are quite accurate and are applicable to cases that we see at the present time. Galen wrote of false membranes in the larynx and pharynx and stated that the former were sometimes removed by coughing and the latter by hawking. During the dark ages diphtheria was recognized here and there and undoubtedly added much to the high death rate of that period. During the sixteenth century epidemies in malignant form occurred from time to time in Spain, France, Switzerland, Holland, and Germany. In 1613 this disease was so widely prevalent and so deadly in Spain that in the chronicles of that time this is known as the "diphtheria year." In the same country and in the same century Heredia recognized the asthenic and suffocative types and wrote concerning diphtheria paralysis. Before the middle of the seventeenth century diphtheria had found its way into the American colonies and we are told that in the year 1659 Samuel Danforth lost four of his eleven children of "a malady of the bladders in the windpipe." As early as 1761 an American physician, Bard, made important contributions to the study of this disease and held, contrary to the teachings of his time, that croup is laryngeal diphtheria. It is to Bretonneau, however, that we owe the beginnings of the modern study of this disease and its present name. He published his first essay on this disease in 1821. Basing his statement upon observations made in an 
outbreak in a garrison at Tours, he showed that diphtheria is an infectious disease and he demonstrated that among the troops the infection was carried in common drinking-cups and other table utensils. $\mathrm{He}$ elaimed that croup is laryngeal diphtheria and that the angina of scarlet fever is quite distinct; in fact, Bretonneau so thoroughly studied the epidemics under his observation that there was but little added to our knowledge of the disease until the discovery of the specific bacillus and its toxin. Bretonneau's views were accepted and emphasized by the great clinician, Trousseau, and were adopted by the profession in France and America but were ignored largely. in Germany until the development of bacteriology rendered their demonstration possible. In England diphtheria was confused with scarlet fever up to the middle of the nineteenth century and the name of this disease appeared for the first time among the classified causes of death in London in the report for the year 1855 . Even after that time this disease was in many instances diagnosed as "cynanche maligna."

During the sixties of the nineteenth century many were busy searching for the particulate cause of this disease and several organisms were presented as claimants for this honor, but at that time bacteriologic methods had not been developed sufficiently to enable the most expert to pick out the one responsible for the disease. In 1883 Klebs called attention to a bacillus with well marked characteristies frequently found in diphtheritic throats and a year later Loeffler continued this work.

The Bacillus.-The Klebs-Loeffler bacillus is a nonmotile rod, generally straight but sometimes slightly bent. Its length is quite variable (from 3 to 5 microns) and its breadth about one-fourth its length. The most characteristic form of the bacillus is the club shape. All the rods do not have this form, but in every field there are many so characteristically clubbed that the expert has no difficulty in identification at sight. This is true, especially of cultures made directly from the throat. Even the presence of other bacteria does not prevent identification. A sterilized cotton swab is drawn over the membrane in the throat and then over the surface of a slant serum tube. After from six to twelve hours in the incubator a stain of the growth on the serum is made. While the diphtheria bacillus takes most basic' stains well, Loeffler's alkaline methylene-blue is most generally employed. Direct microscopic examination of this stain enables the expert to determine with certainty in the great majority of instances the presence or absence of the specific organism and to announce the bacteriologic diagnosis. The bacteriologist, however, does not depend solely upon the presence of club shaped bacilli. The Klebs-Loeffler bacillus has a peculiar segmented appearance when colored with the stain mentioned above. This is more easily recognized than described, but it becomes so familiar to 
one engaged in this work that he is seldom mistaken. The morphologic identification is rendered easier by the fact that, as a rule, the only other bacteria on the slide are streptococci. If the observer satisfies himself that only streptococci are present, he makes a negative report. In a few instances there are diphtheria-like organisms which may leave one in doubt. However, when in doubt the only wise thing to do is to make a positive diagnosis, when the case will be properly isolated and treated with antitoxin.

Subcultures of the diphtheria bacillus show many and varied involution forms. Many attempts have been made to establish a relationship between morphology and virulence, but these have led to no practical results. Loeffler's serum should be carefully prepared and should consist of three parts serum (horse, ox, or sheep) and one part neutral bouillon containing 1 per cent each of peptone and grape sugar. The freshly prepared serum should be shaken for some hours with 3 per cent chloroform before the bouillon is added. On one day the serum is heated from five to six hours at from $70^{\circ}$ to $80^{\circ} \mathrm{C}$. On the next day it should be held at $80^{\circ} \mathrm{C}$. for one hour and for the same time at $90^{\circ} \mathrm{C}$., then for half this time at $100^{\circ} \mathrm{C}$, and then allowed to harden slowly. On this medium the bacillus forms a good growth in the incubator within six hours. The optimum growth temperature is about $37^{\circ} \mathrm{C}$., but growth persists anywhere between $20^{\circ}$ and $41^{\circ} \mathrm{C}$. While air is not absolutely essential growth is most rapid when this is abundantly supplied. In liquid cultures a scum forms on top and the subjacent fluid may remain clear. The sides of the tube may be covered with the growth. On ordinary agar, development is slow and scanty. Gelatin colonies are not characteristic and the medium is not liquefied. In the presence of certain carbohydrates there is a slow production of acid, but this does not occur in bouillon when the muscle-sugar has been removed. In the absence of carbohydrate from the medium the alkalinity is increased.

The resistance of this bacillus to untoward conditions depends upon whether it is still protected by its membrane or has been freed from it. The membrane even when partially dried may retain its virulence for months. Park found vigorous living bacilli in a dried membrane four months after its removal from the throat, and he is convinced that under these conditions diphtheria bacilli may continue alive and virulent for much longer periods. Ordinary cultures kept at incubator temperature usually die after a few weeks, but if the tubes be protected from the light and kept at low temperature the bacilli retain their virulence for years. Freezing does not destroy or lessen the virulence, except for the time being, of the diphtheria bacillus. Under certain other conditions the Klebs-Loeffler organism soon dies. Silk threads dipped in cultures and exposed to direct sunlight soon become inert. Phenol, 
5 per cent and mercuric chlorid 1-1,000 destroy the bacillus within five minutes. Absolute alcohol has but little or no effect, but when the strength is reduced to from 60 to 30 per cent a few minutes suffice to kill the bacilli. According to Meyer, eertain tooth pastes destroy diphtheria bacilli in less than one minute. It must not be inferred from this that a diphtheritic mouth could be easily disinfected by such an agent. The bacilli are protected by the pharyngeal folds and the crypts of the tonsils. However, since the healthy carrier plays a large part in the distribution of this disease, more attention to oral hygiene might be of service in this as well as in other diseases.

The diphtheria bacillus finds a most suitable medium in milk, in which it grows readily and abundantly and, according to Sternberg, at as low a temperature as $20^{\circ} \mathrm{C}$. Its growth in milk causes no recognizable changes in this food. These are matters of grave importance in studying the epidemiology of this disease. The organism, having been introduced into this food, multiplies slowly at low temperatures, rapidly at high temperatures, and in either case causes no change recognizable by sight or taste in the food. It was pointed out by Theobald Smith some years ago that the usual method of heating milk in open vessels will not certainly kill diphtheria bacilli. In this method of heating a scum forms on the surface of the milk and within this membrane diphtheria bacilli may resist a temperature of even $95^{\circ}$ or $100^{\circ} \mathrm{C}$. for an hour. It should be understood that in the proper pasteurization of milk the procedure is carried out in closed vessels, in which diphtheria bacilli are certainly destroyed at much lower temperatures.

Diphtheria Toxin.-Roux and Yersin, having filtered diphtheria cultures one week old or older through porcelain and having in this way removed all bacteria, found that the filtrate, even in minute doses, kills animals. The toxin has not been obtained in a pure state, and we are still in doubt concerning its chemical composition. In some respects it resembles the ferments. It acts slowly and after a period of incubation; it is active in exceedingly small amounts; it is destroyed by heat, and when repeatedly injected into animals in nonfatal, but increasing doses, the animal becomes immune by elaborating an antibody. In these respects diphtheria toxin resembles the ferments. It differs from many ferments in the fact that a given amount produces only a certain effect and apparently goes no further. Different strains of the baeillus show wide variations in their toxin production and the highest toxin producers are not always the most virulent bacilli. What is called diphtheria toxin is an old filtered culture, and the strength of this is determined by ascertaining the minimum amount necessary to kill a guinea pig of from 200 to 250 grams weight within four days. This minimum lethal dose 
(m.l.d.) may be as small as 0.0005 c.c., though that usually employed in the production of antitoxin is much less powerful.

The effect of this toxin on animals has opened up a new field of research, has given a new understanding of disease processes, and has led to one of the most beneficent discoveries-that of diphtheria antitoxin. When a fatal dose of this toxin is injected into an animal, there is a period of incubation during which the animal shows no marked departure from the normal. This incubation period varies with the size of the dose but is never less than about eight hours, even when many times the minimum lethal dose has been used. In our opinion, the significance of this period of incubation has been misunderstood. It should not be inferred that nothing happens during this time. The disturbance simply does not rise to the plane of gross clinical observation. The toxin begins to act soon after its introduction into the animal body. Within an hour or two the temperature begins to rise and proceeds slowly until some hours before death, when it begins to fall, and at death it is several degrees below normal. The skin about the point of injection becomes edematous and later necrotic. The interval between injection and death is the same as after inoculation with the living bacillus. With sublethal doses of the toxin there is often paralysis, beginning in the posterior extremities and extending over the body. The internal organs are hyperemic with hemorrhages in the adrenals, stomach, and intestine. In rare instances a gastric ulcer is found. It is important to note that after the first day there is a fall in the blood pressure. The effects of the toxin on temperature and blood pressure suggest the action of the protein poison. It will probably be found that the toxin is a ferment which slowly disrupts some protein, setting free the poison which in small amount increases, and in larger decreases, the temperature and lowers blood pressure. The toxin apparently has special avidity for neryous tissue. Whether this action is primarily central or peripheral has not been determined. When the toxin is injected into a susceptible animal it soon disappears from the blood current and manifests its activity on certain organs and tissues. It has not been found in the urine except when massive doses are given. In insusceptible animals, it remains for a long time in the blood stream and is, of course, without action on the tissues. It is not able to digest the proteins of insusceptible animals, and it is for this reason the animal is refractory.

In the preparation of diphtheria toxin it is essential that the growing bacilli should be abundantly supplied with oxygen. In his early experiments Roux did this by passing a current of oxygen through the culture media. However, it has been found that this is not necessary and a sufficient supply of oxygen is secured by growing cultures in large necked Erlenmeyer flasks. As has been stated, in the presence 
of an excess of carbohydrates the diphtheria bacillus produces acid and fails to elaborate an efficient toxin. This observation has led some investigators to remove the traces of sugar from their meat preparations by preliminary fermentation, either with yeast or with bacillus coli. Park and Williams, however, have found this precaution unnecessary, provided that the meat broth, after being made neutral to litmus, is treated with 7 c.c. to the liter of normal sodium hydrate solution. The largest yield of toxin is obtained after growth at $37.5^{\circ} \mathrm{C}$. for eight days. Before this time the formation of toxin has not fully progressed and after this time that formed begins to deteriorate.

There has been some difference of opinion as to whether diphtheria toxin is a protein or a nonprotein body. It has never been obtained in a form which does not respond to at least some of the general protein reactions. Most investigators believe that it is a protein body and closely related to the albuminoses. The claim has been advanced by Uschinsky that diphtheria toxin is not a protein substance because it is produced when diphtheria bacilli are grown in media containing no proteins. In our opinion, this argument is of no weight, because it has certainly been demonstrated beyond all possibility of doubt that bacteria may and do synthetize amino acids into proteins. The toxin in filtered cultures is destroyed by a temperature of from $58^{\circ}$ to $60^{\circ} \mathrm{C}$. It may be kept for months without loss in strength in amber bottles in a dark, cold place.

Diphtheria Antitoxin.-We owe the discovery of antitoxin, one of the most beneficent discoveries of all ages, to the labors and genius of Behring and Roux. It is true that others had prepared the way, but this detracts not at all from the debt of gratitude the world owes these menone a distinguished German, the other an equally distinguished Frenchman. To the preliminary work America made two important contributions. Mitchell and Reichert showed that the active principles in the venom of serpents are protein bodies or so closely related to proteins that they have not been separated. Sewall immunized pigeons to the venom of rattlesnakes (Crotalus horridus). Ehrlich immunized animals to the similar vegetable poisons, abrin, ricin, and robin, and demonstrated that passive immunity could be established in other animals by the transference to them of the serum of those actively immunized. Then came the discovery by the Frenchmen, Roux and Yersin, of diphtheria toxin closely resembling snake venom in its action. The main methods now employed in the preparation of diphtheria antitoxin may be stated as follows: Sound, healthy horses are kept under observation and tested with mallein to insure freedom from glanders. Injections of diphtheria toxin, beginning with very small doses, are given these animals at intervals of a few days. It is desirable that the first dose should be so small 
that marked or general reactions are not induced; indeed, it is now customary to give as the first injection a toxin neutralized or nearly neutralized by antitoxin. Horses differ widely in susceptibility to the toxin. From time to time a small amount of blood is drawn from the horse and its protective power against the toxin is tested on guinea pigs. The amount of the serum necessary to protect a guinea pig of 250 grams weight against 100 minimum lethal doses of the toxin is known as the "immunity unit." When the protective value of the serum has risen to the desired point, from four to eight liters of blood are drawn from the horse under aseptic precautions into sterile glass cylinders. These are allowed to stand in a cool dark place until coagulation is complete, when the serum is treated with some preservative, such as 0.5 per cent phenol, 0.4 per cent tricresol, or a small amount of camphor, and placed in containers ready to be injected into the child sick with diphtheria. In some countries no preservative is used, but the serum is heated to $56^{\circ}$ C. In all countries antitoxin is made under governmental supervision and the drug is tested in some laboratory. In this country the U. S. Public Health Service only can license a firm to prepare antitoxin and all batches manufactured are tested in the Hygienic Laboratory at Washington. All horses do not produce the same grade of antitoxin, and sera from different animals are mixed or pooled and the product tested as to its protective value. Some horses continue to produce a high grade of antitoxin for years and from four to six liters of serum may be obtained from such animals monthly. It will be understood that in such cases the injections of toxin must be repeated from time to time. Immediately after an injection of toxin the antitoxin content of the blood falls, then it rises and generally reaches its highest point in ten to twelve days, remaining at that point for a variable time. The horse is immunized by the repeated injections of the toxin. The process of immunization consists in developing in the animal's body an antitoxin, the excess of which is in the blood, and in the serum after coagulation. The antitoxin is capable of neutralizing the toxin both in vitro and in vivo. The diphtheria bacillus is growing in the throat of the child and pouring its toxin into the child's blood. The blood serum of the horse is injected into the child and neutralizes the toxin coming from the throat. The horse has been immunized, and when its blood serum is introduced into the child, the child becomes for the time physiologically a part of the horse and partakes of the horse's immunity just to the extent of the amount and potency of the horse serum injected. It should be understood that the blood serum of the horse containing the antitoxin is not bactericidal. It does not kill the bacilli in the throat, it only neutralizes the toxin in the blood and tissues. It must be evident from this that the earlier in the disease the antitoxin is used the more beneficial it is. When the 
toxin poured from the throat into the blood meets with no antibody it acts on the body-cells and destroys them, and to the extent to which this has been done before the antitoxin has been administered it is without value. Kossel has shown that when the antitoxin is injected on the first sign of the disease the percentage of recoveries is one hundred. In this every hour counts. Of 2,428 cases reported by Hilbert the percentage of deaths varied with the day on which the antitoxin was administered as follows: First day, 2.2 ; second day, 7.6 ; third day, 17.1; fourth day, 23.8; fifth day 33.9 ; sixth day, 34.1 ; after the sixth day, 38.2. According to Wernicke, the saving of lives during the first year of the use of antitoxin in Germany amounted to 20,000, and if the agent is properly and promptly used the saving would amount to 45,000 lives annually. The value of this agent, however, is even greater than is indicated by the lowering in death rate. For every sick child saved, at least five are saved from being sick. While the curative value of diphtheria antitoxin is great its temporary preventive value is greater still. The physician called to a family in which one child has diphtheria gives a curative dose to the one and immunizing doses to all others. Even when the sick one has been neglected so long that the curative value of the agent has been lost its preventive value is still potent. Diphtheria antitoxin has lowered not only the mortality rate, but still more the morbidity rate.

Unfortunately, the immunity induced by diphtheria antitoxin is temporary, lasting only from three to four weeks. From this it frequently happens that the child who has had an immunizing dose may a few months or possibly a few years later need a curative dose. The fear of anaphylactic shock has led many physicians to hesitate about a "reinjection" of horse serum after an interval of ten days or longer, but "reinjection" may be undertaken with safety. In all cases of "reinjection" after an interval of ten days or longer a fraction of a cubic centimeter should be injected. If there are or are not untoward symptoms following this, after an hour or two any amount of the serum may be injected with safety. This procedure should be adopted not only in all cases of reinjection, but when the patient has shown asthmatic symptoms; indeed, it is best to proceed in this way in all cases even of primary injection. With these precautions no physician need fear to use diphtheria antitoxin when it is needed.

When antitoxin is used early in the disease the extension of the membrane usually stops in a few hours. For this reason, laryngeal diphtheria is now rarely seen except in neglected cases. Not only does extension of the membrane stop on administration of the antitoxin, but, as a rule, that already formed begins to recede, becomes detached and fades away. It may be truly said that the discovery of antitoxin has 
robbed diphtheria largely of its terrors, and it might not be amiss to call attention to the fact that without animal experimentation this could not have been done. Hundreds of animals have been sacrificed in order that hundreds of thousands of human lives might be saved from one of the most distressing forms of death man has known.

Notwithstanding the possession of this wonderful and almost miraculous preventive and curative agent, the problem of the eradication of diphtheria remains one of the most difficult still confronting scientific medicine. The bacillus seems well nigh ubiquitous. Thousands of people in health carry these deadly germs in their throats and mouths. If it has breeding-places outside the human body they have not been discovered.

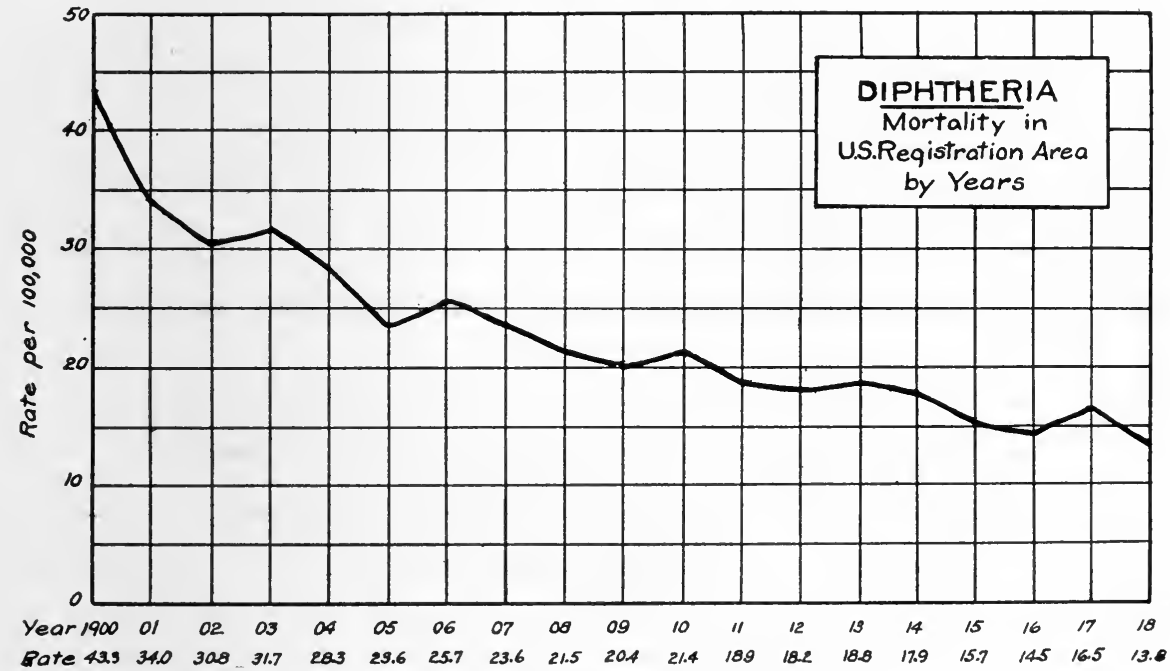

Fig. 24.

Morbidity and Mortality in Recent Years.-It has been 25 years since the medical profession came into the possession of diphtheria antitoxin. It is, therefore, proper for us to ascertain what has been accomplished with this agent. Unfortunately, we have no figures showing the morbidity from this disease either before or after the introduction of antitoxin; indeed, the figures showing the mortality from this disease must be accepted with some reservation. Table XV gives the death rates per 100,000 population from diphtheria in the registration area of the United States since the census of the year 1879-1880.

Diphtheria antitoxin was placed in the hands of the medical profession in 1895. The figure for 1889-1890 (97.8) might be regarded as quite nearly representing the death rate from this disease in preantitoxin days. It will be seen that within five years after the introduction of 
TABLE XV

\begin{tabular}{lccc}
\hline \hline YEAR & DEATH RATE & YEAR & DEA'III RATE \\
\hline $1879-1880$ & 112.6 & 1909 & 20.4 \\
$1889-1890$ & 97.8 & 1910 & 21.4 \\
1999 & 45.2 & 1911 & 18.9 \\
1900 & 43.3 & 1912 & 18.2 \\
1901 & 34.0 & 1913 & 18.8 \\
1902 & 30.8 & 1914 & 17.8 \\
1903 & 31.7 & 1915 & 15.7 \\
1904 & 28.3 & 1916 & 14.5 \\
1905 & 23.6 & 1917 & 16.5 \\
1906 & 25.7 & 1918 & 13.8 \\
1907 & 23.6 & & \\
1908 & 21.5 & & \\
\hline
\end{tabular}

this agent the mortality from the disease in the registration area was reduced more than one-half, and since that time the reduction has on the whole been slow and gradual. In 1917 it was less than one-half what it was in 1902, by which time it might be said that antitoxin was well known and within the reach of the profession throughout the country.

DIPHTHERIA IN THE LARGEST CITIES OF THE U.S.

(1910 RANKING)

AVERAGE ANNUAL DEATH RATE PER 100,000 FOR THE YEARS 1911-17

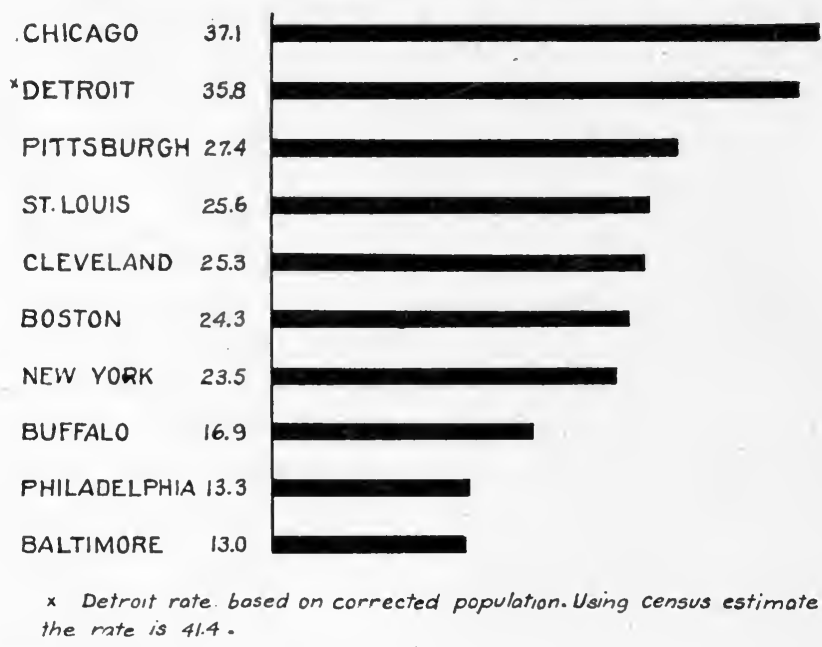

Fig. 25.

The total deaths from diphtheria in the registration area in 1917 were 12,453 , and inasmuch as the registration area at that time held about 75 per cent of the population it is estimated that the total number of deaths in this country in that year was about 16,604 . 
TABLE XVI

Death Rate from Diphtheria per 100,000 Population in the Large Cities of the United States Since 1911

(Using Corrected Mid-year Population Foliowing 1920 Census)

\begin{tabular}{lrrrrrrrrrr}
\hline \multicolumn{1}{c}{ CIтY } & 1911 & 1912 & 1913 & 1914 & 1915 & 1916 & 1917 & 1918 & 1919 & 1920 \\
\hline Boston & 20.0 & 15.8 & 23.0 & 23.8 & 30.6 & 25.9 & 38.1 & 29.1 & 17.8 & 18.6 \\
New York & 26.0 & 22.8 & 26.8 & 29.3 & 24.2 & 19.6 & 21.5 & 22.1 & 22.2 & 18.5 \\
Philadelphia & 31.0 & 23.6 & 21.5 & 19.3 & 18.8 & 22.6 & 26.5 & 21.5 & 24.3 & 22.8 \\
Detroit & 31.8 & 32.2 & 47.2 & 22.8 & 15.3 & 32.0 & 48.3 & 30.0 & 32.2 & 36.5 \\
Chicago & 38.3 & 41.1 & 41.0 & 32.2 & 27.4 & 31.4 & 47.8 & 27.0 & 23.0 & 23.0 \\
St. Paul & 33.1 & 10.9 & 19.4 & 30.8 & 13.3 & 9.6 & 17.9 & 25.0 & 33.4 & 24.6 \\
Minneapolis & 23.8 & 14.1 & 17.1 & 43.1 & 18.3 & 19.6 & 18.9 & 23.9 & 18.1 & 11.7 \\
St. Louis & 17.7 & 21.4 & 29.5 & 35.2 & 26.7 & 21.3 & 29.7 & 16.8 & 33.5 & 33.5 \\
San Francisco & 5.1 & 6.7 & 6.5 & 19.1 & 24.7 & 27.6 & 14.9 & 8.1 & 10.3 & 15.4 \\
New Orleans & 7.2 & 16.6 & 31.8 & 31.1 & 31.0 & 10.0 & 8.3 & 4.7 & 4.4 & 5.6 \\
Washington & 5.5 & 4.2 & 7.4 & 7.7 & 7.0 & 8.8 & 8.3 & 9.5 & 27.8 & 14.5 \\
Atlanta & 14.9 & 15.7 & 13.5 & 10.9 & 8.4 & 15.8 & 8.5 & 6.2 & 8.1 & 11.8 \\
Richmond & 9.9 & 5.7 & 5.6 & 2.7 & 4.0 & 3.2 & 5.6 & 7.9 & 8.8 & 11.5 \\
Pittsburgh & 23.4 & 31.5 & 37.0 & 28.3 & 27.3 & 22.2 & 24.4 & 21.7 & 17.6 & 19.4 \\
\hline
\end{tabular}

When the figures in Table XVII are compared with those in Table XVI, it will appear that diphtheria causes more deaths in urban than in rural populations:

\section{TABLE XVII}

Death Rate from Diphtileria Per 100,000 Population in the Rural Part of Registration States

\begin{tabular}{ccc}
\hline \hline YEAR & DEATH RATE \\
\hline 1911 & 15.1 \\
1912 & 15.5 \\
1913 & 14.7 \\
1914 & 13.8 \\
1915 & 12.9 \\
1916 & 11.7 \\
1917 & 12.3 \\
\hline
\end{tabular}

It is when we come to study the death rates from diphtheria in the smaller cities that we find, even within recent years, the death rate from this disease frequently equals and in some instances surpasses that of preantitoxin days (Table XVIII).

If we add those small cities in which the death rates from this disease have been from two to four times those of our largest cities, this list would be a long one. This emphasizes what we have seen to be true with the other infectious diseases of infancy and childhood-that the most marked failure in the prevention of the infectious diseases is exemplified in the records of our small cities. 


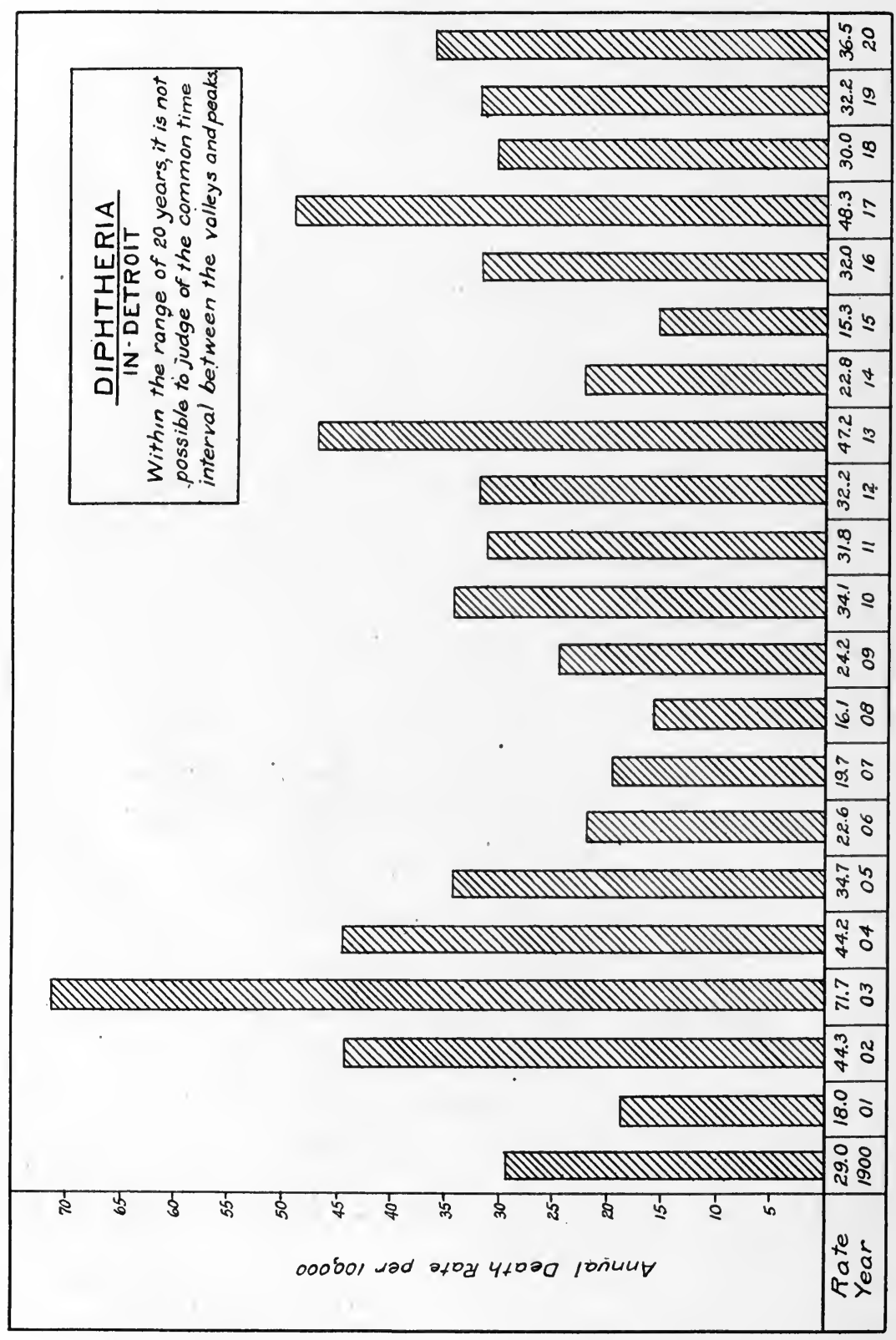

Fig. 26. 
TABLE XVIII

Death Rate from Diphtheria Per 100,000 Population in Certain Small Cities of the United States in Certain Years

\begin{tabular}{lcc}
\hline \hline ClTY & YEAR & DEATH RATE \\
\hline Berlin, N. H. & 1912 & 193.1 \\
Steelton, Pa. & 1911 & 165.6 \\
Windhamtown, Conn. & 1912 & 136.8 \\
Mt. Carmel, Pa. & 1916 & 123.3 \\
Homestead, Pa. & 1912 & 109.7 \\
Shenandoah, Pa. & 1911 & 109.7 \\
McKees Rocks, Pa. & 1913 & 109.1 \\
Hamilton, Ohio & 1913 & 108.0 \\
Vincennes, Ind. & 1912 & 107.1 \\
Batavia, N. Y. & 1912 & 98.8 \\
\hline
\end{tabular}

The Schick Test.-From what has been said concerning the standardization of antitoxin in horse serum, it is evident that it is possible to obtain serum from human beings and not only to test it for antitoxin, but to determine the antitoxin content. This, however, would be a laborious and time-consuming task. In 1913 Schick proposed a simple clinical

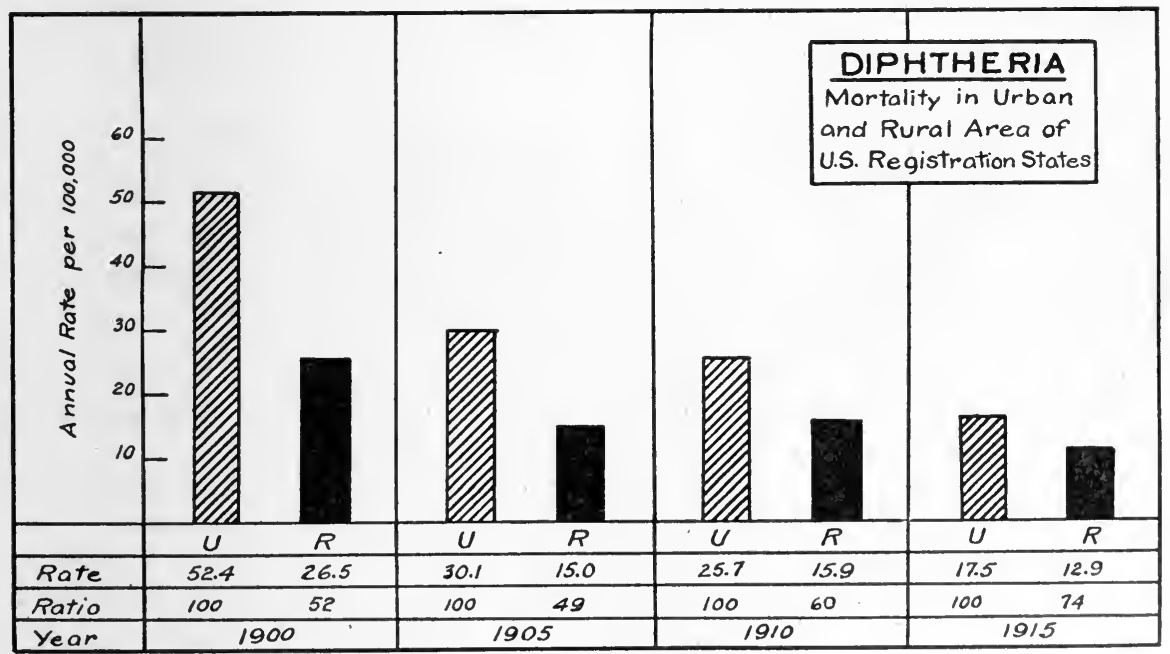

Fig. 27.

method of determining the presence and relative amount of antitoxin in the blood of an individual. This is now known as the Schick test, and it has proved of the greatest value in ascertaining those individuals who have and those who have not antitoxin in protective amount in their blood. Schick prepares a solution of standard, ripened toxin which is equivalent to one-fiftieth of a m.l.d. for a 250 -gram guinea pig. This 
dose of diphtheria toxin is dissolved in 0.2 e.c. of sterile salt solution and this amount is injected intracutaneously on the flexor surface of the forearm or arm. The preparation must be accurately measured and it is injected through a fine steel or platinum-iridium needle. The injection must not be made under the skin, but into the skin. The oval opening in the needle must be on the upper side and must be seen through the outer layer of skin as the needle is introduced and before the toxin is discharged. When this amount of diphtheria toxin is injected into the skin a wheal-like elevation showing prominently the openings of the hair follicles makes its appearance. The result of this test should be ascertained and recorded at the end of $24,48,72$, and 96 hours. The reactions which may follow this injection are divided into the following groups: (1) positive; (2) negative; (3) pseudo or false; (4) combined.

It will be well, in the first place, to understand the nature of these reactions. If there be in the cutaneous lymph of the individual enough antitoxin to neutralize the amount of toxin introduced there will be no reaction. The amount of antitoxin per c.c. of blood or lymph necessary to completely neutralize the one-fiftieth m.l.d. of the toxin introduced is one-thirtieth of a unit and the presence of this amount of antitoxin in the blood of the child is enough to protect it from infection with the diphtheria bacillus. According to Behring, even as small an amount as one one-hundredth of a unit of antitoxin per c.c. of blood is sufficient to protect a child against the disease.

Park, of the New York City Board of Health, to whose investigations we owe the great advance that has been made in our knowledge of the recognition, prevention, and treatment of diphtheria founded upon the Schick test, has recently (March, 1919) issued a bulletin from which we make the following quotation:

"The Schick reaction is a convenient and reliable clinical test, by which the antitoxic immunity of an individual to diphtheria can be determined. A fresh solution of diphtheria toxin is prepared for this purpose of such strength that 0.1 c.e. or 0.2 c.c. represents one-fiftieth of the minimum lethal dose of toxin for a 250 gram guinea pig. This amount is injected with a good syringe, preferably a 1 c.c. 'Record.' * * * The positive reaction represents the action of an irritant toxin upon tissue cells that are not protected by antitoxin. It indicates, therefore, an absence of immunity to diphtheria. A trace of redness appears slowly at the site of injection in from 12 to 24 hours, and usually a distinct reaction in the course of 24 to 48 hours. The reaction reaches its height on the third or fourth day, and gradually disappears, leaving a definitely circumscribed scaling area of brownish pigmentation which persists for three to six weeks. At its height the positive reaction consists of a circumscribed area of redness and slight infiltration, which measures from 1 to $2 \mathrm{~cm}$. in diameter. The degree of redness and infiltration varies to a great extent with the relative susceptibility of the individual. The positive reaction is seen in about 7 to 10 per cent of the newborn, 30 per cent during the first year of life, 35 decreasing to 15 per cent between two and fourteen years, and 10 per cent in adults. 
"In the negative reaction the skin at the site of injection remains normal. Negative reaction definitely indicates an immunity to diphtheria if the test toxin is of full strength, has been freshly diluted, and the injection has been made into the proper layer of the skin. A negative reaction obtained in a child that has reached the age of three years indicates that it has an immunity which is probably permanent. Of 1,000 carefully observed individuals, not one developed clinical diphtheria, even though they were exposed to the disease, and some were carriers of virulent diphtheria bacilli.

"The pseudo reaction represents a local anaphylactic response of the tissue cells to the protein substance of the autolyzed diphtheria bacilli, which are present in the toxic broth used for the test. Like other anaphylactic skin phenomena, the reaction is of an urticarial nature, appears early, within six to eighteen hours, reaches its height in thirty-six to forty-eight hours, and disappears on the third or fourth day, leaving a poorly defined small area of brownish pigmentation and generally no scaling. At its height the pseudo reaction shows varying degrees of infiltration, and appears as a small central area of dusky redness with a second areola, which gradually shades off in the surrounding skin. The reaction may also have a rather uniform red appearance, and be two or three times the size of true reaction. A control test, made by injecting toxin heated to $75 \mathrm{C}$. for five minutes gives a similar reaction which passes through the same elinical course. Individuals who give a pseudo reaction only, have antitoxin and are immune to diphtheria. The false reaction is seen in relatively few of the older children, but in a much larger number of adults, in whom it is of importance to recognize and control it both by the injection of heated toxin and by observing the elinical course of the reaction.

"The combined reaction represents the positive and the pseudo reaction in the same individual. The central area of redness is larger and better defined, the amount of infiltration is also more marked. The reaction is recognized by noting the evidence of a true reaction, a definite area of scaling brownish pigmentation, after the pseudo element has disappeared in the test. In addition, a similar though weaker reaction is obtained in a eontrol test made with heated toxin. The control represents only the pseudo reaction. The combined reaction indicates an absence of immunity to diphtheria.

"The Schick test is of practical value in determining the immunity to diphtheria of the public in general, but especially of the child population in schools, hospitals, institutions, and in homes during an outbreak of diphtheria. It will save a considerable amount of antitoxin and avoid unnecessary sensitization of more than 65 per cent of the exposed individuals. The test is also of distinct value in the active immunization of susceptible individuals against diphtheria with mixtures of toxin-antitoxin, and in the diagnosis of clinically doubtful eases of diphtheria."

Schick found that 7 per cent of the newborn, 43 per cent of those in the first year of life, 63 per cent of those between two and five years, 50 per cent of those between five and fifteen years, and 10 per cent of adults gave positive reactions. Park's figures on susceptibility are given in Table XIX.

It will be seen from these figures that from 85 to 90 per cent of infants under three months of age have enough antitoxin in their blood normally to protect them against infection with the diphtheria bacillus. The antitoxin in the newborn comes from the mother; in fact, for some indefinite period after birth the child is physiologically a part of the 
TABLE XIX

\begin{tabular}{lc}
\hline \multicolumn{1}{c}{ AGE } & PER CENT \\
\hline Under 3 months & 15 \\
3-6 months & 30 \\
6 months-1 year & 60 \\
1-2 years & 70 \\
2-3 years & 60 \\
3-5 years & 40 \\
5-10 years & 30 \\
10-20 years & 20 \\
Over 20 years & 15 \\
\hline
\end{tabular}

mother. Anatomic separation between mother and child is accomplished at birth, but physiologically for a time thereafter the child remains a part of the mother. It has been shown experimentally by Gröer and Kassowitz that 84 per cent of mothers and their newborn children have a

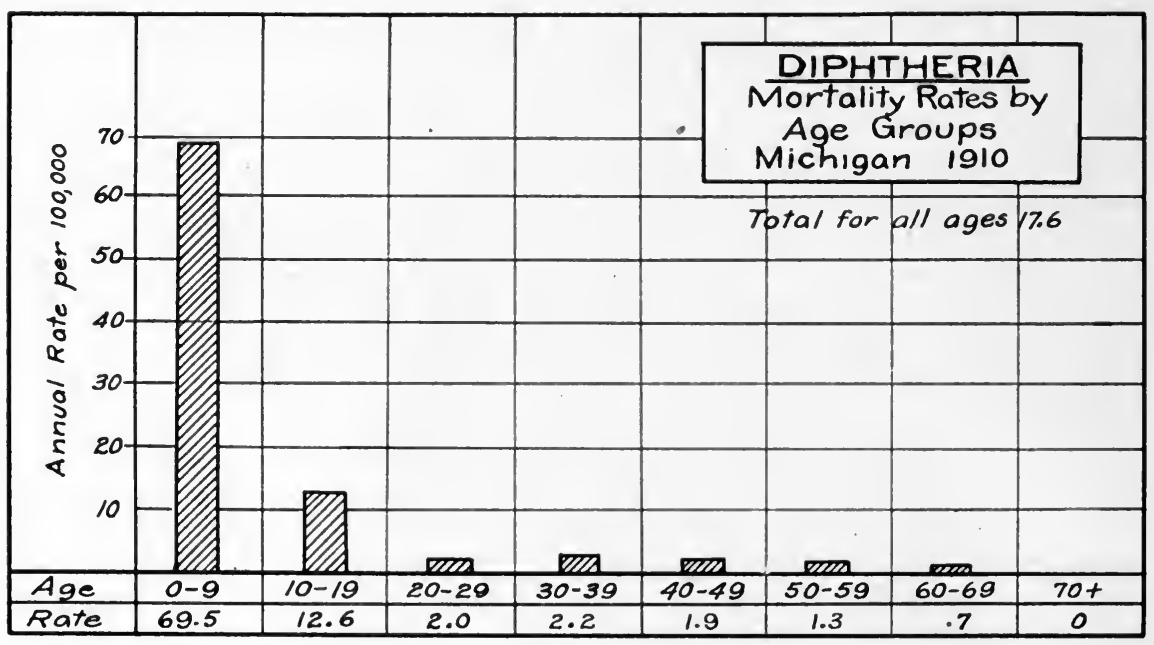

Fig. 28.

large amount of antitoxin in the blood. As the child, with increasing age, gradually loses its physiologic relationship to its mother the antitoxin in its body decreases; in other words, its inherited protection against infection with diphtheria disappears. Later in life it accumulates antitoxin and finally in adult years about 85 per cent have accumulated enough of this protective agent to render them immune to the disease. On this point Park makes the following statement:

"During systematic testing of groups of children according to families, we were impressed with the frequency which all the children of the same family gave a similar reaction. If variations were found, younger children always gave the positive 
reactions. If the youngest child had a negative reaction, all the older children were usually negative. On the other hand, if the oldest ehild in a family gave a positive reaction, the younger children with very few exceptions showed positive reactions. These striking facts are additional proofs that there are factors, possibly hereditary in character, which in the absence of infections with the KlebsLoeffler bacillus give rise to the presence of the so-called natural antitoxins. The large amount of antitoxin which is present in some of the cases is hard to explain; for example, two young children, six and one half and three years of age, who had no history of clinical diphtheria, showed respectively 15 and 19 units of natural antitoxin per c.c. of serum. The large proportion of older children and adult persons having antitoxin adds to the difficulty of considering the natural antitoxin as usually due to a previous infection with diphtheria bacilli either as a case or as a carrier. In a recent investigation published by Gröer and Kassowitz the combining and physical characteristics of the antitoxin present in persons who had never had diphtheria appear to be identical with that due to immunization."

In our opinion, the most probable explanation of the above-mentioned phenomena is that children as they grow into and advance in adult life acquire antitoxin by frequent exposures to the diphtheria bacillus. In this way a large percentage of mothers acquire an immunity to this disease which is passed on to their children, but which is lost in them in a few years and ultimately is restored in the same way that it was acquired by the mothers.

It may be that the development of diphtheria antitoxin with increasing age is a physiologic function and not dependent upon repeated exposure to the bacillus. In a recent (1920) article Zingher says:

"In children under six months of age, toxin-antitoxin should not be used as it usually produces no immunization."

The Schick test has proved of value in enabling the medical officer when a number of children have been exposed to this disease to determine which of these should receive antitoxin injections. Formerly it was customary to give antitoxin to all such children;. now the Schick test is applied to all and antitoxin is administered to only those who give a positive response and in this way demonstrate their susceptibility. This saves large expenses, but what is of more importance, it does away with the unnecessary sensitization of a large number of individuals. The test is of the greatest importance in determining which children should be actively immunized with the toxin-antitoxin mixture.

It has long been observed clinically that one attack of diphtheria does not give immunity to subsequent infections, at least not in all instances. The truth of this observation has been demonstrated by Park by applying the Schick test to patients who had recently recovered from the disease. At the Willard Parker Hospital 32 such patients were subjected to the Schick test from three to four months after recovery from the disease. Of these 19 gave positive reactions, while 13 were negative. Park states: 
"The absence of antitoxic immunity in a large proportion of individuals who have had diphtheria, as seen at all tests in the children who received no therapeutic antitoxin injections and in tests made after the disappearance of the horse antitoxin in those receiving injections, leads us to assume that diphtheria patients develop as a rule an antibacterial inmunity, which is associated in only about onethird the eases with antitoxic immunity. Chronic reinfections with the KlebsLoeffler bacillus, as seen in children who remain a long time in the diphtheria wards, appear to finally lead in the majority of cases to the production of an antitoxic immunity.",

Active Immunization with Toxin-Antitoxin Mixture.-The patient, skilful, and scientific work done by Park and his associates in recent years holds out great promise for the ultimate eradication of diphtheria. It has been known for some years that if properly balanced mixtures of toxin-antitoxin be injected into guinea pigs these animals acquire a high degree of lasting immunity. The application of the Schick test has shown that those who are supplied with a sufficient amount of normal antitoxin are already immune and are not in need of further protection; in other words, those who give a negative Schick reaction are immune to diphtheria, probably for life, and it is not necessary to treat them when exposed to the infection. On the other hand, those who give a positive Schick reaction are susceptible to diphtheria and when exposed should be given antitoxin for immediate and temporary protection and should also be treated with the toxin-antitoxin mixture for more permanent protection. Park has shown that injections of the toxin-antitoxin mixture develop gradually but slowly a high and lasting immunity to the disease. He has made numerous experiments along this line. In one instance 16 susceptibles had the toxin-antitoxin injection. At the end of four weeks five of these, as shown by the Schick reaction, were immune and 11 still susceptible; while after from one year to 18 months, 11 of the 16 were immune, four gave only a faint positive reaction and one a moderate reaction. In another series of 158 cases it was shown that a similar gradual development of immunity took place. Park says:

"From the retest in the naturally immune children in this series and in a larger one at present under observation by us, the rather important fact may be deduced that a great majority of children of school age, who show a negative Schick reaction, probably remain protected for years, and even for life. Such a persistent immunity, as shown with the test, could be successfully utilized in school children. During an outbreak of diphtheria among children previously tested, it would be necessary to immunize with antitoxin only the susceptible children. Class work could then continue without interruption.

"Our results in active immunization up to the present time have led us to the conclusion that, while the toxin-antitoxin injections cannot be depended on to prevent diphtheria in the presence of immediate danger of infection, because of the slow development of immunity in the majority of eases, yet the injection is of value in rendering a community or school population immune to the danger of infection from future exposure up to a period of possibly several years." 
In 1919 Park stated that he and his coworkers had actively immunized with toxin-antitoxin mixtures more than 6,000 susceptibles (including infants under one week old), without the subsequent development of a single case of diphtheria among them. Provided the toxin-antitoxin mixture is properly prepared it is absolutely harmless. In infants no reaction is apparent, while in older children and adults there may be a moderate swelling of the arm at the place of injection lasting from one to three days. Park finds that one injection gives immunity to 80 per cent of those previously susceptible; two injections give immunity to 90 per cent, and three injections to 97 per cent. So far immunity is known to last for four years and it is believed that it continues throughout life.

In 1920 many municipal health departments were administering toxinantitoxin as a routine procedure to all children in the kindergarten and first grade of the public and parochial schools where they were able to get the consent of the parents.

Commercial firms are now preparing and placing on the market toxinantitoxin mixtures with proper facilities for administration. A most unfortunate occurrence happened at Dallas, Tex., in November, 1919. One batch of toxin-antitoxin mixture, as was shown later, contained free toxin and as a result of its use more than 50 severe reactions were reported and six deaths occurred. It is recommended that whenever a medical officer uses this preparation he test each batch on guinea pigs.

Attempts have been made to actively immunize against diphtheria by injection of killed cultures of the diphtheria bacillus. Up to the present time no satisfactory results have been obtained by this method. In our opinion, this is exactly what we should expect. Diphtheria is an intoxication rather than an infection. It is true that the toxin is produced by bacilli growing on some mucous surface, but when the blood contains protective quantities of antitoxin the bacillus is practically harmless. After attempting active immunization by means of killed cultures of diphtheria bacilli, Park wrote:

"These results with the vaccine are rather discouraging, especially in view of the elaims made by several authors of the therapeutic value of such vaceines in carriers. Not only did we fail with these vaceines in ridding carriers of their organisms, but we even failed to immunize about one-quarter of the injected individuals against becoming carriers or developing clinical symptoms.",

Attempts have also been made to induce active immunization by treatment with the toxin-antitoxin mixture and dead bacilli. So far as we can see there is no convincing evidence that this combination has any advantage over the toxin-antitoxin mixture; in other words, we are not convinced that the addition of dead bacteria to the toxin-antitoxin mixture improves the immunizing property of the latter. Until this is shown to be the case we cannot recommend the use of dead diphtheria bacilli either 
alone or together with the toxin-antitoxin mixture in active immunization.

There is much work still to be done on the normal antitoxin of the blood. We are not yet sure as to its origin. As we have said, in infants it certainly comes from the mothers, but this inherited gift is soon dissipated. How does the growing child acquire more normal antitoxin? Is its development dependent upon some deeper inheritance, and would it come if the child never came in contact with diphtheria bacilli? If the bacilli were not so widely prevalent, would the child as it grows into adult life acquire more liberal quantities of this protective substance? Is its formation and accumulation in the body caused in any way by food, physical conditions of life, the acquisition of other infections, etc.? Older authors have written much concerning predisposition to diphtheria. Is there only one predisposing factor and that the absence or paucity of antitoxin in the blood, or are there other conditions which predispose to this disease? Some clinicians are inclined to stress the condition of the mucous membrane of the upper air passages in discussing predisposition to diphtheria. Others are inclined to put much emphasis upon variations in the virulence among different strains of the bacillus. In a recent (1917) most valuable work on pediatrics, Dunn states:

\footnotetext{
"Adults show a much smaller proportion having natural immunity than children, but yet diphtheria is much commoner in childhood. The frequency of the disease in children is not due to greater susceptibility, but to the same reason which causes all of the contagious diseases to attack children more than adults, namely, that the conditions of life in childhood favor the spread of all diseases aiquired by close contact. Diphtheria is most common between the ages of two and five years. It is infrequent after ten years. Among individual predisposing causes are adenoids, enlarged tonsils, and a tendency to catarrhal affections of the mucous membranes. For this reason the disease is seen more often in autumn, winter, and spring than in summer. There are other factors. Certain children seem to have an individual constitutional predisposition. The chances of infection and the severity of the case depend not only on the constitutional predisposition, but upon the virulence of the particular diphtheria bacilli causing the disease."
}

Taking this quotation we are inclined to the opinion that the author is wrong in the first sentence. The Schick test has shown that adults more frequently than children have in their blood protective quantities of antitoxin. This explains satisfactorily the relative immunity of adolescents and adults to this disease. A more serious point, however, in the above quotation is the claim that adenoids, enlarged tonsils, and catarrhal affections predispose to this disease. If a Schick test shows a full amount of antitoxin in the blood of a given child, are we justified, so far as protection against diphtheria is concerned, in neglecting abnormal conditions of the upper respiratory passages? The mucous membrane of the phar- 
ynx and adjacent structures supplies the medium upon which the bacillus grows. This medium is of variable character and must depend for its relative constitution upon the health condition of the parts. It is quite easy to believe that the diphtheria bacillus will grow abundantly upon the medium found in the throat of one child and fail to grow at all on that furnished by another child. The amount of toxin formed in the child's throat is determined by the virulence of the strains of diphtheria bacillus and by the composition of the medium upon which it grows. It may be that if the child's blood contains as much as one-thirtieth of a unit of antitoxin the bacillus will not grow in the throat of that child whatever may be the condition of the mucous membranes. These are questions which quite naturally arise in the discussion of this subject and their satisfactory solution must be left to future investigations.

The Period of Incubation.-Pediatricians are inclined to speak cautiously about the period of incubation in this disease, and we are compelled to adopt the same attitude. A nurse in a diphtheria ward may become infected within a few days after her entrance, while on the other hand she may be in the closest contact with diphtheritic children for months and then unexpectedly develop the disease. In the former instance we can suppose that her blood is lacking in normal antitoxin content. In the second instance there are two possibilities. In the first place, her long residence in the midst of diphtheria and her freedom from the disease may be attributed to the failure of the bacillus to find its way into her throat; or the normal antitoxin content of her blood may have been reduced by fatigue or possibly from some unknown cause. Only in exceptional cases do we know when this bacillus finds its way into the throat. Quite a number of physicians have acquired this disease by heroic efforts to open filled tracheotomy tubes by sucking out their contents; in other words, they have drawn into their mouths rich eultures of the diphtheria bacillus. In every instance of this kind, so far as we know, the disease has developed within a few hours and has proved fatal. In one instance a child put into its mouth a tracheotomy tube which had been recently removed. In this case also the disease developed in a few hours and proved fatal. If we count the period of incubation from the time of entrance of the organism into the throat to the development of the first bit of membrane this period in the majority of instances is undoubtedly short, but taking a great number of cases it would naturally be liable to wide variation.

Unfortunately, there are no characteristic prodromal symptoms in this disease; certainly none that are pathognomonic. The clinical diagnosis is usually made with the appearance of the membrane. The experienced pediatrician seldom hesitates after an examination of the throat; though rarely it turns out that he is wrong. There are mild cases in which the 
membrane is slow in appearing and some in which it never develops. The failure to make an early diagnosis of this disease and the consequent failure to use antitoxin early enough is still the eause of a large number of deaths. Recently the health commissioner of Chicago made an investigation of the cause of death in 75 cases of diphtheria, with the following conclusions:

Deaths due to failure to call a doctor at beginning of illness

Deaths due to failure of physician to make early diagnosis

Deaths probably due to insufficient antitoxin

Deaths not preventable

In a recent article, entitled, "Diphtheria, the Uncontrolled," Carey, epidemiologist of the Massachusetts State Department of Health, reports the investigation of 1,000 deaths from this disease with the intention of ascertaining what factors might be present and common to all these deaths, and to fix a theoretical responsibility and to plan for their prevention in the future. In the 1,000 deaths investigated it was found that in 31.5 per cent the children had been sick one week or more before medical attention was secured; in 11.8 per cent the patients were moribund at the time of the doctor's first visit; in 7.6 per cent the nature of the disease was not recognized until it was too late for antitoxin to be efficacious. The above figures tell in a more striking manner than words can express the reason why the death rate from this disease continues as high as it is while we have in our hands an agent which when promptly given neutralizes the toxin just as certainly as an acid neutralizes an alkali. In the Chicago investigation it appears that the failure to save life is about equally divided between parents and physician, with somewhat more blame resting on the latter, because the physician must have been responsible for the deaths due to the administration of insufficient antitoxin.

In every progressive city, even those of not more than 25,000 inhabitants, there is a bacteriological laboratory where diphtheria swabs are examined. At present many of the smaller cities, including some of more than 25,000 inhabitants, and the rural populations must depend upon state laboratories or utilize the nearest municipal laboratory. It would be interesting to know to what extent the failure to have these laboratory facilities is accountable for the preantitoxin death rates which, as we have shown, still prevail in many localities. While, as we have stated, there are no pathognomonic symptoms in the early stages of this disease, the physician of long experience in its treatment is often able to recognize the fact that, although neither signs nor symptoms are marked, the child is seriously ill. In many cases the temperature is but slightly elevated. While the increase in the pulse rate is subject to great variation, the heart is weak. In most instances the child is indifferent to its sur- 
roundings, is lethargic, and in short, it is evident that it is suffering from some powerful poison. It goes without saying that when the characteristic membrane appears or a bacteriologic examination is positive, the diagnosis is made. In the lower animals, especially in the guinea pig, whether inoculated on an abraded mucous membrane with the bacillus or treated with the toxin, death occurs usually in from three to four days. In the child receiving its infection in the natural way the period of incubation, counting from the time of invasion to the development of signs or symptoms, averages from three to four days, although it may be somewhat shorter and frequently is longer.

Virulency Test.-It is true that there are wide variations in the virulence of different strains of the Klebs-Loeffler bacillus. It is impossible to distinguish between virulent and nonvirulent strains by morphologic or cultural tests. It has been found by Park and his coworkers in New York that from 4 to 8 per cent of individuals in localities where diphtheria is endemic are carriers of bacilli morphologically and culturally not to be distinguished from the diphtheria bacillus, but from 30 to 50 per cent of these are nonvirulent. Wilcox and Taylor found that 4.5 per cent of the children admitted to the scarlet fever wards of the Willard Parker Hospital were carriers of the diphtheria bacillus, but when these organisms were isolated and tested only about 50 per cent were found to be virulent. It has occasionally happened that an individual who is being kept in quarantine on account of a previous attack of this disease and who cannot be released until two or more successive cultures are negative, continues to carry diphtheria bacilli for months. In such a case the matter becomes serious and the question is asked what is to be done with the individual who persists in displaying evidence of his infection. The answer is that the virulence of the organism should be tested and if it is found nonvirulent the carrier should be discharged. In all wellequipped health laboratories there is provision for testing the virulence of diphtheria bacilli. The strain under examination is grown for 48 hours in ascitic broth (one part ascitic fluid plus two parts veal broth) and 1 c.c. of this culture is injected subcutaneously into each of two guinea pigs, one of which has no other treatment while the other receives at the same time 0.5 c.c. of a 100 -unit antitoxin. If the strain is virulent the control pig with the antitoxin will live, while the test pig, receiving the culture only, will die. Recently Zingher and Soletsky, of the New York City Health Laboratories, have devised and described a method in which economy in the use of guinea pigs is secured. The hair over the abdomen of these animals is removed either by the application of a paste of barium hydro-sulphid or by plucking. At as widely separated points as possible over the abdomen of an animal four cultures may be injected. In a second animal the same cultures are injected along 
with the antitoxin. It is necessary, however, to use a bacterial emulsion in this method. The entire surface growth from a fresh 24-hour culture on a Loeffler slant is suspended in 20 c.c. of normal saline. Of this preparation 0.2 c.c. is injected intracutaneously into corresponding areas of the abdominal surfaces of both pigs, one without and the other with antitoxin.

\footnotetext{
"The results of the tests are noted in 24,48 , and 72 hours. Virulent strains produce a definitely circumseribed local inflammatory lesion, which shows superficial necrosis in 48 to 72 hours. In the control pig the skin remains normal if the injections have been accurately carried out. With nonvirulent strains no lesions will be found in either control or test animal.",
}

Diphtheria Carriers.-We have already called attention to the fact that diphtheria bacilli are frequently found in the throats of healthy persons. While it is true that in a certain percentage of these the organism is nonvirulent, there still remain in the tested cases about 50 per cent in which the bacilli are virulent. Moreover, we are not sure what would happen if nonvirulent diphtheria bacilli were introduced, especially in large numbers, into the throat of a child carrying no antitoxin in its blood. Go into almost any school and examine the throat of every child and at almost any time some one will be found carrying this bacillus. If the examination be made at a time when diphtheria is epidemic in the neighborhood the number of carriers will be considerable. Stop every individual on the street and make a swab from the throat of each and some one will be found carrying this organism. If it be in the midst of a prevailing epidemic the number examined will not be large before a positive case is found. The number of carriers detected in Chicago by months from 1911 to 1918 inclusive is shown by the figures in Table $\mathrm{XX}$.

TABLE XX

\begin{tabular}{rrrrrrrrrrrrrr}
\hline \hline YEAR & JAN. & FEB. & MAR. & APR. & MAY & JUN. & JUL. & AUG. & SEPT. & OCT. & NOV. & DEC. & TOTAL \\
\hline 1911 & 82 & 67 & 34 & 18 & 25 & 31 & 1 & 3 & 58 & 311 & 473 & 69 & 1172 \\
1912 & 23 & 23 & 40 & 31 & 29 & 41 & 6 & 19 & 58 & 230 & 172 & 100 & 772 \\
1913 & 204 & 151 & 52 & 30 & 57 & 65 & 18 & 14 & 43 & 111 & 145 & 140 & 1030 \\
1914 & 209 & 98 & 195 & 160 & 196 & 142 & 115 & 85 & 241 & 456 & 387 & 324 & 2608 \\
1915 & 181 & 138 & 187 & 132 & 113 & 137 & 114 & 76 & 739 & 866 & 451 & 284 & 3418 \\
1916 & 226 & 223 & 199 & 109 & 183 & 142 & 64 & 77 & 381 & 575 & 1040 & 1083 & 4302 \\
1917 & 625 & 456 & 498 & 384 & 506 & 425 & 121 & 122 & 662 & 942 & 1016 & 507 & 6262 \\
1918 & 171 & 121 & 134 & 157 & 109 & 225 & 79 & 66 & 152 & 189 & 168 & 148 & 1719 \\
\hline
\end{tabular}

It is to be plainly understood that not one of these individuals showed any clinical symptoms of diphtheria. As large as these figures are, they by no means represent the total number of diphtheria carriers in Chicago. The total includes only those who were examined on account of 
some suspected relation to a clinical case. Several attempts have been made to determine the percentage of healthy carriers among the population at large. Goldberger and Williams, working in Detroit in 1914, made a most complete examination of the situation in that city following a rather severe epidemic of diphtheria. Their results are based upon both individual nose and throat cultures, as well as a combined nose and throat culture. Four thousand and ninety-eight individuals, all apparently healthy, were examined, and of these 38 , or approximately one per cent, were found to harbor B. diphtheriae. Of 19 cultures isolated from 19 carriers, two were found to be virulent, suggesting a possible 0.1 per cent of the individuals examined as carriers of virulent bacilli. Like studies were made in 1902 by a committee of the Association of Massachusetts Boards of Health. Three thousand and ninety-six healthy persons in Eastern United States were examined and 43 carriers found, or 1.4 per cent. Of 1,154 persons in Minnesota, 80 were found to be carriers, or 6.9 per cent. Many similar studies have been made in large American cities. The average number of earriers of the bacilli seems to be in the neighborhood of two per cent, but of this number only comparatively few are found to harbor virulent bacilli. It follows from these facts that any child not protected by antitoxin in its blood is liable to acquire this disease not only from coming in contact with one sick with it, but from a perfectly healthy person. Carey, in the investigation referred to above, was unable to ascertain the source of the infection in 90 per cent of the 1,000 cases of diphtheria studied. As we have already stated, if the diphtheria bacillus naturally grows and multiplies anywhere outside the human body such a breeding-place has never been discovered. While the Klebs-Loeffer bacillus grows easily upon culture medium, in nature it is practically an obligate parasite. An unknown percentage of human beings carry cultures of this organism in their throats. The healthy, as well as those known to be infected, may transmit this organism to others in common drinking-cups, on unsterilized knives, forks, spoons, and in many other ways. It is the ubiquitous nature of this organism which renders the eradication of the disease one of the most difficult problems confronting epidemiologists.

Control and Eradication.-It is self-evident that the individual with diphtheria should be isolated, and this isolation should be complete. If the infected child remains in its home it must be placed with an attendant in a room to which no one save the doctor and nurse has access and from which no one save such attendants has exit. The doctor and the nurse in going to and from the room should take certain precautions. The doctor when he visits the patient should put on his gown and his rubber gloves before entering the room and he should deposit these on leaving the room. Nothing should be taken from the room unless it be 
immersed in some disinfecting solution or in water which is to be boiled with its contents immediately after leaving the room. If isolation in the home is practicable and is carried out as herewith provided there is absolutely no danger of the disease being transmitted from the patient to any one else. If, however, the doctor or the attendant is careless a number of secondary cases will result. The child sick with diphtheria who cannot be cared for in its home under the conditions above mentioned should be taken to the infectious disease hospital. Those children who have already been in contact with the infected person should be given immediately immunizing doses of antitoxin. With these there is no time to wait for the development of a Schick test. The administration of antitoxin even in proper quantity to contacts will not protect in 100 per cent of cases, but it will protect in a large percentage. Failure to protect in a certain number is due to the fact that in these individuals invasion by the bacillus has already taken place. In Chicago in a group of 1,965 cases with 4,460 contacts and with 3,743 of these immediately treated with antitoxin, 33 developed diphtheria notwithstanding the administration of the antitoxin. All of the 33, however, developed the disease on or before the fourth day, indicating that invasion by the bacillus had in all probability taken place before the administration of the antitoxin.

If we are going to utilize the knowledge gained by Park and his associates and confirmed by others, it will be necessary to make a Schick test on every child and to actively immunize with the toxin-antitoxin mixture all those who are susceptible. This involves a heavy task, but one which is not beyond human accomplishment and which in the end will cost a great deal less in money and in labor than diphtheria now costs, to say nothing of the saving in this country of not less than 16,000 lives annually. Byard, in discussing the question of the eradication of diphtheria in New York, says that under present conditions two babies and one child die in that city each day from this disease, and then turning to the question of radical and universal immunization, he says that 141,564 babies are born each year in the city; that there are 610,870 children under five years of age and 527,175 between five and nine years, thus giving a highly susceptible group totaling $1,279,609$. If all susceptibles were immunized with the toxin-antitoxin mixture, we could hope for the ultimate and complete eradication of this disease, provided of course that this method of immunization was carried on for years. We cannot expect any radical procedure of this kind. The average parent, with instincts inherited, quite naturally resents every offer of the medical officer to do anything to the child. The value of immunization against diphtheria must be demonstrated among children in hospitals and other institutions and among those whose parents are sufficiently intelligent to have some comprehension of the process and to appreciate 
its results. In the meantime, the experimental investigator will advance his researches, while the intelligent physician will utilize to the fullest extent the agents of prevention that science has placed in his hands and at the same time will do what he can to educate the public.

The question of terminal disinfection in the private home after the release of a diphtheria patient should be easily settled. Infected articles should be burned, or if they are too valuable, should be disinfected. Sheets, pillow cases, and like articles may be immersed in some disinfecting solution or simply placed in water and subsequently boiled. There is no danger of bacilli being distributed through the air, at least for any time, and the disinfection of the air in the room, as in all other acute infectious diseases, is in our opinion, absolutely unnecessary and without value.

\section{Bibliography}

Byard, Dever S.: Arch. Pediat., 1920, xxxvii, 22.

Carey, Bernard W.: Boston Med. and Surg. Jour., 1919, clxxxi, 92.

Creighton, Charles: History of Epidemies in Britain, Cambridge University Press, 1894.

Duns, C. H.: Pediatries, Troy, N. Y., 1917, Southworth Company.

EDITORIal Texas State Jour. Med., 1920, xv, 312.

PARK, WM. H.: Collected Studies from the Research Laboratory, Department of Health of the City of New York, 1915, viii.

IRobertson, JoHN D.: Report and Handbook of the Department of Health of the City of Chicago, 1918.

Welch, W. M., aNd Schamberg, J. F.: Acute Contagious Diseases, 1905, Lea Bros. Company.

Zingher, A., AND Soletsky, D.: Collected Studies from the Research Laboratory, Do partment of Health of the City of New York, 1915, viii. 


\section{CHAPTER XI \\ SCARLET FEVER}

\section{SCARIATINA}

Definition.-Scarlatina is an acute, specific, infectious disease. In most instances the onset is abrupt and manifest by headache, vomiting, sore throat, chills, or chilly sensations, and rapidly rising temperature. Usually on the second or third day there appears a scarlet rash, which becomes more or less generalized and terminates on recovery in desquamation. The virulence of this disease shows widely marked variations in different outbreaks. As a rule, the case mortality varies from two to ten per cent, and in certain epidemics greatly exceeds the last-mentioned figure. Scarlet fever is frequently accompanied by enlarged lymphatic glands, especially in the neck, and these may suppurate and be slow in healing. Otitis media is a frequent and dangerous accompaniment. In a variable percentage (from fifteen to thirty) nephritis may follow this disease.

History.-An attempt has been made, notably by Malfatti, to ascribe the terrible epidemic, so vividly depicted by Thucydides, that swept Athens about 430 B. C. to scarlet fever, but we can find no reason for such a claim. Others have sought evidences of scarlet fever in the writings of Hippocrates, but the results of these researches have not been satisfactory. There is certainly no description given by the father of medicine of a disease which can be identified as scarlet fever. It appears that the Arabian physician, Rhazes, who lived in the ninth century, records the observation that the coloration of the eruption in his measles cases was in some instances more intense than in others and that those who showed the scarlet rash were more likely to die than those with less highly colored eruptions. Gregory, who has studied the history of the eruptive fevers, concludes that Arabian physicians describe a species of measles which from the extent of the desquamation must have been scarlatina. The first description of the disease sufficiently definite to distinguish between it and measles was written by Ingrassias, of Palermo, (1560). In 1574 Baillou, under the name of rubiolae, described an epidemic in Paris and differentiated it from measles. A similar description by Coythar, of Poitiers, appeared in 1578. This author points out the differential diagnosis between the disease which he describes and smallpox and measles. Early in the seventeenth century a severe epidemie, marked by sore throat and a scarlet eruption, became widely prevalent. in Italy, Spain, and Portugal. For quite two centuries 
this disease appeared in different localities in these countries. It was known under different names, such as, "Naples sore throat," "the disease of strangulation," and by the Spanish word "garrotillo." It is probable that both scarlet fever and diphtheria occurred in these outbreaks.

We are indebted to Sydenham, who, in 1665 named and described this disease, thus giving it a definite and permanent place in the list of specific diseases. However, Sydenham apparently never saw scarlet fever in virulent form. He wrote :

"Scarlet fever (scarlatina) may appear at any season. Nevertheless, it oftenest breaks out towards the end of summer, when it attacks whole families at once, and more especially the infant part of them. The patients feel rigors and shiverings, just as they do in other fevers. The symptoms, however, are moderate. Afterwards, however, the whole skin becomes covered with small red maculæ, thicker than those of measles, as well as broader, redder, and less uniform. These last for two or three days, and then disappear. The cuticle peels off; and branny scales, remain, lying upon the surface like meal. They appear and disappear two or three times.

"As the disease is, in my mind, neither more nor less than a moderate effervescence of the blood, arising from the heat of the preceding summer, or from some other exciting cause, I leave the blood as much as possible to its own despumation, and to the elimination of the peccant materials through the pores of the skin. With this view, I am chary both of bloodletting and of clysters. By such remedies, I hold that a revulsion is created, that the particles inimical to the blood become more intimately mixed therewith, and, finally, that the proper movement of nature is checked. On the other hand, I am cautious in the use of cordials. By them, the blood may be over-agitated, and so unfitted for the regular and equable separation in which it is engrossed. Besides which, they may act as fuel to fever.

"I hold it, then, sufficient for the patient to abstain wholly from animal food and from fermented liquors; to keep always in doors, and not to keep always to his bed. When the desquamation is complete, and when the symptoms are departing, I consider it proper to purge the patient with some mild laxative, accommodated to his age and strength. By treatment thus simple and natural, this ailment-we can hardly call it more-is dispelled without either trouble or danger; whereas, if, on the other hand, we overtreat the patient by confining him to his bed, or by throwing in cordials, and other superfluous and over-learned medicines, the disease is aggravated and the sick man dies of his doctor."

In 1684 a Scottish physician, Sibbald, wrote a book in which, among other things, he mentions scarlet fever and claims that it had recently developed de novo.

"Just as the luxury of man increases every day, so there grow up new diseases, if not unknown to former generations, yet untreated of by them. Nor is this surprising, since new depravations of the humors arise from unwonted diets and from various mixtures of the same. Among the many diseases which owe their origin to this age, there has been most recently observed a fever which is called scarlatina, from the carmine color with which almost the whole skin is tinged. Of this disease the observations are not so many that an accurate theory can be delivered or a method of cure constructed." 
Morton, who practiced in London during the last 30 years of the seventeenth century, published in 1694 a book in which he treats of scarlet fever. However, he says that he uses this term in deference to its adoption by the profession. He believed the disease called scarlet fever to be confluent measles. He reports a number of cases, most of which undoubtedly were measles, but some of which with equal certainty were scarlet fever, and there is no doubt that Morton saw many cases of malignant scarlatina. He wrote of these cases as constituting a veritable plague and speaks of the swollen tonsils, the gorged fauces, the ulcerated lips, and sordid crusts, as well as of suppurating glands. There is no doubt that during the whole of the seventeenth and eighteenth centuries scarlet fever under various local names, such as, angina maligna, angina gangrænosa, anginose sore throat, etc., existed continuously throughout western Europe and the British Isles and contributed not inconsiderably to the mortality of the times.

One of the most classical epidemies known to history is that which occurred in New England in the year 1735, generally known as the "throat distemper." The following description is found in Belknap's History of New Hampshire:

"About this time, the country was visited with a new epidemic disease, which has attained the name of the throat distemper. The general description of which is a swelled throat, with white or ash-colored specks, an efflorescence of the skin, great debility of the whole system, and a strong tendency to putridity. Its first appearance was in May, 1735, at Kingston in New Hampshire, an inland town, situate on a low plain. The first person seized was a child, who died in three days. About a week after, in another family, at the distance of four miles, three children were successively attacked, who also died on the third day. It continued spreading gradually, in that township, throughout the summer, and of the first 40 who had it, none recovered. In August it began to make its appearance at Exeter, six miles northeastward; and in September, at Boston, 50 miles southward, though it was October, before it reached Chester, the nearest settlement on the west of Kingston. It continued its ravages through the succeeding winter and spring, and did not disappear till the end of the next summer.

"The most who died of this pestilence were children; and the distress which it occasioned, was heightened to the most poignant degree. From three to six ehildren were lost out of some families; several buried four in a day, and many lost their all. In some towns, one in three, and in others one in four of the sick were carried off. In the parish of Hampton Falls it raged most violently. Twenty families buried all their children. Twenty-seven persons were lost out of five families; and more than one sixth part of the inhabitants of that place died within thirteen months. In the whole province, not less than 1,000 persons, of whom above 900 were under 20 years of age, fell victims to this raging distemper.

"Since the settlement of this country such a mortality had not been known. It was observed that the distemper proved most fatal, when plentiful evacuations, particularly bleeding, were used; a great prostration of strength being an invariable symptom. The summer of 1735 , when the sickness began, was unusually wet and cold, and the easterly wind greatly prevailed. But it was acknowledged to be, not 
'a creature of the seasons;' as it raged throughout every part of the year. Its extent is said to have been 'from Pemaquid to Carolina;' but with what virulenee it raged, or in what measure it proved fatal, to the southward of New England, does not appear.

"The same distemper has made its appearance at various times since. In 1754 and 1755 , it produced a great mortality in several parts of New Hampshire, and the neighboring parts of Massachusetts. Since that time it has either put on a milder form, or physicians have become better acquainted with it. The last time of its general spreading was in 1784, 5, 6, and 7. It was first seen at Sanford in the County of York; and thence diffused itself, very slowly, through most of the towns of New England; but its virulence, and the mortality which it caused, were comparatively inconsiderable. 'Its remote, or predisposing cause, is one of those mysteries in nature which baffle human inquiry.' ",

One is likely to think that the above quotation from a lay writer is a gross exaggeration, but strange to say, and yet fortunately, in all the parishes of New England at that time, certainly in New Hampshire, accurate records of births and deaths were kept and are still preserved. Furthermore, these records bear out, at least in a general way, the statements quoted. There can be no doubt that such an epidemic originated in the little village of Kingston in New Hampshire in 1735 and during the following years spread throughout the American colonies, causing here and there a high mortality. When it reached Boston it fell under the observation of Douglas, one of the most competent medical men of his time. Douglas has left a valuable report of his observations of this disease. The population of Boston at that time was about 12,000 and Douglas estimates the number of cases of illness at 4,000. However, it is evident that in reaching this large number he counted all who had sore throat. The fatality in Boston was by no means comparable with that which had been experienced in the small towns of New Hampshire. There can be no doubt from the descriptions given by Douglas that he had to deal with scarlet fever, although it is possible there may have also been cases of diphtheria. He calls the eruption miliary. In some the eruption preceded the soreness of the throat, while in others it accompanied or followed the throat trouble. The ordinary course was a chill with shivering, followed by nausea and vomiting, with swelling and redness of the tonsils. The face became flushed and the eruption was accompanied by fever. As the patients recovered there was marked peeling of the extremities and in some instances the nails of the fingers and toes were cast off. Among the after-effects were anasarca, pustules, boils, and sloughing sores.

It is interesting to note that this epidemic, with like instances, was used by Noah Webster in his argument against quarantine. He believed that this and other diseases appearing in the American colonies at that tributed all epidemies to earthquakes, floods, the approach of comets, 
etc., and was led to write his most interesting book on pestilence by his desire to combat the theory of infection. The New England throat distemper gave him, in his opinion, a splendid example of his theory. This disease broke out in a small inland village. It could not possibly have come across the ocean from any direction. Of course, we know that Webster was wrong, but we can probably never tell just where the New England epidemic of throat distemper originated. In this connection, however, it may be interesting to state that at that time the trade of the province of New Hampshire consisted chiefly, according to Belknap, in the exportation of lumber and fish to Spain, Portugal, and the West Indies, while at the same time trade with the mother country continued. We learn from other authorities that the New Englanders of that time shipped lumber and fish not only to England, but to Spain and Portugal, going from the last mentioned place to the west coast of Africa where they took on slaves. These were earried to the West Indies. After discharging its human freight the New England craft carried rum and molasses to the home ports. For years before and at the time of the outbreak at Kingston this disease was known to be constantly present at Plymouth, England, and in the ports of Spain and Portugal; and its presence in the West Indies is a matter of historical knowledge. There would, therefore, be no great strain on the imagination to account for the importation of scarlet fever into the New Hampshire village in $\mathbf{1 7 3 5}$. In the isolated villages of New England at that time the virus of this disease undoubtedly found in the throats of the children rich soil for its development. One hundred years had elapsed since the landing of the Pilgrim Fathers; the isolated settlements had increased in size largely through home growth, and a highly susceptible generation was ready to receive the new virus. When the disease reached Boston its virulence was greatly diminished. We know from the records that there had been more or less scarlet fever in Boston immediately preceding 1735 . Consequently, we are ready to believe that the newly imported virus found less susceptible material to work on.

As we have already stated, scarlet fever for many years showed itself here and there in isolated American villages. Webster's interesting book furnishes many examples, a few of which we shall quote:

"The fatal ulcerous sore throat was contemporary in America and in France in 1737; but that disease did not appear, in its formidable array, in England, until 1742. In 1734-1735 appeared its sister malady, the scarlet fever in Edinburgh.',

"In America the same angina prevailed in 1742.",

"In 1742 a mortal fever prevailed at Holliston, in Massachusetts in which died Mr. Stone, the minister, and 14 of his congregation.",

"In England the angina maligna continued its ravages with increased mortality. The same malady prevailed in France in 1749, and there was an earthquake at London." 
"In America the spring flights of pigeons were unusually large. The dysentery was epidemic and mortal; in the same year at Hartford and New Haven; probably in many other places. With this fatal dysentery prevailed a mortal angina for several years.",

Writing of the year 1755 , Webster says:

"The angina maligna was very mortal in some parts of America. In one town on Long Island two children only, under 12 years of age, survived."

"In 1769 the summer was very hot, and in the autumn appeared a comet with a vast coma. Venus past over the sun's disk on the third of June; there was a small earthquake in New England and a great tempest. Among the diseases in America is mentioned a fatal angina in Boston, and other towns, but I am not furnished with its history. The same distemper prevailed in 1770 and in Jamaica occasioned considerable mortality."

"In 1771 anginas, in some parts of America, occasioned considerable mortality."

"The anginas of the preceding year continued to prevail in 1772."

"But the most mortal disease, was, the cynanche trachealis or bladder in the throat. In general, there was little canker, but an extreme difficulty of breathing; the patient being nearly suffocated with a tough mucus or slime, which no medicine could attenuate of discharge and which finally proved fatal. All medical aid was fruitless, and scarcely a child that was attacked in some towns, survived."

"The year 1774 was more healthy than the preceding and the scarlatina anginosa began to show itself in Edinburgh and in some parts of America, especially at Philadelphia. On the fourth of May was a fall of snow.'

"In 1775 cynanche maligna invaded all the northern parts of America and in many places it continued to be current with dysentery for three years. This was the case in Middletown on Connecticut River."

Epidemiologists have studied and discussed the New England throat distemper of the eighteenth century and are divided in their conclusions as to its nature. Hirsch concludes that it was "an exquisite scarlet fever." On the other hand, Häser believes that it was diphtheria. We have gone over the original records so far as we have had access to them, and we believe that the prevalent disease in New England at the time was scarlet fever, but that there were outbreaks of diphtheria as well. In 1789 Bard, of New York, published a most excellent report of the disease as he saw it in that eity. Bard thinks that the disease in New York was the same as that observed in Boston and reported upon by Douglas in 1736. In this opinion we cannot concur. Whatever may have been the nature of the New England throat distemper the cases described by Bard could, in our opinion, have been nothing else than laryngeal diphtheria, although it must be admitted that extensive membranes are sometimes formed in scarlet fever. Bard made an autopsy on one of his cases, of which the following is a report:

"Upon examining the body, which was done on the afternoon of the day she died, I found the fauces, uvula, tonsils, and root of the tongue, interspersed with sloughs, which still retained their whitish color. Upon removing them, the parts underneath appeared rather pale than inflamed. I perceived no putrid smell from them, nor was 
the corpse in the least offensive. The esophagus appeared as in a sound state; the epiglottis was a little inflamed on its external surface, and on the inner side, together with the inside of the whole larynx, was covered with the same tough white sloughs, as the glands of the fauces. The whole trachea, from the larynx down to its division in the lungs, was lined with an inspissated mucus, in form of a membrane, remarkably tough and firm; which, when it came to the first subdivision of the trachea, seemed to grow thin and disappear; it was so tough as to require no inconsiderable force to tear it, and came out whole from the trachea, which it left with much ease; and resembled more than anything, both in thickness and appearanee, a sheath of thin shammy leather."

Epidemics of malignant sore throat, some of which were probably scarlatina, while others were diphtheria, continued to appear from time to time in England and Ireland. The years 1743-52-55-59-62-86 are given as those in which scarlatina anginosa showed unusual virulence in Ireland; in fact, these epidemics did not cease with the close of the century but continued far into the nineteenth. During these years scarlet fever became very benign and so continued sometimes for a decade or more. Then physicians began to talk of the attenuation which the virus of this disease was evidently undergoing, or they were inclined to attribute its low mortality to the treatment employed. However, congratulations of this kind have not continued indefinitely, because from time to time the virus of scarlet fever has assumed its old malignity. It may be of interest to read what Graves, the great clinician of Dublin, wrote on this point :

"'The long continuance of the period during which the character of searlet fever was either so mild as to require little care, or so purely inflammatory as to yield readily to the judicious employment of antiphlogistic treatment, led many to believe that the fatality of the former epidemic was ehiefly, if not altogether, owing to the erroneous method of cure then resorted to by the physieians of Dublin, who counted among their numbers not a few diseiples of the Brunonian School; indeed, this opinion was so prevalent, that all those whose medical education commenced at a much later period, were taught to believe that the diminished mortality of scarlet fever was entirely attributable to the cooling regimen and to the timely use of the lancet and aperients, remedies interdicted by our predecessors. This was taught in the schools, and searlet fever was every day quoted as exhibiting one of the most triumphant examples of the efficacy of the new doctrine. This I myself learned-this I taught; how erroneously will appear from the sequel. It was argued, that had the eases which proved fatal in $1801-2$ been treated by copious depletion in their very commencement, the fatal debility would never have set in, for we all regarded this debility as a mere consequence of previous excessive reaction. The experience derived from the present (1834-35) epidemic has completely refuted this reasoning, and has proved that, in spite of our boasted improvements, we have not been more successful in 1834-35 than our predecessors in 1801-2.'"

About the middle of the eighteenth century an ulcerous sore throat became a veritable plague in London and in some other English cities. This could scarcely have been other than scarlet fever, as is indicated by the following quotation: 
"It began with a chill and rigor, followed by heat. The throat became sore, and there were nausea, vomiting, and purging. The face turned red and swollen, the eyes were inflamed and watery, the patient was restless, anxious, and prostrated. The seizure was often in the forenoon, and in all instances the symptoms became much worse towards night, to be relieved by a sweat in the morning, as in an intermittent fever. The uvula, tonsils, velum, inside of the cheeks and the pharynx were a florid red, with a broad spot or patch, irregular in figure, of pale white color like the blanched appearance of the gums when they have been pressed by the finger. Usually on the second day of the disease, the face, neek, breast, and hands to the tips of the fingers became of a deep erysipelatous color with perceptible swelling, the fingers in particular being often of so characteristic a tint as at once to suggest an examination of the throat. A great number of small pimples, of a deeper red than the skin around them, appeared on the arms and other parts; they are larger and more prominent in those subjects, and in those parts of the same subjects, where the redness is least intense, which is generally on the arms, the breast and lower extremities. With the coming out of this rash, the sickness, vomiting, and purging cease. The white spot or spots on the throat are now seen to be sloughs. They come first usually in the angles above the tonsils. They are not formed of any foreign matter covering the parts but are real mortifications of substances leaving an ulcer with corrosive discharge behind. The nocturnal exacerbation now takes the form of delirium and incoherent talking. The parotids are commonly swelled and painful; and if the disease be violent, the neck and throat are surrounded with a large edematous tumor threatening suffocation. The pulse is 120, perhaps hard and small. The urine is at first crude and pale like whey; afterwards it is more yellow, as if there were bilc; and towards recovery it is turbid and deposits a 'farinaceous' sediment. The disease has no crisis, but in general, if the patient were to recover, the amendment began on the third, fourth, or fifth day, when the redness disappeared and the sloughs in the throat were cast off.'

For many years the disease above described was known as Fothergill's sore throat with ulcers. It is still a question whether this was an unmixed epidemic of scarlet fever or an epidemic of this disease accompanied by many cases of diphtheria.

Creighton, in his most valuable work on the History of Epidemies in Britain, has collected the main facts about the most pronounced epidemics in the British Islands during the seventeenth, eighteenth, and nineteenth centuries.

During the eighteenth and nineteenth centuries the northern part of the United States has shown a history of scarlet fever almost parallel to, though not quite so highly fatal as, that of the epidemic which "emerged" (as Douglas says) at Kingston, N. H., in 1735; in other words, there has been no break in the continuity of that epidemic. There have been numerous instances when for the time being there has been no scarlet fever in a given locality, but there is no proof at least, that at any time since $\mathbf{1 7 3 5}$ has there been a break in the continuity of scarlet fever in the northern United States. During the eighteenth century this disease, so far as this country is concerned, was most in evidence in New England, and this for two reasons. In the first 
place, scarlet fever has always been more prevalent in the northern than in the southern United States. In the second place, the settled portion of the northern United States was during the eighteenth century largely confined to New England. With the immigrant moving from New England across the Hudson into the region of the Great Lakes and beyond scarlet fever has never lost his trail, and today this disease is most prevalent in Michigan, Wisconsin, and Montana, when we study mortality records for whole states. When we take large cities, we find that on the whole undue prevalence of this disease is found in the same geographical area as is indicated by a study of the states. While in these states there are yearly fluctuations within wide limits, we find abnormal prevalences of the disease during the past 20 years in the

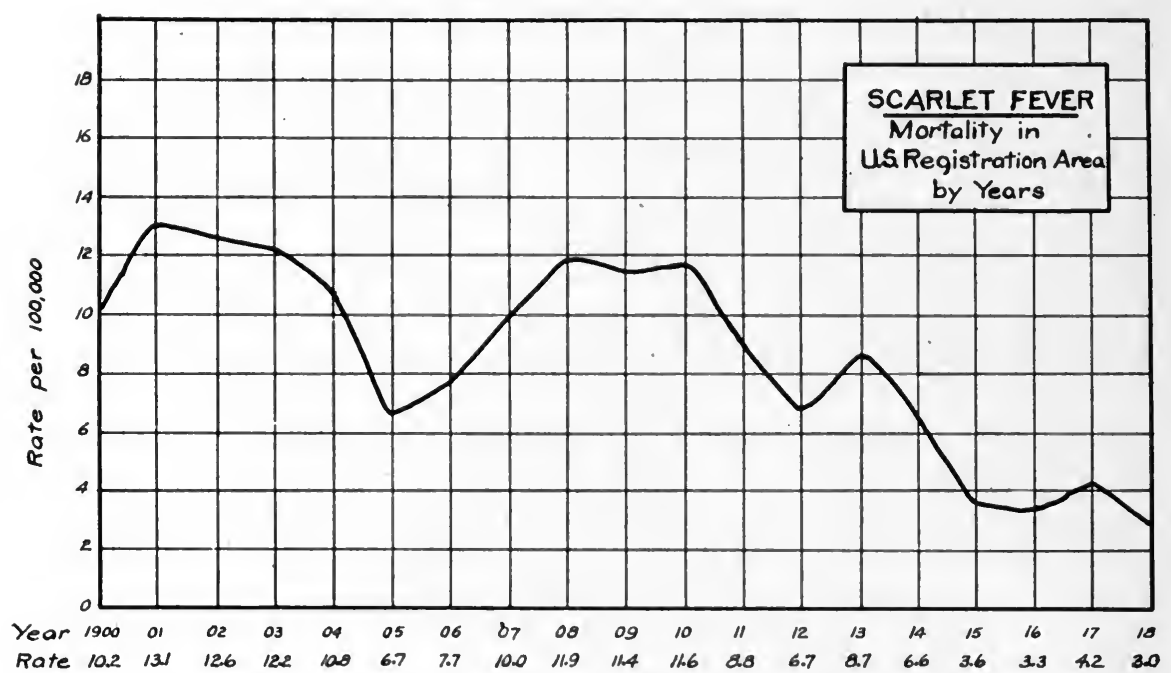

Fig. 29.

region of the Great Lakes and in the mountainous districts of the northwest. In the past 20 years Rochester, Buffalo, Cleveland, Toledo, Detroit, Chicago, Milwaukee, St. Paul, and Minneapolis, have had in one season or another, even in two or more, abnormally high death rate from this disease. If we study the smaller cities, we find that this same region, more frequently than others, supplies the highest death rate from scarlet fever. Taking the registration area as a whole, the death rate from this disease shows a very satisfactory decrease during the past 15 or 20 years. This showing, however, must not mislead us. In the first place, as the registration area has extended the area of its increase has been, partly at least, if not wholly, in the southern portion of this country where there is and always has been less scarlet fever than in the northern part. In the second place, with the extension 
of the registration area into the South, we are increasing the number of negroes in the population and a survey of the vital statistics will show at. a glance that either scarlet fever is less fatal among negroes than among whites or that the deaths among the former are not reported as accurately as they are among the latter. It must be admitted that the statistics for the original registration states show a similar decline. There is much evidence, indicating at least, that the farther south we go the less scarlet fever we have. Rogers, in his work on Fevers in the Tropics, makes the following statement:

"Unlike measles and the other exanthematous diseases of temperate climates, scarlet fever is very rarely met with in India, its very occurrence in the country having been called in question as late as 1871, when a controversy on the subject arose in the columns of the Indian Medical Gazette over a case reported to have been seen in Simla, and several very definite instances of the disease being imported into India by trooping ships, and spreading to a limited extent, were recorded. Thus 13 cases were reported by Gibson with typical rash on the second day, strawberry tongue, inflammation of the tonsils, pharynx, and salivary gland, albuminuria and dropsy in six cases, and discharges from the nose and ears causing deafness in one instance, all occurring anong the children of a British regiment. Again, the disease broke out at both Sialkote and Jullunder in the Punjab among the children who came to India by the same trooper, on whose royage several cases of scarlatina had occurred, the attacks being very similar to those just mentioned, while several fatal cases occurred. Since that time many similar importations of the disease have taken place, some of which appear to have been due to infection from clothes, etc., through the post from infected houses in England; but it only gives rise to very limited outbreaks and rapidly dies out. *** It is clear, then, that scarlatina does occur in India as an imported infection; but that it is usually of a mild nature, and shows no tendency to spread widely, but on the contrary rapidly dies out, especially in the hotter parts of India. There is no evidence that it ever becomes an indigenous disease, and rarely if ever attacks natives of the country."

\section{Castellani says:}

"Scarlet fever has often been introduced into the tropics, but it does not spread, Thus we have seen cases introduced into Colombo from the steamers, but there has never been an epidemic of scarlet fever in that town to our knowledge, nor have we seen an indigenous case."

Recent Prevalence and Virulence.-Since there seems to be a widely prevalent idea among both the profession and the laity that scarlet fever in recent years is losing its importance, we desire to go somewhat more minutely into this question. If we take the figures as furnished by the United States Census Bureau, this prevailing opinion finds substantial support, as is shown in Table XXI.

It will be seen that these figures give very substantial support to the belief that scarlet fever is approaching the negligible point so far as the acute infectious diseases influence the death rate. However, as we have already stated and wish to further emphasize, the registration area has 


\section{RACIAL VARIATION}

\section{IN MORTALITY FROM COMMUNICABLE DISEASE}

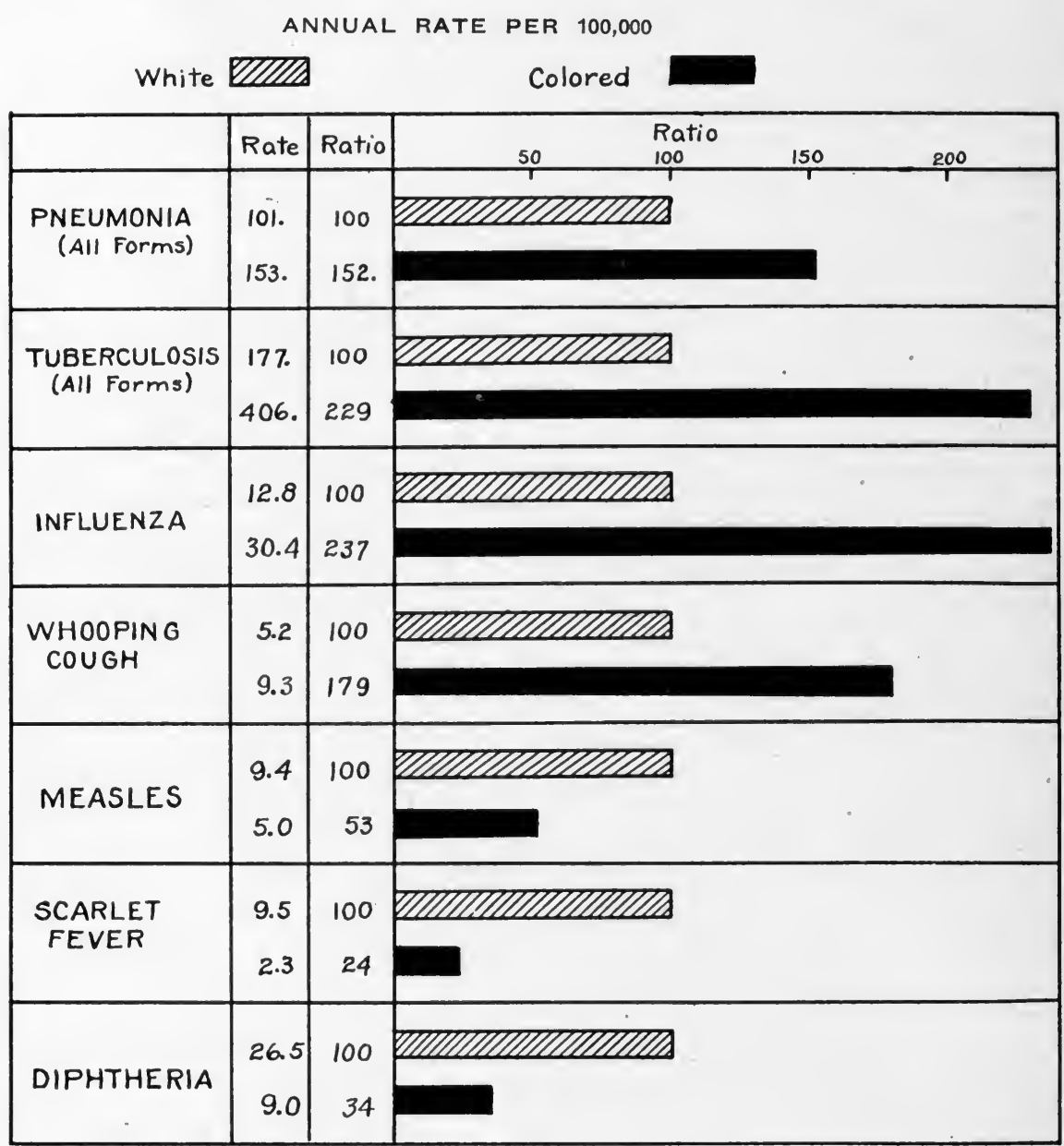

Statistics of Insured Wage Earners Metropolitan Life Insurance Co.

Fig. 30 .

changed greatly since 1880. At that time it included only two states, Massachusetts and New Jersey, together with the District of Columbia, and certain large cities scattered over the country. Moreover the year 1879-1880 was one characterized by an unusual prevalence of this disease in the then registration area. If we go somewhat more minutely into the census figures, we may learn some things concerning this disease which will at least interest us, and possibly we may profit thereby. 
TABLE XXI

Deati Rate from Scarlet Fever Per 100,000 Population in thé Registration Area of the United States since tire Census of 1879-1880

\begin{tabular}{lccc}
\hline \hline YEAR & DEATH RATE & YEAR & DEATH RATE \\
\hline $1879-1$ S80 & 54.0 & 1909 & 11.4 \\
$1889-1890$ & 13.6 & 1910 & 11.6 \\
1899 & 11.6 & 1911 & 8.8 \\
1900 & 10.2 & 1912 & 6.7 \\
1901 & 13.1 & 1913 & 8.7 \\
1902 & 12.6 & 1914 & 6.6 \\
1903 & 12.2 & 1915 & 3.6 \\
1904 & 10.8 & 1916 & 3.3 \\
1905 & 6.7 & 1917 & 4.2 \\
1906 & 7.7 & 1918 & 3.0 \\
1907 & 10.0 & & \\
1908 & 11.9 & & \\
\hline
\end{tabular}

TABLE XXII

Death Rates from Scarlet Fever per 100,000 Population in the Large Cities of tile United States SINCE 1911

(Using Corrected Mid-year Population Folijowing 1920 Census)

\begin{tabular}{lrrrrrrrrrr}
\hline \hline \multicolumn{1}{c}{ CITY } & 1911 & 1912 & 1913 & 1914 & 1915 & 1916 & 1917 & 1918 & 1919 & 1920 \\
\hline Boston & 11.3 & 4.6 & 10.9 & 9.3 & 11.6 & 5.4 & 6.2 & 3.3 & 4.0 & 9.4 \\
New York & 15.3 & 12.7 & 11.3 & 9.4 & 5.9 & 2.3 & 2.6 & 3.4 & 2.6 & 3.9 \\
Philadelphia & 12.4 & 7.6 & 10.5 & 8.1 & 1.6 & 2.5 & 2.0 & 2.7 & 3.4 & 6.3 \\
Detroit & 12.2 & 10.2 & 17.5 & 7.5 & 3.5 & 6.6 & 17.2 & 5.1 & 7.5 & 9.7 \\
Chicago & 21.9 & 27.2 & 39.0 & 10.1 & 3.4 & 6.4 & 24.6 & 2.2 & 4.6 & 6.6 \\
St. Paul & 16.1 & 4.1 & 8.1 & 20.5 & 13.3 & 3.5 & 3.5 & 18.2 & 6.4 & 6.4 \\
Minneapolis & 6.1 & 4.7 & 19.3 & 26.8 & 3.2 & 4.6 & 3.1 & 9.0 & 6.7 & 7.0 \\
St. Louis & 27.8 & 6.5 & 6.1 & 19.4 & 3.0 & 6.5 & 7.5 & 2.8 & 2.6 & 3.1 \\
San Francisco & 3.5 & .7 & 4.0 & 1.1 & 1.9 & 1.7 & 2.5 & 2.2 & 2.3 & 1.9 \\
New Orleans & 1.2 & 2.6 & .6 & .6 & .5 & .3 & .5 & .0 & .8 & 1.8 \\
Washington & 1.2 & 1.1 & 3.8 & .3 & 2.3 & 1.5 & 2.2 & 2.4 & .9 & 1.8 \\
Atlanta & 4.4 & 1.2 & .0 & 1.2 & .6 & 1.6 & 1.1 & 4.2 & 4.6 & 2.9 \\
Richmond & 2.3 & .7 & 3.5 & 1.4 & .7 & 1.9 & .6 & 0. & 1.8 & 0 \\
Pittsburgh & 10.7 & 10.8 & 26.7 & 33.8 & 9.7 & 3.7 & 2.3 & 1.9 & 1.5 & 3.6 \\
\hline
\end{tabular}

With this in view we present Table XXII, the figures of which are taken from the mortality statistics of 1917, and from other sources.

With the exception of New York City there is not a consistent or uniform reduction. High rates reeur every few years, this being especially noticeable with Detroit, Chicago, St. Paul, and Minneapolis.

However, if we wish to get some idea of the recent virulence of scarlet fever, we must take the figures from smaller places. Some of these we give in Table XXIII.

If we include in this table cities with death rates between 30 and 


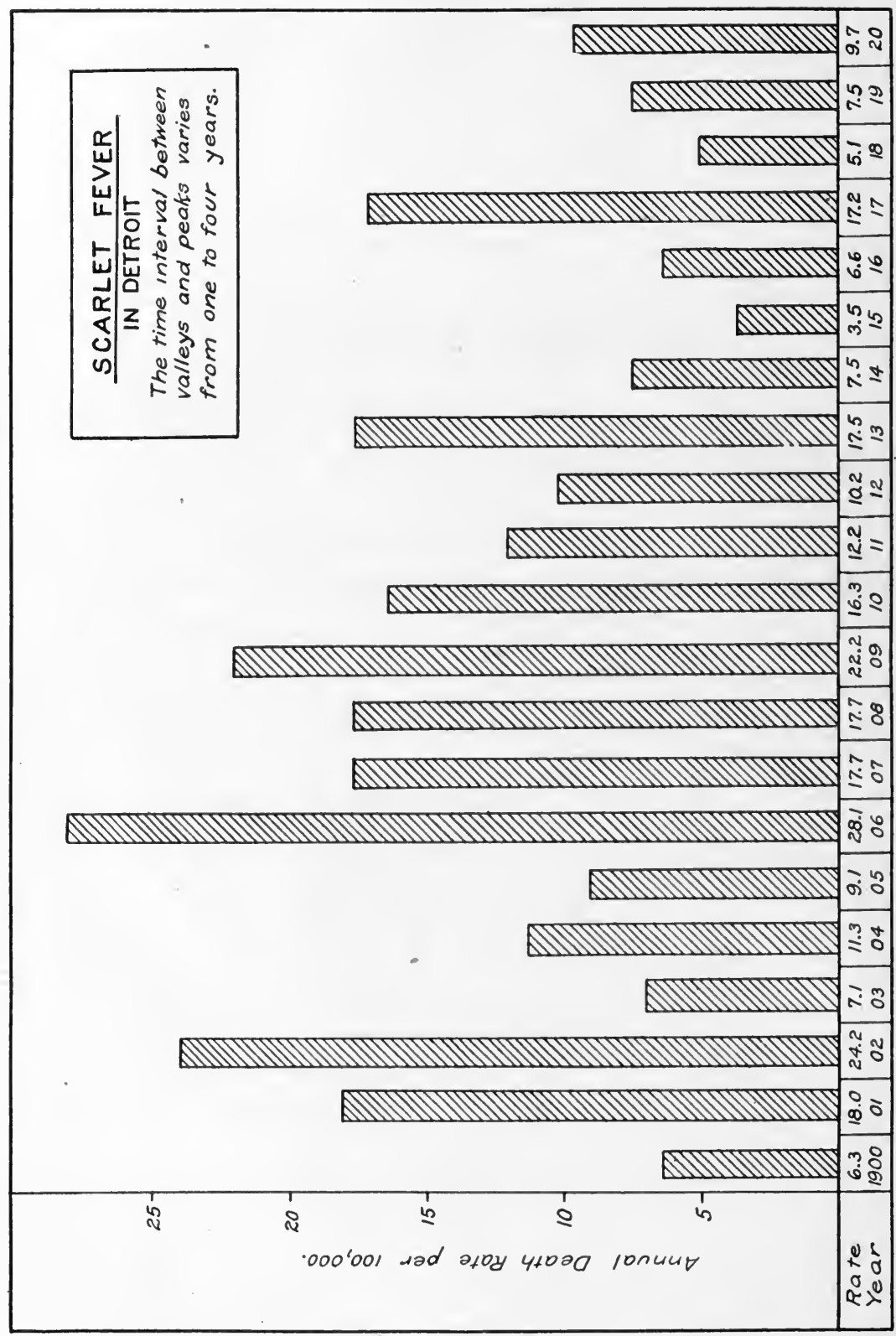

Fig. 31.

100 per 100,000, the list would be a long one. The New England villages in the eighteenth century did not suffer from a more malignant and fatal scarlet fever than have those here listed suffered in the twentieth century. The scarlet fever in Boston in 1736 killed, accord- 


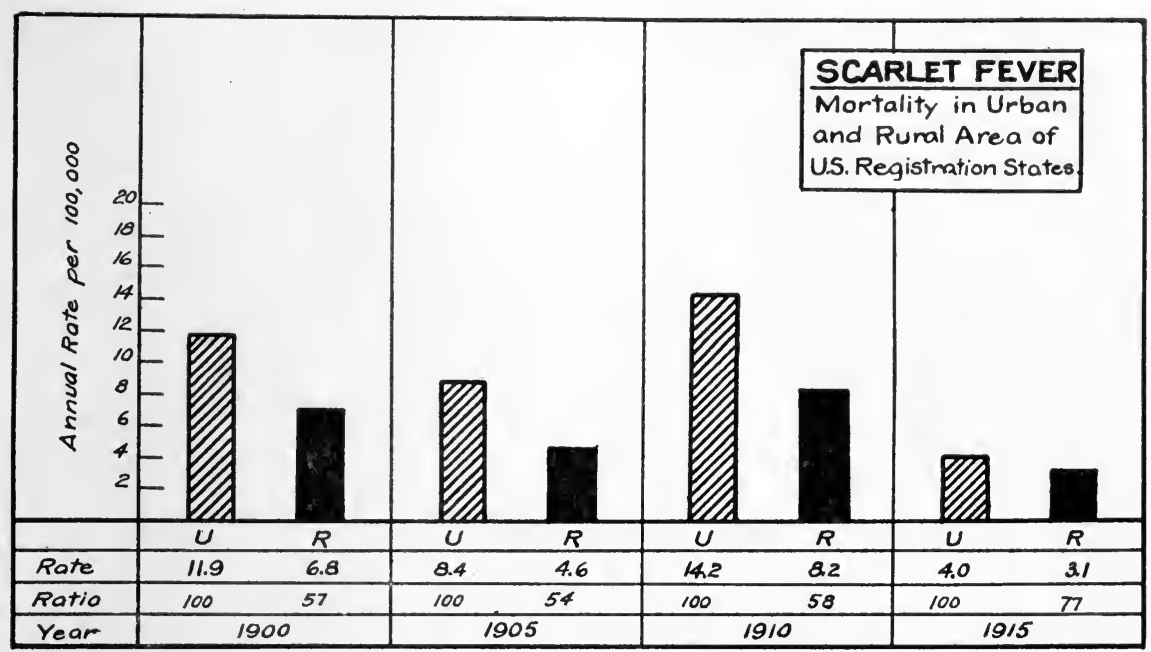

Fig. 32 .

TABLE XXIII

Death Rate from Scariet Fever per 100,000 Population in Certain Small Cities of the United States in Certain Years

\begin{tabular}{ccc}
\hline \hline CITY & YEAR & DEATH RATE \\
\hline Shenandoah, Pa. & 1911 & 219.4 \\
Shenandoah, Pa. & 1912 & 177.8 \\
Shenandoah, Pa. & 1913 & 101.7 \\
Kenosha, Wise. & 1912 & 203.1 \\
Great Falls, Mont. & 1913 & 146.7 \\
Adamstown, Mass. & 1913 & 110.0 \\
Trinidad, Colo. & 1912 & 106.3 \\
\hline
\end{tabular}

TABLE XXIV

Cases and Deaths from SCarlet Fever in Chicago from 1911-1918

\begin{tabular}{crcc}
\hline \hline YEAR & CASES & DEATIIS & CASE MORTALITY \\
\hline 1911 & 7422 & 471 & 6.3 \\
1912 & 8703 & 602 & 6.9 \\
1913 & 10600 & 906 & 8.5 \\
1914 & 3518 & 228 & 6.5 \\
1915 & 3366 & 77 & 2.3 \\
1916 & 8681 & 162 & 1.9 \\
1917 & 13444 & 624 & 4.6 \\
1918 & 1809 & 46 & 2.5 \\
\hline
\end{tabular}

ing to Douglas, one out of thirty-five cases. In 1913 out of 10,600 cases of this disease in Chieago, 906 died, one to eleven. We have no means of aseertaining the ease mortality in either the small New England towns in the eighteenth eentury, nor that in the towns given in the 
above list in the twentieth century; but the former could scarcely have been greater than the latter. We, therefore, have grave doubts about the decrease in the malignity of the scarlet fever virus in recent years. The case mortality in Chicago is shown in Table XXIV.

Robertson, health officer of Chicago, states that a wave of scarlet fever sweeps over Chicago every three to six years.

Transmission.-So long as it was believed that the infection of scarlet fever is carried in the epithelial cells from the desquamating skin, it was held that this disease is air borne. Granted that the infection is contained in these fine scales, there would be no escape from the conelusion that the disease is scattered through the air. Recently, however,

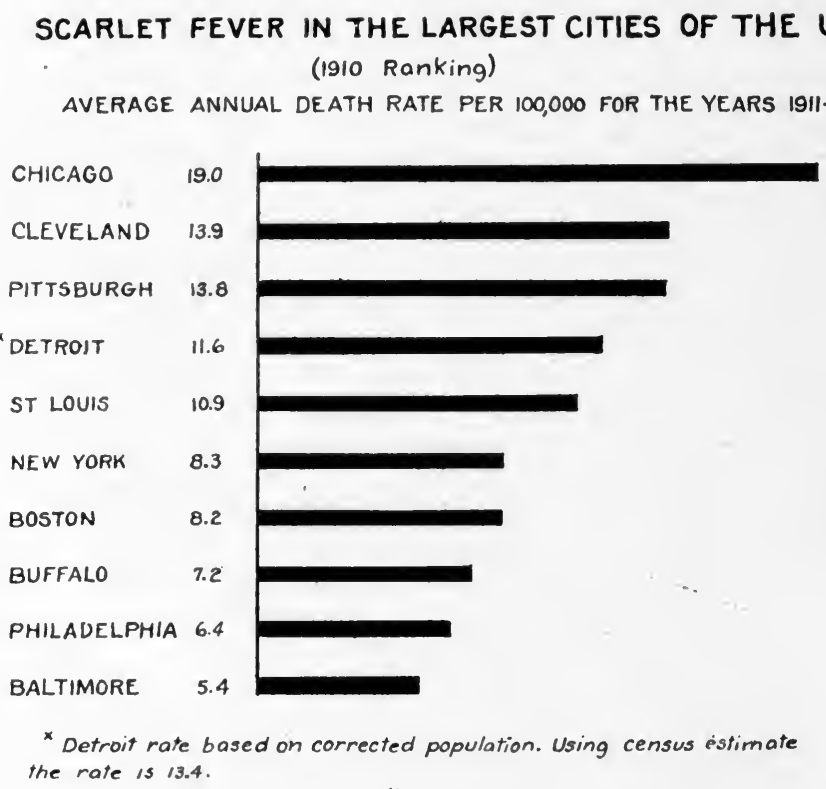

Fig. 33.

much evidence has accumulated against the infectious nature of the epidermal scales; consequently, the possibility of the disease being transmitted through the air has not only become doubtful, but the weight of evidence is heavily against it. There was a period some twenty years ago when reasonable objection could be made against the location of a scarlet fever hospital in the neighborhood of other hospitals, of schools, manufacturing and business houses, or of private residences. However, objection to the location of a hospital for infectious diseases on the supposition that scarlet fever is air borne no longer finds support. The prevalence of scarlet fever in the vicinity of hospitals housing this disease, has been closely studied, both in Europe and in this country. An investigation was made concerning the distribution 
of scarlet fever roundabout the Iospital for infants and the Trousseau Hospital in Paris. This resulted in a complete denial that this disease is spread through the air. Similar studies have been made in Boston, Detroit, and Providence. There were no cases of scarlet fever found within one-eighth of a mile of the Boston City Hospital, while in the next eighth of a mile there were 68 , in the third 71 , and in the fourth 72 ; in other words, it happened in this instance that the farther one went from the hospital the more scarlet fever he found. Hundreds of nonimmune medical students in Philadelphia, according to Welch and Schamberg, visit the scarlet fever wards of the Philadelphia Hospital, remain from 20 minutes to an hour, and not one of these has ever contracted the disease. Forcheimer gives the following evidence against the transmission of scarlet fever through the air:

"In one of two adjacent city houses searlatina broke out. The children affected with scarlatina were in the habit of playing with the children in the other house. As the cases were mild ones the children could not be kept in bed and came to the windows in the rear of the house. These windows were not more than nine feet from the windows of the house in which the noninfected ehildren lived. The distance was too great for the children to touch each other, and while nothing passed from house to house, the sick and well children were constantly conversing with each other, and still not one of the noninfected children became infected."

In fact, it has been demonstrated repeatedly in recent years that scarlet fever patients may be kept in the same ward with other patients without danger of transfer of the disease, provided contact transmission be absolutely avoided.

As long ago as 1893 Dowson advanced the idea that the essential lesion of scarlet fever is in the throat, just as chancre is the primary and essential lesion of syphilis. While this view has much in its favor, it cannot be said to have as yet met with general acceptance. However, the weight of evidence now is that the virus is found in the discharges from the throat and such other discharges as may come from the ears and from suppurating glands. At just what time these discharges are most heavily laden with the virus or contain it in its most active form, there are no data to justify a decision. It seems quite certain, as has been stated, that the virus is not contained in the epidermal scales thrown off in desquamation. The catarrhal symptoms, and consequently the mucous discharges, are most active at the time of the eruption, but the period of quarantine should be determined by the continuance of the mucous and purulent discharges and should not depend upon or be determined by the stage of desquamation; in other words, the danger to other children in the release of convalescents is not indicated by the stage or condition of the cutaneous lesion, but by the continuance of mucous and purulent discharges. There is, in the first place, some difference of opinion concerning the period when the child developing scarlet 
fever first becomes dangerous to others. On this point Forcheimer makes the following statement:

"Comparisons have been drawn between the contagiousness of measles and that of scarlatina during the precruptive period. This must of necessity be fallaeious, as it must be remembered that the enanthem of measles appears from one to four days before the exanthem, while in searlatina it rarely develops longer than 24 hours before the eruption appears. The enanthem really must be looked upon as an eruption, as it shows the primary loeal effeet has been followed by the general constitutional manifestations. It is not, therefore, astonishing that measles is contagious from one to four days before the eruption appears, but it would be quite extraordinary if this were the case in searlatina. If the contagion does exist during the preeruptive period, it must be very slightly developed. In a large experienee with searlet fever, the author has never seen a seeond ease arise from exposure to the patient during the preeruptive stage. Indeed, it is his experienee that the disease is not very contagious even during the first day of the eruption."

Scarlet fever is certainly a typical contact disease and the contact may be immediate or mediate, direct or indirect. In all probability direct contact is responsible for only a small percentage of the cases of this disease. Doctors and nurses very rarely contract it. The virus may be carried short distances through the air in droplets thrown off in coughing. If a patient coughs directly into the face of an attendant, contact is immediate. If, on the other hand, discharges from the throat, nose, ears, or suppurating glands are deposited upon handkerchiefs, dressings, bed clothes, etc., and are from these transferred to the attendant, contact is mediate or indirect. There are a few cases on record in which physicians operating upon scarlet fever patients or making postmortems of those dead from this disease have become infected through accidental wounds and have developed the disease. These are examples of direct contact. Attempts have been made to secure some exact data as to the relative frequency of direct and indirect contact in the transmission of this disease. However, the basis upon which this work has been done is open to criticism. Some have classed as instances of direct contact all those cases in which two or more children in the same family develop the disease at the same time, within a few hours, or within a day or two. In our opinion, this classification is not justified. Two children in the same family become ill of this disease on the same day. The chances are that one did not get it from the other, but that both got it from the same source. There is some doubt about multiple cases occurring in the same family at intervals. Admitted that the child first taken ill has been the source from which the others obtained the virus, it remains undecided whether the transfer from one child to the other was direct or indirect. It seems quite certain that the transfer of this disease from child to child in the great majority of instances is indirect and through the agency of some mediate bearer of 
the virus. The physician, nurse, or other attendant may carry the infection from the sick to the well on his person, on his clothing, or on thermometers, tongue depressors, or in short, on any inanimate thing or things carried.

How long will the virus of scarlet fever retain its vitality extracorporeally? When one recalls the ideas held only a few years ago concerning the transmission of yellow fever by fomites, he hesitates to repeat similar instances concerning the transmission of scarlet fever. However, we no longer believe that yellow fever is transmitted by fomites. We gave up this belief when it was demonstrated that that disease is transmitted only by a certain species of mosquito. Until we have some evidence to the contrary, we must continue to be-

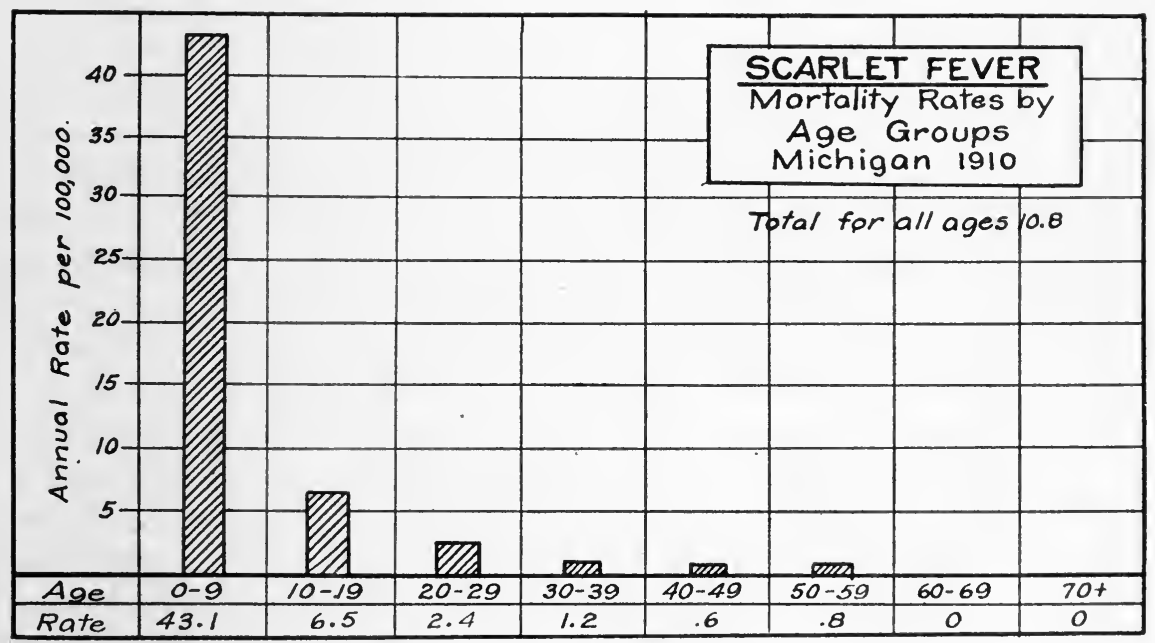

Fig. 34 .

lieve that scarlet fever is disseminated largely by fomites, and this belief must determine our efforts in the control and eradication of the disease. It is reported that a lock of hair cut from a scarlet fever patient caused the disease in a child twenty years later; that the clothes and dolls of a little girl dying from scarlet fever, after having been kept in a closed trunk in the attic, caused the disease in a child eight years later; that the quilts used by a scarlet fever patient in August, and put away without disinfection, caused this disease in November; that books read by scarlet fever patients transmitted the disease to others months later; that the virus of this disease has been carried in letters, packages of gifts, merchandise, etc., through the mail for hundreds of miles. We are ready to admit that in each and every one of these instances the disease may have been transferred in some more direct way, but at present this represents our best information. More- 
over, in our opinion, a study of the epidemiology of this disease justifies the conclusion that it is spread chiefly through fomites. Scarlet fever might not inappropriately be called a "neighborly disease." It is spread from family to family in direct proportion to the intercourse of people and the interchange of things between families.

We have found in our studies that this disease thrives mostly in small cities and villages-in places where all are neighbors and where kindly offices are extended from family to family, where visits between families are frequent, where food dainties are exchanged, and in short, where the entire community makes the nearest approach to the conditions of a large family. Under these conditions, which thrive, and, indeed, exist only in small eities and villages, we find the most distressing epidemies of scarlet fèver. In larger cities these manifestations of neighborly interest are less frequently in evidence. Families related either by blood or by inclination live miles apart. The health officers of some of our larger cities attempt to show the distribution of this disease within their jurisdiction by giving the number of cases in each ward, but this means nothing - the next ward may be just across the street or across the alley. Families living in the same apartment house may be wholly unknown to the one in which a case of scarlet fever exists. There is no exchange of the civilities and amenities of life among people occupying the same building.

Epidemiologists are naturally much concerned about the question of the distribution of scarlet fever, diphtheria, measles, and chickenpox, in schools. In Detroit during 1919 it was found that the greatest number of cases of these diseases occurs in the sixth year of life. Does this mean that there is actually more of these diseases among children just entering school, or that more cases are discovered at this time? Judging from the number of cases discovered by school nurses in the homes, it would seem as though attendance at school does not lead to an increase in disease; it simply reveals more disease. The percentage of each of these diseases by age groups is shown in Table XXV.

In scarlet fever there is a gradual increase in incidence up to the sixth year and then a slightly more abrupt falling off. Increasing motility and traveling radius with advancing age seem to have more influence than the act of entering school, for the increase at six over five or five over four is no greater than the increments below these ages. This applies to diphtheria as well. In measles the increase at five and six is more abrupt. Attendance at school undoubtedly brings more cases to light. There is no reason for believing that it is responsible for spreading measles. Whooping cough is very largely a disease of preschool life. School has no visible effect in distributing this disease. Chickenpox resembles measles in that the reported cases are more nu- 
TABLE XXV

\begin{tabular}{|c|c|c|c|c|c|}
\hline $\mathrm{AGE}$ & $\begin{array}{l}\text { SCARLET } \\
\text { FEVER }\end{array}$ & DIPHTHERIA & MEASLES & $\begin{array}{l}\text { WHOOPING } \\
\text { COUGH }\end{array}$ & $\begin{array}{l}\text { CHICKEN- } \\
\text { POX }\end{array}$ \\
\hline Under 1 year & 0.6 & 1.6 & 4.4 & 10.3 & 3.8 \\
\hline 1 year & 2.4 & 2.6 . & 10.1 & 10.6 & 4.2 \\
\hline 2 years & 4.9 & 7.1 & 12.8 & 12.7 & 7.2 \\
\hline 36 & 7.8 & 8.0 & 12.6 & 14.1 & 6.5 \\
\hline 46 & 10.5 & 7.5 & 11.7 & 13.7 & 7.2 \\
\hline 56 & 10.2 & 8.9 & 16.7 & 12.1 & 12.0 \\
\hline $6 \%$ & 11.1 & 9.5 & 16.8 & 12.3 & 14.5 \\
\hline 76 & 9.5 & 7.5 & 12.5 & 5.6 & 13.4 \\
\hline 66 & 7.5 & 5.4 & 7.6 & 3.3 & 9.5 \\
\hline $9 \quad 66$ & 5.3 & 3.5 & 3.2 & 1.6 & 5.0 \\
\hline \multicolumn{6}{|c|}{$\begin{array}{l}\text { Per cent of disease in } \\
\text { children under } 10\end{array}$} \\
\hline
\end{tabular}

merous at the age of entering school. Here, also, it would seem that school attendance is responsible for discovering rather than spreading disease. Ninety-six per cent of all whooping cough reported occurs in children before they have reached their tenth year. Corresponding figures for measles are 89 per cent, chickenpox 83 per cent, scarlet fever 70 per cent, and diphtheria 62 per cent.

Osborn, epidemiologist of the Massachusetts State Department of Health, states that during the year 1919 scarlet fever showed a notable increase in Massachusetts. This increase was not observable until August after the schools had closed. In 1919 there were in Boston in August about 100 more, cases of scarlet fever than there had been during the same month in any year since 1915. The August increase grew through September and the following months. Osborn states that this increase was not due to milk epidemics. In Massachusetts for the period 19091914,845 cases of scarlet fever were traced to milk, while for the period 1915-1918 only 140 cases were traced to this source. Attempts to control scarlet fever in Massachusetts are based upon the following principles: (1) The incubation period is from two to five days, with a maximum of eight days. (2) The virus is present in the nose, throat, ear, and suppurating gland discharges for about 28 days after the onset of the disease. Quarantine is based upon this principle but must be extended in all cases where discharges are active at the end of the period indicated. (3) Persons who have been in contact with a case of scarlet fever may become sick of this disease within eight days after the last contact, since this is believed to be the maximum period of incubation for practical purposes.

The closing of schools on account of the appearance of scarlet fever among the pupils has been found to be unwise. It is far better that 
school should be continued and that careful medical inspection should be made every day. Moreover, nurses should visit the homes of absentee children. By this inspection and through these visits new cases are found more promptly and more abundantly than is the case when notification must pass through the ordinary channels.

In Chicago out of 6,798 cases tabulated, 2,188 were under school age, while only 920 were over school age. Of course, there is a difference between the number of children of school age and those who are actually in school. No one, so far as we know, has ever had opportunity to make a comparative study of the prevalence of any of the infectious diseases of childhood among those actually in attendance at school and those of the same age and living under like conditions who are not going to school. Since the law requires that all children between certain ages shall attend school, we must assume that this law is complied with; but if we were asked to name the exact percentage of compliance with it we could not answer.

There is a wide difference in severity of the disease at different ages. Taking as an example the experience of Detroit in 1919, we find the following case mortality:

$\begin{array}{lc}\text { AGE } & \text { PER CENT CASE MORTALITY } \\ \text { Under 1 year } & 29.0 \\ \text { 1 year } & 6.0 \\ \text { 2 years } & 8.0 \\ 3 \text { “6 } & 6.0 \\ 4 \text { “6 } & 2.0 \\ \text { 5-9 years } & 1.5\end{array}$

Where 29 children out of a hundred under one year of age die after contracting scarlet fever, but one or two cases out of a hundred are fatal beyond the fifth year.

To those who believe that scarlet fever has become of trifling importance, it may be interesting to know that in our own Army during 19171918 scarlet fever, diphtheria, and mumps had higher rates than during 1861-1862. The death rate for scarlet fever was higher during the World War, as was also that from mumps. In the report of the Surgeon General for the fiscal year ending June 30, 1919, it is stated that there were reported during that year 8,117 cases of searlet fever with 258 deaths, a case mortality of 3.1 per cent. For troops stationed in the various states outside the larger camps, the highest rate was in the State of Montana, it being 63.83 per 100,000. For the entire United States Army the admission rate for white troops was 3.42 and for the colored 0.46. Comparing the nativity rate for the white from the southern rural states with the negro from the same states, a rate of 1.07 for the white is obtained and 0.21 for the colored, or practically five to 
one. The highest prevalence of scarlet fever in the army was among those who came from the north central and northwestern sections of the country. In short, the largest numbers came from those districts in which scarlet fever was, before the war and during it, most prevalent. For every 1,000 soldiers born in Wisconsin 7.35 had scarlet fever, while the ratio for those born in Florida was 0.31. Does this mean that the soldier from Wisconsin is more than twenty-four times as susceptible to the disease as the one from Florida? We think not. We think that the Wisconsin man suffered an exposure to this infection of much more than twenty-four-times that to which the boy from Florida was subjected. It is true that the highest admission for scarlet fever was at Camp Hancock in Georgia, but this epidemic which numbered 388 cases, certainly appeared and was largely prevalent among northern troops. The rate of Camp Hancock per 100,000 was 14.26, then followed Lewis in Washington with 12.69, Sherman in Ohio with 11.85, Dodge in Iowa with 10.62. Camps with exceedingly low rates were Humphreys, Va. (0.12), Beauregard, La. (0.20), and Wheeler, Ga. (0.17). Both the morbidity and mortality from this disease were low among southern troops, both white and black.

Milk-borne scarlet fever frequently occurs and in hundreds of instances epidemics have followed the lines of distribution of milk from certain dairies. At one time it was believed that there is a bovine scarlet fever and that the disease might be transmitted from cow to child. The evidence now in our possession contradicts this belief. Scarlet fever is frequently disseminated through milk, but in all cases the milk is contaminated by a human carrier of the virus. In a recent study made by the health commissioner of Chicago, it was shown that 409 out of 1,132 cases of scarlet fever occurred on the milk routes of two large dairies. One with 664 wagons had 245 cases, a rate of 36.8 cases per 100 wagons; the other with 514 had 164 cases, a rate of 31.9 cases per 100 wagons. Milk-borne scarlet fever can be wholly prevented by proper pasteurization. This, however, does not do away with the desirability of control and inspection by the health authorities of all dairies supplying milk to their communities.

The Virus.-That scarlet fever is due to a specific virus no one doubts, but the nature of that virus no one knows. This failure to know is not due to lack of industry on the part of bacteriologists and protozoölogists, because they have filled volumes with the details of their studies. They have searched the secretions from the throat, the blood, the urine, the perspiration, and they have found an embarrassment of riches in the desquamating epithelial tissue. In the last-mentioned soil most varied and most abundant crops of bacteria of every known form and size have been found. These findings have not been confined, however, 
to bacteria, and varieties of yeast, protozoa, and other organisms, described in long texts, have been brought forth from time to time as possible causes of this disease. It would be without profit to even enumerate either the workers who have delved in this field or the discoveries they have made.

In 1885 epidemiologists were greatly excited about a discovery supposed to have been made in England. In the investigation of a rather extensive epidemic of scarlet fever the infection was traced to a certain dairy at Hendon. It was found that practically all the cows in this dairy were suffering from an eruptive disease which manifested itself about the eyes, nose, and certain places on the skin, especially with vesicles on the udders and teats. Some of the animals evidently had sore throat, as was evidenced not only by inflammation, but by swelling under the jaws. Many had a dry, husky, irritative cough, and in some bronchial râles were easily detected. The milk from these animals after standing for a short time often became "ropy, slimy, or as thick as a pudding." A commission of learned men, with Klein, the bacteriologist at its head, announced that it had discovered in this dairy an epidemic of searlet fever among cows; or, in other words, they had found bovine scarlatina. It seemed for a time as though the mystery of this disease was to be solved. Dogs, eats, guinea pigs, and other animals, large and small, were inoculated with material taken from the various parts of the diseased cows. The results of these inoculations varied with the animal employed, the dose given, the avenue of administration, etc. The specific organism, so it was believed, was isolated and proved to be a streptococcus. Immediately, bacteriologists, pathologists, and epidemiologists, rushed into this field, which promised to be a veritable Eldorado for the pioneer investigator. It was soon shown that the disease among the cows was by no means limited to the Hendon dairy. The same condition was found in herds in various parts of England and there were heated discussions as to its nature, but there was one thing certain; that although the milk from other dairies with infected cows was sold, this was the only farm to which scarlet fever could be traced. This was enough to prove conclusively that the cow disease was not responsible for the scarlet fever. Investigation went further and showed that there was scarlet fever or had been scarlet fever among the laborers on the farm. This solved the question as to why the Hendon milk spread scarlet fever while that from other infected herds did not. From that time, however, there has come down to us and is still prevalent among our best bacteriologists, a belief that there is a streptococcus which in some way is concerned in the causation of scarlet fever. Some go so far as to hold that it is the primary cause of the disease; others that it is a secondary infection. Streptococei have almost innumerable varie- 
ties. The question arises: Is there any special streptococcus that has to do with scarlet fever, either as a primary or a secondary agent?

Moser and Pirquet are convinced that certain streptococei obtainable from cases of scarlet fever are quite different from those found in other diseases. Blood serum from scarlet fever patients agglutinated scarlet fever streptococei, while blood serum from other children did not. Normal horse serum agglutinates streptococei from various sources, but only in low dilutions, while blood serum from horses immunized with scarlet fever streptococei agglutinates these organisms in high dilutions. By means of specific immune serum Meyer differentiated scarlatinal streptococei from those found in other diseases. These and like experiments have been repeated in many laboratories, but the results secured have been quite as wide apart as the laboratories in which the work has been done. Three or four specific sera have been prepared and used in the treatment of scarlet fever. With these agents also, the reported results have differed widely. There has been a tendency within recent years to conclude that if the serum of a patient agglutinates in high dilutions a certain strain of streptococeus, this strain, if found in the body of the patient, may be justly considered as the cause of the disease. In our opinion, such a conclusion is not warranted by sufficient scientific data. Any bacterium that may be growing in the body during the course of any disease may be agglutinated by the serum of the patient. This does not show that such a bacterium is the primary and essential cause of the disease. Streptococci are well known secondary invaders in many diseases. As has been stated, they take part in the last acts of the dramas of tuberculosis, syphilis, and cancer, and yet they are not the primary cause in any of these diseases. If it can be shown that a certain strain of streptococeus is a constant invader in scarlet fever, such a demonstration will be of great practical value. Tunnicliff has recently reviewed the literature of this subject and states her conclusions admirably as follows:

"The serum of sheep immunized with hemolytic streptococei from the throat in the acute stage of searlet fever has been found to contain opsonins and agglutinins for the hemolytic streptococei that prevail in the throat and complicating lesions early in this disease, but not for hemolytic streptococei from other sources, such as erysipelas, mastoiditis, measles, influenza, diphtheria, and the normal throat. The results of absorption tests also indicate that the hemolytic streptocnecus from searlet fever forms a distinct group, scarlatinal streptococei removing the ofsonins and agglutinins for these cocci, while absorption with a hemolytic streptococeus from erysipelas has no such effect. These results suggest that the hemolytic streptococei of scarlet fever form a distinct group from the immunologic point of view. Possibly the serum produced with this scarlatinal streptococeus group may prove of use in the diagnosis and treatment of searlet fever, and eventually, perhaps, in determining the length of infectivity.", 
If a certain strain of streptococcus is an invariable accompaniment of scarlet fever, it is important that it be determined where and how it can be found and identified. In 1903, Hektoen, after reviewing the literature and doing considerable research work on this problem, wrote as follows:

"Streptocoeei may be found in the blood of cases of scarlet fever that run a short, mild, and uncomplicated clinical course; that streptococei occur with relatively great frequency in the more severe and protracted cases of scarlet fever, in which there may also develop local complications and, clinical signs of general infection, such as joint inflammation, but even in the grave cases of this kind spontaneous recovery may take place; and finally that streptococcemia may not be demonstrable in fatal cases of scarlet fever. The theory that scarlet fever is a streptococcus disease does not seem to receive any direct support from this work."

The same investigator in 1907 still held to the above expressed view, as is shown by the following:

"Thus it becomes evident that there are rather definite obstacles to the unrescrved acceptance of the streptococcus theory of scarlet fever. The view that the specific cause of scarlet fever is not known and that the streptococeus is a concomitant or secondary invader for the growth and activity of which the conditions in this discase are peculiarly favorable, it seems to me to harmonize better with the facts now at hand. This view receives strong support from the fact that smallpox when fatal is practically always associated with streptococcus invasion, so that the suggestion has been made that smallpox, too, is a streptococcus disease. Perkins and Pay dispose of this elaim, however, by causing smallpox in the monkey with materials entirely free from streptococei, which by themselves, as expected, have no such effect. It has been said that smallpox would be a relatively harmless disease were it not for the streptococcus invasion, and there are certainly good reasons to look on scarlet fever in the same light. From the fact that there is no evidence in scarlet fever of increased activity on the part of pneumococci and certain related cocei that normally inhabit the throat, we may infer that the conditions in the scarlatinal throat are peculiarly favorable to Streptococcus pyogenes. Indeed, in view of the paramount importance of streptococei in the course and outcome of scarlet fever the chief significance of the pure scarlatinal virus would seem to lie in its power to open the door, so to speak, to streptococci. From this point of view the need for potent antistreptococcus remedies is as urgent and their eventual specific effects as logically explainable as would be the case were scarlet fever considered a streptococcus disease pure and simple. One point'remains. In view of the fact that streptococi grow in virulence in the susceptible animal organism it becomes our duty to guard carefully by adequate isolation against the transfer of espeeially virulent strains from patient to patient. We can understand from what we know of the dissemination of throat and mouth bacteria in coughing and other ways the great chance of such transfer when patients lie side by side in the same room. Perhaps the sad instances of several deaths from scarlet fever of children of the same family, sometimes spoken of as examples of family susceptibility to scarlatina, often result from the passage from child to child of especially virulent streptococci."

The streptococcus is not the only organism which, in recent years, has been believed to be the cause of scarlet fever. Several years ago 
Class described his diplococcus scarlatinae, but this work, so far as we know, has not been confirmed. This organism was found in the throats of scarlet fever patients, where from the very nature of the cases a great variety of microorganisms might be expected to accumulate. The finding of a certain bacterium in the throat in this disease, even though it be found in all cases in a local epidemic, would not be accepted as satisfactory evidence that it is the essential cause of the disease. In 1904 Mallory described, under the name "Cyclasterella scarlatinalis," a protozoan obtained from the skin in scarlet fever. Later, this organism, or a similar one, was found by Field and Duval during life and after death. It has not been shown, however, that this protozoan or anything like it is constantly present in this disease, or that it has any etiologic relation to it. Later still, Mallory and Medlar, apparently having given up the former's ideas concerning the protozoan, put forth a new elaimant under the name "Bacillus scarlatinae." Cantacuzène has given a brief description of an organism which he found in the throat of scarlet fever patients and with which he believed that he had induced a disease in monkeys similar to scarlet fever. One of the latest contributions to this subject is by Pryer and Kelly, whose finding is probably identical with that of Cantacuzène. This list of claims for the prize of being the cause of this disease might be greatly extended, but is sufficient to show that the specific cause of the disease remains unknown.

It is quite certain that the virus, whatever it may be, does not exist in the epidermal scales of the eruption characteristic of the disease. It resides in the secretions from the throat and nose and in the purulent excretions of complicating otitis and adenitis. With fresh or dried preparations of these materials the disease may be transmitted. Moreover, there are cases, small in number it is true, which never exhibit an eruption and consequently never desquamate, and from these, as well as from ordinary cases, scarlet fever may be transmitted. There has been no proof so far that either the feces or the urine is infectious, but we can see no reason why both excretions may not, in some instances at least, bear the virus. Certainly the infected secretions from the throat may be swallowed and pass from the body in the feces, and that the blood does carry the specific virus to more or less distant glands can scareely be a matter of doubt.

At what period in the progress of the disease the virus is most readily transmitted is a question of great importance and unfortunately one about which positive statements cannot be made. We may infer that during the period of incubation and the earliest part of the invasive stage the disease is transmitted to others only under exceptional conditions. It is when the specific secretions are poured out most abun- 
dantly and scattered most widely that there is the greatest danger of transmission to others. It has been found even after six weeks detention in hospital that about two per cent of children discharged carry the infection to their homes. We have no information, however, concerning the actual condition of these children at the time of their discharge. They may have had running ears or suppurating glands, and certainly as long as these conditions continue they must be regarded as disease carriers. Again, we do not know in how many of these instances the virus accompanies the child to its home in fomites. Much more exact information is needed along these lines before definite statements can be made.

We have already referred to the tenacity of life apparently possessed by the searlatinal virus. We are inclined to the opinion that the length of its extracorporeal existence is determined largely by its exposure to heat and light. As we have seen, scarlet fever is less prevalent in the southern than in the northern states and that secondary cases rarely occur in the tropies. The most probable explanation for these well attested facts is that the light and heat of the South are inimical to the eontinuance of the life of the virus outside the human body. It is also possible that the resident of warm climates by reason of his environment, diet and habits, becomes a less favorable culture medium.

Susceptibility.-Either the negro is less susceptible than the white to scarlet fever or the existence of the disease in the former is not so easily recognized and the deaths not so thoroughly reported. This statement is borne out by figures in Table XXVI taken from the U. S. Census of 1917 for the deaths per 100,000 among whites and blacks in certain southern states :

TABLE XXVI

\begin{tabular}{llllllll}
\hline \hline STATE & 1911 & 1912 & 1913 & 1914 & 1915 & 1916 & 1917 \\
\hline $\begin{array}{c}\text { Kentucky } \\
\quad \text { (total) }\end{array}$ & 3.7 & 2.8 & 2.4 & 2.6 & 2.3 & 2.4 & 2.1 \\
white & 4.1 & 3.1 & 2.7 & 2.8 & 2.5 & 2.5 & 2.3 \\
$\quad \begin{array}{c}\text { colored } \\
\text { North Carolina }\end{array}$ & 0.8 & 1.1 & 0.0 & 1.5 & 1.2 & 0.8 & 0.4 \\
$\quad$ (total) & 2.1 & 2.6 & 2.8 & 0.6 & 1.6 & 2.6 & 1.7 \\
white & 2.9 & 3.2 & 3.5 & 0.6 & 2.5 & 3.5 & 2.2 \\
colored & 0.8 & 1.5 & 1.4 & 0.6 & 0.0 & 0.5 & 0.5 \\
\hline
\end{tabular}

There is the same difference in the death rates from this disease among the eolored and white populations of the registration eities of the South as is shown in the states.

In a general way it may be stated that, while searlet fever may show itself at any time of the year, it is more prevalent in cold than in warm weather. We are inclined to attribute this not only to the fact that 
ehildren are kept more closely housed in cold weather, but to the greater destructive action of light and heat on the virus during its extracorporeal existence. The figures in Table XXVII, taken from the report of the health officer of Chicago, give the cases and deaths by months in that city:

TABLE XXVII

\begin{tabular}{lrrrrrrrrrrrr}
\hline \hline YEAR & JAN. & FEB. & MAR. & APR. & MAY & JUNE & JULY & AUG. & SEPT. & OCT. & NOV. & DEC. \\
\hline 1917 & & & & & & & & & & & & \\
Cases & 1734 & 1893 & 2710 & 1893 & 1660 & 1116 & 512 & 228 & 324 & 482 & 470 & 421 \\
Deaths & 68 & 94 & 109 & 119 & 84 & 52 & 31 & 17 & 14 & 13 & 16 & 7 \\
1918 & & & & & & & & & & & & \\
Cases & 323 & 224 & 213 & 188 & 163 & 100 & 92 & 59 & 104 & 104 & 106 & 133 \\
Deaths & 15 & 3 & 3 & 4 & 3 & 3 & 3 & 3 & 0 & 1 & 4 & 4 \\
\hline
\end{tabular}

It should be stated that in the northern United States scarlet fever may appear in severe epidemic form at any season. We have an impression from the literature that epidemies of this disease when occurring in the summer are likely to be more extensive and more virulent if the weather be cold and wet.

We have already shown that most cases of this disease occur between the fifth and tenth years of life, the sixth generally showing the maximum. Donnally, figuring on millions of cases, found that about 50 per cent occur between three and eight years and 90 per cent before the sixteenth year. Susceptibility to scarlet fever is not so universal as that to measles and the spreading of the former is more easily controlled. According to Hoff, when both scarlet fever and measles were introduced into the nonimmune populations of the Faroe Islands 99 per cent of these people developed measles, while only 38.3 per cent contracted scarlet fever. That age, apart from previous attack of the disease, has some protective influence is indicated by the observations made in the Faroe Islands. Among these highly susceptible people 56.3 per cent of the cases of scarlet fever were under twenty years of age, while only 7.6 per cent were over forty. However, too much weight must not be given these figures, because we have no information concerning the age distribution among these people. Second attacks of scarlet fever are rare, less than those of either measles or smallpox, but third attacks have been reported. Forcheimer says :

"Any one with large experience in scarlet fever lasting over a sufficient length of time will have seen at least one case of third attack.',

Animal Inoculation.-Ordinary laboratory animals have proved refractory to all attempts to induce in them any semblance of searlet fever. Experiments on the lower monkeys have been doubtful and for the most part negative. In 1904 Grünbaum reported highly questionable symptoms of this disease in a chimpanzee about which he had placed 
the nightshirt of a scarlet fever patient. However, in addition to the garment the animal's throat had been swabbed with the excretions obtained from the pharynx of a case of scarlet fever. In 1911 Hektoen and Weaver fed Macacus rhesus monkeys on milk to which had been added material taken from the throats of scarlet fever patients and failed to secure any results. Later, Cantacuzène announced that he had succeeded in inducing the disease in lower monkeys by inoculating them with blood, pericardial effusions, and emulsions of lymph nodes from cases of scarlet fever. Later still, Bernhardt reported successful inoculations of Macacus rhesus monkeys by material taken from the throats of scarlet fever patients and transferred to the throats of the monkeys. He also claimed that he had obtained similar results by the filtrates obtained after passing emulsions through a Berkefeld filter. The most promising results were reported in 1911 by Landsteiner, Levaditi, and Prasek. These investigators used three chimpanzees. The material employed for inoculating these animals consisted of throat discharges and blood from scarlet fever patients. In all of these animals symptoms and lesions quite characteristic of scarlet fever were induced. Two of the chimpanzees died, while the third recovered after the development of characteristic angina and the formation of a false membrane in the throat. The streptococcus isolated from the throat cultures taken from the scarlet fever patients did not induce symptoms or lesions of searlet fever in animals.

Incubation and Invasion.-The period of incubation in scarlet fever is usually from two to four days. In case the infection is acquired through a wound, as has happened, after tracheotomy or other surgical procedure, the period of incubation is very short, from twenty-four to fortyeight hours. In case of usual exposure the period may be extended to eight days and, as a rule, this is considered the limit. Contacts are in most communities held in isolation for at least eight days. If at the expiration of this time there is no evidence of infection contacts may be dismissed, although, of course, their clothing should be disinfected at the time the exposure is known to occur. Not only should clothing be disinfected, but a thorough cleansing bath should be administered. Generally the onset of the disease is sudden and accompanied by a distinct chill or chilliness, accompanied by pallor. In the great majority of instances vomiting, sometimes repeated, is an early symptom and may be accompanied by diarrhea. Following the chilly sensations the face becomes flushed and the temperature rises abruptly, often going as high as $104^{\circ}$, and sometimes higher. The pulse is frequent and the child is evidently greatly prostrated and seriously ill. These conditions are usually accompanied by sore throat, although this may not be mentioned by the patient. In all instances where the above-mentioned symptoms 
appear the throat should be carefully examined whether complaint is made of soreness in this locality or not. At the same time careful examination should be made of the cervical glands, which begin to enlarge very early in the development of the disease. On inspection of the throat and mouth the enanthem, or mucous eruption, may be detected. The tonsils are swollen, abnormally red, and bear dark red spots interspersed with whitish ones, the latter consisting of secretions from the crypts. The redness and the macules rapidly spread from the pharynx to the mucous membranes of the gums and the cheeks. As a rule, the tongue is coated and the papules project abnormally and show a deepened red coloration. This condition is known as the "strawberry" tongue. The period which elapses between the first appearance of the enanthem and the exanthem, or cutaneous eruption, is usually less than 24 hours.

The rash of scarlet fever appears first on the anterior aspects of the neck and chest, from which it spreads rapidly over the trunk and the extremities. As a rule, the face is not involved. The forehead and the lips are unusually pale, this effect being enhanced by the scarlatinous appearance of the neck and chest. The eheeks may be flushed with the fever, but are seldom involved in the eruption. When examined superficially it appears that the skin is uniformly colored a deep red, but upon closer examination it will be seen that this appearance is due to innumerable pinhead red points with a base of normal color. Within 24 hours, sometimes earlier, the greater part of the body is involved in the rash. However, it continues to develop for two to three days, during which time the skin is hot, dry, and sometimes swollen, especially over the backs of the hands and the feet. After having reached its maximum intensity the rash continues at this stage from one to three days and then rather rapidly fades away. Peeling or desquamation follows slowly and may be in evidence fór weeks. The frequent involvement of the lymphatic glands, the occasional development of arthritis, and the kidney. complications indicate that the virus is distributed through the body in the blood.

Control.-The successful control of scarlet fever depends upon its early recognition, the complete isolation of the patient, and the thorough disinfection or destruction of all fomites. The law requires the physician or householder to report this disease as soon as it is recognized. We are sorry to say that many physicians fail to recognize the disease, and consequently fail to put into action preventive measures until the latter are of but little avail. In Michigan some years ago a physician was arrested and tried for not reporting a case of scarlet fever under his care. His plea was that he did not recognize the case to be scarlet fever until the child was dying and consequently thought it unnecessary to report the 
case at that late date. A public funeral was held and other eases developed. The Court held, quite wisely, that it was the physician's duty to report the case as soon as he recognized its nature, even though he did not do so until after the child was dead.

Undoubtedly a physician may attend cases of scarlet fever and then visit other patients without changing his clothing and still not disseminate the disease, but, in our opinion, this is due to good luck and is not a consequence of the proper observance of epidemiologic teaching. If the physician in attending his scarlet fever patients does not come in direct contact with the patient or with articles soiled by the discharges, there is no reason that he should bear, on his person or on his clothing, the virus. The same may be said of the nurse. Most physicians, however, prefer to get close enough to their patients to make a careful study of them, and in the majority of instances this means to come in contact either directly with the patient or possibly with infected articles. The same to an even greater extent is true of the nurse. We hold, therefore, that the physician on visiting his scarlet fever patients should wear a gown and that when he leaves the sick room he should thoroughly disinfect his hands. The wearing of a gown while attending patients with this disease may be ridiculed as a formality, pronounced a rite, or otherwise disparaged, but we believe that in the present state of our knowledge concerning the transmission of this disease the conscientious physician will take every possible precaution.

The health officer is often requested to close the schools when scarlet fever appears among the children. We believe this to be unwise. If the children are allowed to continue their attendance at school and a skilful diagnostician inspects them once or twice a day, we believe that cases would be more quickly recognized and preventive measures put into operation more promptly than if dependence is placed wholly upon the family physician. As we have already shown, scarlet fever is most prevalent during school age and during those months when school is in session. It is, therefore, a disease with which the medical inspector of schools should be familiar. Whether the school child found to have this disease should be isolated in its home or removed to an infectious disease hospital is a question that should be answered solely by the judgment of the health official as to which place affords the better opportunity for the care of the individual child and for the protection of others.

How completely shall the sick child be isolated? If the child is cared for in its home it must occupy a room into which no one but the nurse and physician can come. These attendants should use every known precaution to avoid carrying the virus when they leave the room. Nothing should be taken from the room unless it be submerged in some disinfect- 
ing solution. Parts of the house occupied or visited by the child at any time during the days immediately preceding the onset of the disease should be closed to all susceptible members of the family until thoroughly disinfected. Susceptible children in the same household should be stripped, bathed, and their clothing disinfected. Children in the same household who have previously had scarlet fever should be bathed, dressed in sterilized clothing, their school books and other things which they carry with them to school disinfected, and permitted to attend school. Provision for home treatment in all its particulars should be approved by a representative of the health department.

It is expected that infectious disease hospitals be provided with all facilities needed either for the welfare of the individual patient or for the protection of others. Inasmuch as the child with scarlet fever is especially susceptible to secondary infection, it is essential that there should be no contact either direct or indirect between the individual patients in the same hospital. It may be a matter of life or death to have virulent strains of streptococci distributed among the scarlet fever patients in a hospital. If a given hospital or ward contains a dozen scarlet fever children.in only one of whom a highly virulent streptococcus finds lodgment the death rate among the 12 may be greatly increased if an ignorant or careless attendant succeeds in transferring this streptococcus to the others. It may seem strange to argue that one child with scarlet fever should be protected from infection by another with the same disease, but, in our opinion, it is failure to understand and appreciate this point that has led to the high death rates from scarlet fever, measles, pneumonia, and possibly certain other diseases, in hospitals; indeed, attendants in a scarlet fever hospital or ward should take the same care in going from patient to patient as would be done if all suffered from different diseases.

In most municipal regulations children of a quarantined household not protected by a prior attack may be admitted to school if a change of residence is made and seven days have elapsed since date of last exposure, while children in the same household who have had scarlet fever may be permitted to attend school, provided a change of domicile is made and the physician certifies in writing his personal knowledge of the prior attack. These provisions apparently do not take into consideration the possibility of transmission of the disease through fomites and, in our opinion, are defective in this respect.

How long should the scarlet fever patient be held in quarantine? In our opinion, no definite time can be set. In state and municipal regulations, so far as we have examined them, the period of quarantine in this disease varies from three to six weeks. In diphtheria and typhoid fever we continue isolation until cultures from the throat in the former and specific bacilli 
in the urine and feees in the latter are no longer found. We have no such test for scarlet fever. It has happened that when scarlet fever patients have been held in quarantine for six and even for ten weeks they have in a small percentage of cases carried the disease to their homes. Attempts have been made to determine the proper time for quarantine in this disease by ascertaining the number of so-called returned cases. It is easy to understand, however, that in one instance a patient may. be kept in quarantine for ten weeks and still carry the disease to his home, while another kept in quarantine for only three weeks fails to do so. It is quite certain that scarlet fever patients should be kept in quarantine until there is permanent cessation of all discharges due to the disease. In order to be safe, it might be well to provide that no patient shall be discharged from quarantine for this disease until at least the seventh day after all discharges due to the disease have ceased. On being discharged every patient should have a thorough cleansing bath, should be attired in sterilized clothing, and should not carry from the sick room or from the hospital anything that might possibly be infected.

There is a reason, aside from preventing the spread of the disease, for prolonged supervision of the individual convalescing from scarlet fever. We refer to the importance of making every effort to avert the development of nephritis, a too frequent sequel to this disease and one more likely to occur when the patient is prematurely returned to active duties.

\section{Bibliography}

Bard, SaAuel: Trans. Am. Phil. Soc., 1789, i, 388.

Belknap, Jeremy: The History of New Hampshire, ii, 118, Boston, 1791.

BernhardT, M. G.: Deutsch. med. Wchnschr., 1911.

Cantacuzène, M. J.: Compt. rend. de la Soc. de Biologie, 1911.

Castellani, A., and Chalmers, A. J.: Manual of Tropical Medicine, New York, 1919, William Wood \& Co.

Creighton, C.: A History of Epidemies in Britain, 1894, Cambridge University Press. Dunn, C. H.: Pediatries, Troy, N. Y., 1917, The Southworth Co.

Forchemmer, F.: 'Twentieth Century Practice of Medicine, xiv.

Griffith, J. P. C.: The Diseases of Infants and Children, Philadelphia, 1919, W. B. Saunders Co.

Hertoen, L.: Jour. Am. Med. Assu., xl, 685 ; ibid., xlviii, 1158.

Landsteiner, K., Levaditi, C., and Prasek, E.: Am. de l'Inst. Pasteur, 1911, xxv, 754.

Osborn, Stanley H.: Boston Med. and Surg. Jour., 1919, elxxxi, 663.

Robertson, J. D.: Report and Handbook of the Department of Health of the City of Chicago, 1911-1918.

Rogers, Leonard: Fevers in the Tropies, London, 1908, Oxford University Press.

Schamberg, J. F.: Diseases of the Skin and the Eruptive Fevers, Philadelphia, 1917, W. B. Saunders Co.

Sydenham, Thomas: Latham's Translation.

TunNiCliff, Ruth: Jour. Am. Med. Assn., lxxiv, 1386.

Webster, NOAн: A Brief History of Epidemic and Pestilential Diseases, Hartford, 1799.

Welch, W. M., ANd Schamberg, J. F:: Acute Contagious Diseases, Philadelphia, 1905, Lea Brothers \& Co. 


\section{CHAPTER XII}

\section{MUMPS}

\section{EPIDEMIC PAROTITIS}

Definition.-Mumps is a specific, infectious disease, involving the salivary glands, generally the parotid, and oceasionally extending to the testes in the male and the ovaries in the female. Most frequently the two parotids are involved successively, rarely simultaneously. In rare instances the submaxillary or sublingual glands may be involved and the parotids escape. Still more rarely the first evidences of this disease may be in the testes. With the best information we have at the present time we may say that it is due to a filtrable virus. In the great majority of instances one attack gives immunity throughout life.

History.-There is no doubt from the following deseription that Hippocrates was familiar with this disease.

"Swellings appeared near the ears, in many on one side, in most on both, without a fever or any confinement, but in some with a little fever. In all they disappeared without either inconvenience or suppuration, contrary to the custom of such tumors from other eauses. At this particular time they were naturally soft, large, diffused, without inflammation or pain, and went off universally without any visible signs. Children, young persons, adults, especially those who frequented the public places of exercise, were most subject to them. A few women were also affected. The greatest part had dry eoughs, which were soon succeeded by hoarseness. Some again after a while had painful phlegmons upon the testicles, sometimes upon one, sometimes upon both. Some had fevers, others none; most of them trouble and fatigue enough; but with respect to the chirurgical part they did very well.',

It is strange that for many centuries after Hippocrates there appears to be no recognition of this disease, certainly as an epidemic, in any of the medical writings. The great French surgeon, Paré, did not distinguish epidemic from secondary parotitis. This confusion continued until rather late in the eighteenth century, and, indeed, persisted with some authorities up to the early part of the nineteenth century. Thus, Capuron, at the last-mentioned date, wrote as follows:

"We give the name of mumps to the swelling of the parotid glands. Tumors of this sort are almost always produced by the process of dentition, or by certain drying up of ulcerated or suppurating ears; they depend also sometimes upon a scrofulous vice; they are accompanied or not by fever, according as there is a simple swelling or an inflammation of the glands."

One of the first to recognize mumps as a specific disease was Hamilton, of Scotland (1761), who reported an epidemic and noted the occurrence 
of orchitis and atrophy of the testicles. During the latter half of the eighteenth century extensive epidemics occurred in Italy and were reported by Caspari, Tozzetti, and Laghi. The writings of these Italians did much to acquaint the medical world with the epidemic and contagious nature of this disease. In 1752 Pratolongo, of Genoa, in a letter to a fellow physician, made the following statement:

"The only disease which we can regard as epidemic in Genoa at present is that which we call mumps. In addition to the swelling of the parotids we have seen in some patients an enlargement of the testicles together with a violent fever. In others this swelling of the parotids was followed by an anasarca such as sometimes supervenes upon an attack of scarlatina, accompanied by great difficulty in breathing and a sharp fever. Do you think that we could elass this disease among the eruptive fevers?"

The question with which the above quotation closes is still an open one.

There is apparently no part of the world which is continuously free from this disease. It appears from time to time in British and French

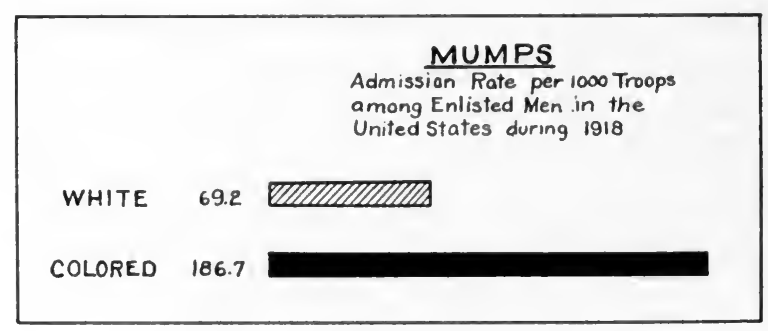

Fig. 35 .

troops stationed in widely separated parts of the world. Laveran has shown that it is quite as common among French garrisons in Algeria as it is in those in the home country. It is common in India, the West Indies, Africa, and South America. It appears from time to time on emigrant and troop ships sailing in all the seven seas.

In armies mumps is an important disease, not on account of its great mortality, but from the number of men attacked and the time lost. Among our own soldiers during the late war there were 213,943 cases of mumps. During the year 1918 alone there were 166,370 and the loss of time caused by this disease aggregated 2,894,074 days. In a general way we may say, taking our troops in this country and in Europe during 1918, that 7,766 men were disabled by this disease for each day of the year. Among the troops in this country the disease interfered rather seriously with drill and other preparation, because when men are exercised while suffering from the mumps orchitis is more likely to occur. Laveran, studying this complication among French soldiers, says that 
it develops in one case out of three. This is a higher percentage than that given by most medical officers. Burns, epidemiologist at Camp Lewis during 1917-1918, also found that 33 per cent of the cases of mumps admitted to his base hospital were complicated by orchitis. This is a higher rate than was observed at any other camp, in most of which this complication was reported as rare. The total number of deaths in all American troops for the year 1918 attributed to this disease was 151, and in these the actual cause of death, in most instances at least, was pneumonia coming on as a sequel to the mumps. Apparently, the negro is more susceptible to this disease than the white man, the rates for white and colored troops in the United States for the year 1918 being respectively 69.16 and 186.72. In Europe the admission rate for white troops was 41.71 and for the colored 210.46. Considering the nativity rates for southern states, that for the white troops was 88.85; that for the colored 170.36. Whether these differences are due to race, or are explainable by the fact that the negroes were more largely rural, is a question. We are inclined to the opinion that the apparent difference in rate is best explained by the fact that a larger proportion of the negroes came from rural homes, and consequently more susceptible material was found in their ranks. Mumps was much more prevalent among enlisted men than among officers. Among the former this disease stood second for admission to hospital, while among the officers it ranked seventh, and no officer died as a result of this infection. We are inclined to explain these facts by the greater average age of officers and the smaller number of officers coming from rural homes; in other words, we doubt not that a large percentage of the officers had secured immunity by a previous attack of the disease. This disease is a further illustration of the fact to which we have frequently called attention, that so far as communicable diseases are concerned the best preparation for army service is previous residence in an urban or densely populated community. Another interesting fact brought out by the study of mumps among our soldiers in the late war is that it was more prevalent in the larger and more crowded camps than in the smaller and less densely populated posts; in other words, like measles, scarlet fever, influenza, and meningitis, mumps prevails in proportion to the density of the susceptible population into which it is introduced. The virus apparently gains strength by the amount and closely packed condition of the fuel upon which it has opportunity to feed.

The Virus.-The first baeteriologic investigations of mumps were made by Capitan and Charrin in 1881. These investigators found in the blood, more rarely in the saliva, micrococci of variable forms and sizes, single, double, or arranged in chains. These findings were apparently confirmed by Bouchard, Ollivier, and others. In 1892 Laveran and Cat- 
rin found in 67 out of 95 cases of mumps micrococci resembling those already described. The testicular fluid obtained by puncture with a hypodermic needle gave positive results in 12 out of 16 cases. No inoculation experiments were made with these bacteria. In 1896 Mecray and Walsh found a diplococeus in the discharges from Steno's duct. Again, no inoculation experiments were made and the sole evidence that any bacterium has a causal relation to this disease rests upon the finding of the organism and its agglutination by the blood of individuals suffering from this disease. In 1908 Granata, for the first time, used filtered saliva in animal inoculation. Since that time work along this line has been practically confined to inoculation experiments. Gordon, and later Nicolle and Conseil, injected material obtained from parotid glands into animals. Granata worked with rabbits and Gordon, Nicolle and Conseil with monkeys. The results obtained by these experiments were, to say the best, doubtful. In 1918 Wollstein tried inoculation experiments on cats. These animals were selected because they are known to be subject to attacks of parotitis. Old animals were found to be unsuitable and the investigations were carried out upon the young. Children with mumps were asked to rinse their mouths with sterile physiologic sodium chlorid solution and the washings were filtered through a new Berkefeld candle $\mathrm{N}$. The injections of these filtrates were made directly into the parotid and the testes. Wollstein reported her experiments as follows:

\footnotetext{
"The animals recovered rapidly from the ether and showed no ill effects from the inoculations. The following day the temperature had usually risen $0.5^{\circ} \mathrm{C}$, but the white blood cells had not increased in number. There was, as a rule, slight tenderness in the inoculated testicle, and often in the parotid as well. This was evidently of mechanical and not of inflammatory origin and always disappearing within another 24 hours, learing the eats apparently well on the second day, although the temperature was from $0.5^{\circ}$ to $0.5^{\circ}$ C. above normal. After six or seven days tenderness returned in the testis, accompanied by swelling, and similar symptoms appeared in the parotid. An increase in the leucocytes became apparent two days after inoculation and reached the maximum in about seven to fourteen days, coinciding with the height of the fever. The swelling and pain in the parotid lasted from two to five days, but the testicular swelling rarely subsided in less than ten to fourteen days. In the third week all the symptoms began to disappear, the leucocytes reaching the normal first, the tenderness disappearing at the same time, and the fever persisting for another week. While tenderness on palpation of the parotids was less marked than that of the testes, and the swelling never reached the stage of marked facial asymmetry, the eats manifested some degree of discomfort in the inoculated parotid. The appetite was only slightly affected and at no period of the experiment did the cats seem especially ill. The disease was not fatal in any instance. Animals killed late in the second or early in the third week showed swelling of the inoculated parotid as well as of the other salivary glands and neighboring lymph nodes. The salivary filtrates from patients ill from one to three-days produced these marked symptoms in the inoculated eats. On the sixth day of the disease the effect of the filtrate injection was much less marked, and inoculation
} 
of material obtained from a patient nine days or longer after onset of the mumps attack was apparently without results; that is, the cats showed a temperature range of only $0.5^{\circ} \mathrm{C}$, , the leucocytes were not even doubled in number, and the mononuclear cells were not increased, but the polymorphonuclears were. At necropsy the parotid and the adjacent salivary glands and lymph nodes showed weither gross nor microscopic changes. It would seem, therefore, that the infective period of the mouth secretions from parotitis is comparatively short, lasting about a week and corresponding to the period of swelling of the parotid gland. A fresh swelling appearing in the opposite parotid gland would naturally prolong the infectious period for a given patient.",

Transmission of the disease from cat to eat was accomplished by means of the saliva of the inoculated eat and with emulsions made from the inoculated glands. The best time for the reinfection was found to be from the fourteenth to the seventeenth day when the testicular swelling, the leucocytosis, and the fever were highest. During the first seven to ten days transfers were less uniformly successful. Atrophy of the inoculated testicle was seen in some cases.

It seems from the results of these investigations that we are justified in concluding that mumps is due to a filtrable virus which is contained in the saliva of the infected individual. This must be borne in mind in all attempts to control the disease.

Susceptibility.-There is no period of life which is free from infection with this disease. It is most prevalent, however, between the fifth and fifteenth years. Orchitis is not common before the fifteenth year and for this reason it is preferable that the disease be acquired before that time, inasmuch as this is the most serious usual complication. It is frequently stated that mumps is more common among males than among females. This is due to the fact, however, that most of the statistics that have been collected concerning this disease have been gathered in boys' schools and in army camps. In civil life and under ordinary conditions there is no evidence that sex has any influence on susceptibility to this disease. The orchitis, however, is much more serious than the ovaritis. The latter is seldom or never a serious complication and the only evidence of its existence is a pain in the region of the ovary. In rare instances the ovary in adolescent or adult females is sufficiently enlarged to be detected. No serious result has ever been known to follow ovaritis due to this disease. As has been stated, as a rule one attack of mumps gives immunity to this disease throughout life. However, there are exceptions and we know a physician who during 20 years of practice had an attack of mumps many times, as he claimed every time he came in contact with the disease. This is probably an exaggeration, but undoubtedly he had many attacks. In the majority of instances the attack is unilateral and the other gland is involved only as the first one begins to subside. This, unfortunately, prolongs the period when the individual is liable to transmit the disease. In 
rere instances the infection is confined to the submaxillary gland, and in still rarer instances to the sublingual gland. In many cases all the salivary glands are involved, though the affection of the parotid is most prominent and receives the greatest attention. In very rare instances the testis is involved before the salivary glands. Cases of this kind are not likely to be recognized, or are prone to be otherwise diagnosed, except in case of a prevailing epidemic. Recurrent cases are occasionally observed in which attacks at intervals of a few weeks may develop in the gland or glands previously involved.

Control.-Some very good authorities, Laveran for instance, insist that no attempt to limit this disease should be made when children between five and fifteen years of age are involved. After the last-mentioned period of life has been reached orchitis becomes an important factor in males. As we have already said, the fact that as a rule one parotid is involved after another, the period of isolation is greatly prolonged and becomes a most serious matter in armies. It is evident that this disease is highly contagious in its early stages and in armies its early recognition becomes a matter of grave importance. We suggest that when this disease appears in troops the men who have not had the disease be lined up every morning and the condition of the parotid gland determined by palpation. Those found in the early stages of the disease should be relieved from duty, not with the intent of preventing or modifying the development of the disease in the parotid gland, but to prevent the development of orchitis. In civil life it is easy enough to isolate a case of mumps for from three to four weeks as is usually recommended, but in armies where there are thousands of cases this may become a matter of the most serious import. Some French authorities, notably Comby, recognizing the difficulty of stamping out this disease by isolation, recommend that infected troops be temporarily removed from their quarters and time given for thorough fumigation and disinfection. So far as we know, this has never been tried, but we are ready to predict that when tried it will not be found successful. While mumps may be, and undoubtedly is, distributed more or less through fomites, the infected men carry the virus with them wherever they go and change in location of camp will, in our opinion, be without benefit. There is one point concerning the control of this disease in armies to which, so far as we know, attention has not been sufficiently called. We refer to the almost surety of transmission through mess kits and table utensils. Undoubtedly the saliva bears the virus. It is a disease which is not characterized by frequent coughing, and the saliva is most likely to be transferred from one individual to others through infected mess kits and table utensils. Thorough sterilization of all these articles in camps where this disease has appeared we regard as essential. It has been pointed out several times by army surgeons that continued 
duty is quite certain to favor development of orchitis. Several years ago a French army surgeon and a special student of mumps, Catrin, wrote as follows :

"A certain number of observers have remarked that fatigue seems to favor the occurrence of orchitis. Chauvin has seen simple congestion become transformed into actual inflammation after a long mareh; Servier recommends that these patients be sent at once to the hospital, and Laveran says that those who are obliged to follow laborious pursuits and who continue their labors after the onset of mumps are more subject than others to orehitis, and swelling of the testicles in such cases assumes greater proportions."

This is all right in an army in time of peace, but when confronting an enemy the situation is altogether different and whatever may be the danger to the individual continuance on duty becomes an absolute necessity. In civil life the control of mumps may be a matter of no great importance, but in armies during periods of active operations it may endanger military success. We have elsewhere suggested, not only with reference to mumps, but also with measles, that when large armies are to be quickly mobilized from raw recruits medical officers should ascertain from these recruits those who have had and those who have not had the disease and should distribute the men in organizations in such a way that no organization contains an undue number of those susceptible to this disease, but that every organization should be made up so far as possible of a mixture of immunes and nonimmunes. If this were done we would not have, so frequently at least, the unfortunate condition of army hospitals being overwhelmed and demoralized by massive outbreaks of these infectious diseases. Every recruit or draft man should bear on his person a card showing what infectious diseases he has had and those he has not had. An overwhelming number of susceptibles in any organization should be avoided so far as is possible. In a way this might be accomplished by a more thorough mingling of urban and rural recruits. To gather into one camp men wholly or largely from rural districts is to insure hospital demoralization, to interfere with drill, and to delay the conversion of raw into seasoned troops.

\section{Bibliography}

Сомву, J.: Twentieth Century Practice of Medicine, xiii, 555.

Grifrith, J. P. C.: The Diseases of Infants and Children, Philadelphia, 1919, W. B. Saunders Co.

Jenkins, WM. A.: Tice's Praetice of Medieine, iii, 311.

Vaughan, V. C., and Palmer, G. T.: Jour. Lab. and Clin. Med., July and August, 1919 , iv.

Wollstein, Martha: Jour. Am. Med. Assn., 1918, lxxi, 639. 


\section{CHAPTER XIII}

\section{WHOOPING COUGH}

\section{PERTUSSIS}

Definition.-Pertussis is an acute, specific disease, recognized by a pathognomonic cough. The specific cause is believed to be the Bordet bacillus, which grows in masses between the cilia of the bronchi. All ages are susceptible, but the highest death rate by far is among children under five years. As a rule, one attack gives life long immunity.

History.-There is no evidence that medical writers of the Greek and Arabic schools recognized this disease. The first paper, so far as we know, on whooping cough was written by Baillou in 1578, in which there is given a very accurate account of the symptoms of this disease. Baillou speaks of it as a well-known disease at that time, but he says, correctly so far as we can ascertain, that no one had previously written concerning it. During the seventeenth century, under various names, such as chincough, kink-cough, and kink-kost, it was known in England and Scotland. It is rather strange that a disease with such a peculiar and striking symptom should not have received earlier recognition. In our opinion, this cannot be accounted for on the supposition that the disease did not exist. It is more probable that the peculiar cough was regarded as a mere freak and not as significant of a distinct and serious disease. On this point, Creighton makes the following statement:

"In Phaer's Booke of Children (1553) chineougl is not named. It is perhaps more singular that the disease should be omitted from the list in Sir Thomas Elyot's Castel of Health (1541), of maladies proper to three periods of ehildhood; for that list has every appearance of being an exhaustive enumeration. Still, it would be erroneous to suppose that the convulsive cough of children which is so common an epidemic incident in our time, and in some impressionable subjects is the almost necessary sequela of a coryza or eatarrh, did not then occur in the same circumstances as now. When Willis, in his Pharmaceutic Rationalis in 1674, remarks that pertussis was left to the management of old women and empirics, he suggests the real reason why so little is said of it in the medical compends. Sydenham mentions it twice, and on both occasions in a significant context. Under the name of pertussis. 'quem nostrates vocant Hooping Cough,' he brings it in at the end of his account of the measles epidemic of 1670 , without actually saying that it was a sequela of the measles. His other reference to it, under the name of the convulsive cough of children, comes in his account of the influenza of 1679. In both contexts it is adduced as an instance of a malady much more amenable to bloodletting than to pectoral remedies, the depletion being a sure means of cutting short an attack that was else very apt to be protracted, if not altogether uncontrollable." 
In 1701 whooping cough was placed in the mortality lists of the parish clerks in London, in which year six deaths were attributed to it. With marked fluctuations this disease has continued on the list up to the present time, and during recent years the death rate from it has shown but little variation, and certainly no prolonged or marked decrease.

In our own country whooping cough runs from year to year a nearly level death rate, as the figures in Tables XXVIII, XXIX, and XXX taken from the mortality statistics of 1917 , show.

TABLE XXVIII

Death Rate from Whooping Cough per 100,000 Population

\begin{tabular}{lrrrrrrr}
\hline \hline & 1911 & 1912 & 1913 & 1914 & 1915 & 1916 & 1917 \\
\hline Registration area & 11.3 & 9.3 & 10.0 & 10.3 & 8.1 & 10.2 & 10.4 \\
Registration states & 11.2 & 9.3 & 10.3 & 10.3 & 8.3 & 10.6 & 10.5 \\
Cities in registration states & 10.7 & 8.1 & 9.3 & 9.2 & 7.9 & 9.7 & 9.4 \\
Rural part of registration states & 11.8 & 10.4 & 11.3 & 11.4 & 8.6 & 11.4 & 11.6 \\
Registration cities in nonregistration states & 11.9 & 9.7 & 6.4 & 10.8 & 5.7 & 4.4 & 9.0 \\
All registration eities & 10.9 & 8.4 & 8.9 & 9.4 & 7.6 & 9.0 & 9.3 \\
\hline
\end{tabular}

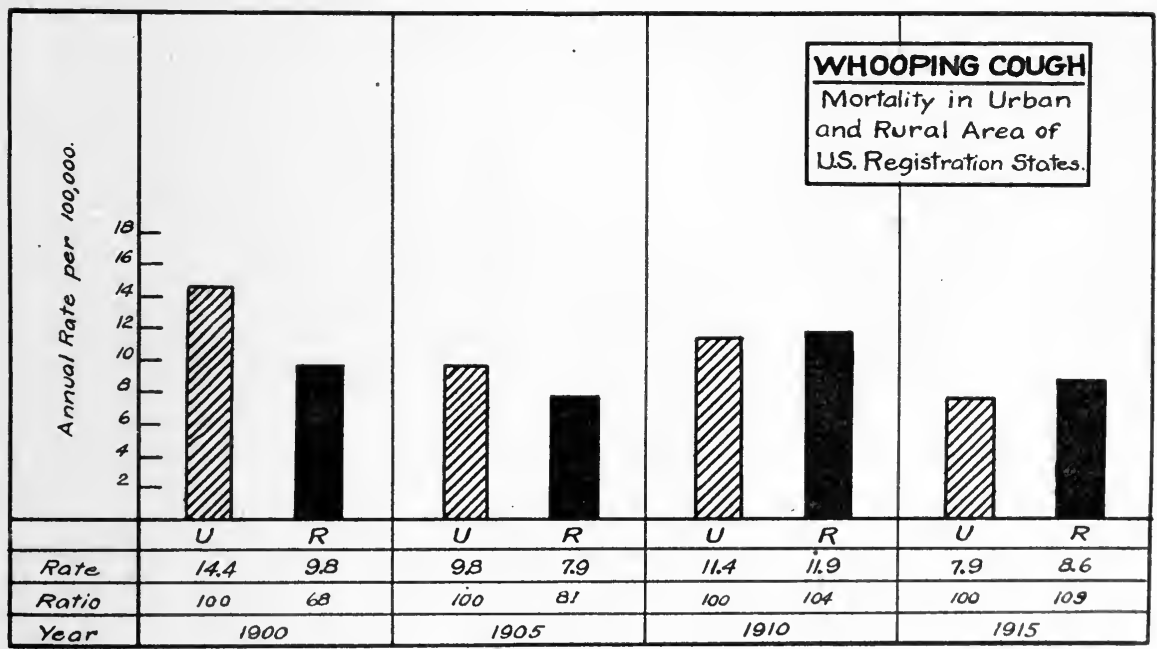

Fig. 36 .

These figures show, as has been shown for measles and scarlet fever, that epidemics of whooping cough take on in certain places and at certain times a high degree of malignity. The census authorities attribute the high rates for the southern states to their large colored population, and assert that the mortality from this disease among negroes is nearly twice as high as among whites. We are not ready from these figures to claim that this difference in mortality between whites and blacks is racial. We 
TABLE XXIX

Death Rate from Whooping Covgi per 100,000 Population in the Large Cities of United States Since 1911

(Using Corrected Mid-year Population Following 1920 Census)

\begin{tabular}{lrrrrrrrrrr}
\hline \multicolumn{1}{c}{ CITY } & 1911 & 1912 & 1913 & 1914 & 1915 & 1916 & 1917 & 1918 & 1919 & 1920 \\
\hline Boston & 15.0 & 11.0 & 13.9 & 6.7 & 15.6 & 10.2 & 5.2 & 20.5 & 8.1 & 20.6 \\
New York & 7.6 & 5.4 & 7.9 & 5.1 & 7.6 & 7.3 & 9.2 & 12.3 & 3.0 & 10.9 \\
Philadelphia & 6.9 & 5.9 & 6.3 & 14.7 & 2.5 & 7.5 & 9.3 & 19.7 & 1.6 & 5.2 \\
Detroit & 5.2 & 13.8 & 15.8 & 12.2 & 9.7 & 8.2 & 17.9 & 17.0 & 5.1 & 13.1 \\
Chicago & 2.3 & 6.0 & 4.0 & 9.3 & 2.7 & 4.0 & 7.8 & 6.9 & 5.4 & 5.5 \\
St. Paul & 10.1 & 5.5 & 5.9 & 4.0 & 5.7 & 7.4 & 2.2 & 16.0 & .9 & 10.6 \\
Minneapolis & 15.4 & 2.8 & 4.6 & 13.7 & 5.0 & 7.7 & 5.0 & 21.5 & 2.9 & 12.5 \\
St. Louis & 4.0 & 9.1 & 3.8 & 12.1 & 3.4 & 10.0 & 4.3 & 22.5 & 0 & 4.0 \\
San Francisco & 6.8 & 5.0 & 3.6 & 12.1 & 4.7 & 3.8 & 5.8 & 7.1 & 2.2 & 5.6 \\
New Orleans & 28.0 & 2.0 & 3.1 & 5.3 & 4.9 & 3.8 & 5.9 & 18.4 & 3.1 & 5.1 \\
Washington & 7.3 & 17.8 & 4.7 & 5.0 & 4.9 & 9.7 & 4.2 & 10.2 & 4.6 & 3.6 \\
Atlanta & 24.3 & 14.0 & 18.2 & 18.4 & 17.8 & 2.7 & 11.1 & 16.1 & 4.5 & 2.5 \\
Richmond & 31.0 & 3.6 & 10.6 & 47.2 & 6.6 & 30.0 & 27.5 & 13.4 & 10.9 & 23.0 \\
Pittsburgh & 18.9 & 10.4 & 15.6 & 9.2 & 8.0 & 19.2 & 10.5 & 24.4 & 8.7 & 13.6 \\
\hline
\end{tabular}

TABLE XXX

Death Rate from Whooping Cough per 100,000 Population in Certain Small Cities of the United States in Certain Years

\begin{tabular}{lcc}
\hline \hline CITY & YEAR & RATE \\
\hline Anaconda, Mont. & 1916 & 75.7 \\
Portsmouth, N. H. & 1916 & 68.6 \\
Butte, Mont. & 1916 & 66.8 \\
Gloucester, Mass. & 1916 & 61.5 \\
Webb City, Mo. & 1916 & 57.9 \\
Hagerstown, Md. & 1913 & 57.3 \\
Wakefield, Mass. & 1915 & 55.9 \\
\hline
\end{tabular}

suspect that it is due to the relative lack of care given to the colored child attacked by this disease.

The Virus.-In 1900 Bordet and Gengou first described the microorganism which they obtained in pure cultures and which is now believed to be the specific eause of this disease. This organism is present in the sputum of patients during the catarrhal stage and during the first week of spasmodic coughing. The evidence of the causal relationship of this organism submitted by its discoverers might be formulated as follows: (1) The bacillus is found usually in large numbers and frequently in pure cultures in the sputum of all early cases. (2) It is not found in the sputum in other diseases. (3) The blood serum of patients convalescing from whooping cough agglutinates this organism in moderate dilutions. (4) The serum of patients with whooping cough gives the complementfixation test specific for this organism. In 1912 Mallory and Horner 
found that in whooping cough there are masses of the Bordet bacillus growing in the eilia of the bronchi. Moreover, they were able to produce a similar condition in puppies by intratracheal inoculation with this organism. It seems, therefore, to be quite definitely settled that this bacillus is the specific cause of pertussis. It has never been found in the blood and is supposed to have its exclusive residence in the mucous membrane of the respiratory tract. It has been suggested by Mallory, and it seems altogether reasonable, that this bacillus acts largely, if not altogether, mechanically. The peculiar cough is undoubtedly an attempt to remove this living, multiplying, foreign body. This is not in accord with the older ideas concerning the nature of whooping cough and some very distinguished authorities are not ready as yet to accept the Bordet bacillus

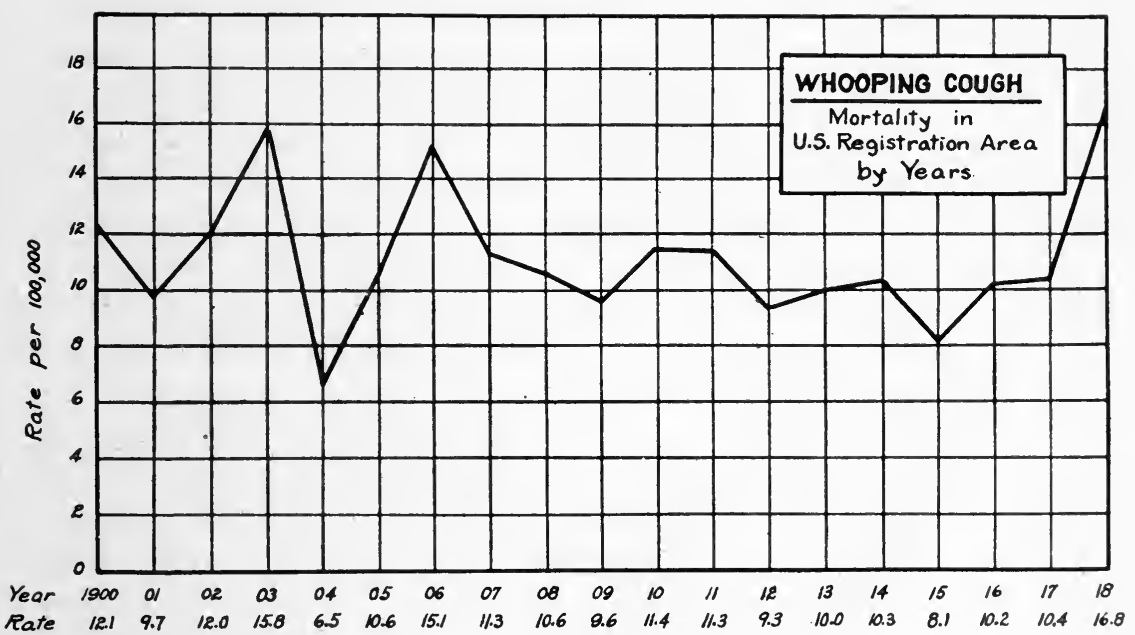

Fig. 37.

as the sole and sufficient cause of this disease. It has long been taught that there is a nervous element in its causation and manifestations. We are reminded by these authorities that pneumonia may be caused by a variety of microorganisms; that the blood serum of patients with scarlet fever may, and often does, agglutinate certain strains of streptococci, and that attempts to induce pertussis in the lower animals have not been entirely satisfactory.

Accepting the Bordet bacillus as the causative agent, another important question arises. Is this organism a single and pure strain, or is it a group containing many strains? We now know that there are at least three distinct strains of the typhoid bacillus and that vaceination with one does not give immunity to the other two. Is this true of the Bordet bacillus? This is a matter of the greatest importance in the consideration 
of the use of this organism as a vaccine either for prevention or cure. To this point we shall return later.

The Bordet organism is a cocco-bacillus, having a diameter of about 0.3 and a length of about 1 micron. It may be found singly, in pairs, and often in groups. It is nonmotile, has neither capsules nor spores, and stains poorly with anilin dyes. Carbol methylene-blue, carbol toluidin and carbolfuchsin stain it promptly. The ends give a deeper stain than the center. When first recovered from sputum some care is required in making an artificial culture, and for this purpose Bordet has recommended a potato-glycerin-blood agar. The sputum is received in a sterile dish, washed with sterile salt solution, spread over the above-mentioned medium, and incubated at $37^{\circ} \mathrm{C}$. Pure cultures of this organism may be obtained and these are used, as we shall later see, as vaccines.

Susceptibility.-There is no age exempt from possible infection with this disease. Several instances have been reported in which infants less than one week old have acquired the disease from their mothers. There is one report of a child developing this disease on the second day after birth. The whoop peculiar to the disease does not develop in very young children. There are explosive coughs, followed by intense cyanosis and the child appears quite lifeless, while its body is often covered with a cold sweat. Abt states that in young children there is usually some bronchitis. The same author gives the following striking report of the development of the disease in older children and adults:

“The patient feels perfectly well, he is playing quietly and is unconcerned; suddenly he ceases to play, his face becomes pale, he holds his breath, very frequently, he attempts to ery. Adults in the same condition eomplain of a tickling in the throat and of the pharynx and larynx and sometimes experience a feeling of pressure or heaviness over the thorax. Sometimes they complain of dizziness. Very often they experience a sense of choking accompanied by a feeling of anxiety. The premonitory symptoms last only a few minutes. The paroxysm begins with a sharp, loud inspiratory sound, which may be singing or whistling in character. This is followed by a number of expiratory eoughs, possibly five or ten. These may repeat themselves several times to be followed by more or less respiratory difficulty and the expectoration of tenacious white mucus. Very often vomiting of food and mucus occurs. Frequently the patients are cyanotic during these attacks, the face and neck being particularly blue. The veins of the neck become prominent, the larynx is raised, the muscles of the neck are contracted, the muscles of the upper part of the body and of the extremities may show spasmodic movements. Urine and feces are sometimes passed involuntarily. Blood may appear from the mouth and nose, older children usually run to a chair, or to mother or nurse to seek support. After the attack is over the children usually lie down prostrated. Frequently they perspire profusely; though in a brief time their misery is forgotten and they resume their play as though nothing had happened."

While no age is exempt from this disease and it is sometimes seen in extreme age, it is still a matter of great importance at what age the child contracts the disease. Luttinger, after an analysis of 10,000 cases, 
says that about 80 per eent of all cases and 97 per eent of all deaths from this disease are in children under five years of age. The significant figures in Table XXXI are taken from Creighton.

TABLE XXXI

Deaths From Whooping Cough per 1,000,000 Living at the Respective Age-Periods

\begin{tabular}{rcc}
\hline \hline & $0-5$ & $5-10$ \\
\hline $1851-60$ & 3624 & 174 \\
$1861-70$ & 3766 & 152 \\
$1871-80$ & 3652 & 135 \\
\hline
\end{tabular}

Luttinger has made a valuable report upon the epidemiology of pertussis as studied in New York City. In making an analysis of 10,000 cases he finds the age distribution to be as shown in Table XXXII.

TABLE XXXII

\begin{tabular}{ccc}
\hline \hline AGE & NUMBer of CASES & PERCENTAGE \\
\hline Under 1 year & 1940 & 19.4 \\
1 year, under 2 & 2019 & 20.1 \\
2 years, under 5 & 4010 & 40.1 \\
5 years, under 15 & 1799 & 17.9 \\
15 years and over & 232 & 2.3 \\
\hline
\end{tabular}

Table XXXIII gives the deaths which occurred in the old eity of New York (Manhattan and Bronx) during the last 50 years.

TABLE XXXIII

\begin{tabular}{|c|c|c|c|c|c|}
\hline \multicolumn{4}{|c|}{$\mathrm{AGE}$} & DEATHS & PERCENTAGE \\
\hline \multicolumn{4}{|c|}{ Under 1 year } & 8668 & 50.8 \\
\hline \multicolumn{4}{|c|}{1 year, under 2} & 4659 & 27.3 \\
\hline \multicolumn{2}{|c|}{2 years, } & ، & 5 & 3213 & 18.8 \\
\hline 5 & 6 & ، & 15 & 471 & \multirow[t]{2}{*}{2.7} \\
\hline 15 & 6 & ، & 25 & $13)$ & \\
\hline 25 & ، & ، & 45 & 10 & \multirow{3}{*}{0.3} \\
\hline 45 & “6 & 6 & 65 & S & \\
\hline \multirow[t]{2}{*}{65} & ، & and $c$ & over & 4 & \\
\hline & & & & 17046 & \\
\hline
\end{tabular}

The figures given in these tables show that, both in ineidence and in deaths, children under five years of age suffer most severely from this disease. This should be borne in mind in all attempts to combat the disease.

Pertussis is the only infectious disease attacking both sexes which shows a marked and constant preponderance, both in incidence and in 
deaths, among girls. This fact has been observed from the time of the earliest writers on the subject. It is believed to be due to differences in the anatomy of the larynx in the sexes. On this point Creighton writes as follows:

"No other epidemic malady has shown the same excess of female deaths in proportion to the numbers of the sexes living, diphtheria being the only other that shows an exeess at all. The excessive deaths by whooping eough among female infants was roughly shown by Watt in 1813, viz., 975 females to 842 males in the registers of the Glasgow High Church, College Church, and the Northwestern Cemetery, the relative numbers of the sexes living at the respective ages being then unknown. In all Seotland in 1889 the ratio was 1,043 male deaths to 1,225 female. The singular difference between the sexes in this respect is almost certainly related to the corresponding differences in the formation and development of the larynx, the organ which gives character,

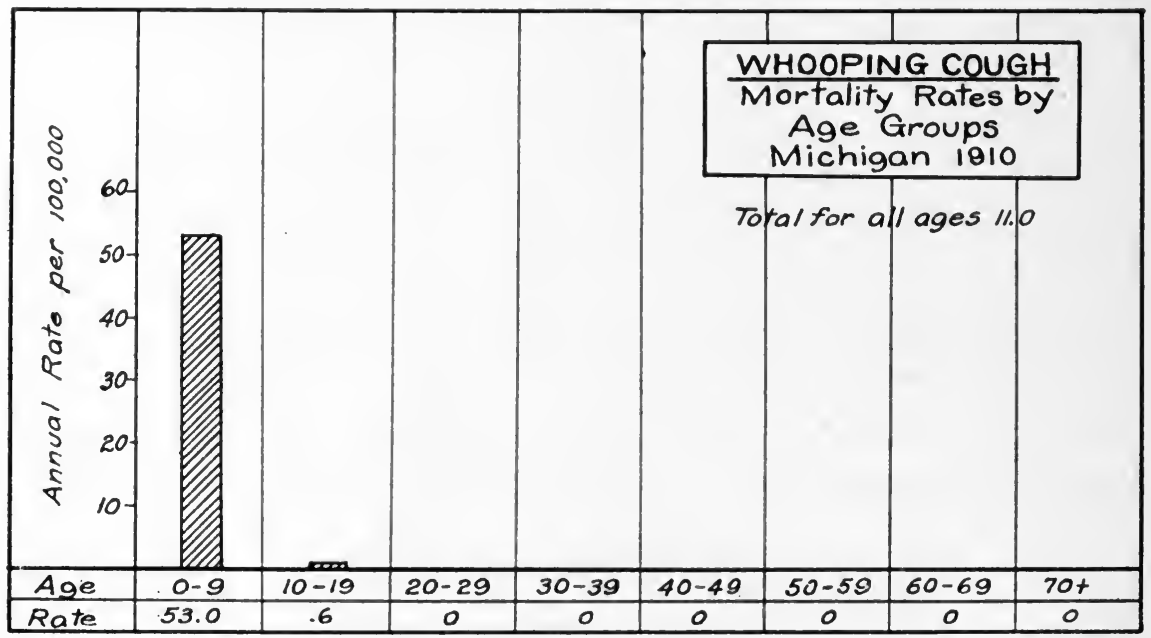

Fig. 38 .

at least, to the convulsive eough of children. The expansion of the larynx in boys, which becomes so obvious at puberty and remains so distinetive of the male sex, is one of those secondary sexual characters which begin to differentiate quite early in life, and are probably congenital to some extent. It is not known whether female children are more often attacked than males, but it is probable that they are predisposed both to aequire coughs of the convulsive and suffocative kind and to have their lives shattered by the attack-for the same anatomic and physiologic reasons, namely, the imperfect development of the posterior space of the glottis with the spasmodic elosure by reflex action."

Of 8,520 cases studied by Luttinger with reference to sex, it was found that 44.8 per cent were among boys, while 55.2 per cent were among girls. Of 17,046 deaths from this disease, 44.1 per cent were among boys and 55.8 per cent among girls. The higher death rate among girls is really greater than is shown by these figures, because there are more male children born. The influence of sex upon both morbidity and mortality 
from this disease apparently holds for all ages. In adult life this might be explained by the greater intimacy existing between women and children. As Luttinger says:

"It seems that with the accentuation of sex differenees there is a higher proportion of deaths among girls as compared with the deaths among boys."

In no other infectious disease of children does this sex difference appear. In measles, as a rule, there are more deaths among males than among females. In scarlet fever there is no constant and appreciable difference as far as sex is concerned.

When we come to study the seasonal variations in pertussis, we are surprised to find that neither the morbidity nor the mortality curve of this disease corresponds with similar curves of the respiratory diseases. We quite naturally expect that the curve for this disease would correspond with that for acute bronchitis, but we actually find that when one of these curves is at its highest point the other is at its lowest. Whooping cough prevails most abundantly and contributes most largely to the death rate during the spring and summer. While both morbidity and mortality vary with the calendar months, this increased prevalence during the spring and summer has been observed at all times and in all places. In his studies Luttinger has given the accompanying table (Table XXXIV), showing the incidence and deaths among 6,868 cases during the year 1915:

TABLE XXXIV

\begin{tabular}{lccc}
\hline \hline MONTH & CASES REPORTED & NEW CASES AT CLINIC & DEATHS \\
\hline January & 341 & 140 & 14 \\
February & 381 & 65 & 16 \\
March & 452 & 91 & 16 \\
April & 462 & 112 & 42 \\
May & 754 & 114 & 43 \\
June & 599 & 231 & 34 \\
July & 843 & 253 & 40 \\
August & 906 & 348 & 51 \\
September & 640 & 295 & 55 \\
October & 543 & 181 & 39 \\
November & 458 & 173 & 15 \\
December & 389 & 157 & 20 \\
& -2160 & 385 \\
\hline
\end{tabular}

The curves for both morbidity and mortality coincide in most years more nearly with those of the diarrheal diseases rather than with those of the respiratory infections. Luttinger makes the following comment on this point: 
"In the faee of these faets, one must ask himself involuntarily whether the fatality of pertussis is not due to something besides the respiratory infeetion; whether a toxin similar to that produeed by the diarrheal diseases is not the direet cause of death. Another view would be that death during the summer is hastened by gastrointestinal complieations and that during the winter bronehopneumonia is the usual complieation."

The health reports of Chicago give the following summary of cases and deaths from whooping cough in that city by months from 1911 to 1918 inclusive (Table XXXV).

TABLE XXXV

YEAR JAN, FEB, MAR, APR. MAY. JUN. JUL. AUG. SEPT. OCT, NOV. DEC. TOTAL CASE MORTALITY

\begin{tabular}{lrrrrrrrrrrrrrr}
\hline 1911 & & & & & & & & & & & & & \\
Cases & 57 & 54 & 91 & 61 & 92 & 143 & 110 & 78 & 46 & 40 & 87 & 92 & 951 & 5.4 \\
Deaths & 3 & 1 & 3 & 3 & 8 & 6 & 7 & 7 & 0 & 2 & 6 & 6 & 52 & \\
1912 & & & & & & & & & & & & & & \\
Cases & 153 & 152 & 270 & 344 & 473 & 287 & 249 & 244 & 115 & 54 & 39 & 82 & 2462 & 5.6 \\
Deaths & 4 & 9 & 12 & 11 & 24 & 22 & 19 & 20 & 11 & 3 & 1 & 4 & 140 & \\
1913 & & & & & & & & & & & & & & \\
Cases & 139 & 97 & 69 & 90 & 71 & 72 & 144 & 230 & 149 & 73 & 143 & 203 & 1480 & 6.8 \\
Deaths & 16 & 12 & 8 & 6 & 9 & 8 & 8 & 12 & 6 & 4 & 4 & 8 & 101 & \\
1914 & & & & & & & & & & & & & & \\
Cases & 490 & 435 & 794 & 592 & 839 & 570 & 485 & 382 & 170 & 82 & 67 & 102 & 5317 & 4.3 \\
Deaths & 8 & 23 & 29 & 48 & 30 & 25 & 20 & 19 & 11 & 7 & 4 & 6 & 230 & \\
1915 & & & & & & & & & & & & & & \\
Cases & 54 & 78 & 130 & 89 & 115 & 112 & 120 & 114 & 124 & 105 & 125 & 138 & 1334 & 4.3 \\
Deaths & 7 & 0 & 5 & 4 & 3 & 9 & 7 & 8 & 2 & 2 & 1 & 10 & 58 & \\
1916 & & & & & & & & & & & & & & \\
Cases & 177 & 268 & 337 & 295 & 287 & 252 & 357 & 367 & 213 & 78 & 78 & 144 & 2853 & 4.0 \\
Deaths & 9 & 10 & 7 & 11 & 9 & 8 & 8 & 16 & 22 & 5 & 5 & 3 & 114 & \\
1917 & & & & & & & & & & & & & & \\
Cases & 221 & 214 & 314 & 275 & 378 & 538 & 821 & 946 & 605 & 245 & 284 & 315 & 5156 & 4.2 \\
Deaths & 12 & 13 & 7 & 15 & 18 & 33 & 32 & 30 & 27 & 13 & 6 & 12 & 218 & \\
1918 & & & & & & & & & & & & & & \\
Cases & 387 & 454 & 581 & 537 & 392 & 500 & 589 & 485 & 200 & 109 & 61 & 50 & 4345 & 4.2 \\
Deaths & 18 & 16 & 35 & 26 & 9 & 8 & 13 & 15 & 16 & 20 & 4 & 5 & 185 & \\
\hline
\end{tabular}

One is compelled to use caution in speaking of the case mortality from this disease. In the first place, not all cases are reported and a considerable number of those reported under this name are really not cases of whooping cough. In the second place, the mortality must be figured from reported cases and consequently is liable to great inaccuracies. In New York City there were 385 deaths out of 6,868 reported cases, showing a mortality of 5.6 per cent. This does not vary greatly from the case mortality as reported in Chicago, which may be seen from the figures given in the above table varies from 4 to 6.8 per cent. Luttinger is of the opinion that if all cases, typical and atypical, both in young and 
adults, were reported the case mortality would fall to 1 per cent, or possibly to half this number.

Should we classify pertussis as a rural or urban disease, or is there any difference in its prevalence and its death rate under urban and rural conditions? If we turn back to the table showing the death rate in the registration area, we shall see that for the rural part of this area it is quite constantly a little higher than that for cities. In our opinion, this would not justify us in concluding that this disease is more common or more deadly on the farms than in the eities. If we knew the truth, we would be more likely to find that the slightly higher death rate in the rural districts is due to the decidedly higher death rates in villages and in small cities. We think that we have some substantiation of this belief in the figures already given showing the excessive mortality from the disease in certain small cities. Moreover, when we study the morbidity and mortality from this disease in large eities, we must bear in mind that, taking the city as a whole, we have crowded areas, and other areas which would compare favorably with those of country life. In New York, Luttinger found the disease most prevalent and the death rate highest in the tenement houses, especially in large families occupying not more than one or two rooms. It seems quite certain that both morbidity and mortality from this disease depend largely upon housing conditions and upon the narrowness of space in which children live. Luttinger is of the opinion that it is not fresh air per se which is so effective, but change of air. He has observed children in New York with this disease greatly improved by changing from one congested district to another. This probably means that by the change the child moves from narrow quarters densely infected with the virus of this disease into equally narrow quarters without the infection. It is quite certain that children with this disease are greatly benefited by more abundant air space and greater opportunity to roam over wider areas. Luttinger has recommended that ferry boats be used as hospitals or rendezvous for children with this disease. We are quite convinced that every city should have a large recreation farm, one part of which should be set aside for the reception of children suffering from whooping cough. The fact that the disease is most prevalent and most dangerous during the summer time would render this proposal relatively easy of adoption. In the tenement districts of New York pertussis is a common and a serious disease. In the boroughs of Richmond and Queens there is but little pertussis and deaths from this cause are exceedingly rare. If all children could be properly housed, with abundant space, both in doors and out of doors, whooping cough might again be dropped from the list of fatal diseases.

Transmission.-Fortunately, an intelligent and energetic health official can ascertain the source of infection in most cases of this disease. In its 
transmission fomites plays a very subordinate rôle. Of course, the common use of pencils and other personal articles in school might serve in the distribution of the disease, but it is not supposed at the present day any teacher is so ignorant of the fundamentals of sanitation that he or she would permit such things to occur. Luttinger has traced the source of infection in 2,310 cases, and the results are given in Table XXXVI.

TABLE XXXVI

\begin{tabular}{lr}
\hline \hline Neighbor & 1311 \\
Relative or friend & 204 \\
Playmate & 203 \\
School & 146 \\
Nursery & 76 \\
Moving pictures & 62 \\
Recreation pier & 15 \\
Roof garden & 9 \\
Ferry & 8 \\
Street ( ( ) & 6 \\
Subway, elevated and street cars & 4 \\
Unknown & 263 \\
& Total 2310 \\
\hline
\end{tabular}

In the great majority of instances this disease is transmitted through the air from an infected to an uninfected child. Direct contact between the two is not necessary. Instances are reported in which children have whooped across street cars into the faces of healthy children and within two weeks the latter developed the disease. Similar exposures are not confined to street cars, but may occur in any vehicle of transportation, waiting room, or in short, in any-place of assembly whether it be fixed or moving.

Adults frequently carry the bacillus of whooping cough from one child to another. "Pertussis Pete" has not won the fame awarded "Typhoid. Mary," but Luttinger has given his history as follows:

"An interesting pertussis carrier was Peter G., who boarded with a sister in Harlem. Two nieces and one nephew eontracted whooping eough from their neighbor and Peter began coughing a few weeks later. About the beginning of March he visited a sister living in Brooklyn with the view of reconciling her to the Harlem sister, from whom she had been estranged. About eight days later one of the children began to cough. Mareh 10 the Brooklyn sister visited her Harlem relative alone, made up with her and told her of her children, who were whooping. Both sisters finally met at the Whooping Cough Clinic a week later. In the meantime, Peter left his Harlem sister and went to live with his brother, who had just come from some Pennsylvania town; Peter was still coughing. A week later one of his brother's children began to cough and a few days later started to whoop and vomit. The other ehild had had whooping eough before. The father now aceused Peter of having given the disease to the ehild and they quarreled, Peter going to live with a cousin (a bachelor) in East Twelfth Street. On 
April 1 one of the neighbor's children began to whoop, and as there were no pertussis cases in that house, we finally traced the source of infection to Peter, who was stiil coughing and asked him for his sputum. This he delayed until April 20, when he sailed for Italy, having enlisted in the army. He sent a sputum jar to the laboratory on that day, but it was found later that it was his cousin's sputum, who was perfectly normal.',

A small percentage of children seem to have a natural immunity to this disease; at least when exposed they do not develop it. In some this is apparently a temporary immunity, because on a second exposure they acquire the disease. In many other instances the supposed immunity is due to the slight and evanescent symptoms of the disease developed in certain children.

Vaccination.-Medical journals devoted to diseases of children have been during the last ten years literally crowded with articles upon the use of vaccines, principally in the treatment, but in part for the prevention, of this disease. For two years the Board of Health of the City of New York operated a whooping cough clinic in which vaccines, especially as curative agents, were employed. In a disease which lasts as long as this any curative agent which will shorten the time of its duration may be regarded also as a protective agent. In the New York clinic 952 cases were treated with specific vaccines, while 149 were treated with drugs. The average duration of the paroxysmal stage of the 952 vaccine cases, irrespective of the time when treatment was begun, was nearly 37 days, while the 149 drug cases had an average duration of over 50 days. In 709 cases in which the vaccine was given before the end of the third week the average duration of the disease was a little over 20 days, while the controls, consisting of 88 patients treated with drugs, whooped for nearly 32 days. It is reported that the best results were obtained when the vaccine was not employed until the second week of the disease. Luttinger, who conducted this clinic, says that he has no explanation for this unless it be the old one of the "negative phase." Besides shortening the disease vaccine is reported to have favorably influenced the severity and frequency of the paroxysms. The appetite becomes normal, sound sleep returns, and in general the condition of the child greatly improves. In chronic cases vaccine was apparently without effect. The vaceine employed at this clinic varied from time to time, but the following procedure was finally adopted.

"To a child over a year old, an initial injection of 500 million bacteria is given; at the end of 48 hours another dose containing 1 billion is administered, and after the lapse of another 48 hours 2 billion are injected subcutaneously. After an interval of five days the child is examined again, and if the paroxysms and the romiting have ceased, is treated with cod liver oil or other tonies. If the paroxysms are much milder but still persist, a dose of 4 billion bacteria is given, followed by 8 billion in three days and 10 billion after another lapse of three days. Should the paroxysms become 
more violent after the first three injections, a week should be allowed to elapse before the second series of three injections is given, and instead of 4 billion this series is started with only 2 billion."'

We have already brought up the question of possible variations in strains of the Bordet bacillus. In order to provide against the possibility of multiple strains, autogenous vaccines have been generally employed. This has an additional advantage in the freshness of the preparation. It is believed, by some, at least who have written rather extensively on this subject, that no vaccine more than a week old should be used.

The results obtained by the prophylactic use of these vaccines are by no means satisfactory. In the last report of the New York clinic it is stated that of over 600 children who were given prophylactic injections, 368 were supposed to have been subsequently exposed to infection. Of those exposed 37 per cent contracted the disease and 63 did not. When we eonsider the possibility of a certain number of children escaping the disease under ordinary conditions of exposure, we can readily understand that these figures are not convincing. If Mallory's teachings concerning the location and modus operandi of the Bordet bacillus be true, it can hardly be expected that a vaccine with the efficiency of those now used in the prevention of typhoid fever will be secured. However, this problem is now in the hands of most competent pediatricians and we have no doubt that it will be settled in the near future.

Diagnosis.-Unfortunately there is no means by which this disease can be positively recognized until the characteristic whoop is in evidence, and this occurs long after the early stages of transmission. The agglutination test at any stage is not marked. Moreover, it is not constant. It cannot be presumed that every coughing child has this disease and the agglutination test, if it were more diagnostic, would not be likely to be employed earlier. Some have attempted by animal experimentation to demonstrate the value of the agglutination test. This is not logical. Of course, if we inject the Bordet bacillus or any other organism subcutaneously or intravenously into rabbits and repeat this a sufficient number of times, in all probability the treated animals will furnish sera which agglutinate the organism employed in their treatment. This, however, is no fair test, because the Bordet bacillus is not present in the blood in pertussis. According to Mallory, it is confined largely, if not altogether, to the ciliated epithelia of the bronchi; therefore, we need not expect to find a high agglutination titer when we employ blood serum. Much work has been done upon the complement-fixation reaction, which undoubtedly is present in a considerable proportion of children ill with, or convalescing from, this disease; but this is not constantly the case, and whatever interest may attach itself to this reaction in experimental work it remains largely scientific and is not practicable. Friedländer claims that he has 
obtained positive complement-fixation reactions in 100 per cent of his cases in the catarrhal stage, but he admits that it is stronger during convalescence and that it often persists for many months. There is a wonderful and exceptional leucocytosis, with both an absolute and a relative increase in the lymphocytes, in the catarrhal stage of whooping cough. The number of white corpuscles often goes up to 30,000 and has occasionally been reported as high as twice this number. However, there are cases, possibly due to complications, in which this form of leucocytosis is not in evidence. It remains true, therefore, that the pathognomonic whoop is the first positive diagnostic evidence of the existence of the disease. For this sign the physician must often rely largely upon the testimony of members of the family. The child will not always whoop in the presence of the physician, though characteristic spasmodic cough may be induced in many instances by pressure upon the back part of the tongue. Too often, we fear, the physician thinks the disease has progressed so far by the time he has made a diagnosis that there is no use in reporting the case. He should remember that because the child has already infected a dozen others is no justification for his failure to prevent its infecting many more. Adults and very young children seldom demonstrate the existence of the disease by the peculiar cough, and still they may serve as sources of infection.

Control.-From what has already been written, it must be evident that the eradication of this disease is almost beyond human effort, but this should not prevent us from making every attempt to limit it, especially among children under six or seven years of age. It goes without saying that children with this disease should not be permitted to attend school. We fear, however, that the desire to protect a school often leads the physician to neglect or ignore the danger of the child carrying the disease to younger members of its own family. The family physician and the medical health officer should always bear in mind that among children under six years of age this is a highly fatal disease, while among those above this age, under proper care, the death rate is low. It has been proposed that more attention should be given to the isolation of young, uninfected children than to the isolation of the child with this disease. If all children under six could be protected, the death rate from whooping eough would fall markedly. The practice in regard to isolation of children with whooping cough varies widely in different cities and communities. Some health officers require the placarding of houses, while others do not. Some demand that the infected child be kept indoors, while others permit greater freedom of movement. It is quite certain that kecping the child with this disease in the house is not good for the child itself. Infected children pass through the disease much more easily and successfully when they are permitted outdoor exereise. New York City main- 
tained a whooping cough clinic for two years, but discontinued it at the expiration of that time. The success of such a plan must be doubtful. Mothers brought their children to the clinic from wide distances, occasionally from areas beyond the city, and it is impossible to tell how many children may have been infected during these journeys. In 1913 the health officer of Chicago put into operation a modified method of dealing with this disease. For the first two weeks the patient is kept in the house or allowed access to the yard if not used by children of other families, provided always that when the child is out of doors it should be under guardianship. For the remaining three weeks of the quarantine period the child is permitted, if accompanied by an attendant, to go on the street, provided that there is fastened around the sleeve of the outer garment a yellow band of material two inches in width and bearing the inscription "whooping cough" in black letters. Such children are not permitted to enter places of assembly or to use public conveyances under penalty of being subjected to restrictions as in the first period of quarantine. One cannot foretell the ultimate result that will be reached by vaccination in this disease, but under present conditions we believe that the death rate would be lowered if children after passing the sixth year, and including only those in good health, should be intentionally submitted to this infection. We admit that there would be some complications and possibly a few deaths under such an arrangement, but it would reduce greatly the number of carriers at large. Certainly, if vaccination be made successful, it would be greatly preferable to voluntary exposure. We cannot close this section without reemphasizing the desirability of limiting the spread of the disease among children under six years of age.

\section{Bibliography}

Creighton, Charles: History of Epidemies in Britain, ii, 1894, Cambridge University Press.

Dunn, Ciras. H.: Pediatries, Troy, N. Y., 1917, The Southworth Co.

FriedLÄNDER, AlFren: Trans. Am. Pediat. Soc., 1914, xxvi, 22.

Hess, Alfred F.: Jour. Am. Med. Assn., 1914, lxiii, 1007.

Huenerens, E. J.: Am. Jour. Dis. Child., 1917, xiv, 283.

LutTinger, Paul: Reprints from the Department of Health of the City of New York, 1915-1917.

Morse, John L.: Trans. Am. Pediat. Soc., 1913, xxv, 6.

RoBertson, JоHN D.: Report and Handbook of the Department of Health of City of Chicago, 1911-1918.

VEEDER, BORDEN S.: Am. Assn. for the Study and Prevention of Infant Mortality; transactions for 1917 , p. 86. 


\section{CHAPTER XIV}

\section{INFLUENZA}

\section{EPIDEIMIC INFLUENZA}

Description.-Influenza is an acute, febrile disease of extreme communicability, characterized by catarrh of the respiratory tract and occasionally of the alimentary canal, and accompanied by pains in the head and musculature. Most significant also is the suddenness of onset and marked prostration. The period of incubation is from two to four days. The duration of the disease varies from a few days to several weeks, depending upon the severity of the attack.

Although the mortality tables show deaths from influenza each year, it is the epidemic variety of influenza which is of chief concern. Influenza in interepidemic periods is a name given to an acute eatarrhal disorder which occasions the death of people in the extremes of lifethe infant and the aged. Endemic influenza is a very general term synonymous with la grippe. It differs from the epidemic influenza mainly in its lesser contagiousness. Endemic influenza occurs chiefly in the winter months and is more sensitive to $a^{a}$ seasonal influence than either pneumonia or bronchitis. During the period 1912-1916, influenza caused an average of 9,926 deaths annually in the registration area. As a cause of death it ranked tenth in magnitude. The average number of deaths from tuberculosis, which stood first, was 96,055. It is epidemic influenza which we shall refer to throughout the text by the shorter term, influenza.

Influenza takes its name from the Italian word "influenz", it being supposed in the seventecnth century to have been caused by certain mysterious astral or telluric influences. The French derivation "la grippe", comes from the verb "agripper", meaning to attack. Other terms at various times applied to this disease are febris catarrhalis epidemica, catarrhe pulmonaire, folette, coqueluche, petit poste, rhume epidemique, Russiche krankheit, eatarro Russo, Spanish influenza.

Although medical history is replete with accounts of epidemies and pandemics, influenza today is in reality a little understood malady. It is not at all strange that the ancients regarded it with awe and ascribed its cause to the supernatural, for even in the present age we are in the dark as to the exact origin and we are mystified by its astounding manifestations.

In 1892 Pfeiffer, a German bacteriologist, announced that he had dis- 
covered a bacterium which was responsible for influenza. Pfeiffer's work was never accepted whole heartedly, for the organism to which he gave his name was found in the throats of healthy people and in those with measles and other respiratory inflammations as commonly as in people sick with influenza. Much bacteriologic work has been done since that time but to no avail so far as actually identifying a specific organism with the disease. A variety of organisms have been recovered from the throats of living patients and from their bodies after death. Fatal influenza is most commonly complicated with pneumonia and the pneumococci have been found with great frequency.

Ignorant though we are of the specific cause of influenza, whether bacterium, coccus, protozoan, or filtrable virus, there is no doubt that the disease is spread by excretions of the respiratory tract. It is through this avenue also and possibly through the eyes and alimentary tract that the infection enters the body. The disease is transmitted from person to person directly by coughing, sneezing and talking, and indirectly through carelessly washed dishes, by handshaking, by touching common articles freshly infected by those coming down with the disease. It is possible at least that the disease may be spread by infected dust.

Influenza knows no boundaries. Apparently it roams the globe at will. Australia, India, China, Europe, the Americas and the islands of the great oceans have experienced its heavy hand. Though generally thought of as a cold weather disease, it has occurred at all seasons of the year. Influenza does not confine its ravages to the weak and infirm. It is partial to the strong and vigorous. It is the young adult in the prime of life who falls before the infection in greatest number. Men and women alike are attacked. In its epidemicity influenza is almost without a parallel. It is such diseases as the plague and yellow fever that recognize in influenza a kindred being. These two diseases have largely bowed before advancing human knowledge. Influenza alone remains unbridled.

A lapse of years finds a great proportion of the population susceptible to epidemic influenza. From 10 to 40 per cent of a community show visible symptoms. From 1 to 5 per cent of this group die. These are approximate figures and wide variations occur. Considering the great number attacked, the mortality is relatively low. Typhoid fever kills 7 to 10 per cent of those attacked. Influenza kills about 5 per cent of those exhibiting definite symptoms of the disease.

In speaking of the epidemiologic resemblance of influenza to other infectious diseases, Vaughan (Warren) says :

"In our comparison of influenza with other infectious diseases we wish to show particularly that the disease is not in a class by itself but that its epidemiologic features are not unlike those of other respiratory infections, that the manner of spread and the 
mode of infection are similar to those of other diseases. *** It is the opinion of the author that the phenomenon which contributes chiefly to the occurrence of influenza in epidemics and pandemics and which causes the characteristic curves of a primary influenza wave is the absence of any permanent immunity. $* * *$ Measles occurring

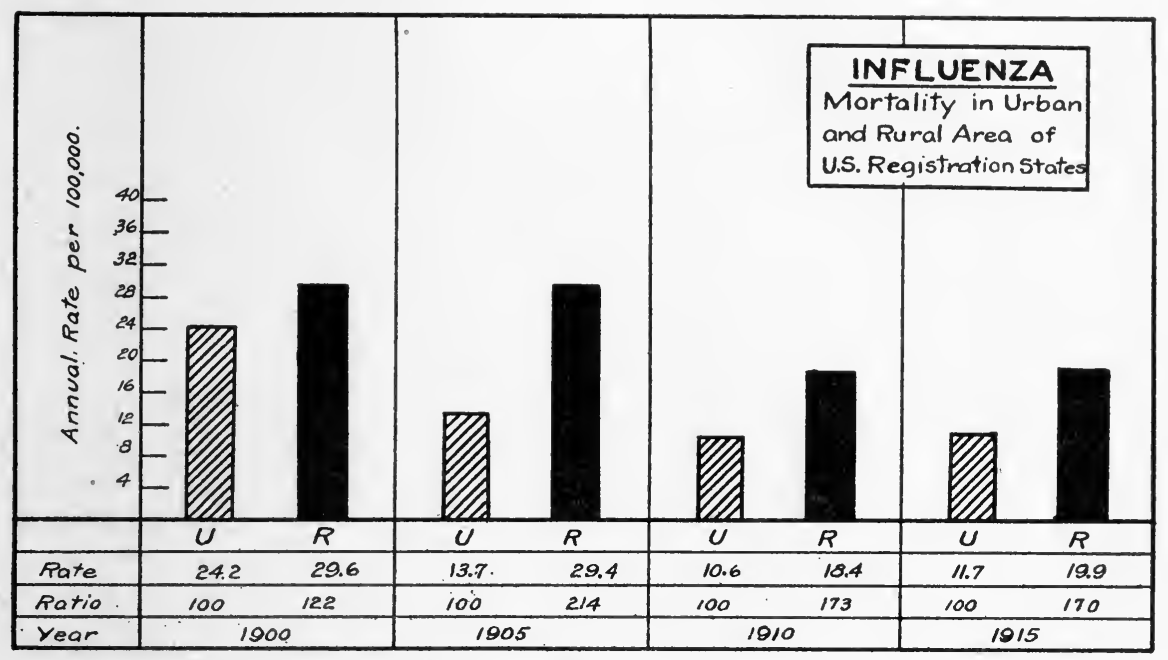

Fig. 39.

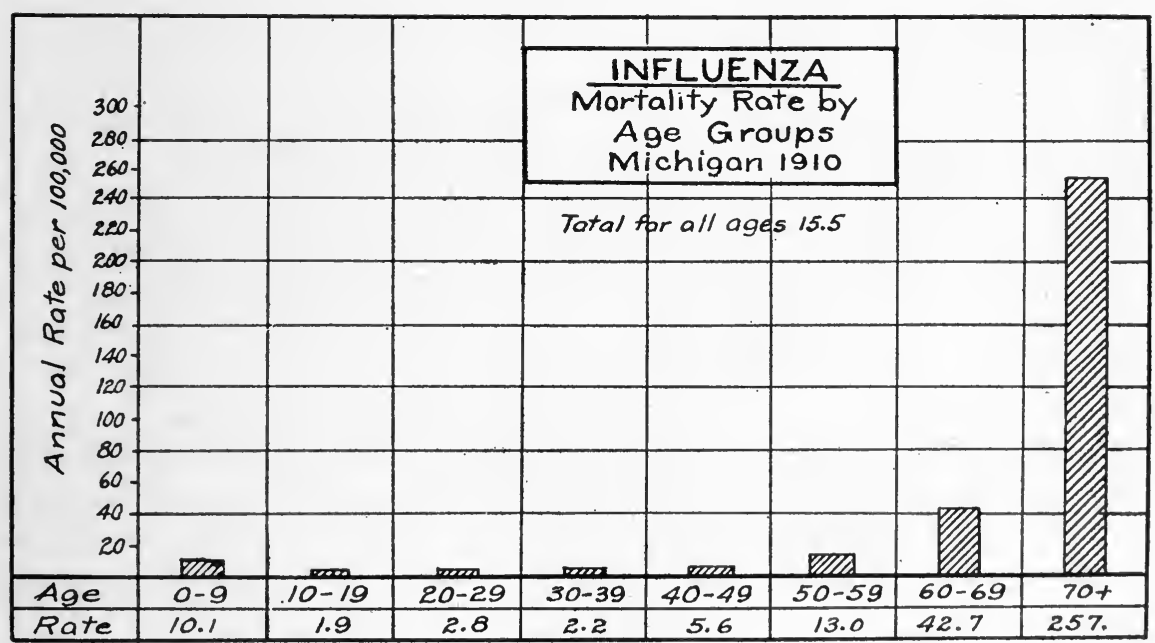

Fig. 40.

in a nonimmune population spreads through that population with the same high rate as does the influenza."

We shall review the epidemics of the disease as they have been handed down to us by medical historians. It is from recent times, however, that what knowledge we possess of the disease is mainly derived. In 
the last thirty years there have occurred two world wide pandemicsin 1889-90, and in 1918-19. Recurrences of the disease also appeared in the years immediately following. With our more complete method of recording such events, it has been possible in these last two outbreaks to learn something of the extent and severity of the disease and its selective action with respect to age, sex, color, etc.

History.-There are many written aceounts of pestilence purporting to be influenza. It is difficult to determine whether or not all of the soealled influenza epidemics are identical in character with.pandemics of recent times. There are many symptoms that sound familiar, but, on the other hand, there are certain rather marked differences in the ramifications of the disease. Common to all are the factors of extreme communicability and well nigh universal susceptibility.

What appears to be the first recorded instance of an influenza epidemic is referred to in Livy in $412 \mathrm{~B}$. C. There is also an early account of influenza in the Athenian army in Sieily in 415 B. C. These early aceounts are not authentic and the best evidenee for ealling them influenza seems to be that they were sudden and widespread.

As one writer puts it: "What other disease than influenza could it have been which occurred, for example, in the year 591 or 592 B. C. which often, when hardly ushered in by headache and coma, rapidly ended in death." The later accounts speak of influenza as a relatively nonfatal disease.

Abbott, writing on "The Influenza of 1889-90," states that influenza epidemics have distributed themselves over the centuries as follows:

$\begin{array}{lr}\text { 14th Century } & 6 \\ 15 \text { th } 6 & 7 \\ 16 \text { th } 6 & 11 \\ 17 \text { th ، } & 16 \\ 18 \text { th } 6 & 18 \\ 19 \text { th } 6 & 11\end{array}$

The most complete description of previous epidemics that we have seen is that given by Finkler in Vol. XV of the Twentieth Century Practice of Medicine, 1898. In giving a résumé of the subject we have drawn largely from this source. We have also made use of descriptions by Parkes and by Hirsch.

Hirsch looks upon the year 1173 as witnessing the first epidemic of which authentic reports are available. The disease at this time spread over Italy, Germany and England. Italy and France were visited again in 1323 and Italy alone in 1328.

In 1387 what is regarded by many as a true influenza epidemic appeared in Italy in January and February. It was also prevalent over Franee and Germany. About 10 per cent of the population were af- 
fected. Old and debilitated persons were the most ready victims and yet very few died. The acute symptoms lasted four or five days.

In 1404 what is believed to be influenza visited the Netherlands and Germany. Hardly any one escaped. The principal symptoms were cough and hoarseness.

In the winter months of 1413-1414, it is believed influenza of a mild form prevailed in France, Italy and Germany. There is some evidence of an epidemic in 1427, the symptoms of which were pain in the kidneys, chilliness, anorexia, insomnia and cough.

The first pandemic occurrence of the disease was in 1510. Introduced into Sicily from the Island of Malta, it invaded Spain, Italy, crossed the Alps to Hungary, Germany, France and England. The attack was sudden and scarcely a family in all Europe was thought to have escaped. The symptomatology included fever, cough, hoarseness, headache, anorexia, insomnia, pains in the stomach, kidneys and legs. The mortality was slight.

In 1557 an epidemic invaded Europe, starting in Sicily in June and passing to Italy in August, Dalmatia, Switzerland, France, England and Spain in September and the Netherlands in October. It is also reported to have visited America. There is a difference of opinion on the fatality of the infection. One author indicates a high fatality in Nimes, France, whereas another states that for the country as a whole the disease was not fatal except to children. Still another author comments on the nonfatal eharacteristic. The description of the disease in Holland corresponds closely with the symptoms exhibited in 1918. The main symptoms, beside violent headache and tormenting cough, were severe dyspnea. ("that the sick seemed dying") accompanied by pain in the loins on walking, "constant severe pain of the loins that they could not walk."

The second pandemic on record was in 1580, when Europe, Asia, Africa and America were invaded. The disease entered Europe either from Africa or Asia in the spring. The stepping stones in succession were the Island of Malta, Sicily, Spain and Italy, Hungary, Germany, the Baltic, France and England. It was especially virulent in England from the middle of August to the end of September, returning again in October and November of the same year. The mortality appears to have been low, one author stating that hardly one out of a thousand persons died. On the other hand, many deaths from it are mentioned in Spain. The heavy mortality in some places is believed to have been due to exhaustion through venesection. Complications are first mentioned in this pandemic and include otitis, parotitis and affections of the bile ducts. The symptoms were similar to those in 1557. The course of the pandemic is not clear, for it is spoken of in France in May and June; 
in Naples in August and September, and in Germany in the late summer and autumn, Silesia having it in September and Saxony in October. This is in contradistinction to its course as outlined in the beginning of the paragraph.

An epidemic is said to have spread from Belgium in 1593 and reached all parts of Europe. The mortality was low. In 1626 there was an epidemic in Italy; in 1647 in America (Massachusetts and Connecticut) and the West Indies. In 1658 it occurred in England, beginning in April. The symptoms were cough, catarrh, fever, anorexia, pain in the back and limbs, occasional nosebleed, bloody expectoration and bloody stools. It was fatal only to the old and debilitated. Another mild epidemic occurred in 1675, attacking Germany in September, Hungary and Austria in October, and England the latter part of October. The old and decrepit died. An epidemic occurred in England and Ireland in 1688. It first appeared in London in May and extended all over England, reaching Ireland in July. The epidemic lasted about seven weeks in a community. The statement is made that only one in fifteen escaped, but that not one in a thousand died that had it. In 1693 it reappeared in Dublin in November, passed to England a month later and invaded Holland and Flanders. Various sections of Europe were affected in 1709 and 1712 .

The epidemic of 1729 and 1730 is the first said to have entered Europe through Russia. Prior to this the entry had been through Constantinople or Italy. Complications mentioned were phthisis, pneumonia, pleuritis, inflammation of the brain, delirium, convulsions, petechiae, miliaria, parotitis and abortion. In 1732 and 1733 the disease was pandemic in character, being reported from both hemispheres. One account gives its beginning in Connecticut in October, 1732. It spread to Massachusetts, Maryland, Newfoundland, West Indies and South America. This was probably independent of the epidemic in Europe which entered Russia through Poland in November and spread over Europe. The disease was mild although pneumonia is mentioned as a complication, as are also orchitis, swelling of the salivary glands and earache. Some of these symptoms are suggestive of mumps.

It is in the accounts of 1742 and 1743 that the names of influenza and la grippe are first used. At this time the disease was reported in Continental Europe and England. In the epidemic in Edinburgh in 1758, it is said that not one out of six or seven escaped. North America and Continental Europe were likewise visited this year. Mention of the disease is made in America in 1761, Philadelphia, Massachusetts and the West Indies being eited. The symptoms were severe pain in the head and limbs, sensation of coldness, shiverings succeeded by great heat, running at the nose, and troublesome cough. The duration of the at- 
tack was from eight to ten days. This deseription does not tally closely with the illness of 1918. Parts of Europe were likewise affected this year. In the epidemic of 1762 in Europe there is mention of pleuritis and pneumonia attacking those who would not take to bed early. In 1767 both Europe and America were visited. In the epidemic of 17751776, old asthmatic persons are said to have suffered most in England. Fothergill, quoted by Finkler, says that he had no knowledge of an epidemic "where so many people were seized, and in so short a time, and with so little comparative mortality." An epizootic among dogs and horses is reported to have occurred at this time also.

The years 1781-82 were pandemic years. The disease first appeared in China and India in the autumn of 1781, passed through Siberia to Russia and invaded St. Petersburg in January, 1782. From here it extended over the rest of Europe. Germany first reported it in March, Denmark and England in April, Scotland in May, Netherlands, Ireland, France and Italy in June, and Spain not until August. The danger of suffocation is mentioned in connection with the symptomatology. Swelling of the glands of the neck and exanthema of the hands and feet, also mentioned, do not sound like our recent outbreak. Whatever this infection was, it left few untouched. It is said that two-thirds of the population of Rome and three-fourths of the population of Munich were affected. Vienna had to close the theaters for eight days. Children were not attacked as frequently as adults. Although the disease was mild, it was observed that the death rate was raised in London at this time. The causes given for this epidemic are curious. The Consilium Medicum at Vienna said it was due to the swallowing of small insects. Air vitiated by noxious insects from China is another cause given. Increased volume of electricity in the air is also offered as an explanation.

The closest resemblance to the present day symptoms of the disease is found in the accounts of the epidemic of 1788-89 in Europe as described by Grill, quoted by Finkler. Among other symptoms given are "a general debility, extraordinary hebetude, a heavy head, a heaviness and numbness of the lower extremities, weakness and confusion of the senses, dull eyes, a sensation of pressure in the region of the stomach, tightness over the chest and difficult respiration." In this year we also read of epidemies in the fall in New England, New York and Philadelphia with a recurrence the following spring.

Hirsch's account mentions epidemics in the West Indies and Europe in 1805-1806, in North America and Great Britain in 1807, in Brazil in 1811, in North America in 1815-1816 and in 1824-1825, in the southern states of North America in 1826, in Russia and Siberia in 1827. In 1830 an epidemic began in Manila, Philippine Islands, in September and took two.months to reach Russia. Five months after this it was in 
Germany and two months later in France. The spread of the disease from Manila to the neighboring islands of Borneo and Sumatra required four months' time, or two months longer than it took to reach Russia. This suggests the influence of accessibility and readiness of communication in the spread of influenza. There was no increased mortality in Paris. Children under 14 and adults over 45 seem to have been spared. This epidemic continued at intervals throughout Europe in 1831 and 1832. The year 1833 seems to have witnessed a higher fatality from the disease. In Königsberg, 145 persons died in the last week in March, whereas the normal mortality did not exceed 50. A case mortality of 14 per cent is also eited by one author. On the other hand, in Bohemia the mortality was said to have been less than usual. India was visited in 1834. The epidemic in Europe in 1836-1837 is reported to have attacked adults from 20 to 40 years of age and of these, more women than men. The physicians of Würtemberg at the same time speak of its great dissemination among children. The most frequent complication in this epidemic was pneumonia following influenza. The brain and spinal cord seemed to have been invariably affected.

Hirsch describes a succession of epidemics after 1837. Iceland and Australia were involved.in 1838 and Abyssinia in 1839. In 1841 Germany was chiefly affected. In 1843 there are accounts of influenza in Europe, Siberia and North America. Parts of Europe were overrun in 1844-45-46 and 47. In 1847 and 1848 the entire eastern hemisphere seems to have been visited, including the Hawaiian Islands and also islands in the West Indies. Both hemispheres are reported to have been affected in 1850-51, Australia in 1852, Cape Colony in 1853, Europe in 1854-1855 and Brazil in 1855.

In 1857-58 there was wide diffusion of the disease over both hemispheres. In 1860 it occurred in Australia and Tasmania, in 1861 in Philadelphia, in 1862 in the Bermudas, Netherlands, Cape of Good Hope, and Iceland, in 1863-1864 in France, California and Switzerland, in 1866 in France and England, in 1867 in France, Germany and Belgium, in 1868 in Constantinople, in 1871 in Cape of Good Hope, in 1873 universally in the United States. A more widespread visitation of both hemispheres is credited to the years 1874 and 1875. Both Europe and America reported outbreaks in 1879 .

In the spring of 1889 there was an epidemic in Greenland and the Hudson Bay territory. By May it had reached Bokhara, Russia. Lasting there until August, it traveled to Siberia and reached St. Petersburg in October. This was the beginning of the greatest pandemic ever witnessed up to this time. The widespread nature of this pandemic and the fact that the mortality statistics of the time are available, make it most suitable for comparison with our most recent epidemics. 
An account of this epidemic in Europe is to be found in an editorial of the British Medical Journal, 1890, i, No. 40. This was written about January, 1890, or before London was seriously affected.

The first European cases were reported in St. Petersburg about October 15. By November 12, the disease had extended to all parts of European Russia. In Tomsk in Central Siberia, 2000 miles from St. Petersburg, the disease was also reported present about October 15 .

It was estimated that 650,000 people in St. Petersburg were attacked. This represented three quarters of the population. At Meso, 500 miles east of the Caucasus, 35 per cent of the garrison were sick by the end of December. Berlin was not affected until about the end of November. It was estimated that one-third of the population were attacked. Within three weeks, or by December 18, influenza was present in nearly every important town from Hamburg in the north to Munich in the south. Tramway service at Mainz was partially suspended, owing to the large number of men sick. By December 23, influenza had reached Dresden and on the 27th it was reported at Prague. It was present in Vienna on December 11, Belgrade the sixteenth, Bucharest and Sophia the twentyfourth. In the north of Europe it appeared in Brussels about December 12, and in Antwerp on the 16th. Schools were closed in these cities. It had begun to decline in Amsterdam by December 24. It was in Copenhagen early in December, 6000 eases being reported the week ending the 21st. In Paris it was observed as early as November 17 and began to assume epidemic proportions by the 26th. In the Pyrenees it was observed on December 12 at Málaga. Madrid reported it on the fourteenth. By December 29 most of the Spanish provinces were affected. It appeared in Lisbon about the third week in December. Italy reported influenza on December 13. Up to the first of the year influenza had made little or no impression in England.

In the same issue of the Journal above referred to, we read a description of the disease as given at a meeting of the Paris Academy of Medicine:

"The epidemic of grippe assumes a more malignant character, and does not confer immunity by an attack; there are many instances of the same person being seized two or three times. The cephalic and muscular pains, which were at the onset of the epidemic the striking symptoms, are now combined with serious pulmonary trouble, bronchitis, pneumonia and bronchopneumonia. Tuberculous conditions become aggravated, dangerous pneumonia and pleurisy set in, and in many instances cause death * * * * Entire loss of appetite habitually attends an attack of grippe, but those not attacked complain of want of appetite."

The Vienna correspondent to the Journal wrote as follows:

"In the majority of cases the disease has nervous and catarrhal character; the gastric symptoms have been observed in a relatively small number of cases." 

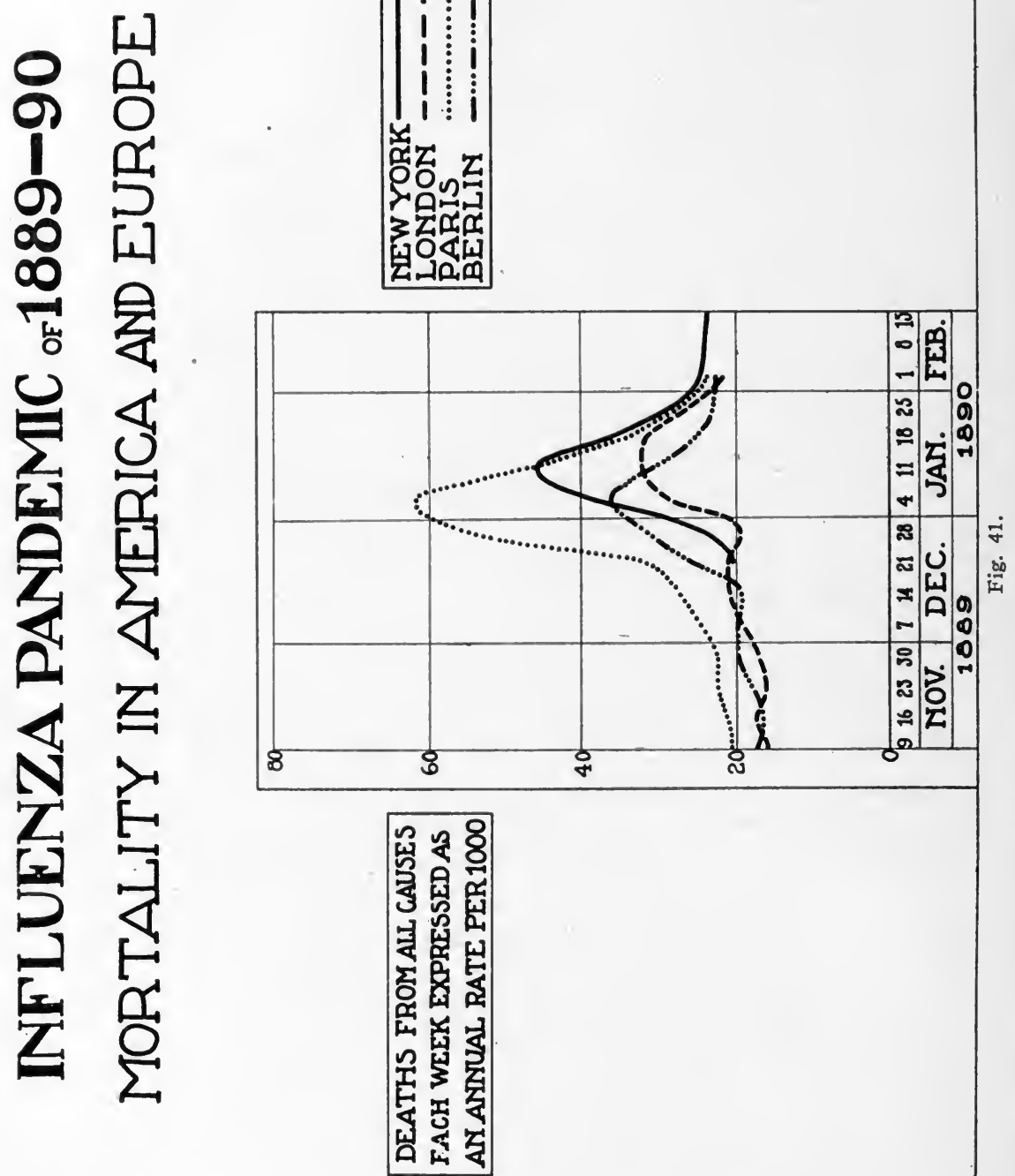

"The epidemic has been very severe among the tramear and postoffice employes; among the latter those whose work exposed them much to the weather suffered most."

The disease is reported as very prevalent among teachers and school children.

The epidemic in Europe, as we have seen from the above, followed the frequented routes of travel. The more accessible the place by rail transportation, the quicker the transmission of the epidemic. Requiring over 
two months to reach St. Petersburg from Bokhara, it took about six weeks to reach Berlin and from Berlin to the other cities of Germany, to Austria Hungary, Holland, Denmark, Italy and Spain was but a matter of a week or two.

In 1830 the pandemic consumed eleven months in passing from Moscow to North America. In 1889 it traveled the same distance in three months.

In the latter part of December, 1889, influenza was well established in England and America. By January, 1890, it was reported in Asia Minor, Constantinople and Athens. It was also present at this time in Morocco, Mexico, South America and Australia.

The mortality by weeks in the cities of New York, London, Paris and Berlin for the period of the epidemic is shown in the chart. (Fig. 41.) Paris appears to have reacted first, Berlin next, then New York and lastly London. The peaks of the total mortality curve are but a week apart. Some rural areas in England were not affected until four months after the cities. The West Indies apparently escaped the infection in these years.

In America the disease evidently first appeared around New York and Boston about the beginning of the second week in December, for the mortality rates showed a distinct rise the week ending the twentyeighth. It will be noted that these coastal cities received their infection as early as Vienna, Belgrade or Copenhagen. With an established shipping service between the German ports and America this is not strange. It is not the lineal distance between places that governs the influenza transmission rate, but rather the degree of intercourse. Judg-

TABLE XXXVII

Influenza Epidemic of 1889-1890; Annual Death Rate Per 1000 from Ali Causes

\begin{tabular}{lccccccccr}
\hline \multicolumn{1}{c}{ CITY } & DEC. 21 & 28 & JAN. 4 & 11 & 18 & 25 & FEB. 1 & 8 & 15 \\
\hline Milwaukee & 15.0 & 13.2 & 15.6 & 18.3 & 23.7 & 23.2 & 30.6 & 23.2 & 16.0 \\
St. Louis & 17.5 & 14.6 & 17.3 & 15.0 & 20.1 & 18.9 & 23.2 & 19.9 & 15.9 \\
Cleveland & 18.3 & 14.0 & 20.1 & 26.9 & 29.7 & 28.7 & 28.7 & 21.9 & - \\
Minneapolis & 12.0 & 12.4 & 13.6 & 18.0 & 23.5 & 21.5 & 18.7 & 15.5 & 13.3 \\
Providence & 13.8 & 18.9 & 28.4 & 39.8 & 41.8 & 27.2 & 27.2 & 25.7 & 22.8 \\
New York & 20.7 & 22.3 & 34.7 & 42.5 & 36.1 & 27.1 & 23.9 & 23.5 & 22.7 \\
Washington & 24.0 & 18.3 & 21.5 & 29.8 & 33.9 & 36.4 & 25.5 & 21.7 & - \\
Fall River & 12.6 & 22.4 & 13.4 & 26.6 & 30.9 & 35.8 & 18.3 & 25.9 & - \\
San Francisco & 17.1 & 22.4 & 16.4 & 21.6 & 34.7 & 35.0 & 37.3 & 30.0 & 23.0 \\
Cambridge & 19.4 & 22.3 & 22.3 & 35.7 & 23.1 & 19.4 & 14.9 & 20.1 & - \\
Boston & 22.4 & 27.0 & 40.5 & 48.3 & 40.1 & 24.9 & 22.3 & 19.7 & 22.3 \\
Lowell & 21.5 & 30.9 & 26.2 & 28.2 & 45.6 & 38.9 & 36.9 & 17.4 & 26.8 \\
\hline 12 cities & 19.5 & 21.2 & 29.1 & 35.4 & 33.6 & 27.0 & 25.1 & 22.7 & 21.3 \\
\hline
\end{tabular}

(These figures are from the 21st Annual Report of the Massachusetts State Board of Health.) 
ing by the dates, New York and Boston were infected from Germany, for the eastern European cities reported the disease concurrently with America.

Along with the increase in the New York and Boston rates in the week ending December 28, there was a similar tendency evidenced in Providence, Fall River, Cambridge and Lowell. In the following week (ending January 4), Cleveland and Washington were visibly affected. In fact Washington's rate seems unusually high a week before the rise at New York and Boston, but instead of continuing to rise, it fell back abruptly the following week. In the week ending January 11, the deaths began to mount in Milwaukee and Minneapolis. St. Louis and San Francisco were affected simultaneously in the week ending January 18, or between three and four weeks later than Boston. By the middle of January, therefore, the United States was widely infected.

It was not until the first part of February that Boston's rate had returned to normal. Altogether the epidemic lasted about seven weeks in Boston and for not over six weeks in the middle western eities. The highest rate reached in Boston was 48.3. This occurred in the third week of the mortality increase.

In the eities at least, the epidemic disappeared with the approach of spring and was not again heard of for over a year. In March and April, 1891, there was a recurrence of the infection of such intensity as to cause as many deaths in places as during the initial appearance.

Abbott sums up his analysis of the 1889-1890 epidemic in Massachusetts with the following significant statements:

1. The ratio of population attacked was 40 per cent $(850,000$ people340,000 cases).

2. People of all ages were attacked, but the ratio of adults was greatest, of old people next and of children and infants least.

3. Males seemed to suffer more than females.

4. The average duration of the acute stage of attack was from three to five days.

5. The predominant symptoms were mainly of three general groups -nervous, catarrhal and enteric, the last being much less common than the others; the special symptoms most observed in the nervous group being extreme depression, pain and weakness; in the catarrhal group, cough, dyspnea and coryza; in the enteric group, nausea, vomiting and diarrhea.

6. Bronchitis and pneumonia were the chief complications.

7. About 27 per cent of all employed persons were absent almost five days because of the disease.

Shattuck writes that 40 per cent of the employes of the Pacific Mills in Lawrence, Massachusetts, were sufficiently affected to cause an ab- 
sence from work of $61 / 2$ days. Among 4242 employes, 1699 had influenza, and of these, eight had pneumonia, two eases resulting fatally. This is a much smaller mortality than reported elsewhere.

The epidemic which began in December, 1889, showed recurrences in the spring of 1891, the winter of 1891-1892, the spring of 1893' and the winter months of 1893-1894. The figures for the state of Massachusetts, as reported by Frost in Public Health Reports for August 15, 1919, are given in Table XXXVIII.

\section{TABLE XXXVIII}

Influenza Death Rates (Annual Per 100,000) in Massachusetts-by Months 1889-1894

\begin{tabular}{lrrrrrr}
\hline \hline MONTH & & & \multicolumn{5}{c}{ YEAR } \\
& 1889 & 1890 & 1891 & 1892 & 1893 & 1894 \\
\hline Jan. & .09 & 10.96 & .39 & 24.33 & .58 & 7.41 \\
Feb. & .09 & 2.90 & .39 & 7.64 & .70 & 3.16 \\
Mar. & .14 & 1.43 & 1.00 & 3.02 & 1.20 & 1.38 \\
Apr. & .14 & .94 & 3.87 & 1.83 & 2.57 & 1.09 \\
May & .05 & .40 & 4.65 & 1.10 & 2.07 & .40 \\
June & .05 & .36 & 1.57 & .72 & .46 & .20 \\
July & .05 & .22 & .52 & .51 & .17 & .16 \\
Aug. & 0. & .04 & .52 & .17 & .12 & .08 \\
Sept. & 0. & .18 & .52 & .21 & .04 & .16 \\
Oct. & \multicolumn{1}{c}{.09} & .22 & .22 & .38 & 0. & .20 \\
Nov. & 0. & .27 & .26 & .68 & .46 & .16 \\
Dec. & .55 & .40 & 10.08 & .47 & 3.90 & .36 \\
\hline
\end{tabular}

The report of the Surgeon General of the United States Army for 1896 contains an account of an influenza outbreak at Plattsburg barracks in that year.

The report of Frost already referred to shows a definite increase in mortality in both New York City and Cleveland in December, 1915, and January, 1916. The increase is stated to have been general over the Registration Area, influenza in epidemic form with little fatality being reported in January, 1916, from twenty-two states. In both New York and Cleveland the mortality from influenza and pneumonia during January, 1916, 1917 and 1918, is distinctly greater than in the years preceding. The annual death rate per 100,000 from influenza and pneumonia in Cleveland was as follows: 
The figures are very suggestive of a gradual increase in the prevalence of this disease for several years prior to the 1918 outbreak.

The variation from year to year since 1887 is illustrated in the Massachusetts experience as reported by Frost.

TABLE XXXIX

Death Rates from Influenza and Pneumonia in Massachusetts-by Years, from 1887 to 1916. (ANNUAL RATE PER 100,000)

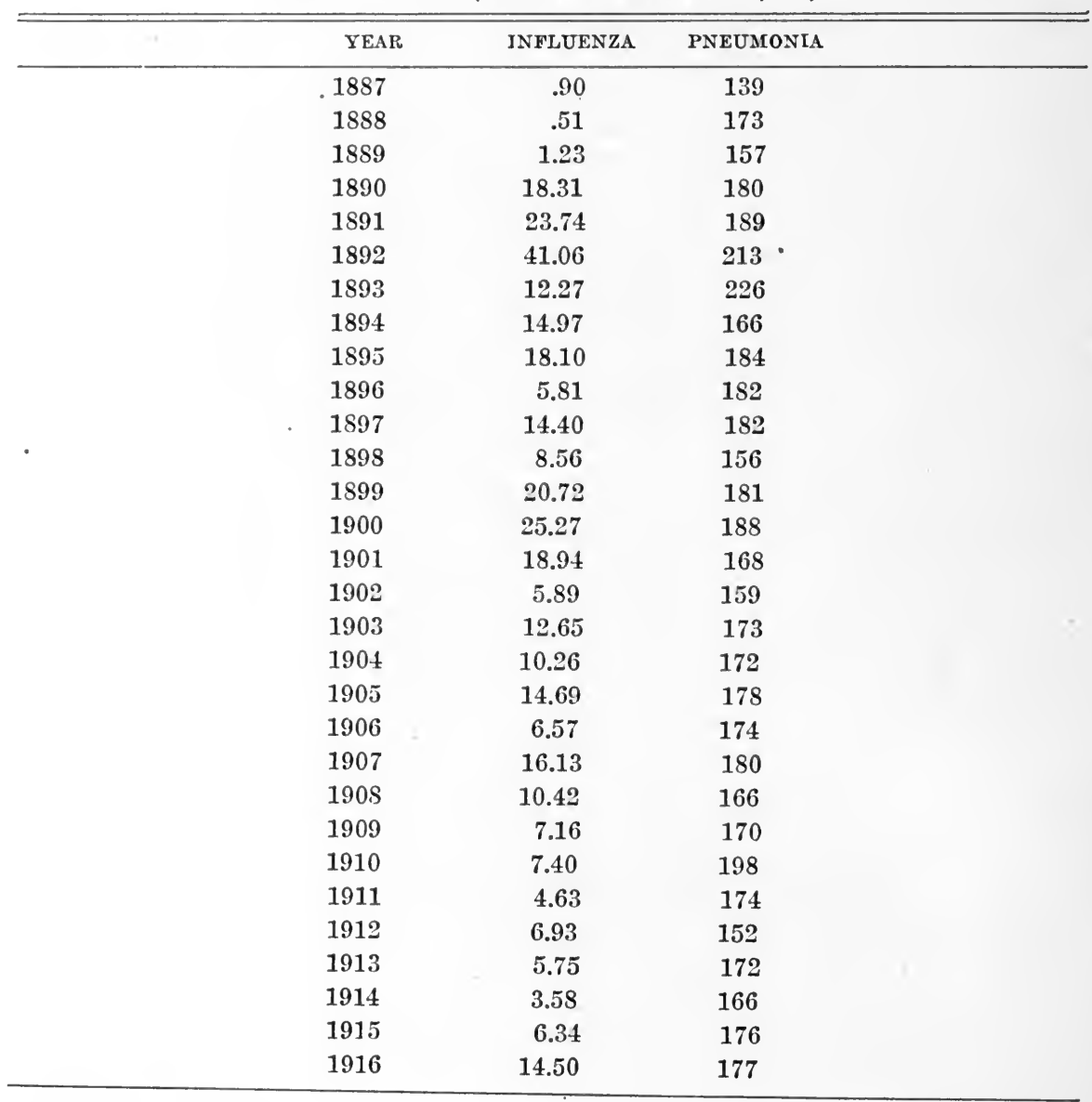

Biehn states that from 38 per cent to 82 per cent of school children in Chicago were attacked. The American Journal of Clinical Medicine quotes figures from the Chicago Health Department showing that up to December 1, 1916, deaths from pneumonia did not exceed 50 per week. The deaths for the weeks following were:

\begin{tabular}{|c|c|}
\hline $\begin{array}{l}\text { December } 11 \\
\text { December }\end{array}$ & 77 \\
\hline
\end{tabular}


An epidemic this same year occurred in Worcester, Mass., and is reported by Coffey.

From the literature of the time, it appears that the 1916 outbreak in this country originated in the west, first attracting attention in Denver. It then spread eastward, Chicago being affected before Massachusetts.

Dublin gives the accompanying tabde (Table XL) in which the deaths from influenza and pneumonia during the months of December, 1914, and January, 1915, are compared with deaths from the same cause during the months of December, 1915, and January, 1916.

TABLE XL

\begin{tabular}{lcccc}
\hline \hline \multirow{2}{*}{ CITY } & \multicolumn{2}{c}{ DEATHS REPORTED AS } & \multicolumn{2}{c}{ DEATHS REPORTED AS } \\
DUE TO INFLUENZA & DUE TO PNEUMONIA \\
\hline Baltimore & $1915-1916$ & $1914-1915$ & $1915-1916$ & $1914-1915$ \\
Cincinnati & 57 & 12 & 219 & 101 \\
New Orleans & 81 & 2 & 105 & 84 \\
New York & 97 & 44 & 35 & 29 \\
Philadelphia & 494 & 62 & 2067 & 1207 \\
Providence & 324 & 62 & 564 & 272 \\
Total & 38 & 3 & 31 & 31 \\
& 1091 & 185 & 3021 & 1733 \\
\hline
\end{tabular}

This writer states that the Industrial Department of the Metropolitan Life Insurance Company covering the entire country and embracing ten millions of people, had the following experience:

In December, 1914, and January, 1915, the number of

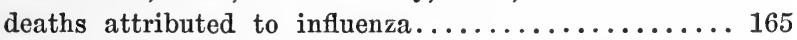

While in the corresponding months of 1915-1916 the deaths attributed to influenza.............. 957

The deaths attributed to pneumonia in December, 1914,

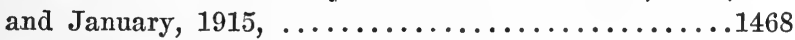

While the number of deaths attributed to the same cause in December, 1915, and January, 1916, .........2563

There are some who believed the excessive incidence of pneumonia in 1917 in the United States Army camps was preceded by influenza, but the records do not bear this out. The Surgeon General's report for 1917 shows that but 19 cases of pneumonia resulted from the 32,248 reported cases of influenza, which is but 0.059 per cent, quite different from the autumn of 1918, when from 15 to 20 per cent of influenza cases terminated in pneumonia.

Looking back over the epidemics that have spread through Europe, we find that six entered through Italy and proceeded westward and to the south-one passed from England or Ireland to the continent; one passed 
eastward and south from France; one passed to Europe eastward from America and one westward from America. These figures are approximately correct and are sufficient to indicate a general, though by no means universal, trend of pandemics or at least of continental pandemics westward through Europe, the starting point being either Asia or Africa or else within Europe itself. Ordinarily these epidemics have affected many people, sometimes the majority of the population, and yet the fatalities have been relatively slight, quite different from the pandemics of 1889-90 and 1918. The fatalities in former epidemics apparently have been mostly confined to the old and the very young. Epidemics have occurred at all seasons of the year, although the pandemics have been associated more frequently with the winter months. Germany is mentioned as the site of epidemics with greater frequency than most countries. This is probably due to more efficient reporting. We have few records of what happened in the Orient and yet there must have been many outbreaks there.

Historical accounts of pandemics, from 1580 include 1732, 1781, 1830, $1833,1836,1847,1850-51,1855,1857-58,1874-75$ and 1889. The intervals between these years, starting with 1510 and including 1557 and 1567, are $47,10,13,152,49,49,3,11,2,4,2,16$ and 14 .

The years intervening between pandemics in this classification vary from 2 to 152. In six instances the interval is 11 years or less.

Considering North America alone we find epidemic years given as $1557,1580,1647,1732,1737,1760,1780,1789,1805,1824,1830,1836$, 1843, 1850, 1860, 1863, 1873, 1874, 1879, 1889, 1891, 1896, 1916, 1918. The yearly intervals in this instance are $23,67,85,5,23,20,9,16,19,6$, $6,7,7,10,3,10,1,5,10,2,5,20,2$.

With these facts as a guide it is quite out of the question to predict what we may expect in the years to come. As to the appearance of the next pandemic, how widely it will be disseminated and what havoc it will play, we can now do little more than speculate.

Coming now to the most recent manifestations of influenza, we shall find that in the pandemic which swept the world in 1918 and 1919 and again in the winter of 1919 and 1920, greater havoc was wrought than in 1889-90. We can scarcely conceive of a more deadly scourge of wide dimensions and yet, with precise figures lacking in the preceding pandemics, it is impossible to state with any assurance whether or not the 1918-1919 outburst was the most destructive of all. It has been so proclaimed and there is much to support the belief.

Entering this country about the first of September, 1918, influenza spread from place to place in a surprisingly short space of time. Great bodies of men were centered in army camps training for overseas service in the World War. Influenza stalked through these temporary cities, 
claiming victims right and left from among the strongest and most virile of America's manhood. Never before had the Army Medical Staff been confronted with such an appalling amount of sickness. Hospital facilities were quite inadequate and accommodations had to be made overnight in barracks, tents, mess halls, auditoria and elsewhere. In some camps 30 per cent of the strength were sick within a period of three weeks. At Devens, a camp of 30,000 men, the Base Hospital normally accommodated 1800 to 2500 . During the epidemic there were housed in this hospital at one time 8,000 patients. This necessitated a degree of crowding which would never have been permitted under normal circumstances. The well were impressed into service, unfamiliar as they were with their duties, as nurses and attendants to the sick. At one time 90 of the 300 regular female nurses at Devens were down with the disease: So rapidly did the deaths mount that many camps were unable to prepare bodies for burial.

We speak first of the army camps, for here the mortality was greatest. In the civilian areas the epidemic played equal havoc with those people of the same age as the soldiers, but taking the population as a whole, the devastation was much less than in the camps. It was the young adult from 20 to 35 who suffered most. The dissemination of the disease was no greater at this age group than at others, but the fatalities were greater. Male and female, the young and the old, the strong and the weak, the well-to-do and the poverty-stricken, all felt the heavy hand of the pestilence. Truly influenza was no respecter of persons.

While we shall think of the epidemic in this country as beginning in September, it will be well to point out certain events which preceded. Influenza was not unknown before this date. In fact many camps and civilian communities had witnessed an outbreak in the spring of no mean proportions and yet relatively mild in fatality.

The annual report of the Surgeon General of the Army gives a record of 32,248 cases of influenza during the year 1917. While this number is impressive, yet the disease was not of sufficient import to attract attention. But nineteen cases of pneumonia resulted from these cases and but seven deaths. However, the sickness caused an average loss of time of 4.13 days per admission. This is in contrast to bronchitis, which caused but 0.59 days' loss per admission.

Influenza occurred sporadically at Camp Kearny, in California, during December, 1917, and Janıary, 1918. Thíere were 537 eases in all and the average time spent in the hospital for each case was ten days. Camp Johnston, in Florida, reported 173 mild influenza cases in January with no deaths. Epidemies of streptococeic character resembling influenza occurred at Camp Lee, in Virginia, in January and February. Camp 
McClellan, in Alabama, reported that during the six winter months, October, 1917, to March, 1918, influenza next to colds and sore throat, was the most prolific cause of sickness. Small epidemics of influenza were reported from Camp Sevier, in South Carolina, during the winter of 1917 and 1918. Bronchopneumonia and empyema likewise increased at such time. During the period from September, 1917, to April, 1918, 321 cases of influenza were diagnosed at the Base Hospital at Camp Cody, in New Mexico. It is said that these diagnoses were not substantiated by laboratory findings and were given for want of a better name. The total number of eases of bronchitis and influenza was much larger than this, as many cases were reported at the regimental infirmaries and many were not reported at all. At Devens, in Massachusetts, during the four winter months, December, January, February and March, there were more than 3,300 cases variously diagnosed acute bronchitis, pharyngitis, laryngitis and tonsillitis, under treatment at the Base Hospital alone, which means a much larger number cared for elsewhere.

In March influenza seemed to pass from the sporadic to the epidemic stage, many camps reporting increases at this time. Lewis, in Washington, reported 69 cases of influenza in January, 40 in February and 577 in March. As a rule influenza was a disease of recruits, but in March the infection spread among the more seasoned troops as well. Soper reports that Camp Forrest, Georgia, was invaded by an influenzal infection on March 18, and within two weeks it reached every organization, including the detached Reserve Officers' Training Camp, but it did not invade the more isolated prison camp. It is estimated that out of a total strength of 28,586 in the different organizations at Ft. Oglethorpe at that time, 2,900 or about 10 per cent of the whole had the disease. The number of cases sick enough to be detained in quarters or sent to hospital was 1,468 . The epidemic apparently reached its height by the end of the third week and then rapidly subsided.

The most striking symptoms as given by Soper were as follows: Headache, pain in the bones and muscles, especially in the back; marked prostration; chills followed by fever, the temperature rising rapidly and often reaching $104^{\circ}$. Redness of the conjunctivæ and inflammation of the nose and throat were almost constant manifestations. In a few cases there was a definite rash; in many there was nausea and in a few vomiting.

Influenza occurred at Camp Bowie, in Texas, in the latter part of March to such an extent that from 75 to 100 cases were reported daily. Many pneumonias, mostly Type IV, followed these infections. The pneumonias were mild in character and of short duration. At least 25 cases of pneumonia during the last week of March may be ascribed to the outbreak of acute catarrhal infections. 
Camp Funston, in Kansas, had a well marked influenza outbreak in March, as will be seen from the chart. Every organization in camp was attacked. Between March 4 and 29, 1127 men were sent to the Base Hospital and many more were cared for in the infirmaries of the camp. The outbreak spent itself in about two weeks. The disease, except in mild cases, was accompanied by fever with temperature from 99 to 103 degrees. Other characteristic symptoms were marked prostration, severe headache, aching pains in the muscles of the back of the neck, lumbar region, arms and legs. Coryza and cough were rare symptoms. The temperature fell in from 24 to 48 hours and the patient felt better. In general the epidemic was mild but it visibly increased the pneumonia incidence. (Fig. 43.)

The prevalence of pneumonia in civil life during March and April is suggested by the elevated mortality rates of numerous cities, notably Kansas City, Jersey City, Newark, New York City and Washington. The disease was not recognized as epidemic influenza, but the definite knowledge of its presence in some cities at this time and the fact that the excess mortality was charged to pneumonia is very suggestive of influenza as the primary cause.

As seen in the Funston chart, a second wave of influenza of lesser proportions appeared in the early part of April, lasting from the third to the 13th. On this occasion the disease was confined to recruits who had arrived in camp a few days before the epidemic began. The March epidemic was not confined to recruits. In fact the units which suffered most severely had been organized six months or more, and the men had had from three to six months' service. On April 28 a third wave of influenza broke out among the very recent draft men and continued for about two weeks. There is a close relation between pneumonia and influenza as disclosed by the rise in the pneumonia rate coincident with or shortly after the influenza rise.

Pneumonia was confined to the organizations then suffering from influenza. The immunity left by the first infection in March is indicated by the distribution of the disease among old and new men. At first the longer service men were attacked and apparently all susceptibles were reached. After that it was only the recruits who were affected.

TABLE XLI

Per Cent Distribution of Influenza Cases Among Recruits and Seasoned Men

\begin{tabular}{lccc}
\hline MONTH & $\begin{array}{c}\text { UNDER 1 Mo. } \\
\text { SERvice }\end{array}$ & $\begin{array}{c}1-3 \text { MOS. } \\
\text { SERVICE }\end{array}$ & $\begin{array}{c}\text { OVER } 3 \text { Mos. } \\
\text { SERVICE }\end{array}$ \\
\hline March & 8.2 & 7.9 & 83.9 \\
April & 70.2 & 13.4 & 16.5 \\
May & 66.8 & 17.1 & 16.1 \\
\hline
\end{tabular}




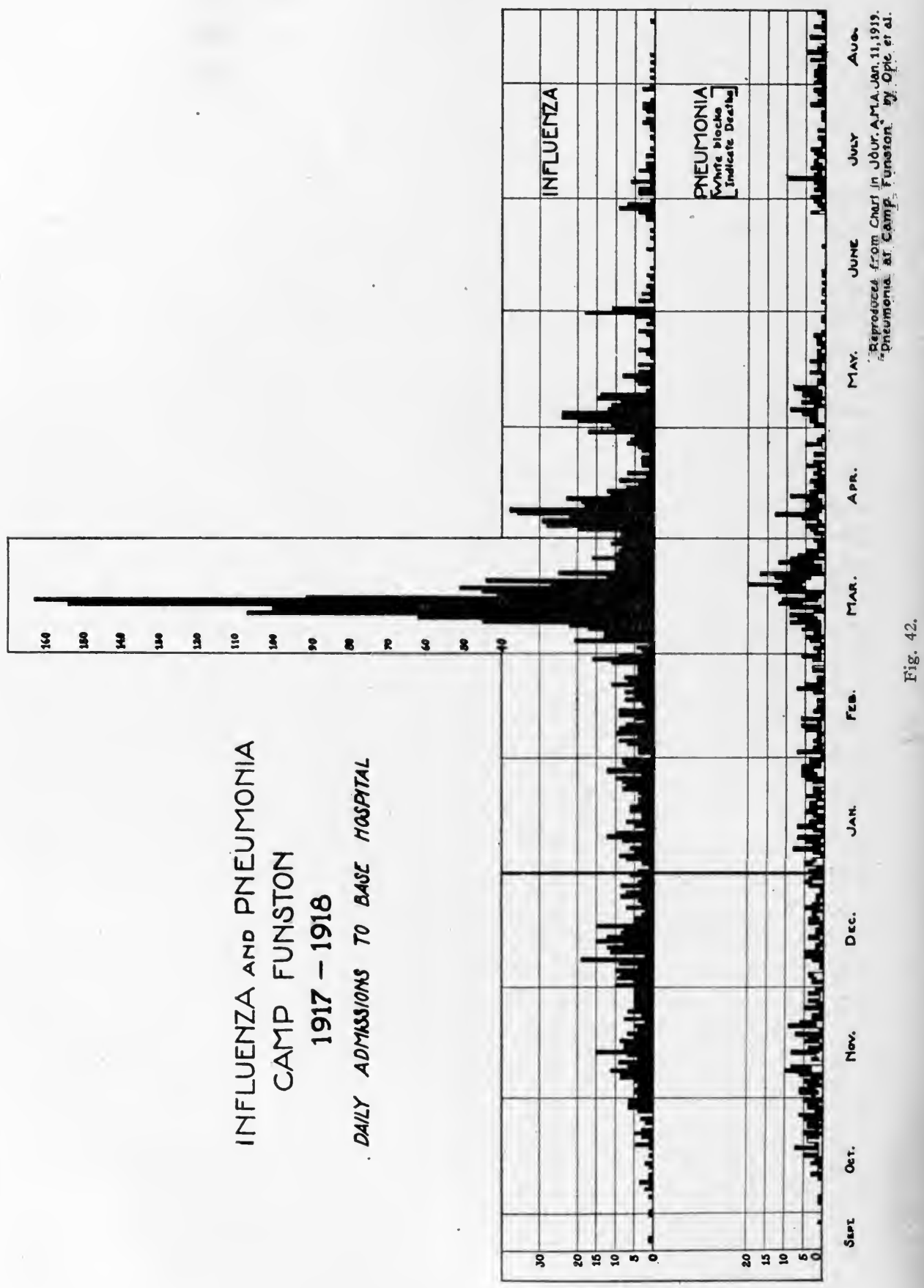


Influenza was the prevailing disease at Camp Beauregard, in Louisiana, in April and practically all cases of lobar pneumonia from April 15th to the end of May were sequelæ to influenza. Numerous cases of empyema followed these pneumonias. Camp Johnston was visited by a mild influenza outbreak in April, 111 cases being reported as against 47 for March, 87 for February and 173 for January. Camp Logan reported an intense form of streptococcic infection in April among 7,000 recruits from Camps Taylor, Grant and Dodge, this illness being confined to the men from Taylor and Dodge. Septic sore throat, scarlet fever and empyema were common.

From April 8 to 13 an influenza outbreak occurred at Camp Shelby, in Mississippi, 643 eases being treated in the hospital. Cases treated elsewhere and undiscovered cases were estimated to be twice this number. The epidemic was explosive in character and attacked all classes of men.

This influenza, though mild, was typical of that occurring later in the year. The symptoms of a specific case, G. E., were as follows:

Symptoms began 36 hours before entering hospital. Started with a continuous frontal headache. The following day had a severe chill, ached all over and had a high fever. Chill was repeated in evening. Entered hospital on second day. Had no more chills, but carried a high fever, and complained a great deal of aching, and a moderate degree of weakness. Bowels were normal before attack, but constipated since. No abdominal cramps. Slight cough and was able to expectorate small amounts of purulent sputum. Appetite good. Urination normal. Temperature 102 , pulse rate 120 , respiration 28 . The physical examination of the patient revealed a marked fetid odor of breath and a marked congestion of conjunctiva. The pharynx showed a high degree of granular type of congestion with a rather definite line of demarcation between hard and soft palate. The tongue showed a heavy white coating. Posterior chain of superficial cervical glands felt shotty. The lungs showed few bronchial rales at the base, otherwise normal. Heart was normal. K. J. and biceps reflex exaggerated; no Babinski; no eruption.

Camp Kearny was visited by epidemic influenza in April and from April 10 to June 1 there were 785 admissions to the Base Hospital from this cause. The disease was mild in character although there were some complicating pneumonias. The infection was believed to have been introduced by the Japanese fleet which was visiting San Diego at this time, and from which several cases of influenza were brought ashore for treatment.

Epidemic influenza during April was reported outside of the army. Two thousand cases occurred among the employes of the Ford Motor Company, Detroit. On April 26 a mild but marked outbreak appeared in the Naval Hospital at Gulfport, Mississippi. 
Increased death rates in the spring are to be noted also in the following cities; Pittsburgh, Milwaukee, Minneapolis, Denver, Worcester, Cleveland, Columbus, Grand Rapids, Toledo, Birmingham, Providence, Syracuse, Atlanta, Cincinnati, Indianapolis. In some cities high mortality rates continued into May.

Although the western coast cities do not show an elevated death rate, it is known that a widespread form of non-fatal influenza prevailed in San Diego and Los Angeles. More than one-fourth of the inmates in the California State Prison at San Quentin had influenza during April.

After April influenza seemed to disappear in the army camps and what little occurred was sporadic. Funston had a small outbreak among recruits as will be seen on the chart. Lewis reported 225 Base Hospital admissions for this cause as against 577 for March and 257 for April.

The U. S. S.— reported an epidemic of influenza commencing on May 6,1918 , with one case, followed by five cases the next morning, and twenty on the following day. In all cases the temperature ranged from $100^{\circ}$ to $105^{\circ}$ with but slight respiratory disturbance. All complained of occipital headache, pains in the back and limbs and malaise. The epidemic occurred during passage from Gibralter to an African port.

On May 25 a Dutch steamer arrived at Pearl Harbor, Hawaiian Islands from the Orient and reported a "grippe" epidemic aboard shortly after leaving Yokohama. It was in this month that influenza is reported to have ravaged Spain, thirty per cent of the population being attacked.

Among the army camps in this country influenza was rare or absent altogether during the summer. Lewis reported the fewest cases since February. Funston had some influenza among recruits as they arrived in camp. Shelby was free from influenza until the latter part of August when an outbreak occurred among summer recruits.

In Europe, Asia and the Pacific Islands and on ships traveling the high seas, June marked either the beginning or the crest of widespread outbreaks of influenza. Belfast was the first of the European cities of which we have record to show a rising death rate. The annual rates per 1000 by weeks for June and July were as follows:

$\begin{array}{rr}\text { Week ending June } 8 & 14.1 \\ \text { June 15 } & 17.1 \\ \text { June 22 } & 25.3 \\ \text { June 29 } & 45.2 \\ \text { July } 6 & 44.4 \\ \text { July } 13 & 28.3 \\ \text { July 20 } & 20.2 \\ \text { July } 27 & 17.4\end{array}$

The rate in Sheffield reached 27.9 in the week ending July 6. In the next weeks peaks were registered in Liverpool, Dublin, London, Leeds, 
Edinburgh, Birmingham, Manchester and Newcastle-on-Tyne. In the week ending July 20, Glasgow showed a slight increase to 15.9, Bristol to 15.7 , Bradford to 21.9.

In Continental Europe increases in mortality were much less pronounced than in England. The rates for June, July and August in several continental cities are given in Table XLII.

TABLE XLII

\begin{tabular}{|c|c|c|c|c|c|c|}
\hline . & \multicolumn{2}{|c|}{ WEEK ENDING } & HAMBURG & COPENHAGEN & CHRISTIANA & VIENNA \\
\hline & \multicolumn{2}{|c|}{1918} & & & & \\
\hline & \multirow[t]{4}{*}{ June } & 8 & 18.8 & 12.7 & 12.9 & 17.9 \\
\hline & & 15 & 23.6 & 11.1 & 11.3 & 20.8 \\
\hline$\theta$ & & 22 & 21.6 & 9.9 & 10.5 & 18.2 \\
\hline & & 29 & 20.9 & 9.9 & 10.9 & 19.0 \\
\hline & \multirow[t]{4}{*}{ July } & 6 & 21.1 & 10.8 & 11.7 & 19.3 \\
\hline & & 13 & 23.3 & 9.2 & 16.2 & 22.7 \\
\hline & & 20 & 22.5 & 10.9 & 24.2 & - \\
\hline & & 27 & 23.5 & 12.8 & 21.2 & - \\
\hline & \multirow[t]{5}{*}{ August } & 3 & 16.4 & 13.9 & 10.4 & - \\
\hline & & 10 & 16.6 & 14.7 & 13.0 & 15.6 \\
\hline & & 17 & 一 & 11.2 & 8.8 & 16.7 \\
\hline & & 24 & 16.6 & 9.7 & 7.0 & - \\
\hline & & 31 & - & 8.2 & 8.8 & - \\
\hline
\end{tabular}

Paris and Berlin show rate elevations, in the former very slight and in the latter a prolonged elevation and not a sharp peak as in the English cities.

Influenza is reported to have been at its height in Germany during June and July and the matter had reached such a critical stage in July that two conferences were held over it-one in Berlin and one in Munich.

In France the disease was prevalent from April through July. Talley, reporting in the Naval Bulletin for November 30, 1919, states that from April 1 to July 31, four months, there were admitted to the U. S. Naval Hospital No. 5 at Brest, fifty cases of influenza and 26 pneumonia. Among the French military and civil personnel there were during the same period 4,838 cases of influenza with 688 hospital admissions. Fiftythree deaths occurred among the latter. In general the infection was mild but at one station among 1018 young French recruits, 68 per cent were admitted to the hospital and 4.8 per cent of the entire personnel died. The severity of the infection in this case may be judged by the fact that in no camp in the United States, to our knowledge, were the morbidity and mortality so high. The epidemic in France had so far declined by the end of July that the enrollment of the class of 1920 was allowed to proceed.

Epidemic influenza is said to have begun in India the first of the sum- 
mer and spreading along the channels of communication soon extended to all parts of the empire. The infection at this time was ever so much milder than the epidemic which occurred in the autumn.

Bombay, India, exhibits a mortality peak of 77.8 the week ending July 6. Rates for other weeks in June and July were as follows:

$\begin{array}{rr}\text { Week ending June 8 } & 37.2 \\ \text { June 15 } & 33.2 \\ \text { June 22 } & 37.2 \\ \text { June 29 } & 54.6 \\ \text { July 6 } & 77.8 \\ \text { July } 13 & 63.0 \\ \text { July 20 } & 40.6 \\ \text { July 27 } & 34.2\end{array}$

In the week ending July 20, Calcutta had a rate of 52.9. In the following week Madras reported a rate of 63.6.

Influenza occurred in the Philippine Islands in June. According to Walker, Camp MeGrath was first visited by influenza on June 13, although the disease had been prevalent among the civilian population for some time. The strength of the command, most of which were Filipino troops, at this time was about 865 . Between June 13 and September 21, there occurred 209 cases indicating an infection of 24 per cent of the command. All but 10 of these cases occurred during June and July. The disease spread slowly, the greatest number of admissions on any one day being 19 on June 25 . The disease was mild but cost each patient about five days loss of time. But two eases of pneumonia developed and there were no fatalities.

Fort William McKinley, in the Philippines, was composed of 2,554 Regular Army officers and enlisted men (white) and 2.050 Philippine Scouts. Phelan, reported that influenza invaded the command on June 13 and lasted about two weeks. About 14 per cent of the command were affected and five deaths resulted. The greatest number of admissions on any one day was 192 on June 17. A second outbreak occurred on November 8 , affecting 8 per cent of the command, the personnel of which had been materially changed since June, and caused 2 deaths. On each occasion the native troops suffered more than the men from the States.

Influenza at Fort Mills, Philippine Islands, was mild, the cases widely distributed and occurring in such small numbers as hardly to warrant the term epidemic. This command consisted of 2,180 Americans and 1,500 Filipinos. The first cases were noted on November 2. The daily admissions for influenza never exceeded 14. During November there were reported 108 cases of influenza, 11 of pneumonia and 3 deaths. This represents an incidence of but 2.9 per cent. About 
10 per cent of the influenza cases contracted pneumonia. The mortality was .08 per cent of the entire command. It is believed that most of the influenza was contracted on visits to Manila.

The U. S. Military Hospital at Tientsin, China, reported that influenza began September 19. It was mild with a single exception, and there were no complications. The command comprised the 15th Infantry with detachments of the Medical, Quartermaster and Signal Corps. The strength averaged 1200. Between September 19 and December 31, 17 per cent had influenza. The soldier who died was believed to have had typical lobar pneumonia from the beginning. The disease was generally distributed throughout North China. The mortality among natives seemed to be greater than among Europeans and Americans.

Influenza was introduced into Hawaii apparently in May as we have noted. It did not reach epidemic proportions until June. The Naval Hospital at Pearl Harbor reports that the infection began about the middle of June and involved the whole personnel of the station. The symptoms were mild. Patients had fever and pain in the back and legs. Recovery followed in three or four days.

It was on June 26 that the epidemic first appeared at Schofield Barracks, Hawaii, a command of 8,456 men, consisting of 2,136 colored, 3,000 Filipinos, 1,500 Americans and 1,820 representing Hawaiians, Japanese, Chinese, Koreans, Portuguese, Spanish and Porto Ricans. Between June 26 and August 15, there were admitted to the Quarantine Camp 647 cases, or 7.6 per cent of the command. Three hundred and seventy of these cases occurred during the last three days in June. It is known that many other cases occurred which were cared for in quarters. There were 70 admissions for pneumonia during this time and 11 deaths. The predominating symptoms were headache, temperature as high as 105 degrees, increased pulse, general weakness, pains in back of neck and lumbar regions, cough with pains of pleurisy, watering of the eyes, rigors and râles. The duration of the disease was from a few hours to ten days. Lobar pneumonia was more frequent among the complications than bronchopneumonia.

The similarity of this Hawaiian epidemic in June with that of the army camps in this country is indicated by the relative immunity of the 2nd Infantry during the epidemic at Camp Dodge in the autumn. This regiment with one exception had the lowest incidence of any unit at Dodge (6.6 per cent). Its mortality was .29 per cent or one-seventh that of the Depot Brigade. The 2nd Infantry left the Hawaiian Islands June 29 and arrived in Dodge about August 1. The men contracted influenza before leaving Hawaii and 300 cases occurred between June 30 and September 7 .

There was a mild epidemic of influenza during the month of June in 
a marine regiment stationed at San Juan, Santiago de Cuba. Symptoms subsided in twenty-four hours.

The British steamship, "City of Exeter," arrived in Philadelphia on June 22, 1918, from Liverpool, having sailed from that port on the ninth of the same month. Upon arrival at quarantine, 27 Lascars and 1 English quartermaster were found to be ill with pneumonia. The pneumonia was post-influenzal. Two steamships from Norway and one from another Scandinavian port arrived in New York August 14 and 15, having on board a number of patients ill with the so-ealled "Spanish" influenza. There was a sharp outbreak of influenza at Fort Morgan, near Mobile, Alabama, during the month of August, 1918. The Norwegian liner "Bergensford," which arrived in New York on August 12, had 200 cases of influenza on board during the voyage. So far as the Navy was concerned, the epidemies during the summer were widespread but not fatal.

It was in the latter part of August that influenza in epidemic form reappeared in the army camps, this time at Shelby. The Shelby epidemic was mild but apparently less so than in the previous April.

We have purposely indulged in a rather detailed description of influenza prevalence prior to September because these facts are not well understood generally. The more severe manifestation of the disease in this country began around Boston about the first of September. Simultaneously this situation was being duplicated in Europe and Asia. There ean be distinguished very definite outbreaks of influenza as judged from the heightened mortality rates. About the first week in July, Belfast, London, Berlin and Bombay were affected. The rate in Calcutta rises the latter part of July. The third week in September witnessed an increased mortality in such widely separated ports as Boston and Bombay. In October the death rate ascends in New York, San Franciseo, London, Paris, Berlin, Stockholm and Calcutta. The second outbreak in Belfast was delayed till November. After this time we will note recurrences as follows: Deeember, Bombay; January, Boston, New York, San Francisco, Berlin, Calcutta; February, Belfast, London, Paris, Bombay. (Table XLIII ; Fig. 43.)

These figures are of the greatest interest in demonstrating that the epidemic did not pursue a linear course around the globe, burning itself out in one locality before proceeding to the next. On the contrary, the influenza infection was well distributed over the earth in the spring and summer of 1918. For some reason, possibly due to a seasonal influence, the disease was relatively non-fatal at this time, although Belfast was affected with a severity nearly as great as in November. The stage was already set upon the advent of colder weather. It was unnecessary for the infection to be re-transmitted from one side of the globe to the other. It was already well seeded in widely scattered points. Cer- 
TABLE XLIII

Mortality in American, European and Asiatic Cities in 1918-1919 AnNual Death Rate Per 1000 by Weeks

(Figures taken for the most part from weekly Census Bureau reports and reports of U.'S. Public Health Service. Rates in U. S. cities slightly in error as they were published before 1920 census.)

\begin{tabular}{|c|c|c|c|c|c|c|c|c|c|c|}
\hline 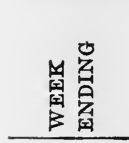 & 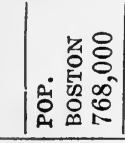 & 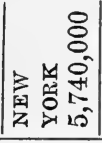 & 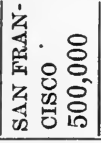 & 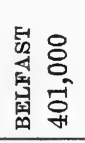 & 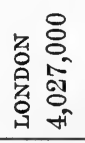 & 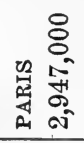 & 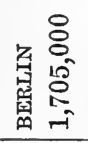 & 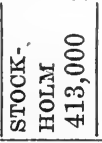 & 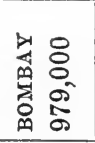 & 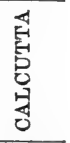 \\
\hline June 8 & & 11.0 & 14.0 & 14.1 & 11.2 & 11.1 & 16.6 & 10.1 & 37.2 & 24.9 \\
\hline June 15 & Did & 11.0 & 15.0 & 17.1 & 11.0 & 11.0 & 16.4 & 13.3 & 33.2 & 26.9 \\
\hline June 22 & not & 10.0 & 15.0 & 25.3 & 12.0 & 11.5 & 15.9 & 10.8 & 37.2 & 25.7 \\
\hline June 29 & & 12.0 & 13.0 & 45.2 & 13.2 & 11.2 & 19.4 & 10.9 & 54.6 & 26.6 \\
\hline July 6 & exceed & 10.0 & 12.0 & 44.4 & 17.3 & 11.5 & 20.9 & 13.3 & 77.8 & 25.6 \\
\hline July 13 & 15 & 10.0 & 12.0 & 28.3 & 18.4 & 13.0 & 16.4 & 8.1 & 63.0 & 31.3 \\
\hline July 20 & from & 11.0 & 15.0 & 20.2 & 15.2 & 11.5 & 18.3 & 9.7 & 40.6 & 52.9 \\
\hline July 27 & & 12.0 & 12.0 & 17.4 & 11.8 & 10.4 & 20.9 & 10.6 & 34.2 & 50.4 \\
\hline Aug. 3 & June & 10.0 & 13.0 & 15.0 & 11.4 & 10.7 & 20.1 & 11.3 & 33.2 & - \\
\hline Aug. 10 & to & 13.0 & $13 \dot{0} 0$ & 13.5 & 10.5 & 9.7 & 22.5 & 18.4 & 33.8 & - \\
\hline Aug. 17 & August & 10.0 & 11.0 & 15.7 & 9.8 & 10.7 & - & 12.6 & - & 25.1 \\
\hline Aug. 24 & & 10.0 & 10.0 & 13.8 & 9.6 & 10.6 & 23.2 & 12.1 & 31.1 & - \\
\hline Aug. 31 & & 10.0 & 10.0 & 12.7 & 10.5 & 12.1 & - & 11.5 & - & 25.4 \\
\hline Sept. 7 & 12.2 & 9.8 & 10.3 & 11.3 & 11.3 & 11.9 & 21.3 & - & 36.4 & 23.5 \\
\hline Sept. 14 & 14.9 & 10.1 & 12.8 & 14.5 & 10.5 & 12.9 & 21.9 & 10.6 & 51.0 & 28.0 \\
\hline Sept. 21 & 33.3 & 10.4 & 14.3 & 14.5 & 11.8 & 15.7 & 21.7 & - & 100.0 & 29.9 \\
\hline Sept. 28 & 67.4 & 11.0 & 12.8 & 10.2 & 12.2 & 15.9 & 20.5 & - & 169.1 & 32.8 \\
\hline Oct. 5 & 100.2 & 16.3 & 13.4 & 9.6 & 12.7 & 17.8 & 21.3 & 16.7 & 257.2 & 34.6 \\
\hline Oct. 12 & 87.3 & 30.1 & 14.6 & 14.1 & 13.9 & 26.0 & 29.2 & 33.5 & 220.4 & 41.6 \\
\hline Oct. 19 & 51.8 & 51.5 & 31.8 & 11.0 & 20.1 & 34.9 & - & 36.7 & 114.2 & 42.5 \\
\hline Oet. 26 & 29.6 & 61.6 & 77.2 & 13.8 & 34.5 & 46.1 & 41.7 & 40.5 & 72.1 & 47.1 \\
\hline Nov. 2 & 21.7 & 52.9 & 89.0 & 17.5 & 55.5 & 43.2 & 37.5 & 33.2 & 54.0 & 46.1 \\
\hline Nov. 9 & 17.5 & 35.6 & 67.2 & 34.0 & 55.5 & 28.4 & 22.0 & 26.4 & 43.3 & 53.2 \\
\hline Nov. 16 & 15.3 & 22.8 & 36.0 & 54.7 & 44.0 & 21.0 & 21.7 & 23.2 & 40.9 & 59.7 \\
\hline Nov. 23 & 17.1 & 17.9 & 23.0 & 65.0 & 36.8 & 17.9 & 21.1 & 22.1 & 40.1 & 58.5 \\
\hline Nov. 30 & 16.0 & 15.8 & 18.4 & 61.4 & 32.8 & 19.4 & 22.7 & 20.1 & 33.1 & 63.3 \\
\hline Dec. 7 & 16.9 & 16.0 & 18.6 & 41.7 & 26.0 & 20.6 & 23.7 & 20.9 & 36.2 & 56.5 \\
\hline Dec. 14 & 19.6 & 16.6 & 24.1 & 24.7 & 18.2 & 19.7 & 23.5 & 19.5 & 55.0 & 56.6 \\
\hline Dec. 21 & 22.2 & 17.1 & 30.6 & 19.2 & 14.4 & 15.9 & 14.7 & 20.1 & 86.5 & 53.3 \\
\hline Dec. 28 & 29.3 & 18.0 & 38.0 & 19.4 & 13.3 & 17.0 & 9.7 & 20.4 & 85.0 & 51.4 \\
\hline Jan. 4 & 32.4 & 19.8 & 40.0 & 17.3 & 16.6 & 17.8 & 22.3 & 23.0 & 101.0 & 47.2 \\
\hline Jan. 11 & 31.7 & 20.9 & 49.8 & 20.4 & 15.8 & 18.8 & 14.6 & 20.1 & 81.0 & 48.6 \\
\hline Jan. 18 & 26.2 & 24.3 & 50.9 & 15.7 & 14.8 & 18.5 & 20.9 & 15.6 & 76.5 & 56.1 \\
\hline Jan. 25 & 26.6 & 25.8 & 32.1 & 18.9 & 14.1 & 18.0 & 19.6 & 14.6 & 180.6 & 65.6 \\
\hline Feb. 1 & 19.3 & 24.5 & 21.9 & 16.4 & 16.9 & 18.1 & - & 15.6 & 237.8 & 51.1 \\
\hline Feb. 8 & 20.0 & 22.1 & 16.0 & 21.8 & 20.2 & 21.8 & - & 12.2 & 160.2 & 47.3 \\
\hline Feb. 15 & 17.4 & 20.9 & 16.5 & 22.8 & 27.2 & 26.2 & - & 13.6 & 92.1 & 45.2 \\
\hline Feb. 22 & - & 21.3 & 17.1 & 28.3 & 34.2 & 34.1 & - & 14.2 & - & - \\
\hline Mar. 1 & 18.5 & 21.6 & 19.8 & 30.9 & 32.4 & 30.3 & - & 14.5 & - & - \\
\hline Mar. 8 & 19.9 & 20.8 & 18.4 & 30.3 & 26.6 & 25.9 & - & 18.6 & - & - \\
\hline Mar. 15 & 18.3 & 19.5 & 16.8 & 33.9 & 21.4 & 20.4 & - & - & - & - \\
\hline Mar. 22 & 15.1 & 18.1 & 16.1 & 30.9 & 18.0 & 17.9 & - & - & - & - \\
\hline Mar. 29 & 15.9 & 17.5 & 15.7 & 26.9 & 17.6 & - & - & - & - & - \\
\hline
\end{tabular}




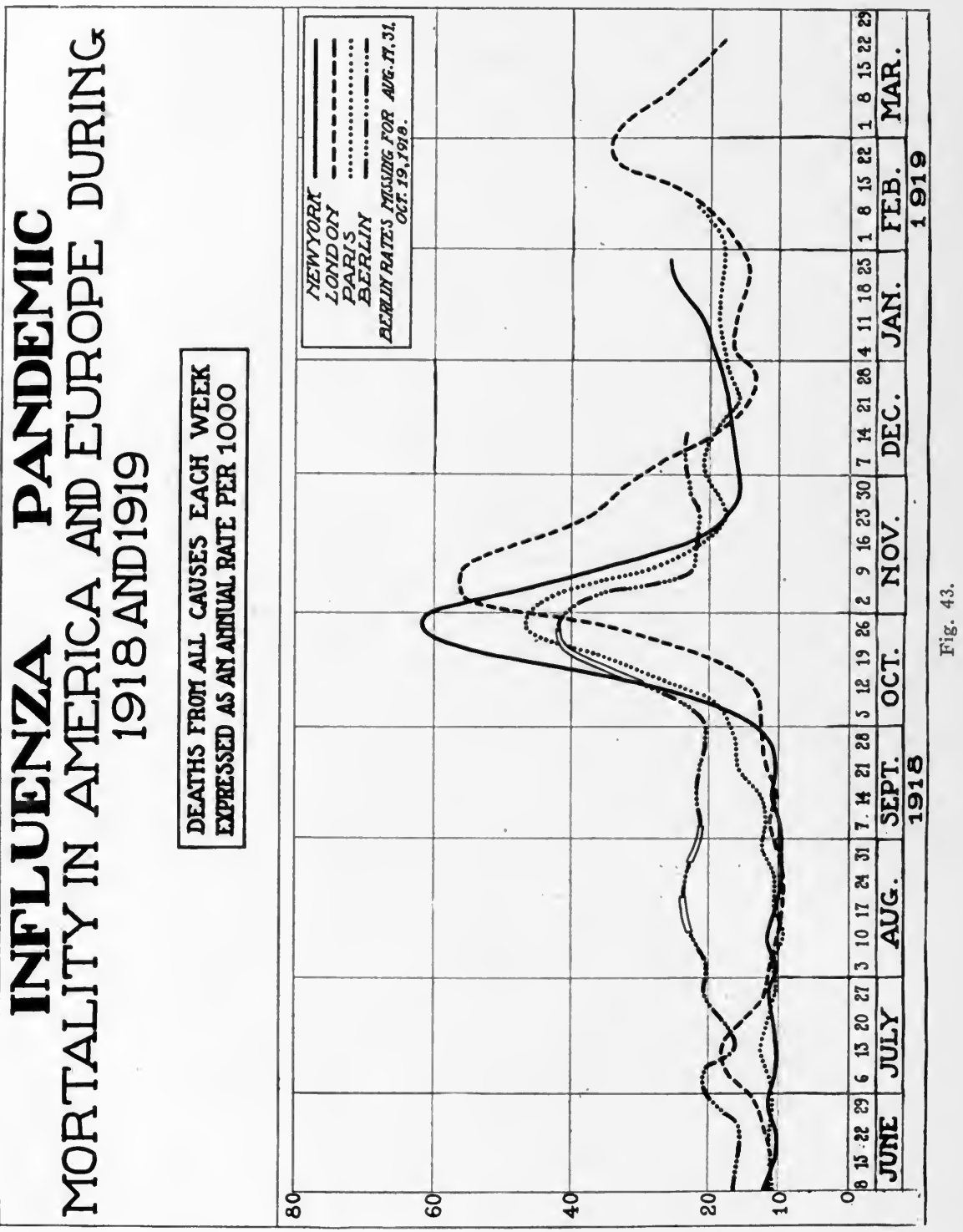

tain universal influences, which we little understand, reactivated the infection simultaneously in Boston and Bombay, causing an outburst of most malignant character.

Vaughan (Warren) believes that the 1918 pandemic had its origin in the United States; that the virulence of the organism was intensified by voyages to and from Europe, and that about the last of August, 1918, a highly virulent strain of the virus was introduced into this country 
from Europe, entering at Boston, possibly at other ports as well, and spreading all over the country.

Within our own country the autumn outbreak began around Boston. On Thursday afternoon, August 28, 50 cases of influenza were transferred from the Naval Receiving Ship at Commonwealth Pier, Boston, to hospitals on shore. The disease spread rapidly within the First Naval District so that by September 11, 2000 cases had been reported.

Early in the first week of September the disease had reached the Second Naval District and outbreaks occurred in the Philadelphia Navy Yard and as far distant as the Naval Station at Pensacola, Florida. By September 13, 420 cases had occurred among the Naval personnel in the vicinity of Newport, R. I. The epidemic made its appearance in the Philadelphia Navy Yard about September 11, the Philadelphia cases being traceable to Boston, several small drafts having been received from Boston about this time.

Keegan, writing in the Naval Bulletin of September 27, describes the serious character of this initial Boston infection in the following words:

"From 5 to 10 per cent of the cases in this epidemic have developed a very severe and massive bronchopneumonia. Of the 95 pneumonias in the hospital complicating influenza, 35 have died, and another 15 or 20 are desperately ill. This indicates a finai mortality of from 60 to 70 per cent.',

Following upon the outbreak in Boston, the infection burst forth in other cities, traveling from the east to the west coast.

Judging by the first significant increase observed in the total mortality, we may group the large cities of the country in their order of influenza occurrence as follows :

Week ending

September 13

September 20

September 27

October 4

October 11

October 18

\section{Cities}

Boston, Cambridge.

New Haven, Providence, Fall River, Syracuse, Worcester, Pittsburgh, Rochester.

Buffalo, Cincinnati, Denver, Grand Rapids, Philadelphia, Washington, Lowell, Baltimore, Toledo.

Atlanta, Birmingham, Chicago, Indianapolis, Kansas City, Louisville, Memphis, Milwaukee, Newark, New Orleans, New York, Richmond, Albany, Dayton, Nashville, Columbus, Omaha.

Minneapolis, Seattle, Cleveland, Los Angeles, Oakland, St. Louis, St. Paul, Spokane.

Portland, (Oregon), San Francisco.

While the general trend westward is clearly shown, it is singular to find the outbreak appearing so late in such eastern cities as New York and Cleveland.

Note: In the charts (44-49) of eities shown in the following pages the first horizontal line above the base represents an annual mortality rate from all causes of 10 per 1000. The succeeding lines are 20, 30, 40, 50, and 100 . 


\section{MORTALITY \\ IN U.S. CITIES DURING 1917-18-19}

WEETLY OCCURPENCE OF DEATHS FROM ALL CAUSES

EXPRESSED AS AN AYYUAL RATE PER 1000

\section{NEW ENGLAND}
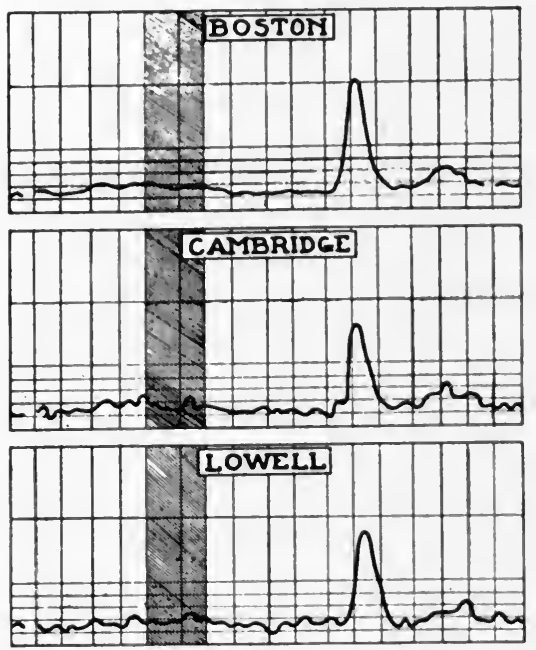
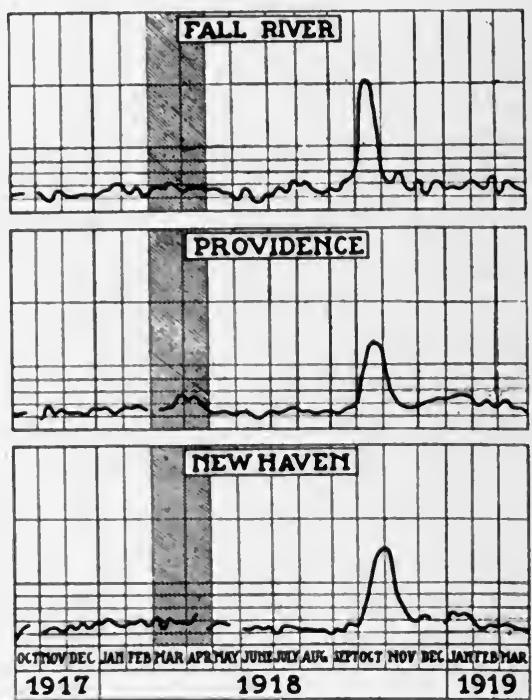

Fig. 44.

As a rule, the mortality peak occurred in the third week of increased mortality. In Boston the mortality rate began to increase in the second week or about fourteen days after the first cases of influenza were recognized. In Philadelphia, Washington, Chicago and New Orleans not even a week elapsed between the recognition of cases among civilians and an increasing mortality rate. The highest rates for any week were:

Philadelphia

Baltimore

New Orleans

Albany

Washington

Nashville

Pittsburgh

Fall River

Boston
Annual Case Rate per 1,000

158

148

120

112

109

104

101

100

' 100
Week ending

October 18

، 18

، 25

، 25

" 18

6 18

November 2

October 11

، 4 


\section{MORTALITY IN U.S. CITIES DURING 1917-18-19. \\ WEEKCY OCCURREYCE OF DEATHS FROM ALL CAUSES
EXPRESSED AS AY AYYUAL RATE PER 1000}

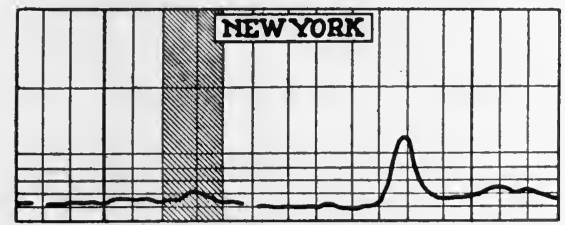

\section{MIDDLE ATLAITIC}
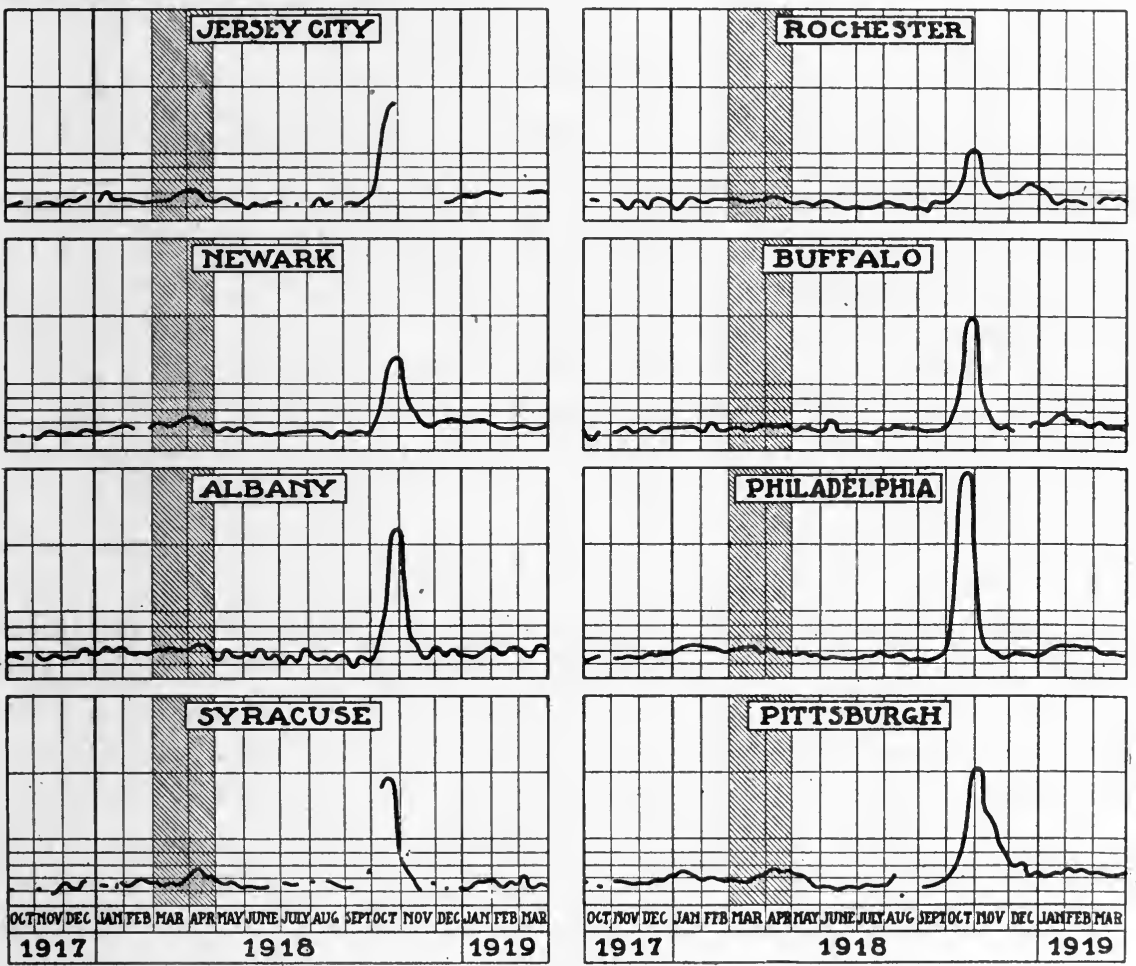

\begin{tabular}{|l|l|}
\hline 1917 & 1918 \\
\hline
\end{tabular}

1919

Fig. 45.

The disease was so widespread by October that every one of nearly 50 large cities which reported their death rates regularly to the Census Bureau manifested an increased mortality. There were marked differences among cities in the size of the mortality rate. The highest peak reached by Grand Rapids was 20.4. Neither Milwaukee nor St. Louis, nor Seattle exceeded 30. Philadelphia's maximum rate of 158 was thus five times that of St. Louis.

As will be observed in the charts, the cities show marked differences 
in the character of the mortality curve. The New England cities are quite similar in having a sharp peak in October and about one well marked recurrence during January. The Middle Atlantic cities present a similar picture although the recurrent period is less clearly defined. The mortality picture of the southern cities is somewhat different. The mortality peaks of Atlanta, Louisville and Birmingham are much less pronounced. Recurrences are shown in each of these cities, the second wave in Birmingham being as high as the first. In the other two eities there are three distinct peaks, the first and third showing a similar configuration. The cities of the east north central region show features in common, the mortality peaks being unusually low when compared to the eastern cities. Grand Rapids barely reveals any unusual mortality until December and even then a rate of 30 is not exceeded. Milwaukee has two distinct peaks of small magnitude, neither exceeding 35. There are two distinct peaks at Indianapolis, the first reading as high as 40 . Cincinnati and Columbus are quite similara rate of 50 not being exceeded. The first wave in October and November, though not great, is distinctly outlined and differentiated from the second of lesser magnitude in Dayton, Cleveland, Toledo and Chicago. In Kansas City and Omaha there are two distinct peaks of mortality of about equal magnitude, the 50 mark being reached both in October and December. St. Louis shows a rise to 30 in October, then a further rise to 45 in December. St. Paul and Minneapolis show an extended period of heightened mortality from October to January rather than a sharp peak.

Farther west we find Denver's experience resembling the double blunt peaks of Kansas City. San Francisco and Oakland show high initial peaks with recurrence in January of some magnitude. These cities differ from other cities in that the second peak is great, even following upon a high initial peak. Los Angeles, Portland and Seattle have two mortality peaks of lesser magnitude. At Spokane there is an elevated mortality from October to February, no weekly peak exceeding 30.

In the cities having a high initial mortality there is either no recurrent wave, as in the case of Fall River, Albany, Syracuse, Pittsburgh and Nashville, or a recurrence but slightly manifested as in the New England, Middle Atlantic and South Atlantic cities. Exceptions to this rule are found in the case of New Orleans, San Francisco and Oakland, all seaports which had high peaks initially followed by a recurrent peak of appreciable size. (Fig. 52.)

Certain inland cities of the south, such as Atlanta and Birmingham, and the eities around the Great Lakes suffered less from the epidemic than the average. In general the more accessible and more traversed cities suffered more keenly than those off the main lines of travel. 


\section{MORTALITY \\ IN U.S. CITIES DURING 1917-18-19 \\ WEEKLY OOCURREMGE OF DEATHS FROMALL CAUSES
EXPRESSED AS AN ANIYUAL RATE PER 1000}

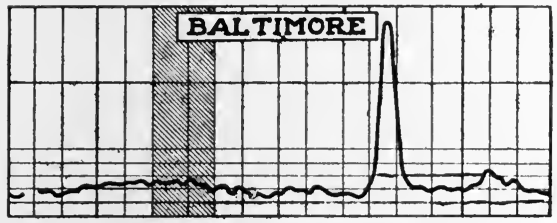

SOUTH ATLANTIC EAST SOOTH CENTRAL WEST SOUTH CENTRAL
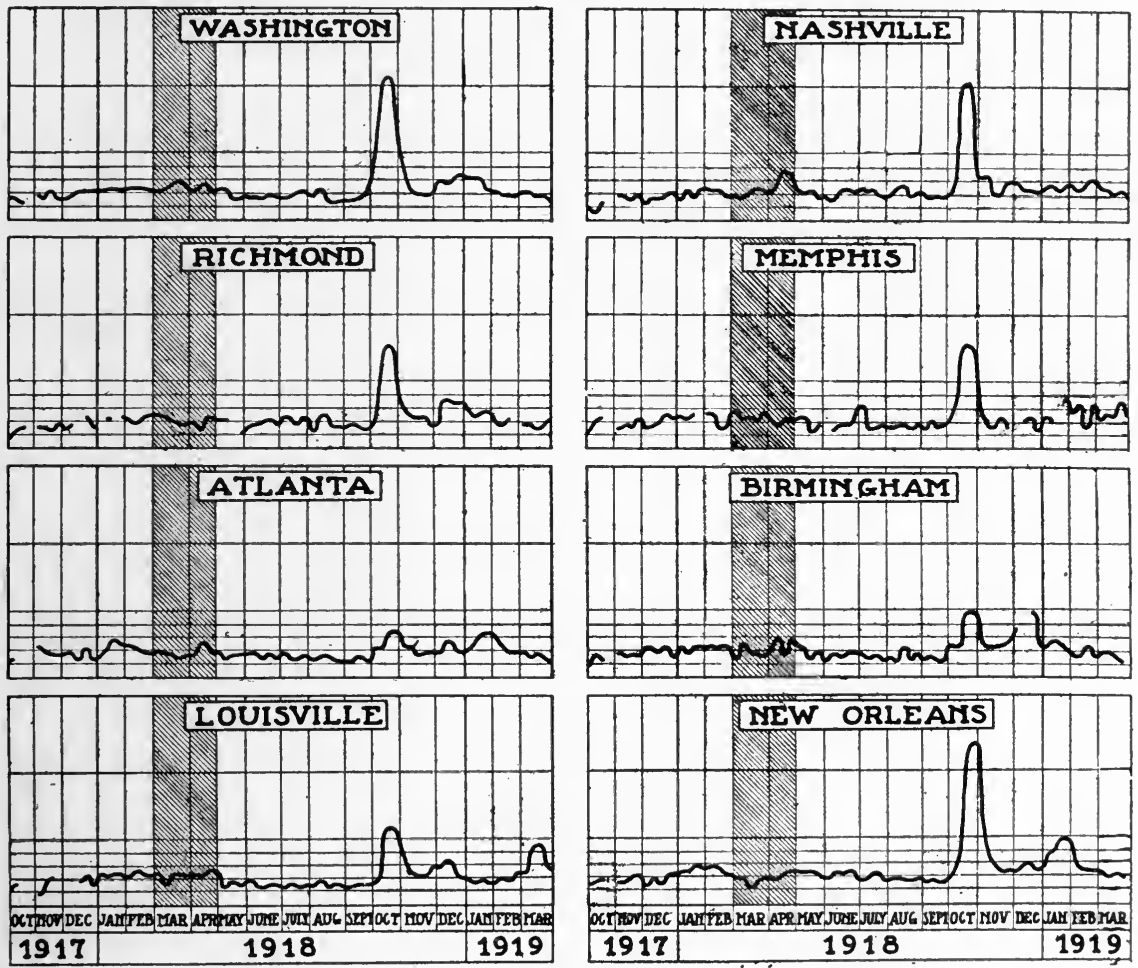

Fig. 46.

The ranking of the sections may be judged also from the claim rate per 1000 policies (annual basis) announced by the Metropolitan Life Insurance Co. from October, 1918 to June, 1919. Middle Atlantic 9.1; West South Central 9.0; New England 8.9; South Atlantic 8.8; East South Central 7.6; East North Central 6.4; West North Central 6.2; Pacific 5.8; Mountain 5.6.

The first army camp to show signs of an influenza epidemic in the autumn of 1918 was Shelby, located at Hattiesburg, Mississippi. Whether 


\title{
MORTALITY \\ IN U.S. CITIES DURING 1917-18-19.
}

\begin{abstract}
WEEKZY OCCURREYCE OF DEATHS FROY ALL CAUSES
EXPRESSED AS AY AYMUAL RATE PER 1000
\end{abstract}

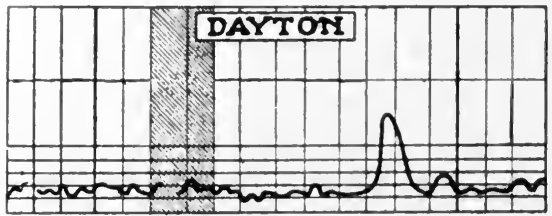

\section{EAST NORTH CENTRAL}
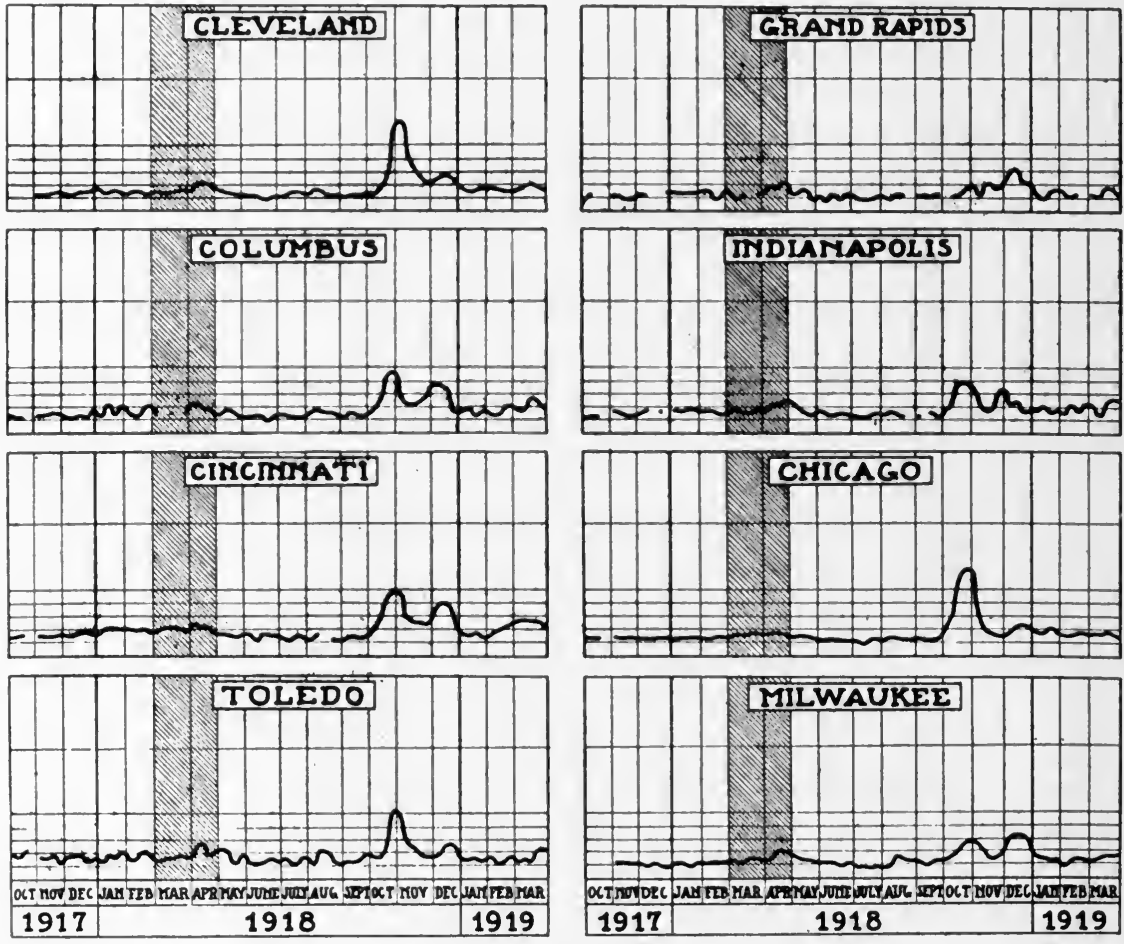

Fig. 47.

this epidemic was identical in every respect with that which broke out a week or so later at Devens we do not know. It appears to have been the same type of disease. There were fatal pneumonic complications and in this respect it differed from the influenza of the spring, and yet the complications did not seem to be as prevalent as a month or so later. This early epidemic at Shelby seemed to be intermediate between the spring disease and the epidemic of later fall. It was confined to summer recruits. There is no reason for believing that Devens contracted 


\section{MORTALITY \\ IN U.S. CITIES DURING 1917-18-19. \\ WEEKLY OCCUPRENGE OF DEATHS FROMALL CAUSES \\ EXPRESSED AS AN AMYOAL RATE PER 1000}

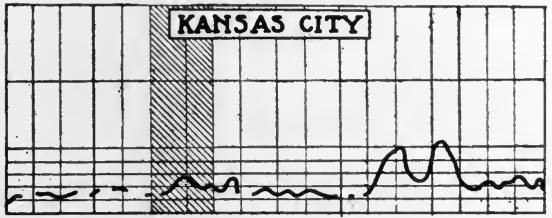

\section{WEST NORTHCENTRAL}
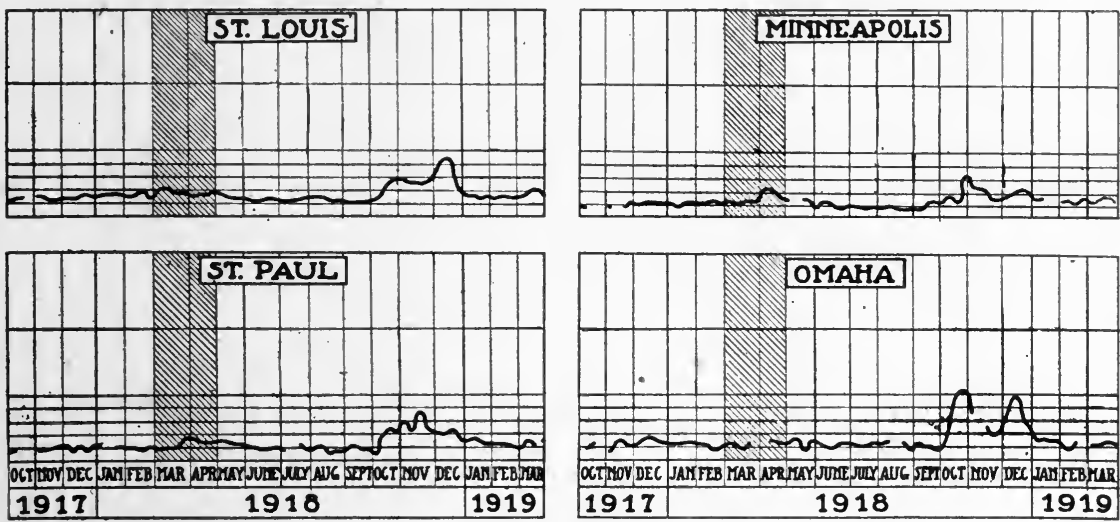

Fig. 48.

it from Shelby. Shelby's August epidemic may have been started by visitors from Boston and vicinity. The chronology of the epidemics will permit this. Shelby may have been infected from overseas through some port other than Boston. These facts we may never know, but it seems quite certain that the outbreaks subsequent to Devens were more or less associated with Devens or the port of Boston rather than with Shelby.

We have illustrated the progress of the pandemic through the army camps in this country. Here we have shown as accurately as possible the date of the first case at each camp. This date is somewhat uncertain in some camps because the epidemic was not recognized as a new entity but as a rapid extension of respiratory affections already in existence. (Fig. 50.)

Camp Lewis had reported influenza throughout the year and the beginning of the epidemic is fixed on the date when the first great increase in cases occurred. The same is true for Pike where from 8 to 89 cases 


\section{MORTALITY \\ IN U.S. CITIES DURING 1917-18-19}

WEEKZY OCCURRENCE OF DEATHS FROM ALL GAUSES

EXPRESSED AS AY AYMUAL RATE PER 1000

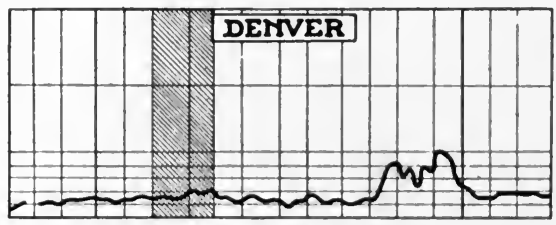

\section{MOUNTAIM ard PACIFIC}
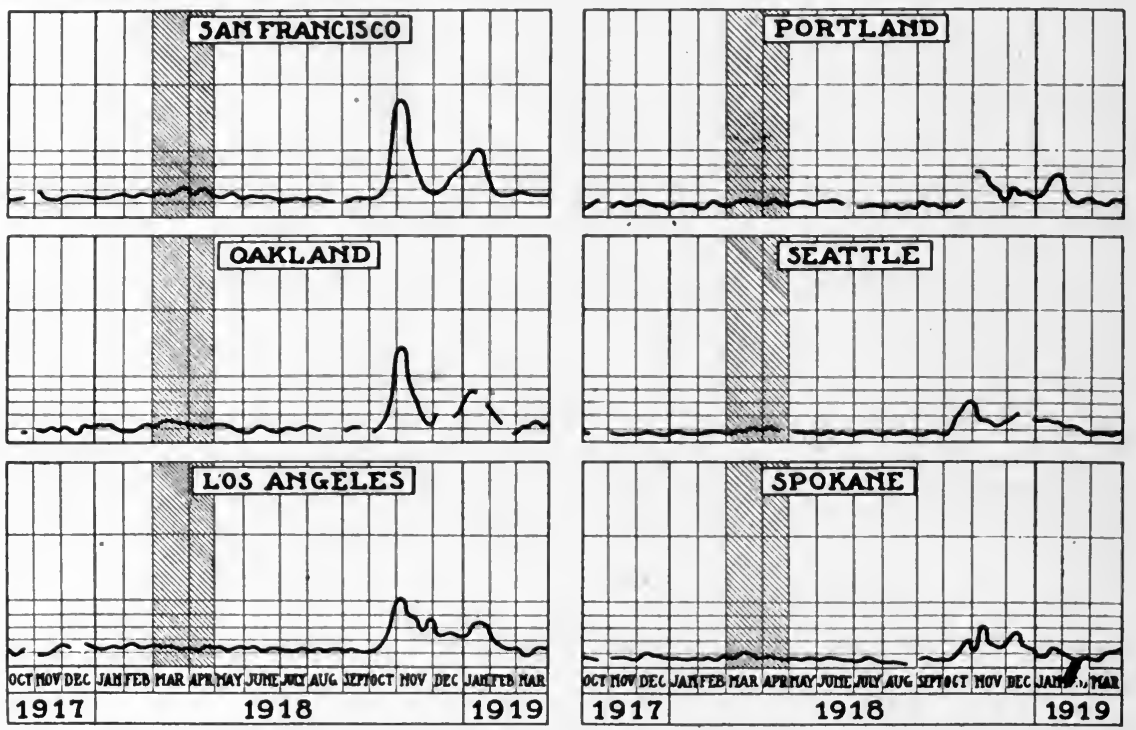

Fig. 49.

a day had been reported since September 1. The epidemic is officially regarded as having begun on September 23. The admissions about this time were as follows:

$\begin{array}{rrr}\text { September } & 19 & 42 \\ 20 & 50 \\ 21 & 57 \\ 22 & 89 \\ 23 & 214 \\ 24 & 436 \\ 25 & 885 \\ 26 & 797 \\ 27 & 1037\end{array}$



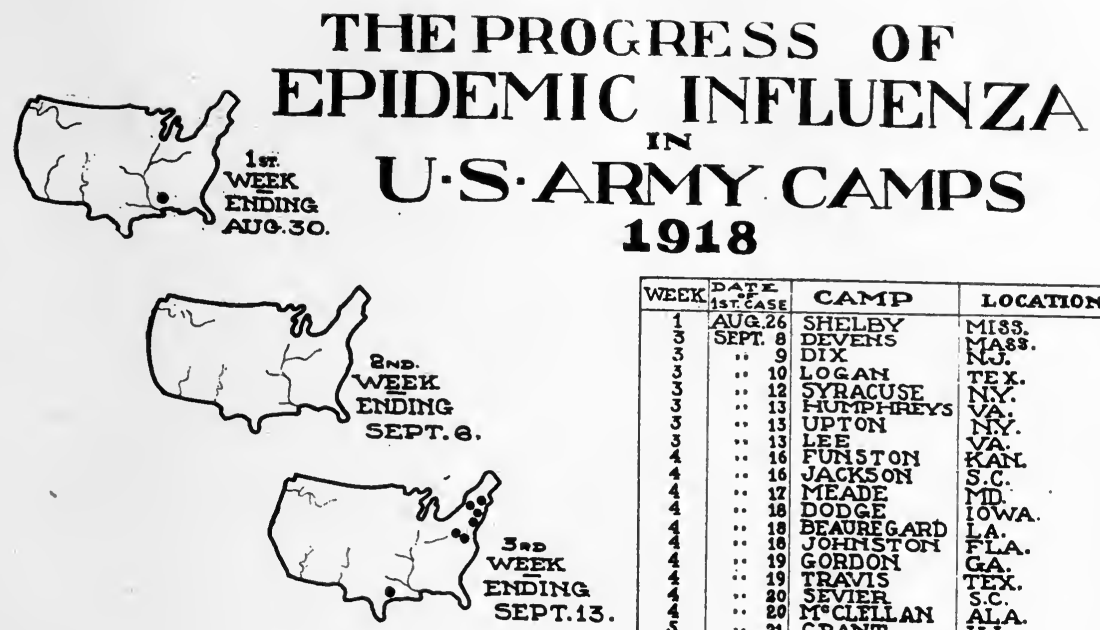

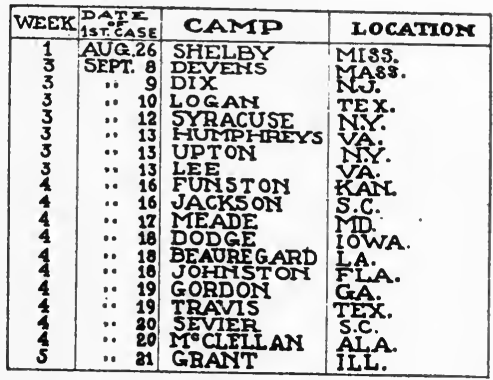
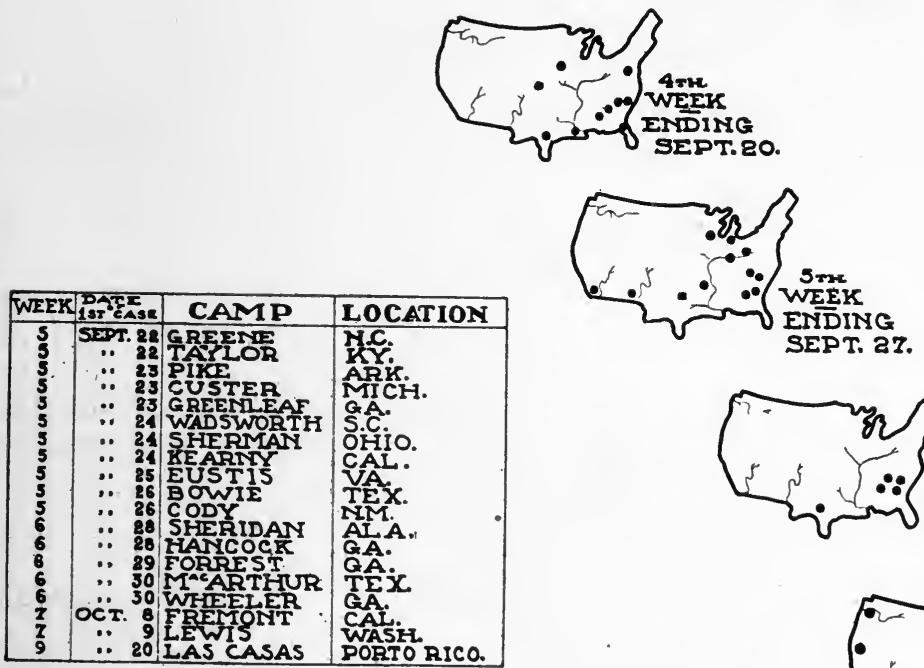

Fig. 50.
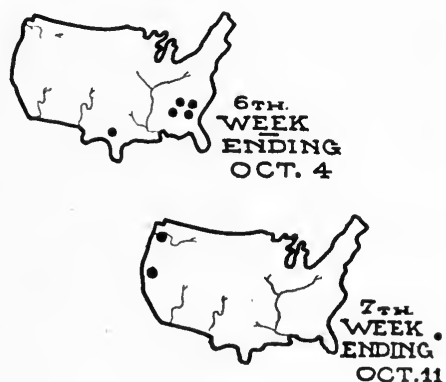

Camp Humphreys had 15 cases of influenza during the first twelve days in September, then 29 cases from the 13th to the 18 th, 56 on the 19 th, 49 on the 21st, 56 on the 22 nd and 163 on the 23rd. The epidemic is regarded as having started at Humphreys on the $13 \mathrm{th}$.

The epidemic is said to have begun at McClellan on September 20, with the admission of one case. During the next twelve days there were never more than 10 admissions daily. On October 3 there were 36 ; on the fourth none; on the fifth 88; and on the eighth 254.

It is quite different at Camp Grant, where influenza appeared as a new 


\section{TABLE XLIV}

Rates of Death From All Causes During Influenza Epidemics 1889-90 AND 1918

(Figures Represent Annual Death Rate per One Thousand)

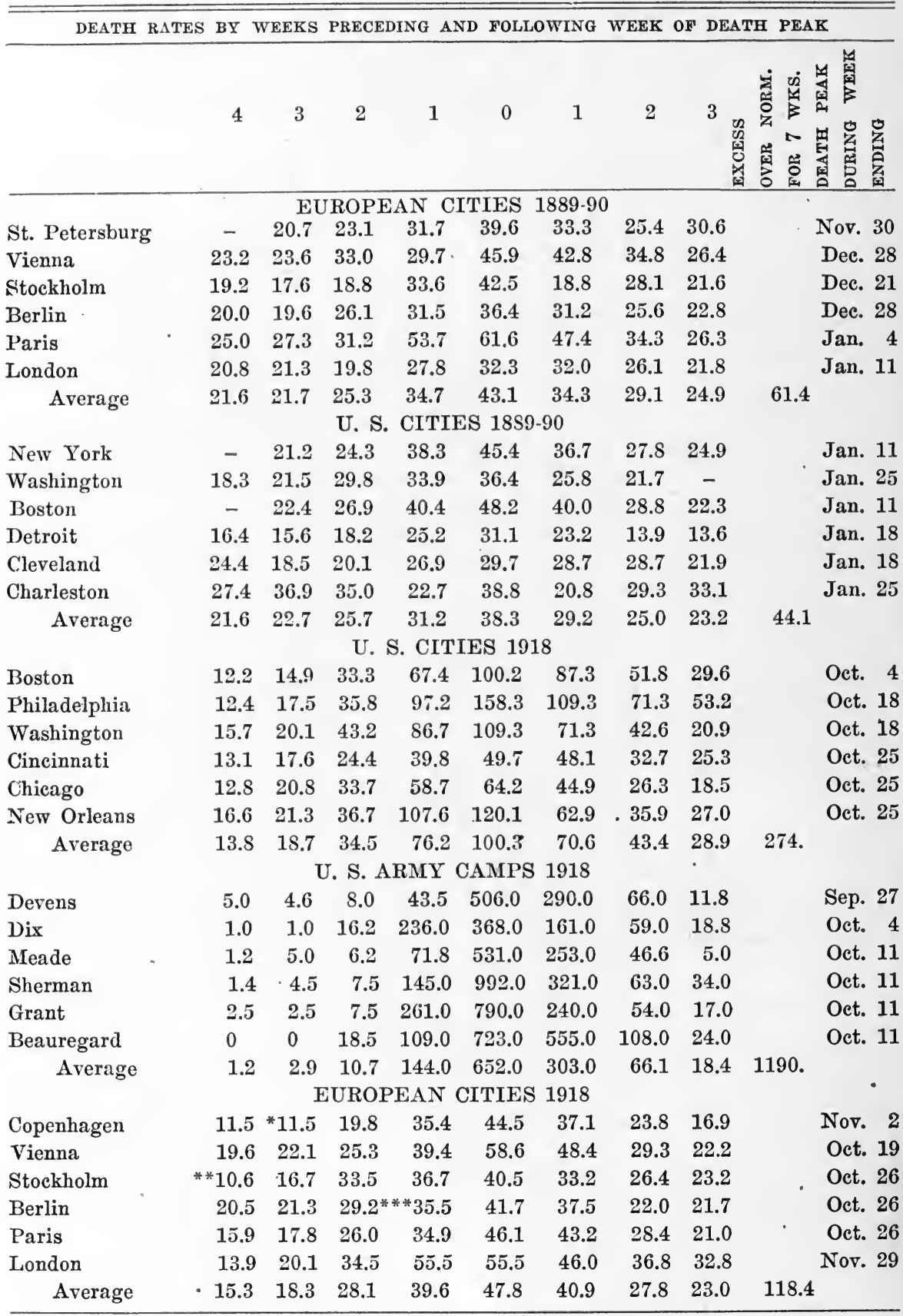

* Data for previous week inserted for completeness. Actual rate missing.

** Data for week ending September 14. Actual rate missing.

***Average for weeks either side. Actual rate missing.

Explanatory notes: The 4th week preceding the death peak is used as a norm in the absence of data for the preceding weeks. Lack of data also explains why identical cities have not been used in 1889.90 and 1918. 


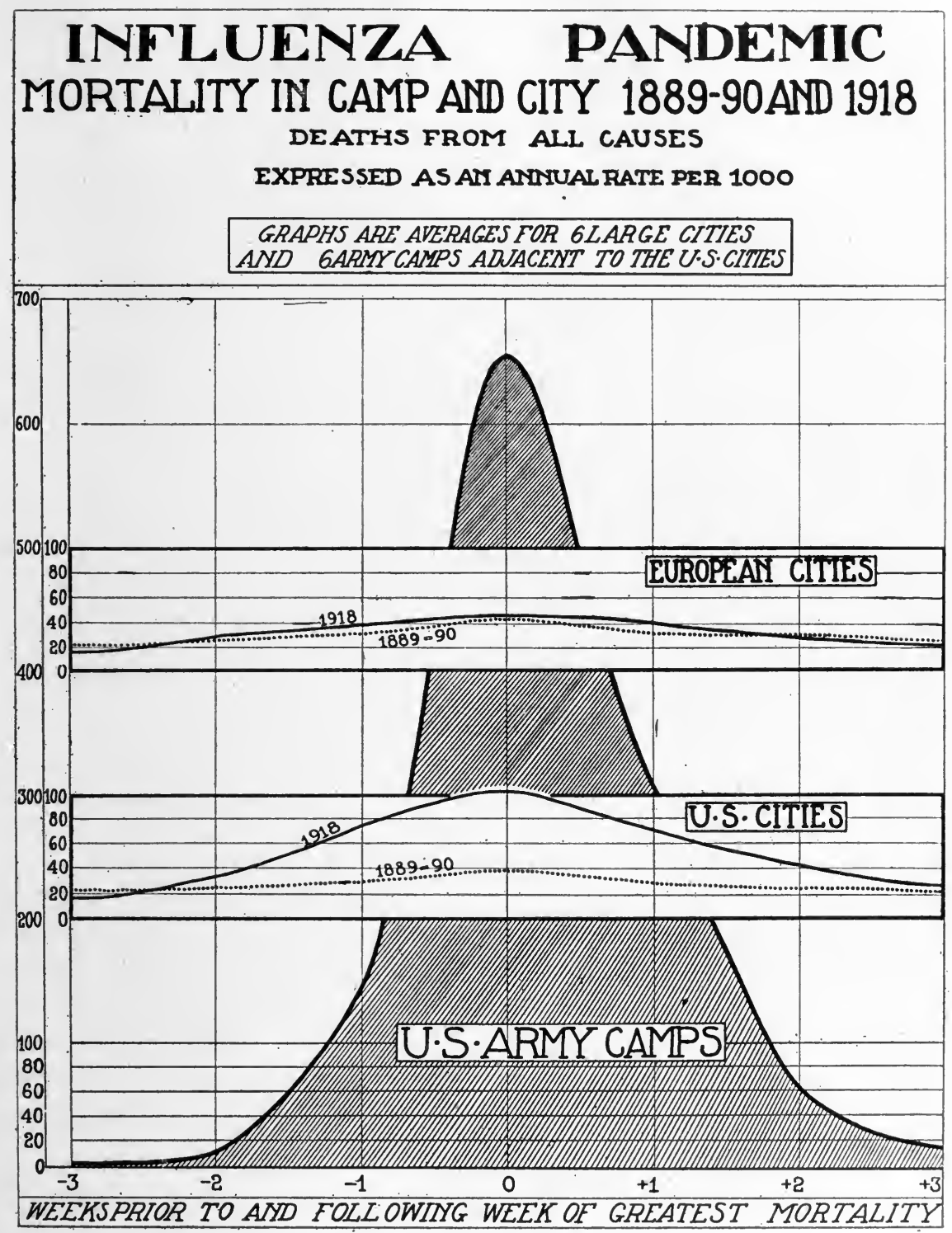

Fig. 51.

infection and was recognized immediately. 'The epidemiologist at Grant is specific in his statement that the epidemic "made its first appearance at this camp on the afternoon of Saturday, September 21, 1918, in one of the barracks of the Infantry Officers Training School.' Camp Taylor is equally certain that the first case of influenza appeared on September 22 .

We mention these facts as there may arise a difference of opinion 
over the exact beginning of the epidemic. In making up the above table the interpretation of the local authorities, who it is believed are best acquainted with the situation in each eamp, has been accepted.

One ean see from the chart that the Devens epidemic is separated from the Shelby outbreak by thirteen days. During the week ending September 13, which included the beginning of the Devens epidemic, six other eamps reported an epidemic under way. With one exception, Logan, which is located in Texas, these eamps are all loeated in the northeastern corner of the eountry within a short distance of Boston. In the week following, which is the fourth, counting Shelby as the first eamp affected, ten eamps reported outbreaks. With the exception of Dodge and Funston all of these were in the Atlantic Coast or Gulf States. In the fifth week the disease made its appearance at twelve camps, five located in the belt bordering the Great Lakes and traversed by the Mississippi River. Kearny on the Pacific Coast reported its first ease at this time, the infection apparently being imported from San Diego. Seven camps located within the states of Georgia, Alabama, Texas and Missouri, reported epidemies in the sixth week. In the seventh week Fremont and Lewis on the west coast reported the disease, and not until the ninth week, or 44 days after the epidemic appeared at Boston, do we find it at Las Casas in Porto Rico.

As in the eities, the disease traveled through the army eamps from the northeastern sector down the Atlantic Coast, through the Middle West and eventually to the Pacific Coast.

A eomparison of the 1918 outbreak with that in 1889-90 ean be made satisfactorily only by computing the excess mortality from all causes over the normal. Inasmuch as the first wave of the epidemic lasted about seven weeks, we may eompute the excess mortality for this period over a normal which is represented by the rate in the fourth week preceding the mortality peak. (Table XLIV.) In the comparison we have used European eities and American in both epidemies, and U. S. Army camps in the 1918 epidemic. Expressed as percentage of population killed in seven weeks' time we have the following:

$\begin{array}{lll}\text { European Cities } & 1889-90 & .12 \\ \text { U. S. Cities } & 1889-90 & .085 \\ \text { European Cities } & 1918 & .23 \\ \text { U. S. Cities } & 1918 & .53 \\ \text { U. S. Army camps } & 1918 & 2.29\end{array}$

The rate in the army camps is, of course, much greater, primarily because they contained the age group most fatally affected by influenza. This high rate is also due to the fact that the epidemic expended all its havoc within this period, there being practically no recurrent wave in the camps; whereas, in the cities the disease was more prolonged and 
recurred at least once and even twice in some cities in the succeeding four months.

Using as a unit of measurement the lowest figure, .085 for the U. S. cities in 1889-90, we may express the epidemies comparatively by stating that European cities in 1889-90 lost 1.4 times as many people, European cities in 1918, 2.7 times; U. S. cities in 1918, 6.2 times; U. S. Army camps, 27 times. For the brief period under consideration this country suffered less than Europe in 1889-90, but more so in 1918. (Fig. 51.)

In the initial fall outbreak in 1918 of seven weeks' duration, the six U. S. cities lost .53' per cent of their population, and in the entire 28 weeks ending March 29, the total was only .59.

From the records of four European cities for which data are available .23 per cent of the population died in the first seven weeks and in the entire 28 weeks ending March 29, the figure rose to .48 per cent. Thus the American cities suffered more acutely in the initial outbreak, the recurrent waves being of relatively little account. In Europe the recurrences following the initial outbreak doubled the mortality of the initial seven weeks' outbreak.

As we shall point out later, the spring and summer epidemic conferred immunity against the fall outbreak. It is very probable that the spring and summer epidemic in Europe immunized a large proportion of the popu-

TABLE XIV

Death Rates (Annual Per 1000) In Large Cities of the U. S. by Weeks During Influenza Epidemic of 1920

\begin{tabular}{|c|c|c|c|c|c|c|c|c|c|c|c|}
\hline 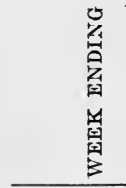 & 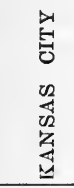 & $\begin{array}{l}0 \\
0 \\
0 \\
0 \\
0 \\
0\end{array}$ & 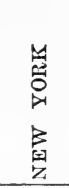 & $\begin{array}{l}\text { 舅 } \\
\text { 总 } \\
\text { 兽 } \\
\end{array}$ & 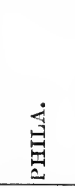 & $\begin{array}{l}\text { 台 } \\
\text { 皇 } \\
\text { O }\end{array}$ & 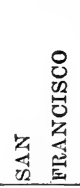 & 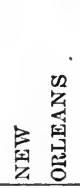 & 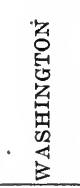 & 莺 & 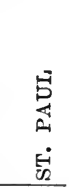 \\
\hline Jan. 3 & 16.3 & 14.4 & 14.0 & 11.6 & 15.6 & 16.8 & 16.5 & 18.8 & 19.2 & 14.7 & 9.7 \\
\hline 10 & 15.8 & 13.8 & 15.3 & 12.5 & 16.7 & 16.9 & 15.4 & 19.6 & 16.0 & 17.8 & 9.1 \\
\hline 17 & 19.3 & 15.1 & 14.6 & 9.0 & 16.2 & 14.1 & 19.6 & 22.6 & 17.8 & 17.8 & 9.1 \\
\hline 24 & 32.7 & 23.5 & 19.5 & 15.6 & 16.8 & 16.8 & 19.2 & 18.8 & 28.6 & 11.4 & 16.2 \\
\hline 31 & 39.5 & 41.3 & 28.0 & 29.4 & 18.3 & 20.3 & 22.9 & 20.9 & 43.4 & 12.9 & 26.9 \\
\hline Feb. 7 & 61.5 & 39.1 & 35.0 & 34.5 & 22.1 & 27.1 & 25.2 & 20.1 & 37.9 & 23.3 & 31.0 \\
\hline 14 & 44.0 & 24.6 & 35.1 & 27.1 & 34.3 & 33.7 & 31.8 & 25.0 & 28.8 & 33.3 & 24.9 \\
\hline 21 & 29.1 & 17.7 & 24.8 & 16.9 & 37.2 & 32.1 & 28.8 & 32.3 & 22.3 & 42.4 & - \\
\hline 28 & 18.4 & 14.7 & 18.2 & 13.6 & 28.9 & 24.2 & 23.2 & 29.9 & 17.5 & 36.2 & 11.7 \\
\hline Mar. 6 & 17.4 & 16.0 & 17.1 & 11.6 & 21.6 & 19.5 & 18.3 & 28.0 . & 16.8 & 27.1 & 11.7 \\
\hline 13 & 17.4 & 15.3 & 16.8 & 10.8 & 19.9 & 16.6 & 19.2 & $28.8^{\circ}$ & 16.1 & 18.9 & 10.1 \\
\hline 20 & .19 .3 & 14.7 & 15.5 & 14.0 & 17.7 & 16.7 & 14.9 & 21.8 & 17.8 & 19.6 & 14.7 \\
\hline 27 & 18.4 & $14 . \overline{5}$ & 15.3 & 14.4 & 16.6 & 17.8 & 17.9 & 21.0 & 16.3 & 19.6 & 14.9 \\
\hline Apr. 3 & 14.8 & 13.9 & 14.4 & 13.8 & 15.7 & 13.9 & 16.9 & 20.3 & 12.5 & 15.3 & 9.8 \\
\hline
\end{tabular}




\section{EXCESS MORTALITY ${ }_{\text {IN USSCITIES }}$ DURING INFLUENZA EPIDEMIC PERCENT OF POPULATIOM DYING}

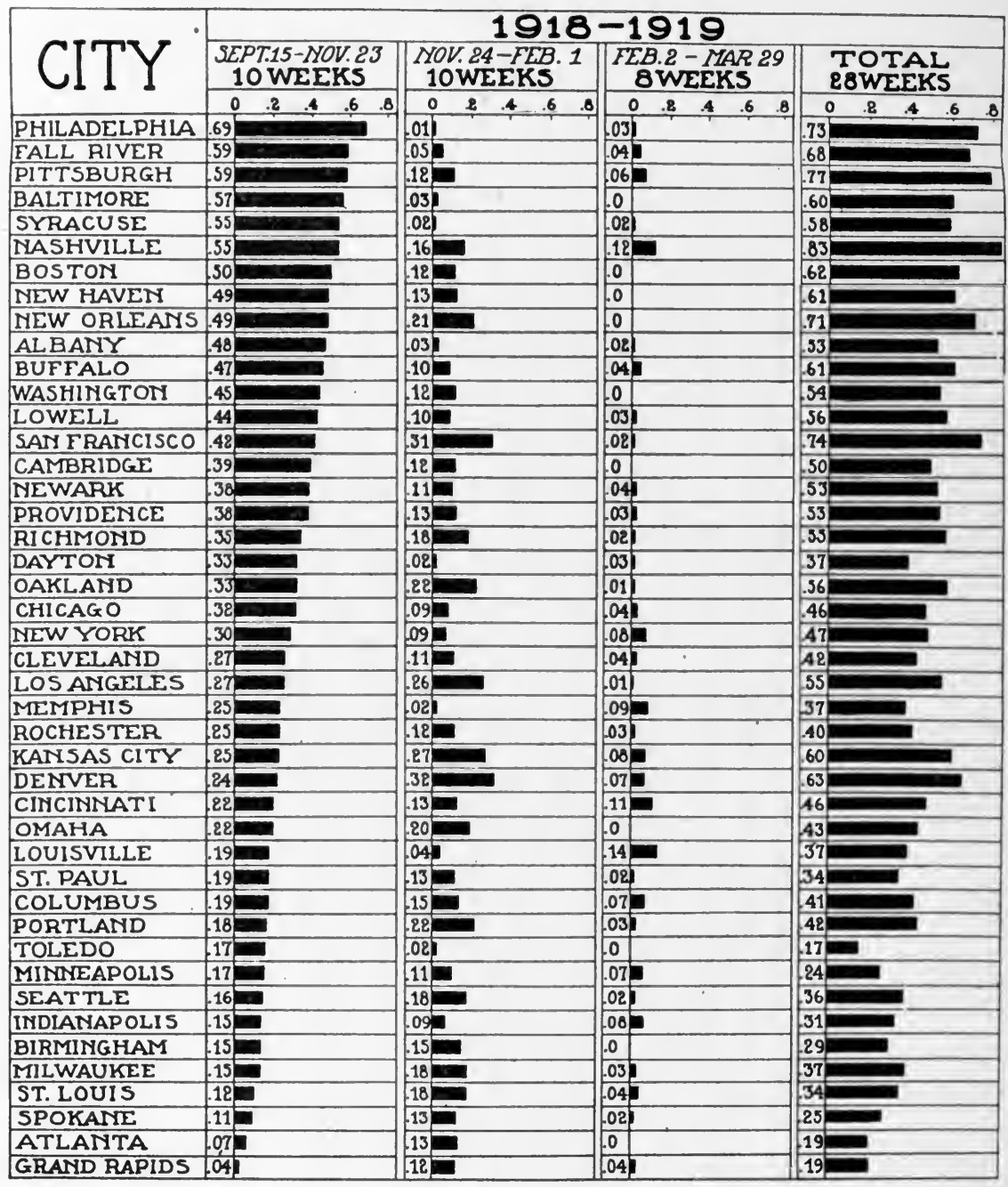

Fig. 52.

lation, thus reducing the susceptible material in the autumn.

Influenza disappeared from the large cities of the United States by spring. We read of its occurrence in the rural districts in this country and elsewhere on the globe during summer and fall. In May it was reported in Queensland, Australia and Argentina; in June in Malta and 
Madagascar; in July in Australia and Argentina; in August in Chili and Australia. China, Spain and Sweden reported cases during the autumn. During September, 1919, 1166 cases were reported to the U. S. Public Health Service from Mississippi. At the same time there were reported from Illinois 597 cases; California 160; New Jersey 133; Louisiana 68; Massachusetts 226, On November 26, epidemic influenza was reported from one district in Taylor County, Kentucky. On January 13, 88 cases were reported at Mountain House, Elsmore County, Idaho, and the town was quarantined. In the week ending January 7 , an increase in the state reports was noted from California, the District of Columbia, Idaho, Illinois and New Jersey.

Kansas City exhibited an increase in total mortality from 15.8 to 19.3 in the week ending January 17. This same week Chicago's death rate was 15.1 against 13.8 for the previous week. The following week the disease had become firmly entrenched in Detroit, New York, Washington and Milwaukee. (Table XLV.)

Nearly all cities affected in 1918 were again visited by the infection in January and February, 1920. The latter outbreak was of brief duration, however, and was far less fatal, although there is an exception to this in the ease of Detroit. In seven weeks of 1920 there occurred 1930 excess deaths attributable to the epidemic, an amount which represented 0.20 per cent of the population. In the first seven weeks of the 1918 outbreak Detroit lost 1520 people, or .17 per cent of the population. In 1918 and 1919 recurrent waves in the succeeding 14 weeks raised Detroit's mortality to .28 per cent. Thus, while the 1920 epidemic caused more damage in seven weeks' time, because of its brevity, it actually resulted in fewer deaths during the winter than the 1918-19 outbreak.

The annual death rates in Detroit by weeks in the two seven-week periods were as follows:

$\begin{array}{lrrrrrrr} & -3 & -2 & -1 & \text { PEAK } & +1 & +2 & +3 \\ \text { Autumn, 1918 } & 13.5 & 21.2 & 36.0 & 38.7 & 27.6 & 19.5 & 14.4 \\ \text { Jan. and Feb., 1920 } & 14.4 & 15.7 & 33.9 & 60.8 & 43.0 & 21.6 & 17.5\end{array}$

Influenza was prevalent in many points other than the United States during the winter of 1919-1920. Cities in India were affected as early as December. From January to June outbreaks of a less severe character than 1918 were reported from Japan, Switzerland, Great Britain, France, Canary Islands, Australia, New Zealand, the Philippines, Sumatra, Bulgaria, Greece, Italy, Sweden, Iceland, China, South America, Mexico and Canada. In fact the recurrent wave was more or less universal over the globe. 


\section{THE CAUSE orMORTALITY DURING THE INFLUENZA EPIDEMIC IN THE LARGE ARM Y CAMPS}

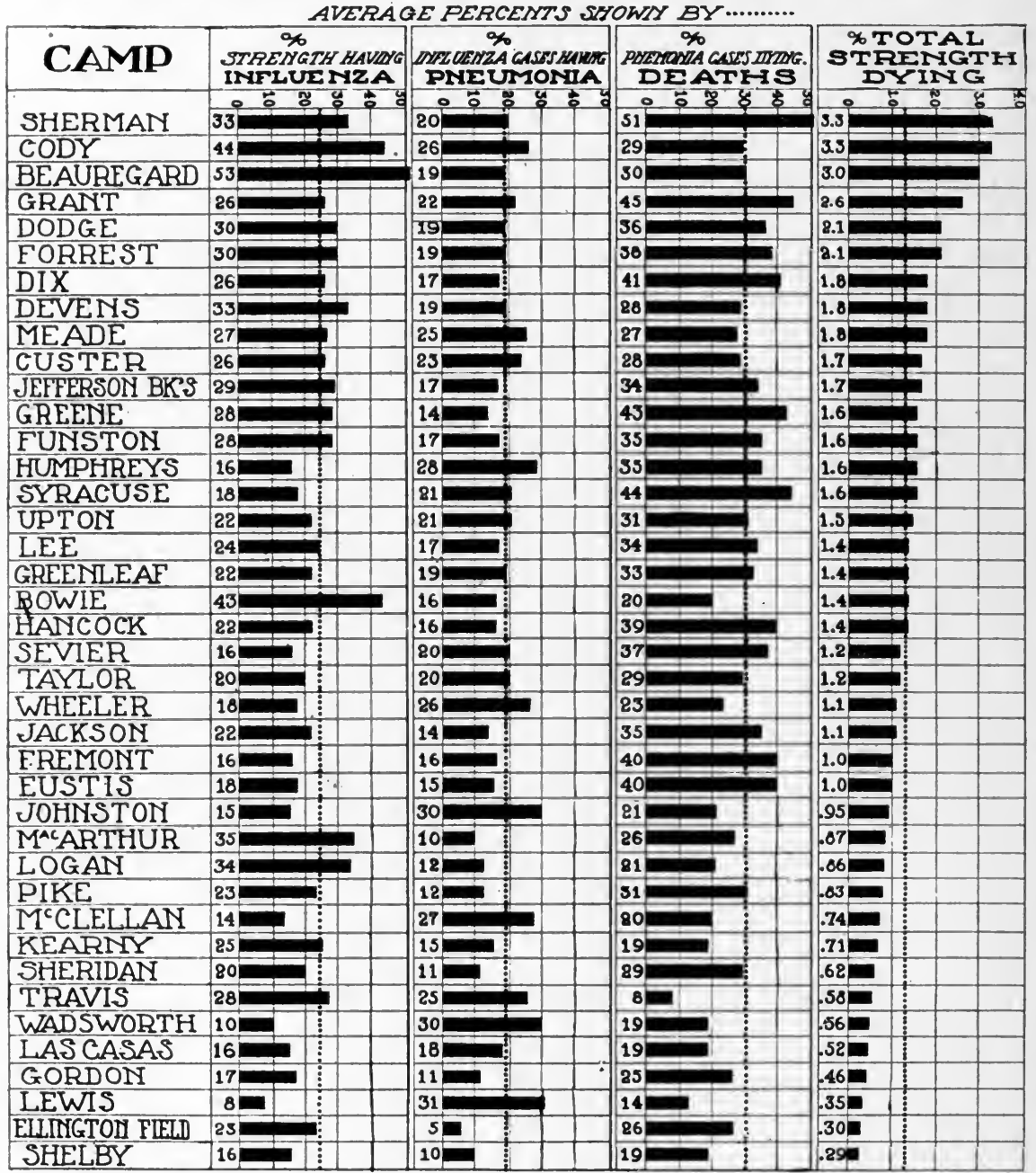

(Data represent seven weeks of maximum prevalence at each camp.)

$$
\text { Fig. } 53 .
$$

In the preceding pages, the features characterizing influenza pandemics have been pointed out. There is much remaining to know, yet our knowledge of the subject has shown definite advance each century. We quote from a contemporary (Vaughan, Warren) in summing up the outstanding points which have been arrived at independently, but which coincide with our own belief. 
"(1) Recurrence of true pandemics at wide intervals, primarily intervals of several decades. (2) The apparent origin of the epidemic in a single place, or perhaps in two, rarely more, localities. (3) The rapid spread over all countries, the rapidity being governed by the facilities for human travel. (4) The rapid evolution of the disease in the communities where ontbreaks occur. (5) The rapid subsidence of the epidemic after several weeks' duration. (6) The apparent lack of connection with differences of wind or weather, with seasons or with climate. (7) The usually low mortality in contrast to the enormous morbidity."

"The salient characteristies of recurrences are: (1) Generally decreased morbidity. (2) Decidedly more circumscribed geographic distribution and absence of definite lines of spread. (3) Gradual evolution and duration throughout several months. (4) Successive decrease in intensity and frequency in successive epidemics."

\section{The Selective Action of Influenza with Respect to Age, Sex and Race.-} Epidemic influenza in the last two great pandemics has been partial to the young adult. While all ages have been attacked, it is the young men and women who have died in greatest numbers.

In the outbreak of 1889-90 Auerbach gives statistics of 200 families in Cologne of whom 75 per cent were attacked. Of 235 persons ill there were 59 men, 95 women and 81 children. The excess among women he explains as due to illness among female servants. It seems quite certain from the numerous data given that young adults were affected most widely. Young children particularly escaped. School children above ten years of age were affected more widely than those below this age.

In the recent epidemics of influenza all ages, both sexes and all races have been attacked, although in varying degree. So far as can be ascertained from various surveys that have been made influenza made little distinction in its age preference up to 40 years. Above this age the prevalence of the disease was less than among those below. There was no consistent sex selection, males and females being attacked about equally. As between the white and colored races, there seems to be quite uniform opinion as to the excess of influenza among the whites.

Frost summarizes the results of surveys made by the Public Health Service in widely separated communities over the country. The attack rate varied from 15 per cent in Louisville to 54 per cent in San Antonio.

TABLE XLVI

Per Cent of Population Attacked by Influenza as Determined by Canvass of Representative Districts

\begin{tabular}{lllr}
\hline \hline Louisville* & 15 & Baltimore & 25 \\
New London & 19 & Augusta & 34 \\
Macon & 21 & Little Rock & 36 \\
Spartansburg & 21 & Minor communities in Maryland & 41 \\
San Francisco & 22 & San Antonio & $5 t$ \\
Des Moines & 23 & All localities & 28 \\
\hline
\end{tabular}

*Canvass concluded before epidemic had run its full course. 


\section{EXCESS MORTALITY DURING INFLUENZA EPIDEMICS IN U.S. CITIES \\ PER CENT OF POPULATION DYING}

\begin{tabular}{|c|c|c|c|c|}
\hline$\frac{1918-1919}{28 \text { weeks Sept.15-Mar.29. }}$ & & CITY & & $\frac{1920}{14 \text { weeks Dec.28-Apr.3. }}$ \\
\hline & .83 & NASHVILLE & 05 & \\
\hline & 77 & PITTSBURG & .20 & E \\
\hline & 74 & SAN FRANCISCO & .23 & 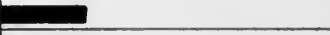 \\
\hline & .73 & PHILADELPHIA & .21 & 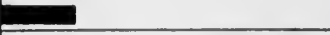 \\
\hline & .71 & NEW ORLEANS & .18 & \\
\hline & 68 & FALL'RIVER & .0 & \\
\hline 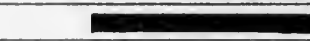 & .62 & BOSTON & .19 & \\
\hline E & .61 & NEW HAVEN & .24 & \\
\hline 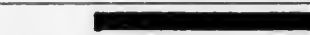 & .61 & BUFFALO & .14 & 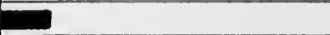 \\
\hline$=$ & 60 & KANSAS CITY & 34 & \\
\hline E & 60 & BALTIMORE & .12 & 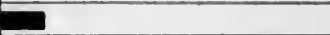 \\
\hline$=$ & 58 & SYRACUSE & .15 & 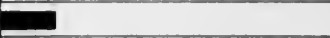 \\
\hline E & 56 & LOWELL & .12 & 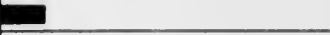 \\
\hline$=$ & 55 & RICHMOND & .08 & 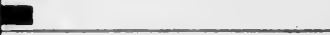 \\
\hline E & .55 & LOS ANGELES & .16 & \\
\hline 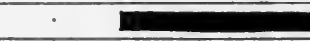 & .54 & WASHINGTON & .17 & 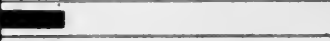 \\
\hline E & .53 & ALBANY & .16 & 0 \\
\hline & .53 & NEWARK & .17 & \\
\hline & .53 & PROVIDENCE & .19 & 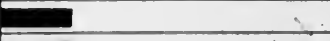 \\
\hline$=$ & .50 & CAMBRIDGE & .22 & 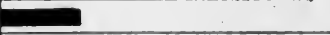 \\
\hline - & .47 & NEW YORK & .27 & 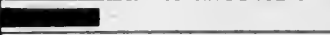 \\
\hline & 46 & CHICAGO & 22 & 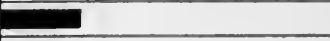 \\
\hline & .46 & CINCINNATI & .15 & 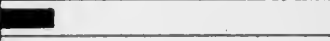 \\
\hline & 43 & OMAHA & .07 & E \\
\hline & .48 & CLEKELAND & .14 & 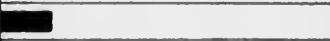 \\
\hline & .41 & COLUMBUS & .19 & \\
\hline & 40 & ROCHESTER & .08 & 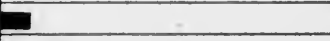 \\
\hline & .37 & MILWAUKEE & 16 & 7 \\
\hline & 37 & DAYTON & .16 & 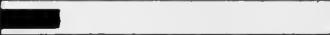 \\
\hline & .37 & LOUISVILLE & .12 & 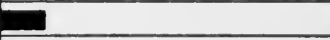 \\
\hline & .34 & ST. PAUL & .18 & $\Longrightarrow$ \\
\hline & .34 & ST. LOUIS & .24 & $\square$ \\
\hline & .31 & INDIANAPOLIS & 20 & 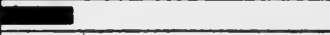 \\
\hline & .29 & BIRMINGHAM & 13 & - \\
\hline & .24 & MINNEAPOLIS & 27 & \\
\hline 드. & .19 & ATLANTA & 27 & $\square$ \\
\hline E & .19 & GRAND RAPIDS & .15 & 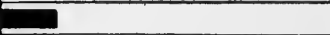 \\
\hline E & .17 & TOLEDO & $: 07$ & 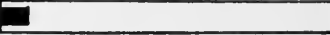 \\
\hline
\end{tabular}

Fig. 54. 
The distribution by age groups of all localities combined is shown in Table XLVII.

TABLE XLVII

\begin{tabular}{ccc}
\hline \hline AGE & $\begin{array}{c}\text { PER CEN'T OF AGE } \\
\text { GROUP ATTACKED }\end{array}$ & $\begin{array}{c}\text { RATIO, ALL } \\
\text { AGES AS } 100\end{array}$ \\
\hline $0-4$ & 29 & 105 \\
$5-9$ & 36 & 130 \\
$10-14$ & 35 & 125 \\
$15-19$ & 31 & 110 \\
$20-24$ & 29 & 105 \\
$25-29$ & 32 & 115 \\
$30-34$ & 31 & 110 \\
$35-39$ & 28 & 100 \\
$40-44$ & 22 & 80 \\
$45-49$ & 20 & 70 \\
$50-54$ & 17 & 60 \\
$55-59$ & 15 & 55 \\
$60-64$ & 14 & 50 \\
$65-69$ & 13 & 45 \\
$70-74$ & 11 & 40 \\
75 and over & 8 & 30 \\
\hline
\end{tabular}

Note: These figures were taken from a graph and are approximately correct.

The greatest prevalence is found between the ages of five and nine, in this case 36 per cent of the group being attacked. Up to forty years the percentage varies from 28 to 36 . Above forty years of age the proportion attacked falls off uniformly to a minimum of 8 at seventy-five years and over.

Surveys were conducted by the New York State Influenza Commission in Oswego among 13,000 people and in Watertown among 20,500 people between November 15, 1918, and January 7, 1919. These figures presented in Table XLVIII indicate a higher attack rate than in the group of eleven communities previously reported.

TABLE XLVIII

Per Cent Population Attacked

\begin{tabular}{cccc}
\hline \hline & & \multicolumn{2}{c}{ ELEVEN COMMUNITIES } \\
& OSWEGO & WATERTOWN & IN U. S. \\
\hline All ages & 46 & 28 & 28 \\
$5-14$ & 49 & 31 & 29 \\
$15-19$ & 58 & 37 & 36 \\
$20-39$ & 50 & 30 & 30 \\
$40-59$ & 33 & 20 & 20 \\
60 and over & 18 & 10 & 12 \\
\hline
\end{tabular}


The distribution of cases in Watertown is almost identical with that in the eleven communities investigated by the Public Health Service. Oswego was more widely affected at all age groups. Jordan, studying the occurrence of the disease among employes of the Chicago Telephone Company, concludes that between ages 16 and 60 the cases were fairly evenly distributed in proportion to the number of people exposed at each age group. In general it may be said that from a quarter to a third of the population below 40 was attacked. Above this age the number was less.

It is only by means of these surveys that a true conception of the extent of the disease is obtained. In most communities influenza was not a reportable disease prior to the 1918 epidemic. Even when made reportable to the local health department, the returns are pitifully small compared to the actual number of cases occurring. This is due primarily to the fact that many people do not call a physician, and also because physicians are so overcome with calls that the reporting of the ease becomes to many of secondary importance. During January and February, 1920, there were reported to the Detroit Health Department 13,255 cases. Among these cases there occurred 854 deaths. In addition to this number there were reported 1302 deaths which had never been reported as cases. If the ratio of deaths to eases were the same for unreported cases, this would mean 20,200 cases which had not been reported. Even this computation would give but 33,455 eases for a city of one million people, or 3 per cent. The surveys in other cities of the 1918 epidemic indicated an attack rate of nearly 30 per cent.

During the 1920 epidemic in Detroit, the distribution of cases by sex and age group was indicated from a count of 5000 cases to be as shown in Table XLIX.

TABLE XLIX

\begin{tabular}{ccc}
\hline \hline & PER CENT OF TOTAL CASES \\
AGE GROUP & MALE & FEMALE \\
\hline $0-9$ & 9.6 & 9.0 \\
$10-19$ & 4.4 & 4.2 \\
$20-29$ & 14.4 & 16.6 \\
$30-39$ & 13.0 & 12.2 \\
$40-49$ & 4.7 & 4.3 \\
$50-59$ & 2.2 & 2.7 \\
$60-69$ & .7 & .9 \\
70 and over & .3 & .3 \\
\hline
\end{tabular}

Frost reports that with few exceptions the attack rate at all ages was somewhat higher in females than in males. Thus he shows that for every 100 males attacked there were- 


$\begin{array}{rll}106 & \text { females } & \text { All localities } \\ 118 & \text { “ } & \text { Macon } \\ 113 & \text { “ } & \text { Minor Maryland communities } \\ 110 & \text { “ } & \text { Spartansburg } \\ 109 & \text { “ } & \text { Baltimore } \\ 104 & \text { “ } & \text { New London } \\ 104 & \text { “ } & \text { San Francisco } \\ 103 & \text { “ } & \text { San Antonio } \\ 102 & \text { “ } & \text { Augusta } \\ 101 & \text { “ } & \text { Des Moines } \\ 99 & \text { “ } & \text { Louisville } \\ 98 & \text { “ } & \text { Little Rock }\end{array}$

Excess of influenza in females was most pronounced between the ages of 15 and 35. In Oswego 47.4 per cent of males were attacked to 46.8 per cent females. Corresponding figures in Watertown were 27.3 per cent for males and 28.9 per cent for females. In Oswego the male rate exceeded the female at ages 5-14, 20-39 and 40-59. In Watertown the male rate was less than the female at all ages. In Baltimore the male rate was greater under five years. At other points in Maryland the male rate exceeded the female under five years and from forty to fifty-nine.

There is a distinction to be noted between morbidity and mortality in their influence upon age. The difference between the distribution of the original infection and distribution of the mortality among the age groups is well brought out in the Baltimore studies. Below age thirty-five there are not great differences in the attack rate.

The mortality chart shows quite a different picture. The similarity of the death rates under thirty-five years of age is entirely lacking. From age five to nine there is a progressive ascent in the rates up to age twenty-five to twenty-nine. The population killed in the first group, (five to nine) is .20 per cent; for the latter (twenty-five to twenty-nine) it is 1.01 per cent. The rate falls abruptly to .62 per cent for age thirty to thirty-four, then with even more abruptness to .24 per cent for age thirtyfive to thirty-nine. From this point the rates fluctuate around .2 per cent and exceed .3 per cent only at age sixty and over. (Fig. 55.)

We have explained that going backward from age twenty-nine to twenty-five toward the early ages, the rates fall progressively until we come to the earliest age group, 0-5, and there the descent ceases, for instead of being below .2 per cent this rate is .79 per cent, or second to the highest rate.

These charts are most instructive and suggest that whereas people below age thirty-five are about equally susceptible to influenza, they are not by any means equally susceptible to fatal influenza. The naturally higher mortality at twenty-five to twenty-nine years in civil life will explain why soldiers have had higher rates than civilians of all ages.

Figures for Oswego and Watertown show an even greater death toll at the same age groups than in Baltimore (Table L). 


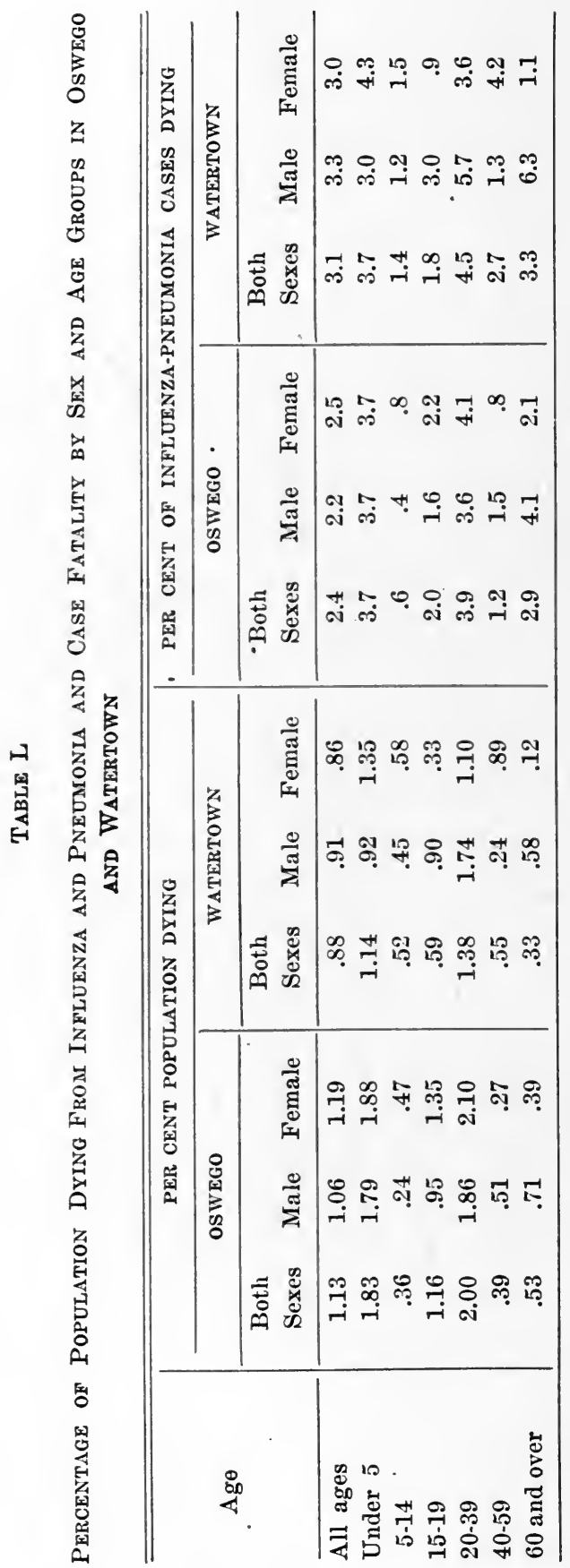




\section{MORBIDITY AND MORTALITY FROM INFLUENZA BY AGE GROUPS AMONC I 6,797 PEOPLE IN BALTIMORE DER CENT OF POPULATION AT. EACH AGE GROUP AFFECTED}
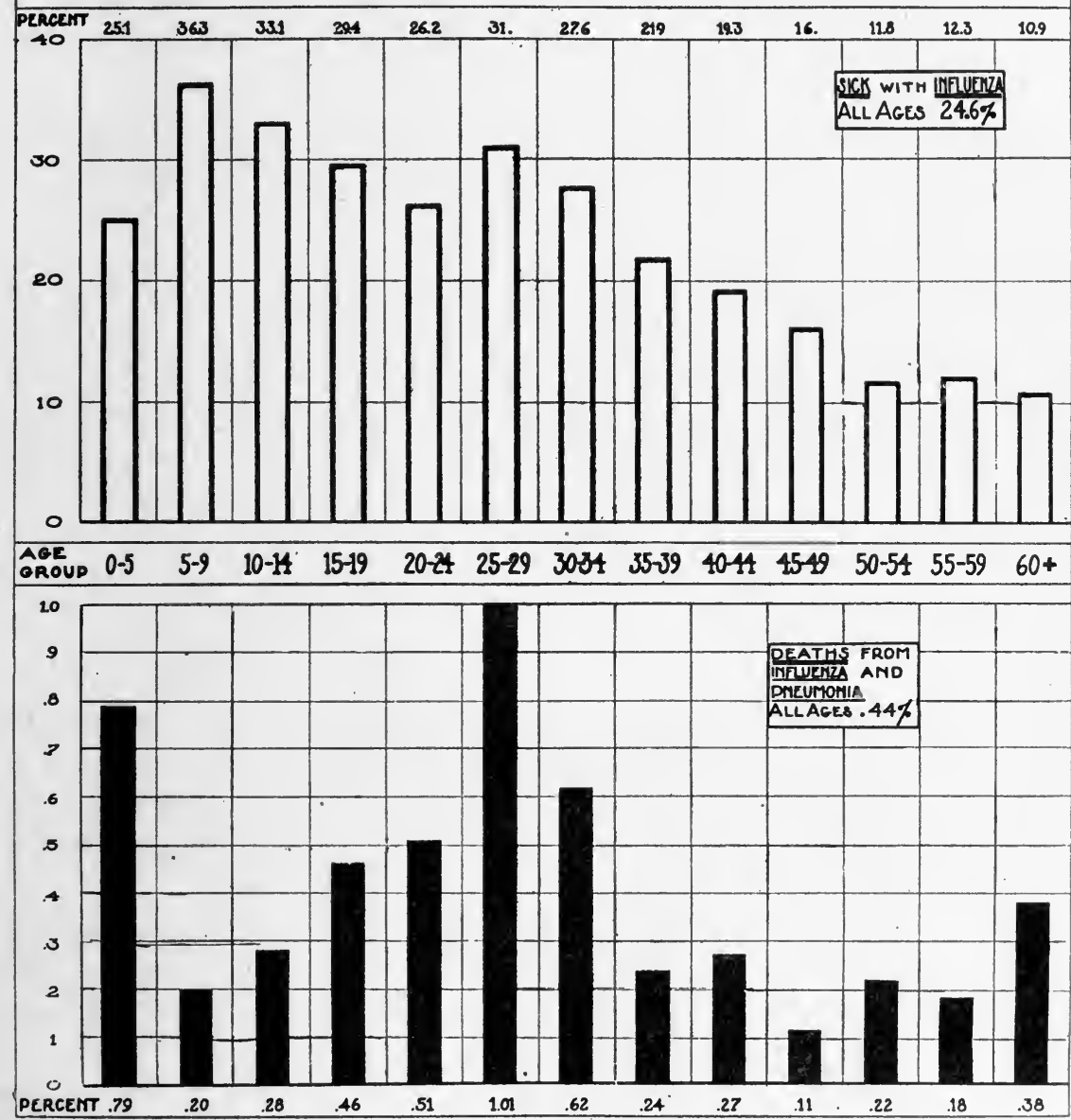

Fig. 55.

The age group twenty to thirty-nine was most severely affected. In Oswego 2 per cent of this group died, and in Watertown 1.38 per cent. In the former place females died in greater numbers than males. In Watertown deaths among males were greater.

As in Baltimore these figures indicate that those under five and those between twenty and thirty-nine succumbed more readily to the epidemic. 
The age group five to fourteen and the groups over thirty-nine suffered much less.

Taking into account the number of influenza cases at the different age groups, we obtain some conception of the varying lethality of the disease. We may state this in the following manner: A person sick with influenza had the best ehance of recovery if between the ages five and fourteen; next best either at fifteen to nineteen, or forty to fiftynine; next if over fifty-nine; next if under five; and the least chance of recovery if between the ages twenty and thirty-nine.

Among young adults, twenty to thirty-nine, about 4 per eent of influenza cases died. In children under five slightly less than 4 per cent of the cases died ( 3.7 per cent). Between five and fourteen about 1 per cent of cases died.

What may be regarded as the most complete data published on influenza and pneumonia mortality is found in a report by the Census Bureau covering the states of Indiana and Kansas and the city of Philadelphia during the last four months of 1918. Influenza and pneumonia, either primary or contributory, were responsible for the death in four months' time of approximately

$$
\begin{array}{llll}
.33 \% & \text { of the people of Indiana } \\
.31 \% & \text { "6 } & \text { Kansas } \\
.78 \% & \text { “ } & \text { " } & \text { Philadelphia }
\end{array}
$$

Death rates by age groups vary in the same manner as those already quoted. The very young and the adult from twenty to forty died in greatest numbers. For single age groups the rate was highest in children under one year in Indiana and Philadelphia. In Kansas age twentynine was the highest.

That we may better understand the extent which each age group suffered, we have prepared a chart for Boston in which the rates for eac' group during the epidemic are contrasted with the deaths from influenza and pneumonia in a normal period. (Fig. 56.)

As the normal we have taken the average deaths from these two causes in the months of September, October and November for the five years 1912-1916 inclusive. At the bottom of the page is a third graph which represents the number of times the death rate in each age group in normal times is contained in the rate during the epidemic period.

In normal times we have an inverted skew curve with the apex at age group ten to nineteen. The highest rates are at the extremes of life.

The second graph presents an entirely different shape, resembling the letter W, with its central peak at the left of the center. The central 


\section{AGE DISTRIBUTION OF DEATHS FROM INFLUENZA ArD PNEUMONIA AT BOSTON}

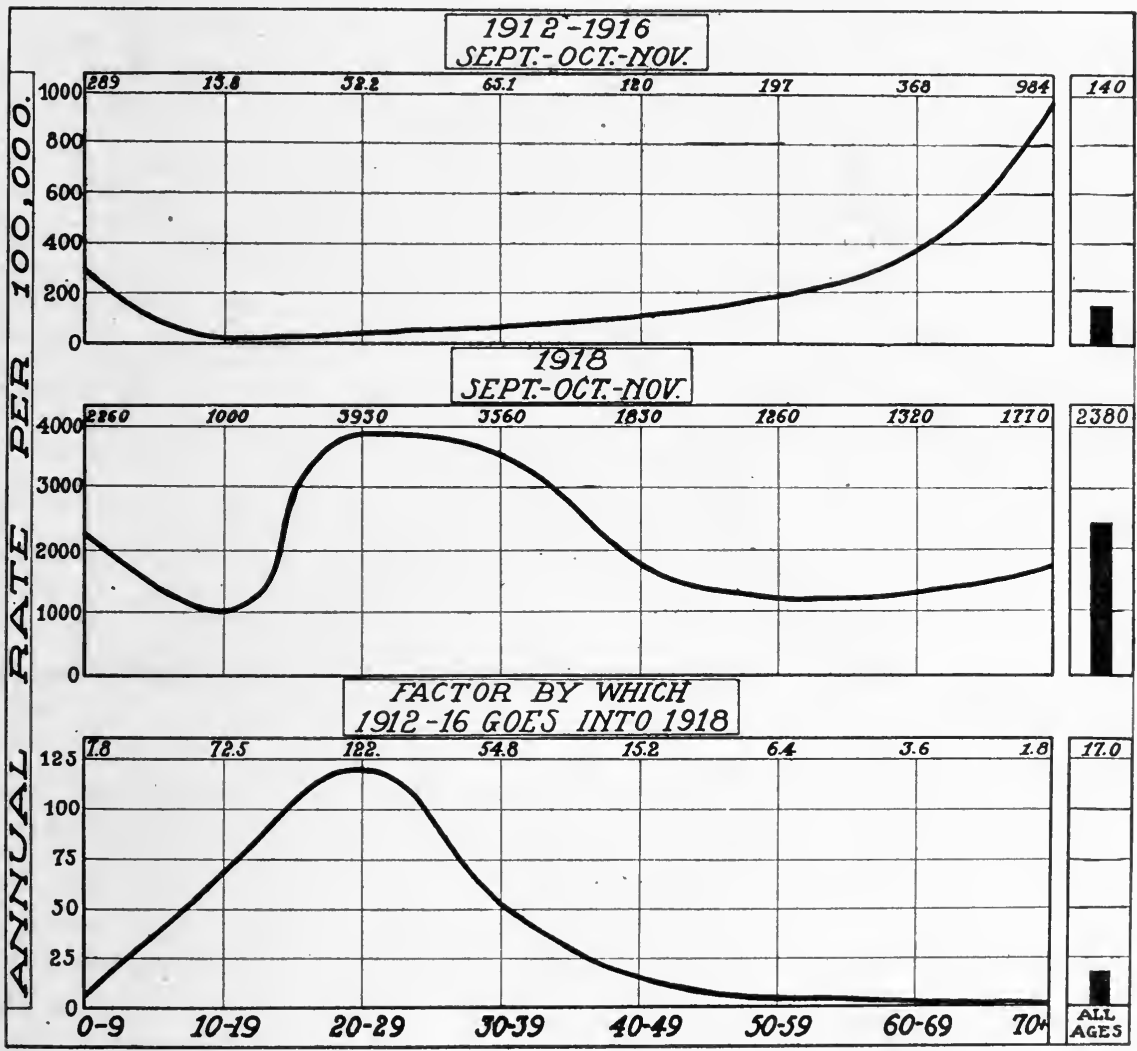

Fig. 56.

peak is the highest point (3930). The valleys are at age ten to nineteen and fifty to fifty-nine. The left wing of the W ascends to 2260. The right wing (1770) rises but slightly from the valley.

The third ehart representing the relation between the upper two is symmetrical about age twenty to twenty-nine as a mode. Either side of the mode, ages ten to nineteen and thirty to thirty-nine have similar rates as do the next removed pair under nine and forty to forty-nine. The three oldest age groups descend in order but the descent is so grad- 


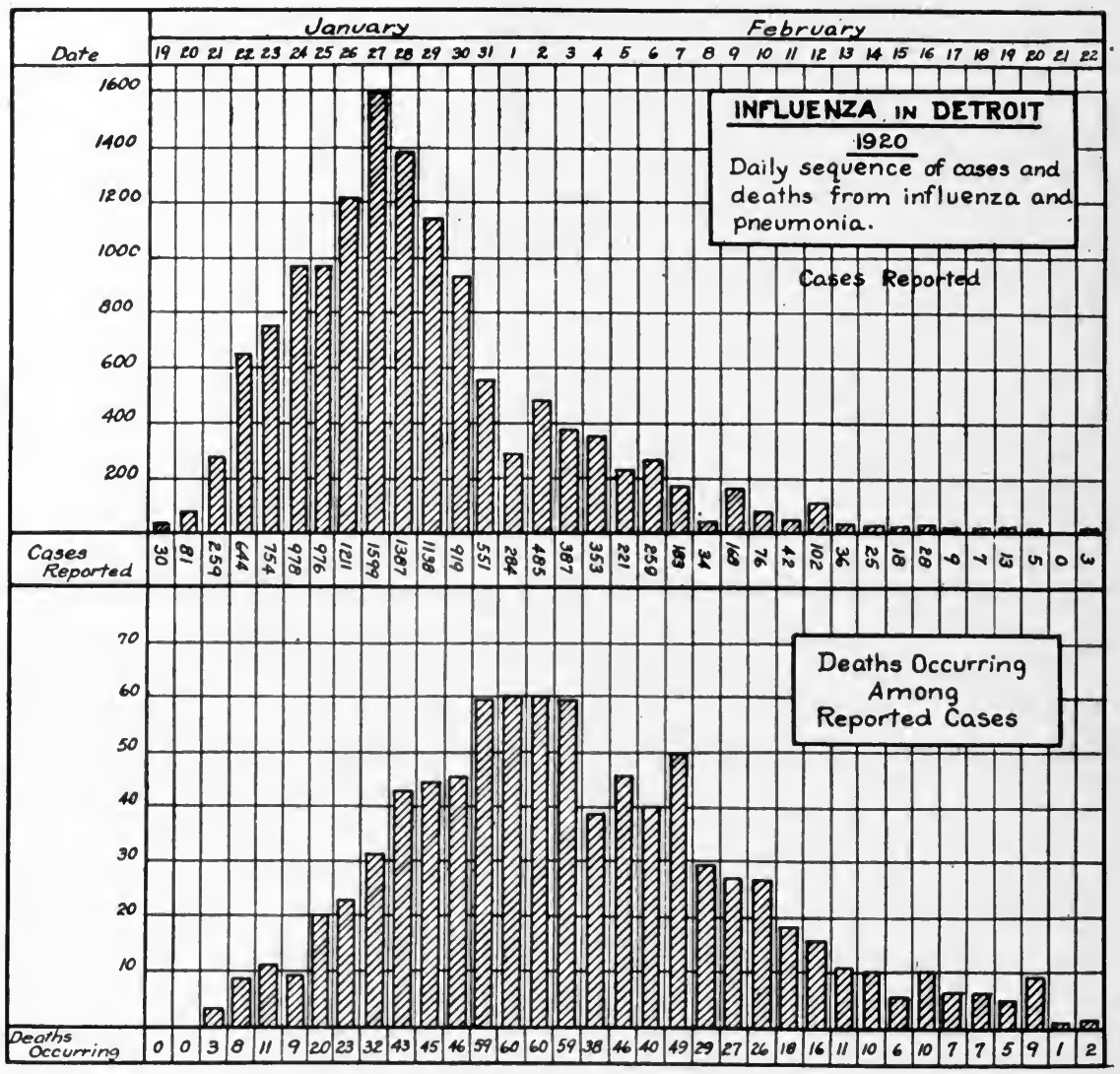

Fig. 57.

ual as to make the curve almost level and parallel with the base line. The effect of age on mortality is indicated by the extremes of the factors. At age twenty to twenty-nine, mortality was 122 times normal. At ages 70 and over, it was only 1.8 times normal.

Studies in Detroit of the 1920 outbreak showed that the disease was more severe with the females of child-bearing age. Above forty, males had greater fatality.

TABLE LI

\begin{tabular}{cccc}
\hline \hline & & PER CENT CASE MORTALITY & \\
AGE GROUP & MALES & FEMALES & TOTAL \\
\hline $0-9$ & 4.9 & 5.1 & 5.0 \\
$10-19$ & 4.0 & 5.0 & 4.5 \\
$20-29$ & 5.3 & 7.1 & 6.3 \\
$30-39$ & 6.4 & 5.8 & 6.1 \\
$40-49$ & 9.6 & 6.5 & 8.1 \\
$50-59$ & 10.0 & 7.3 & 8.5 \\
60 and over & 24.6 & 17.5 & 20.6 \\
\hline
\end{tabular}


We are indebted to Elwood, Secretary of the New York State Hospital Commission, for valuable statistics bearing on influenza in the state institutions. Records were collected from thirteen institutions and represent over 40,000 people. Influenza was more prevalent among females, both employes and patients. In the former it was 60 per cent greater among females. In the latter there were 20 per cent more cases among females. The female was more susceptible to the disease than the male.

Both groups-patients and employes-show from 30 to 40 per cent more pneumonia among male influenza cases than among females.

Among employes fatality among pneumonia cases is 60 per cent greater in males.

Among patients, pneumonia case fatality is 10 per cent greater in females.

Considering the percentage of population dying from the epidemic, the rate among employes is 40 per cent greater among males. Among patients the rate is almost identical for males and females.

\section{TABLE LII}

Influenza in New YORK STATe Hospitals

Perlod-October 1, 1918-January 15, 1919

\begin{tabular}{|c|c|c|c|c|c|c|c|c|}
\hline & \multicolumn{4}{|c|}{ OFFICERS AND EMPLOYES } & \multicolumn{4}{|c|}{ PATIENTS } \\
\hline & \multicolumn{2}{|c|}{ NO. OF CASES } & \multicolumn{2}{|c|}{ RATIO* } & \multirow{2}{*}{$\begin{array}{l}\text { No. of } \\
\text { Males }\end{array}$} & \multirow{2}{*}{$\begin{array}{l}\text { CASES } \\
\text { Females }\end{array}$} & \multicolumn{2}{|c|}{ RATIO* } \\
\hline & Males & Females & Males & Females & & & Males & Females \\
\hline $\begin{array}{l}\text { Average daily } \\
\text { strength }\end{array}$ & 2907 & 2564 & & & 16,200 & 19,037 & & \\
\hline $\begin{array}{l}\text { Per cent hav- } \\
\text { ing influenze }\end{array}$ & 16.1 & 26.2 & & 1.8 & 7.8 & 9.2 & & 1.2 \\
\hline $\begin{array}{l}\text { Per cent influ- } \\
\text { enza patient } \\
\text { having pneu } \\
\text { monia }\end{array}$ & $\begin{array}{ll}- & \\
\text { ts } & \\
\text { u- } & \\
& 16.6\end{array}$ & 11.7 & 1.4 & & 33.0 & 24.7 & 1.3 & \\
\hline $\begin{array}{l}\text { Per cent pneu- } \\
\text { monia patier }\end{array}$ & ints & & & & & & & \\
\hline dying & 37.2 & 22.8 & 1.6 & & 61.4 & 67.0 & & 1.1 \\
\hline $\begin{array}{l}\text { Per cent } \\
\text { strength dyin }\end{array}$ & ng 1.0 & .70 & 1.4 & & 1.57 & 1.52 & 1.03 & \\
\hline
\end{tabular}

*Note: The ratio is based on a value of 1 for the lower figure.

Corresponding figures for the U. S. Army camps are as follows:

Per cent having influenza

23.2

Per cent influenza having pneumonia

Per cent pneumonia cases dying

Per cent strength dying
18.3

31.2

1.33

(See Fig. 53, p. 340, also Fig. 59, p. 401.)

The age distribution of influenza mortality changed as the epidemic progressed. From the Boston experience which we have noted, the 
infection appeared heaviest, first among the young adult twenty to twenty-nine years. In September 32.3 per cent of all influenza and pneumonia deaths were for this age group. In October the percentage was 27.6 and in November 16.2. The age group nine and under represented 13.7 per cent of all influenza and pneumonia deaths in September, 20.1 per cent in October and 20.6 per cent in November. Similarly the percentages for the age of thirty and over was for the three months 6.7, 8.9 and 14.0.

Expressed in another manner, age group twenty to twenty-nine in September saw 2.4 times as many deaths as age nine and under, and 4.8 times as many as age thirty and over. In October age group twenty to twenty-nine was only 1.4 times age nine and under and 3.1 times age thirty and over. In November the epidemic had burned itself out among the young adult and found its sustenance elsewhere. Thus age twenty to twenty-nine was actually less, or .8 as great in deaths as age nine and under and but 1.2 times greater than age thirty and over.

This same phenomenon was observed in the 1920 Detroit outbreak, the young adult (twenty to twenty-nine) being the first attacked. During the first five days these cases were 35 per cent of the total. In the second five days they amounted to 33 per cent; third five-day period twentynine per cent and fourth 28 per cent. The percentage of cases among children under ten years were first 14 per cent of the total. The succeeding percentages was 16,20 and 26 .

TABLE LIII

\begin{tabular}{lccccccc}
\hline \hline PERIOD & $0-9$ & $10-19$ & $20-29$ & $30-39$ & $40-49$ & $50-59$ & 60 AND OVER \\
\hline $\begin{array}{l}\text { 1st 5 days } \\
\text { Jan. 19-23 }\end{array}$ & 14. & 10. & 35. & 26. & 11. & 4. & .8 \\
$\begin{array}{l}\text { 2nd 5 days } \\
\text { Jan. 24-28 }\end{array}$ & 16. & 9. & 33. & 28. & 10. & 5. & 2.6 \\
$\begin{array}{l}\text { 3rd 5 days } \\
\text { Jan. 29-Feb. 2 }\end{array}$ & 20. & 8. & 29. & 24. & 9. & 5. & 3.2 \\
$\begin{array}{l}\text { 4th 5 days } \\
\text { Feb. 3-7 }\end{array}$ & 26. & 7. & 28. & 23. & 8. & 5. & 3.5 \\
\hline
\end{tabular}

These figures indicate that the age groups which mingled most freely with their fellow beings were the first to be attacked. It was then carried into the home and passed on to the less mobile age groups, children under 10 and people over 60. Vaughan (Warren) in the Boston survey observed that the first ease in a family was much more frequently the wage earner.

The negro was apparently less susceptible to influenza than the white, but once attacked, his chances for recovery were not as good. Frost 
gives the case rate and the case fatality among white and colored for various cities (Table LIV).

TABLE LIV

\begin{tabular}{lcccc}
\hline \hline & $\begin{array}{c}\text { PERCENTAGE } \\
\text { White }\end{array}$ & $\begin{array}{c}\text { ATTACKED } \\
\text { COLORED }\end{array}$ & \multicolumn{2}{c}{ PERCENTAGE DYJNG } \\
WHITE & COLORED \\
\hline Augusta & 46 & 21 & 2.1 & 2.1 \\
Minor Maryland Communities & 42 & 39 & 1.7 & 1.2 \\
Little Rock & 36 & 34 & 1.4 & 1.8 \\
Baltimore & 28 & 12 & 1.3 & 3.4 \\
Spartansburg & 22 & 17 & 1.2 & 0.8 \\
Macon & 22 & 14 & 1.0 & 2.2 \\
Louisville & 18 & 5 & 0.9 & 1.2 \\
$\quad$ Mean & 31 & 20 & 1.4 & 1.8 \\
\hline
\end{tabular}

Taking the approximate mean for the various cities as an index, it appears that the white race had about 50 per cent more influenza than the colored. On the other hand, the colored people with influenza died in 30 per cent greater numbers than the whites.

Frankel and Dublin give a very convincing analysis of the racial difference during the epidemic of 1918:

"The second consideration is with reference to color. In this connection, a very clear picture is presented. Normally, the respiratory diseases are much more highly represented among colored persons than among whites, and this applies to both sexes. Thus, in the seven year period, from 1911 to 1917, influenza-pneumonia showed an excess of 72 per cent colored males over white males, and of 56 per cent colored females over white females. But, during. the period of the epidemic, the situation was reversed. The whites suffered from higher rates than the eolored. This is clearly shown during the first three months of the epidemie, when colored males showed a rate of 1,522 per 100,000 , as compared with a rate of 1,844 per 100,000 for white males. The same condition is shown among females, the colored rate being 1,504 and the white rate 1,723. Put in other words, while the rate among white males during this period, October to December, was nearly fifteen times as great as during the period 1911 to 1917 , that of colored males was only seven times as great as the rate during the same seven year period. White females during the height of the epidemie showed a rate more than sixteen times as high as the normal, while colored females experienced a rate only nine times as high. After the first of January, 1919, that is, after the severest period of the epidemic had passed, the colored group showed higher rates than the white, and the amount of excess approximated what had prevailed in normal times, as the distance from the explosive period of the epidemic inereased. In the last quarter, April to June, the excess of colored males over white males was 94 per cent, and of colored females over white females, 83 per cent, figures which are almost identical with those for the twelve months ending September 30,1918. The facts indicate with great clearness that the effect of the epidemic was much greater among white lives than among colored lives. This difference wore off as the epidemic waned and conditions returned to normal, as they virtually did during the last quarter of this period, from April to June, when a marked excess of colored mortality is shown over white mortality. Table 4, 
[Table LV] below, gives the relation of colored to white mortality by quarters for each of the two sexes.

TABLE LV

Influenza Pneumonia Deati Rate Classified by Sex and by Quarters, OCTOBER, 1918, TO JuNE, 1919

\begin{tabular}{ccc} 
PERIOD & $\begin{array}{c}\text { PERCENTAGE } \\
\text { MALE }\end{array}$ & $\begin{array}{c}\text { COLORED OF WHITE* } \\
\text { FEMALE }\end{array}$ \\
\hline October, 1918, to June, 1919 & 97 & 101 \\
October to December & 83 & 87 \\
January to March & 139 & 133 \\
April to June & 194 & 183 \\
Norm : & & \\
October, 1917, to Sept., 1918 & 193 & 185 \\
Total, 1911 to 1917 & 172 & 156 \\
\hline
\end{tabular}

*'This means ratio of colored to white with white taken as 100 .

In certain army camps of this country, where there were appreciable numbers of colored troops, the incidence was found to be greater in general among the white. Thus 25.7 per cent of 426,000 white soldiers had influenza as eompared to 20.3 per cent of 93,700 colored troops. All camps were not alike in this respect. Thus in seven out of twenty camps of which we have record the incidence of the disease was actually greater among colored.

The severity of the disease among the two races varied so widely that we suspect the introduction of other influences, such as fatigue and immunity and length of service. Pneumonia was more pronounced among negroes in nine camps and less pronounced in two. At Johnston pneumonia followed in 21 per cent of the white influenza patients and in but fifteen per cent of the colored.

At Eustis only 12 per cent of the white influenza patients contracted pneumonia as against 40 per cent of the colored.

Grouping the numbers from all camps concerned, we find that 19.6 per cent of white influenza patients contracted pneumonia. Among 13,266 colored influenza patients, 20.5 per cent had pneumonia. Thus for every 100 white influenza patients contracting pneumonia, there were 105 colored.

The case fatality among the colored pneumonia patients was very slightly greater than among the whites. Six camps-Bragg, Eustis, Greene, Wadsworth, Beauregard and Dodge, witnessed greater fatality among the negroes. Six other eamps-Bowie, Devens, Johnston, Pike, Dix and Travis, had less fatality among the negroes. The white fatality was about 2.5 times greater at Dix and more than twice as great at Johnston. In no instance did the colored fatality exceed the white by over 30 per cent. 
Among 12,050 white pneumonia patients, the proportion dying was 24.7 per cent. Among 2771 colored pneumonia patients the mortality was 25.9 per cent. For every 100 white patients dying there were 105 colored. This relation is based on the supposition of an equal number of white and colored soldiers.

The epidemic killed a greater proportion of colored than of white soldiers. The combined effect of these different factors resulting in death indicates that the colored soldier fared worse than the white. The camps, however, were equally divided in this reckoning.

Of 496,000 white soldiers, 1.25 per cent died from the epidemic. Of 97,900 colored soldiers, 1.39 per cent died. If the strength had been the same this would mean that for every 100 white soldiers dying there were '111 colored deaths.

Physical Stamina and Influenza.-We have been accustomed to think that the strong healthy body is more resistant to disease. Barring the question of specific immunity, naturally or artificially acquired, the robust person is better able to withstand the vicissitudes of life than the weak and sickly. So far as influenza is concerned, specific immunity to the disease is a greater protection than general physical health.

There is an interesting illustration in this connection to be found in the history of Camp Dix. The personnel of this camp at the time of the epidemic was made up of the $34 \mathrm{th}$ Division, recently arrived from Camp Cody. These men were recruited from the west and their general bearing and physical condition were such as to command admiration on all sides. The balance of the camp was made up, for the most part, of limited service men from the northeast-men whose physical condition was such as to prevent them from measuring up to all the requirements of a full-fledged soldier. The western men had been at Dix two weeks when influenza began. They lost by death about 1.9 per cent of their strength. Other aggregations in camp lost 1.7 per cent. The men of the 34 th Division had been in service about 5.2 months, the other organizations, 3.1 months. The urban men of the 34 th Division had a pneumonia case mortality of 18 per cent, the rural men 27 per cent. Among the eastern men, whether rural or urban, the pneumonia case mortality was about 10 per cent. In this instance good physical condition did not offer special protection against the disease.

Syracuse had about the poorest grade of men physically of any camp, the men coming from the deferred classes of the draft. Eighty-five per cent were rated as Class C men, which meant unfitness for military duty. The total mortality at Syracuse was just half that of Camp Sherman, made up of physically strong men. The pneumonia case mortality at Syracuse was the third highest among forty large camps. Low morbidity but high case mortality was observed among the limited service 
men of the Quartermaster Corps at Camp Custer. At Upton there was more pneumonia developing among the influenza cases in the development battalions, made up of physically unfit men than in the rest of the camp. Deaths among the pneumonia cases were not, however, unusually high in this camp. Raritan Arsenal, New Jersey, had men of inferior physical development and yet their sickness incidence was but 15 per cent and mortality .31 per cent, or thirty-first among forty-four smaller eamps. (Fig. 53.)

We must, of course, not confuse poor health with physical defects. Class $\mathrm{C}$ of the draft had many healthy men who were unfitted for military duty because of physical injury or missing legs, arms, etc. There is no reason to expect a one-legged man to be more susceptible to disease than a man with two legs.

Although robustness and physique were not a guarantee against a severe attack of influenza, there is ample evidence that the lowering of physical tone by fatigue produced an unfavorable effect. The seasoned soldier weathered the epidemic better than the recruit.

At Camp Hancock we find that next to the two organizations who were massively infected en route from Camp Grant, the units with the most sickness were the 5th and 6th Machine Gun Training Corps, composed of men from Louisiana, Alabama, and North Carolina, selected in the July, 1918 draft. These men were of poor physique, of less than average mentality, and over 50 per cent were infected with hookworm when they entered the service. About 3.3 per cent of these men died; whereas, the mortality for the entire camp was 1.4 per cent.

The recruit, strange to camp life, unused to cold, unaccustomed to severe physical exertion, was the one primarily responsible for sickness in the American army. The seasoned man, hardened by his camp life, was rarely ill. This was the experience in the winter of 1917-18. It was the experience during the influenza epidemic. The seasoning process as much as anything, means improved vasomotor tone. The body learns to respond to changes in temperature, to resist cold without the accompanying nasopharyngeal and intestinal congestion and without undue loss of body heat. It is this change and the regular removal of fatigue and waste products, which make a man a more efficient physiological machine and together with the association in close contact with many others, make him less susceptible to respiratory disease.

Forty-six per cent of Camp Sherman's strength had seen less than one month's service. Sherman was the worst sufferer from the epidemic. Cody lost about the same percentage of strength and 60 per cent of the camp were fresh recruits. Fifty per cent of the men at Beauregard, 40 per cent at Grant, 55 per cent at Forrest, many at Dodge, 30 per cent at 
Devens, 33 per cent at Custer, 50 per cent at Greene, 90 per cent at Syracuse, had been in service less than one month.

These camps stand in the upper half of the mortality table. In the lower half of the mortality table may be mentioned Fremont with no recruits, Travis with 4 per cent, Las Casas with 5 per cent, Sheridan with 6 per cent, Eustis with 7 per cent, McClellan with 10 per cent.

Dividing the camps into four groups, according to mortality we find the first, representing the highest mortality, had 41 per cent recruits, the second 31 per cent, the third 22 per cent and the fourth 16 per cent.

Draft men entering Camp Grant the first week in September lost nearly 4 per cent of their strength from influenza. The colored draft arriving late in September likewise lost about 4 per cent of their strength. The mortality of other units of the camp was around 2 per cent, or half as much.

\begin{tabular}{lccccc}
\multicolumn{3}{c}{ Camp Grant } & \multicolumn{3}{c}{ Per cent } \\
& & Number & Deaths & Mortality & Ratio \\
Men in service more than one month & 17,200 & 297 & 1.7 & 1.0 \\
Men in service less than one month & 16,200 & 625 & 3.9 & 2.8
\end{tabular}

If recruits had not been sent to Grant in September, the camp mortality rate from the influenza epidemic would have been 1.7 per cent. The arrival of over 16,000 recruits raised the camp rate to 2.6 per cent, an increase of 53 per cent.

It was the observation of Kovinsky, epidemiologist at Camp Grant, that the seasoned men had just as much influenza but they did not succumb as readily. In other words, they were not immune to the influenza infection, but they passed through the illness better.

It was the experience at Camp Pike that there was not only greater fatality among recruits, but that there was also more influenza.

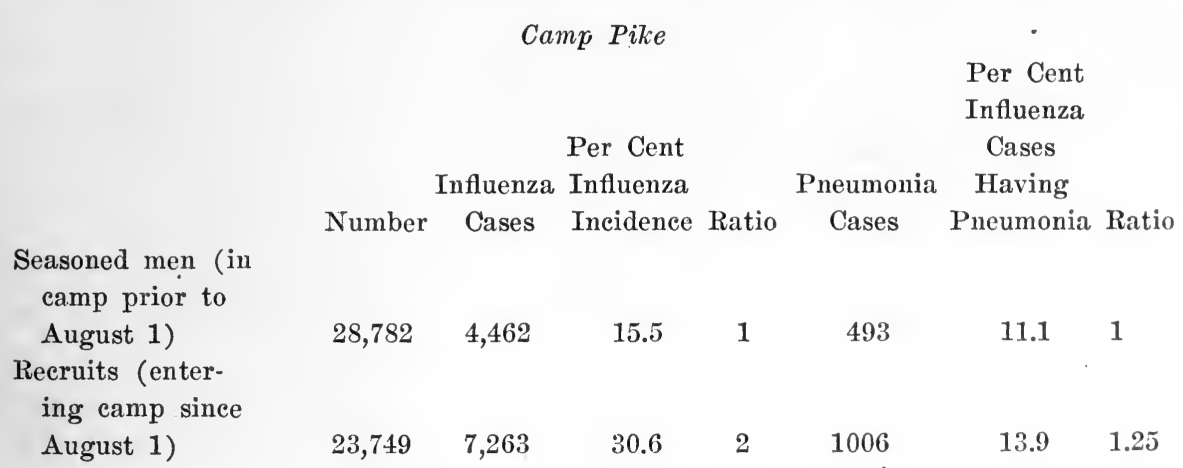

Among the seasoned men having influenza 11 per cent developed pneumonia. Of the recruits sick with influenza 13.8 per cent contracted pneu- 
monia. This is in the ratio of 1 for the seasoned men to 1.25 for the recruits.

Practically all men at Funston had seen more than one month's service. Differences in the term of service existed in various units beyond this time. The 41st Infantry consisted almost entirely of men long in the service. The 20th Infantry comprised one-fourth seasoned men and three-fourths relatively new men. The 70th Infantry and the 69 th were composed for the most part of recent draft men. The 30th Machine Gun Battalion and the 28th Field Artillery were likewise made up of new men. The proportion of sick and the mortality in those units is shown in Table LVI.

TABLE LVI

\begin{tabular}{|c|c|c|c|}
\hline UNITS & PERSONNEL & $\begin{array}{c}\text { PER CENT } \\
\text { ADMITTED } \\
\text { TO HOSPITAL }\end{array}$ & $\begin{array}{l}\text { PER CENT } \\
\text { DYING }\end{array}$ \\
\hline 41st Infantry & Seasoned & 17.3 & .58 \\
\hline 20th Infantry & Seasoned & 22.4 & 1.27 \\
\hline 30 Machine Gun Battalion & New & 61.3 & 4.55 \\
\hline 28th Field Artillery & New & 26 & 2.05 \\
\hline 69th Infantry & New & 28.5 & 1.46 \\
\hline 70th Infantry & New & 34.2 & 4.12 \\
\hline
\end{tabular}

This was during the period of the influenza epidemic. Hospital admissions were not wholly for influenza.

Dividing the Divisional Units into seasoned men and recruits the following relation is shown:

\begin{tabular}{lcccc} 
& & PER CENT & TOTAL PER CENT & \\
& NUSPITA & DYING FROM INFLUENZA & \\
& NUMER & ADMISSIONS & AND PNEUMONIA & RATIO \\
\hline Relatively seasoned men & 16,183 & 33.8 & 1.39 & 1 \\
Relatively new men & 8,972 & 30.3 & 2.29 & 1.65 \\
\hline
\end{tabular}

The difference in morbidity is negligible, the recruits contributing less to the hospital than the seasoned group. In mortality the recruits died in the ratio of 1.65 to 1 . This difference is less than at Grant, where there was greater divergence in the length of service.

It was noted at Camp Custer that the men with only two months or less of service were far more susceptible to influenza than those of longer service.

Camp Lee furnishes interesting information on this question. An examination of 445 deaths from influenza and pneumonia showed 30.1 per cent were among men of less than one month's service and yet not more than 9.2 per cent of the camp strength had been in service less than one month. The figures are given below: 


$\begin{array}{lccc} & \begin{array}{c}\text { LESS THAN } \\ \text { ONE MONTH'S } \\ \text { SERVICE }\end{array} & \begin{array}{c}\text { ONE TO THREE } \\ \text { MONTHS' } \\ \text { SERVICE }\end{array} & \begin{array}{c}\text { MORE THAN } \\ \text { THREE MONTHS } \\ \text { SERVICE }\end{array} \\ \begin{array}{c}\text { Per cent distribution } \\ \text { of camp population }\end{array} & 9.2 & 45.2 & 45.6 \\ \begin{array}{c}\text { Per cent dying from influ- } \\ \text { enza and pneumonia }\end{array} & 30.1 & 46.2 & 23.7\end{array}$

Of the first 4,269 cases of influenza at Sherman, 69 per cent occurred in 15,493 recruits of one month's service or less who comprised less than one-half the camp strength.

Fort Logan, which stood fifth in the mortality table for medium-sized camps lost 2.3 per cent of its strength in the epidemic. Thirty-five per cent of the strength were draft men from Utah who arrived in camp the week preceding the epidemic. Anticipating sickness among these recruits, they were placed in pyramidal tents, three and four men to a tent. They were inspected twice daily by a medical officer. They were kept isolated from the rest of the command and messed separately, and yet these precautions did not prevent the epidemic from exacting a heavy toll.

Raritan Arsenal at Metuchen, New Jersey, composed of 5,000 men lost .36 per cent of its strength. These men came from other posts or camps. None came directly from civil life.

There was much more influenza and pneumonia among recruits at Ft. McDowell, California.

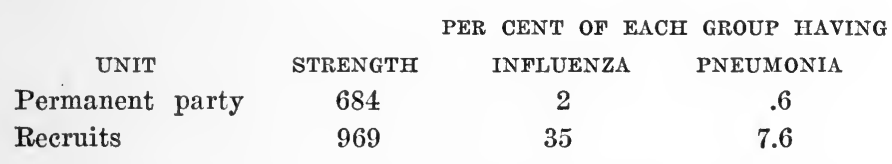

Influenza morbidity and mortality were greater among limited service recruits at Columbus Barracks, Ohio. The average length of service of recruits was two weeks. They were arriving while the epidemic was in progress.

\begin{tabular}{lcccc} 
& \multicolumn{3}{c}{ PER CENT OF EACH GROUP } \\
& WITH & \\
& STRENGTH & INFLUENZA & DYING & RATIO \\
Permanent party & 929 & 3 & .32 & 1.0 \\
Recruits & 1814 & 12 & 3.0 & 9.3
\end{tabular}

Reports from Upton show eight new men dying to one seasoned man. Recruits were scattered among companies of the 2nd Battalion in the proportion of three recruits to two camp men, and yet at least 167 recruits became sick before a case was discovered among the men who had been in camp prior to September 6 . 


\begin{tabular}{ccccc}
\multicolumn{5}{c}{ PER CENT OF EACH GROUP } \\
HAVING \\
UNIT & STRENGTH & INFLUENZA & DYING & DEATH RATIO \\
Camp men & 991 & 12 & .4 & 1 \\
Recruits & 1369 & 45 & 3.3 & 8
\end{tabular}

Using .4 per cent mortality to represent the camp less the recruits and the transient units, it will be seen that Upton's rate was raised 300 percent by recruits (Upton's rate was 1.2 per cent).

Draper made a special study of fatigue and exposure and its relation to disease while at Camp Funston in the spring of 1918. He notes the ease of Company C, 355th Infantry which received 172 new men fresh from three weeks initial stay at the Detention Camp. They joined the Company the latter part of March and after participating in the heavy drill schedule for a week or so, began to develop illness elosely resembling influenza. The first eases appeared on April 8. The admissions at this time were:

\begin{tabular}{|c|c|}
\hline April 8 & 8 \\
\hline & 9 \\
\hline 10 & 0 \\
\hline
\end{tabular}

On the tenth, drill was abandoned and the men were allowed to rest in barracks for four days. The admissions after the tenth were as follows:

$\begin{array}{rr}\text { April } 11 & 8 \\ 12 & 1 \\ 13 & 1 \\ 14 & 2 \\ 15 & 1\end{array}$

This experiment was sufficiently convineing to the authorities at Funston so that the work schedule was immediately reduced for recruits in the Detention Camp. The result of this plan is seen among 10,000 recruits arriving at Funston about this time. A group of 6,500 was quartered at Camp Republican, and another group of 3,500 at Camp Pawnee. The drill schedule was intended to be the same for both groups but it worked out differently. At Pawnee, owing to the congestion at the receiving station, the men coming from the trains were delayed from two to four days in passing through the receiving station. The drill schedule planned was eut in half for these men and no heavy fatigue work was required. This group, therefore, had a much more graduated introduction to the rigors of camp life than had been the custom heretofore.

The progress of the recruits at Camp Republican was more rapid. Arriving at all hours of the day and night they were passed through the 
receiving station without delay. They arrived tired and sleepless after long journeys in crowded cars. Without rest they were registered, unitormed and inoculated with triple typhoid and smallpox vaccines. During this procedure they moved slowly forward in a long line, part of the time naked, keenly alert to their new and strange surroundings. On top of a sleepless journey it was an exhausting process. Several men fainted daily.

From the receiving station this group was driven five miles to Camp Republican and assigned to quarters. Another sleepless night ensued for many as the vaccination reaction set in. There was no drill on the next day's schedule, but in its place there was policing of the grounds. Some of the recruits were observed carrying rock down from a hillside to make paths. It must be remembered that some of these had been at sedentary occupations only a few days before. The pulse readings of a group chosen at random were taken after exertion dyspnea had passed.

$\begin{array}{lc}\text { OCCUPATION } & \text { PULSE RAT:E } \\ \text { Druggist } & 148 \\ \text { Bookkeeper } & 168 \\ \text { Salesman } & 128 \\ \text { Foundryman } & 100 \\ \text { Stationary Fireman } & 132 \\ \text { Dishwasher } & 120 \\ \text { Driver } & 122\end{array}$

The next day's drill commenced under the reduced schedule which incidentally was double that at Camp Pawnee. Three or four days later the men began to report sick with symptoms resembling influenza. Sickness was not absent at Pawnee but the difference in the admissions at the two places is clear cut. Within three weeks from their entrance to camp, the 6,500 men at Republican had sent 427 to the sick list, or 6.5 per cent. Pawnee in the same period added 131 of their 3,500 to the sick list, or 3.7 per cent.

The best demonstration of the harmful effect of fatigue on recruits is to be seen among men in railway transit from one station to another. To this cause Camp Cody owes its advance from thirtieth to second in the mortality table. Beginning September 26 the epidemic had by October 22 practically burned out after affecting 15 per cent of the personnel and causing the death of .8 per cent. The arrival of draft men from Minnesota and Oklahoma reintroduced the disease and it continued to rage until the end of November.. When the epidemic was over, 44 per cent of the entire camp had been afflicted and 3.3 per cent had died. Sick recruits nearly tripled the morbidity rate and quadrupled the mortality rate.

The Cody recruits had been sent from their homes while the epidemic 
was broadcast. Some were probably sick when they boarded the train; others contracted the disease en route. Two to four days travel under these conditions caused men to die on the train. "Hundreds were rushed from the train to the hospital, and in a few hours from the hospital to the morgue."

Forrest had a similar experience. Influenza among 7,800 men in camp during October affected 21 per cent and caused the death of 1.3 per cent. The disease had about disappeared when several thousand draft men from the middle west arrived the latter part of October. This group was thoroughly infected en route. Thirty cases of influenza were removed from the train. The epidemic again broke out with the result that by the middle of November, Forrest had an influenza morbidity of 30 per cent and a mortality of 2.1 per cent. Morbidity was thus raised nearly 50 per cent and mortálity 60 per cent.

Influenza was first noted at Hancock on September 28. On the thirtieth, 3,108 men arrived from Camp Grant. Seven hundred of this group were sick with influenza and went directly to the hospital from the train. Over 700 more followed in the next two days. The fatigue of the journey and the massiveness of the infection made 39 per cent sick and caused the death of 4.6 per cent. The camp as a whole had 22 per cent sick and 1.4 per cent died. Morbidity was 80 per cent greater and mortality more than three times as great among the new men.

The fatigue and exposure of transit on the electric railroad reacted disastrously on influenza patients evacuated from the Syracuse Recruit Camp to Fort Ontario, 35 miles distant. "The rate of pneumonia complication and the death rate were especially high among this group although at the time an endeavor was made to send only those cases that did not seem seriously ill."

The bad condition of recruits arriving at Fort Screven, Georgia, was a subject of comment. It is reported that some recruits were taken from sick beds by the local draft board in order to fill the quota. Travel seriously affected men reporting to Fort Stevens, Oregon. Men sick with influenza were being removed from trains continually.

On October 26 and 27, between 1:30 Р. м. and 3:00 А. м., $780 \mathrm{draft}$ men arrived at Ft. MeArthur, California, from northern Colorado, Wyoming, and Oregon.

"Of these 42 were taken directly to the hospital, suffering from influenza or pneumonia. Twenty-nine were received the following day. By November 3, 122 of these men were admitted to the hospital on account of influenza. During this period a number of these were admitted to the hospital suffering from tonsillitis, bronchitis, ete., which invariably follows a new arrival of men, especially from the draft. An effort was made to differentiate between these conditions and influenza. Of the $122,57 \mathrm{de}$ veloped bronchopneumonia within from one to five days after admission. So virulent was the infection that eleven died within 24 hours of the first symptoms of pneumonia, 
and eight others within $4 \mathrm{~S}$ hours, making 19 who died within 48 hours of the first reeognized symptoms of pneumonia. The appearance of these patients was from the first that of extreme gravity."'

The first cases of influenza at Camp Kearny were of a mild type, and no pneumonia was reported until October 1 .

"This occurred in a detachment of 70 men, which arrived on this date from Camp Taylor, Ky. One soldier having pneumonia and seven having influenza were transferred to the Base Hospital directly from the train on arrival in Camp. The entire detachment was placed in an isolation camp at once. About 20 other cases developed. The influenza occurring in this detachment was of a more severe type than that admitted previously. The first death was reported October 6. This was the soldier who was admitted to the hospital with pneumonia five days previously. The onset of pneumonia began from 24 hours up to five or six days after the onset of influenza. Fatigue was considered a causative factor in the early onset of pneumonia, also in the severity of influenza without pneumonia.',

We have little doubt that the high incidence of disease and death among the 34th Division from Cody which arrived at Camp Dix just prior to the epidemic was influenced by the fatigue of travel.

There are many illustrations of this nature. The sudden transition of civilians to army life, the fatigue incident to traveling to their station, the massive infection acquired as a result of close association with sick or carriers on the trains did a great deal to elevate the mortality in certain army camps. The principal effect of fatigue seems to have been in reducing the body's fighting powers, for an increase in the intensity of the disease is seen to be more pronounced than an increase in the number of those contracting influenza.

Influenza was more severe among certain classes of physically weak in the civil population. The pregnant woman, people with heart trouble and tuberculosis showed a heightened death rate during the epidemic. Figures published by the Metropolitan Life Insurance Company are interesting in this connection (Table LVII).

It is to be noted that this same observation has been made previously. Parkes states that certain other diseases increased in mortality at the time of the epidemic in 1889-1890 in Paris. He mentions especially phthisis, heart disease, apoplexy, paralysis, diabetes, cirrhosis of the liver, and Bright's disease. There was but a slight increase in mortality from measles and whooping cough. In London phthisis deaths diminished following the epidemic.

As further evidence that influenza, in spite of its apparent partiality for the young and robust, did also pick out those weakened by certain diseases, we may mention the conclusions of Pearl who computed the correlation between the mortality in U. S. cities and various factors, such as density of population, geographical location, etc. He found 
TABLE LVII

Death Rate Per 100,000 Among White Industrial Policy Holders

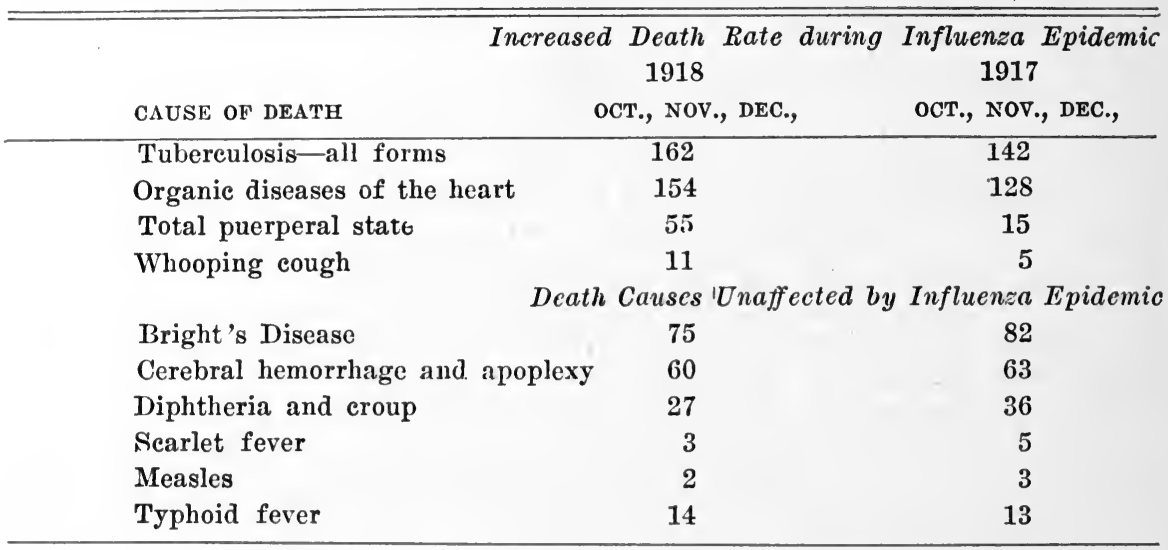

that the explosiveness of the epidemic was greatest in those cities with the highest death rates prior to the epidemics. The figures in Table LVIII are of interest.

\section{TABLE LVIII}

Coffricient of Correlation between Epidemicity Index and Various Demographic Data

\begin{tabular}{lccc}
\hline & & $\begin{array}{c}\text { COEFFICIENT DIVIDED BY } \\
\text { PROBABLE ERROR }\end{array}$ \\
\hline $\begin{array}{ccc}\text { Density of Population } \\
\text { Geographical Position } \\
\text { (Nearness to the orig- }\end{array}$ & +0.092 & \pm 0.107 & .9 \\
inal focus in Boston) & -0.348 & \pm 0.095 & 3.5 \\
Age Distribution & & & \\
(Deviation from a & & & \\
standard population) & -0.262 & \pm 0.101 & 2.6 \\
Growth of Population & -0.327 & \pm 0.096 & 3.4 \\
Total Death Rate in 1916 & +0.661 & \pm 0.061 & 11. \\
Death Rates from & Specific Diseases & in 1916 & \\
Pulmonary Tuberculosis & +0.525 & \pm 0.078 & 6. \\
Organic Heart Disease & +0.567 & \pm 0.073 & 8. \\
Acute Nephritis and & & & \\
Bright's Disease & +0.507 & \pm 0.080 & 6. \\
Influenza & +0.287 & \pm 0.099 & 2.9 \\
Pneumonia (all forms) & +0.388 & \pm 0.092 & 4.2 \\
Typhoid Fever & +0.176 & \pm 0.105 & 1.7 \\
Cancer & +0.198 & \pm 0.104 & 1.9 \\
Measles & +0.069 & \pm 0.107 & .6 、 \\
\hline
\end{tabular}

Interpreted in more familiar terms, this table means that the explosiveness of the epidemic was not influenced by the density of popu- 
lation. There is a fair degree of probability that the virulence of the infection diminished the farther it traveled from Boston. The difference in age distribution in the cities had little influence on the explosiveness of the outbreak. There is some indication that the cities which grew most rapidly since 1910 were less affected than the older and more stable cities. Most distinct, however, is the indisputable fact, from this statistical analysis, that the cities with a high death rate in 1916 were the most ready victims of the epidemic. The cities with high death rates from tuberculosis, organic heart disease, acute nephritis, and pneumonia, were least resistant to epidemic influenza. Endemic influenza, typhoid fever, cancer, and measles did not predispose to epidemic influenza. Pearl concludes with these words:

"The most significant factor yet discovered in eausing the observed wide variation amongst these 39 American cities in respect to the explosiveness of the outbreak of epidemic influenza mortality in the autumn of 1918 was the relative normal liability of the inhabitants of the several cities to die of one or another of the three great eauses of death which primarily result from a functional breakdown of one of the three fundamental organ systems of the animal body, the lungs, the heart, and the kidneys."

This result is indeed an incentive to right living and the development of good general health. Sanitarians may take heart in this finding. It had almost seemed as though influenza did not respect good health but actually preferred the strong and robust. It would appear that the young adult suffered most severely, primarily because he intermingles most freely with his fellow-men and in consequence receives the freshest and most virulent infection.

Susceptibility and Immunity.-From the preceding pages we have seen that all people are not equally susceptible to influenza. The young are attacked more readily than the old. The white race is somewhat more susceptible than the colored. The rural soldier is less immune than the city boy. The more isolated the community, the more susceptible are the inhabitants when the infection is introduced. It is probable that urban life gives to an individual a nonspecific immunity making him somewhat less susceptible to influenza and certainly better able to withstand the disease. One attack conveys a transient immunity against subsequent attack. Immunity thus acquired apparently does not last over a year or two at the most.

Vaughan (Warren) conducted a survey of 10,000 individuals divided among 2117 families in Boston. Forty-five per cent of these families had one or more persons attacked by influenza in the 1918-19 outbreak. In the 1920 recurrence, 27 per cent of the families were attacked. Of these families attacked in the 1920 outbreak, 14 per cent had been visited by the previous epidemic. Thus 58 per cent of the families had 
cases in either one or both outbreaks. The interesting point is that 42 per cent of all families in the survey gave no history of influenza in either outbreak.

Even in families which were attacked, there was most commonly at least one and frequently several individuals in the household that did not contract the disease. In less than 8 per cent of 1236 families was every member of the family stricken. There is thus a natural immunity on the part of many, as is evidenced by their failure to contract the disease in the face of most certain and intimate exposure.

An attack of influenza in the spring of 1918 protected people against the disease in the autumn. The best illustration of this fact is furnished from the experience of the San Quentin Prison in California. Between April 13 and May 26, 1918, influenza affected at least 500 of the 1900 prisoners, seven developed bronchopneumonia and three died. On October 3 the autumn influenza epidemic appeared at the prison. Three and seven-tenths per cent of the 1900 were attacked as compared to 27 per cent in the spring. There were eight pneumonias and two deaths. The important fact is this-

Forty-two of the 69 prisoners attacked in October had entered prison since the April epidemic and not one of the remaining 27 who were there in April had been sick at that time.

On November 25 a third outbreak began and involved 58 cases in all. of this number 32 entered prison since April. Twenty-six were in prison prior to April but had not been sick at that time.

Another illustration is that of the 2nd Infantry Regiment which had influenza in Hawaii and after leaving the islands in June. These men arrived in Camp Dodge about August 1. In September and October when the epidemic occurred at Dodge this organization had an incidence of 7 per cent and a mortality of 0.29 per cent. With one exception this was the lowest incidence among nineteen units, and with seven exceptions in small units this was the lowest mortality. Camp Lewis had influenza continuously throughout the year preceding the epidemic and its rate in the autumn was among the lowest. Las Casas had an outbreak resembling influenza in the summer, and its rate in the autumn was extremely low.

We have been informed that the Regulars who came to Beauregard as the 5th Infantry in August had been through an influenza epidemie at Panama in the spring. These men suffered very much less than the draft men in the autumn epidemic.

Camp Shelby had the lowest mortality of the 40 large eamps and we attribute this largely to the immunizing effect of the spring outbreak when 643 cases were admitted to the Base Hospital and many other cases occurred in camp which, owing to their mildness, were not reported. In 
the autumn outbreak very few, if any, of the seasoned men of the 34th Division who had been in Camp in April were affected. The disease was confined to recruits entering camp during the summer. The 34th Division had left camp by September 20. En route to Camp Mills some influenza developed and still more cases occurred during the three-week encampment there and later on shipboard and after landing in England and France. We are, however, informed by the Division Surgeon that the disease was noticeably less common in this organization than in others. Other camps had a history of influenza in the spring, but, owing to a complete change of personnel, they did not benefit from this protection in the autumn. It is significant that the American Expeditionary Force composed of seasoned troops-men who had come from camps where influenza prevailed in the spring and who had weathered the spring and summer attacks in Europe, exhibited a much lower disease and death rate than the newer soldiers encamped in the United States. The A. E. F. was laboring under the strain of active warfare but by reason of their immunity but 4.5 per cent of the strength had influenza from September to December. In this country the attack rate was 22.6 per cent. In the A. E. F. only .48 per cent of the strength died from all diseases, whereas 1.56 per cent died in this country.

\section{TABLE LIX}

Influenza and Mortality among All Troops in U. S. and A. E. F. Autumn Season-17 weeks (Week ending Sept. 6 to Dec. 28, 1918)

\begin{tabular}{lccccc}
\hline \hline & & & & PER CENT OF STRENGTH \\
& & NUMBER & DEATHS & & $\begin{array}{c}\text { DYING } \\
\text { FORCE }\end{array}$ \\
& AVERAGE & INFLUENZA & FROM ALL HAVING & FROM ALL \\
STRENGTH & CASES & DISEASE & INFLUENZA & DISEASE \\
Troops in U. S. & $1,493,000$ & 338,343 & 23,319 & 22.6 & 1.56 \\
A. E. F.* & $\mathbf{1 , 7 4 5 , 0 0 0}$ & 77,828 & 8,411 & 4.5 & .48 \\
\hline
\end{tabular}

* Influenza data not available in A. E. F. for last thrce weeks and mortality for four weeks in December. This will not affect the influenza rate materially as the influenza incidence had by this time returned to normal or what it was before the epidemic began. The mortality figures in the table are somewhat low as the weekly mortality rates had not returned fully to normal by December 1.

Those who had influenza in September and October were not affected in the recurrent waves. In the recurrence of influenza at Dorr Field during January 1919, Squadron A, which was affected most severely in the first epidemic, had no cases. From Camp McClellan we get a report of a similar incident. Speaking of influenza during December, the report goes on to say that "Battery A, which had very few cases during the October epidemic, sent more cases to the hospital than any of the other units in this organization, while Battery $G$, which was affected most during the epidemic, had very few cases this month." Camp 


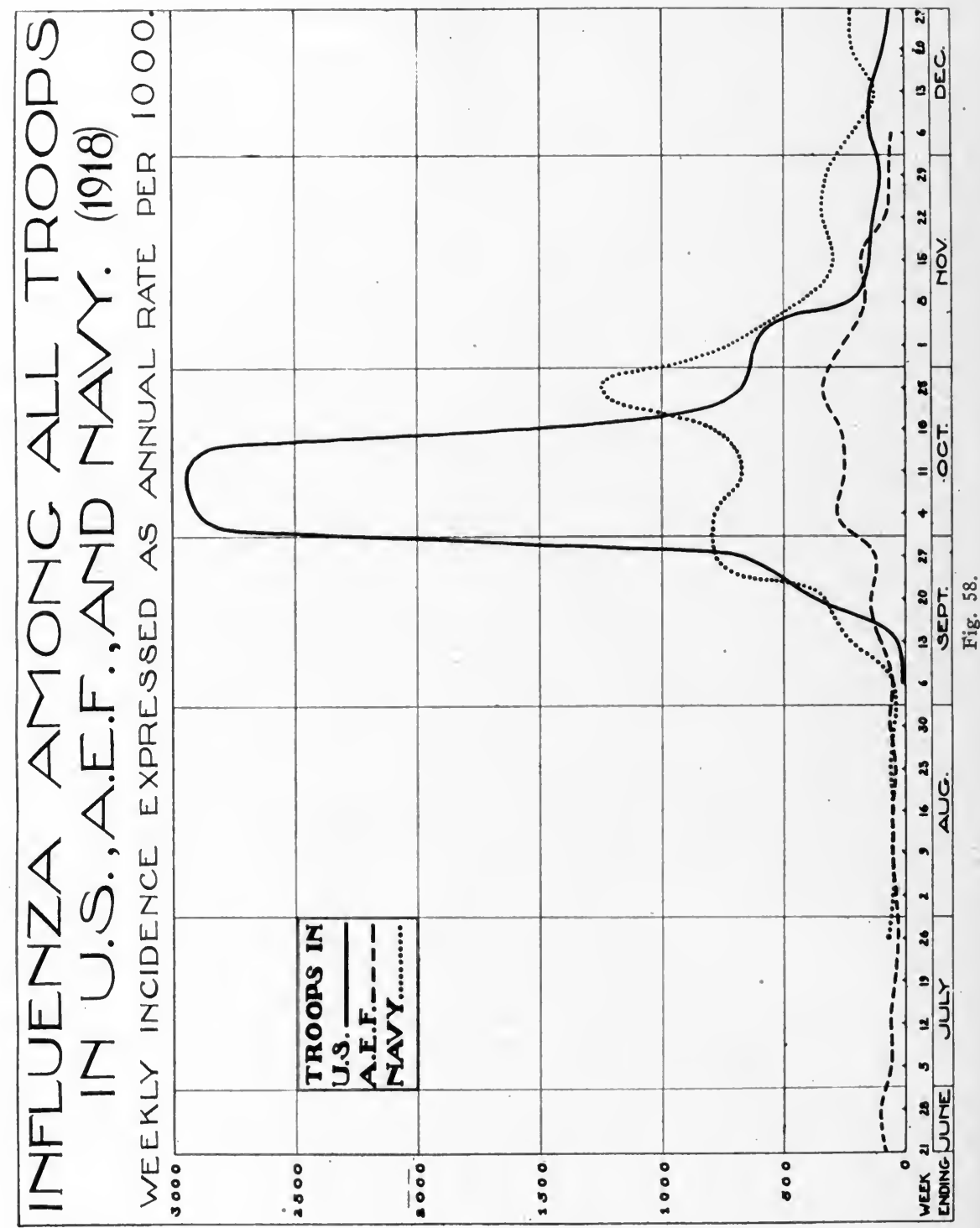

Jackson reported a recurrence of influenza in January, which was localized largely in the 48th Infantry, a regiment that had passed through the autumn epidemic with very few eases at Camp Sevier.

The protective influence of a previous attack apparently does not last much longer than a year. Of 9478 cases studied in the Detroit outbreak in January and February, 1920, 985 reported a previous attack. Of this number 57 claimed to have had the disease prior to September, 1918; 641 between September, 1918 and March 31, 1919; 138 between March, 1919 
and January, 1920; 149, time not stated. Thus 10.4 per cent of cases claimed a previous attack. This leaves 89.6 per cent attacked for the first time in the 1920 outbreak. On its face it appears that a person with a previous attack is less likely to have a second attack, but it must be borne in mind that the susceptible group is probably at least 10 times as large as the number of those who have been attacked previously. Consequently, we should expect many more cases among them even though the attack rates of the two groups are equal.

Vaughan (Warren) found from his Boston survey in 1920 that one attack of the disease gives protection which lasts for a few months. A previous attack contracted on an average of from ten to seventeen months before confers no protection whatever against a second attack. On the contrary, the attack rate was slightly higher in this group than in those who had not previously had the disease.

From a study of the Great Lakes Naval Training Station and Camp Grant, Jordan found that 19.8 per cent of those with influenza in January, 1920, gave a history of a previous attack in the 1918-1919 epidemic. The percentage of January, 1920, cases without previous attack was 17.9. He concludes that no marked immunity exists 12 to 15 months after a previous attack althoúgh it may obtain for a lesser period.

Elsewhere we have pointed out that influenza played more havoc among soldiers hailing from the country than those coming from the city. Thus the rural men of the 34th Division at Camp Dix had a pneumonia case mortality of 27 per cent as against 18 per cent for the urban men. This fact is attributed to nonspecific immunity acquired from contact with many people. Immunity of this character did not protect to any extent against contracting influenza, but it did protect against a severe attack. Soldiers long in the service had lower mortality rates than the recruit. There was less difference in the morbidity rates.

A comparison of influenza mortality among civilians living in the city and country shows a greater mortality in the city. Communities of less than 10,000 in Indiana showed an average annual mortality from influenza and pneumonia during the last four months in 1918 of 750 . In the larger places the rate was 1144. Corresponding figures for Kansas were 800 for the rural and 1233 for the cities. The transmission of the infection is of course much less rapid in the country and this fact would tend to make the disease less fatal. Side by side in camp and exposed to the same environment the rural boy has a poorer chance of weathering the disease.

It was Auerbach's observation during the 1889-90 epidemic in Cologne that rural school children in Cologne had more influenza than eity children. Records of 30,000 children show the following percentages attacked: 


\begin{tabular}{ccc}
\multicolumn{1}{c}{ AGE } & URBAN & SUBURBAN \\
13 to 14 & 34 & 43 \\
11 to 12 & 32 & 38 \\
10 & 31 & 37 \\
9 & 26 & 31 \\
8 & 28 & 28 \\
7 & 22 & 26
\end{tabular}

The influence of nonspecific immunity is seen in the much visited community as compared with the isolated community. In the Union of South Africa it is reported that 40 per cent of the population had influenza in the 1918 outbreak and 2.3 per eent of the entire population died from this cause. This is more than double the mortality in the United States.

The case and death rates among different groups of American Indians is further illustrative of this point. The occupants of the isolated reservations in the west suffered much more severely than those in the more frequented east.

TABLE LXI

INFLUENZA AMONG AMERICAN INDIANS

Oct. 1, 1918-May 31, 1919

\begin{tabular}{|c|c|c|c|c|c|}
\hline LOCATION & POPULATION & PER CENT & INFLUENZA & PER CENT & DEATHS \\
\hline Atlantic States & 8910 & & 18 & 1.43 & \\
\hline Central States & 173,000 & & 19 & 1.19 & \\
\hline Mountain States & 91,500 & & 35 & 3.89 & \\
\hline Pacific States & 31,100 & & 24 & 1.68 & \\
\hline
\end{tabular}

Transmission of Influenza.-There is little doubt that influenza is spread from person to person through coughing, sneezing, laughing and talking, or by other means which make possible the direct transfer of fresh salivary material from the mouth and nose of the infected person to the nasopharynx of the hitherto uninfected. There is a wide difference of opinion over the importance of certain other impersonal modes of infection.

Is influenza spread by the wind? Is influenza spontaneously generated by weather combinations? To these and other queries we may find in the literature conflicting answers. Hirsch is quite dogmatic in his statement that influenza is not a contagious or communicable disease. The fact that influenza has occurred on shipboard far removed from land and that the disease breaks out at times on an isolated island following the arrival of a ship even though no cases have appeared on the ship, is interpreted as controverting the principle of transmission from human to human. The existence of the disease carrier, himself not ill, did not occur to Hirseh, who says: 
"We may hesitate to accept the conclusions $* * * * * * * * *$ when we duly keep in mind that the suspected importers of the morbid poison remain, as we are expressly told, unaffected by it, that they continue untouched by the epidemic.",

This author sums up in the following words:

" These considerations taken along with peculiarities in the incidence and course of influenza epidemics-their occurrence suddenly and without prelude and their attacking the people en masse, their equally sudden and complete extinetion after a brief existence, generally of two to four weeks, and the frequent restriction of the disease to one place, while the whole country around has been completely free from it-all these points are so foreign to the mode of development and the mode of spreading proper to such maladies as originate beyond doubt through the communication of a morbid poison, that we shall find it hard to discover any reason for counting influenza among the contagious or communicable diseases.",

While influenza does occur with great suddenness, it does not attack en masse but rather in succession. Vaughan's (Warren) careful survey in Boston proves this. He states that in the 1920 outbreak in only 5 per cent of families attacked did more than one case develop on the first day of the appearance of the disease in the family. Usually the wage earner contracted the disease first and introduced it into the family.

Hirsch's illustration of outbreaks on ships at sea while interesting, certainly does not preclude the possibility of human transmission of the infection. He refers to experiences described by surgeons of the English navy in the following words:

"Thus in September, 1781, infiuenza attacked the crew of an East Indiaman on the voyage from Malacea to Canton, so generally that searcely a single person escaped; when they left Malacea the disease was not.prevalent there, but when they arrived at Canton it transpired that their outbreak on board, in the China Sea, had happened at the very time when the disease was showing itself with equal intensity at Canton.",

Again, quoting from the Reprint on the Health of the Navy, 1857, the following :

"Influenza broke out on the Monarch while at sea, on the passage from Payta to Valparaiso. She had left the former place on the twenty-third of August and arrived at the latter on the last day of September: About the 12th of the month seven men were placed on the sick list with catarrhal symptoms, and during the following ten days upwards of eighty were added. $*^{*} * * * * *$ On the arrival of the ship at Valparaiso the place was healthy; but in the course of a few days some cases of influenza made their appearance and very soon afterwards the disease extended over the whole town. $* * * * * * * * *$ The Surgeon further observes that the whole coast from Vaneouver's Island southwards to Valparaiso was visited by the epidemic."

There is implication at least in the above accounts of a spontaneous breaking out of the epidemic quite apart from any eommunication between the affected persons. Either this, or else the infection was air borne, both land and sea being equally affected. Without additional 
facts it is idle to offer explanations of these occurrences more in line with our present understanding of the disease.

There is ample evidence in more recent times of the communicability of the disease from person to person and from town to town, eity to eity, and camp to camp through human agency and this alone. The army experience in this country in the fall of 1918 is replete with these illustrations.

Camp Devens was infected by visitors to Boston and by men returning from Boston. Student officers from Devens introduced the infection to Grant.

Camp Grant, Illinois:

"It is the opinion of the Medical Officers of this camp that the disease was brought into this eamp by men who were transferred here with the Infantry Central Officers Training School. Candidates for this school arrived from Camp Devens on September 16 , where this disease had already made its appearance."

\section{Camp MeArthur, Texas:}

"Influenza of the epidemie type was introduced into this camp by the arrival of 500 men (1 troop train) from Camp Grant, Illinois, on September 30, 1918. Upon arrival here 125 of these men, running temperatures, were taken from the train' and sent to the Base Hospital.",

Ft. Leavenworth, Kansas:

"So far as ean be ascertained the contagion was imported by enlisted men arriving at this post from Camp Grant, Illinois, and Camp Dodge, Iowa.,"

Camp Upton, New York:

“The outbreak of influenza at Camp Upton first attracted attention on Friday, Sep. tember 13, 1918, when 38 eases were diagnosed in Companies 5, 6 and 7 of the Depot Brigade. All eases were among a new group of special service men who arrived in eamp on September 6. Two-thirds of the men were from Boston and vicinity where an extensive epidemic of influenza was raging among both civilian and military population. The other third was from other parts of New England. One of the men was sick when he left Boston and was sent to the Base Hospital immediately on his arrival at Camp Upton.",

\section{Camp Jackson, South Carolina:}

"The epidemic started with the men from New York on September 16, 1918, who had been in camp only a short time, averaging about six days. The disease spread rapidly to all troops.",

\section{Camp Greenleaf, Georgia :}

"The disease made its first appearance at the camp on September 23, on which date there was sent to the General Hospital an enlisted man of the Service Company, just returned sick from leave in Massachusetts, where influenza at that time was prevalent.',

Syracuse Recruit Camp, New York:

"On September 4, the first contingent of the second, or September enrollment of the draft began to report at the Camp and from that date until the 10th of the month, approximately 10,000 men reported. On September 12, 14 cases of influenza reported at In- 
firmary No. 5, all the cases coming from eastern Massachusetts and from the 104th and 105th and 106th Recruit Companies. Judging from the facts that the epidemic at that time was raging severely in that state, it may reasonably be concluded that the disease was imported into this camp from that source. These companies were made up largely of men from Massachusetts and from them the disease spread to other companies."

\section{Ft. Wood, New York:}

"The first cases of Spanish influenza appeared here immediately following the transfer to this P'ost of 30 recruits from Syraouse, New York on September 23, 1918."

\section{Aviation General Supply Depot, Pennsylvania:}

" On the evening of September 20, 1918, the first case was picked up at the railroad station of Middletown, from a detachment of Ordnance troops en route to the Ordnance Supply Depot, Middletown, Pa., and brought to the Post Hospital, Aviation General Supply Depot, with a diagnosis of bronchitis. On September 23, 1918, 18 new cases developed in the Ordnance Supply Depot.",

\section{Camp Wheeler, Georgia :}

"First case recognized October 9-that of a Second Lieutenant of Infantry, who arrived in camp in the initial stage of the disease. On this date 75 junior officers arrived from Camp Perry, Ohio, having come from the already infected North. Two of these officers who reached camp ill enough to go to the hospital, gave a history of having stopped at Philadelphia and a third in Baltimore, where he visited his family then ill with the disease. The third developed influenza one day later, the three being officially reported as influenza on October 11.',

\section{Springfield Armory, Massachusetts :}

"The epidemic began on the arrival of the 11th Ordnance Guard Company from Raritan Arsenal, New Jersey, which was on September 14, 1918, at which place they had been quarantined for six weeks.",

\section{Watervliet Arsenal, New York:}

"The first cases of influenza reported at the hospital on September 23rd. The patients were from the 5 th and 7 th Ordnance Guard Company which arrived the day previous from the Raritan Arsenal, Raritan, New Jersey.',

\section{Camp Dodge, Iowa:}

"On September 16, 27 men arrived from Chicago and were assigned to the Motor Convoy. Two of the group became sick immediately after arriving and were sent to the Base Hospital with a diagnosis of bronchitis. All these men were assigned to duties which brought them in contact with men from various parts of the camp."

"On September 21, 30 drivers of ambulances, who had gone to Chicago during the past week with truck ambulances, came back after having stayed over two days. Two of this group were taken sick on the train and were admitted to the Base Hospital on September 22. Ten of the thirty contracted the disease. They were probably the greatest and earliest source of spread in this camp.',

Ft. Crockett, Texas:

"It is evident that this epidemic started from a number of troops from Camp Martin, New Orleans, Louisiana, arriving at this post about the time the first ease was discovered.", 


\section{Camp Moultrie, South Carolina:}

"The epidemic of Spanish Influenza began at this post October 4, 1918, upon the arrival of about 100 men transferred from other camps, part coming from Fort Crockett, Texas, and part eoming from Camp Zachary Taylor, Kentucky."

Fort Worden, Washington:

"Until October 16, 1918, no actual cases of influenza had dereloped within the command. On that date a detachment of 315 men from eastern Washington arrived at the post for duty, coming from a section where the epidemic had already gained a strong foothold and bringing the disease to this fort."

Fort MeArthur, California :

“On October 23, ten draft men arrived from southeastern Colorado. One gave a history of having been ill when he entrained; also influenza in his family at the time of his departure. These men traveled in the same ear and were intimately associated throughout the journey, which consumed three days. All these men were taken from the train to the hospital, suffering from influenza of a few hours duration; nine had apparently contracted it from the one who was ill when he entrained.",

"Eighteen draft men from another section of Colorado trareled on the same train but in another ear. Some of these went into the car and talked to the men in Group 2, but spent little time in the ear. One of these was taken to the hospital from the train, suffering from influenza."

Influenza was reported from Alaska in October, probably being carried from the States. Its transmission through Alaska is suggested by the following excerpts:

Fort William H. Seward, Alaska:

"Influenza appeared October 22, 1918, in newly arrived draft inen from Ketclikan, Alaska.",

Fort Liscum, Alaska:

"It is beliered that the epidemic of influenza at this post during the month of November, 1918, oceurred as a result of infection from a group of inducted men arriving November 4, 1918, from Kodiak, Alaska. These were suspected contacts and were at once isolated in a racant set of officers' quarters, with the exception of two, admitted to the hospital with diagnosis of pharyngitis. In the hospital these two were carefully isolated, the dishes used by them were retained in their room and one attendant was detailed to eare for them, being relieved from all other duties in consequence. Six days afterwards these patients, together with two of the hospital staff and an additional man of the group from Kodiak manifested the characteristic clinical symptoms of influenza."

Schofield Barracks in the Hawaiian Islands reported influenza as early as July, two months before it appeared in epidemic form in this country. The introduction of the disease to Hawaii gives us an insight into its spread from one continent to another.

Schofield Barracks, H. I. :

"July 2, the epidemic had broken out in upper Sehofield, where there were two battalions of the 2nd Hawaiian Infantry stationed. Here the disease was in a lighter form 
and not so many cases were reported. About the same time members of the 32nd Infantry, also stationed at upper Schofield, began to have the disease. This regiment was between the first two cantonments having the disease. On July 5 the epidemic reached the 25th Infantry, which is also stationed between the first two cantonments. A week later the Officers' Training Camp men were taken ill."'

"The history of this epidemic shows that on about May 25th, a Dutch steamer arrived here from the orient and reported a grippe epidemic aboard shortly after leaving Yokohama. Three weeks later the epidemic had started at Pearl Harbor. From Pearl Harbor the disease was carried to Schofield by men of "F"' Company, who visited that district to draw back pay. When these men visited Pearl Harbor the disease was at its height and within five days after, six were sick with the same affection."

The above evidence is sufficient to show how the epidemic was transmitted from place to place in this country, one camp infecting another. The more remote the post or station, the more slowly did the infection travel. The less intimate the contact, the more isolated the camps, the less opportunity for mingling with many people, the more slowly did the epidemic progress.

Hirsch argues against the importance of intercourse in spreading the disease and offers the suggestion that the epidemic "has not spread more quickly in our own times, with their multiplied and perfected ways and means of communication than in former decades or centuries."

On the other hand, Finkler calls attention to the fact that in 1830 the pandemic consumed 11 months in passing from Moscow to North America; whereas in 1889-90 it was there in three months. In the latter years the disease required two months to reach St. Petersburg from Bohkara. In six weeks it reached Berlin and from Berlin to other German eities it was a matter of a few weeks or even days.

With aerial transmission we should expect more or less of a linear course of the disease. Rather, as Hirsch points out, is the transmission by leaps and bounds. In passing across Europe influenza did not proceed as the crow flies, but as the railway service radiated. Small isolated areas about London were not affected for several months, although far removed eities in Great Britain and America were affected in the meantime.

Dust may be a medium in transmitting disease. Tubercle bacilli have been found in street dust. Streptococci have been recovered from floor dust. Americans are profuse spitters. The French peasants complained of this among American soldiers. The epidemiologist at Camp Bowie stated that never before had he seen such promiscuous expectoration indoors and out. One of the authors has seen medical officers spit on the floor of a lounging room adjacent to the mess hall. The streets of every eamp receive quantities of infected sputa. Barrack room floors contain infected sputa and the viability of bacteria indoors must be longer, owing to the warmer temperature and the absence of sunlight. There is 
such a thing as infected dust but there is little statistical evidence of its causing disease in a way that cannot be accounted for by more direct means. Camp Logan reports that "the spread of the disease was aggravated at the beginning of the epidemic by the large amount of dust in company streets." Association of dust with disease is minimized, however, by this sentence which follows: "The rain (referring to a storm on September 19th) settled the dust, but increased the incidence of disease, because of exposure."

The following is taken from a Camp Funston report:

"Severe windstorms seem to have a bad effect on spreading disease. At Detention Camp No. 2, the epidemie increased in intensity until the sixth of October, when 410 men were transferred to the hospital, and on the seventh of October the number was increased by 224 more. On this date a stiff wind blew from the southwest causing a dust storm to prevail during the entire day; on the eighth there was a marked inerease in the number of cases, 675 men being sent to the hospital during this day. The increase was largely attributed to the duststorm during the day.",

The above must have been regarded as nothing more than a coincidence by the Division Surgeon at Funston, who writes that-

"(1) The epidemic spread directly east and west within the camp along the lines of greatest travel. (2) It spread in a direction opposite to that of the prevailing winds. (3) It was not influenced in any way by dust or inclement weather. The largest number of admissions occurred on days when the weather has been and was moderately clear."'

During the epidemie there were many dusty days at Camp Humphreys. "The geographical distribution of the cases does not seem to indieate that dust was an active factor in the production of the disease. The service battalions, which are the elosest to the 3rd Regiment and directly in line to receive their dust, did not suffer as greatly as did the 7th Regiment, which is to the windward of the 3rd Regiment and at a considerable distance."

There were dust storms at Camp Cody, but the high mortality there was due to the admission of sick recruits. There was no marked dustiness at Sherman during September. At Camp Beauregard it was cold and damp.

Syracuse places great emphasis on the extreme precipitation during the epidemic. There was no dustiness whatever. Devens reported but little dust in the camp owing to the frequent rains. At Grant it was cold and damp prior to the epidemic. Later there was a great deal of dust on windy days.

The only grouping of camps at all suggestive of dust transmission of disease is the camps in the dust swept areas of Texas-Bowie, McArthur, Logan, Travis and Ellington Field-all of which had a relatively high incidence but low mortality. This is true to some extent of the smaller posts and stations in Texas.

Finkler states that in 1889-90 in England eight cases of influenza occurred in four places among the 415 inhabitants of the 51 light ships 
and 20 lighthouses along the English coast. Influenza was not carried through the air to unfrequented places.

Until the possibilities of human transmission have been ruled out we can place but little reliance on aerial transmission of disease from one community to another. It is not beyond the realm of probability that influenza was transmitted by infected dust raised into the breathing zone within the limited confines of barracks. While such transmission is probable we are unable to recognize that this was an important factor when compared with the more direct means incidental to intimate human contact.

For the spread of influenza two factors are necessary, first, the presence of the infective agent, bacterial or protozoal or filtrable virus or whatever later generations may find it to be, and, second, the presence of susceptible human beings. The transmission of the disease through a community is dependent upon the rapidity with which the infective material is transmitted from person to person. The more intimate the contact of individuals, susceptible individuals, the more rapid the progress of the disease.

Vaughan (Warren), who made an intensive study of influenza in 1920 in certain selected districts of Boston, arrives at the following conclusions: (1) We find that not only are crowded families more apt to have cases of influenza, but also that crowded families are more apt to have multiple cases. (2) The cleaner the family the less is the likelihood of multiple cases. (3) Families with two or more cases were dirty in a greater proportion than was the standard for all families. Families with no cases and with but one case were dirty in a smaller proportion than the standard. (4) Single cases predominated in the clean, roomy dwellings, while multiple cases predominated among the dirty and crowded. (5) As a rule there was at least one and usually several individuals in each household who did not contract influenza. (6) As a rule, in the 1920 epidemic, cases of influenza developed in families successively and not explosively. The infection is most frequently brought into the house by the wage earners. Certainly it is always imported from the outside. (7) Sleeping contact is more productive of influenza than are the less intimate forms.

Influenza raced through the army camps. The channels of communication from person to person were multitudinous and unbroken. In the cities the infection was forced to follow a more devious course and its progress was retarded. The total mortality rates in Baltimore and Camp Meade will illustrate this fact clearly.

WEEK ENDING

Baltimore

Camp Meade
SEPT. 20

14.3

6.2
SEPT. 27

17.0

71.8
OCT. 4

28.1

531.
oCT. 11

69.5

OCT. 18

148.3 
In Baltimore four weeks elapsed between the normal rate and the mortality peak. At Camp Meade but two weeks intervened.

Ninety per cent of the influenza cases during the entire autumn season of 1918 occurred in some camps within two weeks. In others the epidemic was prolonged by troop arrivals over a period of nine weeks. We may make the generalization that the epidemic took more than twice as long in passing through a city. This statement applies to the early outbreak in September and October. There were recurrences in the cities, in some, one, in others, as many as three, from the time of the first outbreak to the end of March, 1919. Thus the actual duration of the epidemic including the lapses between the recurrent periods was in some cities nearly six months.

Recurrences of small magnitude did happen in a few camps. Taylor may be cited as an example. After reaching a mortality peak of 296 , in the week ending October 18, the rate fell as low as 7.1 on November 29. It then rose to 32.4 on December 13, to 29.3 on January 10, and 56.4 on February 28. The camps were being demobilized after the middle of November and we cannot say with certainty that recurrence of influenza would not have taken place had the men remained in camp. The camps were in a state of flux owing to the reception of overseas men for demobilization. It is entirely possible that the recurrent in-

TABLE LXI

Duration of Camp Epidemics-Time Necessary for 90\% of Total Influenza of Autuarn Season to Occur

(Autumn Season Includes Sept., Oct., Nov. and Dec.)

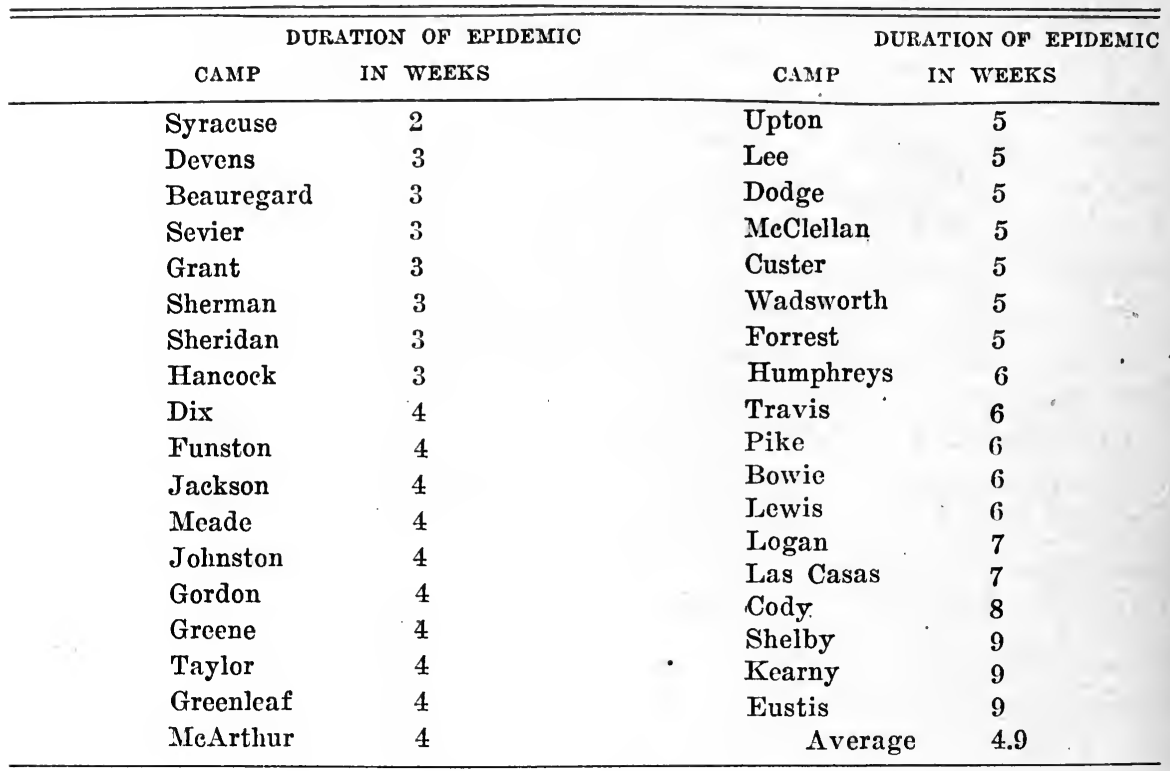


fection may have been introduced or confined to these new increments. At any rate, the recurrences that did occur were insignificant in comparison with certain cities where the recurrent wave ascended even higher than the original outbreak.

Pearl gives a table (Table LXII) for forty U. S. cities showing the length of the epidemic. In this table he regards the epidemic having terminated when the mortality rate returned to normal after the first outbreak, even though a recurrence followed later. Alongside these figures we have placed a similar table for thirty-eight large army camps.

\section{TABLE LXII}

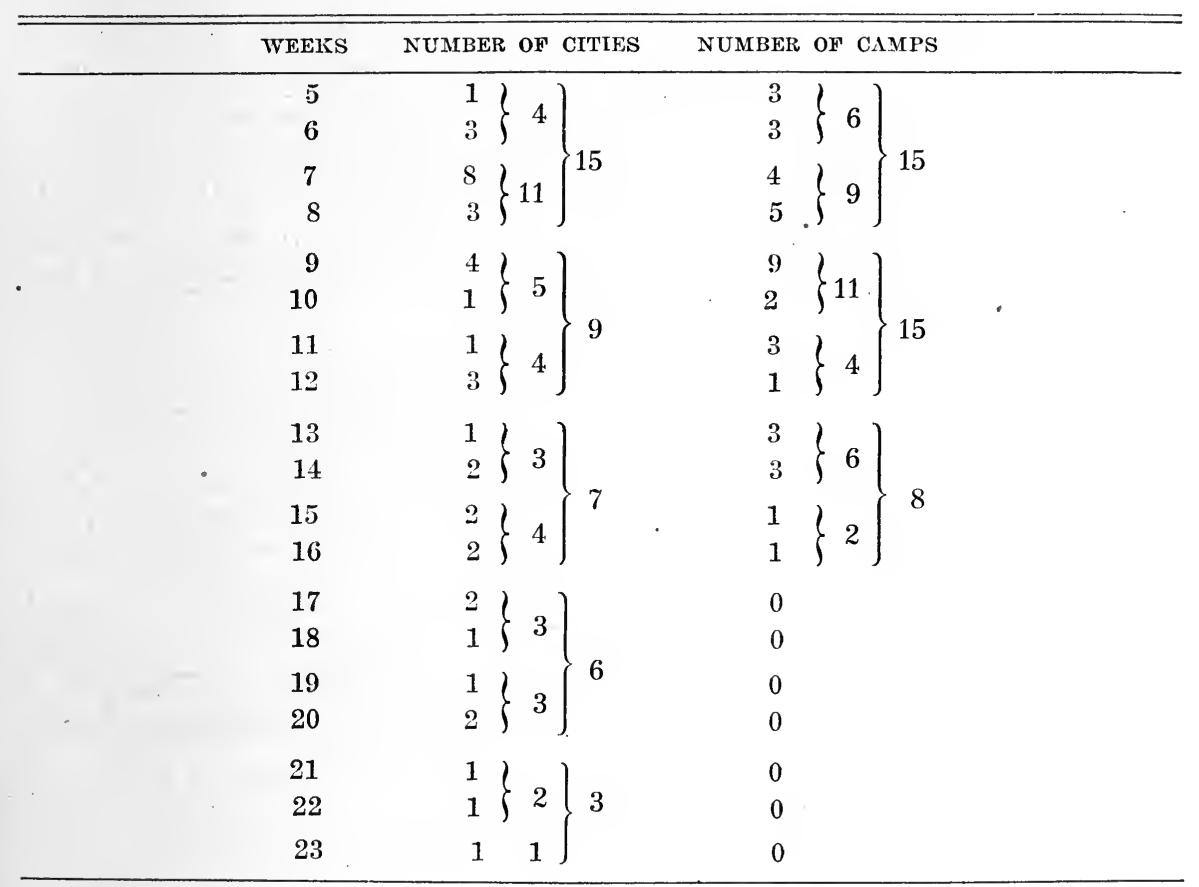

The average duration in the cities was about twelve weeks; in the camps between nine and ten. Pearl also offers an index of epidemicity to measure the explosiveness and extent of the outbreak in different cities. This expression is represented as follows:

$$
\mathrm{I}=\frac{\mathrm{P} \mathrm{M}^{1}}{\mathrm{~T}}
$$

$\mathbf{P}$ denotes the maximum peak of mortality rate observed during the duration $\mathrm{T}$ of the epidemic in weeks, and $\mathrm{M}^{1}$ the mean death rate from July 6,1918 , to the outbreak of the epidemic, in other words, a norm. 
As worked out the values range from .92 for Atlanta to 20.51 for Philadelphia. The median, or 50 per cent value, is around 5.0.

That the difference in explosiveness of the camp and city epidemics was much greater than is indicated in the number of weeks for which the mortality rate was abnormal, is indicated by the epidemicity rate for Camp Devens computed on the above basis. The figure is 62 , or three times as great as the Philadelphia figure, the largest city value. The index for Camp Lewis, where the outbreak proceeded more leisurely, is about 10 , or twice as great as the median for all cities, and eleven times the lowest city index.

In general, the shorter and more abrupt the epidemic, the greater the mortality. This is a general statement to which there are notable exceptions. Sherman passed through the epidemic in three weeks and stood first in mortality. Cody had eight weeks of the epidemic and had about the same mortality as Sherman. We may illustrate the significance of this table by saying that six out of nine camps of brief epidemic duration stood among the first eighteen in the mortality table; whereas but two camps with a prolonged epidemic stood in this division.

TABLE LXIII

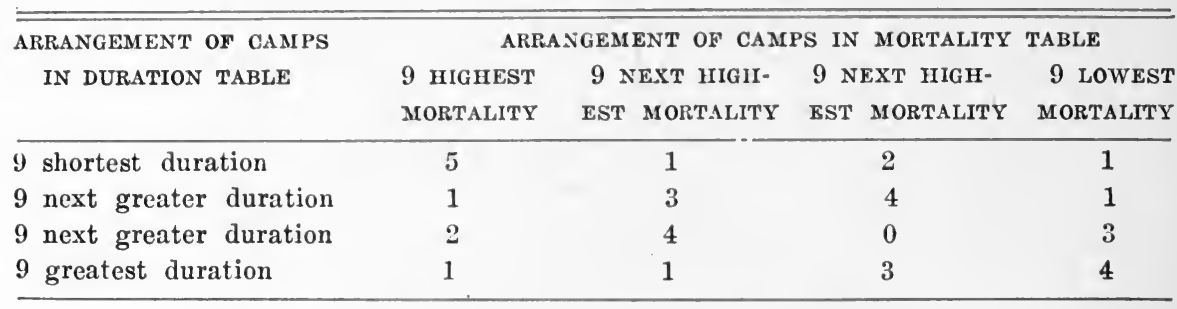

The influenza virus gains access to the body through mouth and nose. It has also been suggested that the eye offers a portal of entry, the nose and body in turn being infected through the conjunctival sac. Acute conjunctivitis was a characteristic symptom frequently mentioned in the 1918 epidemic.

To Lynch and Cumming of the U. S. Army, we are also indebted for very valuable researches emphasizing the part played by infected dishes in spreading influenza. These authors found after a study of influenza incidence in eamps that those organizations using mess kits which were washed by the individual in common wash water of lukewarm temperature had much higher morbidity rates than among the units using tableware or mess kits which were boiled. Thus the case incidence of the former group was 51.1 per thousand and among the latter 252. The ratio is one to five.

Transmission of the infective agent among those using mess kits is facilitated by the wholesale pollution of the hands with washings from 
the hands of others. The soldiers pass in line past the pail of wash water, each dipping his dishes in the water and using his hand as a mop in cleansing. Units of as many as 200 men have used the same wash water without changing. The temperature of the wash water is usually not over $40^{\circ}$ or $50^{\circ} \mathrm{C}$., else the hand cannot be inserted in the water. At this low temperature the bacterial content increases as each man contributes his washings to the pail. As each man contaminates the wash water with his own variety of bacteria, he at the same time contaminates his mess equipment and his own hands with the bacterial contributions of those preceding him.

It is easy to understand how an entire company may quickly acquire a common bacterial flora through this means, the mouth readily becoming infected either from hands or dishes or both. It was found that when tableware is used and is washed by the few men assigned to that duty, the temperature of the wash water is at or near the boiling point and disease producing bacteria readily succumb to this treatment.

These observations, representing almost 66,000 troops distributed among eleven different camps, offer a very convincing argument for the transmission of influenza by the hand to mouth route. The authors regard this as the major route of infection, not only in camps but in civil life as well. Transmission by droplet infection they regard as of minor significance. They state: "Droplet infection is subsidiary *** because explosive outbreaks do not result from droplet infection and as far as mouth pathogens are concerned, eighteen inches is about the maximum distance that plates on a level with the mouth can be infected by droplets expelled through the act of coughing. Moreover, since the weight of these droplets (even the finest spray) is such that they fall rapidly, the probability of infection by the flight of organisms is slight." This experience was confirmed by figures collected from restaurants and department store dining rooms where dishes are washed by both methods.

We are in entire sympathy with the explanation of the infective rôle played by the hands. To this agent probably more than any other is the rapid spread of a disease like influenza due. It has been argued that the sudden explosive character of influenza precludes any such wholesale infection of a population through such a seemingly slow process as person to person transmission. It is thought by some that only by aerial transmission could such sudden widespread contamination result. But is human contact such a slow mode of transmission? Imagine, if you will, and it requires no stretch of the imagination, that each person shakes hands or comes into intimate personal contact with but ten people a day. On the following day each of these ten comes into intimate contact with ten others. This process need be but continued seven days to reach a million people.

And is the human hand such a vicious agent as we would make out? 
Lynch and Cumming have shown that of 340 hands of streptococeus hemolyticus carriers, 37 per cent were positive for this organism. Of 40 hands of pneumonia patients, 15 per cent showed pneumococei. Of 95 hands of diphtheria patients, 6 per cent werè positive. Of hands of carriers of streptococcus viridans, 100 per cent were positive.

Do these hands transmit germs to inanimate objects commonly handled, such as table tops, door knobs, bedposts, etc.? Of 29 inanimate objects within the range of streptococcus carriers, 24 per cent were positive. Of 14 inanimate objects within the range of pneumonia patients, 21 per cent were positive.

He who doubts may further venture the suggestion that the hand is not continuously infected because it is washed several times during the day. Hands of a streptococeus carrier were positive after washing in soap and running water for seven minutes. By serial washing of twenty-three pairs of hands for periods of three minutes in five basins of water, Lynch and Cumming found that during the first three minutes each pair of hands yielded 23 million organisms; the second period 13 million; the third period 8 million; the fifth period 3 million. And who of us makes a practice of spending fifteen minutes at each handwashing?

Influenza virus does not pass into the body through the skin of the hand. The hand may become contaminated without danger to the person so long as the hand does not communicate the infective material to the mouth and nose. Humanity at large makes a common practice of earrying the hand to the mouth and nose many times a day. The human hand enters the mouth on various pretexts-to dislodge food from the teeth, as an unconscious act of nervousness, to carry cigarettes, food, drink, etc. The hand goes to the nose in using the handkerchief.

One of the authors has made an actual count of the opportunities for aequiring infection in the course of a day while living in a large eity:

Touching hands to articles that were or might have been touched by others im-

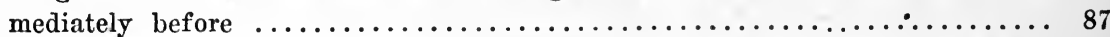

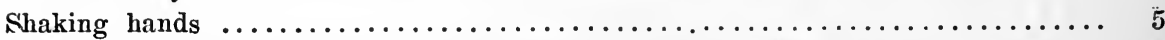

Carrying to mouth articles possibly infected by others................. 17

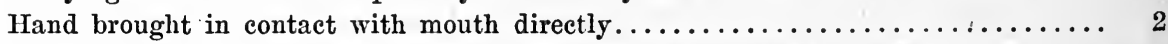

Hand brought in contact with nose indirectly through handkerchief.......... 7

Until our knowledge of the specific causative agent of influenza is far more exact than at present and until definite proof is furnished of other means of transmission, we must regard the spread of epidemic influenza from one hemisphere to the other, from one town to the next, as primarily due to intimate human intercourse.

Bacteriology.-The causative agent of epidemic influenza is unknown. What is referred to as the influenza or Pfeiffer bacillus, is a small gram- 
negative, nonmotile bacillus which grows acrobically at $37^{\circ} \mathrm{C}$. as a transparent colony on blood agar.

It was in 1892 during the recurrent wave following the great pandemic of 1889-90 that Pfeiffer announced the finding of this organism in 31 cases of clinical influenza. The bacillus was noted in smears from the pharynx and sputum. In purulent bronchial secretions it was present in almost pure culture. At the same time Canon claimed to have noted the same organism in stained preparations of the blood of 20 influenza patients. In 1892 also Kitasato is said to have discovered a bacillus answering the same description.

Pfeiffer's main reasons for ascribing the disease to this bacillus were the frequency with which it was found associated with the disease and its absence elsewhere. It was his belief also that the organism disappeared following recovery. He states that he caused the disease irregularly in monkeys and atypically in rabbits and that the organism was recovered from the site of the lesion.

In 1894 Kruse found the Pfeiffer bacillus in 18 influenza patients examined in the hospital in Bonn. He was unable to isolate the organism in patients suffering from other diseases of the respiratory passages. In the same year Richter and Borchardt confirmed Pfeiffer's flndings as to the association of the bacillus with the disease and its absence elsewhere. Huber agreed with this finding in 1893. Weichselbaum, Kowalski, Friedrich, Kruse and others did not find the bacillus in the blood of influenza patients, nor could Pfeiffer himself do so in his later work.

Pfeiffer later found in three cases of bronchopneumonia following diphtheria in children a bacillus larger, though similar in other respects, to which he gave the name pseudoinfluenza bacillus.

Pfeiffer's discovery was never accepted whole-heartedly, particularly because the organism was found by other workers frequently in other than influenza patients. Thus Lord in 1907 found but 15 per cent of cases with clinical influenza showing the Pfeiffer bacillus whereas in an inter-epidemic period he had found the bacillus in as much as 25 per cent of unselected cases. Forcheimer reports that the influenza bacillus has been found not only in the sputum of influenza patients but also at postmortem in measles, scarlet fever, diphtheria, tuberculosis, conjunctivitis, whooping cough, chronic bronchitis, and bronchiectasis.

As Pfeiffer's discovery came after the pandemic of 1889-90 and as there has been no pandemic occurrence of influenza between that date and the vear 1918, our knowledge of the bacteriology of the disease has been formulated largely in the last two years. In this period an immense amount of study has been made of the bacterial life found associated with influenza patients. Nearly all of our large army camps had laboratories where investigations were conducted during 1918-1919. Many governmental agen- 
cies as well as private workers have added materially to our present understanding of the subject.

The work in army camps has been reported upon in communications to the Surgeon General's office. It has been the privilege of the authors to review a great number of these reports, many of which have never been published in the current periodicals.

Records of bacteriological work among uncomplicated cases of influenza were received from twenty-one camps, the data being collected during the autumn outbreak of 1918 .

The prevailing organism in the mouth and nose of uncomplicated influenza cases was as follows:

Pfeiffer bacillus-14 camps-(Shelby, Upton, Funston, Johnston, Kearny, Crane, General Hospital No. 16, McArthur, Garden City Air Field, Devens, Dodge, McClellan, Fremont).

Pneumococcus - 4 camps-Logan, Taylor, Grant, Sherman.

Streptococcus hemolyticus-2 camps-Custer, Wadsworth.

Staphylococcus-1 camp-Syracuse.

At Logan the pneumococcus prevailed in 76 per cent of the 78 examinations as against 73 per cent for the Pfeiffer bacillus.

Fluetuation in the flora as the epidemic progressed was reported from Taylor.

\begin{tabular}{|c|c|c|c|c|}
\hline & \multicolumn{4}{|c|}{ Per Cent of Positive Cultures } \\
\hline & PFEIFFER & PNEUMOCOCCUS & S. NON- & M. \\
\hline & BACILLUS & & HEMOLYTICUS & CATARRHALIS \\
\hline Early in epidemic & 9 & & 65 & 80 \\
\hline 1st week epidemic & $2 s^{\circ}$ & 35 & 34 & 52 \\
\hline $2 d$ week epidemic & 37 & 59 & & 31 \\
\hline $3 d$ week epidemic & 39 & 68 & 34 & 12 \\
\hline
\end{tabular}

Early in the epidemic M. catarrhalis predominated at Taylor. In the second and third weeks it was the pneumococcus. S. nonhemolyticus decreased as the epidemic progressed whereas the Pfeiffer bacillus increased.

In seven camps the Pfeiffer bacillus was invariably present in smears and cultures of sputum and swabbings from the nasopharynx. Nine camps reported the Pfeiffer bacillus present in from 30 per cent to 80 per cent of the cases. In six camps the Pfeiffer bacillus was rarely, if ever, found. The six camps reporting the Pfeiffer bacillus rarely, if ever, found, were Custer, Wadsworth, Wheeler, Grant, Sherman, and Syracuse.

At Camp Custer there were examined 366 specimens from the nose and throat and but 8 per cent were found with Pfeiffer bacilli. A previous study of 357 healthy soldiers had revealed but 1 per cent with Pfeiffer bacillus.. Blood agar media were used throughout.

Two hundred and thirty-two sputum specimens at Wadsworth yielded but 3 per cent positive Pfeiffer bacillus. The method of determination is not stated. 
Suspecting diphtheria, Grant studied 293 cultures on Loeffler's blood agar and reported Pfeiffer bacillus as absent. Seventy-one cultures grown on dextrose blood agar and 86 cultures grown on plain agar did not show the Pfeiffer bacillus more than a few times. Fourteen specimens were grown in broth for enrichment and then plated on plain blood agar. The Pfeiffer bacillus was indicated in one-fifth of the cases.

Sherman, where the epidemic was so severe, was unable to recover the Pfeiffer bacillus except in a very few cases and yet seemingly the most painstaking care was exercised. The laboratory technic followed at Sherman is described as follows by McKnight:

"Media used consisted of plain meat infusion broth for blood and postmortem fluid cultures, plain meat infusion agar for pour plates for blood cultures, and plain blood agar plates, made with $7 \%$ whole human or rabbit blood in meat infusion agar. Culture media were always warmed in the incubator before being inoculated. Examinations included blood, throat, sputum, and postmortem cultures.

" a. Blood cultures were made in duplicate bottles containing 100 c.e. of plain broth, using 2 and 3 c.c. of patient's blood, and in poured plates using 1 to 2 c.c. of patient's blood with 8 to 10 e.c. of melted agar. The cultures were examined every day, and were incubated 3 days before being pronounced sterile.

" b. Throat cultures were made with sterile swabs on bent wires, making nasopharyngeal and tonsillar contacts. Blood plates were inoculated with the swabs and smeared with a platinum loop. The plates were incubated at $37^{\circ} \mathrm{C}$. and examined for the characteristic fine influenza bacillus growth after 24 hours.

"c. Sputum was washed well in sterile salt solution, and a portion slightly larger than a pea introduced into a corked sterile tube containing 1 c.c. of plain broth. The tubes were well shaken to procure an even distribution of the organisms and direct smears and streak cultures of the material were made and examined for influenza bacilli. Attempts were made in some instances to isolate the bacilli from washed sputum by incubating some of the sputum with an equal quantity of $5 \%$ solution of sodium taurocholate at 37 degrees for 15 minutes in order to remove the contaminating pneumococei before streaking the blood plate.

“d. Postmortem cultures of heart's blood, lung exudate, pleural fluid and pericardial fluid were made in bottles containing 100 c.c. of plain meat infusion broth. Streak cultures from swabbing of the trachea were made on blood plates. Small portions of lung, spleen and kidney tissue were aseptically removed and macerated in 3 c.c. of broth. Direct smears and streak cultures on blood plates were made of this material."

Four hundred specimens from the riose and throat were examined at Syracuse without finding the Pfeiffer bacillus.

The bacterial flora in pneumonia cases following an attack of influenza was somewhat varied. In six camps the Pfeiffer bacillus was the predominant organism (Camps Kearny, Crane, McClellan, Dix, Mills, Meade). The pneumococcus was most prominent in 12 camps (Shelby, Upton, Funston, McArthur, Devens, Wadsworth, Wheeler, Sherman, Ft. Barrancas, Lewis, Beauregard, Morrison Air Service Depot). Some form of the strep- 
tococcus predominated in 3 camps [Custer (S. nonhemolyticus), Columbus Barracks, Ft. Leavenworth].

The pneumococcus as we should expect is the predominating organism in the pneumonia cases. Three camps-Dix, Mills, and Meade-which did not report on influenza cases, indicate the Pfeiffer bacillus as the predominating organism in pneumonia. Meade reported S. viridans frequently associated with the Pfeiffer bacillus.

Of the various types of pneumococcus in the pneumonia cases, Type IV was most common. The average of 14 camps shows over 3 per cent Type I, over 8 per cent Type II, about 8 per. cent Type III, and over 53 per cent Type IV. This differs from the measles epidemic period at Funston in the autumn of 1917 only in the greater frequency of the first two types at Funston. The proportion of Type IV is almost identical.

Types I and II are very much less frequent than in the summer pneumonia at Jackson, which camp furnishes a typical bacteriological picture. Summer pneumonia was mainly lobar in character.

Type IV is slightly more frequent in influenza pneumonia than in summer pneumonia.

TABLE LXIV

Distribution of Pneumococcus Types from Sputum of Pneumonia Patients During The Influenza EPIDEMic and at OTHer Trmes

\begin{tabular}{|c|c|c|c|c|c|c|c|c|}
\hline PLACE & PERIOD & $\begin{array}{l}\text { No. } \\
\text { EXAMS. }\end{array}$ & $\begin{array}{r}\text { PER } \\
\text { I }\end{array}$ & $\begin{array}{l}\text { CENT } \\
\text { II }\end{array}$ & $\begin{array}{l}\text { I OF } \\
\text { IIA }\end{array}$ & $\begin{array}{l}\text { TOTA } \\
\text { III }\end{array}$ & $\begin{array}{l}\text { EXAMS. } \\
\text { IV }\end{array}$ & NOTES \\
\hline Shelby & Fall-1918 & 293 & 3 & 5 & & 9 & 83 & \\
\hline Upton & “ & 473 & 3 & 9 & & 7 & 66 & \\
\hline Funston & ، & 500 & 9 & 13 & & 8 & 48 & \\
\hline Kearny & “ & 135 & 2 & 2 & & 12 & 36 & \\
\hline Pike & ، & 69 & 13 & 4 & 22 & 7 & 54 & Early in epidemic \\
\hline MeArthur & 66 & 440 & 1 & 5 & & 1 & 86 & \\
\hline Logan & “6 & 302 & 1 & 12 & & 16 & 90 & \\
\hline Custer & “6 & 1015 & 1 & 2 & & 2 & 17 & \\
\hline Wadsworth & "6 & 103 & 2 & 17 & & 15 & 60 & \\
\hline Meade & "، & 479 & 2 & 1 & & 1 & 10 & \\
\hline Lewis & “" & 684 & 3 & 1 & & 0 & 90 & \\
\hline Beauregard & "، & 723 & 1 & 8 & & 12 & 79 & \\
\hline Morrison, Va. & 16 & 52 & 6 & 23 & & 18 & 12 & \\
\hline Ft. Leavenworth & “" & 155 & 0 & 14 & & 0 & 15 & \\
\hline \multicolumn{3}{|c|}{ Average of per cents } & 3.3 & 8.3 & & 7.7 & 53.3 & \\
\hline \multicolumn{3}{|c|}{ Funston $\quad$ Fall-1917 } & 15 & 13 & & 2 & 50 & $\begin{array}{l}\text { During measles } \\
\text { epidemic. Sept.- } \\
\text { Dec., } 1917\end{array}$ \\
\hline Jackson & nmer-1918 & 142 & 21 & 27 & & 6 & 46 & $\begin{array}{l}\text { June 1-Aug. 31, } \\
\text { Lobar Pneumonia }\end{array}$ \\
\hline Rockefeller Instit & & 454 & 33 & 29 & 4 & 13 & 20 & $\begin{array}{l}\text { Lobar pneumonia } \\
\text { among civilians. }\end{array}$ \\
\hline
\end{tabular}


In observations on 297 healthy civilians, Cole et al found pneumococci present in 39 per cent. They were for the most part Type IV. Types I and II were absent.

Among 454 civilian pneumonia cases these workers found pneumococci in all cases, Types I and II predominating.

Per Cent Distribution

297 Healthy

Civilians

Type I Type II Type II, a, b, x. Type III Type IV Absent

454 Civilian

Pneumonia Cases 33

.30

7

11

22

60

This picture differs from that found among soldiers in 1918. At Camp Funston both in the summer and in the autumn from 61 per cent to 68 per cent of men showed pneumococci present, Types III and IV alone being represented.

In influenza-pneumonia cases, Types I and II of pneumococci are found but to a lesser extent than among civilians.

77 Healthy men at Funston (Summer 1918)

50 Healthy Recruits at Ḣot Springs, Ark., bound for Camp Pike

(Autumn 1918)

500 Post Influenzal Pneumonias at Funston (Autumn 1918)
Per Cent of Total Examinations Type I Type II Type III Type IV $\begin{array}{llll}0 & 0 & 5 & 56\end{array}$

$\begin{array}{llll}0 & 0 & 12 & 56\end{array}$

9 14

8

48

Judging solely by the Funston figures, post influenzal pneumonia in its bacteriology was not unlike post measles pneumonia, both showing increases in the frequency of Types.I and II. These two epidemic periods at Funston exhibit quite different bacteriology from the summer pneumonias, mainly primary lobar pneumonia, the latter, because of the greater prevalence of Types I and II, resembling the pneumonia of civil life.

24 Pneumonia patients, mostly white

Per Cent of Total Examinations

Type I Type II Type III Type IV

(Summer 1918)

38

21

4

33

Looking at the bacteriology of the pneumonia cases as reported from the different camps we fail to detect any distinct association between any single organism and the camp mortality. The camps where the Pfeiffer bacillus predominated are evenly distributed over the mortality table, Kearny and McClellan having low rates and Dix and Meade high rates. The 12 camps where pneumocoeci were predominant are evenly distributed 
throughout the mortality table, Shelby, Wadsworth, Wheeler and Lewis having low rates and Funston, Devens, Sherman and Beauregard high rates. Custer, where the streptococcus was most prevalent, had a high rate. Wadsworth, where the streptococcus was most prominent among the uncomplicated influenza eases, had a low rate.

Seventeen camps reported the results of bacteriological studies made at necropsy. The prevailing organism found in 14 eamps where material from the lungs was observed is shown below:

Pfeiffer bacillus-5 camps-Johnston, Crane, Pike; Devens, Dodge.

Pneumococcus-8 eamps-Kearny, McArthur, Taylor, Grant, Sherman, Syracuse, Greenleaf, Beauregard.

Staphylococcus-Jackson.

Camps high in mortality and others low are seen to harbor what seems to be the identical organism. No single organism stands out as associated universally with high mortality. Of the pneumococei, Type IV usually predominates. This oecurs at Kearny, Pike, Sherman, Syracuse, Greenleaf and Jackson. Exceptions to this rule are to be found at MeArthur where Type III prevails, and Camps Custer and Grant where Type II leads in frequency. Jackson presents a curious result in that a staphylococcus was found in 60 per eent of 207 cases whereas the pneumococeus was found in but 36 per cent. Half of the pneumocoeci were Type IV.

Decreases in pneumocoeci and increases in the number of streptocoeci as the pneumonia cases eame to autopsy are to be observed at Pike.

Post influenzal pneumonia (broncho)

Autopsy, lung specimen (bronchopneumonia)
Pneumococeus $86 \%$

S. hemolyticus $14 \%$

Pneumococeus $64 \%$

S. hemolyticus $71 \%$

This change was not observed at Custer where both pneumocoecus and S. hemolyticus increased slightly. S. nonhemolyticus was nearly halved in amount.

Post influenzal pneumonia

Autopsy

$\begin{array}{ll}\text { Pneumococcus } & 22 \% \\ \text { S. hemolyticus } & 22 \% \\ \text { S. nonhemolyticus } & 41 \% \\ \text { Pneumococcus } & 27 \% \\ \text { S. hemolyticus } & 27 \% \\ \text { S. nonhemolyticus } & 23 \%\end{array}$

The predominance of Type III pneumococci in the cases coming to autopsy at Camp MeArthur is most surprising especially as this organism was so infrequent in the sputum of pneumonia patients.

The blood from influenza patients rarely contained the Pfeiffer bacillus. Three per cent of 80 specimens at Devens were positive. At Dodge blood specimens did not yield the Pfeiffer bacillus but did show the pneumo- 
coccus in 10 per cent and the streptococcus in 2 per cent of the cases. At Custer no growth was found in 140 blood specimens.

In pneumonia patients blood samples indicated somewhat heavier infection. The pneumococcus was found in 18 per cent and the hemolytic streptococcus was recovered in 47 per cent and the staphylococcus in 6 per cent of 223 specimens. Devens examined 118 samples of blood and found no Pfeiffer bacilli, 24 per cent pneumococci, 1 per cent S. hemolyticus. Five hundred and ten blood samples at Custer yielded no Pfeiffer bacillus, 1 per cent pneumococcus and 2 per cent S. hemolyticus.

Twenty per cent of the blood samples at Wadsworth contained the hemolytic streptococcus. One hundred specimens at Sherman showed no Pfeiffer and but 6 per cent pneumococcus. Fifty-four specimens at Beauregard gave the following:

\begin{tabular}{|c|c|c|c|}
\hline Pneumococeus & Type & & $9 \%$ \\
\hline & Type & II & $46 \%$ \\
\hline & Type & III & $22 \%$ \\
\hline & Type & IV & $35 \%$ \\
\hline & S. hemo. & & $5 \%$ \\
\hline
\end{tabular}

This unusual invasion of the blood as shown at Beauregard may have had some bearing on the severity of the disease which was manifested there. On the other hand Sherman with even higher mortality does not show such invasion of the blood stream with pneumococci. Wadsworth with 20 per cent of blood specimens harboring S. hemolyticus had a relatively low mortality.

Blood infection was more pronounced in the postmortem studies. The camps and the distribution of bacteria in blood specimens are shown in Table LXV.

TABLE LXV

Per Cent of Total Examinations

\begin{tabular}{|c|c|c|c|c|c|c|c|c|c|}
\hline \multirow[b]{2}{*}{ CAMP } & \multirow[b]{2}{*}{ PF. } & \multicolumn{4}{|c|}{ PNEUMOCOCCUS } & \multicolumn{2}{|c|}{ STREPTOCOCCUS } & \multirow[b]{2}{*}{ VIR. } & \multirow[b]{2}{*}{$\begin{array}{l}\text { STAPHY- } \\
\text { LOCOCCUS }\end{array}$} \\
\hline & & I & II & III & IV & HEMO. & $\begin{array}{l}\text { NON. } \\
\text { HEMO. }\end{array}$ & & \\
\hline MeArthur & 56 & & & 44 & & 3 & & 3 & 3 \\
\hline Logan & 3 & & & & & & & & \\
\hline Devens & 3 & $(\cdot$ & 65 & & ) & 8 & & & 1 \\
\hline Taylor & $50-15$ & ( & $48-72$ & & ) & $18-2$ & $15-22$ & & \\
\hline Wadsworth & & & & & & 80 & & & \\
\hline Grant & & 7 & 67 & 0 & 25 & & & & \\
\hline Greenleaf & & & & & 77 & ( & 2 & ) & 21 \\
\hline
\end{tabular}

Invasion of the blood by all types of organisms took place after death. The Pfeiffer bacillus was frequently found at McArthur and Taylor: 
pneumococcus at McArthur (Type III), Devens, Taylor, Grant (Type II mainly), Greenleaf (Type IV); the streptococcus hemolyticus in 80 per cent of the cases at Wadsworth and occasionally at Grant, Taylor, and Devens. The staphylococcus was in $21^{\circ}$ per cent of cases at Greenleaf.

Park and Williams, from a survey of influenza cases in New York City in the autumn of 1918, found the Pfeiffer bacillus more constantly present than any other organism. There was also a large number of meningococcus-like organisms and of streptococei of the viridans group. In 24 cases out of 30 autopsies they found the Pfeiffer bácillus in the lungs, in five of which they were in pure culture. The Pfeiffer bacillus was present in large numbers in twenty-six tracheas. Pneumococci (mostly Type IV) were in 15 lungs; streptococcus hemolyticus in nine, gram-negative coccus in one, staphylococeus in two and small gram-negative bacillus in one.

Jordan found the Pfeiffer bacillus present in 64 per cent of cases examined in Chicago. Holman, in 32 autopsies at Pittsburgh, found the Pfeiffer bacillus present in 78 per cent. In direct smears from the nasopharynx in 48 influenza cases Holman found 73 per cent positive for the influenza-like bacillus, 86 per cent pneumococcus-like, and 43 per cent for M. catarrhalis-like.

Comparatively little work was done on the bacteriology of mild influenza in the spring of 1918. Beason, writing from the laboratory of the base hospital at Ft. Riley reported to the sanitary inspector of the 92d Division on the result of bacteriologic studies made on 18 soldiers selected at sick report on March 14, 1918. These men presented symptoms of la grippe, which was widespread at the time. Cultures were made from pharyngeal swabs. The distribution of prevailing organisms in these 18 cases was as follows:

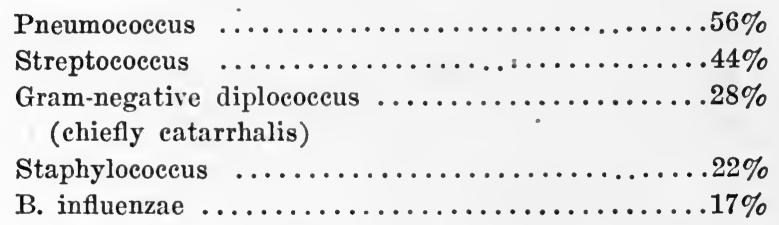

Beason concludes from this that "the percentage of $\mathrm{B}$. influenzae is no greater than has often been found in routine examination of cases of pharyngitis which occurred outside of epidemics of influenza. The indication is that the present trouble-pharyngitis and other respiratory inflammation-is of mixed etiology."

Draper studied 21 recruits at Funston who exhibited all the symptoms of influenza. This was in May, 1918, or some time after the pronounced influenza and pneumonia outbreak which occurred in March. "Throat cultures, made on blood agar plates from 17 cases, showed in 15 the normal and usual mouth flora. In one case the streptococcus hemolyticus, and in 
another a doubtful B. influenzae were found. Blood cultures, 21 by aerobic method and 17 by anaerobic method, were sterile."

Moore reported from Camp Shelby in April: "The Pfeiffer bacillus was easily found, generally in large numbers. The sputum contained clumps made up almost exclusively of the Pfeiffer bacillus."

Laboratory examinations were made of 15 cases of clinical influenza occurring at Camp Bowie in March, 1918. The distribution of organisms was as follows:

$\begin{array}{lr}\text { Pfeiffer bacillus } & 5 \\ \text { Streptococei } & 7 \\ \text { Pneumococei (Type IV) } & 13\end{array}$

Staphylococeus and M. catarrhalis were also found in a few specimens. Pneumococei predominated in the sputa of 6 cases. The influenza bacillus and streptococei predominated in but one specimen. All blood cultures were negative.

The Pfeiffer bacillus has been found among influenza cases more frequently than any other organism and many of the negative results ean undoubtedly be traced to errors in sampling or cultivating.

Evidence of the elusiveness of the organism is furnished from a number of army camps. Camp Kearny at first did not look for growth on blood agar plates after the second day.

"It was found, however, that in some instances B. influenzæ, and in rare cases, pneumococcus, appeared in the plates after the third day's incubation. Consequently we concluded that many organisms, especially B. influenzæ, were probably overlooked in our first cultures. Difficulties in the cultivation of the organism were encountered also in the 221 throat cultures taken at the outbreak of the epidemic. Only after warm plates were taken to the wards and inoculated directly, were the $32 \mathrm{~B}$. influenzæ cultures obtained, in contradiction to absolutely negative results from swabs submitted routinely from the wards."

Camp Taylor reported that the Pfeiffer bacillus was found four times more frequently in the sputa of patients than in the nasal swabbings. Opie's work at Camp Pike showed that by mouse inoculations the Pfeiffer bacillus could be detected in many cases where cultures from the nose and throat were negative. Thus, mice, inoculated with saliva from influenza patients showed 78 per cent positives among 23 patients. Saliva cultures from these same cases showed but 61 per cent positive, throat swab cultures 57 per cent, nose swab cultures 22 per cent.

Park and Williams found that cultures from sputum were unsatisfactory, the influenza bacillus being easily missed. The pharynx at the entrance of the posterior nares was the most likely site of the organisms, although the tonsils yielded results almost as good. The earlier the cases were cultured, the more constantly were the organisms found.

In view of the difficulties of sampling as well as of growing the Pfeiffer 
bacillus it ean well be appreciated that this organism has been passed over many times where a change in technic would have disclosed its presence.

Workers in the laboratory of the New York State Department of Health produced such discordant results that it was felt advisable to send the staff to the laboratory of the eity of New York where previous experience in this line of work had permitted the establishment of a successful technic. As soon as this was mastered, uniform results were attained. The U. S. Hygienic Laboratory likewise sent workers into districts which had been reporting the absence of the Pfeiffer bacillus and they had no difficulty in finding the organism in a great majority of cases.

The methods of Park and Williams in New York and Holman in Pittsburgh were uniformly successful in locating the Pfeiffer bacillus and the reader is referred to these workers for laboratory technic.

Holman added cultures direct to human blood agar plates containing 5 per cent blood. Fresh blood incorporated in the medium or smeared over the plates he regards as, for transfer purposes, inferior to hemoglobin altered after the method of Voges, the blood being added to melted agar at a temperature of about $100^{\circ} \mathrm{C}$. For direct plating the ordinary blood agar is regarded as best, B. influenzae appearing as clear, tiny, pinpoint, inert colonies. Transfers are made from typical colonies to heated blood agar.

Pigeon blood was used originally by Pfeiffer. Human, horse, and rabbit blood is used to even better advantage, as the source of hemoglobin. According to Bell the most desirable hydrogen-ion concentration is $\mathrm{P}_{\mathrm{H}} 7.3$ or 7.4. Avery's medium runs $P_{\mathrm{H}} 7.3$ to 7.5 , that of Winchell and Stillman 7.2 to 7.5 .

The presence of hemoglobin is a necessary agent in the cultivation of influenza bacilli. Attempts have been made to find out what portions of hemoglobin are necessary. Apparently only a small amount is needed and Davis has suggested that it may act as a eatalytic agent. According to Holman altered hemoglobin gives a more luxuriant growth. This medium may be made by heating after the method of Voges in the preparation of the media, fresh blood being added to melted agar at $100^{\circ} \mathrm{C}$. or the hemoglobin may be altered by other bacteria, which explains the favorable symbiotic action noted in connection with the influenza bacillus. Holman states that preparations of hematin agar on which the influenza bacillus refused to grow could be made a favorable medium by the addition of living or freshly killed cultures of staphylococci, and other bacteria. The growth of the influenza bacillus is not aided by other organisms on heated blood agar. This would indicate that other bacteria simply convert hemoglobin into a form more adaptable to the influenza bacillus and when this conversion has been accomplished originally by heat or other agents the symbiosis is missing. Holman reports the influenza bacillus in ordinary blood agar to be greatly stimulated by the presence nearby of colonies of 
staphylococcus pyogenes aureus and albus, pneumococci, streptococci viridans and hemolyticus.

The "emulsions of a staphylococcus killed by boiling for five minutes, when added to ordinary blood agar had a marked stimulating effect although no evidence of hemolysis was present. This effect was practically absent if the emulsion was boiled for 15 minutes or after being killed was left at room temperature for several days. There was no evidence of these stimulating effects by any of these methods when heated blood agar was used, the colonies in this medium growing equally large by themselves."

Scenting the possibility of an anaerobic organism being the real cause of influenza, Norton, in February and March 1919, collected specimens of sputum and material from the tonsils and nasopharynx and of blood from influenza patients, the majority of whom had uncomplicated cases. These were cultured and grown anaerobically according to a number of different procedures. The results failed to yield "any evidence that strict anaerobic organisms are concerned in influenza."

Morphologically the Pfeiffer bacillus is very small, rarely exceeding 1.5 microns in length and 0.3 micron in thickness. There is no spore formation. The ends of the cell are rounded and there is no capsule. The bacillus is nonmotile. Some strains show a tendency to produce threads. Staining is best done with dilute carbolfuchsin for five or ten minutes. Great care is needed to identify this bacillus in direct smears because of its pleomorphism.

We have stated that Pfeiffer's chief reason in singling out this bacillus as the cause of influenza was because of its prevalence among clinical influenza cases and its absence elsewhere. Later work does not bear out this contention. Many authors report the Pfeiffer bacillus from many sources, in normal throats as well as in disease processes quite distinct from clinical influenza. Rivers refers to the findings of numerous workers which we may mention. Auerbach and Davis isolated the organism from the throats of patients with measles, pertussis, diphtheria, and scarlet fever; Klieneberger obtained a pseudoinfluenza bacillus from pus in a gall bladder; Cohn from acute urethritis; Meunier from bronchopneumonia; Slawyk from the blood and spinal fluid of a child with meningitis; Thurofield in septicemia without meningitis; Wollstein from pertussis.

More recently Opic at Camp Pike, examined 384 measles patients finding the Pfeiffer bacillus present in 33 per cent. Wright, at Camp Taylor, found 45 per cent of measles patients showing the Pfeiffer bacillus in cultures from the nasopharynx. In meningitis patients it was present in but 5 per cent.

In the summer of 1918 following the spring epidemic of influenza at Camp Funston, Opie found 35 per cent of a normal group of soldiers showing the Pfeiffer bacillus in their sputum. 
Park and Williams made a very careful bacteriologic survey in the autumn of 1918 at the beginning of the epidemic in New York City. Their findings are quoted below:

TABLE LXVI

INFLUENZA BACILLUS

\begin{tabular}{lccc}
\hline \hline GRouP & PRESENT & ABSENT & $\%$ PRESENT \\
\hline & Influenza cases or associates & & \\
Hospital cases & 160 & 40 & 80 \\
Marines & 30 & 0 & 100 \\
Home for children & 47 & 1 & 98 \\
& Autopsy of Influenza cases & & \\
Lungs & 24 & 6 & 80 \\
Tracheas & 26 & 1 & 96 \\
Heart's blood & 3 & 27 & 10 \\
& Controls & & \\
Nurses-contacts & 4 & 6 & 40 \\
Nurses-noncontacts & 1 & 7 & 9 \\
Measles & 4 & 2 & 67 \\
$\Lambda$ dmission ward & 4 & 5 & 6 \\
Home for girls (isolated) & 2 & 32 & 33 \\
Preventorium (not isolated) & 14 & 25 & \\
\hline
\end{tabular}

While their findings indicate a greater prevalence of the Pfeiffer bacillus among influenza cases, yet its presence elsewhere is quite common.

Jordan states that the Pfeiffer bacillus is much more common among influenza patients than elsewhere. It was.found in 64 per cent of 47 influenza cases, and in but 14 per cent of 28 cases of tonsillitis, sore throat, and common colds.

What is described as the Mather's coccus was found in 66 per cent of cases although quite independent of the Pfeiffer bacillus. The latter was found more frequently in the early stages of the disease, the former more often associated with pneumonia cases. The Mather's coccus was present in those with colds about as frequently as in influenza throats, thus differing from the Pfeiffer bacillus.

Influenza bacilli were present in 13 to 51 per cent of normal throats during the period December, 1918 to May, 1919, according to Winchell and Stillman. These authors found 39 per cent of boys in an asylum with Pfeiffer bacillus present although no influenza had occurred there. Among 52 convalescents from influenza in a girls' home, the organism was found in 38 per cent of cases.

It is these facts which weaken the position of those who believe in the etiologic significance of the Pfeiffer bacillus in epidemic influenza. Recent work in a number of laboratories has shown that the Pfeiffer bacilli are really a group, the different members of which have distinct cultural 
characteristics. It is entirely possible that the true cause of clinical influenza is due to one strain of the group. Granting this, the bacilli found in nonepidemic periods or in normal throats might well be nonvirulent members of the hemophilic group.

As Rivers points out, there is in reality one true B. influenzæ existing in name only, and that is the first one grown and described by Pfeiffer. There are many hemoglobinophylic bacilli. Some produce indol, others do not. This characteristic holds true through many generations. Some reduce amylase, others do not. Some strains always reduce nitrates, others do so irregularly while still others fail to do so at all. In tubes of blood broth milk, Rivers found that some strains made the milk "slightly but definitely acid, others slightly but definitely alkaline, while still others gave doubtful results."

Jordan found that 10 of 13 strains of influenza bacilli form indol. Davis, as far back as 1915 suggested dividing the influenza bacilli into two groups, one showing the phenomenon of symbiosis, the other lacking it. Rivers has shown that B. influenzae can be definitely distinguished from B. pertussis by cultural differences, the latter for instance turning milk alkaline in a manner never observed with the former.

So long as these cultural differences exist, may we not expect to find distinct differences in the behavior of the organism in the human host?

It must be added that so far group differentiation has not added much support to the causal association of the Pfeiffer group with influenza. Presumably the organisms responsible for the severe cases of influenza would be closely related but the reports of numerous workers fail to show this to be the case. Park found from observations on the agglutination reactions of nine autopsy strains that each produced abundant agglutinations only for itself, the absorption test showing absolutely no cross agglutination. "It is apparent then that these autopsy strains are the individual's own influenza bacilli.",

The most toxic strain found by Bell was isolated from a normal throat of a student who had never had influenza. This was fully 6 weeks before the onset of the epidemic in that locality. A person may have in his throat at the same time three different strains of the Pfeiffer bacillus. Bell found that cross agglutination occurred frequently in low dilutions, and in a few instances in high dilutions. It was impossible by these methods to differentiate the organisms isolated from normal healthy throats prior to the epidemic and those isolated from influenza patients.

Utheim found that 11 out of 30 strains isolated from influenza patients agglutinated with the patients' serum but that only one was agglutinated by heterologous serum.

An evidence of infection by an organism is the production in the host of specific antibodies. Cooke examined the serum from a number of in- 
fluenza patients by the complement-fixation test using several strains of B. influenzæ as antigens. While the results indicated that the organisms were pathogenic and infected many if not all patients with influenza, yet this evidence is insufficient to prove that $B$. influenzæ is the sole etiologic agent in influenza.

There can be no question of the pathogenicity of the Pfeiffer bacillus but evidence is still lacking to prove this hemophylic group or any member of it as the initiating cause of influenza in epidemic form.

Attempts have been made to identify the cause of influenza by transmitting the infection to animals and man. Of particular interest in this connection are the works of Rosenau, Keegan, Goldberger, and Lake in Boston, and that of MeCoy and Richey in San Franciseo. In the Boston experiment 68 volunteers were secured from the U. S. Naval Detention Training Camp at Deer Island. These men had been exposed in some degree to influenza either at the eamp or elsewhere. However, 47 men were without history of an attack during the recent epidemic and 39 of these without history of any such attack in their lives. This work was begun in November, 1918. A suspension of freshly isolated culture of Pfeiffer's bacillus was instilled into the nose of each of 3 nonimmunes, and into 3 controls who had a history of a recent attack. None of the volunteers showed any subsequent reaction. Ten presumably nonimmune individuals were inoculated with a suspension of a number of different pure cultures of Pfeiffer's bacillus. No reaction followed. Thirty men were inoculated by means of spray or swab or both, with secretions, both filtered and unfiltered, from the upper respiratory tract of typical cases of influenza in the active stage of the disease. In no instance was an attack of the disease produced. Ten were exposed to cases in the hospital, patients eoughing in their faces, but without acquiring the disease. The patients were in the early stages of the disease. Secretions from 5 typical cases of influenza were filtered and some of the filtrate injected subcutaneously into 10 volunteers. Ten other men were inoculated with the pooled blood of these 5 cases. In no case was the disease transmitted to the well men.

On the west coast simultaneously, experiments were made on volunteers from the Yerba Buena Naval Training Station at San Francisco. These men had not been exposed to influenza in the prevailing pandemic. They had, however, all been vaccinated with large doses of a bacterial vaccine containing Pfeiffer bacilli, the three fixed types of pneumococci and hemolytic streptococci. Five men had instilled into their nostrils a heavy suspension made by emulsifying cultures of 8 strains of Pfeiffer's bacillus without filtration. Five others were similarly treated with material passed through a Berkefeld $N$. candle. No person developed influenza during 7 days' observation. Forty men were inoculated by means of a medicine dropper or atomizer applied to the nose with emulsion of secretions from 
the upper respiratory passages of active eases of influenza from 15 to 48 hours after onset. An equal number of men were similarly treated with filtered material. All results were negative. In 3 eases in which unfiltered material was used, sore throat developed corresponding clinically to acute tonsillitis and in two of these cases an almost pure culture of a hemolytic streptococcus was recovered from throat cultures. A filtered emulsion dropped into the conjunctiva of 2 volunteers did not produce influenza, neither did the same material when injected subcutaneously into one volunteer. One cubic centimeter of blood taken during the active stage of influenza failed to cause influenza when injected subcutaneously into a volunteer. The unfiltered material used in these cases was found to contain living organisms representing the Pfeiffer bacillus, pneumococeus of Type IV and hemolytic streptococci. From 3 to 4 hours elapsed between collection of the sample and inoculation. In Boston the interval was much shorter, in some cases less than a minute. Nicolle and Lebailly are reported in the same issue of Public Health Reports to have successfully transmitted influenza to two persons by using the filtrate of a bronchial expectoration of a patient ill two days. The injection was subcutaneous. The disease could not be transmitted by blood inoculations.

A successful attempt to induce clinical influenza in monkeys with the Pfeiffer bacillus was reported by Blake and Cecil. The strain used was received from an influenzal pneumonia case in a child. The virulence of the organism was increased by successive passages through mice and monkeys. Injections were made intraperitoneally and fluid withdrawn from 8 to 10 hours after injection and cultures prepared. The virulence of the strain was raised so that .01 c.c. of a sixteen-hour blood broth culture injected intraperitoneally killed a white mouse within 48 hours.

The material for inoculation was either (1) a first or second subculture on blood agar or in blood broth of $\mathrm{B}$. influenzæ recovered from the peritoneal exudate or heart's blood of monkeys dying of B. influenzæ, peritonitis and septicemia; (2) first or second subculture of B. influenzæ recovered from the respiratory tract of monkeys killed during the acute stage of the pneumonia produced by intratracheal injection of the organism, or (3) peritoneal exudate of monkeys dying of $\mathrm{B}$. influenzæ peritonitis. Inoculations were made either by direct swabbing or pipette into the nose and mouth or by intratracheal injection.

Twelve monkeys received the nose and mouth inoculation and in every instance there resulted an acute respiratory disease which appeared identical with influenza in man. The onset occurred from 3 to 6 hours after inoculation, the first symptom being prostration in varying degree. This was accompanied by abrupt rise in temperature from 103 to 106 in some cases, and in others less or even no febrile symptoms. Upper respiratory tract infection followed with sneezing, blinking of the eyes and rubbing 
of the nose. In general the disease was self limited, lasting from three to five days. During the active stage of the disease the leucocyte count was either subnormal or normal.

"Five monkeys developed an acute sinusitis of the antrum of Highmore from which B. influenzæ in pure culture or mixed with some other organism was recovered at necropsy." Two animals developed pneumonia and were killed in the early stage of the disease. B. influenzæ in pure culture was reeovered from the lungs of both.

Of the 10 monkeys receiving intratracheal injections, seven developed pneumonia, two a tracheobronchitis and one evidenced no infection. "Of the seven monkeys developing pneumonia, three were killed during the active stage of the disease with the recovery of B. influenzæ in pure culture of all; four were killed immediately after recovery, cultures made at necropsy being sterile." In the other two cases the organism was recovered from one and not from the other.

The pneumonia picture was essentially identical with that ascribed to pure influenza bacillus infection in the lungs of man.

It is the conclusion of the authors that $B$. influenzæ is the specific cause of elinical influenza.

Olitsky and Gates from continued observations made during 1918 and 1919 believe that the causal agent of influenza is a multiplying substance or virus capable of passing through a Berkefeld filter. Pfeiffer bacillus, the pneumococci, viridans and other organisms are looked upon as secondary invaders quite unrelated to the inciting virus, and yet able to produce fatal pneumonias with pleurisies when implanted in a soil previously invaded by the unnamed influenza substance.

Nasopharyngeal saline washings were taken from eight cases of influenza within the first 36 hours of the disease and from 12 eases at later stages. Washings were also taken during an interepidemic period from 14 persons believed never to have had influenza.

Intratracheal inoculations were made in rabbits using the unfiltered washings, the filtered washings, lung tissue suspensions, filtered and unfiltered, from previously inoculated rabbits, and similar lung tissue preserved in sterile 50 per cent glycerin.

Within 24 to 48 hours after inoculation with the material from the early influenza cases, there developed fever and ordinary signs of indisposition. Most striking was definite leukopenia. These symptoms continued for three days and, if uncomplicated by other bacterial infection, the rabbit recovered. The respiratory organs were affected to the exclusion of all other structures. "The lungs were voluminous and edematous, and had a mottled, hemorrhagic appearance." "The edema was pronounced; the alveoli were filled with serum."

These effects were not produced by similar treatment with material 
from twelve cases of influenza collected 48 hours after onset, and in the stage of convalescence or of secondary pneumonia, nor from 14 persons free from influenza tested in the epidemic and interepidemic periods.

It is noteworthy that the typical clinical and pathologic effects were produced in as many as 15 rabbits by successive inoculation of ground lung tissue from a rabbit previously infected.

"In one series of experiments glycerinated material free from ordinary bacteria was passed with typical effects through 10 successive rabbits."

The authors point out that it is extremely difficult to secure these effects with the filtrates obtained directly from the patient's secretions. However, after first passing the material through a series of rabbits, repeated filtration through Berkefeld " $\mathrm{V}$ " and " $\mathrm{N}$ " candles between rabbit passages did not hinder the typical development of the conditions described.

From the facts as above given we find that it is impossible to come to any definite conclusion concerning the etiologic agent in primary influenza. It must be admitted that the Pfeiffer bacillus was found in this disease more frequently and more constantly than any other single organism, but in our opinion its presence, even if this could be shown to be universal, does not prove that this organism is the cause of the disease. Pfeiffer's bacillus seems to be widely distributed and at times is easily found in the throats of healthy people as well as in the throats of those suffering from other diseases than influenza, such as measles, whooping cough, etc. Moreover, this organism has been frequently found when epidemic influenza has not existed. In other words the presence of the Pfeiffer bacillus and clinical influenza are not by any means always concomitant. We must, therefore, come to the conclusion that the real virus of influenza has not yet been identified.

Turning to the other question, as to the etiologic factors in the pneumonias, it must be evident that any one of a number of microorganisms, including the several types of the pneumococcus, certain types of the streptococcus and of the staphylococcus, and possibly other organisms, may cause and do cause the condition which we have designated as pneumonia. It is more proper now to speak of "the pneumonias ;" certainly if we are discussing them from an etiologic standpoint. It is hardly within the province of the epidemiologist to go deeply into this subject; certainly not until the bacteriologist and the pathologist have reached more definite conclusions founded upon more scientific data than can at present be given.

There are most competent medical observers who believe that the changes in the lungs found at autopsy in many cases of deaths from pneumonia, although important, are not sufficient to account for the symptoms or to cause death. These men believe that they are dealing with a bacteremia or toxemia in which the changes in the lungs bear the same relation to the disease as ulcerations in the intestines bear to typhoid fever. There 
has opened up here a wide field in which the epidemiologist, the bacteriologist, the clinician and the pathologist must all work. Much material for the solution of this problem has been accumulated but much more remains to be done.

Combative Measures.-In spite of our intimate experience with epidemic influenza in the last two years, there is little that can be offered in the way of prevention. We may lessen typhoid fever by insuring safe water supplies for our cities. Smallpox may be avoided by vaccination. Against influenza our weapons have so far proved of little avail. This is largely due to the fact that the causative agent of the disease is still unknown. Quarantine is ineffective because practically it cannot be applied in a really efficient manner. Only by interruption of all means of intercourse between communities could we expect much from this measure. Many army camps established quarantine before the disease had gained a foothold, but even with armed guards and physical examination of all entering camp the disease could not be excluded. Camp Wheeler was successful for several weeks in warding off the disease, but eventually it gained entrance. When such a great proportion of the population is susceptible, it is quite out of the question to prevent the disease from being carried into a community by those people who at the time of entrance show no disease symptoms. We have seen from the surveys made by the U. S. Public Health Service that about 28 per cent of the population were attacked. This varied widely for different communities. This figure represents the proportion that were visibly affected. It is quite probable that the number of those infected including those where the attack was so mild as not to occasion evident malaise, is very much larger. A report from the Air Service Depot at Morrison, Virginia, bears directly on this question.

"A sudden outbreak of a highly contagious respiratory infection attacked within a period of twelve days practically one-half of the entire camp and then stopped because probably all who were susceptible had been attacked, and of those not reporting sick, undoubtedly a large number had mild attacks and became immune. The last statement seems borne out by the fact that a considerable number of medical officers, personnel and nurses experienced slight malaise, headache, eto., for one day and then felt better and developed no further symptoms."

Recognizing that the infective agent may be present among those who exhibit no symptoms, it is impossible even by means of rigid physical in. spection to weed out all dangerous persons.

Island communities, such as Fort St. Phillip in Louisiana, could and dir establish an effective quarantine. Up to December 5 there had not been a case of influenza, although the disease was rampant elsewhere. Fort St. Phillip is situated on the Mississippi River 65 miles below New Orleans. By admitting no person from the outside the disease was excluded. 


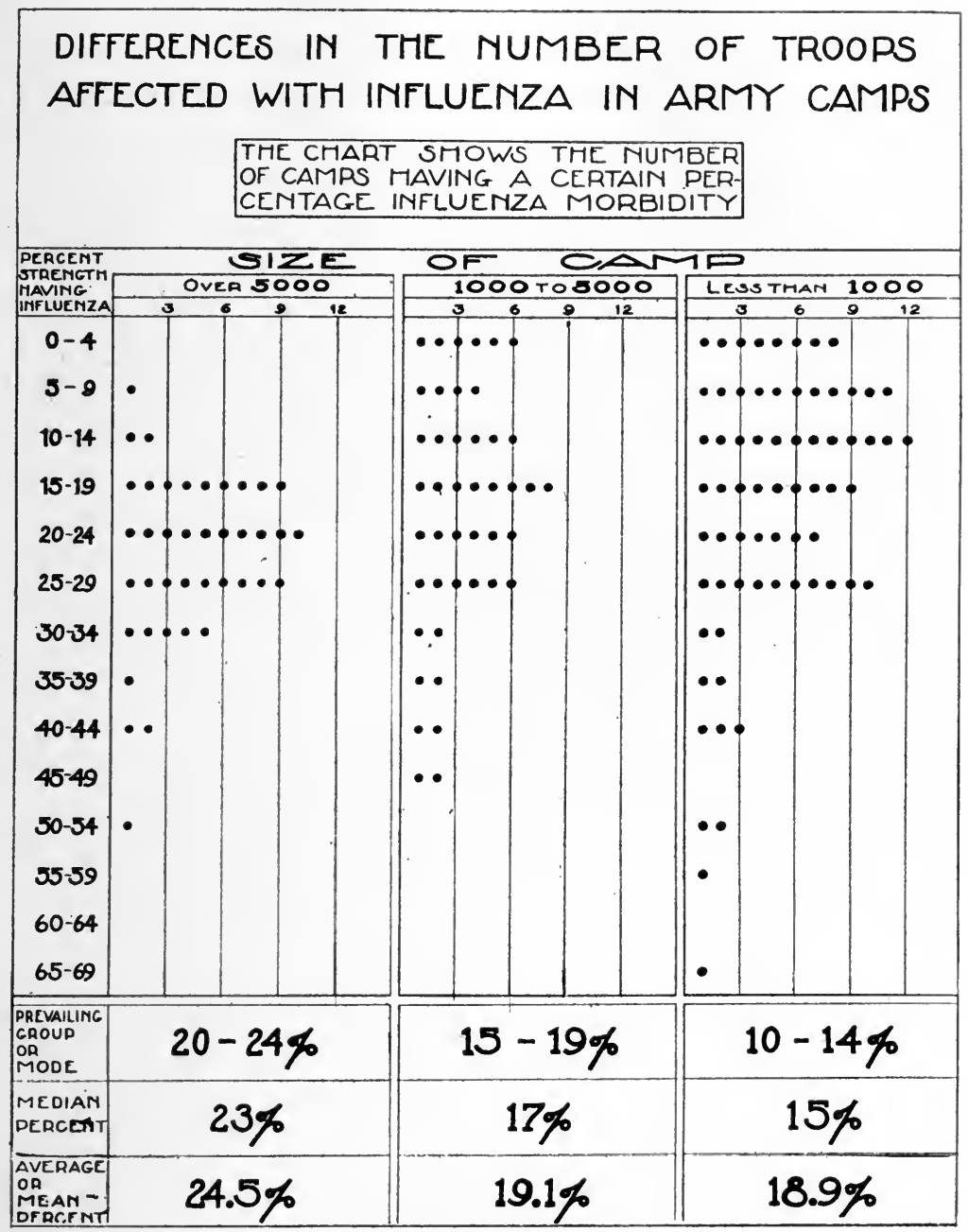

Fig. 59.

Daily medical inspection was used apparently to good effect in the Army camps to separate the incipient cases from the well. This of course is too herculean a task for civilian communities. At Camp Funston men were examined twice daily and 50 per cent of the hospital admissions were discovered in this manner. It was common to find men who had been doing full duty with temperatures over 100. Camp Upton reported that the men of the Development Battalions who were receiving treatment for various chronic ills had about half as much influenza as the rest of the camp. This is attributed to the fact that the men were coming under daily observation of medical officers, and the sick were thus excluded early.

The stoppage of communication between companies within a camp pre- 
vented the spread of influenza to some organizations. The 302nd Auxiliary Remount Depot at Camp Upton was geographically isolated from the rest of the camp and there was also added a vigorous quarantine. Not a case occurred here for 34 days although the epidemic was raging elsewhere in the camp. Eventually it reached the Remount Depot. The 3rd Development Battalion, though equally apart from the camp geographically, did not maintain strict quarantine and had influenza early in the epidemic.

Intercompany quarantine delayed the progress of the epidemic disease at Camp Lee but did not prevent it from ultimately reaching all units.

Of the forty large camps, thirty-four were quarantined, twenty-three had physical examination of all entering camp in addition to quarantine, nineteen had quarantine, physical inspection and placed new men in a detention camp. Eight instituted the above measures and also gave each man entering camp prophylactic treatment of the nose and throat. The names of these eight camps, their influenza incidence and their relative standing in incidence are given in Table LXVII.

TABLE LXVII

\begin{tabular}{lcc}
\hline \hline CAMP & $\begin{array}{c}\text { PER CENT OF STRENGti } \\
\text { Having INFLUENZA }\end{array}$ & RELATtve \\
\hline Cody & 44 & 2 \\
Funston & 28 & 11 \\
Greene & 28 & 12 \\
Meade & 27 & 13 \\
Wheeler & 18 & 28 \\
Gordon & 17 & 31 \\
Fremont & 16 & 32 \\
Johnston & 15 & 37 \\
\hline
\end{tabular}

Lewis, with the lowest rate of the large Army camps (8 per cent), did not give the new arrivals a physical examination, nor did they use the prophylactic treatment. Wadsworth with 10 per cent incidence and MeClellan with 14 per cent did not use the prophylactic spray. Camp Humphreys was at no time under quarantine; nor were the men restricted in their amusements. The influenza incidence was only 16 per cent. The following large camps were not quarantined, and their incidence as a group is no higher than other eamps:

$\begin{array}{ll}\text { Forrest } & 30 \% \\ \text { Humphreys } & 16 \% \\ \text { Jackson } & 22 \% \\ \text { McArthur } & 35 \% \\ \text { Logan } & 34 \% \\ \text { Sheridan } & 20 \%\end{array}$


It has been proposed that people wear face masks to protect the portals of disease entry, namely, the mouth and nose. This measure was thoroughly tried out in many places. The eity of San Francisco adopted this practice, but the comparative mortality figures from American municipalities do not indicate that this eity benefited by the practice. If masking were an effective procedure we should expect the Army camps that masked to have a lower incidence than those that did not mask. Of nineteen camps where masks were used 9 had high incidence, 10 low. In 15 other camps where masks were not used 7 had high incidence, 8 low. Masking was generally ineffective in army camps.

Theoretically, eloth masks of proper thickness and weave will prevent germ infected dust particles or droplets of saliva from entering mouth and nose. They also diminish the number of contacts between the hands and face. Masks do not prevent hand to mouth infection. The purpose of the mask is not sufficiently understood to give real protection. A sentry on a lonely beat has been observed faithfully wearing his mask. Some people have removed the mask in public to avoid being conspicuous. When by themselves it has been replaced. Obviously such use of the mask cannot accomplish what it is intended to accomplish. At Camp Devens the surgeons, nurses and hospital attendants prior to the epidemic wore no masks and yet escaped pneumonia and other diseases consistently. During the epidemic, masks were worn, and yet many suffered severely.

Protective throat sprays and gargles have been tried in many quarters, and the opinion on their value is divided. A test was made at Camp Upton upon 1600 men, 800 being given the nose and throat spray with dichloramine-T 4 times daily together with gargling with Dobel's solution 3 times daily, and the other 800 remained untreated. The percentage of influenza cases developing after a period of 20 days was the same in both groups.

Camp Sherman had 33 per eent of its personnel affected and had the highest mortality of the large camps and yet all members of the command were required to gargle twice daily with a 1-10,000 quinine solution whose pneumococcidal value is well established.

At Camp Funston it was found that 17 out of 25 hospital attendants and influenza contacts contracted influenza even though a protargol spray was used vigorously. Among 25 other men who were not sprayed, but one case developed. All 50 wore masks and worked in the same temporary hospital under the same conditions. It was the feeling here that wholesale spraying actually spread the infection through the common use of an atomizer. A similar experience is recited from Camp Cody.

The Air Service Flying School in Tennessee reports as follows:

"As a prophylactic measure the entire personnel had their nose and throat sprayed with a one to ten-thousand sulphate of quinine aqueous solution, and one drop of a 
twenty per cent argyrol solution instilled in each eye, daily for a period of eight days, the value of which after careful observation was questionable."

We do not believe that the nose and throat prophylaxis is an effective weapon against influenza.

So far as we can ascertain there is nothing to be gained by closing public schools at times of threatened influenza. The disease acquires its first foothold among adults and is thence brought into the home. The exclusion of children from school will not prevent infection being introduced by older members of the family. In fact, school attendance under medical inspection permits the early detection of many cases which would not be revealed otherwise. Vaughan (Warren) quotes Parsons as stating that out of 125 households in the epidemic of 1889-90, the first case was a bread winner in 96 , a housekeeper in 9 , a child in school in 13 , a child not at school in 2. Until all places of public assembly - theatres, street cars, playgrounds, stores, factories, etc.-are closed, it seems quite beside the point to close schools.

The only motive for closing schools would seem to be that it can be done. Much more effective would be the complete stoppage of all business activities and the confinement of people to their homes. This is perhaps impractical and consequently we do not see it seriously urged. Instead, school closing is urged because this is a practical measure. Closing of some assembly places and staggering of the opening and closing hours of stores were tried in many eities, and yet it is not clear that eities which took these steps fared better than others.

Immunization, both as a preventive and a curative treatment of influenza, has been tried, but the success of these measures has not been thoroughly established. Vaccines have been prepared using single strains of the influenza bacillus, and other organisms, as well as polyvalent vaccines containing streptococei, pneumococei, staphylococei and the Pfeiffer bacillus. Much of this work was carried on in the later stages of the epidemic and there is no assurance that the individuals treated were not already immune.

Two commissions were appointed in Massachusetts to report on the value of vaccination. The Commission on Scientific Investigation, composed of Rosenau, McCoy and Gay, concluded that from evidence which they had reviewed there was no basis for regarding prophylactic vaccination against influenza as of value in either preventing the spread of the disease or reducing its severity. In view of the fact that there was some indication of less influenza among the vaccinated the Commission believed that the work should be continued and that further experimental evidence should be collected. No unfavorable effect of vaccines was encountered. The Board of Statistical Investigation, composed of Whipple, Davis and Crum 
concurred in the above conclusions. It was felt that though the value of vaccine therapy was unproved the work should be encouraged.

The Illinois Influenza Commission, composed of O'Neill, Drake and Cobb, found that eleven different vaccines had been used to some extent in 93 counties. As to whether these vaccines had been of value in preventing the spread of the disease, 19 counties were uncommunicative, 29 answered in the affirmative, 27 were of divided opinion, and 16 regarded their use of no preventive value. Twenty-three counties reported no cases after vaccination. Twenty-four counties reported influenza following vaccination. The evidence regarding the value of influenza vaccination was, to say the least, not conclusive.

A most interesting review of the attempts to secure immunization through vaccination has been prepared by Haythorn and appears as a part of the influenza studies made at the University of Pittsburgh. We may mention the work at Dixmont, Pa., where 600 people were inoculated with a mixed vaccine prior to the appearance of the epidemic. The influenza cases among this group amounted to 7.3 per cent, whereas among 700 unvaccinated the incidence was 9.8 per cent. There were no deaths among the vaccinated and 9 deaths among the unvaccinated. None of the vaccinated developed pneumonia although 15 cases occurred among the controls. Vaccination at the institution was begun October 20 and completed November 6 . The first influenza case occurred November 20 . The vaccine contained in each c.c. 200 million each of the Pfeiffer bacillus, M. catarrhalis, B. Friedländer, pneumococci, streptococci and staphylococci both aureus and albus. The administration was in four doses at 4-day intervals. This experiment shows a slight reduction in the severity of the disease among the vaccinated.

McCoy, Murray and Teeter are quoted as vaccinating patients in a San Francisco insane asylum. Three hundred and ninety people were inoculated prior to November 15, and the first case of influenza appeared on the 26th. A vaccine furnished by the Chicago Health Department was used and consisted of the Pfeiffer bacillus, pneumococei of all four types, S. hemolyticus and staphylococei. The results reported below were the reverse of the above in that no protective value of vaccination was shown.

$\begin{array}{lcc} & \text { VACCINATED }(390) & \text { NOT VACCINATED (390) } \\ \text { Per cent having influenza } & 31.0 & 26.0 \\ \text { Per cent having pneumonia } & 5.9 & 4.3 \\ \text { Per cent dying } & 2.6 & 1.8\end{array}$

Vaccination was done on a large scale under the direction of Sherman for the Carnegie Steel and Frick Coke Companies. About 36,000 persons were inoculated with three different types of commercial mixed vaccines. Statistics were collected by having people send in cards with answers to 
certain questions. The diagnosis of influenza and pneumonia could only be estimated as physicians' reports were not available. Altogether an immense amount of work was done but the information collected was without worth so far as establishing the value of the vaccine.

Much the same may be said of the vaccinations made with a mixed preparation sent out by Rosenow of Rochester, Minn. Apparently about 21,000 people were vaccinated by numerous physicians in the vicinity, and from the reports returned by them it appears that the influenza incidence per thousand vaccinated was 14.6 , pneumonia incidence 1.8 , mortality 0.19 in the six weeks following vaccination. Among 62,000 controls Rosenow found an influenza rate per 1000 of 229 , pneumonia 15.7 and mortality 3.4. As no allowance was made for the stage of the epidemic at which vaccination was carried out and as statistics collected in this manner are open to great error, it is not felt that these figures carry the weight that Rosenow would give to them.

Vaccination with Leary's preparation made from several strains of the Pfeiffer bacillus was carried on at many of the Massachusetts State hospitals. At the Morrison State Hospital for Epileptics the influenza incidence among 461 vaccinated was 35 per cent. Among 518 unvaceinated it was 32 per cent. Seventeen per eent of the vaccinated cases died. In the control group 13 per cent died. There is no indication from this that Pfeiffer bacillus vaccine furnished protection against the disease.

In the preceding paragraphs we have offered a somewhat discouraging array of combative measures against influenza. The fact remains that like many of the proposed vaceines, the administrative steps against the disease must continue to be largely of the "shotgun" variety. If there is any element of goodness in a measure, health departments, lacking specific information, cannot be blamed for recommending them, ineffective though they may later prove to be.

Administrative action can best be directed toward adequate relief measures. A plan of campaign should be worked out in advance so as to ensure the adequate reporting of eases, nursing, visitation and attention, and hospitalization of the severe cases. T'o the individual no better advice can be offered than the necessity of taking to bed early in the disease and remaining there for the full time advocated by the physician in attendance. The shortening of hours of labor would assist if people would use this extra time for rest. Best of all as a preventive measure, impractical though it may appear, is for people to remain at home or if this is out of the question to eat home prepared lunches and to walk to work. The reduction of eommingling to the zero point holds out the most hope in combating the spread of the disease.

As to our preparation for future pandemics which are bound to come we must rely upon the diligent research of the epidemiologist and the bac- 
teriologist. They must begin their efforts at once and these efforts must be continuous.

\section{Vaughan (Warren) has the following to say concerning preparation for} future epidemics of this disease:

"To become thoroughly acquainted with epidemic influenza in all its manifestations would require a lifetime of study. Knowledge of the disease would be greatly furthered if competent epidemiologists should see fit to devote their entire time to a study of the disease in its various ramifications. The author suggests a research organization of individuals whose function it would be to become completely acquainted with influenza. The organization should be under the direction of a competent board of epidemiologists. Under them would work several groups composed of epidemiologists, bacteriologists, and others. There should be sufficient groups so that they might be distributed to diverse regions of the earth. They should be equipped for travel, with mobile bacteriologic laboratories and all the. necessary equipment for epidemiologic surveys so that at a moment's notice they could proceed wherever an epidemic of any disease simulating influenza is reported to be prevalent. The working groups would be under the administrative control of the central directors and would make their reports to them. All groups should be so distributed geographically as to have easy and rapid access to any community in which an epidemic might occur. They would keep themselves informed concerning the disease prevalence in all communities under their jurisdiction. This would be done through the cooperation of the civil health authorities and through the utilization of all other available sources of information. The central board should be constantly in touch with the groups so that the infectious disease prevalence in all parts of the world would be known at all times.

" $\mathrm{Had}$ such an organization been in existence during the last thirty years, every one of the so-called influenza epidemics reported in one place or another would have been investigated. Detailed epidemiologic, statistical, demographic, and bacteriologic reports would have been made. It matters little how small or insignificant the outbreak appears to be. Even the smallest have their characteristic features and are worthy of study. If we study epidemic influenza but once in thirty years, we shall never become well acquainted with the disease. We must see it repeatedly and frequently. If it does not exist during the intervals we must study the diseases simulating it. It is surprising how much of the knowledge acquired in 1989 was forgotten by 1918. Even some of the more important features had passed from memory. Thus we find statements in 1918 that the age morbidity was quite different from that in all preceding epidemics. Research into the literature of the past does not corroborate this impression.

"If influenza is scattered throughout the earth in mild form, it would avail us but little to send a commission to Bokhara to study the endemic focus supposed by some to exist in Turkestan. Even though the disease were endemic in that country, one would not expect to discover epidemies there. The general immunity of the population in the endemic area is increased. Nevertheless one unit should surely be stationed in Turkestan, there to study the existing conditions regarding infectious diseases.

"There would be ample work for all groups at all times. The study would not be limited to a consideration of infectious diseases. Sociologic conditions may be of importance. We have recorded instances of this. Wherever there is an unusual concentration of large masses of individuals the investigators should study the results of such concentration. An advantage of this organization would be that the groups through their central bureau would establish an information bureau of infectious disease prevalence 
analogous to the popular weather bureau of today. They would report the presence of a cloud before it had appeared on the loeal horizon.

"In the absence of any epidemies resembling influenza, there would be abundant opportunity for correlated work. We have mentioned the epidemiologic resemblanees between influenza and eertain other infectious diseases. Comparative study of any or all of them is of importanee. The bacteriologist and the immunologist would find plenty of material in the study of measles prevalenees. If the cause of measles can be discovered, another step toward the discovery of the cause of influenza will have been made. The two diseases are so similar in their manner of spread, in the mode of transmission, in their elinical characteristies and in the results of laboratory attempts to transmit the disease, that one must assume that the causative viruses are not dissimilar. Any new facts that we may gain coneerning measles will be of value in the study of influenza. Many years could be well devoted merely to a study of immunity in influenza.

"The results obtained by this proposed organization for the investigation of influenza would be slow in achievement. The study is not of a type calculated to appeal to the popular imagination. Communities in which the dread of an imminent pestilence is not present would subseribe with some hesitation to appeals for pecuniary assistance. Fortunately however there are in existence several organizations already well developed along these lines, organizations chiefly interested in certain other diseases. There can be no doubt but that at the present time the finaneing of such a broad project eould be arranged and that the groups eould be effieiently organized on the basis of experience already gained in similar projects.

"Crookshank well remarks that our present epidemiologie intelligenee serviee is hardly superior to that of a meteorologic office which only gives warning of rain when unfurled umbrellas pass along the street. Influenza will surely return. There will be mild epidemies within the next few years. In time another pandemic will arrive and after it will come pandemic after pandemic. In 1918 as in 1889 we were caught unprepared. Let us do our utmost to prevent the reeurrence of this tragedy. To delay is to lose the valuable information gained during the last two years. The future is not without well grounded hope, but sucess will not be achieved until we have attained a much deeper understanding of the epidemiology of influenza."

\section{Bibliography}

Аввотт, Samuel W.: Massachusetts State Board of Health Report for 1889.

BAKER, GladdeN W.: New York State Heallth Department Bulletin, May 1, 1919.

Finkler, Ditmar: Twentieth Century Practice of Medicine, XV, 1898.

Frost, W. H. and Sxdenstricker, Edgar: Public Health Reports, June 20, 1919.

Hirsch, August: Handbook of Geographical and Historieal Pathology, i, 1883.

Lynch, Charles and Cumming, James G.: American Journal of Public Health, January, 1919 and November, 1919.

Parkes, L. C.: Reynolds System of Medicine, i, 1879.

Pearl, Raymond: Public Health Reports, August 8, 1919:

Smith, A.: Annual Report of New York City Health Department for 1890 and 1891. Special Tables of Mortality from Influenza and Pneumonia in Indiana, Kansas and Philadelphia, Pa., September 1, to Deember 31, 1918, Bureau of the Census, Government Printing Office, Washington.

Studies on Epidemic Influenza, The University of Pittsburgh School of Medicine, 1919. Vaughan, WARren: A detailed review of the epidemiology of influenza, Monograph, No. 1, American Journal of Hygiene, Baltimore, 1821. 


\section{CHAPTER XV}

\section{TUBERCULOSIS}

Descriptive.-Under this name we include all those diseases, the etiologic factor of which is the bacillus tuberculosis. These diseases differ widely among themselves in symptomatology, progress, and end results. Millions of men carry this infection during the greater part of their sojourn on earth without at any time being aware of it. Hundreds of thousands constitute the annual tribute that mankind pays to this, the captain of the hosts of death. There is no other infection so universal among men and but few, if any, others in which the case mortality is so low. Children with tuberculous glands and joints often grow into the lustiest adults. Miliary tuberculosis is a rapid, fulminating disease, surely fatal in the end, and it takes its toll from the vigorous as well as from the frail. The child dying from tuberculous meningitis may be plump, rosy, and the picture of health, while another child in the same family may be scrofulous and still grow into vigorous manhood. Massive infection with virulent bacilli may send the most robust to the grave in a few months. Mild infections are generally unrecognized, and it appears that most individuals are infected before they reach adult life. There are but few tissues in the anatomy of man free from possible infection by the specific organism of this disease. The skin, glands, bones, mucous and serous membranes, the thoracic and abdominal viscera, the brain, and other nervous tissue, may furnish a place of residence and supply food for this undesirable guest. The symptoms and ultimate results are determined, in part at least, by the nature of the tissue in which the invader finds lodgment; but when the infection is in the same tissue in two or more individuals, the progress of the invading organism is subject to wide variations. In the majority of people who come to death no matter what the cause, whether disease or violence, tuberculous lesions are found in the lungs. Calcified and other forms of arrested growth of the bacillus may be the only evidence of infection in man. In other individuals pulmonary invasion is followed by rapid proliferation of the bacillus, extensive destruction of tissue, the development of alarming symptoms and speedy termination in death. In the majority of instances, tuberculous disease does not follow tuberculous infection. The problems of tuberculosis are many and varied and most of them remain without adequate solution.

There are but few, if any, of the vertebrate animals, from the lowest 


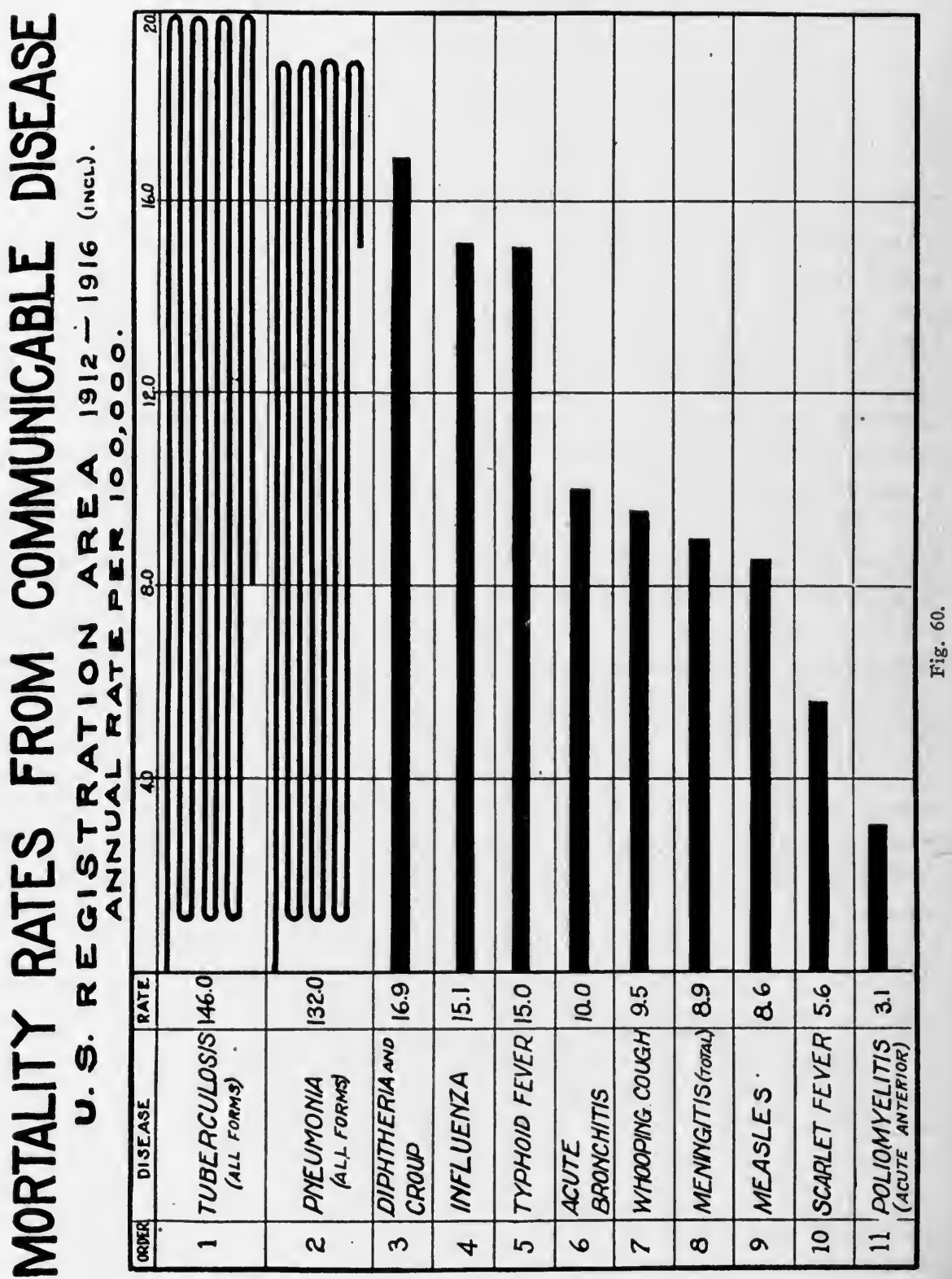

to man, free from possible invasion by this bacillus, which has apparently in its unending warfare against the higher forms of life developed armies especially trained for the invasion and occupation of the individuals constituting animal groups. From this apparent specialization there have resulted several different varieties or species of the bacillus, which 
we now classify, (a) the human, (b) the bovine, (c) the avian, (d) the piscidian, (e) the reptilian bacilli.

History.-Smith and Ruffer have demonstrated the presence of tubercle bacilli in the carious vertebrae of mummies, thus showing that this organism had become a parasite of man when the race was still in its cradle. Hippocrates shows an interesting acquaintance with pulmonary tuberculosis. He believed that this disease might be due to (a) imperfect resolution of a pneumonia, (b) to hemoptysis, (c) purulent pleurisy. This means undoubtedly that Greek physicians recognized the fact that pulmonary tuberculosis frequently follows the conditions mentioned. It is interesting to add that Hippocrates believed that in phthisis there is a growth in the lungs.

The many positive references to consumption found in Greek and Roman writings, ${ }^{1}$ both medical and nonmedical, establish beyond a doubt their full acquaintance with this malady. They manifest a keen observation of the symptoms, causes, development, and dreaded results of the disease and, although we meet with no scientific explanation approaching the modern theory of tuberculosis, it is entirely possible that some of the ancient physicians may have had at least a visual acquaintance with the small rounded nodules produced by the bacillus tuberculosis, as subsequent references may suggest.

Euryphon, a celebrated physician of Gnidos in Asia Minor during the first half of the fifth century в. с. and an older contemporary of Hippocrates, treated consumption with the milk of she-asses and mothers, and likewise employed actual cautery. (See Galen vi, 775; vii, 701; Soranus Vita Hippocr. in Hippocr. Opera, iii). A scene described by Meineke (Fragmenta Comoed. Antiq. $\mathrm{II}_{2}$ 679), may possibly refer to him, and in this passage he is ridiculed for treating the consumptive Cinesias with cautery. Furthermore, if he be the author of the "Gnidiae Sententiae" in the Corpus Hippocraticum (Galen xvii, A, 886, Kuehn Ed.) his knowledge of consumption or phthisis was already well defined, for in the "de affectionibus internis" of this collection appears a lucid discussion of three divisions of phthisis: First, pulmonary infection caused by a flow of putrid phlegm from the head to the lungs, causing death usually within a year's time; second, that eaused by overexertion, in which the patient suffers as in the first case, except that the sputum is of a heavier consistency, the cough more aggravated in the case of older people, the pain in the lungs more marked, and the patient feels as if a heavy weight were resting on his chest; pain also appears in the back, the skin becomes transparent, and at the slightest exertion he gasps for

1The following survey of tuberculosis in Greek and Roman times is by Professor Bruno Meinecke; the entire work, from which this is summarized, has been accepted as a Doctor's Dissertation at the University of Michigar. 
breath. Those afflicted with this kind of consumption live longer, but eventually die within a period of three years. The symptoms of the third form of phthisis are recognized by blood and bile in the spinal marrow, a watery phlegm and bile in the veins; the patient becomes emaciated, his appearance is black and swollen, the parts below the eyes become pale, he experiences difficulty in breathing and in coughing as well, until a temporary relief is secured by vomiting bile and phlegm. This disease is of longer duration than either of the former, but the patient usually dies in nine years.

Hippocrates (c. $460-357$ в. c.) hạs given us a truly excellent description of phthisis in the first book "de morbis." The passage is so interesting and instructive that we quote it in full:

"And from the following causes especially does empyema arise in the lungs; if a person, who is afflicted with inflammation of the lungs, does not relieve himself during the critical days, but retains the sputum and phlegm in his lungs, in such a one there appears empyema. If he receives attention immediately, he usually escapes; but if he is neglected, the lung beeomes infeeted and uleerated from the putrid substance that establishes itself within, and it ean neither take in any nourishment worth mentioning nor relieve itself at all of any part of the substance within, but the patient becomes subject to choking spells, his breathing becomes impaired and is accompanied by a wheezing sound, and thereafter he breathes from the upper part of his chest; but finally by reason of the phlegm the respiratory passage is elosed up and the patient dies. Empyema also arises whenever phlegm flows down from the head of the patient to the lungs. At first this downward flow usually escapes notice and eauses a slight cough; the sputum is a little more bitter than usual, and from time to time there appears a moderate fever. In due time, however, the lungs become irritated; they ulcerate within in consequence of the pituite, which sticks to them and beeomes purulent, and eauses a feeling of heaviness in the chest, a violent pain in the front and in the back; the body becomes hotter as the result of higher temperature, and consequently the lungs draw to themselves phlegm from the entire body, but especially from the head. The head in turn beeomes hot and attraets the phlegm from the body, and this, becoming purulent and of a thick consistency, the patient vomits up. In proportion as the time advanees, the more purulent is the quality of the matter which the patient spits up; the temperature runs up higher, the cough beeomes more frequent and violent, and a lack of appetite harasses the sick man. Finally, however, a digestive disturbance takes place in the lower bowel, a condition which appears as the result of the phlegm that goes down from the head. When it comes to this, the patient dies, as stated above, either because the lungs are purulent and decayed or because the lower part of the belly has disappeared.",

Hippocrates then continues to describe a second kind of phthisis which is caused by the bursting of a blood vessel; the blood is transformed into purulent matter, and the result is pulmonary ulceration. Finally a third kind of consumption may appear as the result of an acute pleuritis or a chronic localization of phlegm and blood in the pleura, a condition which may likewise cause pulmonary suppuration. In all of these cases the phthisis is curable, if treated betimes. 
Hippocrates, however, seemed to recognize another form of phthisis, namely that which arises through phymata, Latin tubercula. Many scholars have accepted this expression as evidence to prove an acquaintance of Hippocrates with tuberculosis. Virchow (Archiv. xxxiv, 1865) entirely rejects this explanation and draws the conclusion that phymata was used synonymously with abscesses. It is possible that an excessive enthusiasm for the medical knowledge of the ancients has tempted and induced some to read too much into the Greek word, especially since it has been regularly transferred to the Latin by tuberculum. An examination of the different passages in which phyma occurs cannot, of course, reveal any direct proof that Hippocrates understood the modern scientific theory of tuberculosis; indeed, this would certainly be too much to expect, for there was no such thing as scientific medicine viewed from the modern standpoint. Daremberg, Fuchs, Hirsch, and others have interpreted the Greek word phyma in the sense of "tubercle" and they have defended their position vigorously. Although space forbids an exhaustive discussion on the arguments advanced by both sides, it may be interesting, as well as instructive, to enumerate a few passages from Hippocrates in which he employs the word phyma.

In the "Praenotiones Coacae" Bk. I appears the statement that "those in whose lungs phymata (Latin tubercula) are formed spit up purulent matter within a period of forty days, and if they survive this time, they become consumptive in most cases." Another reference occurs in "De Morbis" Bk. I: "A phyma (Latin tuberculum) is formed in the lung in the following manner: whenever there is a gathering of phlegm or bile, it becomes foul and as long as it is yet practically unripe, it causes but slight pain and a dry cough; but as soon as it becomes mature, a severe pain arises in the front and in the back, and fever as well as violent cough attack the patient. But when the bursting, maturing, and purging have taken place very quickly, and the inside moreover cannot dry up, but the phyma gives off pus from itself, such a condition proves fatal; for the phlegm flowing down from the head and the rest of the body to the phyma becomes purulent, is transformed into pus and spewed up, as a result of which the patient dies." Another interesting passage is found in the book "De Articulis:" "And such persons (speaking of those whose vertebrae are deformed) usually have hard and unconeocted phymata (Latin tubercula) in the lungs." And farther on, "The most of such cases are associated with abscesses (or tubercles) within the spine-but these will be discussed among, the chronic pulmonary diseases." Hippocrates likewise uses the word in speaking of inflammation of the tonsils and the mouth, where it seems to have no further connotation than a pus formation or abscess. It is however, interesting to 
remark that when he mentions pulmonary ulceration as prevalent amongst consumptives, he distinguishes between phthisis caused by "empyema" and that caused by "phymata." (See De Morbis I Ch. xi Introd.) The difference may be neither a serious nor a scientific .one, but it is possible that Hippocrates may have based the use of his word upon a visual acquaintance with nodular elements found either in the lungs of animals or men. But whatever interpretation we may put upon different passages, we may safely conclude from the evidence at present available that Hippocrates neither knew nor understood any scientific medical theory of tuberculosis as that word is today explained. We may also say that the exegesis of several passages is purely a relative matter, since the Greek readings are not always uniform; hence, in the words of Horace "adhuc sub iudice lis est."

The contagious aspect of consumption does not seem to have been touched upon by Hippocrates. An interesting reference, however, occurs in the Aegineticus of Isocrates, a celebrated orator, contemporary with Hippocrates. In chapter fourteen of this work there is an allusion to a man who takes care of a consumptive that had named him his heir. "As many of my friends," he says, "as came to me, expressed their fear that I also would die, and advised me to look out for myself, asserting that most of those who have taken eare of such a disease have themselves likewise perished."

Aristotle, Problemata, Sec. vii, 8, includes consumption among the communicable diseases because it vitiates the air in the manner of a pestilence. "Why is it," he asks, "that persons are stricken when they come near to consumptives? The contagion of consumption arises for the reason that it infects the breath and makes it heavy; moreover, all diseases, which originate from a corrupt exhalation, such as are pestilential, attack us very readily. For he, who approaches, inhales air of that kind."

Allusions to consumption by Greek nonmedical authors are not of frequent occurrence, for such references would naturally be only incidental. Herodotus (484-408 ? в.c.) Hist. vii, 88, tells us the following:

"For as he was riding, a dog ran beneath the feet of the horse; and the horse, not seeing it, was frightened, reared up and threw Pharnuches, and after he had fallen, he spit blood, and the.disease turned into consumption."'

Plato (428-347 B.c.) Legg. 916A, also touches upon phthisis. He says:

"If a person sells a slave who is ill with consumption-or with some other lingering and incurable disease not noticeable to the average man, in case he is sold to a physician or trainer, there shall be no restitution."

Pausanias 10, 2, 4, (second century A.D.) interestingly describes the dream of a certain Phayllus in the following words: 
Among the votive offerings to Apollo there was an ancient bronze statue of a man whose flesh had wasted away and whose bones alone remained. It was said by the Delphians that this was a votive offering of Hippocrates, the physician. Phayllus fancied that he himself resembled this image, and forthwith consumption seized him and fulfilled the prediction of his dream."

Another very famous Greek physician was Aretaeus, of Cappadocia, who probably prasticed medicine in the first century A. D. during the time of Nero. In many respects he follows up the principles of the Father of Medicine, but his description of phthisis is even more clear and definite. According to him this disease is caused (1) by an abscess in the lungs, (2) by a chronic cough, (3) by hemoptysis. (See Bk. I, caput viii, "De causis et signis diut. morb.") In the same passage he.also makes a distinction between real phthisis and empyema, and further on in chapter ten he gives a comprehensive description of pulmonary abscesses as a result of which the lungs become ulcerous and finally phthisical. In liber ii, caput II "De causis et signis acutorum morborum" we have a further excellent description of phthisis as caused by hemoptysis, and the symptoms of this disease resultant from chronic catarrh are accurately set forth in the first named passage above. His discussions are all convincing and clear, and evince a thorough medical knowledge. What a lucid picture he portrays here:

"Those who try the fluids either with fire or water seem to me not to know consumption very well; for the sight is more to be trusted than every other sense, not only relative to that which is brought up, but also with regard to the appearance of the sick person. For if one of the people should see a man pale, weak, coughing, and emaciated, he rightly divines consumption. But those who have no ulcer in the lungs, yet have wasted away with chronic fevers, cough frequently, hard, and fruitlessly, bringing up nothing, such persons are likewise called consumptive, and that too advisedly. . . Their voice is hoarse, the neck somewhat bent, slender, not easy to control . . the nose is sharp, and slender; cheeks protruding and red; eyes hollow, glittering and bright; they are swollen, and pale, or livid in countenance. The thin parts of the jaws rest upon the teeth resembling a smile, in other respects corpse-like. So also in all other respects they are slender without flesh; it is not only easy to tell the number of their ribs, but also to know where they terminate. . . The old are not frequently afflicted, but they seldom recover; the young till the prime of life become phthisical from blood-spitting, and get well, though not readily; children continually coughing even till they become consumptive, yet recover."

Galen (130-201 A. D.), a native of Pergamus in Mysia, fully corroborates the statements of his predecessors, though his discussions on consumption are generally inferior in clearness and perspicuity to those of Hippocrates and Aretaeus. He treats of pulmonary consumption in several different places, notably in the fourth book "De locis affectis" and the three books "De difficultate respirationis," where his version contributes nothing new. The much discussed phyma of Hippocrates likewise reappears. Particularly in the fourth book "De locis affectis" and in the first 
book "De difficultate respirationis" Galen seems to supply further material for the adherents of the Hippocratic theory of tubercles by characterizing the phyma as hard and crude-Latin tuberculum coctu difficile and tuberculum crudum-a statement, which seems to confirm the Hippocrates passage in the book "De articulis," already referred to. We may safely say, however, that Galen adds absolutely nothing definite whereon either the one faction or the other can base a logical or convincing argument.

We may now seek for further basic evidence concerning consumption among Roman writers. As was the case respecting literature and art, so also in medicine the Greeks exerted a great influence over the Roman mind and left the impress of their knowledge in unmistakable terminology. The Roman word for consumption is tabes, which signifies merely a wasting away; other words used to designate consumption are phthisis, tussis sicca, syntexis, atrophia, cachexia and their various derivatives. These words are practically all of Greek origin. That fact, however, does not invalidate the evidence which we find in Roman writers.

A most interesting treasure of a medical character, that seems to be distinetly Italian, was found in $\mathbf{1 8 3 6}$ in a small lake located on Monte Falterona, a summit of the Apennines. (See G. Dennis, Cities and Cemeteries of Etruria, ii, 97). Here were brought to light bronzes of a votive character (ex-votos), probably offerings at some ancient shrine. Some have been clearly identified as being representations of persons suffering from some disease. Among others there was a frame wasted away by consumption or atrophy. Dr. Lmil Braun, the eminent archaeologist, offers certainly a novel theory to explain the presence of these ex-votos in a lake at the top of a mountain, and, fanciful though it may seem, one can yet find in it a possible solution of a curious problem. He suggests that this lake was formed by volcanic action prior to the period when the bronzes were deposited. Then the waters acquired a medicinal quality from the trees the lake contained, yielding a property which modern chemistry extracts from creosote. He calls attention to the fact that the diseases shown in the ex-rotos are just such as might be alleviated by that medicine and that a base of creosote is used by medical men in modern times to curb attacks of phthisis. Patients laboring under consumption seem especially to have frequented the lake. He points to similar lakes in China and the editor's footnote refers to several ancient examples. These bronzes may date from a very early period.

The modern medical world has been only too ready to cast aside one of the greatest medical writers of antiquity in the person of the Roman writer Celsus. Two things are chiefly responsible for this conditionthe lack of a modern English translation and the mistaken notion that his testimony seems to have no value because external proof cannot be 
adduced that he was a professional or even a practicing physician. An idle statement on the latter notion, however, would hardly suffice to convince the skeptical; a conclusion on that score can be impartially reached only by a thorough study of his works, and they everywhere give evidence of a seasoned medical knowledge. Whether physician or not, his testimony cannot be disregarded.

Celsus, who may have flourished between 30-50 A. D., wrote a superb description on consumption and its different species in Bk. 'III, Chapter xxii of his De Medicina. "The tabes (consumption)," he says, "often keeps those, whom it attacks, longer in a state of continued danger. And of this also there are several species. One of them occurs when the body is not nourished, and, since something is naturally ever leaving it, while nothing succeeds in its place, a most extreme emaciation takes place; and, unless an attempt is made to cure it, death ensues. This the Greeks call atrophia. It is wont to happen from two causes. For either from excessive fear a person takes less, or from excessive desire he takes more than he ought to; thus either too little weakens or too much vitiates. There is a second species, which the Greeks call cachexia, where the condition of the body is bad; and on this account all of the nutriment becomes putrid. *** Besides the consumption it sometimes also happens that the surface of the skin is made rough and irritated by frequent pimples or ulcers, or some parts of the body swell. The third, and by far the most dangerous is that species which the Greeks call phthisis. It generally starts from the head; thence it trickles down into the lungs; then follows an exulceration, and as a result there occurs a slight fever; and even though this subsides, nevertheless it returns; there is also a frequent cough; the patient spits out pus, and sometimes bloody matter. Whatever the patient spits out by coughing, has a bad smell, if placed upon a fire; and so those who are not sure of the disease, use this means of identification." The remainder of the chapter is taken up with remedial suggestions, foremost among which Celsus advocates change of climate, sleep and rest, milk and various palliatives. The disease generally comes on at the strongest age, that is, from the eighteenth to the thirtyfifth years.

In the Natural History of Pliny, the Elder (23-79 A. D.), we find many interesting discussions on curative medicine, and among other diseases consumption receives some attention. Alica, he tells us in Book XXII, 25,61 , is distinctly a Roman remedy which is especially useful when there is a tendency to consumption after a long illness. It should be used in a proportion of three cyathi to one sextarius of water. This mixture is to be boiled till all the water has evaporated, after which one sextarius of sheep's or goats' milk is to be added. Pliny prescribes 
its daily use, and suggests that honey be added later. According to Galen, however, alica was known in the days of Hippocrates, for the Greek used the word chondros to signify the same spelt-grits. Another highly prized remedy for pulmonary diseases and cases of incipient phthisis is the root of consiligo, which was also used for a like affection among swine and cattle by passing it through the ear of the animal. Pliny also recommends change of climate and more particularly the benefit of a sea-voyage. Milk is very useful for all kinds of internal ulcerations. In Books XXV, 53, and XXVIII, 9, 33, he says that in Arcadia cows' milk is prescribed for phthisis, consumption, and cachexy (Latin phthisici, syntectici, eachectae). By far the most interesting cure is mentioned in Book XXVIII, 17, 67:

"Consumptives are cured by a wolf's liver boiled in thin wine, the bacon of a sow fed upon herbs, and the flesh of a she-ass taken with broth. In this manner especially the people in Achaia effect the eure of this malady. It is said, too, that the smoke of dried dung (that from eattle which feed upon green fodder) is benefieial for phthisis, if inhaled through a reed"-Bostock and Riley suggest that this is an instanee of smoking, though not a tempting one-"'and likewise the tips of eows' horns, when burned, with an admixture of honey in a proportion of two spoonfuls, the whole being swallowed in the form of pills. Not a few people say that goat suet in a pottage of aliea or freshly melted with honey-wine in the proportion of an ounce to a eyathus, the mixture being stirred with a twig of rue, eures both phthisis and cough. An author of standing assures us that a patient whose condition seemed hopeless, regained his health by taking one cyathus of wild goat suet with a like quantity of milk. Other writers have said that the ashes of swine-dung in raisin-wine, and the lung of a deer, especially of a hart, dried in smoke and beaten up in wine, are helpful.'"

What a novel preseription! Pliny enumerates other cures for consumption, and in his discussions employs the nomenclature mentioned above. The tabes is also mentioned among the diseases of trees, and its close resemblance to the human species is well defined in the following (Bk. XVII, $24,37)$ :

"Undique primo dolor, mox et macies earum partium fragilis, postremo tabes morsque, non intrante suco aut non perveniente, maxumeque id fici sentiunt."

"At first the tree suffers pain everywhere, then its parts become emaciated and brittle, and finally consumption and death follow, for no sap ean enter nor yet go through the parts. Fig-trees especially are thus affected."

In Book XXVIII, 8, 24, we read that "elephanti sanguis et syntecticis prodest iecurque comitialibus morbis."

"The blood and liver of elephants are beneficial to those who suffer from consumption and epilepsy."

Subsequent Latin medical writers merely confirm the evidence which has been presented. We may mention Theodorus Priscianus (fl. 400 A.D.) and Caelius Aurelianus (fl. ? 420 A.D. ), both of whom drew at 
least some of their material from Galen and Soranus (Greek physicians, second century A.D.). The former (Bk. II, Ch. XXI) seems to regard phthisis as so well known that he concerns himself mainly with the symptomatology of the disease, which consists in a characteristic fever that appears especially towards evening, and sometimes as a quotidian and then again as intermittent. Cough and consumption of the body are an invariable concomitant, and if this condition becomes chronic, convalescence is hopeless; the physician must then content himself with an effort to console the patient in the manner of a friend. It may not be altogether amiss to remark that the latter suggestion is decidedly worthy of emulation.

In similar manner the semiotic description of Aurelianus is singularly complete (De morbis chronicis, Bk. II, 14). The origin of the malady he ascribes to several causes, such as hemoptysis, chronic cough, and pulmonary ulceration. It is interesting to note that he states, "corporis tenuitas sequitur, quae nudatis membris proditur magis, quam ex aspectu vultus" (a wasting away of the body comes on which is more apparent when the limbs are uncovered, than from the appearance of the face). Atrophy of the lungs without cough and empyema he differentiates from phthisis.

Latin nonmedical literature provides further interesting material. Horace (65-8 B.c.) S. 1, 9, 32, refers to consumption as a "cough" (tussis) and mentions it in one breath with pleurisy. Vitruvius (fl. B.c. 14) includes cough, pleuritis, and phthisis among the diseases that are curable only by invigorating remedies, and hence proper climatic and atmospheric conditions must be sought where the air is uniform, calm, sufficiently dense and not subject to frequent draughts and changes.

Seneca (4 B.c.-65 A.D.), the great Stoic plilosopher, was deeply interested in things medical because he was beset by constant tendencies to consumption; he was a veritable "Schwindsuchtskandidat" if we may believe Dio Cassius (59-19): “After Gaius had ordered him to be put to death, he let him go because he believed one of his concubines who said that he (Seneca) was ill with consumption and would die ere long." He himself also testifies (Ep. 50, 10) that during his youth he had been subject.to frequent catarrhal affections, which, through lack of attention, became chronic and, as a result, he became later on emaciated. This experience he expresses more generally in Ep. 75, 12, where he regards phthisis as the inevitable result of a chronic cough that is of long duration or standing. "The most temperate," he says in Ep. 90, 5, "are attacked by disease, the strongest by phthisis."

Gellius (fl. second century A.D.) 4, 24, designates consumption as a morbus of the whole body. Martial (c. 43-104 A.D.) XI, 21, 7, refers 
to a consumptive (phthisico) wench; and Juvenal (54-134 A.D.) XIII, 95 , with stinging satire suggests that some men will bear any punishments of the gods if they can but keep their ill-acquired gain, even though they may incur consumption (phthisis) and ulcerous sores. Vegetius (fl. fifth century A.D.) Vet. 5, 54, and Columella (fl. 50 A.D.) R.R. $6,14,1 ; 7,5,14$, both tell of the symptoms and cure of consumption among cattle, sheep, and swine. The latter clearly refers to pulmonary consumption when he writes:

"Ulceration of the lungs is also very baneful; thereafter cough and emaciation come on and finally phthisis attacks them.",

Apollinaris Sidonius, a Christian writer of the fifth century, Bk. V, Ep. 14, speaks of Baiae as a place where "the baths are beneficial for those who are consumptive (phthisiscentibus) and suffer in the liver."

This chain of evidence may be safely interpreted in certain specific and well-defined conclusions: First, that consumption was one of the common diseases among the Greeks and Romans; second, that the delineation of its ravages by both Greek and Roman writers, as well as the conspicuous attention which is bestowed upon it in a general descriptive way, bears testimony to a prevalence at least proportionately as great as in our day, and all the more so when we consider the fact that proper prophylactic measures were not understood; third, that the contagious aspect of consumption was probably observed but not understood; fourth, that not only human but also bovine consumption was known; fifth, that the Romans probably gained their knowledge of the disease from the Greeks; sixth, that the etiology, symptomatology, prognosis, diagnosis, and therapeutics of consumption were well understood; seventh, that tuberculosis as the modern physician knows it was not an established scientific fact, but that the ancient consumption must have been the result produced by the same phenomena.

Petersen has shown that pulmonary tuberculosis was well known to Hindoo physicians many centuries before our era. They also apparently were able to distinguish between early and advanced stages, because they undertook to treat the former but declared the latter hopeless. The treatment of incipient cases was very similar to that employed today and it consisted in feeding with fats, especially butter fats obtained from the milk of cows, goats, sheep, mares, and elephants. Apparently, the consumptive was allowed to eat meat, and the flesh of asses, mules, horses, camels, elephants, wild-cats, rabbits, and birds, was recommended. These meats were boiled in oil, accompanied with peas and other vegetables. Even wine was allowed the consumptive. It appears that the Hindoo physician permitted his consumptive patients to have flesh and wine, although these articles of food were forbidden by the legal code of the 
Hindoos. The period of the later Roman and Byzantine Empires added nothing to the knowledge of the ancients on this subject.

During the seventeenth and eighteenth centuries there was much discussion concerning tuberculosis, but no experimental investigation into its nature. The term "tubercle" was first employed by the anatomists of the seventeenth century and referred to nodules of various sizes in diverse tissue. It was first applied to pulmonary tuberculosis by Sylvius (1614-1672), who believed that the nodules in the lungs consisted of glandular tissue breaking down and becoming ulcerous by suppuration. The glandular nature of pulmonary tuberculosis was denied by Reid (1785) and by Baillie (1793). The former taught that tubercles had their

TUBERCULOSIS (all forms) IN THE LARGEST CITIES OF THE U.S.

(1910 RANKING)

AVERAGE ANNUALDEATH RATE PER 100,000 FOR THE YEARS 1911-17.

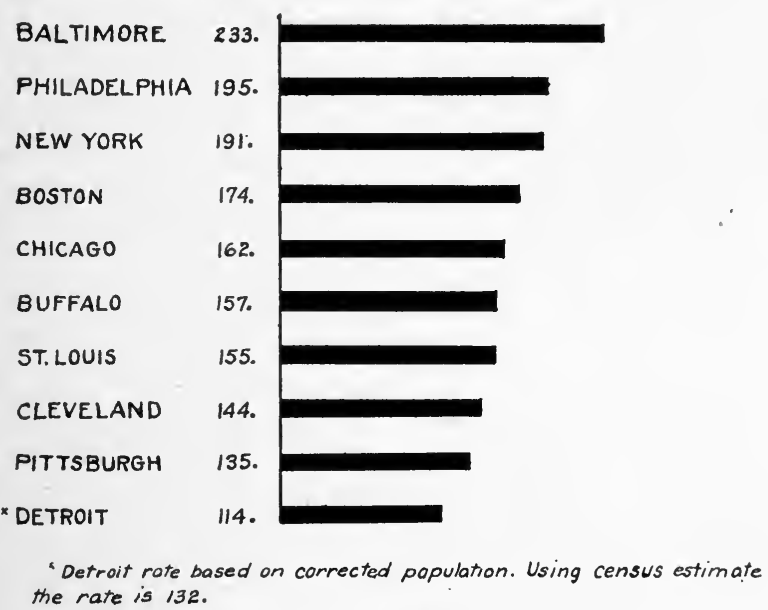

Fig. 61.

origin in the thickening of lymphatic tissue, while the latter attributed them to proliferation in the connective tissue. It is interesting to note that Bayle (1774-1816) wrote a book in which he claimed that pulmonary phthisis is not a local disease, but the result of a general infection. He was the first author to describe and name miliary tuberculosis.

Laennec (1781-1826), who did so much to develop auscultation as an aid to diagnosis in diseases of the lungs and heart, taught the unity of tuberculosis. He demonstrated that the anatomic lesions might consist of sharply defined, isolated nodules or of a tuberculous infiltration. In both there is at first a grey, transparent, new formation, which gradually becomes opaque, yellow, and then softens. He pointed out the resemblance between the softened matter and cheese. From this, the term caseation came into use. 
Beginning with the middle of the nineteenth century, Virchow became a great authority in cellular pathology. He developed this new subject with so much originality and vigor that his dictum for a while became law throughout the medical world. He richly deserved this honor, but, unfortunately, his views on tuberculosis did much to offset the teachings of Laennec, and for a short time retarded the progress of our knowledge in this matter. He combated the unity of this disease and attempted to make a sharp distinction between true tuberculosis and those processes in which caseation is, according to his teaching, due to inflammatory and hyperplastic processes. The result of this teaching was that tuberculosis came to be regarded as a progressive process, in which small nodular growths were formed, and which only exceptionally terminated otherwise than in death. Nicmeyer founded his clinical teaching upon Virchow's pathology, and in doing so he employed statements more forcible in diction than true in fact. "'The end of tubereulosis, as a rule, is death," is one of these statements which for many years determined the attitude of the medical profession towards the disease. "The greatest misfortune that can happen to one with phthisis is that he should become tubercular" had its effect upon medical teachers and practitioners for many years after scientific proof of the unity of tuberculosis had been firmly established.

Villemin (1865) established once for all the unity of tubereulosis and its transmissibility from one animal to another; indeed, Villemin should be regarded as the founder of modern knowledge conecrning tuberculosis. He inoculated many and varied animals in diverse ways with the sputum and other products from tuberculous lesions in man and eattle, and in all tuberculosis developed. He established not only the unity of various tuberculous manifestations in man, but demonstrated its existence in other animals. He went further and showed that certain nodular diseases which had been confounded with tuberculosis were distinct from it. $\mathrm{He}$ proved that the inoculation of animals with other than tuberculous products did not lead to the development of this disease. He overthrew the theory of heredity in this disease and established the fact that it does not result from colds or other ills. He went so far as to suggest house infection as an important factor in the dissemination of tuberculosis. $\mathrm{He}$ was the first to indicate differences between the human and the bovine species of the bacillus. He noticed that rabbits inoculated with human tuberculous material were not so quickly nor so generally affected as when the inoculation was made with material from the cow. He stated:

"At first we were inclined to regard this as accidental, but subsequent experiment led us to conclude that the tubercle from the cow inoculated into rabbits possesses a much greater activity than that obtained from man. It may be supposed that like all 
virulent matter, tuberculous material is the more virulent the more closely related are the animals supplying and those receiving the virus."

This statement seems to have been overlooked or forgotten until Nocard and Roux observed similar results in their experiments. It remained, however, for Theobald Smith (1898) to establish morphologic, cultural, and pathogenic differences between the two species of tuberculosis bacilli. We wish to emphasize the fact that Villemin insisted that the home is the place in which there is greatest opportunity for infection.

Quite naturally, Villemin's results stimulated much work along these lines. All kinds of foreign materials were injected into guinea pigs and other animals, and the lesions following such injections were closely studied. At first many claimed that any foreign body thus introduced might lead to the development of tuberculosis. More exact experiments, however, hushed all criticism and the findings of Villemin have stood the test of time.

In 1882 Robert Koch, after long and patient study, succeeded in isolating from tuberculous material the specific bacillus, which he grew in pure culture and demonstrated its pathogenicity. That there is no tuberculosis without this specific organism has long since been established as a truism. The finding of the specific bacillus was an essential step in the progress of the study of the disease, but at first and, indeed, for many years, it led us quite astray. It was believed, quite naturally, that whenever any one became infected with this organism he was sure to develop the disease. Again it was found that we had overlooked some investigations which had been made many years before, or had at least failed to appreciate their importance. As early as 1831 Lombard and Papavoine, making autopsies in the Children's Hospital at Paris, had pointed out the large number of children bearing this infection, many of whom had not developed the disease. In the examination of 910 children, these investigators found that about three-fifths of the bodies examined were tuberculous. Some ten years later, Chambers, of London, found tuberculous lesions in 26.1 per cent of 2,046 autopsies. However, the wide prevalence of tuberculous infection did not attract attention until 1900, when Naegeli reported that in 500 autopsies made at the Pathological Institute in Zurich he found seventy-one per cent showed tuberculous lesions. In individuals under 18 years of age only twenty-five per cent showed such lesions, but in older people the percentage of infection was found to reach as high as ninety-eight. This report upset the ideas of pathologists, and in different parts of the world examinations with the purpose of detecting tuberculous lesions at autopsy have been made. These investigations have shown that, while the percentage of tuberculous infection differs somewhat according to density of population, it is now safe to say that among civilized people who have reached adult 
life from fifty to one hundred per cent have been infected at some time and show tuberculous lesions at autopsy, whatever the cause of death may have been. Furthermore, these studies have shown that tuberculosis at the time of birth, or congenital tuberculosis, is exceedingly rare. Practically no child is born into the world infected with this bacillus. The few cases of congenital tuberculosis found are not enough to detract in any material way from the truth of this statement. With increasing age the percentage of infection grows, and practically every one who dies after reaching adult life has more or less tuberculous infection at the end, it matters not how robust and free from actual disease the individual may have been. For a while the claim was made that many of the small and healed lesions found after death were due either to infection with highly attenuated cultures of the tuberculosis bacillus or possibly by some closely related organism possessed of no pathogenic properties. However, inoculations of guinea pigs with the organisms found in these small and healed lesions disproved this supposition. About 1891 Koch prepared his first tuberculin and found that small doses of this substance produced widely different effects in tuberculous and nontuberculous animals. In the former, such infections were followed by a marked elevation of temperature accompanied by local reaction in the tuberculous area. In nontuberculous animals, similar injections had no effect upon the temperature. At first Koch believed that he had found a specific eure for this disease in the products of the bacillus itself. This hope proved without foundation, but tubereulin furnishes a most reliable agent for the detection of tuberculous infection in man and the lower animals. With this agent we have a means of determining the frequency of tuberculous infection in the living, and it is no longer necessary to await autopsy to find out whether specific infection exists or not. Using tuberculin, Pollak found that the children of tuberculous parents in Vienna were infected to the extent of ninety-six per cent before reaching the fourth year. In New York, Fishberg found that eighty-four per cent of children living with tuberculous parents are infected before reaching the fourteenth year. Calmette, testing 1,226 persons of all ages from all social conditions and all apparently healthy, found that during the first year of life only nine per cent were infected, but at the age of fifteen the infection involved more than eighty-seven per cent.

The Pirquet modification of the tuberculin test has given a ready, safe, and reliable method of determining the presence of tuberculous infection, and its use has confirmed the older findings by autopsy and by the use of the tuberculin of Koch. The universality of tuberculous infection, especially in crowded cities, is apparently no longer a matter of dispute. The findings are so constant and uniform that they must be accepted. 
It is impossible to foretell what observations and researches in the future may bring forth, but at present (1921) all epidemiologic studies and all attempts to eradicate tubereulosis must be made with the understanding that the following are fundamental, demonstrable facts:

Without the specific bacillus there is no tuberculosis. (2) Children are born free of this disease. (3) Tuberculous infection increases with age and when adult life is reached between fifty and one hundred per cent of the population in eivilized countries carry this infection. (4) Tuberculous infection in the great majority of individuals is not followed by tuberculous disease.

The Bacillus.-The specific causal agent of tuberculosis is a rod-like organism, whose length varies from one-fourth to one-half the diameter of a red blood corpuscle (1.5 to 4.12 microns). Its diameter is about one-fifth its length. It was formerly believed that this, like other bacteria, should be classed among microscopic plants, but the most exact chemical studies have failed to show the presence of cellulose in their structure. This organism does not take the ordinary basic anilin stains readily, but when heated with certain mordant containing stains, such as earbolfuchsin, it takes on and retains the color even after washing with dilute mineral acid. For this reason it is known as an "acid-fast" bacillus, and it has been found that this group contains a number of microorganisms whose relationship is a matter of great interest. By special staining the tubercle bacilli are easily detected in sputum and other excretions from tuberculous lesions. In sputum it is most frequently found in clumps in which the bacilli lie side by side. Occasionally two rods form an angle like the letter "V." For some time there was doubt whether or not this bacillus forms spores. The low temperature at which it is destroyed and the fact that it is quickly killed by sunlight, renders spore formation highly improbable. In old cultures, branched forms are occasionally seen. It is nonmotile, possesses no flagella, and forms no capsule, but is inclosed in a waxy coating which gives it unusual resistance to desiccation and to chemical disinfectants. When the fats and waxes are thoroughly removed by prolonged extraction with alcohol and ether, the bacilli take the basic anilin stains readily and are no longer acid-fast. Young bacilli before they have accumulated their waxy protection are stained more easily and are not acid-fast. The mature organisms are not only acid-fast, but are also alcohol- and alkali-fast. Occasionally rods are seen to consist of nodules which stain more deeply than the intervening spaces, thus giving the rod a beaded appearance and causing it to resemble a chain of micrococei. Such forms may be seen in old artificial growths and more rarely in the sputum.

Sometimes, especially in old cavities, in lupus lesions, in cold abscesses, and in fibroid pulmonary tuberculosis, tubercle bacilli cannot be demon- 
strated by the ordinary staining method. Instead of rods one finds granules. Frequently these are arranged so as to appear as rods made up of granules. It is more than probable that this is a further development of the beaded form. It was customary a few years ago to say that pus from such tubereulous eavities and from the other sourees mentioned above is sterile, but if some of this granular material be injected into a guinea pig tuberculosis develops and in the tuberculous areas and tissues of the pig normal tubercle bacilli are found. These are known as the granules of Much, who first described them. There has been some controversy as to the nature of these granules, and this problem has not yet reached a satisfaetory conclusion. According to Meader, they may be regarded as the results of a stage, either in the evolution or in the degeneration of the bacillus. In our opinion, they are more probably degenerative forms. Some have suggested that they are spores, but the fact that they are less resistant than the baeilli to ehemical agents renders this supposition highly doubtful.

It is difficult to make artificial cultures directly from sputum or from tuberculous tissues. This difficulty is due to the more ready growth of other bacteria usually accompanying the tuberele bacillus in these materials and to the relative slowness with which the tubercle bacillus adapts itself to the artificial medium. The method usually adopted in seeuring artificial cultures is to inoculate guinea pigs intra-abdominally with tubereulous sputum and await the development of the disease in the organs of this animal. The accompanying saprophytic bacteria have been destroyed in the guinea pig and the tuberculous tissue of that animal contains only the specific bacillus. The animal is posted and some of the tissue removed aseptically and distributed over the slanted surface of Smith's dog serum or Dorset's egg medium. Preparations thus made and placed in the ineubator at $37^{\circ} \mathrm{C}$. generally show an initial growth after about ten days and a plainly characteristic development may be expected within four or five weeks. The preparation of subcultures on dog serum or egg medium is comparatively easy, and as the bacillus is grown generation after generation in this way its development on artificial media becomes more rapid and vigorous. It was found early in the study of this bacillus that the addition of from five to six per cent of glycerol to the culture medium hastens the growth of the human tubercle bacillus. It is said that the addition of this substance to media retards the growth of the bovine bacillus. To obtain massive quantities of tubercle bacilli it is best to grow them on glycerol broth. A sterilized cork is floated on the broth and a small bit of bacillary growth placed on the cork. The bacilli multiply, cover the surface of the medium, and, as the growth matures, floccules drop from the film and float through the subjacent fluid or rest upon the bottom. From growths of this kind, after 
from six weeks to three months, massive quantities of bacillary substance may be obtained by filtration through sterilized paper. The liquid part of the medium passes through perfectly clear and all the bacilli are collected upon the paper, where they may be washed with alcohol, dried, and kept indefinitely. A study of the food upon which these bacilli thrive is a matter of great importance. It has been shown, especially by Kendall, Day, and Walker, that they may grow in media whose only nitrogen content is in the form of ammonium salts. Even upon this medium the bacillus develops normally, but slowly, and builds up its characteristic and specific tissue.

The optimum temperature for the growth of the bacillus is that of the human body, from $37^{\circ}$ to $38^{\circ} \mathrm{C}$. However, its range of possible growth lies between $30^{\circ}$ and $41^{\circ} \mathrm{C}$. Low temperatures do not kill it. Milk containing these bacilli may be kept frozen quite indefinitely, but when thawed the organisms will be found without change in structure or in virulence. Moist heat at $50^{\circ} \mathrm{C}$. must be continued for ten or twelve hours to kill these organisms. At $55^{\circ} \mathrm{C}$. four hours are necessary; at $60^{\circ} \mathrm{C}$. one hour; at $65^{\circ}$ fifteen minutes; at $70^{\circ}$ ten minutes; at $80^{\circ}$ five minutes; at $90^{\circ}$ two minutes; at $95^{\circ}$ one minute. It should be understood, however, that the temperature required to kill these organisms depends largely upon the medium in which they are found. It is of special interest to know the temperature to which milk should be heated to destroy tubercle bacilli. Many experiments have been made on this point, and it has been found that much depends upon whether the milk is heated in an open or a closed vessel. In an open vessel a pellicle forms on the surface and thus protects entangled bacilli. However, Park has shown that the upper part of the fluid is not heated to the same degree as the lower portion. It is quite important that the pasteurization of milk should be done in closed vessels and that a temperature of not less than $60^{\circ} \mathrm{C}$. be maintained for 30 minutes. Fairly clean milk containing no lumps of foreign matter pasteurized in this way can be considered safe. Centrifugalization preceding pasteurization makes a cleaner milk and adds to the certainty of the destruction of tubercle bacilli. The question is asked whether the new method of powdering milk destroys tubercle bacilli. We know of no experiments on this point, but since the ferments in the milk are not destroyed we are inclined to doubt the destruction of all bacilli. Tuberculous sputum and all infected discharges should be received into receptacles which can be burned. The bacilli are much more resistant to dry heat and in this condition they will bear a temperature of $100^{\circ} \mathrm{C}$. for an hour.

Tubercle bacilli are highly resistant to the ordinarily employed chemical disinfectants. They are not destroyed, with certainty at least, within 24 hours by absolute alcohol, mercuric chlorid (1 to 500), carbolic 
acid (five per cent), formalin (five per cent), and cresol (ten per cent). In short, it is quite dangerous to rely upon the chemical disinfection of tuberculous sputum and other infected excretions unless a strong alkali or acid be used and the exposure to the disinfectant be continued for many hours. The stools of individuals suffering from chronic pulmonary tuberculosis should be disinfected with chlorin water or should be buried.

The effect of desiccation and of exposure to sunlight upon tuberculous discharges is a matter of the greatest importance to epidemiologists. Desiccation in the absence of sunlight is not to be relied upon, since it has been shown that tuberculous sputum spread upon glass and kept at room temperature may remain virulent for one year. Stone found tubercle bacilli in sputum which had been standing for three years. He had no difficulty in finding the bacilli. He inoculated this material into rabbits and concluded from the results secured that the organisms were alive and virulent. Burns examined tuberculous sputum which had stood in corked wide-mouthed bottles for 22 years and found the bacilli normal in appearance and in staining properties, but without effect upon guinea pigs. An important question is how long will these organisms live in dust. The answer to this depends upon many conditions. They have been found alive and virulent in pulverized sputum for from four to seven days, in finely divided eloth fibers from five to ten days, and in street dust from three to eight days. The length of life in dust depends upon the fineness of the particles and the exposure to light. Direct sunlight, even for a few minutes, kills the tubercle bacillus. However, the rapidity and thoroughness of this action is dependent upon the surface exposure and the intensity of the light. Artificial cultures exposed to diffuse daylight near windows survive from five to seven days. On the other hand, sputum deposited upon linen, dried, and exposed to diffuse daylight may retain its virulence for a month or longer. It seems that direct sunlight decomposes the waxy and fatty substances in the bacilli and kills them within an hour or two. Again, this depends upon the physical composition of the sputum. Abundant mucus and lumpy material protect the contained bacilli. It is quite evident from what has been said that it is of importance to enforce the anti-spitting regulation and not depend upon the uncertain destruction of the organisms by either desiccation or sunlight.

The influence of putrefaction upon the viability of the tubercle bacillus is a matter of importance. The undisinfected stools of thousands of advanced cases of consumption are being thrown every day into our sewers. Many of these sewers discharge into water-supplies. Brown and his associates found virulent tubercle bacilli in every sample of water taken from below the surface in the Saranac River from the outlet of the sewer to a distance of three and one-half miles down the stream. No 
such organisms were found in the river above the outlet and none were found twelve miles below the sewer. Saranac is a village occupied largely by consumptives and their feces undisinfected are thrown into the sewer. Brown concludes that the presence of the acid-fast bacilli in the water of the river was due to infected feces; indeed, where he found the bacilli he also found particles of feces. It has been found that the putrefaction of tuberculous discharges does not destroy tubercle bacilli with any promptness. Highly virulent organisms, as well as in some cases attenuated forms, have been found in sewage six months and longer after they were placed therein; indeed, virulent tubercle bacilli have been reported in the sewage used to irrigate a field in which vegetables were grown to supply a tuberculosis sanatorium.

The cadavers of men or animals may contain living virulent bacilli for months and possibly for a year or more. When the body of one dead from this disease is properly prepared for the grave and encased in a wooden or metallic coffin there is no danger of the spread of the disease from this source. However, proper precaution is not always made, especially in bodies which have been autopsied. Occasionally one sees blood and other fluids from the dead body soaking through the coffin and soiling the outside. We see consumptives in every stage of the disease on nearly every train on which we travel. The educated among these move among their fellow-men taking proper precautions, while the ignorant and careless deposit their infected sputum in all public conveyances. The dangers of spreading the infection from the last mentioned are incomparably greater than those that arise from the bodies of those who die from the disease.

The Chemistry of Tubercle Bacilli.-There is no evidence from any source, so far as we know, that this bacillus produces either exo- or endotoxin, using the word toxin as it is generally understood. The word "toxin" has come to have a definite meaning and includes all those poisons which, when introduced into animals in nonlethal and increasing doses, cause the body cells to elaborate antitoxins.

Koch's work on tuberculin gave an impetus to the study of the chemistry of this organism. He took old glycerol bouillon cultures, evaporated them to about one-tenth the original volume, and removed from this concentrated fluid any remaining unbroken, undisintegrated bacilli by filtration. The filtrate containing a large percentage of glycerol is stable and may be kept and used indefinitely. It is evident that this preparation contains the soluble products which have come not only from the tubercle bacilli, but from other constituents of the culture medium. It in no sense is anything like a pure extract of the cellular substance of the tubercle bacillus. It contains all those substances which have passed into solution during the growth of the organism in the medium; also any 
substances that have been extracted from the cellular substance by the processes used. Moreover, it contains disintegrated and split products from the peptone and from the material used in the preparation of the bouillon. Following this method a great number of so-ealled tuberculins have been prepared, and it is no wonder that most diverse results have been obtained by the use of these preparations. Patients who have been sensitized by these products are found to respond to injections of various proteins. In 1908 Vaughan and Wheeler made the first report on their chemical studies of the cellular substance of the tubercle bacillus and of the filtrate obtained from old bouillon cultures. The organism used was one which had been grown in artificial culture media for many years and had lost all pathogenieity, even for guinea pigs. This strain was grown in glyeerol bouillon for from one to six months, after which the cellular substance was easily separated from the fluid portion by filtration. The cell substance was thoroughly extracted with alcohol and ether to remove fats and waxes. Next it was rubbed up in mortars and passed through fine mesh sieves furnishing a powdered bacillary substance. On microscopic examination this was seen to consist of bacilli which were readily stained with earbolfuchsin, but were no longer acidfast. This cellular substance was broken up with a two per cent solution of sodium hydroxid in absolute alcohol at $78^{\circ} \mathrm{C}$. This treatment splits the cell substance into a soluble and insoluble portion. The former is designated as the "cell poison" and the latter as the "cell residue." The cell poison kills guinea pigs with exactly the same symptoms as are induced by similar poisons obtained from other proteins; in other words, the tuberculo-poison produces the symptoms and postmortem appearances seen in guinea pigs after anaphylactic shock. White and Avery prepared this poison in the same manner and found that a certain degree of tolerance could be established. This is in harmony with the findings of Vaughan and Wheeler in their experiments with the protein poison from other sourees. The last mentioned investigators found that the administration of this poison to tuberculous animals intensified the tuberculous processes and that in all cases the treated animals died before the controls. When given to healthy animals in small repeated doses it has no visible effect; in larger repeated doses it causes a chronic intoxieation characterized by loss of flesh and general marasmus. Howes used a tubereulo-poison prepared by Vaughan's method, but obtained from tuberculous sputum. With this preparation he tried immunizing guinea pigs by treatment with repeated doses of the cell poison and subsequent inoculation. In other animals he inoculated first with virulent tubercle bacilli and then tried the effects of treatment with repeated doses of the eell poison. His conclusion is stated as follows: 
"The resistance in pigs 15,10 , and 17 , to tubercle bacilli was apparently increased by immunizing doses of cell poison. In pigs 9 and 13 cell poison was discontinued too early to establish any degree of immunity.',

His conclusion concerning the treatment of previously inoculated guinea pigs is as follows:

"At autopsy pigs, 5, 6, 4, 3, 2, and 1, showed fewer pathologic findings than did control pigs 8 and 19 in receiving treatment, suggesting the possible beneficial action of cell poison in supporting the combating forces. Cell poison in small repeated doses and in large doses had similar deleterious effects upon control pigs. The results of these experiments warrant a more thorough investigation, especially of the therapeutic value of cell poison obtained from sputum."

Vaughan and Wheeler found that the cell residue or nonpoisonous portion of the cellular substance of the bacillus is without recognizable effect upon the health of animals, either in single or repeated doses, large or small. They did find, however, that this substance sensitized guinea pigs to subsequent injections with either virulent or avirulent cultures, dead or alive; in other words, treatment with the cell residue produced much the same condition as has been observed in tuberculous animals on attempts to reinfect or superinfect them. The cell residue was used by Vaughan, Jr., in the treatment of several hundred cases of tuberculosis and he came to the conclusion that in advanced cases it did no good and was probably harmful, while in early cases it apparently was beneficial. That it did induce symptoms of protein sensitization in human beings with tuberculosis there could be no doubt. This product certainly deserves further study. The method of splitting the tubercle cell substance into poisonous and nonpoisonous parts should be varied. It seems quite evident at present that in neither of these cleavage products have we found any specific cure for tuberculosis. Very recently attempts have been made in England to produce detoxicated vaccines. Thomson has reported favorably on such vaccines which he has employed in the treatment and prevention of certain acute respiratory diseases, and his method of detoxicating the bacteria is the same as that used by Vaughan and Wheeler. Still more recently the use of detoxicated tuberculin has been reported.

Agnew hydrolyzed the cell residue and found that it contained 4.59 per cent of nitrogen and yielded the following amino-acids: glutamic acid, alanin, valin, leucin, and phenylalanin; while the cell poison contains 9.89 per cent of nitrogen and yields alanin, valin, and phenylalanin, but no glutamic acid or leucin, thus indicating quite a radical difference in the chemical composition of the two portions of the cellular substance of the tubercle bacillus.

We shall not go into the chemistry of the filtrate obtained after the removal of the cell substance by filtration of the cultures, for the reason 
that we are interested at present only in the chemistry of the tubercle bacillus. It seems probable that the acid-fast characteristic of this bacillus is due to the presence of higher alcohols which are constituents of the fats and waxes.

The Bovine Bacillus.-These organisms are shorter and thicker than the human variety; indeed, certain strains are so short that they may be mistaken for cocei. They take the carbolfuchsin stain more readily and heat is not necessary. The addition of glycerol to the culture medium does not hasten, but apparently retards their growth. Granulation and the beaded formations are more common than in the human bacillus. They are cultivated with more difficulty, but grow fairly well on serum and egg medium. They are pathogenic not only to cattle, but to guinea pigs, rabbits, sheep, goats, hogs, dogs, cats, rats, and mice. According to Smith, one millionth of a milligram injected under the skin of a guinea pig kills, while one-tenth of a milligram of the human bacillus is necessary to produce the same result. Rabbits, which are quite resistant to the human bacillus, die within from 17 to 21 days after intravenous injection of the bovine variety. The lungs are found to be filled with small grey tubercles, rich in bacilli. Small nodules abound in the spleen, liver, and kidneys. It is said that the application of a culture of the bovine bacillus to the shaved abdomen of a rabbit is sufficient to induce general tuberculosis. Cattle are highly susceptible to this organism, while they are practically immune to the human bacillus. After intravenous injection cows die within from three to four weeks with generalized tuberculosis. After inhalation of this organism easeous pneumonia results. Feeding with infected material causes intestinal tuberculosis with extension to the lymph glands and abdominal organs, and in most instances the lungs are involved. After subcutaneous injection there is a local swelling followed by infection of the regional glands. Finally generalized tuberculosis follows and the animal dies within a few months. Practically all the tuberculosis of our domestic animals is due to infection with the bovine bacillus, with the exception that chickens, and possibly other domestic fowl, owe their infection to the avian variety. It is safe to say that tuberculosis in our domestic mammals is due largely to the bovine bacillus.

Having ascertained that our domestic animals do not run great risks in being infected with the human variety, it is well to inquire what danger there is to man from infection with the bovine variety. Is it wise to follow the advice of Koch and neglect milk as a factor in the causation of tuberculosis in man? This important question has been investigated in this country by Park and Krumwiede, by an English Commission, by two German Commissions, and by many individuals in various parts of the world. In 1,441 deaths from tuberculosis in man the variety of the 
bacillus found in the body has been determined. In 117 of these 8.1 per cent) the bovine variety alone has been found. In 7 cases $(0.5$ per cent) the infection has been found to be a mixed one, both varieties being present.

In pulmonary tuberculosis in adults the bovine variety is rare (4 cases out of 732). In tuberculosis of the bones and joints, at all ages, the bovine variety occurs more frequently ( 5 cases out of 98 ). In meningeal tuberculosis, at all ages, the frequency is still greater (3 cases out of 32 ). In general tuberculosis, at all ages, there is a further increase (33 cases out of 172). In tuberculosis of the glands of the neck, the proportion is still higher (45 cases out of 157). In tuberculosis of the abdominal organs, the presence of the bovine variety reaches its highest point (30 cases out of 99 ).

When we study the proportion of the two varieties in adults and in children we get more practical information. In children 23.8 per cent of generalized tuberculosis, 40 per cent of tuberculosis of the cervical glands, and 49 per cent of tuberculosis of the abdominal organs, are due to infection with the bovine variety. It is quite natural that the greater number of cases of bovine tuberculosis should be found among children. These figures demonstrate that in childhood a considerable proportion of infection comes from milk. At the same time they show that the chief source of infection in man is the human variety and that, as a rule, tuberculosis is transferred from man to man.

In 1917 Griffith summarized the bacteriologic findings of the British Commission up to that time. He states that the main objects were as follows: (1) To determine by examination of unselected series of cases the relative proportions of the human and the bovine types of tubercle bacilli in different kinds of human tuberculosis. (2) To ascertain the frequency of occurrence and the distribution in the human body of variant strains of tubercle bacilli. The summary is shown in Table LXVIII.

Cobbett believes that, while human, bovine, and avian tubercle bacilli have probably descended from a common ancestral type, they have acquired special characteristics and are now stable. He holds that the human variety is responsible for ninety-four per cent of the fatal cases of tuberculosis in man, leaving only six per cent to be charged to the bovine bacillus. On the other hand, he states that fifty per cent of the nonfatal cases in man is due to the bovine bacillus. The latter organism infects most commonly in childhood and the organism involves most frequently the cervical and mesenteric glands.

Adami states that the evidence in our possession indicates that quite a large proportion of cases of active tuberculosis in adult life is due to the recrudescence of tuberculosis acquired in childhood. He asks whether there may not be a fallacy in the argument. that cases showing 
TABLE LXVIII

\begin{tabular}{|c|c|c|c|c|c|c|c|}
\hline \multirow[b]{2}{*}{ SERIES } & \multirow[b]{2}{*}{ AGE PERIODS } & \multirow[b]{2}{*}{$\begin{array}{c}\text { NUMBER } \\
\text { OF } \\
\text { CASES }\end{array}$} & \multicolumn{4}{|c|}{ TYPE OF INFECTION } & \multirow[b]{2}{*}{$\begin{array}{l}\text { PLR CENT } \\
\text { OF BOVINE* } \\
\text { INFECTIONS }\end{array}$} \\
\hline & & & HUMAN & BOVINE & $\begin{array}{c}\text { MIXTURES } \\
\text { (HUMAN } \\
\text { AND } \\
\text { BOVINE) }\end{array}$ & ATYPICAL & \\
\hline \multirow[t]{2}{*}{$\begin{array}{l}\text { British Commis } \\
\text { sion on tuber- } \\
\text { culosis }\end{array}$} & $\begin{array}{l}0 \text { to } 5 \text { years } \\
5 \text { ‘ } 10 \\
10 \text { “ } 16 \\
16 \text { and upwards }\end{array}$ & $\begin{array}{r}35 \\
11 \\
6 \\
27\end{array}$ & $\begin{array}{r}19 \\
8 \\
6 \\
25\end{array}$ & $\begin{array}{r}14 \\
3\end{array}$ & 2 & & $\begin{array}{r}45.71 \\
27.27 \\
7.40\end{array}$ \\
\hline & All ages & $80^{* *}$ & 59 & 17 & 4 & & 26.25 \\
\hline \multirow[t]{2}{*}{$\begin{array}{l}\text { Postmortem } \\
\text { cases in chil- } \\
\text { dren }\end{array}$} & $\begin{array}{l}0 \text { to } 5 \text { years } \\
5612\end{array}$ & $\begin{array}{l}61 \\
52\end{array}$ & $\begin{array}{l}48 \\
45\end{array}$ & $\begin{array}{r}13 \\
5\end{array}$ & 1 & 1 & $\begin{array}{l}21.31 \\
11.53\end{array}$ \\
\hline & All ages & 113 & 93 & 18 & 1 & $I$ & 16.81 \\
\hline \multirow[t]{2}{*}{ Sputum } & $\begin{array}{l}10 \text { to } 16 \text { years } \\
16 \text { and upwards }\end{array}$ & $\begin{array}{r}6 \\
206\end{array}$ & $\begin{array}{r}6 \\
199\end{array}$ & 3 & & 4 & 1.45 \\
\hline & All ages & 212 & 205 & 3 & & 4 & 1.41 \\
\hline \multirow[t]{2}{*}{$\begin{array}{l}\text { Bone and joint } \\
\text { tuberculosis }\end{array}$} & \begin{tabular}{|l}
0 to 5 years \\
56610 ، \\
$10 ، 16$ \\
16 and upwards
\end{tabular} & $\begin{array}{r}84 \\
167 \\
89 \\
52\end{array}$ & $\begin{array}{r}57 \\
121 \\
76 \\
46\end{array}$ & $\begin{array}{r}25 \\
42 \\
8 \\
3\end{array}$ & & $\begin{array}{l}2 \\
4 \\
5 \\
3\end{array}$ & $\begin{array}{r}29.76 \\
25.15 \\
8.98 \\
5.76\end{array}$ \\
\hline & All ages & 392 & 300 & 78 & & 14 & 19.90 \\
\hline \multirow[t]{2}{*}{$\begin{array}{c}\text { Genitourinary } \\
\text { tuberculosis }\end{array}$} & $\begin{array}{l}10 \text { to } 16 \text { years } \\
16 \text { and upwards }\end{array}$ & $\begin{array}{r}2 \\
15\end{array}$ & $\begin{array}{r}2 \\
12\end{array}$ & 3 & & & 20.00 \\
\hline & All ages & 17 & 14 & 3 & & & 17.65 \\
\hline \multirow[t]{2}{*}{$\begin{array}{l}\text { Cervical gland } \\
\text { tuberculosis }\end{array}$} & \begin{tabular}{|l}
0 to 5 years \\
56610 \\
10 ' 16 \\
16 and upwards
\end{tabular} & $\begin{array}{l}14 \\
29 \\
22 \\
37\end{array}$ & $\begin{array}{r}2 \\
10 \\
13 \\
27\end{array}$ & $\begin{array}{r}12 \\
19 \\
8 \\
9\end{array}$ & & $\begin{array}{l}1 \\
I\end{array}$ & $\begin{array}{l}85.71 \\
65.51 \\
36.36 \\
24.32\end{array}$ \\
\hline & All ages & 102 & 52 & 48 & & 2 & 47.05 \\
\hline \multirow[t]{3}{*}{$\begin{array}{l}\text { Miscellaneous } \\
\text { cases }\end{array}$} & 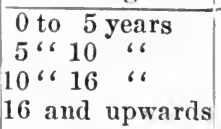 & $\begin{array}{l}2 \\
6 \\
2 \\
4\end{array}$ & $\begin{array}{l}1 \\
5 \\
2 \\
4\end{array}$ & $\begin{array}{l}1 \\
1\end{array}$ & & & \\
\hline & All ages & 14 & 12 & 2 & & & 14.30 \\
\hline & Grand Total & 930 & 735 & 169 & 5 & 21 & 18.70 \\
\hline
\end{tabular}

*Including the cases of mixed infection.

**Including one case the age of which was not stated.

human bacilli have been infected by other human beings. The relative rarity of bacilli of the bovine type in adults, according to Adami, demands one of the following explanations: (a) The bovine infection acquired in childhood is so fatal that all affected die in their early years, and this is wholly contrary to the evidence at our disposal. (b) The bovine bacillus which infects in childhood dies out and is replaced at a later date by infection with the human type, and of this there is no clear evidence. (c) Through long residence in the human body the bovine form takes on the characteristics of the human strain. Adami is quite certain that from time to time we encounter intermediate strains, and this favors 
the opinion that the bovine bacillus may, on account of long residence in the human body, take on the characteristics of the human type.

Behring thinks that all active tuberculosis in adult life is due to infection with the bovine bacillus in infancy. In this way he accounts for pulmonary tuberculosis, or phthisis, which he believes to be a late manifestation of the tuberculosis acquired in infancy from infected milk. He holds that the bovine bacilli, after passing through the walls of the alimentary canal, take up their residence in the lymph glands where they remain in an avirulent or a slightly virulent state until awakened by some intercurrent disease or other untoward incident in later life. Having acquired in this way greater virulence they cause phthisis. $\mathrm{He}$ says: "Phthisis is but the last verse of the song, the first verse of which was sung to the infant in its cradle." This theory has become widely prevalent since it was first advocated by Behring in 1903, and it must be admitted that there are many facts which, at least seemingly, support it. Pulmonary phthisis, the most frequently fatal form in adult life, is very rare in children. On the other hand, glandular and bone tuberculosis are common in childhood.

The fundamental facts concerning tuberculosis in cattle are very much the same as those of this disease in man. Calves, even from mothers in advanced tuberculosis, come into the world free from the disease; indeed, as we shall see later, in Denmark tuberculous cows are used for breeding purposes. They are isolated from nontuberculous animals and as soon as the calves are dropped they are separated from the mothers and allowed to suck nontuberculous animals or are fed upon the boiled milk of their tuberculous mothers. None of the young thus cared for develop the disease unless they are subsequently exposed. We see, therefore, that both children and calves show congenital tuberculosis so very rarely indeed that it may be stated that it does not exist. In both species tuberculosis is an acquired and not a hereditary disease. Whether it be acquired or not after birth depends upon exposure to the infection. According to Moore, tuberculosis is rarely found among the beef cattle brought from the West to the slaughter houses of Kansas City and Chicago. On the other hand, it is widely prevalent in many of the dairy herds in widely separated regions of this country.

The Avian Bacillus.-The barnyard fowl is frequently tuberculous. Much of the poultry that comes to market when properly inspected shows enlarged livers filled with yellowish tuberculous nodules. This variety of the tubercle bacillus can be distinguished morphologically and culturally from other forms. Chickens are easily infected by feeding, developing hepatic tuberculosis accompanied by loss of weight. Intravenous injection, even of a minute quantity, leads to rapid emaciation and death may result before macroscopic changes are in evidence. In 
less rapid eases the liver shows the characteristic enlargement and nodules. Intraperitoneal and intramuscular inoculations are slower, but in time develop the disease. Ducks and pigeons may be infected, but in nature the disease is much less frequent than in chickens.

The testimony concerning the susceptibility of the guinea pig to this variety of the tubercle bacillus is conflicting. Evidently, it is less than that shown by this animal to either the human or bovine varieties. Rabbits seem to be more susceptible, though typical tubercular nodules are not always developed in these animals, and many die from septicemia. Calves fed on the avian bacillus may develop local tuberculosis in the intestinal and mesenteric glands, but this seems to have little effect upon the growth and health of the animal. Subcutaneous injections in cattle eause an inflammatory reaction, which may extend to neighboring glands but fail to develop general tuberculosis. In the studies of the varieties of tubercle bacilli found in man already referred to, three cases of infection with avian bacilli were reported. However, the rarity indicated by these figures casts doubt upon the accuracy of the observations. In a study of the epidemiology of tuberculosis in man the avian bacillus may be wholly disregarded. That the flesh of tuberculous fowls frequently comes to the table is beyond dispute. The methods of cooking probably kill all the bacilli. Besides, the appearance of the liver is so striking that this organ is removed before dressed fowl comes under the eye of the consumer. The meat man is quite sure to attend to this matter, and if he fails no observant cook could overlook so abnormal an organ as the liver in a well-developed case of tuberculosis gallinaceus.

It is worthy of comment that domestic parrots have tuberculosis due to the human bacillus.

While man is free from the danger of acquiring infection from the avian bacillus, it is also interesting to note that neither the human nor the bovine type infects chickens. Chickens pick up and eat infected sputum deposited by careless men and probably occasionally feed upon the matter coughed up by the tuberculous cow, but no amount of feeding with human or bovine bacilli produces tuberculosis in chickens. Even subcutaneous and intravenous inoculations are practically harmless in the barnyard fowl. The bacilli thus introduced may retain their vitality in these birds for a long time, but they fail to develop tuberculosis in their hosts.

The Piscidian and Reptilian Types.-The bacilli which cause tuberculosis in cold-blooded animals are not capable of growth at the temperature of the body in mammalians, and therefore cannot be a source of infection in man. One would not knowingly eat a tuberculous fish, but if he unknowingly does so, tuberculosis will not result. It may be recalled that one Dr. Friedmann came over from Germany a few years ago and 
offered to treat American consumptives with bacilli obtained from turtles. His claims were wholly without foundation.

Other Acid-Fast Bacilli.-There are several well-known and fairly well studied saprophytic bacteria which have a close resemblance to tubercle bacilli in their reactions towards certain stains. They are not easily stained by ordinary dyes, but when thoroughly stained with carbolfuchsin or similar agents the color is not removed by washing with dilute mineral acid. These are classed with tubercle bacilli as "acidfast" organisms. These have been especially studied by Moeller, who has defined several species or varieties. One point of interest is that they may be mistaken for tubercle bacilli. This is likely to occur because one of them is found on timothy hay and is known as "Moeller's timothy" bacillus. Another is found in cow dung. So far as they have been tested they are not pathogenic to any animal.

Whether or not all of these acid-fast organisms have been evolved from a common ancestor is a fruitful field for theoretic discussion. No one has as yet converted one of these into a pathogenic organism, although this has been tried by frequent passage through animals. They may produce local reactions which closely resemble those developed by inoculation with one of the pathogenic types, but they do not cause general tuberculosis. That their cellular structure is similar, chemically at least, to that of the virulent types is shown by the following: (1) Their similar behavior towards stains. (2) Their local effects. (3) Animals sensitized to one of these saprophytic forms are partially sensitized to the virulent types.

Many attempts have been made to convert not only avirulent into virulent organisms, but one virulent type into another. The human and avian types have been carried through many cows and the human and bovine varieties have been carried through chickens. The results of this work have been by no means uniform. Some investigator's have convinced themselves that a transformation from one type to another is easily and quickly secured, while another man, equally competent, repeats the work and is sure that such transformation never results. The conditions under which such work must be done render mistakes easily possible, and to obtain satisfactory and convincing proof seems quite impossible. For instance, one man attempts to carry the human type successively through many calves in order to transform it into the bovine variety. At last he inoculates a calf and later finds in that animal a typical bovine organism. The experimenter claims that he has demonstrated a transformation, but his critic says that the tubcreulosis found in the last cow is not a result of the inoculation, but is a true bovine tuberculosis from a wholly different source.

One of the most convincing experiments in favor of transformation in 
type is that of Nocard, who placed a culture of the human type in a collodion sack and introduced the sack into the abdominal cavity of a chicken; after some weeks he transferred the sack to the abdominal cavity of a second chicken, and after another interval to a third. After this, he found that the type had been changed to avian. This has been repeated by two investigators, one of whom confirms and the other denies Nocard's claim. The solution of the question concerning the relation of the saprophytic acid-fast bacilli to the pathogenic, and of the different types of the latter to one another, must await further investigation.

Avenues of Infection.-When tuberculosis is transferred from man to man it is believed that this occurs chiefly through inhalation. The nose serves as a filter. Its secretions are to some extent germicidal and its. tissue is apparently quite immune to this infection. It is not uncommon to find virulent tubercle bacilli in the nasal cavities of those who attend tuberculous patients, but primary tuberculosis of this region is practically unknown; and even secondary infection is exceedingly rare. In the nose there is a free exit for accumulations, and the ease with which sneezing is induced by slight irritation and air ean be forced through it by blowing the nose tend to keep this cavity clean. Mouth breathing increases the chances of infection. The retronasal pharynx is less easily kept clean and is more frequently the site of infection, but this also is generally secondary. The tonsils frequently, and even in nontuberculous persons, are found to contain tubercle bacilli. Whether these glands do more than retain these organisms is not known. The larynx is more frequently the seat of primary infection and is involved sooner or later in about one-fourth the cases of pulmonary tuberculosis. Infection of the trachea and large bronchi is rare.

Concerning the primary site of the thoracic lesion in pulmonary phthisis, there is still difference of opinion. One theory is that the lesion is usually primary in the lymph glands, especially the glands around the hilus of the lungs, and that involvement of the lung parenchyma is due to spreading from these primary foci, usually along the bronchi or the peribronchial tissues. The opposite view is held by some,- that the glandular involvement is usually or always secondary to invasion of the lung parenchyma. Ghon believes that the focus is primary in the lung and that at autopsy it is found to be small, varying in size from pinhead dimensions to those of a cherry, being most often about the size of a pea. It is often smaller than the "secondary" focus in the glands, the glandular material being particularly prone to hyperplastic changes; but Ghon explains that the apparent age of a lung focus is always as great or greater than that of the glandular process. It is chiefly on this pathologic distinction that he bases his theory of parenchymatous pri- 
ority. He states that the mediastinal glands which one finds to be tuberculous are those that would become so from a primary focus in the lungs, and that on following out the drainage area from the involved glands into the lung tissue a tuberculous lesion is almost always found in the latter area. The changes in the glandular strueture never appear to be older than do those in the parenchyma. The primary lung focus is described as usually single (72 per cent), rarely occurring as more than two or three lesions, usually situated immediately beneath the pleura, which frequently becomes involved, and rarely located at the apices. It may occur in any lobe, being present in 31 per cent in the right upper lobe, in 23 per cent in the left upper lobe, in 22.5 per cent in the right lower, and least frequently in the right middle lobe. The most common site is on the anterior surface of the upper lobes midway between the apex and interlobar fissure. In the lower lobes the most frequent site is near the lower edge posteriorly. The lymphatic drainage from these areas passes through the bronehopulmonary glands to the tracheobronchial group. The inferior tracheobronchial glands, those beneath the angle made by the right and left bronchi, receive the greater portion of the drainage from the lower portions of the lungs, while the right and left superior tracheobronchial systems, located in the angles between the bronchi and the trachea, receive from the upper portions. Ghon shows that the diseased glands lie in the drainage system collecting from the regions of the primary lung foci. According to him, numerous anastomoses connecting the various sets of glands explain apparent deviations from this rule.

Canti reaches conelusions similar to those presented by Ghon. In sixteen cases studied, single lung foci were discovered in eight and multiple in two. Six "no lung foei" were found, but in all these abdominal tuberculosis was present. Tuberculosis of the mediastinal glands was found in twelve of the sixteen. In ten the glands were located in anatomic relationship with a "lung focus" and in two no focus could be found. In the latter there was extensive accompanying abdominal tuberculosis. The lung focus was usually caseous or fibrocaseous and averaged the size of a pea; one was calcareous, one was liquefying, and one showed eavitation. The most severely infected mediastinal glands were recorded as being in a caseous or fibrocaseous state. Canti, in elaiming that the lung focus when present is the original tuberculous lesion in the body, emphasizes the fact that the focus is usually single; that when it is present there is in the majority of cases no other lesion in other organs indicating another portal of entry; that when the portal of entry appears to be elsewhere, as in the intestines, there is usually no lung focus, and that there is a quite eonstant relationship between the occurrence of lung foei and tuberculons mediastinal glands. 
The work of Ghon and Canti does not prove conclusively the priority of lung infection, but it does serve to remind us that the mode and site of invasion of the tubercle bacillus in pulmonary tuberculosis is still a debated question. It remains to be proven that in two such different structures as lung parenchyma and glandular tissue, a caseous or fibrocaseous process in the latter is necessarily of more recent duration than a calcified or cavitated lesion in the former. Ghon found only one case with lung focus which showed no involvement of the mediastinal glands, while there were seven showing mediastinal glands without lung foci and with no evidence of a source of infection outside the lung. This appears somewhat contradictory to his theory.

The transmission of tuberculosis from one animal to another in our laboratories is exceedingly rare. In more than 30 years of experimentation with the tubercle bacillus, involving hundreds of guinea pigs and rabbits, in our own laboratory we have never observed the transmission of the disease from inoculated animals to controls, although often they have been kept in the same eages. Koch found such transfer of the infection rare in his work. However, he reported seventeen guinea pigs and eight rabbits naturally infected in this way. In all of these there developed a pulmonary tuberculosis resembling that which occurs in man. In the laboratory of the Department of Agriculture at Washington only one such case of transmission has been reported. However, laboratories are generally kept cleaner than many homes.

The development of tuberculosis in laboratory animals by means of the inhalation of pulverized infected sputum has been frequently reported. One of the most classical and frequently quoted instances of this kind is the experiment made many years ago by Cornet. In a room of seventysix cubic meters capacity this investigator placed 48 guinea pigs in cages arranged at various heights above the floor. The carpet in this room was impregnated with tuberculous sputum, and after drying was vigorously swept and shaken. This process was repeated on four consecutive days. It was found that of the 48 animals thus exposed $47 \mathrm{de}$ veloped the disease within two months. The same investigator sponged the walls of a room occupied by a tuberculous patient who expectorated on the floor, and the material absorbed by the sponge was injected into guinea pigs with positive results. It has been claimed that this and similar experiments do not represent actual conditions and the theory of the transmission of the disease through inhaled dust is overestimated. Some investigators have found that inhaled bacteria decrease greatly from the nose backward and deny that tuberculosis is commonly disseminated in this way. However, all admit that the inhalation of infected dust may be one of the methods by which tuberculosis is disseminated. Bartel and Spieler placed 12 guinea pigs in locked cages in a house oc- 
cupied by tuberculous patients and at the same time allowed 16 other guinea pigs to run loose in the house and to be handled by the infected individuals. This experiment was continued for from two to three weeks. Of the 12 guinea pigs in the cages 3 developed tuberculosis, and of the 16 allowed to run at large 10 developed the disease. There seems to have been in this instance no attempt to take care of the sputum expectorated by the tuberculous individuals. In another house in which a careful tuberculous patient resided, 8 guinea pigs were allowed to run at large and only one of these developed tuberculosis. Chapin placed 36 guinea pigs in cages in a fairly clean laborer's house in which there was a consumptive whose sputum was rich in bacilli. Sixteen of these animals were fed by the consumptive and the others by an employee free from disease. The exposure was continued from February 11, 1908, to May 14. Most of these animals died of nontuberculous disease or were starved or killed by rats after removal from the house. Of the 11 surviving, 8 were shown to be tuberculous and 3 not. Chapin states:

"It seems impossible that the test animals could have been infected otherwise than through the air. That it was mouth spraying rather than dust infection seems likely, for the patient took much interest in the animals and was often seen with his face close to the netting of those in the eage, talking to them and coughing at them only a few inches distant.',

Interesting experiments along this line have been made in studying the spread of tuberculosis among cattle. Schroeder and Cotton placed seven uninfected cows in a stable with three tuberculous animals. The stalls occupied by the infected and the uninfected were changed from time to time. Of the seven healthy animals thus exposed, six developed the disease. In the same experiment, 100 guinea pigs were exposed in cages, one-half being placed below the mangers while the other half were hung on the walls. Only one of these guinea pigs, and that one in a cage under the manger, became infected. In another experiment, 35 guinea pigs were placed in cages which were hung on the walls of stalls occupied by tuberculous cattle. Two of these developed tuberculosis. The exposure was continued for 135 days. It is doubtful in these experiments whether the tuberculosis was spread from cow to cow through the air, but there is little doubt that this was the method of transmission so far as the guinea pigs are concerned. Klein placed guinea pigs in the ventilation shaft of the Brompton Hospital for Consumptives and most of the animals thus exposed developed tuberculosis. LeNoir and Camus made an interesting experiment in a tuberculous ward. Four guinea pigs were placed in a cage on the floor and were fed by the patients. One of these developed tuberculosis. Five guinea pigs were kept in a cage on the floor but out of reach of the patients. One of these developed tuberculosis. A cage with a guinea pig and three 
little ones was hung near the ceiling so that it was necessary to use a ladder in feeding them. Two of the young developed tuberculosis, while the third died of an intercurrent disease. From these and similar experiments, which might be multiplied, there can be no doubt that tuberculous infection may be and is disseminated through dust containing infected sputum.

Flügge has contended that infection through dust is not the usual method of the transmission of tuberculosis and he has suggested that this disease is most commonly transferred from man to man through "droplet infection." He and his students have shown that as small a number as 62 bacilli is sufficient to cause this disease when transmitted by droplet infection, while several million are required to produce the disease when taken by the stomach. They have infected guinea pigs by causing them to breathe in front of coughing tuberculous patients. No infection took place when the distance was more than one meter. When the distance was not more than 45 centimeters and the exposure lasted for ten minutes or longer the infection was generally transmitted. Bernheim was able to infect guinea pigs at a distance of not over 25 centimeters, but did detect tubercle bacilli on agar plates held one meter from the mouth. Whether the bacilli are carried directly into the lungs by the inhaled air or find lodgment and grow in the bronchial glands from which they are subsequently transferred to the lungs is a question. This, however, is a matter of scientific and academic interest. It has been abundantly demonstrated that droplet infection is one form of transmission of tuberculosis infection from man to animal, and it is fair to assume that this is also a frequent occurrence in the transmission of the disease from man to man.

It should be plainly understood that in ordinary breathing, even from the lungs of an individual in the last stages of pulmonary consumption, the exhaled air is bacteria free. It would be very unfortunate indeed for any one to get the idea that whatever precaution is used it is not safe to associate with a tuberculous individual. As was shown by Tyndall many years ago, bacteria are not given off in the air from moist surfaces. The old theory that disease is transmitted in gaseous form has no foundation in scientific fact. Under proper precautions every necessary attention may be given to tubereulous persons without danger of infection. The sputum should be collected and burned, and the floors and walls of hospitals and sanatoria oceupied by tuberculous patients have been found generally to be free from the bacilli.

In the great majority of instances man becomes infected with the bovine bacillus through milk and by way of the alimentary canal. However, it should not be forgotten that the human bacillus may be transferred in like manner. It was a eustom years ago for the mother to chew 
the food given her children or for the nurse to taste the milk or other food on which the child was to be fed. It is still a custom, unfortunately, to give but little attention to the sterilization of tableware, especially forks, spoons, cups, and glasses. Fortunately, in most intelligent communities the common drinking cup has disappeared, but in restaurants, boarding-houses, soft drink establishments, etc., there is still inadequate attention to these matters. In the larger hotels those who handle food are examined carefully in order to ascertain whether they are typhoid carriers or have tuberculosis or syphilis, but in many of the smaller and cheaper eating places these diseases are not excluded either in the kitchen or in the dining room. In most advanced cases of pulmonary tuberculosis the feces carry tubercle bacilli and there is some danger of infecting the food, as exists with typhoid earriers. The stools of the typhoid patient during the active stage of the disease are disinfected. This is not true in the case of the consumptive. Probably a still greater danger is to be found among the guests. The transmission of tuberculosis from man to man through food and drink remains a big and unsolved problem. There may be and is some doubt as to whether infection with the bovine bacillus in infancy or childhood is an unmixed curse, but there can be no doubt about the possibility of the human bacillus finding its way from man to man through the alimentary canal of the recipient. Moreover, there is no doubt that even pulmonary tuberculosis may and often does develop from infection by way of the alimentary canal. Hogs fed on tuberculous material develop pulmonary tuberculosis. Ravenel found tubercle bacilli in the lungs within a few hours after placing them in the stomach, even when they were so placed not by way of the mouth but by direct surgical opening into that viscus. Even the introduction of tubercle bacilli into the intestines has been followed by pulmonary tuberculosis; indeed, this manifestation of the disease may result whatever the avenue by which the organism is introduced. There is the house-fly, which feeds upon tuberculous sputum and feces and then visits the dining room where it walks over solid food and often drowns itself in the milk which the child is soon to drink. There may be some question about our unduly magnifying inhalation in the dissemination of this disease, but there can be no doubt that we have placed too little emphasis upon infection by ingestion.

Brown and Cumming independently have shown that forks, spoons, and cups used by people with open tubereulosis bear the bacilli. Cumming has shown that when spoons used by individuals with open tubereulosis are washed as they ordinarily are in the home, both the wash water and the rinse water, and the spoon after washing, contain tubercle bacilli.

Having been taken into the mouth, what becomes of the tuberele bacil- 
lus? It is thought by some, with good reason, that carious teeth provide a port of entry and that the infection introduced in this manner finds its way into the adjacent glands. Sore and raw gums, due to deposits of tartar and the fermentation of lodged particles of food, offer additional points of entry not only for the tubercle bacillus, but for other pathogenic organisms; in fact, tubercle bacilli have been detected in carious teeth, in tartar, and in the coating ${ }^{\circ}$ on the tongue. The function of the tonsils in regard to this infection has not been satisfactorily determined. Tubercle bacilli are found in a large percentage of these organs when removed. The food quickly passes the esophagus and the acidity of the stomach contents probably affords some protection to that organ. In most cases of infection by way of the alimentary tract, the bacilli find lodgment in the small intestine, through the walls of which they may pass without leaving recognizable lesions. It has been claimed by some that the healthy mucous membrane of the digestive tract is impermeable to this organism. Others hold that tubercle bacilli are killed by the juices and during the digestive processes of the stomach and intestine. That neither contention holds has been proved by direct experiment. Ravenel introduced tubercle bacilli, made into an emulsion with melted butter, into the stomachs of dogs which had been kept on soft food and were purged before the experiment in order to remove any bits of bone or other material that might injure the mucous membrane. Three and onehalf hours later and during full digestion these animals were killed and material from the mesenteric glands was injected with positive results into guinea pigs.

The third avenue for the introduction of tubercle bacilli into the body is interesting, though quite uncommon. We refer to wounds. In the oldfashioned circumcision, as practiced by the Jewish Rabbi, this official sought to stop the flow of blood by applying his lips to the wound. It has occasionally happened that the operator was in an advanced stage of pulmonary tuberculosis and his saliva was teeming with tubercle bacilli. The records show several cases of the transmission of tuberculosis in this way. Since eircumcision is performed soon after birth, the child operated upon is nontuberculous and is in a condition most susceptible to the rapid spread of this infection. In the majority of such instances miliary tuberculosis, followed by death, occurred. On the other hand, the surgeon, the medical student in the dissecting room, and the butcher, all occasionally handling tuberculous tissue, may receive wounds and acquire infection in this way. In most instances the disease thus acquired in adult life remains a local affection, or at most involves some of the nearest glands. In these it is supposed that the effect of inoculation is relatively trivial, because in adult life most men are already infected with tuberculosis. 
There are three forms of tuberculous skin affections, tuberculous verrucosa, lupus, and tuberculous ulcers. These may be due to scratching with infected finger nails, euts with infected knives, or wounds with other infected instruments.

Latent Tuberculosis.-Before the work of Villemin it was largely believed by medical men that tuberculosis is an inherited disease. At that time much was written concerning the inheritance of a disposition to tuberculosis. Of course, nothing was known about the bacillus, but the medical men of the time observed that the disease was inclined to run in families. The children of tuberculous parents grew to adult life, wandered near and far from the parental roof, took up their residence ofttimes in most distant countries, established their own homes and began the accumulation of property, when in early adult life many of them developed the disease and died therefrom. What was more natural than to attribute these well attested facts to the inheritance of à disposition to develop the disease? Since the work of Villemin and the identification and isolation of the causal bacillus by Koch, there has been practically only one man, whose work has enabled him to speak with authority, who has claimed that tuberculosis is inherited. We refer to Baumgarten, who has been a close, intelligent, and productive investigator in the realm of tuberculosis; indeed, he announced the discovery of the bacillus only a few days after Koch had presented the results of his investigations in 1882. Baumgarten has continued to hold that tuberculosis is an inherited disease. However, his idea of inheritance is different from that of his predecessors. He believes that the child is born into the world from tuberculous parents already infected with the specific bacillus and that this organism lies latent in the tissue of the child until it is awakened into activity by some intercurrent disease or some other untoward condition of life, such as constant want of proper food or existence of insanitary surroundings. Notwithstanding the authority with which this great man has spoken, it must be admitted that every fact that has accumulated and stood the test of time since 1865 contradicts him in no uncertain terms. Thousands of autopsies have been made on newly-born infants, many of them from tuberculous parents, and, as we have already stated, congenital tuberculosis is so exceedingly rare that its existence may be totally ignored. None of these children have shown during their few days of life any signs or symptoms of tuberculosis, nor has the most careful and minute examination been able to reveal any lesion characteristic of this disease in their bodies. Moreover, many of these children soon after birth have been subjected to the tuberculin test, which hunts out and reveals the most hidden tuberculous lesions in the body, and no newly-born child has responded to this test, which, when properly applied, is as nearly infallible as human evidence ean be. Thousands of ealves from 
tuberculous mothers have been autopsied and exposed to the tuberculin test with like negative results; indeed, tuberculous cows are used for breeding purposes, and when the young are removed from the mothers directly after birth and placed where subsequent infection is impossible no one of them has ever developed the disease. In our laboratories tuberculous guinea pigs and rabbits bear offspring and when postnatal infection is prevented never show infection. Skilled obstetricians in various parts of the word attending thousands of mothers, many of whom are tuberculous, in confinement, have sought most diligently for evidence of congenital tuberculosis and the finding of this condition has been so rare that it may be said not to exist. It seems that if there is anything certain about the development of this disease, we are justified in saying that every child comes into the world free from it. It is not in any sense an inherited disease. Neither the disposition to tuberculosis nor the specific bacillus of the disease is earried in any tissue of the child at birth. Every case of tuberculous infection is acquired and in no instance is it inherited.

In infancy the chance of infection depends upon the environment in which this period of life is passed. During the first year infection depends upon the immediate surroundings in which the infant is placed. During this time it leads a vegetative life, as it were. A careless tuberculous mother, nurse, or other person brought in close contact with the child may infect it. If artificially fed it may receive its infection through the milk. Later it begins to erawl about on the floor, picking up all kinds of particles and often placing them in its mouth. It crawls over the carpet or the naked floor, where possibly tuberculous material has been deposited by a careless consumptive or brought in from the street on the feet of father, mother, some other member of the family or a visitor. On its hands and knees as it grows older and stronger, it visits every corner of the house and is likely to try the taste and digestibility of anything it may find. As it grows older and stronger still, its range of activity widens and its chances of infection increase. It frequently applies its fingers alternately to its mouth, the leaves of dusty picture books, unbrushed shelves in kitchen and cupboard, the paper on the wall, and in fact every material thing which comes within its reach. The youth goes to school, comes in contact with the clean and unclean, swaps bites of apple and exchanges sticks of candy, and thus in a thousand ways multiplies the chances of taking into the body the ubiquitous tubercle bacillus. It does not matter whether the child be from tuberculous or nontuberculous parents; neither does it matter whether at the time of receiving the infection the child be in robust health or be frail in body. The infection may be planted in the tissue under either condition. As the range of activity widens still farther, the chances of infection multiply. It is 
not strange, therefore, that the percentage of tuberculous infection depends upon age and range of activity. The application of the tuberculin test shows that, while none are infected at birth, at two years of age the percentage of the infected reaches about ten; at four about twenty-five; at ten about fifty; at fifteen about sixty, and at twenty-one from seventyfive to eighty. At later periods in life the percentage is so great that it may be said that every one who reaches old age is infected. In a general way, we may say, taking deaths at all ages, that fifty per cent or more at death are infected but that only one-seventh to one-tenth die from tuberculosis. These facts compel us to distinguish between tuberculous infection and tuberculous disease. In other infectious disease we expect symptoms to follow infection within a relatively short and more or less definite time. The period of time elapsing between infection and the first symptoms of the disease we denominate the period of incubation. What is the period of incubation in tuberculosis? It may be a few weeks, it may extend to many years, and in the majority of instances death from other cause results before tuberculosis manifests itself as an active disease. Most infectious diseases run their course, end in recovery or death, and we say that the disease is self-limited. Tuberculosis is not a self-limited disease. In this respect it resembles syphilis. There is no definite period of incubation. The active manifestations of the disease may continue through years. As a rule, in the infectious diseases one attack gives more or less marked immunity to subsequent attacks, but in tuberculosis symptoms may vacillate, with recessions and exacerbations alternating for years, while the infection remains in the body through all these vicissitudes. In other infectious diseases the source of infection is pretty well defined. In typhus fever the bearer of the infection is the body-louse; in the bubonic form of the plague it is the rat-flea; in yellow fever it is the stegomyia, and in malaria the anopheles. In any of these diseases an individual may come in closest contact with the infected man and no harm results unless the special distributor of the infection plays its part. In typhoid fever, Asiatic cholera, and dysentery, the infections are confined to the discharges of those ill. In rabies it is only the rabid animal that is capable of transferring the disease. In the venereal diseases closest contact is required. The spread of tuberculosis is not limited in a similar way. The tubercle bacillus comes from some infected animal. Practically, we may say that man is infected with this disease only from fellow-men and from cows. The tubercle bacillus does not multiply outside the body, but it is widely distributed and retains its power of infection for a time which varies from a few hours in direct sunlight to two or three years in dark, moist places. Of all the bacteria that infect man this organism is most universally distributed. It lies in wait for its host in the home, on the 
street, in the school, in the church, in every place of assembly. We can avoid insects, we can boil our drinking water, we can kill our rabid dogs, we can deny ourselves the intimate contact necessary to contract the venereal diseases, but we cannot disinfect every part of every house, all the dust on the street, neither can we kill all the cows that are tuberculous or avoid coming in contact, either directly or indirectly, with the excretions of tuberculous men. For diphtheria and tetanus we have antitoxins, for smallpox, typhoid fever, and cholera, we have vaccines, but up to the present time we have neither antitoxin nor vaccine with which to combat tuberculosis. We might make our residence in the most malarial region, but we ean shut out the mosquito and be safe. We have no screens which exclude the tubercle bacillus, and, besides, we would not know where to place them did we have them.

From fifty to one hundred per cent of us have tuberculous infection, while there are not more than from three to five per cent of us in whom this disease has actively developed. Animals are the culture flasks in which the tubercle bacillus grows and it cannot develop or multiply outside the animal body. There is no intermediate host, as in malaria, typhus fever, or yellow fever. The tubercle bacilli which are dangerous to man grow in his own body and in those of eattle. It has been so long a parasite in the tissues of these animals that it has apparently in a large percentage of cases established a modus vivendi with its host. In the great majority of instances the relationship of host and guest gives no great discomfort to either, but when the host becomes unsatisfactory to the guest the latter commits murder. It has been assumed by many that bacteria are of simple structure and exceedingly limited in their functions. It is true that they are unicellular organisms and morphologically simple in structure, but in fact the tuberele bacillus has as wide a range of function as any cell in our lungs. It has gone through an evolutionary development measured by years as long as that of man. It has learned to protect its own life from adverse environmental influences.

In many laboratories men skilled in this line of work have attempted to trace the path of this bacillus from the moment it enters the body uritil it establishes itself in some tissue and manifests its presence there by its activity. Up to the present time (1921) these investigations have led to diverse and often contradictory conclusions. In 1905 Baumgarten stated:

\footnotetext{
"Tubercle bacilli cannot enter the body anywhere without bringing about tuberculous changes at the portal of entry.",
}

Were this true, the task of tracing the path of the bacillus through the body would be relatively easy, but the falsity of this statement was proved many years before it 'was made. As early as 1878, four years before Koch isolated the organism, Langeron fed tuberculous material to rab- 
bits and subsequently examined these animals with great care. He concluded :

"Tuberculosis is transmissible from rabbit to rabbit by way of the digestive tract, and this transmission is brought about by a virulent principle without any disease occurring in the organs of digestion."

In 1885 Wesener repeated this experiment on the same species of animal and found tuberculous changes in the mesenteric lymph nodes in all of them, while only half exhibited any evidence of tuberculosis in the intestine itself. In these instances, there could be no doubt that in all the animals the bacilli invaded the body through the intestinal wall and that in many instances they did so without leaving even microscopic evidence of their passage through the wall. In 1879 Orth introduced tuberculous material into the mouths of rabbits, taking great eare not to injure the mucous membrane, and later found that the cervical glands became tuberculous. Experiments of this kind have been repeated so often that there ean be no doubt that tubercle bacilli may pass through the mucous membrane of the alimentary canal without leaving any recognizable lesion in that tissue. Theoretically it may still be claimed that these organisms are not able to penetrate unbroken mucous membrane without leaving a lesion, but what mucous membrane continues for any length of time without microscopic breaks in its continuity? Minute wounds in the mouth or in the intestine may occur from rough particles of indigestible material without disturbing in any way the health of the individual; therefore, the discussion whether or not the tubercle bacillus may pass through perfectly healthy mucous membrane without leaving its mark becomes academic and for all practical purposes without value. As Krause points out, the mother brings her child to the physician on account of the enlarged lymph nodes in the neck, and neither the mother nor the physician ean recognize any abnormality in the mucous membrane of the mouth or throat. That the bacillus has passed through this tissue and reached the cervical glands there can be no question, and still the mucous membrane is, apparently at least, perfectly healthy. It seems so far as infection through the alimentary canal occurs, that the bacilli pass through the mucous membrane with or without leaving a lesion and are carried to the lymph nodes which receive the material absorbed from that region. Many years ago Cornet attempted to formulate a law of localization, which may be briefly stated as follows: After passing through the alimentary wall the bacilli are earried to the nearest lymph nodes, where they invariably cause histologic changes which can be recognized grossly. Following this so-called law, attempts have been made to trace the invader as it proceeds through the body. In his postmortem study of experimental animals and of men dying from tuberculosis, the pathologist has attempted to determine the oldest lesions. What 
must he find in order to decide whether one lesion is older than another? The only guide that he can follow in deciding this matter is the degree of destructive change that has occurred and is revealed on examination of different tissues. That tubercle bacilli may pass through lymph nodes without leaving easily recognizable changes is also certain. Many years ago Orth found after experimental inoculation, extensive tuberculous changes in the lungs and kidneys, while there were none or but slight alterations in the intervening lymph nodes. In 1890 Loomis, of New York, produced tuberculosis in animals by inoculating them with material from lymph nodes in which no evidence of tuberculosis could be found. This finding has been abundantly confirmed and even carried further. Bartel found that lymph nodes may contain tubercle bacilli and be infectious for more than 100 days after inoculation and with no sign of tuberculosis in the nodes. It is also certain that bacilli may pass through one lymph node without leaving trace of so doing and set up characteristic and even extensive changes in other nodes or in other tissues beyond. Krause, of Baltimore, a most careful and skilful investigator, makes the following statement on this point:

"It was my habit to introduce the germs into guinea pigs underneath the skin of the right groin. The bacilli were deseendants of a eulture that had been isolated many years before and that had, during years of growth in an incubator, lost much of their invasive eapacity for laboratory animals. They therefore set up disease in guinea pigs, but it was disease of a kind which, while it spread, did not kill. Animals would survive the inoculation indefinitely-for several years. I could, therefore, follow the course of the discase locally, at the site of inoculation, from month to month. I could see and record how the lymph nodes of the groin enlarged and became firmer as disease in them progressed. But as time went on and the animals continued to live I could also note how they would invariably again beeome smaller and return to their more normal size and consistency. When, after about two years I would kill and examine my animals, I always found the same things had happened. There was tuberculosis in many places in the body. There was practically none to be seen in the various organs, but many widely seattered groups of nodes were affected with tubercle. And the one significant finding was that there was, often at least, one remote group of glands that had far more tubercle than had the nodes that drained the point of inoculation. This group was the far-away tracheobronchial chain that receive their lymph from the lungs and the lower half of the windpipe and from nowhere else. In other words, germs started from the site of inoculation had by a very circuitous route arrived at distant points that bore no organic relation whatever to the point of introduction and had here set up the most prominent changes in the body. At the same time, the inguinal nodes, those that were really connected with the groin, after the lapse of a year or two, appeared to the naked eye to be almost normal-so much so that it. frequently required microscopic examination to make sure that there was any tubercle in them. Now, had I not been familiar with the entire tuberculous history of my animals-had I not had this under uninterrupted observation, and, as it were, under control, and had I not applied Cornet's criterion of interpretation of the portal of entry, with such animals before me, I should have had no other recourse than to conclude that the respiratory tract was the portal of entry, because manifestly here the tracheobronchial nodes bore 
the brunt of the infection. Yet, such a conclusion was absolutely unwarranted, for the all-sufficient reason that here I had myself artificially brought about the infection and I had done this at a place where entrance of germs by way of the lungs could not possibly come into question."

In a final analysis of a study of the period of incubation in tuberculosis, we may say that the active disease begins when the invading organism establishes itself in any tissue in the body and when reaction between the invading cell and the body-cell begins. This fight between invading cells and body-cells is so localized and so few are the combatants engaged that the result of the skirmish is imperceptible to the body as a whole. The battle field is microscopic and the killed and maimed are so few that no impression, at least no recognizable impression, is made upon the animal body as a whole. The symptoms, if there be any, do not rise to the level of clinical recognition, either by the patient himself or the most skilful physician who might examine him. To the pathologist, the period of incubation is the time which elapses between the entrance of the bacilli into the body to the appearance of the first characteristic changes which may be recognized either by the unaided eye or by the microscope. To the clinician, the period of incubation covers the time between the entrance of the bacilli into the body and the time when the body as a whole is first disturbed sufficiently for the disturbance to be recognized. We think that we now have a clear understanding so far as is possible of what the period of incubation in tuberculosis covers. Since the microscopist or the pathologist eannot make his examinations except in the rare instances of superficial tuberculosis, like that of the skin, for all practical purposes the period of incubation in this disease exists from the time of entrance of the invading organism into the body until the time when the destructive changes induced by the invader are sufficient to disturb the health and well-being of the individual as a whole. This may cover a few weeks, it may extend through years, it may and often does not develop even though the individual lives to a ripe age. The period of incubation may be cut short and the active development of the disease hastened by any untoward incident that occurs to the individual as a whole. It will be seen from this that there are two big problems before those who are seeking to combat this disease. One is to limit the spread of the infection, the other to prevent the development of the disease in those already infected. So long as those who are infected are not discharging from their bodies in any secretion or excretion the specific microorganism they are not a danger to others. We see, therefore, that one problem is involved in the other. If we could prevent all latent eases developing into open ones we could soon eradicate the disease. Until we succeed in doing this we shall never eradicate tuberculosis. To the extent to which we reduce the development of latent 
cases into open ones to that extent do we reduce the sources of infection. To limit our efforts to the prevention of infection without effort to prevent the development of latent into open tuberculosis is to undertake a task which in its very nature is such that it can never be accomplished. So far as the infection of man through tuberculous milk is concerned, this appears to us to be relatively easy to prevent. To prevent infection from man to man will require a remaking of the whole fabric of our social existence. The eradication of tuberculosis is bound up with the control of other diseases, with improved housing, with better feeding, with education, with the abolition of poverty, and with the slow evolution of the race.

It is said that wide-spread tuberculosis is a product of civilization, and so it is. Savage man is largely free from this disease. The North American Indian scarcely knew it until the white man came, and even among our hardy grandfathers, who were the pioneers in the development of this country, tuberculosis played no great part. The white man is carrying civilization with syphilis and tuberculosis into every part of the world. Tuberculosis may be, and undoubtedly is, a product of our civilization, but its continuance with us abundantly proves that our civilization is as yet imperfect and incomplete. There are more intelligent people in the world than at any other period in its existence, but the majority of mankind is still in a state of dense ignorance. Even in our own country with its boasted civilization there are millions who cannot read or write, there are hundreds of thousands living in insanitary houses, there are many inadequately supplied with proper food, there are hundreds of thousands under the stress and strain of making ends meet. The eradication of tuberculosis is a problem involved in the evolution of civilization. To attempt to detach the tuberculosis problem from the general public health movement is a mistake. It has been shown by figures that the introduction of pure water-supplies and improved sewerage has not only reduced deaths from typhoid fever, but also saved thousands from tuberculosis. A large number of typhoid patients carry latent tuberculosis and even when they recover from the more acute infection they fall victims to the more insidious and pertinacious invader. Each has his special panacea for doing away with tuberculosis. One thinks that the great cause of this disease is alcoholism; another that it is insanitary housing, yet another that it is need of fresh air. Underfeeding, insufficient clothing, imperfect lighting and ventilation in homes and in factories, infected dust, and many other things are held responsible for the wide prevalence of this disease. Yes, it is each and every one, and all of these untoward conditions of life which convert the latent tuberculous case into an open one, remove the seal from the culture tube, and scatter its contents broadcast. 
Measles, scarlet fever, diphtheria, influenza, and various other acute infections prostrate many of those with latent tuberculosis, possibly taking their death toll from many, but even those who recover find that their span of life has been shortened materially, because the intercurrent disease has so weakened bodily resistance that sleeping tubercle bacilli have awakened and have fallen more earnestly and more effectively to their work of the destruction of vital organs. War, famine, and pestilence have in the last few years materially reduced the population of certain areas, but the deaths caused by these agencies directly will be greatly increased by the wider prevalence of tuberculosis among those who have survived. The death rate from tuberculosis is climbing already in every country that has felt the effects of the World War and it will probably be many years before this rate falls to the pre-war level. It may possibly be that the World War will ultimately give the peoples who engaged in it better government and better conditions of living, but for some years to come we must expect the tuberculosis death rate to go up in each country in direct proportion to the increased stress and strain of living which have been imposed upon its people. A wise Frenchman said many years ago that the best government is that which gives to its citizens the greatest freedom from disease and the greatest average of life.

It is an old and repeated observation that tuberculosis is a frequent sequel to measles, scarlet fever, whooping cough, and other acute infectious diseases. Whether these diseases cause lesions, thus affording more ready entrance of tubercle bacilli into the body, or whether the sequence is due to an awakening of already established tuberculous foci, may be a matter for debate. Probably both things happen. Measles, for instance, is accompanied by marked alterations, with the production of many minute lesions, in the mucous membrane of the respiratory tract. Through these breaks in continuity tubercle bacilli may more easily than under normal conditions find their way into the body. However, there are reasons for believing that measles not only opens up new portals for infection, but that in the majority of instances where the sequence between these diseases is noted, it is due to previous infection with tuberculosis. The tuberculous processes develop so quickly after the measles that it is hardly possible, at least in the majority of instances, to suppose that infection with tubercle bacilli has not preceded the acute attack of measles. Moreover, there is scientific testimony which, if we interpret aright, bears upon this question with great weight. It has been frequently observed in measles cases where the tuberculin test has been made that a prompt and vigorous response has followed, showing undoubtedly the existence of tuberculous infection. When cases of this kind are attacked by measles, both during the attack and for some time thereafter, they fail either wholly or partially to respond to the 
tuberculin test. Response to the tuberculin test indicates, as we understand it, that the body-cells have been sensitized and are pouring out secretions, the purpose of which is to counteract the multiplication of tubercle bacilli in the body. When measles comes on in a tuberculinpositive individual these specific secretions evidently lose, for the time being at least, something of their specificity and are utilized in the combat with the measles virus. Consequently, when the Pirquet test is made, the cellular secretions fail partially or altogether to have any digestive action on the tuberculin introduced in the scarified tissue. This is a result which we can see, and we assume that a similar phenomenon is taking place in the one or more tuberculous foci which may exist in the body; in other words, the body cells being threatened with subjugation by the virus of measles turn their every energy into combat with this foe, and even go so far as to withdraw those agencies whose specific purpose it has been to combat the tubercle bacilli. During the continuance of the attack of measles these antituberculosis secretions are exhausted. They have spent every energy not in combat with that enemy against which they have been trained, but against one which threatened speedier and more wide-spread destruction; in other words, the offensive weapons of the body-cells have been for the time withdrawn from the siege of the tuberculosis forces and turned against a new enemy which is advaneing over wider areas and possibly threatening points of great strategic importance. If this be true, and there can be no question about the frequency and promptness with which tuberculosis develops after measles, there can be no question about the importance of preventing, controlling, and modifying the attacks of measles in our effort to secure a reduction in the number of eases and deaths from tuberculosis. Not only is this true, but the matter has even a wider significance. Before the attack of measles comes on the individual in the case had latent tuberculosis. He was not a source of danger; he could go among his fellowmen, although he had tuberculous infection, without disseminating this infection in any way. The attack of measles converts this individual into an open case of tuberculosis and not only shortens his life, but renders him so long as he does live a distributor of the disease. Could some effective vaccine for measles be found it would be a most effectual aid in stamping out tuberculosis. In the absence of such a protective agent it behooves both the physician and the patient to consider measles a more important disease than it is usually credited and to throw around the patient every protective influence possible. It may be just as dangerous, both to the individual and to the community, to expose a case of latent tuberculosis to measles, scarlet fever, or whooping cough, as to again expose such an individual to tuberculosis.

Concerning the relation between influenza and tuberculosis there seems 
to be some difference of opinion. It has been reported by some that cases of influenza show like failure to respond to the tuberculin test that has been observed in measles. On this point Bloomfield and Mateer make the following statement:

"While the literature on alterations of the skin test during acute infeetious diseases is meager, it is generally believed that measles stands out as the only instance in which constant depression occurs. It is of interest, therefore, to find somewhat similar results in epidemic influenza. Beyond pointing out the fact it seems difficult to associate the phenomena in the two diseases, despite certain rather striking analogies whieh exist between them. It would seem wise, however, to restudy in detail the changes in skin sensitiveness in other acute febrile diseases, as similar conditions may perhaps be found to exist.",

These observers applied the Pirquet test to 19 cases of tuberculosis during the febrile period of influenza and found that at that time only one reacted, while after the febrile stage of influenza had passed 17 reacted to the skin test.

During the 1918 epidemic of influenza much attention was given to the effects of this disease on patients in tuberculosis hospitals. Fishberg, writing in November, 1919, has collected data upon this subject and has stated his conclusions as follows:

"Epidemic influenza has no etiologie relation to tuberculosis, and is not to be eonsidered as a reactivator of dormant tubereulous lesions. Tubereulous patients are no more likely to suffer from influenza than persons with healthy lungs, and when phthisical patients contract influenza the acute complicating disease is not likely to run a more acute, severe, and fatal course than others. The tuberculous process in the lungs has not been observed to assume an acute and progressive course after an attack of influenza. Vital statisties and reports from sanatoriums show that during the past and current years the mortality from tubereulosis has not increased, despite the fact that influenza has been raging during this period, nor has the number of tubereulous patients seeking admission to institutions inereased during the past twelve months. The pulmonary sequelae remaining after influenza are almost without exeeption nontuberculous in character and do not require the treatment aceorded to phthisieal patients."

The question might be raised whether or not the data upon which Fishberg writes his conclusions were collected too early after influenza epidemics to furnish positive information concerning the influence of the epidemic influenza of 1918 upon the mortality from tuberculosis. It is generally admitted, we believe, that there was a recrudescence of influenza in this country in 1916. The last volume of the U. S. Mortality Statistics that we have at hand is that for 1917. In this the following statement occurs:

"The number of deaths in 1917 from tuberculosis of the lungs, ineluding acute miliary tubereulosis, is 97,047 . The death rate (128.9 per 100,000 population) is considerably higher than the rate for 1916 (123.8), and an inerease is evident in all the subdivisions of the registration area.', 
We make no claim that the increased mortality from tuberculosis in 1917 was due to the influenza of 1916. However, we do question the statement that the tuberculosis death rate did not increase in 1917 and 1918. Inasmuch as we are now (1920) having another severe epidemic of influenza, we must await further and more exact information concerning the relationship between these two diseases.

There seems to be no doubt that increased stress and strain of living, whether it be manifest in the greater prevalence of acute infections, in greater difficulty in securing abundant and proper food, or whatever it may be, do increase the mortality from tuberculosis. While exact figures are yet wanting, the general report comes to us that in the countries most seriously disturbed by the World War and where these disturbances have been most continuous and persistent the death rate from tuberculosis has greatly increased. Speaking of this matter so far as England is concerned, Cobbett says:

"Tuberculosis has increased during the war; and the annual numbers of deaths from pulmonary tuberculosis in the years 1913-14-15-16, have been in the proportion of $100,104,112$, and $112 . "$

This, as we understand it, refers to the increase in this disease in the civilian population of England and does not concern the soldier. It is a well-known fact that during and immediately following the siege of Paris (1870-1871) the death rate from pulmonary tuberculosis rose enormously.

Vaughan (Warren) has collected and discussed the recent literature concerning the relation between influenza and tuberculosis. From this paper we make the following abstract: After the epidemics of 18891893 Leichtenstern stated that the mortality tables of all countries agree in showing considerable rise in the mortality from pulmonary tuberculosis in influenza periods. The clinicians of that time made the frequent observation that the course of tuberculosis is markedly and unfavorably influenced by influenza and its pneumonic complications. Latent cases often became active and arrested cases broke out anew. Afebrile cases were changed to the hectic type and frequently hemoptysis followed. During the height of the 1889 epidemic the weekly death rate from tuberculosis in London rose to double the average. The increased death rate during the epidemic was, however, not limited to tuberculosis, but there was almost a doubling of deaths from all acute respiratory infections. Jordan remarks that in New York City in 1918 during the two weeks of maximum epidemic mortality, the deaths reported from pulmonary tuberculosis numbered 430 as compared with 264 for the corresponding weeks of 1917 . Vaughan and Palmer found that the deaths from tuberculosis in the army were higher in the autumn of 1918 than they had been any time since mobilization began. The death rate from 
tuberculosis in the autumn of 1917 in the army was 15 . In the fall of 1918 it was 46 . This is, however, in part accounted for by the retention in the army of cases of tuberculosis which had been accumulating since the first draft. Newsholme in reviewing the relationship between influenza and tuberculosis in England remarks that so far as the national statistics in that country are concerned deaths from tuberculosis are undoubtedly hastened during an influenza epidemic. Of the epidemic of 1889 in Massachusetts, Abbott thought the chief diseases which followed in its train and were intimately associated with it were bronchitis and pneumonia and that tuberculosis when already existing in the victim of the attack was undoubtedly aggravated and in many cases a fatal termination was hastened. Baldwin says that influenza is a frequent and important agent in bringing latent tuberculosis to life. Amberson and Peters take sharp exception to the view of Fishberg, and have collected evidence on the other side. They point out that a comparison of the incidence of 5.4 per cent among hospitalized tuberculous patients at Chicago cannot be compared with a much higher incidence of the epidemic in military camps. Furthermore, the incidence at some sanatoria was low, while at others it was high, nearly as high as for the community at large. Hawes in discussing the epidemic among Massachusetts sanatoria says that Lakeville escaped entirely, while Rutland which consisted chiefly of ambulatory cases, less easily controlled, had an influenza incidence of 18.3 per cent among the patients and 21.3 per cent among the employes. At the Montefiore Home, the proportion of tuberculous patients and employes contracting influenza was practically the same as among the nontuberculous employes and about the same percentage of both groups developed bronchopneumonia. There is a fallacy in the comparison of incidence in the different institutions, and this is shown by the work of Jordan, Reed and Fink, who found that in the various Chicago telephone exchanges the attack rate varied from five per cent to twenty-seven per cent although the working conditions were approximately the same. The influenza rate in one section of the Students Army Training Corps in Chicago was 3.9 per cent, while in another section it was 39.8. In like manner Frost found the incidence in Louisville, Ky., to be fifteen per cent, and in San Antonio, Tex., 53.3 per cent. These figures show the difficulty of comparing rates for various institutions and various groups of individuals. Although Fishberg quoted Rickmann in support of his claim that influenza has no effect upon tuberculosis, Amberson and Peters used Rickmann's work in support of their contention and call attention to the fact that in 30 out of 40 tuberculous persons reported by him who had contracted the influenza, the attack did not produce any aggravation of the lung condition. It is to be presumed that it did in the other ten, and this means that twenty- 
five per cent of the tuberculous patients who contracted influenza had their pulmonary condition aggravated. This should be regarded as a considerable number. According to Stivelman, 11.4 per cent of tuberculous influenza cases died at the Montefiore Home. In a survey of convalescents from the Loomis Sanatorium, Amberson and Peters found that 70 had contracted influenza, or 5.7 per cent of the number surveyed, and that 11.4 per cent of these had relapses in their pulmonary condition, apparently due to the influenza, while 22.9 per cent had died from the intercurrent infection. Tubercle bacilli have been found in the sputa of convalescent influenza patients whose sputum had previously been negative, by Amberson and Peters, as well as by Berghoff, at Camp Grant. The last mentioned found that fifty per cent of his cases showed a reactivation and a positive sputum after an attack of influenza. Amberson and Peters, agreeing with Fishberg in the observation that there has been no increase in the general mortality from tuberculosis within recent months, suggest as a possible explanation that during the epidemic enough of the old cases were carried off to account for a temporary lull. Leichtenstern observed this same phenomenon following the 1889-1890 epidemic. The state of our knowledge concerning the relation between influenza and tuberculosis is clouded by divergent opinions. To further complicate the picture, there are authors who assume a middle ground and contend that there is some truth in both lines of the question. Amelung believes that the morbidity among patients with pulmonary tuberculosis is slight and that influenza takes a milder course in such patients than in the nontuberculous unless the disease is far advanced, but that pulmonary tuberculosis may and sometimes does follow the disease in patients whose lungs were previously sound. Peck finds that in some tuberculous patients the disease has been aggravated, but in the majority the intercurrent influenza did not appear to have been the causative factor in the acute exacerbation of the tuberculosis. Debré and Jacquet state that, as a rule, in Europe tuberculous individuals have been less severely attacked by influenza, but they admit that there are exceptions and that in certain sanatoria in France the morbidity and mortality from influenza have been great. They say the first explanation that comes to mind is that the tuberculous are isolated in hospitals where general hygienic conditions are good. Having concluded that tuberculosis does protect in some measure against influenza, Debré and Jacquet discuss whether the latter has increased the severity of tuberculosis in the subjects who were already tuberculous. They review the literature and arrive at their conclusions, not from statistical records, but from general observations. They consider first those cases of tuberculosis which are open when attacked, and second, latent tuberculosis. Their conclusion concerning the first group is that influenza does not have 
any effect upon the rapidity of evolution of the tuberculous process, except in very rare instances, such as an occasional case of miliary tuberculosis following influenza. They believe, however, that latent tuberculosis is intensified and dormant conditions awakened. It seems difficult to reconcile these ideas. If one type of tuberculous individual is rendered more susceptible to his disease, it would seem reasonable to expect that all types would be likewise affected. Armstrong, studying this matter at Framingham, Mass., finds that sixteen per cent of the entire population had influenza, but only four per cent of the tuberculous group in the community was so affected. Most of the latter were of the arrested type and were going about the community taking their part in industry and exposed to the same degree of contact as was the case with the normal population. The fatality was equally in contrast. Armstrong concludes that there appears to be a relative degree of protection for the highly tuberculized. If we accept these figures at their face value we must conclude either that tuberculosis offers some degree of protection in acute influenza or that the tuberculous of Framingham have been so well trained in sanitation and personal hygiene that they are able to protect themselves against influenza. Certainly this did play a part, to the extent at least that individuals knowing themselves to be infected with tuberculosis, and knowing themselves to be in the presence of a pandemic, became more wary of crowd contact, and in case they did become ill they undoubtedly went to bed at the earliest opportunity. If, on the other hand, this is a true demonstration of relative immunity in a chronically infected individual, the explanation must be sought elsewhere. Does a chronic respiratory infection confer a relative degree of immunity to an acute respiratory disease? Do the germs already on the premises exert, so to speak, "squatters' rights"? Are we observing an example of nonspecific immunity due to local preceding infection? Still another factor must play an important rôle, the factor of race stock. The excess of tuberculosis in negroes, for instance, over that in whites, is in some localities double or treble, while various observers, as Frost, Brewer, and Frankel and Dublin, report that the influenza incidence and mortality among negroes was decidedly less than among the whites. Winslow and Rogers state that in Connecticut the proportion of influenza-pneumonia deaths is lower than would be expected among persons of native Irish, English, and German stock, and higher than was to be expected among Russian, Austrian, Canadian, and Polish stock; while it was enormously high among the Italians. Italians notably have a low death rate from tuberculosis, while the Irish have a high death rate from this disease. In Framingham where the tuberculosis incidence rate for the entire population was 2.16 , the rate in the Italian race stock was .58 and in the Irish 4.80. In Framingham 
there was about four times as much influenza among the Italians as among the Irish. Is this apparent insusceptibility of certain race stock an inherent condition, or is it dependent chiefly on differences in living conditions and in age prevalence in the different races? Probably, it is chiefly the former, since Frost found that among negroes the incidence of influenza was lower even though the living conditions were much poorer than among the whites. Armstrong's survey has thrown light upon the effect of the influenza upon previously tuberculized individuals. In a survey of 700 who had the acute disease there were ten arrested eases of tuberculosis, or 1.4 per cent. All these had been known to be arrested cases previous to the epidemic and in none of them did the disease appear to have been actively and permanently lighted up. On the other

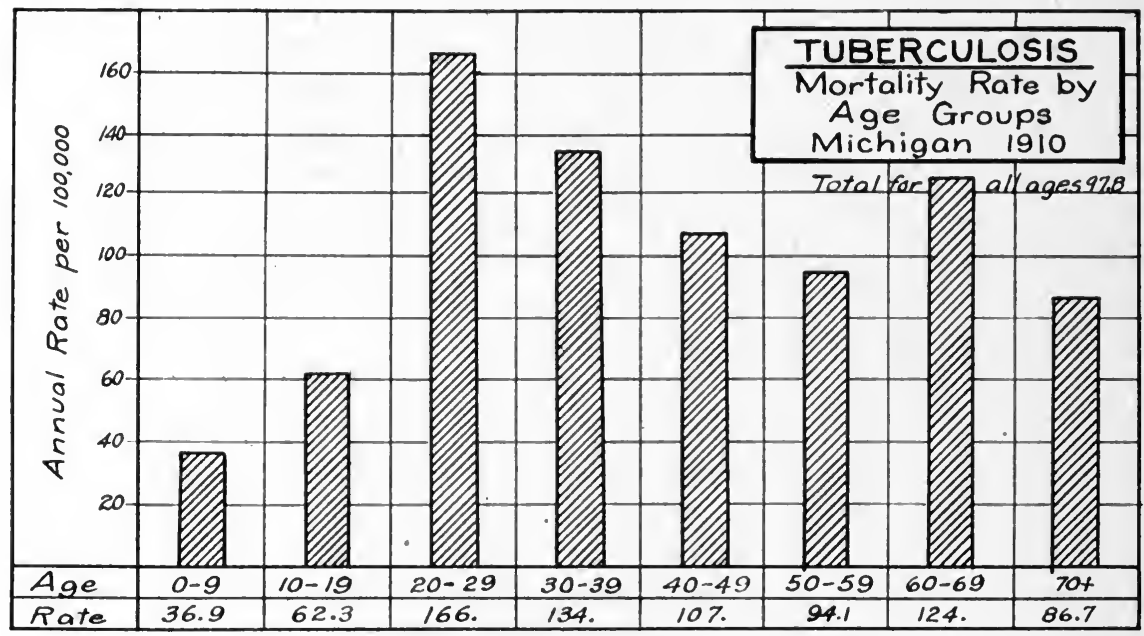

Fig. 62 .

hand, 13 cases, or two per cent of the 700, were found to have active tuberculosis which had hitherto been undiagnosed, and there were eight additional cases with indefinite bronchopulmonary signs. These were believed to be cases of incipient tuberculosis. Vaughan (Warren) concludes his paper with the following:

"If we may judge merely by the balance of evidence and risk any conclusions from such conflicting testimony, we may sum up as follows: (1) Great variation in the interaction of tuberculosis and influenza must be expected, because of the many stages at which the tuberculous may be attacked, because of the altered mode of living of known consumptives, and because of the protected life of most of them. (2) Phthisical patients as a group, are relatively insusceptible to influenza infection. This may be due to the tuberculous process itself or to some extrinsic but nearly related cause. (3) But many individuals with pulmonary tuberculosis do get influenza. (4) And the disease, having been contracted, in many cases hastens the fatal termination of the tuberculous process. (5) It may be that this phthisical exacerbation occurs more fre- 
quently in individuals with latent tuberculosis, individuals who are not at the time mobilizing their protective antibodies."

Reinfection in Tuberculosis.-Koch observed that a tuberculous guinea pig reacts quite differently on the second inoculation. When tubercle bacilli are injected under the skin of a healthy guinea pig the wound closes and apparently heals perfectly within two or three days. There seems to be no abnormality for from 10 to 14 days, after which a hard nodule appears at the point of inoculation. This softens and may leave an ulceration which continues as long as the animal lives. A little later the nearest lymphatic glands become enlarged and hardened. The effects of the inoculation may spread widely throughout the animal body. When a guinea pig already tuberculous receives a pure culture under the skin the wound heals, but a few days later the area around the point of inoculation becomes dark and indurated; gradually, but within a few days, this area becomes necrotic, sloughs, and leaves a flat ulcerated surface which usually heals quickly and permanently. This experiment has been modified and repeated upon many animals. The conclusion which some have reached from these experiments is that superinfection of a tuberculous animal is difficult. Römer and others have tried to reinfect tuberculous animals by feeding, by inhalation, by means of dust in spraying, and in short, by every known method, and have concluded that superinfection of an already tuberculous animal is not so easy as primary infection and is accompanied by different phenomena. An analogy is drawn between tuberculosis and syphilis. As long as a man has syphilis he cannot be superinfected whatever the degree of exposure to which he submits himself. If he has entirely recovered from syphilis he may be reinfected. Granting the difficulty, and even the impossibility, of superinfecting one who already is infected with tuberculosis, should we eall this condition one of immunity? In other words, is it proper to say that one who has tuberculous infection, whether it be quiescent or active, is immune to tuberculosis? It seems to us that we have come to use the word immunity rather loosely. If we understand the meaning to be attached to the word in these loose connections there possibly is no objection, but we should clearly understand just what we mean. Some prominent investigators go so far as to hold that it is a good thing for a child to become infected with bovine tuberculosis. It is admitted that this strain of the bacillus is less fatal to human beings than the human type. Some hold that one who has tuberculous infection in latent form need not concern himself about possible subsequent infection. It is a matter of indifference to him whether he inhales or ingests more tubercle bacilli, they can do him no harm. Since the majority of adults already carry tuberculous infection all precautions looking to the prevention of reinfection are unnecessary; therefore, the destruction of 
bacilli, the burning of sputum, laws against spitting on the streets, in short all care we have given to the spread of the infection, is without value. Is this conclusion valid? Should we in our attempts to eradicate this disease, inoculate our children with bovine tubercle bacilli and rest assured that, while the majority of them may develop glandular, bony, or other forms of bovine infection and while many of them will be more or less crippled for life and still others will die, those who survive childhood can go through life defiant of the more deadly human type? We do not think that any one is prepared to make such a recommendation. It is true that superinfection is more difficult than primary infection and that the animal already infected more stoutly resists a second infection. As we understand it, the animal carrying a tuberculous infection is not immune to tuberculosis. In no proper sense can this be true. Such an animal is sensitized to tuberculosis but is by no means immune to it. The body cells of such an animal are pouring out special secretions, which, in an ineffective way are destroying the tubercle bacilli already established in the body. When an attempt is made to reinfect such an animal, the tubercle bacilli, before they can become implanted in the body and find a suitable place in which to multiply, are destroyed. Koch showed that while it is difficult to superinfect an animal already carrying tuberculous infection, large or virulent doses of bacilli lead to the speedy death of the animal. A healthy animal without any tuberculous infection bears without recognizable disturbance large quantities of tuberculin, while in the tuberculous a much smaller amount causes a high fever and may lead to speedy death. The bacilli that are used in attempts to superinfect are destroyed before they accommodate themselves to the tissue in which they are placed. In their destruction, poisons are set free and if they be introduced in sufficient quantity the animal dies more or less promptly from the effects of their disintegration. In our opinion, sensitization to tuberculosis is evidence of an attempt on the part of the body cells to destroy the bacilli in the body and incidentally to destroy reenforcement from without.

There is a wide difference of opinion after nearly 20 years of trial as to the value of tuberculin in the treatment of this disease, but even its most ardent advocate would not recommend that it be administered in uncontrolled doses without any reference to the amount or activity of the preparation. Granting that the individual with latent tuberculosis is able through the secretions of his body cells to destroy all the living tubercle bacilli that may be introduced into the body, it must be admitted that in so doing a poison to which this individual is especially susceptible is set free in the body. It may be that we have overestimated the danger of reinfecting one who already carries tuberculous infection, but to say that such a person may take into his body indiscriminately, whether it 
be by inhalation or ingestion, any number of any strain of tubercle bacilli, is to preach a doctrine which we believe is wholly at variance with scientific facts and would lead to most disastrous results.

In answer to the statement made above, we are told that the animal with tuberculous infection is immune not only to exogenous bacilli, but to those taken from its own lesions. We grant the truth of this, but it does not alter in the least, so far as we can see, our opinion. To take bacilli which have established themselves by building around themselves protective structures, in the lymph node for instance, and place them. naked, as it were, in some other tissue of the body, is certainly to greatly increase the chances of their destruction. With all pathogenic bacilli there is undoubtedly a critical period when they are establishing themselves in the midst of the cells of the body. In inoculating a nontuberculous animal, we are sending invaders into unprotected, unarmed, and unsuspecting communities. In inoculating a tuberculous animal, we are sending like invaders into a territory, every citizen of which is armed and prepared to fall upon the enemy. The conditions are wholly unlike and quite naturally the phenomena manifested are dissimilar.

All admit that autogenous reinfection or superinfection takes place. In this way the disease extends from lymph node to lymph node, from organ to organ, by routes fairly direct or more or less circuitous, until the most distant and vital parts of the body may be reached. It is probably true that in the great majority of instances tuberculosis begins as a strictly localized infection and the augmentation of its forces is due to multiplication within the body and not by accession from without, but at the same time it is a demonstrated fact that even dead and disintegrated tubercle bacilli, broken beyond recognition, thrown into the tuberculous animal do an injury which would not be felt by a nontuberculous animal.

We regard the phenomena of superinfection in tuberculosis of so much importance that we shall go somewhat into detail. The announcement of the results of his experiments on this point by Koch induced many to take up the matter experimentally. At first, Baumgarten, Arloing, and others obtained results which were apparently a direct contradiction of those reported by Koch. These investigators found that the injection of large amounts of tubercle bacilli into tuberculous guinea pigs caused death within from six to twenty hours; in other words, their work confirmed a previous finding of Koch that the injection of relatively small doses of even dead tubercle bacilli produces speedy death in tuberculous guinea pigs. Römer and Hamburger studied the phenomena of superinfection with great care and skill. From their studies it was found that the following conditions must be secured before the phenomena can be called into existence: (1) The primary affection must be slight and 
the tuberculosis resulting therefrom must be pursuing a chronic course. (2) The time of reinfection should be as long as possible after the primary infection. The increase in resistance becomes greater the older the tuberculous process in the animal. (3) The dose on reinfection must be small, although it may be large enough to produce the disease in a fresh animal.

Hamburger exposed guinea pigs with chronic tuberculosis to the inhalation of dust sufficiently rich in bacilli to invariably cause the disease in fresh animals. In this experiment he found that he was unable to develop tuberculosis in the lungs of the tuberculous guinea pigs thus exposed. Of course, he could not tell what effect the superinfection had upon the tuberculous processes already under way in the animals subjected to this experiment. Schiek induced tuberculosis in the anterior chamber of one eye in rabbits. Thirty-seven days later the other eye was inoculated, and 36 days later the most carcful examination revealed no tuberculosis, but tubereulous changes did occur after 60 days. Finally, Kraus and Hofer apparently explained the phenomena of superinfection. Guinea pigs which had been primarily infected subcutaneously, intraperitoneally, intracutaneously, or intratracheally, received intraperitoneal injections of tubercle bacilli. From time to time some of these injected bacilli were removed from the peritoneal cavity and examined. It was found that within from 15 to 30 minutes after the introduction of living tubercle bacilli into the peritoneal cavity of an animal with chronic tuberculosis, changes in the bacilli were evident on microscopic examination. Even after this short time the bacilli under the double stain showed blue granules and many of them resembled diphtheria bacilli.

In some bacilli the number of granules was small; in others they were numerous. Other bacilli showed the appearance of splinters, and others still were stained red, with apparently an uncolored surface. The blue granules, showing blue because they were no longer acid-fast, were found to be of very variable size; some were barely visible under the highest powers, while others appeared like cocci. These granules recall the observations made by Pfeiffer in his classical experiment on digestion of cholera vibrios in the peritoneal cavity of an animal which had previously been sensitized to this organism; in other words, Kraus and Hofer apparently demonstrated that fresh tubercle bacilli injected into an animal with chronic tuberculosis suffer partial digestion. In the peritoneal cavity, these bacilli are not engulfed in phagocytes but undergo an extracellular digestion. This process may persist until certain bacilli are completely digested, while others are only partially affected. Another interesting point was brought out in these experiments. The secretions of animals having either human or bovine infection acted in like manner upon these two varieties; in other words, the human type sensitized 
animals to the bovine type and vice versa, while neither of these types sensitized to avian bacilli nor do the avian sensitize to mammalian bacilli. The study of the phenomena of superinfection in tuberculosis discloses one of the methods used by the body cells in their combat with invading tubercle bacilli. When we bear in mind that practically all adults are infected with tuberculosis and that only from one-seventh to one-tenth die of this disease, we must admit that the body cells wage against the invading cells a warfare which in the majority of instances saves, at least, the life of the individual. In some, the body defenses prove inefficient and death results. In others, the body cells hold the invading cells in check, oppose their advance, but finally end in defeat. In other cases, the body cells with their defensive secretions never allow the invading cells to pass beyond the area in which they first entrench themselves. In tuberculosis the body cells furnish no antitoxin, because the invading cells contain no toxin. When the body cells are sensitized they pour out a specific digestive fluid which acts upon the tubercle bacilli. This digestive fluid splits the tubercle-cell substance into parts, liberating a poison which, as in other infectious diseases, causes the symptoms of the disease. Since tuberculosis is in the great majority of instances, at the beginning of invasion, at least, a strictly local disease, only the body cells in the invaded locality are at first sensitized. As the area invaded by the tubercle bacilli widens sensitization proceeds in like manner. It, therefore, follows that the development of active tuberculosis is due to the failure of the body cells to cope with the invading organisms. If man could discover some harmless agent which, injected into the bodies of children, would generally sensitize their cells to pour out a specific secretion against tubercle bacilli, the prevalence of this disease would be greatly curtailed. We hare already shown that the nonpoisonous part of the tubercle bacillus may sensitize guinea pigs. However, the amount of work done along this line has not been sufficient to enable one to speak with authority. The evidence we have should encourage further research.

The tubercle bacillus, alive or dead, when injected into the body sensitizes only those body cells with which it comes in contact. The sensitizing agent which we seek must be a soluble substance. It must be readily diffusible, and in all probability permeation of body cells is necessary to their sensitization. On account of their resistance to destructive agents unbroken tubercle bacilli are not suitable as sensitizing bodies. Up to the present time most attempts to find, in the tubercle-cell substance, an agent with which this disease may be combated have been confined to the poisonous groups in the molecule of the tuberculoprotein. Undoubtedly, the repeated injection of tuberculin in well-regulated doses does give increased tolerance to this poison, but that immunity, or even increased resistance marked enough to be of great value in bacterial in- 
vasion, can be secured in this way is not probable. Many men have spent much time in trying to immunize animals to tuberculosis. Koch made the following statement concerning his efforts in this direction:

"All attempts with the unaltered, living or dead tubercle bacilli to secure absorption by the tissue after subcutaneous, intraperitoneal, or intravenous injections, have failed me and others who have worked along the same line. When dead tubercle bacilli are injected subcutaneously they invariably cause pustules, forming abseesses which continue for months and in the discharges from which tubercle bacilli easily recognized by staining may be obtained. I did secure somewhat better results by injecting dead tubercle bacilli into the peritoneal cavity, but invariably such injections are followed by circumscribed inflammatory processes which terminate in the majority of instances in occlusion of the intestines and the death of the animal. When dead tubercle bacilli are intravenously introduced into rabbits there are formed in the lungs tuberculous nodules resembling those caused by living bacilli and these nodules contain unaltered tubercle bacilli. In this case also, absorption does not proceed as we would wish. Having found that unbroken tubercle bacilli are not suitable for purposes of immunization, we have tried their disintegration by chemical agents. Tubercle bacilli can be broken up and their constituents carried into solution only through the action of strong alkalies or dilute mineral acids at boiling temperature. By these agencies the bacillary substance is so altered and so deeply disintegrated that it has no immunizing effect." .

So-called antibodies, such as agglutinins, precipitins, and complement fixation substances, are easily brought into existence by inoculation with the tubercle bacillus. Löwenstein injected intravenously into goats slightly virulent and then highly virulent tubercle bacilli. The serum of the animals thus treated showed a high agglutination titer ( 1 to 5,000$)$, but did not manifest the slightest protective action even when the bacilli were allowed to stand in contact with the serum for 24 hours at $37^{\circ}$ C. These results confirm what we have long held concerning these socalled antibodies. In our opinion, they are products of infection and are not measures of the degree of immunity.

A volume might be filled with citations of the work done in attempting to establish immunity to tuberculosis. The list of tuberculins prepared and used numbers more than 100, but in practically all of these the poisonous part of the bacillus has been selected. Tubercle bacilli, exposed to the sunlight, to the action of the $x$-ray, and to a great variety of chemical agents, have been employed in these attempts, but up to the present time (1920) no preparation has proved efficient. The last word has not been said concerning the importance of phagocytosis in the combat against the invasion with this bacillus. It was believed by Metchnikoff that in animals highly resistant to this infection phagocytes envelop and destroy the bacilli, but even Wright admits that his former claims concerning the prognostic value of the opsonic index are overstated. In the natural course of tuberculosis in man we so seldom find tubercle bacilli in the phagocytes that it is not believed phagocytosis plays any important rôle in resistance to this disease. 
Bushnell believes that we are finally to be saved from tuberculosis by being thoroughly tuberculized. He divides the peoples of the world into tuberculized and nontuberculized races. In the former, tuberculosis is a chronic, slowly progressive, relatively benign, disease. In nontuberculized races it is an acute, epidemic, highly fatal, disease. Certain localities, especially in tropical and semi-tropical countries, have had the reputation of being free from tuberculosis, but when an attempt has been made to use these places as resorts for those afflicted with this disease the natives have quickly developed tuberculosis and the death rate has been high. In our own country we have in large numbers, so far as races are concerned, the white, the negro, and the Indian. Bushnell shows, especially by statistics of deaths in Charleston, S. C., that before the Civil War the tuberculosis death rate among whites and blacks was about the same. He concludes from this that even then the negro race was already tuberculized. Following the Civil War the death rate among negroes rapidly increased, soon exceeded, and has continued to exceed, the death rate from this disease among the whites. The great increase in the death rate from tuberculosis among the blacks after the Civil War is attributed by Bushnell to poverty and bad housing conditions. He says:

"The facts adduced as to the tuberculosis of the negro justify the following conclusions: the negroes as a race in the United States have long been in contact with the virus of tuberculosis. They are probably as well or nearly as well tuberculized as the white race. This is shown by the fact that when they were slaves, when their masters gave regular employment, provided food and to some extent looked after their health, their tuberculosis rates differed little from those of the whites. When their emancipation thrust them unprepared into the struggle for existence their sufferings and errors were revealed in an enormously increased mortality not only from tuberculosis but from other diseases."

Bushnell is quite certain that tuberculized races acquire a certain degree of immunity. This is in no sense inherited, but must be acquired by each individual, and the danger lies in the acquisition of this immunity. He says:

"Civilized man can never escape the dangers of infection with the tubercle bacillus. But if we did escape the dangers of infection, we should also lose the benefits of tuberculization. Supposing that with extraordinary energy and sagacity we banish all tuberculosis from our town and rear an absolutely uninfected group of children. Having passed childhood under the irksome restrictions that would be necessary, the time must come when they shall be permitted to enter the outside world, for the fear of disease cannot remain the paramount consideration during life. As soon as, now adults or adolescents, they leave the sheltering confines of their native town, they will be exposed to the dangers of primary tubereulous infection and that at an age when the world beckons most invitingly and when prudence is least developed. In fact they would be in a hardly less dangerous situation than the tropical native when he first enters a civilized community. Prophylaxis has simply resulted in exchanging the danger 
of a chronic and usually relatively benign infection for the danger of an acutely fatal infection."

Bushnell is of the opinion that our Indians are just now becoming tuberculized and that in consequence of this the birth rate is exceeding the death rate and the Indian promises not to disappear from the earth but to multiply and reproduce his kind. In 1891 an effort was made to try the Indian as a soldier. Authorization was obtained for eight troops of Indian Cavalry and nineteen companies of Indian Infantry. In the years immediately following, over eight hundred Indians were enlisted in the army and made up exclusively Indian organizations, but on account of the wide and deadly prevalence of tuberculosis among them this plan was given up. It has been proposed by those having in charge Indian schools that all children sent to such schools should be tested with the tuberculin test and that those giving response to this test should not be sent to school. Bushnell is of the opposite opinion and says :

"In the writer's judgment, children should not be allowed to attend the larger schools unless they have a positive skin reaction to tuberculin. There is danger here that those who react positively may be on the point of breaking down with manifest tuberculosis of severe type, so that long railway journeys will lead to disaster. We would therefore make the further suggestion that no Indian ehild be sent to boarding school unless he is in apparently good health, shows at least no marked glandular involvement, gives no physieal signs of tubereulosis of the lungs and has been positive for the Pirquet reaction for at least one year. In other words, a tuberculous vaceination should be required as well as a vacination against smallpox."

Bushnell is of the opinion that we should give special attention to the protection of our children, and this protection should consist in endeavoring to prevent massive infection among them.

Individual Contact.-In advanced cases of pulmonary tuberculosis the saliva and the moist lips bear the bacilli, often in great numbers. If an individual of this type press his moist lips against a glass slide and the spot be properly prepared and stained tubercle bacilli will often be demonstrated. Some years ago we saw professionally a lusty drummer of about 30 years of age who acknowledged that he had spent many nights with a prostitute in an advanced stage of tuberculosis. Within a few weeks he developed miliary tuberculosis and died within three months. This is undoubtedly an exceptional occurrence, but it illustrates the great danger that lies in too intimate contact with tuberculous individuals. Even the well-trained, highly intelligent; and most careful consumptive leaves his infection on those articles at the table which come in contact with his mouth, such as forks, spoons, cups, and glasses. His napkin and his handkerchief generally bear the bacilli, even when they are used only for wiping his lips. His fingers frequently carry the infection; his tooth brush invariably, his pencils and penholder usually. If properly instructed he holds a rag or handkerchief in front of his 
nose and mouth when he sneezes or coughs. The former he burns before it dries and the latter he immerses in some disinfectant until it can be boiled. If he fails to provide this protection when he sneezes or coughs and the spray strikes directly in the face of some one else, infection through nose or mouth may result. The extent to which the consumptive may scatter his infection on his clothing, his bedding, and throughout the room, depends upon the care exercised. In ordinary breathing the exhaled air is free from bacilli and in ordinary talking the danger of infecting those about him is negligible. On the other hand, the careless, ignorant, untrained consumptive, scatters his infection over his clothing, bedding, and about his room. We once visited a man with advanced pulmonary tuberculosis. We found him sitting in an invalid chair spitting at the stove. Two little children were crawling over the ingrain carpet which covered the floor, picking up and tasting of whatever they found. These children died of tuberculous meningitis before the less rapidly progressing disease of their father killed him. There is some risk in living in the same room even with a careful consumptive. This danger multiplies as his carelessness increases. The careful consumptive expectorates into paper cups which are burned; the careless man expectorates wherever he finds it convenient.

Infected Homes.-Flick; of Philadelphia, and others have pointed out that certain houses become infected and that new families coming into such houses, even those without previous history of the disease, are likely to become infected. This is true not only of the slums and the homes of the poor in the eity, but applies also to the homes of the better-to-do and even of the rich, both in city and in country. Quite naturally, bacilli. deposited in dark, damp places retain their vitality for a longer time than those which find their resting places in dry, well-ventilated, sunny places. The laws of many states and cities provide that when a home is vacated by a tuberculous family the rooms and fixtures be thoroughly sterilized before a new family can move in. Williamson, of Edinburgh, finds that the number of cases of pulmonary tuberculosis in any district is almost precisely in direct proportion to the number of houses of one and two rooms which exist in it. He states that the City of Edinburgh is divided into wards and that the number of cases of tuberculosis in the city follows almost precisely in direct proportion the number of one and two-roomed houses which are found in the wards. For 1912 the morbidity rates of pulmonary tuberculosis per 1,000 inhabitants according to the number of rooms in the house in Edinburgh, are as follows:

$\begin{array}{ll}\text { One room } & 6.9 \\ \text { Two rooms } & 5.6 \\ \text { Three rooms } & 3.5\end{array}$

Four rooms and over 1.4 
Like conditions are shown in Glasgow and Greenock. On the question of housing and tuberculosis, Williamson makes the following statement:

"The key to the situation lies unquestionably in the housing question. If statisties prove anything, they prove to the hilt that the disease originates and thrives in direct proportion to the unsatisfactory nature of the house and its surroundings; and they prove invariably that an improvement on these conditions is followed, as by the law of cause and effect, by an immediate fall in the tuberculosis rate. One almost wonders why we are so slow to learn these facts. Every local authority knows them well, and every medical officer of health, I am satisfied, is intimately acquainted with them; but it must be admitted that the progress in housing reform is, to say the least of it, not in proportion to our knowledge in regard to these matters. Innumerable illustrations exist of the improvement which follows the outlay of some expenditure in improving the housing conditions, which ought to serve to convince many of us who might be slow to believe. Possibly every local authority can furnish its own statisties. We in Edinburgh certainly ean. Wherever improvement schemes have been earried out, an immediate improvement has primarily been noticed in the tubereulosis death rate. In every large center of population precisely the same experience has been met."

The same authority is quite insistent that insanitary houses should be pulled down, for he holds that as long as they exist they will be occupied. It is largely, but not altogether, a matter of poverty that leads to the occupation of these houses. So long as these slum dwellings exist there will be people who will oceupy them and spend otherwise their money which should be legitimately laid out in rent. He is quite convinced that so far as tuberculosis is concerned preventive measures center largely in the housing question and that irrespective of cause there is an urgent need of dealing more effectively than has heretofore been the case with congested areas, dense foci of population, and insanitary dwellings.

About 20 years ago much was said concerning the "lung blocks" in New York City. In 1903 Biggs stated that in the most overcrowded districts of New York City the mortality from consumption was more than three times that obtaining in the less crowded districts. In 1898 more than thirty-five per cent of the people in certain districts in New York lived more than two persons in a room. The death rate, not only from tuberculosis but from all causes, was from two to three times greater in these areas than in districts in which less than ten per cent of the population lived more than two in a room. From 1900 to 1910 all deaths in New York City were charged to the borough in which they occurred. This quite naturally gave an excessive death rate to those boroughs in which large hospitals are located. Beginning with 1911, deaths were distributed to boroughs of residence. In the last-mentioned year the death-rate from all forms of tuberculosis in Manhattan Borough was 233.3 per 100,000, being the highest death rate from this eause exhibited by any borough in the city at that time. The death rate from tuberculosis in Manhattan Borough has, according to the U. S. Census, gradually fallen until in 1917 
it stood at 180.4 per 100,000 . How much the improvement in the tenement houses in this borough has contributed to the fall in the death rate it is quite impossible to say. In the Bronx Borough the death rate from tuberculosis is higher by a considerable percentage than it was in 1911 .

Writing in 1919, Younger says that 471,000 people in Glasgow are living in houses of one and two apartments, with the following percentage distribution :

55.7 live more than two in a room

27.9 live more than three in a room

10.7 live more than four in a room

3.4 live more than five in a room

The same author states that an investigation made in Glasgow in 1911 showed that in houses of one, two, and three apartments there were 2,852 children and 6,543 adults living in regular contact with 2,275 persons suffering from phthisis; 1,244 persons were sleeping in the same room with patients, while actually 1,596 persons were sleeping in the same bed with 1,143 patients, which means that in some cases two persons were sleeping in the same bed with a phthisical patient. In a subsequent report of October, 1916, it was found that of 1,228 cases registered between January and June, 1916, twenty-four per cent only had a separate room for sleeping, while in the case of fifty per cent the bed had to be shared with another person. In Edinburgh for the years 1911-1915, while 112 children under five years of age died in one of the better-class wards, no less than 797 died in one of the thickly congested wards; and in these two wards the deaths of children under five years of age from tuberculous diseases numbered 12 and 64 respectively.

Chalmers, studying conditions as they existed in Scotland in 1918, found that out of 631 male cases discharged from sanatoria with disease "arrested" or "improved" before May 31, 1916, it appeared that after an average period of five and one-half years from the date of discharge from the sanatorium less than half these cases survived. All had been benefited materially by the instruction and treatment which they had received in the sanatorium, yet more than half of them succumbed under the conditions to which they returned. A study was made of 753 households to which patients had returned from sanatoria and it was found that in only thirty-three per cent of the cases the patient had a separate sleeping room, while in the case of every 100 persons 60 others shared their beds. The death rate from phthisis per 1,000 in the old insanitary areas in Liverpool fell from 4 to 1.9 when the new housing for these people went into effect. According to Younger, the rule still holds in Scotland that a room and a kitchen constitute a house. The same author adds that in one of the southern counties in Scotland in the years 1914- 
1915 out of 263 eases of tuberculosis which were reported 159 occurred in houses of one, two, and three apartments. In a small, badly-ventilated house of two rooms there lived father, mother, and nine children. Of these the mother and three children died from tuberculosis and two other ehildren became infected.

To find evidence of insanitary housing it is not necessary to go to Europe, or even to New York or other of our own great eities. There is not a city of 25,000 or more inhabitants in this country in which considerable numbers of people will not be found to be living in most insanitary abodes; indeed, we might truthfully go farther and say that in most villages and even in isolated farmhouses tuberculosis is largely

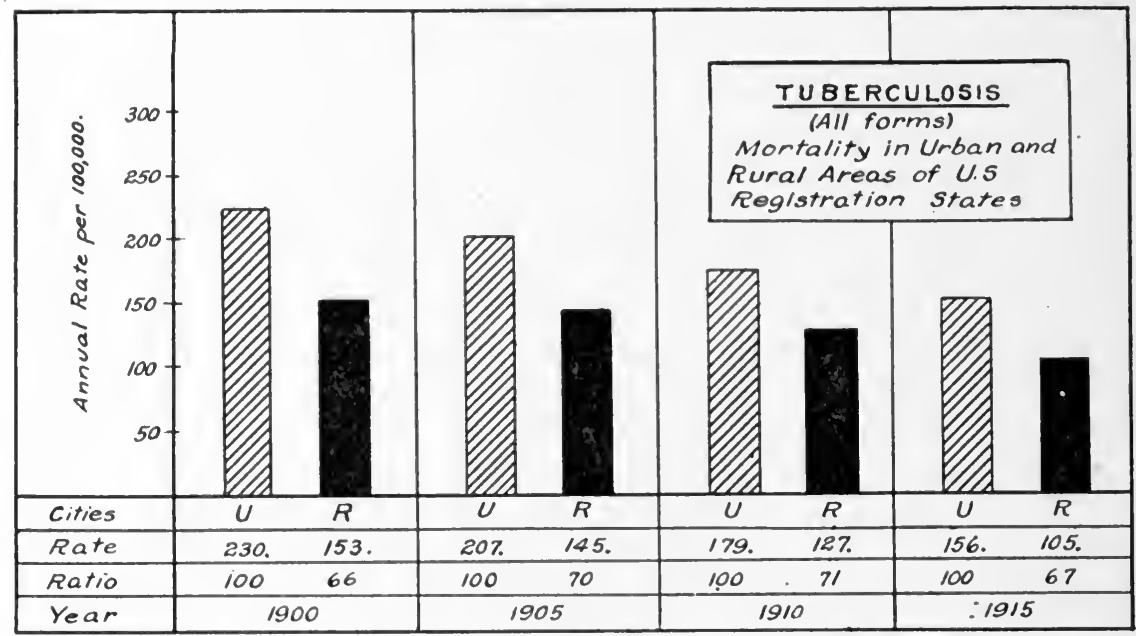

Fig. 63.

a matter of housing. In 1916 the Michigan State Board of Health made an investigation into the housing problem in some of the smaller cities of the state. In one of these, justly famed for its pleasing exterior, there were found 43 buildings with 176 interior rooms occupied by families large and small. The number of these rooms receiving a fair amount of sunlight was 55. Of those in which the light was too poor to enable one to read on a cloudy day was 31 . In 41 it was impossible to read ordinary type on a bright day, and in 49 there was absolutely no sunlight. In another small eity there were 46 houses with 259 interior rooms. Eightyfour of these had no ventilation, 63 only that secured from transoms, 68 had some but insufficient air, thus making a total of 215 rooms with inadequate ventilation and 44 with a fair amount. The following is an illustration of some of the human abodes in the smaller cities of this state: 
"An overcrowded, poorly kept, rooming house occupied two upper floors of a threestory building. Its left wall is next to another building and is not useful for light. On the other side of the building there were once four rooms on each of the two floors, lighted only by 'lot-line' windows. Eleven years ago these windows were wiped out by a three-story building on the next lot and ever since, light housekeeping couples have tried to use the building. The hall is so dark that one must grope his way in it. The water-closet on the second floor is a windowless pocket that was in foul condition when seen at three different times. The water-closet on the third floor, off the dark hall, has no light and contains a wash bowl that is the only sink for 11 persons who live in five groups. The occupants of these windowless rooms cook and eat, as well as sleep, in them. They have no place for storing food and almost no equipment for washing anything."

Many volumes and innumerable papers have been written concerning the relationship between housing and tuberculosis with the almost unanimous agreement that insanitary housing is a most important factor in the breeding and dissemination of this disease. The individual physician runs against this problem almost daily in his practice. Some have demonstrated great ability in improving home conditions. In many instances sleeping porches have been added to tenement quarters, while in others fire escapes, flat roofs, tents in the yard, and other means have been employed in order that the tuberculous patient may live, at least part of the time, out of doors, and in order that other members of the family might have a greater degree of protection against infection. The housing problem is one which the medical profession alone cannot control. It involves poverty, ignorance, and indifference on the part of the tenant and the greed and heartlessness of the owner. No country can claim any great degree of eivilization so long as it permits the insanitary housing now in evidence in practically every city and village and is not unknown even in isolated farmhouses. Building codes have been presented to municipal and state authorities and in many localities protection against insanitary housing has been provided for by legal enactment. There has been great improvement in this direction during the last 20 years, but we have hardly started on the long road which we must travel before satisfactory results are actually secured. While the burden of seeking this improvement is too heavy to be borne by the medical profession, it is the duty of every individual whose intelligence permits him to see the need to call loudly and continuously for help. The housing problem concerns not only tuberculosis, but many other diseases, some of which are intellectual and moral as well as physical. The eradication of tuberculosis is largely an economic and industrial problem. It should be understood that the housing problem concerns not only the home, but the school, the business house, the factory, our penal and charitable institutions, in fact every building in which man plays or works, sleeps or eats, or passes any part of his existence. 
Garden Cities.-The slowly lengthening period of average life and the still more slow retrogression in morbidity among the civilized peoples of the world owe much to the innate esthetic sense which has always been a strong compelling force in the nature of man. Man has always striven for the beautiful, at least what he thinks beautiful. The discovery and utilization of window glass admit sunlight, the most deadly foe to bacterial life, into the home, shop, factory, and other places of rest, play, or work. Improved methods of heating and ventilation have enabled man to crowd together with less danger than in past times. It would be impossible to control infectious diseases in our great cities of today had not the much abused plumber acquired his present skill. Improved means of transportation have rendered it possible for congested areas to at least partially empty themselves. The skill of the architect has enabled him to build sanitary houses many stories in height and thus to increase density of population without endangering life or health. Most of our cities have grown up by chance, as it were, without supervision from any one. It is only within the past few years, in this country at least, that the location and conduct of slaughter houses came under city control and their owners compelled to submit to sanitary regulation. Even at the present day manufacturing establishments, discharging from their chimneys tons of unburned coal, pollute the air of cities, soil the furniture and furnishings of homes, and even penetrate the lungs of citizens. The careless and indifferent still expectorate on our sidewalks. These and many other ill conditions offend the esthetic sense of a community, and for this reason, rather than from a desire to protect health, their abatement is being demanded more persistently year by year. The city designer and the landscape gardener desire to incorporate in their plans much open space, which they seek. to beautify by the esthetic products of both nature and art. One of the oldest Garden Cities in the world is our own Capital-Washington. Here the great French engineer, L'Enfant, had a free hand. It is said that he took a gridiron and placing a wheel on top of it made this his model. He certainly succeeded in making a beautiful city. The amount of land and air space included in the open areas is greater than that found in any other national capital; but it must be borne in mind that disease and death may lurk in flowery places. Of course, we cannot know whether a thought of improving public health influenced in any way the design so successfully worked out by L'Enfant. In all human probability he had no thought of tuberculosis or of any infectious disease, but he desired to make a beautiful city. There is an uncertain rumor which has come down through the century or more since these plans were made that the designer, having had recent experience during the French Revolution in the barricaded streets of Paris, arranged the intersections of 
his radiating avenues with his rectangular streets with the idea that guns placed in these circles could sweep the city in every direction. Whether his ideal of beauty or his thought of armed protection in case of revolution were the only motives that led to his design we eannot say, but we do feel safe in saying that in all human probability the control of tuberculosis never entered his mind. He desired long vistas with imposing facades, with ample width of street, and frequent opportunity for adornment. If the health of the coming citizens had been uppermost in his mind we dare say he would have made a different eity. As was shown years ago by Kober, Washington has its proportion of slums, of insanitary homes, of buildings in which tuberculosis breeds and from which it disseminates. Since the beginning of the present century much attention has been given to the planning of Garden Cities and in doing this a new element, the idea of preserving the health of the people, is dominant. In this country many so-called Garden Cities have come into existence-they have not been altogether successful-there has been the usual quarrel between owner and tenant, but, on the whole, this marks a move in the right direction. In England even greater attention has been given to the designing and building of model villages and cities. The movers in this matter deserve our heartiest support, but they must never forget that insanitary abodes are still possible in beautiful cities. The spread of tuberculosis is dependent upon something besides the density of population-the number per acre. We have in mind a Scotch house, one room and a kitchen, the only home on a farm of 1,500 acres in the mountains of Tennessee, and in this home tuberculosis has grown as in an incubator and without doubt the seed has been carried by members of the family to distant homes. We desire beautiful cities, beautiful homes, substantial school houses, properly placed workshops, etc., but even above beauty we must demand that there be in none of these a breeding-place for that arch enemy to mankind, the tubercle bacillus, that microscopic unicellular organism which has slain more than the sword. In our opinion, even the best cities of the world today are only milestones in the progress of civilization and that the best of them will soon be past and forgotten. In England the tendeney seems to be to provide for the excess of population in the old cities by spreading the people out over the country. In America the plan seems to be to provide for the excess in the congested districts by building skyscrapers. Possibly the Englishman, having so little land in his small island, desires to be near the soil, while the American, having such vast acres, desires to get away from it as far as possible. Time alone will tell the direction in which improved housing will be most satisfactory. Personally we must admit that in this matter we are inclined to the English view. The housing problem, as well as the designing of model villages and cities, fall within 
the province of the engineer, the architect, and the landscape gardener. It may be worthy of note that the Garden Cities already in operation in England cover areas running from 40 to nearly 4,000 acres. Of these, Letehworth is the largest, with a death rate just before the war of 4.8 per 1,000, while that of old eities of about the same population ran from 18 to 20 per 1,000 . It must not be inferred from this, however, that the low death rate at Letchworth could be ascribed altogether to the improved conditions of living. It must be evident that improved housing is in all cases a matter for local and individual consideration. The plan adopted will depend upon many things, such as, the site available, the population to be accommodated, the occupations of the citizens, the propinquity of laborers to their workshops, etc.

In many places in this country we are building Garden Cities, although it is rarely that one hears this name applied to them. There is no uniformity in their planning and it is the esthetic sense in man, coupled, in the more intelligent, with the desire to improve the health conditions under which they live that leads to these settlements. They are to be found in the vicinity of many of our large cities. It is probably fortunate that there is great diversity in these material attempts to improve living conditions. These are manifestations of man's desire to smooth out the wrinkles on the face of Nature and to make of this earth a more beautiful, as well as a more healthful, habitation for himself. We ean rest assured that sooner or later man's desire for the beautiful and his abhorrence of the ugly will cause him to desert the apartment and the tenement and find for himself a more desirable and a more healthful location.

Home Care of the Tuberculous.-The great majority of the 110,285 persons who died in 1917 from tuberculosis in this country were cared for in homes by private physicians. This condition will continue for many years and there is no greater obligation falling upon the practitioner of medicine than that imposed by this disease. In the home he must do what he can for the welfare of his patient and at the same time he must, so far as possible, protect other members of the family from infection. As a rule, there is no better place for the consumptive than his home, provided he be rich; but, as we have already seen, the prevalence of tuberculosis is in inverse ratio to the possession of wealth. It has been said with truth that health is a purehasable commodity, and this is certainly true so far as the consumptive is concerned. In many instances it is not a question whether the home is the most suitable place for the patient, for it is the only place. No open case of this disease should be cared for in the home unless supervision and isolation can be secured. This is a sound statement, but how effective the isolation can be made and to what degree the supervision can be exercised are questions which must be settled in each individual case. In the homes of the well-to-do the effective isola- 
tion of the patient is usually not a matter of difficulty. He can have a bedroom to himself, the doctor can order whatever food he desires his patient to have, and can demand rest to such an extent as his wisdom may indicate. One of the first duties of the physician in treating a case of pulmonary tuberculosis in the home is to take charge of the sanitation of the premises. He must educate the family in the bacteriology and epidemiology of this disease. Where there is sufficient room in the yard or garden sleeping accommodation for the patient can be provided out of doors. When this is not possible the patient should have a room to himself, and when this is not possible he should have a bed to himself, and the physician or the trained nurse should decide about the fixtures, furniture, and all that pertains to the room. If some one else must oceupy the room and there is a choice between an adult and a child, the former should be selected. Of course, the sputum should be burned and in advanced eases of pulmonary tubereulosis the feces should be disinfected, preferably with chlorin, before discharged into the sewer. The trained nurse, is, as a rule, better prepared to take charge of the details of the house than the physician. On the bed an oversheet should protect blankets, and sheets, pilloweases, night dresses, and handkerchiefs used by the consumptive should be kept in a disinfecting solution until they can be boiled.

In Berlin the dust from rooms occupied by cases of pulmonary phthisis was collected and inoculated into guinea pigs. Of 28 rooms to which this test was applied 19 gave positive results. In Manchester of 31 rooms tested 19 gave positive results. The efficiency of different methods of disinfecting rooms occupied by phthisical patients has been tested by Delepine. He used first one pound of sulphur to 825 cubic feet of space and exposed his tubereulous material for four hours. Out of six experiments the bacilli remained virulent in four. In another series he tried chlorin gas produced by the action of hydrochloric acid on chlorid of lime. In 13 rooms tested by this method the bacilli remained virulent in 11. So far as we know, disinfection of rooms with liquid ehlorin has not been tried. Delepine found formaldehyd the most efficient gaseous disinfectant. On this point he makes the following statement:

"Formaldehyd has, therefore, penetration properties greater than those of most used gaseous disinfectants. The bacillus tuberculosis was killed whether in dry or moist state even when placed in deep, narrow recesses or imbedded in a thick layer of sputum."

For washing walls and scrubbing floors Delepine recommends a solution of chlorinated lime containing one and one-half ounces to the gallon. The wall paper is thoroughly saturated with this solution applied with a brush and is then stripped from the walls. The bare walls, ceiling, and floors, are scrubbed with the solution. The investigation of Rideal 
and Slater confirmed the findings of Delepine so far as the use of formaldehyd is concerned.

The English health laws make provision for the domiciliary treatment of tuberculosis. Holden states that as at present carried out this form of care of the tuberculous is a serious danger to the health of the community. General practitioners cannot give sufficient time to instruct patients and families in all the precautions that must be carried out. Nursing and feeding when left to the family, however much they have been instructed, are neglected or are irregularly attended to. Something may be gained by sending the patient for a time to a sanatorium for educational purposes, but in the majority of instances such patient must return to the conditions existing at home, be they favorable or unfavorable. At present (1920) there are in this country approximately 550 sanatoria, hospitals, and day camps, with a total of not more than 35,000 beds devoted to tuberculosis. The annual death toll in the registration area is about 110,000 and the number of clinically active cases is estimated at between 600,000 and 700,000 . It must be evident from these figures that the great majority of advanced cases pass their last days in their own homes or in those of some relative or friend, and that so far as medical attention is concerned it must come from the private practitioner; in other words, the heaviest burden of the tuberculosis problem is still being carried by the general practitioner. At least during some part of their career more than ninety per cent of those with active tuberculosis pass through the hands of the private physician.

Dispensary Care.-The "tuberculosis dispensary" has come to have a definite meaning and is doing in many places a most excellent work. By this name we designate institutions which consist of laboratory and other diagnostic rooms rather than places where drugs are dispensed. Boards of health in our large cities are establishing these dispensaries in selected places where they will be most convenient to the homes of the working classes. Here anybody may come and undergo an examination including microscopic test for the bacilli, "and in some places x-ray examination, and ascertain whether or not he has this disease. A nurse from the dispensary is sent to the home of each patient and gives to the family instruction in the methods of preventing the spread of the disease. The nurse carries to the family food in case of need and sees to it that the one for whom the food is intended gets it. The patient if not in an advanced stage reports to the physician at the dispensary from time to time, and such patients as may need such care are sent to sanatoria and hospitals. In homes where open cases are found other members of the family are subjected to the Pirquet or some other modification of the tuberculin test and at the same time undergo careful physical examination. In some dispensaries the $\mathrm{x}$-ray as a diagnostic agent is 
used with marked success. We regard the tuberculosis dispensary as one of the most effective agents in our combat with this disease. Every day we come in contact most closely with those classes among which this disease is most prevalent. Those in charge of these dispensaries should realize the importance of their functions and should study each and every case with the greatest care, employing every scientific aid within their reach. There is always a tendency in such work to lapse into perfunctory and even vicious habits. Those in charge of these institutions should clearly understand the difference between tuberculous infection and tuberculous disease. The man who is not feeling well goes to the dispensary. It is found that he has clinical tuberculosis; it may be latent still or it may be open. The nurse visits the home and induces all other members of the family to come to the dispensary. Here they are examined with the purpose not only of ascertaining whether they have tuberculous disease, but the question of tuberculous infection must be also investigated: If the latter condition exists without the former, such patients must be watched both by the nurse who goes to the home and by the physician at the dispensary who repeats his examination from time to time.

Tuberculosis Sanatoria.-Eighty or more years ago a country practitioner in England, Bodington by name, did much for his tuberculous patients by insisting that they live quite continuously out of doors. This original, long-headed country doctor. did much for those who placed themselves under his care, and at the same time succeeded in bringing down upon himself the ridicule of the medical profession of that time. In 1862 a German physician, Brehmer, established a tuberculosis sanatorium in the mountains near the village of Goerbersdorf not far from Breslau. This man succeded in building up an institution, acquiring somewhat of a fortune, and making for himself a reputation. One of his students, Dettweiler, withdrew from his preceptor, modified the treatment to some extent, and also achieved quite a reputation. However, the scientific, sane, altruistic apostle of the outdoor life was Edward L. Trudeau. Born in New York, the son of a New Orleans physician, educated in Paris and New York, this young man, with brilliant social prospects, had barely started as a practitioner in New York when he found that he had consumption, a disease from which a brother had recently died. Trudeau went to the Adirondacks and began that contest with the tubercle bacillus which was to continue throughout life and which ended in 1915. The story of the years spent by Trudeau in the then wilderness is the history of the development of the tuberculosis campaign in this country. For many years with admirable fortitude he kept the invader of his own body at bay and at the same time ministered to hundreds who sought him out amid the snows of Saranac, and he contributed more 
than any other man of his time to the scientific knowledge of this disease. He built in his shack a crude laboratory in which he did most effective work. In the summer of 1890 at a meeting of the International Medical Congress in Berlin, Koch made a most sensational statement. He said that he had found "a substance, which when injected into a guinea pig renders this animal insusceptible to inoculation of the tuberculosis virus. This same substance, in guinea pigs already afflicted with advanced and generalized tubereulosis, brings about a complete arrest of the development of the disease although the animal organism does not suffer the least harmful effect under the influence of the remedy." At the time Koch declined to tell the nature of this wonderful discovery. The whole world, both lay and medical, was thrown into a state of violent exaltation. Specialists in the treatment and care of this disease flocked to Berlin and jostled one another in their efforts to secure this remedy, more precious than gold. Fabulous sums were offered for this new elixir of life. Patients on their death beds in distant parts of the earth sent messengers to Berlin to secure the magic remedy. For months Koch gave no definite information concerning this wonderful preparation. He disclosed neither its source nor its chemical composition. Finally in January, 1901, he made the following statement:

"The remedy with whieh we aim to eure tubereulosis is a glyeerin extract of pure cultures of tuberele bacilli.",

Later it was found that Koch's tuberculin was a concentrated glycerin bouillon culture from which the unbroken bacilli had been removed by filtration. Thirty years have elapsed since Koch made his remarkable statement to the medical congress at Berlin. No evidence has been secured during these years which gives any adequate justification of the claims that he made at that time. Koch's announcement was made in August, 1890. In November of the same year Trudeau published in the Medical Record protocols showing that he had used dead tubercle bacilli and filtered and sterilized cultures of tubercle bacilli in attempts to immunize animals and that his results had been uniformly negative; in other words, at the time Koch made his announcement, Trudeau from his little laboratory at Saranac announced to the world the failure of tuberculin to immunize animals.

In his autobiography Trudeau tells that the idea of building a tuberculosis sanatorium came to him in 1882 after reading an account of Brehmer's institution, the work this man had done at Goerbersdorf, and that of his former pupil Dettweiler, at Falkenstein. Brehmer believed that tuberculosis was in some way dependent upon or related to a small heart and he attached much importance to graded climbing exercise for his patients to strengthen the heart. On the other hand, Dettweiler was an 
ardent advocate of complete rest and did not believe that a small heart had any relation to pulmonary tuberculosis.

Trudeau writes concerning the plan for his sanatorium, saying that, although at that time tuberculosis was not regarded as a transmissible disease, he felt that aggregation should be avoided, and he determined upon the cottage plan. In 1915, at the time of Trudeau's death, his sanatorium had grown into a picturesque village consisting of 30 buildings scattered over the hillside, with the cottages grouped about the administration building. In addition to the patients' cottages, there were a nurses' home, an infirmary for bed-ridden patients, a post office, a handsome library, and a fireproof laboratory.

When the announcement of Koch's discovery of the bacillus reached him, Trudeau hastened to New York and under the instruction of Prudden and his assistants soon learned how to stain the organism. He went back to his little laboratory, filled with energy and enthusiasm, and for many years he was the only man in this country who prepared pure cultures of the bacillus and the medical schools depended largely upon him for this product. By placing boxes within boxes and packing the interspaces with wool and sawdust and by getting up on cold nights to replenish the fire, he kept his incubator going with sufficient evenness of temperature to secure growth. He says:

"After some practice I grew quite expert in keeping my thermostat near the right heat, and indeed, it was with this little home-made apparatus that I first sueeeded in growing the germ in pure cultures outside of the body."

After being convinced that tuberculosis is always due to specific infection Trudeau seems to have been puzzled about the relation of environment to the disease, and, like the sane man he was, he determined to investigate experimentally. He tells the story as follows:

"I naturally began to wonder, if tuberculosis was a germ disease and the germs had already gained access to the body, how a change of climate, rest, fresh air, and food, could influence the disease. In seeking an experimental answer to this question I decided on the following experiment: Lot one of five rabbits were inoculated with pure cultures and put under the best surroundings of light, food, and air attainable. Lot two of five rabbits inoeulated at the same time in the same way were put under the worst conditions of environment I could devise. Lot three of five rabbits were put under similar bad conditions without being inoculated. Lot one I turned loose on a little island in front of my camp at Paul Smith's, where they ran wild all summer in the fresh air, sunshine, and were provided with abundant food. Lot two and lot three were put in a.dark, damp place where the air was bad, confined in a small box and fed insufficiently. The results showed that of the rabbits allowed to run wild under good conditions, all, with one exception, recovered. Of lot two, the same as lot one, but put in unfavorable surroundings, four rabbits died within three months and the organs showed extensive tubereulosis. Lot three, uninoculated animals were then killed and, although emaciated, they showed no tubereulous diseaso.' 
These simple experiments made under most primitive conditions are absolutely convincing and show more forcibly than volumes of arguments the relation, on the one hand of the bacillus, and on the other of environment, to the development and control of this disease.

Whenever Trudeau was in a quandary about anything he sought the light in the most direct way possible. He wanted to know what success he was having in preventing the infection of the cottages occupied by his patients. He, therefore, collected the dust from the walls of each of the cottages and injected this into separate lots of guinea pigs. The dust proved free from bacilli in all the buildings, even in the infirmary, which had constantly been occupied by the most advanced cases, with the exception of one cottage in which a careless patient had been housed.

For the first few years Trudeau and his little cottage sanatorium were recognized only by his personal friends, but gradually his good work became known. In 1891 Bowditch opened a tuberculosis hospital at Sharon, Mass. The first state tuberculosis sanatorium was that at Rutland, Mass., and was opened in 1898. At present (1920) every state in the union, nearly every large city, and many smaller cities and many counties, have public tuberculosis sanatoria, while the number of private institutions along this line grows larger year by year. The tuberculosis sanatorium has demonstrated its value in the combat against this disease.

The officers of sanatoria differ quite widely in the class of patients which they admit. Usually these institutions desire to secure only incipient or moderately advanced eases. The National Tuberculosis Association has adopted the following classification: (1) Incipient: Slight infiltration limited to the apex of one or both lungs or a small part of one lobe. No tuberculous complications. Constitutional symptoms (including gastric or intestinal disturbance, or rapid loss of weight) may be slight or absent. There may be slight or no elevation of temperature or acceleration of pulse at any time during 24 hours. The expectoration is usually small in amount or absent. Tubercle bacilli may be present or absent. (2) Moderately Advanced: Marked infiltration more extensive than under incipient, with little or no evidence of cavity formation. No serious tuberculous complications and no marked impairment of function, either local or constitutional. (3) Far advanced: Extensive localized infiltration or consolidation in one or more lobes, or disseminated areas of cavity formation. Serious tuberculous complications, with marked impairment of function, local or constitutional. (4) Acute Miliary Tuberculosis: In this classification, each of the three stages are based on certain specific pathologie findings and clinical symptoms. Unfortunately, however, lesions and symptoms are not interchangeable, but are immobilized in each of the three stages. This is confusing, for occa- 
sionally an advanced case may present only incipient symptoms or vice versa.

In the diagnosis of pulmonary and glandular tuberculosis in childhood the following definitions are given. (1) Loss of Weight: By loss of weight should be understood an unexplainable loss of at least five per cent below normal limits for that particular child or an unexplainable failure to gain weight over a period of four months. (2) Loss of Strength: By loss of strength in its pathologic sense is meant ease of tire and lack of staying power which are unusual for that individual child and which cannot be satisfactorily explained. (3) Fever: In children rectal temperatures alone are dependable. To constitute fever in its pathologic sense in childhood there must be more or less constant elevation of temperature over $100^{\circ}$, taken at various times during the day and lasting over a period of one week. (4) Pulse: No definite standard can be laid down. Observation should be over a longer period and a wider latitude than in adults before attaching significance to this as a symptom in childhood. (5) Hemorrhage: As in adults, any amount of blood, with or without sputum, requires investigation as to its source. This is a rare symptom in childhood. (6) Family History: An occasional case of tuberculosis in the patient's uncles, aunts, cousins, etc., should not be considered of importance, unless there has been intimate exposure and personal contact with such a case. It is an important fact, however, when the patient's immediate relations, as mother, father, brother, sister, nurse, attendants, etc., have been tuberculous and especially so when there has been prolonged and intimate contact. (7) Exposure: Exposure to tuberculosis, no matter how slight, from a human source or from milk, is of great importance. Prolonged exposure, especially under unhygienic habits or surroundings is of greater importance. (8) Cough: No cough is characteristic of tuberculosis in childhood. Persistent cough for six weeks requires investigation. Tuberculosis can and often does exist without any cough whatever. In certain cases of bronchial gland tuberculosis, there may be a brassy, strident, paroxysmal cough, resembling that of pertussis. (9) Sputum: Sputum, if present, should be examined. It is comparatively rare in tuberculosis in childhood. (10) Hoarseness: Any huskiness or persistent hoarseness requires investigation. This is rare in childhood. (11) Rales : Rales are not to be regarded as essential in diagnosis and are not in themselves evidence of tuberculosis; in fact, by the time rales are found in tuberculosis in childhood the disease is usually advanced and the diagnosis only too evident. (12) Dulness: Only very light percussion should be used. Dulness is not to be looked for at the apices as in adults, but over both sides of the sternum (parasternal dulness), and between the scapulae (interscapular dulness). (13) Altered Voice and Breath Sounds: Pure 
bronchial breathing and egophony are comparatively rare in tuberculosis in childhood. Harsh, prolonged, high-pitched expiration and an intense whispered voice are often present. The whispered voice and not the spoken voice should be used. (14) D'Espine's Sign: Intense whispered voice heard below the third dorsal vertebra is considered by many as abnormal and indicates the presence of enlarged bronchial glands. Such glands are not necessarily tuberculous though.

For adults the following definitions are given. (1) Loss of Weight: By this should be understood an unexplained loss of at least five per cent below normal limits for that particular individual within four months' time. (2) Loss of Strength: In its pathologic sense, by loss of strength is meant undue fatigue and a lack of staying power which are unusual for the individual patient and which cannot be satisfactorily explained. (3) Fever: An occasional temperature of 99 should not be considered fever. A temperature which persistently runs over 99.4 when taken at least four times a day over a period of one week (by mouth five minutes) should be considered of significance to constitute a fever. (4) Elevation of Pulse: Where the average normal pulse of the patient is already known, an elevation of fifteen beats per minute when the pulse is taken quietly at home during various periods of the day should be considered abnormal. In cases where the average pulse is not known, and this constitutes the majority of cases, one should consider an average pulse of 85 or over in men and 90 or over in women to be abnormal. The combination of subnormal temperature and an elevated pulse as defined here should be considered of great importance. (5) Hemorrhage: Any amount of expectorated blood, with or without sputum, may mean that tuberculosis is present and requires careful and thorough medical investigation as to its source. Blood streaks, blood spots, etc., may or may not mean tuberculosis. On the other hand, hemorrhage of one or two teaspoonfuls is presumptive evidence of the disease. (6) Family History: An occasional case of tuberculosis in the patient's uncles, aunts, cousins, etc., should not be considered important, unless there has been intimate exposure and personal contact with such a case. It is an important factor when the patient's immediate relatives, such as brother, sister, father, mother, or grandparents, have been tuberculous, and especially so when there has been prolonged and intimate contact. (7) Exposure: Childhood exposure is of the greatest importance. Moderate exposure among normal, healthy adults of cleanly habits is of less importance. Prolonged contact with unhygienic habits or surroundings may be a dangerous factor at any age. (8) Cough: There is no cough characteristic of tuberculosis. Every cough that persists for six weeks or over requires investigation. Tuberculosis may 
exist without any cough. (9) Sputum: The presence of sputum is not necessary for a positive diagnosis. The constant raising of sputum, with or without cough, requires investigation. Absence of bacilli in the sputum after one or several laboratory examinations is not necessarily proof against the presence of active tuberculosis. (10) Hoarseness: Any hoarseness or persistent huskiness demands investigation.

When the building of tuberculosis sanatoria was first begun there was much objection among the laity, sometimes found among medical men as well, to the location of such institutions near residences. This was due to the old and deeply founded belief in the miasmatic dissemination of disease. So great were these objections 20 years ago that frequent lawsuits resulted therefrom. It was claimed by the owners of property in the neighborhood that the value of their holdings would be greatly depreciated by the sanatorium. Experience and education have done much to remove these objections, and now there is seldom any complaint. It is unnecessary to add that in the selection of a location for tuberculosis sanatoria healthfulness must be borne in mind. The soil should be dry and well drained. There should be plenty of sunlight, an abundance of fresh air, with shade available. From remote times there has come down to us a tradition that pine forests offer especially desirable places for recuperation from this disease. This is probably due to the fact that such locations cannot be densely settled; otherwise there would be no pine forests. However, even among distinguished experts in the treatment of this disease there is an idea that the aromatic exhalations from pine trees have some direct beneficial effect upon pulmonary phthisis. The selection of a place where severe winds are broken, either by hills or forests, is desirable. Of course, such an institution should be far removed from the dust and smoke of cities, roads, and industrial plants. In order to save money tuberculosis sanatoria are frequently placed on poor farms and in close proximity to the county almshouse. To do this is to treat the tuberculous individual as though he were a pauper and it has a depressing influence. Such a selection should never be made, unless the institution be especially for the benefit of the occupants of the almshouse.

As its name implies, a sanatorium is a place for recuperation from disease. It is not a mere rest house, nor should it be a place to which people resort for pleasure or dissipation. It is a place where the workman who has developed active symptoms of tuberculosis on account of undue stress and strain may find much needed rest, which will aid greatly in his restoration to health. It is a place where the tired female clerk who, working on starvation wages, has developed active tuberculosis may find proper food, well prepared, and invitingly served. It is a place where 
all of those who for any reason have developed active symptoms of the disease may be educated and disciplined in those procedures necessary to enable them to care for themselves and to avoid infecting others. The success of a given sanatorium depends largely upon the personnel of its administration. Of course, all construction must be such that there is abundant opportunity to rest and to live day and night with an abundance of fresh air bathing the body and entering the lungs at all times. The three great forces which are of telling value in tuberculosis sanatoria are-(1) education, (2) rest, (3) food. Some one has truthfully said that whether an individual in whom incipient tuberculosis has been detected recovers or not depends upon that individual's intelligence and character. Without intelligence to appreciate the instruction given in such an institution, one can receive but little benefit. Without character strong enough to follow the rules and regulations inculcated in such an institution there is but little hope of permanent benefit.

The tubereulosis sanatorium has proved a most valuable agent in limiting the dissemination of the disease. In it the patient is taught how to take eare of his sputum or other excretions without endangering others. The influence of example is of the greatest importance. The less intelligent will be instructed by the more intelligent among his fellow patients; the one who is disinelined to deny himself every wish will be impressed by the strength of character of those about him. Like every other condition in life the influence of environment, including the behavior of our fellows, is of importance.

The sanatorium should, of course, be manned by a personnel of the highest scientific skill. Every sanatorium should possess a well-equipped laboratory, manned by competent, enthusiastic men, and its clinicians should be skilled in diagnostic art and most humane in their attitude towards their patients. We think it fortunate that the personnel of most of our sanatoria is recruited from among those who have recovered from the disease. The attempt to eliminate tuberculosis by the establishment of sanatoria is a wise move and in the right direction, but without supplementary agencies its limitations are great. It would be well if every incipient case could pass some months in a properly conducted sanatorium, but this cannot be because the number of such cases is too great. However, the most distressing thing is that the majority of individuals receiving sanatorium benefit and improving therefrom must go back to home conditions which, unfortunately, in the great majority of instances, mean relapse, redevelopment of active symptoms, progress in the destructive processes, and ultimately death.

Tuberculosis Colonies.-There is no sharp line of demarkation between tuberculosis sanatoria and colonies. In a general way it may be said 
that the former are for periods of short residence during which the value of rest is secured and training in modes of living is given. On the other hand, a colony is supposed to be occupied for a longer time and to supply suitable employment of a productive nature but without the fatigue which might prove harmful. The colonies are supplementary to the sanatoria. Sanatorium treatment fails largely because in the majority of instances it is necessary for the individual to return after a short residence and on temporary improvement to his former conditions of living, both in home and in workshop. This results in a large percentage of cases in a speedy return of the active symptoms of the disease; therefore, the colony seems to be a necessary adjunct and supplement to the sanatorium. It is true that in certain sanatoria the routine life of the patient involves regulated exercise, but ordinarily in sanatoria there is no attempt to offer the patient any productive occupation. Tuberculosis colonies differ widely one from the other in provision for occupational exercise. Cobbett, who has had in charge one of the most successful colonies in England, writes as follows:

"The long period of residence contemplated will make it necessary to provide for the occupation of the inmates in ways not yet attempted. Games and recreation will not suffice, but serious work will be demanded to which a man may give himself daily without violence to his self-respect, and which even may be brought to contribute materially to the eost of his living. The new sanatoria, then, starting from small beginning, should aim at becoming industrial colonies, furnished with workshops, where handicrafts, as varied as possible, and including those, if any, which are characteristie of the district, may be followed and provided, above all, with ample grounds for gardening, and agriculture. At the same time the needs of those with advanced disease must be met by liberal provision so that they can pass their days as pleasantly as is possible. In such institutions it seems possible to make a new beginning to deal directly with the problem of prevention of tuberculosis by attacking the disease at its very source."

The great number of tuberculous soldiers returning to their home countries from the World War has kindled great enthusiasm for the tuberculosis colony. Varrier-Jones, who has built up the Papworth Hall Colony in England, writes as follows:

"What is the remedy? What but to graft on to our existing sanatoria the colony principle of employing patients at their own or allied trades. There is not the slightest doubt that soldiers will stay in a village community, always provided that the amenities of life are there. Social surroundings, healthy environment, steady but not too laborious work, freedom of eonscience, home-life remodeled on sound and healthy lines, all these things should be within their reach. The scheme is an ambitious one, but this is the day of great enterprises. We hear a good deal about the establishment of village communities for disabled men. For no class of the disabled is a life sheltered and yet active, proteeted and yet self-respecting, so necessary as for the tuberculous. The man who has suffered the loss of a limb, or the loss of sight, may still find a place in his old surroundings, if properly assisted and trained; the man suffering from shell shock may need 
long convaleseent treatment away from the stress of town life, but eventually he will probably be able to resume his old occupation; the consumptive, alone, if seriously attacked by disease, ean never hope for even a modicum of health and strength in crowded streets and ordinary workshops; the consumptive, alone, of all this sad procession of broken lives, ean never return to normal eonditions without endangering his family and his fellow-workmen, without involving not only himself but others in disaster. Let communities be started in which our consumptive soldier can live in his own home, sheltered from the fieree eompetition of the outside world, a self-respecting worker, an economic asset. Let employment be found, the model factory ereeted, the hours of toil properly regulated, a fair wage paid. In such villages his ehildren ean be earefully protected from infection by open-air schools supervised by a competent health visiting staff. The man's leisure hours ean be made glad by recreation arranged by the colonists' own committee, and his life agreeably spent. 'A mere utopian dream,' they say, 'or how do you know that the consumptive ex-soldier will submit even to such conditions?' I say that he has already done so, that the requests for admission to the Papworth Colony now far exceed the accommodation. 'In that case,' the eritic answers, 'the demand will always be greater than you ean possibly meet.' The demand is great. Is that a reason why we should not try to meet it? We sineerely want to do well by these men; shall we stop at wanting, and put forward no practical solution? 'But the expense will be ruinous.' So is the evil; so too the cost of the evil. If one village community of this kind can be shown to carry within it the germ of sueeess, the idea will surely fructify and similar communities will spring up around existing Eanatoria or in other suitable spots. A large country house surrounded by gardens and grass-lands, as at Papworth, forms an excellent nucleus, and such houses often come into market in these days of ehange. The village can to a large extent be built by the colonists themselves. They begin by building shelters, they can go on to building cottages - specially designed shelters and cottages; and then to making the furniture for these shelters and cottages. The joy of seeing useful and beautiful things growing under their hands, the development of musele and vitality which comes with work earried out without fatigue, the cheerful surroundings, the sense of comradeship, and best of all, the prospect of a return to a happy family life, are most powerful aids to nature in driving out the ravager, where this is still possible, or in limiting his ravages when he is too securely entrenehed to be dislodged.'

Those who are deeply interested in the tuberculosis problem did hope that our federal government would point out the way in the treatment of the thousands of tuberculous soldiers who are now pensioners on the country. However, it must be admitted that up to the present time (1921) those in charge of the tuberculous soldier have done nothing noteworthy and very little that is even commendable.

The enthusiasm that has been displayed, most notably in England, concerning the great good to be accomplished by tuberculosis colonies is encouraging, but the weakness inherent in all such plans must be. evident to every thinking person. It is easy enough to say to the tuberculous clerk, to the tradesman, to the skilled or unskilled worker, to the student, to the professional man, and in fact to any man or woman in whom tuberculosis is found to be progressing, "you must go into the country; you must live in the open air; you must drop your trade, your 
calling, your profession, your present means of making a living, and your life hereafter must be spent out-of-doors."' It is easy to give this kind of advice, but, who can follow it? What man or woman, trained in some other occupation, can go into the country and compete with the farmer, the dairy man, the fruit grower, the stock man, or the members of any other outdoor profession who have grown up in their own line of work? What employer of labor, with the legal complications that are now placed upon him in regard to sickness and accident insurance, is going to employ, under any conditions, the man or woman who not only has had no training in that line of work, but on account of physical inability is unable to do the duties that fall upon the most unskilled man employed in the works? What kind of a gardener would the young clergyman, who has recently married and settled down as an assistant in a given parish, and who is found to have active tuberculosis, make? The same might be asked of any and every other trade, calling, or profession. The establishment of tuberculosis colonies, like that of tubereulosis sanatoria, marks a milestone in the long road of effort which the race must make before it gets rid of this disease; but it is only a mere milestone and a long distance must be traveled before the goal is reached. Individuals are being benefited and will continue to be benefited by tuberculosis sanatoria and colonies, but that either of these can ever become an important factor in the eradication of this disease is a wild and unfounded dream. As indications of man's humanity and his sympathy toward his fellow-man, these institutions are highly creditable, but as effective agents, even in our combat with this widely disseminated and almost universally prevalent disease their value will be largely futile. The agents to be employed in the eradication of this disease must have a more fundamental grip upon our civilization before they can be effective.

We are not pronouncing sanatoria and colonies worthless. To the contrary, we are convinced that they have served and are serving most useful purposes. Thousands of cases of tuberculosis are saved through these agencies annually. They are worth not only what they cost, but their multiplication should be urged. We cannot have too many of them, but we must not expect to reach final results through these agencies. Because we cannot at once close every leak in our treasury, is no reason that we should not close those we are able to reach. To build sanatoria and establish colonies are the duties of today, but we must constantly hold in mind the fact that in the progress of the race there are endless tomorrows and each day will bring its own task which must be performed with ardor and enthusiasm. The race has scarcely left the cradle in which it has been rocked by the nurse, civilization. Under the guidance of this nurse, who still is woefully lacking in intelligence and skill, the 
child is barely beginning to perceive that the world is large and its own range of vision extremely.limited. The race must struggle through childhood, youth and adolescence before it reaches maturity. It is to be hoped that its faltering steps will grow steadier and firmer, its muscles will become stronger, its bones will furnish a firmer support, its pulse send a richer blood through its veins, and its brain grow clearer and clearer, as it approaches adult life. We are probably doing the best that we can today, but we must realize that we must grow in strength and accomplishment before any great reward is won. To eradicate tuberculosis is one of the biggest problems that man has ever undertaken, and to accomplish it he must possess a higher degree of intelligence and a greater sense of responsibility than are now possessed by even the wisest and the most humane citizens of the earth. It is said that tubereulosis is a result of our civilization, and we have no doubt that this is the true explanation of the wide prevalence of this disease. To admit this is to acknowledge that our civilization has gone wrong. We have strayed from the right road and have been led by some ignis fatuus into a jungle. We must ascertain in what direction the right road lies. We must find it and follow it, although it is more than probable that our descendants through many generations will do as our ancestors and we have done-follow some false trail, and like ourselves, find themselves in the mire. We do not pretend to be wise enough to point the way back to the right road, but we do know that we shall not gain it by standing still. We must use our best intellectual gifts, follow the direction they indicate, and if we find that they lead us farther and farther from the road, we must retrace our steps. Find the right road we must; because it is a matter of life and death, not so much for the individual as for the race.

Tuberculosis Hospitals.-A tubereulosis hospital is distinguished from a sanatorium or a colony by the degree of severity of the disease manifesting itself at the time in those sent to the institution. While sanatoria and colonies are filled with incipient eases, those in advanced stages are sent to hospitals. Quite naturally, among incipient cases there will be acute exacerbations, and it follows that every sanatorium and colony must be supplied with an infirmary. Moreover, whatever the stage of tuberculosis, those suffering from this disease are not immune to other diseases and hospital treatment must be provided for all communities. One of the first special hospitals for the tuberculous was the Brompton Hospital, which was built for the care and treatment of advanced cases of this disease found in London. Considering the time when the first building connected with this hospital was constructed, it was wonderfully adapted to the special purpose for which it was intended. It has been 
enlarged and modified from time to time in accord with advances in our knowledge of the nature of the disease. It has been copied all over the civilized world and today practically every large city has one or wore special hospitals for tuberculous patients. In some instanees these are connected directly with sanatoria, while in others they are quite apart from other institutions. The removal of open cases of tuberculosis from homes, from shops, from communities, and their segregation in special hospitals, have probably been important factors in the decrease in the death rate from this disease. As has been indicated, England has set the pace in the building and maintenance of tuberculosis hospitals, and in England before the war the death rate from this disease was lower than that in any other country. There are those who are inclined to attach a causal relationship between these facts. It may be well to go a little more fully into the subject of English hospitals. So far as we can ascertain, the first special hospital for tuberculosis in England was at Margate. It was opened in 1791 and had 162 beds in all, 16 only being devoted to tuberculosis. The Royal Hospital for Diseases of the Chest, with 80 beds and located on the eity road in London, was opened in 1814. Then followed the Brompton Hospital for Consumptives with 321 beds in 1841; the City of London Hospital for Diseases of the Chest with 164 beds in 1848; the Bournemouth Hospital with 71 beds in 1855; the Mt. Vernon Hospital for Consumptives at Hampsted with 120 beds in 1860 ; the Royal National Hospital at Ventnor with 155 beds in 1869. Since the last-mentioned date tuberculosis hospitals have multiplied in England and the result of this has been the isolation of open cases. Whether the establishment of these hospitals and the isolation of open cases, which their existence has rendered possible, have been factors in the reduction of the disease in England, no one ean say with certainty. However, it seems reasonable to believe that the withdrawal of centers of infection-and each open case is a center of infection-from homes, workshops, and communities, has probably had some influence. There are eminent tuberculosis experts in England today who seem to be thoroughly convinced that attention to the advanced eonsumptive is the most effective weapon that ean be employed in the combat with this disease. For instanee, in a recent diseussion, Lumsden stated that the war against tuberculosis in Great Britain and Ireland has been largely a failure and that much public money has been spent without corresponding results. He holds that all cases of open tubereulosis should be segregated and so placed that the spread of the disease through these people is impossible. He writes as follows:

"We have been traveling in a circle-first we send unsuitable cases to sanatoria, informing the patient we hope to cure him, and we then permit him to return to his old environment without effort to improve it or to properly supervise his home and its 
unhealthy devitalizing condition. Recrudescence or reinfection commonly oceurs, the patient once more becomes a focus of infection for his family and the community, and he returns from the sanatorium for the tragedy to be played out to its disastrous end. Until this vicious circle is broken tuberculosis will surely remain with us, and public money will be spent without any finality being attained. The tragic feature of this urgent and colossal problem is that we now know how the disease ean be stamped out, but for sentimental reasons we lack the pluck to tackle it by putting into force the necessary machinery. In my humble judgment a thoroughly efficient and strong tuberculosis department, with all the necessary powers. should be instituted under the new Ministry of Health. Housing, provision of a clean milk supply, tuberculosis and other dispensaries, school inspection, etc., are all essential factors no doubt, but one of the first efforts should be to secure the segregation or control of all tuberculous cases of an infectious nature. The disease should be made compulsorily notifiable, the home should be inspected by the local authority, and one of the two following courses adopted: (1) Proper sleeping and living conditions established so that infection shall be reduced to a minimum, which we know can be done in most cases with small expense, and generally with the family's eooperation, once the position is made elear to them. (2) If this course cannot be accomplished the patient should be compulsorily removed to a home where he can no longer spread the infection."

In every community in our land of a few hundred or more there are one or more open cases of pulmonary tuberculosis going about among the people. The extent to which these carriers are infecting the community depends upon their consideration for others and their intelligence. Take the most conscientious and the best trained man with tuberculosis and it is impossible for him to eat in a restaurant, at a hotel, in a private dining-room, or anywhere, without infecting the forks, spoons, cups, and glasses with which his lips come in contact. Then there are at large thousands of careless and indifferent men and women with advanced pulmonary tuberculosis who are infecting the sidewalks and floors of churches, theaters, and other places of assembly, and even homes-not only their own but those which they visit. We have ordinances against spitting on the sidewalk, there are notices posted in all public buildings, "Do not spit on the floor; it may spread disease," but one does not have to be a keen observer or to pry closely into the behavior of those about him without seeing that these injunctions are today largely without effect. No community would permit a man with smallpox eruption to walk its streets and visit its public halls, theaters and churches, and still we can protect ourselves against smallpox by vaccination. Certainly it is true that the careless consumptive is a greater danger to the community in which he lives than is a man covered with the eruption of smallpox. We are aware of the fact that the regulations of health departments of all our cities caution those with open tuberculosis as they mingle among others and advise certain protective procedures, but we are also cognizant of the fact that these regulations are daily and hourly disregarded in nearly every community in this land. We fail to deal 
effectively with the advanced consumptive because he is too numerous and our sympathy for many of his class leads us to disregard the danger he is to others. We would not be alarmists in this direction; we have no desire to awaken phthisiophobia, but we are convinced that greater attention should be given to advanced eases of this disease, both for their own sake and for the protection of the public. Many of them are poor, have dependents, must work, are not able to properly house, clothe, or feed themselves and their families. Let the public do these things for them. This will be a practical charity, or better still, it will be the performance of an actual duty. It will result in improving conditions under which the consumptive and his dependents live, and at the same time lessen the danger to the public. Let us build more sanatoria and establish more colonies, but at the same time let us make more ample provision for advanced cases. There is another important point in this connection. It has been found that when advanced cases are admitted to sanatoria and hospitals a considerable percentage of them show wonderful recuperative power, and in some instances it can be shown by statistics that the percentage of improvement is quite as great among moderately advanced eases as among incipient ones when both are placed under the same improved environment. From May, 1912, to May, 1914, 1,056 patients were discharged from the Massachusetts State Sanatoria. In December, 1916, 312 of these patients were found in good condition and at work. On admission to the sanatorium thirty-five per cent of these were classified as incipient, forty-three per cent as moderately advanced, and twenty-two per cent as far advanced. Most persons who develop clinical tuberculosis do so on account of some untoward condition in their lives. They are working too hard, are living in insanitary houses, have not enough of the right kind of food. Had it not been for one or more of these untoward conditions their tuberculous infection would never have developed into tuberculous disease. The tuberculous disease has advanced slowly or rapidly according to the weight of the untoward condition to which the individual has been subjected. Remove the unfavorable, debilitating condition of life, and the individual who is pronounced in a moderately advanced or even in an advanced stage often recovers with striking rapidity and to a surprising extent. A wage earner in a family develops tuberculous disease because he has to work too hard to support those relying upon him or he underfeeds himself that he might adequately feed his dependents. These represent the stress and strain which have brought active illness upon this man. Common humanity demands that we lift these burdens from his shoulders and place him under most hygienic conditions. It will surprise us how often and how quickly men and women of this class regain at least some moderate degree of health. We are too much inclined to look upon the 
advanced consumptive as hopeless. There is no other grave disease from which so many recover. It has been truthfully said that tuberculosis is largely a disease of poverty. This is certainly more generally true of this disease than of any other prevalent among us. A large proportion of those who die from tuberculosis die because they are poor. To permit any one to die of poverty in this land, not only of abundance but of riches, is a disgrace to the community and a blot upon the escutcheon of a nation which proclaims that all men are born free and equal. In no other disease is it so generally true that health is purchasable. Just at this point we are making a plea for the better eare of the advanced case, and we are making this plea in behalf of both the individual and the community at large. Certainly in a campaign against this disease we eannot afford to neglect even the seriously wounded. The wonderful surgery which proves so efficacious in many of these cases consists merely in improvement in environment, and proper feeding.

It must be self-evident that the tuberculosis hospital should be a special institution and that the treatment of cases of this disease in general hospitals should be avoided so far as possible unless the general hospital is provided with special wards for the tuberculous. There must be special provision for an abundance of air, for sleeping porches, and in the best hospitals of this kind there should be a separate room for each patient. Fortunately, a good example was set in some of the early English hospitals. Brompton was famous for its large and luxurious wards and for the excellence of its arrangement. The Ventnor Hospital is admirably situated on the Isle of Wight. Its grounds cover more than 20 acres and it is protected from northern winds by being located under the cliff. Not more than one patient is permitted in a room, the air-space of which affords from 34 to 51 eubic meters, and 140 eubic meters of air per head per hour at a uniform temperature of $62^{\circ} \mathrm{F}$. pass through the building. Four meals per day of most nutritious food prepared in most appetizing manner are supplied. Of course, it is not easy to draw a sharp line between sanatoria and hospitals. Every sanatorium must have its infirmary or hospital. It must be evident that with all our tuberculous institutions, by whatever name we designate them, the accommodation afforded is insufficient and we must expend in the future thousands of dollars where we are expending tens at present.

In most states the laws permit each county, or two or more counties combined, to build tuberculosis hospitals. In some states this law is not merely permissive but is mandatory. In Massachusetts all cities of over 50,000 population are required to provide hospital accommodation independently of the counties in which they are located. In most of the states there is a charge for those who are able to pay, while in others all treatment and eare at state and county tuberculosis hospitals are free. 
The rich have no difficulty in finding accommodations in private institutions. The county hospital, whether it be for the treatment of tuberculosis or any other disease, provides for the very poor, while those who have difficulty in making ends meet under ordinary conditions and who cannot be classed as paupers are the ones for whom it is most difficult to provide.

Educational Propaganda.-While there have been through all the centuries from Hippocrates to the present time a few keen observers who have held that tuberculosis is a transmissible disease, there was no positive information upon which this claim could be based until it was furnished by the experiments of Villemin, first published in 1865 . The virus, whose existence Villemin's experiments firmly established, was identified, isolated, and tested by Koch in 1882. Taking Villemin's work in the sixties of the last century as a basis, many men in various parts of the world began to teach not only in medical schools, but in public lectures, that tuberculosis is both transmissible and preventable. Propaganda of this kind has continued to the present time, and since, during these years, at least to the beginning of the World War, the death rate from tuberculosis was gradually decreasing, we are inclined to ascribe this decrease to the spread of information bearing upon the nature and transmission of the disease. At the present time we are not willing to admit that this work has not been a factor in the decrease in the death rate. We may differ among ourselves as to how great a factor it has been, but that it has borne some causal relation we are inclined to admit. In our own country all intelligent people now know the fundamental facts concerning the nature and transmission of tuberculosis. It was necessary to diffuse this information through the public before we could induce legislatures to enact bills providing for the reporting of cases, the disinfection of houses, the erection of sanatoria, etc. City councils had to be educated before they would provide free dispensaries, supply visiting nurses, and furnish the needy with food, build hospitals, sanatoria, etc. In addition to these things, it has been necessary to educate the medical profession, especially the older members who were thoroughly imbued with the idea that tuberculosis is a hereditary disease and many of whom resented any other view of the matter. The profession still needs education in the early recognition of the disease. The proof of this may be found in the large number of early cases accepted for army service and passed upon sometimes by experts as sound men. Educational propaganda has done much both specifically concerning tuberculosis and generally in regard to sanitation. The attitude of the public towards health problems is quite different in 1920 from that of 1880 , and this has been due largely to educational propaganda. It might be said that there is no truer index 
to the intellectuality of a given community than the provision which exists for the preservation of the health of its members. This is a measure not only of the intellectuality of the community, but at the same time of its sense of moral obligation.

The means employed in public health education have varied widely in form and in effectiveness. At first it was necessary to direct the educational forces to the adult population, because it was desirable that something be done promptly. Quite naturally, it happened that the portion of the adult population aimed at and reached was the more intelligent, including neither the idle rich nor the indolent poor. Influential thinking, progressive people were reached by lectures, the holding of sanitary conventions, the distribution of leaflets and pamphlets, appeals for a fairer distribution of the comforts of life, etc. Looking back on the past 40 years, we must say that this work was fairly well done and it has been rewarded with tangible and far-reaching results. Some far-seeing educators appreciated the fact that the surest way to reach the greatest number of people would be through the public schools. In 1895 the legislature of Michigan, prompted by the state board of health, enacted a law making it obligatory upon the teachers of the state that the nature and modes of transmission of communicable diseases be taught in every public school of the state. In order that the teachers might have information on these subjects to impart, the law furthermore provided that the state board of health distribute among the teachers from time to time leaflets and pamphlets upon which the required instruction be based. The effect of this law has been farreaching and there is not a student in a high school in Michigan who has not had instruction in the nature, causation, and prevention of disease. At first the law was largely ignored by the teachers, simply because most of them were too ignorant to comply with the provisions. of the law. With proper prodding from the state board of health this law has become highly effective. Similar laws have been adopted in other states and we think it is safe to say that today among our nativeborn population who have passed through the primary and secondary schools there is a fair degree of intelligence on these subjects. Of course, this does not hold good in our congested industrial centers filled with those who cannot speak or read our language; neither does it apply to certain sparsely settled areas where no adequate school instruction of any kind is within the reach of the children. In our opinion, there is nothing more fundamental, nothing more powerful for good, nothing more beneficial to the race, than proper instruction of the children in all matters pertaining to public health. A wise German many years ago said: "Permit me to educate the children of two or more generations 
and I will change the world." We think that this statement, made by Leibnitz, has been verified in the unwise training of two generations of German children and we are confident that equally potent results are obtainable when instruction in righteousness is given. It is certain that the evolution of the race, and with it the eradication of tuberculosis, lies in the hands of future generations, whose guardians, mentors, and directors we are. Of course, we are handicapped by the fact that we do not always know just what to teach. We are never exactly sure of the truth. We cannot with a great deal of confidence map out for our children a road we have never traveled, and since this road lies before them and we stop before it is reached, the directions that we give are sure to be faulty. However, future generations must keep their eyes open, must rely in their final decision upon themselves, and fortunately will be prone to make merry over the misinformation given them by their elders and predecessors. We are making a practical point that the most powerful agency, in fact the only effective agency, in the ultimate eradication of tuberculosis lies in the justified hope and expectation that succeeding generations are to possess a greater intelligence and be moved by a deeper sense of moral obligation than we possess or experience. Let us insist that the soundest possible instruction in public health matters, including the eradication of every communicable disease, be properly prepared, properly adjusted to the expanding mind, and be demonstrated to every future citizen as he or she climbs the educational stair to civic maturity.

Tuberculosis Surveys.-Just as oral and written instruction is helped by being supplemented by practical demonstration, so the educational campaign against tuberculosis has been greatly benefited by surveys. The most noteworthy and extensive of these in this country was that inaugurated and carried into effect by the Michigan State Board of Health (1915-1917). The legislature of the state made an appropriation of $\$ 100$,000 for this survey. The purposes held in view were: (1) The finding, by physical examination, so far as possible, of all cases in the state. (2) The instruction of each tuberculous individual, so far as found, and his family in the methods of living best suited to prevent the spread of the disease to others and to arrest the progress of the disease in the individual. (3) The placing of each tuberculous individual under the care of a local physician. (4) The awakening of each community to the desirability of concentrated action in the endeavor to restrict the disease.

The area unit of the work was the county and the time devoted to each county was three weeks. In some of the more sparsely inhabited sections two counties were taken as a unit. During the first week two visiting nurses went into the county and, first of all, they visited the local 
physicians and secured their cooperation. This was most willingly given and no physician failed to give to the work most hearty support and personal aid. The nurses then went to the teachers of the public schools and asked them to advise all scholars who seemed under par in health to come to the clinies to be held next week. Along with the nurses the publicity agent went. He secured the cooperation of the local newspapers which printed articles furnished by the agent, advertised the free clinics, and advised all to go. Thousands of columns of information concerning tuberculosis were published by the newspapers of the state without cost. Ministers were asked to preach about tubereulosis on the Sunday at the close of the first week. Manufacturers were asked to advise all men under them and who were in any way out of health, to go to the clinic.

During the second week elinies were held. The diagnosticians were selected for their special skill in the detection of the disease in its early stages. They were men who had the respect and confidence of the medieal profession. Clinies were held simultaneously in each important city or village in the county. In each place three rooms were used, a waiting room, a dressing room, and a clinic room. In the first the trained nurse took the histories and the temperatures, making triplicate records. These records accompanied the patient into the elinie room where the expert made his examination in the presence of local physicians; indeed, the expert gave a continuous diagnostic clinic on tuberculosis. It was estimated that more than ninety-five per cent of the local physicians attended these clinies, and some became so deeply interested in the work that they followed the experts from county to county and became so skilled that they were added to the staff. The clinician continued the records begun by the nurse and turned over one copy to the family physician of the individual, who was asked to report to the state board of health from time to time the further history of the case.

The third week was given to edueational work and was devoted to both the community as a whole and to the individual. Every positive ease found by the expert was visited in his home by a trained nurse who gave practical lessons in house sanitation, food, sleeping quarters, etc. Talks were given in the schools, factories, churches, clubs, etc. The county legislators, or supervisors, as they are called in this state, were seen and the county's needs and duties were pointed out. The local physicians were urged to provide weekly free clinies for the detection of early cases of tuberculosis. The need of a county sanatorium for the cure and edueation of the positive cases was made evident. In all these directions the work bore excellent fruit. Many full-time health officers have since that time, largely stimulated by the work, been provided in small cities and many counties have made appropriation for sanatoria. 
The state board of health was convinced that the training of the physicians in the early diagnosis of tuberculosis will prove of the greatest value. The people in general may soon forget about the campaign against tuberculosis, but the local physicians will not forget and with their weekly free clinics they will keep the work going, will prove its value to the community, and will secure for themselves a higher appreciation of the nature and value of their services.

The total population of the area surveyed during the first year was 1,319,283. The number examined was 11,528; positive, 2,914; suspicious, 2,231 ; arrested, 375 . There was no claim that all cases of tuberculosis in the area covered were found; indeed, the physicians in their weekly clinics have constantly added to the number. It would not be fair to suppose that if all the inhabitants of the area had been examined the percentage of positive cases would be nearly so high as that found among those actually examined, because these were selected for examination either because they were known to have been unusually exposed to this infection or because of the evident fact that they were not in robust health. Although tuberculosis had been by law a reportable disease since 1910, of the positive cases found only two per cent had previously been reported; in other words, ninety-eight per cent were cases which had not been recognized until these clinics were held. The positive cases were distributed by stages of the disease as follows:

$\begin{array}{lr}\text { Advanced } & 6.8 \text { per cent } \\ \text { Moderately advanced } & 27.2 \text { per cent } \\ \text { Incipient } & 52.6 \text { per cent } \\ \text { Arrested } & 12.9 \text { per cent } \\ \text { Not determined } & 0.5 \text { per cent }\end{array}$

Among 2,923 cases in which the sex was indicated there were 1,786 females and 1,137 males. With regard to age, 764 or 26.1 per cent were 15 years or less; 1,041 or 35.6 per cent were between 16 and 30 years inclusive; 747 or 25.5 per cent were between 31 and 45 inclusive; 283 or 9.6 per cent were between 46 and 60 inclusive, and 89 or .3 per cent were between 61 and 80 years. Of all cases detected, 61.1 per cent were between the ages of 16 and 45-the most productive age of life.

It was found that when there had been only one case of open tuberculosis in the family, during the first 15 years of life the source of infection in the majority of instances is either the father or mother, while beyond 20 years of age a history of tuberculosis in brother or sister predominates, the brother line reaching its maximum at 25 and the sister line at 30 years. It was furthermore found that until 10 years of age the female predominates as a source of infection, this being undoubtedly due to the 
close association between mother and child at this period of life, irrespective of difference in sex. From 10 to 15 years in boys the female line drops abruptly, while the male becomes the greater source of infection. From 15 on, the male source of infection generally predominates. It is interesting to note that below the age of 10 no marked difference in the two sexes is notable, although here again the mother, as one would expect, represents the greater source of infection. After 10 years the part played by intimate association between members of the same sex is apparent.

Many valuable diagnostic facts were ascertained. Among 2,957 cases there were 605 that gave a definite history of pleuritic involvement at some time previous to, or coincident with, the examination, there being 46 cases in which no information in regard to this point was obtained. A history of pleuritic involvement was, therefore, obained in 20.8 per cent of all cases questioned with regard to this point. Owing to the definite character of pleuritic pain it is believed that these statistics are most reliable. Among 2,906 eases in which information with regard to the occurrence of pulmonary hemorrhage was obtained, 428 or 14.7 per cent had been afflicted at some time previous to the examination. Mere streaking of sputum was not considered, nor was the rusty sputum associated with pneumonic attacks.

This survey was completed in 1917 and its extension was interrupted by the war. The statistics for the second year have not as yet been satisfactorily tabulated. It is to be hoped that similar surveys may be made in different sections of the country, as they are most valuable from an educational and from a statistical standpoint.

The Framingham Experiment.-In 1916 the Metropolitan Life Insurance Company sent the following letter to the National Tuberculosis Association:

"The Metropolitan Life Insurance Company is much interested by reason of the fact that over sixteen per cent of the deaths in its industrial department are due to tuberculosis. In 1915 , the eompany paid claims of over $\$ 4,000,000$ on the lives of 14 ,325 policy holders dying from this disease. The company believes that an intensive experiment might well be made in the United States to determine whether it is possible to substantially reduce the mortality and morbidity of tuberculosis in the hope that the disease may eventually be eradicated. To this end, we are prepared to place at the disposal of the National Association for the Study and Prevention of Tuberculosis the sum of $\$ 100,000$ for the purpose of conducting a community experiment over a period of three years in the control of tuberculosis, on condition that the association selects a community of approximately 5,000 inhabitants, preferably in New York or Massachusetts, in which conditions would be favorable for such an experiment and that a special committee of the association be appointed, on which the company shall be represented, to whom full power shall be given to institute the necessary preliminary survey and to conduct the experiment along the lines finally determined upon by this committee. We further suggest that in making the experiment, stress should be placed 
on: (1) Periodic medical examination of all members of the community. (2) Medical and nursing care of all cases of tuberculosis. (3) Sanatorium or hospital care for such cases as may need it. (4) A tuberculosis clinic or dispensary. (5) Cooperation of local and state health officers, employers, labor unions, school authorities, etc. It is our hope that this experiment will be a practical contribution towards the study of the etiology of tuberculosis and that the results obtained may indicate a method for the prevention and elimination of the disease."

This offer was accepted by the National Tuberculosis Association and after a conference the limit of population in the place to be selected was raised to 15,000 or 16,000 . In accepting this gift and undertaking the work, the National Tuberculosis Association proposed the following questions: (1) Is it possible to discover and to place under adequate medical, nursing and relief supervision all of the cases of tuberculosis, incipient and advanced, active and arrested, in a normal industrial community? (2) Is it possible to ascertain with some degree of definiteness, the responsible social and economic factors in disease causation, particularly as regards tuberculosis? (3) What is the most efficient utilization of the existing means available for the discovery and treatment of the disease? What percentage of theoretically preventable disease is practically preventable with the use of known instruments? (4) How can social forces best be used to prevent unnecessary sickness and death, particularly from tuberculosis? (5) Practically, what is the best community health machinery for meeting these ends, and what are the essential factors in an adequate, preventive, detective, and remedial mechanism?

After studying the conditions prevailing in certain small cities in Massachusetts and New York, Framingham was selected. This town had in 1916. a population of approximately 16,000 people. It is situated in eastern Massachusetts, 21 miles from Boston. It recommended itself to the committee as an average community, with mixed industries, varying racial groups, a good local health organization, linked with an excellent state department of health, a normal amount of disease, particularly tuberculosis, well-trained physicians, good hospitals, and a sufficient promise of cooperation from medical, industrial, commercial, and social organizations to give reasonable assurance of success.

Armstrong, a skilled expert in tuberculosis, was placed in charge of this work and began its operation in December, 1916. When the time limit of three years was nearing expiration the National Tubereulosis Association appointed a board of experts to review the work and report upon its success and advise concerning its continuation. This committee reported in December, 1919, and from this report we make the following quotation:

"The success of this organized campaign for the discovery of tuberculosis is indicated by the fact that nearly 200 cases are now under observation in Framingham as com- 
pared with 27 at the beginning of the demonstration, and it is particularly encouraging to note that during the first year forty-two per cent of the new reported cases were of an advanced type, while in the second year only sixteen per cent of the cases were advanced, and in the first five months of 1919 only twenty-three.

"The most important of all the practical contributions made by the demonstration is the working out of a plan for medical consultation service which is clearly the most promising means yet devised for securing a reasonably complete knowledge of tuberculosis existing in a given community. This work has been earried on by Dr. P. C. Bartlett with admirable scientific skill and exceptional tact and judgment. The physicians of the community almost without exception avail themselves freely of this service and aside from the direct results in the detection of early and doubtful cases, the stimulating effect upon the local medical profession constitutes an invaluable service to the cause of public health in Framingham.

"Believing that the control of tuberculosis is closely connected with all conditions which tend to build up the health of the community, the officers of the demonstration have devoted a considerable share of their attention to the formulation of a general health program beyond the specific field of tuberculosis itself. The medical examination drives have yielded some of the most complete data in regard to the prevalence of disease of all sorts in a random section of the population that have ever been collected in this country. These data are of much value as indicating the prevalence of such conditions and the unit costs involved in such examinations. Even more important from the practical standpoint has been the notable development of public health work in Framingham along a wide variety of lines, which has resulted directly or indirectly from the activities of the demonstration. The amount of money spent for health protection in Framingham has been increased during the life of the demonstration from 39 cents to $\$ 2$ per capita. Half of the latter sum is contributed from public sourees by the department of health and the department of education, and the other half from private sources through the Civic League, and the industrial clinies established by public-spirited manufacturers. The local health department has grown to be a strong and effective one and the system of medical inspection of school children organized under the department of edueation represents one of the best examples of such service to be found in the United States. Machinery for protecting the health of infants and children of the pre-school age has been worked out under the auspices of the Civic League, and with the advances made during the present summer includes four infant clinics and a pre-school clinic. On the whole, this work seems somewhat less satisfactory than the other phases of the local health movement, but a foundation has been laid upon which progress will no doubt be made in the future.

"The object of the gift made by the Metropolitan Life Insurance Company to the National Tuberculosis Association was not as we understand it, primarily to benefit Framingham or any other local community, but to determine the practicability of the control of tuberculosis by the full application of the best known methods. The really important question before your committee is not, therefore, the extent to which Framingham has benefited by the development of an admirable scheme of local health organization, but the degree to which this organization has been effective in controlling tuberculosis. Success along this line might obviously be measured in two ways, by a decrease in new cases, or by a decrease in the tuberculosis mortality. With the progress of the demonstration and the improvement of machinery for finding early cases, the recorded morbidity at first materially increased, but it is interesting to notice that during the first five months of 1919 only 40 new cases came to light, as against 55 new cases for the corresponding months of 1918. Obviously, however, no conclusion can be drawn from this isolated comparison. From the standpoint of mortality, the tubercu- 
losis death rate (after eliminating nonresidents and adding deaths of residents occurring outside of the town) has fallen from 93 per 100,000 in 1917 to 79 in 1918 and to a rate corresponding to 76 for the first five months of 1919. This is an encouraging showing (in view of the fact that the tuberculosis death rate in similar Massachusetts communities has in general materially increased); but again the period is so short and the actual number of deaths so small that no reliable conclusions can be drawn. The member of your committee representing statistics has prepared a computation of the probable errors involved in the problem and concludes after fitting the rates for 19071916 to the curve of probable error, and allowing for the mean error in any given rate projected for 1919-1923, that in order to warrant reasonably safe conclusions one or the other of the following combinations of tuberculosis death rates must be reached during the latter period:
A rate of 30 or less for any 1 year
A rate of 45 or less for any 2 years
A rate of 60 or less for any 3 years
A rate of 75 or less for any 4 years
A rate of 90 or less for all 5 years

"It is obvious that the rates between 70 and 80 for 1918 and 1919 cannot possibly be considered as significant, and it could scarcely have been expected in dealing with a disease of the nature of tuberculosis that any conclusive results could be reached in a period so short as three years."

The committee states its conclusions:

"It seems clear that if the Framingham demonstration should cease on January 1, 1920 , the local community will have benefited materially and many important contributions will have been made to the practical control of tuberculosis, but that the main question for which the demonstration was endowed will remain unanswered. In the judgment of your committce this would be a most unfortunate contingency. Our best authorities on tuberculosis have been subject during the past few years to a growing skepticism in regard to the adequacy of methods which have been advocated for the control of this disease and have turned eagerly to the Framingham demonstration as the first real opportunity to set their doubts at rest. If the experiment should be abandoned at this time it would be necessary to begin all over again somewhere else, for a test of the best methods now available is absolutely essential for future progress in this field. * * * In addition to the fundamental conclusions as to the practicability of the control of tuberculosis by the methods used in Framingham, a continuation of the demonstration should throw immediate light upon the theoretic factors which contribute to the development of tuberculosis as a disease, and should therefore make it possible to modify our program along more purposeful lines in the future. The investigations made at Framingham have as yet led to no far-reaching conclusions in regard to these factors. The most suggestive point that has been brought out is perhaps the variation in disease incidence among different race stocks. The combination of a very high proportion of tubereulin reactions with a low mortality among Italians and the precisely opposite condition among persons of Irish race stock is of considerable significance. The study of the subsequent history of a large number of arrested eases now under observation of the demonstration constitutes, in itself, an invaluable opportunity to determine some of the factors which convert latent into active disease. In the continuation of the work of the demonstration, we would urge that special attention be paid to the intensive study of environmental conditions, in the home, the factory, and the community, with a view to throwing more light than has yet been obtained 
upon the possible effect of each condition upon the spread of tuberculous infection and the development of active disease. We are inclined to believe that the detailed study of even a few hundred cases should yield results of real value in this connection; -if correlated with observation of the environmental conditions surrounding a properly selected control group of families free from tuberculosis.'

The committee recommended the continuance of the demonstration and the Metropolitan Life Insurance Company has appropriated $\$ 30,000$ for the work during 1920.

The National Tuberculosis Association.-This Association was organized in 1904, and since that time it has served as the center for all voluntary effort in the combat with tuberculosis in this country. Its revenue is derived from the sale of Christmas seal stamps. The liberality with which this organization has been supported is exceedingly gratifying, inasmuch as it indicates the extent to which interest in this matter pervades the people as a whole. In December, 1919, the gross revenue from the sale of Christmas seal stamps was about $\$ 4,200,000$. Of this about ninety per cent is spent in the communities making the contribution, while ten per cent goes to the national organization, which furnishes the seals, supports the American Review of Tuberculosis, now publishes Outdoor Life, distributes leaflets and pamphlets, makes contributions for special researches, and serves as a central bureau for the state tuberculosis associations. In every state there is a local association with a full-time executive secretary who directs the work in his territory. Any excess of money which the national association may find in its possession is distributed in localities where the sale of stamps has not been large and where need for work is great. The state organizations serve in stimulating municipalities and counties to build and equip sanatoria and to employ visiting nurses. Under the guidance of the national organization important advances have been made in dealing with this disease since 1904. The educational value of the December campaign made in each year is of incalculable benefit and the money so liberally donated by all classes of people has been economically and judiciously expended. Before the World War there was an International Tubereulosis Association, with headquarters at Berlin and holding its sessions every third or fourth year in some capitol. Many of the contributions made at these meetings were of great scientific and practical importance.

Some idealists went so far as to dream, and even to tell the dream, that in our common combat with tuberculosis the civilized nations of the earth would never fight among themselves. We now know that this was an idle dream. The great war has materially retarded the combat against tuberculosis in every nation involved. This does not mean that we are to be discouraged; it means that we are to redouble our efforts, hoping that in some way the price paid by the present generation will 
prove a paying investment to the generations that are to follow. The politicians who framed the League of Nations apparently forgot to lay any stress upon the most valuable asset of each and every nation-its health.

Open-Air Schools.-In the combat against tuberculosis searcely any provision has been made more satisfactory than the open-air school. The first of these in this country, so far as we know, was established in Boston in 1908. The demonstration of the value of this institution was so positive that many cities have followed the example and numerous openair schools, especially in our larger cities, are now in operation. They differ quite markedly in requirements for admission, in buildings, and in management. The Parker-Hill Open-Air School in Boston at first consisted of a lean-to used as a kitchen, toilet rooms with shower baths, and a large tent. Three meals per day were served the children, who spent practically all their time during favorable weather in the vegetable and flower garden adjacent. Open cases were excluded. On entering the school each child was furnished with a reclining chair, blankets, a long warm overcoat, overshoes, a bag made of paraffined canvas to keep out the rain and wind, and supplied with a detachable blanket lining. Woolen caps or hoods, mittens, etc., were furnished each child. The number was originally limited to 20 . The daily routine was something as follows: The children arrive at the school at 8:30 and have their breakfast. After breakfast all but four are ready to begin lessons. These four remain in the dining-room, clear the tables, wash and dry the dishes. The time between breakfast and dinner is devoted to regular periods of 20 minutes, divided between study and exercise. At 12:30 dinner is served, and again the children help set the tables and some of them wait on the others. After dinner they are required to remain quiet, and sleep possibly, for one hour. Then the regular school work is taken up. At 4 o'clock a light supper is served and at 5 the children return home. The first open-air school in Chicago began operation in the summer of 1909. The equipment was simple, consisting of a large shelter tent and 30 steamer chairs. Children were admitted only after medical examination and were carefully watched by physician and nurse. During the winter this organization was moved into a four-story brick structure with a roof 40 by 70 feet. On the roof was an open-air tent 14 by 24 feet made of asbestos board. The roof was also provided with a toilet, with a shower bath, and a storeroom. Sixty-five per cent of the children in this school came from families in which some member had died from or was in an advanced stage of tuberculosis. No open cases were admitted. Every child was given a cold shower bath each morning. This lasted for about ten seconds, after which the child was 
given a brisk rub with a Turkish towel. At first the children objected to these baths, but in a few days learned to like them and objected to going without them. An order was issued that if a child did not react properly after a cold bath this procedure was to be discontinued. However, it was not found necessary to discontinue the bath in any instance. All children gained in weight, at least normally, and most of them showed a decided lowering of temperature when fever was present. In some institutions the children live out of doors during favorable weather, doing their studying and going through their exercises on the open lawn. Open-air schools have certainly demonstrated their value. Their multiplication should be encouraged; indeed, tubereulous children have done so well in these institutions that we may ask the question why their beneficial effects should not be provided for more liberally than they have been in the past in all our public schools. If tubereulous children are so greatly benefited by such institutions, why should they not be used more widely in the prevention of the disease?

Present Schemes for Combating Tuberculosis.-In April, 1919, the League of Red Cross Societies held a conference at Cannes, Franee. The experts at that meeting formulated the following statement concerning methods to be recommended and employed in the antituberculosis campaigns :

"(1) We believe that in any organized campaign against tubereulosis the following factors are fundamental and indispensable: (a) Dispensaries on an adequate seale, furnished with laboratories and appropriate equipment and affording provisions for early diagnosis, including the examination of contacts by expert physicians, and with especially trained visiting nurses, who will carry into the homes of patients the neeessary care, assistance, instruction and advice, who will especially consider the needs of children, and who will direct the patient to appropriate agencies for this purpose. (b) Provision for the careful, regular inspection of school ehildren with a view to the early detection of tuberculosis. (c) Hospital treatment, on an adequate scale, of acute, advanced and hopeless eases of tuberculosis, separated from other cases not infeeted with tuberculosis. (d) Sanatorium facilities for all suitable cases. (e) Continuous popular education regarding tuberculosis, its causes and prevention, by all suitable means and agencies.

"(2) It is evident that tuberculosis is inextricably associated with the general living and working conditions of the people, and we therefore recommend the encouragement of legitimate efforts directed towards the improvement of these conditions. We regard of particular importance in this connection the eare of children and the problems of housing, eleanliness, nutrition and alcoholism.

"(3) We recommend the institution of appropriate measures to prevent the transmission of tubereulosis through infected milk.

"(4) We approve the establishment of open-air schools for the accommodation of children already affected by, or suspected of, tuberculosis, and measures should be taken to protect children against contagion in the household by placing them with healthy families in the country or in special asylums, when it is not practicable to remove the infected patient from his family, 
"(5) We call attention to the importance of the extension of the open-air principles to all institutions and places where many individuals are housed together, such as barracks, orphanages, workhouses, penitentiaries, and the like.

"(6) Experience has shown us the importance of careful supervision of the tuberculous patient during the entirc period of his illness. We, therefore, urge the need for close cooperation between the several institutional factors (dispensaries, hospitals, sanatoria, etc.), and the more extended development of skilled social service under medical direction.

"(7) We think that attention should be drawn to the great risk to which tuberculous patients are exposed through exploitation of alleged cures without scientific authority.

"(8) Inasmuch as a problem of particular difficulty is that of providing suitable accommodations for those patients with tubereulosis, able to perform a certain amount of work under favorable conditions, we recommend the eneouragement of efforts for the establishment of agricultural and other colonies and the organization of suitable industries which should be linked with the dispensaries and sanatoria under medical supervision.

"(9) Recognizing that accurate knowledge of the distribution of tuberculosis is an essential preliminary to its control by public authorities in any community, we approve the principle of compulsory notification of tuberculosis to the health authorities under appropriate regulations.

"(10) We call special attention to the capital importance of scientific researeh in the field of tuberculosis and the collection of information as to all factors bearing upon the prevalence and distribution of the disease."

Suggestions for Future Work.-In the first place, we believe that scientific work on the biology and chemistry of the tubercle bacillus should be encouraged. From our own experience and labors in this field, we believe that it is within the range of possibility to discover among the split products of the cellular substance of the bacillus some fraction which, when properly used, will increase resistance to infection. There is a tendency among tuberculosis experts to look upon infection with the bovine bacillus in childhood as affording some degree of immunity to subsequent infection with the human variety. However, it would be unwise, unscientific and altogether unreasonable, to leave this matter to chance. We do not look forward very hopefully to the discovery of any agent which will give anything like absolute immunity to this disease, but there is much in the history of scientific work that has been done along these lines to encourage us in the belief that, while absolute immunity eannot be secured, increased resistance, even to a slight degree, would be a powerful help in our combat with tuberculosis.

Another line along which scientific research should be encouraged is that of chemotherapy. It must be admitted that up to the present time nothing very hopeful has come out of this work, but the beneficial discoveries in the treatment of syphilis should give us encouragement.

The work of Pollak in Vienna, of Fishberg in New York, and of Calmette at Lille, seems to demonstrate that a large percentage of tuberculous 
infections occurs before the fifteenth year of age. Since prolonged exposure seems to be necessary to infection with this organism, it seems justifiable to conclude that the home is preeminently the place where the infection is acquired. We believe that the most important problem involved in our combat with tuberculosis is that of housing. During the past 50 years there has been a tendency all over the civilized world to migrate to the cities. There has been a general and almost universal movement from rural to urban communities. Tuberculous infection is most frequent in congested areas. The dense throngs that crowd our cities must be dispersed. To secure this movement "back to the land" social, economic, and industrial conditions must undergo a change. This movement cannot be forced. No argument which appeals to better health conditions will succeed in securing it. Manufacturers have located their plants in great cities in order that they might be near railroad and shipping terminals. Cars and boats bring in their raw material and carry out the finished product. It has been, therefore, a matter of great economic importance that factories be located in cities. This was especially true so long as the transport to and from these factories was carried on by means of animal-drawn vehicles. Now that motor trucks are so largely used, differences in distance are of but little importance; indeed, a motor truck on an open country road may travel miles in the same time that would be required to move blocks in a congested part of the eity. If we interpret our observations correctly, the time has already come when the manufacturer loses rather than gains by having his shops situated in a crowded part of a large city. Already certain manufacturers are retiring to rural localities. This movement will be slow, because the profitable disposition of property owned in the city is not always quickly and satisfactorily secured.

There are social reasons for the great migration from rural to urban communities. These probably constitute the strongest forces in the pull from the farm to the city. The isolated farmer feels the necessity of moving to the city in order to educate his children and to secure for his family in general many of the comforts and luxuries of life unobtainable on the farm. "Back to the land" will not play an important part in the reduction of tuberculosis unless we "go back" to proper housing and generally improved conditions of life. In a general way, the country is preferable to the city as a place of residence for one who wishes to escape infection with this disease, or, having been infected, wishes to suffer the least possible from its consequences. According to the census of 1917, the mortality from tuberculosis in Bronx Borough, of New York City, was 221.8 per 100,000, while in Rochester it was 119.7 and in Kansas, 56.5. However, tuberculosis will not be eradicated by transfer of the population from insanitary urban quarters to equally insanitary 
rural habitations. Ireland on the whole is an agricultural country with scattered villages and farmhouses, but tuberculosis eauses a much higher death rate in rural Ireland than in industrial England. If tuberculosis is to be eradicated it will be necessary, in our opinion, to disperse the condensed populations of our cities, but in this dispersal there must be adequate sanitary habitations provided for those who are dispersed. Whether the crowds of our cities are to be dispersed by the erection of more and taller skyscrapers or by provision for garden cities, as in England, time alone will determine. From an esthetic standpoint certainly, and from an utilitarian standpoint probably, we are inclined to the opinion that the garden eity is a move in the right direction. We believe that the world in general, and our nation especially, would be improved physically, intellectually, and morally, if the ownership of sanitary homes and immediately adjacent garden spaces could be secured, for all citizens. As we have already insisted, a garden city does not necessarily mean splendid wide avenues, public parks, monuments, etc., however much these may be desirable, but it means that there shall be as a part of every home a garden and preferably not more than six residences per acre.

A very strong factor in the pull from the country to the city lies in the fact that man is distinctly a gregarious animal. He lives, or prefers to live, in flocks and herds. Up to the present time the average man has not within himself enough intelligence to make of himself an interesting companion. He finds his highest enjoyment in assemblies of some kind and he is somewhat indifferent to the pabulum that is furnished him at these assemblies. As man grows more intelligent, we are inclined to the belief that he will find a growing enjoyment in his library, his laboratory, or his workshop, and at the same time a lessening pleasure in the club, the lodge, and the movie. In our opinion, the mass of mankind is not yet fitted for the highest form of family life in contradistinction to herd life.

We are inclined to the opinion that a growing, scientifically conducted, physical education of the young must precede any great reduction in morbidity and mortality from tuberculosis. Every boy and girl should have a manual, as well as a mental, training. Each citizen should learn to do something with his hands and his muscles and to do that something so well that it will be a pride to himself and a benefit to others. This physical training must begin in our primary schools and extend through our universities, must embrace all and be adjusted to each. There should be a physical examination made of every one between five and twenty years of age. This should be repeated annually, and the results should serve in directing the line of work the individual is to follow. There should be in every community a health center, with its hospital, its pre- 
ventorium, its laboratories, its trained expert physician, visiting nurses, and such other additions to its personnel as may be necessary to keep the health of the community at the highest possible point. We do not dream that such conditions as we have in mind are to be reached in the near future, but we do hope that we are moving towards them, however slowly we may make progress. As it now is, we wait until some young man or woman has reached maturity, is able to do one thing and nothing else, and finding this individual developing tuberculosis, we say to him or her-you must cease doing the only thing you can do, try to do something else, become a farmer, a market gardener, or something of the kind. Under present conditions this is the best we can do, but all must see that very little is to be accomplished in this way. We do not mean to insist that we stop doing this little, but while we are doing it let us learn to do something bigger and better. Every individual should be able on reaching adult life to do something with his hands sufficiently well to enable him to live thereby.

The labor unions are demanding shorter working days and fewer of them. The tuberculosis expert tells us that the infection is often awakened into disease by overwork, by physical exhaustion, by overstraining, by neglecting rest and recreation. The labor union is, therefore, seeking that which may benefit the race. If every individual really worked and worked earnestly in some productive capacity six, or even four, hours a day, the world would produce much more than it could use; but we are not ready for the six-hour day, because we are not sufficiently intelligent to properly spend the remaining 18 hours. Even now, as a rule, the unskilled laborer spends his working hours under better hygienic conditions than those surrounding him during the rest of the day. In this connection we wish to make a quotation from Lyman, who, as superintendent of a large sanatorium, has had much experience with the laboring classes:

"'But, one thing we have never yet emphasized enough is, what I would call the loafing conditions of our people, how they spend their leisure hours, their pleasure hours. When we face, as we do now, some of the demands from labor that they are discussing, the six-hour day or a five-day week, when we hear them making the demand, as now in one of our great industries, for a five-day week with a six-hour day, and we realize that they are only planning to work for 30 hours a week, one asks in the name of public health generally, what are they going to do with all those other hours? I have had boys come into my sanatorium, and I ask 'what is the matter with you?' 'The shop has killed me.' 'How many hours in the shopq' 'Ten.' 'What did you do with the other 14\%' That is the first time it occurred to him that there were other 14 hours in the day. Then I told him what he did with them. He dashed out of the shop, watching that clock till it got to the last hour, went home, changed his clothes and went out. Maybe he came in for supper; maybe he did not. He usually came in at midnight or one o'clock in the morning. He had either been to a dance or a movie show up to that hour; then he got up in the 
morning too late to get breakfast. He only grabbed a piece of bread in his hand and pushed it clear as he went out to work. That is what he has done with his loafing hours, and nine times out of ten that had contributed very much more to the man's downfall than the ten hours put in at the shop."

All the questions pertaining to public health are involved in the tuberculosis problem. The majority of people who have measles, whooping cough, typhoid fever, and in short, any of the communicable diseases, are at the time of acquiring these infections already infected with tuberculosis. Most acute, infectious diseases are intercurrent to tuberculosis and many of them furnish the additional straw which is to convert tuberculous infection into tuberculous disease. It has been remarked that the re-

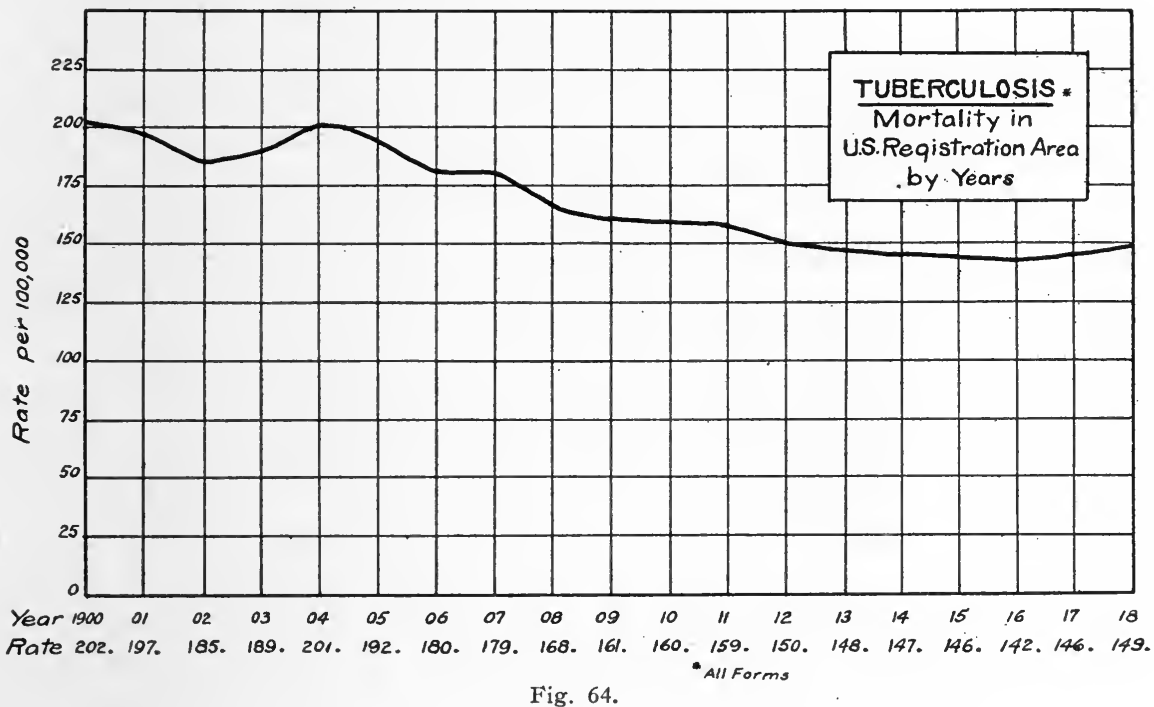

duction in morbidity from typhoid fever has already shown a recognizable reduction in mortality from tuberculosis. In all probability the same is true of other acute communicable diseases. Such diseases render those who have not already acquired tuberculous infection more susceptible to such infection, and among those who already have tuberculous infection the intercurrent disease tends to awaken this infection and convert it into active disease. It follows that the reduction of tuberculosis is wrapped up in general and in detail with the reduction of the more acute infectious diseases. The tuberculosis problem is one which cannot be solved by the medical profession alone. It involves economic, social, and industrial conditions, and the ultimate eradication of this disease must await the more complete and mature development of civilization as a whole. 
Prevalence and Distribution of Tuberculosis in the United States.The following figures give the death rate per 100,000 from all forms of tuberculosis in the United States beginning with the census of the year 1879-1880:

\begin{tabular}{lc}
\multicolumn{3}{c}{ DeATHS PER 100,000} \\
YEAR & 326.2 \\
$1879-1880$ & 264.4 \\
$1889-1890$ & 205.2 \\
1899 & 201.9 \\
1900 & 196.9 \\
1901 & 184.5 \\
1902 & 188.5 \\
1903 & 200.7 \\
1904 & 192.3 \\
1905 & 180.2 \\
1906 & 178.5 \\
1907 & 167.6 \\
1908 & 160.8 \\
1909 & 160.3 \\
1910 & 158.9 \\
1911 & 149.5 \\
1912 & 146.6 \\
1913 & 146.8 \\
1914 & 145.8 \\
1915 & 141.6 \\
1916 & 146.4 \\
1917 & 149.0 \\
1918 &
\end{tabular}

The highest incidence of this disease is in Arizona, New Mexico, Colorado, and California. This is evidently due to the fact that these states are widely resorted to by those having the disease. Of the registrants for the draft from Arizona 10.2 per cent were found to have tuberculosis or to be suspicious cases; in New Mexico the ratio was 6.6 per cent. Next to the four mentioned come Maryland, Rhode Island, Kentucky, North Carolina, Maine, Virginia, Tennessee, and Connecticut. From three to five per cent of the registrants from these states were found to have tuberculosis or to be suspicious cases. The high incidence of tuberculosis in southern New England is believed to be due to the large number of Irish employed in manufacturing establishments in that region. Possibly the number of invalids attracted to Maine and New Hampshire may increase the tuberculosis death rate in those states. In Kentucky, North Carolina, Maryland, Virginia, and Tennessee, there is a high tuberculosis death rate among the negro population. For instance, in 1917 the death rate from this disease among the whites in Kentucky was 165.6, while that among the colored people was 481.2 per 100,000 . During the same 
year in Maryland the relative rates were $\mathbf{1 5 4 . 1}$ for the whites and 433.8 for the colored. In 1917 the states with the lowest rates were Utah (43.3), Kansas (56.5), Washington (81.7), Vermont (90.4), and Wisconsin (98.3). According to the United States census for 1917, the lowest rates among the large eities appear for Spokane (62.1), Portland, Oregon (64.8), Seattle (76.1), Scranton (94.3), and Grand Rapids (95.6). However, we wish to express caution concerning the acceptance of the figures in this group. It is possible that one or more of these cities have tuberculosis hospitals outside the eity limits where patients die and the deaths are not figured against the city. We are somewhat inclined to be skeptical concerning some of the figures furnished by our census. For instance, the states of Kansas and Utah are credited with very low death rates. We have no doubt that in these states comparatively low death rates do prevail, but Kansas was not admitted to the registration area until 1914 and all estimates of death rate have been figured on a calculated population. An overestimate of population reduces the death rate markedly.

Love and Davenport, in a study of the defects found in draft men, show that the highest incidence of this disease is in the desert states of Arizona and New Mexico and the adjacent states of Colorado and California. They attribute this fact, as has been indicated, to the migration of tuberculous people to these states. New England has long been known for its high death rate from this disease, and this is attributed largely to the great number of recent immigrants. The smaller amount of tuberculosis is found in the great plains region and northern part of the Rocky Mountain Range. The high rate in southern states may be due to the presence of susceptible negroes, especially mulattoes. The draft boards detected 55,631 frank cases and 17,119 cases of suspected tuberculosis of the lungs. In addition, there were reported 9,312 cases of tuberculosis of other organs, making a total of 82,000 of draft age in whom tuberculosis was found to be a more or less active condition. Besides these, there were reported in the Army during the year 1918, 23', 178 cases of this disease. During the year 1918 the death rate from tuberculosis, all forms, in our Navy was 26 per 100,000. There were 2,398 admissions to the sick list from this disease, with 131 deaths.

Effects of the War on Morbidity and Mortality Rates in Germany and Allied Countries.-We owe the following facts to the International Health Board, which has gathered them after most careful and exact investigation. Germany suffered from the war less than any of its allied countries. In the first place, the population of Germany, comparing its present territory with the same sections of the former empire, increased during the war. The population in 1910 (exclusive of Alsace Lorraine and other ceded provinces) was 58,859,857; the population in 1918 (including all plebiscite areas) accoruing to minutely carcful official estimate 
was $59,667,729$. There was an actual improvement in infant mortality in Germany during the war. In 26 of its leading cities, with the single exception of Frankfurt, the annual death rate per 1,000 children under one year of age decreased and the average annual rate for the 26 cities as a whole dropped from 153 in 1914 to 119 in 1919 . While the tuberculosis rate in Berlin increased during the war the rate of increase was much less than in the large cities of the German allies. The death rate from tuberculosis in Berlin increased fifty-two per cent from 1913 to 1918 but dropped twelve per cent in 1919, while the increase in Vienna for the same period was 65.7 per cent and that in Budapest was eightyseven per cent.

In Vienna the birth rate per 1,000 dropped from 19.1 in 1912 to 7.6 in 1918 and 13.0 in 1919; the death rate increased from 15.4 in 1912 to 20.4 in 1918 and 22.1 in 1919. In Poland the birth rate of 36.0 per 1,000 before the war dropped to 11.0 per 1,000 in 1918; the death rate increased from 20.0 per 1,000 before the war to 24.0 per 1,000 in 1918 . In Warsaw, for every 100 children born, 73.1 persons died in 1913 and 364.4 in 1918. In Vienna, for every 100 children born, 86.4 persons died in 1913 and 267.0 in 1918. In Budapest, for every 100 children born, 74.4 persons died in 1913 and 203.0 in 1918.

The following figures give births and deaths in the places mentioned in 1913 and 1918:

\begin{tabular}{|c|c|c|c|c|}
\hline & . $\mathrm{R}$ & Per 100,000 & & \\
\hline & BIRTHS & DEATHS & BIRTHS & DEATHS \\
\hline Warsaw & 2377 & 1737 & 739 & 2674 \\
\hline Cracow District & 4045 & 2360 & 1820 & 2910 \\
\hline Vienna & 1767 & 1527 & 763 & 2041 \\
\hline
\end{tabular}

\section{Bibliography}

AdAMI, J. G., AND MCCrae, J.: Sixth International Congress on Tuberculosis, 1908, i, 325.

Armstrong, D. B.: Various Reports on the Framingham Experiment, Mon. Ser. Nos. 1 to 3,1918 .

Baumgarten, P. C.: Lehrbuch der pathogenen Mikroorganismen, Leipzig, 1911.

Bushneld, George E.: A Study in the Epidemiology of Tuberculosis, New York, 1920, William Wood \& Co.

Coвbetт, L.: The Causes of Tuberculosis, London, 1917.

DelePINe, S.: Jour. State Med., 1920, xxviii, 107.

Fishberg, M.: Pulmonary Tuberculosis, Philadelphia, 1919, Lea \& Febiger.

FLICK, L. F.: Consumption a Curable and Preventable Disease, Philadelphia, 1908, D. McKay.

FlüGGE, C.: Zeit. f. Hygiene, 1909, xxx, 125.

HIPPOCRATES: Adams' Translation, Sydenham Soc., London, 1849.

KOCH, ROBERT: Klin. Wehnschr., 1882, xxi, 221 to 230 ; Tenth International Med. Congress, Berlin, $1890, \mathrm{i}, 35$ to 47 ; Deutsch. Med. Wehnschr., 1890, xvi, 1029; xvii,
101 and 1189 .

Krause, A. K.: Am. Rev. Tuber., ii, 718.

LAENNEC, $R$. T. H.: Traité de l'Auscultation médiate et des maladies des poumons et du coeur, M. Bayle, Brussels, 1828. 
NAEgeli, O.: Arch. f. Path. Anat., 1900, clx, 426.

Ravenel, M. P.: Jour. Am. Med. Assn., 1916, lxvi, 613.

SENNETT, A. R.: Garden Cities in Theory and Practice, London, 1905, Bemrose \& Sons. TrudeaU, E. L.: An Autobiography, Philádelphia, 1916, Lea \& Febiger.

VARrier-Jones, P. C.: Jour. State Med., 1920, xxviii, 12.

Vaughan, V. C., AND Wheeler, Sybil M.: Protein Split Products, Philadelphia, 1913, Lea \& Febiger.

VAughan, WARren: Jour. Lab. and Clin. Med., 1919, iv, 123.

Villemin, J. A.: De la virulence et de la spécificité de la Tuberculose, Paris, 1868, Masson et fils; Etudes sur la tuberculose; preuves rationelles et experimentales de sa spécificité et de son inoculabilité, Paris , 1868, J. B. Bailliere.

Williamson, R. M.: British Jour. Tuber., 1915, ix, 111.

Younger, W.: British Jour. Tuber., 1919, xiii, 155. 


\section{CHAPTER XVI}

\section{LEPROSY}

Definition.-Leprosy is an exceedingly chronic, general disease, with characteristic lesions, involving the skin and nerves. It is believed to be due to the Bacillus leprae, discovered by Hansen.

History.-Leprosy undoubtedly prevailed among the Egyptians as early as 2,400 years before Christ. "The records show that it existed in India and China at least 1,000 years B.c. Whether the word "leprosy" as used in the Old Testament designated only this disease or included other loathsome conditions, has been a matter of dispute among scholars and remains to date without definite settlement. It is claimed by some that the Israelites beeame so badly infected with this disease while in Egypt that they were for sanitary reasons driven out of that country. The Persian wars apparently introduced leprosy into Greece and with the Roman subjugation of Greece it was diffused over Italy, in parts of which it had long existed. During the first and second centuries of our era it became quite common in Italy and gradually spread over the greater part of Europe. It was so common and had awakened so much apprehension in France in the sixth and seventh centuries that the segregation of lepers was begun. At least from the time of the Caesars to the thirteenth century leprosy greatly increased in Europe and became so widespread that repressive measures seemed absolutely necessary.

With the general scientific awakening of the fifteenth and sixteenth centuries, more attention was given to the restriction of this disease. In various eountries the establishment of Lazarettos, or what we now call leprosaria, began and extended rapidly. It is said that the number of these institutions in. France reached 1,500 and that in the whole of Western Europe there were as many as 19,000 . This is probably an exaggeration, but that special institutions for the housing of those afflicted with this disease became numerous there can be no doubt. Lepers were generally known as Christ's poor and the leprosaria were under the speeial eare of the Order of St. Lazarus, and were directed and managed chiefly by priests, who were themselves lepers. The attempt was made, and it must have been on the whole successful, to segregate all lepers in these institutions. Vigorous measures were enforeed in order to secure the complete isolation of the afflicted and the protection of others. When a leper went abroad he wore a peculiar garb and at night he carried a bell. He could indieate what he desired to purchase in the market only by pointing at it with a stick. Lepers were strongly forbidden to 
speak to healthy persons above a whisper or to eat with any other than a leper. Recognized by day by his peculiar garb and at night by the tinkle of his bell, the leper was shunned. During the seventeenth century the number of lepers in Europe rapidly decreased until the disease practically disappeared, "except on the fringe of the continent." In some localities the church is said to have gone so far that it performed a burial service over a person as soon as he was pronounced a leper. However, traces of this disease have continued up to the present time, especially in Norway and in parts of Russia. Moreover, it has never disappeared in Asia and in Africa. Whether the North American Indians or the Aztecs of Mexico were afflicted with the disease in pre-Columbian days is still a matter of dispute, with the evidence clearly in favor of the negative side. It appears to have been introduced into Madeira and the Canary Islands about the end of the fifteenth century and probably came into what is now included in the territory of the United States through slaves brought from Africa in the last quarter of the eighteenth century. So far as records go it first appeared in Florida. Since that time and even up to the present there are occasional fresh importations from Norway, China, Russia, and the Islands of the Pacific. In the distant Orient-India, Japan, and China, leprosy was noted in the earliest records. From China and possibly from India it spread until it reached the smallest and most distant islands of the Pacific Ocean. From the earliest historical time it has been known in Northern Africa, but there is no record of it in South Africa until it was introduced about the middle of the eighteenth century by the Dutch from Java. However, concerning the period of its introduction into South Africa there is some difference of opinion. Hippocrates describes a disease which may have been leprosy, but more probably was psoriasis. In medical literature the first clearcut and unequivocal description of this disease was furnished in the first century of our era by Aretaeus of Cappadocia.

Present Distribution.-This ancient disease is still widely distributed over all tropical and temperate regions. There is no nation wholly free from it. There are colonies or nurseries here and there and every century some seed from at least one of these finds growth in some other locality. Some of these nurseries are known to have been in continuous existence since the dark ages. Such is the colony at Bergen, Norway, where, according to Hansen, there was leprosy as early as the eleventh century and probably much earlier. In this locality the seeds of the disease have never been entirely destroyed and the number of lepers has fluctuated from time to time with the effectiveness of the restrictive measures. From 1836-1856 the number of lepers in Bergen multiplied more than three times. This increase led to stricter regulations and by 1907 the number had fallen to about two-thirds of what it was in 
1836. From this nursery at Bergen seed has been scattered to the furthermost parts of the earth and daughter colonies have been established, especially in Russia and the United States.

In some countries after its introduction it has spread with great rapidity. It appears that leprosy was scarcely known in the Sandwich Islands in 1859 but by 1881 no less than 800 lepers had been isolated in these islands. It is believed to have been introduced into New Caledonia in 1860 and it is said now to be quite prevalent. The Islands of Mauritius and Rodrigues are believed to have been infected by single lepers.

The guess as to the number of lepers now living in the world runs from one to two million. These are mere guesses and it is not probable that the exact number of lepers in any country is known. Certainly we do not know how many there are in the United States. According to Brinkenhoff, there were in 1909, 146 officially reported cases; but this includes only 50 in Louisiana where, according to Dyer, there are at least 300 . Pollitzer estimates the number in this country at 530, while Ashmead thinks it reaches 3,000 . The former is undoubtedly too low and the latter probably too high. There has been some discussion as to the need of more restrictive measures in this country. Some think that there may be a rapid increase in the prevalence of this disease, while others are inclined to regard this matter as of less importance. Certainly we should have a national leprosarium, one or more. These should be provided even if there is no danger in the spread of the disease. They are needed in order that the number already infected might be properly cared for. To longer neglect to provide a leprosarium is not only shortsighted, but well-nigh criminal. It is true that state laws provide that lepers shall be isolated, but this is only on paper and without the possibility of enforcement. Neither state nor nation has made any provision for the isolation of these unfortunates, and the way in which some of them have been tossed from pillar to post is not a credit to our civilization. Even in Louisiana where there is a state colony, isolation is by no means effectively enforced.

In 1921 Boyd and Fox, of the U. S. Public Health Service, made an intensive study of the presence of leprosy in a Texas city. Although this city is not named in their report, it is quite evident from the description that it is Galveston. They say:

"The endemic area is a moderate-sized subtropical city, located at the eastern end of a long, narrow island in the Gulf of Mexico, two or more miles distant from the mainland. Industrially the city is a sea-port of considerable importance, while manufacturing is of relatively minor importance. Demographically the city differs from most southern cities in the rather small proportion of negroes and in the large proportion of foreign-born whites, or native whites of foreign-born parentage, composing the population. The site of the city has been settled about 100 years, but the information that we have been able to collect regarding the incidence of leprosy does not cover a 
period of over 30 years. This is not to be taken as a suggestion that leprosy was unknown prior to that time, but is due to the absence of any official data regarding its early occurrence. The earliest case of leprosy of which we have been able to learn was recognized in 1886, and shortly thereafter permanently left the area. It was apparently of local origin and is not included in our series. In 1889 Dock reported two cases of local origin, which are included in our series. In a personal communication Dock states that he made at the time a 'pretty thorough survey of the Mexican and Chinese quarters, but did not find any other eases.' Only one Chinese case of leprosy has ever been known there, and of our series only two are Mexican. In addition to the 45 cases of our series we have learned of 25 additional cases of leprosy that have existed in this focus; but owing to the lapse of years and the absence of recorded data, we have been unable to secure many particulars and have, accordingly, omitted these cases. Our series of 45 cases occurred during the past 30 years. We have divided these cases into three groups as follows: Class A: living cases at present residing in the area (26); Class B: cases presumably alive, but who have removed from the area (6); and Class C: cases known to be dead (13).

"It is apparent that cases have occurred among whites and negroes only in proportion to their distribution in the population of this area. On the other hand, it is to be noted that cases are more numerous among those whites of foreign-born parentage than among native-born whites. The incidence among those of German parentage seems to be proportionately higher than among those of other descent. Thus, in 1910 the native born whites of foreign-born German parents were 2,365 or six per cent of the total population, whereas thirty-five per cent of the present cases are among their number. The significance of this is not elear. It may indicate greater susceptibility among those of German ancestry or the strain of leprosy prevalent here may be of Teutonic origin.",

The insects in the eity which might be suspected of transmitting the infection are: Musca domestica, stegomyia fasciata, and cimex lectularius. Boyd and Fox conclude that it is not probable that any one of these insects is responsible for the transmission of the disease. In their plague work they examined 23,000 rats, in only seven of which rat leprosy, as indicated by the detection of acid-fast bacilli, was found. The leprous rats were all Norways.

These authors conclude their report with the following statements:

"(1) The majority of the known cases of leprosy in this focus have acquired infection locally. (2) There is apparently a greater incidence of the disease among persons native born of foreign-born German parents than among any other group in the population. (3) There is a preponderance of cases among males. (4) More cases have developed during the second decade of life than in any other age period, though no age group appears to be immune. (5) The length of residence within the area appears to be a more important factor than age in determining the onset. The majority of cases develop the disease during the second decade of residence. (6) It is uncertain whether the area of incidence of the disease is undergoing any increase. (7) In proportion to the population of the area, there is probably a higher incidence of leprosy in the area studied than elsewhere in Continental United States. (8) The eases within the area are grouped into several distinct foci. (9) A major proportion of the cases give a history of contact or association with a case of leprosy prior to the onset of their own infection. Known contact transfer will not explain the origin of all the eases or the peculiar grouping into foci of a small radius. (10) The hypothesis of insect 
transmission is inadequate to explain the grouping of the eases. More likely, contact with unrecognized cases or unrecognized contact with known lepers is responsible for the forty per cent of cases from whom a contact history was unobtainable. In concluding, we should call attention to the fact that absolutely no official control or supervision in any form has ever been exercised over the cases of leprosy within this area."

The Bacillus.-The bacillus discovered by Hansen, of Bergen, is believed to be the specific cause of leprosy, although all the conditions necessary to establish this fully have not been complied with. This organism is a long (1.5-6 microns), slender (0.2-0.5 micron) rod, which is acid-fast and belongs to the same group as the tubercle bacillus. It takes fuchsin and similar stains more readily than the tubercle bacillus and loses the stain more easily when washed with dilute mineral acid. However, these differences are so slight and vary so much with different strains of both organisms, that they do not give a means of positive differentiation. The leprosy bacillus is oftener found in bundles and these are larger than those of the tubercle bacillus. This is not always distinctive, however. Much effort has been made to find some certain method by which the microseopic distinction between the two organisms may be determined. An expert in this work may distinguish these in many instances, but ordinarily it is not easy. The bacillus of leprosy may be mistaken not only for the tubercle bacillus, but it may be confounded with nonpathogenic acid-fast organisms, such as the smegma bacillus. The leprosy organism seems more truly parasitic than the tuberele bacillus and so far it has not been grown satisfactorily on artificial media, unless the method of Clegg be an exception. This American investigator uses a bouillon to which amoebae from dysenterie stools have been added. This preparation is inoeulated with leprosy tissue and some observers have succeeded in obtaining a growth which at the last report had been carried to the tenth generation. Attempts to inoculate the lower animals with leprosy material have not been convineing. A number of men have submitted themselves to this experiment and still the result is left in doubt. In several eases this test has been negative. In others it has been positive, but in all of the latter the tested individuals have been associated more or less intimately with lepers. The Hawaiian convict, who had his choice between suffering the death penalty and inoculation, developed local lesions but these soon disappeared. For nearly two years he remained apparently well and then developed the disease, of which he died after four years. This test cannot be considered final, because the disease had affected some of his family.

The fact that the Hansen bacillus is found in all lepers, when a thorough examination is made, must for the present be accepted as a justification of the belief that it is the causal agent. However, many problems in the etiology of this disease remain unsolved. This bacillus may 
be aided by some other causal agent or it may be infective only in certain conditions or states of health or in certain deviations from health. It seems safe to say that the evidence available at present indicates that leprosy is a transmissible disease, but that it requires more intimate contact for its transmission than most of the other bacterial affections. Its history, even when studied most superficially, indicates its contagiousness. Such a colony as that at Bergen has brought it down continuously for at least 800 years. When a leper finds his way into a region where the disease has never been known, it slowly spreads. In this way, cases have developed in Minnesota, Michigan, Iowa, and elsewhere in this country. From Norway the disease has been transplanted to the Baltic provinces of Russia, where the number at present is estimated at more than 1,000. In Finland there is a leprosarium which was established in 1355 and in 1908 it housed 87.

Avenues of Infection.-Supposing that the disease first manifests itself at the site of inoculation (which is not always true), there is reason for believing that the bacillus finds its entrance through wounds on the surface. It is a common observation in leprous countries that those who go barefooted are more frequently attacked than those who wear shoes, and that the first recognizable lesions are on the feet. Two French physicians report that among 2,437 cases under their observation in CochinChina, 526 were general from the start, 550 showed the first lesions on the feet, 420 on the hands, 321 simultaneously on feet and hands, and 337 on the face. It is an old belief that infection frequently develops in the nose. Heiser found nasal ulceration in 799 out of 1,200 eases, and others have reported even larger percentages. In recognizing this disease, one of the first things to do is to stain the nasal secretion of the suspected person for the bacilli. Among the healthy, associated with lepers, the bacilli are frequently found in the nose. There is no reason for thinking that it develops from deep breathing-in of the bacillus, because the lungs are never primarily involved.

That the disease is transmitted from man to man there can be no doubt; in fact, there are many notable instances of such transmission. Father Damien, a Belgian missionary, went to the Molokai Leper Asylum in the Sandwich Islands in 1873 to minister to the afflicted inhabitants. The disease was first recognized in him in 1882 and he died from its effects in 1889.

A case is reported by Benson of an Irishman who had acquired leprosy in the West Indies, returned to Ireland and lived for 11 months in the closest intimacy with a brother. Both occupied the same bed and after his death, which occurred 11 months after his return to Ireland, the brother wore his elothes. In the fifth year after the return of this leper from the West Indies the brother showed typical signs of the disease, 
although he had not been out of Ireland except once and then only to visit England. A Frenchman who had acquired the disease in CochinChina, returned to Strasbourg and lived intimately with a nephew, the nephew subsequently developing the disease. While the transmission from man to man must be admitted as a demonstrated fact, it appears that this disease possesses low infectivity and that it is transmitted only after prolonged and intimate contact. Further evidence of this is found in the ready and marked decrease in the incidence of the disease as a result of the partial and imperfect isolation practiced in Western Europe about 200 years ago. In Ceylon there is a large leprosarium and it is said that no nurse in attendance on the patients in this institution has ever acquired the disease. It is certainly not hereditary. It is equally true of this disease as of tuberculosis, that no child is born into the world afflicted with it. Leprous mothers give birth to healthy children, although about ten per cent of such children later in life develop the disease.

Many and varied attempts have been made to transfer the disease to the lower animals. Occasionally some experimenter has reported positive results, but under longer observation the supposed leprotic nodules have proved to be simply temporary swellings, which have ultimately disappeared without leaving any trace. It seems that we are justified in saying that all attempts to induce the disease in laboratory animals have failed. Nicolle injected Macacus monkeys with lepra bacilli and inserted into their tissue excisions from leprous growths. In some instances indolent tumors appeared and these were found to contain leprosy bacilli, but the disease was never unquestionably induced. Marchoux and Bourret made similar experiments with like results on chimpanzees. A peculiar skin disease resembling leprosy in some particulars occurs spontaneously in rats and it is a popular opinion, in some countries at least, that leprosy is transmitted by the bite of this rodent.

The possibility of the transmission of this disease through the agency of insects has been considered and many species have fallen under suspicion. Goodhue reports that he has demonstrated the presence of lepra bacilli in a mosquito, Culex pungens. Marchoux and Bourret think that some carrier is likely to be found among the gnats (simulidae). Ticks, flies, lice,-in fact almost every known biting insect has been under suspicion. It is claimed by some that since direct inoculation from man to man has failed, transmission through insects is highly improbable. This, however, does not necessarily follow. The bacillus may be modified, having its virulence increased or decreased while a resident in the body of the insect. It seems more than probable that poverty, filth, and hunger are predisposing causes. However, all these conditions favor the development and multiplication of vermin, while at the same time they 
may lower body resistance. Having found entrance into the body the bacilli apparently take up their abode in certain lymphatics, where they multiply and from which point they are disseminated by the blood and lymph to the most distant parts of the body. In many instances of this disease the bacilli are found in great numbers in diverse tissues; in fact, there are but few diseases in which the infecting agent becomes more numerous. It follows from what has been said that nothing definite is known concerning the period of incubation. It is generally given as from a few weeks to many years. There is, moreover, some difference of opinion as to what marks the appearance of the active disease. Some regard the eruption as the first evidence of active disease, but in a large majority of instances a fever, which is generally intermittent and irregular in character, precedes the first eruption and is accompanied by malaise, headache, pains in different parts of the body, sensations of cold, formication, numbness, etc.; in fact, these attacks of leprotic fever, without any recognizable lesions, may occur at intervals for years before there are characteristic eruptions. This fever seems to be due to dissemination of the bacilli through the body, and indeed, it is regarded as a septicemia. The bacilli apparently multiply in broods and the dissemination of these irregularly developing new generations is accompanied by the intermittent and irregular fever.

The tubercles or nodules which appear in the skin and are known as leproma may be pigmented or nonpigmented, ulcerated or nonulcerated. They are for the most part intradermal, but may lie in. the subcutaneous tissue.

Tubercular Leprosy.-Leprosy is generally regarded or classified as occurring in two forms according to the skin lesions. One of these is known as tubercular or nodular leprosy, while the other is known as maculo-anesthetic or smooth leprosy. Of course, it will be understood that this division is artificial and that probably in the majority of instances both forms will be found in the same individual, though one may predominate. After one or more attacks of the irregular septicemic fever, which has been spoken of, erythematous or macular eruptions appear, most frequently on the face and limbs. The maculae may disappear, or thicken and become nodules or tubercles, which are dermal lesions projecting from the skin or from the mucous membrane. For a while they may come and go, but sooner or later they become permanent structures. There are subsequent attacks of fever and some of these are likely to be followed by new erops of tubercles. While these nodules are most common on the face and limbs, they may appear anywhere in the skin and may be in great numbers. Those appearing on the face, forehead, lips, ears, etc., may become quite prominent and may quite 
obliterate normal facial lines and give to the patient an abnormal, grotesque or hideous appearance. Often the eyelids become involved and may be quite destroyed by the ulceration of the nodules. The nose is a frequent point of attack; as a rule, first through the mucous membrane, the cartilage ulcerates and breaks down. The nose may be quite destroyed and on account of ulceration and cicatrization the countenance may be so contorted as to leave but little resemblance to the human face.

Anesthetic Leprosy.-In this form of the disease pathologic changes are most marked in the nervous system. The first manifestation is one of irritation of the nerves, resulting in pain, vasomotor disturbances, and motor alterations, manifesting themselves in muscular twitching. Macular eruptions appear on the skin. Some of these may grow into tubercles, while others may remain as smooth red spots, varying greatly in shape and in size. These spots may grow larger and as they do so the centers become pale, while the peripheries are usually marked by papules. The skin in these areas becomes more or less rapidly anesthetic, while at the same time it becomes wrinkled and scaly. As has been stated, there are many mixed forms, the tubercular and the anesthetic manifestations existing in the same individual. It is the disfiguration of the human form, and especially of the face, that has made the victims of this disease in all ages so repulsive and has awakened so much apprehension as to its spread.

Eradication.-Our ancestors some centuries ago pointed out the way for the extermination of this disease. At that time it had become so common that it was continually in evidence and the desirability of restrictive measures permitted of no discussion. The people of Western Europe did in a fairly humane way a splendid piece of sanitation. Unfortunately, unsterilized spots were left here and there and in these the disease has continued its existence, reaching out into new areas whenever opportunity was offered. The work done at that time demonstrated that the infectivity of the virus of this disease for man is not great and that its complete eradication is easily possible. The reduction in the disease was so great that succeeding generations have looked upon its appearance here and there as a curiosity and as something deserving of no great attention. We should complete the work begun by our ancestors. The motives that should lead us to do this are at least two fold. In the first place, those who have this disfiguring disease should be cared for in a humane way. The state says that the leper must be isolated and kept in this condition, but there is no provision for isolation. The result is that the local authorities where the leper is found pretend to provide for him, but at the same time they are sure to suggest to the leper that he is an 
undesirable guest and that the door for his escape will always stand unlocked. In the second place, we should completely eradicate the disease which our ancestors handled so well, in order that the world might be absolutely free from this disfigurement. While leprosy is not likely to become widely disseminated in the United States, there is no reason that we should have a few cases, or that we should have one. One wellequipped leprosarium would provide a home for every leper in Continental United States. We should provide such a home, sparing no pains to make it beautiful, comfortable, and even sumptuous, and in this institution the last leper in our country could pass his days.

Since the above was written (1920) the Federal Government has purchased the leprosarium hitherto maintained by the State of Louisiana. This institution will be under the care of the U. S. Public Health Service, which has done so much for the lepers in Hawaii. 'The hope expressed in the above paragraph seems approaching realization. Not only have we a national leprosarium, but within the past few months reports concerning the treatment of this disease with certain products obtained from chaulmoogra oil have been most encouraging. The success of the leprosarium will depend wholly upon its management, and especially upon the provisions made for taking care of victims of this disease whenever found in the United States. We have decided to allow our statements about the need of a national leprosarium to stand as they were written a few weeks before the purchase of the Louisiana property by the Federal Government. We shall await the future before we express any opinion as to the accomplishments of this advance. Up to the present time, (January, 1921) we understand that the National Leprosarium is not functioning, inasmuch as there has been some trouble about the title.

Apparently, the segregation of lepers has been of value in the eradication of the disease in proportion to the thoroughness with which it has been carried out. In 1856 the number of lepers per 100,000 population in Norway was 191.3, the percentage of lepers segregated being 8.2. In 1910 the number of lepers per 100,000 in Norway was 13.5, and the percentage of lepers segregated was 63.0. McCoy gives the following figures concerning the total admission rate per 10,000 population at Molokai for the time covered:

YEAR

1870-79

1880-89

1890-99

1900-10

1915
TOTAL ADMISSIONS 1495 1968 1276 805 49
ADMISSIONS PER 10,000 POPULATION 23.8 24.3 11.7 4.5 2.1
TOTAL LEPERS PER 1,000 POPULATION IN COLONY

10.6

9.3 10.2

4.9

2.3 
Some years ago, under the direction of Heiser, an island colony for lepers was put into operation in the Philippines. A compulsory act of segregation was put into operation at the same time and an intensive educational campaign was carried out through the Islands. The number of lepers segregated at that time was about 8,000. After ten years the number had been reduced to 3,000 by the natural process of mortality among them and by the nearly complete cessation of new infections. In speaking of this, Rogers, who has had large experience with leprosy in India makes the following statement:

"Such a rapid reduction within one decade cannot be explained away on any grounds except the success of the measures adopted in removing the sources of infection from the midst of the general population, and so proves conclusively the value of segregation efficiently carried out and sets an example to other affected countries which they should not delay following, as far as the local conditions permit. The success of the Philippine measures is, in my opinion, the greatest advance which has been made in the history of leprosy, and one which opens up vast possibilities in the near future."

As these sheets were passing through the press we received, through the kindness of Dr. José Albert, a member of the Philippine Health Service, the following figures concerning the yearly admissions to the Culion Leper Colony.

\begin{tabular}{|c|c|c|c|c|}
\hline \multirow{3}{*}{ July to } & & & ADMITTED & REMAININ \\
\hline & December & 1906 & 802 & 546 \\
\hline & Year & 1907 & 690 & 780 \\
\hline & 6 & 1908 & 1603 & 1126 \\
\hline & 66 & 1909 & 1378 & 1726 \\
\hline & "، & 1910 & 930 & 2172 \\
\hline & ، & 1911 & 889 & 2511 \\
\hline & "6 & 1912 & 964 & 2991 \\
\hline - & 66 & 1913 & 795 & 3298 \\
\hline & "6 & 1914 & 859 & 3602 \\
\hline & "6 & 1915 & 555 & 3680 \\
\hline & 6 & 1916 & 966 & 4265 \\
\hline & 6 & 1917 & 613 & 4485 \\
\hline & "6 & 1918 & 973 & 4692 \\
\hline & “، & 1919 & 551 & 4706 \\
\hline January & $y$ to May & 1920 & 225 & 4736 \\
\hline
\end{tabular}

It is evident from these figures that the enthusiasm expressed by Rogers in the above quotation, is hardly justified. Albert writes that it is evident, in spite of the fact that many cases are hidden, that the number of annual admissions has oscillated within relatively narrow limits without any great noticeable decrease.

In 1920 a conference of leprosy experts was held in India, at which time the following propositions were unanimously passed: (1) Leprosy is contagious, but slowly, with a long incubation period, through the escape of the causative bacillus in the nasal discharges of the majority of cases. 
(2) The disease is not directly hereditary, children being free from actual infection at birth, but they are especially susceptible to contagion from an early age, children as a class being more susceptible than adults. (3) In view of the preceding opinions, segregation is the most effective measure for reducing the prevalence of leprosy. (4) The Committee, therefore, unanimously endorses the memorandum regarding the amendment of the Indian Lepers Act of 1898. (5) The Committee recommends that steps be taken to provide facilities for the training of medical assistants in the diagnosis and in the treatment of leprosy. (6) The members of the Committee are of the opinion that, in view of the considerable degree of fecundity of lepers, especially of females, and the excessive danger of contagion to the children of lepers, which play a great part in maintaining the prevalence of the disease, the separation of the sexes is desirable as far as possible. (7) The treatment of leprosy with the salts of fatty acids introduced by Rogers has been lately tested by 14 medical officers and assistants in leper asylums throughout India with most favorable results, seventy-two per cent showing marked improvement, in spite of the fact that most of the cases were advanced and the period of treatment has been comparatively short.

If a national leprosarium is finally established in this country and put into full operation, there must be some special provision for the transportation of lepers from places where they are found to the national leprosarium. With the present dread of the disease this would be quite impossible. It will do us no good to have a national leprosarium unless there is legal provision by means of which a leper detected in Massachusetts may be transferred to the national institution.

In June, 1921, we were told by the Surgeon General of the U. S. Public Health Service that he is now prepared to transport a leper from any part of the United States to the leprosarium in Louisiana. 


\section{CHAPTER XVII}

\section{CEREBROSPINAL MENINGITIS}

\section{CEREBROSPINAL FEVER}

Description.-This is a feebly infectious, highly fatal disease, due to the Diplococcus intracellularis meningitidis, more simply and properly designated as the meningococcus. The predilection site of this organism is in the meninges of the brain and cord where it incites inflammatory and suppurative processes eausing a high mortality, reaching in some epidemics seventy per cent and even higher. Man is the only known earrier and distributor of this mieroorganism and it may be found in the nasopharynx of healthy persons, the percentage of carriers varying from time to time and reaching its highest point when cases of the disease are present in the community. Evidently the majority of people are immune to this infection. Why many carry the meningococcus while relatively few become actively infected with it, is one of the unsolved problems in epidemiology. Like certain other infections it strikes down the most vigorous and robust and the highest mortality is among these. It may kill within a few hours, but the most fatal period is from the second to the fifth day, more than half the deaths occurring during this time. Convalescence may begin as early as the third day but is usually slow and often requires many weeks for complete restoration. The most destruc. tive epidemics have been among young soldiers in barracks and among other young people living in crowded conditions, though many small epidemics have been reported in the most sanitary villages and sporadic cases occur in sparsely settled communities and in isolated farmhouses. The disease is distributed in most instances by healthy carriers and the difficulty in tracing its course is great.

The onset may be sudden or the development may be more gradual. In instances of the former kind there is scarcely any other disease more tragic in its manifestations and more speedy in inducing death. The most common of the early symptoms is headache, which may be frontal, vertical, oceipital, or general. As a rule, it is acute and violent. The patient, elasping his head between his hands, utters eries and groans that cannot be repressed. It has been described as lancinating, boring, or erushing, "as if the head were in a vise, or nails or screws were forced into the brain." It eomes on in paroxysms and in infants the frontal fontanelle may open during one of these paroxysms. The headache of cerebrospinal meningitis is easily distinguished from that of typhoid 
and typhus fevers on account of its intensity and by its earlier appearance. The headache of typhoid and typhus is later in making its appearance, is dull, constant, and never so acutely paroxysmal as in cerebrospinal meningitis. The early headache of this disease is due to inflammation of the cranial nerves, while that which persists and characterizes the later stages is due to the pressure caused by the accumulation of fluid in the cerebrospinal cavity.

In many instances vertigo is an early and continuous symptom. It varies greatly in the degree with which it manifests itself. The young soldier standing in line or reporting for duty may become suddenly unconscious and fall to the ground. In other instances, after reporting at sick call he goes staggering like a drunken man to his quarters. This symptom was noted in the earliest reports of the disease. Concerning the Strasbourg epidemic (1840-1841) Tourdes says :

"Vertigo was among the earliest symptoms, sometimes it confused the mind and rendered walking impossible. In two eases the patients were seized with a giddiness, which compelled them to whirl round, when they fell and did not rise again."

In most instances, however, vertigo is less striking. It is accompanied by a sense of depression at the stomach, with nausea, acceleration and irregularity of the pulse and is increased by raising the head. Ames, one of the early American reporters on this disease, speaks of the excessive debility, giddiness, and dimness of sight and mentions these among the initial symptoms, but not as constant.

Vomiting is an early symptom in the majority of cases of cerebrospinal meningitis, especially when the onset is sudden. In these cases the usual sequences are rigors, headache, vertigo, and vomiting. The vomiting is due to cerebral disorder, and is not in any way dependent upon fermentative changes in the food in the stomach. Generally it is explosive and the matter ejected consists of unaltered or partially digested food without evidence of putrefactive or fermentative changes.

One of the earliest manifestations of the disease is a sudden general weakness or debility. From the moment of attack the patient finds himself completely prostrated and often he is unable to raise his hand before he is fully conscious of being ill. Foot says :

“The pulse was asthenic, and every symptom indicated a great and sudden prostration of the energy of the brain and nervous system. I have never witnessed a disease in which the powers of life are so suddenly and entirely prostrated."'

The prostration accompanying the development of cerebrospinal meningitis differs in degree from that which is evident in typhoid and typhus fevers and besides it is earlier and more complete in its development.

Delirium in one form or another is a frequent, though not a constant, symptom of this disease. It may be of the wild, maniacal form, manifest 
in attempts to rise from the bed and escape and accompanied with the display of great muscular strength. In a report of early epidemies among French soldiers, Tourdes says:

"It was rarely that delirium was absent during the course of the disease. Frequently it was transient; it ceased and was renewed, and it alternated with other symptoms, as pain and coma. Excited and furious the patients struggled with their attendants and endeavored to escape; others contented themselves with complaints and groans or incoherent words and were restless without any object; others still remained sombre and silent."

In some epidemics, for instance in that at Montgomery, Ala., in 1848, the delirium presented remarkable contrasts. In some it was wild and maniacal, while in others it was only desponding and apprehensive and in still others it was playful and jocose. In some epidemics, for instance that at Sturgis, Mich., in 1863, delirium was not observed in any case, and in his report of the epidemic in Philadelphia of about the same time, Stille says that there were not more than two or three cases of maniacal delirium.

The earlier reporters on this disease gave much attention to the expression of the face, or, as they called it, the facies. This, however, is widely variable depending upon the degree and persistence of pain. On this point Stillé says :

"In the apoplectic form, i. c., with sudden or rapid loss of consciousness the expression is fixed and stupid, like that of a person dead drunk; but such terms do not imply that the features are turgid and purplish. In this disease we observe neither the dark, dull, swollen and duskily flushed face of typhus, nor the languid, sleepy expression and circumscribed flush of the cheek which are so characteristic of typhoid fever. Except during absolute insensibility occurring in rapidly fatal cases-and even then the face is more frequently sunken than bloated,-there is a look of greater intelligence than belongs to the diseases mentioned, more mobility of the eyelids, more life in the eye, a less degree of decomposition of the natural expression of the features. Upon this very characteristic and distinetive facies Hirsch remarks, that the pale and sunken countenance at the beginning of the attack is altogether remarkable. Forget asserts that paleness of the face and skin generally was perhaps the most ordinary condition. So says Corbin, who observed it in 18 out of 20 cases; and Mayne compares the appearance in the worst cases to that of cholera. Hirseh, also, adds, that the condition in question was observed by him, not in the gravest eases only, but in a considerable number of patients who were less dangerously ill."

The deep, unbroken, prolonged stupor of typhoid fever is rarely seen in cerebrospinal meningitis. Both American and French observers point out, however, that the mental disturbance is more or less profound and they call attention to the fact that in many instances on convalescence there is no memory of the ordeal through which the patient has passed. Tourdes says that most of his patients on recovery had no remembrance of the commencement of their illness and were greatly surprised to find themselves in a hospital. 
Hyperesthesia of the skin, sometimes of that of the whole body, is not an uncommon phenomenon in this disease. Strong remarks that in some few of his cases the nerves acquired such a degree of morbid sensibility that the whole surface of the body was painful to the touch. The patient sometimes objects on account of this hyperesthesia to any change in position whether it be secured by his own movements or by those of the attendant. Pressure of the bedclothes may be painful. BurdonSanderson reported this as a marked and frequent symptom in the cases on the Lower Vistula which he was sent from England to investigate in the sixties of the nineteenth century. The same symptom has been observed and reported upon by Strong and other American authors.

Pain in the shoulders, back, and limbs, is graphically described by many of the early American authors. Fiske says:

"In some, a pain resembling the sensation felt from the stinging of a bee, seizes the extremity of a finger or toe; from thence it darts to the foot or hand, or some other part of the limbs, sometimes in the joints, and sometimes in the muscles, carrying a numbness or prickling sensation in its progress. After traversing the extremities, generally on one side only, it seizes the head, and flies with the rapid sensation of electricity over the whole body, occasioning blindness, faintings, sickness at the stomach, with indescribable distress about the precordia; a numbness or partial loss of motion in one or both limbs on one side, with great prostration of strength. The horrible sensation of this process no language can describe."

One would suspect the above quotation as being an exaggeration were it not confirmed by many other writers, not only in this country, but in France, Portugal, and England; indeed, Tourdes claimed that the spinal pains manifest in this disease are truly pathognomonic.

One of the most characteristic, though not a constant, symptom of this disease is muscular rigidity, especially that of the muscles of the neck, accompanied by more or less marked opisthotonos. While this symptom does not develop in every case of the disease, when it is found and when tetanus is excluded, the symptom is certainly pathognomonic; indeed, the position of the patient as he lies in bed with the head drawn back and with evidence of pain when an attempt is made to approximate the chin to the chest, is in most instances sufficient to justify a diagnosis of the disease. To the epidemiologist this is a matter of importance, because in the study of epidemics reported under different names we would often be at a loss to recognize the disease were it not that we find among statements of the more unusual symptoms, the following: "A drawing back of the head, with a kind of clonic spasm of the muscles of the neck and a corpse-like rigidity of the limbs." Most of the early epidemies in this country were reported by country doctors, often in the most out of the way places and under a variety of names; though "spotted fever" is the 
designation most frequently employed. Today, as we read these reports with the purpose of determining whether or not the disease was cerebrospinal meningitis, we would often be left in doubt were it not for the statement in one form or another, that more or less opisthotonos was in evidence. A few instances of this kind may serve as illustrations. In 1847 Chester, of Union County, Ark., reported four cases in all of which opisthotonos was markedi. In 1813 Dunbar, in the Valley of Virginia, described an epidemic in which the head was drawn back and the spine curved even more than in the worst eases of tetanus. In 1847 Hicks and Taylor, of Whitesville, Tenn., described an epidemic in which rigidity of the posterior cervical muscles and retraction of the head, as in tetanus, were in evidence. In 1857 Kendall, of Onondaga County, N. Y., reported an epidemic in which he says among other things: "Others lie in a torpid state, the head drawn back upon the nuchae." Numerous other instances along this line will be brought out when we come to discuss the history of the disease.

Clonic convulsions, less common than tetanoid symptoms, are sometimes in evidence. These, especially in children, have been reported from the first known epidemic down to the present time. Forget gives a graphic picture of these symptoms and writes of the "distressing spectacle presented by young men who but a short time before were full of health and strength, struggling desperately in frightful eonvulsions which were the mournful harbingers of inevitable death." The muscular twitching, tremors, and jerking of the tendons so frequently observed in typhus and typhoid are less in evidence in cerebrospinal meningitis. Paralysis, though infrequent, sometimes results and may involve any limb, a great part of the trunk, and, indeed, in at least one instance, involved the muscles of deglutition. As a rule, these paralyses are temporary and muscular power is regained during convalescence.

As we shall see when we come to discuss its history, cerebrospinal meningitis during the first half of the nineteenth century was most frequently designated in this country as "spotted fever," and this name continues as the popular designation among the laity in many sections of the country up to. the present time. Some of the earliest reporters on epidemics of this disease recognize that eruptions are by no means necessary attendants, nor indeed either constant or frequent manifestations, of the disease; in fact, North made just these statements in his book written in 1811. Undoubtedly, spots on the skin were much more frequently observed and showed a greater variety in the early epidemies than in later ones. Fish, writing of this disease as it appeared at Hartford, Conn., states: 
" In 1807 when the spotted fever first appeared at Hartford there were but few cases without petechiae or livid spots; in the following years they were less common, and since then they have not been seen in that place, before death.",

Every possible variety of cutaneous eruption has been reported as in evidence in this disease, but there is no eruption characteristic of it and, as we have seen, there have been epidemics in which no case has shown any form of eruption. Within recent years the most common eruption is herpes labialis. Quite naturally, a disease which involves such vital tissues as the meninges of the brain and cord may be accompanied by extravasations, of varying degree, of blood into the cutaneous and subcutaneous tissue. It has been suggested that the more frequent appearance of spots in the earlier epidemics was due to the methods of treatment then employed. Spanish fly blisters of great size were frequently applied to various parts of the body. Heroic methods were employed in some instances to produce profuse perspiration. Billets of wood were dipped in boiling water and placed under the bedelothing. Those of us who have employed hot water bottles for a similar purpose know how easy it is to blister a half conscious patient in this way. Although our hard-headed, well informed, country doctors of the first half of the nineteenth century gave the wrong name to the disease, they soon recognized that this was a mistake and it did not in any way affect the value of their contributions. As Sargent wrote in 1849 in reporting an epidemic at Millbury, Mass., and referring to the name: "They gave the name (spotted fever) to the epidemic only because its lesion was not known, and the name was picturesque." In recent years and when it is known that the treatment has nothing to do with the cutaneous eruptions, it has been observed that the rashes that may appear in cerebrospinal fever are subject to wide variation. They may be erythematous and transient, occurring at any time in the progress of the disease. The most common rash is maculopapular and is usually in evidence, if it occurs at all, some time during the first five days of illness. The color varies from scarlet to dusky and the eutis is distinctly elevated so that it can be easily felt and the color does not disappear on pressure. These lesions vary greatly in size and the most common location is over the abdomen, although they may appear on the extensor surfaces of the arms, legs, and occasionally upon the backs of the hands and on the feet. The rash may be petechial, consisting of reddish or dusky papules which do not fade on pressure. This form of rash may be in evidence on the first day and is more abundant upon those parts of the body most subject to pressure; for instance, about the knees, elbows, and shoulders. A purpuric rash is more ominous and may consist of spots as large as marbles. This form of rash is generally regarded as an omen of ill.

The duration of the disease is subject to wide variation. Strong says: 
"The disease appears in every shape, from the dreadful plague, which baffles all medical skill, and destroys life in five hours, down to the mild disease, which, if properly treated will confine the patient only a few days."'

Of twenty fatal cases reported by this author, seven died within twenty-four hours, five within forty-eight hours, three in seventy-two hours, and the remaining five from the third to the seventh day. Fish says that death took place usually between eighteen hours and seven days, but he reports instances of patients dying after twelve days. In reporting an epidemic in Vermont, Fassett states that in no case did recovery take place in less than from three to five weeks, and Ketchum, reporting on other patients in the same epidemic, states that several died after five or six weeks. Tourdes says that the disease is distinguished by the slowness of recovery and the rapidity of fatal issue, and Hirsch adds that its duration is between a few hours and several months. Relapses are not infrequent. A number of cases have been reported of second attacks after apparently complete recovery. In one of these reported by Warshauer, five years elapsed between attacks. Complications are frequent and varied, including those which involve the cardiovascular system, such as endocarditis, myocarditis, and pericarditis ; those involving the respiratory system, such as bronchitis, lobar and bronchopneumonias; those involving the genitourinary system, such as nephritis, pyelitis, and eystitis; those involving the articular system, such as inflammation of the joints; those involving the nervous system, including the special senses, such as hemiplegia, paraplegia, conjunctivitis, keratitis, and various other affections of the eye; also otitis media, labyrinthine disturbances and temporary or permanent deafness.

Hydrocephalus or an accumulation of an undue amount of cerebrospinal fluid is an invariable accompaniment, especially in those cases which survive a few days or longer. The hydrocephalus may be general or internal and is, partially at least, responsible for the headache, spinal pain, vomiting, and other nervous manifestations. Generalized hydrocephalus is partially and temporarily relieved by lumbar puncture. In internal hydrocephalus there is partial or complete blockage of the secretions from the ventricles into the subarachnoid space, due to inflammatory reaction. This, of course, is not relieved by lumbar puncture. Internal hydrocephalus is most frequently seen in children and leads to a low delirium deepening into a comatose condition which, according to French authors, is often indicated by a high pitched tone of voice known as the hydrocephalic ery.

Without autopsy the differentiation of cerebrospinal meningitis from typhus fever could not have been made, at least at the time it was made. As the name indicates, the lesions are practically confined to the meninges of the brain and cord. At autopsy this inflammatory condition 
may be found at all stages in the development and progress of the disease. It may be in evidence solely by an acute congestion with increased formation and turbidity of the cerebrospinal fluid. Later the process becomes suppurative. A thorough and exhaustive study of the lesions of this disease was made by Councilman, Mallory and Wright, at the request of the Massachusetts State Board of Health in 1898.

It may be thought that we have gone too deeply into the symptomatology and pathology of this disease for a work on its epidemiology, but it must be remembered that we have been compelled to decide whether certain reports made many years ago concerned this disease or some other, and it is important that our readers in order to follow us have some clear conception of the nature of the disease including its symptomatology and pathology. In discussing these subjects we have been as brief as possible, but have considered it obligatory upon us to refresh the reader's mind with a statement of the most salient points.

History.-One who attempts to trace the history of our knowledge of epidemic diseases must be impressed with the fact that a large part of the progress so far made has been dependent upon the differentiation of diseases; in other words, the fundamental corner stone in all medical studies has been the discovery of correct methods of diagnosis. In the earliest annals of epidemiology all infections were lumped together under such general terms as the plague and the pestilence. Quite naturally, certain diseases have at all times presented such distinctive symptoms or such characteristic lesions that they were easily recognized. For instance, the earliest description of what we now know as the plague is so clear cut and so distinctive that there is no question concerning the nature of the disease described. The emaciation, cough, and hectic flush of pulmonary consumption were frequently observed and easily recognized by Egyptian, Hindoo, and Greek authorities. There is, therefore, no question as to the great antiquity of tuberculosis. The Hebrews grouped under the general name of leprosy every disfiguring and loathsome form of skin disease and today it is quite impossible for us to determine how many different diseases were included under. the general term of leprosy. The Greeks, as is shown by the writings attributed to Hippocrates, gave considerable attention to differentiation among diseases, or to what we now term correct diagnosis. Arabian physicians did splendid work in the recognition of chickenpox, measles, and scarlet fever, and their differentiation from smallpox, with which they had previously been confounded. From the decline of Greek civilization, including Greek medicine, up to about the beginning of the nineteenth century, European physicians made but little progress in unraveling the tangled threads of those diseases which were grouped together under the general names of typhus, fever, or synochus. It is 
true that by the time mentioned they had learned to draw certain lines between intermittent and continued fevers. This, in and of itself, was a great work. European physicians had learned, notwithstanding the clouds of superstition that filled the atmosphere in which they lived, to recognize that intermittent fevers prevailed in certain localities, notably in swampy regions. Furthermore, they had tested the value of cinchona bark in all fevers and had found it to be of service in the intermittent fevers but without effect upon the continued fevers. Anatomists, painters, and sculptors had practiced dissection of the human body in their special studies and now and then some rare man had resorted to the dead room in order to find out something about the nature of the disease which had killed his patient, but autopsy for diagnostic purposes was rare even up to the beginning of the nineteeth century. French physicians took the lead in the development of pathologic studies and the early part of the nineteenth century witnessed the differentiation of cerebrospinal meningitis and typhoid from the group which had hitherto been designated as typhus. Neither of these advances could have been made without autopsy.

The honor of recognizing cerebrospinal meningitis as a disease sui generis and distinct from typhus fever must be given to a practitioner in Geneva by the name of Vieusseux. On the fourteenth of February in the year 1805 he saw in the more squalid quarters of Geneva a case which, on account of its striking symptoms, attracted his attention. Within eight days there were other eases in the same quarter and the disease spread rapidly throughout the eity, entering the mansions of the rich as well as the hovels of the poor. At first he was inclined to believe that the disease owed its origin to the insanitary conditions under which his first patients lived, but its rapid extension to the best parts of the city convinced him that this explanation was not correct. He observed that the fever was slight but was accompanied by apoplectic symptoms and had its seat in the brain. He states that the onset was sudden, accompanied by complete debility, severe pain in the head, vomiting and rigidity of the muscles of the neck, with the head drawn backwards. In children there were convulsions, the facies was expressive of pain, the pulse feeble, small, frequent, and in many scarcely perceptible. In fatal cases the patient lost consciousness and death resulted within from twelve hours to five days. All those who lived longer recovered. In nonfatal cases the disease also ran a rapid course and recovery resulted in a few days or the disease passed into a common bilious fever. The epidemic was confined to children and adults under thirty years of age. There were only two eases among older people. With the assistance of a colleague, Mathey by name, Vieusseux found that the lesions were confined to the meninges of the brain and cord, 
the vessels of which were congested, while a blood-stained, gelatinous exudate covered the surface of the brain. The choroid plexus was reddened, the ventricles distended with fluid, and the posterier lobe contained purulent material-there was no abnormality in any other part of the body. The inhabitants of Geneva became alarmed and the report went abroad that there was an epidemic of yellow fever in the city. Vieusseux was modest in his report and stated that from the small number of cases the disease scarcely should be called an epidemic and he attributes the fear among his townsmen to the lightning-like rapidity with which strong men were struck down and the high mortality among those who developed the disease. In his opinion the disease was not contagious, because in most houses visited by it there was only one case. Attendants and neighbors remained uninfected. In the few instances in which two cases occurred in the same house both developed the disease so nearly simultaneously that he did not believe one acquired it from the other. In the City Hospital where there were ninety patients only one developed the disease. With the approach of spring the disease quickly and completely disappeared, leaving 26 deaths attributable to it. It is interesting to note that when these bodies were buried the coffins were first covered with powdered charcoal and then with earth. Some of the houses were fumigated. Vieusseux attributed the disease to some special disposition of the air, delay of vegetation, cold nights and the great heat of the days.

One year later (1806) a similar epidemic appeared in the village of Medfield, Mass., and was investigated by two local practitioners, Danielson and Mann. It is worthy of remark that at the time neither of these Americans had read, or had any knowledge, of the epidemic at Geneva. Fortunately, they made five autopsies and it was afterwards proved that in both symptomatology and pathology the disease observed at Geneva and that seen in New England were identical. Recently an attempt has been made to trace some relation between these two epidemics. Foster and Gaskell conclude their remarks on this subject with the following statement:

"As to whether any emigration from Switzerland took place there is no evidence, but there has always been interchange between Geneva and North America.'”

Other writers say that the epidemic spread from Medfield over New England and finally over the entire United States. In our opinion, the reasons for which will be given later, cerebrospinal fever was already widely distributed throughout the world before.the epidemics at Geneva and Medfield were reported. Councilman and his colleagues call attention to the fact that in the same volume of the "Medical and Agricultural Register", which contains the description by Danielson and Mann, there is a letter from Indiana describing a severe epidemic which 
appeared at Vincennes in that State. The disease as reported in Indiana was strongly suggestive at least, of meningitis, and the editor of the magazine asks the question whether these two reports do not concern the same disease. Because a disease is not named or reported does not signify that it does not prevail or has not existed previously or at some other place. We are now ready to submit evidence that cerebrospinal meningitis existed long before it was recognized by Vieusseux and Mathey in Switzerland and Danielson and Mann in Massachusetts. According to Radmann, there is in the burial register in the village church of Ueckermunder a footnote attached to certain names, stating that these persons died in $\mathbf{1 7 2 2}$ of 'a disease which had been known in the village since 1702. This disease is briefly described in the register. It was known locally as "Die Steifnis" and its chief symptoms were severe pain in the head, vomiting, vertigo, and muscular rigidity, especially marked in the muscles of the neck. People were struck down suddenly and many died on the second, third, or fourth days. The note further states that the medical men of the time believed the disease had come from a poisonous cloud which had fallen upon the earth and which not only struck down the people, but also poisoned the growing grain. In a history of epidemies written by Ozanam and published in France in 1835 it is stated that epidemics of cerebral fever appeared in Germany, Holland, France and Spain about the middle of the sixteenth century. In German Switzerland this disease was known as "hauptwehe" (pain in the head). It was highly fatal, especially among children. The same authority mentions a disease which was observed in England in 1661 in which autopsy showed congestion of the blood vessels of the brain and diffusion into the cavities of the brain and cord. A similar epidemic was reported by Saalmann in Westphalia in 1788. In this, certain autopsies were made and it is distinctly stated that inflammation of the meninges of the brain and cord was found. According to Bascome, a disease known as epidemic cephalalgia was reported in Europe in 1503. The most usual symptoms consisted of violent delirium, convulsions, contractions of the limbs, followed by prostration and coma. Death occurred speedily-sometimes in a few hours, more often during the third or fourth day. According to the same author, there was a similar epidemic in upper Italy in 1505.

It will be best to briefly review the reports on epidemics of this disease pertaining to our own country and then we shall briefly discuss the prevalence of the disease in other lands. As we have already seen, the first epidemic reported was that at Medfield, Mass., in 1806. Fish saw similar cases at Hartford, Conn., in 1807. The next report comes from Minisinck, Orange County, N. Y. (1808-1809). This report, by Arnell, adds nothing to our knowledge concerning the nature of the disease or 
the manner of its spread. In 1810 epidemic meningitis was reported in York County, Me., and sporadic cases continued to appear in that locality during the following five years. In 1810 the disease was reported from so many villages in Massachusetts that the State Medical Society appointed a committee to investigate and report. The most interesting parts of this report are concerned with the symptoms, the eruptions, and the postmortem appearances. So far as symptoms are concerned headache is given as the first and the most universal. Delirium was generally mild, though at times furious and maniacal. No stress is placed upon tetanoid symptoms, although these were observed in many instances. Special attention was given to the spots which, however, were found in comparatively few cases. They were believed to be more portentous in proportion as they were more darkly colored. Coagulated lymph was found in the subarachnoid space. The lateral ventricles contained serum and the choroid plexus was harder and thicker than usual. In 1811 North published his monograph on "Spotted Fever" in which he included all the literature up to that time.

During our war with England (1812-1814) cerebrospinal meningitis was widely distributed throughout the country and made its appearance among our troops, especially in the cantonments. Mann, the medical historian of that war, gives an interesting and valuable report in which he shows that both bronchopneumonia and cerebrospinal meningitis were in evidence. He says, among other things :

"It may be necessary to observe, the winter epidemic of 1812-1813 was a form of disease distinct from that which in the northern districts of the eastern states the preceding winters had been known by the name of spotted fever, although the exciting causes may have been similar. In the spotted fever, mental derangement was an almost general concomitant of the disease. In many instances, this affection of the brain was the first symptom of morbid action. Whereas, pneumonia, especially among the troops was never accompanied with mental derangement, at its first attack, and but seldom in its more advanced stages; nor until the laborious respiration which was a most prominent symptom, at its first attack, had somewhat subsided or the patient was at the point of death. This epidemic appeared under the forms of both sthenic and asthenic diathesis; although under the last it was often, if not always deceptive. In many of the first cases at Burlington, the disease proved fatal in two, three, and four days, by the violence of the first attack; in some instances, in less than 24 hours after the first symptoms of indisposition supervened. *** This epidemic was widespread in its influence, prevailing from Lake Erie down to Lake Champlain, over Vermont, the northern countries of Connecticut, Massachusetts, and New Hampshire."

The same authority, after speaking more fully on the bronchopneumonia, adds:

"There were a few cases of a disease which assumed the form of spotted feverin which the brain seemed to be the seat of the disease; a mental derangement having been the first alarming symptom, without any pneumonie affection. All these died within the first twenty-four hours; two of the number within six hours." 
In this, as in other wars, cerebrospinal meningitis was a barrack, rather than a field disease, and it was especially observed at one of the eantonments known as Greenbush which occupied a location on the east bank of the Hudson directly opposite Albany. It may be of interest to quote Mann's description of this camp:

"The cantonment is on an elevated plain, one mile east of the Hudson. Here are barracks for the troops of the United States Army, suffieiently eapacious to accommodate $4000 \mathrm{men}$, with adequate quarters for their officers. On an eminence 60 feet higher, is the hospital, which may aceommodate 100 patients. The wards of this hospital are too small in their dimensions, both for health and convenience, being only 20 feet by 16, and 9 feet in height. The elevation of the hospital is so great above the surface of the river, that the fogs, which during the hot season, are suspended from the flats and villages on the banks, seldom rise to its summit. The temperature of the climate on the Hudson is more regular than in the same latitude on the Atlantic shores; where are experienced greater and more sudden transitions of weather than here. The cantonment, at Greenbush, has the reputation of being healthful; and the eountry in its vicinity salubrious. The City of Albany, the eapital of the State of New York, is situated one mile in length on the west bank of the Hudson. It rises from the river by a gradual aseent nearly 200 feet to the elevated plain. The width of this eity is from one-quarter to one-half a mile. On the margin of the river, the lands are alluvial and rich; while those more elevated and uneven, are a mixture of elay and sand, and barren. That part of the eity, on the alluvial flats has the reputation of being less salubrious than that on the hill. The want of a rigid health police is manifested by the filthiness of some of the streets; more especially of the backyards connected with stables and kitchens.",

It is evident from Mann's writings that bronchopneumonia was the most prevalent disease at the cantonment at Greenbush, but that cases of eerebrospinal meningitis did occur there and at other army stations there ean be no doubt. Mann gives the following brief statement concerning the condition of the brain in a postmortem:

"On removing the skull eap, the dura mater and brain were distended with blood; on making an incision into any part of the brain, the cut surface was instantly covered with the blood which oozed from its vessels; the right lateral ventricle contained about a tablespoon of serum."

Fuller, an army surgeon stationed on the Niagara frontier, reports much pneumonia and adds:

"In a few instances, the inflammation has attacked the brain and its meninges, producing phrenitis."

Other cases were observed and reported upon by Mann at Malone, N. Y., and Sharon, Mass. Southwick, of Albany, reported a few cases, the first one of which came from the cantonment at Greenbush. One cannot read the medical history of the war of 1812-1814 without being impressed with the belief that the camps at that time presented in miniature exactly the same conditions seen in greatly magnified form in our 
cantonments in 1917-1918. It seems that our Navy at that time, as in later wars, did not wholly escape this disease. Hunt reports cases from Washington in which there was pain sufficient to keep the patient in "a state of constant agony."

In 1813 Dunbar, of Winchester, Va., reported a small local epidemic confined largely to the ages of from 12 to 20 years. That this was cerebrospinal meningitis there can be no doubt, for he states that within a few hours after the attack the head was drawn back and the spine curved. Petechiae and vibices were observed over the greater part of the body.

In 1815 Wilson, of New Hampshire, wrote a small book, entitled, "Nature and Treatment of Spotted Fever." In our opinion, this author included both bronchopneumonia and cerebrospinal meningitis in his cases. Some are clearly the one, while some are certainly the other. The author himself seems to have had an idea that there were certain lines of demarkation running through his case reports, because he says:

" In 1812 the lungs were almost universally affected, and death was caused by suffocation, etc. In all the cases which I witnessed in 1813, the lungs were not so generally affected; the head appeared to be the part which suffered most, and the patients appeared to die in convulsions, apoplexy, palsy, etc."'

His postmortem reports bear out the suspicion that he was dealing with both bronchopneumonia and cerebrospinal meningitis. In one report he says that the upper portion of the lung was heavy and sank in water and that the air cells were filled with a yellowish lymph. In another case he found more or less extravasations in the membranes of the brain and deposits of lymph on its surface. About the same time Utley, of Lyme, Conn., recognized two varieties of spotted fever, and in this instance also there can be no doubt that he had seen both bronchopneumonia and cerebrospinal meningitis. Kercheval, of Bardstown, Ky., wrote:

"It happens in a certain proportion of the eases that the head is the primary seat of the disease; the chest at the same time remaining free from any local disorder."

Indeed, it is evident from a number of reports made from different sections of this country that the physicians of the first quarter of the nineteenth century under the denomination of "spotted fever" included cases of both bronchopneumonia and cerebrospinal meningitis. We are in doubt as to which of these diseases predominated in reports made from 1812 to 1815 by Bascome, of Granville, N. Y., Vaughan, of Hallowell, Me., Hazeltine, of the same state, Trent, of Richmond, Va., Hereford, of Middlebury, Va., Davis, of Milledgeville, Ga., Kerr, of Natchez, Miss., and others. In 1815-1816 Davis, of Columbia, S. C., made a report on spotted fever in that state and this has been accepted by some as an epidemic of cerebrospinal meningitis, but we admit that we are more inclined to the opinion that many of his cases at least, were pneumonia. 
In 1815 Gallup published a book, entitled, "Epidemic Diseases in the State of Vermont," in which he devotes a section to spotted fever. Some of his cases undoubtedly were cerebrospinal meningitis, because he states that the seat of the trouble was found to be in the head where there was congestion and exudation. In 1816-1817 cerebrospinal meningitis, generally reported under the name of spotted fever, was in evidence in North Carolina, South Carolina, Virginia, Ohio, Connecticut, and Illinois.

In 1823 there was an epidemic in Middletown, Conn., which is reported in a most interesting monograph published two years later by Miner, a practitioner of that village. Miner designated this disease as "Typhus Syncopalis or Sinking Typhus." It is of considerable epidemiologic importance that we determine as nearly as possible whether the epidemic reported by this author was one of cerebrospinal meningitis, either in part or altogether. There were 360 cases between the last of March and the last of December, 1823, in the village of Middletown. We have not been able to find any estimate of the population of this place at that time, but it could not have exceeded 3,000; in fact, Middletown has up to the present date (1920) not reached a population of more than 10,000. It seems, therefore, that about one-tenth of the entire population was involved in this epidemic. If this inference be true, all the cases could not, in our opinion, have been cerebrospinal meningitis. We know of no epidemic of this disease in which so large a proportion of the inhabitants of a community has been involved. We do not believe, judging from the history of this disease, that ten per cent of the people in any place are susceptible to it. In the second place, there was no distinction in age among those attacked. "Persons of all ages, from one year old to seventy and of both sexes, were the subjects of the disease." Cerebrospinal meningitis shows a partiality for children and young adults and strikes down most frequently the more robust and vigorous. In the third place, the epidemic lasted too long for one of cerebrospinal meningitis - the last of March to the last of December. It is said, however, in the paper, that one-half the mortality happened in November. The summer of this year, according to the paper, was an exceedingly hot one. While this fact does not completely negative the claim that the disease was cerebrospinal meningitis, it is not probable that this disease continued to spread in a sparsely settled village during the open months of a hot summer when crowding was reduced to a minimum and there was the least possible opportunity for direct transfer of the infection from one individual to another. In a large city cerebrospinal meningitis may continue in epidemic form for months, and possibly for years, but this is not its usual behavior in small communities. Finally, we are not convinced that any large number of these cases were cerebrospinal meningitis, because the case mortality was too low. Among the 
360 cases there were only 12 deaths, 6 children and 6 adults, a rate of 3.3 per cent. So far as we know, there has never been an epidemic of cerebrospinal meningitis with a case mortality as low as this.

There are paragraphs in Miner's paper which seem to point clearly to cerebrospinal meningitis, as the following selections indicate:

"In nearly every instance, whether severe or moderate, the disease began with more or less pain in the head, especially about the forehead. Vertigo was also a symptom almost universal. In some, a sudden faintness, dizziness, or sense of extreme exhaustion, so that the patient could neither sit nor stand upright was the first symptom. In others, the attack resembled a severe concussion of the brain, or a sudden stroke of palsy or apoplexy. Indeed, everything seemed to show that the brain or nervous system was the primary seat of the disease. *** Indeed, vomiting or nausea, in this disease, seemed ordinarily, to be entirely symptomatic of an affection of the brain, or was obviously the effect of an emetic or cathartic, the sympathies being the reverse of sick headache. *** The condition of the brain, in this disease, was usually very similar to that state which is produced by exposure to carbonic acid gas, or which is occasioned by excessive doses of some preparation of lead, or by certain essential oils, or of active and pure narcotics. In many cases it resembled concussion, or apoplexy, or palsy. In all the severe, and in a majority of the mild cases, there was from the very access, before a particle of medicine had been taken a peculiar deficiency of vital energy in the brain, and the whole nervous system. So that raising the patient into an erect posture, would generally produce the same sort of vertigo, anxiety at the stomach, acceleration and irregularity of the pulse, nausea, and even fainting, which result from a similar position after a great loss of blood. In almost every instance, the patient was more comfortable when the head was low; and much inconvenience, and even danger was almost invariably occasioned from any other than a horizontal posture. * * * Many had most of the usual symptoms of hydrocephalus internus."

These are the strongest points in the description of the symptomatology of the disease for suspecting that it was cerebrospinal meningitis. To us, they are not convincing for the reasons already stated. Unfortunately, no autopsy was made, and a similar omission makes many interesting reports on spotted fever and under other names about that time difficult when one attempts to determine the real character of the disease described. As has been indicated, Miner did not call the disease "spotted fever" but "typhus syncopalis." He discusses at some length the nature of the disease which he had under his care and comes to the conclusion that it was a mild form of the disease described by Fish in Hartford in 1809, and which undoubtedly was cerebrospinal meningitis.

"From these considerations, it is believed, no one will hesitate to admit that this disease, at least as respects the severest and most sudden cases, was a genuine spotted fever, and that it was essentially different from common typhus. Indeed, the two diseases differ so much, especially during several of the first days, that the best treatment of common nervous fever, would prove fatal in every severe case of spotted fever. * * * It is true, that in a great majority of the cases of this year, the disease was less violent, and more manageable than in the spotted fever of 1809, and, indeed, than it was this same season in several adjoining towns. The only difference appears to be, 
that the mild and insidious eases, were in a much greater proportion this year than formerly. The eases of sudden attack, were identically the same as those of 1809 , and all the others were clearly varieties of the same epidemic. But this is a point of difference which existed to an equal degree between several of the different eases of both epidemies. Why may there not be prominent varieties of spotted fever, as well as of common typhus, yellow fever, cynanche-maligna, pneumonia typhodes, dysentery, etc.?",

In the last quotation, we imagine that we can determine with some degree of positiveness the nature of the disease described by Miner. He probably had a few cases of cerebrospinal fever, and like many of us who have practiced medicine, he saw in every patient something of the disease which made the deepest impression upon him. Sydenham wrote of the "epidemic constitution." Later authors spoke of the scrofulous and other diatheses. Even within our own generation some able authorities have seen the "uric acid diathesis" in practically every patient and every disease under observation. There can be no doubt that Miner had seen cerebrospinal meningitis before 1823. This is shown in the book, entitled, "Essays on Fevers and Other Medical Subjects" by Miner and Tully. Miner was a man of erudition and of deservedly good repute, and the monograph which we have had under consideration was most influential and beneficial in halting the depletion treatment so largely employed at that time. In his paper he emphasizes the desirability of avoiding everything which might "waste the vital powers." He opposed not only bleeding, but purging and oversweating as well. "It is as safe to leave a wounded artery to nature, as in this disease to administer a cathartic, without directing its free operation to be constantly checked by opium." He speaks repeatedly of the subsidentia, by which he mcans sinking, faintness, and a low ebb of vitality. The monograph probably did harm in the unstinted recommendation of opium and alcohol in the treatment of spotted fever. It is true that Miner himself used some discretion in the employment of these agents, but the immense doses of these poisons given by other physicians in the treatment of so-called spotted fever appall us of the present day. We are inclined to the opinion, however, that alcohol and opium were less harmful than the depletion measures which had been formerly used.

We have already suggested that the more frequent appearance of spots as recorded in early reports of cerebrospinal meningitis might be explained by the treatment. Permit us to quote briefly what Miner says about treatment:

"Warm or tepid bathing, was rather dangerous. The water should be so hot, as to make as strong an impression as can be borne, without danger of sealding. When strong, and at the same time quick rubefacients were required, bottles of hot water, heated bricks or wood, hot spirits, mustard, liquor of ammonia, capsicum, and oil of turpentine, were important agents. Epispastics of eantharides would produce no effect, in many cases, till the skin had been first excited in this way; but as soon as the sus- 
ceptibility of the patient was raised by internal and external means, to the blistering point, the freest application and repetition of them, was attended with the most obvious beneficial effects. They were more especially useful to the shaved vertex, forehead, temples, spine, epigastrium, and extremities. In every case (the very mildest are not with safety excepted), the forehead, and in the severe, the vertex should be immediately blistered. Shaving the head, and blistering it early, is more serviceable than any other external application. Torpor of the bladder almost invariably yielded to sinapisms, or more especially, to blisters, on the inside of the thighs. So sensible were those patients who had their reason, of the beneficial effect of blisters, that they frequently begged to have them repeated. To one patient, more than twenty blisters, and to another, more than thirty were applied during the course of the disease, and evidently with the greatest advantage. Oil of turpentine, warmed, and confined to prevent evaporation, was the speediest epispastic, and was of much benefit in coma. In a very few cases, there supervened such a peculiar irritation in the latter stage, that blisters required to be repeated with caution, on account of strangury, as this symptom, in irritable cases, sometimes increased the morbid action. It always lessened it in cases of torpor. In no other disease is it so essential to distinguish counteracting from coinciding agents. But after all, in the severest forms of the disease, opium, alcohol, arsenic, aromatics, and external applications, with no other medication, might be depended upon."'

When we read the above, we are quite convinced that the treatment had something to do with the wide distribution, remarkable size, and great diversity shown by the spots and which gave the erroneous name of spotted fever to many of the epidemics of cerebrospinal meningitis reported in this country in the first half of the nineteenth century. This is a matter of importance, because, even so great an authority as Hirsch, in one of his earlier writings, states that the spotted fever of America had nothing to do with cerebrospinal meningitis.

Small epidemics of cerebrospinal meningitis, generally under the name of spotted fever, continued to be reported during the third decade of the nineteenth century. These epidemics were by no means confined to New England, but involved the South and the West, at least as far as Arkansas and Missouri, and the Northwest. Hirsch, in speaking of this disease in the United States, says:

"The year 1816 forms the close of that time of sickness, with the exception of two quite localized epidemics, one at Middletown, Conn., in 1823, and the other in Trumbull County, Ohio, in 1828.

The United States were absolutely free from the epidemic meningitis from that date down to the year 1842, so far at least, as we may infer from the silence of the epidemiographical authorities.",

Are we justified in concluding that a disease has disappeared from so large an area as that covered by the United States, even in 1830 , because we find in the medical literature of the time no reports of its existence? The assumption which Hirsch makes in the above quotation is invalid for two reasons. In the first place, there are reports of epidemics 
of meningitis during the time he suspects the United States to be entirely free from this disease, and which he did not find. The most notable is that which occurred in New London, Conn., in 1832, and is reported by Savage, who says:

"It was unanimously identified, by the physicians of New London, with a disease which appeared epidemically at different periods, throughout the New England States. the earliest account of which, it is said, is given by Drs. Danielson and Mamn, in 1806."

The second invalidating factor in this assumption lies in the fact that it is the unusual and exceptional physician who reports cases or epidemics of any kind for publication.

In 1845-1846 an epidemic was reported in Clark County, Ill. This disease went under the popular name of "black tongue," a name quite as inappropriate as that of "spotted fever." Fortunately, the doctor in attendance, McCoy, made autopsies and called the disease "cerebrospinal arachnitis," a very proper name. In 1847 , when soldiers were being mobilized and sent to Mexico, cerebrospinal meningitis was widely disseminated, being reported in the District of Columbia, Mississippi, Tennessee, Missouri, and Arkansas. The only account of it among the soldiers is that by Love, who reported it in the Second Mississippi Rifles assembled and quartered for a time at New Orleans. Love calls attention to the fact that this regiment had been subject to severe drill in inclement weather and that, although in the quarters at New Orleans it was next to a Pennsylvania regiment, the latter remained free from the disease. Love also calls attention to the fact that the affected regiment was placed in crowded quarters. After the Mexican War reports show that this disease was widely distributed among the civil population. At a meeting of the Boston Society for Medical Improvement in 1849 cases were reported by at least three New England village doctors. Corlew reported cases, which he had been wise enough to autopsy from Millbury, Mass. Paine made a report from Sutton, Mass., and Green, from Worcester County, Mass. There can be no doubt about the reality of cerebrospinal meningitis in these cases. Both in symptomatology and pathology they conform to the classical picture. The disease struck down the strong and vigorous and death occurred in some instances within a few days. Opisthotonos was observed and was found to be marked in many instances. The following is a report on an autopsy made by Corlew :

“The body was mottled by large ecchymoses. Two or three inches of adipose substance over abdomen, and nearly as much over thorax. Viscera of thorax and abdomen examined carefully present no abnormal appearance. Lungs, healthy and erepitating. Valves of heart normal; spleen small; liver, of usual size and appearance; kidneys, the same. All the abdominal organs inlaid in adipose substance; no elevation of Peyer's patches. Upon opening the cranium, there was no unusual adhesion of the dura mater, which presented its ordinary appearance, and preserved its polish on the arachnoid 
side. Under the cerebral arachinoid, over the upper surface of both hemispheres was seen a whitish deposit, as of purulent matter. On attempting to remove the arachnoid, the pia mater came with it, the two cohering so closely as not to admit of separation; and the purulent deposit was between them. On both sides of the cerebellum there was a considerable intermeningeal deposit of pus and lymph, also. The membranes having been detached from the cerebellum, it had not its usual whiteness and polish, but was dull and punctate, like the inside of a strawberry, and on being seraped was found to be somewhat softened. The cut surface presented an unusual number of red points. The cerebellum seemed healthy; no unusual fluid in ventricles. No pus seen along medulla oblongata. The spinal marrow being exposed from the front, at commencement of dorsal vertebrae, a considerable deposit of pus and lymph was found without softening of marrow. At middle of dorsal vertebrae there was another similar deposit with softening of the marrow.',

At the beginning of the Civil War cerebrospinal meningitis was widely distributed throughout both the North and the South, and during the war it was reported among soldiers at Hall's Hill, Va., near Washington, by Frothingham; at the General Hospital, at Louisville, Ky., by Leber; in the Seventy-Fourth Ohio Infantry by Wirth, at Nashville, Tenn.; at Brandon, Vt., by Woodward; at Newbern, N. C., by Upham; at Chillicothe, Mo., by Prewitt; at Benton Barracks, Mo., among colored troops, by Russell; at Galloupe's Island, Boston Harbor, Mass., by Lyman; among recruits at Concord, N. H., by Crosby; on the James River, by Clark; at Kansas City, Mo., by Thorne; among recruits at Brattleboro, Vt., by Phelps; at Ft. Monroe, Va., by Bontecou, and by army surgeons in many other localities. Among the Confederate Troops, it was reported by Haywood at Raleigh, N. C.; by Moses at Mobile, Ala.; by Banks at Fredericksburg, Va.; by Jones at Augusta, Ga., and by other surgeons at different places. It would not be profitable to go into detail concerning cases observed in either army during the Civil War. These are well covered, with reports of many autopsies, in the Medical and Surgical History of the War of the Rebellion.

During and after the Civil War this disease was widely disseminated throughout the United States. No doubt the mobilization of troops carried the infection into the army. The discharge of sick and wounded during the war and the demobilization at the close of the war greatly aided in the wide dissemination of the disease. Again, we must call attention to an error on the part of Hirsch: He says:

"In 1865 the sickness would appear to have been in abeyance (in the United States) if we may judge from the absence of all reference to it."

Cases were reported in January, 1865, by McGill; in February, 1865, by Jones; in March, 1865, by Doty, Whitcomb, Brown, and others ; indeed, a cursory examination of the Medical and Surgical History of the War of the Rebellion will show that there is searcely a month in the year 
of 1865 in which cerebrospinal meningitis is not reported among either soldiers awaiting discharge or those recently discharged.

It is of epidemiologic interest that from the time of the first recognition of this disease by Danielson and Mann at Medfield, Mass., in 1806, up to the Civil War, we have found no account of the existence of the disease in any large city in the country. This raises an important question. Is cerebrospinal meningitis a rural disease? Certainly every important contribution to its symptomatology, epidemiology, and pathology, found in American medical literature during the time mentioned comes from a country doctor. Did our large cities have no cases, or were they unrecognized, or at least unreported? Since the Civil War there have been epidemics in nearly every large city in the country. Quite naturally, no positive evidence bearing on this question can be adduced. We must state, however, that in collecting material from which this sketch so far has been prepared, our esteem and respect for the country doctor of that time have been greatly increased. Moreover, we can trace the influence of one wide-awake, intelligent, inquiring physician in a community upon his colleagues and upon those who follow him. It is not probable that a small place, like Montgomery, Ala., for instance, has been a breedingplace for this disease since 1842, and yet, three important contributions concerning the disease have come from that place. The first was by Boling in 1842. Since that time, independently, and in different years, Ames and Baldwin, of the same small community, have made interesting and valuable contributions to our knowledge of cerebrospinal meningitis. Had Danielson and Mann been satisfied by doing their best for their patients and had they not been led by a desire for further information which they attempted to secure by autopsy, the history of this disease in this country would have been quite different from what it is.

As has been said, after the Civil War, cerebrospinal meningitis was recognized in the large eities and came within the cognizance of the most astute clinicians of the time. In 1866-1867 Stille had opportunity to study 98 cases in the Philadelphia Hospital, and he wrote a monograph, entitled, "Epidemic Meningitis." We are mostly interested in knowing what conclusion was reached by this keen observer concerning the infectiousness of the disease. On this point he speaks with eaution, but he leaves the reader with the impression that he does not believe the disease is contagious. He says:

"'It' may be said, in general terms, that epidemic meningitis has occurred in all portions of the temperate zone inhabited by European races and their descendants; in all sorts of localities, among all ranks and conditions of society, at all ages, in both sexes, and that it is therefore in the strongest sense of the word a pandemic disease. When the statement is added that the disease is not disseminated by contagion, it evidently falls in the eategory of the diseases referred to, of whose occurrence no other 
explanation is at present possible than that they are directly produced by a specific atmospheric poison.',

Upon one point, however, Stillé was very positive. He was sure that cerebrospinal meningitis is a disease sui generis and that it is not a form or modification of typhus fever. On this point he was, as we shall attempt to show later, far in advance of Murchison, the great English authority on fevers at that time.

In 1866 Webber, of Boston, wrote a very valuable paper on cerebrospinal meningitis, which was awarded the Boylston prize. This paper is one of the most valuable and exhaustive monographs on the history of the disease. It is to be regretted, however, that this author did not sufficiently appreciate the differences between the lesions of typhus fever and those of cerebrospinal meningitis. His concluding paragraph reads as follows:

"There is almost an exact agreement between these two diseases in the symptoms and postmortem appearances, except in reference to those dependent upon lesions of the cerebrospinal system. The causes and treatment are essentially the same. We must conclude, however, then that epidemic cerebrospinal meningitis is only epidemic typhus, wherein, from some cause, the cerebrospinal system is the principal seat of the attack. By this classification the great variety in the symptoms is easily explained and the epidemies in which the pneumonic form was most frequently seen were true typhus, less frequently complicated with lesions of the brain."

It is quite evident from this quotation that Webber made not only the mistake of believing that cerebrospinal meningitis is a form of typhus, but he confounded pneumonia with both typhus and meningitis. This confusion is seen in many places in his essay where he accepts as cerebrospinal meningitis many reported epidemics which were, in part at least, plainly bronchopneumonia.

In 1873 Smith (J. Lewis) studied an epidemic in New York City and in his conclusions made the following statement concerning the contagiousness of this disease:

"My statistics, therefore, harmonize with the doctrine of noncontagiousness, but it is obviously very difficult to determine from clinical experience whether an epidemic constitutional disease is absolutely noneontagious, or contagious in a very low degree. Cerebrospinal fever is one or the other, but if contagious it is apparently less so than either typhoid fever or Asiatic cholera."

These wise words express the most logical conclusion which one could reach from a study of the literature available at that time; also from present observation among his patients.

Again, we must call attention to a statement made by Hirsch. Writing in 1886 and after referring to the wide distribution of the disease in the United States for some years after the Civil War, he adds: 


\footnotetext{
"Subsequent to 1874 the United States authorities are silent about epidemic meningitis; so that the disease as a widespread malady would appear to have ended for the present on American soil with the extinction of the epidemic last mentioned."
}

According to Councilman and his colleagues, the number of deaths reported from this disease in Massachusetts alone from 1878 to 1896 inclusive was 2,909 , an average of nearly 150 per year, with a minimum of 78 in 1878 and a maximum of 171 in 1888. Looking over the reports of the United States Marine Hospital Service we find this disease reported in Indiana in 1889; in Kentucky in 1891; in Louisiana in 1892; in Kentucky in 1895; in many places in Pennsylvania in 1896; in Iowa, Maryland, Massachusetts, and 22 localities in Michigan in 1898; in Connecticut, Illinois, Mississippi, New Hampshire, New Jersey, Pennsylvania, and Wisconsin in 1898 and 1899 ; in Florida in 1899. In 1899 it is stated that extensive epidemics have prevailed during the past two years, in which the mortality ranged from fifty-nine to eighty per cent; in 1901 in Michigan, Indiana, and New York; in 1902 "a few cases reported;" in 1903 "eight cases at Navy Yards, few eases elsewhere;" in 1905 "179 deaths;" in 1907 " 12 deaths in San Francisco; an epidemic in Sacramento Valley, Cal.; 20 cases in Portland, Ore.; 125 cases in Philadelphia in April and 23 ' in May;" in 1912 "scattered cases, increasing, having greatest number but average number of deaths small; disease has become epidemic in localities in Arkansas;" in 1913 "disease in Tennessee and Southern Illinois, sporadic cases occurring almost continuously throughout the country; only occasionally does it become acutely epidemic;" in 1917 prevalence for 1916 gives the case rate per 1,000 population ranging from 0.001 in Maine to 0.059 in Connecticut. Disease more prevalent, evidently due to mobilization of troops; reported at St. Louis, Hartford, Minneapolis ; 237 cases in Philadelphia, 779 in St. Louis, 116 in New York, 103 in Minnesota; from March 1 to May 9 there were reported in Connecticut 48 cases; in the District of Columbia, 10 ; in Illinois, 73 ; in Indiana, 22; in Kansas, 40 ; in Maryland, 18; in Mississippi, 30 ; in Michigan, 10; in Minnesota, 169; in New York State, 123; in Ohio, 79; in Pennsylvania, 284; in Rhode Island, 17; in Wisconsin, 36.

These figures taken from the weekly reports of the Public Health Service suffice to prove that during the period covered, this disease was constantly present and widely distributed throughout our country; but they do not adequately show the extent to which the disease existed during that time. From 1904 to 1907 cerebrospinal meningitis was more widely spread throughout the world and killed more people than in any previous period of its history. It was during this time that epidemics prevailed in New York, Boston, Glasgow, Belfast, Cologne, and other large cities in this country and in Europe. In 1905 the morbidity from this disease in New York City reached eighty per 100,000; in Glasgow 
in 1907 it was 84.7, and in 1909 it was 10 in Paris. So far as we know, these are the highest morbidity rates among civilians authentically reported since the diagnosis of this disease has been rendered certain by lumbar puncture. As a rule, the percentage of individuals attacked by this disease in any community is small.

From the U. S. Mortality Statistics we learn that the death rate from this disease in the registration area, per 100,000 inhabitants, was, in $1890,6.3$; in $1900,7.1$; in 1911, 12.3; in 1913, 10.4; in 1914, 8.8; in 1917, 9.1; in 1918, 9.8. As nearly as we can ascertain, the number of deaths in the registration area reported as due to this disease has varied from 2,039 in 1900 to 8,064 in 1918. Assuming that the case mortality averages about fifty per cent, this means that there are about 16,000 cases annually in the registration area.

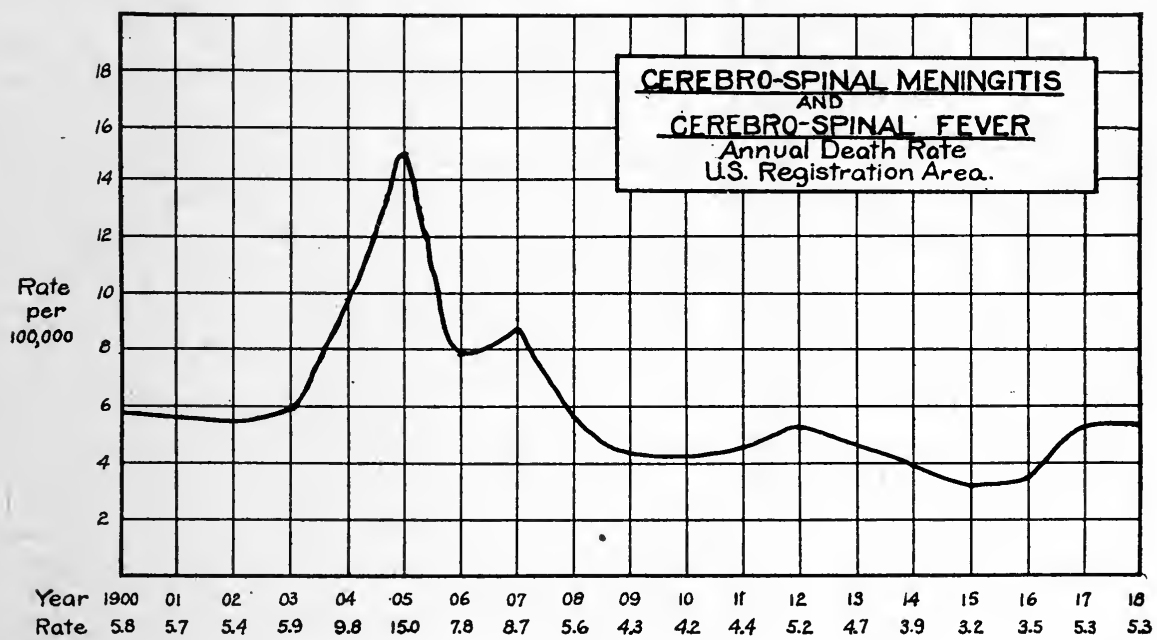

Fig. 65 .

Possibly these figures are too high and may include, probably they do, deaths due to other forms of meningitis, although tuberculous meningitis is excluded from this group in the figures given above.

With the infection spread so widely, as indicated by the above, it is quite evident that the method of mobilization put into operation when we entered the war in 1917 would drag this disease into every cantonment. This was plainly foreseen by medical officers before a cantonment was laid out, a barrack constructed, or an organization of draft men assembled. In considering the limit of space to be allotted each man in barrack and tent, a statement of the epidemiologic characteristics of cerebrospinal meningitis was made to the Secretary of War. This received intelligent and sympathetic attention, and rules and regulations were formulated accordingly. The exigencies attendant upon hasty mobilization did not, however, in all instances permit the strict enforcement of these regulations. 


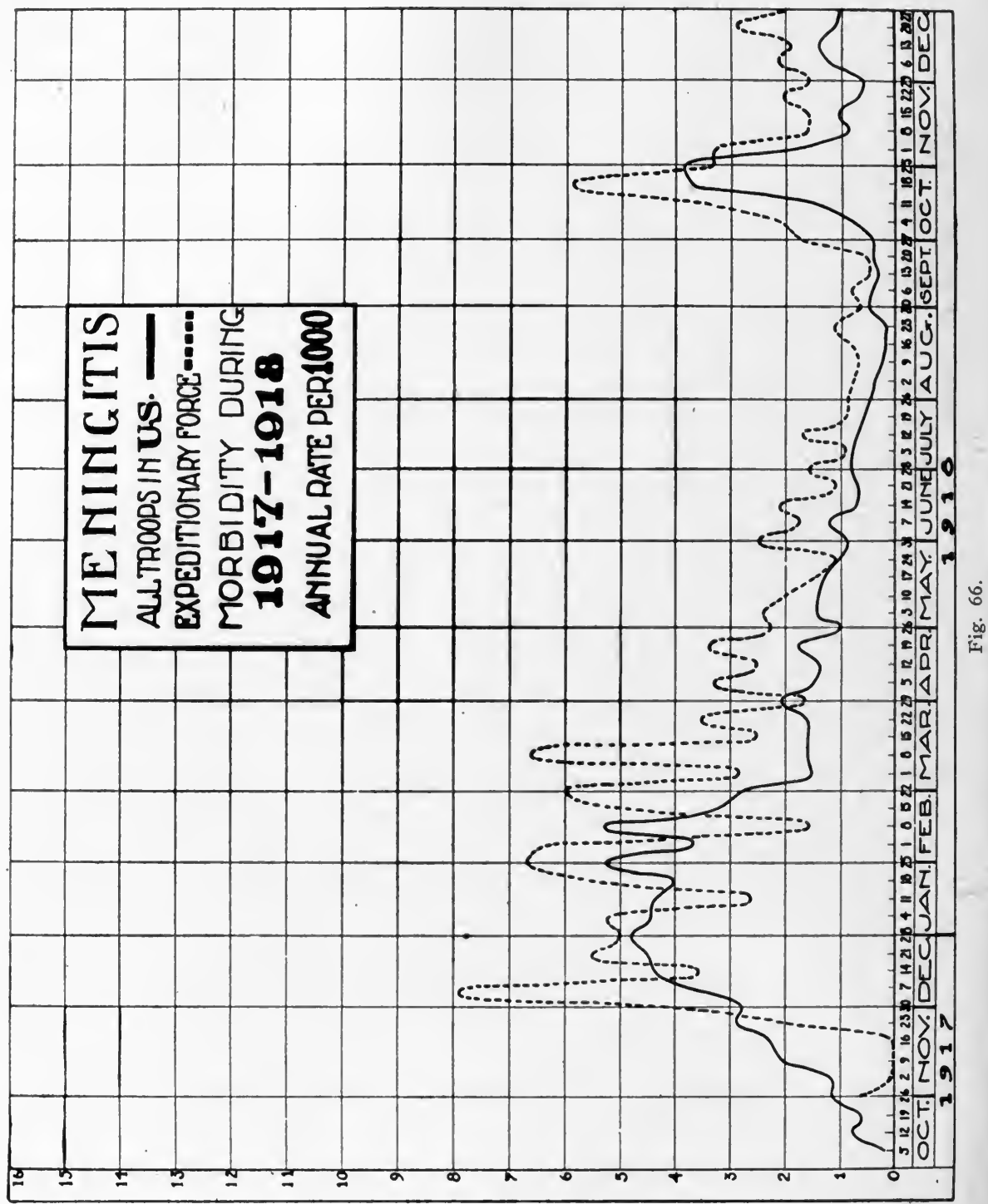

Vaughan and Palmer, in a report on communicable diseases in the National Guard and National Army of the United States during the six months from September 29, 1917, to March 29, 1918, make the following statement:

"Meningitis has appeared in every camp during the six winter months. The prevalence of this disease at Camp Jackson, however, has stood out above that of all other camps. Next to Jackson stand Beauregard and Funston. The rates are 25.7, 12.8, and 9.8, respectively. Next comes Doniphan with 5.0 and the other camps follow with rates at close intervals, the figures receding gradually from this point. 
TABLE LXIX

Meningitis in National Guard and National Army Camps; Figures Represent ANNUAl RATE PER 1,000

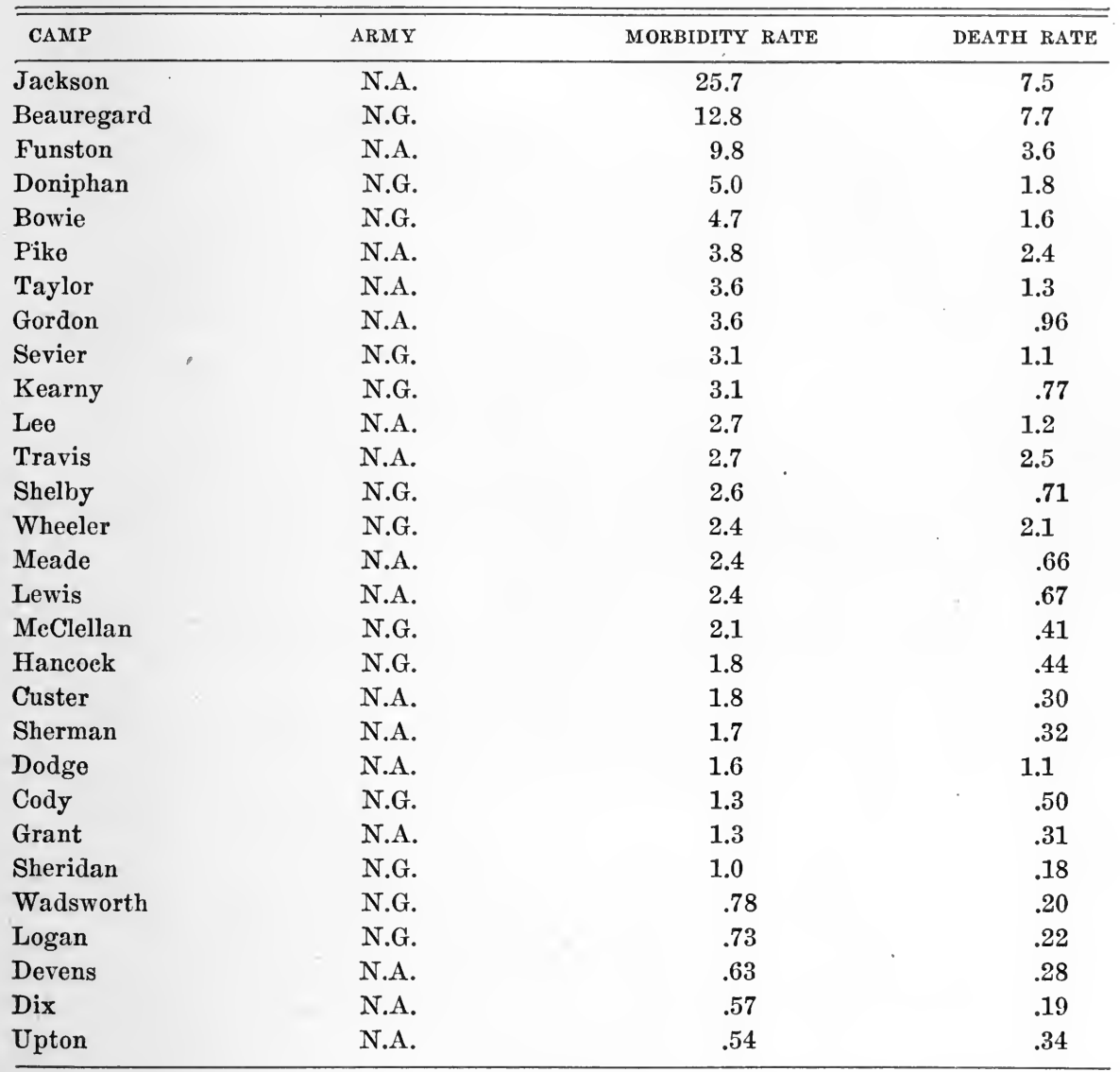

"The three camps with the highest morbidity rate likewise have the highest death rate. Beauregard, however, shows a death rate even greater than Jackson. Again, it is the southern troops who have suffered most severely from this disease. Those escaping it are the troops from the northeastern and northern sections of the country.

"Meningitis, next to pneumonia, has been the most serious disease that the Medical Corps of the Army has had to meet. It is serious by reason of its high fatality and also beeause this of all diseases shows the greatest excess over the disease in eivilian communities. It will be recalled from the early part of this report that meningitis was estimated to be 45 times as prevalent in the Army as in civilian life, whereas the figure for measles was 19 and for pneumonia 12 . For this past winter we have the figures only for New York City and Pittsburgh, within the age group 20 to 29 years. The annual death rate for meningitis per 1,000 was .038 for New York and .047 for Pittsburgh. The death rate for all ages in New York City was .043. The rate for age group 20 to 29 years is thus eighty-eight per eent of that at all ages. Applying this factor to the rates in the various states we ean obtain a rough approximation of the rates for the specific age group in these states. 
TABLE LXX

Meningitis in Civil Communities

Figures Represent Annual Death Rate Per 1,000

\begin{tabular}{llc}
\hline \hline \multicolumn{1}{c}{ PLACE } & RATE FOR ALL AGES & $\begin{array}{c}\text { RATE FOR AGE 20 TO 29 YEARS } \\
\text { (COMPUTED BY USING NEW } \\
\text { YORK CITY FACTOR OF 88\%) }\end{array}$ \\
\hline \hline Vermont & .0 & .0 \\
Virginia & .16 & .14 \\
Delaware & .12 & .11 \\
Connecticut & .038 & .034 \\
Massachusetts & .042 & .037 \\
Colorado & .12 & .11 \\
Michigan & .096 & .085 \\
St. Louis, Mo. & .047 & .042 \\
New York City & .043 & .038 \\
\hline
\end{tabular}

"Accepting the rates thus obtained for what they are worth, we may contrast these figures with those for various army camps, as shown in the following table:

\section{TABLE LXXI}

Meningitis in Civil Life (Age 20 to 29 Years) and Army Camps Annual Denth Rate Per 1,000, Six Winter Monthis, 1917-1918

\begin{tabular}{|c|c|c|c|c|c|c|}
\hline PLACE & & RATE & & RATB & CAMP & SOURCE OF TROOPS \\
\hline Vermont & .0 & & & & & \\
\hline Connecticut & .034 & .024 & & .28 & Devens & New England States \\
\hline Massachusetts & .037 & & & & & \\
\hline Virginia & & .14 & & 1.2 & Lee & $\begin{array}{l}\text { Virginia, West Virginia, } \\
\text { Pennsylvania }\end{array}$ \\
\hline Delaware & & .11 & & .19 & Dix & $\begin{array}{l}\text { Delaware, New Jersey, } \\
\text { New York }\end{array}$ \\
\hline Colorado & & .11 & & 3.6 & Funston & $\begin{array}{l}\text { Colorado, Arizona, Kan- } \\
\text { sas, Missouri, Nebraska, } \\
\text { New Mexico, South Dako- } \\
\text { ta }\end{array}$ \\
\hline Michigan & & .085 & & .30 & Custer & Michigan, Wisconsin \\
\hline New York City & & .038 & 97 & .20 & Wadsworth & New York \\
\hline Pittsburgh & & .047 & & $\begin{array}{l}.34 \\
.44\end{array}$ & $\begin{array}{l}\text { Upton } \\
\text { Hancock }\end{array}$ & $\begin{array}{l}\text { New York } \\
\text { Pennsylvania }\end{array}$ \\
\hline
\end{tabular}

"It is evident from this table that there is an excess of meningitis even in those camps which have been unusually free from pneumonia and measles. Camp Dix has next to the lowest death rate of all the camps, namely, .19 per 1,000. People of age group 20 to 29 years in the State of Delaware show a rate for the past six months of .11 per 1,000. The camp rate is nearly twice as great as a comparable civil community in this instance. Camp Devens has more than ten times the rate of certain New England States. Camp Lee has nearly ten times the rate of Virginia. Camp Funston has more than thirty times the rate of Colorado. Custer has three and a half times the rate of Michigan. Camps Wadsworth and Upton are seren times greater than New York City. 
Camp Hancock has nearly ten times the rate of Pittsburgh. If the rate for Louisiana. is at all like that for Virginia, namely, .14 per 1,000, we find that Camp Beauregard's rate of 7.7 would be in the ratio of 55 to 1 .

"It seems quite evident that meningitis has been most prevalent in those camps whose soldiers come from areas in which this disease has been endemic. For some years meningitis has been widely scattered in South Carolina and adjoining states and it appeared in the families of the workmen engaged in building the barracks at Camp Jackson before the troops began to assemble and during the period covered it was reported in many places in South Carolina. Meningitis has been endemic in Missouri and Kansas for some years and this accounts for its presence at Funston. Here also it appeared in the workmen and their families and in villages near Funston. We have no data concerning meningitis in the civil communities from which the soldiers at Beauregard came. The behavior of this disease is a strong argument for a reserve army and for culturing throats before the draft men leave their homes.

"In the last three months some thirty or more localities in South Carolina have reported meningitis to the State Board of Health. During this time men from all parts of the State are being sent to Camp Jackson. It must happen that many of them from the infected localities are meningitis carriers. These are less likely to develop the disease than some of those with whom they come in contact. If the draft men, especially those from the infected localities, were assembled in small groups at or near their homes and held until cultures were made and no carriers sent to camp, in our opinion this disease would be greatly reduced among our soldiers. As it is, one carrier transmits this infection to others and in a short time there are many carriers."

In reporting on this disease among the troops in this country during the summer and fall of 1918 with an average strength of about 1,493,000, Vaughan and Palmer say:

"Meningitis prevailed most extensively during the winter months, December, January, and February, 1917-1918. The weekly rates from April to December, 1918, have approached the previous winter rates in only two weeks, namely, in the last weeks of October, or just following the peak of the influenza epidemic. During the summer period meningitis was reported from 31 of the 38 large camps, the admissions varying from 1 to 34 for the period. Camp Pike stands at the top of the list with an annual admission rate per 1,000 of 2.2. Pike is followed closely by Gordon, Sevier, and Funston. In two camps there are more deaths than cases, showing that even in cantonments all cases of this disease are not recognized during life. Grant had a case mortality of seventy-nine per cent, Dodge sixty-three, and Sevier fifty-three. On the other hand, Meade had a case mortality of but twenty-one per cent, while Johnston had 11 cases with no deaths. In the other camps the case mortality runs from thirty-eight to fiftythree per cent. During the autumn months (1918) meningitis increased, Beauregard heading the list with an admission rate of 4.9. At least seven camps had rates higher than the highest for the summer season. During the winter of 1917-1918 meningitis was most prevalent at Jackson, Beauregard, and Funston. During the summer season these camps were within the first twelve on the list, and during the autumn season the same camps were in the first thirteen. For the other camps there is apparently no persistent rate, as the standing of each fluctuates. Our figures do not indicate any relation between influenza and meningitis. Beauregard, Devens, and Meade, stand high in influenza, but the other camps in which meningitis was prominent were not unusually high in influenza. Taking the first nineteen eamps during the autumn season, all of which have at least twelve cases of meningitis, we find the case mortality varying from 
six per eent at Sherman to fifty-five per cent at Pike. At Funston where meningitis was more or less prevalent throughout the fifteen months, the ease mortality was fifty per cent; at J'ackson it was thirty-one, and at Beauregard eighteen.

"Meningitis is a disease apparently to which few people are susceptible. It has not oceurred epidemically in the army. The cases are sporadic. Of twelve eases at Cody during 1917-1918 not one was associated with any other. Meningitis is kept alive by the existence of earriers, people who may or may not have had the disease. At Cody in the spring of 1918 , among 20,000 men 268 earriers were found. In no instance did these give a previous history of meningitis and yet they were dangerous to their comrades, for all of the active eases admitted elose association with one or more carriers. One hundred and thirty-two eases oceurred at Beauregard from September, 1917, to May, 1918. These were widely distributed over the camp and occurred independently of one another. At Lewis, for the fifteen months from October, 1917, to December, 1918, there were 54 eases. In this division 18,998 men were cultured and 273 , or 1.4 per cent, found to be positive. The ratio of carriers to eases is about five to one. No elinical cases developed among these earriers. It was the general opinion that the cases fell off as the cultural method of control, followed by isolation of carriers, was put into effect. Again, we are struck with the nonepidemic nature of the disease from the fact that with three exceptions no secondary cases developed within an organization."

The same reporters give the following figures concerning the seasonal distribution of this discase among troops in this country:

$\begin{array}{lccc}\text { SEASON } & \text { CASES } & \text { DEATHS } & \text { PER CENT FATALITY } \\ \text { Winter } & 2264 & 604 & 26.6 \\ \text { Summer } & 497 & 248 & 50.0 \\ \text { Autumn } & 619 & 244 & 39.5\end{array}$

Cerebrospinal meningitis was, on the whole, more prevalent in the American Expeditionary Force than among the troops in this country. This raises two questions which we are not able to answer. Was it due to closer contact with the civil population in France, or was it due to exposure to a different strain of the meningococcus?

The Surgeon General, reporting on the disease for the calendar year of 1918 and including all troops in this country and in France, states that there were 2,988 cases with 1,179 deaths, a fatality of 39.4 per cent. For enlisted men as a cause of death it stood fifth in the United States and fourth in Europe. In this country the mortality was 34.8 per cent, while in Europe it was 43.31. For colored troops serving in the United States the admission rate was 2.44 and for the white 1.20. The death rate among colored troops was 0.98 and among the whites 0.41 . For the troops serving in Europe the admission rate for colored was $\mathbf{1 . 6 6}$ and for the white 0.36. The case mortality for all American troops, enlisted men, was among the colored 45.01 and among the white 35.84 . The highest admission rate for the calendar year, both in this country and in Europe, was during the month of January. However, in the Expeditionary Force the January rate was closely approximated in March. 
From this time during the remainder of the calendar year the rate in the Expeditionary Force continued above that in the troops at home. In October the rate, both at home and abroad, showed a marked ascent and approached closely that of January. The lowest rate reached in this country was in August and that in Europe in September.

Cerebrospinal fever was first recognized in Canada in 1819, and since that time its history in that country has practically paralleled that in the United States. We, therefore, deem it unnecessary to go into detail concerning the prevalence of this disease in our neighbor dominion. Both countries have alike borne the odium of being breeding-places for the meningococcus and it is more than probable that in neither country, at least since the beginning of the nineteenth century, a year has passed without paying small tribute to this highly fatal microorganism.

The history of cerebrospinal meningitis in France is similar to that in the United States with the exception that in the former country most of the early epidemics were reported by army surgeons. The first was an outbreak in the garrison at Grenoble in the spring of 1814 and was reported by Comté. In this year a communication was sent to all the prefects of the Empire, stating that there are two forms of typhus.

" In ordinary typhus the disease begins with a sense of heaviness along the spine; followed by lumbar pain, vomiting, pain in the head, convulsions, regular exacerbations, nocturnal delirium, and difficult deglutition. The other takes the form of an encephalitis, with pain in the head, extending from the vertex to the occiput, and continuing along the spine. This form is accompanied by a maniacal delirium and in some eases with symptoms of general tetanus.',

There can be no doubt that calling the attention of medical officers to these two forms of so-called typhus made them alert in the recognition of cerebrospinal meningitis and accurate reports concerning this disease have been made in the French Army from that time to this. At least one French officer, Boudin, went so far as to claim that all the so-called typhus in the French Army during the Napoleonic Wars was cerebrospinal meningitis. As Netter says, in doing this Boudin went in the opposite direction to that followed by Murchison in England, who claimed that all cases were typhus.

It would hardly be profitable to follow all the epidemies in France either in the military or civil population from 1814 down to the present time. It will be better to dwell upon special points. French Army surgeons who studied this disease in individual garrisons quite generally came to the conclusion that it was not contagious. Some of them reached the conclusion that while not contagious it was transportable. Small bodies of troops were moved from place to place, but it was found that the disease accompanied them. Mistler wrote that he did not believe the disease was contagious, but that the morbid epidemic principle attached 
itself to the men or to their effects and in this way accompanied military organizations. This is a matter of great epidemiologic interest. The experience of French officers in the first half of the nineteenth century in trying to rid organizations of epidemic disease by moving from post to post has been repeated in other armies with like failure since that time. During our war with Spain an army corps was moved from Alger, Va., to Bristol in the same state and later to Camp Meade, Pa., in order to get rid of typhoid fever. The same tactics were tried at Chickamanga Park. Some of the badly infected regiments at this place were sent to Lexington, Ky., others to Knoxville, Tenn., and still others to Huntsville, Ala.; indeed, this method of attempting to get rid of typhoid fever was tried in many instances in our army during the summer of 1898. During the World War it was proposed by a medical officer that a large eantonment, the construction of which had cost millions of dollars, should be evacuated and the troops moved elsewhere in order to get rid of cerebrospinal meningitis and pneumonia. This error has been repeated often enough to demonstrate its futility. The moving troops carry the meningococcus in their throats and the typhoid bacillus in their intestines and gall bladders and do not get rid of them by being transferred from one location to another.

French officers demonstrated that cerebrospinal meningitis is especially a disease of soldiers and that among soldiers it is a disease of barrack life rather than of field life. Time and time again it was shown not only that this disease accompanied troops in their transfer from barrack to barrack, but that it disappeared when they were seattered in the field. In the majority of the early epidemics in the French Army the disease was confined to soldiers in barracks and only rarely spread to the civilian population. When it did so it first appeared among those eivilians who eame in closest contact with the soldiers. During the first part of the nineteenth century, of 62 epidemics in France the disease was confined in 43 to the soldiery; in six it prevailed most extensively among the troops but involved certain townspeople; only in five did it prevail equally among soldiers and civilians. Even when two organizations were barracked with only a short distance intervening the disease was confined wholly or nearly altogether to one organization. Sometimes it was an infantry regiment; at others a cavalry troop, and in still others a marine organization.

Another thing of importance was perfectly evident to French officers. Among soldiers the disease was more eommon in recruits than in seasoned men. This fact was confirmed in our Civil War.

French officers saw plainly that this disease is favored in its development by overerowding in badly ventilated barracks. Gasté not only was convinced of this in the epidemic at Metz in 1839-1840, but he scat- 
tered his troops in order to abate the disease and found this method apparently successful. Writing of the epidemic of Strasbourg in 1841, Tourdes states that the disease originates in crowded quarters.

\footnotetext{
"Given a poorly ventilated barrack in which recruits are accumulated and you have the best possible condition for the development and spread of the disease."
}

Corbin not only confirmed this opinion in his study of the epidemic at Orleans in 1847, but he went further and got a little nearer the truth when he wrote that crowded, overheated, quarters best favor the development of this disease. It is desirable to here point out that possible harm may be done by scattering an infected military organization. The wider distribution and the provision of more space for these men are highly desirable, but the scattering should not be done by sending the men from infected organizations to uninfected ones, and especially by sending them to recruit depots.

As has been stated, most of the early French officers whose observations were confined to local epidemics were quite sure that the disease was not contagious, but some of those who followed it from place to place became convinced of its contagiousness. For many years Boudin stood almost alone not only among French observers, but those in all parts of the world, in contending for the infectious nature of this disease. One of the most convincing instances cited by Boudin in his contention for the infectious nature of the disease is the experience of the Eighteenth Infantry. Cerebrospinal meningitis had been epidemic among the eivil population in the Department of Landes in the Lower Pyrenees adjacent to Spain for some years prior to 1836 . This regiment was stationed at Bayonne in this part of France and was in part recruited from the population of this section. Cerebrospinal meningitis appeared in two garrisons in the Department of Landes, Bayonne and Dax, in which this regiment was quartered. The regiment moved first to Bordeaux and then to Rochefort, but the disease not only continued with the regiment, but spread to some slight extent outside the garrison, especially at Rochefort where the inmates of a prison became involved. This regiment was stationed at Bayonne in 1836. It left Rochefort at the end of 1838 and went to Versailles. In February, 1839, six men of the regiment, all occupying the same room, came down with cerebrospinal meningitis at intervals of a few days. At Versailles the disease extended to other regiments, but out of a total of 160 cases at that place 116 occurred in the.Eighteenth, while the remainder were distributed through four other infantry regiments and three troops of cavalry. The Eighteenth regiment was subsequently transferred to Chartres and later distributed to Metz, Strasbourg, Nancy, Schlestadt, and Colmar, in all of which places cerebrospinal meningitis not only continued in the 
Eighteenth Infantry, but especially attacked the recruits to this regiment, and spread to other military organizations with which the Eighteenth came in contact and in some places to the civil population. There is some reason for believing, though the proof is not clear, that the disease was spread by troops from the Department of Landes towards the Mediterranean. From 1841 to 1849 nearly every garrison in France from Lille in the North to Marseilles in the South and from Brest on the Atlantic Coast to Strasbourg on the Rhine reported small epidemics of this disease. Not only was this true, but cerebrospinal meningitis was carried by French soldiers to Algerian garrisons.

This disease was first reported in Italy in 1839 at the village of Ancona and it is believed that it was carried to that place by French soldiers who had garrisoned this village since 1832 . By 1845 it was known that the disease was widely scattered over Italy, being reported not only in the northern part but as far south as Naples. The epidemiologist writes that it passed from Italy across the Adriatic and reached Corfu in 1840. In our opinion, it is more than probable that the disease had existed in all these countries long before it was first recognized at Geneva in 1805 . Germany apparently was slow to recognize this disease, although as we have shown, it is quite clear that it existed in that country as far back as 1722. In the early part of the nineteenth century there were small epidemics, reported in some instances as encephalitis and in others as acute hydrocephalus, which were undoubtedly cases of cerebrospinal meningitis; in fact, French authors supply the earliest positive information concerning cerebrospinal meningitis in that section of Europe now included in the German Empire. They state that a village in Baden became infected from Strasbourg in the year 1841. Hirsch writes:

"A number of typical eases of meningitis epidemica were seen at Würzburg in June, 1851 ; perhaps that was also the nature of the epidemic which was prevalent in the summer of 1853 at some other places in Lower Franconia, although I am unable to speak positively about it."'

From 1863 to 1866 cerebrospinal meningitis was widely recognized in practically every part of Germany. During this time the notable epidemic which appeared in scattered places in East and West Prussia, Posen, Brandenburg, and Pomerania, attracted the attention of the world and various countries sent experts to investigate the nature of the disease. The most valuable report that resulted was that of BurdonSanderson, the English commissioner. From that time up to the present, epidemics of this disease have been reported from Germany and valuable contributions to the bacteriology and pathology of the disease have been made by German savants.

During the sixties of the nineteenth century cerebrospinal meningitis had come to be recognized in every European country. 
In Ireland this disease was recognized in 1845, when it occurred among the Royal Irish Constabulary at Dublin. It was first reported in Denmark in 1848. In Norway sporadic cases were noted early in the nineteenth century, but the first epidemic was recognized about 1866. Probably the most widespread epidemic of that time was seen in Sweden beginning in 1854 and extending to 1861. The records for these years are given in the following table:

$\begin{array}{lcc}\text { YEAR } & \text { CASES } & \text { DEATHS } \\ 1855 & 3000 & 886 \\ 1856 & 2000 & 428 \\ 1857 & 3051 & 1387 \\ 1858 & 1909 & 779 \\ 1859 & 1415 & 582 \\ 1860 & 347 & 148 \\ 1861 & 91 & 27\end{array}$

The above figures impress one with the idea of a rather extensive and highly fatal epidemic. On studying them in detail we find that cerebrospinal meningitis manifested its characteristics in this epidemic as well as in others, inasmuch as it traveled by leaps and with halts. As a rule, it practically disappeared each summer and awakened into new life and vigor with the coming of the cold months.

In 1840 it was reported in Brazil and Paraguay. From 1840 to 1847 it became widely distributed over Algiers. In 1876 it was first reported in the Fiji Islands, where it became epidemic in 1885. Corney gives an account of its invasion of these Islands, but it is not so clear cut as that which the same author gives of the introduction of measles. This is necessarily the case, because measles is transmitted exclusively from the sick to the well, while cerebrospinal meningitis is, for the most part, distributed by carriers who are in apparent health. According to Corney, in the Fiji Islands the disease was first diagnosed as tetanus and sunstroke and its true character was not recognized until it became epidemic.

Cerebrospinal meningitis was first reported in India by Carter in 1878, although it was quite widely distributed over Asia before that time, as is shown by reports from Smyrna in 1872 and from Persia in 1874. Concerning its existence in Africa, Chalmers and O'Farrell make the following statement:

"Cerebrospinal meningitis was reported from the West Coast of Africa by the Williams in 1900 and from then onwards from time to time. In 1905 there was a severe epidemic in Northern Nigeria, which was reported upon by Twomey and Davidson, and in 1906-1908 in the northern territories of the Gold Coast, where it was ably investigated by Horn. In Northern Nigeria it was said that cattle were attacked, and in the Lorha district of the northern territories of the Gold Coast the natives are said to have noted a great mortality among fowls prior to the epidemic of the disease. Accord. ing to Bargy and Horn, the disease was marked at this time in French West Afriea to the 
west of the Black Volta. In 1909 it was reported as being present in 1907 in the northern districts of Togoland by Jaffe, when it was known to cause 300 deaths. Jaffe obtained the true diplococcus of Weichselbaum from his cases. Epidemic cerebrospinal meningitis has been recorded in British East Africa since 1906, when Haran noted five cases, and has been ably described by Shicore and Ross in 1913. In 1913 Butler drew attention to a curious feature of the disease, in that there are places in East and Central Africa where it has always been endemic and seldom epidemic, and he quotes Uganda in general, whilst in the highlands of British East Africa the endemic form is seldom seen but epidemics of greater or less virulence are known. He says that in one tribe alone between 20,000 and 40,000 deaths are attributed to this disease, a mortality which sleeping sickness can hardly be said to rival.",

The last statement in this quotation is highly important if true. We know of no authentically reported epidemic of cerebrospinal meningitis in which such large death rates can be attributed to this disease and we doubt very much the aceuracy of the statement.

Chalmers and O'Farrell state that eerebrospinal meningitis has been known in Anglo-Egyptian Sudan as far back as living man ean remember and it is said that in the days of the Khalifa it caused 900 deaths per annum in the City of Omdurman. Although these authors have not been able to prove the existence of this disease in the Sudan through past centuries, they state that the people have a distinct name for the disease. This in and of itself suggests that cerebrospinal meningitis has been distinguished from other diseases, at least through more than one generation. Chalmers and O'Farrell suggest that the real home of this disease might be Central Africa, from which it long ago passed to Egypt where French soldiers in Napoleon's expedition may have come in eontact with it and carried it back to France. This is an interesting suggestion, but there can be no doubt that eerebrospinal meningitis existed in Europe long before the Corsican went to Egypt.

We now wish to say a few brief words concerning cerebrospinal meningitis in England. Many English medical men of repute state that this disease has never been widely distributed in that country. If this be true there must be some reason for it, either climatic, racial, or in manner of living, and it is the duty of the epidemiologist to inquire into these points. Murchison was the great English authority on continued fevers during the period centering about the middle of the nineteenth century and at a time when this disease was being widely discussed in France and America. It may be well to eall attention to the fact that Murchison for a long time disputed the French and American claims for the differentiation between typhus and typhoid fevers. He held that the so-called spotted fever reported from America was typhus fever. When Burdon-Sanderson returned from his mission to Danzig and laid before the Pathological Society of London, May 2, 1865, the specimens 
taken from a child that had died from the epidemic disease and demonstrated the lesions in the meninges, Murchison claimed that his belief that the disease was typhus fever was not shaken by the demonstration. He stated that he had found such lesions in typhus fever. The position held by Murchison is ably discussed by Stillé in his monograph on Epidemic Meningitis published in 1867. In the early editions of his great work on Fevers, Murchison did not give meningeal inflammation as a finding in typhus fever. Later he did admit that such postmortem appearance may be seen after death from typhus. The whole matter hinged upon the diagnosis founded upon the lesions after death. On this point Stillé wrote:

"A typhus fever with a special direction to the cerebrospinal meninges, appears to us a very pathological monstrosity. The meningeal inflammation is evidently due to quite another element than that which originates the typhus symptoms. The one is specific, peculiar, and characteristic of this disease alone; the others are incidental, nonessential, and common to this disease and many other diseases. That which distinguishes epidemic meningitis is the concentration of the morbid action of the disease upon the membranes of the nervous centers, and the tendency of that morbid action to purulent or plastic exudation. If those who die of it in the first stage do not always exhibit inflammatory exudation, it is simply because death arrests the incohate process in its earliest steps. If the patient survives for several days and then perishes, the characteristic lesions are revealed. But it is not so in typhus."

In 1829 Smith (Southwood) published, "A Treatise on Fevers" and he cites a number of cases which were clearly cerebrospinal meningitis. In these cases, in speaking of the arachnoid, he says:

"It is seldom or never in a healthy condition. It is always either more vascular than natural, or when in this respect unchanged, it is altered in structure, being thick, opaque, and milky; when in this latter state a gelatinous fluid is usually effused beneath it."

In 1839 Roupell published a book, entitled, "A Short Treatise on Typhus Fever," in which he says of a postmortem finding in a certain epidemic:

"In all the cases an affection of the membranes of the brain was found, either turgescence of vessels, increased vascularity, effusion of serum or pus, or the deposition of lymph, varying from the thickness of a line to a quantity only sufficient to render the arachnoid opaque."

These certainly were cases of cerebrospinal meningitis.

In 1830 a third English physician, Tweedie, wrote a monograph, entitled, "Clinical Illustrations of Fever, Comprising a Report of the Cases Treated at the London Fever Hospital, 1828-29," in which he records a long list of autopsies. It is evident that this author recognized that he was dealing, under the name of typhus, with marked modifications of the disease and he makes the following classification: (1) Simple typhus. (2) Typhus with inflammation of the brain. (3) Typhus with ulceration 
and in some instances perforation of the intestine. (4) Typhus with pulmonary inflammation. A present day reading of the records of his autopsies will, we think, convince any one that this author during the time covered by the dates given in the title of his book was seeing typhus, typhoid, bronchopneumonia, and cerebrospinal meningitis. In one place he uses the word "Typhoid" and he reports 16 cases with ulceration of the intestine, in two of which there was perforation. He states that there were 12 cases with inflammation of the brain, and some of his autopsy records supply classical pictures of the findings in cerebrospinal meningitis, although at least one of these may have been tuberculous meningitis.

Low has recently (1916) reported on the distribution of cerebrospinal meningitis throughout the world, bringing the report of Hirsch, which closed with 1886, up to date.

As Stillé points out, Murchison most undoubtedly saw .cases of cerebrospinal meningitis and he wrote a fairly correct differentiation between this disease and typhus fever, as the following quotation shows:

"At the commencement of this century, the symptoms of typhus were referred to cerebral inflammation; and at the present day, typhus is not uncommonly designated 'brain fever.' The chief points of distinction between typhus and inflammation of the brain are the following: in inflammation, the headache is much more intense, and of a throbbing, darting, bursting, or constricting character; in typhus, the patient rarely describes it by such terms. The delirium of inflammation is more violent and acute than that of typhus, and aceompanies or alternates with the headache; whereas the headache has almost always ceased in typhus before the delirium commences. The loud eries and sereams observed in the delirium of meningitis do not occur in typhus. In inflammation there is great intolerance of light and sound; but in typhus the senses are obtuse, and deafness is more common. In both diseases the face is flushed, and the conjunctivae are injected; but in typhus the flush is more dusky, and the blood in the conjunctival vessels of a darker tint than in inflammation. In both diseases there may be general convulsions, followed by coma, but typhus never commences in this way, as meningitis sometimes does. Strabismus and partial palsy are far more common in inflammation than in typhus. The physiognomy of meningitis is anxious and expressive of pain or wild and defiant; in typhus it is oftener blank and stupid. In typhus there is much more muscular prostration from the first than in inflammation. The pulse in inflammation is usually firm; in typhus it is soft and compressible. Nausea and urgent vomiting are common in inflammation; rare, in typhus. Lastly, in typhus, there is a peculiar eruption appearing about the fourth or fifth day."

Disregarding the small epidemics and sporadic cases of cerebrospinal meningitis, reported under its proper name in England from time to time during the nineteenth century, we are led to conclude that during none of that time was England free from this disease and that there is no reason for suspecting that the Englishman for any reason is less susceptible to it than the Frenchman or the American. Since the recognition of the meningococcus as the specific cause of the disease and re- 
sort to lumbar puncture has been employed in diagnosis, English physicians have not been behind their fellows in any country in contributing to the symptomatology, pathology, and epidemiology of cerebrospinal meningitis. It happened, however, that when the Canadian Expeditionary Force reached England in the early part of the World War and some cases of this disease appeared among civilians brought in close contact with Canadian soldiers, British medical officers advanced the claim that the disease had been brought into England from Canada where its existence and wide distribution had long been known. It is undoubtedly true that Canadian soldiers carried meningococei to England, but in doing so they were only carrying coals to Newcastle.

In studying the literature from.which we have compiled this brief history of cerebrospinal meningitis we have been led to the following conclusions: (1) This disease has existed from remote times. (2) It has been and is widely scattered throughout the known world. It appears in sporadic form and in small limited epidemics. (4) Only a small proportion of any community is susceptible to this disease. Its epidemic characteristics have not changed since the first evidence of its existence was recorded, whatever name the recorder has attached to it.

Hirsch divides epidemics of cerebrospinal meningitis into the following four cycles: (1) 1805-1830, North America and Europe; (2) 1837-1850, Europe, North America, and Algiers; (3) 1854-1875, Europe, North America, Africa (parts), Western Asia, and South America; (4) 18761884, small epidemics in former areas; to which Chalmers and O'Farrell add, (5) 1885 to present time, recognition of the disease in the Tropies, West Indies, Fiji Islands, Anglo-Egyptian Sudan, West Africa, Western Australia, and Mexico; severe epidemies in America and Europe; i.e., this is the period of the first pandemic.

We see only one reason for dividing epidemics of this disease into cycles. It is convenient for the historian to do so. Some epidemiologists, like the pacifists, prophesy when one epidemic is over that there will never be another. With the pacifist, the war just finished or the war in progress is the last the world is to see. The epidemiologist, with the mind of the pacifist, is always ready to say that the world will never see another great epidemic. There is no harm in assertions of this kind, unless they lead to the abandonment of methods of preparation. The world has not seen its last war nor its last epidemic of this disease, and the same can be safely predicted of many other diseases.

The Meningococcus.-Although hungry, predatory, saprophytic, bacteria fall upon the dying man and penetrate his most impregnable parts before the heart actually ceases to beat, the meningococcus, having found its way into the bony cavity of the head and spine, is always in 
evidence when an autopsy after death from this disease is made within a reasonable time. It is true that in such autopsies the diseased tissues and other exudates may supply the investigator with mixed cultures, but the meningococcus is easily and constantly found. The first man who made smears from the meningeal exudate after death from this disease and examined it after proper preparation and under a sufficiently high power, undoubtedly saw the meningococcus. Picking out the specific organism under these conditions is an easy task compared with that of finding the constant and causative bacterium in sputum, saliva, or intestinal contents. So far as our investigations have gone, it appears that Klebs in 1875 saw cocci in the cerebrospinal fluid after death from this disease and suggested that these microorganisms might be the causative agents. In 1881 Gaucher certainly made a painstaking bacteriologic study not only of the exudate in the brain and spinal cord, but of the urine and blood as well and in all he found cocci, the description of which leads to the belief that they were identical with the organisms which we now know as meningococci. In 1883 similar observations and similar findings were reported by Ughetti and a little later by Marchiafava and Celli. These investigators reported the micrococei both as intra- and extracellular and in this they were right, as subsequent investigations have shown. These findings were confirmed by numerous bacteriologists; Leyden and Leichstenstern in 1885; in 1886 by Foà and Bordoni, Uffreduzzi and Lemoine, and Frankel and Weichselbaum. In 1887 Weichselbaum published a paper on "The Etiology of Acute Cerebrospinal Meningitis," in which he described his findings in eight cases of meningitis without pneumonia. In two of these pneumococeus was found, and in six he found a diplococeus, resembling the gonococcus, often contained in leukocytes and gram-negative. To this organism he gave the name, Diplococcus intracellularis meningitidis. This name has been accepted and to Weichselbaum is generally given the credit of having established the specific causal relationship of this organism to cerebrospinal meningitis.

The meningococcus has a diameter of about one micron and appears as a diplococcus with the adjacent sections flattened, presenting the appearance of a kidney bean. In artificial cultures, especially those more than 24 hours old, spherical forms may predominate; rarely this organism is seen in the form of tetrads, but never in chains. In morphology the micrococcus is fairly constant, but in size there are more or less marked variations. It takes the basic anilin dyes readily and for the most part uniformly, but the larger involution forms are likely to over stain. The demonstration that the organism should be gram-negative is regarded as essential in the identification of the meningococcus. So far as we know, all recent contributors to the study of this organism 
agree on this point with the exception of Chalmers and O'Farrell, who claim (1916) that there is found, though they admit very rarely, a gram-positive strain which may cause meningitis.

The meningococcus is an obligate aerobe, requires special media for its artificial growth, and is especially susceptible to changes in temperature. It will grow under certain conditions artificially at between $25^{\circ}$ and $42^{\circ} \mathrm{C}$. It speedily dies at or below $22^{\circ} \mathrm{C}$. Primary cultures can be obtained, at least certainly, only on media containing blood and nearly every laboratory has its favorite medium. Unfortunately, many of these are known by outlandish names which mean nothing to those not provided with the formula. It fails to grow in artificial culture media, or develops but slowly, when the temperature is as low as $23^{\circ}$ C.; in fact, in some laboratories failure to grow at this temperature is regarded as a means of identification. This test alone certainly cannot be considered sufficient and there are certain strains which develop slowly at this low temperature. Outside the body this organism is very delicate and fragile. It is easily killed by drying and exposure to sunlight. Some experiments indicate that when brought to the point of actual desiccation it dies within less than five minutes, but in the presence of moisture it may survive ordinary daylight for a few hours.

The meningococcus belongs to a group of gram-negative cocci, any or all of which may be found in the nasopharynx. This group includes: (1) Meningococcus; (2) micrococcus flavus; (3) micrococcus catarrhalis; (4) micrococcus pharyngis siccus; (5) diplococcus mucosus capsulatus. The gonococcus belongs to the same group, but of course its natural habitat is not the nasopharynx. The members of this group may be differentiated by studying their reactions with various sugars. A great amount of splendid work has been done along this line and we are impelled to call especial attention to the report made by EIser and Huntoon (1909). The following principal fermentation tests are relied upon for the differentiation of members of this group: The meningococcus ferments glucose and maltose.

(2) The gonococcus ferments glucose only. (3) The micrococeus flavus is chromogenic and in its fermentation reactions may be divided into three groups; group one ferments glucose, maltose, saccharose, and levulose; group two ferments glucose, maltose, and levulose ; group three ferments glucose and maltose slowly. (4) Micrococeus catarrhalis does not ferment any of the ordinary sugars. (5) Micrococcus pharyngis siceus ferments glucose, maltose, saccharose, and levulose quickly. (6) The diplococcus mucosus capsulatus is encapsulated, while none of the others are. In the laboratories of the base hospitals during the World War, the question was asked: "Is the finding of a gram-negative coccus in the nasopharynx sufficient to proclaim the individual a carrier"? It was neces- 
sary to answer this question in the negative. On the other hand, the finding of a gram-negative coccus in the cerebrospinal fluid is sufficient to justify a diagnosis of cerebrospinal meningitis and to distinguish this from all other forms of meningitis.

After a study of the cocei found in the nasopharynx Griffith (1916) wrote as follows :

"All strains of gram-negative cocei, obtained from the nasopharynx, identical microscopically, culturally and in fermentation tests with meningococci, must, in default of a specific test for virulence and until the serological relations have provided definite evidence to the contrary, be considered to be meningococci.",

Another question of great importance is, "Are there different strains of the meningococcus"? This is of the greatest importance, because, if there be different strains, it may be necessary to produce specific sera for the treatment of eases due to different strains. Work along this line has been done by application of the agglutination test. Elser and Huntoon in this country, Dopter in France, and Gordon and Murray in England, as well as others; have worked upon this subject. At the present time (1920) the classification worked out by Gordon and Murray and known under the former name is generally followed. Gordon's classification makes four strains, and when a patient is ill with cerebrospinal meningitis it can be assumed that the strain found in his nasopharynx is identical with that which is causing the meningeal inflammation and which will be found in the spinal fluid on puncture; therefore, when the puncture is made the specific serum suitable for that case is at hand and ready to be injected into the spinal cavity.

In 1909 Dopter described his parameningococcus and reported 12 cases of eerebrospinal meningitis due to this organism. These individuals were not benefited by the ordinary antimeningococcal serum but did improve when a special parameningococcal serum was prepared and used. The term "parameningococcus" is, in our opinion, unfortunate. It means, if it means anything, that this organism is like but is not a meningococcus. It is now quite generally admitted that Dopter's parameningococcus is a strain of meningococcus and is identical with No. 1 of Gordon's group.

For the purpose of the rapid identification of meningococci a polyvalent serum is prepared. This will agglutinate all strains of the meningococcus in dilutions of from 1-200 to 1-2,000. By the addition of proper preservatives this serum can be kept quite indefinitely. It having been determined that the microorganism under study is a meningococcus the special strain can be determined by the use of specific sera.

We have already called attention to the fact that the meningococcus and the gonococcus belong to the same group. It has been suggested 
that about the beginning of the nineteenth century the gonococcus underwent a mutation resulting in the production of an organism with a special affinity for. the meninges. This idea originated in the brain of some bacteriologic dreamer. It has no support in reality, and it has been demonstrated that the injection of cultures of the meningococcus into the urethra does not induce gonorrhea. The gonococcus mutation theory is a little more absurd than that advanced by Job Wilson to account for the appearance of cerebrospinal meningitis in New England. Wilson wrote:

"Our climate (particularly the climate of New England) though at best very changeable, for many years prior to 1804, has been comparatively mild and steady. But since 1804 a new era has commenced. The changes of our climate have been greater and more frequent. The effects of these changes have been very remarkable both in the animal and vegetable kingdoms. To many individuals of each of these kingdoms, they have proved immediately fatal. The late changes, or some other cause, have produced a disease or rather diseases, which our mother country (England) appears never to have been acquainted, as she never suffered so great and sudden extremes of heat and cold.',

Hamer jocosely says that the meningococcus was not born in 1805 and since it is convenient to connect a date with so important an event as the birth of a micrococcus, he prefers to attach to it the year 4004 B.c. when, according to his childhood training in chronology, all the trouble in this previously peaceful world was started by the creation of Adam and the placing of him and his bride in the Garden of Eden.

While the meningococcus can be induced to grow on certain artificial media, it is found in nature only in the body of man. The question of animals carrying this microorganism has been investigated but it has never been answered with perfect satisfaction. There is a disease known as epizoötic cerebrospinal meningitis, which has appeared among horses in this country and in Germany. A similar disease among cattle was reported by Meyer in 1867 and in lambs by Rolloff in 1868. Similar observations in dogs have been reported from Russia and the disease has been induced in healthy animals by injecting the micrococcus into the meninges. In 1909 the question of meningitis in animals was investigated by Christiani, who came to the conclusion that the disease is due to a coccus closely resembling Weichselbaum's organism; but he leaves the question, as he states, for settlement to future workers. Studies by Hutyra and Marek in 1913 led these investigators to the conclusion that epizoötic meningitis is not an etiologic entity. There is no convincing evidence that man has ever acquired cerebrospinal meningitis by association with animals.

In 1896 Kieffer found meningococei in the nasopharynx of those ill with cerebrospinal meningitis. This finding was confirmed with a large number by Councilman, Mallory and Wright in 1898. It is now a recog- 
nized fact that the meningococcus is present in the nasopharynx of all those ill with this disease, especially during the first few days. As we have already stated, the strain in the nasopharynx and that found in the spinal fluid after puncture is always the same. In 1901 Albrecht and Ghon found the meningococcus in the nasopharynx of perfectly healthy individuals who, so far as known, had never been in contact with those ill of the disease, at a time when the active disease was not present in the community. From an epidemiologic standpoint it is highly important that we get all the information we can concerning noncontact carriers. In 1906 Kutscher found the organism in 7.14 per cent of 56 noncontacts; in 1906 Kolle and Wassermann found two carriers (1.8 per cent) among 114 people in Berlin, and in the same year Droba and Kucera examined 160 children in a community free from the disease and found all negative. In 1907 Huebner and Kutscher found eight carriers (two per cent) among 400 soldiers. There was at the time no cerebrospinal meningitis in this regiment, but two months previous it had been stationed near another regiment in which the disease existed. These eight earriers had not been in contact with one another. In 1908 Schumacher and Aumann found two per cent of carriers among 1,500 soldiers in the absence of any ease of the disease. In the same year Lingelsheim failed to find any carriers among 311 persons examined. In 1909 Lieberknecht found eight per cent of carriers among 150 noncontact school children. In 1916 Eastwood and Griffith detected 10.2 per cent of carriers in a hospital of 480 patients where there was no case of the disease. In the same year Scott found 13.7 per cent of carriers in a hospital of 138 patients. During the war Worster-Drought and Kennedy made nasopharyngeal swabs from 1,018 soldiers, drawing from two units $-\mathrm{X}$ and $\mathrm{Y}$. Nineteen (1.86 per cent) were found to be carriers. Four of these furnished Type I; nine, Type II; one, Type III, and three, Type IV of Gordon's strains; while two earried strains which were nonagglutinable with any of the sera. Of the $\mathrm{X}$ unit 684 men were examined and 2.6 per cent found positive; of the $\mathrm{Y}$ unit 334 were examined and only one ( 0.3 per cent) was found positive. The $\mathrm{X}$ men were billeted in empty dwelling houses, while the $\mathrm{Y}$ men lived in barracks. The authors say:

“The higher percentage of carriers among the $\mathrm{X}$ unit may be explained by the fact that the men of this unit, as a rule, are constantly moving from place to place and consequently are more liable to meet with infection. The $\mathrm{Y}$ men, on the other hand, usually remain in the same place for a considerable time."

Chalmers and O'Farrell examined 847 British soldiers stationed at Khartoum, finding 86 carriers (10.1 per cent). The strain found in all of these belonged to Group I. Other investigators examined noncontacts and found from 0.0 to 30.0 per cent of carriers. These findings explain 
some of the characteristics of epidemics of cerebrospinal meningitis observed in its early history and at that time unexplainable. In 1886 Hirsch wrote:

"In the manner of its outbreak, incidence, and diffusion as an epidemic, meningitis reveals many peculiarities which are not without significance for estimating the effect that exterior circumstances seem to have in the pathogenesis. In many instances, as the foregoing historical sketch makes quite clear, the outbreak of the disease was a perfectly isolated incident in a locality that had been altogether free from it before; it has appeared at one or more places, not infrequntly far apart, while the country round the affected villages or towns, as well as the whole country lying between them, has remained quite untouched not merely while the sickness lasted, but in many cases also after it had died out in its original seats. Even in its general diffusion in Italy, in the United States at a later period, and in Sweden and Germany, in which countries the disease did in fact overrun wide tracts of territory, the places affected by it were only a few and far apart, while the intervening, or the immediate neighborhood, was touched in an extremely slight degree or spared altogether. A second peculiarity of its epidemic incidence comes out in the fact that in the epidemics, such as those just mentioned, which overran considerable tracts of country, the extension of the disease was much more rarely continuous or along certain radial lines than per saltum or quite irregularly, neither following the highways of traffic nor revealing any other obvious predilection. We find, no doubt, some exceptions to this, and very remarkable exceptions, in the diffusion of the epidemic through Central Franconia, where it traveled somewhat regularly from northeast to southwest; also in its progress during several of the French epidemies, in which it could be shown to have followed the movements of troops; and again in Sweden, where one can trace the regularity and uniformity in the progress of the epidemic from South to North. But if we go into the details of its course in these and other instances, we shall find reason to believe that in them also its progress was for the most part per saltum."

Since we know that this disease is spread chiefly by healthy carriers the explanation of the above-mentioned epidemic peculiarities needs no further elucidation.

When active cases of cerebrospinal meningitis are present in a community or a military organization the number of carriers is greatly increased, the percentage varying with the intimacy with which the healthy come in contact with the sick. In 1908 Bruns and Hohn found among close contacts 36.7 per cent of carriers; among less intimate contacts 22.5 ; among noncontacts 7.9. The proportion of carriers to those actually ill with the disease has been found to vary in different epidemics, the carriers being from two to forty times the number of cases. As the number of cases in an epidemic increases the number of carriers among the healthy also increases; as the number of cases decreases the number of carriers decreases. Black found this to be true in the epidemic at Dallas in 1912. Studying the disease as it prevailed at Camp McClellan in 1917-1918 Robey found nineteen per cent of carriers in companies in which meningitis occurred and thirteen per cent among 5,717 men who 
were swabbed on returning from furlough. According to Glover, the carrier rate among soldiers in the absence of active disease is normally under five per cent, but when the earriers reach twenty per cent active cases are likely to develop and the latter figure should be regarded as the danger line.

The Path of Invasion.--There seems to be no possible doubt that the meningococcus is earried into the body with the inspired air. There is doubt, however, whether this is the sole avenue of invasion. It must be admitted that it may reach its normal habitat in the nasopharynx through the mouth in food or drink, or by using infected tableware, especially including drinking cups, glasses, spoons, and forks. Having found its way either through the nose or mouth the meningococcus takes up its residence in the nasopharynx. Here it apparently is without marked pathogenic action. Kieffer while working with this organism accidentally introduced it into his nose. He reports that a slight catarrh and a transient headache followed, but otherwise the introduction of the organism was without effect. At one time, Dopter held that the development of cerebrospinal meningitis occupies three distinct and definite stages, as follows: (1) Catarrhal; (2) septicemic; (3) meningeal; and that in a given individual the development of the disease might be confined to the first of these stages or extend to the first and second, terminating at the latter, or it might extend to the third when the active disease state is reached. This view has received support, especially among English observers. In 1915 Lundie, Thomas and Fleming reported that all carriers examined by them showed nasopharyngeal catarrh. WorsterDrought and Kennedy have gone rather fully into this subject. They say :

"The greater number of carriers that we have dealt with in the past three years have shown no nasopharyngeal eatarrh, and in the remainder the evidence that it was due to the meningoeoccus was most indefinite. Nasopharyngeal eatarrh, tonsillitis, ete., was present in a fair proportion of contacts found negative. For instance, of 56 positive contacts found by us during the first half of 1917, one complained of a 'cold' and 'sore throat' with pharyngitis; another had enlarged tonsils, and a third was suffering from influenza. Among the 546 negative contacts with whom these carriers were associated, five were suffering from 'colds,' 'sore throat' or pharyngitis, and nine had enlarged tonsils.",

The report of the British Medical Research Committee is adverse to any causal relationship between catarrh and carriers of the meningococcus. The view now generally accepted is that in the nasopharynx, where it is so frequently found that we may designate this region as the normal habitat of the meningococcus, it is without pathogenic action. By what route does the micrococcus pass from the nasopharynx to the meninges, and why does it successfully make this journey in a few while it fails 
to do so in the great majority? The following possible routes have been suggested: (1) Through the sphenoidal sinuses; (2) by way of the ethmoid; (3) through the Eustachian tube to the middle ear and thence to the brain; (4) through the lymphatics; (5) by way of the blood current.

The view that meningococci pass through the sphenoidal sinuses was advanced by Westenhoffer as early as 1905 and was based upon 29 autopsies, in one-third of which he found inflammation of the sphenoidal sinuses. Later, however, failing to discover meningococci in the substance of the sphenoid bone, he gave up this theory. The absence of meningococci from the sphenoid bone was confirmed by Elser and Huntoon in 1909. These investigators failed to find meningococci in the walls of the sphenoidal sinus and in the hypophyseal region of the skull. More recently this view has met with some support in reports made by Embleton and Peters and independently by Holden, who found in the sphenoidal sinus pus containing meningococci. The latest investigation of this route of invasion comes from Worster-Drought and Kennedy who state:

"In 28 autopsies we found nothing to support the view of direct extension via the sphenoidal sinus. Although direct infection of the meninges by the meningococeus may possibly occur in rare instances, just as in pneumococcal or streptococcal meningitis secondary to foci in the nasal fossae, it is certainly not the usual mode of infection in cerebrospinal fever. The occasional occurrence of sphenoidal sinusitis, in view of Westenhoffer's later observations, is more than probably secondary to the meningitis, rather than occupying a causal relationship to it."

The transethmoidal route was suggested by André and was based upon experiments which he made with tissue stains, but this demonstration has not been confirmed by the finding of meningococei along this route.

The passage of the meningococcus along the Eustachian tube to the middle ear and thence to the brain is apparently negatived by the rarity of otitis media in this disease. This complication is exceedingly rare and occurs late when seen.

It has been suggested by Greenfield, McDonald, and others, that the meningococcus passes along the lymphatics of the spinal nerve routes, either in the cervical, thoracic, or abdominal regions, thus reaching the cord and subsequently extending to the brain. Fowler and others suggest that the meningococcus is due to abdominal infection, on the following grounds: (1) The lesions in the cord are the oldest. (2) The abdominal reflexes are abolished, showing that the lower part of the cord is affected. (3) There is only slight cerebral disturbance in some cases. (4) Infants fed exclusively at the breast do not have the disease. The last statement is not in harmony with the teaching of certain French experts on this disease. 
The belief that the meningococcus is transported by the blood from the nasopharynx to the meninges is at present generally accepted; however, there is still room for questioning this as the only path of invasion. As we have already seen, the onset of this disease may be sudden, coming like a thunder clap from a clear sky, or it may be gradual and accompanied by an indefinite period of general malaise. In the latter cases, the meningococcus has been found, at least in some of them, in the blood before meningeal symptoms are in evidence. This is not a new observation, because, as we have already stated, Gaucher as early as 1881 reported the finding of the coccus not only in the blood, but also in the urine. Worster-Drought and Kennedy summarize the evidence bearing on the path of invasion as follows: "(1) The mode of entry by the meningococcus is almost certainly by the blood stream. (2) In the ordinary type of cases the coccus is carried to the meninges by the blood within a few hours, without definitely infecting the blood itself in the sense of causing a true septicemia. (3) In some cases definite blood infection occurs (true septicemia), e.g., in most fulminating cases, prior to the involvement of the meninges; in such cases the septicamia often overshadows the meningitis, which may be comparatively slight. (4) In rare cases the organism may remain infecting the blood alone for a considerable period before finally reaching the meninges, or the patient may die from such septicemia before meningitis occurs. (5) In other instances the meningococcus infecting the blood may invade structures other than the meninges, e.g., the cardiac valves or various joints." Herrick, who had large experience in the clinical observation of meningitis at Camp Jackson during the war obtained positive blood cultures in from fifty to eighty per cent of his cases when cultures were made early. This led him to employ antimeningococcal serum intravenously in the early stages of the disease, and he believed that the results obtained amply justified this procedure.

In 1917 Flexner wrote:

"The meningococcus enters and leaves the body by way of the seeretions of the nasopharyngeal membrane. Since no other avenue of entrance or exit is known, the bacteriological control of epidemic meningitis centers upon those membranes. The meningococcus passes from the mucous membrane to the meninges in which it multiplies still further and thus sets up an acute inflammation called meningitis. It is not established whether the microorganism passes directly to the nervous system by way of the lymphatic connections between the nasopharyngeal mucosa and the meninges, which extend along the olfactory nerves, or indirectly by way of the blood. The former route is the one probably taken; but it not infrequently happens that the meningococcus may be cultivated from the general blood early in the course of the infection. Moreover, the occasional occurrence during the meningitis of metastatic meningococcal inflammation, such as arthritis, panophthalmitis, and endocarditis, indicates the ability of the microorganism 
to survive in and be implanted from the blood. However this may be, there is reason to believe that the meningococcus multiplies in the nasopharyngeal mucosa before it invades the meninges and the interval between the two processes may be brief or long, which is equivalent to stating that every case of epidemic meningitis develops out of a carrier. Two classes of persons harbor the meningococcus in the nasopharyngeal mucous membranes; First, persons suffering from epidemic meningitis; second, healthy carriers of the microorganism. The potential dangers from the two sources are unequal. The ill patient is usually confined in bed; he does not tend to move about and come into contact with many persons. While confined, he is dangerous to the doctor and the sickroom attendants and if in a hospital ward, to other patients. It is only when convalescent that, if not restrained, he menaces others. The healthy carrier, being usually unsuspected, is unhampered in his movements. He is a menace, therefore, to a wide and indefinite number of persons.',

No one has as yet satisfactorily answered the question why the meningococcus reaches the meninges in the minority of persons and fails to do so in the majority. Chalmers and O'Farrell have offered an explanation which they find in autovaccination. They believe that in the majority of carriers the meningococcus finds its way in small numbers into the blood current where it leads to the production of protective bodies by which immunity is secured. They further believe that this immunity can be demonstrated by the increased opsonic index in the blood. In accordance with this belief they have used micrococci killed by exposure to a temperature of $50^{\circ} \mathrm{C}$. for 20 minutes, both for protective and curative purposes. To adults they give 200 millions at the first dose and this is followed within two days by a second dose of 500 millions. We shall return to the question of vaccination in this disease. In our opinion, the explanation of freedom from the active disease in carriers as offered by Chalmers and O'Farrell is open to serious question. While the disease seldom develops in chronic carriers this is known to occur occasionally. We are not in a position, however, to speak with any finality upon this point. The suggestion that certain strains are more pathogenic than others and that those in whom the active disease develops have been unfortunate in getting into their nasopharynx especially virulent strains certainly does not hold. It has been made quite certain by frequent observation that the same strain is present in the carrier who has transmitted the disease and in the sick person who has received the infection. Many instances of this kind were recorded during the World War. A soldier carrying in his nasopharynx the meningococcus has on furlough transmitted the disease in its most virulent form to one or more members of his family, and the presence of the same strain in the carrier and in the sick persons has been demonstrated. In most epidemies and in practically every country and under all conditions of life it has been generally observed that the disease manifests itself most frequently 
and with the highest fatality among the strong and vigorous. During the epidemic in New York in 1904 only six per cent of those who developed the disease were reported to have been previously in poor health or in any way under par. We have seen that this disease is one especially of soldiers and children. Soldiers are selected because of their good physiques and among children cerebrospinal meningitis shows no preference for weaklings; in other words, it cannot be claimed that it improves the race by killing off the unfit.

It has been suggested by no less an authority than the English Research Committee that the meningococcus as it grows in the nasopharynx of carriers is a saprophyte with parasitic potentialities. There might be some debate as to whether a microorganism which, so far as is known, grows and multiplies only in man's body, can be called a saprophyte. Etymologically a saprophyte is something that grows only on lifeless, decaying, rotten matter; on the other hand, a parasite is an organism which lives and feeds upon or at the expense of some other living thing. Every parasitic bacterium is not necessarily pathogenic. It requires more than parasitism to produce disease. Of course, we are free to set aside all rules of etymology and change the meaning of a word to suit our convenience, at least men have frequently done this, but we should know what is meant when we state that the meningococcus, growing only in man, is a saprophyte; i. e., not that it is feeding on rotten matter but that it is not causing active disease. We prefer to say that, so far as we know at present, the meningococcus is in nature always a parasite. It is nonpathogenic to the structures of the nasopharynx; it is highly pathogenic to the meninges. Besides, calling the meningococcus when found only in the nasopharynx a saprophyte, and then calling it a parasite when it sets up a disease in the meninges, does not explain why its activities in one man's body are wholly saprophytic while in another's body it becomes parasitic.

Temporary and Permanent Carriers.-No sharp line of demarkation can be drawn between these classes. It has become a custom among those who have studied carriers to classify as temporary those in whose nasopharynx the meningococcus is found at one examination and is absent a week or ten days later and in all subsequent examinations; on the other hand, those in whom the results are positive at two or more examinations a week or ten days apart are called ehronic earriers. Most carriers are temporary, but in some the results have been positive for varying periods extending up to three months. Carriers often clear up when living and weather conditions improve. Of course, every man who develops cerebrospinal meningitis is a carrier before there is any mani- 
festation of the disease and, as a rule, he continues to carry the meningococcus in his nasopharynx during the first six or eight days of the active disease, provided he lives that long. It is interesting to note that very few cases of the disease develop among recognized carriers. During the war there was abundant opportunity to make observations on this point, because it was the custom in all armies to search for and isolate carriers. The English Research Committee report the development of the disease in only two cases of 690 carriers held under observation in 1915, and one of these cases was of doubtful diagnosis. Fildes and Baker report no case of the disease among 485 carriers under their observation. WorsterDrought and Kennedy report one case, and this only suggestive of meningitis, among 140 carriers. Experiences reported by military and naval surgeons on the relation between carriers and cases vary widely. Mink, who had charge of this work at the Great Lakes Naval Training Camp in 1917-1918, makes the following statement:

"It was possible during the epidemic to make cultures of practically every one of the 24 barracks connected with regiments. We found barracks in which meningitis developed that contained eight or nine per cent of carriers. On the other hand, we found barracks in which no meningitis developed that contained twenty-five or thirty per cent of carriers. In the instances in which a large percentage of carriers was found, carriers were removed; and in other instances the carriers were left in the barracks. New cases failed to develop in both instances. It was early found impracticable to remove all the carriers without producing overcrowding among the earriers, which resulted in additional cases. There seems little doubt that the oft repeated statement that carriers rarely develop the disease is erroneous, as the incident rate among carriers is higher than the rate for the general station. It is not the intention to maintain that carriers do not transmit the disease, but it is maintained that to combat this disease by the removal and segregation of all carriers is absolutely impracticable.'

Period of Incubation.-Observations made during the World War have shown that susceptible people may develop this disease within a very short time-from one to five days-after coming in contact with a carrier; on the other hand, the disease may develop in an individual who is already a recognized carrier and may have been in this class for many days before there was any sign or premonition of active disease. Indeed, short periods of incubation were reported before the war, but the circumstances surrounding them were not always convincing. In 1911 Sophian stated that a New York physician who had not previously been exposed to this disease and who made an autopsy on a Greek dying on an incoming steamer, developed a malignant form of the disease 24 hours later. Steiner and Ingraham tell of a hospital orderly who, having been in attendance on a ease of cerebrospinal meningitis, went to his home and four days later one of his children developed the disease. 
Richter tells of a woman who visited two children suffering from meningitis and four days later developed the disease. During her convalescence she was visited by a young man who showed symptoms of meningitis two days later. In turn a co-worker of this young man developed the disease four days later. Fairley and Steward tell of two soldiers who went home on three days' sick leave. One of these developed cerebrospinal fever on the last day of his leave. His mother nursed him and developed the disease five days after her son reached home. The second soldier did not develop the disease while on leave and at the end of the three days returned to his post, but on the day of his return, that is three days after his arrival, a member of the household he had visited developed this disease. Six days after his return this soldier showed symptoms of meningitis. Flack tells of a soldier who returned from France on leave; two days after reaching home one of his children and three days after his arrival another child, developed the disease. The soldier was then swabbed and Type II of the meningococcus was found in his throat, and the same type in the throats of the children. Worster-Drought and Kennedy have collected a number of instances which show that the period of incubation in this disease may be as short as from two to three days; on the other hand, the disease has been seen to develop in recognized earriers from a few days to six weeks after isolation. It would seem from these facts that in some individuals meningocoeci pass with scarcely a halt directly into the meninges, while in others these organisms are arrested and detained in the nasopharynx. In some instances there is an escape from the detention eamp and the objective point in the meninges is reached. In most instances, however, the barriers around the detention eamp prove sufficient and the prisoners die. From these facts the most reasonable assumption is that susceptibility to this infection is determined by the condition of the tissues of the nasopharynx. If there be a break in the continuity of the protective membranes of this region the micrococcus finds ready entrance and quick conveyance to the meninges. The meningococcus apparently passes a commensal, not a saprophytic, existence in association with the tissue cells in the nasopharynx. In this locality there is no actual combat between the body cells and the invading organisms, and consequently no active disease. In the nasopharynx the meningococeus is a parasitic but not a pathogenic organism. When it reaches the meninges its behavior is quite different. The cells of the meninges are sensitized by the invading organism. The development of sensitization requires a certain timethe period of incubation,-after which the meningeal cells begin the destruction of the invading organisms, the meningococcal proteins are 
broken down, poisons are set free, and meningeal inflammation and suppuration result. To us, this is the most satisfactory explanation of demonstrable facts connected with meningococcal invasion of the body. It seems to be established beyond a doubt that in some persons-a minority of those exposed-the meningococcus meets with no opposition in its movement towards that tissue for which it has a predilection-the meninges. One man is susceptible to this disease, while another becomes a carrier only, and the difference, as we see it, between the two lies wholly in the degree of permeability of the walls of the nasopharynx. These differences between individuals may lie in inherited anatomic peculiarities of the region of the nasopharynx. This view is supported by the fact that, while as a rule only one member of a family is successfully invaded by the microorganism, there are instances in which practically every member of the family develops the disease. In some individuals susceptibility may depend upon the presence or absence of lesions in the nasopharynx at the time the meningococcus reaches this locality, or upon the development of lesions at any time during the occupation of this region by meningococei. The wounds in the nasopharynx may be microscopic or they may be gross. In the literature one finds quite a list of eases in which injuries to the skull have been followed by meningitis. Quite naturally, this list is not a long one, because the injury cannot cause specific meningitis unless the organism is present. Again, the injury must be of such a nature, even when meningococei are present in the nasopharynx, that a path to the brain and cord is opened up. It has been suggested that the meningococcus is assisted in finding its way through the walls of the nasopharynx by some hitherto unrecognized microorganism. It may also happen that permeability of the wall of the nasopharynx has been made possible by the activity of some bacterium already present in this region when the meningococcus arrives. Netter suggests the simultaneous activity of some unknown cosmic agent. It seems to us that this assumption is not necessary. If there be in the community some cosmic condition which renders people susceptible to the disease it should affect all alike, but even in the most severe epidemic the percentage of those in the community who develop the disease remains small. We think. that the facts which have been brought out in the study of this disease indicate quite conclusively that the meningococcus does not break its way through the nasopharyngeal wall by its own efforts and that it finds its way through these structures only under exceptional conditions. In a nasopharynx which is in normal condition on the arrival of the meningococeus and which remains in this condition so long as the organism tarries in this locality, the meningococcus is a harmless guest. When the way to the brain and cord is 
open on the arrival of the meningococeus or is opened during its stay in this locality it becomes a most deadly agent.

Seasonal Incidence.-In the temperate zone most epidemics of cerebrospinal meningitis occur in the winter or spring. It must be admitted, however, that low temperature in and of itself has no influence on the prevalence of this disease. This was demonstrated many years ago. Some of the epidemics reported in the early part of the nineteenth century occurred in warm weather and others in mild winters. Concerning the epidemic observed in Paris in 1847 Levy wrote:

"Neither cold nor heat, neither rain nor sunshine had anything to do with the prevalence of this disease. Our statisties show two maxima which correspond, one with the greatest heat of the summer and the other with the lowest temperature of the winter."

Many of the outbreaks in French garrisous and in isolated villages in this country occurred in normal weather and some appeared in the height of summer and decreased with the approach of winter. Still, as we have said, the majority of epidemics, taking the temperate zone as a whole, have appeared in winter. The winter of 1917-1918 in this country east of the Rocky Mountains was the coldest known since temperature records have been kept, and it was during this period that cerebrospinal meningitis was most in evidence in our cantonments. Certainly cold alone cannot be even a predisposing factor in the development of this disease, because, as Chalmers and O'Farrell show, it is often prevalent and highly fatal in tropical Africa. Cold and inclement weather probably contributes to the spread of this disease in several ways. In the first place, bad weather conditions drive people indoors where they congregate and come into close contact. In the second place, in such weather catarrhal conditions are more prevalent; sneezing, coughing, and spitting are indulged in more frequently, and through these agencies the meningococci growing in the nasopharyngeal cavities of carriers are expelled and scattered more widely. In the third place, and this is a matter of great importance in cold weather, it is the tendency of man not only to congregate indoors and to crowd, but to keep the temperature indoors abnormally high. So far as we have information, the meningococcus dies in a few minutes when expelled from the body into an atmosphere the temperature of which is below $22^{\circ} \mathrm{C}$. (71.6 F.).

Influence of Crowding.-We have already called attention to the fact that French medical officers long ago were convinced that crowding favors the development and spread of this disease. Our medical officers in the World War had ample confirmation of this fact. Glover found that the number of carriers among English soldiers increased as the barrack beds were brought closer together; and what is of more importance, 
he noticed, quite naturally, that extending the distance between beds did not immediately decrease the number of carriers. With the beds one foot four inches apart there was a carrier rate of ten per cent. If the beds were crowded to one foot the number of carriers rose to twenty per cent, and with further reduction in the space between beds the carriers went as high as thirty per cent. It should be understood that this refers to noncontact carriers. Glover writes:

"Whilst overcrowding produced a rise in the carrier rate very quickly, a return to mobilization standard was not sufficient to reduce the carrier rate markedly, but the carrier rate was reduced with remarkable success by spacing out the beds to two and one-half feet distance between each, although not at so fast a rate as overcrowding will raise it. 'To raise the carrier rate by overcrowding was both easier and quicker than to diminish it by spacing out."

From observation in our own camps we came to the conclusion that the transfer of meningococci, pneumococci, and streptococei from person to person does not occur so much during sleeping hours as during waking hours. However, it is well to take the number of beds in a barrack as the best measure of the extent to which crowding is carried, and for this reason we insist on a space between beds of at least two and one-half feet, and preferably three feet. The smallest crowd in which cerebrospinal meningitis can be transferred consists of two-one a carrier, the other a recipient. If a successful transfer between these two is made they must be close enough so that the spray thrown out in coughing, sneezing, and loud talking from the donor may reach the recipient. Multiply both donors and recipients in the crowd and you multiply the number of successful transfers. It should be plainly understood that the transfer of the meningococcus from one person to another does not require for its success a bedroom or a barrack. As we have had occasion to say in discussing pneumonia and other respiratory infections, men may be dangerously crowded while out of doors. The advantage in a successful transfer of the microorganism, however, lies within doors, especially in overheated quarters. Glover, after making a careful study of the spread of cerebrospinal meningitis among English soldiers, states his conclusions as follows:

“(1) A high carrier rate usually denotes overcrowding and dangerously unhygienic conditions, even though no cases of the disease may have recently occurred. (2) Whilst sporadic cases may occur in a military as in any other community with any carrier rate, anything approaching an epidemic of cerebrospinal fever is heralded by a warning rise of considerable height in the carrier rate. (3) Severe overcrowding will probably be accompanied by a carrier rate of at least twenty per cent. This percentage is indicated as the danger line in the War Office Memorandum on cerebrospinal fever. A carrier rate of this height will usually imply that the mobilization standard of forty square feet per man has been infringed, and that beds in the unit examined are less than one foot apart. It should be regarded as a signal for prompt and effective action 
to diminish overcrowding, and to improve ventilation. (4) The distance between beds is of paramount importance. (5) Carrier rates between ten and twenty per cent are unsatisfactory, and imply a certain amount of overcrowding; they must be watehed with suspicion. (6) Carrier rates from two to five per cent may be considered usual under the best conditions obtainable in barracks and hutments. (7) Under the same conditions of overcrowding (noncontacts) carrier rates agree substantially with contact carricr rates. (8) Quite a moderate degree of spacing out of beds, combined with simple methods for improving ventilation, are highly efficient agents in reducing high carrier rates. (9) When a unit shows a high carrier rate, a distance of at least two and one-half feet between the beds should be enforced. The 'peace' standard would, of course, be even more effective. (10) Recruits should be specially spaced out during their first three months of service."

In searching the literature of this disease we have found reports of small epidemies in prisons, orphanages, almshouses, and hospitals, but we have seen no report of epidemics eonfined to public sehools. In most schools the excessive erowding essential to the development of an epidemie of this disease does not exist, at least not often. Certainly no child who has been in contact with the disease should be permitted to attend school unless shown by examination not to be a carrier; but the conditions existing in our public schools are, in our opinion, not favorable to the development and spread of this disease. The greater prevalence of cerebrospinal meningitis among negroes and whites in narrow, overcrowded apartments finds its explanation in the greater ease with which the meningococeus is transferred from one to another under these conditions.

Influence of Location.-Epidemies of cerebrospinal meningitis have been reported from marsh and from mountain; from sea coast and inland; from cold and hot countries; in times of flood and in times of drouth; at the level of the sea and at high altitudes; among the poor and among the rich. The meningococeus seems to be satisfied to take up its residence in any individual of the Genus homo and it is always seeking to reach the highly protected eentral nervous system of its host. Improved sanitation, meaning by this the removal of excreta, garbage, and waste, has no influence upon this disease. It is in no sense a filth disease. There is no evidence that the meningococeus even lives for any length of time, to say nothing of growing and multiplying its kind, outside the body of man and it will grow in the body of a elean man just as readily as in that of a filthy individual. There is no hope of ridding the world of this disease through the skill of the sanitary engineer. When man becomes sufficiently well trained in personal hygiene that he is able to avoid receiving all consignments of bacterial flora from the upper air passages of his neighbors the last of the meningococei will die.

Relation of Fatigue.-There is no ground for suspecting that fatigue, 
or any other bodily condition so far as that is concerned, has any determining influence upon the reception by an individual of the meningococcus and its entertainment in his nasopharynx; in other words, fatigue certainly has nothing to do with the number of carriers unless it be that it brings on more violent respiration accompanied by coughing, sneezing, etc. These procedures may aid in the distribution of the organism, but they can have nothing to do with increasing the susceptibility of the receiver. Fatigue, however, is believed, and with good reason, to be an important factor in influencing the susceptibility of man to this organism, provided it has already been taken into his air passages. Under fatigue the meningococcus is more likely to penetrate the walls of the nasopharynx and reach the brain. This has been so frequently observed and under conditions so well understood that it has all the force of an animal experiment. We have seen that cerebrospinal fever is a disease of soldiers, and especially of recruits. Moreover, among recruits it prevails mostly under heavy drill. As shown by Mink, who had charge of the bacteriologic investigations at the Great Lakes Naval Training Camp during the War, there were as many as twenty-five per cent of carriers among the recruits coming to that station. For three weeks these recruits were kept in what was known as the "incoming detention camp" and here they were tenderly cared for. They received their vaccination, various medical examinations, donned their sailors' garb, and were permitted to take life easy. Mink says that no case of meningitis ever developed in the incoming detention eamp. At the end of three weeks of this kind of life the embryo sailor was transferred to "Camp Dewey." Here a life of comparative hardship fell upon him rather suddenly. He was drilled, asked to keep all the walks about the camp free from snow during the heavy falls of January, 1918, and other burdens were placed upon him. During this period cerebrospinal meningitis appeared. In the French, English, and American Armies the highest incidence of this disease occurred during the first year in which the nation engaged in the war. In the French troops there were in $1915,1,075$ cases or 4.3 per 10,000; in 1916 there were 451 cases or 1.8 per 10,000; in 1917, 406 or 1.5 per 10,000. In the British Army the number of cases in 1915 per 1,000 admissions was more than twice what it was in 1916. In our own army, as we have already shown, this disease reached its maximum within a few months after mobilization. During the summer of 1918 it greatly receded, with some accessions in the fall. When the disease does appear among seasoned soldiers, as it sometimes does, it is more prevalent and more fatal among those upon whom heavy burdens are imposed. This was commented upon by Love in the Mexican War and by Frothingham in the Civil War. The latter, writing concerning a small epidemic in the Army of the Potomac during the winter of 1861-1862, says: 
"Here was a large army, living under almost exactly the same circumstances as to food, beds, ventilation, clothing, etc., and within a few miles of space; beyond a few cases (enough to indicate some common cause) an unusual disease occurred in one brigade (as I did not hear of any other cases, I take it for granted there were none). Malarious and continued fevers prevailed in all the camps throughout the Army. The only circumstance influencing disease in which this brigade differed from others, was the severity of its labors. Under General Daniel Butterfield, a stern disciplinarian, who, always, sick or well, did his own entire duty and would receive no less from his subordinates, the men were drilled to the full extent of their powers-often to exhaustion.',

Diagnosis.-Before the identification of the meningococcus and the employment of lumbar puncture the diagnosis of cerebrospinal meningitis depended upon symptoms and autopsy findings, and where autopsies were not made it is often difficult to decide as to the nature of the disease described. Now the diagnosis of cerebrospinal meningitis, including certain differentiation from other forms of meningitis, is rendered easy and certain. The meningococcus is found in the nasopharynx and in the spinal fluid and, as we have stated, the organisms obtained from both sources belong to the same group. Puncture of the spinal cord and the removal of fluid is a beneficial and therapeutic measure in and of itself, inasmuch as it relieves the pressure on the brain and cord. Add to this the specific effects of antimeningococcic serum introduced directly into the seat of the disease and it is evident that, while diagnosis is rendered certain, treatment is doubly beneficial.

Sporadic Cases.-Scattered cases of typical meningitis are occurring from time to time in every part of the known world. In the majority of these, autopsies are not made, and for a long while epidemiologists were at a loss to determine whether or not they were due to the meningococcus. It will be understood that there are other forms of meningitis and some of the sporadic cases are due to one cause and some to another, but the method of diagnosis now resorted to makes identification positive. It has been shown, especially in this country and in France, that many of the sporadic cases of meningitis are caused by the meningococeus, and from what we have learned concerning the avenues through which this disease is distributed we can easily understand why sporadic cases are occurring. An individual, a carrier, may from any direction or any distance be received in a household and fondle and caress the baby in such a way as to transfer meningococci which she has brought with her to the child. It is in this or similar ways that the disease appears from time to time without giving rise to an epidemic. Fortunately, methods for the recognition of meningitis and the differentiation between etiologically different forms of this disease have so improved that at present it is not necessary to await autopsy for a correct diagnosis. In all eases of suspected meningitis lumbar puncture may be resorted to and the ex- 
amination of the fluid obtained in this way will show not only whether the disease is meningitis, but, also, if it is, to what it is due.

Distribution of Cases.-Usually there is not more than one case of this disease in a home in civil life or in a barrack or company among soldiers. This does not contradict the fully attested fact that this infection depends for its distribution upon crowding or intimacy of contact. It is due to the fact that of those who receive the infection only a small percentage develop the disease. In this respect cerebrospinal meningitis differs from measles. Put a soldier in the infectious stage of measles on a troop train, in a barrack, or on a transport, and every one of his comrades who is not protected by having had the disease is likely to develop it. Put one who is a carrier of the meningococcus under like conditions; many of his comrades may become carriers, but only a few, possibly none, will develop the disease. But any one who has in this way become a carrier may distribute meningococci in other crowds, in homes or communities, and this continues until some one in whom the disease can develop has been reached. Every one who has not had measles is susceptible to the disease. Only small percentages of communities are in a condition to develop cerebrospinal meningitis. Among those who have not had measles one hundred per cent are susceptible, while in any group of men, whatever their previous experience may have been, there are only a few who develop cerebrospinal meningitis after becoming carriers.

While, as a rule, active cases of this disease are found singly in homes, in barracks, and in companies, there are exceptional instances in which it strikes down large numbers in certain groups. In an epidemic in a French garrison in 1839 six out of eight soldiers occupying the same room were attacked practically at the same time. The case of the "Brown" family, reported by Sewall, of New York, in 1872, is a classical illustration of how this disease sometimes goes through a family. On Tuesday, January 30, Albert, a school boy, age seven, was kicked by a school fellow and fell striking his head against an iron railing. This occurred about 1 P. м. He made no complaint until about 7 P. M., when he was seized with intense pain in his head. He died at 4 A. M. on Wednesday, no physician having seen him. An autopsy was made and a thin layer of extravastated blood was found covering the surface of the brain, extending to its base, and the ventricles contained bloody serum. The verdict of the autopsy was that the boy died from concussion of the brain due to the injury to his head. On Sunday, February 4, a brother, four years old, had spasms, with vomiting, and died at 6 A. M. Monday the fifth. On February 6, Theresa, age thirteen, complained of severe pain in the head. This patient passed through a typical course of cerebrospinal meningitis, but finally made a complete recovery. On Wednesday, February 7 , Berthold, age eleven years, developed the disease and died within 
eighteen hours. An autopsy was made in this case. The entire brain surface was intensely congested, the sinuses being tense with blood. On Saturday, February 10, the baby, age fifteen months, began vomiting and having loose stools and died at nine the next morning. Autopsy showed the dura mater congested, but no effusion of lymph or pus on or under the pia mater or arachnoid. On March 16, Henry, the only remaining child, age five years, was seized with vomiting and convulsive symptoms. On the twentieth he was taken to the hospital and there died on April 8. Sewall says:

"Thus perished five out of six children in one family by this fearful malady. They are Germans, the mother a stout very healthy appearing woman, the father pale and thin and somewhat intemperate, yet not a sickly man. He is a tinman by trade, and they occupied the first floor of an Eleventh Avenue apartment, the front room being used as his shop. They lived poorly, were crowded and badly nourished and ventilated. Beneath them in the cellar, from a loose elbow in the sewage pipe, communicating with the privy in the yard, were continually rising, for no one knows how long, the foul effluvia peculiar to this drainage, and which must have strongly contaminated their blood, thus rendering them an easy prey to this active destroyer.',

Mérand, of Nozay (France), reports that cerebrospinal meningitis appeared in a family consisting of father, mother, and ten children living in the greatest poverty in a small room containing four beds. Six of these developed the disease, the exact nature of which was determined after lumbar puncture. At Alexandria, La., near which city Camp Beauregard was located, in a family of nine all save the father developed this disease and four died. Instances of multiple cases in families might be continued, though they are not numerous, and they incline us to give more attention to the claim that the passage of the meningococcus from the nasopharymx to the brain may be facilitated by some unusual anatomic structure which may predominate in certain families. Symmers, who has made many autopsies in Bellevue Hospital, states that he has found the status lymphaticus in sixty per cent of the necropsies on cerebrospinal meningitis cases, while the incidence of this condition in cases of death due to other diseases does not exceed eight per cent. Many years ago Westenhoffer found this condition in a series of 29 necropsies. This is a matter which deserves further investigation.

Age Incidence.-There is some difference of opinion among authorities as to the influence of age on this disease. Griffith says:

"Age is a powerful etiological factor, children and adolescents being especially susceptible, and infants in the first year being in no way exempt. Of 2,916 eases in the Silesian epidemic in 1905 reported by Flatten eight per cent occurred in the first year, forty-seven per cent from birth to five years, and twenty-nine per cent from five to ten. Of 2,179 cases in the epidemic in New York City in 1904 and 1905 fifteen per cent were under one year and sixty-seven per cent under ten years of age. It has been observed even in the newborn.'" 
The deaths from cerebrospinal meningitis in Massachusetts from 1887 to $\mathbf{1 8 9 5}$ have been distributed by age. These figures show a large number of deaths in infants under one year of age. In commenting on these figures Councilman, Mallory and Wright say:

"It appears that 316 deaths from this disease, or 26+ per cent of the whole number, were reported as having occurred among children under one year. This fact necessarily vitiates the accuracy of the returns to a considerable degree, since the disease is extremely rare among infants as well as among those of advanced years."

On the other hand, Netter and Debré claim that this disease is highly prevalent among infants and they quote the Massachusetts figures, criticised by Councilman and his colleagues in support of their statement. In

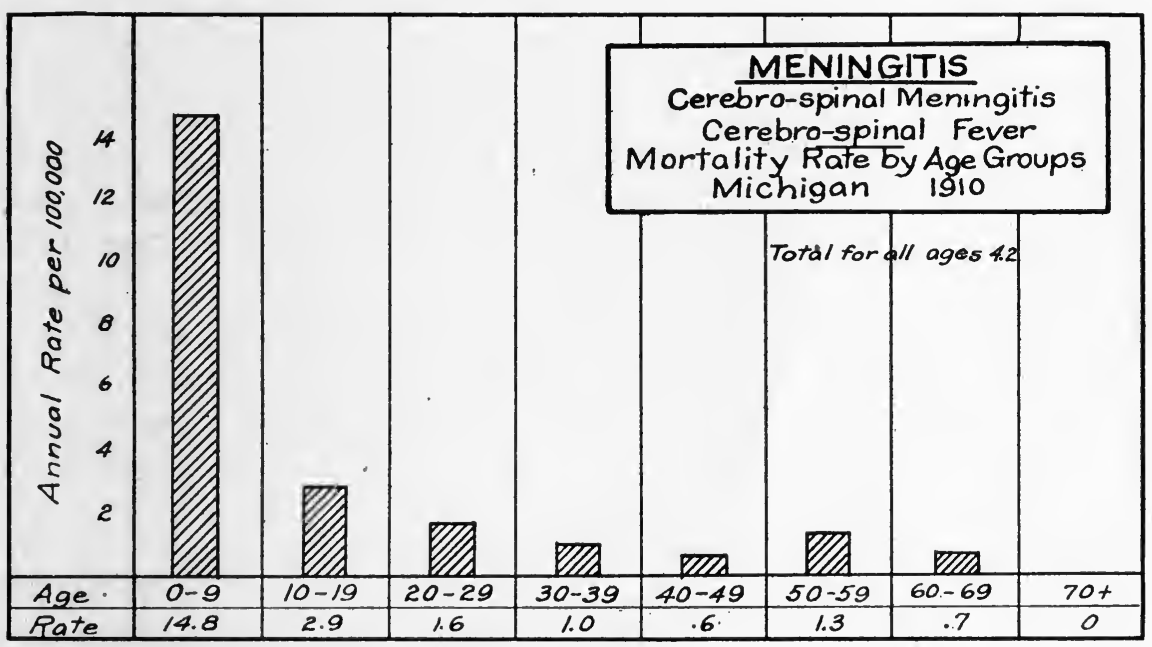

Fig. 67.

the New York epidemic (1905-1907 inclusive) the following figures in regard to age incidence were given: In 1905, 2,180 cases, with sixtyseven per cent under ten years; in 1906, 993 eases, with sixty-five per cent under ten years; in 1907, 828 cases, with sixty-eight per cent under ten years. In German epidemics covering the same period, there were 8,198 cases, with 80.12 per cent below sixteen years of age. In many epidemics, for instance at Montgomery, Ala., in 1848, and in Australia in 1915, less than one-third the cases occurred in children under ten. As Compton has pointed out, all these figures are of but little value. Before any justifiable decision ean be reached we must know the age distribution in the community in which the disease occurs. Figuring it for all outbreaks in England and Wales, Compton concludes that the most susceptible age is under five years and the least susceptible between 35 and 40 years. 
We have gathered from a study of the epidemies the opinion that the age has varied widely in different epidemics and that this is partially explained by the fact that the crowding upon which the spread of the disease has depended has been among different age groups. Quite naturally, in military epidemics there are no children and no old people; on the other hand, in civilian epidemics the age incidence varies with the age groups most frequently participating in and becoming a part of crowd life. Necessarily, military life in eantonments and barracks is more congested than that of our most populous eities. In the mobilization of an army young men from diverse sections, including localities in which there are many carriers, are brought into camp and thrown into most intimate contact. These conditions are most favorable to the development of cerebrospinal meningitis. Every fall thousands of young people from diverse and widely separated sections of the country, including localities in which carriers are numerous, assemble at our large universities. But we know of no instance of an outbreak of cerebrospinal meningitis following these assemblies of students, and for the very good reason that they are not brought into the intimate contact experienced in camps and eantonments. In the organization of the Student Army Training Corps in 1918 the sanitarian was not consulted. Had he been, he would not have recommended the placing of students in crowded barracks when they might have been billeted and continued the procedure ordinarily followed in these schools. Of course, this statement about the Student Army Training Corps concerns influenza and pneumonia rather than cerebrospinal meningitis.

Vaccination.-Cerebrospinal meningitis is relatively so rare that universal vaccination against it would hardly be justified even if we had a safe and sure method of earrying out this procedure. Vaccination against this disease may be considered in civil life in the presence of an epidemic and in military' life in the mobilization of troops whether active cases of the disease be present or not. The vaccine is easily prepared by heating cultures to $50^{\circ} \mathrm{C}$. (possibly higher) for from 20 to 30 minutes. This vaccine has been tested scientifically by determining the extent agglutinins, complement-fixation bodies, etc., are developed in those receiving the vaccine. It will be understood that either polyvalent vaccine or one for each special strain must be employed and its efficiency must be determined on polyvalent or special strain cultures. In 1912 Sophian and Black treated ten medical students with vaccines. Five received an initial dose of 500 million cocci and after an interval of seven days a second dose of 1,000 million; the second five received a first dose of 1,000 million and after an interval of seven days a second dose of 2,000 million, and after a further interval of seven days a third dose of 2,000 million. Agglutination and complement-fixation tests demonstrated 
that these vaccines were efficient in producing so-called immune bodies. The local and constitutional symptoms resulting from the employment of these vaccines are of about the same significance as of those that follow typhoid vaccination.

The clinical evidence concerning the value of vaccination in this disease remains to be determined and it will be a difficult task to make this determination. During the Dallas epidemic of 1912 Sophian reported the vaccination of about 100 persons. Two of these, nurses in attendance on cases of cerebrospinal meningitis, developed the disease some weeks after inoculation but recovered. Several reports on vaccination, both in civil and in military life, in which no cases have developed among the vaccinated have been made; but with a disease to which so few people are naturally susceptible, these reports do not amount to much, unless it be to show that vaccination is harmless and does not increase susceptibility to the disease by developing a negative phase. As has been stated, Chalmers and O'Farrell used the vaccine both in a preventive and a curative way and they claim that it is of value in both instances and that its value depends upon an increase in the opsonic index.

Passive Immunization.-It was pointed out by Jockmann in 1906 that the specific serum for this disease might be used in establishing temporary immunity in the presence of an epidemic. It should be understood, however, that in case such immunity is secured it is only of temporary duration, probably as in the case of diphtheria antitoxin, lasting not more than three or four weeks. In the Dallas epidemic in 1912 Sophian injected antimeningococcal serum into physicians, nurses and attendants. None of those thus treated developed the disease within a month and only 'one, an attendant, developed it within six weeks. There are, of course, objections to this method of securing passive immunity, inasmuch as it renders the individual thus treated susceptible to the dangers of serum disease in case of subsequent treatment with serum for any disease. So far as we know, this method was not employed among soldiers in the late war.

Control.-Control measures in this disease must be directed to the individual and to masses. It should always be borne in mind that in order to become a carrier, which is essential to the development of this disease, the individual must come into close contact with another carrier under certain conditions of ventilation or lack of it, under certain conditions of temperature, and degrees of proximity. If the common habit of sneezing, coughing, and throwing saliva in a spray into the faces of others could be stopped the existence of carriers, and consequently of cases, would be greatly decreased. As has been stated, the meningococcus soon ceases to exist when cast out into an atmosphere with a temperature below $22^{\circ} \mathrm{C} . \quad\left(71.6^{\circ} \mathrm{F}\right.$.). The temperature of rooms 
in which men congregate should, if possible, not be permitted to reach this point. . It may be that there has been less of this disease in England than in America, because of the difference in temperature which is usual indoors in the two countries. In winter we overheat our houses. It cannot be said that we pay less attention to ventilation than the English, but it certainly is true that in our homes, churches, schools, places of assembly in civil life, and in barracks in military life, we have overheated rooms. It is a truth not appreciated generally by medical officers that if the temperature of the barrack is kept as low as it should be the soldier needs heavier clothing when indoors and resting than he does when at drill out of doors. Notwithstanding the liberality with which we fitted out the barracks in our cantonments, our soldiers were often cold at night in these barracks because the blankets were deficient in number or unsuitable in texture. It is idle to expect men to sleep in cold rooms with insufficient bedding; on the other hand, with sufficient bedding the ordinary man, certainly after some experience, prefers to sleep in a cold room and even out of doors.

If it ever be necessary to again mobilize a large army, we hope that greater and more scientific methods for the prevention of this disease will be put into practice than were employed in our latest experience. So far as this disease is concerned, and as we have pointed out it applies to eertain other diseases, the draft men should be assembled in the vicinity of their homes in groups of not more than 30 . Here they should be bathed, barbered, furnished uniforms, and held in quarantine for at least 14 days. During this time they should receive their vaceines and every man cultured for the meningococcus, and only those who are not carriers should be sent to the cantonment. They should not proceed to camp on crowded troop trains, but each group should be kept to itself and should be isolated for at least seven days after arrival at camp. No barrack should accommodate more than 30 men. Under these conditions, if the disease does occur or if carriers multiply and approach the danger limit, the control of the disease will be within the range of human possibility, while under the conditions that prevailed during the mobilization of 1917 this was not true. In eamp from time to time groups of men, including whole companies, possibly battalions and regiments, should be cultured and when the number of carriers approaches the danger line of twenty per cent, the fact that overerowding is likely to give trouble should be recognized and steps to spread out the men should be taken without waiting for the development of an active case of the disease. Recruits should be held in incoming detention camps in groups of not more than 30 and every man in each should be cultured for the meningococcus before he is given a permanent assignment. It seems to us that our military men have gone too far in insisting that all soldiers, under all conditions, 
must spend their nights in barracks. In December, 1917, Jefferson Barracks, near St. Louis, was designated as the place of rendezvous for those who might volunteer from the Southwest. No one could tell how great a number this might be and none could predict that the first half of December in that region would show a zero temperature. The number who did volunteer and arrive at Jefferson Barracks was much greater than anticipated. The temperature was much lower than any one expected. Accommodations were inadequate and thousands of men spent the nights shivering with cold and in the morning found only a cold breakfast awaiting them, while in the City of St. Louis there are hundreds of public, semi-public, and thousands of private buildings that would have been gladly opened to these soldiers for the night. The billeting of troops may not be the most satisfactory way of handling them, but it seems to us that there are times when it might be resorted to and result in greater personal comfort to the soldier and at the same time be a means of preserving his health.

As we have seen, it is well nigh the universal opinion of medical officers who have observed it, that exhaustive drill increases susceptibility to this disease. The transition from the slow, quiet life of the farmer's boy to that of the alert, severely drilled soldier should be less abrupt than it was in our camps in 1917-1918. At that time line officers quite properly felt that time was an important element in making an army, and there can scarcely be any doubt that many lives were lost in consequence of our long delayed preparation for war and the suddenness with which we entered it. There is no need and no benefit in regretting past deeds, but there is reason why we should not forget them, lest we repeat them. Universal military training or proper physical training for all young people in our high schools, colleges, and universities would unquestionably improve the race, physically, mentally, and morally, and at the same time would place the nation in a better position for defending itself promptly and efficiently by force of arms should it be compelled to do so.

The individual who develops this disease should be isolated and those who attend him should practice every known measure to prevent the spread of the infection. It will, of course, be understood that the meningococci that are in the cerebrospinal canal are not being discharged from the body, but that every man ill with this disease has a batch of meningococci in his nose and throat and these are being discharged in coughing, sneezing, spitting, and in the secretions, especially from the nose, that may flow out from these cavities.

With the patient taken care of, attention must be given contacts. This word has come to have in this disease a rather definite and distinct meaning. "Contacts" are those who have been sleeping, eating, living, 
visiting, in the same house as that occupied by the patient. It will readily be seen that under these terms the number of contacts is subject to wide variations, all should be cultured. Those who are negative should be permitted to go about their business, while the carriers need special attention.

According to Worster-Drought and Kennedy, the following is the gist of the regulations pertaining to this disease adopted in the British Army:

“On the patient's removal to an isolation hospital or ward, disinfection of all his clothing, bedding, feeding utensils and quarters is carried out. All contacts are then segregated and afforded ample space in freely ventilated quarters. At the earliest possible moment a bacteriological examination of the nasopharynx of each contact is made. While awaiting result, it is recommended that the isolated men, if physically fit, be drilled daily in the open air. Those in whom the swab proves negative are returned to duty, provided the throat is normal. Carriers are transferred to isolated quarters in hospital and treated until two successive swabs, taken from the nasopharynx at several days interval, are reported as negative."

In military life the segregation of carriers is generally easy, even if the number mounts to the hundreds. In civil life the number of contacts is likely to be small in the majority of instances and they, also, can be properly handled. Under certain industrial conditions, even the detention of a large number of contacts long enough to take swabs and make cultures is impracticable. The period during which an individual carries the meningococcus is widely variable. During the war many attempts were made to relieve carriers of their undesirable guests by subjecting them to certain procedures either as individuals or in masses. As early as 1910 Vincent and Bellot, of the French Army, proposed the inhalation of a solution consisting of thymol, 35 centigms.; guaiacol, 2 gms. ; iodin, 12 gms., and sixty per cent alcohol, 200 gms. This preparation more or less modified was largely employed in the French Army during the war. In our own and in the British Armies much attention was given to the employment of solutions of chloramine, two of which, chloramine-T and dichloramine- $\mathrm{T}$, were employed. These are chemicals which contain chlorin linked to nitrogen and possess high disinfecting coefficients. These and various other preparations were used by the individual soldier with a hand atomizer and were thrown by steam atomizers into large rooms occupied by many soldiers at one time. In some camps the gas house where the soldier first familiarized himself with the necessity for wearing a mask, was employed with highly diluted vapors of chlorin to clear the throat of meningococci. Notwithstanding the many experiments resorted to for this purpose nothing definite has come out of them, and from a fairly large experience and observation with these procedures during the War we are inclined to the conclusion that there was no demonstration of their value. When the 
number of carriers in an organization approaches the danger line troops should be spread out. Contacts who are found to be carriers should be segregated; but crowding among these should be avoided. Carriers in health should be moderately drilled in the open air, supplied with sufficient bedding, and quartered in barracks the temperature of which should never rise above $65^{\circ} \mathrm{F}$, and preferably not above $62^{\circ} \mathrm{F}$. It will be understood that soldiers occupying such quarters will need extra garments during the day and extra bedding at night. There is one thing that clears up the carrier, and that is life in uncontaminated, not overheated, air.

Antimeningococcal Serum.-About the same time (1905) Flexner in this country and Jochmann in Germany prepared a specific serum for the treatment of cerebrospinal meningitis. During the War this serum was prepared in large quantities by the Rockefeller Institute in this country, by the Lister Institute in England, and by the Pasteur Institute in France. The serum is made by injecting intravenously into horses increased doses, first of cultures of the meningococcus killed by heat and later of living cultures. Immunization of the horse is a slow process and requires about six months in order to furnish an efficient preparation. When a horse is once immunized the animal continues to supply a reliable product for as long as six years, possibly longer. Some preparations are made by the injection of polyvalent cultures, while special sera are prepared by the employment singly of each of Gordon's four strains. The preservative used for the most part is tricresol, which is added to the extent of from 0.15 to 0.2 per cent. Thus prepared, the serum is fairly stable and can be used for weeks and months after its preparation.

When lumbar puncture is resorted to for the removal of the fluid the serum is injected directly into the cavity and without the withdrawal of the needle. The removal of portions of the fluid relieves the tension within the cavity and consequently mitigates some of the distressing symptoms, especially the headache; while the introduction of the specific serum directly into the tissues involved in the disease aids greatly in the cure. In pre-serum days the case mortality from this disease ran in different countries at different times from about fifty to as high as eighty and even ninety per cent. Since the resort to specific serum the mortality has been greatly decreased, depending upon the time treatment - is begun. When this occurs within the first three days the mortality has been known to fall as low as eighteen per cent; when first given between the fourth and seventh days, about twenty-seven per cent; when not resorted to until after the seventh day the fatality averages about 36.5 per cent. It will be understood that the fluid withdrawn from the spinal cavity in this disease is highly infectious and those who take part in the procedure, as well as those who handle the fluid in later bacterio- 
logic studies, should exercise all the precautions known to one skilled in this line of work.

The specific serum, employed in the manner indicated, apparently has a direct action upon the meningococcus. This is shown by the rapidity with which the number of microorganisms in the fluid is reduced, by alterations in the size and staining property of the cocci, and by the fact that phagocytic action is more or less increased. The fluid after injection becomes less turbid and contains a smaller number of pus cells and ultimately returns to a normal, limpid state. Flexner believes that the serum possesses bacteriologic, opsonic, and antiendotoxic properties. After studying 1,300 eases of the disease treated with the specific serum, Flexner states his conclusions as follows:

"It is our belief that the analyses of histories of eases of epidemic meningitis which have been presented, furnish convineing proof that the antimeningococeic serum, when lised by the subdural method of injection, in suitable doses and at proper intervals, is capable of reducing the period of illness; of preventing, in large measure, the chronic lesions and types of the infection; of bringing about complete restoration to health, in all but a very small number of the recovered, thus lessening the serious, deforming, and permanent consequenees of meningitis; and of greatly diminishing the fatalities due to the disease."

\section{OTHER FORMS OF MENINGITIS}

Tuberculous Meningitis.-It is important that we recognize the fact that meningitis may be caused not only by the meningococcus, but by other organisms as well. In all eases of meningitis the causative agent can be determined, sometimes with absolute certainty and in the remaining instances with great probability. This can be done by lumbar puncture and a bacteriologic and microscopic examination of the fluid thus obtained. It is highly desirable that this be done in all cases, especially in sporadic cases. Quite evidently, no good ean be accomplished by injecting antimeningococcal serum into the spinal canal when the meningitis is due to some other cause. In most cases of tuberculous meningitis the presence of tuberculosis in some other part of the body is easily recognized; in fact, is already known before meningeal symptoms develop. This, however, is not always the case. Generally there is tuberculosis in some other part of the body, but it may be so limited and so inaccessible on examination that it is not detectable. If the meningococcus is not found in the withdrawn cerebrospinal fluid, careful examination should. be made not only for the tubercle bacillus, but for other microorganisms. In cases of tuberculous meningitis the tubercle bacillus may be found as a rule on careful examination, and as a rule, there is no other microorganism present. This was found to. be true in 25 cases studied by Elser and Huntoon; in 44 examined by Libman; in more than 200 studied by Sophian, and in 20 reported by Worster-Drought and Kennedy. 
Tuberculous meningitis is by no means unknown among soldiers; indeed, the following figures taken from Netter and Debré, showing the distribution of different forms of meningitis among French soldiers from 1882 to 1908, are surprising in the large number of eases of tuberculous meningitis:

TABLE LXXII

\begin{tabular}{lccccc}
\hline \hline YEAR & $\begin{array}{c}\text { CEREBROSPINAL } \\
\text { CASES }\end{array}$ & $\begin{array}{c}\text { MENINGITIS } \\
\text { DEATHS }\end{array}$ & $\begin{array}{c}\text { SIMPLE } \\
\text { CASES }\end{array}$ & $\begin{array}{c}\text { MENINGITIS } \\
\text { DEATHS }\end{array}$ & $\begin{array}{c}\text { TUBERCULOUS MENINGITIS } \\
\text { DEATHS }\end{array}$ \\
\hline $1882-87$ & $q$ & 9 & $q$ & 116 & $?$ \\
$1888-92$ & 30 & 21 & $q$ & 54 & 64 \\
$1893-97$ & 30 & 21 & $q$ & 44 & 94 \\
1898 & 25 & 14 & $q$ & 32 & 109 \\
1899 & 28 & 20 & $q$ & 40 & 87 \\
1900 & 48 & 28 & 8 & 55 & 103 \\
1901 & 42 & 28 & 66 & 41 & 109 \\
1902 & 48 & 33 & 62 & 40 & 87 \\
1903 & 46 & 24 & 46 & 32 & 76 \\
1904 & 33 & 20 & 38 & 33 & 69 \\
1905 & 77 & 47 & 39 & 37 & 77 \\
1906 & 111 & 57 & 40 & 39 & 108 \\
1907 & 108 & 39 & 51 & 26 & 112 \\
1908 & 111 & 59 & 72 & 46 & \\
\hline
\end{tabular}

Pneumococcic Meningitis.-Meningitis occurs in about two per cent of cases of pneumococcic pneumonia. It may also occur as a secondary infection in otitis media and sinusitis, and with still greater frequency it appears as a primary disease. On examination of the spinal fluid gram-positive diplococci are found. There are cases reported in which both the meningococcus and the pneumococcus are found. In instances of this kind one infection is probably secondary to the other and it is generally believed that the pneumococcic invasion is the secondary one. In 1917 Netter and Salanier reported 300 cases of cerebrospinal fever in four of which there was a secondary pneumococcal invasion of the meninges. Pneumococcic meningitis is believed to be always fatal. In traumatic meningitis a pneumococeus may be the invading organism.

Streptococcic Meningitis.-Rarely, the streptococcus becomes a secondary infecting agent in cerebrospinal fever. Rolleston found two instances of this in 247 cases of cerebrospinal meningitis developing in the British Navy during the three years following August, 1914. He believes that one of these was due to otitis media, while the other resulted from direct infection on lumbar puncture. The possibility of introducing such an organism as the streptococeus while making a lumbar puncture should always be borne in mind and the procedure should be so conducted as to render this impossible. When the fluid drawn at the first 
puncture contains only meningococei while that drawn at a later puncture contains a mixed eulture, it is generally assumed that the secondary organism was introduced in making the puncture and the man who did it receives the blame. In our opinion, this is not always justified, because a secondary infection does occur in some instances where no lumbar puncture has been made and the mixed culture is not demonstrated until autopsy. Before an operator is condemned on the appearance of a secondary infection of the spinal fluid a most eareful search for other possible sources of secondary invasion should be made. The streptococcus may be the primary and sole cause of meningitis. In a study made on cases in which lumbar puncture was not practiced, Councilman and his colleagues report eight cases of streptococcic meningitis and in all of these they believe the infection to be secondary. In four the primary infection was in the ear; in one it appeared as an erysipelas of the face and scalp; in another as an acute endocarditis, and in still another as an alveolar abscess; the last one following a fracture of the base of the skull extending across the cribriform plate of the ethmoid bone.

Anthrax Meningitis.-The fact that this disease is a bacteremia indicates that any part of the tissue of the infected animal may be invaded. A highly inflamed condition of the meninges with hemorrhages throughout the brain may be found after death from anthrax meningitis.

Syphilitic Meningitis.-Syphilitic meningitis when it occurs, which it does rarely in the secondary stage of this disease and simultaneously with a cutaneous eruption, may be mistaken for cerebrospinal fever. The spinal fluid obtained by lumbar puncture is clear; no bacteria are found either in smears or in eultures, and both the blood and cerebrospinal fluid give a Wassermann reaction. It is possible for a syphilitic to develop cerebrospinal meningitis, but in such case the meningococcus will be found in the spinal fluid.

Experimental Cerebrospinal Meningitis.-The first suceessful production of typical meningitis in animals induced by cultures of the meningococeus was reported by Councilman, Mallory and Wright in 1898. A goat received in the spinal canal one cubic centimeter of a bouillon suspension of a pure culture of the meningococcus from an acute case at the Massachusetts Hospital. The injection was made in the afternoon and the animal was found dead at ten o'clock the next mornng and evidently had been dead for some hours. On opening the brain and cord, macroscopically nothing could be seen except intense injection of the meninges of both brain and cord. Microscopically the blood vessels were deeply injected and there was found in the meninges an exudate containing pus cells; "the tissue of the meninges was swollen, the fibers softened and separated, the cells were swollen, and there seemed to be beginning proliferation, although no nuclear figures were found." Experiments 
on goats were repeated without success in Austria and France. In 1906 successful inoculations through the spinal cord in monkeys were reported by Lingelsheim and Leuchs, and about the same time and quite independently Flexner succeeded in reproducing the disease in monkeys by intrathecal injections of cultures of the meningococcus. Since that time many other investigators have confirmed the possibility and, in fact, the relative ease with which this disease may be developed in monkeys when the meningococcus is introduced directly into the brain or into the cord. In the experimentally induced disease the lesions found after death are practically the same as those observed in man. It should be clearly understood that, while these experiments demonstrate that cerebrospinal meningitis may be induced in monkeys and in goats, possibly in other animals, by the introduction of cultures of the meningococcus, it has also been shown that other bacteria introduced in the same way into the same species produce like lesions. Meningitis is, therefore, so far as its pathology is concerned, a disease which may be due to any one of a considerable number of microorganisms; but cerebrospinal meningitis, characterized by its epidemiology, is a specific disease and is due to the meningococcus.

Meningitis Due to Other Organisms.-Undoubtedly, there are many bacteria which are pathogenic to the meninges and if they find their way to these tissues meningitis may result. Among those which have been identified as occurring in meningitis on examination at autopsy, we may mention Pfeiffer's. bacillus, Friedländer's bacillus, Gaertner's bacillus, and Micrococeus flavus. It has been shown by recent investigations that the meninges of different species of animals react differently when inoculated with bacteria. It is more than probable that one or more bacteria, which under ordinary conditions are absolutely without pathogenic action on man, would prove highly infectious and possibly markedly fatal if they should find their way to the meninges. This may occur as a result of fracture of the base of the skull.

\section{Bibliography}

Ames, S.: New Orleans Med. and Surg. Jour., November, 1848.

Arnell, D. R.: American Medical \& Philosophical Register, i.

Baldwin, W. O.: Am. Jour. Med. Se., October, 1866, lii, 321.

Boling, W. M.: New Orleans Med. and Surg. Jour., May, 1847.

Boudin, C. M.: Histoire du Typhus Cérébro-Spinal, Paris, 1854.

Purdon-SAnderson, J.: Report of the Results of an Inquiry into the Epidemics of Cerebrospinal Meningitis prevailing about the Lower Vistula, London, 1865.

Chalmers, A. J., ANd O'Farrell, W. R.: Jour. Trop. Med. and Hygiene, May, 1916, xix, 101, 117.

Councllman, W. T., Mallory, T. B., and Wright, J. H.: Epidemic Cerebrospinal Meningitis and its Relation to other forms of Meningitis; a Report of the State Board of Health of Massachusetts, 1898.

Danielson, L., and Mann, E.: Medical \& Agricultural Register, 1806; Medical Communications to Massachusetts Medical Society, 1809. 
Fish, H.: Transactions of the Physico-Medical Soeiety of New York, 1817.

FleXNER, S.: Jour. Exper. Med., Mareh, 1907, ix, 142.

HIRSCH, A.: Handbook of Geographieal and Historical Pathology, Vol. III, New Sydenham Society, London, 1886.

Love, T. N.: New Orleans Med. and Surg. Jour., July, 1848.

Low, BRUCE: Reports of the Loeal Government Board of Public Health and Medical Subjects, New Series, No. 110, 1916.

MANN, J.: Medical Sketehes of the Campaigns of 1812, '13 and '14, H. Mann \& Co., Dedham, Mass., 1816.

Mathey, C.: Jour. de Méd. Chirurg. \& Pharm., T. xi.

Miner, Thomas: Typhus Syncopalis, Middletown, Conn., 1825.

Murchison, C.: Lancet, 1865 , i, 417.

NetTer, A., and Debré Robert: La Méningite Cérébro-Spinale, Masson et Cie, Paris, 1911.

North, E. A.: A Treatise on a Malignant Epidemic commonly called Spotted Fever, T. \& F. Swords, New York, 1811.

Rolleston, H. D.: Lumleian Leeture, 1919.

Roupelil, G. L.: A Short Treatise on Typhus Fever, Waldie, Philadelphia, 1840.

SAVAGE, T. S.: Med. and Surg. Jour., 1833, viii.

Smith, Southwood: A Treatise on Fever, Carey \& Lee, 1830.

Sophinn, A.: Epidemic Cerebrospinal Meningitis, C. V. Mosby Company, St. Louis, 1913.

Stillé, Alfred : Epidemic Meningitis, Lindsay \& Blakiston, Philadelphia, 1867.

Strong, N.: Medical and Philosophical Register, New Series, 1811, i.

Tourdes, G.: Histoire de L'êpidémic de Méningite-cérébro-spinale observée à Strasbourg en 1840 et 1841, Derivaux, Strasbourg, 1842.

TweEdie, A.: Clinical Illustrations of Fever, Hurst, Chance \& Co., London, 1830.

Vieusseux, C.: Jour de Méd. \& Pharm., xi.

Worster-Drought, C., And Kennedy, A. M.: Cerebrospinal Fever, A. and C. Black, 1919. 


\section{CHAPTER XVIII}

\section{POLIOMYELITIS}

\section{INFANTILE PARALYSIS}

Description.-Poliomyelitis is a systemic, infectious disease due to a specific filtrable virus. So far as is known man is the only carrier and distributor of this disease. It manifests itself in acute form and in some of those who recover in various paralytic manifestations. The virus produces its characteristic lesions in the central nervous system. It may affect every part of the cord, and the symptoms which are manifest depend largely upon the part of the cord involved and the extent of injury done. At one time it was supposed that this virus acts only on the anterior horns, affecting the roots of the motor nerves and leading to motor paralysis. Further researches have shown that, while the anterior horns are frequently and seriously involved, the virus is not limited to these tissues and may affect slightly and temporarily, or more seriously and permanently, every part of the cord. In very rare instances the posterior horns are so seriously affected as to lead to sensitory disturbances and possibly anesthesia. In every fatal case of poliomyelitis brought to autopsy changes have been found in the medulla oblongata, and the action of the virus on the structure and function of this part is responsible for some of the symptoms. It was first pointed out by Redlich that there may be in this disease evidence of inflammatory reaction in the brain. This has been confirmed by subsequent observers, but, as a rule, changes in this organ are minute. The basal part of the brain is most frequently involved and the appearances indicate congestion of the cerebral meninges. Symptoms which are sometimes in evidence, especially spastic paralysis, indicate meningeal inflammation.

It will be understood that there is great variety in the location and intensity in action of the virus in the central nervous system. The process may stop with congestion and edema. Any paralyses resulting from these stages will be temporary.

Acute poliomyelitis most usually attacks those who are in apparent health. Rarely, there is a chill. Fever, accompanied by malaise, is an early manifestation. Headache, stiffness of the muscles of the neck, spontaneous pains in various parts of the body, and general muscular tenderness are common, but in no wise distinetive manifestations. Unfortunately, there is nothing in the early symptomatology of this disease which is even indicative of its specific nature. The first characteristic 
sign is paralysis. It is true that in the majority of instances paralysis is preceded by indefinite, widely variable, symptoms. There may be coryza, cough, lassitude, and every symptom consistent with beginning influenza. In other cases, nausea, vomiting, and purging may suggest some gastric or intestinal disorder. In young children drowsiness is often marked. Mothers state that their children were sleeping too much and on awakening them in the morning they found them with some limb paralyzed. Paralysis generally begins in the lower extremities involving one or both legs. In other cases it begins in the arms, and in still others its first and possibly only evidence is in some muscles of the neck or trunk. The progress of the paralytic development may be upward or downward and when the muscles of respiration are involved death becomes imminent. The involvement of the respiratory muscles may be confined to the intercostals and in these it may be uni- or bilateral. Breathing may be carried on exclusively by the diaphragm. Necessarily, involvement of the respiratory muscles threatens death from asphyxiation.

History.-No doubt infantile paralysis infected the race from its earliest times, but the first medical man to call attention to it as possibly a disease was an Englishman, Underwood, who wrote upon this subject in 1784. We think it will be interesting to ascertain what was said about this disease by this, apparently the earliest, observer and recorder of its symptoms. Underwood wrote a book, entitled, "A Treatise on the Diseases of Children," the first edition of which was published in 1784. We have before us a copy of the fourth edition with the imprint of 1799. The section in which we are interested is entitled, "Debility of the Lower Extremities." Under this caption the author, in part, speaks as follows:

"The disorder intended here is not noticed by any medical writer within the compass of my reading, or is not so deseribed as to aseertain the disease. It is not a common disorder anywhere, I believe, and seems to oecur seldomer in London than in some other parts. Nor am I enough aequainted with it to be fully satisfied, either in regard to the true cause, or seat of the disease, either from my own observation, or that of others, except in the instance of teething or foul bowels; and have not myself had an opportunity of examining the body of any child who has died of this complaint. I shall therefore only deseribe its symptoms, and mention the several means attempted for its cure, in order to induce other practitioners to pay attention to it. If it arises from teething or foul bowels the usual remedies should be employed; and have always effected a cure. But the complaint more commonly seems to arise from debility, and usually attacks children previously redueed by fever, seldom those under one, or more than four or five years old. It is then a chronical complaint, and not attended with any affection of the urinary bladder, nor with pain, fever, nor any manifest disease; so that the first thing observed is a debility of the lower extremities which gradually become more infirm, and after a few weeks are unable to support the body. Children so affeeted have gone off their feet, as it is ealled, or dragged them only very 
feebly, after having been able to walk stoutly for some time. They have become in every respect weak and languid; their head has grown large and their limbs become emaciated, but do not feel cold to the touch, though of deep leaden-blue color from the fingers and toes to the elbows and knees; with the face almost equally discolored, and like that of adults in a fit of asthma. A fall, or such like accident, has sometimes been suspected as the cause of these symptoms, but unsupported by any appearance on the vertebræ or other parts. * * * Nothing has seemed to do any good but irons to the legs, for the support of the limbs, and enabling the patient to walk. At the end of four or five years, some have by this means got better, in proportion as they have acquired general strength."

It seems to be generally agreed by those who have studied the history of poliomyelitis that Underwood was the first to appreciate the fact that this form of paralysis is the result of a disease. In another section of his book he clearly distinguishes between infantile paralysis and other forms of paralysis, especially those due to Pott's disease.

The first German physician, so far as we can learn, to call attention to this disease was Jörg (1816) who briefly described a case; and from this description we make the following abstract: This little girl was born sound although her mother was a sickly woman. When the child was about six weeks of age it suffered from a severe disease due to catching cold, possibly typhus fever. The child was very ill for many weeks and its parents despaired of its recovery. In the meantime the mother was taken sick and the child was transferred to the eare of another family. On the recovery of the mother and return to her of the child she found that it could not use its legs and that its feet had become club feet.

In 1836 another English physician, Badham, observed and recorded cases of infantile paralysis and in his paper he made inquiry concerning the cause of the paralysis and was inclined apparently to attribute it to lesions in the central nervous system. He appealed to the physicians of all lands to observe and report similar eases.

Badham reported four cases of acute poliomyelitis which he observed in children in the same neighborhood within a few days, each child being about two years of age. The symptoms in these cases were practically the same and are quite characteristic of the disease. A child who had been fairly well and playful during the day, although the mother remembered later that it had complained of thirst and showed some unusual drowsiness, was put to bed by its mother without any suspicion that the child was at all ill. On dressing the child the next morning the mother first noticed that the eyes were turned in. Having dressed the child the mother's apprehension was heightened by finding that it could not stand. A physician's advice was immediately sought. "No disturbance of the constitution could be traced from teething, or from torpor of the bowels; nor, on examining the spine attentively, could I detect 
any tenderness on pressure." After a few days the drowsiness disappeared and after a longer time, the eyes recovered their normal position. Some months later the paralyzed limb showed some return to sensibility, but at the same time it was found to be smaller than the other leg. "The exercise of the will over the affected extremity, though entirely abolished at first, has now partially returned, inasmuch as she no longer drags the limb after her, as she at first did, but projects or flings it forward with a jerk, the direction and force of which she seems not to have the slightest power to moderate or control." Badham makes the following observations in reporting these cases:

“(1) The extraordinary youth of the patients is to be noticed. It will be observed that the age in all the above cases eorresponded within a few months. (2) Although each case was either preeeded by or ushered in by some apparent cerebral symptomviz., in two by drowsiness, in the others by an abnormal state of the pupil-yet (3) it is remarkable that in no one instance has the health been in any degree impaired. (4) If the case in which the remarkable strabismus occurred could lead us to expect a cerebral complication, rather than a spinal one, there is other suspicion of congested, oppressed, or irritated brain."

It is evident from these quotations that Badham in 1836 not only saw cases of acute poliomyelitis, but correctly decided that the lesions of this disease, certainly those upon which paralytic symptoms depend, were in the cord and brain.

In 1839 Brück reported instances of infantile paralysis in which all the extremities were involved. In 1840 Heine wrote a monograph on the spinal paralysis of infants. This close observer and deep thinker had charge of what we would now call an "orthopedic institute." Naturally, many of his patients owed the condition which brought them to him to what we now know as poliomyelitis. There is no evidence that Heine ever saw this disease in its acute form and he certainly never made an autopsy. From a careful study of the history of his cases he came to the conclusion that many of them at least, were due to the same cause and that the chief lesion was in the spinal cord. Following the first edition of Heine's monograph there were many contributors to this subject and evidence of the rather wide prevalence of infantile paralysis, especially in France and England, was accumulated in the medical journals of that time.

The first time that attention was called to the existence of infantile paralysis in this country was in the fall of 1841 when Colmer published a short note on the occurrence of ten cases of paralysis in infants within a radius of a few miles in the Parish of West Feliciana, La. Colmer states that all these children were under two years of age and he believed that the cause of paralysis in all was teething. From 1840 until the latter part of the nineteenth century much good work was done from 
time to time in the study of paralytic cases, but the acute form of the disease remained without recognition. French pathologists studied the cord in paralytic cases long after the acute stage had passed. In 1865 Prévost and Vulpian found the anterior horns in the cord in paralytic cases markedly atrophied. About the same time Charcot and Joffroy explained the paralyses by the degenerative changes which they found in the anterior horns, and Duchenne demonstrated the behavior of paralyzed muscles to the faradic eurrent. In this way both pathologists and physiologists came to the conclusion that the lesions of the disease exist in the cord and they confined these lesions to the anterior horns. This was the best they could do with the material at hand. They had no opportunity to study acute cases; in fact, at that time acute poliomyelitis was not recognized. The disease was not known except in the paralytic manifestations possessed by its survivors.

In 1868 Bull, of Sweden, reported an epidemic involving 14 persons, which he had treated under the diagnosis of cerebrospinal meningitis. On reading this report, it is easy to see that Bull made a mistake in his diagnosis and that his patients really suffered from poliomyelitis. While temporary paralysis may occur in cerebrospinal meningitis, the extensive and permanent paralyses reported by Bull can occur only in poliomyelitis. Twelve of Bull's 14 cases were in children between four and ten years, the other two being in a girl of fifteen and a boy of twenty. Five died and the nine survivors were all more or less paralyzed. This epidemic, however, was not regarded as one of poliomyelitis until the acute form of the disease received recognition.

The first recognition of poliomyelitis as an acute infectious disease is attributed to Bergenholtz, a Swedish physician, who described an epidemic in 1881. Since that time epidemics have been reported in nearly every part of the civilized world. We do not think that there is any adequate justification in the claim that acute poliomyelitis is a new disease. As we have stated, before the eighties of the last century the medical world knew poliomyelitis only by the paralytics which the acute disease left. In previous ages there was no reason for believing that cases of infantile paralysis, which must have been in evidence everywhere, owed their infirmity to an acute infectious disease. Mitchell claims to have found positive evidence of infantile paralysis in Egyptian mummies. The paralysis was attributed to teething, convulsions, accident, or to some other indefinite, indefinable, unknowable, cause.

Space will not permit us to list the epidemics that have occurred even in our own country since 1893. Fortunately, such a list has been prepared by the U. S. Public Health Service and by other writers on this subject. We must content ourselves with taking some of the most notable epidemies and gathering from them the mass of facts which have been 
learned concerning the nature of the disease, its epidemic characteristics, and the avenues through which it is transported.

An epidemic in Sweden in 1887 gave Medin opportunity to make a thorough study of acute poliomyelitis and he did this so successfully that the term "Heine-Medin's Disease" has been and is still used as a synonym for infantile paralysis. Heine studied the paralytic form; Medin studied the acute form and showed the connection between the two.

Were we inclined to be disputatious, we could find more than one point for controversy with most authors who have written upon the history of the development of our knowledge of this disease. The literature of infantile paralysis is voluminous and much of it is scattered through little known provincial journals. It would require many months of close application, and library facilities rarely possessed, for one to go over and properly appraise each contribution made to this subject. We do not pretend to have done this, but we find some statements by learned authors that are so grossly at variance with what we read that we feel compelled to make a few remarks along this line. As we read the history, the time from Heine to Medin was by no means a vacuum, as Römer states. Before Heine, both Underwood and Badham had recognized infantile paralysis as the result of a disease and not of accident. Moreover, they and others had correctly located the lesions of the disease in the spine and cord. During the time from Heine to Medin the great and important contributions to the pathology and histology of this disease were made by Rilliet and Barthez, by Prévost, by Charcot, and others. Medin was not the first to recognize the fact that acute poliomyelitis is an infectious disease. Cordier wrote very definitely on this point in describing an epidemic which occurred in Sainte-Foy-l'Aargentière in 1885. In this report Cordier gives a detailed history of 13 acute eases, and in commenting upon them he says that the disease is a malady just as specific as typhoid fever or smallpox. Moreover, he adds that the paralytic cases observed resulted from an epidemic of this disease which was "specific, infectious, and properly speaking, microbial." From Heine to Medin there were many reports of cases of infantile paralysis, but in none of these have we found such clear cut and positive statements, justified by his observations, as those made by Cordier. It is possible that a more thorough search of the literature would reveal other instances of the recognition of infantile paralysis as resulting from an acute infectious disease. Be this as it may, the propriety of designating poliomyelitis as Heine-Medin's Disease might be seriously questioned.

In his now classical paper on an epidemic of infantile paralysis read by Medin at the Tenth International Medical Congress in Berlin in 1890 the author refers to Cordier's paper three times. In the first place, he states that Cordier reported an epidemic of 13 cases in the vicinity of 
Lyon in June and July, 1885, but that Cordier saw none of these cases in the acute stage (which is true) and that his report consisted of notes taken one year later. Medin further states that he had no knowledge of Cordier's paper until a year after the Stockholm epidemic, which Medin described.

The second reference by Medin to Cordier's paper is as follows:

"Infantile paralysis is without doubt a specific infectious disease. As observations during the last ten years have shown it may appear as an epidemic. Cordier believes that the disease is contagious, but upon this point I have come to no positive conclusion although I have twice seen two sisters in the same family come down with the disease at intervals of a few days."

The third reference is as follows:

"Cordier recommends that children sick with this disease should be isolated and that healthy children should be prevented contact with those ill. Whether this be necessary or useful I cannot say.",

We believe it true that Medin was the first to publish an extensive account of the symptoms of acute poliomyelitis, but he certainly was not the first to teach that this disease is infectious and that it does occur in epidemic form.

The Scandinavian Peninsula has long borne the odium of being the birth place and nursery of poliomyelitis. There is, however, nothing that convinces us that this disease has existed more abundantly in Norway and Sweden than in the United States or France. Acute poliomyelitis has been recognized for a longer time and by a larger proportion of the physicians in Norway and Sweden than in any other country and, in our opinion, this accounts for the supposedly wider prevalence of the disease in these lands. Harbitz says that acute poliomyelitis has been recognized in Norway as a distinct disease ever since 1820 and that cases were exhibited in the Medical Society of Christiana in 1842 and again in 1853.

Ager concludes a study of the history of infantile paralysis with the following statements :

"(1) Some form of paralysis of unknown origin was sufficiently common in Asia to have attracted the attention of Boerhaave before 1761. (2) Infantile paralysis appeared in England some time between 1784 and 1799, judging from the writings of Underwood. (3) It did not appear on the continent until 15 or 20 years later. The disease prevailed to some extent among Anglo-Indian children about 1800, if not earlier, and its victims returned to England at least as early as that date."

The Swedish epidemic of 1905 gave Wickman opportunity to make valuable contributions to our knowledge of the pathology and symptomatology of this disease; but we are especially concerned with Wickman's contributions to its epidemiology. In 1899 Leegaard, of Norway, demonstrated that in its epidemic progress poliomyelitis shows a marked ten- 
dency to follow lines of travel. Leegaard came to the conclusion that infantile paralysis is infectious but not contagious. This conclusion was quite justifiable at the time, because it could not be shown, scarcely in any instance, that a person developing the disease had been in recent contact with another in the active stage of the disease. Wickman, reporting on the Swedish epidemic of 1905, in which there were 1,031 cases, was the first to prove that this disease is conveyed from person to person partly by those who have had the arrested form of the disease, never developing paralytic. symptoms, and largely by people apparently in perfect health serving as carriers. So long as it was believed necessary before a disease could be placed in the contagious class that it be shown that every one developing the disease had been in recent contact with an active case, the contagious nature of poliomyelitis could not be demonstrated. In the study of the epidemic of 1905 Wickman showed that the distribution of the cases was irregular. There were groups of four, five, or more, cases scattered here and there. In the intervening regions there was no case or only isolated ones. The infected localities showed no relation to the density of population. The rural districts were largely scourged, while many of the cities were but lightly visited. Wickman went into out of the way places in Sweden and showed how the disease is introduced generally by carriers establishing in the community some center of infection, from which these carriers spread the disease throughout the community. Isolated school houses became centers of infection. The virus was brought to the school house not necessarily by one ill or one who subsequently became ill, but by a carrier. From the school house it was transported in like manner to the homes of the patrons of the school; in short, the recognition of carriers of this virus was an essential step in the study of the epidemiology of the disease. In the 1911 Swedish epidemic Kling, Pettersson and Wernstedt enlarged upon, confirmed, and made more certain the epidemiologic work which Wickman had inaugurated. They demonstrated by experiments upon monkeys that in the bodies of persons dead from acute poliomyelitis the virus of the disease exists not only in the spinal cord, but in the intestines, trachea, mouth, and nose. Specimens from all these localities in the bodies of persons dead from the disease were inoculated into monkeys and out of 32 such experiments 25 , or 78 per cent, were positive.

The presence of the virus in the intestines and in the mouths of persons ill at the time with the disease was demonstrated by like experiments. "We consider ourselves fully justified in drawing the conclusion from the results obtained, that the virus in all probability is always present on the mucous membranes of the nose, mouth, pharynx, and intestine of persons affected by infantile paralysis, during the acute stage of the disease. It is obvious that the knowledge of this fact is of the greatest 
importance in formulating our views regarding the mode of transmission of the disease."

By experiments on monkeys they demonstrated the presence of the specific virus in the secretions from the mouths of three persons who had not been in contact with patients showing definite pareses, but who were suffering from an illness believed to be the abortive form of acute poliomyelitis.

The presence of the virus was demonstrated in the mouths of other members of the family in which a single case of the disease existed. "In six families, the experimental investigations have thus demonstrated the existence of carriers of the virus. They were always found where their presence could be suspected. In the three families where a greater number of healthy persons were examined, several virus-carriers were found in each family, three in each of the families $I$ and $K$ and two in the family M. In each of the families only one typical case with paralysis occurred. One is therefore no doubt justified in assuming that virus-carriers are very common and often in number greatly exceed the clinically positive cases."

The long persistence of the virus in those recovering from the disease is shown by the following:

"From this it will be gathered that the secretion from the mucous membrane of the mouth and intestine of persons who have recovered has had the power of infecting monkeys still several months, in one case 204 days (nearly seven months), after the onset of the illness, giving rise to an experimental poliomyelitis with fatal issue. It cannot very well be denied that a possibility of an infection also of human beings through these secretions must exist. It was only in one case that we did not succeed in demonstrating the presence of the virus after the comparatively short time of 30 days. The subsequent examination of this case at the end of 190 days gave still a negative result. It seems therefore possible that the organism in solitary cases relatively quickly gets rid of the infectious agent or that this at least passes into a nonvirulent stage."

Experiments along this line indicate that, while the virus persists or may persist for many months in the secretions of those recovering from the disease, it rather quickly shows evidence of loss in infecting power. "The experiment shows, that the microbe rather quickly-or after eight to fourteen days-loses its power of causing inflammatory exudations in the inoculated animals. This fact is of very great importance from a practical point of view since it perhaps gives us right to assume that the virus, possibly rather soon after the termination of the acute stage, gets weaker."

The possibility of spreading this disease through infected inanimate objects was tested by Josefson. Handkerchiefs used by children sick with acute poliomyelitis, a piece of fancy work done by these children, a pic- 
ture book handled by them, and flies eaught and handled by the children, were all extracted with physiologic salt solution and this extract injected intraperitoneally and intraneurally into monkeys. It was found that the animals receiving extracts from the handkerchiefs and the fancy work developed the disease, while those receiving extracts from the picture book and the flies did not. Josefson makes the following statement:

"My experiments have thus demonstrated that the virus adheres to lifeless objects and can remain virulent, but I was unable to show this in the case of flies."

\section{Kling, Pettersson and Wernstedt state their conclusions as follows:}

"We have succecded in demonstrating the presence of the microbe of infantile paralysis on the mucous membranes of the mouth, nose, pharynx, trachea, and the intestine of persons who have died of infantile paralysis. We have also done this on the corresponding mucous membranes of typical cases during life, excepting in the ease of the trachea, which has not been subjected to an investigation, and we have further demonstrated its presence in the same places in patients who were only very slightly attacked and presented no characteristic symptoms; some of these later lived in the surroundings of positive eases of infantile paralysis, others again under conditions where, as far as could be ascertained, no intercourse with eases of paralysis had taken place. Finally we have demonstrated the presenee of the virus in persons who were themselves perfectly well but had been in contact with eases of infantile paralysis. There occur as has been pointed out by Wickman, Zappert, Müller, Netter, and others, in times of infantile paralysis a large number of cases that do not show the symptoms hitherto considered as characteristic of this disease. With regard to these the clinical diagnosis cannot with certainty determine the nature of the disease in more than quite a minimal proportion of the eases. To these sourees of infection must further be added the existence of an undoubtedly large number of virus carriers. Since the microbe is known to exist on the above mentioned nucous membranes in persons who have never been ill, it must be presumed that it has got there from outside and in all probability through infectious secretions from other individuals. Under such circumstances there is no reason for doubting this manner of transmission also with regard to those affected by the disease, no matter whether these are typical or atypical cases. The presence of the virus on the mucous membranes of the patients must therefore not necessarily be the result of an excretion from the interior of the organism, but may instead be due to a proliferation of microbes which have been transported to the mucous membrane from outside. That the former, a discharge of microbes, so to speak, may take place in man is very probable, since Landsteiner, Levaditi and Danulesco as well as Levaditi and Danulesco have succeded in demonstrating the existence of the microbe in the nasal mucus of monkeys which had been intracerebrälly infected, but how often and to what extent an excretion of this kind takes place is yet quite unknown.

"According to our experience the virus carriers and the abortive cases in a family may be four or five times as numerous as the eases showing typical symptoms. The abortive cases are naturally, in their turn, surrounded by other abortive cases and virus carriers. Under these conditions it is not surprising that we have up to the present time not succeeded in finding any connection between the different cases. In order to be able to follow the path of infection some traces of it must first be found and it is just this that has hitherto proved impossible. The picture given by Jäger of cerebrospinal meningitis from an epidemiological point of view ean with utility be applied also to the case of infantile paralysis. The former disease has hitherto, according to Jäger, 
presented itself as a mountainous landscape covered by a dense fog, out of which only the peaks have projected far apart, but as the fog disperses the ridges conneeting the peaks also become discernible.

"When we discover methods that admit of an etiological diagnosis instead of the symptomatological, the fog now covering the unknown in infantile paralysis will disappear and allow us to see clearly the mode of infection. Then we shall probably find that infantile paralysis is a common disease of childhood, generally displaying only very slight symptoms, but sometimes acquiring a more virulent character giving rise to disturbances in the nervous system. The same applies also to other infectious diseases. Searlet fever, for instance, in certain epidemies, shows an inclination to cause changes in the kidneys or other organs. We shall then perhaps find the cireumstance that grown-up persons only very seldom are attacked by infantile paralysis explained (as it is in scarlet fever) by the fact that adults have already had the disease in their childhood. Finally we may discover that the reason why greater towns seem to have been relatively spared the disease lies in the fact that the nonmalignant form, which we have hitherto never taken into consideration, is, owing to more lively communications, much more common in the towns than in the country, in this respect resembling a number of other diseases, e. g., measles."

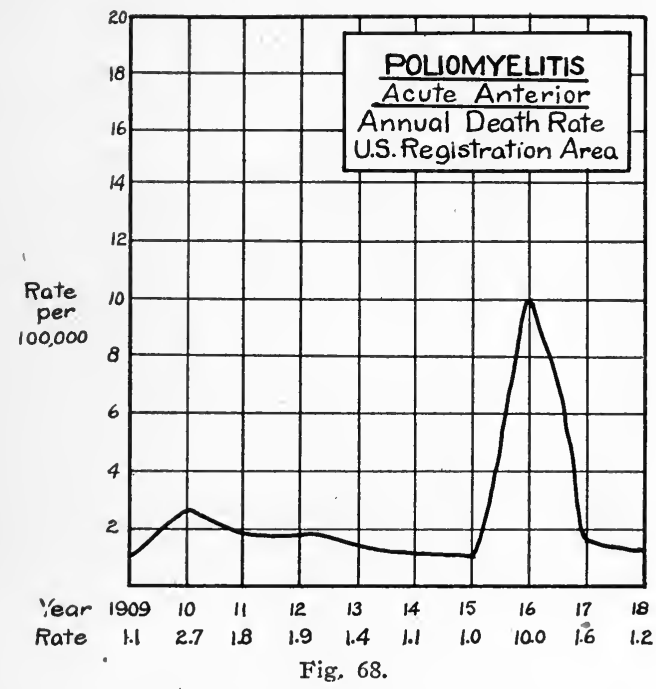

Up to 1907 there are no statistics concerning poliomyelitis in the City of New York. Bogardus has given the following list of eases applying to the New York Orthopedic Hospital for treatment of the sequelae of infantile paralysis during the ten years preceding 1907:

\begin{tabular}{|c|c|c|c|}
\hline YEAR & CASES & YEAR & CASES \\
\hline 1897 & 79 & 1903 & 108 \\
\hline 1898 & 65 & 1904 & 90 \\
\hline 1899 & 78 & 1905 & 82 \\
\hline 1900 & 56 & 1906 & 106 \\
\hline 1901 & 98 & 1907 & 215 \\
\hline 1902 & 110 & & \\
\hline
\end{tabular}


Since all these cases should be regarded as the result of an attack of acute poliomyelitis and since it has been quite satisfactorily determined that the number of nonparalytic cases in an epidemic of the acute disease is about five times the number of paralytic cases, in our opinion the conclusion is justified that acute poliomyelitis was endemic in the City of New York at least from 1897 to 1907 and that the number of cases of the acute disease each year in that city was at least five times the numbers given in the above list. This makes no allowance for deaths in paralytic cases nor recoveries from transient paralyses. Taking these into consideration, we do not think that we are going far wrong when we say that in all probability there existed in the years included in the above list in the City of New York at least ten times as many cases of acute poliomyelitis as is shown by the figures giving the cases of permanent paralysis.

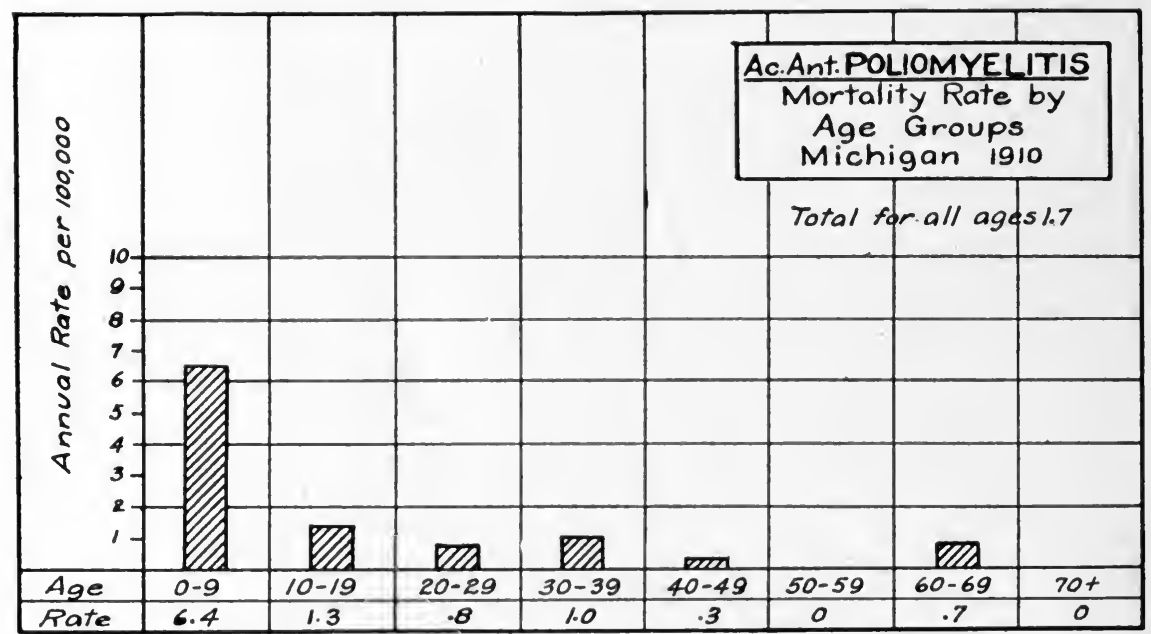

Fig. 69.

We conclude, therefore, that acute poliomyelitis, unrecognized except in paralytic cases, has been endemic and quite widely prevalent in New York City at least since 1897, and there is no reason for believing that it came into existence in that year, because the recognition of chronic paralyses runs back through centuries. Be this as it may, in $1907 \mathrm{New}$ York City experienced its first recognized epidemic of acute poliomyelitis. A committee of medical men appointed to investigate this epidemic secured positive information concerning 800 cases, although they believed that at least 2,500 had occurred in the city during the summer. This apparently was a mild epidemic and the committee estimated the case mortality at five per cent. Compared with the mortality in other cities about the same time, this is low. In our opinion, however, no one knows 
the average case mortality from acute poliomyelitis in any epidemic, because the figure reached will vary greatly with the number of cases classed as abortive and, of course, the larger this number the smaller the death rate. Death rates in epidemies of this disease should be figured in two ways: first, on the total number of cases, including abortive cases; second, on the number of cases showing positive evidence of paralysis. In the 1907 epidemic in a study of 1,762 families in which the disease appeared there were only 18 with two cases in the same family and two with three cases. The committee figured that the period of incubation was approximately from three to seven days. From 1907 to 1915 only sporadic cases of this disease were reported in New York City and during the last three of these years such cases show a gradual decline.

Beginning May 12, 1916, sporadic eases of poliomyelitis were reported in Brooklyn. By the beginning of June there were one or more cases in every borough in Greater New York. During the month of May and the first ten days in June 108 cases of this disease had their onset, but only 15 were reported during this period. From this it is evident that there were 93 centers of infection spreading the disease between May 1

TABLE LXXIII

Cases of Poliomyelitis by Week of Onset

\begin{tabular}{lrrrrrr}
\hline \hline WEEK ENDING & MANHATTAN & THE BRONX & BROOKLYN & QUEENS & RICHMOND & CITY \\
\hline June 3 & 5 & 1 & 25 & 1 & 1 & 33 \\
June 10 & 8 & 0 & 39 & 0 & 2 & 49 \\
June 17 & 6 & 0 & 84 & 1 & 8 & 99 \\
June 24 & 17 & 3 & 204 & 4 & 5 & 233 \\
July 1 & 50 & 8 & 351 & 8 & 28 & 445 \\
July 8 & 118 & 26 & 491 & 52 & 29 & 716 \\
July 15 & 119 & 29 & 466 & 99 & 46 & 759 \\
July 22 & 192 & 54 & 482 & 117 & 54 & 899 \\
July 29 & 305 & 47 & 538 & 145 & 41 & 1076 \\
Aug. 5 & 318 & 82 & 573 & 218 & 15 & 1206 \\
Aug. 12 & 325 & 90 & 345 & 150 & 25 & 935 \\
Aug. 19 & 293 & 85 & 250 & 119 & 12 & 759 \\
Aug. 26 & 226 & 52 & 151 & 72 & 4 & 505 \\
Sept. 2 & 173 & 61 & 87 & 38 & 8 & 367 \\
Sept. 9 & 104 & 34 & 68 & 28 & 4 & 238 \\
Sept. 16 & 91 & 35 & 45 & 20 & 0 & 191 \\
Sept. 23 & 59 & 30 & 31 & 17 & 2 & 139 \\
Sept. 30 & 46 & 23 & 25 & 8 & 3 & 105 \\
Oct. 7 & 38 & 27 & 8 & 13 & 0 & 86 \\
Oct. 14 & 16 & 8 & 10 & 7 & 0 & 41 \\
Oct. 21 & 17 & 7 & 4 & 5 & 0 & 33 \\
Oct. 28 & 14 & 2 & 5 & 3 & 0 & 24 \\
Nov. 4 & 6 & 4 & 1 & 3 & 0 & 14 \\
Nov. 11 & 4 & 0 & 1 & 0 & 0 & 5 \\
Nov. 18 & 3 & 1 & 2 & 0 & 0 & 6 \\
\hline & & & & & &
\end{tabular}


and June 10 which were not reported and which constituted a serious menace to the public health, as no isolation measures were employed.

The spread of the epidemic in the different boroughs is indicated by the accompanying figures (Table LXXIII).

In commenting upon the above table Emerson, then health commissioner of the city, makes the following remarks:

“These figures present several interesting epidemiological points. We have, in each of the five boroughs of the eity, an example of the typical course of the epidemic covering a period of time from four to six months, the number of eases rising steadily until about the middle of the period, when it steadily declines. Under the same conditions of temperature, rainfall, humidity, cloudiness, sunshine, wind, dust, ete., we have the outbreak progressing in one part of the eity and subsiding in another. In the Boroughs of Prooklyn and Queens, the epidemic starting about the same time reaches its maximum in the same week, then begins to decline. In Manhattan and the Bronx also starting near together, it reaches its maximum in the week following the Brooklyn and Queens maximum. In the Borough of Richmond, on the other hand, starting later than the others, it reaches its maximum two weeks earlier."

The percentages of deaths distributed according to week of illness show the following figures: Died during the first week, 1,510 or 81.7 per cent of total deaths; died during the second week, 211 or 11.4 per cent; died during the third week, 60 or 3.2 per eent; died later than the third week, 67 or 3.6 per cent.

From June 1 to November 1 there were reported in Greater New York 8,928 eases of poliomyelitis with 2,407 deaths, a case mortality rate of 26.96 per cent. This is the largest epidemic, both in number of cases and in fatality rate, ever known. The case rate per 1,000 population was distributed among the boroughs as follows: In Manhattan, .94; in the Bronx, 1.16; in Brooklyn, 2.24; in Queens, 3.22; in Richmond, 2.92. The number of persons per acre in the several boroughs, as shown by the census of 1910, was as follows: Manhattan, 176 ; the Bronx, 16; Brooklyn, 42; Queens, 3.5; Richmond, 2.3. It will be seen from these figures that the case rate per 1,000 population was highest in the least densely populated areas.

Every possible effort was made to restrict the disease and to properly care for cases. Physicians were instructed as to the nature of the disease, its early diagnosis and the preventive measures that should be used. Every case was taken to hospital unless the family could meet the following requirements: (1) Secure the daily attendance of a physician. Provide a special attendant who must observe quarantine regulations, do no cooking and avoid contact with other children of the household. (3) Provide a special room for patient and attendant. (4) Screen windows in patient's room. (5) Provide separate toilet. (6) Sterilize everything that left the room occupied by patient and attendant. Disinfect and renovate the room after removal of patient. The final sta- 
tistics show that the death rate among hospital patients was less than among those treated in their homes.

In the few instances in which the family refused to comply with the above given requirements and also refused to consent to the sick person being carried to hospital, transfer was made by the health officials and this was sustained by the Court.

The health commissioner provided a sufficient force of inspectors who could be called upon at any time when the diagnosis was in doubt and when, as it rarely happened, one of these inspectors was denied admission to the house an officer of the law accompanied him and the examination of the patient proceeded. Probably never in the history of an epidemic from any disease were the agencies and officers of a great city so united and so thoroughly cooperative as they were in New York in 1916. An effort was made to ascertain how the disease was spread, a special committee being appointed for this purpose. Members of this committee visited 5,496 homes in which the disease occurred and attempted to learn in what way the infection had been brought into the home. In a group of 250 families in which more than a single child was attacked, no instance was encountered in which the first child affected was less than six months old. In 15 of these families the first child attacked was under two years of age; in 49 , between two and three years of age; in 60 , above three years. From this it is inferred that in the great majority of families the disease was brought into the home by, and first attacked, some child old enough to come ir contact with people outside the home. It is worthy of note that the average interval of time between the onset of the first symptoms and the date upon which the cases were reported to the Department in the 5,496 families was five and one-half days. This means that in the great majority of these instances during the time mentioned there was no efficient protection taken against the spread of the disease. The committee appointed to ascertain the avenues through which the disease was spread summarizes its report as follows:

"First, information of practical daily import was obtained by the physicians and nurses who visited premises in which cases of poliomyelitis occurred and the relatives and friends of the affected families, which was turned over immediately to the Department of Health for its use and guidance. Second, the data thus collected and recorded were subsequently collated and analyzed in the hope that light might be thrown on the important questions of source of infection, period of incubation, types of disease, significance of food, of diseases among domestic animals, insects, and some other subsidiary topics. This second line of inquiry yielded information which led us to regard the disease as one (a) communicated by personal contact, (b) in which the slight and abortive cases are the most frequent sourees of the contagion, and (c) in which the incubation period varies between three and ten days. We were not able to make a study of the question of the healthy carrier, but we think it probable that he plays a less conspicuous part in disseminating the infection than does the mild and often unrecognized case of the disease. We gave especial attention to the working out of the in- 
cubation period on the basis of the data collected. Recognizing the difficulties and fallacies of the undertaking in a large, miseellaneous population, such as exists in Greater New York, we eannot assert that our eonelusion is absolute. We think it probable, however, that taken together with the conclusions of previous investigators, it is virtually correet. We could, finally, find no substantial evidence to support the notion of food, lower animal or insect carriage of the infection, although in regard to those subjects our investigations were incidental rather than essential."

The 8,928 cases were distributed among 8,635 families; in 8,287 of these, or ninety-six per cent, there was only one case. The total children in these families were 24,883 . This shows that in these families there were about 16,500 children intimately exposed to the infection who did not contract the disease. In 313 families, 3.6 per cent of all those involved, there were two cases; in 28 families, or .3 per cent, there were three cases; in 6 families, or .06 per cent, there were four cases. Total children in these three groups were 1,516, while the total cases were 736 . This means that $48+$ per cent of the children exposed in this group of families, while in those in which there was only one case in each, $33+$ per cent, were affected. Commenting upon these facts, Emerson makes the following statement:

"The investigation showed that, in nearly all instances where more than one case occurred in a family, the onsets were so close as to suggest simultaneous infection. Some cases were found to have been attaeked with the disease at a later date, indicating that they were probably secondary infections, but such eases decreased in number, as a rule, with increasing length of time from the first case. Very exceptionally were seeondary cases separated from the primary case by a longer interval than two weeks. Similar results were obtained in the study of multiple cases in one house; the majority of the cases would appear to have had a simultaneous infeetion. * * * Of 1,500 of the total cases in which the matter of previous exposure was studied in detail by a rep. resentative of the Department of Health, it was found that in 29 cases there had been direct exposure to another ease in the same family; in 25 instances the fatal case had been exposed to cases out of the family; in 13 eases a member of the family had visited active cases of poliomyelitis within a week of the onset of the disease in the fatal case."

The health commissioner made a study of the comparative communicability of poliomyelitis with diphtheria, scarlet fever, and measles and reached the following conclusion:

"These figures would seem to indicate that poliomyelitis is certainly no more readily communieable than are diphtheria, scarlet fever and measles, and one might fairly conclude that with immunity to this disease as evident as that recognized in the other so-ealled contagious diseases, in a fixed population, its communicability is decidedly less."

While this disease usually affects only one child in a family, it rarely goes through a large family attacking every child. In this poliomyelitis resembles cerebrospinal meningitis. The largest number of children affected in any one family, so far as we have found in the literature, is seven. This was reported by Pasteur, of Much Hadham, England, who wrote: 
"There are seven children in the family; the eldest eleven years, the youngest eighteen months old. Their home lies on high ground in a healthy neighborhood, and they live in good eircumstances. Their previous health has been exceptionally good. Early in July (1896) the whole seven were attacked in rapid succession within the space of ten days by an acute febrile disorder of two to four days duration, which was eharacterized mainly by headache and general malaise. Within a few days of the onset paralytie symptoms supervened in three out of the seven, whilst in two more there was some temporary disturbance of nervous equilibrium."

In a study of 1,000 cases in Brooklyn it was found that 241 occurred in private houses, 12 in boarding houses, and 747 in tenement houses. Throughout the city it was found that the smallest proportion of cases occurred in the larger tenements of from 30 to 90 apartments. There was observed a tendency for cases to be grouped in city blocks and not to cross the street. "The completed block in a city forms a more or less definite entity, which differs in many respects from a mere aggregation of dwellings. It is completely walled in on its four external sides and usually encloses a large common space which is divided into yards apportioned to the dwellings. Over quite a considerable part of the areas of Manhattan, Brooklyn, and the Bronx where poliomyelitis has been prevalent, the dwellings form entirely, or almost entirely completed blocks of this kind. When districts of this kind are plotted for poliomyelitis, it is seen that the distribution of cases is by no means regular; such could hardly be expected with so few cases occurring over a small area. There is shown, however, quite a distinct tendency for the eases to group themselves more or less definitely in certain blocks while others of similar construction, and supporting a similar population, remain entirely free from the disease. *** It is not easy to put such data upon a statistical basis, but so many groupings like these have occurred that it does not seem possible to ascribe them to chance. It is very evident that they do not correspond with the more common movements of children which would bring them into contact with other children. Such association in playing is more apt to happen between neighbors across the street than it is with those living down the block, around the corner or on the next street. It is also not easy to believe that any human carrier or any flying insects would show the type of movement or migration necessary to produce case distribution of this kind. It is, however, easier to believe that rats, or even domestic cats might easily migrate in this fashion by the way of yards or back fences. It may be mentioned here that dogs are not generally present in the tenement house districts."

An expert entomologist, Brues, studied the possibility of insects playing a part in the distribution of the virus and states his conclusions as follows:

"Some new facts of interest and importance relating to the possible transmission of poliomyelitis by insects have resulted from the present study, but these are not so 
definite or complete as had been hoped. They are also to some extent of apparently conflicting nature, and require at least one unproven assumption to combine them into any working hypothesis. They do not completely disprove the idea that the stable fly (Stomoxys calcitrans) is implicated, although the behavior of the present epidemic does not favor this view. To discard it is to east aside evidence derived from two sets of experiments, however, and it seems very unwise to do this at the present time of uncertainty. As has been shown, it is possible with one assumption to form a working hypothesis based upon rats and fleas which seems to fit the epidemiological observations in general features and in many details as well. The assumption that the rat ean act as a reservoir for the virus of poliomyelitis should be capable of experimental proof, and it would seem that such experiments should be attempted. This by no means precludes the association of some other insect or warm blooded animal, or both. In fact, there is a continual appearance of circumstantial evidence that suggests a population other than the human one, acting as an undereurrent and influeneing the progress of the epidemic. When the many factors so far in doubt are gradually made known, it will be possible to attack the entomological side of the question with better promise of suceess. Among the unknown factors which eause the greatest confusion in interpreting epidemiological evidenee are (1) the length of the period of incubation; (2) the number of mild, abortive or unrecognizable eases and carriers of the virus and their relation to infection and immunity; (3) the duration of infectivity in clinically recognized eases; and (4) the positive knowledge of the presence of the infective agent in insects, domestic animals, or other possible intermediate hosts or reservoirs of the virus."

There are two points which the health commissioner regards as indisputably established by the New York experience of 1916 . He states these as follows: (1) That hospitalization, when prompt and applied to all but moribund eases, probably checks the spread of the disease and determines a lower incidence under similar housing conditions than where removal to hospitals is delayed and many cases are left in their homes. (2) Isolation of groups of children from contact with other children or adults, even when carried out in the midst of areas where the disease is prevalent, suffices to protect such children almost absolutely from infection, in spite of the use of identical water and food supplies, exposure to the same atmospheric conditions and winged insects.

In Manhattan ninety-six per cent of the suitable cases were hospitalized; in Brooklyn only forty-six per cent of such eases were removed from their homes. The death rate per 1,000 population in Manhattan was .94, while in Brooklyn it was 2.24. These facts would be more convincing if the death rates were based on the number of children under five years of age in the two boroughs rather than upon the total population.

So far as the isolation of groups of children is concerned there appears to be no shadow of doubt concerning the conclusion reached by the health comissioner. In New York at the time of the epidemic there were 93 institutions for the permanent care of children with a total census of 21,746 , and 76 for the temporary care of children with a census of 6,365 . As soon as the epidemic condition of the city was 
recognized all these institutions were quarantined and, with the exception of a few instances in which infection undoubtedly occurred before quarantine measures were put into effect, poliomyelitis did not occur in these institutions. There were two other illustrations of the great value of isolation and fortunately these were radically different, one from the other, in their sanitary condition. On Governor's Island at the U. S. Military Post there were between 80 and 90 children living during the entire summer under most sanitary local conditions. Among these children there was not a case of the disease. The other instance is that of Barren Island in Jamaica Bay. On this Island all the city garbage is worked up in the rendering plants. Flies, mosquitoes and other winged insects are abundant; rats are numerous. In 1916 the population was about 1,300 , consisting of negroes, Italians, and Poles of the lowest strata of society. During the summer of 1916 there were on Barren Island 350 children under 16 years of age. There was not a case of poliomyelitis among these. Certainly, we are justified in the face of these facts in concluding (1) that complete isolation from infected areas protects against invasion by the virus of this disease, and (2) that such isolation protects communities from this infection whether the local conditions be sanitary or insanitary.

We have searched the literature of this disease hoping to find some definite statement concerning the proportion of nonparalytic to paralytic cases. We have failed in this search, although Wickman thinks that nonparalytic cases constitute from twenty-five to fifty-six per cent of total cases and he thinks that if all nonparalytic cases could be recognized these figures would be far too low. Müller believes that the nonparalytic in most epidemics outnumbers the paralytic. Up to the present time there is no certainty in the diagnosis until paralysis manifests itself. It is customary, therefore, to include among the nonparalytic eases those in which the paralytic symptoms are transient. Speaking of his experience on this point in New York, Emerson says:

"As early symptoms may be mentioned, fever, vomiting, slight diarrhea or constipation, listlessness, unusual fretfulness or drowsiness. P'erhaps muscular tremors or spinal pain may be present. If carefully observed it is noticed that the child develops slight paralysis of one or more groups of muscles, but instead of continuing, the paralysis disappears within a few hours. Many eases, however, develop no paralysis at all. These cases, nevertheless, are believed to be the causes of infection, and it is obvious that their recognition is of extreme importance in eontrolling the spread of the disease. The diagnosis of poliomyelitis, when paralysis is no longer present or has never been present, may be greatly facilitated by the examination of the spinal fluid and by the use of the biological test for immunity. The spinal fluid, when examined, macroseopically, micro. scopically and chemically gives helpful evidence. The biological or so-called immunity test is less practical or reliable, involving, as it does considerable time, and the not altogether constant performanee of a monkey when virus, even of high virulence is exhibited. If the blood serum of a true ease is mixed with virus of known strength anc 
the combined material inoculated into a monkey, failure to develop the disease in such a monkey is considered presumptive proof that the patient's blood serum contained a substance which neutralized the virus and rendered it inert. If, however, the monkey develops the disease, one cannot positively conclude that the patient has not or has not had poliomyelitis. Both the examination of the spinal fluid and the 'immunity test' leave much to be desired in the way of specificity and eonstancy of results."

From the New York report we take the following statement, which is the most complete that we have been able to find concerning the existence and persistence of paralysis in this disease:

"Of 3,441 cases treated in the four department hospitals, 716 died, giving a mortality of sixteen per cent. Of the eases discharged after treatment, as wholly or partially recovered, 1,223 cases were 'discharged with 'no visible paralysis,' and 2,526 with 'visible paralysis.' In other words, 32.6 per cent of the eases treated showed complete functional recovery, or had not shown paralysis at any time in the course of the discase, while 67.4 per cent showed remaining paralysis in some degree. Of 2,715 cases followed up earefully in the homes it was found that 1,885 had a serious paralysis of one or both lower limbs, and were unable to walk; 530 more were partially paralyzed in in the lower limbs, although still able to walk; 273 had one or both arms totally paralyzed. It should be noted, however, that the average number of days per patient during whieh these eases were under treatment at the hospitals was 32.4 or scarcely more than a month; none was under treatment for two months. It is now known that marked or great improvement may be induced in. paralyzed cases by correct treatment, at a mueh later period than was formerly credited. We have every reason to believe, therefore, that the eases which still remained paralyzed after such a short treatment in the hospitals would, with a prolonged treatment for a period of six months or more, show a larger proportion of complete recoveries of function than here given, and that the deformities, at least, in these cases would be greatly lessened. This belief is all the more reasonable beeause the large majority of the patients treated were under ten years of age-as indeed were most of the cases occurring in the eity-and according to all authorities on the disease (Wiekman, Leegaard, Lovett, et al), age is an important faetor in prognosis; the prognosis as to recovery of function is considerably more favorable in young children than in older children and adults. What has been stated concerning the prognosis of the cases treated in the department hospitals may be considered as approximately true for all eases occurring in the eity in which there was suitable treatment. The results of the 1916 epidemic have taught us many lessons, but one of the most valuable of these relates to the prognosis of poliomyelitis, namely, that though this is undoubtedly a serious epidemic affection, the prognosis of the disease, both as to life and as to function, is by no means so unfavorable as is the case in several other acute infectious diseases with whieh we have been more familiar, but which on this account, we have held in less fear and respect."

We have sought diligently for data concerning crippled children which might give us some idea of the prevalence of poliomyelitis in places and at times where and when the disease has not been recognized. There was a survey made of the State of Massachusetts in 1905 and in this 17,134 persons were enumerated as lame, maimed, and deformed. This gives a ratio of 5.7 for each 1,000 of the population, but there is not the slightest hint as to how many of these crippled people might 
owe their condition to poliomyelitis. In 1915-1916 a similar survey was made of the City of Cleveland and 4,186 crippled persons found. This gives a ratio of six cripples to each 1,000 inhabitants. It is interesting to note the closeness of the ratios found in the State of Massachusetts and in the City of Cleveland. In Cleveland it was estimated by those who made the survey that among all cripples one-eighth and among children more than one-third owed their condition to poliomyelitis. "An analysis of 382 cases of infantile paralysis among crippled children under 15 , by years of occurrence, shows that an average of 27 crippled children a year were added to the group from this one cause during the past 14 years. The greatest number of crippled survivors of any given years are 43 from the 1911 group, 60 from 1912, and 74 from 1915." We think we are justified in concluding from these figures that acute poliomyelitis has for years been prevalent in Cleveland.

In the New York epidemic it was found that a large number of children developing this disease had hypertrophied tonsils or adenoids, or both. Furthermore, it was found that the disease was rare among those whose tonsils had been removed. After the epidemic it was found that among 1,404 children in the public schools whose tonsils had been removed there had not been a case of poliomyelitis, notwithstanding the fact that in 18 instances there had been a case in the same family and in 93 a case in the same house. This is interesting, but we must not jump to the conclusion that the removal of tonsils and adenoids from the throats of children will eradicate poliomyelitis. It is the present belief that the virus of this disease finds port of entry through the nose and throat and in passing these portals it finds lodgment in the local tissue and causes gross disturbances.

So thoroughly was New York cleaned, so carefully were food supplies protected, so constantly was child welfare guarded from every standpoint that, notwithstanding this extensive epidemic and its unusually high fatality, the death rate, both for the first five years of life and for the total population, was lower in the summer of 1916 than it was during the summer of 1915 when there was no epidemic.

Attempts were made to prevent the extension of poliomyelitis from New York City to other parts of the country. The U. S. Public Health Service demanded of children under fifteen years of age who were intending to go to some other state, a certificate signed by an official of the Health Department stating that there had been no case of infantile paralysis in the residence of the child. No attempt was made to regulate in any way the movement of adults. Certain localities in the State of New York demanded that similar certificates be in the possession of those under fifteen years of age coming from New York City. At first there was a considerable exodus of children from the city. If one 
of these ehildren developed poliomyelitis within two weeks after leaving the city it could be brought back, provided it traveled in a private conveyance and was immediately carried to a hospital authorized by the Department of Health to care for cases of poliomyelitis. When a case developed among residents of the city two weeks or more after departure from home the case could not be returned to the city within less than eight weeks, unless it could be shown that proper care and treatment could not be secured at the place of temporary residence. Under this condition the child could be returned to the city if transported in a private conveyance and carried directly to hospital.

There was a notable increase in the number of cases of poliomyelitis reported in many of the large cities of this country during the summer of 1916 and there can scarcely be a reasonable doubt that this increase in number of cases was due to infection imported from the metropolis, though, so far as we know, in no case was this connection demonstrated conclusively. By August 19, poliomyelitis was quite as prevalent in proportion to population in Newark as in New York. While in the intermediate region of Jersey City there was an increase amounting to epidemic proportion, it was not so great as in either New York or Newark. Philadelphia reached the crest of the epidemic wave during the week ending August 26, at which time 132 cases were reported. Baltimore had from ten to twelve times as many cases as Washington. Detroit showed more cases than Cleveland, and Toledo many more than either Detroit or Cleveland. The infection apparently reached Chicago earlier than it did Boston, but it continued longer in the latter. From July 14 to November 10, 1916, there were 254 cases with 34 deaths reported in Chicago, a mortality of thirteen per cent. It may be stated, however, that during the summer and fall of 1917 there were officially reported in Chicago 527 cases with 177 deaths, a mortality slightly in excess of thirty-three per cent. It may be well to make some inquiry concerning the existence of poliomyelitis and the deaths from this disease in Chicago prior to 1916. Although this disease was placed upon the reportable list in 1911, we have no official figures before 1913. Cases and deaths since that time are shown in the following figures:

$\begin{array}{cccccc}\text { YEAR } & \text { CASES } & \text { DEATHS } & \text { YEAR } & \text { CASES } & \text { DEATHS } \\ 1913 & 24 & 4 & 1916 & 254 & 34 \\ 1914 & 53 & 10 & 1917 & 527 & 177 \\ 1915 & 31 & 9 & 1918 & 97 & 27\end{array}$

During the summer of 1916 the chief efforts of the health commissioner of Chicago were directed against the introduction of poliomyelitis, especially from New York, into the eity. Daily reports were received from the Health Department of New York City, giving the names and addresses 
of all new cases. As has been stated, the U. S. Public Health Service demanded certificates of health from children who were leaving the City of New York and bound for points in other states. The Health Commissioner of Chicago was informed of all children coming from New York to Chicago. During the summer the disease appeared in certain localities in the State of Illinois and in adjoining states. These were also reported to the Health Commissioner of Chicago. Health inspectors entered all trains coming into Chicago some miles outside the city and made an inspection, with proper inquiries, of all children on the train. During a part of the season like inspection was carried out on the boats coming into Chicago. Health Commissioner Robertson states that these inspectors during the months of July, August, and September, 1916, examined 38,953 children, of whom 1,199 remained in Chicago and were placed under watch. There is no evidence that any one of these children after reaching Chicago developed the disease. Those who were simply passing through the city were permitted to continue their journey without restriction. Cases developing in Chicago were treated very much as they were in New York. In all instances where the family could not comply with the home requirements as demanded in New York the patient was taken to a hospital and there detained for at least six weeks. Homes in which patients remained were, after recovery or death, disinfected with formaldehyd and received a thorough cleaning, scrubbing, and airing; all articles which had come in contact with the patient were burned or disinfected. The following sanitary measures were recommended to the public: Absolute cleanliness in the homes and premises; thorough screening against flies and mosquitoes; wiping all woodwork with damp cloths; no dry dusting or sweeping; prompt removal of all garbage, manure and other forms of dangerous and offensive refuse; destruction of all kinds of vermin; special attention to bodily cleanliness; keeping children from congregating in playgrounds, movies, and other places of assembly.

In the spring of 1917 the authorities in Chicago passed an ordinance requiring that screens be provided for all doors and windows of houses, tenements or other places of domicile, and on all places where foodstuffs were kept or stored. The health commissioner states that "this is the first fly screen ordinance pertaining to residences or tenements passed by any city council." During the summer of 1917 an effort was made to thoroughly clean Chicago in anticipation of a renewal of the epidemic. The health commissioner states that vacant lots which had been filled with rubbish were cleaned and converted into gardens to the extent of 8,000 acres. As we have already stated, the number of cases in Chicago in 1917 was larger than that of the preceding year. It is the belief of the health commissioner that the sanitary preparation did mueh to keep 
down the number of cases. The report from the department contains the following:

"Chicago's outbreak of infantile paralysis in no way compared in severity and loss of life with that of other cities in the country with much less population. It is confidently believed that the city's clean-up movement, out of which came the city-wide gardening campaign, had much to do with mitigating the severity of the outbreak of the disease in Chicago. It is not intended either to deny or ignore the fact that there were considerations other than those of cleanliness and health that entered into the city gardening campaign. Chief among these was that of reducing the high cost of living by increasing the food supply and to aid in the then nation-wide campaign of food conservation."

Lavinder, Freeman and Frost, of the U. S. Public Health Service, made a valuable study of the extension of poliomyelitis in the summer of 1916 from New York City to the state and to adjoining states. Taking the location in Brooklyn, where the first group of cases occurred, as a center and placing on this radii of $50,100,150,200,250$, and over 250 , miles, they drew circles dividing the territory into concentric zones and studied the extension of poliomyelitis to each of these zones and its prevalence after reaching the locality. They have given us the results of this study in Table LXXIV.

\section{TABLE LXXIV}

Incidence of Poliomyeuitis, By Zones, Northeastern United States 1916

(50-mile zones, with Brooklyn as a center)

\begin{tabular}{r|c|c|c|c|c}
\hline \hline zONES & $\begin{array}{c}\text { Distance From } \\
\text { BRoOKLYN, IN MIles }\end{array}$ & $\begin{array}{c}\text { POPUla- } \\
\text { TION }\end{array}$ & CASES & $\begin{array}{c}\text { RATE PER } \\
100,000\end{array}$ & $\begin{array}{c}\text { DATE OF MID- } \\
\text { DLE CASE }\end{array}$ \\
\cline { 2 - 3 } & Under 50 & $3,296,677$ & 5820 & 176.5 & Aug. 17 \\
II & 50 to 100 & $1,417,160$ & 1296 & 91.5 & Aug. 22 \\
III & 100 to 150 & $1,591,880$ & 1054 & 66.2 & Sept. 12 \\
IV & 150 to 200 & $5,897,338$ & 2591 & 43.9 & Sept. 27 \\
V & 200 to 250 & $1,328,415$ & 384 & 28.9 & Sept. 11 \\
VI & Over 250 & $2,521,808$ & 385 & 15.3 & Sept. 14 \\
\hline
\end{tabular}

They make the following comment on these figures:

"As will be seen from the above, the disease exhibits strikingly, characteristics of wave-like spread seen in its dissemination through New York City. The decreasing incidence with added distance from New York City is quite regular, while the delay in the date of the middle ease, or erest, is quite regular until the last two zones are reached."

Evidently, it was quite necessary for them in this study to take into consideration the lines of travel. They, therefore, cut these zones into sections. Division I included the whole of New England or that section of the Northeastern United States to which the New York, New Haven 
\& Hartford Railroad leads. Division II included the State of New York, to which and through which travel for the most part is served by the New York Central Railroad and minor lines. Division III included New Jersey, Delaware, Maryland, and the District of Columbia. The results obtained in this way are shown in Table LXXV.

\section{TABLE LXXV}

Incidence of Poliomyelitis by Zones Around New York City (Divided According to the Main Lines of Travel Out of New York)

(Rates are per 100,000 population)

\begin{tabular}{|c|c|c|c|c|c|c|}
\hline \multirow{2}{*}{ ZONES } & \multicolumn{2}{|c|}{$\begin{array}{c}\text { DIVISION I-NORTH- } \\
\text { EAST }\end{array}$} & \multicolumn{2}{|c|}{$\begin{array}{c}\text { DIVISION II-NORTH- } \\
\text { WEST }\end{array}$} & \multicolumn{2}{|c|}{ DIVISION III-SOUTII } \\
\hline & $\begin{array}{l}\text { RATE OF } \\
\text { INCIDENCE }\end{array}$ & $\begin{array}{l}\text { DATE OF MID- } \\
\text { DLE CASE }\end{array}$ & $\begin{array}{c}\text { RATE OF } \\
\text { INCIDENCE }\end{array}$ & $\begin{array}{l}\text { DATE OF MID- } \\
\text { DLE CASE }\end{array}$ & $\begin{array}{c}\text { RATE OF } \\
\text { INCIDENCE }\end{array}$ & $\begin{array}{l}\text { DATE OF MID- } \\
\text { DLE CASE }\end{array}$ \\
\hline I & 89.4 & Aug. 16 & 251.5 & Aug. 18 & 163.6 & Aug. 14 \\
\hline II & 64.7 & Aug. 27 & 240.9 & Aug. 15 & 64.9 & Aug. 27 \\
\hline III & 85.0 & Sept. 13 & 45.1 & Aug. 24 & 63.2 & Sept. 17 \\
\hline IV & 41.7 & Sept. 29 & 75.7 & Sept. 3 & 22.5 & Sept. 29 \\
\hline $\mathrm{V}$ & 15.6 & Sept. 23 & 54.9 & Sept. 18 & 9.5 & Sept. 12 \\
\hline VI & 16.3 & Sept. 27 & 14.6 & Aug. 28 & - & - \\
\hline
\end{tabular}

Their comment on these figures is as follows:

"As will be seen from this summary, the progress of the epidemic along these main lines of travel shows in general the characteristics displayed by the tabulation of the zones as a whole, though with somewhat greater irregularity, as would be expected from the smaller units under consideration. The early ineidence in Zone .II, Division II, is in part due to the epidemic in Hudson which eame much earlier than that in the neighborhood. With this exception, the chronology is quite regular until the marginal zones with low rates are reached, when in all zones the chronology becomes irregular.'

The same investigators made a study of cases of poliomyelitis that developed among those who left New York City in the early part of the epidemic. In Massachusetts, Rhode Island, Connecticut, and New Jersey, there were 7,041 cases of poliomyelitis with only 99 among those who had fled from New York City; in other words, those who had come from the City of New York furnished only 1.4 per cent of the total cases that developed in the states above mentioned. Of these 99 cases, 63 occurred in localities in which the disease was already prevailing when the individual from New York manifested the first symptoms. This leaves only 36 localities in which the first case was in a person from the City of New York. They conclude their study on this point with the following statement:

"In general, the foregoing study does not show that the eases actually in persons from New York City were a very large factor in the spread of the epidemic, since such 
cases were the first known souree of infection in only nine per eent of the infeeted towns, and constituted only 1.4 per cent of the total cases which oceurred in the states included. The time of development of eases in persons going out from New York City is quite irregular, but if only those cases are considered which were probably infected prior to leaving the eity, it is scen that in a large proportion of them the disease developed quite definitely before the end of the seventh day after their departure."

During the months of October and November, 1911, there was an epidemic of poliomyelitis in Cincinnati and adjacent cities and villages on both sides of the Ohio River. This epidemic was studied by Frost, who has brought out some important contributions to the epidemiology of this disease. The most interesting is that the oldest patient was twelve and eighty-eight per cent of the cases were under five years of age. Frost calls attention to the fact, previously observed both in this eountry and in Sweden, that epidemics in large cities are eonfined almost exclusively to young children, while those in rural communities embrace a mueh larger number of adults. In the Cineimnati epidemic 97 families furnished 98 cases of the disease; i. e., there was only one family in which two eases occurred. In these 97 families there were 35 children under one year of age and 20 developed the disease; 132 from one to five years with 71 cases; 48 from six to ten years with six eases; 27 from eleven to fifteen years with one case; 243 over fifteen years with no eases.

Nicoll has shown the age distribution both in morbidity and mortality in this disease in New York City, in other cities in the State, and in rural districts. The percentages of cases of over 16 years of age were as follows: New York City, 1.7; up state cities, 7.4; rural districts, 10.0. The percentages of deaths over 16 years of age were distributed as follows: New York City, 3.0; up state cities, 10.2; rural distriets, 19.1. These figures show quite convincingly that both in morbidity and in mortality the higher age groups suffered more severely in small cities and in rural districts than they did in the metropolis. Similar percentages appear in all comparisons between urban and rural epidemies and suggest the probability that in the larger cities the higher age groups have been partly immunized by previous, for the most part unrecognized, epidemics.

The epidemic in New York began about the time of the closing of the schools. The date set for the opening of the schools was September 11. At this time the epidemic, although on the wane, was still furnishing a large number of fresh cases. It was, therefore, a burning question whether or not the opening of the public schools should be delayed. It was delayed until September 25. There was no increase after the opening of the schools. Health Commissioner Emerson says:

"In the public and parochial schools the number of cases occurring after school opened was practically negligible, and, after careful inquiry it was determined that no secondary eases developed as a result of infection among children of school age.", 
In Cincinnati the epidemic appeared during the school term. The great majority of the patients were too young, however, to attend school; in fact, only ten were attending school immediately prior to their illness. These ten children were distributed among nine schools. In 27 families in which the disease oeeurred, other members of the family were attending school when the disease appeared in the household. Quite naturally, these were not permitted to continue in sehool. On this point Frost says:

"In no instance was there any substantial evidence of a case having been contracted at school by contact with another recognized ease. On the other hand, the fact that there were apparently no secondary cases in any of these nine schools with an approximate attendance of 6,000 children, is rather eonvineing evidence that the danger of contracting poliomyelitis by such contact as takes place at school was at most very small, even among ehildren in this epidemic."

We do not think that Frost intends us to understand that in all epidemics of poliomyelitis or in epidemies of this disease in all communities, the danger of transmission of poliomyelitis among sehool chldren should be ignored. In the Cincinnati epidemic, as has been stated, the disease was largely confined to those under school age, and we are inclined to regard this as at least suggestive that the immunity manifest in that epidemic by the older children was due to the fact that a short time before there had been one or more epidemics of this disease in Cincinnati and the older children owed their immunity to having had the disease. Frost found that the ratio of incidence in the Cincinnati epidemic among children under five years of age was thirteen times as great as in children from five to fourteen years. As in other epidemics, the incidence of the disease was smaller in the more densely populated territory; that for Cincinnati being 26 per 1,000; that for Madisonville 77 per 1,000; for Reading, 100.4; for Covington, 73,2. Frost could find no evidence that the transmission of the disease took place through domestic animals or insects.

In the epidemic in Buffalo, N. Y., in 1912, Frost found that out of 274 cases 81.4 per cent occurred in children under five years of age; 13.9 among those between six and ten years; 1.2 between eleven and fifteen; 2.2 between sixteen and twenty; .7 over twenty years. The Buffalo epidemic appeared about the time of the opening of the public schools. Frost, after studying the influence of the opening of the schools on the spread of the disease, makes the following statement:

"The increase after September 3 in the percentage of cases among school children and of families having members at school suggests that the schools, after this date, played an appreciable part in the spread of infection."

Probably acute poliomyelitis has been watehed and studied as closely in Vermont as in any other part of our country. For the most part 
these observations have been earried out by Caverly, of the State Board of Health, who recognized an epidemic of this disease in the summer of 1894. This epidemic prevailed in a population of about 26,000 . There were 132 recognized cases with a mortality rate of eight per cent. In only one family was there more than one case recognized. The cases were for the most part in children under six years of age, these making up sixty-seven per cent of the total. There were nine adults and one, a man of seventy years of age, had a relatively mild type of the disease with paralysis of both legs, which, however, passed off in ten days. The epidemic apparently started in the small eity of Rutland which is the commercial and geographical center of the area involved. In Rutland there were 55 recognized cases, whiie the remainder were scattered over rural districts through 14 towns. This epidemic was not recognized anywhere in the State except in Otter Creek Valley, which lies on the western slope of the Green Mountains. From 1894 to 1910, although isolated cases were recognized each year somewhere in Vermont, there was nothing like an epidemic. In the latter year the disease apparently ascended the Connecticut Valley from Massachusetts. Of the 69 cases reported, 51 occurred on the east side of the Green Mountains. In 1911 there was a small epidemic in which 27 eases were reported, 22 of these being on the west side of the Green Mountains and 19 in the same valley in which the epidemic of 1894 occurred. In 1912 only 13 cases, widely seattered, were found in the State. Of these, eight were on the east side and five on the west side of the Green Mountains. In 1913, 47 cases were observed in the northern part of the State, extending the entire width of the State, but with 40 out of the 47 cases in the northeastern section. Caverly calls attention to the fact that the last cases reported in 1913' were in the town of Barton, only 25 miles from the town of Hardwick which furnished the largest number of eases in 1913. In 1914 there occurred the severest epidemic that the State had ever known. It began in the village of Barton. There were 306 cases recognized and nearly ninety per cent of these oceurred in the northern part of the State, but there were scattered cases all over the State. Although there was, as a rule, but one ease in a family, in one family four brothers were attacked, the days of onset being October 22, October 31, November 1 , and November 2. One case occurred in a person who it was said had the disease 18 years previously. In 1915, 44 scattered eases were found in the State; in 1916, 64; in 1917, 171; in 1918, 7; in 1915, 15.

The history of this disease in Massachusetts runs something as follows: In 1893 Putnam called attention to the fact that it was unusually prevalent in Boston and reported 26 cases. This writer, however, failed to recognize the fact that poliomyelitis was endemic in Boston and he merely called attention to its unusual prevalence in 1893. In 1900 Pain- 
ter reported 32 cases in Gloucester, Mass.; in the summer of 1907 Lovett made a record of 234 cases scattered over the State; in the following year Lovett and Emerson reported 136 cases; in 1909, 923 cases, and in 1910, 843.

In the summer of 1908 there was an extensive epidemic of poliomyelitis in Wisconsin and during the next year or two it spread widely over Minnesota, Iowa, and Nebraska. We do not expect the virus of this disease or of any other disease to have any regard for state or county lines, and this should be known as the epidemic of the northwestern states. It was studied in Wisconsin by Manning; in Minnesota by Hill; in Iowa by Frost, and in Nebraska by Orr, McClanahan, and others.

The disease was recognized and reported in Alabama in 1896 by Bondurant; in San Francisco in the same year by Sherman; in Maine in the same year by Taylor; in Pennsylvania in 1898 by Taylor; in Illinois in 1905 by Norbury; in Florida in 1907 by Efird; in Michigan in 1907 by Griffin and in 1908 by Manwaring; in Iowa in 1908 by Bierring. Since 1908 there have been epidemics in practically every state in the union and in every province in Canada. These epidemics, as well as those in European countries, showed quite uniformly certain characteristics. The disease, instead of involving at one time a large and continuous area, is dotted here and there; one, two or three cases forming a center, and a like manifestation somewhere miles distant. There may be one, two, or three cases in every county in the State, but there are likely to be certain localities in which the incidence of the disease is greater. Taking into consideration only recognized cases, it has been found in the majority of instances impossible to trace any connection between the cases. Another characteristic feature of this disease is that its greatest incidence in proportion to the population is in the small villages and rural communities. It requires but a few cases of recognized poliomyelitis to justify the health officer in proclaiming an epidemic. There may be only one case per 1,000 of population or only one per 2,000 — an incidence which would be negligible in any other communicable disease-and, still, under these conditions, poliomyelitis should be regarded as epidemic. It will be understood that there are epidemics in which the incidence is much higher. In 1916 the incidence in Greater New York was about one in 750 of population and in the more rural Borough of Richmond, one to 450. Another characteristic of an epidemic of poliomyelitis is that after having visited one region even in the scattered way we have indicated, it apparently spares the same neighborhood for a period of from one to four years thereafter. We are practically forced to conclude that the number of persons susceptible to this disease in any community is small. However, in making this statement we should be cautious. It may be-and there are reasons for believing this-that all 
the apparently insusceptible in a given community are really immune and owe their immunity to having had at some previous time an unrecognized attack of the disease. Acute poliomyelitis is either a disease to which only a very small percentage of people are susceptible or it is a disease to which practically all are susceptible, and at the present time, in our opinion, no one has the data upon which a decision on this point can be based. There is no way of positively and certainly recognizing a case of this disease in which paralytic symptoms are not in evidence. In urban epidemics the disease is confined largely to children under five years of age. In rural epidemics it has happened that fifty per cent of the cases were in adults. With these facts before us, the most reasonable explanation that suggests itself is that in urban populations the higher age groups are not involved in epidemics because they have had the disease and are immune. Before the discovery of Reed and his colleagues some of the best physicians in Cuba believed that the natives of that Island never had yellow fever, because it was never seen in the higher ages among the natives of the Island. Now they know that practically all infant Cubans of that time did have yellow fever and consequently the higher age groups were not, properly speaking, insusceptible, but were immune and owed their immunity to infection during infancy. We are much inclined to form a like opinion concerning poliomyelitis. Such a view conforms most satisfactorily with the epidemiologic data we now have concerning the disease. In our opinion, this disease is endemic the world over and shows its moderate epidemic manifestations only when by some accident enough of the population have escaped the disease in infancy to acquire it in later life.

We have already called attention to the fact that in most instances it is impossible to trace any contact, either direct or indirect, between two cases. A child on an isolated farm-a child who has never been a mile from its home-comes down with acute poliomyelitis. The next case may be ten miles away and may occur in a child under conditions of isolation quite as stringent as those prevailing in the first case. In this way it may happen that there may be from one to four or five cases of the disease in every county in the state. How can the distribution of the virus under these conditions be accounted for? To us, there appears only one possibility, and that is that the carrier of the disease is man. The virus must be transported by people in apparent health. Some, in trying to explain the epidemiologic data we have concerning this disease, find it necessary to conclude that domestic animals or insects are the carriers of the virus. In our opinion, there is no dog, cat, or any other domestic animal, no insect, creeping or flying, which travels so widely, so promiscuously, so ubiquitously, as man.

The Virus.-It was shown in 1909 by Landsteiner and Popper, and 
later in the same year by Flexner and Lewis, that the virus of this disease is present in the spinal cord of a child recently dead from it. If a section of such a cord be rubbed up with physiologic salt solution and injected intracerebrally, intrathecally, intraneurally, intraperitoneally, or intravenously into a monkey, the disease may be transmitted to this animal. If emulsions of the diseased cord be filtered through porcelain and the clear filtrate injected into monkeys by any of the avenues just mentioned, the disease develops. It is, therefore, demonstrated that the virus is a filtrable one.

Flexner and his coworkers in 1907 tried to induce poliomyelitis in monkeys by inoculation with spinal fluids obtained by lumbar puncture in this disease, but even when introduced directly into the central nervous system this fluid was without effect. In 1909 they obtained specimens of the spinal cords of two children who had died of this disease. Emulsions of these cords when inserted directly into the brain quite invariably produced typical symptoms, followed by death in many instances, in monkeys. From the monkeys thus killed, sections of the cord were taken and by similar manipulation the disease was passed from animal to animal through an indefinite number of generations, thus demonstrating that the virus in the cord is not a chemical poison, but a living, growing, self-perpetuating thing. The virus remains virulent and retains its virulency in the cord for a long time after removal from the body and under what are apparently adverse conditions. The infected cord may be kept frozen for weeks or it may be permitted to become contaminated with, and over grown by, molds, and even to undergo autolytic changes, and still the contained virus apparently loses nothing in its strength. When the cord is suspended over caustic potash and subjected to drying for many days, after the manner employed in modifying the virus of rabies, it becomes brittle, but the virus loses nothing in its activity.

In 1913 Noguchi and Flexner announced that they had obtained globoid bodies appearing in pairs, short chains, and masses. Cultures of these were obtained from the filtered virus, and monkeys inoculated with the cultures developed the disease. These investigations have not been confirmed, and furthermore, it has not been shown that monkeys can be immunized by these bodies. It is evident, therefore, that the relationship of these globoid bodies to the disease remains as an incomplete problem.

Following the epidemic of 1916 in New York, Rosenow, Mathers, Herzog and Nuzum, and others, did a large amount of bacteriologic work; including animal experimentation, and several preliminary announcements concerning the nature of the organism which causes this disease were made. This also must be considered an unsettled problem. 
We have followed Rosenow's work along this line with great interest and, although his results have not been uniform, he has apparently opened up a line of investigation which promises much.

We can say with certainty that acute poliomyelitis is due to a living virus which, in part at least, passes through the coarser porcelain filters. When injected into monkeys in proper amount and through certain avenues, it induces a disease almost identical in symptomatology and pathology with that observed in man. It should be stated, however, that the behavior of the monkey to this virus is not always clear cut and definite. It is easy to produce lesions in the brain and cord in the rabbit by intrathecal inoculations with cultures of streptococei, but no one has satisfactorily demonstrated that the results thus obtained are identical with the lesions of acute poliomyelitis in man. As we have previously seen, the virus is present in the saliva, nasal secretions, and intestinal excreta of persons suffering from this disease. Whether the virus which has already reached the cord is ever returned to the intestinal tract or to the upper respiratory passages and eliminated, is a question. In one instance, after the disease had been developed in a monkey by intracerebral inoculation, the virus was found in the mouth and nose. This experiment, however, needs to be repeated. There is no instance, so far as we know, in which the disease has been naturally transmitted from one monkey to another, although inoculated monkeys have been allowed to live in the same cage with their normal fellows. On this point Frost says:

"There is no evidence of spread in this manner, and monkeys have been assoeiated by scores with infeeted animals without taking the disease. * * * In one or two instances guinea pigs isolated with monkeys have developed suspicious paralysis that was not definitely ascertained to be poliomyelitis."

Outside the human body the virus has been found to be highly resistant to drying, to low temperatures, and to standard disinfectants. It is readily killed at a temperature of $50^{\circ} \mathrm{C}$. As we have already seen, its presence has been demonstrated on handkerchiefs, pieces of embroidery, and on other similar things used and handled by patients. It has been found in the dust of rooms occupied by ehildren with acute poliomyelitis.

Transmission.-It is generally believed that the virus of this disease is transmitted from one individual to another through the upper respiratory tract. Monkeys have been inoculated successfully by placing the virus in the nasopharynx, especially after slight scarification of the tissue in this locality. There ean scarcely be a doubt that transfer among human beings may occur in the same way, but we are inclined to the opinion that the virus is more frequently introduced into the 
digestive tract. It has been shown that monkeys can be infected through this avenue, but the amount of virus that must be introduced is much larger than that required when the introduction is more direct, as it is intracranially or intrathecally. As we have stated, the virus is found in the sick in the saliva and in the bowels, and in the dead it has been found in the trachea. It might be debated whether the virus seen in these localities during illness found its way to these localities in passing to the spinal cord or in being eliminated from the spinal cord. We are inclined to the former opinion, but, of course, we have no proof bearing upon this matter.

While the mucous membranes of the mouth, pharynx, and nose usually show evidence of inflammatory reaction in those ill with this disease, it cannot be said that the secretions and discharges from these membranes are in any large proportion of cases abnormally large. On this point Regan, who has studied the upper respiratory passages in more than 800 cases, makes the following statement:

"This brings up the point that secretions and discharges from the mucous membranes are not common in poliomyelitis, in fact one may say that while the causative agent of the disease usually excites an inflammatory reaction of mucous membranes, associated with increased blood supply, this inflammation rarely goes on to the stage of exudation. So that while we often have conjunctival infection and redness of the nasal mucosa, as well as of the upper portion of the drum membrane, we find it unusual to have a discharge from any of these mucous surfaces. An exception to this is occasionally observed in the case of the eyes, for we have noticed a slight and transient mucopurulent secretion from the conjunctiva. This has been most constant in bulbar cases."

We are inclined to the opinion that "hand to mouth" infection, so justifiably emphasized by Lynch and Cumming in influenza and pneumonia, has not been sufficiently recognized in the transmission of poliomyelitis. We all know how universal is the tendency among children to taste of everything. Even today, children may be seen taking alternate bites of apples, swapping sticks of candy, and in the school room, even under the eye and without the disapproval of the teacher, children hold pencils in their mouths and these at night are gathered into a common receptacle and distributed promiscuously the next morning. Spoons, forks, plates, in short eating utensils generally, are not adequately disinfected either in homes, boarding houses, restaurants, or hotels. The visitor to the isolated farmhouse is likely to carry something to the home which may bear an infection transmissible by the "hand to mouth" method to the children of the family.

Path of Invasion.-In the experimental production of this discase in monkeys the most certain method and the one requiring the smallest amount of virus is direct inoculation into the brain. The next certain method is injection into some large nerve. The disease may be induced 
in monkeys, as we have already seen, hy application to the nasopharynx, especially when this is preceded by scarification of the local tissue, by introduction into the stomach and by intraperitoneal inoculation. These experimental data, however, do not give us positive information concerning the pathway of invasion in the natural transmission of the disease. Of course, in nature there is no direct inoculation of the central nervous system. The virus introduced from without must travel by way of the blood, the lymph, or along a nerve, to the cord and brain. That the virus is pathogenic to the mucous membrane of the upper respiratory passages, to lymphatic tissue, and to the central nervous tissue, is evident. In abortive or nonparalytic cases it is assumed that the virus does not reach the central nervous system or reaches it in such small amount that the symptoms and lesions indueed in these localities are slight in extent and transient in duration. We are in great need of some reliable diagnostic method for this disease before the central nervous system is touched. The best contribution to this subject is the report made by Regan on the skin and throat manifestations of acute poliomyelitis. So important is the early recognition of this disease, that we are led to give Regan's summary, which is as follows:

"(1) Congestion of the throat is an almost constant symptom of poliomyelitis during the early acute stage of the malady. It is as a rule limited to the faucial mucosa, and the pharynx, while the soft palate assumes a deep red color, and often in addition a distinct violaeeous tinge, but the surface blood vessels of its mueosa are not usually congested to any very noticeable extent. This violaceous tinge which the soft palate assumes varies in degree, and while not by any means typical, it is somewhat distinctive of poliomyelitis, when it is marked. The eapillary congestion of the mucous membrane of the throat in scarlatina is more intense than it is in Heine-Medin's Disease, and involves a mueh more extensive area. In addition to this there is a punctiform rash on the soft palate and the throat is a bright red eolor.

"(2) A mild degree of inflammation of the tonsils is very common; but follicular exudation is very rare, and true membrane formation was never eneountered.

"(3) The uvula has often appeared unusually small for the ages of the patients.

"(4) The buceal mucosa varies only slightly in eolor during the acute stage, and the blood vessels are only occasionally so markedly eongested as in measles and scarlet fever. Rarely a macular rash is seen on its surface and in such eases Koplik's spots may be elosely simulated.

“(5) The tongue is usuaily heavily eovered with a grayish or yellowish-white coating, the edges and the tip being devoid of covering. Frequently the coating is geographieal in its outline. The papillae are not found to be especially prominent or enlarged. The tongue of poliomyelitis differs definitely in its eharaeteristies from that of scarlet fever.

"(6) Gingivitis occurred in a small proportion of the patients. The epithelial debris covering the gums was only rarely as marked as that often seen in measles.

“(7) Rashes oeeurred so frequently, (in approximately ten per eent of the eases), that it would appear they must not be regarded merely as eoineidental, but rather should be classed under the symptomatology of the disease. Eruptions were most fre- 
quent in the younger children. They may appear as early as the second day or as late as the fifth or sixth week. Their average duration is about four days. In distribution, the rashes showed a decided tendency to localize to the trunk, being more prominent on the neck and chest, and to a lesser extent on the back, abdomen, and face. Bulbar eases rarely showed an eruption while meningitic cases were frequently so accompanied. The type most commonly encountered was the pin-point to pin-head papular variety. It was rather frequently preceded by a scarlatiniform eruption. When purely scarlatiniform it may closely resemble that of searlet fever, but not to such an extent that a differentiation cannot be drawn between the two. We rarely encountered a true maculopapular type of eruption, and in the few instances in which this occurred, there was no difficulty in making a differential diagnosis from measles. The pin-head papular rash would seem to resemble most closely, if it is not identical with, that variety of miliaria called miliaria papulosa. It seems probable that the rashes are due either to the sweating, which is so prominent in the disease, or else to the toxemia arising from the infection of the intestinal tract.

“(8) Herpes labialis are very rare in poliomyelitis,-an important point in differential diagnosis from cerebrospinal meningitis, in which they are common. True herpes zoster was not seen in our cases, but herpetic-like lesions were not uncommonly encountered but they were distinct in their characteristics from the true variety.

“(9) Desquamation is rather frequent in poliomyelitis. It is usually furfuraceous, but not infrequently starts as minute pin-point papules which rupture at their summit, and thence involve the neighboring cutaneous surface, and in such cases resemble closely scarlatinal peeling. It is noted earliest and is more pronounced on the neck and chest. Besides true desquamation, there is 'cutaneous exfoliation' which is more common, more persistent and often exceedingly pronounced. It is probably due to a trophic change."

Flexner and his co-workers have made valuable contributions to the pathogenesis of epidemic poliomyelitis in their experiments upon monkeys. They find that the most constant results are secured when the virus is brought directly in contact with the central nervous system by intracerebral and intraspinal injections. Less constant effects followed the injection of the virus into the peripheral nerves and this they explain by the fact that the virus is compelled to travel along the nerve before it reaches the central organs and is therefore subject to some mishap. When an emulsion containing the virus is injected into the blood a large amount is required to cause the disease in the treated animal and even large amounts are not always successful in doing this. Of a given emulsion a few tenths of a cubic centimeter suffice to cause the disease when injected directly into the brain, while the amount necessary to induce the disease when injected into the blood exceeds 50 cubic centimeters and sometimes even 200 c.c. fail. When injected into the blood, the virus soon leaves the circulating fluid and is deposited in certain tissues. These investigators showed that the virus is carried directly along the nerves to the central system. A small quantity of the vitreous humor was aspirated and replaced by an equal amount of an emulsion of a virulent cord. Within a short time the local cloudiness caused by the injection disappears and the vitreous body returns to its normal ap- 
pearance. After a variable period of incubation the animal develops symptoms of the disease and dies.

Flexner and Amoss state:

"Thus the experimental evidence, which is upheld by observations in human cases of poliomyelitis, supports the view that epidemic poliomyelitis is caused by the entrance into the body of its specific microbic cause or virus, through the upper respiratory mucous membrane to the olfactory lobes of the brain, from which by means of the cerebrospinal fluid it is distributed throughout the substance of the nervous organs, but, since the virus may reach the brain by way of any nervous channel, and even, although with great difficulty, from the blood, it is, of course, possible that in exceptional instances other modes of infection may arise."

The Schick Test in Acute Poliomyelitis.-Zingher found in the New York epidemic of 1916 that the Schick test is positive between one and four years of age in thirty-two per cent of normal children; in a slightly larger proportion of cases of measles; in twice as many cases of scarlet fever, and in nearly three times as many cases of poliomyelitis. Experimentation shows that this increased susceptibility to the Schick reaction is not due to temporary loss of the natural antitoxin, but the same condition continues after convalescence from acute poliomyelitis. These observations lead Zingher to conclude that "a susceptibility to one of the less contagious diseases, like poliomyelitis, indicates that the child is more apt to be susceptible to other contagious and infectious diseases." This is an interesting point and will justify further observation and research.

Vaccination.-As early as 1910 Flexner and Lewis demonstrated that monkeys could be immunized against poliomyelitis by repeated subcutaneous injections with increasing amounts of saline suspensions of the unmodified virus. Two monkeys were treated in this way for a period of about two and a half months, and ten days after the last injection these animals received without evident effect intracerebrally 2 c.c. of a virus, one-tenth c.c. of which proved fatal to untreated animals. In more extended experiments carried on later, they found that the production of active immunity in monkeys is uncertain and the results obtained are quite variable. Landsteiner and Levaditi tried drying the cord after the manner of procedure in rabies. Some cords were dried for twenty days, but again the results were variable and uncertain. Some animals thus treated appeared to be immune, while in others no trace of immunity could be detected. Römer and Joseph tried to produce active immunity by intracerebral injections of a virus mixed with the serum of an animal that had recovered from the disease. These attempts to establish active immunity also gave inconstant results. Recently, attempts to induce active immunity in this disease have been made by Abramson and Gerber, who have employed the virus, modified by chemicals and by heat. Fairly 
good results were obtained by five successive inoculations with the virus modified by heat. The first injection consisted of a virus heated to $55^{\circ}$ C. for one-half hour; the second heated to $55^{\circ} \mathrm{C}$. for one-half hour; the third heated to $45^{\circ} \mathrm{C}$. for one-half hour; the fourth heated to $37^{\circ} \mathrm{C}$. for one-half hour. The fifth injection consisted of glycerolated material from recently paralyzed animals and was not submitted to heat. These injections were administered subcutaneously on successive days. Five out of eight animals thus tested showed no effect when inoculated intracerebrally with a virus, one-tenth c.c. of which had killed untreated monkeys. These authors conclude that active immunity can in this way be obtained within five days and that the method would be of value in an epidemic. In our opinion, this matter should receive further study on animals before it is tried on human beings.

Transmission by Insects.-In studying the distribution of poliomyelitis in Massachusetts from 1907 to 1912 Richardson was led to suspect that the common stable fly (Stomoxys calcitrans) plays a part in the spread of this disease. The experimental investigation of this subject was turned over to Rosenau and later to Anderson and Frost. A monkey, after being inoculated with the cord of a child dead from the disease or from another monkey dead of the disease, was placed in a cage and exposed for several hours to the bites of 300 stable flies. The flies remained in the cage with the monkey from the time of inoculation until death. The same flies had an opportunity to feed upon a second monkey from the time of its inoculation until its death. The flies which had fed upon the two inoculated monkeys were subsequently placed in a cage with two fresh monkeys and before the completion of the experiment a third fresh monkey was added. All three monkeys developed symptoms of poliomyelitis. This positive experiment seemed for a time to explain the transmission of the virus in this disease, and at the same time it appeared to be in harmony with the widely held belief that acute poliomyelitis is a rural disease. Repetition of these experiments however, in the hands of the most competent investigators who first made them, have failed, and the general opinion now is that either the control monkeys became inoculated in some unknown way or that the symptoms and pathologic lesions which they displayed, resembled, but were not, those of poliomyelitis. As Robb says:

"After an exhaustive inquiry the stable fly has now left the court without this stain upon its character.",

One experiment apparently incriminated the ordinary bedbug (Cimex lectularius) and made this pest responsible for the transmission of this disease. Repetition of this experiment has also met with failure. Undoubtedly, insects and flies of almost any kind may mechanically trans- 
mit the virus of this disease. The house-fly, if permitted to feed upon expectorated matter or the dejecta from cases of acute poliomyelitis, may carry the infection to the milk or other food of the child, much as the house-fly transports the typhoid bacillus or the tubercle bacillus; but there is no proof that any insect serves as an essential host to the virus of poliomyelitis. Indeed, the epidemic characteristies of this disease which we have already discussed are such as to convince us that man is the chief, if not the sole, distributor of this infection.

Domestic Animals as Carriers of the Virus.-It has happened in the investigation of certain epidemies of poliomyelitis that paralytic diseases among domestic animals have prevailed at the same time and in the same loeality. Much work has been done on this subject. Animals dead of paralytic symptoms have been autopsied in great numbers. Microscopic studies of the cords of many of these animals have been made and in some instances monkeys have been inoculated with emulsions of these eords. As a result of this work it has been found that paralytic disease among domestic animals is very common, but, so far, there is no evidence that there is any relation between the paralytic diseases of domestic animals and acute poliomyelitis. Conway, who has reported on the outbreak of poliomyelitis in Tompkins County, N. Y., in 1916, makes a foreeful aceusation against the eat as a distributor of this disease. This animal might serve this purpose either by carrying the virus on its coat or by the activity of its fleas. There is, however, no experimental evidence as to either of these possibilities. Like flies, domestic animals may mechanically transport material containing the virus of poliomyelitis; in other words, such an animal may serve the purpose, frequently performed by inanimate objects and be considered as fomites, but there is not the slightest evidence that any domestic animal plays an important role as a carrier of this disease. Man wanders far more irregularly and more diversely than any domestic animal. There is no place where man lives that is inaccessible to other men and there is no home however isolated that does not receive an occasional visitor, and it often happens the more isolated the home the more intimate the reception of the visitor.

The Cerebrospinal Fluid in Poliomyelitis.-Whenever there are symptoms indicating inflammatory reaction in the central nervous system, lumbar puneture should be resorted to and the condition of the spinal fluid may be of great importance in making a diagnosis. As a rule, the fluid flows through the needle under pressure, though seldom so marked as that manifest in cerebrospinal meningitis. In poliomyelitis the spinal fluid has usually what is known as the "groundglass" appearance, which is due to a haziness eaused by the large number of suspended lymphocytes. This appearance is best brought out by examining the fluid through a strong ray of transmitted light. This examination is best 
made in a dark room with the tube containing the fluid held against an artificial light. Red blood cells interfere with this test and in all instances microscopic examination should be made and the number of white blood cells should be determined. It is well to make a differential count. It has been observed that when a test tube partly filled with spinal fluid from a case of poliomyelitis is shaken a heavy foam results and holds for some time. This, of course, is due to the unusually large quantity of albumin and globulin in the fluid. A rough estimate of the amount of albumin can be made with the nitric acid ring test, and for the globulin Noguchi's butyric acid test is employed. The spinal fluid in acute poliomyelitis promptly reduces Fehling's solution. The number of cells found on microscobic examination varies greatly but is usually above normal, ranging anywhere from 20 to 1,000 or even more. There is a preponderance of mononuclears in most cases, but occasionally fifty per cent of polymorphonuclears will be found.

Examination of the spinal fluid will lead to proper differentiation between this disease and cerebrospinal meningitis, tuberculous meningitis, pneumococcic and streptococcic meningitis, and in most instances from syphilitic meningitis. The examination of the spinal fluid, notwithstanding its value, can be of little service in the early recognition of the disease. Quite naturally, we can expect nothing distinctive in this fluid until the central nervous system has been reached and quite deeply affected by the specific virus. The examination of the cerebrospinal fluid would not enable us to recognize acute poliomyelitis at a time when the disease is limited to the mucous membranes and to the lymphatic tissue.

Second Attacks.-Both observation during epidemies and experiments upon monkeys seem to show quite conclusively that in the majority of instances one attack of acute poliomyelitis gives permanent immunity. Francis and Moncreiff have collected from the literature fourteen reported cases of two attacks and have made an analysis of these. Apparent relapses in the acute form of this disease, after intervals of a few days are quite common, but these, of course, are not second attacks. The authors just mentioned state that there are but eight instances reported in which a second attack has occurred after an interval of more than four months and in two of these the nature of the second attack seems doubtful. As we have already stated, it was shown by Kling, Pettersson and Wernstedt that the virus of this disease may persist on the mucous membrane for months, and it is possible that second attacks may be due to autoinfection. The number of these, however, is so small that we are justified in saying that in the great majority of instances one attack gives immunity for life.

Control.-Most writers upon poliomyelitis believe that this disease is of relatively recent origin. It is, apparently, much more prevalent than 
formerly. Whether this is more apparent or real we cannot say. The only possible index of value is to be found in the number of cases of infantile paralysis. Have, or have not, these increased in proportion to the number of inhabitants? So far as we know, there is no satisfactory answer to this question. At the conference held in Washington in $\mathbf{1 9 1 6}$ to discuss this disease and its prevention, Stiles made the following statement:

"Have we not in poliomyelitis a disease which is in the making? There are certain strong arguments in favor of this view. Have we possibly in poliomyelitis a disease which is due to some baeterial or other parasite of an insect which is absolutely nonpathogenic for that insect, but which when transmitted to the human being becomes pathogenie? We have eases of this kind. There are some among us who believe rather strongly that there are parasites of insects which are absolutely or relatively nonpathogenic to the insect, but which may be transmitted to man under eertain eonditions and become pathogenic. For instanee, malaria, in all probability, is an aequired disease in man. The biology of the parasite seems to indicate rather strongly that the malaria organism was originally of the coceidial group and nonpathogenie to insects, and that upon being transmitted to man it became pathogenie. Kala-azar is another disease of the same general eategory, a disease due to a protozoan parasite that is apparently nonpathogenie to insects. Transmitted to man, it changes its habits and becomes a pathogenic parasite."

We are by no means convinced that poliomyelitis is a new disease or that it is much more widely prevalent than in former centuries. Some one has said epigrammatically: "Poliomyelitis is common; infantile paralysis is rare." We predict that when the riddle of poliomyelitis is solved, the usual manifestations of this disease will be found to be mild and that its relation to infantile paralysis is similar to that of searlet fever to acute nephritis. Before Underwood wrote, everybody, and after his writing, nearly everybody, believed that infantile paralysis was due to some accident or that it fell upon some child as a chastisement for the wickedness of its family. It is only within recent years that we have looked for and hunted out poliomyelitis. Much of this work has been done by state boards of health, frequently and wisely assisted by the U. S. Public Health Service. Whether this disease has long prevailed or has only recently come into existence, its control, with our present lack of knowledge, will continue a difficult thing. The New York experience quite positively demonstrated the desirability of hospitalization of the sick and the renovation or disinfection of the homes in which the disease has occurred. It seems to us now, as it did at the time, that the way in which this epidemic was handled is above criticism. There was no concealment, the fullest information was given to the public every day, and this resulted in a united effort which, while it probably did nothing to cut short the epidemic of poliomyelitis, did much to reduee the death rate from all causes in the city to a point never before reached. We do not believe that the re- 
striction placed upon those under 16 years of age who desired to leave the city was either wise or beneficial. If man is the sole distributor of the virus of this disease, there is no reason for believing or assuming that the child is more likely to carry the virus than is the adult. Certainly no one can draw an age limit in restrictions of this kind. Children with this disease and children who have been exposed to the disease should be isolated and, of course, not allowed to travel; but we do not believe that the interference with travel under the age limit imposed at that time would be justified should a similar epidemic appear in the same or some other great city.

In 1916 poliomyelitis appeared in a farmhouse in Tompkins County, N. Y., 16 miles south of Ithaca. The first case was in a child who was brought directly from the infected district in Brooklyn and who was ill when it reached the farm. Six other children in the family living on this farm and in intimate contact with the case did not develop the disease, nor did any one in a group of 30 children who dined with the family the second day after the onset of the illness in the child and who were in more or less intimate contact with the child for the greater part of the day. When the first case appeared in Ithaca (a place of about 17,000) an attempt was made to keep at home all children under 16 years of age. This request was issued by the health authorities and was placarded over the county. For over two weeks guards were stationed night and day on all roads leading to and from the city in order to prevent children from coming and going. All cases and all contacts under 16 years of age were quarantined, the former for six weeks and the latter for two weeks. There was no case reported among contacts. Notwithstanding these precautions, for weeks cases continued to develop not only in Ithaca, but on isolated farms in distant parts of the county. Children who had not been off the farm for weeks came down with the disease. So far as could be ascertained there were only two possible carriers of the virus to these isolated farms, - cats and adult men and women, and there is no proof, and hardly the probability, that eats visited all the farms on which cases developed, but it is certain that there was communication between every one of these farms and the infected localities through adult human beings.

Manning suggests that in communities where acute poliomyelitis is known to exist every death should be followed by an autopsy with special examination of the cord and brain. He thinks it likely that in this way we would get information concerning atypical cases. We have no doubt that medical science would be much advanced by this procedure, but we have an idea that most of the nonparalytic cases of acute poliomyelitis recover.

It should not be forgotten that articles soiled by the excretions of the 
sick and those handled by the sick may bear the infection, and all these should be burned or disinfected. It has been definitely demonstrated that individuals who show no manifestations of having the disease themselves may carry the virus in their upper respiratory passages. It has not been experimentally shown, either for or against the possibility of healthy people carrying the virus in or on their elothing. Until we have definite information on this point, in our judgment it would be wise for the health authorities to demand the thorough sterilization of the clothing worn by those who come in contact with active cases. The presenee of the virus in the stools has been demonstrated, and for this reason we reeommend the thorough disinfection of these discharges.

\section{Bibliography}

Badham, John: Paralysis in Childhood, London Med. Gaz., 1836, xvii, 215.

Caverly, Charles S.: Bull. Vermont State Poard of Health, xvi, No. 4.

Cordier, M.: Lyon Med., January, 1888.

Emerson, Haven: A Monograph on the Epidemic of Poliomyelitis in New York City in 1916.

Flexner, Simon, and Amoss, Harold L.: Jour. Exper. Med., 1914, xx, 249.

Flexner, Simon, and Lewis, Paul A.: Jour. Exper-Med., 1910, xii, 227.

Frauentilat, H. W., ANd Manning, J. V.: A Manual of Infantile Paralysis, F. A. Davis Company, Philadelphia, 1914.

Frost, WADE H.: Epidemiologic Studies of Acute Anterior Poliomyelitis, Hygienic Lab., Bull. No. 90, 1913.

Herne, J.: Beobachtungen über Lähmungszustände der untern Extremitäten, Stuttgart, F. H. Köhler, 1840.

Kling, C., Pettersson, A., and Wernstedt, W.: Report from the State Medieal Institute of Sweden to the XV International Congress of Hygiene and Demography, Washington, 1912.

Lavinder, C. H., Freeman, A. W., and Frost, Wade H.: Epidemiologic Studies of Poliomyelitis in New York City and the Northeastern United States during the year 1916, Public Health Bull. No. 91.

Medin, O.: Tenth International Medical Congress, Berlin, 1890.

Robertson, J. D.: Report and Handbook of the Department of Health of Chicago, 1919.

Römer, P. H.: Die Epidemisehe Kinderlähmung, Springer, Berlin, 1911.

Underwood, Michael: A Treatise on the Diseases of Children, Vol. ii, Fourth Edition, J. Mathews, London, 1799.

Wickman, Ivan: Die Akute Poliomyelitis, Springer, Berlin, 1911.

Zingher, A.: Bull No. 52, Department of Health of New York City. 


\section{CHAPTER XIX}

\section{GLANDERS}

Description.-This disease, caused by a specific microorganism, Bacillus mallei, is most frequent in horses, but is transmissible from the nasal cavity of the horse and may be thrown directly into man's face or may be deposited elsewhere and transferred to man indirectly.

History.-It is an interesting fact that early man was more interested in those diseases which killed his animals than in those which killed himself and members of his family. He believed that disease among human beings was due to the wrath of God, but he seems to have had an idea that the spread of disease among his animals might result from other agencies. It is, therefore, not surprising to learn that as early as the fourth century the infective nature of this disease was held by' a few observing men. In the seventeenth century Solleysel taught that glanders is highly infectious, may extend from stall to stall, and that the air may become impregnated with the infection. In the following century certain French veterinarians recommended that diseased horses be isolated, observed, and if found to have glanders should be killed. In the last decennium of the eighteenth century a Danish veterinarian, Viborg, inoculated sound horses with pus, blood, nasal secretions, saliva, urine, and perspiration from glandered animals, and succeeded in transmitting the disease. Moreover, he demonstrated that heat destroys the infective agent. Later, it was shown experimentally that donkeys, goats, sheep, dogs, eats, rabbits, and guinea pigs, are susceptible. These demonstrations led to experiments with the view of disinfecting the discharges and the stalls which had been occupied by diseased animals. Chaveau showed that the infection is carried, for the most part at least, in the corpuscular elements of the discharges. In 1881, Babes and Havas of Roumania, found bacilli in the pus from an abscess on a man who had this disease. Bouchard and others found the same organism, but its isolation and identification are usually credited to the work of Löffler and Schütz (1882). Certain cases of glanders remain difficult of diagnosis because of the organs involved, there being no available discharge. The discovery of mallein by two Russian physicians in 1890 and the demonstration that this is a valuable diagnostic agent, as tuberculin is in tuberculosis, made the recognition of these occult cases possible and has aided greatly in the eradication of the disease. Acute glanders, which is seen both in man and horses, is a rapidly fatal disease. After a short 
incubation period (from two to five days) the temperature becomes very high $\left(106-107^{\circ} \mathrm{F}.\right)$, there is marked prostration and great pain in the joints and limbs. Local ulceration deepens rapidly and boils and abscesses appear on various parts of the body, rapidly destroy skin, subcutaneous tissue and muscle, exposing blood vessels, tendons, and bones. Death usually occurs in from one to three weeks. The most frequent primary lesion is in the nose, though it may occur in the larynx, lungs, or from a wound on any part of the body. In about ninety per cent of cases, the course is chronic and may continue for years. The chronic form in man is by no means always fatal, the percentage of recovery given by Bollinger being as high as fifty; but no one agrees with this estimate.

Bacillus.-Bacillus mallei is a slightly bent rod, with rounded ends, whose length varies from one to two-thirds the diameter of red blood corpuscles. It is sporeless, nonmotile and nonliquefying. It takes the ordinary basic stains, as methylene-blue and gentian-violet, fairly well, but when intensive coloring is desired, it is better to select a stain containing a mordant, such as carbolfuchsin. The stain is easily removed with dilute mineral acid. This bacillus grows on all ordinary culture media without characteristic appearances except on potato. On the second day a typical potato culture shows a hard, yellowish transparent growth. The general appearance, which has been likened to that of a layer of liquid honey, continues from the sixth to the eighth day, when the transparence gradually disappears and the color takes on a reddish tint resembling that of cuprous oxid. The boundaries of the growth show a slightly greenish tint, while the uncovered surface of the potato is a grayish-white. While the growth on potato is quite unusual, it is not sufficiently characteristic to form the sole basis of identification. For a sporeless organism, it may, when protected from the light, retain its vitality for an unusually long time. Wladmiroff states that the organisms from glycerin bouillon tubes, with the ordinary cotton plug, were found viable after four years. Direct sunlight destroys this organism within from a few hours to a few days, depending upon the thickness of the layer and the intensity of the light. Bacillus mallei is rather resistant to mercuric chlorid, but is easily destroyed by phenol (two per cent), zinc chlorid (two per cent), and lime water (saturated solution). The disinfection of stables with gaseous agents is, as a rule, not feasible on account of the openness of barns. Saturated lime water is the best thing to use and should be freely applied with a brush to all stall furniture.

Avenues of Infection.-Many experimenters have tested the possibility of glanders infection through the unbroken skin, and while results are negative so long as the skin remains without a break, a minute lesion, 
enough to offer an atrium to a bacillus, is easily made. Babes and Cornil rubbed an ointment containing the bacilli on the shaved abdomen of guinea pigs and succeeded in infecting them, probably through the hair follicles or some lesion not recognizable. It seems also probable that the unbroken mucous membranes of the nasal cavities exclude the bacilli, but in neither the man nor the horse is this structure likely to remain without microscopic lesions for any great length of time. This is especially true of the horse on account of the character of its food. Beards, husks, and dust are constantly being inhaled while the animal eats, and injury to the mucous membrane of the upper air passages is a frequent occurrence. The mucous membrane of the mouth is scarcely less exposed to slight but frequent insult. That the glanders bacillus may find its way through the intestinal walls, leaving no recognizable lesion, and first manifest itself in the lungs has been demonstrated by the researches of Nocard. Although Löffler reports a case of human infection through milk, the incident is questioned because other sources of infection were not excluded. Under Viborg's efforts, 100 army horses found to be infected, were slaughtered in Copenhagen and their flesh used as food without ill effects. Similar instances on a smaller scale have been reported elsewhere. The possibility of intrauterine infection in case of a diseased mother is recognized, and indeed, such cases have been reported in both France and Italy. Of all domestic and menagerie animals cows and the house-rat are the only ones that are known to be immune to glanders, although others differ much in degree of susceptibility.

Eradication.-The mallein test is easily applied, is a satisfactory diagnostic agent, and renders the early recognition of the disease in the horse easy. It should be applied to all new horses brought into a herd or purchased by the farmer. Glandered horses should be slaughtered. Transmission from an infected horse or man, when the infection is known to exist, is easily preventable. Stables from which glandered horses have been taken should be treated, as has already been recommended, with saturated lime water.

According to Hobday, in Great Britain glanders has decreased almost to the vanishing point. In 1901, 2,370 horses were destroyed on account of glanders in that country and 1,828 of these were found in London. The Veterinary Department of the Ministry of Agriculture shows that during the year 1920 only 15 outbreaks of this disease occurred in the whole of the British Isles, with a total destruction of only 22 animals, and it is well to remark that these occurred after the sale and distribution of more than 150,000 horses and mules gathered together from various countries and employed in the war. Twenty years ago it was a daily occurrence for a veterinarian to be called to one of the municipal stables in London to inspect the horses, and within one year in one stable of 2,000 
horses 90 glandered ones were found. At the present time the use of mallein and its repeated use is compulsory. When an outbreak of this disease is reported in any stable every animal in the stable is quarantined and tested. All ponies sent into the mines and all horses and mules used for special purposes are given the mallein test. During the war special caution was taken to isolate and test all purchased animals. All units were malleined at regular intervals and each individual animal was tested before being sold, even though its destination was the slaughter house and its flesh was to be served as food for man. Hobday claims that in the whole allied forces there was no army whose animals were so free from glanders as the British. 


\section{CHAPTER XX}

\section{WEATHER AND DISEASE}

Discussion.-While living microorganisms are recognized as the causative agents of the respiratory diseases, there are a number of factors which influence the course and severity of an infection. Of these, none is more interesting, both from a scientific and a popular standpoint, than the weather, and in using the term weather we speak broadly, including the extended phases of weather, such as season and climate. The relation of weather to disease has never been well understood, and yet the mystical possibilities of their relationship have fascinated sanitarians of all times. We have but to recall the origin of the word influenza, which the Italians assigned to a disease supposedly caused by a peculiar combination of astral or terrestrial influences. In Short's History of the Air, published in London in 1749, we read that the influenza epidemic of 1510 "was preceded by a large moist air." In referring to the epidemic of 1580, the following comment is offered: "The weather for some years past having been extraordinarily moist, wet and rainy, wind south, at the rising of the Dog Star, came a cold dry north wind. From the middle of August to the end of September raged a malignant epidemic catarrh." Noah Webster was so wedded to the idea that disease was entirely a product of the weather that he wrote a long treatise on the subject and offered it as proof that because of this uncontrollable weather influence, quarantine was quite an unwarrantable and ineffective procedure in stamping out disease. It is not uncommon in the present day to hear people with bad colds remark that they are "under the weather."

We shall make no effort to eatalog the historical references to the association of the atmosphere with respiratory disease. Suffice it to say that works, both ancient and modern, are replete with such comments. Prior to the discovery of bacteriology and protozology it is not strange that weather influences on health should have been so thoroughly believed. Disease was not understood. What is more natural than that disease should be credited to the elements or regarded as a punishment from an angry god?

Within the last few years interest in this problem has been reawakened by the most stimulating and suggestive studies and writings of Huntington. Approaching the subject from the standpoint of a geographer, this author has been greatly impressed by the apparent influence which cli- 


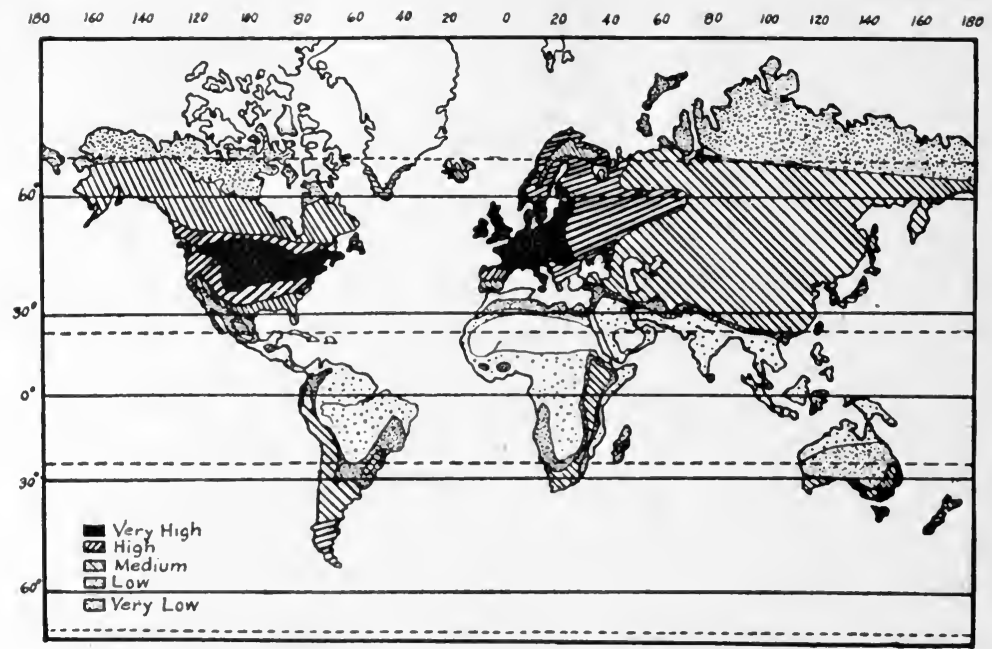

Distribution of Human Enengy on the Basis of Climate

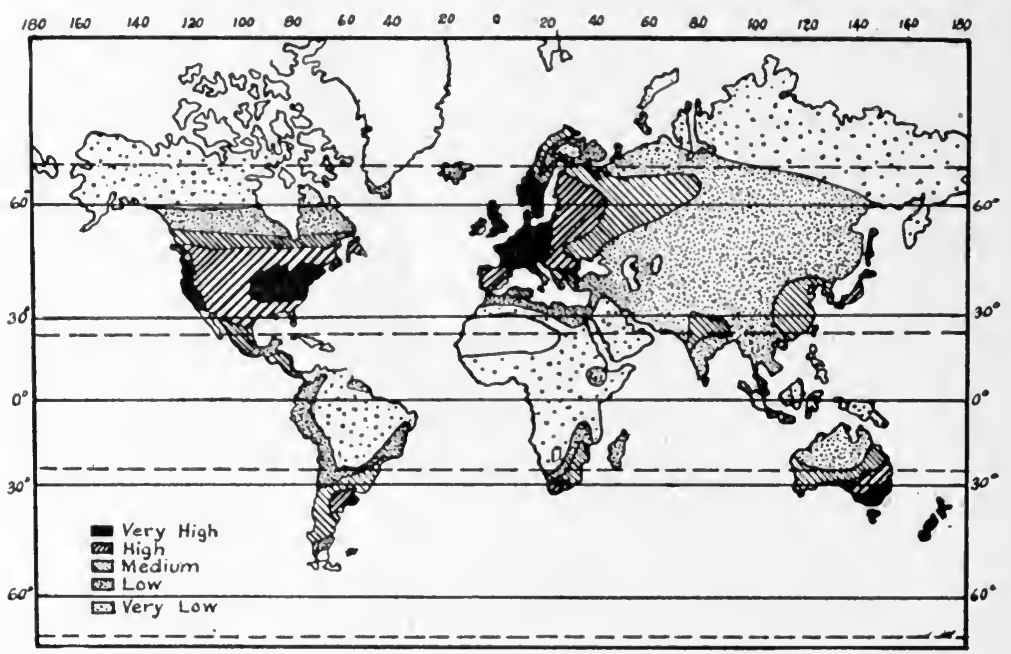

Distribution of Civilization

From Huntington, "Civilization and Climate"

Fig. 70.

mate has played in directing the whole course of civilization. Climate controls human progress through its direct influence on health. The healthiest races have survived; others have fallen behind or perished altogether. Civilization has shown its greatest advance in the temperate zone on the north shore of the Mediterranean and not in the equatorial belt of Africa. From Huntington's work, "Civilization and Climate," we reproduce two maps of the earth, one of which depicts the energizing 
value of different climates, as determined by experimental data, and the other pictures the distribution of civilization. One cannot fail to be struck by a certain similarity in the two maps. Civilization is highest in the areas of optimum climate. Is this a coincidence, or is climate the cause and civilization the effect?

Civilization has shown greatest advancement in the temperate zones. In the north temperate zone we find Europe and North America. There is but little land in the south temperate zone, but what there is, namely, New Zealand, Southern Australia, the tip of Africa and the lower half of South America, rank on a higher plane than the territory to the north within the torrid zone.

The map of human energy is based on the mean temperature, seasonal variation of temperature and the number of storms, the optimum conditions being chosen from the results of numerous mental and physical tests on students and factory workers in eastern United States. The climate which is ideal for the stimulation of human progress is one in which the mean temperature does not fall below a mental optimum of $38^{\circ}$ Fahrenheit or rise above the physical optimum of 60 to $65^{\circ}$. This ideal climate must have variability from, say $19^{\circ} \mathrm{F}$. in the coldest month of the winter to $73^{\circ}$ in the warmest month of the year. A further indication of variability is the cyclonic storms, and Huntington assumes 20 storm centers a year as an optimum condition. Relative humidity is important, the extremes of humidity and dryness being unfavorable. Within these limits moisture is more favorable than dryness.

The map of civilization was prepared from the opinions of 54 geographers, ethnologists, historians, diplomats, colonial officials, travellers and others located all over the world, who independently graded the various countries of the globe on the basis of their progressiveness, leadership and all around ability.

We may call attention to four other maps (Figs. 71 and 72), each of the United States, showing respectively (1) climatic energy (2) mortality reported from insurance experience (3) civilization (4) mortality reported from the army experience. The first three are from Huntington, the fourth from Vaughan and Palmer. The first map is similar to that showing human energy over the world. The most stimulating areas are in the northeast quarter. The third map shows the ranking of sections of the country in civilization, as determined by 23 eminent contributors, all but one, Americans. The second map is drawn from the actuarial experience of three large insurance companies. The figures indicate the actual number of deaths compared with the average of the entire country, which is taken as 100. The fourth map shows the death rate among soldiers from different parts of the country during the winter of 1917-18.

These four measures of human activity are alike in their main features, 
the most favorable section in all instances being in the northeast, the least favored, the south and arid regions of the west. It is of unusual interest that the fourth map, constructed by different delineators quite oblivious to the existence of the other three maps, should bring out so distinctly the greater disease resisting powers of the youth from the colder

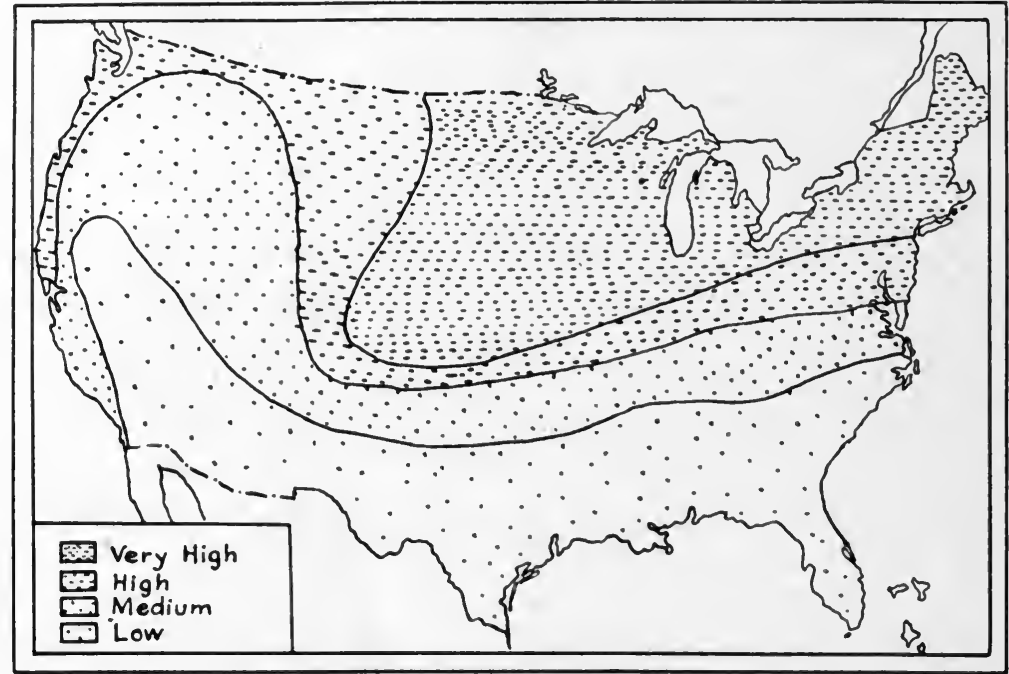

Climatic Energy in the United States.

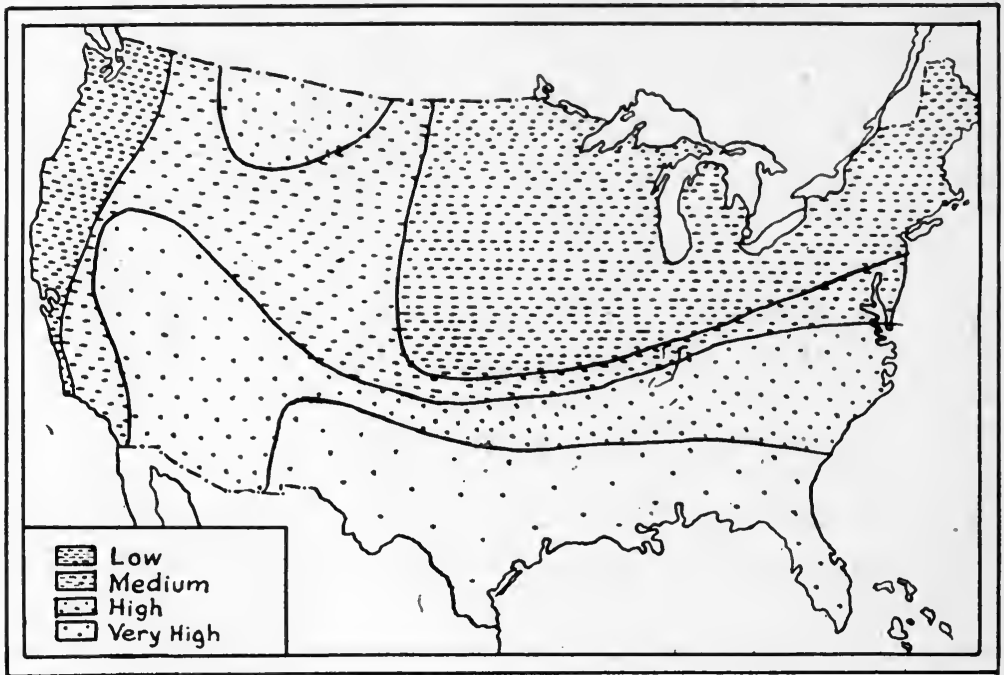

Mortality in the United States

From Huntington, "Civilization and Climate" Fig. 71. 
and more stimulating sections of the country. The existence of a climatic influence on health can scarcely be denied in the face of these facts.

Climate and Disease.-There is no portion of the globe free from dis-

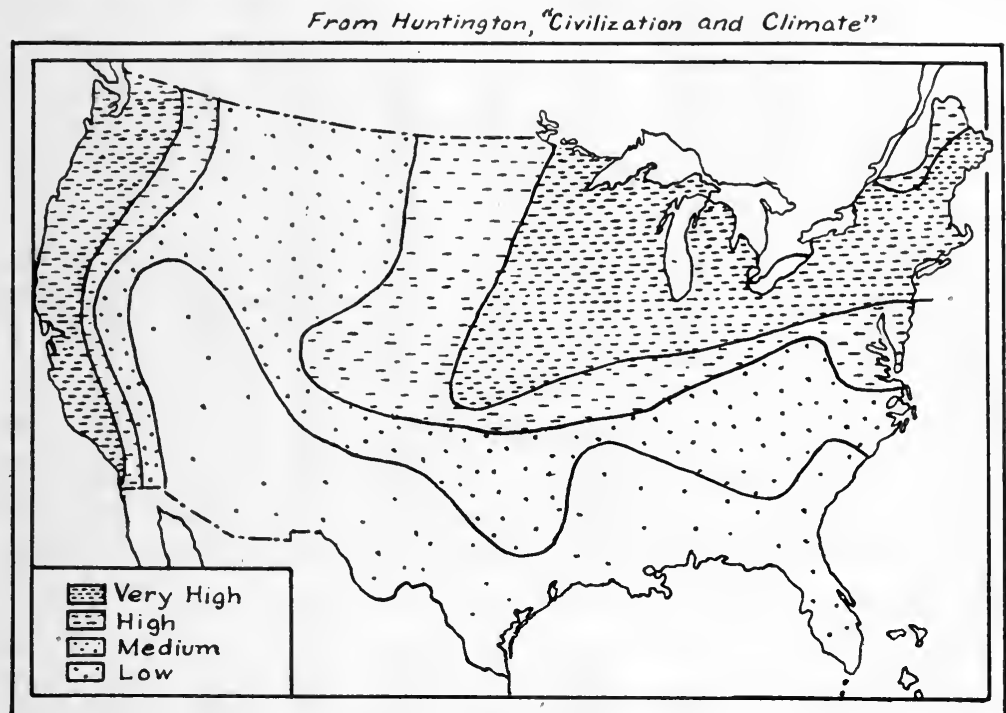

Civilization in the Unifed States

From Vaughan and Palmer

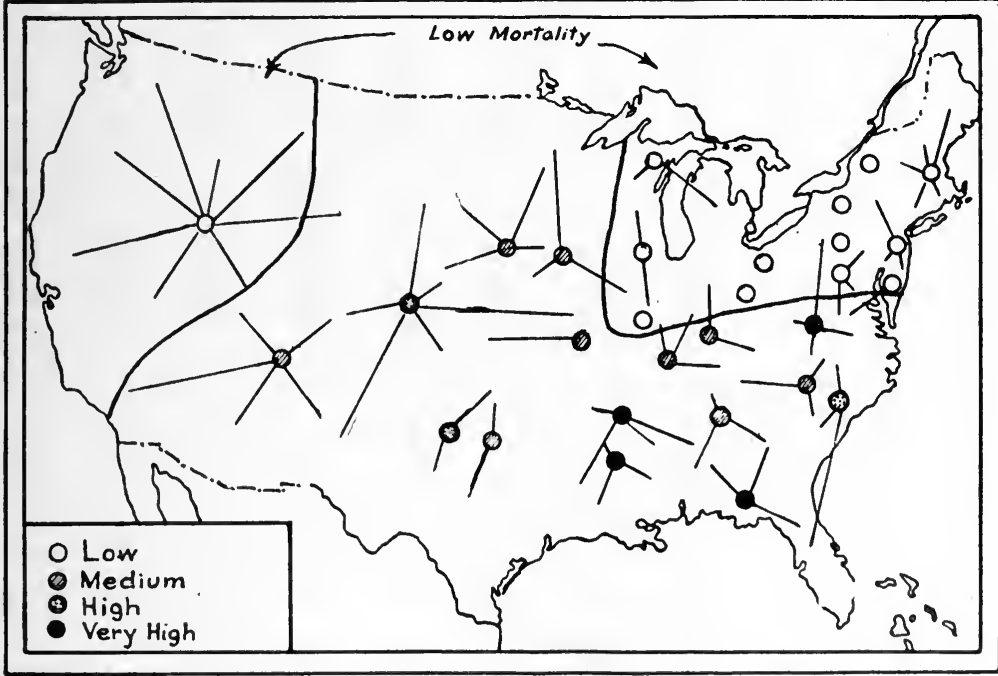

Mortality Among Soldiers in U.S. Army Camps 1917-1918

Note - The circle is located not at the Camp site but in the centre of the district from which the men came.

Fig. 72. 
ease. The type of disease varies, however, with the latitude and longitude. In general it is the diseases of the respiratory tract that flourish in the colder regions, whereas the warmer areas are given more to intestinal and parasitic fevers. Thus malaria was long the scourge of Panama until the heroic military measures of Gorgas made it habitable for the white and black alike. The State of Michigan has extremely little malaria.

Minneapolis has a great deal of scarlet fever; New Orleans very little; and this disease does not even appear in the annual report of the chief health officer of the Panama Canal Zone for 1917.

One may distinguish certain differences in disease prevalence from the death rates in certain American cities of diverse climates. The mean annual temperature of Minneapolis is 44 degrees, Buffalo, 46.4. In ascending order follow Detroit, Boston, Seattle, Kansas City, Atlanta, Los Angeles and finally New Orleans with a mean temperature of 69.1 degrees.

As will be seen in the chart (Fig. 73) pneumonia bears only an indirect relation to the mean temperature. The warm cities, considering the rate for whites only, have less pneumonia than cold cities, but the warmest city does not have the lowest rate, nor does the coldest city have the highest rate. The death rate in Minneapolis, the coldest eity, is only slightly greater than that of Los Angeles, which is next to the warmest eity. Boston and Seattle, with almost identical mean temperatures, have very different pneumonia rates, that for Boston being the highest of all the cities mentioned, while Seattle has the lowest rate. It is the cities of the northeast and central plains region that have the highest rates. The influence of race on pneumonia is well illustrated in Atlanta and New Orleans. Differences in age distribution also affect the rates to some degree. The western cities have a greater proportion of young adults, among whom the death rate is naturally low.

Scarlet fever shows a distinct relation to temperature. With the exception of Seattle this disease is high in the cold cities and almost absent in Atlanta, Los Angeles and New Orleans.

Diphtheria differs materially from scarlet fever in that it prevails in warm as well as cold regions. The death rate in New Orleans is fully as great as in some of the northern cities. Seattle and Los Angeles on the west coast have the lowest rates.

Measles caused nearly as many deaths in the four warmer cities as in the five to the north. Whooping cough is least on the Pacific Coast. Atlanta has as much as Minneapolis.

Malaria and typhoid reveal a marked temperature influence, the reverse of scarlet fever. It is in the warm regions that malaria and typhoid are greatest. In the colder northern cities malaria is almost nonexistent. 

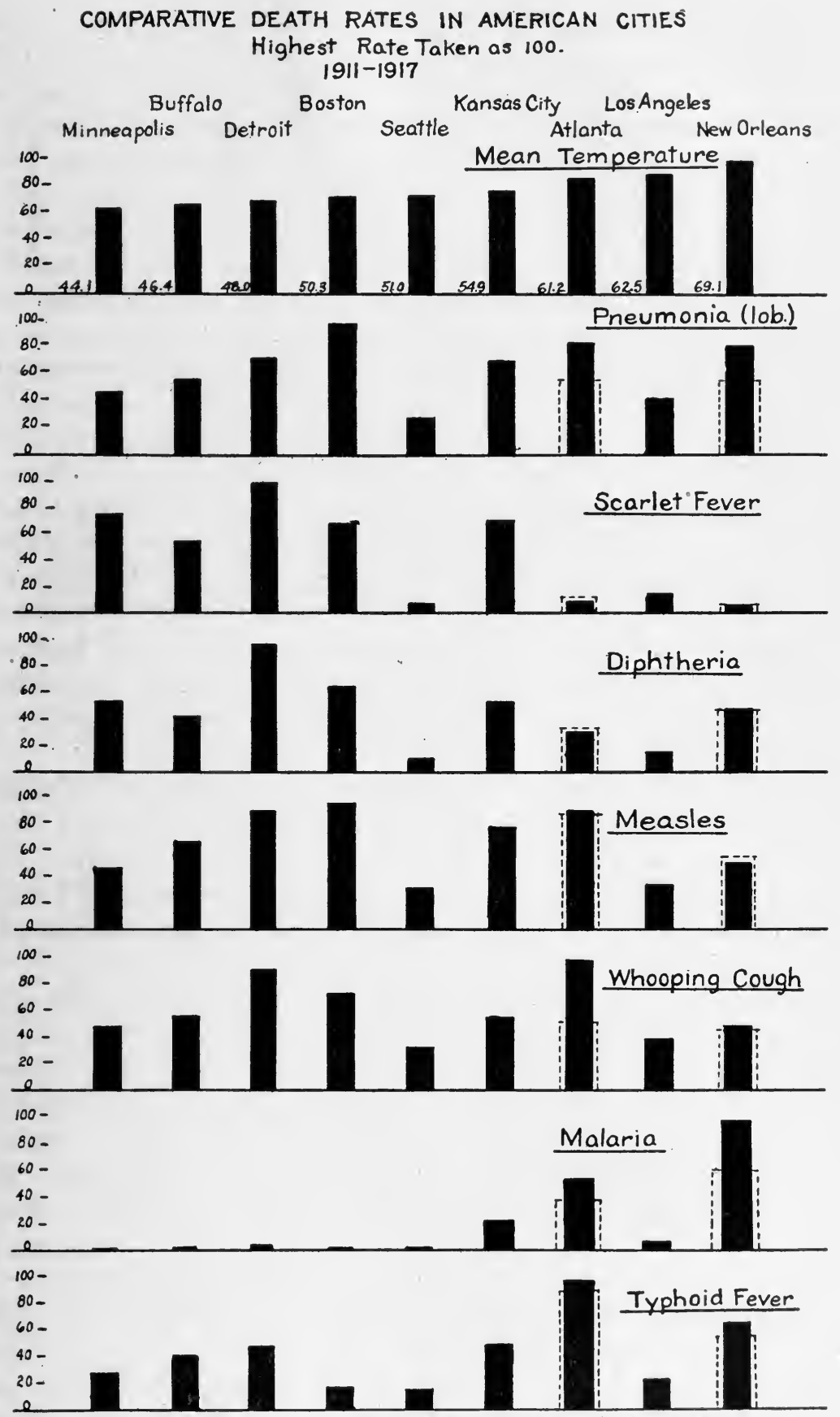

Dash line shows rate for whites alone

Fig. 73. 
The difference between the east and west coast cities is due in part to age differences, as we have mentioned. Thus, in 1910 the age group 20 to 49 represented 60 per cent of Seattle's population, and only 51 per cent of Boston. Los Angeles has many more older people than Seattle, in the first instance 17 per cent of the population being 50 or over, and in the latter but 13 per cent.

Apart from this age difference, however, there is a difference which is unmistakably due to climate, and by climate we mean not only temperature, but fluctuations in temperature, moisture, rainfall, wind action and storms. Two cities with the same mean annual temperature may differ widely in other climatic respects. From 1911 to 1917 inclusive, the mean temperature of Boston was 50.3, or very similar to that of Seattle, which was 51.0. Boston, however, is subject to wide seasonal variations, whereas Seattle is not. The mean temperature for January in.Boston is around the freezing point. In Seattle it is nearer 40. Boston gets hot in midsummer, around 70 in July. Seattle's summer temperature does not rise so high. Boston has its heaviest precipitation in the summer or autumn months. Seattle is unusually arid at this time of year, its heaviest precipitation occurring in December and January. The differences of these two cities in temperature and humidity throughout the year are pictured graphically in Fig. 74, the abscissæ representing humidity and the ordinates temperature.

Recognizing the wide differences that exist in the climates of different localities, there naturally occur several questions. What are the essentials of a healthy climate? Where are the healthiest climates? Does climate exercise a general influence on health, or can a specific climate be antagonistic to one disease and favorable for the propagation of another?

The literature is rich in contradictions on these points. One author states that colds depend for their development "primarily on an excess of moisture in the air we inhale." Another writer states that "catarrhal conditions and bronchitis as a rule are most favorably influenced in a climate of moderate humidity." The truth of the matter is that the answers to these questions are only in the course of framing. Much laborious study must be expended to work out the correlation of these factors. A promising beginning is being made, however, and some day we shall be able to utilize this knowledge for the benefit of mankind.

The Climograph.-Huntington gives us a conception of ideal climate based on experimental observations in his map shown on page 644 . In his later publication, "World Power and Evolution," Huntington analyzes the effect of temperature and humidity on the death rate by means of climographs. Over nine million deaths in the United States, France and Italy have been studied in this manner. The results are, to say 


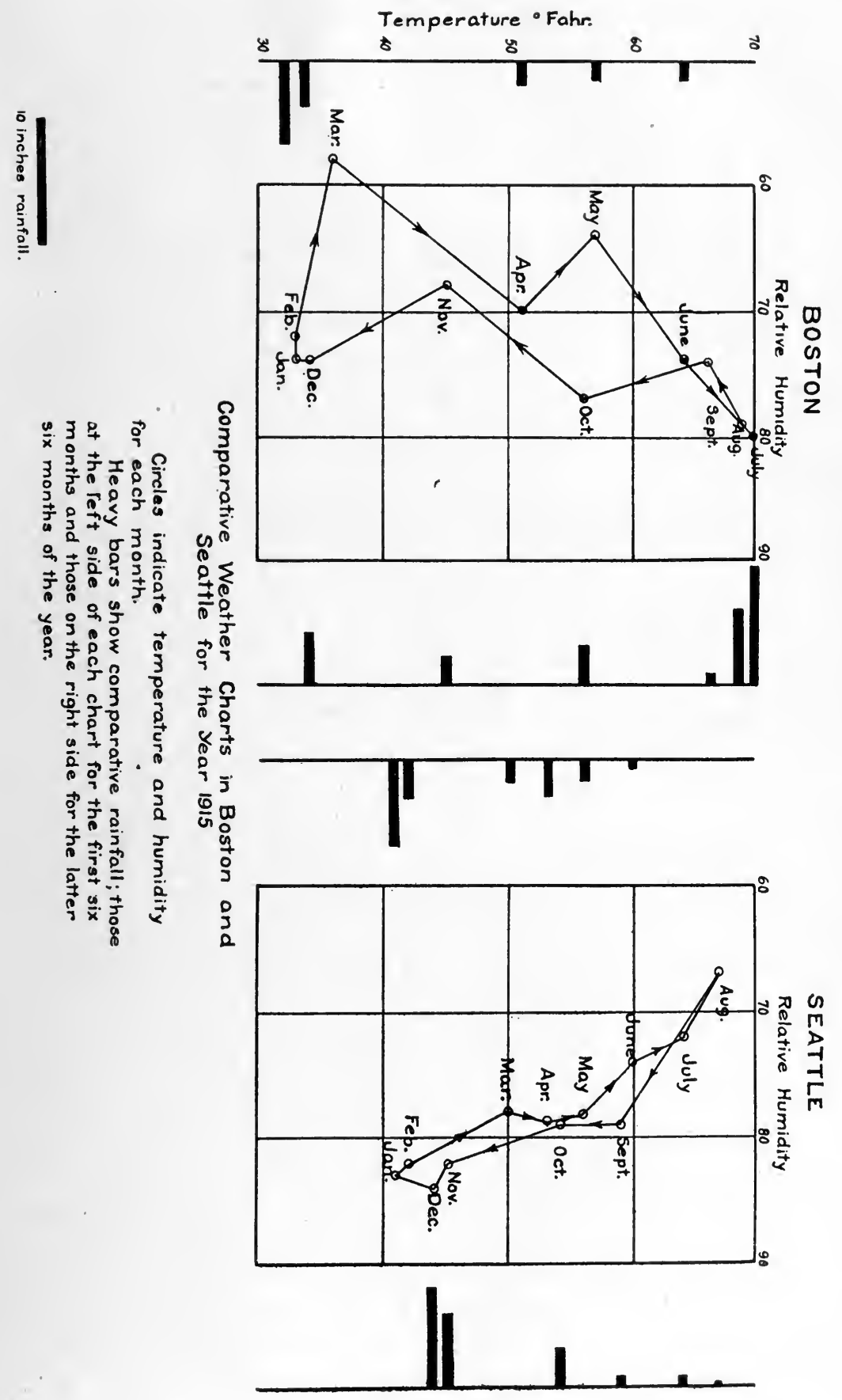

Fig. 74. 
the least, striking. Huntington concludes that there is no such thing as acclimatization. Just as there is an optimum temperature for the growth of infusoria and plants, so there is an optimum for man (Fig. 75).

The construction of the climograph begins with the tabulation of deaths by months of occurrence (Table LXXVI). A corrected figure is

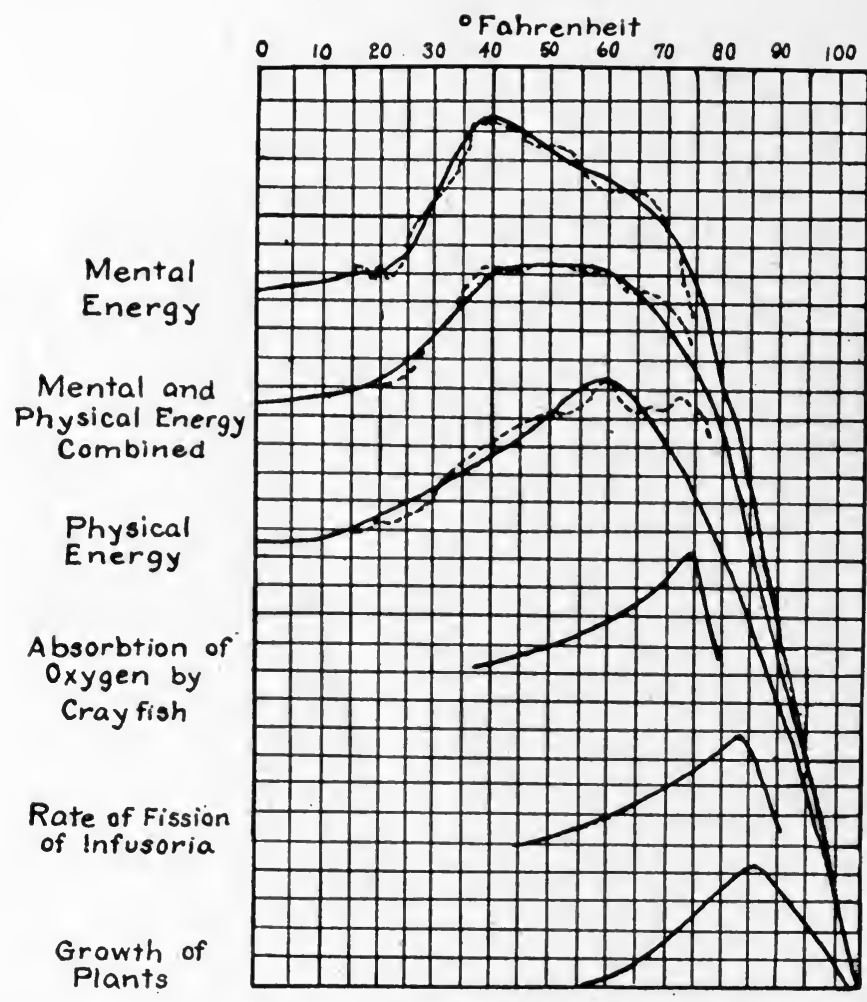

Mean Temperature and Vital

Processes in Plants, Animals and Man

From Huntington

Fig. 75.

"Civilization and Climate"

then obtained for each month by multiplying the February deaths by $31 / 28$ and for months of 30 days, by $31 / 30$. The corrected yearly totals are then plotted and a smooth curve drawn through the annual points (Fig. 76). This is the normal curve for the entire period and represents what would take place if the fluctuations from year to year did not occur. The numbers on this smooth curve show the annual normal mortality and when divided by 12 there is obtained the monthly normal mortality. The 
actual monthly mortality expressed as a percentage of the monthly normal mortality is then computed for each month (Table LXXVII). The next step is to group these percentages by the mean monthly temperature and relative humidity, page 655 . Thus, for temperatures from 70 to 74 , the percentages are averaged first for humidities of 55 to 59 , then 60 to 64 , then 65 to 69 , and so on. This is done for each of the five-degree groups of temperature. We may then insert the average percentage in the center of the squares formed by vertical lines representing relative humidity and horizontal lines representing temperature (Fig. 77). Percentages of equal magnitude are then connected by lines similar to lines of equal elevation on a topographical map.

The method may be illustrated with the pneumonia deaths of Detroit.

TABLE LXXVI

\begin{tabular}{|c|c|c|c|c|c|}
\hline \multirow[b]{3}{*}{ MONTH } & \multicolumn{2}{|r|}{1900} & \multicolumn{2}{|c|}{1901} & \\
\hline & ACTUAL & CORRECTED & ACTUAL & CORRECTED & \\
\hline & DEATHS & DEATHS & DEATHS & DEATHS & \\
\hline Jan. & 38 & 38 & 36 & 36 & \\
\hline Feb. & 36 & 40 & 47 & 52 & \\
\hline Mar. & 47 & 47 & 36 & 36 & \\
\hline Apr. & 66 & 68 & 67 & 69 & \\
\hline May & 34 & 34 & 35 & 35 & (Continue for each of \\
\hline June & 11 & 11 & 12 & 12 & the years under ob \\
\hline July & 12 & 12 & 14 & 14 & servation.) \\
\hline Aug. & 8 & 8 & 7 & 7 & \\
\hline Sept. & 23 & 24 & 9 & 9 & \\
\hline Oct. & 16 & 16 & 25 & 25 & \\
\hline Nov. & 36 & 37 & 39 & 40 & \\
\hline Dec. & 38 & 38 & 48 & 48 & \\
\hline Total & 365 & $\overline{373}$ & 375 & 383 & \\
\hline
\end{tabular}

The corrected totals for the year after equalizing the length of each month are then plotted as shown in the chart (Fig. 76) and a smooth curve drawn between the points.

The monthly normal mortality is determined by dividing the annual normal mortality, taken from the smoothed curve, by 12 . It is the relation of the actual corrected monthly mortality to this figure which

TABLE LXXVII

PNEUMONIA MORTALITY IN DETROIT

\begin{tabular}{|c|c|c|c|c|c|c|c|c|}
\hline \multirow[t]{2}{*}{ YEAR } & \multirow[t]{2}{*}{$\begin{array}{l}\text { ANNUAL } \\
\text { MORTALITY } \\
\text { RECORDED }\end{array}$} & \multirow[t]{2}{*}{$\begin{array}{c}\text { ANNUAL } \\
\text { NORMAL } \\
\text { MORTALITY }\end{array}$} & \multirow[t]{2}{*}{$\begin{array}{l}\text { MONTHLY } \\
\text { NORMAL } \\
\text { MORTALITY }\end{array}$} & \multicolumn{5}{|c|}{$\begin{array}{l}\text { PERCENTAGE OF MONTHLY NOR- } \\
\text { MAL MTORTALITY }\end{array}$} \\
\hline & & & & JAN. & FEB. & MAR. & APR. & \\
\hline 1900 & 373 & 405 & 34 & 112 & 118 & 138 & 200 & \\
\hline 1901 & 383 & 405 & 34 & 106 & 153 & 106 & 203 & (Continued \\
\hline 1902 & 416 & 405 & 34 & 165 & 126 & 162 & 147 & for each \\
\hline $\begin{array}{l}1903 \\
\text { etc. }\end{array}$ & 449 & 410 & 34 & 150 & 212 & 198 & 112 & $\begin{array}{l}\text { month of } \\
\text { the year.) }\end{array}$ \\
\hline 1917 & 1124 & 900 & 75 & 175 & 178 & 225 & 190 & \\
\hline
\end{tabular}




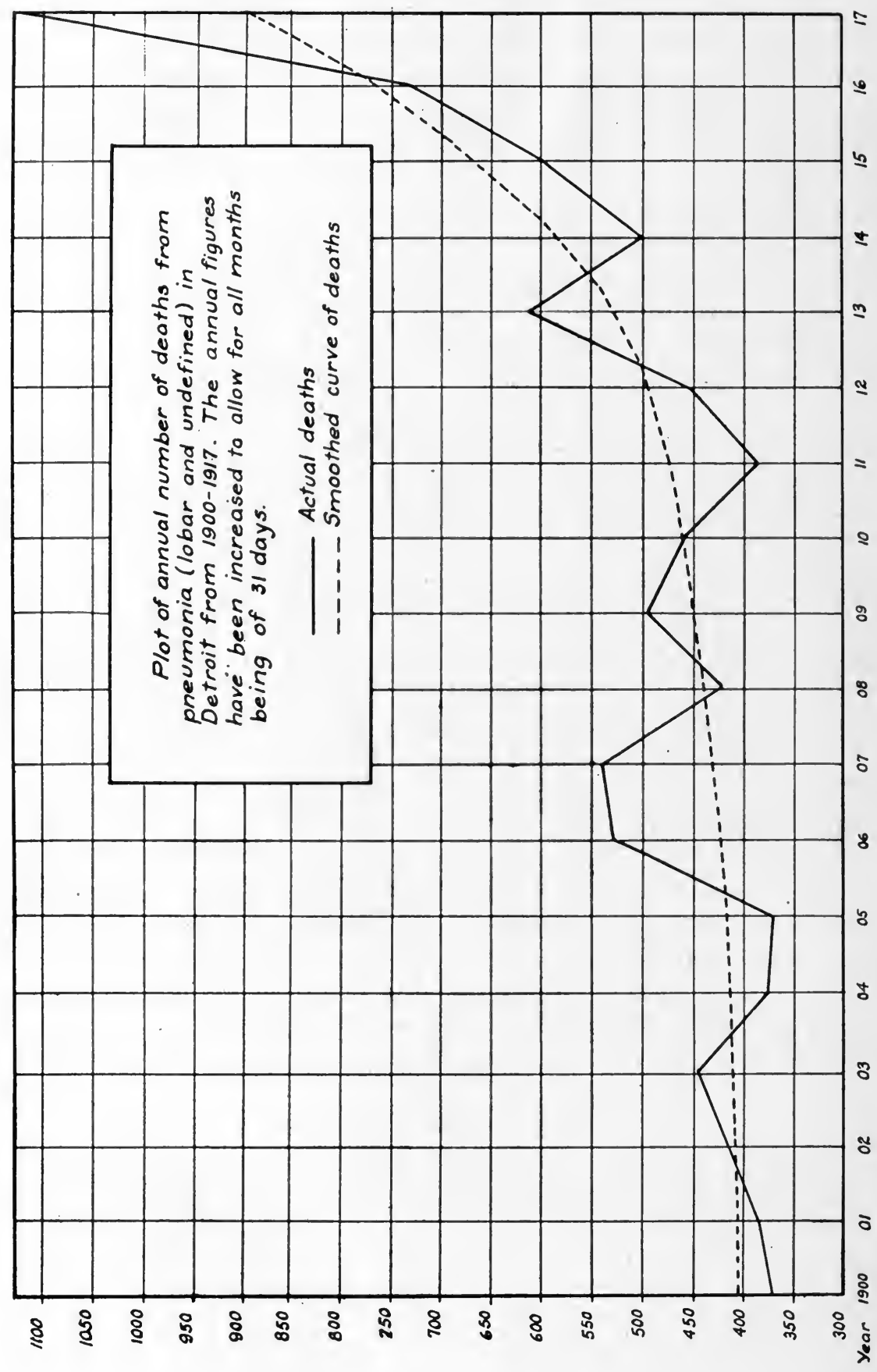

Fig. 76. 
appears in Table LXXVII, expressed as a ratio, the normal mortality being taken as 100 .

Next there are assembled the mean temperatures, mean relative humidities and mortality ratios by five-degree temperature groups. In the illustration we have taken the coldest months, grouping them as below $20^{\circ}$.

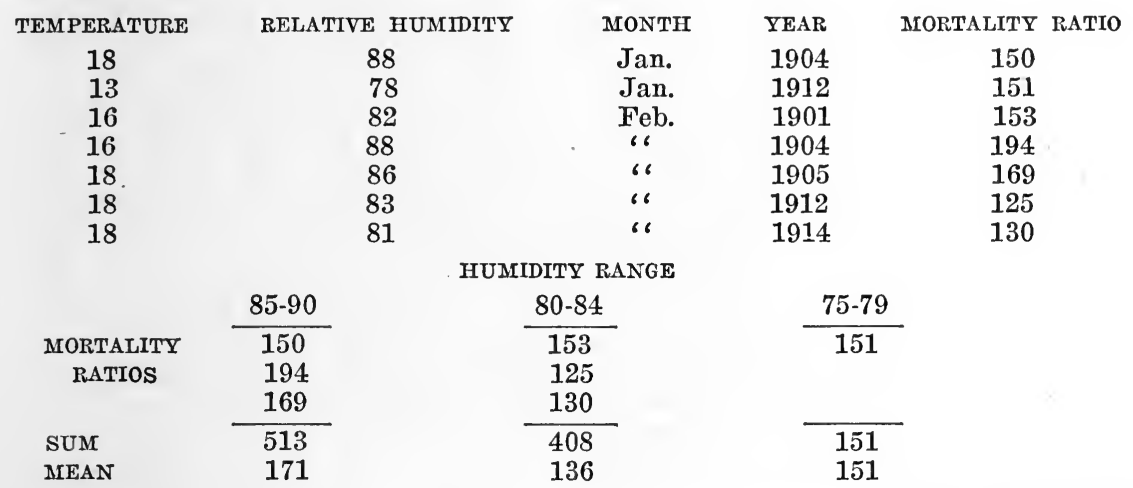

The plot (Fig. 77) is made from the mean mortality ratios derived as shown above.

The plot of pneumonia deaths in Detroit is made up of too few deaths to permit of a regular outline. However, the description, together with the chart, illustrates the method followed. We have used the Detroit figures as we shall return to them shortly in discussing the humidity influence on the death rate.

We reproduce two of Huntington's charts (Fig. 78), the one showing deaths from noncontagious diseases among white people in the United States and the other for deaths from all causes in South Italy. The lines in these climographs have been smoothed somewhat to give regular outlines.*

The healthiest temperature selected by Huntington from experimental studies is 64 degrees, the optimum humidity 80 per cent. Reference to these charts shows that this point nearly coincides with the point of lowest mortality both for South Italy and for the United States. The same holds true for France, Belgium, Finland, Sweden, Japan, Austria, Russia, Scotland, and Germany. To quote from Huntington, "The human race seems to have the best health when the average temperature for day and night together is $64^{\circ} \mathrm{F}$., that is, when the thermometer rises to about $70^{\circ}$ at mid day and drops to perhaps $55^{\circ}$ at night. One would suppose that the Swedes of the far north would have become wonted to a temperature colder than that which is best for the Sicilians

\footnotetext{
*In Figure 77 the lines of equal health, or isopracts, are labeled 25, 50, 75, 100, 125, etc. In Fig. 78 these lines are expressed differently as a plus or minus value above or below the mean. Zero in Fig. 78 corresponds to 100 in Fig. 77 . Minus 5 in Fig. 78 is the same as 95 in Fig. 77, plus 5 the same as 105 .
} 


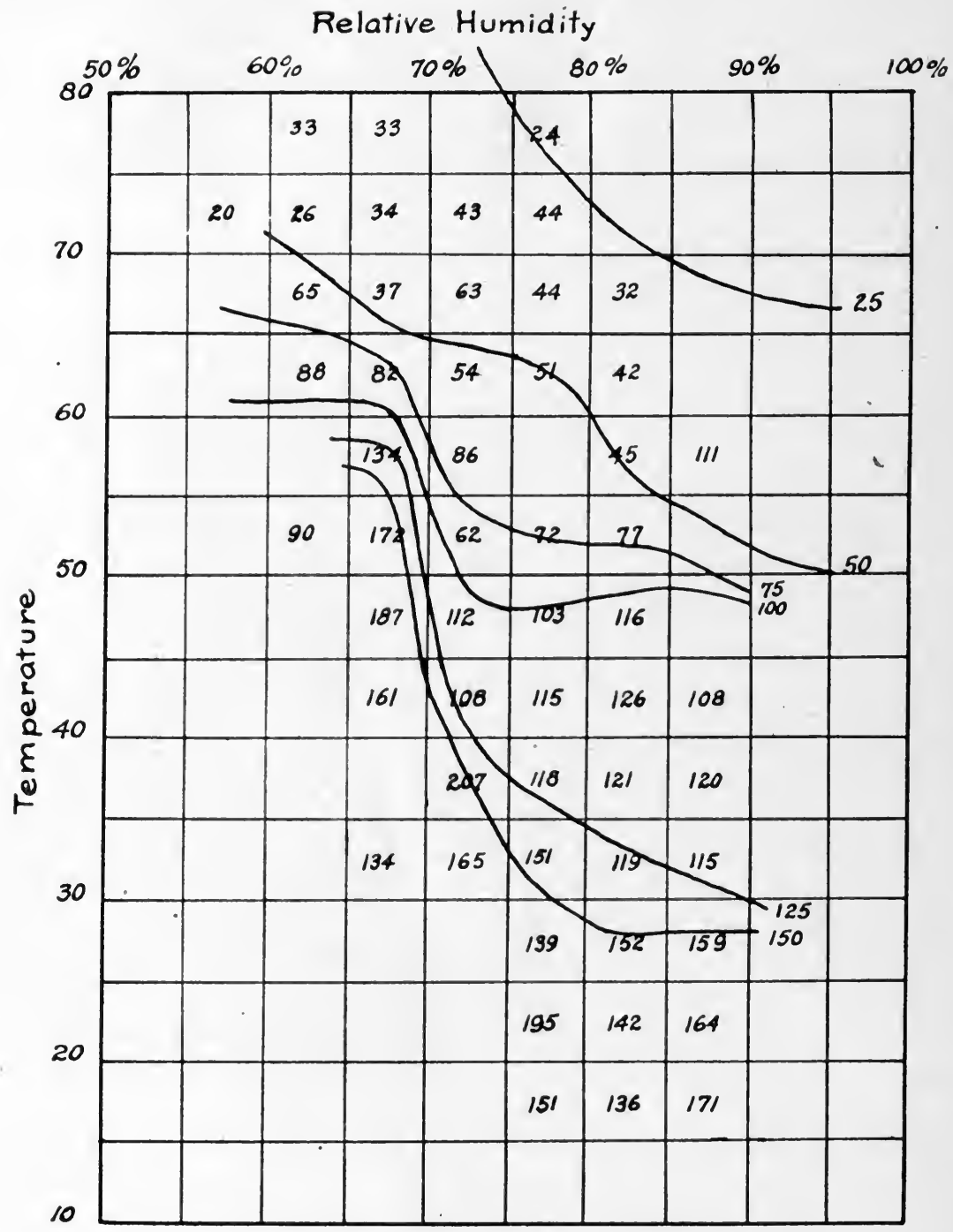

Pneumonia Deaths in Relation to Temperature and Humidity Detroit (1900-1917) 9000 deaths

The monthly mean is 100 . High mortality is indicated by figures above 100. The mortality is greater where the temperature and relative humidity are low.

Fig. 77. 
of the sunny south, but such is not the case. Even the dusky American negroes, whose ancestors have lived for unnumbered ages in the tropical heat of Africa and who still live where it is fairly warm, are at their best in a temperature scarcely higher than that which is most favorable for the blond Finns under the shadow of the Arctic Circle."

That the people of the Hawaiian Islands, where the temperature is
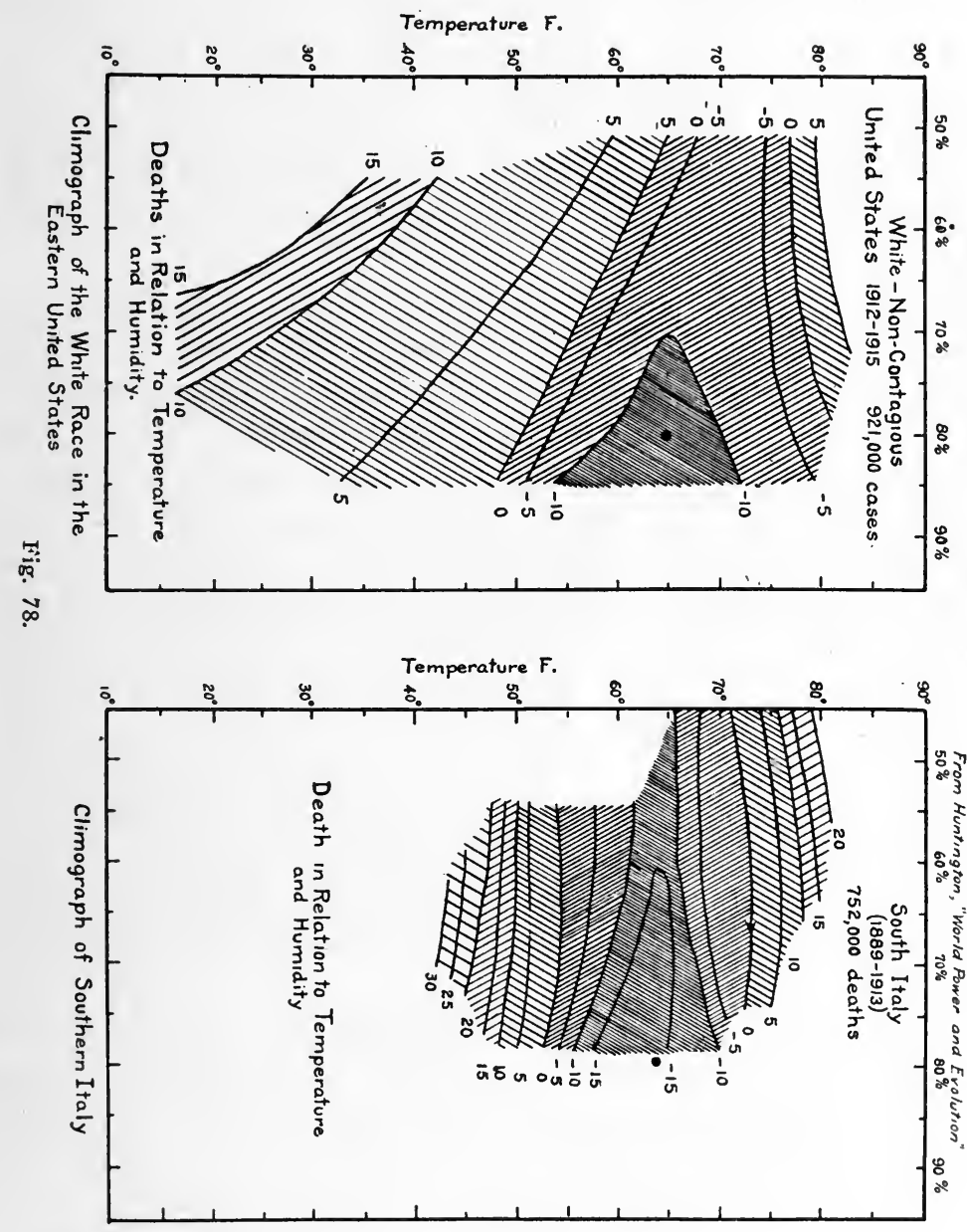

just about $64^{\circ}$, have not led the world in civilization is explained first by the fact that the mental optimum is much lower than the physical optimum, or around $40^{\circ}$, and the temperature in Hawaii is not the most favorable for mental advancement. The most advanced nations have temperatures ranging between the mental and physical optimums. "The best climate would apparently be one in which the winters are cool enough so that there are several months with frost, while the summers 
are warm enough so that there is a considerable period when the thermometer approaches $70^{\circ}$ at noon."

Referring again to the charts, it will be noticed that the topographic lines, or "isopracts" (lines of equal efficiency or equal health) converge at the left. This indicates, according to Huntington, that at a given temperature a high humidity is more favorable than a dry condition. This is more pronounced at low temperatures. It is only in Southern Italy and Southern Japan where dry winter weather appears no more harmful than moist. This, Huntington explains, is due to the fact that houses here are not heated in winter. In the United States and France winter necessitates heat, and it is this which accentuates the dryness and inclines the isopracts below $64^{\circ}$ at a greater angle from the horizontal than those above.

It appcars then that the death rate increases as the temperature departs in either direction, cold or hot, away from the optimum, which is around $64^{\circ}$. Further than this, dry weather increases the death rate, moisture lowers it. At $64^{\circ}$, humidity has the least influence. This is indicated for the total death rate, for deaths from noncontagious diseases and for pneumonia, as worked out by Greenberg for certain eastern cities and by the present authors for Detroit. There is a difference to be noted with contagious diseases among whites in the United States. In this case Huntington reports that above the optimum temperature dryness is more favorable than moisture. This corresponds with our general experience, humid days in summer being more devitalizing than dry days.

Huntington explains that the high death rate in dry periods is due to the dryness per se and not to a seasonal influence. Taking the months by themselves he shows that the dry Januarys are less healthful than the wet Januarys, and the dry Februarys and Marches less than the wet Februarys and Marches. There is but little difference when the Decembers are compared.

We are not convinced that the seasonal influence is ruled out, as Huntington states. We reproduce here the table from which the pneumonia climograph for Detroit was prepared (Table LXXVIII). In general the death rate is higher at the left coincident with lower humidity, just as Huntington states. However, take the temperature group 50 to $55^{\circ}$. The highest rates at the left are made up from records of April and May (spring months). The lower rates at the right are made up from the records of October (autumn month). The same is true of the next lower temperature group 45 to 50 . The highest rate at the left represents 6 Aprils (spring months). As the autumn months of October and November become mixed with the Aprils, the rate is less. The same tendency continues as we go down the temperature scale. The spring months as a rule have lower relative humidity than the autumn months. It is at this time that the death rate is higher. Is the death rate higher in the spring because it is dry or 
because people are more fatigued by the strain of winter and are, as a result, less resistant to pulmonary infections?

TABLe LXXVIII

Grouping of Months by Mean Temperature and Humidity and Mean Mortality RATio for PNeUMONIA For EACH GrouP

DETROIT-1900-17

RELATIVE HUMIDITY

\begin{tabular}{|c|c|c|c|c|c|c|c|c|}
\hline & & & 65 & 70 & 5 & 0 & 85 & 90 \\
\hline 80 & & $\begin{array}{c}\text { I July } \\
33\end{array}$ & $\begin{array}{c}1 \text { July } \\
1 \text { Aug. } \\
33\end{array}$ & & $\begin{array}{c}1 \text { Aug. } \\
24 \\
\end{array}$ & & & \\
\hline 70 & $\begin{array}{c}1 \text { July } \\
20 \\
\end{array}$ & $\begin{array}{c}1 \text { July } \\
26\end{array}$ & $\begin{array}{l}6 \text { July } \\
3 \text { Aug. } \\
34\end{array}$ & $\begin{array}{c}5 \text { July } \\
6 \text { Aug. } \\
43\end{array}$ & $\begin{array}{c}3 \text { July } \\
1 \text { Aug. } \\
44\end{array}$ & & & \\
\hline & & $\begin{array}{cc}1 & \text { May } \\
3 & \text { June } \\
1 & \text { Sept. } \\
65 & \end{array}$ & $\begin{array}{c}2 \text { June } \\
1 \text { Aug. } \\
37 \\
\end{array}$ & $\begin{array}{c}7 \text { June } \\
2 \text { Aug. } \\
2 \text { Sept. } \\
64\end{array}$ & $\begin{array}{c}3 \text { Aug. } \\
3 \text { Sept. } \\
\\
44 \\
\end{array}$ & $\begin{array}{cc}1 & \text { June } \\
1 & \text { Sept. } \\
& 32 \\
\end{array}$ & & \\
\hline 60 & & $\begin{array}{r}1 \text { May } \\
88 \\
\end{array}$ & $\begin{array}{c}1 \text { May } \\
1 \text { Sept. } \\
\\
82 \\
\end{array}$ & $\begin{array}{cc}1 & \text { May } \\
3 & \text { June } \\
5 & \text { Sept. } \\
54\end{array}$ & $\begin{array}{cc}2 & \text { June } \\
2 & \text { Sept. } \\
1 & \text { Oct. } \\
& 51 \\
\end{array}$ & $\begin{array}{c}3 \text { Sept. } \\
42 \\
\end{array}$ & . & \\
\hline & & & $\begin{array}{c}6 \text { May } \\
134 \\
\end{array}$ & $\begin{array}{l}3 \text { May } \\
1 \text { Oct. } \\
86 \\
\end{array}$ & & $\begin{array}{r}1 \text { Oct. } \\
\quad 45 \\
\end{array}$ & $\begin{array}{c}1 \text { May } \\
111\end{array}$ & \\
\hline 50 & & $\begin{array}{c}1 \text { April } \\
1 \text { May } \\
90\end{array}$ & $\begin{array}{c}2 \text { May } \\
172 \\
\end{array}$ & $\begin{array}{c}11 \text { May } \\
4 \text { Oct. } \\
64 \\
\end{array}$ & $\begin{array}{r}5 \text { Oct. } \\
72 \\
\end{array}$ & $\begin{array}{c}3 \text { Oct. } \\
71 \\
\end{array}$ & & \\
\hline & & & $\begin{array}{c}6 \text { April } \\
187 \\
\end{array}$ & $\begin{array}{l}4 \text { April } \\
1 \text { Oct. } \\
112 \\
\end{array}$ & $\begin{array}{l}2 \text { April } \\
2 \text { Oct. } \\
1 \text { Nov. } \\
103\end{array}$ & $\begin{array}{r}1 \text { Nov. } \\
116 \\
\end{array}$ & & \\
\hline 40 & & & $\begin{array}{cl}1 & \text { Mar. } \\
1 & \text { April } \\
161\end{array}$ & $\begin{array}{l}1 \text { April } \\
3 \text { Nov. } \\
108\end{array}$ & $\begin{array}{c}2 \text { April } \\
3 \text { Nov. } \\
115 \\
\end{array}$ & $\begin{array}{c}2 \text { Nov. } \\
126 \\
\end{array}$ & $\begin{array}{c}1 \text { Mar. } \\
198\end{array}$ & \\
\hline & & & & $\begin{array}{ll}1 \text { Mar. } \\
1 \text { April } \\
207\end{array}$ & $\begin{array}{l}3 \text { Mar. } \\
4 \text { Nov. } \\
118 \\
\end{array}$ & $\begin{array}{cl}1 & \text { Mar. } \\
3 & \text { Nov. } \\
121\end{array}$ & $\begin{array}{c}1 \text { Nov. } \\
120\end{array}$ & \\
\hline 30 & & & $\begin{array}{l}1 \text { Mar. } \\
134 \\
\end{array}$ & $\begin{array}{l}3 \text { Mar. } \\
\quad 165 \\
\end{array}$ & $\begin{array}{ll}2 & \text { Mar. } \\
1 & \text { Dec. } \\
& \\
& 151 \\
\end{array}$ & $\begin{array}{ll}3 & \text { Jan. } \\
1 & \text { Feb. } \\
1 & \text { Mar. } \\
4 & \text { Dec. } \\
& 119 \\
\end{array}$ & $\begin{array}{ll}1 & \text { Jan. } \\
1 & \text { Dec. } \\
& \\
& \\
& 115 \\
\end{array}$ & \\
\hline & & & & & $\begin{array}{cc}1 & \text { Feb. } \\
2 & \text { Mar. } \\
& \\
& 139 \\
\end{array}$ & $\begin{array}{ll}6 & \text { Jan. } \\
2 & \text { Feb. } \\
2 & \text { Mar. } \\
5 & \text { Dec. } \\
& 152 \\
\end{array}$ & $\begin{array}{cc}3 & \text { Jan. } \\
1 & \text { Feb. } \\
2 & \text { Dec. } \\
& \\
& \end{array}$ & \\
\hline 20 & & & & & $\begin{array}{c}2 \text { Feb. } \\
195 \\
\end{array}$ & $\begin{array}{ll}2 & \text { Jan. } \\
5 & \text { Feb. } \\
4 & \text { Dec. } \\
139 \\
\end{array}$ & $\begin{array}{ll}1 & \text { Jan. } \\
1 & \text { Feb. } \\
1 & \text { Dec. } \\
164\end{array}$ & \\
\hline & & & & & $\begin{array}{c}\text { Jan. } \\
151 \\
\end{array}$ & $\begin{array}{c}3 \text { Feb. } \\
136\end{array}$ & $\begin{array}{ll}1 & \text { Jan. } \\
2 & \text { Feb. } \\
171\end{array}$ & \\
\hline 10 & & & & & & & & 1 \\
\hline
\end{tabular}


We have made another test of the humidity influence by dividing the records of pneumonia for each month during the 18 year period of 1900 to 1917 into three groups, the six driest, the six intermediate as to humidity and the six wettest. According to Huntington the driest months will have the highest death rate. We do not find from Detroit figures any ground for such an inclusive statement.

TARLE LXXIX

Pneumonia Mortality in Detroit

\begin{tabular}{ccccccc}
\hline \hline Month & \multicolumn{2}{c}{$\begin{array}{c}\text { Pneumonia Mortality } \\
\text { Ratio }\end{array}$} & \multicolumn{2}{c}{ Relative Humidity } & \multicolumn{2}{c}{ Temperature } \\
& $\begin{array}{c}\text { 6 driest } \\
\text { Months }\end{array}$ & $\begin{array}{c}\text { Months } \\
\text { Months }\end{array}$ & $\begin{array}{l}\text { Dry } \\
\text { Months }\end{array}$ & $\begin{array}{c}\text { Wet } \\
\text { Months }\end{array}$ & $\begin{array}{l}\text { Dry } \\
\text { Months }\end{array}$ & $\begin{array}{c}\text { Wet } \\
\text { Months }\end{array}$ \\
\hline Oct. & 61 & $71^{*}$ & 72 & 80 & 52 & 51 \\
Nov. & 91 & $116^{*}$ & 75 & 82 & 39 & 41 \\
Dec. & $121^{*}$ & 117 & 80 & 85 & 29 & 27 \\
Jan. & 145 & 145 & 81 & 86 & 24 & 25 \\
Feb. & 164 & $166^{*}$ & 79 & 86 & 23 & 20 \\
Mar. & $181^{*}$ & 159 & 71 & 82 & 35 & 33 \\
Apr. & $161^{*}$ & 139 & 66 & 75 & 48 & 45 \\
\hline
\end{tabular}

(High mortality indicated hy figures above 100 . Asterisk indicates higher rate.)

In October and November the pneumonia mortality is greater in the wet months. In March and April the reverse is true. There is no difference between wet and dry Januarys and scarcely any difference between wet and dry Februarys or Decembers.

A moist July in Detroit is coincident with a higher death rate than a dry July. The same is true with August and September.

TABLE LXXX

\begin{tabular}{cccccccc}
\hline \hline Month & \multicolumn{2}{c}{$\begin{array}{c}\text { Pneumonia Mortality } \\
\text { Ratio }\end{array}$} & \multicolumn{2}{c}{ Relative } & Humidity & \multicolumn{2}{c}{ Temperature } \\
& $\begin{array}{c}\text { 6 driest } \\
\text { Months }\end{array}$ & $\begin{array}{c}\text { 6 wettest } \\
\text { Months }\end{array}$ & $\begin{array}{l}\text { Dry } \\
\text { Months }\end{array}$ & $\begin{array}{c}\text { Wet } \\
\text { Months }\end{array}$ & $\begin{array}{c}\text { Dry } \\
\text { Months }\end{array}$ & $\begin{array}{c}\text { Wet } \\
\text { Months }\end{array}$ \\
\hline May & $107^{*}$ & 97 & 64 & 75 & 59 & 57 \\
June & 63 & $64^{*}$ & 66 & 76 & 67 & 66 \\
July & 36 & $43^{*}$ & 64 & 75 & 74 & 71 \\
Aug. & 29 & $40^{*}$ & 68 & 77 & 72 & 64 \\
Sept. & 42 & $46^{*}$ & 70 & 80 & 65 & 64 \\
\hline
\end{tabular}

Averaging the entire 12 months we find the proportion of deaths in the driest and wettest months to be identical.

In view of the above we do not feel that Huntington has satisfactorily established the harmfulness of low humidity. Of course we are influenced by the records of one eity, whereas Huntington has many more data to draw upon. However, if low humidity is such a vicious factor as is intimated, we should expect to find evidences of it in Detroit as well as other places. 
The highest pneumonia mortality in Detroit from 1900 to 1917 occurred in 1917, and this year has the lowest mean annual temperature. The lowest pneumonia mortality was in 1911, and this year is the warmest in 18 years. Dividing the years into the six coldest, six intermediate and six warmest, we find the mortality ratio in the first, 108, the intermediate 97 and the warmest, 101. The coldest years thus tend to have the greatest pneumonia. The exceptions to the general rule will be found in the following table.

TABLE LXXXI

Pneumonia Mortality and Mean Annual Temperature in Detroit

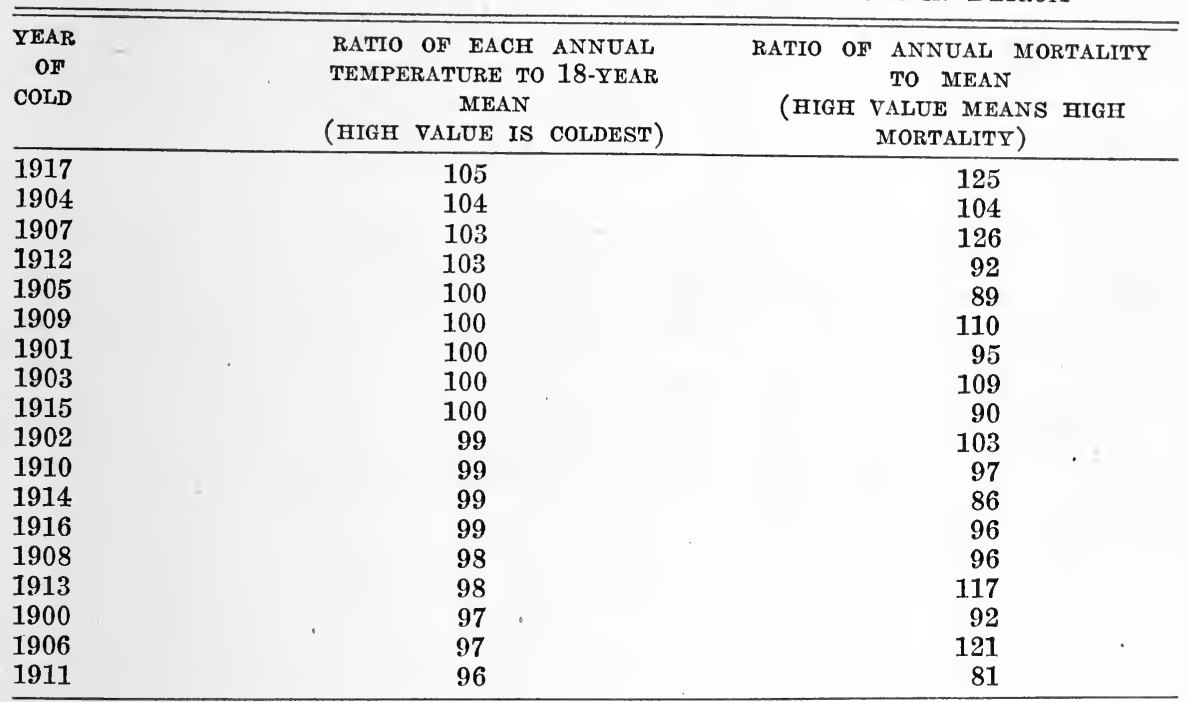

The mortality ratio of the six coldest Januarys in Detroit is 152 (mean temperature 21), the six warmest Januarys 130 (mean temperature 30). The six coldest Marches, however, have a mortality ratio of 147 , the six hottest Marches, 177. For eight months of the year the pneumonia mortality is greatest in the coldest months. In March and April pneumonia is markedly greater when the weather is warm. In November and September there is little difference either way, although the cold months are very slightly more favorable to health (Table LXXXII).

From this single instance of Detroit it would seem as though pneumonia is least prevalent when winter comes on gradually and works up to its coldest point in February and March. Conversely, a winter which begins with cold damp weather and is followed by warm dry weather in March and April is apt to lead to heavy pneumonia mortality. Incidentally, it may be noted that these averages are not the result of extreme figures for one or two years, but are representative of the general tendency and for that reason are to be given considerable weight. 
TABLE LXXXII

Pneumonia Mortality Ratios in Relation to Monthly Temperatures. DETROIT-1900-17

(High value means high pneumonia, 100 being a mean value for the year)

\begin{tabular}{lccc}
\hline \hline MONTH & 6 WARMEST MONTHS & 6 COLDEST MONTHS & MOST FAVORABLE \\
\hline Oct. & 57 & 76 & Warm \\
Nov. & 100 & 95 & Cold* \\
Dec. & 121 & 139 & Warm \\
Jan. & 130 & 152 & Warm \\
Feb. & 152 & 158 & Warm \\
Mar. & 177 & 147 & Cold \\
Apr. & 176 & 139 & Warm \\
May & 96 & 132 & Warm \\
June & 58 & 67 & Warm \\
July & 33 & 44 & Warm \\
Aug. & 34 & 44 & Cold* \\
Sept. & 49 & 48 & \\
\hline
\end{tabular}

${ }^{*}$ Slight difference

Season and Disease.-Much clearer than the relation of health to mean annual atmospheric conditions is its relation to the seasons. Pneumonia in the Northern United States is always greater in February than in August. This never fails. August is always hotter than February. There is a certain temperature range for each month which is peculiar to that month. To a lesser degree this is noticeable with humidity and other atmospheric manifestations.

Continuing with Detroit for illustration, February is the coldest month of the year. It is also the month of greatest temperature variation. (Fig. 79). The highest relative humidity is found in January, the lowest in May. In actual amount of moisture present in the air there is the least in February and the most in July. February is the month when we shall find the lowest relative humidity or the greatest aridity indoors. July is the month with the greatest precipitation (rain and snow), while November has the least (Table LXXXIII).

As shown by the chart (Fig. 80), pneumonia mortality follows very closely the seasonal temperature curve, and this is even more marked if the temperature graph is set one month to the left. It would seem from this that the pneumonia mortality in any month is closely bound up with the temperature in the month preceding. On plotting the graph for vapor pressure or for relative humidity at an indoor temperature of $70^{\circ}$ there is much less resemblance to pneumonia mortality, the monthly variation from the annual mean being far greater than the variation of pneumonia or temperature from their annual mean. Temperature exercises a greater influence on pneumonia than moisture.

The seasonal variation in pneumonia differs materially from certain other diseases. Taking Detroit for illustration the pneumonia curve is quite regular, the peak occurring in March, the lowest point in August. 


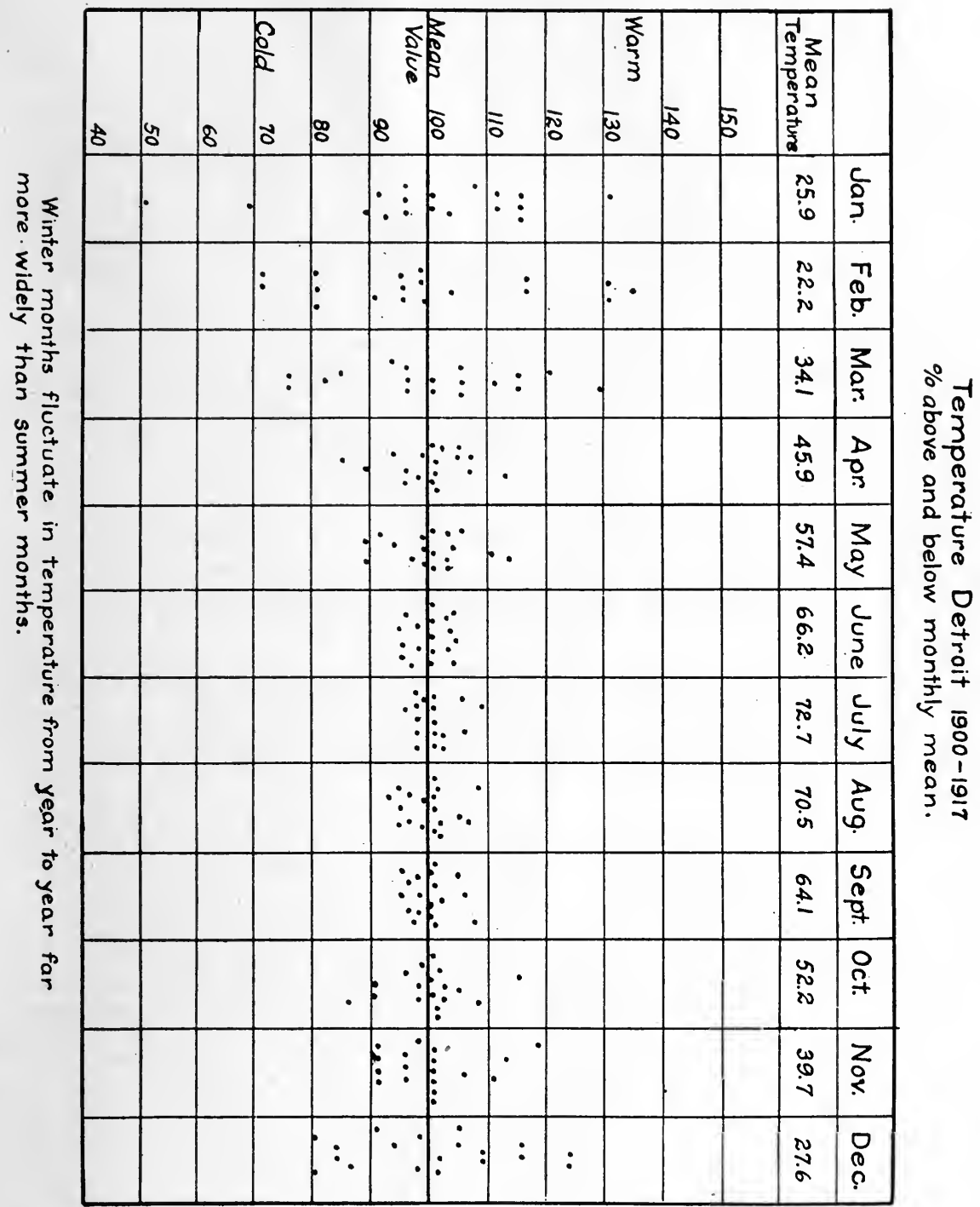

Fig. 79.

Pulmonary tuberculosis is less affected by the seasons than most diseases. The peak occurs in March, the amount being but 17 per cent in excess of the mean for the year. The low points are in September and October. The graph is fairly uniform, the mortality rising with the onset of cold weather and declining with warm weather (Table LXXXIV).

Diphtheria and scarlet fever are similar in general although the graphs cross each other, and there are some rather distinct differences. Diphtheria is highest in November, scarlet fever in January. The low point 


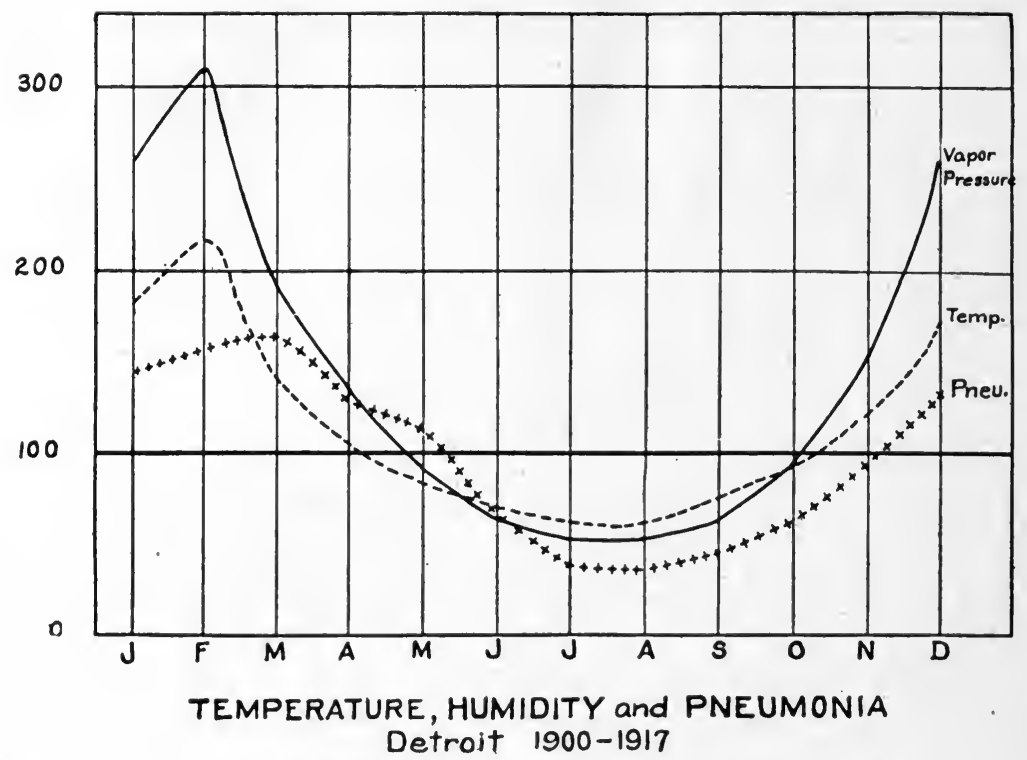

Graphs show relation of monthly values to mean, those for temperature and vapor. pressure being inverted so as to parallel the pneumonia graph.

Fig. 80.

TABLE LXXXIII

Monthly Weather Averages for Detroit-1900-17

\begin{tabular}{|c|c|c|c|c|c|c|c|}
\hline MONTH & $\begin{array}{l}\text { MEAN } \\
\text { TEMP. }\end{array}$ & $\begin{array}{l}\text { MEAN REL. } \\
\text { HUMIDITY }\end{array}$ & $\begin{array}{l}\text { MEAN VAPOR } \\
\text { PRESSURE } \\
\text { (INCHES } \\
\text { MERCURY) }\end{array}$ & $\begin{array}{l}\text { REL. HUM IDITY } \\
\text { INDOORS AT } \\
70^{\circ} \text { TEMP. }\end{array}$ & $\begin{array}{l}\text { MEAN PRECIPI } \\
\text { TATION }\end{array}$ & $\begin{array}{l}\text { NO. M } \\
\text { WITH } \\
\text { I- TEMP. } \\
\text { ING } \\
\text { FROM }\end{array}$ & $\begin{array}{l}\text { MONTHS } \\
\text { I IEAAN } \\
\text { DEPART- } \\
10 \% \\
\text { MEAN }\end{array}$ \\
\hline Jan. & 25.9 & 83.3 & .113 & 15 & 2.49 & & 9 \\
\hline Feb. & 22.2 & 82.1 & .094 & 13 & 2.04 & & 11 \\
\hline Mar. & 34.1 & 76.9 & .150 & 21 & 2.30 & & 9 \\
\hline Apr. & 45.9 & 70.5 & .218 & 30 & 2.64 & & 3 \\
\hline May & 57.4 & 69.0 & .326 & 45 & 3.28 & & 3 \\
\hline June & 66.2 & 71.3 & .458 & 63 & 3.42 & & 0 \\
\hline July & 72.7 & 69.3 & .556 & 76 & 3.62 & & 0 \\
\hline Aug. & 70.5 & 72.2 & .538 & 74 & 2.90 & & 0 \\
\hline Sept. & 64.1 & 74.8 & .446 & 61 & 2.81 & & 0 \\
\hline Oct. & 52.2 & 76.3 & .298 & 41 & 2.19 & & 2 \\
\hline Nov. & 39.7 & 78.7 & .192 & 26 & 1.85 & & 3 \\
\hline Dec. & 27.6 & 82.6 & .121 & 17 & 2.13 & & 9 \\
\hline
\end{tabular}

for diphtheria is July, for scarlet fever, August. Diphtheria starts its ascent in August and rises precipitously to November. It then declines and by February is below the annual mean. It declines but slowly until June and then with more abruptness to July. 
TABLE LXXXIV

Seasonal Variation in Mortality for Certain Diseases, Detroit-1900-17

(Figures are ratios, the monthly mean for the year being 100)

\begin{tabular}{cccccc}
\hline \hline MONTH & $\begin{array}{c}\text { PNEUMONIA } \\
\text { (LOBAR AND UN- } \\
\text { DEFINED) }\end{array}$ & $\begin{array}{c}\text { TUBERCULOSIS } \\
\text { (PULMONARY) }\end{array}$ & DIPHTHERIA & $\begin{array}{c}\text { SCARLET } \\
\text { FEVER }\end{array}$ & MEASLES \\
\hline TOTAL & & & & & \\
NO. OF & 9100 & 8895 & 2691 & 1097 & 705 \\
DEATHS & & & & & \\
\hline Jan. & 145 & 95 & 117 & 154 & 94 \\
Feb. & 158 & 110 & 94 & 131 & 114 \\
Mar. & 163 & 117 & 95 & 135 & 145 \\
\hline Apr. & 153 & 111 & 94 & 151 & 160 \\
May & 118 & 110 & 89 & 131 & 177 \\
June & 69 & 100 & 85 & 86 & 128 \\
\hline July & 40 & 95 & 61 & 57 & 94 \\
Aug. & 37 & 91 & 71 & 42 & 79 \\
Sept. & 45 & 88 & 90 & 58 & 14 \\
\hline Oct. & 67 & 88 & 124 & 71 & 20 \\
Nov. & 97 & 92 & 155 & 104 & 55 \\
Dec. & 132 & 103 & 128 & 103 & 123 \\
\hline
\end{tabular}

Scarlet fever rises in September and ascends more slowly, crossing the mean in November and rising abruptly to a peak in January. It falls off in February but again rises to a secondary peak in April almost as high as in January. From this point it descends abruptly to the low point in August.

Measles presents the most variable outline, the peak in May being nearly 13 times the low point in September. Measles rises in October and with great abruptness during November and December. It falls below the mean in January and then ascends with less abruptness than in the autumn to its peak in May. The decline from May to September is abrupt.

It is believed by many that the school is responsible for the rise in acute contagion in the autumn. We have already pointed out elsewhere that the age distribution of cases does not indicate an unusual increase in disease when a child enters school. The seasonal curves of disease mortality likewise implicate the weather more than the school. Lobar pneumonia is a disease of adults. The pneumonia mortality is extremely sensitive to temperature, following it very closely. Pneumonia starts its increase in September with the beginning of cooler weather, and its rise parallels the declining temperature. This relationship exists quite independent of any school influence. Diphtheria is much more of an adult disease than scarlet fever, and yet diphtheria rises more abruptly with declining temperature and the opening of school than scarlet fever. In spite of the opening of the schools, scarlet fever does not rise to its peak until January and secondarily in April. Measles is even more 
deliberate and fails to reach its maximum till May. Measles deaths are mostly among babies, and as August is a bad month for infants, the measles deaths do not reach the minimum until September. There is nothing in the behavior of these graphs which involves the school as a breeder of disease. It is the weather influence in the long run which controls the form of the curves.

TABLE LXXXV

MoRtality in Detroit-1900-17

\begin{tabular}{|c|c|c|c|}
\hline DISEASE & MAXIMUM & MINIMUMI & $\begin{array}{l}\text { RATIO, MAXIMUM TO } \\
\text { MINIMUM (AS 1) }\end{array}$ \\
\hline $\begin{array}{l}\text { Tuberculosis } \\
\text { (pulm.) }\end{array}$ & 117 (Mar.) & 88 (Sept., Oct.) & 1.3 \\
\hline Diphtheria & 155 (Nov.) & 61 (July) & 2.5 \\
\hline Scarlet fever & 154 (Jan.) & 42 (Aug.) & 3.7 \\
\hline Pneumonia & 163 (Mar.) & 37 (Aug.) & 4.4 \\
\hline Measles & 177 (May) & 14 (Sept.) & 12.6 \\
\hline
\end{tabular}

We have been measuring disease prevalence with deaths in place of cases, because the death records are much more complete. The objection may be raised that the severity of a disease may be much greater at one season than another, and for this reason the mortality is not a proper measure of its prevalence. The morbidity records in Detroit, so far as they are available, closely parallel the mortality. Morbidity is high when mortality is high.

The seasonal peculiarities of the diseases mentioned have been interpreted from the mean or average, and there are departures to be noted in individual years. Thus, while for the entire 18 years, March averages as the worst month for pneumonia, the highest mortality actually occurred in March only 5 times. It occurred in February 6 times, April and December 3 times each and January once.

\section{TABLE LXXXVI}

Mortality in Detroit-1900-17

\begin{tabular}{|c|c|c|c|c|c|c|c|c|c|c|}
\hline \multirow[t]{2}{*}{ DISEASES } & \multicolumn{2}{|c|}{$\begin{array}{l}\text { MORTALITY } \\
\text { PEAK FOR } \\
\text { ENTIRE }\end{array}$} & \multicolumn{4}{|c|}{ MORTALITY PEAKS IN } & \multicolumn{2}{|c|}{ INDIVIDUAL } & YEARS & \multirow[b]{2}{*}{ MAT } \\
\hline & PERIOD & SEPT. & OCT. & nov. & DEC. & JAN. & FEB. & MAR. & APR. & \\
\hline Pneumonia & Mar. & 0 & 0 & 0 & 3 & 1 & 6 & 5 & 3 & 0 \\
\hline Tuberculosis* & Mar. & 2 & 1 & 1 & 1 & 1 & 2 & 3 & 3 & 2 \\
\hline Diphtheria & Nov. & 1 & 1 & 6 & $\mathbf{3}$ & 4 & 2 & 1 & 0 & 0 \\
\hline Scarlet Fever & Jan. & 1 & 0 & 3 & 3 & 5 & 3 & 2 & 5 & 2 \\
\hline Measles** & May & 0 & 0 & 1 & 4 & 1 & 3 & 1 & 2 & $\overline{2}$ \\
\hline
\end{tabular}

*July 1, Aug. 1. * *July 1, June 2, Aug. 1.

The mean for diphtheria is greatest in November, but there is a wide seasonal range. An outbreak of the disease may occur at any time of 
year, but it is far more likely to occur in the early winter than at other times.

Scarlet fever is more variable in its occurrence than diphtheria. The mean happens to fall in January, but high points have been registered in all months from November to May inclusive. It is rare in summer.

Measles shows wide variation also. The high point occurs sometimes in December, again in February and even in the summer months of June, July and August. It is more frequently associated with the spring than the autumn months.

There are opposing points of view with reference to the control of the acute contagions. There are some who look upon these diseases as natural events in a child's life which it is impossible to escape. The sooner contracted the better, for immunity is then established for life. This applies particularly to measles and to a lesser extent to scarlet fever. The objection to this plan is that these diseases often leave permanent injury. If childhood can be passed over without contracting scarlet fever, a person may escape altogether, for natural immunity appears with advancing age. This is likewise true with diphtheria.

It is not impossible to escape contagion. By proper daily inspection in the schools it is possible to upset the natural seasonal occurrence of disease. This is well illustrated in the ease of Detroit. From 1900 to 1904, 27 per cent of the mortality from scarlet fever in Detroit occurred in the autumn months of October, November and December. From 1905 to 1909 this percentage was 34; from 1910 to 1914, 14; and from 1915 to 1917,16 . Normally the disease would appear in the fall with increasing cold weather. As daily inspection by nurses, physicians and teachers becomes more general, its progress is being made increasingly difficult, and the disease has thus been postponed from the autumn to the spring months. From 1900 to 1904, 21 per cent of scarlet fever mortality occurred during April, May and June. From 1905 to 1909 this percentage was 23 ; from 1910 to 1914 , 35; and from 1915 to $1917,38$.

The same shifting of disease from the autumn toward the winter and spring is noted with diphtheria.

$\begin{array}{ccc}\text { Period } & \begin{array}{c}\text { Autumn } \\ \text { (Oct., Nov., Dec.) }\end{array} & \begin{array}{c}\text { Spring } \\ \text { (Apr., May, June) }\end{array} \\ 1900-04 & 40 & 18 \\ 1905-09 & 35 & 20 \\ 1910-14 & 33 & 20 \\ 1915-17 & 30 & 27\end{array}$

These figures are most suggestive and should give encouragement to adequate school inspection. $\mathrm{To}^{\circ}$ curb the spread of disease the case must be removed from his fellows early or before he has a chance to 
SEASON and DISEASE MORTALITY 1911-1917
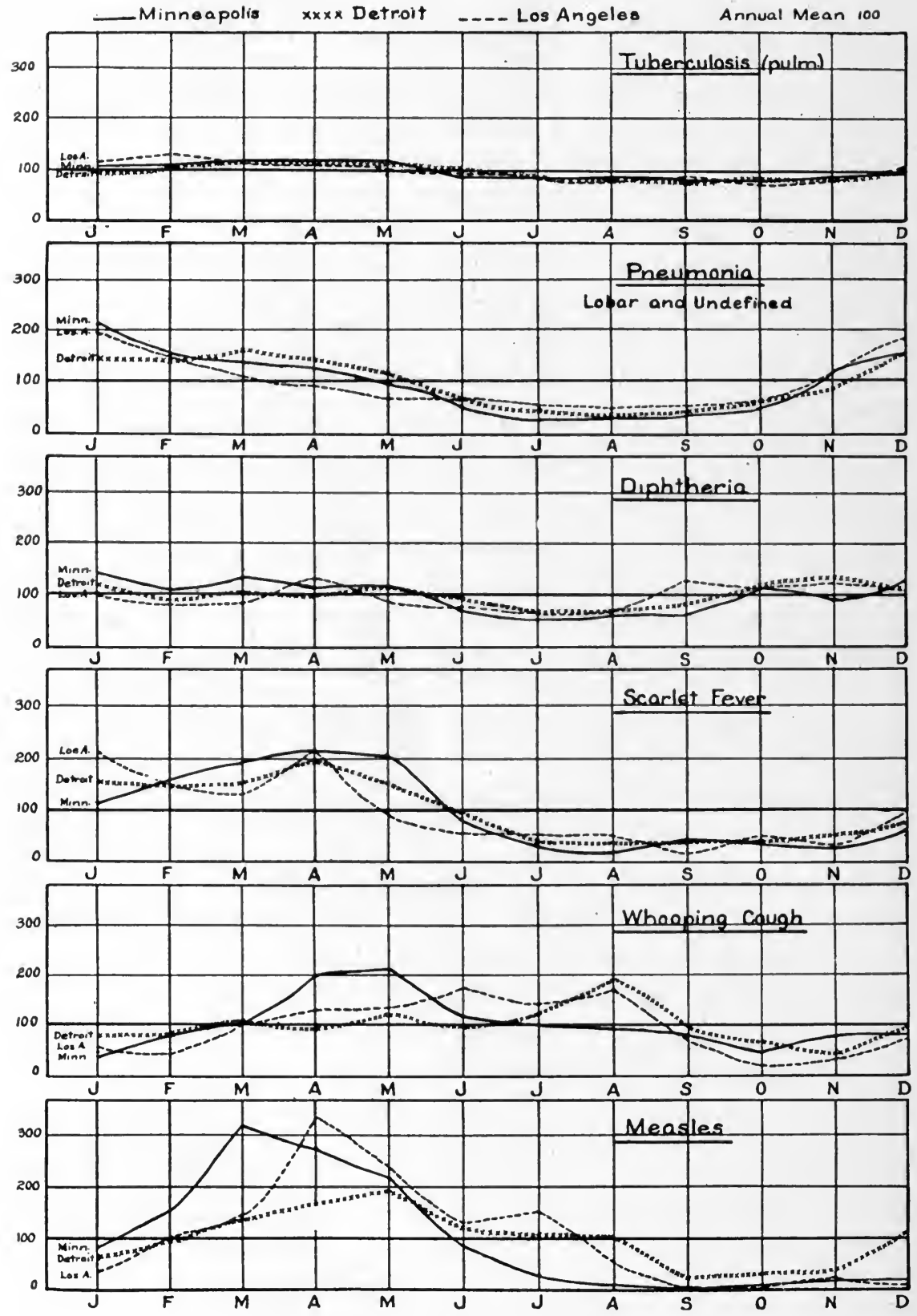

Fig. 81 . 
spread the infection. The sooner he is removed, the fewer the exposures. To really accomplish results there should be a brief inspection by teachers every morning and every child with suspicious symptoms should be referred to physician or nurse.

What is most interesting in the study of seasonal influence on disease is the agreement to be noted in cities widely separated and of diverse climates. This will be seen in the charts comparing Detroit, Minneapolis and Los Angeles (Fig. 81). The graphs for tuberculosis are very similar, and this is true of pneumonia. There are some minor differences for diphtheria, Los Angeles showing a sudden rise in the early fall, Detroit in the late fall and Minneapolis still later. Minneapolis has whooping cough and measles somewhat earlier in the year than the other two cities.

Just as the acute contagions have their seasonal fluctuations so do the minor throat affections to which we may apply the general term colds. Palmer has shown from a study of New York City school children that colds increase with the onset of cold weather in the autumn and decline with the oncoming of warm weather in the spring. Weather factors other than temperature exert only a minor influence on the prevalence of colds so far as can be ascertained. It is significant to follow the sequence of colds and pneumonia in the New York study. First to reach their peak are mild colds not sufficient to cause absence from school. Three weeks later, or about the middle of December, there occurs the peak of severe colds sufficient to keep children home from school. Last to rise is the curve of pneumonia mortality. This relationship, together with the close relationship previously shown between pneumonia and temperature, suggests that pneumonia, more than other diseases, is an outcome of lowered vitality. Due to the stress of increased heat production and metabolism, the body becomes weakened as the winter season continues, and pneumonia continues to increase until the advent of warmer weather in the spring.

Influenza and the Weather.-The influence of weather on the influenza pandemic of 1918 finds a divided opinion. Nichols finds nothing unusual in the weather except the heavy rainfall in September, and this was true prior to the epidemic in 1890 . He attributes the severity of the epidemic to overcrowded living conditions during the cold winter of 1917-18. He regards a "certain concentration of germ laden air" as necessary for the spread of certain diseases like influenza. Business depressions, strikes and other causes which bring about congested living conditions he believes to be equally as damaging as climatic changes.

Huntington, from a study of records for New York and Boston, concludes that the "virulence of epidemic influenza appears to be closely dependent on the weather," although he remarks that "epidemics of this 
character may break out at any season and that once started, they run their course in spite of weather or climate."

It was the privilege of the authors to review the disease prevalence in the army camps during the autumn of 1918. In some camps the weather was unusually severe, in others mild. Of the 37 large camps, 31 reported mild weather and only six severe weather. Of those reporting mild weather, 14 had high mortality and 17 low mortality. Of those reporting severe weather 4 had high mortality and 2 low mortality. This finding is supported by the smaller camps. Severe weather was rare. It was not notably associated with high mortality.

Sherman and Cody, with the greatest mortality, reported mild weather, Hancock and Johnston, with relatively low mortality, reported severe weather. Camp Funston specifically states that the disease "was not influenced in any way by * * * inclement weather." Camp Humphreys is equally positive about this, " ** * There is apparently no connection between incidence of new cases and the weather conditions. On the days on which there was the lowest temperature and the greatest range of temperature, there were only a small number of new cases, nor was there an increase on the following days." Wooley, at Devens, believed inclement weather may have been indirectly responsible in spreading the disease because it gave men an excuse for staying indoors and thereby favored contact infection. The organizations, such as the Quartermaster Corps and Remount Depots, which were most exposed to the weather, suffered the least during the epidemic.

The low incidence of the disease among these same organizations is mentioned by other camps as well. Beauregard reports that of 11 camp organizations, the Remount Station stood ninth in influenza incidence (38 per cent), tenth in pneumonia incidence (3.5 per cent) and tenth in mortality (.5 per cent). The Ambulance Company, also an outdoor organization, was the only unit to have lower mortality than the Remount Depot. Camp Hancock reported that the Auxiliary Remount Depot of 537 men had 11.3 per cent sick with influenza (11th among 17 units), but not a death occurred. No other organization eseaped in this manner. The above is significant, but it must be interpreted with caution, for these units were subject to less intimate contact as well as being exposed to the weather.

Syracuse reports that there was no period of twenty-four hours without rainfall during the two weeks preceding the epidemic and "from September 9 to the end of October there was no interval of forty-eight hours without rainfall. The weather was unseasonably damp and cold." Syracuse stood 15th in the mortality table.

Altitude is mentioned in connection with the epidemic at Fort Lawton, Washington. It is said that patients sent to the hospital from a higher 
altitude and a colder climate seemed to have a more severe form of the disease. This was noticed especially in men coming to Camp Lewis from Alaska. In all probability the Alaska men were more susceptible to respiratory infections generally because of their isolation.

Bad weather seems to have been of some consequence at Beauregard, which had a mortality out of all proportion to the other southern camps. "At night the weather was extremely cool. There is a heavy dew in this climate, which keeps bed clothing constantly damp unless it is sunned every day. The cold was entirely out of proportion to readings of the thermometer. There was no frost, and it is probable that the temperature was not lower than 50 degrees. The nights, however, were very uncomfortably cool."

At McClellan about one-half of the command arrived in camp during the two weeks preceding the epidemic, and many reached camp during a cold rain and had to make camp without a change of clothing, owing to the delay of the baggage. McClellan stood 31st in mortality.

Logan's first case was September 10. The daily admissions did not exceed 68 until the 19th, when a cold rain known as a "Texas Norther" set in and continued throughout the next day. The temperature dropped from 92 on the 19th to 53 on the 21st. The admissions for the 19th were 144; for the 20th, 101; 21st, 105; 22nd, 207. Logan stood 29th in the mortality table and 5 th in morbidity.

Despite these differences of opinion in camps, there did occur a most unusual sequence of weather for the entire country in 1917 and 1918, which is worth noting. In the first place the winter of 1917-18 east of the Rocky Mountains was the coldest in the history of the Weather Bureau. West of the Rockies there was experienced the warmest winter on record. In the autumn of 1918 there again occurred an unprecedented weather manifestation. East of the Rockies the abrupt drop in temperature between August and September was universal. August was unusually hot, September unseasonably cool. The drop in temperature from August to September was 13.6 degrees at Chicago, and 11.6 degrees at New York City. This depression at Chicago was the greatest that had occurred since 1871. The nearest approach was in 1876, 1896 and 1916, when the drop was 12.2 degrees. The depression for New York was the greatest since 1872. In 1871 the fall was 12.2.

The fall in temperature at this season was greater than the mean for previous years at every camp with the exception of Cody, Lewis, Kearny and Las Casas. Here we are struck with another coincidence. The epidemic at these camps did not begin until the latter part of September or October. Cody's first case was on September 26, but the eases following this were few in number and occurred irregularly until well into October. Kearny's first case was reported on September 24, but there 
was no marked rise in the admissions until the middle of October. Lewis recognized the beginning of an epidemic on October 9, although as many as 40 cases a day had been reported previous to this. The epidemic at Las Casas began abruptly on October 20.

The drop in temperature from September to October was not unusual for either Cody or Kearny. The latter had a mild epidemic and the former would have fared likewise but for the admission of large numbers of sick recruits. The drop from September to October was greater than usual for both Lewis and Las Casas.

Coupled with the abrupt temperature change in September was a heavy rainfall. The Weather Bureau reports that during August precipitation was below normal in the West Gulf and Plains Region, and in the Middle and South Atlantic States. September was characterized by unusually heavy rainfall.

So far as the army camps were concerned it is felt that the severity of the weather did play a perceptible part in the severity of the infection. The Southern camps suffered less than those in the North. As a dividing line between North and South, we have taken the northern border of North Carolina, Tennessee, Arkansas, Oklahoma, New Mexico, Arizona and through the upper part of California so as to include Fremont at Palo Alto in the Southern group. The relation between geographical location and incidence of influenza may be judged by the fact that of the 16 large Northern eamps-

10 had high incidence

6 had low incidence

Of the 24 Southern camps-

9 had high incidence

15 had low incidence.

Analyzing the large camps on the basis of their mortality, we find that of the Northern camps-

13 had high mortality

3 had low mortality

Of the 24 Southern camps-

7 had high mortality

17 had low mortality.

Influenza was more prevalent in Northern camps, and the mortality was appreciably greater.

Unfortunately, at least for the support of our contention, the mortality in the cities does not follow the order shown by the camps. The camps in the east and west north central states were the hardest hit, but the cities in this region suffered less than other sections of the country. 
Camp Grant had a high mortality. Chicago, nearby, had a relatively low mortality. Camp Sherman had heavy mortality; yet Ohio cities had relatively a low mortality. The camps undoubtedly reflect the effect of weather upon a cosmopolitan group of men gathered from all sections of the country. There are many other influences in the cities which would react differently with the weather influences. Thus, in spite of the fact that a weather or climatic influence is not reflected by the cities of the country, we feel that the army experience is sufficient to convince one that weather was not without its effect on the severity of the disease.

TABLE LXXXVII

Geographical Location of Camp and Its Relation to MoRtality from Influenza and PNeUMonia

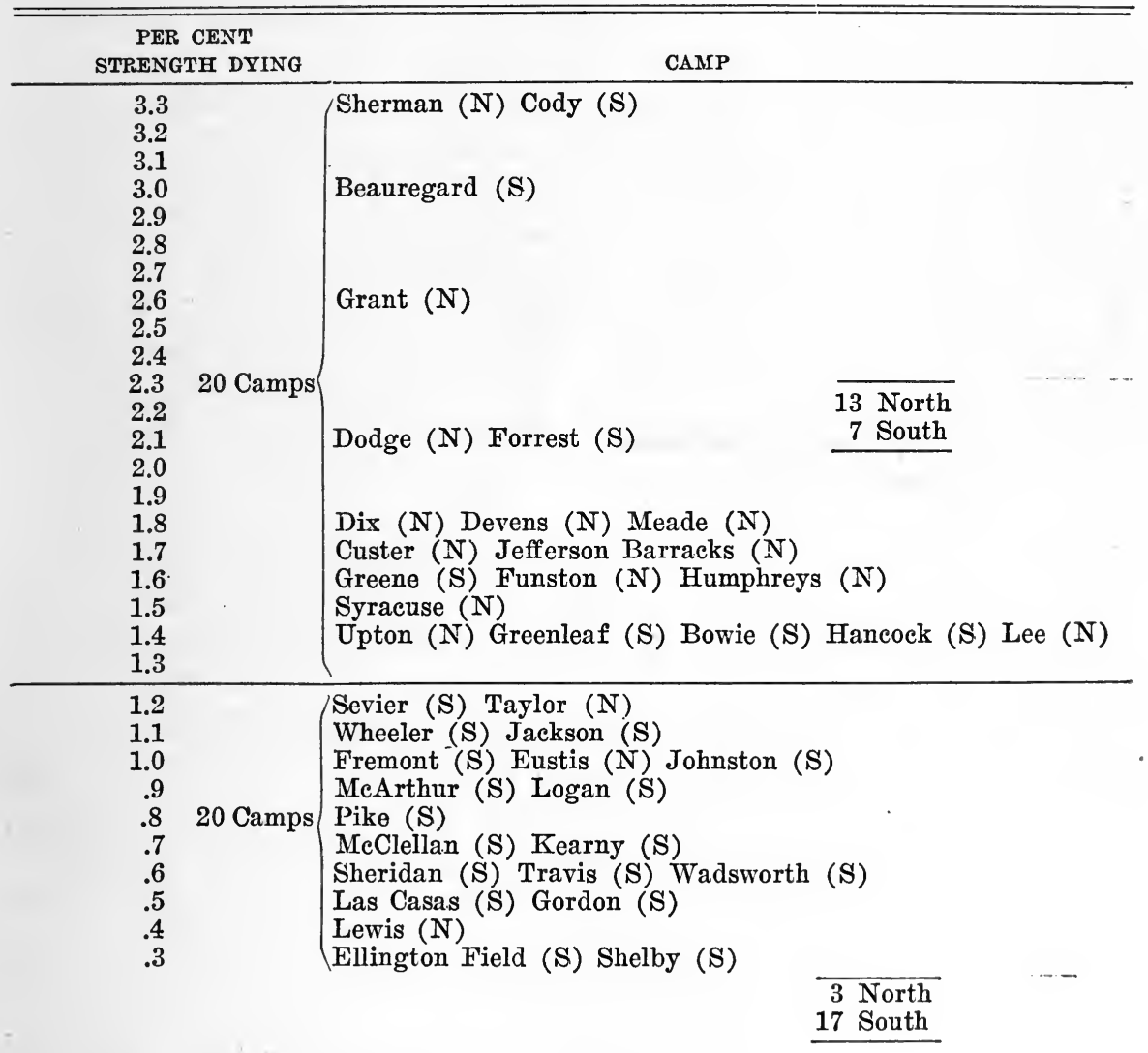

What Starts Influenza Epidemics?-This is the most puzzling question of all, and its solution is hindered by the paucity of records prior to the epidemic of 1889-90. Did a winter of unprecedented cold, followed by a most unseasonable drop in temperature in the autumn, have any effect in initiating the outbreak in this country? 
There are two observations that have a bearing on this matter. Brownlee has pointed out that after an epidemic has appeared there will be recurrences at intervals of 33 weeks, provided the thirty-third week does not fall between June and December. If so, the recurrence will come in 66 or 99 weeks. This sequence was largely true in 1889, '90 and '91 and also in 1918 and 1920, the recurrent wave in the latter year being just about 66 weeks after the outbreak in the autumn of 1918. A second 66 week interval ended in April, 1921, without general recurrence.

Brownlee's theory is of particular interest when taken in conjunction with a suggestion made by Huntington that epidemies may be stirred into being by atmospheric conditions which alter the rate of propagation of germ life, just as Woodruff has noted with paramecium. With this infusorium the rate of sexual reproduction is subject to marked rhythms or cycles ranging in length from 10 to 60 days. At the lowest point in the cycle there takes place a rearrangement of the cell structure, termed "endomixis." This is not sexual and yet it serves as a stimulus analagous to conjugation, for there follows a rapid increase in the rate of reproduction. Endomixis occurs simultaneously in unrelated races of paramecium even though kept under markedly different conditions of temperature and nutrition. If there are forces in nature capable of altering vital processes independently of such intimate influences as temperature and nutrition, would not this phenomenon acting on pathogenic germ life help to explain the beginnings of epidemics and the irregular fluctuations in disease prevalence? It is known that a weak galvanic current in the nutrient media of bacteria, yeast and other plants accelerates the rate of reproduction markedly. Perhaps this unknown vital force is of an electrical nature. Richter suggests that an excess of ozone associated with periods of high pressure gives life to an influenza pandemic by poisoning the respiratory tract.

We must look at disease prevalence as the resultant of many forcesindividual susceptibility, facility for germ exchange and atmospheric combinations. When the unfavorable features of each occur simultaneously, we must expect far more disease than when the combinations are favorable. The greater the number of such influences, the greater the possible combinations and the greater the variation in the results.

Daily Weather Changes and the Death Rate.-Just as climate and season affect health, so does the change in weather from day to day. Huntington has associated the changes in mortality in New York City from 1877 to 1884 with the changes in temperature from one day to the next. Altogether about 300,000 deaths and 3,000 days are concerned. This period is chosen because at this time deaths were recorded as of the day of death instead of the usual custom of day of report. We reproduce the graphs illustrating the study (Fig. 82). At the left of the median line are 


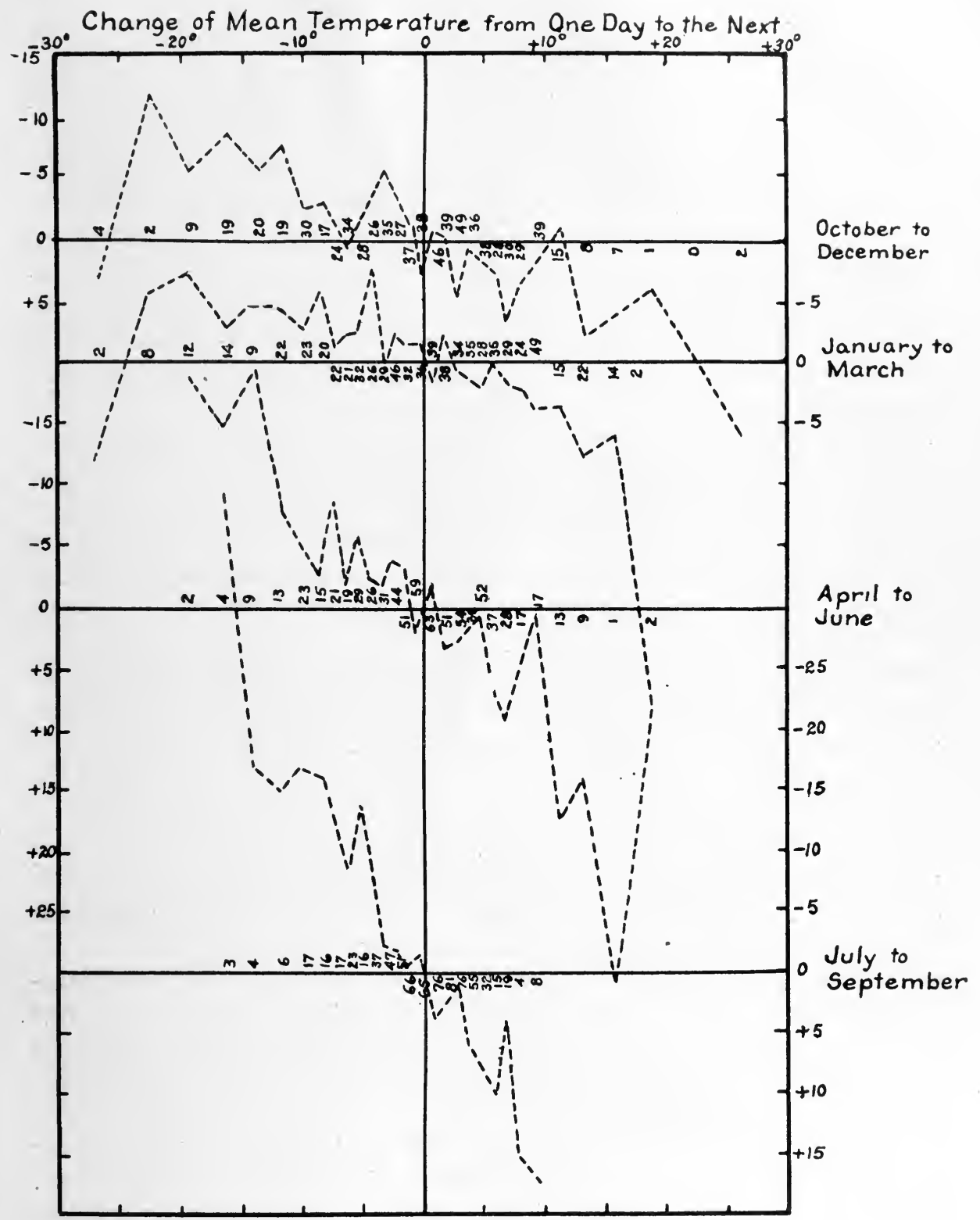

Daily Changes in Health compared with Changes of Mean Temperature at New York City 1877-1884

\section{From Huntington}

"World Power and Evolution"

Fig. 82 . 
the deaths on days when the temperature declined from the day preceding. At the right of the line are the deaths on days of rising temperature over the preceding day. The year has been divided into four seasons. The figures have been inverted so that good conditions of health may be represented by high positions and bad health by low. In general a drop in temperature is associated with good health, a rise in temperature, with poor health.

This graph demonstrates in no unmistakable terms the close relationship of weather to death rate. Huntington explains that a fall of temperature acts like a stimulant. Persons near death are precariously balanced. A drop in temperature is sufficient stimulus to carry them over the critical period. A rise in temperature, on the other hand, acts as a depressant sufficient in a crisis to cause death.

There has been considerable criticism of Huntington's explanation, many believing that the air temperature on a particular day cannot possibly govern the deaths on that day, inasmuch as no account is taken of the conditions on the days preceding and also because a sick person indoors is not exposed to the wide variations out of doors. The facts are before us in this chart and, skeptical as we may feel inclined to be, it is difficult to ignore these figures.

Severe drops in temperature (more than 25 degrees) from October to March are unfavorable to health, but below this point there is a distinct advantage to health when the temperature falls. A rise in temperature is unfavorable. The days on which temperature neither rises nor falls are neutral, or without effect on the death rate.

It will be noted that fluctuations. in temperature affect health to a much greater degree in warm weather than in cold weather. Thus a drop of 15 degrees from the previous day reduces the mortality about 5 per cent in the autumn, 3 per cent in winter, 15 per cent in the spring and 22 per cent in summer. Rises in temperature cause increased mortality in about the same proportions. In studying the work of factory operatives Huntington has found that a change in temperature either up or down is more stimulating than uniformity. Of the two, a fall is more stimulating than a rise.

In this connection we may point out that with the onset of cold weather in the autumn there are more daily drops in temperature than rises. With oncoming warm weather in the spring there are more rises than drops. Does not this help explain why our autumn mortality is less than that in the spring?

How Does Weather Influence Health? -In the preceding pages we have dealt with the associated facts of weather and disease as we find them. The relationship is apparent. The reasons for this relationship are yet to be explained. Does the natural immunity of the body retreat before 
these unfavorable weather manifestations, or do some germs grow better in cold weather? Several explanations have been proposed.

Pneumonia is by no means confined to cold climates. Gorgas found pneumonia in an excessive degree in a warm country such as Panama. This was aggravated by overcrowded living conditions, and pneumonia declined when the men were scattered. It is not absolute temperature then which governs pneumonia. It is not that germs grow better in cold weather. Rather is the disease controlled by the general health of the individual and the accessibility to disease germs. In our northern cities cold causes people to hibernate. The skin loses its power of accommodation and is less able to control the loss of heat from the body. Domestic animals turned to pasture grow heavier coats than those kept in the stable, and they are less sensitive to weather as a result.

It is not so much the cold of cold weather that affects us as our semicivilized responses to this cold. Outdoor cold drives us to live in overheated atmospheres indoors. It is this fact which helps explain the apparent contradiction in the effect of weather on pneumonia. Huntington's charts of daily deaths and the rise and fall of temperature indicate that a drop is favorable to a lessened mortality. On the other hand, it is during the cold season that pneumonia is greatest. Physiologically, cold is stimulating, heat is depressing. Practically, cold weather places a greater strain on the body in metabolism and in waste elimination. The body is more exacting. Working under a heavier schedule, it must not be denied its rest. If given a chance-ample sleep, living in cool rooms-the body responds to the stimulation of winter.

It is said that tuberculosis patients do much better in the cold season. Sick people so care for themselves as to counteract the unfavorable concomitants of cold. By so doing they are in a position to reap in full the benefits of cold. Arctic explorers are not prone to pneumonia. It is thus the habits of life which cold weather induces, rather than the weather itself, which leads to pneumonia. It is for this reason that we may regard much of the pneumonia as humanly preventable.

That overheating indoors measurably weakens health is well brought out in the studies of the New York State Commission on Ventilation. Slight overheating $\left(75^{\circ}\right)$ increases heart action and body temperature. It reduces the inclination to do physical work. It brings on fatigue prematurely. Miller and Cocks report that workers in warm moist atmospheres, such as laundries, exhibited atrophic rhinitis in 46 per cent of all those examined. Among workers in hot dry atmospheres, boiler room men, stationary engineers and the like, 35 per cent were found with atrophic rhinitis. Among outdoor workers, drivers, teamsters, postmen, etc. the percentage was 19. Among young college students it was 3 per cent. These same authors show that exposure to heat causes swelling of the 
turbinate bones of the nose, an increase in secretion and redness of the mucous membrane. Exposure to cold air causes a reduction in swelling, moisture and color. Winslow, Miller and Noble found that a moderately high atmospheric temperature tended to decrease the power of agglutinin formation in the rabbit. These reactions are all indications of lessened health, or perhaps we should say of defensive responses in the face of dangers which threaten health. We see a verification of the above tendency in ventilation studies of New York City school children reported at different times by Baker and Palmer. It was the pupils in the cool and cold ventilated rooms that had fewer colds than the pupils in the warmer, more uniform, fan ventilated rooms.

Miller and Noble reported that infection of rabbits with Bacillus bovisepticus was readier after extremes of chilling and heating. The change from low to high temperature proved more unfavorable than the reverse. In the influenza epidemic of 1889-90 in Germany it was the indoor workers, according to Finkler, that were most freely attacked by influenza.

$$
\text { A-IN THE Ópen AIR }
$$

\begin{tabular}{lc}
\multicolumn{1}{c}{ A-IN THE OPEN AIR } \\
& \\
& PER CENT ATTACKED \\
WY INFLUENZA
\end{tabular}

Mudd, Grant and Goldman have carried on most interesting studies which have a direct bearing on the mechanism of infection, but space will not permit a recital of these facts.

To a lesser degree are the acute contagions, such as scarlet fever, diphtheria and measles, dependent on lowered vitality. Rather is it the combination of infective material and susceptible people which governs the prevalence of these diseases; but back of that, judging from our graphs, there is still a seasonal influence which causes the peak of diphtheria deaths in the autumn, scarlet fever in winter, measles in spring and whooping cough in summer.

Some Possible Uses of a Better Understanding of Weather and Disease.-The study of weather and disease is a fascinating pastime, but we 
hear the voice of the skeptic asking what use we expect to make of the knowledge gained. The weather is given to us. We cannot change it. If we are dissatisfied with a climate, we can move elsewhere, but as for neutralizing bad weather, it is out of the question.

It is true that we cannot alter the outdoor weather, but we can alter our habits of life and our indoor environment if it is proved worth while. The practical value of an accurate understanding of weather and disease lies in its application to ventilation of buildings and the treatment of sickness. It has been pointed out to us that cool variable temperature

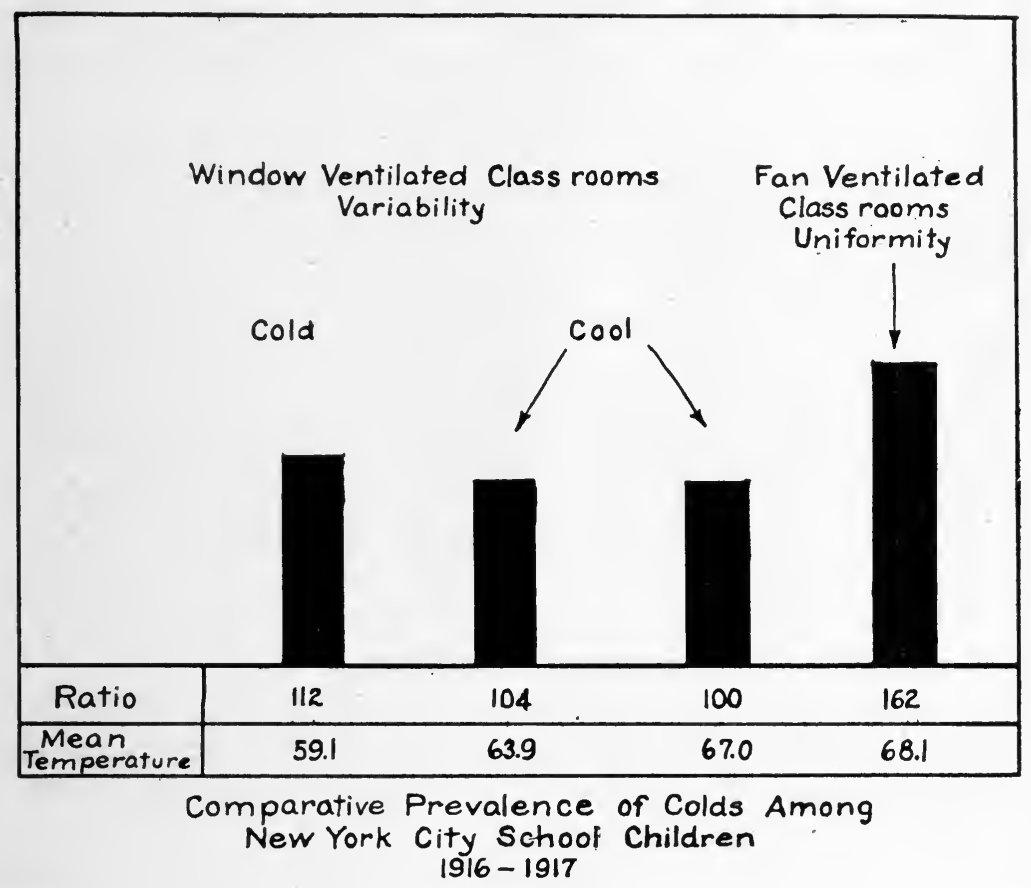

Fig. 83.

with somewhat high humidity is best for health. We have the partial verification of this conclusion in a study of respiratory disease among New York City school children, as reported by Baker and more recently by Palmer. Records were collected of 5,000 school children distributed in three types of ventilated rooms. One group occupied cold open window rooms, the temperature averaging around 59 degrees. Another group was in window ventilated rooms at a higher temperature, between 64 and 67 . The third group was in fan ventilated rooms, whose temperature ran quite uniformly from 68 to 70 degrees. Off hand we would say that the children in the cold rooms were risking their health 
at such low temperatures. Again we might think that the children in the fan ventilated rooms would be better off because of more complete air circulation and hence chemically purer air. The result is quite different. Colds were most frequent in the warmer, more uniform fan ventilated rooms. They were least in the mild window ventilated rooms, but the difference between this and the cold window rooms was negligible. This study suggests a method of attacking pneumonia, namely by accustoming ourselves to rooms of low variable temperature. By so doing we reduce the extremes in temperature. It is this hardening process which the body needs in order to maintain a vasomotor tone sufficient to counteract the depressing effect of the winter season. The morning cold bath exerts a similar action, namely, that of keeping the skin in training to cope with temperature changes.

These studies have shown that pneumonia increases as physical vitality decreases. When this fact is fully sensed we shall adopt the habit of easing up in February, resting in order to counteract fatigue. A week's rest or vacation or shorter hours of labor at this season will be the means by which this may be brought about.

We are spending our winter days in temperatures between 70 and 80 degrees. Houses are overheated, theatres are overheated, offices and stores are overheated. Happily the temperature of school rooms is gradually being reduced. This situation continues because the unfavorable effect of warm rooms on health is not widely sensed. When the machinery for popularizing the relation of weather to disease is better organized, we shall begin to take advantage of this knowledge, and then may we expect a reduction in the pneumonia toll.

If low humidity is bad and high humidity is good, as Huntington believes, we shall adapt this fact to building ventilation. Some day we shall understand how best to utilize the air environment in warding off other diseases as well as pneumonia.

In addition to preserving health by taking advantage of the optimum air conditions indoors we shall utilize the weather-disease relationship in restoring health. It is within 50 years that Trudeau demonstrated the value of the open air in the treatment of tuberculosis. Now our cities have special open air classes for undernourished and tuberculously inclined children. Formerly great care was taken lest cold air reach a pneumonia patient. Now pneumonia is fought with cold air. Brooks used tents to good advantage in treating influenza patients in Massachusetts.

Nephritis demands a warm moist atmosphere to favor perspiration. Warmth and moisture seem to bring relief to the child suffering with croup. Huntington has produced evidence that the mortality in the operating room may be reduced 20 per cent by proper air conditioning. 
Lastly, we must expect the epidemiologist after he has worked out the intimate relationship of the weather to disease, to predict our health outlook in the months immediately ahead of us. This will allow us to prepare in advance for eventualities. We were not conscious of the approach of the influenza epidemic until it was upon us. A more thorough knowledge of weather combinations will place us on guard in the future.

\section{Bibliography}

Greenberg, D.: Jour. Am. Med. Assn., 1919, p. 252.

Huntington, Ellsworth: Civilization and Climate, Yale University Press, 1915. World Power and Evolution, Yale University Press, 1919. Ecology, January, 1920 , p. 6.

PALmer, G. T.: Ventilation, Weather and the Common Cold. Jour. Lab. and Clin. Med., August, September and October, 1921.

Richter, C. M.: Arch. Inter. Med., 1921, p. 361. 



\section{INDEX}

Agglutinins, 52

Albuminal Diseases, anaphylaxis in, 46 bacterial proteins in, 26

cellular proteins, action of, in, 34 classification of, 60

discussion of, 25

fever, acute fatal, in, 38

continued, in, 39

protein, in, 36

protein poison, action of, in, 34

vaccination in, 50

Anaphylaxis in the infectious diseases, 46

\section{B}

Bacillus, action of dead cellular, 34 of living, 32

Bacteria, nature of, 55

pathogenicity of, 58

Bacterial proteins, 26 chemistry of, 28

\section{C}

Cerebrospinal meningitis, age incidence in, 586

annual death rate from, 551

antiserum for, 593

carriers of, 576

control of, 589

crowding, influence of, on, 580

cutaneous eruptions in, 533

death rate from, in civil life, 554

description of, 528

diagnosis of, 584

distribution of cases of, 585

epidemics of, in French army, 557

fatigue, relation of, to, 582

history of, 535

location, influence of, on, 582

mortality from, by age groups in Michigan, 587

passive immunization in, 589

path of invasion of, 572

period of incubation of, 577

prevalence of, among U. S. troops

and Expeditionary Force, 552

in Africa, 562

in England, 562

in Middletown, Conn., 542

in Civil War, 547

in the Fiji Islands, 561

in the Mexican War, 546

in the War of 1812,539

in the U. S. camps, 553

seasonal variations in, 556 sporadic cases of, 584

vaccination for, 588

virus of, 565

Chickenpox, definition of, 212

diagnosis of, 214

history of, 212

period of incubation of, 213

Climate and civilization, 649

and disease, 648

Climograph, the, 652

Colds, common, 79 discussion of, 79

Colon protein, chemistry of, 28 discussion of, 26

fever, production of, by, 36

immunity to, 41

poison in, 31

Coryzas, the acute, anaphylaxis in, 83

bacteriology of, 79

chilling the body surface, influence of, on, 82

description of, 79

virus, a filtrable, in, 80

Coughs, epidemic, 79

\section{D}

Death rate, influence of weather conditions on, 676

Death rates in American cities, 651

Deaths in Detroit from pneumonia, 656

Diphtheria, antitoxin for, 220

bacillus of, 216

carriers of, 238

control and eradication of, 239

definition of, 215

history of, 215

immunization in, with toxin-antitoxin mixture, 232

morbidity and mortality from, 223

mortality from, by age groups in Michigan, 230

period of incubation of, 235

present prevalence of, in largest cities of the U. S., 224

prevalence of, in Detroit, 226

in registration area, 223

in urban and rural areas, 227

Schick test for, 227

toxin of, 218

virulency test for, 237

Diseases, albuminal, 25

epidemic, contagion in, 20

germ theory of, 20

miasmatic theory of, 18

Pettenkoffer's theory of, 18

theories of, 17 
Diseases, epidemic-Cont'd.

work of Pasteur on, 21

infectious, 25

protein, 25

Fever, scarlet, 242

pneumonic, 85

Foreign proteins, parenteral disposition of, 45

G

German Measles, control of, 182

definition of, 179

exanthem of, 181

history of, 179

invasion by, 181

period of incubation of, 181

transmission of, 182

Glanders, avenues of infection by, 642

bacillus of, 642

description of, 641

history of, 641

\section{$\mathrm{H}$}

Health, influence of weather on, 678

\section{I}

Infectious Diseases, discussion of, 25

Influenza, and the weather, 671

bacteriology of, 382

combative measures against, 400

death rates from, by weeks, 337

in cities and camps, 335

in Massachusetts in epidemics from 1889-94, 309

deaths from, by age groups in Boston, 349

description of, 297

distribution of, among recruits and seasoned men, 315

duration of epidemies of, 378

epidemic of, in 1915-16, 311

in 1920 in Detroit, 350

in 1918, progress of, 333

in state hospitals, 351

fatigue, effects of, on, 360

history of, 300

morbidity from, by age, sex and race, 341

and mortality from, in Baltimore, 347

mortality from, among troops in U. S. and A. E. F., 367

by age groups in Michigan, 299

excess of, in U. S. cities, 338

in America and Europe, 324

in East North Central cities, 330

in large army camps, 340

in Middle Atlantic cities, 327

in Mountain and Pacific cities, 332

in New England cities, 326

in South Atlantic, East South Central and West South Central cities, 329 in the great cities of the world, 323

in urban and rural areas, 299

in West North Central cities, 331 pandemic of, in 1889-90, 306

per cent of population attacked by, 343

dying from, 346

physical stamina, influence of, on epidemics of, 355

prevalence of, at Camp Funston, 316

in U. S. camps, 313

production of, in animals, 397

susceptibility and immunity to, 365

transmission of, 370

use of protective sprays in, 403

of vaccines against, 404

\section{$\mathbf{L}$}

Leprosy, anesthetic form of, 524

avenues of infection by, 521

bacillus of, 520

definition of, 516

eradication of, 524

present distribution of, 517

tubercular form of, 523

$\mathrm{M}$

Measles, Brownlee's theory of, 163

control of, 176

Creighton's theory of, 147

death rates from, 154

definition of, 144

enanthem of, 169

epidemies of, periodicity of, 161

exanthem of, 170

experimental, 170

German (see German Measles, 179)

history of, 144

mortality from, 145

by age groups in Michigan, 169

in Aberdeen and Glasgow, 168

in Chicago, 168

in complicated, 167

in uncomplicated, 167

periodicity of epidemics of, 161

pneumonia, relation of, to, 160

prevalence of, at Camp Wheeler, 156

in army camps, 155

in Detroit, 166

in home camps and Expeditionary Forces, 159

in the Civil War, 160

in the Faroe Islands, 149

in the Fiji Islands, 151

present, 153

production of, in monkeys, 172

transmission of, 175

virus of, 174

Watt's theory of, 146

Meningitis, anthrax, 596

due to other organisms, 597

experimental, 596

pneumococcic, 595 
Meningitis-Cont'd.

streptococcic, 595

syphilitic, 596

tuberculous, 594

Morbilli, 144

Mumps, admission rates for, 276

animal inoculation with, 278

control of, 280

definition of, 275

history of, 275

prevalence of, in armies, 276

susceptibility to, 279

virus of, 277

\section{$\mathbf{P}$}

Pertussis, 282

Pneumococcus, description of, 111 types of, 114

Pneumonias, anthrax, 129

broncho, 118

control of, 141

death rates from, in Michigan and Detroit, 99

description of, 85

distribution of, among urban and rural troops, 97

by race and age, 106

in army camps, 93, 94

in cities, 101

experimental, 126

history of, 86

in urban and rural communities, 99

lobar, 111

nonspecific immunity in, 134

other, 129

phenomena of, 132

plague, 128

pneumococeus, 111

predisposing causes in, 130

prevalence of, at Camp Beauregard, 104

at Camp Devens, 107

at Camp Shelby, 137.

at Camp Sherman, 137

in the Civil War, 90

in the War of 1812, 89

in the World War, 91

recent, 108

seasonal variation in, 139

spirochetal, 127

staphylococcus, 127

streptococcus, 118

traumatic, 129

typhoid, 128

vaccination in, 116

Poliomyelitis, annual death rates from, 609

carriers of, domestic animals as, 636 cerebrospinal fluid in, 636

description of, 599

domestic animals as carriers of, 636 history of, 600 incidence of, around New York, 622

insects, agency of, in, 615

mortality from, by age groups in Michigan, 610

New York epidemic of, 611

path of invasion of, 631

prevalence of, around New York, 622

in Cincinnati, 624

in Ithaca, N. Y., 639

in Massachusetts, 626

in Sweden, 603

in Vermont, 625

in Wisconsin, 627

Schick test in, 634

second attacks of, 637

transmission of, 630,635

vaccination for, 634

virus of, 628

Pollinosis, cutaneous test for, 75

desensitization in, 76

discussion of, 61

history of, 61

prevention of, 78

sensitization in, 67

Precipitins; 51

Protein diseases, discussion of, 25

Protein fever, acute fatal, 38 continued, 39

discussion. of, 36

Protein poison, action of, 34 discussion of, 31

Rubella, 179

R

Rubeola, 144

Scarlet fever, animal inoculation for, 269

antisera for, 265

control of, 271

definition of, 242

epidemic of, Hendon, 264

New Hampshire, 244

epidemics of, in England and Ireland, 248

milk-borne, 263

Webster's report on, 246

Hendon epidemic of, 264

history of, 242

hospital treatment of, 273

incubation and invasion of, 270

inoculation for, animal, 269

milk-borne epidemics of, 263

New Hampshire epidemic of, 244 mortality from, by age groups in

Michigan, 259

in registration area, $\mathbf{2 5 0}$

in urban and rural areas, 255

racial variations in, 252

prevalence of, in Chicago, 255

in Detroit, 254

in largest cities of the U. S., 256 recent, 251 
Scarlet fever-Cont'd.

racial variations in mortality from, 252

streptococcus, relation of the, to, 265

susceptibility to, 268

transmission of, 256

virus of, 263

Webster's report on epidemics of, 246

Season and disease, 664

and disease mortality, 670

Smallpox, control of, 209

definition of, 183

history of, 183

inoculation, effect of, on the progress of, 189

for, 185

practice of, by Jesty for, 193

procedures of, for, 190

incubation and development of, 203

Jenner's work on vaccination for, 194

Kilpatrick's method in, 188

prevalence of, in Bostonn in 1721, 187

in Charleston in 1738,187

in London in seventeenth century, 184

present, 207

vaccination for, 192

Jenner's work on, 194

theory of, in, 202

vaccines, preparation of, in, 198

supervision of, in, 200

varieties of, 205

varioloid, 206

virus of, 203

Streptococcus, description of, 118

in milk, 124

in septic sore throat, 124

mutations in, 121

strains of, 119

vaccines for, 123

$\mathrm{T}$

Temperature, effects of, on disease, 658

Tuberculosis, ancient prevalence of, 411

antibodies, production of, in, 466

avenues of infection by, 438

bacillus of, 425

avian, 435

bovine, 432

chemistry of, 429

colonies for, 486

death rates from, in largest cities of the U. S., 421

description of, 409

detection of, surveys for, 497

dispensary care of, 478

educational propaganda against, 495

Framingham experiment in, 500

future work, suggestions for, against, 507
Garden Cities, protection afforded by, against, 474

history of, 411

home care in, 476

hospitals for, 490

infection, avenues of, by, 438

by individual contact with, 468

latent, with, 445

permanent, of homes, with, 469

influenza, effect of, on, 455

measles, effect of, on, 453

morbidity and mortality rates from, in Germany and allied countries, 513

mortality from, by age groups in Michigan, 460

in urban and rural areas, 472

mortality rates from, 410

National Association against, 504

Open-air schools in the prevention of, 505

permanent infection of homes with, 469

piscidian and reptilian types of, 436

present distribution of, in U. S., 512

schemes of combating, 506

researches of Koch on 423

of Villemin on, 422

sanatoria for, 479

superinfection in, 463

surveys for detection of, 497

tuberculization, effect of, on progress of, 467

$\mathrm{V}$

Vaccination in the infectious diseases, 50

Varicella, 212

Varioloid, 206

Variola, 183

Ventilation, effects of, on health, 680

W

Weather and disease, discussion of, 645 influence of, on civilization, 646

Weather and influenza, 671

Whooping Cough, carricrs of, 292

cases of, and deaths from, in Chicago, 290

control of, 295

death rate from, 283

death rates from, by age groups in Michigan, 288

in age groups, 287

in cities, 284 .

in urban and rural areas, 283

definition of, 282

diagnosis of, 294

history of, 282

seasonal death rates from, 289

susceptibility to, 286

transmission of, 291

vaccination for, 293

virus of, 284 



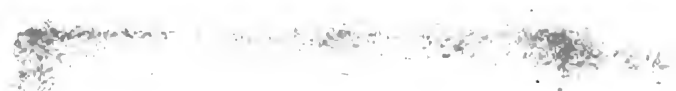




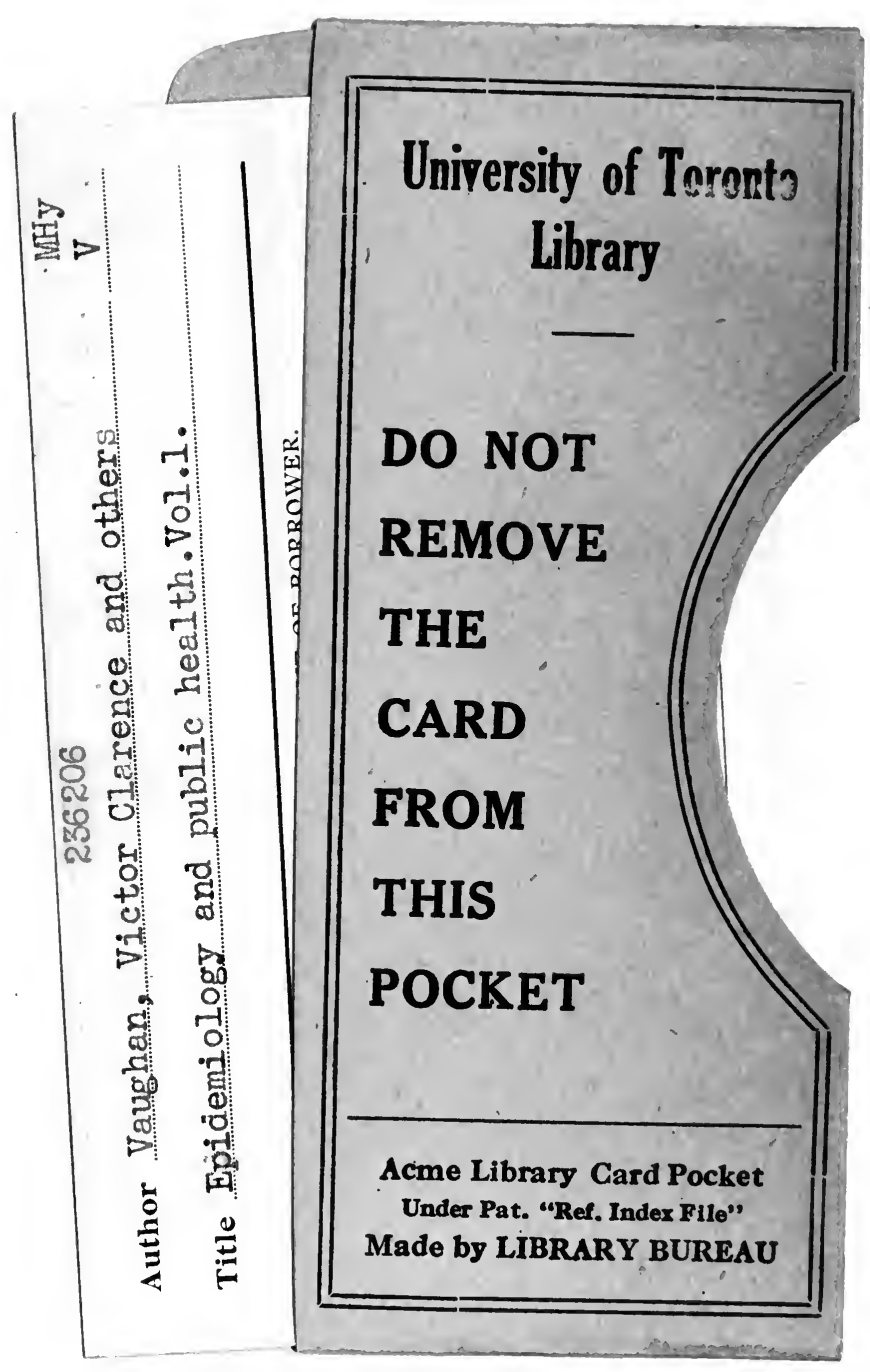


